\title{
A Palladium Complex as an Asymmetric $\pi$-Lewis Base Catalyst for Activating 1,3-Dienes
}

Ben-Xian Xiao, ${ }^{\dagger}$ Bo Jiang,,${ }^{\dagger}$ Ru-Jie Yan,${ }^{\dagger}$ Jian-Xiang Zhu, ${ }^{\dagger}$ Ke Xie,${ }^{\dagger}$ Xin-Yue Gao, ${ }^{\dagger}$ Qin Ouyang, ${ }^{*}$ Wei Du,,${ }^{\dagger}$ and Ying-Chun Chen*广,t;

$\dagger$ Key Laboratory of Drug-Targeting and Drug Delivery System of the Ministry of Education and Sichuan Research Center for Drug Precision Industrial Technology, West China School of Pharmacy, Sichuan University, Chengdu 610041, China

$\$$ College of Pharmacy, Third Military Medical University, Shapingba, Chongqing 400038, China

E-mail: ouyangq@tmmu.edu.cn; duweiyb@scu.edu.cn; ycchen@scu.edu.cn.

\section{Supporting Information}

Table of Contents

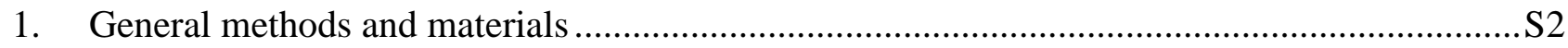

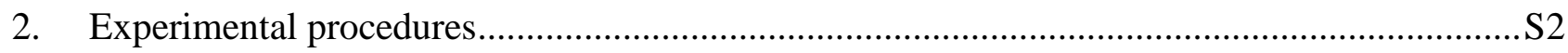

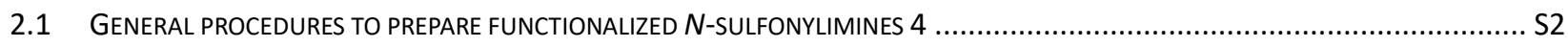

2.2 MORE SCREENING CONDITIONS FOR THE HYDRODIENYLATION REACTION OF 1,3-DIENES WITH N-SULFONYLIMINES UNDER PALLADIUM

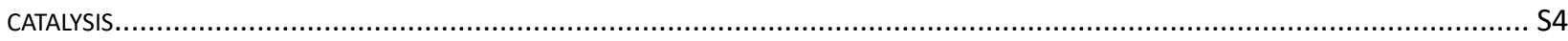

2.3 GENERAL PROCEDURE OF THE ASYMMETRIC HYDRODIENYLATION REACTION OF 1,3-DIENES WITH $N$-SULFONYLIMINES ................ S7

2.4 OPTIMIZATION CONDITIONS FOR THE ASYMMETRIC CASCADE VINYLOGOUS ADDITION/ALLYLIC ALKYLATION OF 1,3-DIENES WITH NSULFONYLIMINES

2.5 GENERAL PROCEDURE OF THE ASYMMETRIC CASCADE VINYLOGOUS ADDITION/ALLYLIC ALKYLATION OF 1,3-DIENES 1 WITH NSULFONYLIMINES 4

2.6 ASYMMETRIC REACTION ON A GRAM SCALE..

2.7 GENERAL PROCEDURE OF ASYMMETRIC CASCADE VINYLOGOUS ADDITION/ALLYLIC ALKYLATION REACTION OF 1,3-DIENES 1 WITH SALICYLALDEHYDE-DERIVED $N$-SULFONYLIMINES 6

2.8 FRIEDEL-CRAFTS REACTION OF DEACTIVATED HETEROARENES S51

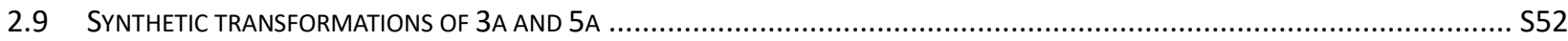

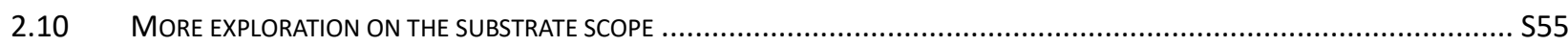

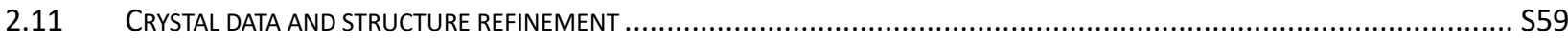

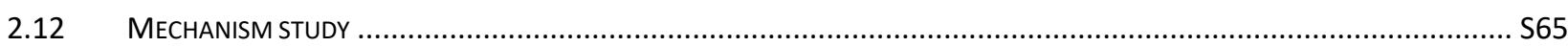

NMR, HRMS Spectra and HPLC Chromatograms ...........................................................S84

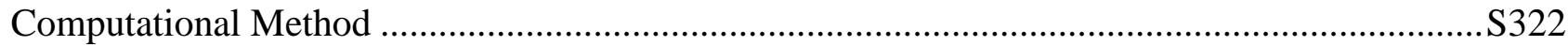

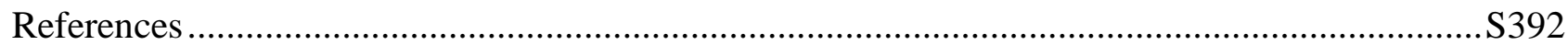




\section{General methods and materials}

When the reactions required heating, the heat source was oil bath. ${ }^{1} \mathrm{H} \mathrm{NMR}(400$ or $600 \mathrm{MHz}),{ }^{13} \mathrm{C}$ NMR (100 or $150 \mathrm{MHz}$ ) spectra were recorded on Varian INOVA-400/54, Agilent DD2-600/54 or Bruker Ascend $^{\mathrm{TM}} 400$ instruments (Chemical shifts were reported in ppm from tetramethylsilane with the solvent resonance as the internal standard in $\mathrm{CDCl}_{3}$ solution, unless otherwise noted). The following abbreviations were used to explain the multiplicities: $\mathrm{s}=$ singlet, $\mathrm{d}=$ doublet, $\mathrm{t}=$ triplet, $\mathrm{dd}$ $=$ double doublet, $\mathrm{dt}=$ double triplet; $\mathrm{td}=$ triple doublet $; \mathrm{m}=$ multiplet, $\mathrm{br}=$ broad, and coupling constants $(J)$ are reported in Hertz $(\mathrm{Hz})$. High resolution mass spectra (HRMS) were recorded on a Waters SYNAPT G2, Agilent G1969-85000 or Shimadzu LCMS-IT-TOF using a time-of-flight mass spectrometer equipped with electrospray ionization (ESI) source. X-ray diffraction experiments were carried out on an Agilent Gemini or Bruker APEX-II CCD diffractometer, and the data obtained were deposited at the Cambridge Crystallographic Data Centre (CCDC 2031429, 2031430 and 2050827). In each case, diastereomeric ratio was determined by ${ }^{1} \mathrm{H}$ NMR analysis and enantiomeric excess was determined by HPLC (Agilent Technologies: 1220 Infinity II, 1200 Series, 1260 Infinity) analysis on a chiral column in comparison with authentic racemate, using a Daicel Chiralpak AD-H Column (250 $\times 4.6 \mathrm{~mm})$, Chiralpak IA Column $(250 \times 4.6 \mathrm{~mm})$, Chiralpak IB Column $(250 \times 4.6 \mathrm{~mm})$, Chiralpak ID Column $(250 \times 4.6 \mathrm{~mm})$, Chiralpak IE Column $(250 \times 4.6 \mathrm{~mm})$, Chiralpak IG Column $(250 \times 4.6$ $\mathrm{mm})$, Chiralcel OD-H Column $(250 \times 4.6 \mathrm{~mm})$. UV detection was monitored at $254 \mathrm{~nm}$. The specific optical rotation was obtained from Rudolph Research Analytical Autopol I automatic polarimeter in $\mathrm{CHCl}_{3}$ solution at $25^{\circ} \mathrm{C}$. The melting point was obtained from WRX-4 Mel-Temp apparatus. Column chromatography was performed on silica gel (200-300 mesh) eluting with ethyl acetate (EtOAc) and petroleum ether or dichloromethane (DCM)/methanol (MeOH). TLC was performed on glass-backed silica plates. UV light, $\mathrm{I}_{2}$, and solution of potassium permanganate were used to visualize products or starting materials. All chemicals were used without purification as commercially available unless otherwise noted. Petroleum ether $\left(60-90{ }^{\circ} \mathrm{C}\right)$ was redistilled. 1,3-Dienes $\mathbf{1},,^{1,2} \mathrm{~N}$-sulfonylimines 2 and $\mathbf{6},{ }^{3,4}$ and ligands $\mathbf{L}$ are commercially available or were prepared according to the literature procedures. 5

\section{Experimental procedures}

\subsection{General procedures to prepare functionalized $N$-sulfonylimines 4}

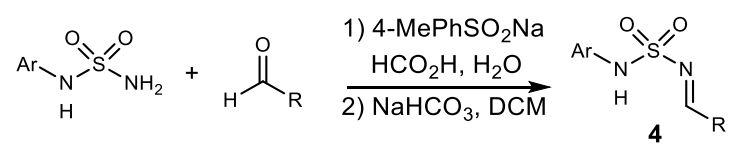

A mixture of aldehyde $(1.0 \mathrm{mmol})$, sulfonamide $(1.0 \mathrm{mmol})$ and sodium 4-methylbenzenesulfinate 
(1.1 mmol) in formic acid $(1.6 \mathrm{~mL})$ and $\mathrm{H}_{2} \mathrm{O}(1.6 \mathrm{~mL})$ was stirred at room temperature. The resulting white precipitate was filtered, and then washed with $\mathrm{H}_{2} \mathrm{O}$ and pentane successively. The resulting solid was dissolved in $\mathrm{CH}_{2} \mathrm{Cl}_{2}$, and a saturated $\mathrm{NaHCO}_{3}$ solution was added. The mixture was stirred at $\mathrm{rt}$ for $2 \mathrm{~h}$. The organic phase was separated and the aqueous phase was extracted with $\mathrm{CH}_{2} \mathrm{Cl}_{2}$. The combined organic layers were dried over anhydrous $\mathrm{Na}_{2} \mathrm{SO}_{4}$ and evaporated under reduced pressure. The residue was purified by column chromatography (petroleum ether/EtOAc = 4/1) or recrystallization from EtOAc/hexane to give the pure imine.

\section{For selected imine substrates}<smiles>O=S(=O)(N=Cc1ccccc1)N=NP</smiles>

(4a) Pale yellow solid, mp 103-105 ${ }^{\circ} \mathrm{C} ;{ }^{1} \mathbf{H}$ NMR (400 MHz, $\left.\mathrm{CDCl}_{3}\right): \delta(\mathrm{ppm})$ $8.72(\mathrm{~s}, 1 \mathrm{H}), 7.85(\mathrm{~d}, J=7.6 \mathrm{~Hz}, 2 \mathrm{H}), 7.60(\mathrm{t}, J=7.2 \mathrm{~Hz}, 1 \mathrm{H}), 7.48-7.45(\mathrm{~m}, 2 \mathrm{H})$, $7.20(\mathrm{~d}, J=8.8 \mathrm{~Hz}, 2 \mathrm{H}), 6.82-6.70(\mathrm{~m}, 3 \mathrm{H}), 3.75(\mathrm{~s}, 3 \mathrm{H}) ;{ }^{13} \mathrm{C}$ NMR $(100 \mathrm{MHz}$, $\mathrm{CDCl}_{3}$ ): $\delta$ (ppm) 170.8, 158.2, 134.7, 132.1, 131.0, 129.1, 128.5, 126.0, 114.5, 55.4; HRMS (ESITOF) m/z: $[\mathrm{M}+\mathrm{Na}]^{+}$Calcd for $\mathrm{C}_{14} \mathrm{H}_{14} \mathrm{~N}_{2} \mathrm{O}_{3} \mathrm{SNa} 313.0617$; Found 313.0619.

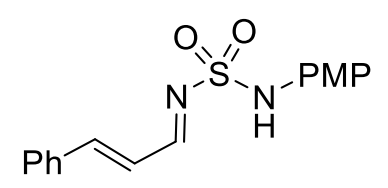

(4l) Yellow semisolid, ${ }^{1} \mathbf{H}$ NMR (400 MHz, $\left.\mathrm{CDCl}_{3}\right): \delta(\mathrm{ppm}) 8.45(\mathrm{~d}, J=9.6$ $\mathrm{Hz}, 1 \mathrm{H}), 7.53-7.52(\mathrm{~m}, 2 \mathrm{H}), 7.44-7.41(\mathrm{~m}, 3 \mathrm{H}), 7.34(\mathrm{~d}, J=15.6 \mathrm{~Hz}, 1 \mathrm{H})$, $7.20(\mathrm{~d}, J=8.8 \mathrm{~Hz}, 2 \mathrm{H}), 6.90(\mathrm{dd}, J=15.6,9.6 \mathrm{~Hz} 1 \mathrm{H}), 6.84(\mathrm{~d}, J=8.8 \mathrm{~Hz}$, 2H), $6.53(\mathrm{~s}, 1 \mathrm{H}), 3.77$ (s, 3H); ${ }^{13} \mathbf{C}$ NMR (100 MHz, $\left.\mathrm{CDCl}_{3}\right): \delta$ (ppm) 171.6, 158.2, 153.4, 134.1, 131.6, 129.1, 128.7, 128.6, 125.8, 124.2, 114.5, 55.4; HRMS (ESI-TOF) m/z: [M + Na $]^{+}$Calcd for $\mathrm{C}_{16} \mathrm{H}_{16} \mathrm{~N}_{2} \mathrm{O}_{3} \mathrm{SNa} 339.0774$; Found 339.0777.

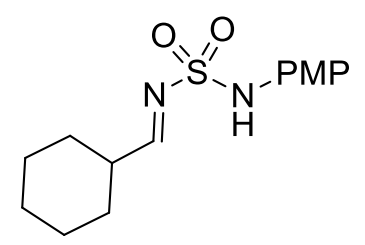

(4m) Yellow semisolid, ${ }^{1} \mathbf{H}$ NMR (400 MHz, $\left.\mathrm{CDCl}_{3}\right): \delta(\mathrm{ppm}){ }^{1} \mathrm{H}$ NMR (400 $\left.\mathrm{MHz}_{\mathrm{CDCl}}\right): \delta(\mathrm{ppm}) 8.13(\mathrm{~d}, J=4.4 \mathrm{~Hz}, 1 \mathrm{H}), 7.15(\mathrm{~d}, J=8.8 \mathrm{~Hz}, 2 \mathrm{H}), 6.84$ $(\mathrm{d}, J=8.8 \mathrm{~Hz}, 2 \mathrm{H}), 3.78(\mathrm{~s}, 3 \mathrm{H}), 2.58-2.15(\mathrm{~m}, 1 \mathrm{H}), 1.97-1.49(\mathrm{~m}, 5 \mathrm{H}), 1.41-$ $0.96(\mathrm{~m}, 5 \mathrm{H}) ;{ }^{13} \mathrm{C}$ NMR $\left(100 \mathrm{MHz}, \mathrm{CDCl}_{3}\right): \delta(\mathrm{ppm}) 182.3,158.2,128.5$, 125.9, 114.4, 55.5, 43.3, 28.4, 25.6, 25.0; HRMS (ESI-TOF) m/z: [M - H] Calcd for $\mathrm{C}_{14} \mathrm{H}_{19} \mathrm{~N}_{2} \mathrm{O}_{3} \mathrm{~S}$ 295.1122; Found 295.1117. 
2.2 More screening conditions for the hydrodienylation reaction of 1,3-dienes with $\mathrm{N}$ sulfonylimines under palladium catalysis

Table S1. Condition screening for the hydrodienylation reaction between 1,3-diene 1a and $N$ sulfonylimine 2 a under palladium catalysis ${ }^{a}$

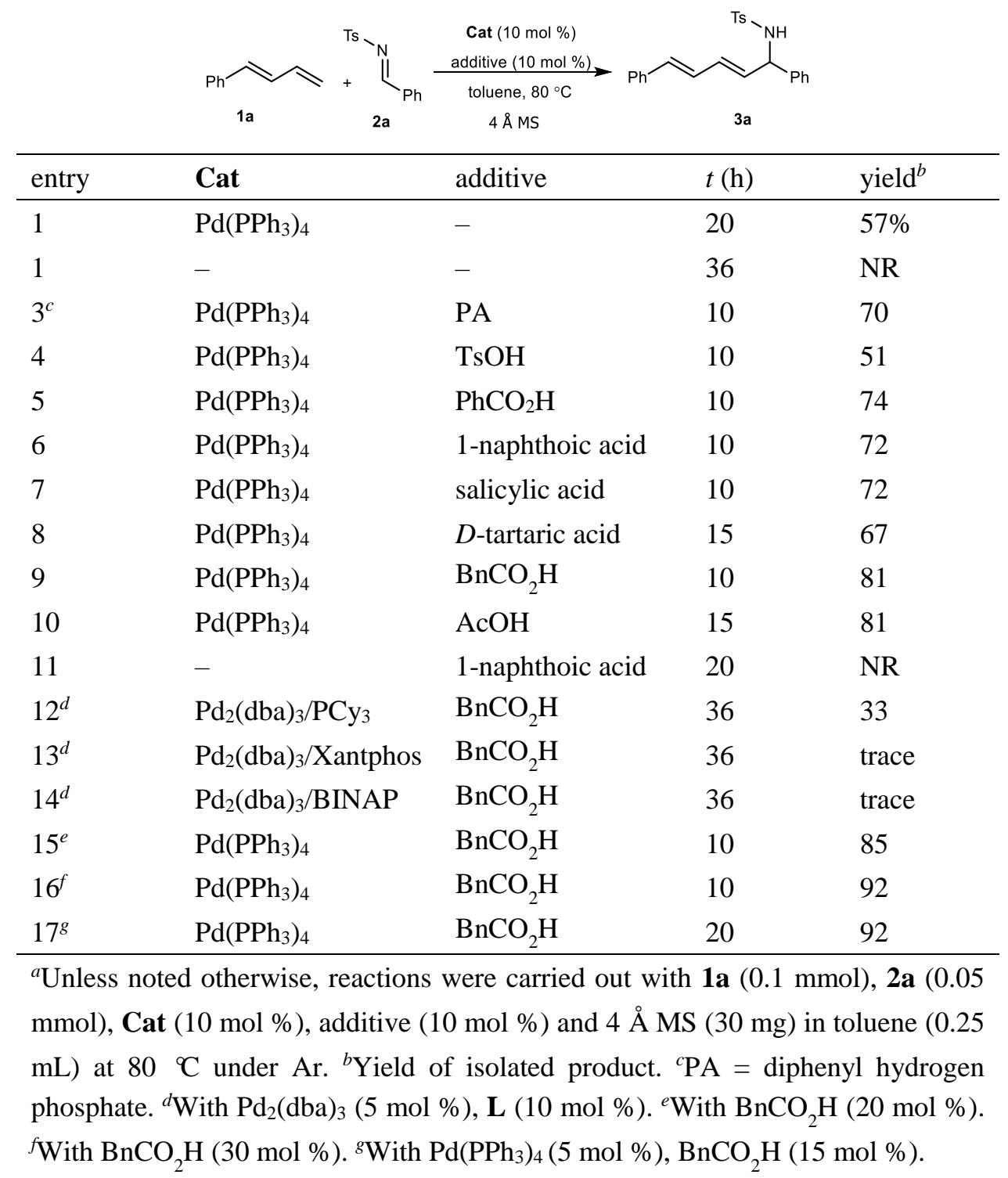


Table S2. Ligand screening for the asymmetric hydrodienylation reaction between 1,3-diene 1a and $N$ sulfonylimine 2 a under palladium catalysis ${ }^{a}$

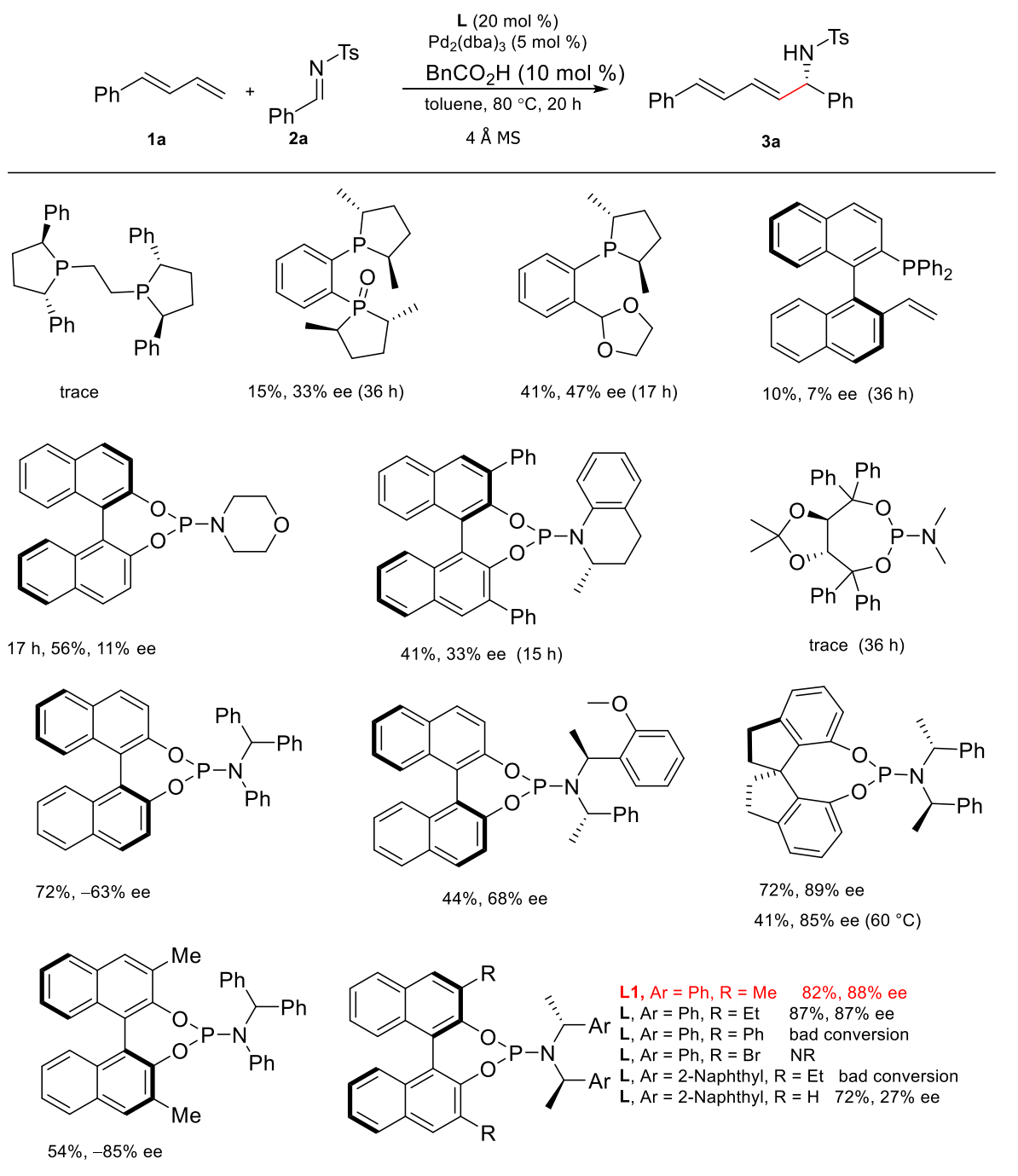

${ }^{a}$ Reactions were carried out with $1 \mathbf{a}(0.05 \mathrm{mmol}), \mathbf{2 a}(0.025 \mathrm{mmol}), \mathrm{Pd}_{2}(\mathrm{dba})_{3}(5 \mathrm{~mol} \%)$, L $(20 \mathrm{~mol} \%), \mathrm{BnCO}_{2} \mathrm{H}(10 \mathrm{~mol} \%)$ and $4 \AA \mathrm{MS}(15 \mathrm{mg})$ in toluene $(0.25 \mathrm{~mL})$ at $80^{\circ} \mathrm{C}$ for $20 \mathrm{~h}$ under Ar. 
Table S3. Additive screening for the asymmetric hydrodienylation reaction between 1,3-diene 1a and $N$ sulfonylimine 2a under palladium catalysis ${ }^{a}$
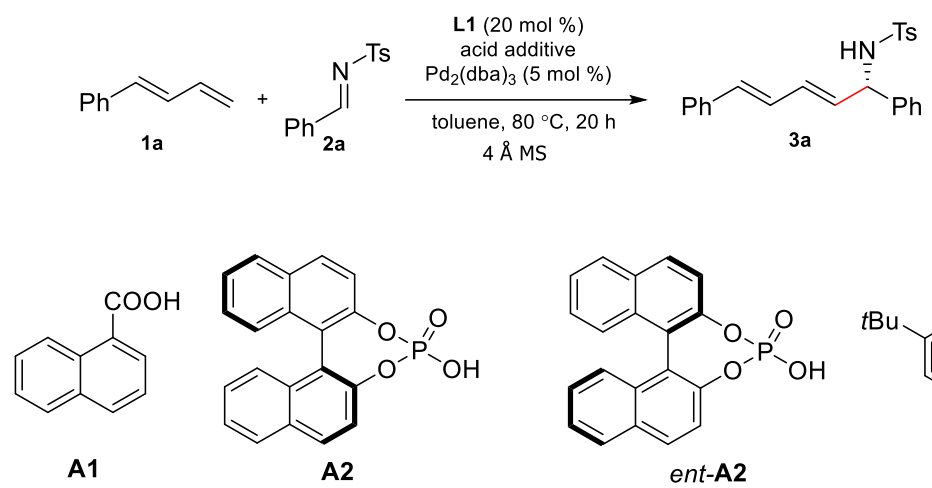<smiles>CC(C)(C)c1cc(C(=O)O)cc(C(C)(C)C)c1</smiles>

A3<smiles>O=C(O)c1ccccc1C(=O)O</smiles><smiles>O=C(O)C(O)c1ccccc1</smiles><smiles>O=C(O)C(O)c1ccccc1</smiles><smiles>O=C(O)C1Cc2ccccc2N1</smiles>

A4

A5

ent-A5

A6

\begin{tabular}{lllll}
\hline entry & solvent & additive $(\mathrm{X} \mathrm{mol} \mathrm{\% )}$ & yield $(\%)^{b}$ & ee $(\%)^{c}$ \\
\hline 1 & toluene & $\mathrm{BnCO}_{2} \mathrm{H}(10 \mathrm{~mol} \%)$ & 82 & 88 \\
2 & toluene & - & 52 & 37 \\
3 & DCE & $\mathrm{BnCO}_{2} \mathrm{H}(10 \mathrm{~mol} \%)$ & 62 & 90 \\
$4^{d}$ & $1,4-\mathrm{Dixone}$ & $\mathrm{BnCO}_{2} \mathrm{H}(10 \mathrm{~mol} \%)$ & trace & - \\
5 & toluene & $\mathrm{BnCO}_{2} \mathrm{H}(20 \mathrm{~mol} \%)$ & 85 & 90 \\
6 & toluene & $\mathrm{BnCO}_{2} \mathrm{H}(30 \mathrm{~mol} \%)$ & 87 & 89 \\
7 & toluene & $\mathrm{PhCO}_{2} \mathrm{H}(30 \mathrm{~mol} \mathrm{\% )}$ & 85 & 92 \\
8 & toluene & $\mathbf{A 1}(30 \mathrm{~mol} \%)$ & 92 & 92 \\
9 & toluene & $\mathbf{A 2}(30 \mathrm{~mol} \%)$ & 65 & 80 \\
11 & toluene & ent-A2 $(30 \mathrm{~mol} \%)$ & 30 & 85 \\
12 & toluene & $\mathbf{A 3}(30 \mathrm{~mol} \%)$ & 65 & 92 \\
13 & toluene & $\mathbf{A 4}(30 \mathrm{~mol} \%)$ & 31 & 85 \\
14 & toluene & $\mathbf{A 5}(30 \mathrm{~mol} \%)$ & 59 & 94 \\
15 & toluene & ent-A5 $(30 \mathrm{~mol} \%)$ & 44 & 94 \\
16 & toluene & $\mathbf{A 6}(30 \mathrm{~mol} \%)$ & 47 & 92 \\
$17^{e, f}$ & toluene & $\mathbf{A 1}(30 \mathrm{~mol} \%)$ & 85 & 94 \\
$18^{e, g}$ & toluene & $\mathbf{A 1}(30 \mathrm{~mol} \%)$ & 40 & 94 \\
$19^{d, e, h}$ & toluene & $\mathbf{A 1}(30 \mathrm{~mol} \%)$ & 62 & 95 \\
$20^{d, i}$ & toluene & $\mathbf{A 1}(30 \mathrm{~mol} \%)$ & 79 & 92 \\
\hline
\end{tabular}

${ }^{a}$ Unless noted otherwise, reactions were carried out with $1 \mathbf{a}(0.05 \mathrm{mmol}), \mathbf{2 a}(0.025 \mathrm{mmol})$, $\mathrm{Pd}_{2}(\mathrm{dba})_{3}(5 \mathrm{~mol} \%), \mathbf{L 1}(20 \mathrm{~mol} \%)$, acid additive and $4 \AA \mathrm{MS}(15 \mathrm{mg})$ in toluene $(0.25 \mathrm{~mL})$ at $80{ }^{\circ} \mathrm{C}$ for $20 \mathrm{~h}$ under Ar. ${ }^{b}$ Yield of isolated product. ${ }^{c}$ Determined by HPLC analysis on a chiral stationary phase. ${ }^{d}$ For $36 \mathrm{~h} .{ }^{e}$ On a $0.1 \mathrm{mmol}$ scale at $0.2 \mathrm{M} .{ }^{f}$ With $\operatorname{Pd}_{2}(\mathrm{dba})_{3}(2.5$ mol \%), L1 (10 mol \%). ${ }^{g}$ With $\mathrm{Pd}_{2}(\mathrm{dba})_{3}(1.25 \mathrm{~mol} \%), \mathbf{L 1}(5 \mathrm{~mol} \%) .{ }^{h}$ At $100{ }^{\circ} \mathrm{C} .{ }^{i}$ With L1 (10 mol \%). 
2.3 General procedure of the asymmetric hydrodienylation reaction of 1,3-dienes with $N$ sulfonylimines

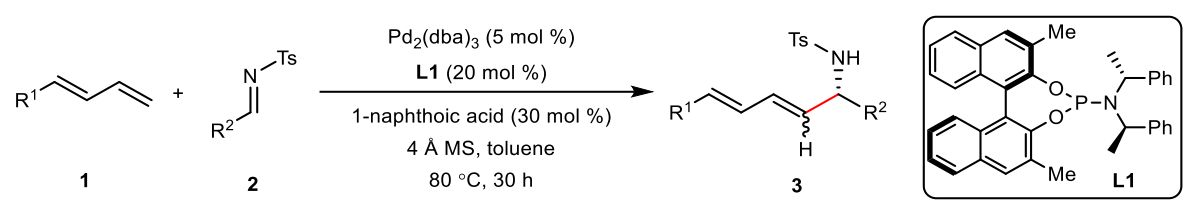

An oven-dried $5 \mathrm{~mL}$ test-tube equipped with a septum and a magnetic stir bar was charged with $\mathrm{Pd}_{2}(\mathrm{dba})_{3}(4.6 \mathrm{mg}, 5 \mathrm{~mol} \%)$ and ligand $\mathbf{L 1}(11.3 \mathrm{mg}, 20 \mathrm{~mol} \%)$. The tube was then evacuated and filled with argon. This cycle was repeated three times, and toluene $(0.5 \mathrm{~mL})$ was added via syringe. The resulting solution was stirred at room temperature for $30 \mathrm{~min}$. A second oven-dried test-tube was charged with diene 1 (2.0 equiv), $N$-sulfonylimine 2 (1.0 equiv), 1-naphthoic acid (5.2 $\mathrm{mg}, 30 \mathrm{~mol} \%$ ) and $4 \AA$ MS (60.0 mg), and the system was placed under an atmosphere of argon (balloon). Then the catalyst solution was added at room temperature. The resulting mixture was stirred at $80{ }^{\circ} \mathrm{C}$ for $30 \mathrm{~h}$. After completion, the product $\mathbf{3}$ was obtained by flash chromatography on silica gel (petroleum ether/EtOAc). The racemic 3 was obtained under the catalysis of $\mathrm{Pd}\left(\mathrm{PPh}_{3}\right)_{4}(5 \mathrm{~mol} \%)$ and $\mathrm{BnCO}_{2} \mathrm{H}$ $(30 \mathrm{~mol} \%)$.

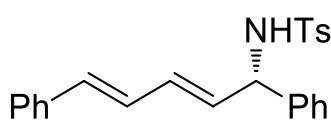

\section{$N$-((S,2E,4E)-1,5-Diphenylpenta-2,4-dien-1-yl)-4-methylbenzene}

sulfonamide (3a): An oven-dried $5 \mathrm{~mL}$ test-tube equipped with a septum and a magnetic stir bar was charged with $\mathrm{Pd}_{2}(\mathrm{dba})_{3}(4.6 \mathrm{mg}, 5 \mathrm{~mol} \%)$ and ligand L1 (11.3 mg, $20 \mathrm{~mol} \%)$. The tube was then evacuated and filled with argon. This cycle was repeated three times, and toluene $(0.5 \mathrm{~mL})$ was added via syringe. The resulting solution was stirred at room temperature for $30 \mathrm{~min}$. A second oven-dried test-tube was charged with diene 1a (26.0 $\mathrm{mg}, 2.0$ equiv), $N$-sulfonylimine $2 \mathbf{a}(26.0 \mathrm{mg}, 1.0$ equiv), 1-naphthoic acid (5.2 mg, $30 \mathrm{~mol} \%)$ and $4 \AA \mathrm{MS}(60.0$ $\mathrm{mg}$ ), and the system was placed under an atmosphere of argon (balloon). Then the catalyst solution was added at room temperature. The resulting mixture was stirred at $80{ }^{\circ} \mathrm{C}$ for $30 \mathrm{~h}$. After completion, purification by flash chromatography on silica gel (petroleum ether/EtOAc $=10 / 1$ ) gave the product 3a: $34.0 \mathrm{mg}$, as a white solid, $94 \%$ yield; $E / Z>19: 1$; $\mathrm{mp} 148-151{ }^{\circ} \mathrm{C}$; $[\alpha]^{25} \mathrm{D}=+30.4(c=0.25$, in $\mathrm{CHCl}_{3}$ ); $91 \%$ ee, determined by HPLC analysis [Chiralpak column ID, $i \mathrm{PrOH} / n \mathrm{Hexane}=20 / 80$, flow rate: $1.0 \mathrm{~mL} / \mathrm{min}, 254 \mathrm{~nm}, \mathrm{t}$ (major) $=29.32 \mathrm{~min}, \mathrm{t}($ minor $)=37.99 \mathrm{~min}] ;{ }^{1} \mathbf{H} \mathbf{~ N M R}\left(400 \mathrm{MHz}, \mathrm{CDCl}_{3}\right)$ : $\delta(\mathrm{ppm}) 7.57(\mathrm{~d}, J=8.0 \mathrm{~Hz}, 2 \mathrm{H}), 7.25-7.06(\mathrm{~m}, 12 \mathrm{H}), 6.52(\mathrm{dd}, J=15.6,10.4 \mathrm{~Hz}, 1 \mathrm{H}), 6.32(\mathrm{~d}, J=$ $15.6 \mathrm{~Hz}, 1 \mathrm{H}), 6.05$ (dd, $J=14.8,10.4 \mathrm{~Hz}, 1 \mathrm{H}), 5.64$ (dd, $J=15.2,6.4 \mathrm{~Hz}, 1 \mathrm{H}), 5.12$ (brs, 1H), 4.94 $(\mathrm{t}, J=6.8 \mathrm{~Hz}, 1 \mathrm{H}), 2.26(\mathrm{~s}, 3 \mathrm{H}) ;{ }^{13} \mathbf{C} \mathbf{N M R}\left(100 \mathrm{MHz}, \mathrm{CDCl}_{3}\right): \delta(\mathrm{ppm}) 143.2,139.6,137.6,136.9$, $133.5,132.4$, 132.0, 129.4, 128.6, 128.6, 127.72, 127.70, 127.5, 127.3, 127.0, 126.3, 59.5, 21.4; HRMS (ESI-TOF) m/z: [M + Na $]^{+}$Calcd for $\mathrm{C}_{24} \mathrm{H}_{23} \mathrm{NO}_{2} \mathrm{SNa} 412.1342$; Found 412.1344 . 


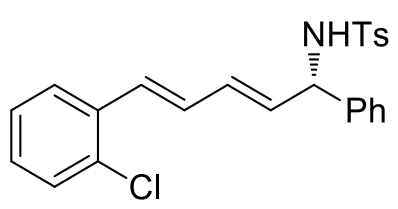

$N$-((S,2E,4E)-5-(2-Chlorophenyl)-1-phenylpenta-2,4-dien-1-yl)-4-

methylbenzenesulfonamide (3b): An oven-dried $5 \mathrm{~mL}$ test-tube equipped with a septum and a magnetic stir bar was charged with $\mathrm{Pd}_{2}(\mathrm{dba})_{3}(4.6 \mathrm{mg}$, $5 \mathrm{~mol} \%$ ) and ligand $\mathbf{L 1}(11.3 \mathrm{mg}, 20 \mathrm{~mol} \%)$. The tube was then evacuated and filled with argon. This cycle was repeated three times, and toluene $(0.5 \mathrm{~mL})$ was added via syringe. The resulting solution was stirred at room temperature for $30 \mathrm{~min}$. A second oven-dried test-tube was charged with diene $\mathbf{1 b}$ (33.0 mg, 2.0 equiv), $N$-sulfonylimine $\mathbf{2 a}$ (26.0 mg, 1.0 equiv), 1-naphthoic acid (5.2 mg, $30 \mathrm{~mol} \%$ ) and $4 \AA \mathrm{MS}(60.0 \mathrm{mg})$, and the system was placed under an atmosphere of argon (balloon). Then the catalyst solution was added at room temperature. The resulting mixture was stirred at $80{ }^{\circ} \mathrm{C}$ for $30 \mathrm{~h}$. After completion, purification by flash chromatography on silica gel (petroleum ether/EtOAc $=10 / 1$ ) gave the product $\mathbf{3 b}: 35.6 \mathrm{mg}$, as a colorless semisolid, 84\% yield; $E / Z>19: 1 ;[\alpha]_{\mathrm{D}}{ }^{25}=+28.2\left(c=1.66\right.$ in $\left.\mathrm{CHCl}_{3}\right) ; 84 \%$ ee, determined by HPLC analysis [Chiralpak column IB, $i \mathrm{PrOH} / n$ Hexane $=40 / 60$, flow rate: $1.0 \mathrm{~mL} / \mathrm{min}, 254 \mathrm{~nm}, \mathrm{t}$ (minor) $=7.18 \mathrm{~min}, \mathrm{t}$ (major) $=11.17 \mathrm{~min}] ;{ }^{1} \mathbf{H}$ NMR $\left(400 \mathrm{MHz}, \mathrm{CDCL}_{3}\right): \delta(\mathrm{ppm}) 7.66(\mathrm{~d}, J=8.0 \mathrm{~Hz}, 2 \mathrm{H}), 7.48(\mathrm{~d}, J=7.2 \mathrm{~Hz}$, $1 \mathrm{H}), 7.34(\mathrm{~d}, J=7.6 \mathrm{~Hz}, 1 \mathrm{H}), 7.25-7.15(\mathrm{~m}, 9 \mathrm{H}), 6.81(\mathrm{~d}, J=15.6 \mathrm{~Hz}, 1 \mathrm{H}), 6.59$ (dd, $J=15.6,10.4$ $\mathrm{Hz}, 1 \mathrm{H}), 6.18(\mathrm{dd}, J=14.8,10.4 \mathrm{~Hz}, 1 \mathrm{H}), 5.78$ (d, $J=14.8 \mathrm{~Hz}, 1 \mathrm{H}), 5.03$ (brs, 2H), 2.36 (s, 3H); ${ }^{13} \mathrm{C}$ NMR (100 MHz, $\left.\mathrm{CDCl}_{3}\right): \delta$ (ppm) 143.4, 139.4, 137.5, 134.8, 133.2, 133.2, 132.3, 129.9, 129.8, 129.5, 129.2 128.7, 128.6, 127.9, 127.3, 127.0, 126.8, 126.2, 59.4, 21.5; HRMS (ESI-TOF) m/z: [M $+\mathrm{Na}]^{+}$Calcd for $\mathrm{C}_{24} \mathrm{H}_{22} \mathrm{ClNO}_{2} \mathrm{SNa} 446.0957\left({ }^{35} \mathrm{Cl}\right), 448.0928\left({ }^{37} \mathrm{Cl}\right)$; Found $446.0950\left({ }^{35} \mathrm{Cl}\right)$, $448.0921\left({ }^{37} \mathrm{Cl}\right)$.

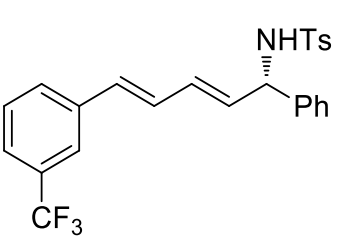

4-Methyl- $N$-((S,2E,4E)-1-phenyl-5-(3-(trifluoromethyl)phenyl)penta-2,4dien-1-yl)benzenesulfonamide (3c): An oven-dried $5 \mathrm{~mL}$ test-tube equipped with a septum and a magnetic stir bar was charged with $\operatorname{Pd}_{2}(\mathrm{dba})_{3}(4.6 \mathrm{mg}, 5$ mol \%) and ligand $\mathbf{L 1}$ (11.3 mg, $20 \mathrm{~mol} \%$ ). The tube was then evacuated and filled with argon. This cycle was repeated three times, and toluene $(0.5 \mathrm{~mL})$ was added via syringe. The resulting solution was stirred at room temperature for $30 \mathrm{~min}$. A second oven-dried test-tube was charged with diene $\mathbf{1 c}$ (39.6 mg, 2.0 equiv), $N$-sulfonylimine 2a (26.0 mg, 1.0 equiv), 1-naphthoic acid (5.2 mg, $30 \mathrm{~mol} \%$ ) and $4 \AA$ MS (60.0 mg), and the system was placed under an atmosphere of argon (balloon). Then the catalyst solution was added at room temperature. The resulting mixture was stirred at $80{ }^{\circ} \mathrm{C}$ for $30 \mathrm{~h}$. After completion, purification by flash chromatography on silica gel (petroleum ether/EtOAc $=10 / 1$ ) gave the product 3c: $34.8 \mathrm{mg}$, as a colorless semisolid, 76\% yield; $E / Z=17: 1 ;[\alpha]^{25} \mathrm{D}=+23.7\left(c=1.18\right.$, in $\left.\mathrm{CHCl}_{3}\right) ; 95 \%$ ee, determined by HPLC analysis [Chiralpak column IB, $i \mathrm{PrOH} / n \mathrm{Hexane}=40 / 60$, flow rate: $1.0 \mathrm{~mL} / \mathrm{min}, 254 \mathrm{~nm}, \mathrm{t}$ (minor) $=6.35 \mathrm{~min}$, $\mathrm{t}$ (major) $=11.44 \mathrm{~min}] ;{ }^{1} \mathbf{H}$ NMR $\left(400 \mathrm{MHz}, \mathrm{CDCl}_{3}\right): \delta(\mathrm{ppm}) 7.66(\mathrm{~d}, J=8.0 \mathrm{~Hz}, 2 \mathrm{H}), 7.56(\mathrm{~s}, 1 \mathrm{H}), 7.49-7.39$ (m, 3H), 7.26-7.14 (m, 7H), $6.67(\mathrm{dd}, J=15.6,10.4 \mathrm{~Hz}, 1 \mathrm{H}), 6.42(\mathrm{~d}, J=15.6 \mathrm{~Hz}, 1 \mathrm{H}), 6.19(\mathrm{dd}, J$ 
$=14.8,6.4 \mathrm{~Hz}, 1 \mathrm{H}), 5.81(\mathrm{dd}, J=14.8,6.4 \mathrm{~Hz}, 1 \mathrm{H}), 5.29-5.25(\mathrm{~m}, 1 \mathrm{H}), 5.04(\mathrm{t}, J=6.8 \mathrm{~Hz}, 1 \mathrm{H}), 2.35$ $(\mathrm{s}, 3 \mathrm{H}) ;{ }^{13} \mathrm{C}$ NMR $\left(100 \mathrm{MHz}, \mathrm{CDCl}_{3}\right): \delta$ (ppm) 143.3 139.4, 137.7, 137.6, 133.6, 131.7, $131.0(\mathrm{~J}=$ $32.2 \mathrm{~Hz}), 129.42,129.4,129.3,129.1,128.7,127.8,127.3,127.0,124.1(\mathrm{~J}=3.6 \mathrm{~Hz}), 124.0(\mathrm{~J}=270.7$ $\mathrm{Hz}), 122.8(\mathrm{~J}=3.8 \mathrm{~Hz}), 122.7,59.43,21.39$; HRMS (ESI-TOF) $\mathrm{m} / \mathrm{z}:[\mathrm{M}+\mathrm{Na}]^{+}$Calcd for $\mathrm{C}_{25} \mathrm{H}_{22} \mathrm{~F}_{3} \mathrm{NO}_{2} \mathrm{SNa} 480.1216$; Found 480.1221.

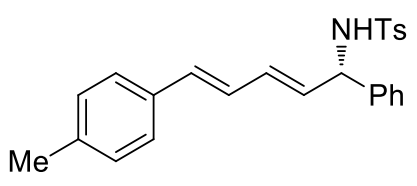

4-Methyl- $N$-((S,2E,4E)-1-phenyl-5-(p-tolyl)penta-2,4-dien-1-yl)

benzenesulfonamide (3d): An oven-dried $5 \mathrm{~mL}$ test-tube equipped with a septum and a magnetic stir bar was charged with $\mathrm{Pd}_{2}(\mathrm{dba})_{3}(4.6 \mathrm{mg}, 5 \mathrm{~mol} \%)$ and ligand $\mathbf{L 1}$ (11.3 mg, $20 \mathrm{~mol} \%$ ). The tube was then evacuated and filled with argon. This cycle was repeated three times, and toluene $(0.5 \mathrm{~mL})$ was added via syringe. The resulting solution was stirred at room temperature for $30 \mathrm{~min}$. A second oven-dried test-tube was charged with diene 1d (28.8 mg, 2.0 equiv), $N$-sulfonylimine $2 \mathbf{a}$ (26.0 mg, 1.0 equiv), 1-naphthoic acid (5.2 mg, $30 \mathrm{~mol} \%$ ) and $4 \AA$ MS (60.0 mg), and the system was placed under an atmosphere of argon (balloon). Then the catalyst solution was added at room temperature. The resulting mixture was stirred at $80{ }^{\circ} \mathrm{C}$ for $30 \mathrm{~h}$. After completion, purification by flash chromatography on silica gel (petroleum ether/EtOAc $=10 / 1$ ) gave the product 3d: Colorless semisolid, $36.2 \mathrm{mg}$, 90\% yield; $E / Z=16: 1 ;[\alpha]^{25} \mathrm{D}=+23.8(c=1.07$, in $\mathrm{CHCl}_{3}$ ); $94 \%$ ee, determined by HPLC analysis [Chiralpak column IB, $i \mathrm{PrOH} / n \mathrm{Hexane}=40 / 60$, flow rate: $1.0 \mathrm{~mL} / \mathrm{min}, 254 \mathrm{~nm}, \mathrm{t}$ (minor) $=7.53 \mathrm{~min}, \mathrm{t}$ (major) $=11.42 \mathrm{~min}] ;{ }^{1} \mathbf{H} \mathbf{~ N M R}(400 \mathrm{MHz}$, $\left.\mathrm{CDCl}_{3}\right): \delta(\mathrm{ppm}) 7.65(\mathrm{~d}, J=8.0 \mathrm{~Hz}, 2 \mathrm{H}), 7.26-7.10(\mathrm{~m}, 11 \mathrm{H}), 6.57(\mathrm{dd}, J=15.6,10.4 \mathrm{~Hz}, 1 \mathrm{H}), 6.39$ $(\mathrm{d}, J=15.6 \mathrm{~Hz}, 1 \mathrm{H}), 6.12(\mathrm{dd}, J=14.8,10.4 \mathrm{~Hz}, 1 \mathrm{H}), 5.70(\mathrm{dd}, J=15.2,6.4 \mathrm{~Hz}, 1 \mathrm{H}), 5.04-4.96$ (m, 2H), 2.36 (s, 3H), 2.34 (s, 3H); ${ }^{13} \mathbf{C}$ NMR (100 MHz, $\left.\mathrm{CDCl}_{3}\right): \delta(\mathrm{ppm})$ 143.3, 139.6, 137.7, 137.6, 134.1, 133.6, 132.7, 131.3, 129.4, 129.3, 128.6, 127.8, 127.3, 127.0, 126.5, 126.3, 59.5, 21.5, 21.2; HRMS (ESI-TOF) m/z: [M + Na $]^{+}$Calcd for $\mathrm{C}_{25} \mathrm{H}_{25} \mathrm{NO}_{2} \mathrm{SNa} 426.1498$; Found 426.1492.

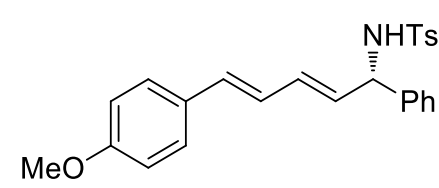

$N$-((S,2E,4E)-5-(4-Methoxyphenyl)-1-phenylpenta-2,4-dien-1-yl)-4methylbenzenesulfonamide (3e): An oven-dried $5 \mathrm{~mL}$ test-tube equipped with a septum and a magnetic stir bar was charged with $\mathrm{Pd}_{2}(\mathrm{dba})_{3}(4.6 \mathrm{mg}, 5 \mathrm{~mol} \%$ ) and ligand $\mathbf{L 1}(11.3 \mathrm{mg}, 20 \mathrm{~mol} \%$ ). The tube was then evacuated and filled with argon. This cycle was repeated three times, and toluene $(0.5 \mathrm{~mL})$ was added via syringe. The resulting solution was stirred at room temperature for $30 \mathrm{~min}$. A second oven-dried test-tube was charged with diene 1 e (32.0 mg, 2.0 equiv), $N$-sulfonylimine 2a (26.0 mg, 1.0 equiv), 1-naphthoic acid (5.2 mg, $30 \mathrm{~mol} \%$ ) and $4 \AA$ MS (60.0 mg), and the system was placed under an atmosphere of argon (balloon). Then the catalyst solution was added at room temperature. The resulting mixture was stirred at $80{ }^{\circ} \mathrm{C}$ for $30 \mathrm{~h}$. After completion, purification by flash chromatography on silica gel (petroleum ether/EtOAc $=10 / 1$ ) gave the product $\mathbf{3 e}: 34.0 \mathrm{mg}$, as a colorless semisolid, $81 \%$ yield; 
$E / Z>19: 1 ;[\alpha]^{25} \mathrm{D}=+15.5\left(c=1.3\right.$, in $\left.\mathrm{CHCl}_{3}\right) ; 96 \%$ ee, determined by HPLC analysis [Chiralpak column IB, $i \mathrm{PrOH} / n$ Hexane $=40 / 60$, flow rate: $1.0 \mathrm{~mL} / \mathrm{min}, 254 \mathrm{~nm}, \mathrm{t}$ (minor) $=9.76 \mathrm{~min}, \mathrm{t}$ (major) $=14.47 \mathrm{~min}] ;{ }^{1} \mathbf{H}$ NMR (400 MHz, $\left.\mathrm{CDCl}_{3}\right): \delta(\mathrm{ppm}) 7.64(\mathrm{~d}, J=8.2 \mathrm{~Hz}, 2 \mathrm{H}), 7.28-7.14(\mathrm{~m}, 9 \mathrm{H})$, $6.83(\mathrm{~d}, J=8.2 \mathrm{~Hz}, 2 \mathrm{H}), 6.48(\mathrm{dd}, J=15.6,10.4 \mathrm{~Hz}, 1 \mathrm{H}), 6.36(\mathrm{~d}, J=15.6 \mathrm{~Hz}, 1 \mathrm{H}), 6.10(\mathrm{dd}, J=$ 15.2, 10.0 Hz, 1H), 5.66 (dd, $J=15.2,6.4 \mathrm{~Hz}, 1 \mathrm{H}), 5.00-4.96(\mathrm{~m}, 2 \mathrm{H}), 3.80(\mathrm{~s}, 3 \mathrm{H}), 2.35(\mathrm{~s}, 3 \mathrm{H}) ;{ }^{13} \mathrm{C}$ NMR $\left(100 \mathrm{MHz}, \mathrm{CDCl}_{3}\right) \delta 159.3,143.2,139.8,137.7,133.1,132.7,130.8,129.7,129.4,128.6,127.6$, 127.6, 127.3, 127.0, 125.5, 114.0, 59.6, 55.2, 21.4; HRMS (ESI-TOF) m/z: $\left[\mathrm{M}+\mathrm{Na}^{+}\right.$Calcd for $\mathrm{C}_{25} \mathrm{H}_{25} \mathrm{NO}_{3} \mathrm{SNa}$ 442.1447; Found 442.1444.

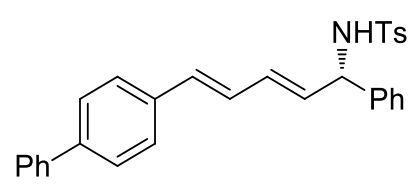

$N-((S, 2 E, 4 E)-5-([1,1 '-B i p h e n y l]-4-y l)-1-p h e n y l p e n t a-2,4-d i e n-1-y l)-4-$ methylbenzenesulfonamide (3f): An oven-dried $5 \mathrm{~mL}$ test-tube equipped with a septum and a magnetic stir bar was charged with $\mathrm{Pd}_{2}(\mathrm{dba})_{3}(4.6 \mathrm{mg}$, $5 \mathrm{~mol} \%$ ) and ligand $\mathbf{L 1}(11.3 \mathrm{mg}, 20 \mathrm{~mol} \%)$. The tube was then evacuated and filled with argon. This cycle was repeated three times, and toluene $(0.5 \mathrm{~mL})$ was added via syringe. The resulting solution was stirred at room temperature for $30 \mathrm{~min}$. A second oven-dried test-tube was charged with diene $\mathbf{1 f}$ (41.2 mg, 2.0 equiv), $N$-sulfonylimine $\mathbf{2 a}$ (26.0 mg, 1.0 equiv), 1-naphthoic acid (5.2 mg, 30 mol \%) and $4 \AA$ MS (60.0 mg), and the system was placed under an atmosphere of argon (balloon). Then the catalyst solution was added at room temperature. The resulting mixture was stirred at $80{ }^{\circ} \mathrm{C}$ for $30 \mathrm{~h}$. After completion, purification by flash chromatography on silica gel (petroleum ether/EtOAc $=10 / 1)$ gave the product 3f: $40.0 \mathrm{mg}$, as a white solid, $86 \%$ yield; $E / Z=13: 1 ; \mathrm{mp}=75-78{ }^{\circ} \mathrm{C} ;[\alpha]^{25} \mathrm{D}$ $=+19.1\left(c=1.1\right.$, in $\left.\mathrm{CHCl}_{3}\right) ; 95 \%$ ee, determined by HPLC analysis [Chiralpak column AD-H, $i \mathrm{PrOH} / n$ Hexane $=40 / 60$, flow rate: $1.0 \mathrm{~mL} / \mathrm{min}, 254 \mathrm{~nm}, \mathrm{t}$ (minor) $=18.38 \mathrm{~min}, \mathrm{t}$ (major) $=21.52$ min]; ${ }^{1} \mathbf{H}$ NMR (400 MHz, $\left.\mathrm{CDCl}_{3}\right): \delta(\mathrm{ppm}) 7.66$ (d, $\left.J=8.4 \mathrm{~Hz}, 2 \mathrm{H}\right), 7.60-7.53(\mathrm{~m}, 4 \mathrm{H}), 7.45-7.14$ $(\mathrm{m}, 12 \mathrm{H}), 6.65(\mathrm{dd}, J=15.6,10.4 \mathrm{~Hz}, 1 \mathrm{H}), 6.45$ (d, $J=15.6 \mathrm{~Hz}, 1 \mathrm{H}), 6.16(\mathrm{dd}, J=14.8,10.4 \mathrm{~Hz}$, $1 \mathrm{H}), 5.75(\mathrm{dd}, J=14.8,5.6 \mathrm{~Hz}, 1 \mathrm{H}), 5.08-5.03(\mathrm{~m}, 2 \mathrm{H}), 2.36(\mathrm{~s}, 3 \mathrm{H}) ;{ }^{13} \mathbf{C} \mathbf{N M R}\left(150 \mathrm{MHz}, \mathrm{CDCl}_{3}\right)$ : $\delta(\mathrm{ppm}) 143.3,140.5,140.4,139.6,137.6,135.9,133.1,132.5,132.1,129.4,128.8,128.7,127.8$, 127.5, 127.3, 127.3, 127.3, 127.0, 126.8, 126.8, 59.5, 21.5; HRMS (ESI-TOF) m/z: [M + Na] ${ }^{+}$Calcd for $\mathrm{C}_{30} \mathrm{H}_{27} \mathrm{NO}_{2} \mathrm{SNa} 488.1655$; Found 488.1658.

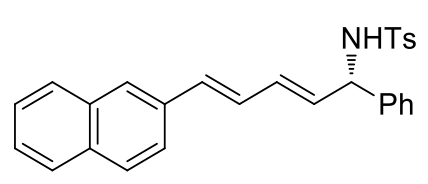

4-Methyl- $N$-((S,2E,4E)-5-(naphthalen-2-yl)-1-phenylpenta-2,4-dien1-yl)benzenesulfonamide (3g): An oven-dried $5 \mathrm{~mL}$ test-tube equipped with a septum and a magnetic stir bar was charged with $\mathrm{Pd}_{2}(\mathrm{dba})_{3}(4.6 \mathrm{mg}$, $5 \mathrm{~mol} \%)$ and ligand $\mathbf{L 1}(11.3 \mathrm{mg}, 20 \mathrm{~mol} \%)$. The tube was then evacuated and filled with argon. This cycle was repeated three times, and toluene $(0.5 \mathrm{~mL})$ was added via syringe. The resulting solution was stirred at room temperature for $30 \mathrm{~min}$. A second oven-dried test-tube was charged with diene $\mathbf{1 g}$ (36.0 mg, 2.0 equiv), $N$-sulfonylimine $\mathbf{2 a}$ (26.0 mg, 1.0 equiv), 1-naphthoic acid (5.2 $\mathrm{mg}$, 
$30 \mathrm{~mol} \%$ ) and $4 \AA$ MS (60.0 mg), and the system was placed under an atmosphere of argon (balloon). Then the catalyst solution was added at room temperature. The resulting mixture was stirred at $80{ }^{\circ} \mathrm{C}$ for $30 \mathrm{~h}$. After completion, purification by flash chromatography on silica gel (petroleum ether/EtOAc $=10 / 1)$ gave the product $3 \mathrm{~g}: 35.3 \mathrm{mg}$, as a colorless semisolid, $80 \%$ yield; $E / Z=16: 1 ;[\alpha]^{25} \mathrm{D}=+33.7$ ( $c=0.35$, in $\mathrm{CHCl}_{3}$ ); $96 \%$ ee, determined by HPLC analysis [Chiralpak column $\mathrm{IB}, i \mathrm{PrOH} / n \mathrm{Hexane}$ $=40 / 60$, flow rate: $1.0 \mathrm{~mL} / \mathrm{min}, 254 \mathrm{~nm}, \mathrm{t}$ (minor) $=17.72 \mathrm{~min}, \mathrm{t}$ (major) $=32.67 \mathrm{~min}]$; ${ }^{1} \mathbf{H}$ NMR $\left(400 \mathrm{MHz}, \mathrm{CDCl}_{3}\right): \delta(\mathrm{ppm}) \delta 7.73-7.69(\mathrm{~m}, 3 \mathrm{H}), 7.63-7.60(\mathrm{~m}, 3 \mathrm{H}), 7.48(\mathrm{~d}, J=8.8 \mathrm{~Hz}, 1 \mathrm{H}), 7.41-$ $7.35(\mathrm{~m}, 2 \mathrm{H}), 7.20-7.10(\mathrm{~m}, 7 \mathrm{H}), 6.68(\mathrm{dd}, J=15.6,10.4 \mathrm{~Hz}, 1 \mathrm{H}), 6.52(\mathrm{~d}, J=15.6 \mathrm{~Hz}, 1 \mathrm{H}), 6.15$ $(\mathrm{dd}, J=14.8,10.6 \mathrm{~Hz}, 1 \mathrm{H}), 5.71(\mathrm{dd}, J=14.8,6.4 \mathrm{~Hz}, 1 \mathrm{H}), 5.01-4.92(\mathrm{~m}, 2 \mathrm{H}), 2.28(\mathrm{~s}, 3 \mathrm{H}) ;{ }^{13} \mathrm{C}$ NMR (100 MHz, $\left.\mathrm{CDCl}_{3}\right): \delta$ (ppm) 143.3, 139.6, 137.6, 134.4, 133.7, 133.6, 133.0, 132.6, 132.2, 129.4, 128.7, 128.3 128.0, 127.8, 127.7, 127.3, 127.0, 126.6, 126.4, 126.0, 123.3, 59.6, 21.5; HRMS (ESI-TOF) m/z: [M + Na] $]^{+}$Calcd for $\mathrm{C}_{28} \mathrm{H}_{25} \mathrm{NO}_{2} \mathrm{SNa} 462.1498$; Found 462.1504.

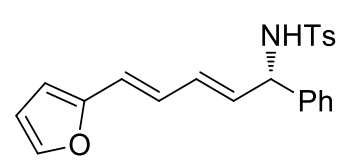

$N$-((S,2E,4E)-5-(Furan-2-yl)-1-phenylpenta-2,4-dien-1-yl)-4-methyl

benzenesulfonamide (3h): An oven-dried $5 \mathrm{~mL}$ test-tube equipped with a septum and a magnetic stir bar was charged with $\mathrm{Pd}_{2}(\mathrm{dba})_{3}(4.6 \mathrm{mg}, 5 \mathrm{~mol} \%)$ and ligand $\mathbf{L 1}$ (11.3 mg, $20 \mathrm{~mol} \%$ ). The tube was then evacuated and filled with argon. This cycle was repeated three times, and toluene $(0.5 \mathrm{~mL})$ was added via syringe. The resulting solution was stirred at room temperature for $30 \mathrm{~min}$. A second oven-dried test-tube was charged with diene $\mathbf{1 h}$ (24.0 mg, 2.0 equiv), $N$-sulfonylimine $\mathbf{2 a}$ (26.0 mg, 1.0 equiv), 1-naphthoic acid (5.2 mg, $30 \mathrm{~mol} \%$ ) and $4 \AA$ MS (60.0 mg), and the system was placed under an atmosphere of argon (balloon). Then the catalyst solution was added at room temperature. The resulting mixture was stirred at $80{ }^{\circ} \mathrm{C}$ for $30 \mathrm{~h}$. After completion, purification by flash chromatography on silica gel (petroleum ether/EtOAc $=10 / 1$ ) gave the product $3 \mathbf{h}: 35.5 \mathrm{mg}$, as a faint yellow semisolid, 93\% yield; $E / Z=11: 1 ;[\alpha]^{25} \mathrm{D}=+11.2(c=$ 0.25 , in $\left.\mathrm{CHCl}_{3}\right) ; 88 \%$ ee, determined by HPLC analysis [Chiralpak column IB, $i \mathrm{PrOH} / n \mathrm{Hexane}=$ 40/60, flow rate: $1.0 \mathrm{~mL} / \mathrm{min}, 254 \mathrm{~nm}, \mathrm{t}$ (minor) $=6.79 \mathrm{~min}, \mathrm{t}$ (major) $=8.45 \mathrm{~min}] ;{ }^{1} \mathbf{H} \mathbf{~ N M R}(400$ $\left.\mathrm{MHz}, \mathrm{CDCl}_{3}\right): \delta(\mathrm{ppm}) 7.61(\mathrm{~d}, J=8.0 \mathrm{~Hz}, 2 \mathrm{H}), 7.32(\mathrm{~s}, 1 \mathrm{H}), 7.24-7.10(\mathrm{~m}, 7 \mathrm{H}), 6.49$ (dd, $J=15.6$, $10.8 \mathrm{~Hz}, 1 \mathrm{H}), 6.35-6.34(\mathrm{~m}, 1 \mathrm{H}), 6.22(\mathrm{~d}, J=3.2 \mathrm{~Hz}, 1 \mathrm{H}), 6.18(\mathrm{~d}, J=15.6 \mathrm{~Hz}, 1 \mathrm{H}), 6.04(\mathrm{dd}, J=$ $14.2,10.8 \mathrm{~Hz}, 1 \mathrm{H}), 5.67(\mathrm{dd}, J=15.2,6.8 \mathrm{~Hz}, 1 \mathrm{H}), 5.01-4.92(\mathrm{~m}, 2 \mathrm{H}), 2.34(\mathrm{~s}, 3 \mathrm{H}) ;{ }^{13} \mathbf{C} \mathbf{N M R}(100$ $\mathrm{MHz}_{\mathrm{CDCl}}$ ): $\delta$ (ppm) 152.7, 143.3, 142.3, 139.6, 137.6, 132.1, 132.0, 129.4, 128.7, 127.8, 127.3, 127.0, 126.0, 121.0, 111.6, 108.8, 59.5, 21.4; HRMS (ESI-TOF) m/z: $[\mathrm{M}+\mathrm{Na}]^{+}$Calcd for $\mathrm{C}_{22} \mathrm{H}_{21} \mathrm{NO}_{3} \mathrm{SNa}$ 402.1134; Found 402.1126.

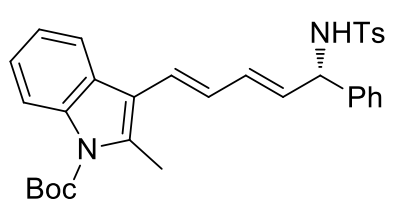

tert-Butyl-2-methyl-3-((S,1E,3E)-5-(4-methylphenylsulfonamido)-5phenylpenta-1,3-dien-1-yl)-1H-indole-1-carboxylate (3i): An oven-dried $5 \mathrm{~mL}$ test-tube equipped with a septum and a magnetic stir bar was charged 
with $\mathrm{Pd}_{2}(\mathrm{dba})_{3}(4.6 \mathrm{mg}, 5 \mathrm{~mol} \%)$ and ligand $\mathbf{L 1}(11.3 \mathrm{mg}, 20 \mathrm{~mol} \%)$. The tube was then evacuated and filled with argon. This cycle was repeated three times, and toluene $(0.5 \mathrm{~mL})$ was added via syringe. The resulting solution was stirred at room temperature for $30 \mathrm{~min}$. A second oven-dried test-tube was charged with diene $\mathbf{1 i}$ (56.6 mg, 2.0 equiv), $N$-sulfonylimine $\mathbf{2 a}$ (26.0 mg, 1.0 equiv), 1-naphthoic acid (5.2 mg, $30 \mathrm{~mol} \%$ ) and $4 \AA$ MS (60.0 mg), and the system was placed under an atmosphere of argon (balloon). Then the catalyst solution was added at room temperature. The resulting mixture was stirred at $80{ }^{\circ} \mathrm{C}$ for $30 \mathrm{~h}$. After completion, purification by flash chromatography on silica gel (petroleum ether/EtOAc $=10 / 1$ ) gave the product 3i: $46.0 \mathrm{mg}$, as a white solid, 85\% yield; $E / Z>13: 1$; $\mathrm{mp}=58-60{ }^{\circ} \mathrm{C} ;[\alpha]^{25} \mathrm{D}=+15.7\left(c=0.6\right.$, in $\left.\mathrm{CHCl}_{3}\right) ; 81 \%$ ee, determined by HPLC analysis [Chiralpak column IB, $i \mathrm{PrOH} / n$ Hexane $=20 / 80$, flow rate: $1.0 \mathrm{~mL} / \mathrm{min}, 254 \mathrm{~nm}, \mathrm{t}$ (minor) $=11.85 \mathrm{~min}, \mathrm{t}$ (major) $=14.52 \mathrm{~min}] ;{ }^{1} \mathbf{H}$ NMR (400 MHz, $\left.\mathrm{CDCl}_{3}\right): \delta(\mathrm{ppm}) 8.11(\mathrm{~d}, J=8.0 \mathrm{~Hz}, 1 \mathrm{H}), 7.67-7.64(\mathrm{~m}, 2 \mathrm{H})$, 7.26-7.16 (m, 9H), 6.55-6.64 (m, 2H), $6.20(\mathrm{dd}, J=15.2,9.6 \mathrm{~Hz}, 1 \mathrm{H}), 5.67(\mathrm{dd}, J=15.2,6.8 \mathrm{~Hz}$, 1H), $5.05(\mathrm{t}, J=6.8 \mathrm{~Hz}, 1 \mathrm{H}), 4.88(\mathrm{brs}, 1 \mathrm{H}), 2.58(\mathrm{~s}, 3 \mathrm{H}), 2.34(\mathrm{~s}, 3 \mathrm{H}), 1.67(\mathrm{~s}, 9 \mathrm{H}) ;{ }^{13} \mathbf{C}$ NMR $(100$ $\mathrm{MHz}_{\mathrm{CDCl}}$ ): $\delta$ (ppm) 150.5, 143.2, 139.7, 137.7, 136.1, 135.9, 133.7, 130.4, 129.4, 128.7, 128.5, 127.8, 127.8, 127.4, 127.0, 125.4, 123.8, 122.9, 119.2, 116.3, 115.4, 84.1, 59.7, 28.2, 21.5, 14.3; HRMS (ESI-TOF) m/z: [M + Na $]^{+}$Calcd for $\mathrm{C}_{32} \mathrm{H}_{34} \mathrm{~N}_{2} \mathrm{O}_{4} \mathrm{SNa}$ 565.2131; Found 565.2133.

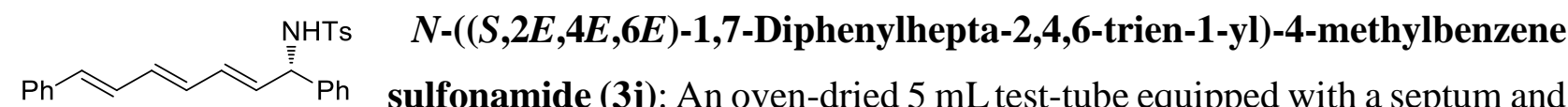
a magnetic stir bar was charged with $\mathrm{Pd}_{2}(\mathrm{dba})_{3}(4.6 \mathrm{mg}, 5 \mathrm{~mol} \%)$ and ligand $\mathbf{L 1}$ (11.3 $\left.\mathrm{mg}, 20 \mathrm{~mol} \%\right)$. The tube was then evacuated and filled with argon. This cycle was repeated three times, and toluene $(0.5 \mathrm{~mL})$ was added via syringe. The resulting solution was stirred at room temperature for $30 \mathrm{~min}$. A second oven-dried test-tube was charged with diene $\mathbf{1 j}$ (31.0 $\mathrm{mg}, 2.0$ equiv), $N$-sulfonylimine $\mathbf{2 a}$ (26.0 mg, 1.0 equiv), 1-naphthoic acid (5.2 mg, $30 \mathrm{~mol} \%$ ) and $4 \AA \mathrm{MS}$ (60.0 mg), and the system was placed under an atmosphere of argon (balloon). Then the catalyst solution was added at room temperature. The resulting mixture was stirred at $80{ }^{\circ} \mathrm{C}$ for $30 \mathrm{~h}$. After completion, purification by flash chromatography on silica gel (petroleum ether/EtOAc $=10 / 1$ ) gave the product $\mathbf{3 j}$ : $35.3 \mathrm{mg}$, as a faint yellow oil, $85 \%$ yield; $E / Z=7: 1 ;[\alpha]^{25} \mathrm{D}=+17.8\left(c=0.55\right.$, in $\left.\mathrm{CHCl}_{3}\right) ; 80 \%$ ee, determined by HPLC analysis [Chiralpak column AD-H, $i \mathrm{PrOH} / n \mathrm{Hexane}=20 / 80$, flow rate: $1.0 \mathrm{~mL} / \mathrm{min}, 254 \mathrm{~nm}$, $\mathrm{t}($ minor $)=16.45 \mathrm{~min}, \mathrm{t}($ major $)=18.11 \mathrm{~min}] ;{ }^{1} \mathbf{H} \mathbf{~ N M R}\left(400 \mathrm{MHz}, \mathrm{CDCl}_{3}\right): \delta(\mathrm{ppm}) 7.60(\mathrm{~d}, J=8.0$ $\mathrm{Hz}, 2 \mathrm{H}), 7.36-7.34(\mathrm{~m}, 2 \mathrm{H}), 7.30-7.26(\mathrm{~m}, 2 \mathrm{H}), 7.24-7.17(\mathrm{~m}, 6 \mathrm{H}), 7.11-7.09(\mathrm{~m}, 2 \mathrm{H}), 6.72(\mathrm{dd}, J=$ $15.8,10.0,1 \mathrm{H}), 6.53(\mathrm{~d}, J=15.6 \mathrm{~Hz}, 1 \mathrm{H}), 6.26-6.01(\mathrm{~m}, 3 \mathrm{H}), 5.62(\mathrm{dd}, J=14.8,6.4 \mathrm{~Hz}, 1 \mathrm{H}), 5.00$ $4.93(\mathrm{~m}, 2 \mathrm{H}), 2.35$ (s, 3H); ${ }^{13} \mathbf{C}$ NMR (100 MHz, $\left.\mathrm{CDCl}_{3}\right): \delta(\mathrm{ppm}) 143.3,139.6,137.6,137.1,134.2$, 133.2, 132.4, 131.8, 131.6, 129.4, 128.7, 128.6, 128.6, 127.8, 127.7, 127.3, 127.0, 126.4, 59.5, 21.5; HRMS (ESI-TOF) m/z: [M + Na $]^{+}$Calcd for $\mathrm{C}_{26} \mathrm{H}_{25} \mathrm{NO}_{2} \mathrm{SNa} 438.1498$; Found 438.1499. 


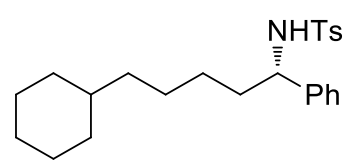

(S)-N-(5-Cyclohexyl-1-phenylpentyl)-4-methylbenzenesulfonamide (3k'):

An oven-dried $5 \mathrm{~mL}$ test-tube equipped with a septum and a magnetic stir bar was charged with $\mathrm{Pd}_{2}(\mathrm{dba})_{3}(4.6 \mathrm{mg}, 5 \mathrm{~mol} \%)$ and ligand $\mathbf{L 1}(11.3 \mathrm{mg}, 20$

mol \%). The tube was then evacuated and filled with argon. This cycle was repeated three times, and toluene $(0.5 \mathrm{~mL})$ was added via syringe. The resulting solution was stirred at room temperature for $30 \mathrm{~min}$. A second oven-dried test-tube was charged with diene 1k (27.2 mg, 2.0 equiv), $\mathrm{N}$ sulfonylimine $2 \mathbf{a}$ (26.0 mg, 1.0 equiv), 1-naphthoic acid (5.2 mg, $30 \mathrm{~mol} \%)$ and $4 \AA \mathrm{MS}$ (60.0 mg), and the system was placed under an atmosphere of argon (balloon). Then the catalyst solution was added at room temperature. The resulting mixture was stirred at $80{ }^{\circ} \mathrm{C}$ for $30 \mathrm{~h}$. After completion, purification by flash chromatography on silica gel (petroleum ether/EtOAc $=10 / 1$ ) gave the product $3 \mathbf{k}$ as a mixture of double bond migration, $26.1 \mathrm{mg}$, as a colorless oil, $66 \%$ yield.

3k was dissolved in $\mathrm{MeOH}$, then 10\% $\mathrm{Pd} / \mathrm{C}$ (5.2 mg, $20 \mathrm{wt} \%$ ) was added.The resulting solution was stirred at room temperature under an atmosphere of $\mathrm{H}_{2}$ (balloon) for $12 \mathrm{~h}$. After completion, purification by flash chromatography on silica gel (petroleum ether/EtOAc $=10 / 1$ ) gave the pure product 3k': $25.6 \mathrm{mg}$, as a colorless oil, $98 \%$ yield; $[\alpha]^{25} \mathrm{D}=-54.6\left(c=1.08\right.$, in $\left.\mathrm{CHCl}_{3}\right)$; $98 \%$ ee, determined by HPLC analysis [Chiralpak column IB, $i \mathrm{PrOH} / n \mathrm{Hexane}=20 / 80$, flow rate: $1.0 \mathrm{~mL} / \mathrm{min}$, $220 \mathrm{~nm}, \mathrm{t}($ minor $)=4.99 \mathrm{~min}, \mathrm{t}$ (major) $=6.23 \mathrm{~min}] ;{ }^{1} \mathbf{H} \mathbf{N M R}\left(400 \mathrm{MHz}, \mathrm{CDCl}_{3}\right): \delta(\mathrm{ppm}) 7.53(\mathrm{~d}$, $J=8.4 \mathrm{~Hz}, 2 \mathrm{H}), 7.16-7.10(\mathrm{~m}, 5 \mathrm{H}), 7.02-6.99(\mathrm{~m}, 2 \mathrm{H}), 4.80(\mathrm{~d}, J=7.2 \mathrm{~Hz}, 1 \mathrm{H}), 4.27-4.22(\mathrm{~m}, 1 \mathrm{H})$, $2.35(\mathrm{~s}, 3 \mathrm{H}), 1.75-1.60(\mathrm{~m}, 7 \mathrm{H}), 1.25-1.02(\mathrm{~m}, 10 \mathrm{H}), 0.83-0.78(\mathrm{~m}, 2 \mathrm{H}) ;{ }^{13} \mathrm{C}$ NMR (100 MHz, $\left.\mathrm{CDCl}_{3}\right): \delta$ (ppm) 142.9, 141.1, 137.7, 129.3, 128.4, 127.3, 127.0, 126.5, 58.3, 37.6, 37.5, 37.2, 33.4, 33.3, 26.7, 26.4, 26.3, 26.1, 21.4; HRMS (ESI-TOF) m/z: $[\mathrm{M}+\mathrm{Na}]^{+}$Calcd for $\mathrm{C}_{24} \mathrm{H}_{33} \mathrm{NO}_{2} \mathrm{SNa}$ 422.2124; Found 422.2126.

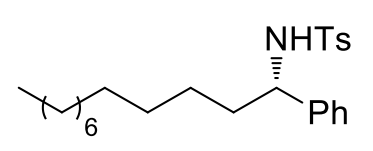

(S)-4-Methyl-N-(1-phenyldodecyl)benzenesulfonamide (3l'): An ovendried $5 \mathrm{~mL}$ test-tube equipped with a septum and a magnetic stir bar was charged with $\mathrm{Pd}_{2}(\mathrm{dba})_{3}(4.6 \mathrm{mg}, 5 \mathrm{~mol} \%$ ) and ligand L1 (11.3 mg, $20 \mathrm{~mol} \%$ ). The tube was then evacuated and filled with argon. This cycle was repeated three times, and toluene $(0.5 \mathrm{~mL})$ was added via syringe. The resulting solution was stirred at room temperature for $30 \mathrm{~min}$. A second oven-dried test-tube was charged with diene $\mathbf{1 l}$ (30.4 mg, 2.0 equiv), $N$-sulfonylimine $\mathbf{2 a}$ (26.0 mg, 1.0 equiv), 1-naphthoic acid (5.2 mg, $30 \mathrm{~mol} \%$ ) and $4 \AA \mathrm{MS}(60.0 \mathrm{mg})$, and the system was placed under an atmosphere of argon (balloon). Then the catalyst solution was added at room temperature. The resulting mixture was stirred at $80{ }^{\circ} \mathrm{C}$ for $30 \mathrm{~h}$. After completion, purification by flash chromatography on silica gel (petroleum ether/EtOAc $=20 / 1$ ) gave the product $\mathbf{3 l}$ as a mixture of double bond migration, $25.1 \mathrm{mg}$, as a colorless oil, $61 \%$ yield.

31 was dissolved in $\mathrm{MeOH}$, then $10 \% \mathrm{Pd} / \mathrm{C}(5.0 \mathrm{mg}, 20 \mathrm{wt} \%)$ was added. The resulting solution was stirred at room temperature under an atmosphere of $\mathrm{H}_{2}$ (balloon) for $12 \mathrm{~h}$. After completion, 
purification by flash chromatography on silica gel (petroleum ether/EtOAc $=20 / 1$ ) gave the pure product 3l': $24.5 \mathrm{mg}$, colorless oil, $98 \%$ yield; $[\alpha]^{25} \mathrm{D}=-20.4\left(c=0.85\right.$, in $\left.\mathrm{CHCl}_{3}\right) ; 86 \%$ ee, determined by HPLC analysis [Chiralpak column, $i \mathrm{PrOH} / n$ Hexane $=10 / 90$, flow rate: $1.0 \mathrm{~mL} / \mathrm{min}, 254 \mathrm{~nm}, \mathrm{t}$ (minor) $=6.05 \mathrm{~min}, \mathrm{t}$ (major) = $7.82 \mathrm{~min}] ;{ }^{1} \mathbf{H}$ NMR $\left(400 \mathrm{MHz}, \mathrm{CDCl}_{3}\right): \delta(\mathrm{ppm}) 7.53(\mathrm{~d}, J=8.0 \mathrm{~Hz}$, 2H), 7.15-7.10 (m, 5H), 7.02-7.00 (m, 2H), $4.81(\mathrm{~d}, J=7.2 \mathrm{~Hz}, 1 \mathrm{H}), 4.28-4.23(\mathrm{~m}, 1 \mathrm{H}), 2.35(\mathrm{~s}, 3 \mathrm{H})$, $1.73-1.63(\mathrm{~m}, 2 \mathrm{H}), 1.29-1.07(\mathrm{~m}, 18 \mathrm{H}), 0.88(\mathrm{t}, J=6.8 \mathrm{~Hz}, 3 \mathrm{H}) ;{ }^{13} \mathbf{C}$ NMR $\left(100 \mathrm{MHz}, \mathrm{CDCl}_{3}\right): \delta$ (ppm) 142.9, 141.1, 137.7, 129.2, 128.4, 127.3, 127.0, 126.5, 58.3, 37.6, 31.9, 29.6, 29.5, 29.4, 29.3, 29.1, 25.8, 22.7, 21.4, 14.1; HRMS (ESI-TOF) m/z: $[\mathrm{M}+\mathrm{Na}]^{+}$Calcd for $\mathrm{C}_{25} \mathrm{H}_{37} \mathrm{NO}_{2} \mathrm{SNa} 438.2437$, found 438.2436 .

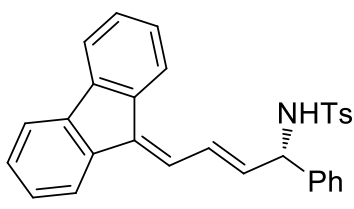

(S,E)- $N$-(4-(9H-Fluoren-9-ylidene)-1-phenylbut-2-en-1-yl)-4-methyl benzenesulfonamide (3m): An oven-dried $5 \mathrm{~mL}$ test-tube equipped with a septum and a magnetic stir bar was charged with $\mathrm{Pd}_{2}(\mathrm{dba})_{3}(4.6 \mathrm{mg}, 5 \mathrm{~mol} \%)$ and ligand $\mathbf{L 1}$ (11.3 mg, $20 \mathrm{~mol} \%)$. The tube was then evacuated and filled with argon. This cycle was repeated three times, and toluene $(0.5 \mathrm{~mL})$ was added via syringe. The resulting solution was stirred at room temperature for $30 \mathrm{~min}$. A second oven-dried test-tube was charged with diene $\mathbf{1 m}$ (40.8 $\mathrm{mg}, 2.0$ equiv), $N$-sulfonylimine $\mathbf{2 a}$ (26.0 mg, 1.0 equiv), 1-naphthoic acid (5.2 mg, $30 \mathrm{~mol} \%$ ) and $4 \AA \mathrm{MS}(60.0 \mathrm{mg}$ ), and the system was placed under an atmosphere of argon (balloon). Then the catalyst solution was added at room temperature. The resulting mixture was stirred at $80{ }^{\circ} \mathrm{C}$ for $30 \mathrm{~h}$. After completion, purification by flash chromatography on silica gel (petroleum ether/EtOAc $=10 / 1$ ) gave the product 3m: $36.2 \mathrm{mg}$, as a faint yellow solid, 78\% yield; $E / Z=15: 1 ; \mathrm{mp}=117-119{ }^{\circ} \mathrm{C} ;[\alpha]^{25} \mathrm{D}=+33.7\left(c=0.63\right.$, in $\left.\mathrm{CHCl}_{3}\right) ; 89 \%$ ee, determined by HPLC analysis [Chiralpak column IB, $i \mathrm{PrOH} / n$ Hexane $=40 / 60$, flow rate: $1.0 \mathrm{~mL} / \mathrm{min}, 254 \mathrm{~nm}, \mathrm{t}($ minor $)=$ $8.02 \mathrm{~min}, \mathrm{t}$ (major) = $11.70 \mathrm{~min}] ;{ }^{1} \mathbf{H}$ NMR $\left(400 \mathrm{MHz}, \mathrm{CDCl}_{3}\right): \delta(\mathrm{ppm}) 7.71-7.57(\mathrm{~m}, 7 \mathrm{H}), 7.37-$ $7.23(\mathrm{~m}, 8 \mathrm{H}), 7.14-7.07(\mathrm{~m}, 3 \mathrm{H}), 6.95(\mathrm{~d}, J=11.2 \mathrm{~Hz}, 1 \mathrm{H}), 6.06(\mathrm{dd}, J=14.4,6.8 \mathrm{~Hz}, 1 \mathrm{H}), 5.23-$ $5.16(\mathrm{~m}, 2 \mathrm{H}), 2.17$ (s, 3H); ${ }^{13} \mathbf{C}$ NMR (100 MHz, $\left.\mathrm{CDCl}_{3}\right): \delta$ (ppm) 143.6, 140.9, 139.20, 139.17, 139.1, 137.8, 137.4, 136.7, 135.9, 129.5, 128.9, 128.19, 128.18, 128.1, 128.08, 127.3, 127.1, 127.0, 126.9, 125.1, 124.9, 120.1, 119.8, 119.6, 59.6, 21.2; HRMS (ESI-TOF) m/z: [M + Na $]^{+}$Calcd for $\mathrm{C}_{30} \mathrm{H}_{25} \mathrm{NO}_{2} \mathrm{SNa} 486.1498$; Found 486.1496

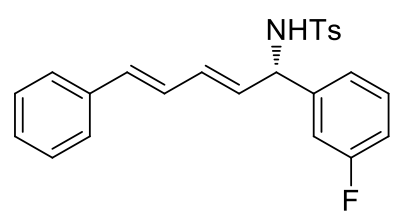

$N$-((S,2E,4E)-1-(3-Fluorophenyl)-5-phenylpenta-2,4-dien-1-yl)-4methylbenzenesulfonamide (3n): An oven-dried $5 \mathrm{~mL}$ test-tube equipped with a septum and a magnetic stir bar was charged with $\operatorname{Pd}_{2}(\mathrm{dba})_{3}(4.6 \mathrm{mg}$, $5 \mathrm{~mol} \%$ ) and ligand $\mathbf{L 1}(11.3 \mathrm{mg}, 20 \mathrm{~mol} \%)$. The tube was then evacuated and filled with argon. This cycle was repeated three times, and toluene $(0.5 \mathrm{~mL})$ was added via syringe. The resulting solution was stirred at room temperature for $30 \mathrm{~min}$. A second oven-dried test-tube was 
charged with diene $\mathbf{1 a}$ (26.0 mg, 2.0 equiv), $N$-sulfonylimine $\mathbf{2 b}$ (27.7 $\mathrm{mg}, 1.0$ equiv), 1-naphthoic acid (5.2 mg, $30 \mathrm{~mol} \%$ ) and $4 \AA$ MS (60.0 mg), and the system was placed under an atmosphere of argon (balloon). Then the catalyst solution was added at room temperature. The resulting mixture was stirred at $80{ }^{\circ} \mathrm{C}$ for $30 \mathrm{~h}$. After completion, purification by flash chromatography on silica gel (petroleum ether/EtOAc = 10/1) gave the product 3n: $34.0 \mathrm{mg}$, as a colorless oil, 84\% yield; E/Z>19:1; $[\alpha]^{25} \mathrm{D}=+36.2\left(c=0.90\right.$, in $\left.\mathrm{CHCl}_{3}\right) ; 96 \%$ ee, determined by HPLC analysis [Chiralpak column IB, $i \mathrm{PrOH} / n$ Hexane $=40 / 60$, flow rate: $1.0 \mathrm{~mL} / \mathrm{min}, 254 \mathrm{~nm}, \mathrm{t}$ (minor $)=6.86 \mathrm{~min}, \mathrm{t}($ major $)=11.62 \mathrm{~min}]$; ${ }^{1} \mathbf{H}$ NMR (400 MHz, $\left.\mathrm{CDCl}_{3}\right): \delta(\mathrm{ppm}) 7.64(\mathrm{~d}, J=8.4 \mathrm{~Hz}, 2 \mathrm{H}), 7.35-7.28(\mathrm{~m}, 4 \mathrm{H}), 7.25-7.17$ (m, $4 \mathrm{H}), 6.96(\mathrm{~d}, J=7.6 \mathrm{~Hz}, 1 \mathrm{H}), 6.92-6.82(\mathrm{~m}, 2 \mathrm{H}), 6.59(\mathrm{dd}, J=15.6,10.4 \mathrm{~Hz}, 1 \mathrm{H}), 6.42(\mathrm{~d}, J=15.6$ $\mathrm{Hz}, 1 \mathrm{H}), 6.11(\mathrm{dd}, J=14.8,10.4 \mathrm{~Hz}, 1 \mathrm{H}), 5.68(\mathrm{dd}, J=15.2,6.8 \mathrm{~Hz}, 1 \mathrm{H}), 5.12(\mathrm{~d}, J=7.2 \mathrm{~Hz}, 1 \mathrm{H})$, $5.02(\mathrm{t}, J=7.2 \mathrm{~Hz}, 1 \mathrm{H}), 2.35(\mathrm{~s}, 3 \mathrm{H}) ;{ }^{13} \mathbf{C ~ N M R}\left(100 \mathrm{MHz}, \mathrm{CDCl}_{3}\right): \delta(\mathrm{ppm}) 162.8(J=245.4 \mathrm{~Hz})$, 143.5, $142.1(J=6.8 \mathrm{~Hz}), 137.4,136.7,134.0,133.0,131.1,130.2(\mathrm{~d}, J=8.2 \mathrm{~Hz}), 129.5,128.6,127.9$, 127.2, 127.19, 126.4, $122.7(J=3.0 \mathrm{~Hz}), 114.6(J=21.0 \mathrm{~Hz}), 114.1(\mathrm{~d}, J=22.1 \mathrm{~Hz}), 59.0(\mathrm{~d}, J=1.4$ $\mathrm{Hz}$ ), 21.4; HRMS (ESI-TOF) m/z: [M + Na $]^{+}$Calcd for $\mathrm{C}_{24} \mathrm{H}_{22} \mathrm{FNO}_{2} \mathrm{SNa}_{4} 430.1247$; Found 430.1249.

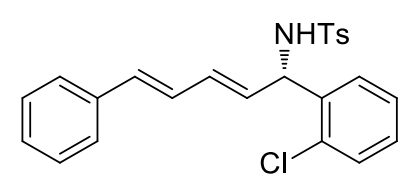

$N-((S, 2 E, 4 E)-1-(2-C h l o r o p h e n y l)-5-p h e n y l p e n t a-2,4-d i e n-1-y l)-4-$ methylbenzenesulfonamide (3o): An oven-dried $5 \mathrm{~mL}$ test-tube equipped with a septum and a magnetic stir bar was charged with $\operatorname{Pd}_{2}(\mathrm{dba})_{3}(4.6 \mathrm{mg}$, $5 \mathrm{~mol} \%$ ) and ligand $\mathbf{L 1}$ (11.3 $\mathrm{mg}, 20 \mathrm{~mol} \%$ ). The tube was then evacuated and filled with argon. This cycle was repeated three times, and toluene $(0.5 \mathrm{~mL})$ was added via syringe. The resulting solution was stirred at room temperature for $30 \mathrm{~min}$. A second oven-dried test-tube was charged with diene $1 \mathrm{a}$ (26.0 mg, 2.0 equiv), $N$-sulfonylimine $2 \mathrm{c}$ (29.4 mg, 1.0 equiv), 1-naphthoic acid (5.2 mg, $30 \mathrm{~mol} \%$ ) and $4 \AA \mathrm{MS}(60.0 \mathrm{mg})$, and the system was placed under an atmosphere of argon (balloon). Then the catalyst solution was added at room temperature. The resulting mixture was stirred at $80{ }^{\circ} \mathrm{C}$ for $30 \mathrm{~h}$. After completion, purification by flash chromatography on silica gel (petroleum ether/EtOAc $=10 / 1$ ) gave the product 3o: $34.0 \mathrm{mg}$, as a white solid, $79 \%$ yield; $E / Z>19: 1$; $\mathrm{mp}=122-124{ }^{\circ} \mathrm{C} ;[\alpha]^{25} \mathrm{D}=+23.9\left(c=1.4\right.$, in $\left.\mathrm{CHCl}_{3}\right) ; 96 \%$ ee, determined by HPLC analysis [Chiralpak column IB, $i \mathrm{PrOH} / n$ Hexane $=40 / 60$, flow rate: $1.0 \mathrm{~mL} / \mathrm{min}, 254 \mathrm{~nm}, \mathrm{t}$ (minor) $=6.24 \mathrm{~min}$, $\mathrm{t}$ (major) = $9.96 \mathrm{~min}] ;{ }^{1} \mathbf{H}$ NMR $\left(400 \mathrm{MHz}, \mathrm{CDCl}_{3}\right): \delta(\mathrm{ppm}) 7.64(\mathrm{~d}, J=8.0 \mathrm{~Hz}, 2 \mathrm{H}), 7.33-7.18(\mathrm{~m}$, 7H), 7.15-7.09 (m, 4H), $6.62(\mathrm{dd}, J=15.6,10.4 \mathrm{~Hz}, 1 \mathrm{H}), 6.41(\mathrm{~d}, J=15.6 \mathrm{~Hz}, 1 \mathrm{H}), 6.14-6.07$ (m, $1 \mathrm{H}), 5.76(\mathrm{dd}, J=15.2,6.0 \mathrm{~Hz}, 1 \mathrm{H}), 5.50-5.42(\mathrm{~m}, 2 \mathrm{H}), 2.33(\mathrm{~s}, 3 \mathrm{H}) ;{ }^{13} \mathbf{C} \mathbf{N M R}\left(100 \mathrm{MHz}, \mathrm{CDCl}_{3}\right)$ : $\delta(\mathrm{ppm}) 143.2,137.2,136.9,136.8,133.9,132.7,132.5,130.7,129.8,129.4,128.9,128.8,128.6$, 127.8, 127.4, 127.2, 127.1, 126.4, 56.9, 21.4; HRMS (ESI-TOF) m/z: $[\mathrm{M}+\mathrm{Na}]^{+}$Calcd for $\mathrm{C}_{24} \mathrm{H}_{22} \mathrm{ClNO}_{2} \mathrm{SNa} 446.0952\left({ }^{35} \mathrm{Cl}\right), 448.0922\left({ }^{37} \mathrm{Cl}\right)$; Found $446.0958\left({ }^{35} \mathrm{Cl}\right), 448.0934\left({ }^{37} \mathrm{Cl}\right)$. 


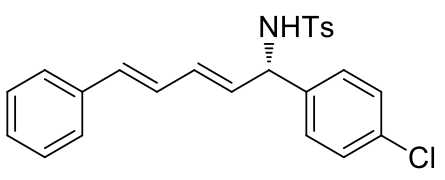

$N-((S, 2 E, 4 E)-1-(4-C h l o r o p h e n y l)-5-p h e n y l p e n t a-2,4-d i e n-1-y l)-4-$ methylbenzenesulfonamide (3p): An oven-dried $5 \mathrm{~mL}$ test-tube equipped with a septum and a magnetic stir bar was charged with $\mathrm{Pd}_{2}(\mathrm{dba})_{3}(4.6 \mathrm{mg}, 5 \mathrm{~mol} \%$ ) and ligand $\mathbf{L 1}(11.3 \mathrm{mg}, 20 \mathrm{~mol} \%$ ). The tube was then evacuated and filled with argon. This cycle was repeated three times, and toluene $(0.5 \mathrm{~mL})$ was added via syringe. The resulting solution was stirred at room temperature for $30 \mathrm{~min}$. A second oven-dried test-tube was charged with diene $\mathbf{1 a}$ (26.0 mg, 2.0 equiv), $N$-sulfonylimine $\mathbf{2 d}$ (29.4 mg, 1.0 equiv), 1-naphthoic acid (5.2 mg, $30 \mathrm{~mol} \%$ ) and $4 \AA \mathrm{MS}(60.0 \mathrm{mg})$, and the system was placed under an atmosphere of argon (balloon). Then the catalyst solution was added at room temperature. The resulting mixture was stirred at $80{ }^{\circ} \mathrm{C}$ for $30 \mathrm{~h}$. After completion, purification by flash chromatography on silica gel (petroleum ether/EtOAc $=10 / 1$ ) gave the product 3p: $40.6 \mathrm{mg}$, as a white solid, $96 \%$ yield; $E / Z=$ 16:1; $\mathrm{mp}=110-112{ }^{\circ} \mathrm{C} ;[\alpha]^{25} \mathrm{D}=+23.5\left(c=0.8\right.$, in $\left.\mathrm{CHCl}_{3}\right) ; 95 \%$ ee, determined by HPLC analysis [Chiralpak column IB, $i \mathrm{PrOH} / n$ Hexane $=40 / 60$, flow rate: $1.0 \mathrm{~mL} / \mathrm{min}, 254 \mathrm{~nm}, \mathrm{t}$ (minor) $=7.78 \mathrm{~min}$, $\mathrm{t}$ (major) = $12.46 \mathrm{~min}] ;{ }^{1} \mathbf{H}$ NMR $\left(400 \mathrm{MHz}, \mathrm{CDCl}_{3}\right): \delta(\mathrm{ppm}) 7.62(\mathrm{~d}, J=8.4 \mathrm{~Hz}, 2 \mathrm{H}), 7.35-7.28(\mathrm{~m}$, 4H), 7.26-7.17 (m, 5H), 7.09 (d, $J=8.4 \mathrm{~Hz}, 2 \mathrm{H}), 6.60(\mathrm{dd}, J=15.6,10.4 \mathrm{~Hz}, 1 \mathrm{H}), 6.42(\mathrm{~d}, J=15.6$ $\mathrm{Hz}, 1 \mathrm{H}), 6.10(\mathrm{dd}, J=15.2,10.4 \mathrm{~Hz}, 1 \mathrm{H}), 5.69(\mathrm{dd}, J=15.2,6.4 \mathrm{~Hz}, 1 \mathrm{H}), 5.10(\mathrm{~d}, J=7.2 \mathrm{~Hz}, 1 \mathrm{H})$, $5.01(\mathrm{t}, J=6.8 \mathrm{~Hz}, 1 \mathrm{H}), 2.37(\mathrm{~s}, 3 \mathrm{H}) ;{ }^{13} \mathrm{C}$ NMR (100 MHz, $\left.\mathrm{CDCl}_{3}\right): \delta(\mathrm{ppm}) 143.5,138.0,137.4$, 136.7, 134.0, 133.6, 133.0, 131.2, 129.5, 128.7, 128.6, 128.5, 127.9, 127.2, 127.2, 126.4, 58.9, 21.5; HRMS (ESI-TOF) m/z: $[\mathrm{M}+\mathrm{Na}]^{+}$Calcd for $\mathrm{C}_{24} \mathrm{H}_{22} \mathrm{ClNO}_{2} \mathrm{SNa} 446.0952\left({ }^{35} \mathrm{Cl}\right), 448.0922\left({ }^{37} \mathrm{Cl}\right)$; Found $446.0956\left({ }^{35} \mathrm{Cl}\right), 448.0936\left({ }^{37} \mathrm{Cl}\right)$.

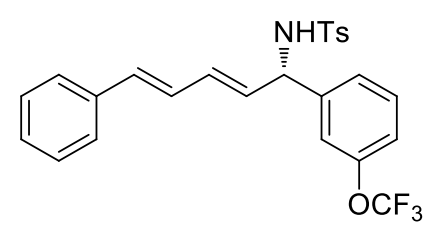

4-Methyl-N-((S,2E,4E)-5-phenyl-1-(3-(trifluoromethoxy)phenyl) penta-2,4-dien-1-yl)benzenesulfonamide (3q): An oven-dried $5 \mathrm{~mL}$ testtube equipped with a septum and a magnetic stir bar was charged with $\mathrm{Pd}_{2}(\mathrm{dba})_{3}(4.6 \mathrm{mg}, 5 \mathrm{~mol} \%)$ and ligand $\mathbf{L 1}(11.3 \mathrm{mg}, 20 \mathrm{~mol} \%)$. The tube was then evacuated and filled with argon. This cycle was repeated three times, and toluene $(0.5 \mathrm{~mL})$ was added via syringe. The resulting solution was stirred at room temperature for $30 \mathrm{~min}$. A second oven-dried test-tube was charged with diene $\mathbf{1 a}(26.0 \mathrm{mg}, 2.0$ equiv), $N$-sulfonylimine $\mathbf{2 e}(34.3 \mathrm{mg}$, 1.0 equiv), 1-naphthoic acid (5.2 mg, $30 \mathrm{~mol} \%)$ and $4 \AA \mathrm{MS}(60.0 \mathrm{mg})$, and the system was placed under an atmosphere of argon (balloon). Then the catalyst solution was added at room temperature. The resulting mixture was stirred at $80{ }^{\circ} \mathrm{C}$ for $30 \mathrm{~h}$. After completion, purification by flash chromatography on silica gel (petroleum ether/EtOAc $=10 / 1$ ) gave the product 3q: $37.7 \mathrm{mg}$, as a colorless semisolid, $80 \%$ yield; $E / Z=11: 1 ;[\alpha]^{25} \mathrm{D}=+26.3\left(c=1.4\right.$, in $\left.\mathrm{CHCl}_{3}\right)$; $93 \%$ ee, determined by HPLC analysis [Chiralpak column IB, $i \mathrm{PrOH} / n$ Hexane $=40 / 60$, flow rate: $1.0 \mathrm{~mL} / \mathrm{min}, 254 \mathrm{~nm}, \mathrm{t}$ $($ minor $)=5.12, \mathrm{t}($ major $)=7.78 \mathrm{~min}] ;{ }^{1} \mathbf{H} \mathbf{~ N M R}\left(400 \mathrm{MHz}, \mathrm{CDCl}_{3}\right): \delta(\mathrm{ppm}) 7.62(\mathrm{~d}, J=8.0 \mathrm{~Hz}, 2 \mathrm{H})$, 7.35-7.20 (m, 6H), $7.18(\mathrm{~d}, J=8.0 \mathrm{~Hz}, 2 \mathrm{H}), 7.14-7.11(\mathrm{~m}, 1 \mathrm{H}), 7.05(\mathrm{~d}, J=8.4 \mathrm{~Hz}, 1 \mathrm{H}), 6.96(\mathrm{~s}$, 
1H), $6.60(\mathrm{dd}, J=15.6,10.4 \mathrm{~Hz}, 1 \mathrm{H}), 6.43(\mathrm{~d}, J=15.6 \mathrm{~Hz}, 1 \mathrm{H}), 6.13(\mathrm{dd}, J=15.2,10.4 \mathrm{~Hz}, 1 \mathrm{H})$, $5.69(\mathrm{dd}, J=15.2,6.8 \mathrm{~Hz}, 1 \mathrm{H}), 5.17-5.14(\mathrm{~m}, 1 \mathrm{H}), 5.05(\mathrm{t}, J=6.8 \mathrm{~Hz}, 1 \mathrm{H}), 2.34(\mathrm{~s}, 3 \mathrm{H}) ;{ }^{13} \mathbf{C ~ N M R}$ (100 MHz, $\left.\mathrm{CDCl}_{3}\right): \delta$ (ppm) 149.3, 143.6, 142.0, 137.3, 136.7, 134.2, 133.2, 130.8, 130.0, 129.5, 128.6, 127.9, 127.2, 127.1, 126.4, 125.5, 120.0, 119.7, 118.7 (q, $J=255.8 \mathrm{~Hz}$ ), 59.0, 21.4; HRMS (ESI-TOF) m/z: [M + Na] ${ }^{+}$Calcd for $\mathrm{C}_{25} \mathrm{H}_{22} \mathrm{~F}_{3} \mathrm{NO}_{3} \mathrm{SNa} 496.1165$; Found 496.1166 .

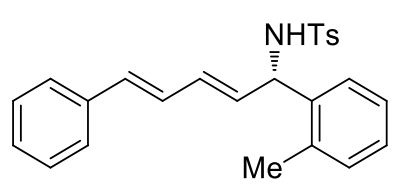

\section{4-Methyl- $N-((S, 2 E, 4 E)-5-p h e n y l-1-(o-t o l y l) p e n t a-2,4-d i e n-1-y l)$} benzenesulfonamide (3r): An oven-dried $5 \mathrm{~mL}$ test-tube equipped with a septum and a magnetic stir bar was charged with $\mathrm{Pd}_{2}(\mathrm{dba})_{3}(4.6 \mathrm{mg}, 5$ mol \%) and ligand $\mathbf{L 1}(11.3 \mathrm{mg}, 20 \mathrm{~mol} \%)$. The tube was then evacuated and filled with argon. This cycle was repeated three times, and toluene $(0.5 \mathrm{~mL})$ was added via syringe. The resulting solution was stirred at room temperature for $30 \mathrm{~min}$. A second oven-dried test-tube was charged with diene 1a (26.0 mg, 2.0 equiv), $N$-sulfonylimine $\mathbf{2 f}$ (27.3 mg, 1.0 equiv), 1-naphthoic acid (5.2 $\mathrm{mg}, 30 \mathrm{~mol} \%$ ) and $4 \AA$ MS (60.0 mg), and the system was placed under an atmosphere of argon (balloon). Then the catalyst solution was added at room temperature. The resulting mixture was stirred at $80{ }^{\circ} \mathrm{C}$ for $30 \mathrm{~h}$. After completion, purification by flash chromatography on silica gel (petroleum ether/EtOAc $=10 / 1$ ) gave the product 3r: $36.4 \mathrm{mg}$, as a semisolid, 90\% yield; $E / Z>19: 1 ;[\alpha]^{25} \mathrm{D}=+22.3\left(c=1.3\right.$, in $\left.\mathrm{CHCl}_{3}\right)$; 97\% ee, determined by HPLC analysis [Chiralpak column IB, $i \mathrm{PrOH} / n \mathrm{Hexane}=40 / 60$, flow rate: 1.0 $\mathrm{mL} / \mathrm{min}, 254 \mathrm{~nm}, \mathrm{t}$ (minor) $=6.58 \mathrm{~min}, \mathrm{t}($ major $)=11.68 \mathrm{~min}] ;{ }^{1} \mathbf{H} \mathbf{~ N M R}\left(400 \mathrm{MHz}, \mathrm{CDCl}_{3}\right): \delta(\mathrm{ppm})$ $7.63(\mathrm{~d}, J=8.0 \mathrm{~Hz}, 2 \mathrm{H}), 7.34-7.05(\mathrm{~m}, 11 \mathrm{H}), 6.61(\mathrm{dd}, J=15.6,10.4 \mathrm{~Hz}, 1 \mathrm{H}), 6.38(\mathrm{~d}, J=15.6 \mathrm{~Hz}$, $1 \mathrm{H}), 6.06(\mathrm{dd}, J=14.8,10.4 \mathrm{~Hz}, 1 \mathrm{H}), 5.74(\mathrm{dd}, J=14.8,6.4 \mathrm{~Hz}, 1 \mathrm{H}), 5.28(\mathrm{t}, J=6.4 \mathrm{~Hz}, 1 \mathrm{H}), 5.10$ $(\mathrm{d}, J=6.8 \mathrm{~Hz}, 1 \mathrm{H}), 2.35$ (s, 3H), 2.26 (s, 3H); ${ }^{13} \mathbf{C ~ N M R}\left(100 \mathrm{MHz}, \mathrm{CDCl}_{3}\right): \delta(\mathrm{ppm})$ 143.2, 137.7, 137.5, 136.9, 135.4, 133.4, 132.3, 131.9, 130.7, 129.3, 128.6, 127.7, 127.6, 127.5, 127.2, 126.8, 126.3, 56.1, 21.4, 19.2; HRMS (ESI-TOF) m/z: $[\mathrm{M}+\mathrm{Na}]^{+}$Calcd for $\mathrm{C}_{25} \mathrm{H}_{25} \mathrm{NO}_{2} \mathrm{SNa}$ 426.1498; Found 426.1501 .

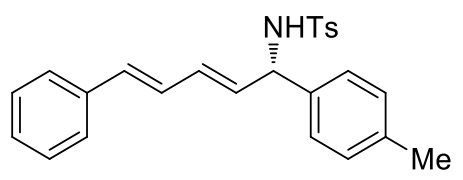

4-Methyl- $N$-((S,2E,4E)-5-phenyl-1-(p-tolyl)penta-2,4-dien-1-yl) benzenesulfonamide (3s): An oven-dried $5 \mathrm{~mL}$ test-tube equipped with a septum and a magnetic stir bar was charged with $\mathrm{Pd}_{2}(\mathrm{dba})_{3}(4.6 \mathrm{mg}, 5$ mol \%) and ligand $\mathbf{L 1}(11.3 \mathrm{mg}, 20 \mathrm{~mol} \%)$. The tube was then evacuated and filled with argon. This cycle was repeated three times, and toluene $(0.5 \mathrm{~mL})$ was added via syringe. The resulting solution was stirred at room temperature for $30 \mathrm{~min}$. A second oven-dried test-tube was charged with diene 1a (26.0 mg, 2.0 equiv), $N$-sulfonylimine $2 \mathrm{~g}$ (27.3 mg, 1.0 equiv), 1-naphthoic acid (5.2 $\mathrm{mg}, 30 \mathrm{~mol} \%$ ) and $4 \AA$ MS (60.0 mg), and the system was placed under an atmosphere of argon (balloon). Then the catalyst solution was added at room temperature. The resulting mixture was stirred at $80^{\circ} \mathrm{C}$ for $30 \mathrm{~h}$. After completion, purification by flash chromatography on silica gel (petroleum ether/EtOAc $=10 / 1$ ) 
gave the product 3s: $35.3 \mathrm{mg}$, semisolid, $87 \%$ yield; $E / Z=12: 1 ;[\alpha]^{25} \mathrm{D}=+26.0\left(c=1.3\right.$, in $\left.\mathrm{CHCl}_{3}\right)$; 94\% ee, determined by HPLC analysis [Chiralpak column IB, $i \mathrm{PrOH} / n \mathrm{Hexane}=40 / 60$, flow rate: 1.0 $\mathrm{mL} / \mathrm{min}, 254 \mathrm{~nm}, \mathrm{t}$ (minor) $=6.27 \mathrm{~min}, \mathrm{t}($ major $)=8.41 \mathrm{~min}] ;{ }^{1} \mathbf{H} \mathbf{N M R}\left(400 \mathrm{MHz}, \mathrm{CDCl}_{3}\right): \delta(\mathrm{ppm})$ $7.65(\mathrm{~d}, J=8.4 \mathrm{~Hz}, 2 \mathrm{H}), 7.33-7.13(\mathrm{~m}, 7 \mathrm{H}), 7.03(\mathrm{~s}, 4 \mathrm{H}), 6.59$ (dd, $J=15.6,10.4 \mathrm{~Hz}, 1 \mathrm{H}), 6.39$ (d, $J$ $=15.6 \mathrm{~Hz}, 1 \mathrm{H}), 6.13(\mathrm{dd}, J=15.2,10.4 \mathrm{~Hz}, 1 \mathrm{H}), 5.71(\mathrm{dd}, J=15.2,6.8 \mathrm{~Hz}, 1 \mathrm{H}), 5.10-5.09(\mathrm{~m}, 1 \mathrm{H})$, $4.97(\mathrm{t}, J=6.8 \mathrm{~Hz}, 1 \mathrm{H}), 2.34(\mathrm{~s}, 3 \mathrm{H}), 2.28(\mathrm{~s}, 3 \mathrm{H}) ;{ }^{13} \mathbf{C ~ N M R}\left(100 \mathrm{MHz}, \mathrm{CDCl}_{3}\right): \delta(\mathrm{ppm}) 143.2$, 137.7, 137.6, 136.9, 136.62, 133.4, 132.3, 132.2, 129.4, 129.3, 128.6, 127.7, 127.6, 127.3, 126.9, 126.3, 59.3, 21.5, 21.3; HRMS (ESI-TOF) m/z: $[\mathrm{M}+\mathrm{Na}]^{+}$Calcd for $\mathrm{C}_{25} \mathrm{H}_{25} \mathrm{NO}_{2} \mathrm{SNa} 426.1498$; Found 426.1506.

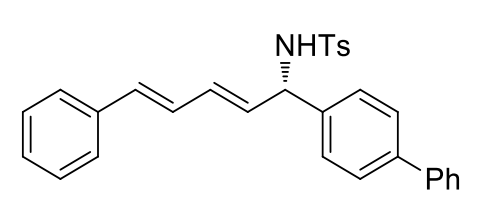

$N-((S, 2 E, 4 E)-1-([1,1 '-B i p h e n y l]-4-y l)-5-p h e n y l p e n t a-2,4-d i e n-1-y l)$ -4-methylbenzenesulfonamide (3t): An oven-dried $5 \mathrm{~mL}$ test-tube equipped with a septum and a magnetic stir bar was charged with $\mathrm{Pd}_{2}(\mathrm{dba})_{3}(4.6 \mathrm{mg}, 5 \mathrm{~mol} \%)$ and ligand $\mathbf{L 1}(11.3 \mathrm{mg}, 20 \mathrm{~mol} \%)$. The tube was then evacuated and filled with argon. This cycle was repeated three times, and toluene $(0.5$ $\mathrm{mL}$ ) was added via syringe. The resulting solution was stirred at room temperature for $30 \mathrm{~min}$. A second oven-dried test-tube was charged with diene $\mathbf{1 a}(26.0 \mathrm{mg}, 2.0$ equiv), $N$-sulfonylimine $\mathbf{2 h}$ (33.5 mg, 1.0 equiv), 1-naphthoic acid (5.2 mg, $30 \mathrm{~mol} \%$ ) and $4 \AA \mathrm{MS}$ (60.0 mg), and the system was placed under an atmosphere of argon (balloon). Then the catalyst solution was added at room temperature. The resulting mixture was stirred at $80{ }^{\circ} \mathrm{C}$ for $30 \mathrm{~h}$. After completion, purification by flash chromatography on silica gel (petroleum ether/EtOAc $=10 / 1$ ) gave the product 3t: $46.0 \mathrm{mg}$, as a white solid, $99 \%$ yield; $E / Z=13: 1 ; \mathrm{mp}=124-126^{\circ} \mathrm{C} ;[\alpha]^{25} \mathrm{D}=+26.7\left(c=0.45\right.$, in $\left.\mathrm{CHCl}_{3}\right) ; 96 \%$ ee, determined by HPLC analysis [Chiralpak column IB, $i \mathrm{PrOH} / n \mathrm{Hexane}=40 / 60$, flow rate: $1.0 \mathrm{~mL} / \mathrm{min}$, $254 \mathrm{~nm}, \mathrm{t}($ minor $)=13.71 \mathrm{~min}, \mathrm{t}($ major $)=17.56 \mathrm{~min}] ;{ }^{1} \mathbf{H} \mathbf{~ N M R}\left(400 \mathrm{MHz}, \mathrm{CDCl}_{3}\right): \delta(\mathrm{ppm}) 7.64$ $(\mathrm{d}, J=8.0 \mathrm{~Hz}, 2 \mathrm{H}), 7.50(\mathrm{~d}, J=7.2 \mathrm{~Hz}, 2 \mathrm{H}), 7.43-7.38(\mathrm{~m}, 4 \mathrm{H}), 7.33-7.26(\mathrm{~m}, 5 \mathrm{H}), 7.22-7.14(\mathrm{~m}$, $5 \mathrm{H}), 6.61(\mathrm{dd}, J=15.6,10.4 \mathrm{~Hz}, 1 \mathrm{H}), 6.41(\mathrm{~d}, J=15.6 \mathrm{~Hz}, 1 \mathrm{H}), 6.16(\mathrm{dd}, J=15.0,10.6 \mathrm{~Hz}, 1 \mathrm{H})$, $5.75(\mathrm{dd}, J=15.1,6.6 \mathrm{~Hz}, 1 \mathrm{H}), 5.18(\mathrm{~d}, J=7.2 \mathrm{~Hz}, 1 \mathrm{H}), 5.06(\mathrm{t}, J=6.8 \mathrm{~Hz}, 1 \mathrm{H}), 2.30(\mathrm{~s}, 3 \mathrm{H}) ;{ }^{13} \mathrm{C}$ NMR (150 MHz, $\left.\mathrm{CDCl}_{3}\right): \delta$ (ppm) 143.2, 140.7, 140.5, 138.5, 137.6, 136.8, 133.7, 132.6, 131.8, 129.4, 128.8, 128.6, 127.8, 127.5, 127.5, 127.4, 127.3, 127.3, 126.9, 126.4, 59.3, 21.4; HRMS (ESITOF) $\mathrm{m} / \mathrm{z}:[\mathrm{M}+\mathrm{Na}]^{+}$Calcd for $\mathrm{C}_{30} \mathrm{H}_{27} \mathrm{NO}_{2} \mathrm{SNa} 488.1655$; Found 488.1660.

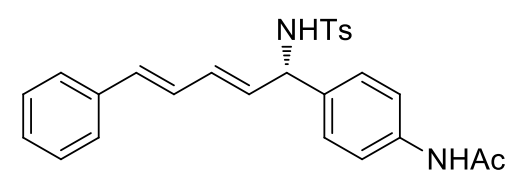

$N$-(4-((S,2E,4E)-1-(4-Methylphenylsulfonamido)-5-phenylpenta2,4-dien-1-yl)phenyl)acetamide(3u): An oven-dried $5 \mathrm{~mL}$ test-tube equipped with a septum and a magnetic stir bar was charged with $\mathrm{Pd}_{2}(\mathrm{dba})_{3}$ (4.6 mg, $5 \mathrm{~mol} \%$ ) and ligand $\mathbf{L 1}(11.3 \mathrm{mg}, 20 \mathrm{~mol} \%$ ). The tube was then evacuated and filled with argon. This cycle was repeated three times, and toluene $(0.5 \mathrm{~mL})$ was added via syringe. 
The resulting solution was stirred at room temperature for $30 \mathrm{~min}$. A second oven-dried test-tube was charged with diene $1 \mathbf{a}$ (26.0 mg, 2.0 equiv), $N$-sulfonylimine $2 \mathbf{i}$ (31.6 mg, 1.0 equiv), 1-naphthoic acid (5.2 mg, $30 \mathrm{~mol} \%$ ) and $4 \AA \mathrm{MS}(60.0 \mathrm{mg}$ ), and the system was placed under an atmosphere of argon (balloon). Then the catalyst solution was added at room temperature. The resulting mixture was stirred at $80{ }^{\circ} \mathrm{C}$ for $30 \mathrm{~h}$. After completion, purification by flash chromatography on silica gel (petroleum ether/EtOAc = 3/1) gave the product 3u: $35.0 \mathrm{mg}$, white semisolid, $78 \%$ yield; $E / Z=14: 1$; $[\alpha]^{25}{ }_{\mathrm{D}}=+22.7\left(c=1.35\right.$, in $\left.\mathrm{CHCl}_{3}\right) ; 95 \%$ ee, determined by HPLC analysis [Chiralpak column IE, $i \mathrm{PrOH} / n$ Hexane $=40 / 60$, flow rate: $1.0 \mathrm{~mL} / \mathrm{min}, 254 \mathrm{~nm}, \mathrm{t}$ (major) $=23.59 \mathrm{~min}, \mathrm{t}$ (minor) $=30.94$ min]; ${ }^{1} \mathbf{H}$ NMR (400 MHz, $\left.\mathrm{CDCl}_{3}\right): \delta(\mathrm{ppm}) 7.82(\mathrm{~s}, 1 \mathrm{H}), 7.66(\mathrm{~d}, J=8.0 \mathrm{~Hz}, 2 \mathrm{H}), 7.38(\mathrm{~d}, J=8.4$ $\mathrm{Hz}, 2 \mathrm{H}), 7.30-7.17(\mathrm{~m}, 7 \mathrm{H}), 7.08(\mathrm{~d}, J=8.4 \mathrm{~Hz}, 2 \mathrm{H}), 6.54(\mathrm{dd}, J=15.6,10.4 \mathrm{~Hz}, 1 \mathrm{H}), 6.33(\mathrm{~d}, J=$ $15.6 \mathrm{~Hz}, 1 \mathrm{H}), 6.01(\mathrm{dd}, J=15.6,10.4 \mathrm{~Hz}, 1 \mathrm{H}), 5.67-5.59$ (m, 2H), 4.94 (t, $J=7.2 \mathrm{~Hz}, 1 \mathrm{H}), 2.33$ (s, 3H), 2.12 (s, 3H); ${ }^{13} \mathbf{C}$ NMR (100 MHz, $\left.\mathrm{CDCl}_{3}\right): \delta(\mathrm{ppm}) 168.9,143.4,137.5,136.8,135.5,133.5$, $132.5,131.7,129.5,128.6,127.7,127.6,127.5,127.2,126.3,120.1,59.0,24.4,21.4$; HRMS (ESITOF) $\mathrm{m} / \mathrm{z}:[\mathrm{M}+\mathrm{Na}]^{+}$Calcd for $\mathrm{C}_{26} \mathrm{H}_{26} \mathrm{~N}_{2} \mathrm{O}_{3} \mathrm{SNa} 468.1556$; Found 469.1558 .

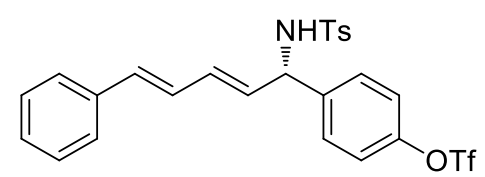

4-((S,2E,4E)-1-(4-Methylphenylsulfonamido)-5-phenylpenta-2,4dien-1-yl)phenyl trifluoromethanesulfonate (3v): An oven-dried 5 $\mathrm{mL}$ test-tube equipped with a septum and a magnetic stir bar was charged with $\mathrm{Pd}_{2}(\mathrm{dba})_{3}(4.6 \mathrm{mg}, 5 \mathrm{~mol} \%$ ) and ligand $\mathbf{L 1}(11.3 \mathrm{mg}, 20 \mathrm{~mol} \%$ ). The tube was then evacuated and filled with argon. This cycle was repeated three times, and toluene $(0.5 \mathrm{~mL})$ was added via syringe. The resulting solution was stirred at room temperature for $30 \mathrm{~min}$. A second oven-dried test-tube was charged with diene $\mathbf{1 a}(26.0 \mathrm{mg}, 2.0$ equiv), $N$-sulfonylimine $\mathbf{2 j}$ ( $40.7 \mathrm{mg}, 1.0$ equiv), 1-naphthoic acid (5.2 mg, $30 \mathrm{~mol} \%)$ and $4 \AA \mathrm{MS}(60.0 \mathrm{mg})$, and the system was placed under an atmosphere of argon (balloon). Then the catalyst solution was added at room temperature. The resulting mixture was stirred at $80{ }^{\circ} \mathrm{C}$ for $30 \mathrm{~h}$. After completion, purification by flash chromatography on silica gel (petroleum ether/EtOAc $=10 / 1$ ) gave the product 3v: $46.4 \mathrm{mg}$, as a white solid, $84 \%$ yield; $E / Z>19: 1 ; \mathrm{mp}=141-143{ }^{\circ} \mathrm{C}[\alpha]^{25} \mathrm{D}=+42.6\left(c=1.76\right.$, in $\left.\mathrm{CHCl}_{3}\right) ; 95 \%$ ee, determined by HPLC analysis [Chiralpak column IB, $i \mathrm{PrOH} / n \mathrm{Hexane}=40 / 60$, flow rate: $1.0 \mathrm{~mL} / \mathrm{min}$, $254 \mathrm{~nm}, \mathrm{t}($ minor $)=6.53, \mathrm{t}$ (major) $=10.31 \mathrm{~min}] ;{ }^{1} \mathrm{H} \mathrm{NMR}\left(400 \mathrm{MHz}, \mathrm{CDCl}_{3}\right): \delta(\mathrm{ppm}) 7.61(\mathrm{~d}, J=$ $8.2 \mathrm{~Hz}, 2 \mathrm{H}), 7.34-7.09$ (m, 11H), 6.59 (dd, $J=15.6,10.4 \mathrm{~Hz}, 1 \mathrm{H}), 6.42$ (d, $J=15.6 \mathrm{~Hz}, 1 \mathrm{H}), 6.09$ $(\mathrm{dd}, J=15.2,10.4 \mathrm{~Hz}, 1 \mathrm{H}), 5.67(\mathrm{dd}, J=15.2,6.8 \mathrm{~Hz}, 1 \mathrm{H}), 5.38-5.36(\mathrm{~m}, 1 \mathrm{H}), 5.08(\mathrm{t}, J=6.8 \mathrm{~Hz}$, 1H), 2.34 (s, 3H); $\left.{ }^{13} \mathrm{C} \mathrm{NMR} \mathrm{(100} \mathrm{MHz,} \mathrm{CDCl}_{3}\right): \delta(\mathrm{ppm}) 148.7,143.7,140.2,137.3,136.6,134.4$, 133.3, 130.7, 129.5, 129.0, 128.6, 128.0, 127.1, 127.0, 126.4, 121.42, 118.7 (q, $J=336.1 \mathrm{~Hz}), 58.7$, 21.4; HRMS (ESI-TOF) m/z: [M + Na $]^{+}$Calcd for $\mathrm{C}_{25} \mathrm{H}_{22} \mathrm{~F}_{3} \mathrm{NO}_{5} \mathrm{~S}_{2} \mathrm{Na} 560.0784$; Found 560.0784. 


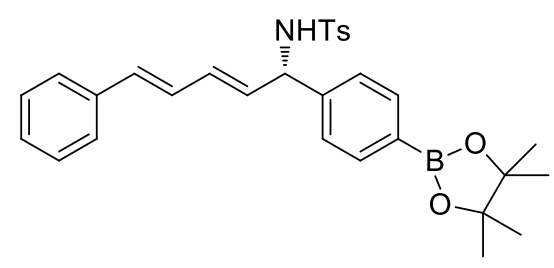

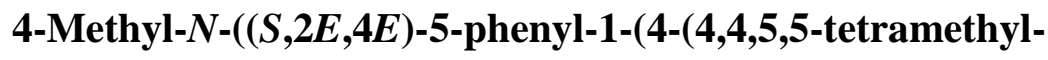
1,3,2-dioxaborolan-2-yl)phenyl)penta-2,4-dien-1-yl)benzene sulfonamide (3w): An oven-dried $5 \mathrm{~mL}$ test-tube equipped with a septum and a magnetic stir bar was charged with $\mathrm{Pd}_{2}(\mathrm{dba})_{3}(4.6 \mathrm{mg}$, $5 \mathrm{~mol} \%)$ and ligand $\mathbf{L 1}(11.3 \mathrm{mg}, 20 \mathrm{~mol} \%)$. The tube was then evacuated and filled with argon. This cycle was repeated three times, and toluene $(0.5 \mathrm{~mL})$ was added via syringe. The resulting solution was stirred at room temperature for $30 \mathrm{~min}$. A second oven-dried test-tube was charged with diene $1 \mathbf{a}$ ( $26.0 \mathrm{mg}, 2.0$ equiv), $N$-sulfonylimine $\mathbf{2 k}$ (38.5 mg, 1.0 equiv), 1-naphthoic acid (5.2 mg, $30 \mathrm{~mol} \%$ ) and $4 \AA \mathrm{MS}(60.0 \mathrm{mg})$, and the system was placed under an atmosphere of argon (balloon). Then the catalyst solution was added at room temperature. The resulting mixture was stirred at $80{ }^{\circ} \mathrm{C}$ for $30 \mathrm{~h}$. After completion, purification by flash chromatography on silica gel (petroleum ether/EtOAc $=10 / 1$ ) gave the product 3w: $29.0 \mathrm{mg}$, as a colorless semisolid, $57 \%$ yield; $E / Z>19: 1 ;[\alpha]^{25} \mathrm{D}=+14.6\left(c=0.52\right.$, in $\left.\mathrm{CHCl}_{3}\right) ; 96 \%$ ee, determined by HPLC analysis [Chiralpak column IA, $i \mathrm{PrOH} / n \mathrm{Hexane}=10 / 90$, flow rate: $1.0 \mathrm{~mL} / \mathrm{min}, 254 \mathrm{~nm}, \mathrm{t}$ $($ minor $)=25.12 \mathrm{~min}, \mathrm{t}($ major $)=27.75 \mathrm{~min}] ;{ }^{1} \mathbf{H}$ NMR $\left(400 \mathrm{MHz}, \mathrm{CDCl}_{3}\right): \delta(\mathrm{ppm}) 7.66(\mathrm{~d}, J=8.0$ $\mathrm{Hz}, 2 \mathrm{H}), 7.62(\mathrm{~d}, J=8.0 \mathrm{~Hz}, 2 \mathrm{H}), 7.35-7.28(\mathrm{~m}, 4 \mathrm{H}), 7.24-7.11(\mathrm{~m}, 5 \mathrm{H}), 6.61(\mathrm{dd}, J=15.6,10.2 \mathrm{~Hz}$, $1 \mathrm{H}), 6.42(\mathrm{~d}, J=15.6 \mathrm{~Hz}, 1 \mathrm{H}), 6.13(\mathrm{dd}, J=15.2,10.4 \mathrm{~Hz}, 1 \mathrm{H}), 5.73(\mathrm{dd}, J=15.2,6.4 \mathrm{~Hz}, 1 \mathrm{H}), 5.04$ $(\mathrm{t}, J=6.8 \mathrm{~Hz}, 1 \mathrm{H}), 4.93(\mathrm{~d}, J=7.2 \mathrm{~Hz}, 1 \mathrm{H}), 2.35(\mathrm{~s}, 3 \mathrm{H}), 1.33(\mathrm{~s}, 12 \mathrm{H}) ;{ }^{13} \mathbf{C ~ N M R}\left(100 \mathrm{MHz}, \mathrm{CDCl}_{3}\right)$ $\delta 143.3,142.4,137.5,136.9,135.1,133.7,132.7,131.8,129.4,128.6,127.8,127.4,127.3,126.4$, 126.3, 83.8, 59.6, 24.8, 21.4; HRMS (ESI-TOF) $\mathrm{m} / \mathrm{z}$ : $[\mathrm{M}+\mathrm{Na}]^{+} \mathrm{Calcd}$ for $\mathrm{C}_{30} \mathrm{H}_{34} \mathrm{BNO}_{4} \mathrm{SNa} 538.2194$; Found 538.2193.

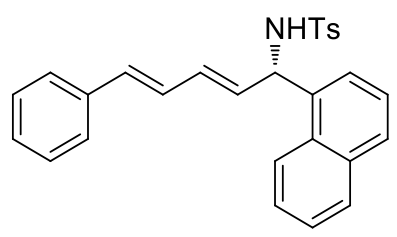

4-Methyl- $N$-((S,2E,4E)-1-(naphthalen-1-yl)-5-phenylpenta-2,4-dien-1yl)benzenesulfonamide (3x): An oven-dried $5 \mathrm{~mL}$ test-tube equipped with a septum and a magnetic stir bar was charged with $\operatorname{Pd}_{2}(\mathrm{dba})_{3}(4.6 \mathrm{mg}, 5$ mol \%) and ligand L1 (11.3 mg, $20 \mathrm{~mol} \%)$. The tube was then evacuated and filled with argon. This cycle was repeated three times, and toluene $(0.5 \mathrm{~mL})$ was added via syringe. The resulting solution was stirred at room temperature for $30 \mathrm{~min}$. A second oven-dried test-tube was charged with diene $\mathbf{1 a}$ (26.0 mg, 2.0 equiv), $N$-sulfonylimine $\mathbf{2 l}$ (30.9 mg, 1.0 equiv), 1-naphthoic acid (5.2 mg, $30 \mathrm{~mol} \%$ ) and $4 \AA$ MS (60.0 mg), and the system was placed under an atmosphere of argon (balloon). Then the catalyst solution was added at room temperature. The resulting mixture was stirred at $80{ }^{\circ} \mathrm{C}$ for $30 \mathrm{~h}$. After completion, purification by flash chromatography on silica gel (petroleum ether/EtOAc $=10 / 1$ ) gave the product 3x: $41.7 \mathrm{mg}$, as a white solid, 95\% yield; $E / Z=$ 16:1; $\mathrm{mp}=181-183{ }^{\circ} \mathrm{C} ;[\alpha]^{25} \mathrm{D}=-31.3\left(c=1.2\right.$, in $\left.\mathrm{CHCl}_{3}\right) ; 97 \%$ ee, determined by HPLC analysis [Chiralpak column IB, $i \mathrm{PrOH} / n$ Hexane $=40 / 60$, flow rate: $1.0 \mathrm{~mL} / \mathrm{min}, 254 \mathrm{~nm}, \mathrm{t}$ (minor) $=10.90$ min, $\mathrm{t}$ (major) = $17.01 \mathrm{~min}] ;{ }^{1} \mathbf{H}$ NMR $\left(400 \mathrm{MHz}, \mathrm{CDCl}_{3}\right): \delta(\mathrm{ppm}) 7.99-7.97(\mathrm{~m}, 1 \mathrm{H}), 7.83-7.80(\mathrm{~m}$, 
1H), 7.74-7.72 (m, 1H), $7.58(\mathrm{~d}, J=8.4 \mathrm{~Hz}, 2 \mathrm{H}), 7.49-7.45(\mathrm{~m}, 2 \mathrm{H}), 7.34-7.19(\mathrm{~m}, 7 \mathrm{H}), 7.07(\mathrm{~d}, J=$ $8.4 \mathrm{~Hz}, 2 \mathrm{H}), 6.63(\mathrm{dd}, J=15.6,10.4 \mathrm{~Hz}, 1 \mathrm{H}), 6.35$ (d, $J=15.6 \mathrm{~Hz}, 1 \mathrm{H}), 6.18$ (dd, $J=15.2,10.4 \mathrm{~Hz}$, 1H), $5.94(\mathrm{dd}, J=15.2,6.4 \mathrm{~Hz}, 1 \mathrm{H}), 5.80(\mathrm{t}, J=6.4 \mathrm{~Hz}, 1 \mathrm{H}), 5.09$ (d, $J=7.2 \mathrm{~Hz}, 1 \mathrm{H}), 2.31$ (s, 3H); ${ }^{13} \mathrm{C}$ NMR (100 MHz, $\left.\mathrm{CDCl}_{3}\right): \delta$ (ppm) 143.1, 137.4, 136.9, 135.0, 134.0, 133.5, 132.7, 132.0, 130.3, 129.2, 128.8, 128.61, 128.56, 127.7, 127.5, 127.2, 126.5, 126.3, 125.8, 125.5, 125.1, 123.3, 56.4, 21.4; HRMS (ESI-TOF) m/z: [M + Na $]^{+}$Calcd for $\mathrm{C}_{28} \mathrm{H}_{25} \mathrm{NO}_{2} \mathrm{SNa} 462.1498$; Found 462.1503 .

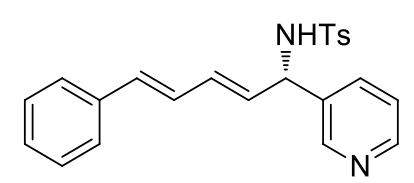

\section{4-Methyl- $N$-((S,2E,4E)-5-phenyl-1-(pyridin-3-yl)penta-2,4-dien-1-yl)}

benzenesulfonamide (3y): An oven-dried $5 \mathrm{~mL}$ test-tube equipped with a septum and a magnetic stir bar was charged with $\operatorname{Pd}_{2}(\mathrm{dba})_{3}(4.6 \mathrm{mg}, 5$ mol \%) and ligand L1 (11.3 mg, $20 \mathrm{~mol} \%)$. The tube was then evacuated and filled with argon. This cycle was repeated three times, and toluene $(0.5 \mathrm{~mL})$ was added via syringe. The resulting solution was stirred at room temperature for $30 \mathrm{~min}$. A second oven-dried test-tube was charged with diene 1a (26.0 mg, 2.0 equiv), $N$-sulfonylimine $2 \mathrm{~m}$ (26.0 mg, 1.0 equiv), 1-naphthoic acid (5.2 mg, $30 \mathrm{~mol} \%$ ) and $4 \AA$ MS (60.0 mg), and the system was placed under an atmosphere of argon (balloon). Then the catalyst solution was added at room temperature. The resulting mixture was stirred at $80{ }^{\circ} \mathrm{C}$ for $30 \mathrm{~h}$. After completion, purification by flash chromatography on silica gel (petroleum ether/EtOAc $=3 / 1$ ) gave the product $3 \mathbf{y}: 36.0 \mathrm{mg}$, as a white semisolid, $92 \%$ yield; $E / Z>19: 1 ;[\alpha]^{25} \mathrm{D}=+27.5(c=0.87$, in $\mathrm{CHCl}_{3}$ ); $91 \%$ ee, determined by HPLC analysis [Chiralpak column IB, $i \mathrm{PrOH} / n \mathrm{Hexane}=40 / 60$, flow rate: $1.0 \mathrm{~mL} / \mathrm{min}, 254 \mathrm{~nm}, \mathrm{t}$ (minor) $=11.70 \mathrm{~min}, \mathrm{t}$ (major) $=14.75 \mathrm{~min}] ;{ }^{1} \mathbf{H} \mathbf{~ N M R}(400 \mathrm{MHz}$, $\left.\mathrm{CDCl}_{3}\right): \delta(\mathrm{ppm}) 8.45(\mathrm{~d}, J=4.0 \mathrm{~Hz}, 1 \mathrm{H}), 8.39(\mathrm{~s}, 1 \mathrm{H}), 7.64(\mathrm{~d}, J=8.0 \mathrm{~Hz}, 2 \mathrm{H}), 7.53(\mathrm{~d}, J=7.6 \mathrm{~Hz}$, $1 \mathrm{H}), 7.34-7.15(\mathrm{~m}, 8 \mathrm{H}), 6.60(\mathrm{dd}, J=15.6,10.4 \mathrm{~Hz}, 1 \mathrm{H}), 6.42(\mathrm{~d}, J=15.6 \mathrm{~Hz}, 1 \mathrm{H}), 6.10(\mathrm{dd}, J=$ 14.8, 10.4 Hz, 1H), 5.73-5.68 (m, 2H), $5.07(\mathrm{t}, J=6.8 \mathrm{~Hz}, 1 \mathrm{H}), 2.35(\mathrm{~s}, 3 \mathrm{H}), 2.23(\mathrm{~s}, 1 \mathrm{H}) ;{ }^{13} \mathbf{C ~ N M R}$ $\left(100 \mathrm{MHz}, \mathrm{CDCl}_{3}\right): \delta$ (ppm) 148.9, 148.6, 143.6, 137.4, 136.6, 135.4, 134.8, 134.4, 133.4, 130.6, 129.6, 128.6, 128.0, 127.2, 127.0, 126.4, 123.5, 57.3, 21.5; HRMS (ESI-TOF) m/z: [M + H] Calcd for $\mathrm{C}_{23} \mathrm{H}_{23} \mathrm{~N}_{2} \mathrm{O}_{2} \mathrm{~S}$ 391.1475; Found 391.1479.

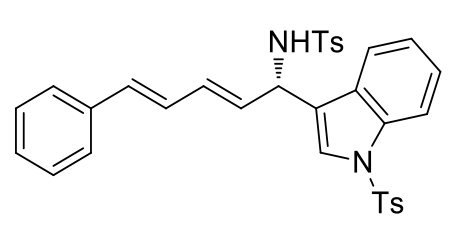

4-Methyl- $N$-((S,2E,4E)-5-phenyl-1-(1-tosyl-1H-indol-3-yl)penta-

2,4-dien-1-yl)benzenesulfonamide (3z): An oven-dried $5 \mathrm{~mL}$ test-tube equipped with a septum and a magnetic stir bar was charged with $\mathrm{Pd}_{2}(\mathrm{dba})_{3}(4.6 \mathrm{mg}, 5 \mathrm{~mol} \%)$ and ligand $\mathbf{L 1}(11.3 \mathrm{mg}, 20 \mathrm{~mol} \%)$. The tube was then evacuated and filled with argon. This cycle was repeated three times, and toluene $(0.5$ $\mathrm{mL}$ ) was added via syringe. The resulting solution was stirred at room temperature for $30 \mathrm{~min}$. A second oven-dried test-tube was charged with diene $\mathbf{1 a}(26.0 \mathrm{mg}, 2.0$ equiv), $N$-sulfonylimine $\mathbf{2 n}$ (45.3 mg, 1.0 equiv), 1-naphthoic acid (5.2 mg, $30 \mathrm{~mol} \%$ ) and $4 \AA \mathrm{MS}$ (60.0 mg), and the system was placed under an atmosphere of argon (balloon). Then the catalyst solution was added at room 
temperature. The resulting mixture was stirred at $80{ }^{\circ} \mathrm{C}$ for $30 \mathrm{~h}$. After completion, purification by flash chromatography on silica gel (petroleum ether/EtOAc $=10 / 1$ ) gave the product 3z: $49.8 \mathrm{mg}$, as a white solid, $86 \%$ yield; $E / Z>19: 1 ; \mathrm{mp}=87-89{ }^{\circ} \mathrm{C}$; $[\alpha]^{25} \mathrm{D}=-24.2\left(c=1.0\right.$, in $\left.\mathrm{CHCl}_{3}\right) ; 87 \%$ ee, determined by HPLC analysis [Chiralpak column AD-H, $i \mathrm{PrOH} / n \mathrm{Hexane}=40 / 60$, flow rate: 1.0 $\mathrm{mL} / \mathrm{min}, 254 \mathrm{~nm}, \mathrm{t}$ (minor) $=18.49 \mathrm{~min}, \mathrm{t}$ (major) $=37.70 \mathrm{~min}] ;{ }^{1} \mathbf{H} \mathbf{~ N M R}\left(400 \mathrm{MHz}, \mathrm{CDCl}_{3}\right): \delta$ (ppm) $7.88(\mathrm{~d}, J=8.4 \mathrm{~Hz}, 1 \mathrm{H}), 7.73(\mathrm{~d}, J=8.4 \mathrm{~Hz}, 2 \mathrm{H}), 7.59$ (d, $J=8.0 \mathrm{~Hz}, 2 \mathrm{H}), 7.45(\mathrm{~d}, J=8.0 \mathrm{~Hz}$, $1 \mathrm{H}), 7.37-7.05(\mathrm{~m}, 12 \mathrm{H}), 6.59(\mathrm{dd}, J=15.6,10.4 \mathrm{~Hz}, 1 \mathrm{H}), 6.37(\mathrm{~d}, J=15.6 \mathrm{~Hz}, 1 \mathrm{H}), 6.16(\mathrm{dd}, J=$ 14.8, 10.4 Hz, 1H), 5.77 (dd, $J=14.8,6.8 \mathrm{~Hz}, 1 \mathrm{H}), 5.27$ (t, $J=6.8 \mathrm{~Hz}, 1 \mathrm{H}), 5.15$ (d, $J=7.2 \mathrm{~Hz}, 1 \mathrm{H})$, $2.33(\mathrm{~s}, 3 \mathrm{H}), 2.30(\mathrm{~s}, 3 \mathrm{H}) ;{ }^{13} \mathrm{C}$ NMR $\left(100 \mathrm{MHz}, \mathrm{CDCl}_{3}\right): \delta(\mathrm{ppm}) 145.1,143.4,137.3,136.7,135.2$, 135.1, 133.9, 133.1, 129.9, 129.8, 129.3, 128.6, 128.2, 127.9, 127.2, 127.2, 126.8, 126.4, 124.9, 124.2, 123.3, 120.8, 120.3, 113.5, 52.5, 21.5, 21.4; HRMS (ESI-TOF) m/z: $[\mathrm{M}+\mathrm{Na}]^{+}$Calcd for $\mathrm{C}_{33} \mathrm{H}_{30} \mathrm{~N}_{2} \mathrm{O}_{4} \mathrm{~S}_{2} \mathrm{Na}$ 605.1539; Found 605.1540.

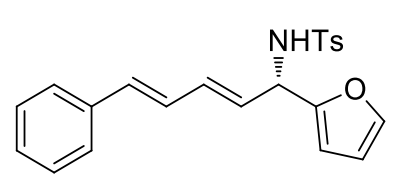

$N$-((S,2E,4E)-1-(Furan-2-yl)-5-phenylpenta-2,4-dien-1-yl)-4-methyl benzenesulfonamide (3aa): An oven-dried $5 \mathrm{~mL}$ test-tube equipped with a septum and a magnetic stir bar was charged with $\mathrm{Pd}_{2}(\mathrm{dba})_{3}(4.6 \mathrm{mg}, 5 \mathrm{~mol} \%)$ and ligand $\mathbf{L 1}$ (11.3 mg, $20 \mathrm{~mol} \%$ ). The tube was then evacuated and filled with argon. This cycle was repeated three times, and toluene $(0.5 \mathrm{~mL})$ was added via syringe. The resulting solution was stirred at room temperature for $30 \mathrm{~min}$. A second oven-dried test-tube was charged with diene 1a (26.0 mg, 2.0 equiv), $N$-sulfonylimine 20 (24.9 mg, 1.0 equiv), 1-naphthoic acid (5.2 mg, $30 \mathrm{~mol} \%$ ) and $4 \AA$ MS (60.0 mg), and the system was placed under an atmosphere of argon (balloon). Then the catalyst solution was added at room temperature. The resulting mixture was stirred at $80^{\circ} \mathrm{C}$ for $30 \mathrm{~h}$. After completion, purification by flash chromatography on silica gel (petroleum ether/EtOAc $=10 / 1$ ) gave the product 3aa: $37.2 \mathrm{mg}$, as a faint yellow semisolid, $97 \%$ yield; $E / Z=13: 1 ;[\alpha]^{25} \mathrm{D}=+16.2(c$ $=0.98$, in $\left.\mathrm{CHCl}_{3}\right) ; 94 \%$ ee, determined by HPLC analysis [Chiralpak column $\mathrm{IB}, i \mathrm{PrOH} / n \mathrm{Hexane}=$ 40/60, flow rate: $1.0 \mathrm{~mL} / \mathrm{min}, 254 \mathrm{~nm}, \mathrm{t}$ (minor) $=6.89 \mathrm{~min}, \mathrm{t}$ (major) $=9.17 \mathrm{~min}] ;{ }^{1} \mathbf{H} \mathbf{N M R}(400$ $\left.\mathrm{MHz}_{\mathrm{CDCl}}\right): \delta(\mathrm{ppm}) 7.68(\mathrm{~d}, J=8.0 \mathrm{~Hz}, 2 \mathrm{H}), 7.35-7.20(\mathrm{~m}, 8 \mathrm{H}), 6.63(\mathrm{dd}, J=15.6,10.4 \mathrm{~Hz}, 1 \mathrm{H})$, $6.46(\mathrm{~d}, J=15.6 \mathrm{~Hz}, 1 \mathrm{H}), 6.24-6.18(\mathrm{~m}, 2 \mathrm{H}), 6.05(\mathrm{~d}, J=3.2 \mathrm{~Hz}, 1 \mathrm{H}), 5.74(\mathrm{dd}, J=15.2,6.4 \mathrm{~Hz}$, 1H), 5.16-5.06 (m, 2H), 2.36 (s, 3H); $\left.{ }^{13} \mathbf{C ~ N M R ~ ( 1 0 0 ~ M H z , ~} \mathrm{CDCl}_{3}\right): \delta$ (ppm) 151.6, 143.2, 142.4, 137.6, 136.8, 134.0, 133.1, 129.4, 129.1, 128.6, 127.8, 127.3, 127.2, 126.4, 110.2, 107.48, 53.3, 21.4; HRMS (ESI-TOF) m/z: [M + Na $]^{+}$Calcd for $\mathrm{C}_{22} \mathrm{H}_{21} \mathrm{NO}_{3} \mathrm{SNa} 402.1134$; Found 402.1136 .

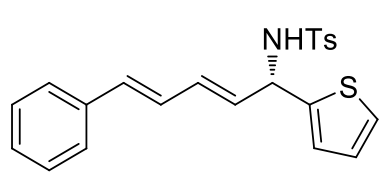

4-Methyl- $N$-((S,2E,4E)-5-phenyl-1-(thiophen-2-yl)penta-2,4-dien-1-yl) benzenesulfonamidesynthesis (3ab): An oven-dried $5 \mathrm{~mL}$ test-tube equipped with a septum and a magnetic stir bar was charged with $\operatorname{Pd}_{2}(\mathrm{dba})_{3}$ (4.6 mg, $5 \mathrm{~mol} \%$ ) and ligand $\mathbf{L 1}(11.3 \mathrm{mg}, 20 \mathrm{~mol} \%$ ). The tube was then evacuated and filled with 
argon. This cycle was repeated three times, and toluene $(0.5 \mathrm{~mL})$ was added via syringe. The resulting solution was stirred at room temperature for $30 \mathrm{~min}$. A second oven-dried test-tube was charged with diene $1 \mathbf{a}$ (26.0 mg, 2.0 equiv), $N$-sulfonylimine $\mathbf{2 p}$ (26.5 mg, 1.0 equiv), 1-naphthoic acid (5.2 $\mathrm{mg}$, $30 \mathrm{~mol} \%)$ and $4 \AA \mathrm{MS}(60.0 \mathrm{mg})$, and the system was placed under an atmosphere of argon (balloon). Then the catalyst solution was added at room temperature. The resulting mixture was stirred at $80{ }^{\circ} \mathrm{C}$ for $30 \mathrm{~h}$. After completion, purification by flash chromatography on silica gel (petroleum ether/EtOAc $=10 / 1)$ gave the product 3ab: $32.8 \mathrm{mg}$, faint yellow oil, $83 \%$ yield; $E / Z=11: 1 ;[\alpha]^{25} \mathrm{D}=+16.6(c=$ 0.35, in $\mathrm{CHCl}_{3}$ ); 94\% ee, determined by HPLC analysis [Chiralpak column IB, $i \mathrm{PrOH} / n \mathrm{Hexane}=$ 40/60, flow rate: $1.0 \mathrm{~mL} / \mathrm{min}, 254 \mathrm{~nm}, \mathrm{t}$ (minor) $=7.23 \mathrm{~min}, \mathrm{t}$ (major) $=9.69 \mathrm{~min}] ;{ }^{1} \mathbf{H} \mathbf{N M R}(400$ $\left.\mathrm{MHz}, \mathrm{CDCl}_{3}\right): \delta(\mathrm{ppm}) 7.70(\mathrm{~d}, J=8.4 \mathrm{~Hz}, 2 \mathrm{H}), 7.36-7.17(\mathrm{~m}, 8 \mathrm{H}), 6.89-6.86(\mathrm{~m}, 1 \mathrm{H}), 6.82(\mathrm{~d}, J=$ $3.6 \mathrm{~Hz}, 1 \mathrm{H}), 6.62(\mathrm{dd}, J=15.6,10.4 \mathrm{~Hz}, 1 \mathrm{H}), 6.46(\mathrm{~d}, J=16.0 \mathrm{~Hz}, 1 \mathrm{H}), 6.24$ (dd, $J=15.2,10.4 \mathrm{~Hz}$, 1H), $5.74(\mathrm{dd}, J=15.2,6.8 \mathrm{~Hz}, 1 \mathrm{H}), 5.31-5.27(\mathrm{~m}, 1 \mathrm{H}), 5.00-4.98(\mathrm{~m}, 1 \mathrm{H}), 2.36(\mathrm{~s}, 3 \mathrm{H})$; ${ }^{13} \mathbf{C} \mathbf{N M R}$ (100 MHz, $\left.\mathrm{CDCl}_{3}\right): \delta$ (ppm) 143.6, 143.4, 137.6, 136.8, 134.1, 132.9, 131.0, 129.5, 128.6, 127.9, 127.3, 127.2, 126.9, 126.4, 125.5, 125.4, 55.2, 21.5; HRMS (ESI-TOF) m/z: $[\mathrm{M}+\mathrm{Na}]^{+}$Calcd for $\mathrm{C}_{22} \mathrm{H}_{21} \mathrm{NO}_{2} \mathrm{~S}_{2} \mathrm{Na}$ 418.0906; Found 418.0909.

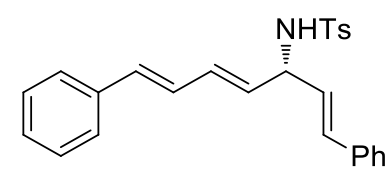

$N$-((S,1E,4E,6E)-1,7-Diphenylhepta-1,4,6-trien-3-yl)-4-methylbenzene sulfonamide (3ac): An oven-dried $5 \mathrm{~mL}$ test-tube equipped with a septum and a magnetic stir bar was charged with $\mathrm{Pd}_{2}(\mathrm{dba})_{3}(4.6 \mathrm{mg}, 5 \mathrm{~mol} \%)$ and ligand L1 (11.3 mg, $20 \mathrm{~mol} \%)$. The tube was then evacuated and filled with argon. This cycle was repeated three times, and toluene $(0.5 \mathrm{~mL})$ was added via syringe. The resulting solution was stirred at room temperature for $30 \mathrm{~min}$. A second oven-dried test-tube was charged with diene $\mathbf{1 a}(26.0 \mathrm{mg}$, 2.0 equiv), $N$-sulfonylimine $N$-sulfonylimine $\mathbf{2 q}$ (28.5 mg, 1.0 equiv), 1-naphthoic acid (5.2 mg, 30 mol \%) and $4 \AA$ MS (60.0 mg), and the system was placed under an atmosphere of argon (balloon). Then the catalyst solution was added at room temperature. The resulting mixture was stirred at $80{ }^{\circ} \mathrm{C}$ for $30 \mathrm{~h}$. After completion, purification by flash chromatography on silica gel (petroleum ether/EtOAc $=10 / 1$ ) gave the product 3ac: $30.0 \mathrm{mg}$, as a white solid, $72 \%$ yield; $E / Z=12: 1 ; \mathrm{mp}=136-138{ }^{\circ} \mathrm{C}$; $[\alpha]^{25}{ }_{\mathrm{D}}=+14.8\left(c=0.85\right.$, in $\left.\mathrm{CHCl}_{3}\right) ; 88 \%$ ee, determined by HPLC analysis [Chiralpak column IB, $i \mathrm{PrOH} / n$ Hexane $=40 / 60$, flow rate: $1.0 \mathrm{~mL} / \mathrm{min}, 254 \mathrm{~nm}, \mathrm{t}$ (minor) $=11.18 \mathrm{~min}, \mathrm{t}$ (major) $=20.04$ min]; ${ }^{1} \mathrm{H}$ NMR (400 MHz, $\left.\mathrm{CDCl}_{3}\right): \delta(\mathrm{ppm}) 7.75(\mathrm{~d}, J=8.0 \mathrm{~Hz}, 2 \mathrm{H}), 7.35-7.15(\mathrm{~m}, 12 \mathrm{H}), 6.62(\mathrm{dd}$, $J=15.6,10.4 \mathrm{~Hz}, 1 \mathrm{H}), 6.46(\mathrm{~d}, J=15.6 \mathrm{~Hz}, 1 \mathrm{H}), 6.36(\mathrm{~d}, J=16.0 \mathrm{~Hz}, 1 \mathrm{H}), 6.23(\mathrm{dd}, J=15.2,10.4$ $\mathrm{Hz}, 1 \mathrm{H}), 5.91(\mathrm{dd}, J=16.0,6.8 \mathrm{~Hz}, 1 \mathrm{H}), 5.63(\mathrm{dd}, J=15.2,6.4 \mathrm{~Hz}, 1 \mathrm{H}), 4.88(\mathrm{~d}, J=7.5 \mathrm{~Hz}, 1 \mathrm{H})$, 4.67-4.63 (m, 1H), 2.30 (s, 3H). $\left.{ }^{13} \mathrm{C} \mathrm{NMR} \mathrm{(100} \mathrm{MHz,} \mathrm{CDCl}_{3}\right): \delta$ (ppm) 143.4, 137.9, 136.9, 136.1, 133.6, 132.5, 132.1, 131.0, 129.5, 128.6, 128.5, 127.9, 127.8, 127.5, 127.4, 127.1, 126.5, 126.4, 57.5, 21.4; HRMS (ESI-TOF) m/z: [M + Na] ${ }^{+}$Calcd for $\mathrm{C}_{26} \mathrm{H}_{25} \mathrm{NO}_{2} \mathrm{SNa} 438.1498$; Found 438.1500. 


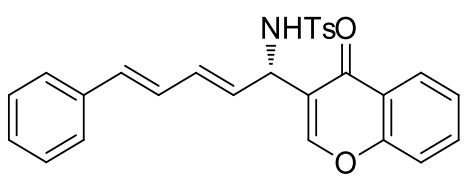

4-Methyl- $N$-((S,2E,4E)-1-(4-oxo-4H-chromen-3-yl)-5-phenyl penta-2,4-dien-1-yl)benzenesulfonamide (3ad): An oven-dried 5 $\mathrm{mL}$ test-tube equipped with a septum and a magnetic stir bar was charged with $\mathrm{Pd}_{2}(\mathrm{dba})_{3}(4.6 \mathrm{mg}, 5 \mathrm{~mol} \%)$ and ligand $\mathbf{L 1}(11.3 \mathrm{mg}, 20 \mathrm{~mol} \%)$. The tube was then evacuated and filled with argon. This cycle was repeated three times, and toluene $(0.5 \mathrm{~mL})$ was added via syringe. The resulting solution was stirred at room temperature for $30 \mathrm{~min}$. A second oven-dried test-tube was charged with diene $1 \mathbf{a}$ ( $26.0 \mathrm{mg}, 2.0$ equiv), $N$-sulfonylimine $N$-sulfonylimine $2 \mathbf{r}$ ( 32.7 $\mathrm{mg}, 1.0$ equiv), 1-naphthoic acid (5.2 mg, $30 \mathrm{~mol} \%)$ and $4 \AA \mathrm{MS}(60.0 \mathrm{mg})$, and the system was placed under an atmosphere of argon (balloon). Then the catalyst solution was added at room temperature. The resulting mixture was stirred at $80^{\circ} \mathrm{C}$ for $30 \mathrm{~h}$. After completion, purification by flash chromatography on silica gel (petroleum ether/EtOAc $=10 / 1$ ) gave the product 3ad: $43.2 \mathrm{mg}$, white semisolid, $94 \%$ yield; $E / Z=12: 1 ;[\alpha]^{25} \mathrm{D}=+27.5\left(c=1.44\right.$, in $\left.\mathrm{CHCl}_{3}\right) ; 94 \%$ ee, determined by HPLC analysis [Chiralpak column AD-H, $i \mathrm{PrOH} / n \mathrm{Hexane}=40 / 60$, flow rate: $1.0 \mathrm{~mL} / \mathrm{min}, 254 \mathrm{~nm}$, $\mathrm{t}($ minor $)=30.79 \mathrm{~min}, \mathrm{t}$ (major) $=53.51 \mathrm{~min}] ;{ }^{1} \mathbf{H} \mathbf{~ N M R}\left(400 \mathrm{MHz}, \mathrm{CDCl}_{3}\right): \delta(\mathrm{ppm}) 8.06(\mathrm{dd}, J=$ 8.4, $1.2 \mathrm{~Hz}, 1 \mathrm{H}), 7.73(\mathrm{~s}, 1 \mathrm{H}), 7.67-7.60(\mathrm{~m}, 3 \mathrm{H}), 7.39-7.18(\mathrm{~m}, 7 \mathrm{H}), 6.99(\mathrm{~d}, J=8.0 \mathrm{~Hz}, 2 \mathrm{H}), 6.60$ (dd, $J=15.6,10.4 \mathrm{~Hz}, 1 \mathrm{H}), 6.44(\mathrm{~d}, J=15.6 \mathrm{~Hz}, 1 \mathrm{H}), 6.25(\mathrm{dd}, J=15.2,10.4 \mathrm{~Hz}, 2 \mathrm{H}), 5.93(\mathrm{dd}, J=$ $15.2,6.4 \mathrm{~Hz}, 1 \mathrm{H}), 4.85-4.81(\mathrm{~m}, 1 \mathrm{H}), 2.06(\mathrm{~s}, 3 \mathrm{H}) ;{ }^{13} \mathbf{C} \mathbf{~ N M R}\left(100 \mathrm{MHz}, \mathrm{CDCl}_{3}\right): \delta(\mathrm{ppm}) 176.7$, 155.9, 153.0, 143.0, 137.7, 136.8, 133.9, 133.8, 132.5, 130.2, 129.2, 128.5, 127.7, 127.4, 127.2, 126.4, 125.6, 125.3, 123.9, 121.6, 117.9, 55.4, 21.1; HRMS (ESI-TOF) $\mathrm{m} / \mathrm{z}:[\mathrm{M}+\mathrm{Na}]^{+}$Calcd for $\mathrm{C}_{27} \mathrm{H}_{23} \mathrm{NO}_{4} \mathrm{SNa} 480.1240$; Found 480.1243 .

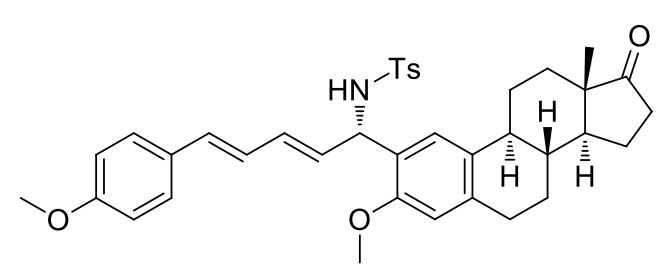

$N-((S, 2 E, 4 E)-1-((8 R, 9 S, 13 S, 14 S)-3-M e t h o x y-13-m e t h y l-$ 17-oxo-7,8,9,11,12,13,14,15,16,17-decahydro-6Hcyclopenta $[a]$ phenanthren-2-yl)-5-(4-methoxyphenyl) penta-2,4-dien-1-yl)-4-methylbenzenesulfonamide (3ae):

An oven-dried $5 \mathrm{~mL}$ test-tube equipped with a septum and a magnetic stir bar was charged with $\operatorname{Pd}_{2}(\mathrm{dba})_{3}(4.6 \mathrm{mg}, 5 \mathrm{~mol} \%)$ and ligand $\mathbf{L 1}(11.3 \mathrm{mg}, 20 \mathrm{~mol} \%)$. The tube was then evacuated and filled with argon. This cycle was repeated three times, and toluene $(0.5 \mathrm{~mL})$ was added via syringe. The resulting solution was stirred at room temperature for $30 \mathrm{~min}$. A second oven-dried test-tube was charged with diene 1 e (32.0 mg, 2.0 equiv), $N$-sulfonylimine $2 \mathrm{~s}$ (46.6 mg, 1.0 equiv), 1-naphthoic acid (5.2 mg, $30 \mathrm{~mol} \%)$ and $4 \AA$ MS (60.0 mg), and the system was placed under an atmosphere of argon (balloon). Then the catalyst solution was added at room temperature. The resulting mixture was stirred at $80{ }^{\circ} \mathrm{C}$ for $30 \mathrm{~h}$. After completion, purification by flash chromatography on silica gel (petroleum ether $/ \mathrm{EtOAc}=10 / 1$ ) gave the product 3ae: $36.2 \mathrm{mg}$, as a white solid, 59\% yield; $\mathrm{mp}=64-66{ }^{\circ} \mathrm{C} ;[\alpha]^{25} \mathrm{D}=+36.0\left(c=0.3\right.$, in $\left.\mathrm{CHCl}_{3}\right) ; 10: 1$ d.r.; ${ }^{1} \mathrm{H}$ NMR (400 MHz, $\left.\mathrm{CDCl}_{3}\right): \delta(\mathrm{ppm}) 7.54(\mathrm{~d}, J=8.4 \mathrm{~Hz}, 2 \mathrm{H}), 7.27-7.25(\mathrm{~m}, 2 \mathrm{H}), 7.07(\mathrm{~d}, J=7.6 \mathrm{~Hz}, 2 \mathrm{H})$, 
6.83-6.81 (m, 3H), $6.48(\mathrm{dd}, J=15.6,10.4 \mathrm{~Hz}, 1 \mathrm{H}), 6.41-6.34(\mathrm{~m}, 2 \mathrm{H}), 6.11(\mathrm{dd}, J=14.8,10.4 \mathrm{~Hz}$, 1H), 5.80 (dd, $J=15.6,6.4 \mathrm{~Hz}, 1 \mathrm{H}), 5.60-5.57$ (m, 1H), 4.99 (t, $J=8.0,1 \mathrm{H}), 3.79$ (s, 3H), 3.70 (s, $3 \mathrm{H}), 2.84-2.80(\mathrm{~m}, 2 \mathrm{H}), 2.50(\mathrm{dd}, J=18.4,8.4 \mathrm{~Hz}, 1 \mathrm{H}), 2.32(\mathrm{~s}, 3 \mathrm{H}), 2.19-1.93(\mathrm{~m}, 5 \mathrm{H}), 1.51-1.35$ (m, 7H), $0.88(\mathrm{~s}, 3 \mathrm{H}) ;{ }^{13} \mathrm{C} \mathrm{NMR}\left(100 \mathrm{MHz}, \mathrm{CDCl}_{3}\right): \delta$ (ppm) 220.8, 159.2, 154.2, 142.5, 137.9, 137.2, $132.3,131.8,131.7,131.1,129.9,128.9,127.5,127.2,126.2,126.0,124.7,114.0,111.3,58.6,55.3$, 50.3, 48.0, 43.9, 38.2 35.8, 31.5, 29.7, 29.6, 26.6, 25.9, 21.5, 21.5, 13.8; HRMS (ESI-TOF) m/z: [M $+\mathrm{Na}]^{+}$Calcd for $\mathrm{C}_{38} \mathrm{H}_{43} \mathrm{NO}_{5} \mathrm{SNa}$ 648.2754; Found 648.2753.

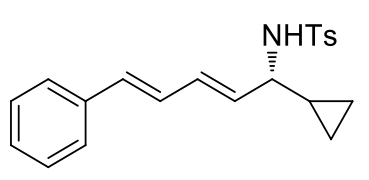

$N$-((R,2E, $\boldsymbol{E} E)-1-C y c l o p r o p y l-5-p h e n y l p e n t a-2,4-d i e n-1-y l)-4-m e t h y l$
benzenesulfonamide (3af): An oven-dried $5 \mathrm{~mL}$ test-tube equipped with a
septum and a magnetic stir bar was charged with $\mathrm{Pd}_{2}(\mathrm{dba})_{3}(4.6 \mathrm{mg}, 5 \mathrm{~mol} \%)$ and ligand $\mathbf{L 1}$ (11.3 mg, $20 \mathrm{~mol} \%$ ). The tube was then evacuated and filled with argon. This cycle was repeated three times, and toluene $(0.5 \mathrm{~mL})$ was added via syringe. The resulting solution was stirred at room temperature for $30 \mathrm{~min}$. A second oven-dried test-tube was charged with diene 1a (26.0 mg, 2.0 equiv), $N$-sulfonylimine $2 t$ (22.3 mg, 1.0 equiv), 1-naphthoic acid (5.2 mg, $30 \mathrm{~mol} \%$ ) and $4 \AA$ MS (60.0 mg), and the system was placed under an atmosphere of argon (balloon). Then the catalyst solution was added at room temperature. The resulting mixture was stirred at $80^{\circ} \mathrm{C}$ for $30 \mathrm{~h}$. After completion, purification by flash chromatography on silica gel (petroleum ether/EtOAc $=10 / 1$ ) gave the product 3af: $10.4 \mathrm{mg}$, colorless oil, $29 \%$ yield; $E / Z=12: 1 ;[\alpha]^{25} \mathrm{D}=+2.4\left(c=0.9\right.$, in $\left.\mathrm{CHCl}_{3}\right)$; $73 \%$ ee, determined by HPLC analysis [Chiralpak column AD-H, $i \mathrm{PrOH} / n$ Hexane $=10 / 90$, flow rate: $1.0 \mathrm{~mL} / \mathrm{min}, 254 \mathrm{~nm}, \mathrm{t}$ (major) $=24.63 \mathrm{~min}, \mathrm{t}$ (minor) $=29.86 \mathrm{~min}] ;{ }^{1} \mathbf{H} \mathbf{~ N M R}\left(400 \mathrm{MHz}, \mathrm{CDCl}_{3}\right): \delta$ (ppm) $7.74(\mathrm{~d}, J=8.0 \mathrm{~Hz}, 2 \mathrm{H}), 7.36-7.23(\mathrm{~m}, 7 \mathrm{H}), 6.57(\mathrm{dd}, J=15.6,10.4 \mathrm{~Hz}, 1 \mathrm{H}), 6.43(\mathrm{~d}, J=15.6$ $\mathrm{Hz}, 1 \mathrm{H}), 6.16(\mathrm{dd}, J=15.2,10.4 \mathrm{~Hz}, 1 \mathrm{H}), 5.50(\mathrm{dd}, J=15.2,6.8 \mathrm{~Hz}, 1 \mathrm{H}), 4.62(\mathrm{~d}, J=6.4 \mathrm{~Hz}, 1 \mathrm{H})$, 3.32-3.27 (m, 1H), 2.39 (s, 3H), 0.92-0.88 (m, 1H), 0.53-0.44 (m, 2H), 0.28-0.15 (m, 2H); ${ }^{13}$ C NMR $\left(150 \mathrm{MHz}, \mathrm{CDCl}_{3}\right): \delta$ (ppm) 143.3, 138.1, 137.0, 133.0, 131.9, 131. 8, 129.5, 128.6, 127.8, 127.7, 127.3, 126.3, 60.0, 21.5, 16.4, 3.6, 3.2; HRMS (ESI-TOF) $\mathrm{m} / \mathrm{z}:[\mathrm{M}+\mathrm{Na}]^{+}$Calcd for $\mathrm{C}_{21} \mathrm{H}_{23} \mathrm{NO}_{2} \mathrm{SNa}$ 376.1342; Found 376.1339.

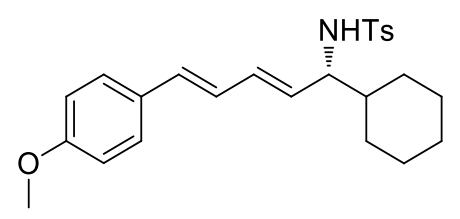

$N$-((R,2E,4E)-1-Cyclohexyl-5-(4-methoxyphenyl)penta-2,4-dien-1yl)-4-methylbenzenesulfonamide (3ag): An oven-dried $5 \mathrm{~mL}$ test-tube equipped with a septum and a magnetic stir bar was charged with $\mathrm{Pd}_{2}(\mathrm{dba})_{3}(4.6 \mathrm{mg}, 5 \mathrm{~mol} \%$ ) and ligand $\mathbf{L 1}(11.3 \mathrm{mg}, 20 \mathrm{~mol} \%)$. The tube was then evacuated and filled with argon. This cycle was repeated three times, and toluene $(0.5$ $\mathrm{mL}$ ) was added via syringe. The resulting solution was stirred at room temperature for $30 \mathrm{~min}$. A second oven-dried test-tube was charged with diene $\mathbf{1 e}(32.0 \mathrm{mg}, 2.0$ equiv), $N$-sulfonylimine $\mathbf{2 u}$ (26.5 mg, 1.0 equiv), 1-naphthoic acid (5.2 mg, $30 \mathrm{~mol} \%$ ) and $4 \AA \mathrm{MS}$ (60.0 mg), and the system 
was placed under an atmosphere of argon (balloon). Then the catalyst solution was added at room temperature. The resulting mixture was stirred at $80{ }^{\circ} \mathrm{C}$ for $30 \mathrm{~h}$. After completion, purification by flash chromatography on silica gel (petroleum ether/EtOAc $=10 / 1$ ) gave the product 3ag: Colorless semisolid, $39.1 \mathrm{mg}, 92 \%$ yield; $E / Z>19: 1 ;[\alpha]^{25} \mathrm{D}=+39.6\left(c=1.69\right.$, in $\left.\mathrm{CHCl}_{3}\right) ; 74 \%$ ee, determined by HPLC analysis [Chiralpak column IB, $i \mathrm{PrOH} / n$ Hexane $=10 / 90$, flow rate: $1.0 \mathrm{~mL} / \mathrm{min}, 254 \mathrm{~nm}, \mathrm{t}$ $($ minor $)=13.47$ min, $\mathrm{t}($ major $)=14.80 \mathrm{~min}] ;{ }^{1} \mathbf{H}$ NMR $\left(400 \mathrm{MHz}, \mathrm{CDCl}_{3}\right): \delta(\mathrm{ppm}) 7.72(\mathrm{~d}, J=8.4$ $\mathrm{Hz}, 2 \mathrm{H}), 7.26-7.22(\mathrm{~m}, 4 \mathrm{H}), 6.84(\mathrm{~d}, J=8.8 \mathrm{~Hz}, 2 \mathrm{H}), 6.35(\mathrm{dd}, J=15.6,10.0 \mathrm{~Hz}, 1 \mathrm{H}), 6.24(\mathrm{~d}, J=$ $15.6 \mathrm{~Hz}, 1 \mathrm{H}), 5.81(\mathrm{dd}, J=14.8,10.0 \mathrm{~Hz}, 1 \mathrm{H}), 5.30$ (dd, $J=15.2,8.0 \mathrm{~Hz}, 1 \mathrm{H}), 4.79$ (d, $J=8.4 \mathrm{~Hz}$, $1 \mathrm{H}), 3.80(\mathrm{~s}, 3 \mathrm{H}), 3.64-3.59(\mathrm{~m}, 1 \mathrm{H}), 2.32(\mathrm{~s}, 3 \mathrm{H}), 1.78-1.59(\mathrm{~m}, 5 \mathrm{H}), 1.41-1.38(\mathrm{~m}, 1 \mathrm{H}), 1.19-1.07$ (m, 3H), 1.00-0.91 (m, 2H); $\left.{ }^{13} \mathbf{C ~ N M R ~ ( 1 0 0 ~ M H z , ~} \mathrm{CDCl}_{3}\right): \delta(\mathrm{ppm}) 159.2,143.1,138.1,132.6,132.1$, 130.0, 129.8, 129.4, 127.4, 127.3, 125.8, 114.0, 61.1, 55.3, 42.9, 29.0, 28.9, 26.2, 26.0, 25.9, 21.4; HRMS (ESI-TOF) m/z: [M + Na $]^{+}$Calcd for $\mathrm{C}_{25} \mathrm{H}_{31} \mathrm{NO}_{3} \mathrm{SNa} 448.1917$; Found 448.1913.

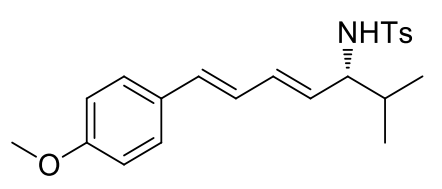

\section{$N$-((R,4E,6E)-7-(4-Methoxyphenyl)-2-methylhepta-4,6-dien-3-yl)-4-} methylbenzenesulfonamide (3ah): An oven-dried $5 \mathrm{~mL}$ test-tube equipped with a septum and a magnetic stir bar was charged with $\mathrm{Pd}_{2}(\mathrm{dba})_{3}$ (4.6 mg, $5 \mathrm{~mol} \%$ ) and ligand $\mathbf{L 1}(11.3 \mathrm{mg}, 20 \mathrm{~mol} \%$ ). The tube was then evacuated and filled with argon. This cycle was repeated three times, and toluene $(0.5 \mathrm{~mL})$ was added via syringe. The resulting solution was stirred at room temperature for $30 \mathrm{~min}$. A second oven-dried test-tube was charged with diene $\mathbf{1 e}(32.0 \mathrm{mg}, 2.0$ equiv), $N$-sulfonylimine $\mathbf{2 v}$ (22.5 mg, 1.0 equiv), 1-naphthoic acid (5.2 mg, $30 \mathrm{~mol} \%$ ) and $4 \AA$ MS (60.0 mg), and the system was placed under an atmosphere of argon (balloon). Then the catalyst solution was added at room temperature. The resulting mixture was stirred at $80{ }^{\circ} \mathrm{C}$ for $30 \mathrm{~h}$. After completion, purification by flash chromatography on silica gel (petroleum ether/EtOAc $=10 / 1$ ) gave the product 3ah: $36.2 \mathrm{mg}$, as a white solid, 94\% yield; $E / Z>19: 1 ; \mathrm{mp}=102-104{ }^{\circ} \mathrm{C} ;[\alpha]^{25}{ }_{\mathrm{D}}=+40.8\left(c=1.6\right.$, in $\left.\mathrm{CHCl}_{3}\right) ; 50 \%$ ee, determined by HPLC analysis [Chiralpak column IB, $i \mathrm{PrOH} / n$ Hexane $=10 / 90$, flow rate: $1.0 \mathrm{~mL} / \mathrm{min}, 254 \mathrm{~nm}, \mathrm{t}($ minor $)=$ $13.69 \mathrm{~min}, \mathrm{t}$ (major) = $16.30 \mathrm{~min}] ;{ }^{1} \mathbf{H}$ NMR $\left(400 \mathrm{MHz}, \mathrm{CDCl}_{3}\right): \delta(\mathrm{ppm}) 7.73(\mathrm{~d}, J=8.0 \mathrm{~Hz}, 2 \mathrm{H})$, $7.27-7.23(\mathrm{~m}, 4 \mathrm{H}), 6.84(\mathrm{~d}, J=8.4 \mathrm{~Hz}, 2 \mathrm{H}), 6.36(\mathrm{dd}, J=15.6,10.0 \mathrm{~Hz}, 1 \mathrm{H}), 6.25$ (d, $J=15.6 \mathrm{~Hz}$, $1 \mathrm{H}), 5.86(\mathrm{dd}, J=15.2,10.4 \mathrm{~Hz}, 1 \mathrm{H}), 5.32(\mathrm{dd}, J=15.2,7.6 \mathrm{~Hz}, 1 \mathrm{H}), 4.83(\mathrm{~d}, J=8.0 \mathrm{~Hz}, 1 \mathrm{H}), 3.80$ (s, 3H), 3.65-3.60 (m, 1H), 2.33 (s, 3H), 1.79-1.67 (m, 1H), 0.89-0.84 (m, 6H); ${ }^{13}$ C NMR (100 MHz, $\mathrm{CDCl}_{3}$ ): $\delta$ (ppm) 159.2, 143.1, 138.0, 132.8, 132.2, 129.8, 129.6, 129.4, 127.4, 127.3, 125.8, 114.0, 61.6, 55.3, 33.1, 21.4, 18.4, 18.2; HRMS (ESI-TOF) m/z: $[\mathrm{M}+\mathrm{Na}]^{+}$Calcd for $\mathrm{C}_{22} \mathrm{H}_{27} \mathrm{NO}_{3} \mathrm{SNa}$ 408.1604; Found 408.1607. 


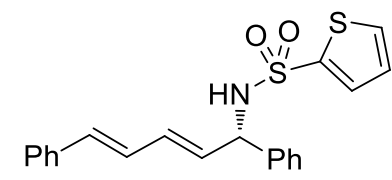

$N-((S, 2 E, 4 E)-1,5-D i p h e n y l p e n t a-2,4-d i e n-1-y l)$ thiophene-2-

sulfonamide (3ai): An oven-dried $5 \mathrm{~mL}$ test-tube equipped with a septum and a magnetic stir bar was charged with $\mathrm{Pd}_{2}(\mathrm{dba})_{3}(4.6 \mathrm{mg}, 5 \mathrm{~mol} \%)$ and ligand L1 (11.3 mg, $20 \mathrm{~mol} \%)$. The tube was then evacuated and filled with argon. This cycle was repeated three times, and toluene $(0.5 \mathrm{~mL})$ was added via syringe. The resulting solution was stirred at room temperature for $30 \mathrm{~min}$. A second oven-dried test-tube was charged with diene $\mathbf{1 a}(26.0 \mathrm{mg}$, 2.0 equiv), $N$-sulfonylimine $2 \mathbf{w}$ ( $25.1 \mathrm{mg}, 1.0$ equiv), 1 -naphthoic acid (5.2 mg, $30 \mathrm{~mol} \%$ ) and $4 \AA$ MS $(60.0 \mathrm{mg})$, and the system was placed under an atmosphere of argon (balloon). Then the catalyst solution was added at room temperature. The resulting mixture was stirred at $80{ }^{\circ} \mathrm{C}$ for $30 \mathrm{~h}$. After completion, purification by flash chromatography on silica gel (petroleum ether/EtOAc $=10 / 1)$ gave the product 3ai: $36.4 \mathrm{mg}$, as a white semisolid, $96 \%$ yield; $E / Z=13: 1 ; \alpha]^{25} \mathrm{D}=+22.2(c=1.8$, in $\mathrm{CHCl}_{3}$ ); 95\% ee, determined by HPLC analysis [Chiralpak column IB, $i \mathrm{PrOH} / n \mathrm{Hexane}=40 / 60$, flow rate: $1.0 \mathrm{~mL} / \mathrm{min}, 254 \mathrm{~nm}, \mathrm{t}$ (minor) $=6.80 \mathrm{~min}, \mathrm{t}$ (major) $=9.38 \mathrm{~min}] ;{ }^{1} \mathrm{H} \mathrm{NMR}\left(400 \mathrm{MHz}, \mathrm{CDCl}_{3}\right)$ : $\delta(\mathrm{ppm}) 7.49-7.47(\mathrm{~m}, 2 \mathrm{H}), 7.37-7.17(\mathrm{~m}, 10 \mathrm{H}), 6.94(\mathrm{t}, J=4.0 \mathrm{~Hz}, 1 \mathrm{H}), 6.65(\mathrm{dd}, J=15.6,10.4 \mathrm{~Hz}$, $1 \mathrm{H}), 6.46(\mathrm{~d}, J=15.6 \mathrm{~Hz}, 1 \mathrm{H}), 6.22(\mathrm{dd}, J=15.2,10.4 \mathrm{~Hz}, 1 \mathrm{H}), 5.79(\mathrm{dd}, J=15.2,6.4 \mathrm{~Hz}, 1 \mathrm{H}), 5.26$ $(\mathrm{d}, J=7.2 \mathrm{~Hz}, 1 \mathrm{H}), 5.12(\mathrm{t}, J=6.8 \mathrm{~Hz}, 1 \mathrm{H}) ;{ }^{13} \mathbf{C} \mathbf{N M R}\left(100 \mathrm{MHz}, \mathrm{CDCl}_{3}\right): \delta(\mathrm{ppm}) 141.6,139.3$, 136.8, 133.7, 132.6, 132.5, 131.9, 131.7, 128.7, 128.6, 127.9, 127.8, 127.4, 127.1, 127.0, 126.4, 59.8; HRMS (ESI-TOF) m/z: [M + Na] ${ }^{+}$Calcd for $\mathrm{C}_{21} \mathrm{H}_{19} \mathrm{NO}_{2} \mathrm{~S}_{2} \mathrm{Na}$ 404.0749; Found 404.0750.

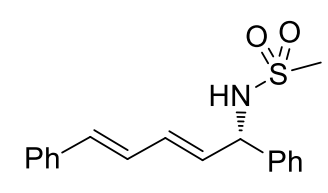

$N$-((S,2E,4E)-1,5-Diphenylpenta-2,4-dien-1-yl)methanesulfonamide (3aj): An oven-dried $5 \mathrm{~mL}$ test-tube equipped with a septum and a magnetic stir bar was charged with $\mathrm{Pd}_{2}(\mathrm{dba})_{3}(4.6 \mathrm{mg}, 5 \mathrm{~mol} \%)$ and ligand $\mathbf{L 1}$ (11.3 mg, 20 mol \%). The tube was then evacuated and filled with argon. This cycle was repeated three times, and toluene $(0.5 \mathrm{~mL})$ was added via syringe. The resulting solution was stirred at room temperature for $30 \mathrm{~min}$. A second oven-dried test-tube was charged with diene $\mathbf{1 a}$ (26.0 mg, 2.0 equiv), $\mathrm{N}$ sulfonylimine $2 \times 18.3 \mathrm{mg}, 1.0$ equiv), 1-naphthoic acid (5.2 mg, $30 \mathrm{~mol} \%)$ and $4 \AA \mathrm{MS}$ (60.0 mg), and the system was placed under an atmosphere of argon (balloon). Then the catalyst solution was added at room temperature. The resulting mixture was stirred at $80{ }^{\circ} \mathrm{C}$ for $30 \mathrm{~h}$. After completion, purification by flash chromatography on silica gel (petroleum ether/EtOAc $=10 / 1$ ) gave the product 3aj: $30.1 \mathrm{mg}$, as a colorless semisolid, 96\% yield; $E / Z=10: 1 ;[\alpha]^{25} \mathrm{D}=+10.1\left(c=1.5\right.$, in $\left.\mathrm{CHCl}_{3}\right) ; 87 \%$ ee, determined by HPLC analysis [Chiralpak column IB, $i \mathrm{PrOH} / n \mathrm{Hexane}=40 / 60$, flow rate: 1.0 $\mathrm{mL} / \mathrm{min}, 254 \mathrm{~nm}, \mathrm{t}$ (minor) $=7.00 \mathrm{~min}, \mathrm{t}$ (major) $=7.92 \mathrm{~min}] ;{ }^{1} \mathbf{H} \mathbf{N M R}\left(400 \mathrm{MHz}, \mathrm{CDCl}_{3}\right): \delta(\mathrm{ppm})$ 7.36-7.14 (m, 10H), 6.68 (dd, $J=15.6,10.4 \mathrm{~Hz}, 1 \mathrm{H}), 6.49$ (d, $J=15.6 \mathrm{~Hz}, 1 \mathrm{H}), 6.32$ (dd, $J=14.8$, $10.4 \mathrm{~Hz}, 1 \mathrm{H}), 5.85$ (dd, $J=15.2,6.8 \mathrm{~Hz}, 1 \mathrm{H}), 5.13(\mathrm{t}, J=7.2 \mathrm{~Hz}, 2 \mathrm{H}), 4.98$ (d, $J=7.2 \mathrm{~Hz}, 2 \mathrm{H}), 2.67$ $(\mathrm{s}, 3 \mathrm{H}) ;{ }^{13} \mathbf{C}$ NMR $\left(100 \mathrm{MHz}, \mathrm{CDCl}_{3}\right): \delta$ (ppm) 139.8, 136.7, 134.1, 132.8, 132.1, 129.0, 128.6, 128.2, 127.9, 127.3, 127.1, 126.4, 59.5, 42.2; HRMS (ESI-TOF) $\mathrm{m} / \mathrm{z}:[\mathrm{M}+\mathrm{Na}]^{+}$Calcd for $\mathrm{C}_{18} \mathrm{H}_{19} \mathrm{NO}_{2} \mathrm{SNa}$ 


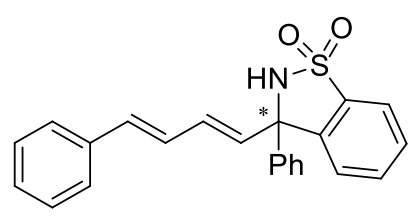

3-Phenyl-3-((1E,3E)-4-phenylbuta-1,3-dien-1-yl)-2,3-dihydrobenzo

[d]isothiazole 1,1-dioxide (3ak): An oven-dried $5 \mathrm{~mL}$ test-tube equipped with a septum and a magnetic stir bar was charged with $\operatorname{Pd}_{2}(\mathrm{dba})_{3}(4.6 \mathrm{mg}$, $5 \mathrm{~mol} \%$ ) and ligand $\mathbf{L 1}(11.3 \mathrm{mg}, 20 \mathrm{~mol} \%)$. The tube was then evacuated and filled with argon. This cycle was repeated three times, and toluene $(0.5 \mathrm{~mL})$ was added via syringe. The resulting solution was stirred at room temperature for $30 \mathrm{~min}$. A second oven-dried test-tube was charged with diene $\mathbf{1 a}$ (26.0 mg, 2.0 equiv), ketimine $\mathbf{2 y}$ (18.3 mg, 1.0 equiv), 1-naphthoic acid (5.2 $\mathrm{mg}, 30 \mathrm{~mol} \%)$ and $4 \AA$ MS (60.0 mg), and the system was placed under an atmosphere of argon (balloon). Then the catalyst solution was added at room temperature. The resulting mixture was stirred at $80{ }^{\circ} \mathrm{C}$ for $30 \mathrm{~h}$. After completion, purification by flash chromatography on silica gel (petroleum ether/EtOAc $=10 / 1$ ) gave the product 3ak: $26.0 \mathrm{mg}$, as a white solid, $70 \%$ yield; $E / Z>19: 1 ; \mathrm{mp}=65-68{ }^{\circ} \mathrm{C} ;[\alpha]^{25} \mathrm{D}=+4.6\left(c=0.7, \mathrm{in}_{\mathrm{CHCl}}\right) ; 55 \%$ ee, determined by HPLC analysis [Chiralpak column IB, $i \mathrm{PrOH} / n \mathrm{Hexane}=40 / 60$, flow rate: $1.0 \mathrm{~mL} / \mathrm{min}, 254 \mathrm{~nm}, \mathrm{t}$ (minor) $=8.45 \mathrm{~min}$, $\mathrm{t}$ (major) = $10.03 \mathrm{~min}] ;{ }^{1} \mathbf{H}$ NMR $\left(400 \mathrm{MHz}, \mathrm{CDCl}_{3}\right): \delta(\mathrm{ppm}) 7.83(\mathrm{~d}, J=7.8 \mathrm{~Hz}, 1 \mathrm{H}), 7.66-7.56(\mathrm{~m}$, 2H), 7.43-7.22 (m, 11H), $6.85(\mathrm{dd}, J=15.6,10.4 \mathrm{~Hz}, 1 \mathrm{H}), 6.75-6.53(\mathrm{~m}, 2 \mathrm{H}), 6.23(\mathrm{~d}, J=14.8 \mathrm{~Hz}$, 1H), 4.85 (s, 1H); ${ }^{13} \mathbf{C}$ NMR (100 MHz, $\left.\mathrm{CDCl}_{3}\right): \delta(\mathrm{ppm}) 142.7,141.7,136.7,135.0,134.9,133.5$, 133.2, 132.4 129.6, 129.0, 128.8, 128.6, 128.0, 127.3, 127.0, 126.5, 125.6, 121.5, 69.6; HRMS (ESITOF) m/z: [M + Na] ${ }^{+}$Calcd for $\mathrm{C}_{23} \mathrm{H}_{19} \mathrm{NO}_{2} \mathrm{SNa}$ 396.1029; Found 396.1026.

2.4 Optimization conditions for the asymmetric cascade vinylogous addition/allylic alkylation of 1,3-dienes with $\mathrm{N}$-sulfonylimines

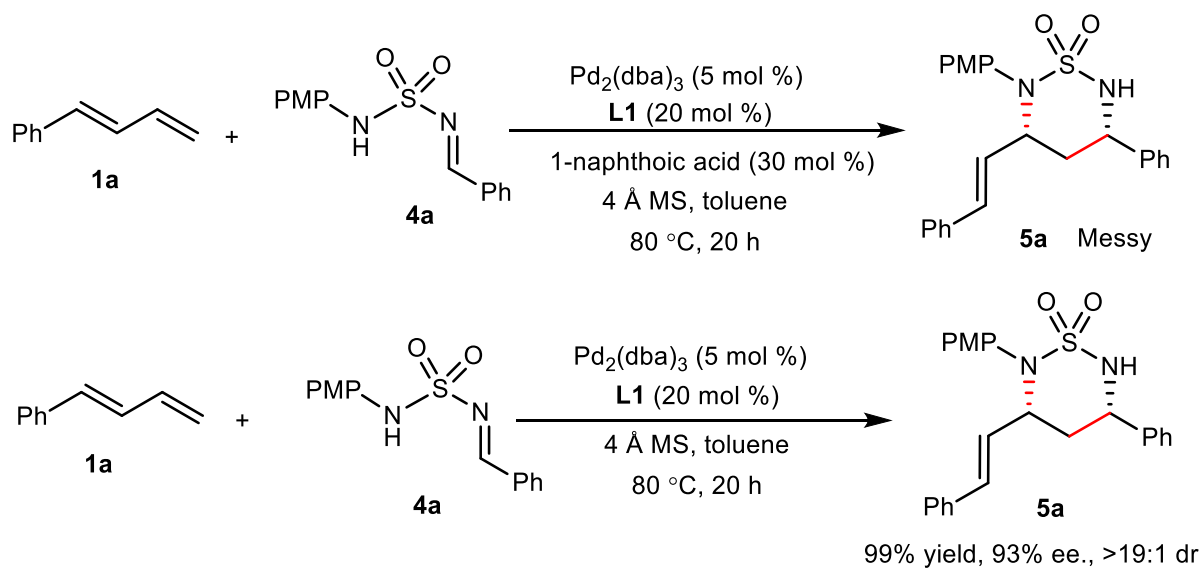

Scheme S1. The effection of acid additive in the cascade reaction

Under the standard conditions of the asymmetric hydrodienylation reaction, it was found the cascade reaction between 1a and $\mathbf{4 a}$ gave a complex mixture, but the single product could be smoothly obtained with excellent stereoselectivity without the acid additive. 
2.5 General procedure of the asymmetric cascade vinylogous addition/allylic alkylation of 1,3dienes 1 with $\mathrm{N}$-sulfonylimines 4

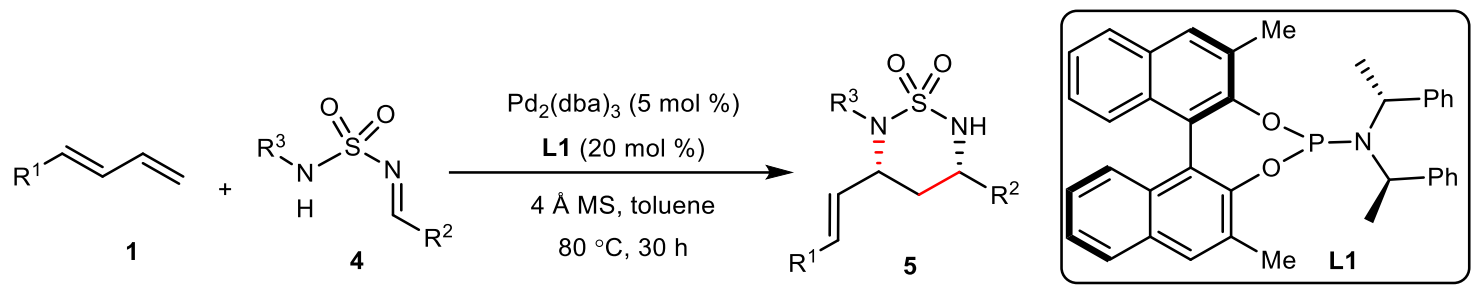

An oven-dried $5 \mathrm{~mL}$ test-tube equipped with a septum and a magnetic stir bar was charged with $\mathrm{Pd}_{2}(\mathrm{dba})_{3}(4.6 \mathrm{mg}, 5 \mathrm{~mol} \%$ ) and ligand $\mathbf{L 1}(11.3 \mathrm{mg}, 20 \mathrm{~mol} \%)$. The tube was then evacuated and filled with argon. This cycle was repeated three times, and toluene $(0.5 \mathrm{~mL})$ was added via syringe. The resulting solution was stirred at room temperature for $30 \mathrm{~min}$. A second oven-dried test-tube was charged with diene 1 (2.0 equiv), functionalized $N$-sulfonylimine 4 (1.0 equiv), and $4 \AA$ MS (60.0 $\mathrm{mg}$ ), and the system was placed under an atmosphere of argon (balloon). Then the catalyst solution was added at room temperature. The resulting mixture was stirred at $80{ }^{\circ} \mathrm{C}$ for $20-60 \mathrm{~h}$. After completion, the product 5 was obtained by flash chromatography on silica gel (petroleum ether/EtOAc $=6 / 1$ to 3/1). The racemic 5 was obtained under the catalysis of $\mathrm{Pd}\left(\mathrm{PPh}_{3}\right)_{4}(5 \mathrm{~mol} \%)$.

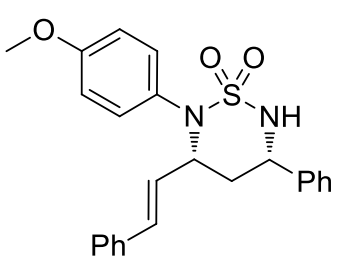

(3R,5S)-2-(4-Methoxyphenyl)-5-phenyl-3-((E)-styryl)-1,2,6-thiadiazinane 1,1-dioxideSynthesis of (5a): An oven-dried $5 \mathrm{~mL}$ test-tube equipped with a septum and a magnetic stir bar was charged with $\mathrm{Pd}_{2}(\mathrm{dba})_{3}(4.6 \mathrm{mg}, 5 \mathrm{~mol} \%)$ and ligand $\mathbf{L 1}(11.3 \mathrm{mg}, 20 \mathrm{~mol} \%)$. The tube was then evacuated and filled with argon. This cycle was repeated three times, and toluene $(0.5 \mathrm{~mL})$ was added via syringe. The resulting solution was stirred at room temperature for $30 \mathrm{~min}$. A second ovendried test-tube was charged with diene 1 (2.0 equiv), functionalized $N$-sulfonylimine $4 \mathbf{a}$ (29.0 mg, 1.0 equiv), and $4 \AA$ MS (60.0 mg), and the system was placed under an atmosphere of argon (balloon). Then the catalyst solution was added at room temperature. The resulting mixture was stirred at $80{ }^{\circ} \mathrm{C}$ for $20 \mathrm{~h}$. After completion, purification by flash chromatography on silica gel (petroleum ether/EtOAc $=6 / 1)$ gave the product 5a: $41.5 \mathrm{mg}$, as a white solid, $99 \%$ yield; $>19: 1 \mathrm{dr} ; \mathrm{mp}=79-82{ }^{\circ} \mathrm{C} ;[\alpha]^{25} \mathrm{D}=$ +69.7 ( $c=0.7$, in $\mathrm{CHCl}_{3}$ ); 93\% ee, determined by HPLC analysis [Chiralpak column AD-H, $i \mathrm{PrOH} / n$ Hexane $=40 / 60$, flow rate: $1.0 \mathrm{~mL} / \mathrm{min}, 254 \mathrm{~nm}, \mathrm{t}($ minor $)=19.48 \mathrm{~min}, \mathrm{t}$ (major) $=25.14$ min]; ${ }^{1} \mathbf{H}$ NMR (400 MHz, $\left.\mathrm{CDCl}_{3}\right): \delta(\mathrm{ppm}) 7.41-7.36$ (m, 7H), 7.26-7.14 (m, 5H), 6.83 (d, $J=8.8$ $\mathrm{Hz}, 2 \mathrm{H}), 6.42(\mathrm{~d}, J=15.6 \mathrm{~Hz}, 1 \mathrm{H}), 5.80(\mathrm{dd}, J=15.6,8.4 \mathrm{~Hz}, 1 \mathrm{H}), 5.05-4.96(\mathrm{~m}, 1 \mathrm{H}), 4.78-4.73(\mathrm{~m}$, $1 \mathrm{H}), 4.48(\mathrm{~d}, J=10.4 \mathrm{~Hz}, 1 \mathrm{H}), 3.75(\mathrm{~s}, 3 \mathrm{H}), 2.33-2.16(\mathrm{~m}, 2 \mathrm{H}) ;{ }^{13} \mathrm{C}$ NMR $\left(100 \mathrm{MHz}, \mathrm{CDCl}_{3}\right): \delta$ (ppm) 159.2, 138.7, 136.0, 133.5, 130.6, 130.5, 129.0, 128.5, 128.0, 127.5, 126.4, 126.2, 114.2, 63.9, 57.9, 55.3, 39.5; HRMS (ESI-TOF) m/z: [M + Na $]^{+}$Calcd forC ${ }_{24} \mathrm{H}_{24} \mathrm{~N}_{2} \mathrm{O}_{3} \mathrm{SNa}$ 443.1400; Found 


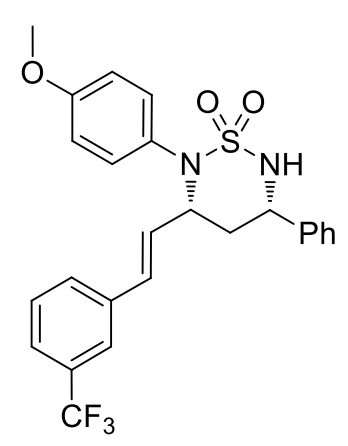

(3R,5S)-2-(4-Methoxyphenyl)-5-phenyl-3-((E)-3-(trifluoromethyl)styryl)1,2,6-thiadiazinane 1,1-dioxide (5b): An oven-dried $5 \mathrm{~mL}$ test-tube equipped with a septum and a magnetic stir bar was charged with $\operatorname{Pd}_{2}(\mathrm{dba})_{3}(4.6 \mathrm{mg}, 5$ mol \%) and ligand $\mathbf{L 1}(11.3 \mathrm{mg}, 20 \mathrm{~mol} \%)$. The tube was then evacuated and filled with argon. This cycle was repeated three times, and toluene $(0.5 \mathrm{~mL})$ was added via syringe. The resulting solution was stirred at room temperature for $30 \mathrm{~min}$. A second oven-dried test-tube was charged with diene 1c (39.6 mg, 2.0 equiv), functionalized $N$-sulfonylimine $4 \mathbf{a}$ (29.0 mg, 1.0 equiv), and $4 \AA \mathrm{MS}$ (60.0 mg), and the system was placed under an atmosphere of argon (balloon). Then the catalyst solution was added at room temperature. The resulting mixture was stirred at $80{ }^{\circ} \mathrm{C}$ for $20 \mathrm{~h}$. After completion, purification by flash chromatography on silica gel (petroleum ether/EtOAc $=6 / 1$ ) gave the product $\mathbf{5 b}: 45.0 \mathrm{mg}$, as a colorless semisolid, $92 \%$ yield; $>19: 1 \mathrm{dr} ;[\alpha]^{25} \mathrm{D}=+63.3\left(c=1.1\right.$, in $\left.\mathrm{CHCl}_{3}\right) ; 90 \%$ ee, determined by HPLC analysis [Chiralpak column AD-H, $i$ PrOH $/ n$ Hexane $=40 / 60$, flow rate: $1.0 \mathrm{~mL} / \mathrm{min}, 254$ $\mathrm{nm}, \mathrm{t}($ minor $)=13.13 \mathrm{~min}, \mathrm{t}($ major $)=21.09 \mathrm{~min}] ;{ }^{1} \mathbf{H} \mathbf{~ N M R}\left(400 \mathrm{MHz}, \mathrm{CDCl}_{3}\right): \delta(\mathrm{ppm}) 7.46-7.30$ $(\mathrm{m}, 11 \mathrm{H}), 6.83(\mathrm{~d}, J=8.8 \mathrm{~Hz}, 2 \mathrm{H}), 6.45(\mathrm{~d}, J=15.6 \mathrm{~Hz}, 1 \mathrm{H}), 5.89(\mathrm{dd}, J=15.6,8.4 \mathrm{~Hz}, 1 \mathrm{H}), 5.03$ (td, $J=11.6,3.2 \mathrm{~Hz}, 1 \mathrm{H}), 4.80-4.75(\mathrm{~m}, 1 \mathrm{H}), 4.63-4.59(\mathrm{~m}, 1 \mathrm{H}), 3.75(\mathrm{~s}, 3 \mathrm{H}), 2.23-2.22(\mathrm{~m}, 2 \mathrm{H})$; ${ }^{13} \mathrm{C}$ NMR (100 MHz, $\left.\mathrm{CDCl}_{3}\right): \delta(\mathrm{ppm})$ 159.3, 138.6, 136.7, 132.2, 130.9 (q, $\left.J=32.1 \mathrm{~Hz}\right), 130.5$, $130.4,129.5,129.4,129.0,128.5,126.2,124.6$ (q, $J=3.8 \mathrm{~Hz}), 123.9$ (q, $J=270.5 \mathrm{~Hz}), 123.1(\mathrm{q}, J=$ $3.8 \mathrm{~Hz}$ ), 114.3, 63.8, 57.9, 55.3, 39.4; HRMS (ESI-TOF) $\mathrm{m} / \mathrm{z}$ : $[\mathrm{M}+\mathrm{Na}]^{+}$Calcd for $\mathrm{C}_{25} \mathrm{H}_{23} \mathrm{~F}_{3} \mathrm{~N}_{2} \mathrm{O}_{3} \mathrm{SNa}$ 511.1274; Found 511.1267.

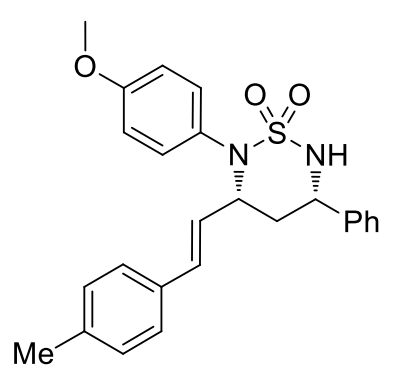

(3R,5S)-2-(4-Methoxyphenyl)-3-((E)-4-methylstyryl)-5-phenyl-1,2,6thiadiazinane 1,1-dioxide (5c): An oven-dried $5 \mathrm{~mL}$ test-tube equipped with a septum and a magnetic stir bar was charged with $\operatorname{Pd}_{2}(\mathrm{dba})_{3}(4.6 \mathrm{mg}$, $5 \mathrm{~mol} \%$ ) and ligand L1 (11.3 mg, $20 \mathrm{~mol} \%$ ). The tube was then evacuated and filled with argon. This cycle was repeated three times, and toluene (0.5 $\mathrm{mL}$ ) was added via syringe. The resulting solution was stirred at room temperature for $30 \mathrm{~min}$. A second oven-dried test-tube was charged with diene 1d (28.8 $\mathrm{mg}, 2.0$ equiv), functionalized $N$-sulfonylimine $\mathbf{4 a}$ (29.0 mg, 1.0 equiv), and $4 \AA$ MS (60.0 mg), and the system was placed under an atmosphere of argon (balloon). Then the catalyst solution was added at room temperature. The resulting mixture was stirred at $80{ }^{\circ} \mathrm{C}$ for $20 \mathrm{~h}$. After completion, purification by flash chromatography on silica gel (petroleum ether/EtOAc $=6 / 1$ ) gave the product 5c: $41.3 \mathrm{mg}$, as a white solid, $95 \%$ yield; $>19: 1 \mathrm{dr} ; \mathrm{mp}=86-88{ }^{\circ} \mathrm{C}$; $[\alpha]^{25} \mathrm{D}=+71.2\left(c=0.84\right.$, in $\left.\mathrm{CHCl}_{3}\right) ; 90 \%$ ee, determined by HPLC analysis [Chiralpak column AD-H, $i \mathrm{PrOH} / n \mathrm{Hexane}=40 / 60$, flow rate: 1.0 
$\mathrm{mL} / \mathrm{min}, 254 \mathrm{~nm}, \mathrm{t}$ (minor) $=16.21 \mathrm{~min}, \mathrm{t}$ (major) $=21.17 \mathrm{~min}] ;{ }^{1} \mathbf{H} \mathbf{N M R}\left(400 \mathrm{MHz}, \mathrm{CDCl}_{3}\right): \delta$ (ppm) 7.40-7.35 (m, 7H), 7.05-7.04 (m, 4H), $6.82(\mathrm{~d}, J=9.2 \mathrm{~Hz}, 2 \mathrm{H}), 6.40(\mathrm{~d}, J=16.0 \mathrm{~Hz}, 1 \mathrm{H})$, $5.76(\mathrm{dd}, J=16.0,8.8 \mathrm{~Hz}, 1 \mathrm{H}), 5.05-4.99(\mathrm{~m}, 1 \mathrm{H}), 4.78-4.72(\mathrm{~m}, 1 \mathrm{H}), 4.63(\mathrm{~d}, J=10.4 \mathrm{~Hz}, 1 \mathrm{H})$, 3.74 (s, 3H), 2.36-2.16 (m, 5H); ${ }^{13} \mathbf{C}$ NMR (100 MHz, $\left.\mathrm{CDCl}_{3}\right): \delta$ (ppm) 159.1, 138.8 137.9, 133.4, 133.2, 130.6, 130.5, 129.2, 128.9, 128.4, 126.4, 126.3, 126.2, 114.1, 64.0, 57.9, 55.2, 39.5, 21.1; HRMS (ESI-TOF) m/z: [M + Na] ${ }^{+}$Calcd for $\mathrm{C}_{25} \mathrm{H}_{26} \mathrm{~N}_{2} \mathrm{O}_{3} \mathrm{SNa} 457.1556$; Found 457.1557.

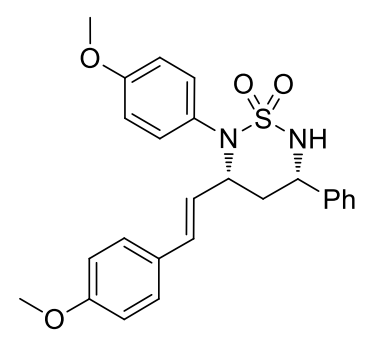

(3R,5S)-2-(4-Methoxyphenyl)-3-((E)-4-methoxystyryl)-5-phenyl-1,2,6thiadiazinane 1,1-dioxide (5d): An oven-dried $5 \mathrm{~mL}$ test-tube equipped with a septum and a magnetic stir bar was charged with $\mathrm{Pd}_{2}(\mathrm{dba})_{3}(4.6 \mathrm{mg}, 5 \mathrm{~mol} \%)$ and ligand $\mathbf{L 1}$ (11.3 mg, $20 \mathrm{~mol} \%)$. The tube was then evacuated and filled with argon. This cycle was repeated three times, and toluene $(0.5 \mathrm{~mL})$ was added via syringe. The resulting solution was stirred at room temperature for $30 \mathrm{~min}$. A second oven-dried test-tube was charged with diene 1 e ( $32.0 \mathrm{mg}, 2.0$ equiv), functionalized $N$-sulfonylimine $4 \mathbf{a}$ (29.0 mg, 1.0 equiv), and $4 \AA \mathrm{MS}(60.0 \mathrm{mg})$, and the system was placed under an atmosphere of argon (balloon). Then the catalyst solution was added at room temperature. The resulting mixture was stirred at $80{ }^{\circ} \mathrm{C}$ for $20 \mathrm{~h}$. After completion, purification by flash chromatography on silica gel (petroleum ether/EtOAc $=6 / 1$ ) gave the product $\mathbf{5 d}$ : $47.0 \mathrm{mg}$, as a colorless semisolid, 99\% yield; $>19: 1 \mathrm{dr} ;[\alpha]^{25} \mathrm{D}=+75.3\left(c=1.65\right.$, in $\left.\mathrm{CHCl}_{3}\right)$; $91 \%$ ee, determined by HPLC analysis [Chiralpak column AD-H, $i \mathrm{PrOH} / n \mathrm{Hexane}=40 / 60$, flow rate: $1.0 \mathrm{~mL} / \mathrm{min}, 254 \mathrm{~nm}$, $\mathrm{t}($ minor $)=20.83 \mathrm{~min}, \mathrm{t}($ major $)=26.62 \mathrm{~min}] ;{ }^{1} \mathbf{H} \mathbf{N M R}\left(400 \mathrm{MHz}, \mathrm{CDCl}_{3}\right): \delta(\mathrm{ppm}) 7.41-7.32(\mathrm{~m}$, $7 \mathrm{H}), 7.09(\mathrm{~d}, J=8.6 \mathrm{~Hz}, 2 \mathrm{H}), 6.83(\mathrm{~d}, J=8.8 \mathrm{~Hz}, 2 \mathrm{H}), 6.77(\mathrm{~d}, J=8.4 \mathrm{~Hz}, 2 \mathrm{H}), \quad 6.37$ (d, $J=15.6$ $\mathrm{Hz}, 1 \mathrm{H}), 5.66(\mathrm{dd}, J=15.6,8.8 \mathrm{~Hz}, 1 \mathrm{H}), 5.04-4.98(\mathrm{~m}, 1 \mathrm{H}), 4.76-4.70(\mathrm{~m}, 1 \mathrm{H}), 4.62-4.53(\mathrm{~m}, 1 \mathrm{H})$, 3.76 (s, 3H), 3.74 (s, 3H), 2.31-2.15 (m, 2H); $\left.{ }^{13} \mathbf{C ~ N M R ~ ( 1 0 0 ~ M H z , ~} \mathrm{CDCl}_{3}\right): \delta(\mathrm{ppm})$ 159.5, 159.1, 138.8, 133.0, 130.6, 130.5, 128.9, 128.7, 128.4, 127.6, 126.2, 125.2, 114.1, 113.9, 64.0, 57.9, 55.3, 55.2, 39.56; HRMS (ESI-TOF) m/z: [M+Na] ${ }^{+}$Calcd for $\mathrm{C}_{25} \mathrm{H}_{26} \mathrm{~N}_{2} \mathrm{O}_{4} \mathrm{SNa} 473.1505$; Found 473.1508.

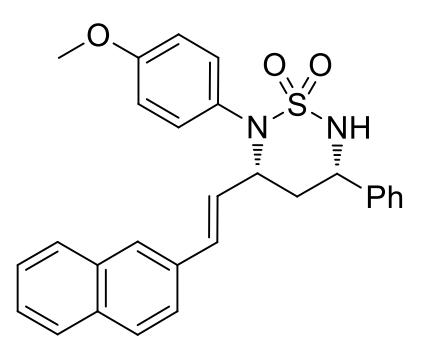

(3R,5S)-2-(4-Methoxyphenyl)-3-((E)-2-(naphthalen-2-yl)vinyl)-5phenyl-1,2,6-thiadiazinane 1,1-dioxide (5e): An oven-dried $5 \mathrm{~mL}$ testtube equipped with a septum and a magnetic stir bar was charged with $\mathrm{Pd}_{2}(\mathrm{dba})_{3}(4.6 \mathrm{mg}, 5 \mathrm{~mol} \%$ ) and ligand $\mathbf{L 1}(11.3 \mathrm{mg}, 20 \mathrm{~mol} \%)$. The tube was then evacuated and filled with argon. This cycle was repeated three times, and toluene $(0.5 \mathrm{~mL})$ was added via syringe. The resulting solution was stirred at room temperature for $30 \mathrm{~min}$. A second oven-dried test-tube was charged with diene $\mathbf{1 g}$ (36.0 mg, 2.0 equiv), functionalized $N$-sulfonylimine $4 \mathbf{a}$ (29.0 mg, 1.0 equiv), and $4 \AA$ MS (60.0 mg), and the system was placed under an atmosphere of argon (balloon). Then the catalyst solution was 
added at room temperature. The resulting mixture was stirred at $80{ }^{\circ} \mathrm{C}$ for $20 \mathrm{~h}$. After completion, purification by flash chromatography on silica gel (petroleum ether/EtOAc $=6 / 1$ ) gave the product 5e: $45.0 \mathrm{mg}$, as a white solid, $96 \%$ yield; $16: 1 \mathrm{dr} ; \mathrm{mp}=132-135^{\circ} \mathrm{C} ;[\alpha]^{25} \mathrm{D}=+69.5\left(c=0.4\right.$, in $\left.\mathrm{CHCl}_{3}\right)$; $85 \%$ ee, determined by HPLC analysis [Chiralpak column AD-H, $i \mathrm{PrOH} / n \mathrm{Hexane}=40 / 60$, flow rate: $1.0 \mathrm{~mL} / \mathrm{min}, 254 \mathrm{~nm}, \mathrm{t}$ (minor) $=15.67 \mathrm{~min}, \mathrm{t}$ (major) $=25.67 \mathrm{~min}] ;{ }^{1} \mathbf{H} \mathbf{~ N M R}\left(400 \mathrm{MHz}, \mathrm{CDCl}_{3}\right): \delta$ (ppm) 7.66-7.59 (m, 3H), $7.45(\mathrm{~s}, 1 \mathrm{H}), 7.36-7.23(\mathrm{~m}, 10 \mathrm{H}), 6.73(\mathrm{~d}, J=8.8 \mathrm{~Hz}, 2 \mathrm{H}), 6.49(\mathrm{~d}, J=$ $15.8 \mathrm{~Hz}, 1 \mathrm{H}), 5.84(\mathrm{dd}, J=15.6,8.4 \mathrm{~Hz}, 1 \mathrm{H}), 4.98-4.92(\mathrm{~m}, 1 \mathrm{H}), 4.75-4.69(\mathrm{~m}, 1 \mathrm{H}), 4.52(\mathrm{~d}, J=$ $10.4 \mathrm{~Hz}, 1 \mathrm{H}), 3.62$ (s, 3H), 2.26-2.11 (m, 2H); $\left.{ }^{13} \mathbf{C ~ N M R ~ ( 1 5 0 ~ M H z , ~} \mathrm{CDCl}_{3}\right): \delta$ (ppm) 159.2, 138.7, 133.6, 133.3, 133.3, 133.0, 130.6, 130.5, 129.0, 128.5, 128.2 127.9, 127.7, 127.6, 126.6, 126.4, 126.2, 126.1, 123.2, 114.2, 64.0, 57.9, 55.3, 39.5; HRMS (ESI-TOF) m/z: $\left[\mathrm{M}+\mathrm{Na}^{+}\right.$Calcd for $\mathrm{C}_{28} \mathrm{H}_{26} \mathrm{~N}_{2} \mathrm{O}_{3} \mathrm{SNa}, 493.1556$; Found 493.1558.

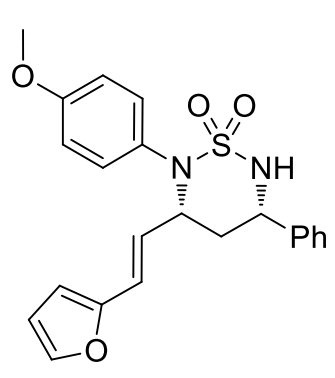

(3R,5S)-3-((E)-2-(Furan-2-yl)vinyl)-2-(4-methoxyphenyl)-5-phenyl-1,2,6thiadiazinane 1,1-dioxide (5f): An oven-dried $5 \mathrm{~mL}$ test-tube equipped with a septum and a magnetic stir bar was charged with $\operatorname{Pd}_{2}(\mathrm{dba})_{3}(4.6 \mathrm{mg}, 5 \mathrm{~mol} \%)$ and ligand $\mathbf{L 1}$ (11.3 $\mathrm{mg}, 20 \mathrm{~mol} \%$ ). The tube was then evacuated and filled with argon. This cycle was repeated three times, and toluene $(0.5 \mathrm{~mL})$ was added via syringe. The resulting solution was stirred at room temperature for $30 \mathrm{~min}$. A second oven-dried test-tube was charged with diene $\mathbf{1 h}$ ( $24.0 \mathrm{mg}, 2.0$ equiv), functionalized $N$-sulfonylimine 4a (29.0 mg, 1.0 equiv), and $4 \AA$ MS (60.0 mg), and the system was placed under an atmosphere of argon (balloon). Then the catalyst solution was added at room temperature. The resulting mixture was stirred at $80{ }^{\circ} \mathrm{C}$ for $20 \mathrm{~h}$. After completion, purification by flash chromatography on silica gel (petroleum ether/EtOAc $=6 / 1$ ) gave the product 5f: $39.0 \mathrm{mg}$, as a faint yellow semisolid, 95\% yield; $7: 1 \mathrm{dr} ;[\alpha]^{25} \mathrm{D}=+86.2\left(c=0.23\right.$, in $\left.\mathrm{CHCl}_{3}\right) ; 88 \%$ ee, determined by HPLC analysis [Chiralpak column AD-H, $i \mathrm{PrOH} / n \mathrm{Hexane}=40 / 60$, flow rate: $1.0 \mathrm{~mL} / \mathrm{min}, 254 \mathrm{~nm}$, $\mathrm{t}($ minor $)=33.27 \mathrm{~min}, \mathrm{t}($ major $)=38.25 \mathrm{~min}] ;{ }^{1} \mathbf{H} \mathbf{N M R}\left(400 \mathrm{MHz}, \mathrm{CDCl}_{3}\right): \delta(\mathrm{ppm}) 7.36-7.24(\mathrm{~m}$, $8 \mathrm{H}), 6.81(\mathrm{~d}, J=8.4 \mathrm{~Hz}, 2 \mathrm{H}), 6.23-6.12(\mathrm{~m}, 3 \mathrm{H}), 5.76(\mathrm{dd}, J=15.6,8.4 \mathrm{~Hz}, 1 \mathrm{H}), 5.00-4.95(\mathrm{t}, J=$ $9.4 \mathrm{~Hz}, 1 \mathrm{H}), 4.74-4.66(\mathrm{~m}, 2 \mathrm{H}), 3.74(\mathrm{~s}, 3 \mathrm{H}), 2.27-2.17(\mathrm{~m}, 2 \mathrm{H}) ;{ }^{13} \mathbf{C}$ NMR $\left(100 \mathrm{MHz}, \mathrm{CDCl}_{3}\right): \delta$ (ppm) 159.2, 151.3, 142.3, 138.7, 130.6, 130.4, 128.9, 128.4, 126.2, 125.8, 121.5, 114.2, 111.2, 108.8, 63.8, 57.9, 55.3, 39.7; HRMS (ESI-TOF) $\mathrm{m} / \mathrm{z}$ : $[\mathrm{M}+\mathrm{Na}]^{+}$Calcd for $\mathrm{C}_{22} \mathrm{H}_{22} \mathrm{~N}_{2} \mathrm{O}_{4} \mathrm{SNa}$ 433.1192; Found 433.1196. 


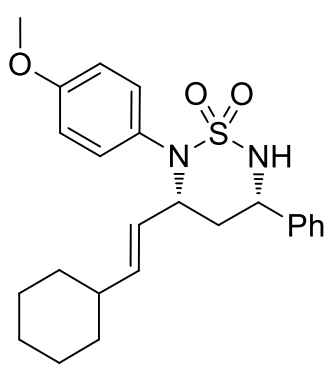

(3R,5S)-3-((E)-2-Cyclohexylvinyl)-2-(4-methoxyphenyl)-5-phenyl-1,2,6thiadiazinane 1,1-dioxide (5g): An oven-dried $5 \mathrm{~mL}$ test-tube equipped with a septum and a magnetic stir bar was charged with $\mathrm{Pd}_{2}(\mathrm{dba})_{3}(4.6 \mathrm{mg}, 5 \mathrm{~mol} \%)$ and ligand $\mathbf{L 1}$ (11.3 mg, $20 \mathrm{~mol} \%$ ). The tube was then evacuated and filled with argon. This cycle was repeated three times, and toluene $(0.5 \mathrm{~mL})$ was added via syringe. The resulting solution was stirred at room temperature for $30 \mathrm{~min}$. A second oven-dried test-tube was charged with diene $1 \mathbf{k}(27.2 \mathrm{mg}, 2.0$ equiv), functionalized $N$-sulfonylimine 4a (29.0 mg, 1.0 equiv), and $4 \AA$ MS (60.0 mg), and the system was placed under an atmosphere of argon (balloon). Then the catalyst solution was added at room temperature. The resulting mixture was stirred at $80{ }^{\circ} \mathrm{C}$ for $20 \mathrm{~h}$. After completion, purification by flash chromatography on silica gel (petroleum ether/EtOAc $=6 / 1$ ) gave the product $\mathbf{5 g}: 36.0 \mathrm{mg}$, as a white semisolid, $85 \%$ yield; $>19: 1 \mathrm{dr} ; \mathrm{mp}=123-124{ }^{\circ} \mathrm{C}$; $[\alpha]^{25} \mathrm{D}=+42.8\left(c=1.25\right.$, in $\left.\mathrm{CHCl}_{3}\right)$; $95 \%$ ee, determined by HPLC analysis [Chiralpak column ID, $i \operatorname{PrOH} / n$ Hexane $=20 / 80$, flow rate: $1.0 \mathrm{~mL} / \mathrm{min}$, $220 \mathrm{~nm}, \mathrm{t}$ (major) $=25.54 \mathrm{~min}, \mathrm{t}$ (minor) $=28.98 \mathrm{~min}] ;{ }^{1} \mathbf{H} \mathbf{N M R}\left(400 \mathrm{MHz}, \mathrm{CDCl}_{3}\right): \delta(\mathrm{ppm}) 7.40$ $7.47(\mathrm{~m}, 7 \mathrm{H}), 6.84(\mathrm{~d}, J=8.8 \mathrm{~Hz}, 2 \mathrm{H}), 5.41(\mathrm{dd}, J=15.4,6.8 \mathrm{~Hz}, 1 \mathrm{H}), 5.02-4.91(\mathrm{~m}, 2 \mathrm{H}), 4.53-4.46$ (m, 2H), 3.79 (s, 3H), 2.23-2.04 (m, 2H), 1.75-1.68 (m, 1H), 1.56-1.43 (m, 4H), 1.29-1.02 (m, 4H), 0.86- $0.72(\mathrm{~m}, 2 \mathrm{H}) ;{ }^{13} \mathbf{C}$ NMR (100 MHz, $\left.\mathrm{CDCl}_{3}\right): \delta(\mathrm{ppm}) 159.1,141.8,138.9,130.8,130.7,128.9$, 128.4, 126.2, 125.5, 113.9, 64.1, 57.9, 55.4, 39.9, 39.5, 32.3, 32.1, 25.9, 25.52, 25.49; HRMS (ESITOF) m/z: $[\mathrm{M}+\mathrm{Na}]^{+}$Calcd for $\mathrm{C}_{24} \mathrm{H}_{30} \mathrm{~N}_{2} \mathrm{O}_{3} \mathrm{SNa} 449.1869$; Found 449.860 .

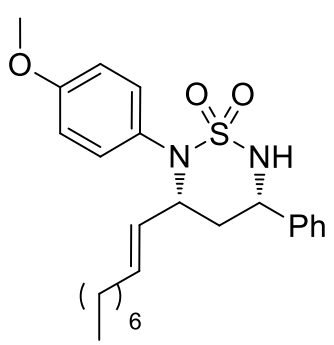

$(3 R, 5 S)-2-(4-M e t h o x y p h e n y l)-3-((E)-n o n-1-e n-1-y l)-5-p h e n y l-1,2,6-$ thiadiazinane 1,1-dioxide (5h): An oven-dried $5 \mathrm{~mL}$ test-tube equipped with a septum and a magnetic stir bar was charged with $\operatorname{Pd}_{2}(\mathrm{dba})_{3}(4.6 \mathrm{mg}, 5 \mathrm{~mol} \%)$ and ligand $\mathbf{L 1}(11.3 \mathrm{mg}, 20 \mathrm{~mol} \%)$. The tube was then evacuated and filled with argon. This cycle was repeated three times, and toluene $(0.5 \mathrm{~mL})$ was added via syringe. The resulting solution was stirred at room temperature for $30 \mathrm{~min}$. A second oven-dried test-tube was charged with diene 11 (30.4 mg, 2.0 equiv), functionalized $N$-sulfonylimine $4 \mathbf{a}$ (29.0 mg, 1.0 equiv), and $4 \AA$ MS (60.0 mg), and the system was placed under an atmosphere of argon (balloon). Then the catalyst solution was added at room temperature. The resulting mixture was stirred at $80{ }^{\circ} \mathrm{C}$ for $20 \mathrm{~h}$. After completion, purification by flash chromatography on silica gel (petroleum ether/EtOAc $=6 / 1$ ) gave the product $\mathbf{5 h}: 37.0 \mathrm{mg}$, as a white solid, $81 \%$ yield; $7: 1 \mathrm{dr} ; \mathrm{mp}=113-115^{\circ} \mathrm{C} ;[\alpha]^{25} \mathrm{D}=+15.1\left(c=0.65\right.$, in $\left.\mathrm{CHCl}_{3}\right) ; 80 \%$ ee, determined by HPLC analysis [Chiralpak column AD-H, $i \mathrm{PrOH} / n$ Hexane $=40 / 60$, flow rate: $1.0 \mathrm{~mL} / \mathrm{min}, 220$ $\mathrm{nm}, \mathrm{t}$ (minor) $=5.45 \mathrm{~min}, \mathrm{t}$ (major) = $6.75 \mathrm{~min}]{ }^{1}{ }^{\mathbf{H}} \mathbf{N M R}\left(400 \mathrm{MHz}, \mathrm{CDCl}_{3}\right): \delta(\mathrm{ppm}) 7.39-7.24(\mathrm{~m}$, $7 \mathrm{H}), 6.83(\mathrm{~d}, J=8.4 \mathrm{~Hz}, 2 \mathrm{H}), 5.50-5.43(\mathrm{~m}, 1 \mathrm{H}), 5.08-4.92(\mathrm{~m}, 2 \mathrm{H}), 4.55-4.49(\mathrm{~m}, 2 \mathrm{H}), 3.78(\mathrm{~s}$, $3 \mathrm{H}), 2.22-2.04(\mathrm{~m}, 2 \mathrm{H}), 1.84-1.72(\mathrm{~m}, 2 \mathrm{H}), 1.25-0.84(\mathrm{~m}, 13 \mathrm{H}) ;{ }^{13} \mathbf{C} \mathbf{N M R}\left(100 \mathrm{MHz}, \mathrm{CDCl}_{3}\right) \delta$ 
159.2, 138.9, 136.1, 130.7, 130.7, 129.0, 128.4, 128.0, 126.2, 114.0, 63.9, 57.9, 55.3, 39.6, 31.9, 31.6, 29.0, 28.7, 28.5, 22.7, 14.1; HRMS (ESI-TOF) m/z: $[\mathrm{M}+\mathrm{Na}]^{+}$Calcd for $\mathrm{C}_{25} \mathrm{H}_{34} \mathrm{~N}_{2} \mathrm{O}_{3} \mathrm{SNa} 465.2182$; Found 465.2176.

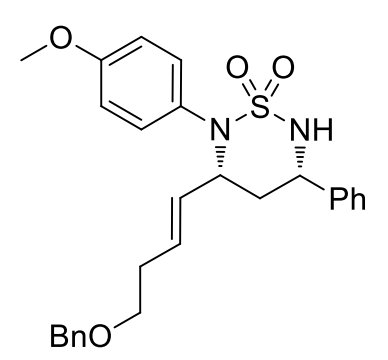

$(3 R, 5 S)-3-((E)-4-(B e n z y l o x y) b u t-1-e n-1-y l)-2-(4-m e t h o x y p h e n y l)-5-$ phenyl-1,2,6-thiadiazinane 1,1-dioxide (5i): An oven-dried $5 \mathrm{~mL}$ test-tube equipped with a septum and a magnetic stir bar was charged with $\operatorname{Pd}_{2}(\mathrm{dba})_{3}$ (4.6 $\mathrm{mg}, 5 \mathrm{~mol} \%$ ) and ligand $\mathbf{L 1}(11.3 \mathrm{mg}, 20 \mathrm{~mol} \%)$. The tube was then evacuated and filled with argon. This cycle was repeated three times, and toluene $(0.5 \mathrm{~mL})$ was added via syringe. The resulting solution was stirred at room temperature for $30 \mathrm{~min}$. A second oven-dried test-tube was charged with diene $\mathbf{1 n}$ (37.6 mg, 2.0 equiv), functionalized $N$-sulfonylimine $4 \mathbf{a}(29.0 \mathrm{mg}, 1.0$ equiv), and $4 \AA \mathrm{MS}(60.0 \mathrm{mg})$, and the system was placed under an atmosphere of argon (balloon). Then the catalyst solution was added at room temperature. The resulting mixture was stirred at $80{ }^{\circ} \mathrm{C}$ for $20 \mathrm{~h}$. After completion, purification by flash chromatography on silica gel (petroleum ether/EtOAc $=6 / 1$ ) gave the product 5i: $39.2 \mathrm{mg}$, as a colorless oil, $82 \%$ yield; $9: 1 \mathrm{dr} ;[\alpha]^{25} \mathrm{D}=+15.1\left(c=1.59\right.$, in $\left.\mathrm{CHCl}_{3}\right) ; 81 \%$ ee, determined by HPLC analysis [Chiralpak column IB, $i \mathrm{PrOH} / n$ Hexane $=40 / 60$, flow rate: $1.0 \mathrm{~mL} / \mathrm{min}, 220 \mathrm{~nm}, \mathrm{t}$ $($ major $)=10.68 \mathrm{~min}, \mathrm{t}($ minor $)=14.27 \mathrm{~min}] ;{ }^{1} \mathbf{H} \mathbf{~ N M R}\left(400 \mathrm{MHz}, \mathrm{CDCl}_{3}\right): \delta(\mathrm{ppm}) 7.38-7.25(\mathrm{~m}$, $14 \mathrm{H}), 6.80(\mathrm{~d}, J=8.8 \mathrm{~Hz}, 2 \mathrm{H}), 5.58-5.51(\mathrm{~m}, 1 \mathrm{H}), 5.15(\mathrm{dd}, J=15.4,8.6 \mathrm{~Hz}, 1 \mathrm{H}), 4.97-4.89$ (m, 1H), 4.57-4.47 (m, 2H), $4.36(\mathrm{~s}, 3 \mathrm{H}), 3.74(\mathrm{~s}, 3 \mathrm{H}), 3.22-3.17(\mathrm{~m}, 2 \mathrm{H}), 2.22-2.03(\mathrm{~m}, 4 \mathrm{H}) ;{ }^{13} \mathbf{C ~ N M R}$ $\left(100 \mathrm{MHz}, \mathrm{CDCl}_{3}\right): \delta$ (ppm) 159.1, 138.8, 138.2, 132.2, 130.7, 130.6, 129.9, 128.9, 128.4, 128.3, 127.6, 127.5, 126.2 114.0, 72.7, 69.3, 63.8, 57.8, 55.3, 39.4, 32.4; HRMS (ESI-TOF) m/z: [M + Na] ${ }^{+}$ Calcd for $\mathrm{C}_{27} \mathrm{H}_{30} \mathrm{~N}_{2} \mathrm{O}_{4} \mathrm{SNa} 501.1818$; Found 501.1814.

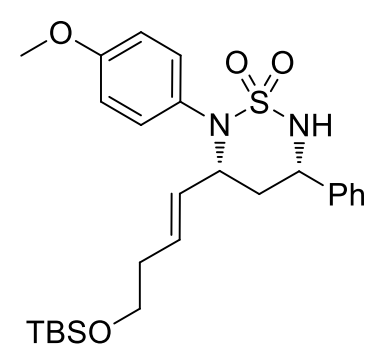

(3R,5S)-3-((E)-4-((tert-Butyldimethylsilyl)oxy)but-1-en-1-yl)-2-(4methoxyphenyl)-5-phenyl-1,2,6-thiadiazinane 1,1-dioxide (5j): An ovendried $5 \mathrm{~mL}$ test-tube equipped with a septum and a magnetic stir bar was charged with $\mathrm{Pd}_{2}(\mathrm{dba})_{3}(4.6 \mathrm{mg}, 5 \mathrm{~mol} \%)$ and ligand $\mathbf{L 1}(11.3 \mathrm{mg}, 20 \mathrm{~mol} \%)$. The tube was then evacuated and filled with argon. This cycle was repeated three times, and toluene $(0.5 \mathrm{~mL})$ was added via syringe. The resulting solution was stirred at room temperature for $30 \mathrm{~min}$. A second oven-dried test-tube was charged with diene 10 (42.4 mg, 2.0 equiv), functionalized $N$-sulfonylimine $4 \mathbf{a}$ (29.0 mg, 1.0 equiv), and $4 \AA$ MS $(60.0 \mathrm{mg})$, and the system was placed under an atmosphere of argon (balloon). Then the catalyst solution was added at room temperature. The resulting mixture was stirred at $80{ }^{\circ} \mathrm{C}$ for $20 \mathrm{~h}$. After completion, purification by flash chromatography on silica gel (petroleum ether/EtOAc $=6 / 1$ ) gave the product 5j: $35.0 \mathrm{mg}$, as a white solid, $70 \%$ yield; 5:1 dr; $\mathrm{mp}=105-107{ }^{\circ} \mathrm{C} ;[\alpha]^{25} \mathrm{D}=+17.0(c=$ 
1.32, in $\mathrm{CHCl}_{3}$ ); $65 \%$ ee, determined by HPLC analysis [Chiralpak column $\mathrm{IB}, i \mathrm{PrOH} / n \mathrm{Hexane}=$ 40/60, flow rate: $1.0 \mathrm{~mL} / \mathrm{min}, 220 \mathrm{~nm}, \mathrm{t}$ (major) $=5.39 \mathrm{~min}, \mathrm{t}$ (minor) $=6.22 \mathrm{~min}] ;{ }^{1} \mathbf{H} \mathbf{~ N M R}(400$ $\left.\mathrm{MHz}, \mathrm{CDCl}_{3}\right): \delta(\mathrm{ppm}) 7.37-7.24(\mathrm{~m}, 7 \mathrm{H}), 6.80(\mathrm{~d}, J=9.2 \mathrm{~Hz}, 2 \mathrm{H}), 5.52-5.45(\mathrm{~m}, 1 \mathrm{H}), 5.14-5.06$ (m, 1H), 4.96-4.81 (m, 1H), 4.52-4.43 (m, 2H), $3.76(\mathrm{~s}, 3 \mathrm{H}), 3.30-3,26(\mathrm{~m}, 2 \mathrm{H}), 2.22-1.93(\mathrm{~m}, 4 \mathrm{H})$, 0.82 (s, 9H), -0.04 (s, 6H); ${ }^{13} \mathbf{C}$ NMR (100 MHz, $\left.\mathrm{CDCl}_{3}\right): \delta(\mathrm{ppm}) 159.2,138.8,132.1,130.7,130.6$, $129.9,128.9,128.4,126.2,114.0,63.9,62.4,57.8,55.3,39.5,35.6,25.9,18.2,-5.4$; HRMS (ESITOF) m/z: $[\mathrm{M}-\mathrm{H}]^{-}$Calcd for $\mathrm{C}_{26} \mathrm{H}_{37} \mathrm{~N}_{2} \mathrm{O}_{4} \mathrm{SSi}$ 501.2249; Found 501.2246.

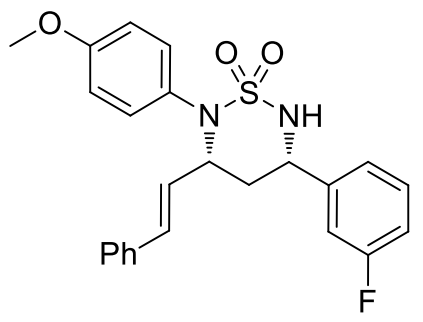

(3R,5S)-5-(3-Fluorophenyl)-2-(4-methoxyphenyl)-3-((E)-styryl)-1,2,6thiadiazinane 1,1-dioxide (5k): An oven-dried $5 \mathrm{~mL}$ test-tube equipped with a septum and a magnetic stir bar was charged with $\operatorname{Pd}_{2}(\mathrm{dba})_{3}(4.6 \mathrm{mg}$, $5 \mathrm{~mol} \%$ ) and ligand L1 (11.3 mg, $20 \mathrm{~mol} \%$ ). The tube was then evacuated and filled with argon. This cycle was repeated three times, and toluene ( 0.5 $\mathrm{mL}$ ) was added via syringe. The resulting solution was stirred at room temperature for $30 \mathrm{~min}$. A second oven-dried test-tube was charged with diene $1 \mathrm{a}(26.0 \mathrm{mg}, 2.0$ equiv), functionalized $\mathrm{N}$ sulfonylimine $4 \mathbf{b}$ (30.8 mg, 1.0 equiv) and $4 \AA$ MS (60.0 mg), and the system was placed under an atmosphere of argon (balloon). Then the catalyst solution was added at room temperature. The resulting mixture was stirred at $80{ }^{\circ} \mathrm{C}$ for $20 \mathrm{~h}$. After completion, purification by flash chromatography on silica gel (petroleum ether/EtOAc $=6 / 1$ ) gave the product 5k: $40.0 \mathrm{mg}$, white solid, 91\% yield; >19:1 dr; $\mathrm{mp}=74-76^{\circ} \mathrm{C} ;[\alpha]^{25} \mathrm{D}=+70.5\left(c=0.8\right.$, in $\left.\mathrm{CHCl}_{3}\right) ; 94 \%$ ee, determined by HPLC analysis [Chiralpak column AD-H, $i$ PrOH $/ n$ Hexane $=40 / 60$, flow rate: $1.0 \mathrm{~mL} / \mathrm{min}, 254$ $\mathrm{nm}, \mathrm{t}($ minor $)=14.34 \mathrm{~min}, \mathrm{t}($ major $)=19.46 \mathrm{~min}] ;{ }^{1} \mathbf{H} \mathbf{~ N M R}\left(400 \mathrm{MHz}, \mathrm{CDCl}_{3}\right): \delta(\mathrm{ppm}) 7.38-7.32$ (m, 3H), 7.26-7.11 (m, 7H), 7.06-7.02 (m, 1H), $6.81(\mathrm{~d}, J=8.4 \mathrm{~Hz}, 2 \mathrm{H}), 6.42(\mathrm{~d}, J=15.6 \mathrm{~Hz}, 1 \mathrm{H})$, $5.79(\mathrm{dd}, J=15.6,8.8 \mathrm{~Hz}, 1 \mathrm{H}), 5.03-4.97(\mathrm{~m}, 1 \mathrm{H}), 4.77-4.71(\mathrm{~m}, 1 \mathrm{H}), 4.63-4.61(\mathrm{~m}, 1 \mathrm{H}), 3.73(\mathrm{~s}$, $3 \mathrm{H}), 2.32-2.27(\mathrm{~m}, 1 \mathrm{H}), 2.21-2.12(\mathrm{~m}, 1 \mathrm{H}) ;{ }^{13} \mathbf{C ~ N M R}\left(100 \mathrm{MHz}, \mathrm{CDCl}_{3}\right): \delta(\mathrm{ppm}) 163.0(\mathrm{~d}, J=$ $245.7 \mathrm{~Hz}), 159.3,141.1(\mathrm{~d}, J=7.0 \mathrm{~Hz}), 135.9,133.7,130.6(\mathrm{~d}, J=8.1 \mathrm{~Hz}), 130.43,130.39,128.5$, 128.1, 127.2, 126.4, $121.8(\mathrm{~d}, J=2.8 \mathrm{~Hz}), 115.4(\mathrm{~d}, J=20.7 \mathrm{~Hz}), 114.2,113.5$ (d, $J=22.3 \mathrm{~Hz}), 63.8$, 57.4, 55.3, 39.3; HRMS (ESI-TOF) $\mathrm{m} / \mathrm{z}$ : $[\mathrm{M}+\mathrm{Na}]^{+}$Calcd for $\mathrm{C}_{24} \mathrm{H}_{23} \mathrm{FN}_{2} \mathrm{O}_{3} \mathrm{SNa}$ 461.1306; Found 461.1309.

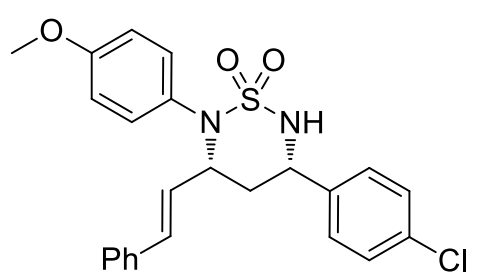

(3R,5S)-5-(4-Chlorophenyl)-2-(4-methoxyphenyl)-3-((E)-styryl)1,2,6-thiadiazinane 1,1-dioxide (5l): An oven-dried $5 \mathrm{~mL}$ test-tube equipped with a septum and a magnetic stir bar was charged with $\mathrm{Pd}_{2}(\mathrm{dba})_{3}$ (4.6 mg, $5 \mathrm{~mol} \%$ ) and ligand $\mathbf{L 1}$ (11.3 mg, $20 \mathrm{~mol} \%$ ). The tube was then evacuated and filled with argon. This cycle was repeated three times, and toluene $(0.5 \mathrm{~mL})$ was added via syringe. The resulting solution was stirred at room 
temperature for $30 \mathrm{~min}$. A second oven-dried test-tube was charged with diene 1a (26.0 mg, 2.0 equiv), functionalized $N$-sulfonylimine $4 \mathbf{c}$ (32.8 mg, 1.0 equiv) and $4 \AA \mathrm{MS}(60.0 \mathrm{mg})$, and the system was placed under an atmosphere of argon (balloon). Then the catalyst solution was added at room temperature. The resulting mixture was stirred at $80{ }^{\circ} \mathrm{C}$ for $20 \mathrm{~h}$. After completion, purification by flash chromatography on silica gel (petroleum ether/EtOAc $=6 / 1$ ) gave the product 5l: $28.3 \mathrm{mg}$, as a white semisolid, $62 \%$ yield; $>19: 1 \mathrm{dr} ;[\alpha]^{25} \mathrm{D}=+49.4\left(c=0.32\right.$, in $\left.\mathrm{CHCl}_{3}\right)$; $94 \%$ ee, determined by HPLC analysis [Chiralpak column AD-H, $i \mathrm{PrOH} / n \mathrm{Hexane}=40 / 60$, flow rate: $1.0 \mathrm{~mL} / \mathrm{min}, 254 \mathrm{~nm}$, $\mathrm{t}($ minor $)=13.01 \mathrm{~min}, \mathrm{t}($ major $)=16.93 \mathrm{~min}] ;{ }^{1} \mathbf{H} \mathbf{~ N M R}\left(400 \mathrm{MHz}, \mathrm{CDCl}_{3}\right): \delta(\mathrm{ppm}) 7.38-7.33(\mathrm{~m}$, $6 \mathrm{H}), 7.25-7.13(\mathrm{~m}, 5 \mathrm{H}), 6.81(\mathrm{~d}, J=8.8 \mathrm{~Hz}, 2 \mathrm{H}), 6.41(\mathrm{~d}, J=16.0 \mathrm{~Hz}, 1 \mathrm{H}), 5.79(\mathrm{dd}, J=16.0,8.8$ $\mathrm{Hz}, 1 \mathrm{H}), 5.01-4.95(\mathrm{~m}, 1 \mathrm{H}), 4.76-4.71(\mathrm{~m}, 1 \mathrm{H}), 4.57(\mathrm{~d}, J=10.0 \mathrm{~Hz}, 1 \mathrm{H}), 3.74(\mathrm{~s}, 3 \mathrm{H}), 2.31-2.12$ $(\mathrm{m}, 2 \mathrm{H}) ;{ }^{13} \mathbf{C}$ NMR $\left(100 \mathrm{MHz}, \mathrm{CDCl}_{3}\right): \delta(\mathrm{ppm}) 159.3,137.2,135.9,134.4,133.7,130.4,130.4$, 129.1, 128.5, 128.1, 127.7, 127.2, 126.4, 114.2, 63.9, 57.3, 55.3, 39.3; HRMS (ESI-TOF) m/z: [M + $\mathrm{Na}]^{+}$Calcd for $\mathrm{C}_{24} \mathrm{H}_{23} \mathrm{ClN}_{2} \mathrm{O}_{3} \mathrm{SNa} 477.1010\left({ }^{35} \mathrm{Cl}\right)$, $479.0981\left({ }^{37} \mathrm{Cl}\right)$; Found $477.1006\left({ }^{35} \mathrm{Cl}\right), 479.0981$ $\left({ }^{37} \mathrm{Cl}\right)$.

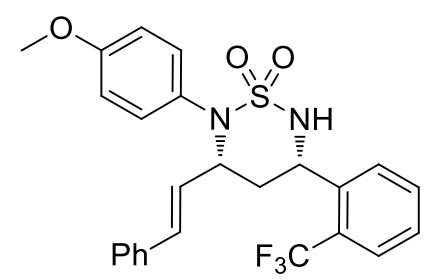

(3R,5S)-2-(4-Methoxyphenyl)-3-((E)-styryl)-5-(2-(trifluoromethyl) phenyl)-1,2,6-thiadiazinane 1,1-dioxide (5m): An oven-dried $5 \mathrm{~mL}$ testtube equipped with a septum and a magnetic stir bar was charged with $\operatorname{Pd}_{2}(\mathrm{dba})_{3}(4.6 \mathrm{mg}, 5 \mathrm{~mol} \%)$ and ligand $\mathbf{L 1}(11.3 \mathrm{mg}, 20 \mathrm{~mol} \%)$. The tube was then evacuated and filled with argon. This cycle was repeated three times, and toluene $(0.5 \mathrm{~mL})$ was added via syringe. The resulting solution was stirred at room temperature for $30 \mathrm{~min}$. A second oven-dried test-tube was charged with diene $\mathbf{1 a}(26.0 \mathrm{mg}, 2.0$ equiv), functionalized $N$-sulfonylimine $4 \mathbf{d}$ (35.8 mg, 1.0 equiv) and $4 \AA \mathrm{MS}(60.0 \mathrm{mg})$, and the system was placed under an atmosphere of argon (balloon). Then the catalyst solution was added at room temperature. The resulting mixture was stirred at $80{ }^{\circ} \mathrm{C}$ for $20 \mathrm{~h}$. After completion, purification by flash chromatography on silica gel (petroleum ether/EtOAc $=6 / 1$ ) gave the product $5 \mathrm{~m}$ : $32.4 \mathrm{mg}$, as a white semisolid, $66 \%$ yield; $14: 1 \mathrm{dr} ;[\alpha]^{25} \mathrm{D}=+25.2\left(c=0.62\right.$, in $\left.\mathrm{CHCl}_{3}\right) ; 94 \%$ ee, determined by HPLC analysis [Chiralpak column AD-H, $i \mathrm{PrOH} / n \mathrm{Hexane}=40 / 60$, flow rate: $1.0 \mathrm{~mL} / \mathrm{min}, 254 \mathrm{~nm}$, $\mathrm{t}($ minor $)=17.52 \mathrm{~min}, \mathrm{t}($ major $)=37.90 \mathrm{~min}] ;{ }^{1} \mathbf{H} \mathbf{~ N M R}\left(400 \mathrm{MHz}, \mathrm{CDCl}_{3}\right): \delta(\mathrm{ppm}) 7.70(\mathrm{~d}, J=7.9$ $\mathrm{Hz}, 1 \mathrm{H}), 7.66-7.53(\mathrm{~m}, 2 \mathrm{H}), 7.50-7.39(\mathrm{~m}, 3 \mathrm{H}), 7.30-7.09$ (m, 5H), $6.84(\mathrm{~d}, J=8.9 \mathrm{~Hz}, 2 \mathrm{H}), 6.44(\mathrm{~d}$, $J=15.6 \mathrm{~Hz}, 1 \mathrm{H}), 5.79(\mathrm{dd}, J=15.6,8.5 \mathrm{~Hz}, 1 \mathrm{H}), 5.45-5.33(\mathrm{~m}, 1 \mathrm{H}), 5.12(\mathrm{~d}, J=9.2 \mathrm{~Hz}, 1 \mathrm{H}), 4.93$ $(\mathrm{d}, J=9.3 \mathrm{~Hz}, 1 \mathrm{H}), 4.85-4.73(\mathrm{~m}, 1 \mathrm{H}), 3.74(\mathrm{~s}, 3 \mathrm{H}), 2.32-2.09(\mathrm{~m}, 2 \mathrm{H}) ;{ }^{\mathbf{1 3}} \mathbf{C} \mathbf{~ N M R}\left(100 \mathrm{MHz}, \mathrm{CDCl}_{3}\right)$ $\delta(\mathrm{ppm}) 159.3,137.7,135.9,133.8,132.8,130.6,130.5,128.5,128.1,127.8$ (q, $J=30.2 \mathrm{~Hz}), 127.1$, 127.0, 126.4, 126.3 (q, $J=5.9 \mathrm{~Hz}), 124.0(\mathrm{q}, J=272.5 \mathrm{~Hz}), 114.21,63.9,55.3,54.3,40.3$; HRMS (ESI-TOF) m/z: $[\mathrm{M}+\mathrm{Na}]^{+}$Calcd for $\mathrm{C}_{25} \mathrm{H}_{23} \mathrm{~F}_{3} \mathrm{~N}_{2} \mathrm{O}_{3} \mathrm{SNa}$ 511.1274; Found 511.1277. 


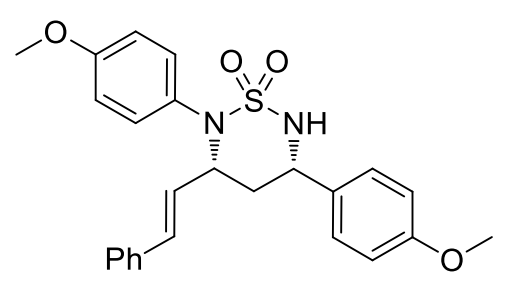

(3R,5S)-2,5-Bis(4-methoxyphenyl)-3-((E)-styryl)-1,2,6thiadiazinane 1,1-dioxide (5n): An oven-dried $5 \mathrm{~mL}$ test-tube equipped with a septum and a magnetic stir bar was charged with $\mathrm{Pd}_{2}(\mathrm{dba})_{3}$ (4.6 mg, $5 \mathrm{~mol} \%$ ) and ligand $\mathbf{L 1}$ (11.3 mg, $20 \mathrm{~mol} \%$ ). The tube was then evacuated and filled with argon. This cycle was repeated three times, and toluene $(0.5 \mathrm{~mL})$ was added via syringe. The resulting solution was stirred at room temperature for $30 \mathrm{~min}$. A second oven-dried test-tube was charged with diene $\mathbf{1 a}(26.0 \mathrm{mg}$, 2.0 equiv), functionalized $N$-sulfonylimine $4 \mathbf{e}(32.0 \mathrm{mg}, 1.0$ equiv) and $4 \AA$ MS (60.0 mg), and the system was placed under an atmosphere of argon (balloon). Then the catalyst solution was added at room temperature. The resulting mixture was stirred at $80{ }^{\circ} \mathrm{C}$ for $20 \mathrm{~h}$. After completion, purification by flash chromatography on silica gel (petroleum ether/EtOAc $=6 / 1$ ) gave the product $5 \mathbf{n}: 43.0 \mathrm{mg}$, as a colorless semisolid, $96 \%$ yield; $>19: 1 \mathrm{dr} ;[\alpha]^{25} \mathrm{D}=+95.8\left(c=0.33\right.$, in $\left.\mathrm{CHCl}_{3}\right) ; 91 \%$ ee, determined by HPLC analysis [Chiralpak column AD-H, $i \mathrm{PrOH} / n$ Hexane $=40 / 60$, flow rate: $1.0 \mathrm{~mL} / \mathrm{min}, 254$ $\mathrm{nm}, \mathrm{t}($ minor $)=20.36 \mathrm{~min}, \mathrm{t}$ (major) $=29.78 \mathrm{~min}] ;{ }^{1} \mathbf{H} \mathbf{~ N M R}\left(400 \mathrm{MHz}, \mathrm{CDCl}_{3}\right): \delta(\mathrm{ppm}) 7.37-7.14$ $(\mathrm{m}, 9 \mathrm{H}), 6.91(\mathrm{~d}, J=8.4 \mathrm{~Hz}, 2 \mathrm{H}), 6.82(\mathrm{~d}, J=8.4 \mathrm{~Hz}, 2 \mathrm{H}), 6.41(\mathrm{~d}, J=15.6 \mathrm{~Hz}, 1 \mathrm{H}), 5.80(\mathrm{dd}, J=$ 15.6, 8.4 Hz, 1H), 4.98-4.92 (m, 1H), 4.76-4.70 (m, 1H), 4.50-4.48 (m, 1H), 3.81 (s, 3H), 3.74 (s, $3 \mathrm{H}), 2.28-2.15(\mathrm{~m}, 2 \mathrm{H}) ;{ }^{13} \mathrm{C}$ NMR (100 MHz, $\left.\mathrm{CDCl}_{3}\right): \delta$ (ppm) 159.6, 159.2, 136.0, 133.5, 130.9, 130.6, 130.5, 128.5, 128.0, 127.6, 127.5, 126.4, 114.3, 114.2, 63.9, 57.4, 55.3, 55.3, 39.5; HRMS (ESI-TOF) $\mathrm{m} / \mathrm{z}$ : $[\mathrm{M}+\mathrm{Na}]^{+}$Calcd for $\mathrm{C}_{25} \mathrm{H}_{26} \mathrm{~N}_{2} \mathrm{O}_{4} \mathrm{SNa} 473.1505$; Found 473.1503.

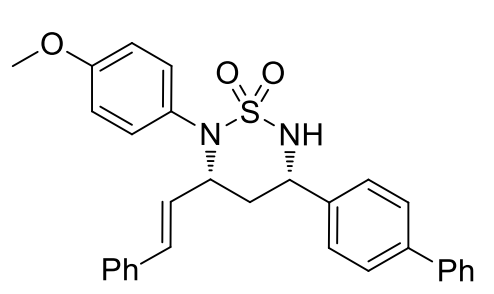

$(3 R, 5 S)-5-([1,1 '-B i p h e n y l]-4-y l)-2-(4-m e t h o x y p h e n y l)-3-((E)-$ styryl)-1,2,6-thiadiazinane 1,1-dioxide (5o): An oven-dried $5 \mathrm{~mL}$ test-tube equipped with a septum and a magnetic stir bar was charged with $\mathrm{Pd}_{2}(\mathrm{dba})_{3}$ (4.6 mg, $5 \mathrm{~mol} \%$ ) and ligand L1 (11.3 mg, $20 \mathrm{~mol} \%$ ). The tube was then evacuated and filled with argon. This cycle was repeated three times, and toluene $(0.5 \mathrm{~mL})$ was added via syringe. The resulting solution was stirred at room temperature for $30 \mathrm{~min}$. A second oven-dried test-tube was charged with diene $\mathbf{1 a}(26.0 \mathrm{mg}$, 2.0 equiv), functionalized $N$-sulfonylimine $\mathbf{4 f}$ (32.0 mg, 1.0 equiv) and $4 \AA$ MS (60.0 mg), and the system was placed under an atmosphere of argon (balloon). Then the catalyst solution was added at room temperature. The resulting mixture was stirred at $80{ }^{\circ} \mathrm{C}$ for $20 \mathrm{~h}$. After completion, purification by flash chromatography on silica gel (petroleum ether/EtOAc $=6 / 1$ ) gave the product 5o: $50.0 \mathrm{mg}$, white solid, $99 \%$ yield; >19:1 dr; $\mathrm{mp}=176-179{ }^{\circ} \mathrm{C} ;[\alpha]^{25} \mathrm{D}=+81.8\left(c=1.9\right.$, in $\left.\mathrm{CHCl}_{3}\right) ; 88 \%$ ee, determined by HPLC analysis [Chiralpak column AD-H, $i \mathrm{PrOH} / n \mathrm{Hexane}=40 / 60$, flow rate: 1.0 $\mathrm{mL} / \mathrm{min}, 254 \mathrm{~nm}, \mathrm{t}$ (minor) $=24.33 \mathrm{~min}, \mathrm{t}$ (major) $=42.25 \mathrm{~min}] ;{ }^{1} \mathbf{H} \mathbf{~ N M R}\left(400 \mathrm{MHz}, \mathrm{CDCl}_{3}\right): \delta$ (ppm) 7.63-7.59 (m, 4H), 7.49-7.36 (m, 8H), 7.27-7.16 (m, 4H), 6.83 (d, J = 8.8 Hz, 2H), 6.45 (d, $J$ $=16.0 \mathrm{~Hz}, 1 \mathrm{H}), 5.84(\mathrm{dd}, J=16.0,8.4 \mathrm{~Hz}, 1 \mathrm{H}), 5.10-5.05(\mathrm{~m}, 1 \mathrm{H}), 4.84-4.74(\mathrm{~m}, 2 \mathrm{H}), 3.71(\mathrm{~s}, 3 \mathrm{H})$, 
2.35-2.24 (m, 2H); ${ }^{13} \mathbf{C}$ NMR (150 MHz, $\left.\mathrm{CDCl}_{3}\right): \delta(\mathrm{ppm})$ 159.2, 141.3, 140.2, 137.7, 135.9, 133.5, 130.6, 130.5, 128.8, 128.5, 128.0, 127.6, 127.4, 127.0, 126.7, 126.4, 114.2, 63.9, 57.7, 55.2, 39.5; HRMS (ESI-TOF) m/z: [M + Na] $]^{+}$Calcd for $\mathrm{C}_{30} \mathrm{H}_{28} \mathrm{~N}_{2} \mathrm{O}_{3} \mathrm{SNa} 519.1713$; Found 519.1718.

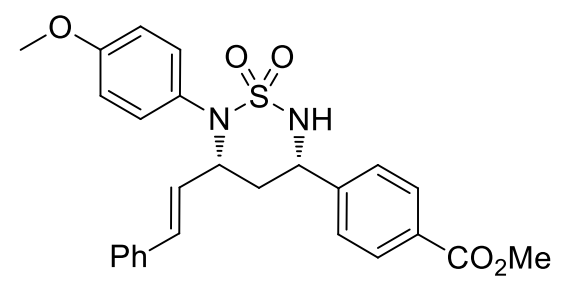

Methyl-4-((3S,5R)-6-(4-methoxyphenyl)-1,1-dioxido-5-((E)styryl)-1,2,6-thiadiazinan-3-yl)benzoate (5p): An oven-dried 5 $\mathrm{mL}$ test-tube equipped with a septum and a magnetic stir bar was charged with $\mathrm{Pd}_{2}(\mathrm{dba})_{3}(4.6 \mathrm{mg}, 5 \mathrm{~mol} \%$ ) and ligand $\mathbf{L 1}$ (11.3 mg, $20 \mathrm{~mol} \%)$. The tube was then evacuated and filled with argon. This cycle was repeated three times, and toluene $(0.5 \mathrm{~mL})$ was added via syringe. The resulting solution was stirred at room temperature for $30 \mathrm{~min}$. A second oven-dried test-tube was charged with diene $1 \mathbf{a}$ (26.0 mg, 2.0 equiv), functionalized $N$-sulfonylimine $\mathbf{4 g}$ (34.8 mg, 1.0 equiv) and $4 \AA$ MS (60.0 mg), and the system was placed under an atmosphere of argon (balloon). Then the catalyst solution was added at room temperature. The resulting mixture was stirred at $80{ }^{\circ} \mathrm{C}$ for $20 \mathrm{~h}$. After completion, purification by flash chromatography on silica gel (petroleum ether/EtOAc $=6 / 1$ ) gave the product 5p: $42.0 \mathrm{mg}$, as a white solid, $85 \%$ yield; $16: 1 \mathrm{dr} ; \mathrm{mp}=100-102{ }^{\circ} \mathrm{C} ;[\alpha]^{25} \mathrm{D}=+66.5(c=$ 0.43, in $\mathrm{CHCl}_{3}$ ); $88 \%$ ee, determined by HPLC analysis [Chiralpak column AD-H, $i \mathrm{PrOH} / n \mathrm{Hexane}$ $=40 / 60$, flow rate: $1.0 \mathrm{~mL} / \mathrm{min}, 254 \mathrm{~nm}, \mathrm{t}($ minor $)=14.67 \mathrm{~min}, \mathrm{t}($ major $)=28.50 \mathrm{~min}] ;{ }^{1} \mathbf{H} \mathbf{~ N M R}$ $\left(400 \mathrm{MHz}, \mathrm{CDCl}_{3}\right): \delta(\mathrm{ppm}) 8.06(\mathrm{~d}, J=8.4 \mathrm{~Hz}, 2 \mathrm{H}), 7.49(\mathrm{~d}, J=8.0 \mathrm{~Hz}, 2 \mathrm{H}), 7.34(\mathrm{~d}, J=8.8 \mathrm{~Hz}$, 2H), 7.24-7.20 (m, 3H), 7.15-7.13 (m, 2H), $6.82(\mathrm{~d}, J=8.8 \mathrm{~Hz}, 2 \mathrm{H}), 6.42(\mathrm{~d}, J=15.6 \mathrm{~Hz}, 1 \mathrm{H}), 5.79$ (dd, $J=15.6,8.4 \mathrm{~Hz}, 1 \mathrm{H}), 5.10-5.04(\mathrm{~m}, 1 \mathrm{H}), 4.79-4.73(\mathrm{~m}, 1 \mathrm{H}), 4.62-4.59(\mathrm{~m}, 1 \mathrm{H}), 3.93(\mathrm{~s}, 3 \mathrm{H})$, $3.74(\mathrm{~s}, 3 \mathrm{H}), 2.35-2.32(\mathrm{~m}, 1 \mathrm{H}), 2.24-2.15(\mathrm{~m}, 1 \mathrm{H}) ;{ }^{13} \mathbf{C ~ N M R}\left(100 \mathrm{MHz}, \mathrm{CDCl}_{3}\right): \delta(\mathrm{ppm}) 166.5$, 159.3, 143.5, 135.8, 133.8, 130.43, 130.36, 130.2, 128.5, 128.1, 127.1, 126.4, 126.2, 114.3, 63.9, 57.6, 55.3, 52.3, 39.3; HRMS (ESI-TOF) $\mathrm{m} / \mathrm{z}$ : $[\mathrm{M}+\mathrm{Na}]^{+}$Calcd for $\mathrm{C}_{26} \mathrm{H}_{26} \mathrm{~N}_{2} \mathrm{O}_{5} \mathrm{SNa} 501.1455$; Found 501.1457.

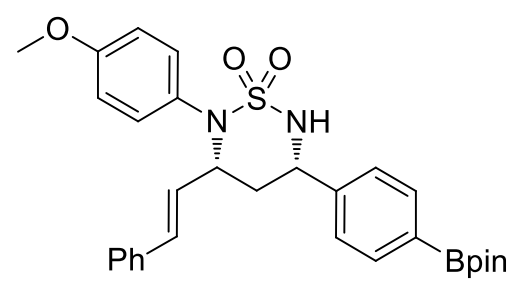

(3R,5S)-2-(4-Methoxyphenyl)-3-((E)-styryl)-5-(4-(4,4,5,5tetramethyl-1,3,2-dioxaborolan-2-yl)phenyl)-1,2,6thiadiazinane 1,1-dioxide (5q): An oven-dried $5 \mathrm{~mL}$ test-tube equipped with a septum and a magnetic stir bar was charged with $\mathrm{Pd}_{2}(\mathrm{dba})_{3}(4.6 \mathrm{mg}, 5 \mathrm{~mol} \%$ ) and ligand $\mathbf{L 1}$ (11.3 mg, $20 \mathrm{~mol} \%$ ).

The tube was then evacuated and filled with argon. This cycle was repeated three times, and toluene $(0.5 \mathrm{~mL})$ was added via syringe. The resulting solution was stirred at room temperature for $30 \mathrm{~min}$. A second oven-dried test-tube was charged with diene 1 a $(26.0 \mathrm{mg}, 2.0$ equiv), functionalized $N$ sulfonylimine $4 \mathrm{~h}$ (41.6 mg, 1.0 equiv) and $4 \AA$ MS (60.0 mg), and the system was placed under an atmosphere of argon (balloon). Then the catalyst solution was added at room temperature. The 
resulting mixture was stirred at $80{ }^{\circ} \mathrm{C}$ for $20 \mathrm{~h}$. After completion, purification by flash chromatography on silica gel (petroleum ether/EtOAc $=6 / 1$ ) gave the product 5q: $38.8 \mathrm{mg}$, white solid, $71 \%$ yield; $14: 1 \mathrm{dr} ; \mathrm{mp}=159-161^{\circ} \mathrm{C} ;[\alpha]^{25} \mathrm{D}=+50.1\left(c=1.11\right.$, in $\left.\mathrm{CHCl}_{3}\right) ; 80 \%$ ee, determined by HPLC analysis [Chiralpak column ID, $i \mathrm{PrOH} / n$ Hexane $=40 / 60$, flow rate: $1.0 \mathrm{~mL} / \mathrm{min}, 254 \mathrm{~nm}, \mathrm{t}$ $($ minor $)=16.77 \mathrm{~min}, \mathrm{t}($ major $)=26.28 \mathrm{~min}] ;{ }^{1} \mathbf{H} \mathbf{~ N M R}\left(400 \mathrm{MHz}, \mathrm{CDCl}_{3}\right): \delta(\mathrm{ppm}) 7.84(\mathrm{~d}, J=8.0$ $\mathrm{Hz}, 2 \mathrm{H}), 7.42-7.33(\mathrm{~m}, 5 \mathrm{H}), 7.24-7.14(\mathrm{~m}, 4 \mathrm{H}), 6.83(\mathrm{~d}, J=8.8 \mathrm{~Hz}, 2 \mathrm{H}), 6.42(\mathrm{~d}, J=15.6 \mathrm{~Hz}, 1 \mathrm{H})$, $5.80(\mathrm{dd}, J=15.6,8.4 \mathrm{~Hz}, 1 \mathrm{H}), 5.06-4.99(\mathrm{~m}, 1 \mathrm{H}), 4.78-4.72(\mathrm{~m}, 1 \mathrm{H}), 4.49(\mathrm{~d}, J=10.4 \mathrm{~Hz}, 1 \mathrm{H})$, $3.75(\mathrm{~s}, 3 \mathrm{H}), 2.34-2.15(\mathrm{~m}, 2 \mathrm{H}), 1.35(\mathrm{~s}, 12 \mathrm{H}) ;{ }^{13} \mathbf{C} \mathbf{N M R}\left(100 \mathrm{MHz}, \mathrm{CDCl}_{3}\right): \delta(\mathrm{ppm}) 159.2,141.6$, 135.9, 135.4, 133.6, 130.5, 130.5, 128.5, 128.0, 127.4, 126.4, 125.5, 125.4, 114.2, 84.0, 63.9, 57.9, 55.3, 39.4, 24.8; HRMS (ESI-TOF) m/z: $[\mathrm{M}+\mathrm{Na}]^{+}$Calcd for $\mathrm{C}_{30} \mathrm{H}_{35} \mathrm{BN}_{2} \mathrm{O}_{5} \mathrm{SNa}$ 569.2252; Found 569.2258 .

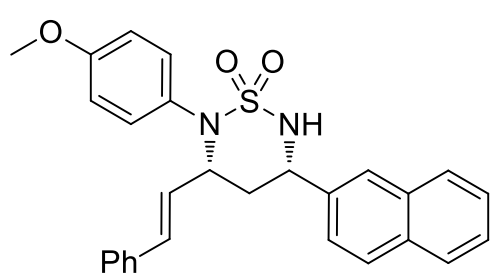

(3R,5S)-2-(4-Methoxyphenyl)-5-(naphthalen-2-yl)-3-((E)-styryl) -1,2,6-thiadiazinane 1,1-dioxide (5r): An oven-dried $5 \mathrm{~mL}$ test-tube equipped with a septum and a magnetic stir bar was charged with $\operatorname{Pd}_{2}(\mathrm{dba})_{3}(4.6 \mathrm{mg}, 5 \mathrm{~mol} \%)$ and ligand $\mathbf{L 1}(11.3 \mathrm{mg}, 20 \mathrm{~mol} \%)$. The tube was then evacuated and filled with argon. This cycle was repeated three times, and toluene $(0.5 \mathrm{~mL})$ was added via syringe. The resulting solution was stirred at room temperature for $30 \mathrm{~min}$. A second oven-dried test-tube was charged with diene $\mathbf{1 a}(26.0 \mathrm{mg}$, 2.0 equiv), functionalized $N$-sulfonylimine $4 \mathbf{i}(34.0 \mathrm{mg}, 1.0$ equiv) and $4 \AA$ MS (60.0 mg), and the system was placed under an atmosphere of argon (balloon). Then the catalyst solution was added at room temperature. The resulting mixture was stirred at $80{ }^{\circ} \mathrm{C}$ for $20 \mathrm{~h}$. After completion, purification by flash chromatography on silica gel (petroleum ether/EtOAc $=6 / 1$ ) gave the product $5 \mathrm{r}: 46.0 \mathrm{mg}$, as a white solid, $96 \%$ yield; $>19: 1 \mathrm{dr} ; \mathrm{mp}=116-118{ }^{\circ} \mathrm{C} ;[\alpha]^{25} \mathrm{D}=+91.3\left(c=0.48\right.$, in $\left.\mathrm{CHCl}_{3}\right) ; 91 \%$ ee, determined by HPLC analysis [Chiralpak column IB, $i \mathrm{PrOH} / n \mathrm{Hexane}=40 / 60$, flow rate: 1.0 $\mathrm{mL} / \mathrm{min}, 254 \mathrm{~nm}, \mathrm{t}$ (major) $=16.07 \mathrm{~min}, \mathrm{t}($ minor $)=20.63 \mathrm{~min}] ;{ }^{1} \mathbf{H} \mathbf{~ N M R}\left(400 \mathrm{MHz}, \mathrm{CDCl}_{3}\right): \delta$ (ppm) 7.86-7.82 (m, 4H), 7.53-7.49 (m, 3H), $7.36(\mathrm{~d}, J=8.4 \mathrm{~Hz}, 2 \mathrm{H}), 7.23-7.14(\mathrm{~m}, 5 \mathrm{H}), 6.78(\mathrm{~d}, J$ $=8.4 \mathrm{~Hz}, 2 \mathrm{H}), 6.43(\mathrm{~d}, J=16.0 \mathrm{~Hz}, 1 \mathrm{H}), 5.82(\mathrm{dd}, J=16.0,8.8 \mathrm{~Hz}, 1 \mathrm{H}), 5.19-5.14(\mathrm{~m}, 1 \mathrm{H}), 4.81-$ $4.76(\mathrm{~m}, 1 \mathrm{H}), 4.69-4.66(\mathrm{~m}, 1 \mathrm{H}), 3.69(\mathrm{~s}, 3 \mathrm{H}), 2.41-2.28(\mathrm{~m}, 2 \mathrm{H}) ;{ }^{13} \mathrm{C} \mathrm{NMR}\left(100 \mathrm{MHz}, \mathrm{CDCl}_{3}\right): \delta$ (ppm) 159.2, 136.1, 136.0, 133.5, 133.2, 133.1, 130.6, 130.5, 128.8, 128.5, 128.04, 128.00, 127.7, 127.5, 126.6, 126.5, 126.4, 124.9, 124.3, 114.2, 57.9, 55.2, 39.4; HRMS (ESI-TOF) m/z: [M + Na] ${ }^{+}$ Calcd for $\mathrm{C}_{28} \mathrm{H}_{26} \mathrm{~N}_{2} \mathrm{O}_{3} \mathrm{SNa}$ 493.1556; Found 493.1561. 


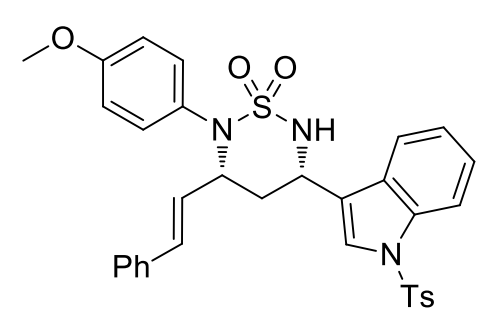

(3R,5S)-2-(4-Methoxyphenyl)-3-((E)-styryl)-5-(1-tosyl-1H-indol-3yl)-1,2,6-thiadiazinane 1,1-dioxide (5s): An oven-dried $5 \mathrm{~mL}$ testtube equipped with a septum and a magnetic stir bar was charged with $\mathrm{Pd}_{2}(\mathrm{dba})_{3}(4.6 \mathrm{mg}, 5 \mathrm{~mol} \%)$ and ligand $\mathbf{L 1}(11.3 \mathrm{mg}, 20 \mathrm{~mol} \%)$. The tube was then evacuated and filled with argon. This cycle was repeated three times, and toluene $(0.5 \mathrm{~mL})$ was added via syringe. The resulting solution was stirred at room temperature for $30 \mathrm{~min}$. A second oven-dried test-tube was charged with diene $1 \mathbf{a}$ ( $26.0 \mathrm{mg}, 2.0$ equiv), functionalized $N$-sulfonylimine $\mathbf{4 j}$ ( $48.4 \mathrm{mg}, 1.0$ equiv) and $4 \AA$ MS $(60.0 \mathrm{mg})$, and the system was placed under an atmosphere of argon (balloon). Then the catalyst solution was added at room temperature. The resulting mixture was stirred at $80^{\circ} \mathrm{C}$ for $60 \mathrm{~h}$. After completion, purification by flash chromatography on silica gel (petroleum ether/EtOAc $=6 / 1$ ) gave the product 5s: $46.2 \mathrm{mg}$, as a white solid, $75 \%$ yield; $>19: 1 \mathrm{dr} ; \mathrm{mp}=103-105{ }^{\circ} \mathrm{C} ;[\alpha]^{25} \mathrm{D}=+56.9(c$ $=0.13$, in $\mathrm{CHCl}_{3}$ ); $92 \%$ ee, determined by HPLC analysis [Chiralpak column IB, $i \mathrm{PrOH} / n \mathrm{Hexane}=$ 40/60, flow rate: $1.0 \mathrm{~mL} / \mathrm{min}, 254 \mathrm{~nm}, \mathrm{t}$ (major) $=14.47 \mathrm{~min}, \mathrm{t}($ minor $)=21.98 \mathrm{~min}] ;{ }^{1} \mathbf{H}$ NMR $(400$ $\left.\mathrm{MHz}, \mathrm{CDCl}_{3}\right): \delta(\mathrm{ppm}) 8.00(\mathrm{~d}, J=8.4 \mathrm{~Hz}, 1 \mathrm{H}), 7.82-7.77(\mathrm{~m}, 3 \mathrm{H}), 7.55(\mathrm{~s}, 1 \mathrm{H}), 7.40-7.18(\mathrm{~m}, 11 \mathrm{H})$, $6.81(\mathrm{~d}, J=8.8 \mathrm{~Hz}, 2 \mathrm{H}), 6.45(\mathrm{~d}, J=15.6 \mathrm{~Hz}, 1 \mathrm{H}), 5.84(\mathrm{dd}, J=15.6,8.4 \mathrm{~Hz}, 1 \mathrm{H}), 5.31-5.22(\mathrm{~m}$, $1 \mathrm{H}), 4.82-4.76(\mathrm{~m}, 1 \mathrm{H}), 4.80-4.75(\mathrm{~m}, 1 \mathrm{H}), 4.36(\mathrm{~d}, J=11.2 \mathrm{~Hz}, 1 \mathrm{H}), 3.75(\mathrm{~s}, 3 \mathrm{H}), 2.48-2.30(\mathrm{~m}$, 2H), 2.35 (s, 3H); ${ }^{13} \mathbf{C}$ NMR (100 MHz, $\left.\mathrm{CDCl}_{3}\right): \delta(\mathrm{ppm}) 159.3,145.3,135.9,135.2,134.9,133.8$, 130.5, 130.4, 130.1, 128.6, 128.5, 128.1, 127.3, 126.8, 126.4, 125.5, 123.6, 122.9, 120.6, 114.2, 113.6, 63.9, 55.3, 51.2, 38.1, 21.6; HRMS (ESI-TOF) $\mathrm{m} / \mathrm{z}:[\mathrm{M}+\mathrm{Na}]^{+} \mathrm{Calcd}$ for $\mathrm{C}_{33} \mathrm{H}_{31} \mathrm{~N}_{3} \mathrm{O}_{5} \mathrm{~S}_{2} \mathrm{Na}$ 636.1597; Found 636.1597.

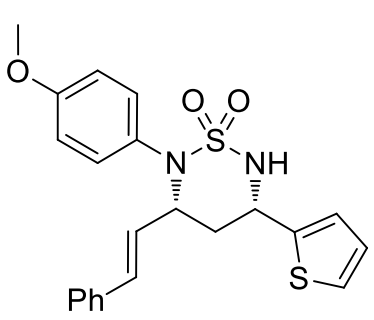

(3R,5S)-2-(4-Methoxyphenyl)-3-((E)-styryl)-5-(thiophen-2-yl)-1,2,6thiadiazinane 1,1-dioxide (5t): An oven-dried $5 \mathrm{~mL}$ test-tube equipped with a septum and a magnetic stir bar was charged with $\mathrm{Pd}_{2}(\mathrm{dba})_{3}(4.6 \mathrm{mg}, 5$ mol \%) and ligand $\mathbf{L 1}(11.3 \mathrm{mg}, 20 \mathrm{~mol} \%)$. The tube was then evacuated and filled with argon. This cycle was repeated three times, and toluene $(0.5$ $\mathrm{mL}$ ) was added via syringe. The resulting solution was stirred at room temperature for $30 \mathrm{~min}$. A second oven-dried test-tube was charged with diene 1a $(26.0 \mathrm{mg}, 2.0$ equiv), functionalized $\mathrm{N}$ sulfonylimine $4 \mathbf{k}$ ( $29.6 \mathrm{mg}, 1.0$ equiv) and $4 \AA \mathrm{MS}(60.0 \mathrm{mg}$ ), and the system was placed under an atmosphere of argon (balloon). Then the catalyst solution was added at room temperature. The resulting mixture was stirred at $80{ }^{\circ} \mathrm{C}$ for $20 \mathrm{~h}$. After completion, purification by flash chromatography on silica gel (petroleum ether/EtOAc $=6 / 1$ ) gave the product $5 \mathrm{t}: 32.0 \mathrm{mg}$, as a semisolid, 78\% yield; >19:1 dr; $[\alpha]^{25} \mathrm{D}=+90.6\left(c=1.3\right.$, in $\left.\mathrm{CHCl}_{3}\right) ; 82 \%$ ee, determined by HPLC analysis [Chiralpak column AD-H, $i \mathrm{PrOH} / n \mathrm{Hexane}=40 / 60$, flow rate: $1.0 \mathrm{~mL} / \mathrm{min}, 254 \mathrm{~nm}, \mathrm{t}$ (minor) $=21.24 \mathrm{~min}, \mathrm{t}($ major $)=24.11 \mathrm{~min}] ;{ }^{1} \mathbf{H} \mathbf{~ N M R}\left(400 \mathrm{MHz}, \mathrm{CDCl}_{3}\right): \delta(\mathrm{ppm}) 7.40-7.32(\mathrm{~m}, 3 \mathrm{H}), 7.27-$ 
$7.19(\mathrm{~m}, 3 \mathrm{H}), 7.18-7.16(\mathrm{~m}, 2 \mathrm{H}), 7.10(\mathrm{~d}, J=3.6 \mathrm{~Hz}, 1 \mathrm{H}), 7.02(\mathrm{dd}, J=4.8,4.0 \mathrm{~Hz}, 1 \mathrm{H}), 6.83(\mathrm{~d}, J$ $=8.8 \mathrm{~Hz}, 2 \mathrm{H}), 6.44(\mathrm{~d}, J=16.0 \mathrm{~Hz}, 1 \mathrm{H}), 5.80(\mathrm{dd}, J=16.0,8.8 \mathrm{~Hz}, 1 \mathrm{H}), 5.30-5.22(\mathrm{~m}, 1 \mathrm{H}), 4.77-$ $4.72(\mathrm{~m}, 1 \mathrm{H}), 4.47(\mathrm{~d}, J=10.4 \mathrm{~Hz}, 1 \mathrm{H}), 3.75(\mathrm{~s}, 3 \mathrm{H}), 2.47-2.43(\mathrm{~m}, 1 \mathrm{H}), 2.31-2.22(\mathrm{~m}, 1 \mathrm{H}) ;{ }^{13} \mathrm{C}$ NMR (100 MHz, $\left.\mathrm{CDCl}_{3}\right): \delta$ (ppm) 159.3, 142.0, 135.9, 133.8, 130.4, 130.4, 128.5, 128.1, 127.2, 127.0, 126.4, 125.6, 124.8, 114.2, 63.7, 55.3, 54.1, 40.1; HRMS (ESI-TOF) m/z: $[\mathrm{M}+\mathrm{Na}]^{+}$Calcd for $\mathrm{C}_{22} \mathrm{H}_{22} \mathrm{~N}_{2} \mathrm{O}_{3} \mathrm{~S}_{2} \mathrm{Na} 449.0964$; Found 449.0970.

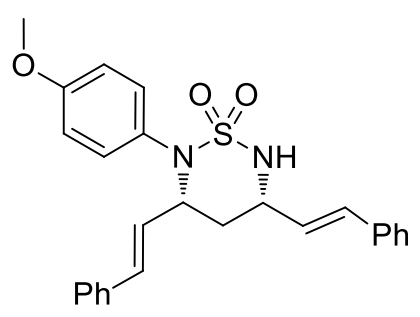

\section{(3R,5S)-2-(4-Methoxyphenyl)-3,5-di((E)-styryl)-1,2,6-thiadiazinane} 1,1-dioxide (5u): An oven-dried $5 \mathrm{~mL}$ test-tube equipped with a septum and a magnetic stir bar was charged with $\mathrm{Pd}_{2}(\mathrm{dba})_{3}(4.6 \mathrm{mg}, 5 \mathrm{~mol} \%)$ and ligand $\mathbf{L 1}$ (11.3 mg, $20 \mathrm{~mol} \%$ ). The tube was then evacuated and filled with argon. This cycle was repeated three times, and toluene $(0.5 \mathrm{~mL})$ was added via syringe. The resulting solution was stirred at room temperature for $30 \mathrm{~min}$. A second ovendried test-tube was charged with diene $1 \mathbf{a}(26.0 \mathrm{mg}, 2.0$ equiv), functionalized $N$-sulfonylimine $4 \mathbf{l}$ (31.6 mg, 1.0 equiv) and $4 \AA$ MS (60.0 mg), and the system was placed under an atmosphere of argon (balloon). Then the catalyst solution was added at room temperature. The resulting mixture was stirred at $80{ }^{\circ} \mathrm{C}$ for $20 \mathrm{~h}$. After completion, purification by flash chromatography on silica gel (petroleum ether/EtOAc $=6 / 1$ ) gave the product 5u: $37.2 \mathrm{mg}$, as a white solid, 83\% yield; 7:1 dr; $[\alpha]^{25} \mathrm{D}=+80.0\left(c=0.35\right.$, in $\left.\mathrm{CHCl}_{3}\right) ; 73 \%$ ee, determined by HPLC analysis [Chiralpak column IA, $i \mathrm{PrOH} / n$ Hexane $=40 / 60$, flow rate: $1.0 \mathrm{~mL} / \mathrm{min}, 254 \mathrm{~nm}, \mathrm{t}$ (minor) $=9.68 \mathrm{~min}, \mathrm{t}$ (major) $=22.32 \mathrm{~min}]$; ${ }^{1} \mathbf{H}$ NMR (400 MHz, $\left.\mathrm{CDCl}_{3}\right): \delta(\mathrm{ppm}) 7.39-7.14(\mathrm{~m}, 12 \mathrm{H}), 6.81(\mathrm{~d}, J=9.2 \mathrm{~Hz}, 2 \mathrm{H}), 6.68(\mathrm{~d}, J=16.0$, $1 \mathrm{H}), 6.40(\mathrm{~d}, J=16.0 \mathrm{~Hz}, 1 \mathrm{H}), 6.19(\mathrm{dd}, J=16.0,5.2 \mathrm{~Hz}, 1 \mathrm{H}), 5.78(\mathrm{dd}, J=16.0,8.8 \mathrm{~Hz}, 1 \mathrm{H}), 4.71-$ $4.61(\mathrm{~m}, 2 \mathrm{H}), 4.26(\mathrm{~d}, J=10.8 \mathrm{~Hz}, 1 \mathrm{H}), 3.73(\mathrm{~s}, 3 \mathrm{H}), 2.23-2.21(\mathrm{~m}, 1 \mathrm{H}), 2.02-1.93(\mathrm{~m}, 1 \mathrm{H}) ;{ }^{13} \mathrm{C}$ NMR (100 MHz, $\left.\mathrm{CDCl}_{3}\right): \delta$ (ppm) 159.2, 136.0, 135.8, 133.5, 131.9, 130.6, 130.4, 128.7, 128.5, 128.3 128.0, 127.5, 126.6, 126.5, 126.4, 114.2, 63.8, 55.9, 55.3, 39.1; HRMS (ESI-TOF) m/z: [M + $\mathrm{Na}]^{+}$Calcd for $\mathrm{C}_{26} \mathrm{H}_{26} \mathrm{~N}_{2} \mathrm{O}_{3} \mathrm{SNa} 469.1556$; Found 469.1554 .

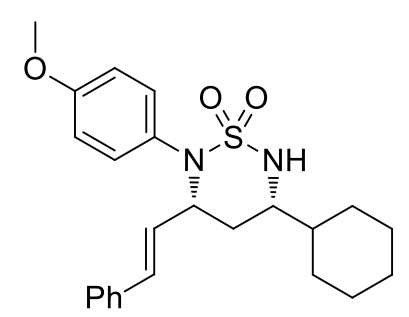

(3R,5S)-5-Cyclohexyl-2-(4-methoxyphenyl)-3-((E)-styryl)-1,2,6thiadiazinane 1,1-dioxide (5v): An oven-dried $5 \mathrm{~mL}$ test-tube equipped with a septum and a magnetic stir bar was charged with $\operatorname{Pd}_{2}(\mathrm{dba})_{3}(4.6 \mathrm{mg}$, $5 \mathrm{~mol} \%$ ) and ligand $\mathbf{L 1}$ (11.3 $\mathrm{mg}, 20 \mathrm{~mol} \%$ ). The tube was then evacuated and filled with argon. This cycle was repeated three times, and toluene $(0.5$ $\mathrm{mL}$ ) was added via syringe. The resulting solution was stirred at room temperature for $30 \mathrm{~min}$. A second oven-dried test-tube was charged with diene $1 \mathbf{a}(26.0 \mathrm{mg}, 2.0$ equiv), functionalized $\mathrm{N}$ sulfonylimine $\mathbf{4 m}$ (29.6 mg, 1.0 equiv) and 4 A MS (60.0 mg), and the system was placed under an atmosphere of argon (balloon). Then the catalyst solution was added at room temperature. The 
resulting mixture was stirred at $80{ }^{\circ} \mathrm{C}$ for $20 \mathrm{~h}$. After completion, purification by flash chromatography on silica gel (petroleum ether/EtOAc $=6 / 1$ ) gave the product 5v: $30.0 \mathrm{mg}$, as a colorless semisolid, $70 \%$ yield; $>19: 1 \mathrm{dr} ;[\alpha]^{25} \mathrm{D}=+66.0\left(c=1.3\right.$, in $\left.\mathrm{CHCl}_{3}\right) ; 77 \%$ ee, determined by HPLC analysis [Chiralpak column AD-H, $i \mathrm{PrOH} / n \mathrm{Hexane}=40 / 60$, flow rate: $1.0 \mathrm{~mL} / \mathrm{min}, 254 \mathrm{~nm}$, $\mathrm{t}($ minor $)=8.94 \mathrm{~min}, \mathrm{t}$ (major) $=11.73 \mathrm{~min}] ;{ }^{1} \mathbf{H} \mathbf{N M R}\left(400 \mathrm{MHz}, \mathrm{CDCl}_{3}\right): \delta(\mathrm{ppm}) 7.35-7.13(\mathrm{~m}$, $7 \mathrm{H}), 6.80(\mathrm{~d}, J=8.4 \mathrm{~Hz}, 2 \mathrm{H}), 6.35(\mathrm{~d}, J=16.0 \mathrm{~Hz}, 1 \mathrm{H}), 5.76(\mathrm{dd}, J=16.0,8.4 \mathrm{~Hz}, 1 \mathrm{H}), 4.56-4.51$ (m, 1H), 4.08-4.06 (d, $J=8.0 \mathrm{~Hz}, 1 \mathrm{H}), 3.73(\mathrm{~s}, 3 \mathrm{H}), 3.68-3.61(\mathrm{~m}, 1 \mathrm{H}), 2.02-1.94(\mathrm{~m}, 2 \mathrm{H}), 1.78-$ $1.62(\mathrm{~m}, 5 \mathrm{H}), 1.43-1.02(\mathrm{~m}, 6 \mathrm{H}) ;{ }^{13} \mathbf{C}$ NMR (100 MHz, $\left.\mathrm{CDCl}_{3}\right): \delta$ (ppm) 159.1, 136.1, 133.1, 130.9, 130.3, 128.5, 128.1, 127.9, 126.3, 114.1, 64.1, 59.5, 55.3, 42.1, 37.1, 29.0, 28.8, 26.2, 25.9, 25.8; HRMS (ESI-TOF) m/z: [M + Na] ${ }^{+}$Calcd for $\mathrm{C}_{24} \mathrm{H}_{30} \mathrm{~N}_{2} \mathrm{O}_{3} \mathrm{SNa} 449.1869$; Found 449.1874.

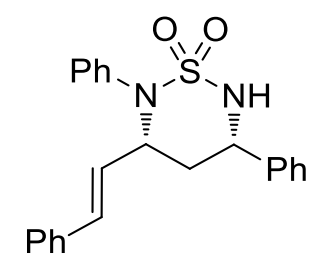

$(3 R, 5 S)-2,5-D i p h e n y l-3-((E)$-styryl)-1,2,6-thiadiazinane 1,1-dioxide $(5 \mathrm{w})$ : An oven-dried $5 \mathrm{~mL}$ test-tube equipped with a septum and a magnetic stir bar was charged with $\operatorname{Pd}_{2}(\mathrm{dba})_{3}(4.6 \mathrm{mg}, 5 \mathrm{~mol} \%)$ and ligand $\mathbf{L 1}$ (11.3 mg, 20 mol \%). The tube was then evacuated and filled with argon. This cycle was repeated three times, and toluene $(0.5 \mathrm{~mL})$ was added via syringe. The resulting solution was stirred at room temperature for $30 \mathrm{~min}$. A second oven-dried test-tube was charged with diene $1 \mathbf{a}$ (26.0 mg, 2.0 equiv), functionalized $N$-sulfonylimine $4 \mathbf{n}$ (26.0 mg, 1.0 equiv) and $4 \AA$ MS $(60.0 \mathrm{mg})$, and the system was placed under an atmosphere of argon (balloon). Then the catalyst solution was added at room temperature. The resulting mixture was stirred at $80{ }^{\circ} \mathrm{C}$ for $20 \mathrm{~h}$. After completion, purification by flash chromatography on silica gel (petroleum ether/EtOAc $=6 / 1$ ) gave the product 5w: $35.5 \mathrm{mg}$, as a white solid, 91\% yield; >19:1 dr; $\mathrm{mp}=72-74{ }^{\circ} \mathrm{C} ;[\alpha]^{25} \mathrm{D}=+80.4(c=$ 1.43, in $\mathrm{CHCl}_{3}$ ); $91 \%$ ee, determined by HPLC analysis [Chiralpak column AD-H, $i \mathrm{PrOH} / n \mathrm{Hexane}$ $=40 / 60$, flow rate: $1.0 \mathrm{~mL} / \mathrm{min}, 254 \mathrm{~nm}, \mathrm{t}$ (minor) $=13.93 \mathrm{~min}, \mathrm{t}($ major $)=18.63 \mathrm{~min}] ;{ }^{1} \mathbf{H} \mathbf{~ N M R}$ $\left(400 \mathrm{MHz}, \mathrm{CDCl}_{3}\right): \delta(\mathrm{ppm}) 7.45-7.09(\mathrm{~m}, 15 \mathrm{H}), 6.40(\mathrm{~d}, J=16.0 \mathrm{~Hz}, 1 \mathrm{H}), 5.76(\mathrm{dd}, J=16.0,8.4$ $\mathrm{Hz}, 1 \mathrm{H}), 5.04-4.98(\mathrm{~m}, 1 \mathrm{H}), 4.81-4.76(\mathrm{~m}, 1 \mathrm{H}), 4.70-4.65(\mathrm{~m}, 1 \mathrm{H}), 2.30-2.15(\mathrm{~m}, 2 \mathrm{H}) ;{ }^{13} \mathbf{C} \mathbf{~ N M R}$ $\left(100 \mathrm{MHz}, \mathrm{CDCl}_{3}\right): \delta$ (ppm) 138.7, 138.1, 135.9, 133.7, 129.5, 129.0, 128.9, 128.5, 128.3, 128.0, 127.3, 126.3, 126.2, 63.8, 57.9, 39.4; HRMS (ESI-TOF) m/z: $[\mathrm{M}+\mathrm{Na}]^{+}$Calcd for $\mathrm{C}_{23} \mathrm{H}_{22} \mathrm{~N}_{2} \mathrm{O}_{2} \mathrm{SNa}$ 413.1294; Found 413.1303.

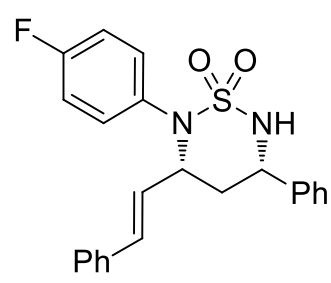

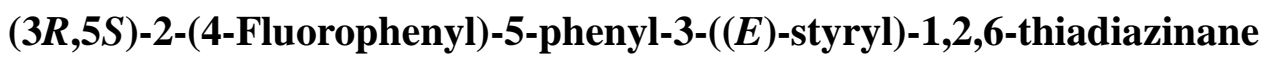
1,1-dioxide (5x): An oven-dried $5 \mathrm{~mL}$ test-tube equipped with a septum and a magnetic stir bar was charged with $\mathrm{Pd}_{2}(\mathrm{dba})_{3}(4.6 \mathrm{mg}, 5 \mathrm{~mol} \%)$ and ligand $\mathbf{L 1}$ (11.3 mg, $20 \mathrm{~mol} \%)$. The tube was then evacuated and filled with argon. This cycle was repeated three times, and toluene $(0.5 \mathrm{~mL})$ was added via syringe.

The resulting solution was stirred at room temperature for $30 \mathrm{~min}$. A second oven-dried test-tube was 
charged with diene 1a (26.0 mg, 2.0 equiv), functionalized $N$-sulfonylimine 4o (27.8 $\mathrm{mg}, 1.0$ equiv) and $4 \AA$ MS (60.0 mg), and the system was placed under an atmosphere of argon (balloon). Then the catalyst solution was added at room temperature. The resulting mixture was stirred at $80{ }^{\circ} \mathrm{C}$ for $20 \mathrm{~h}$. After completion, purification by flash chromatography on silica gel (petroleum ether/EtOAc $=6 / 1$ ) gave the product 5x: $39.0 \mathrm{mg}$, as a faint semisolid, $96 \%$ yield; $>19: 1 \mathrm{dr}$; $[\alpha]^{25} \mathrm{D}=+81.6(c=1.95$, in $\mathrm{CHCl}_{3}$ ); 93\% ee, determined by HPLC analysis [Chiralpak column AD-H, $i \mathrm{PrOH} / n \mathrm{Hexane}=20 / 80$, flow rate: $1.0 \mathrm{~mL} / \mathrm{min}, 254 \mathrm{~nm}, \mathrm{t}$ (minor) $=18.80 \mathrm{~min}, \mathrm{t}$ (major) $=21.19 \mathrm{~min}] ;{ }^{1} \mathbf{H} \mathbf{~ N M R}(400 \mathrm{MHz}$, $\left.\mathrm{CDCl}_{3}\right): \delta(\mathrm{ppm}) 7.43-7.33(\mathrm{~m}, 7 \mathrm{H}), 7.24-7.20(\mathrm{~m}, 3 \mathrm{H}), 7.13(\mathrm{~d}, J=7.2 \mathrm{~Hz}, 2 \mathrm{H}), 7.01-6.97$ (m, 2H), $6.42(\mathrm{~d}, J=15.6 \mathrm{~Hz}, 1 \mathrm{H}), 5.76(\mathrm{dd}, J=15.6,8.4 \mathrm{~Hz}, 1 \mathrm{H}), 5.05-4.99(\mathrm{~m}, 1 \mathrm{H}), 4.76-4.72(\mathrm{~m}, 1 \mathrm{H})$, 4.60-4.54 (m, 1H), 2.33-2.17 (m, 2H); $\left.{ }^{13} \mathbf{C ~ N M R ~ ( 1 0 0 ~ M H z , ~} \mathrm{CDCl}_{3}\right): \delta(\mathrm{ppm}) 162.1(\mathrm{~d}, J=246.5$ $\mathrm{Hz}), 138.5,135.7,134.1,134.0$ (d, $J=3.0 \mathrm{~Hz}), 131.1$ (d, $J=8.7 \mathrm{~Hz}), 129.0,128.6,128.2,127.0$, 126.3, 126.2, 116.0, 115.8, 64.1, 57.9, 39.4; HRMS (ESI-TOF) m/z: $[\mathrm{M}+\mathrm{Na}]^{+}$Calcd for $\mathrm{C}_{23} \mathrm{H}_{21} \mathrm{FN}_{2} \mathrm{O}_{2} \mathrm{SNa} 431.1200$; Found 431.1199.

\subsection{Asymmetric reaction on a gram scale}

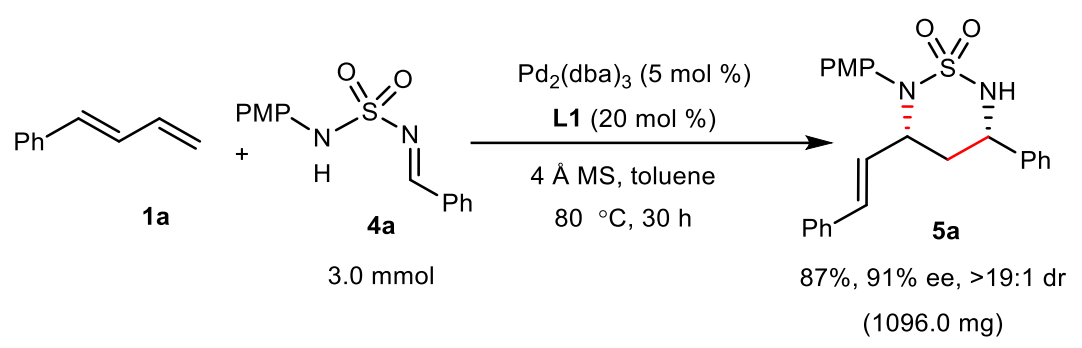

An oven-dried $50 \mathrm{~mL}$ flask equipped with a septum and a magnetic stir bar was charged with $\mathrm{Pd}_{2}(\mathrm{dba})_{3}(137.0 \mathrm{mg}, 5 \mathrm{~mol} \%)$ and ligand $\mathbf{L 1}(339.0 \mathrm{mg}, 20 \mathrm{~mol} \%)$. The flask was then evacuated and filled with argon. This cycle was repeated three times, and toluene $(15.0 \mathrm{~mL})$ was added via syringe. The resulting solution was stirred at room temperature for $30 \mathrm{~min}$. A second oven-dried flask was charged with diene $1 \mathbf{1 a}$ (780.0 mg, 2.0 equiv), functionalized $N$-sulfonylimine 4a (780.0 mg, 3.0 mmol) and $4 \AA$ MS (1800.0 mg), and the system was placed under an atmosphere of argon (balloon). Then the catalyst solution was added at room temperature. The resulting mixture was stirred at $80{ }^{\circ} \mathrm{C}$ for $30 \mathrm{~h}$. After completion, the mixture was filterted, and the solvent was evaporated under reduced pressure. The residue was purified by flash chromatography on silica gel (petroleum ether/EtOAc $=$ 6:1) to give the product 5a, $1096.0 \mathrm{mg}, 87 \%$ yield, $91 \%$ ee, $>19: 1 \mathrm{dr}$. 
2.7 General procedure of asymmetric cascade vinylogous addition/allylic alkylation reaction of 1,3-dienes 1 with salicylaldehyde-derived $N$-sulfonylimines 6

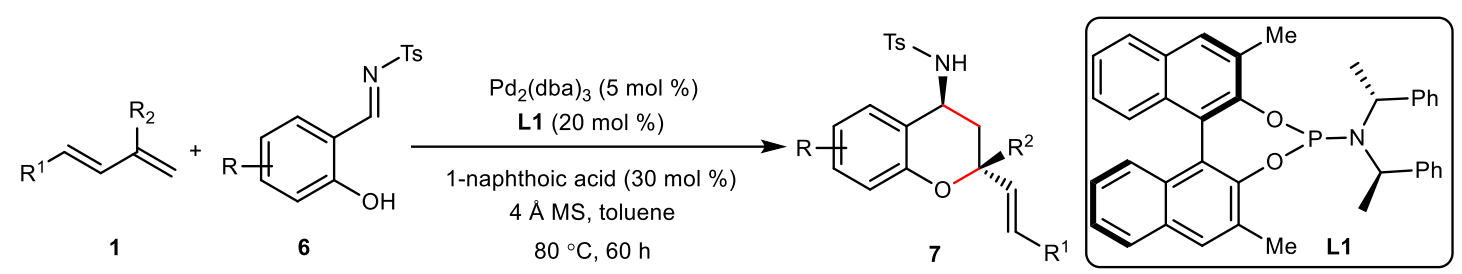

An oven-dried $5 \mathrm{~mL}$ test-tube equipped with a septum and a magnetic stir bar was charged with $\mathrm{Pd}_{2}(\mathrm{dba})_{3}(4.6 \mathrm{mg}, 5 \mathrm{~mol} \%$ ) and ligand L1 (11.3 mg, $20 \mathrm{~mol} \%$ ). The tube was then evacuated and filled with argon. This cycle was repeated three times, and toluene $(0.5 \mathrm{~mL})$ was added via syringe. The resulting solution was stirred at room temperature for $30 \mathrm{~min}$. A second oven-dried test-tube was charged with diene 1 (2.0 equiv), salicylaldehyde-derived $N$-sulfonylimine 6 (1.0 equiv), 1-naphthoic acid (5.2 mg, $30 \mathrm{~mol} \%$ ) and $4 \AA$ MS (60.0 mg), and the system was placed under an atmosphere of argon (balloon). Then the catalyst solution was added at room temperature. The resulting mixture was stirred at $80{ }^{\circ} \mathrm{C}$ for $60 \mathrm{~h}$. After completion, the product 7 was obtained by flash chromatography on silica gel (petroleum ether/EtOAc). The racemic 7 was obtained under the catalysis of $\operatorname{Pd}\left(\mathrm{PPh}_{3}\right)_{4}(5$ $\mathrm{mol} \%)$ and $\mathrm{BnCO}_{2} \mathrm{H}(30 \mathrm{~mol} \%)$.

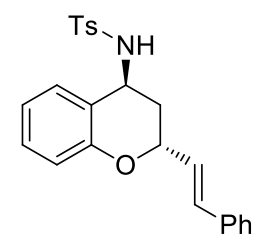

4-Methyl- $N-((2 R, 4 S)-2-((E)$-styryl)chroman-4-yl)benzenesulfonamide (7a): An oven-dried $5 \mathrm{~mL}$ test-tube equipped with a septum and a magnetic stir bar was charged with $\mathrm{Pd}_{2}(\mathrm{dba})_{3}(4.6 \mathrm{mg}, 5 \mathrm{~mol} \%)$ and ligand L1 (11.3 mg, $20 \mathrm{~mol} \%$ ). The tube was then evacuated and filled with argon. This cycle was repeated three times, and toluene $(0.5 \mathrm{~mL})$ was added via syringe. The resulting solution was stirred at room temperature for $30 \mathrm{~min}$. A second oven-dried test-tube was charged with diene 1a (26.0 mg, 2.0 equiv), salicylaldehyde-derived $N$-sulfonylimine $6 \mathbf{6}$ (27.5 mg, 1.0 equiv), 1-naphthoic acid (5.2 $\mathrm{mg}, 30 \mathrm{~mol} \%$ ) and $4 \AA$ MS (60.0 mg), and the system was placed under an atmosphere of argon (balloon). Then the catalyst solution was added at room temperature. The resulting mixture was stirred at $80^{\circ} \mathrm{C}$ for $60 \mathrm{~h}$. After completion, purification by flash chromatography on silica gel (petroleum ether/EtOAc $=10 / 1$ ) gave the product 7a: $20.3 \mathrm{mg}$, as a white solid, $50 \%$ yield; $>19: 1 \mathrm{dr} ; \mathrm{mp}=156-158{ }^{\circ} \mathrm{C} ;[\alpha]^{25} \mathrm{D}=-$ $22.1\left(c=0.86\right.$, in $\left.\mathrm{CHCl}_{3}\right)$; 96\% ee, determined by HPLC analysis [Chiralpak column IA, $i \mathrm{PrOH} / n$ Hexane $=40 / 60$, flow rate: $1.0 \mathrm{~mL} / \mathrm{min}, 254 \mathrm{~nm}, \mathrm{t}$ (major) $=6.46 \mathrm{~min}, \mathrm{t}$ (minor) $=8.62 \mathrm{~min}$ ]; ${ }^{1} \mathbf{H}$ NMR (400 MHz, $\left.\mathrm{CDCl}_{3}\right): \delta(\mathrm{ppm}) 7.85(\mathrm{~d}, J=8.0 \mathrm{~Hz}, 2 \mathrm{H}), 7.41-7.25(\mathrm{~m}, 6 \mathrm{H}), 7.19-7.15(\mathrm{~m}$, $1 \mathrm{H}), 6.87(\mathrm{~d}, J=8.4 \mathrm{~Hz}, 1 \mathrm{H}), 6.78(\mathrm{t}, J=7.6 \mathrm{~Hz}, 1 \mathrm{H}), 6.66(\mathrm{~d}, J=16.0 \mathrm{~Hz}, 1 \mathrm{H}), 6.61(\mathrm{~d}, J=7.6 \mathrm{~Hz}$, 2H), $6.26(\mathrm{dd}, J=16.0,6.0 \mathrm{~Hz}, 1 \mathrm{H}), 4.78-4.75(\mathrm{~m}, 2 \mathrm{H}), 4.39$ (brs, 1H), 2.47 (s, 3H), 2.35 (d, $J=14.0$ $\mathrm{Hz}, 1 \mathrm{H}), 1.96-1.90(\mathrm{~m}, 1 \mathrm{H}) ;{ }^{13} \mathbf{C ~ N M R}\left(100 \mathrm{MHz}, \mathrm{CDCl}_{3}\right): \delta$ (ppm) 154.7, 143.9, 137.4, 136.2, 132.1, 130.0, 129.9, 129.3, 128.6, 128.0, 127.5, 127.3, 126.6, 120.9, 119.8, 117.6, 71.9, 47.8, 35.0, 21.6; 
HRMS (ESI-TOF) m/z: [M + Na $]^{+}$Calcd for $\mathrm{C}_{24} \mathrm{H}_{23} \mathrm{NO}_{3} \mathrm{SNa} 428.1291$; Found 428.1294.

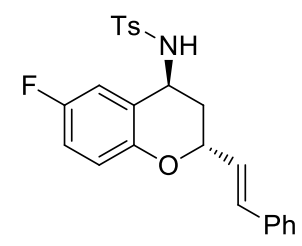

$N$-((2R,4S)-6-Fluoro-2-((E)-styryl)chroman-4-yl)-4-methylbenzene sulfonamide (7b): An oven-dried $5 \mathrm{~mL}$ test-tube equipped with a septum and a magnetic stir bar was charged with $\mathrm{Pd}_{2}(\mathrm{dba})_{3}(4.6 \mathrm{mg}, 5 \mathrm{~mol} \%)$ and ligand $\mathbf{L 1}$

(11.3 mg, $20 \mathrm{~mol} \%)$. The tube was then evacuated and filled with argon. This cycle was repeated three times, and toluene $(0.5 \mathrm{~mL})$ was added via syringe. The resulting solution was stirred at room temperature for $30 \mathrm{~min}$. A second oven-dried test-tube was charged with diene 1a (26.0 mg, 2.0 equiv), salicylaldehyde-derived $N$-sulfonylimine $6 \mathbf{b}$ (29.3 mg, 1.0 equiv), 1-naphthoic acid (5.2 mg, $30 \mathrm{~mol} \%$ ) and $4 \AA$ MS (60.0 mg), and the system was placed under an atmosphere of argon (balloon). Then the catalyst solution was added at room temperature. The resulting mixture was stirred at $80{ }^{\circ} \mathrm{C}$ for $60 \mathrm{~h}$. After completion, purification by flash chromatography on silica gel (petroleum ether/EtOAc = 10/1) gave the product 7b: $32.3 \mathrm{mg}$, as a white solid, 76\% yield; >19:1 dr; $\mathrm{mp}=204-206{ }^{\circ} \mathrm{C} ;[\alpha]^{25} \mathrm{D}=-29.1\left(c=0.54\right.$, in $\left.\mathrm{CHCl}_{3}\right) ; 95 \%$ ee, determined by HPLC analysis [Chiralpak column IA, $i \mathrm{PrOH} / n$ Hexane $=20 / 80$, flow rate: $1.0 \mathrm{~mL} / \mathrm{min}, 254 \mathrm{~nm}, \mathrm{t}$ (major) $=8.78 \mathrm{~min}$, $\mathrm{t}($ minor $)=12.12 \mathrm{~min}] ;{ }^{1} \mathbf{H}$ NMR $\left(400 \mathrm{MHz} \mathrm{CDCl}_{3}\right): \delta(\mathrm{ppm}) 7.84(\mathrm{~d}, J=8.4 \mathrm{~Hz}, 2 \mathrm{H}), 7.39-7.26$ (m, 7H), 6.90-6.80 (m, 2H), 6.65 (d, $J=16.0 \mathrm{~Hz}, 1 \mathrm{H}), 6.29-6.20(\mathrm{~m}, 2 \mathrm{H}), 4.91(\mathrm{~d}, J=6.0 \mathrm{~Hz}, 1 \mathrm{H})$, 4.74-4.70 (m, 1H), 4.37-4.34 (m, 1H), 2.47 (s, 3H), 2.30 (dt, $J=14.4,2.4 \mathrm{~Hz}, 1 \mathrm{H}), 1.95-1.88$ (m, 1H); $\left.{ }^{13} \mathbf{C ~ N M R ~ ( 1 0 0 ~ M H z , ~} \mathrm{CDCl}_{3}\right): \delta(\mathrm{ppm}) 156.7(\mathrm{~d}, J=238.3 \mathrm{~Hz}), 150.7,144.1,137.3,136.1$, 132.3, 130.0, 128.6, 128.1, 127.2, 127.1, 126.6, 120.6 (d, $J=7.0 \mathrm{~Hz}), 118.8$ (d, J = 7.9 Hz), $117.2(\mathrm{~d}$, $J=23.3 \mathrm{~Hz}), 114.9(\mathrm{~d}, J=22.9 \mathrm{~Hz}), 72.1,47.6,35.0,21.6$; HRMS (ESI-TOF) m/z: $\left[\mathrm{M}+\mathrm{Na}^{+} \mathrm{Calcd}\right.$ for $\mathrm{C}_{24} \mathrm{H}_{22} \mathrm{FNO}_{3} \mathrm{SNa} 446.1197$; Found 446.1194.

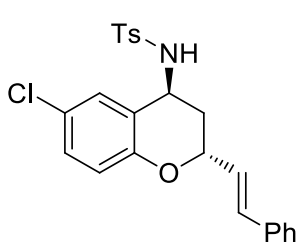

$N$-((2R,4S)-6-Chloro-2-((E)-styryl)chroman-4-yl)-4-methylbenzene sulfonamide (7c): An oven-dried $5 \mathrm{~mL}$ test-tube equipped with a septum and a magnetic stir bar was charged with $\mathrm{Pd}_{2}(\mathrm{dba})_{3}(4.6 \mathrm{mg}, 5 \mathrm{~mol} \%)$ and ligand $\mathbf{L 1}$ (11.3 mg, $20 \mathrm{~mol} \%)$. The tube was then evacuated and filled with argon. This cycle was repeated three times, and toluene $(0.5 \mathrm{~mL})$ was added via syringe. The resulting solution was stirred at room temperature for $30 \mathrm{~min}$. A second oven-dried test-tube was charged with diene 1a (26.0 mg, 2.0 equiv), salicylaldehyde-derived $N$-sulfonylimine $6 \mathbf{c}$ (31.0 mg, 1.0 equiv), 1-naphthoic acid (5.2 mg, $30 \mathrm{~mol} \%$ ) and $4 \AA \mathrm{MS}(60.0 \mathrm{mg})$, and the system was placed under an atmosphere of argon (balloon). Then the catalyst solution was added at room temperature. The resulting mixture was stirred at $80{ }^{\circ} \mathrm{C}$ for $60 \mathrm{~h}$. After completion, purification by flash chromatography on silica gel (petroleum ether/EtOAc = 10/1) gave the product 7c: $25.7 \mathrm{mg}$, as a white solid, 54\% yield; >19:1 dr; $\mathrm{mp}=213-215{ }^{\circ} \mathrm{C} ;[\alpha]^{25} \mathrm{D}=-40.0\left(c=0.31\right.$, in $\left.\mathrm{CHCl}_{3}\right) ; 96 \%$ ee, determined by HPLC analysis [Chiralpak column IA, $i \mathrm{PrOH} / n$ Hexane $=10 / 90$, flow rate: $1.0 \mathrm{~mL} / \mathrm{min}, 254 \mathrm{~nm}, \mathrm{t}$ (major) $=15.08$ 
min, $\mathrm{t}($ minor $)=26.66 \mathrm{~min}] ;{ }^{1} \mathbf{H}$ NMR $\left(400 \mathrm{MHz}, \mathrm{CDCl}_{3}\right): \delta(\mathrm{ppm}) 7.84(\mathrm{~d}, J=8.4 \mathrm{~Hz}, 2 \mathrm{H}), 7.41-$ $4.26(\mathrm{~m}, 6 \mathrm{H}), 7.10(\mathrm{dd}, J=8.8,2.4 \mathrm{~Hz}, 1 \mathrm{H}), 6.80(\mathrm{~d}, J=8.8 \mathrm{~Hz}, 1 \mathrm{H}), 6.66(\mathrm{~d}, J=15.6 \mathrm{~Hz}, 1 \mathrm{H}), 6.39$ $(\mathrm{d}, J=2.4 \mathrm{~Hz}, 1 \mathrm{H}), 6.23(\mathrm{dd}, J=16.0,6.0 \mathrm{~Hz}, 1 \mathrm{H}), 4.80(\mathrm{~d}, J=6.0 \mathrm{~Hz}, 1 \mathrm{H}), 4.76-4.71(\mathrm{~m}, 1 \mathrm{H})$, 4.36-4.34 (m, 1H), $2.48(\mathrm{~s}, 3 \mathrm{H}), 2.31$ (dt, $J=14.0,2.4 \mathrm{~Hz}, 1 \mathrm{H}), 1.95-1.89(\mathrm{~m}, 1 \mathrm{H}) ;{ }^{13} \mathbf{C} \mathbf{N M R}(100$ $\mathrm{MHz}_{\mathrm{CDCl}}$ ): $\delta$ (ppm) 153.2, 144.3, 137.3, 136.1, 132.4, 130.1, 130.1, 128.9, 128.6, 128.1, 127.2, 126.9, 126.6, 125.5, 121.3, 119.0, 72.3, 47.5, 35.0, 21.6; HRMS (ESI-TOF) m/z: [M + Na $]^{+}$Calcd for $\mathrm{C}_{24} \mathrm{H}_{22} \mathrm{ClNO}_{3} \mathrm{SNa} 462.0901\left({ }^{35} \mathrm{Cl}\right), 464.0872\left({ }^{37} \mathrm{Cl}\right)$; Found $462.0901\left({ }^{35} \mathrm{Cl}\right), 464.0868\left({ }^{37} \mathrm{Cl}\right)$.

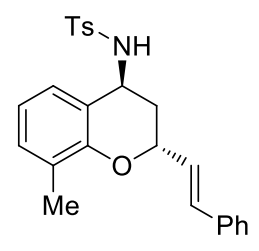

4-Methyl- $N$-((2R,4S)-8-methyl-2-((E)-styryl)chroman-4-yl)benzene sulfonamide (7d): An oven-dried $5 \mathrm{~mL}$ test-tube equipped with a septum and a magnetic stir bar was charged with $\mathrm{Pd}_{2}(\mathrm{dba})_{3}(4.6 \mathrm{mg}, 5 \mathrm{~mol} \%)$ and ligand $\mathbf{L 1}(11.3$

$\mathrm{mg}, 20 \mathrm{~mol} \%)$. The tube was then evacuated and filled with argon. This cycle was repeated three times, and toluene $(0.5 \mathrm{~mL})$ was added via syringe. The resulting solution was stirred at room temperature for $30 \mathrm{~min}$. A second oven-dried test-tube was charged with diene $\mathbf{1 a}(26.0 \mathrm{mg}$, 2.0 equiv), salicylaldehyde-derived $N$-sulfonylimine $6 \mathbf{d}$ ( $28.9 \mathrm{mg}, 1.0$ equiv), 1-naphthoic acid (5.2 $\mathrm{mg}, 30 \mathrm{~mol} \%)$ and $4 \AA$ MS (60.0 mg), and the system was placed under an atmosphere of argon (balloon). Then the catalyst solution was added at room temperature. The resulting mixture was stirred at $80{ }^{\circ} \mathrm{C}$ for $60 \mathrm{~h}$. After completion, purification by flash chromatography on silica gel (petroleum ether/EtOAc $=10 / 1$ ) gave the product 7d: $18.0 \mathrm{mg}$, as a white solid, $43 \%$ yield; >19:1 dr; $\mathrm{mp}=153-156{ }^{\circ} \mathrm{C} ;[\alpha]^{25} \mathrm{D}=-9.1\left(c=0.70\right.$, in $\left.\mathrm{CHCl}_{3}\right) ; 91 \%$ ee, determined by HPLC analysis [Chiralpak column IA, $i \mathrm{PrOH} / n$ Hexane $=10 / 90$, flow rate: $1.0 \mathrm{~mL} / \mathrm{min}, 254 \mathrm{~nm}, \mathrm{t}$ (major) $=10.96$ min, $\mathrm{t}($ minor $)=17.48 \mathrm{~min}] ;{ }^{1} \mathbf{H}$ NMR $\left(400 \mathrm{MHz}, \mathrm{CDCl}_{3}\right): \delta(\mathrm{ppm}) 7.87(\mathrm{~d}, J=8.4 \mathrm{~Hz}, 2 \mathrm{H}), 7.43-$ $7.27(\mathrm{~m}, 6 \mathrm{H}), 7.05(\mathrm{~d}, J=7.6 \mathrm{~Hz}, 2 \mathrm{H}), 6.71-6.67(\mathrm{~m}, 2 \mathrm{H}), 6.43(\mathrm{~d}, J=7.6 \mathrm{~Hz}, 1 \mathrm{H}), 6.31(\mathrm{dd}, J=$ 16.0, 6.4 Hz, 1H), 4.82-4.78 (m, 1H), 4.69 (d, J=4.8 Hz, 1H), 4.39-4.38 (m, 1H), 2.49 (s, 3H), 2.41$2.37(\mathrm{~m}, 1 \mathrm{H}), 2.22(\mathrm{~s}, 3 \mathrm{H}), 1.94-1.86(\mathrm{~m}, 1 \mathrm{H}) ;{ }^{13} \mathbf{C} \mathbf{N M R}\left(100 \mathrm{MHz}, \mathrm{CDCl}_{3}\right): \delta(\mathrm{ppm}) 152.9,143.8$, 137.4, 136.4, 131.3, 131.0, 129.9, 128.6, 128.0, 127.9, 127.3, 126.9, 126.7, 126.6, 120.3, 119.2, 71.7, 48.2, 35.1, 21.6, 16.0; HRMS (ESI-TOF) m/z: $[\mathrm{M}+\mathrm{Na}]^{+}$Calcd for $\mathrm{C}_{25} \mathrm{H}_{25} \mathrm{NO}_{3} \mathrm{SNa}$ 442.1447; Found 442.1447.

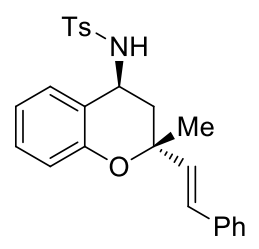

4-Methyl- $N$-((2R,4S)-2-methyl-2-((E)-styryl)chroman-4-yl)benzene sulfonamide (7e): An oven-dried $5 \mathrm{~mL}$ test-tube equipped with a septum and a magnetic stir bar was charged with $\mathrm{Pd}_{2}(\mathrm{dba})_{3}(4.6 \mathrm{mg}, 5 \mathrm{~mol} \%)$ and ligand $\mathbf{L 1}$ (11.3 $\mathrm{mg}, 20 \mathrm{~mol} \%)$. The tube was then evacuated and filled with argon. This cycle was repeated three times, and toluene $(0.5 \mathrm{~mL})$ was added via syringe. The resulting solution was stirred at room temperature for $30 \mathrm{~min}$. A second oven-dried test-tube was charged with diene $\mathbf{1 p}(28.8 \mathrm{mg}$, 2.0 equiv), salicylaldehyde-derived $N$-sulfonylimine $\mathbf{6 a}(27.5 \mathrm{mg}, 1.0$ equiv), 1-naphthoic acid (5.2 
mg, $30 \mathrm{~mol} \%)$ and $4 \AA$ MS (60.0 mg), and the system was placed under an atmosphere of argon (balloon). Then the catalyst solution was added at room temperature. The resulting mixture was stirred at $80{ }^{\circ} \mathrm{C}$ for $60 \mathrm{~h}$. After completion, purification by flash chromatography on silica gel (petroleum ether/EtOAc = 10/1) gave the product 7e: $25.9 \mathrm{mg}$, as a colorless semisolid, $62 \%$ yield; >19: $1 \mathrm{dr} ;[\alpha]^{25} \mathrm{D}=-9.1\left(c=0.70\right.$, in $\left.\mathrm{CHCl}_{3}\right) ; 91 \%$ ee, determined by HPLC analysis [Chiralpak column ID, $i \mathrm{PrOH} / n$ Hexane $=20 / 80$, flow rate: $1.0 \mathrm{~mL} / \mathrm{min}, 254 \mathrm{~nm}, \mathrm{t}$ (major) $=9.95 \mathrm{~min}, \mathrm{t}$ (minor) $=11.11 \mathrm{~min}] ;{ }^{1} \mathbf{H}$ NMR $\left(400 \mathrm{MHz}, \mathrm{CDCl}_{3}\right): \delta(\mathrm{ppm}) 7.81(\mathrm{~d}, J=8.4 \mathrm{~Hz}, 2 \mathrm{H}), 7.31-7.22(\mathrm{~m}, 7 \mathrm{H})$, $7.15(\mathrm{t}, J=7.6 \mathrm{~Hz}, 1 \mathrm{H}), 7.06(\mathrm{~d}, J=7.6 \mathrm{~Hz}, 1 \mathrm{H}), 6.88(\mathrm{~d}, J=7.6 \mathrm{~Hz}, 1 \mathrm{H}), 6.80$ (t, $J=7.6 \mathrm{~Hz}, 1 \mathrm{H})$, $6.39(\mathrm{~d}, J=16.4 \mathrm{~Hz}, 1 \mathrm{H}), 6.08(\mathrm{~d}, J=16.4 \mathrm{~Hz}, 1 \mathrm{H}), 4.70-4.68(\mathrm{~m}, 1 \mathrm{H}), 4.46-4.39(\mathrm{~m}, 1 \mathrm{H}), 2.43(\mathrm{~s}$, $3 \mathrm{H}), 2.30(\mathrm{dd}, J=13.2,6.0 \mathrm{~Hz}, 1 \mathrm{H}), 1.85(\mathrm{dd}, J=13.2,11.6 \mathrm{~Hz}, 1 \mathrm{H}), 1.48(\mathrm{~s}, 3 \mathrm{H}) ;{ }^{13} \mathbf{C} \mathbf{N M R}(150$ $\mathrm{MHz}, \mathrm{CDCl}_{3}$ ): $\delta$ (ppm) 153.9, 143.7, 137.7, 136.2, 131.4, 129.9, 129.7, 129.3, 128.5, 127.8, 127.1, 126.9, 126.5, 121.4, 120.6, 117.0, 77.6, 47.3, 40.7, 28.9, 21.6; HRMS (ESI-TOF) m/z: [M + Na $]^{+}$ Calcd for $\mathrm{C}_{25} \mathrm{H}_{25} \mathrm{NO}_{3} \mathrm{SNa} 442.1447$; Found 442.1445.

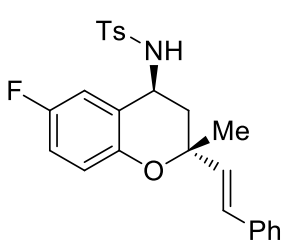

\section{$N$-((2R,4S)-6-Fluoro-2-methyl-2-((E)-styryl)chroman-4-yl)-4-methylbenzene} sulfonamide (7f): An oven-dried $5 \mathrm{~mL}$ test-tube equipped with a septum and a magnetic stir bar was charged with $\mathrm{Pd}_{2}(\mathrm{dba})_{3}(4.6 \mathrm{mg}, 5 \mathrm{~mol} \%)$ and ligand $\mathbf{L 1}$ $(11.3 \mathrm{mg}, 20 \mathrm{~mol} \%)$. The tube was then evacuated and filled with argon. This cycle was repeated three times, and toluene $(0.5 \mathrm{~mL})$ was added via syringe. The resulting solution was stirred at room temperature for $30 \mathrm{~min}$. A second oven-dried test-tube was charged with diene $\mathbf{1 p}$ (28.8 mg, 2.0 equiv), salicylaldehyde-derived $N$-sulfonylimine $6 \mathbf{b}$ (29.3 mg, 1.0 equiv), 1-naphthoic acid (5.2 mg, $30 \mathrm{~mol} \%$ ) and $4 \AA \mathrm{MS}(60.0 \mathrm{mg})$, and the system was placed under an atmosphere of argon (balloon). Then the catalyst solution was added at room temperature. The resulting mixture was stirred at $80{ }^{\circ} \mathrm{C}$ for $60 \mathrm{~h}$. After completion, purification by flash chromatography on silica gel (petroleum ether/EtOAc = 10/1) gave the product 7f: $33.0 \mathrm{mg}$, as a white semisolid, $76 \%$ yield; $>19: 1$ $\mathrm{dr} ;[\alpha]^{25} \mathrm{D}=-2.4\left(c=1.25\right.$, in $\left.\mathrm{CHCl}_{3}\right) ; 95 \%$ ee, determined by HPLC analysis [Chiralpak column IG, $i \mathrm{PrOH} / n$ Hexane $=10 / 90$, flow rate: $1.0 \mathrm{~mL} / \mathrm{min}, 254 \mathrm{~nm}, \mathrm{t}$ (major) $=17.40 \mathrm{~min}, \mathrm{t}($ minor $)=20.05$ min]; ${ }^{1} \mathbf{H}$ NMR $\left(400 \mathrm{MHz}, \mathrm{CDCl}_{3}\right): \delta(\mathrm{ppm}) 7.80(\mathrm{~d}, J=8.0 \mathrm{~Hz}, 2 \mathrm{H}), 7.32-7.23(\mathrm{~m}, 7 \mathrm{H}), 6.87-6.76$ $(\mathrm{m}, 3 \mathrm{H}), 6.36(\mathrm{~d}, J=16.4 \mathrm{~Hz}, 1 \mathrm{H}), 6.04(\mathrm{~d}, J=16.4 \mathrm{~Hz}, 1 \mathrm{H}), 4.85-4.79(\mathrm{~m}, 1 \mathrm{H}), 4.41-4.34(\mathrm{~m}, 1 \mathrm{H})$, $2.44(\mathrm{~s}, 3 \mathrm{H}), 2.25(\mathrm{dd}, J=13.2,5.6 \mathrm{~Hz}, 1 \mathrm{H}), 1.81(\mathrm{t}, J=12.4 \mathrm{~Hz}, 1 \mathrm{H}), 1.46(\mathrm{~s}, 3 \mathrm{H}) ;{ }^{13} \mathbf{C} \mathbf{N M R}(100$ $\left.\mathrm{MHz}, \mathrm{CDCl}_{3}\right): \delta(\mathrm{ppm}) 156.8(\mathrm{~d}, J=238.8 \mathrm{~Hz}), 149.9(\mathrm{~d}, J=1.9 \mathrm{~Hz}) 143.9,137.5,136.0,131.0$, 123.0, 129.9, 128.6, 127.9, 127.0, 126.4, $122.6(\mathrm{~d}, J=6.9 \mathrm{~Hz}), 117.9(\mathrm{~d}, J=7.8 \mathrm{~Hz}), 116.2(\mathrm{~d}, J=$ $23.3 \mathrm{~Hz}), 113.3(\mathrm{~d}, J=24.2 \mathrm{~Hz}), 77.8,47.3,40.2,28.9,21.6$; HRMS (ESI-TOF) m/z: $[\mathrm{M}+\mathrm{Na}]^{+}$ Calcd for $\mathrm{C}_{25} \mathrm{H}_{24} \mathrm{FNO}_{3} \mathrm{SNa}$ 460.1353; Found 460.1353 . 


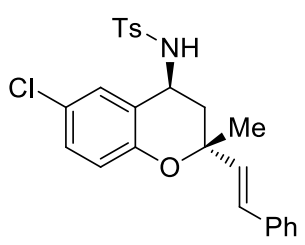

$N$-((2R,4S)-6-Chloro-2-methyl-2-((E)-styryl)chroman-4-yl)-4-methyl benzenesulfonamide (1g): An oven-dried $5 \mathrm{~mL}$ test-tube equipped with a septum and a magnetic stir bar was charged with $\mathrm{Pd}_{2}(\mathrm{dba})_{3}(4.6 \mathrm{mg}, 5 \mathrm{~mol} \%)$ and ligand $\mathbf{L 1}$ (11.3 $\mathrm{mg}, 20 \mathrm{~mol} \%$ ). The tube was then evacuated and filled with argon. This cycle was repeated three times, and toluene $(0.5 \mathrm{~mL})$ was added via syringe. The resulting solution was stirred at room temperature for $30 \mathrm{~min}$. A second oven-dried test-tube was charged with diene 1p (28.8 mg, 2.0 equiv), salicylaldehyde-derived $N$-sulfonylimine $6 \mathbf{c}$ (31.0 mg, 1.0 equiv), 1naphthoic acid $(5.2 \mathrm{mg}, 30 \mathrm{~mol} \%)$ and $4 \AA \mathrm{MS}(60.0 \mathrm{mg})$, and the system was placed under an atmosphere of argon (balloon). Then the catalyst solution was added at room temperature. The resulting mixture was stirred at $80{ }^{\circ} \mathrm{C}$ for $60 \mathrm{~h}$. After completion, purification by flash chromatography on silica gel (petroleum ether/EtOAc $=10 / 1$ ) gave the product $7 \mathrm{~g}: 32.9 \mathrm{mg}$, as a colorless semisolid, $73 \%$ yield; $>19: 1 \mathrm{dr} ;[\alpha]^{25} \mathrm{D}=-43.68\left(c=1.25\right.$, in $\left.\mathrm{CHCl}_{3}\right) ; 94 \%$ ee, determined by HPLC analysis [Chiralpak column IG, $i \mathrm{PrOH} / n \mathrm{Hexane}=10 / 90$, flow rate: $1.0 \mathrm{~mL} / \mathrm{min}, 254 \mathrm{~nm}, \mathrm{t}$ $($ major $)=15.18 \mathrm{~min}, \mathrm{t}($ minor $)=16.18 \mathrm{~min}] ;{ }^{1} \mathbf{H} \mathbf{~ N M R}\left(400 \mathrm{MHz}, \mathrm{CDCl}_{3}\right): \delta(\mathrm{ppm}) 7.80(\mathrm{~d}, J=8.0$ $\mathrm{Hz}, 2 \mathrm{H}), 7.33-7.22(\mathrm{~m}, 7 \mathrm{H}), 7.08(\mathrm{dd}, J=8.4,1.2 \mathrm{~Hz}, 1 \mathrm{H}), 6.94(\mathrm{~s}, 1 \mathrm{H}), 6.80$ (d, $J=8.8 \mathrm{~Hz}, 1 \mathrm{H})$, $6.35(\mathrm{~d}, J=16.0 \mathrm{~Hz}, 1 \mathrm{H}), 6.04(\mathrm{~d}, J=16.0 \mathrm{~Hz}, 1 \mathrm{H}), 4.78(\mathrm{~d}, J=9.6 \mathrm{~Hz}, 1 \mathrm{H}), 4.41-4.35(\mathrm{~m}, 1 \mathrm{H})$, $2.44(\mathrm{~s}, 3 \mathrm{H}), 2.24(\mathrm{dd}, J=13.2,5.6 \mathrm{~Hz}, 1 \mathrm{H}), 1.81(\mathrm{t}, J=12.4 \mathrm{~Hz}, 1 \mathrm{H}), 1.47(\mathrm{~s}, 3 \mathrm{H}) ;{ }^{13} \mathbf{C} \mathbf{N M R}(100$ $\left.\mathrm{MHz}, \mathrm{CDCl}_{3}\right): \delta(\mathrm{ppm}) 152.5,144.0,137.5,135.9,130.8,130.0,129.95,129.3,128.6,127.9,127.0$, 126.9, 126.5, 125.4, 123.2, 118.3, 78.1, 47.1, 40.2, 28.9, 21.6; HRMS (ESI-TOF) m/z: [M + Na $]^{+}$ Calcd for $\mathrm{C}_{25} \mathrm{H}_{24} \mathrm{ClNO}_{3} \mathrm{SNa} 476.1058\left({ }^{35} \mathrm{Cl}\right), 478.1028\left({ }^{37} \mathrm{Cl}\right)$; Found $476.1056\left({ }^{35} \mathrm{Cl}\right), 478.1026\left({ }^{37} \mathrm{Cl}\right)$.

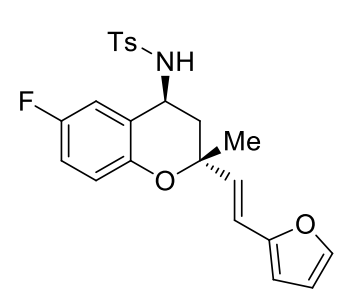

$N$-((2R,4S)-6-Fluoro-2-((E)-2-(furan-2-yl)vinyl)-2-methylchroman-4-yl)4-methylbenzenesulfonamide (7h): An oven-dried $5 \mathrm{~mL}$ test-tube equipped with a septum and a magnetic stir bar was charged with $\mathrm{Pd}_{2}(\mathrm{dba})_{3}(4.6 \mathrm{mg}, 5$ mol \%) and ligand $\mathbf{L 1}(11.3 \mathrm{mg}, 20 \mathrm{~mol} \%)$. The tube was then evacuated and filled with argon. This cycle was repeated three times, and toluene $(0.5 \mathrm{~mL})$ was added via syringe. The resulting solution was stirred at room temperature for $30 \mathrm{~min}$. A second oven-dried test-tube was charged with diene 1 q $(26.8 \mathrm{mg}, 2.0$ equiv), salicylaldehyde-derived $N$ sulfonylimine $6 \mathbf{b}$ (29.3 mg, 1.0 equiv), 1-naphthoic acid (5.2 mg, $30 \mathrm{~mol} \%$ ) and $4 \AA \mathrm{MS}$ (60.0 mg), and the system was placed under an atmosphere of argon (balloon). Then the catalyst solution was added at room temperature. The resulting mixture was stirred at $80{ }^{\circ} \mathrm{C}$ for $60 \mathrm{~h}$. After completion, purification by flash chromatography on silica gel (petroleum ether/EtOAc $=10 / 1$ ) gave the product 7h: $34.0 \mathrm{mg}$, as a yellow semisolid, $80 \%$ yield; >19:1 dr; $[\alpha]^{25} \mathrm{D}=-12.8\left(c=1.64\right.$, in $\left.\mathrm{CHCl}_{3}\right) ; 87 \%$ ee, determined by HPLC analysis [Chiralpak column IG, $i \mathrm{PrOH} / n \mathrm{Hexane}=10 / 90$, flow rate: 1.0 $\mathrm{mL} / \mathrm{min}, 254 \mathrm{~nm}, \mathrm{t}$ (major) $=18.49 \mathrm{~min}, \mathrm{t}$ (minor) $=20.29 \mathrm{~min}] ;{ }^{1} \mathbf{H} \mathbf{~ N M R}\left(400 \mathrm{MHz}, \mathrm{CDCl}_{3}\right): \delta$ (ppm) $7.81(\mathrm{~d}, J=8.0 \mathrm{~Hz}, 2 \mathrm{H}), 7.35-7.33(\mathrm{~m}, 3 \mathrm{H}), 6.87-6.77(\mathrm{~m}, 3 \mathrm{H}), 6.35(\mathrm{dd}, J=2.8,1.8 \mathrm{~Hz}, 1 \mathrm{H})$, 
$6.17(\mathrm{~d}, J=16.0 \mathrm{~Hz}, 1 \mathrm{H}), 6.15(\mathrm{~s}, 1 \mathrm{H}), 5.97(\mathrm{~d}, J=16.0 \mathrm{~Hz}, 1 \mathrm{H}), 4.83(\mathrm{~d}, J=9.6 \mathrm{~Hz}, 1 \mathrm{H}), 4.40-4.34$ (m, 1H), $2.46(\mathrm{~s}, 3 \mathrm{H}), 2.13(\mathrm{dd}, J=13.2,5.6 \mathrm{~Hz}, 1 \mathrm{H}), 1.77(\mathrm{t}, J=12.4 \mathrm{~Hz}, 1 \mathrm{H}), 1.43(\mathrm{~s}, 3 \mathrm{H}) ;{ }^{13} \mathrm{C}$ NMR (100 MHz, $\left.\mathrm{CDCl}_{3}\right): \delta(\mathrm{ppm}) 156.8(\mathrm{~d}, J=238.8 \mathrm{~Hz}), 151.7,149.8,143.9,142.1,137.6,130.0$, 129.4, 127.0, $122.7(\mathrm{~d}, J=6.9 \mathrm{~Hz}), 118.4,117.9(\mathrm{~d}, J=7.8 \mathrm{~Hz}), 116.2(\mathrm{~d}, J=23.4 \mathrm{~Hz}), 113.4(\mathrm{~d}, J=$ $24.3 \mathrm{~Hz}), 111.4,108.6,77.5,47.3,40.1,28.9,21.6$; HRMS (ESI-TOF) m/z: [M + Na $]^{+}$Calcd for $\mathrm{C}_{23} \mathrm{H}_{22} \mathrm{FNO}_{4} \mathrm{SNa}$ 450.1146; Found 450.1146.

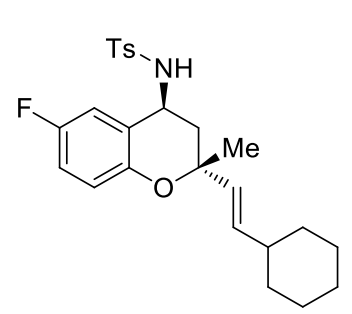

\section{$N$-((2R,4S)-2-((E)-2-Cyclohexylvinyl)-6-fluoro-2-methylchroman-4-yl)-4-} methylbenzenesulfonamide (7i): An oven-dried $5 \mathrm{~mL}$ test-tube equipped with a septum and a magnetic stir bar was charged with $\operatorname{Pd}_{2}(\mathrm{dba})_{3}(4.6 \mathrm{mg}, 5$ mol \%) and ligand $\mathbf{L 1}(11.3 \mathrm{mg}, 20 \mathrm{~mol} \%)$. The tube was then evacuated and filled with argon. This cycle was repeated three times, and toluene $(0.5 \mathrm{~mL})$ was added via syringe. The resulting solution was stirred at room temperature for $30 \mathrm{~min}$. A second oven-dried test-tube was charged with diene $1 \mathbf{1 r}(26.8 \mathrm{mg}, 2.0$ equiv), salicylaldehyde-derived $\mathrm{N}$ sulfonylimine $\mathbf{6 b}$ (29.3 mg, 1.0 equiv), 1-naphthoic acid (5.2 mg, $30 \mathrm{~mol} \%$ ) and $4 \AA \mathrm{MS}$ (60.0 mg), and the system was placed under an atmosphere of argon (balloon). Then the catalyst solution was added at room temperature. The resulting mixture was stirred at $80{ }^{\circ} \mathrm{C}$ for $60 \mathrm{~h}$. After completion, purification by flash chromatography on silica gel (petroleum ether/EtOAc $=10 / 1$ ) gave the product 7i: $33.0 \mathrm{mg}$, as a colorless oil, 74\% yield; >19:1 dr; $[\alpha]^{25} \mathrm{D}=-36.3\left(c=1.47\right.$, in $\left.\mathrm{CHCl}_{3}\right)$; $98 \%$ ee, determined by HPLC analysis [Chiralpak column IG, $i \mathrm{PrOH} / n \mathrm{Hexane}=5 / 95$, flow rate: $1.0 \mathrm{~mL} / \mathrm{min}$, $220 \mathrm{~nm}, \mathrm{t}($ major $)=20.98 \mathrm{~min}, \mathrm{t}($ minor $)=23.26 \mathrm{~min}] ;{ }^{1} \mathbf{H} \mathbf{N M R}\left(400 \mathrm{MHz}, \mathrm{CDCl}_{3}\right): \delta(\mathrm{ppm}) 7.81$ $(\mathrm{d}, J=8.0 \mathrm{~Hz}, 2 \mathrm{H}), 7.34(\mathrm{~d}, J=8.0 \mathrm{~Hz}, 2 \mathrm{H}), 6.83-6.79(\mathrm{~m}, 2 \mathrm{H}), 6.73-6.69(\mathrm{~m}, 1 \mathrm{H}), 5.36(\mathrm{dd}, J=$ 16.0, 6.4 Hz, 1H), $5.23(\mathrm{~d}, J=16.0 \mathrm{~Hz}, 1 \mathrm{H}), 4.77(\mathrm{~d}, J=9.6 \mathrm{~Hz}, 1 \mathrm{H}), 4.35-4.29(\mathrm{~m}, 1 \mathrm{H}), 2.46(\mathrm{~s}$, $3 \mathrm{H}), 2.04(\mathrm{dd}, J=13.2,5.6 \mathrm{~Hz}, 1 \mathrm{H}), 1.85-1.83(\mathrm{~m}, 1 \mathrm{H}), 1.70-1.54(\mathrm{~m}, 6 \mathrm{H}), 1.34(\mathrm{~s}, 3 \mathrm{H}), 1.29-1.06$ (m, 3H), 0.99-0.86 (m, 2H); ${ }^{13} \mathbf{C}$ NMR (100 MHz, $\left.\mathrm{CDCl}_{3}\right): \delta(\mathrm{ppm}) 156.6(\mathrm{~d}, J=238.3 \mathrm{~Hz}), 150.1$, 143.9, 137.6, 136.8, 129.9, 128.9, 127.1, $122.7(J=6.6 \mathrm{~Hz}), 117.8(J=7.8 \mathrm{~Hz}), 116.0(J=23.2 \mathrm{~Hz})$, $113.1(J=24.0 \mathrm{~Hz}), 77.5,47.2,40.0,39.9,32.9,32.6$, 28.9, 26.0, 25.8, 25.8, 21.6; HRMS (ESI-TOF) $\mathrm{m} / \mathrm{z}:[\mathrm{M}+\mathrm{H}]^{+}$Calcd for $\mathrm{C}_{25} \mathrm{H}_{31} \mathrm{FNO}_{3} \mathrm{~S}$ 444.2003; Found 444.2002.

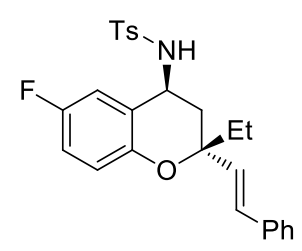

$N$-((2R,4S)-2-Ethyl-6-fluoro-2-((E)-styryl)chroman-4-yl)-4-methylbenzene sulfonamide (7j): An oven-dried $5 \mathrm{~mL}$ test-tube equipped with a septum and a magnetic stir bar was charged with $\mathrm{Pd}_{2}(\mathrm{dba})_{3}(4.6 \mathrm{mg}, 5 \mathrm{~mol} \%)$ and ligand $\mathbf{L 1}$ (11.3 mg, $20 \mathrm{~mol} \%)$. The tube was then evacuated and filled with argon. This cycle was repeated three times, and toluene $(0.5 \mathrm{~mL})$ was added via syringe. The resulting solution was stirred at room temperature for $30 \mathrm{~min}$. A second oven-dried test-tube was charged with diene $\mathbf{1 s}$ (26.8 mg, 2.0 equiv), salicylaldehyde-derived $N$-sulfonylimine $6 \mathbf{b}$ (29.3 mg, 1.0 equiv), 1-naphthoic 
acid (5.2 mg, $30 \mathrm{~mol} \%$ ) and $4 \AA \mathrm{MS}(60.0 \mathrm{mg})$, and the system was placed under an atmosphere of argon (balloon). Then the catalyst solution was added at room temperature. The resulting mixture was stirred at $80{ }^{\circ} \mathrm{C}$ for $60 \mathrm{~h}$. After completion, purification by flash chromatography on silica gel (petroleum ether/EtOAc $=10 / 1$ ) gave the product $\mathbf{7 j}: 21.3 \mathrm{mg}$, as a white semisolid, $47 \%$ yield; $10: 1$ $\mathrm{dr} ;[\alpha]^{25} \mathrm{D}=+10.6\left(c=1.01\right.$, in $\left.\mathrm{CHCl}_{3}\right) ; 97 \%$ ee, determined by HPLC analysis [Chiralpak column $\mathrm{IG}, i \mathrm{PrOH} / n$ Hexane $=5 / 95$, flow rate: $1.0 \mathrm{~mL} / \mathrm{min}, 254 \mathrm{~nm}, \mathrm{t}($ minor $)=13.59 \mathrm{~min}, \mathrm{t}$ (major) $=15.78$ min]; ${ }^{1} \mathbf{H}$ NMR (400 MHz, $\left.\mathrm{CDCl}_{3}\right): \delta(\mathrm{ppm}) 7.81$ (d, $\left.J=8.2 \mathrm{~Hz}, 2 \mathrm{H}\right), 7.32-7.23(\mathrm{~m}, 7 \mathrm{H}), 6.87-6.74$ (m, 3H), 6.33 (d, $J=16.4 \mathrm{~Hz}, 1 \mathrm{H}), 5.95(\mathrm{~d}, J=16.4 \mathrm{~Hz}, 1 \mathrm{H}), 4.73$ (d, $J=9.6 \mathrm{~Hz}, 1 \mathrm{H}), 4.34-4.37$ (m, $1 \mathrm{H}), 2.44(\mathrm{~s}, 3 \mathrm{H}), 2.20(\mathrm{dd}, J=13.2,5.6 \mathrm{~Hz}, 1 \mathrm{H}), 1.80-1.69(\mathrm{~m}, 2 \mathrm{H}), 1.60(\mathrm{~s}, 1 \mathrm{H}), 0.95(\mathrm{t}, J=7.2$ $\mathrm{Hz}, 3 \mathrm{H}) ;{ }^{13} \mathrm{C}$ NMR (100 MHz, $\left.\mathrm{CDCl}_{3}\right): \delta(\mathrm{ppm}) 156.7$ (d, $\left.J=238.5 \mathrm{~Hz}\right), 150.0,143.9,137.6,136.1$, 130.9, 130.0, 129.9, 128.6, 127.8, 127.1, 126.4, $122.9(J=6.6 \mathrm{~Hz}), 117.9(J=7.9 \mathrm{~Hz}), 116.1(J=$ $23.3 \mathrm{~Hz}), 113.1(J=24.2 \mathrm{~Hz}), 80.2$, 47.3, 38.3, 34.5, 21.6, 7.4; HRMS (ESI-TOF) m/z: [M - H] Calcd for $\mathrm{C}_{26} \mathrm{H}_{25} \mathrm{FNO}_{3} \mathrm{~S} 450.1545$; Found 450.1555 .

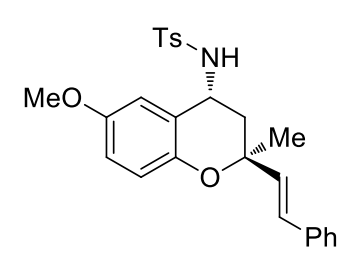

$N$-((2S,4R)-6-Methoxy-2-methyl-2-((E)-styryl)chroman-4-yl)-4-methyl benzenesulfonamide (ent-7k): An oven-dried $5 \mathrm{~mL}$ test-tube equipped with a septum and a magnetic stir bar was charged with $\mathrm{Pd}_{2}(\mathrm{dba})_{3}(4.6 \mathrm{mg}, 5 \mathrm{~mol} \%)$ and ligand $\mathbf{L 1}$ (11.3 $\mathrm{mg}, 20 \mathrm{~mol} \%$ ). The tube was then evacuated and filled with argon. This cycle was repeated three times, and toluene $(0.5 \mathrm{~mL})$ was added via syringe. The resulting solution was stirred at room temperature for $30 \mathrm{~min}$. A second oven-dried test-tube was charged with diene $1 \mathbf{p}$ (28.8 mg, 2.0 equiv), salicylaldehyde-derived $N$-sulfonylimine $\mathbf{6 b}$ (29.3 mg, 1.0 equiv), 1-naphthoic acid (5.2 mg, $30 \mathrm{~mol} \%)$ and $4 \AA \mathrm{AS}(60.0 \mathrm{mg})$, and the system was placed under an atmosphere of argon (balloon). Then the catalyst solution was added at room temperature. The resulting mixture was stirred at $80{ }^{\circ} \mathrm{C}$ for $60 \mathrm{~h}$. After completion, purification by flash chromatography on silica gel (petroleum ether/EtOAc $=10 / 1$ ) gave the product $\boldsymbol{e n t} \mathbf{- 7 \mathbf { j }}: 26.8 \mathrm{mg}$, as a white solid, $60 \%$ yield; >19:1 dr; $[\alpha]^{25} \mathrm{D}=+25.5\left(c=1.35\right.$, in $\left.\mathrm{CHCl}_{3}\right)$; 93\% ee, determined by HPLC analysis [Chiralpak column IG, $i \mathrm{PrOH} / n$ Hexane $=10 / 90$, flow rate: $1.0 \mathrm{~mL} / \mathrm{min}, 254 \mathrm{~nm}, \mathrm{t}($ minor) $=$ $31.49 \mathrm{~min}, \mathrm{t}$ (major) = $39.30 \mathrm{~min}] ;{ }^{1} \mathbf{H} \mathbf{N M R}\left(400 \mathrm{MHz}, \mathrm{CDCl}_{3}\right): \delta(\mathrm{ppm}) 7.81(\mathrm{~d}, J=8.4 \mathrm{~Hz}, 2 \mathrm{H})$, $7.33-7.22(\mathrm{~m}, 7 \mathrm{H}), 6.81(\mathrm{~d}, J=8.8 \mathrm{~Hz}, 1 \mathrm{H}), 6.73(\mathrm{dd}, J=8.8,2.4 \mathrm{~Hz}, 1 \mathrm{H}), 6.51(\mathrm{~d}, J=2.4 \mathrm{~Hz}, 1 \mathrm{H})$, $6.39(\mathrm{~d}, J=16.0 \mathrm{~Hz}, 1 \mathrm{H}), 6.09(\mathrm{~d}, J=16.0 \mathrm{~Hz}, 1 \mathrm{H}), 4.81$ (d, $J=9.2 \mathrm{~Hz}, 1 \mathrm{H}), 4.44-4.38(\mathrm{~m}, 1 \mathrm{H})$, $3.58(\mathrm{~s}, 3 \mathrm{H}), 2.44(\mathrm{~s}, 3 \mathrm{H}), 2.29(\mathrm{dd}, J=13.2,5.6 \mathrm{~Hz}, 1 \mathrm{H}), 1.88-1.82(\mathrm{~m}, 1 \mathrm{H}), 1.48(\mathrm{~s}, 3 \mathrm{H})$; ${ }^{13} \mathbf{C} \mathbf{N M R}$ $\left(100 \mathrm{MHz}, \mathrm{CDCl}_{3}\right): \delta$ (ppm) 153.4, 147.8, 143.7, 137.8, 136.2, 131.4, 129.9, 129.7, 128.5, 127.7, 127.0, 126.4, 121.8, 117.8, 116.2, 110.7, 77.4, 55.5, 47.5, 40.7, 28.9, 21.5; HRMS (ESI-TOF) m/z: $[\mathrm{M}-\mathrm{H}]^{-}$Calcd for $\mathrm{C}_{26} \mathrm{H}_{26} \mathrm{NO}_{4} \mathrm{~S} 472.1553$; Found 472.1554. 


\subsection{Friedel-Crafts reaction of deactivated heteroarenes}

(1) Friedel-Crafts reaction of deactivated furan $\mathbf{1 2}$

1) With $\mathrm{Pd}\left(\mathrm{PPh}_{3}\right)_{4}$
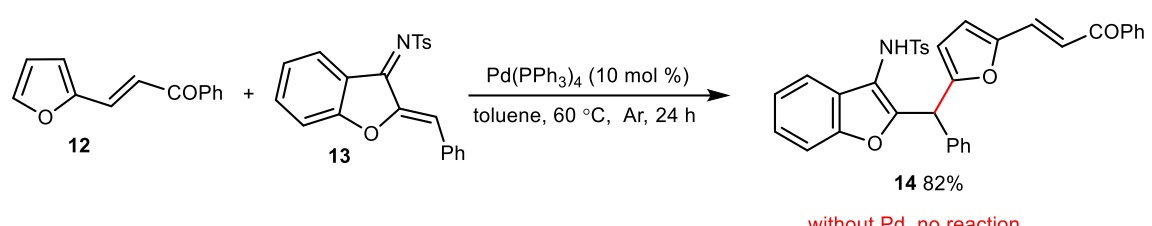

(E)-4-Methyl-N-(2-((5-(3-oxo-3-phenylprop-1-en-1-yl)furan-2-yl)(phenyl)methyl)benzofuran-

3-yl)benzenesulfonamide (14): An oven-dried $5 \mathrm{~mL}$ test-tube equipped with a septum and a magnetic stir bar was charged with $\mathrm{Pd}\left(\mathrm{PPh}_{3}\right)_{4}(11.5 \mathrm{mg}, 10 \mathrm{~mol} \%)$, (E)-3-(furan-2-yl)-1-phenylprop2-en-1-one 12 (23.8 mg, $0.12 \mathrm{mmol}$ ), 1-azadiene 13 (37.5 mg, 1.0 equiv). The tube was then evacuated and filled with argon. This cycle was repeated three times, and toluene $(1.0 \mathrm{~mL})$ was added via syringe. The resulting mixture was stirred at $60{ }^{\circ} \mathrm{C}$ for $24 \mathrm{~h}$. After completion, purification by flash chromatography on silica gel (petroleum ether/EtOAc $=5 / 1$ ) gave the product 14: $46.9 \mathrm{mg}, 82 \%$ yield; as a yellow solid, mp: $198-200{ }^{\circ} \mathrm{C} ;{ }^{1} \mathbf{H}$ NMR (400 MHz, DMSO-d $)$ : $\delta$ (ppm) $10.21(\mathrm{~s}, 1 \mathrm{H})$, $8.03(\mathrm{~d}, J=7.6 \mathrm{~Hz}, 2 \mathrm{H}), 7.69(\mathrm{t}, J=7.2 \mathrm{~Hz}, 1 \mathrm{H}), 7.64-7.51(\mathrm{~m}, 6 \mathrm{H}), 7.44-7.26(\mathrm{~m}, 6 \mathrm{H}), 7.25-7.17$ $(\mathrm{m}, 5 \mathrm{H}), 7.10(\mathrm{~d}, J=3.2 \mathrm{~Hz}, 1 \mathrm{H}), 6.28(\mathrm{~d}, J=3.2 \mathrm{~Hz}, 1 \mathrm{H}), 5.79(\mathrm{~s}, 1 \mathrm{H}), 2.28(\mathrm{~s}, 3 \mathrm{H}) ;{ }^{13} \mathbf{C} \mathbf{N M R}(100$ MHz, DMSO-d6): $\delta$ (ppm) 188.6, 156.1, 153.0, 150.6, 150.4, 143.4, 137.9, 137.6, 136.7, 133.1, 130.6, 129.6, 128.9, 128.7, 128.3, 128.2, 127.5, 126.9, 125.4, 125.0, 123.1, 120.2, 118.3, 118.2, 114.8, 111.6, 111.5, 41.1, 21.1; HRMS (ESI-TOF) m/z: [M + Na $]^{+}$Calcd for $\mathrm{C}_{35} \mathrm{H}_{27} \mathrm{NO}_{5} \mathrm{SNa}$ 596.1502; Found 596.1499.

2) With $\mathrm{Pd}_{2}(\mathrm{dba})_{3} / \mathbf{L} \mathbf{2}$
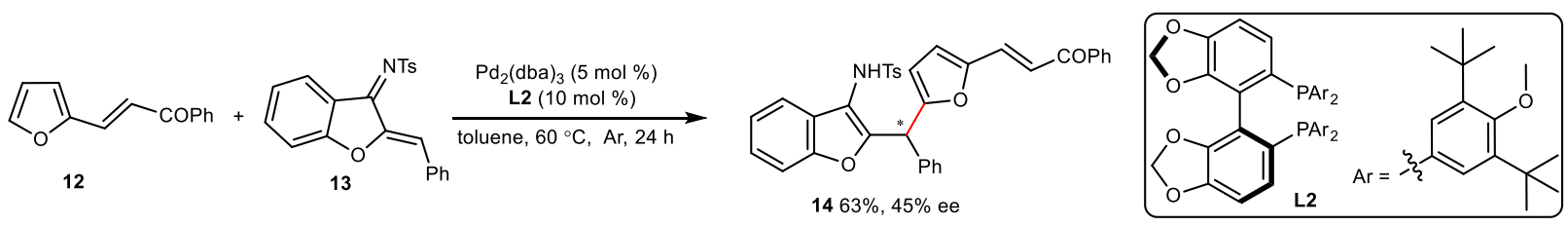

To an oven dried Schlenk tube equipped with a stir bar were added $\operatorname{Pd}_{2}(\mathrm{dba})_{3}(4.6 \mathrm{mg}, 0.005 \mathrm{mmol})$ and $\mathbf{L} 2$ (11.8 $\mathrm{mg}, 0.01 \mathrm{mmol}$ ). The tube was evacuated followed by back-filled with argon for five times. Then distilled and degassed toluene $(1.0 \mathrm{~mL})$ was added via syringe and stirred for $30 \mathrm{~min}$ at room temperature. And the catalytic solution was transferred to the mixture of (E)-3-(furan-2-yl)-1phenylprop-2-en-1-one $12(23.8 \mathrm{mg}, 0.12 \mathrm{mmol})$ and $N$-((Z)-2-benzylidenebenzofuran-3(2H)ylidene)-4-methylbenzenesulfonamide $13(37.5 \mathrm{mg}, 0.1 \mathrm{mmol})$ in one portion by syringe. The mixture was allowed to stir at $60{ }^{\circ} \mathrm{C}$ for $24 \mathrm{~h}$. The crude product was directly purified by flash chromatography on silica gel $($ EtOAc/petroleum ether $=1 / 5)$ to afford cycloadduct 14: $36.1 \mathrm{mg}, 63 \%$ 
yield; $[\alpha]_{\mathrm{D}}{ }^{25}=+20.6\left(c=0.65\right.$ in $\left.\mathrm{CHCl}_{3}\right) ; 45 \%$ ee; determined by HPLC analysis [Daicel Chiralpak $\mathrm{AD}-\mathrm{H}, n$-hexane $/ i$-PrOH $\left.=60 / 40,1.0 \mathrm{~mL} \mathrm{~min}^{-1}, \lambda=254 \mathrm{~nm}\right]: \mathrm{t}($ minor $)=11.23 \mathrm{~min}, \mathrm{t}$ (major) $=19.88$ $\min$.

\subsection{Synthetic transformations of $3 a$ and $5 a$}

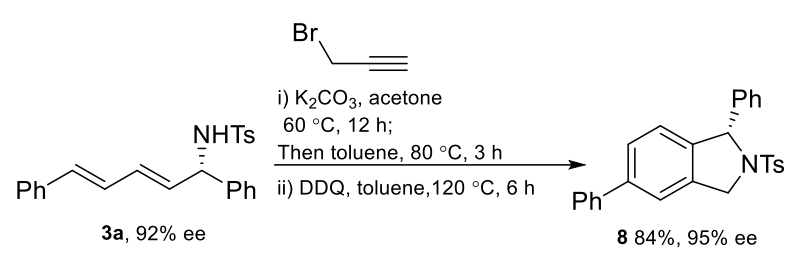

A solution of compound 3a (39.0 mg, $0.1 \mathrm{mmol}, 1.0$ equiv), 3-bromopropyne (23.8 mg, $0.2 \mathrm{mmol}$, 2.0 equiv) and potassium carbonate $(27.6 \mathrm{mg}, 0.2 \mathrm{mmol}, 2.0$ equiv) in acetone $(1.0 \mathrm{~mL})$ was stirred at $60{ }^{\circ} \mathrm{C}$ for $12 \mathrm{~h}$. After completion, the solvent was removed under reduced pressure, and the residue was dissolved in toluene $(1.0 \mathrm{~mL})$. The solution was stirred at $80{ }^{\circ} \mathrm{C}$ for $3 \mathrm{~h}$. After completion, purification by flash chromatography on silica gel $($ EtOAc/petroleum ether $=1 / 10)$ gave the intermediate. Subsequently, the intermediate was dissolved in anhydrous toluene $(1.0 \mathrm{~mL})$ and DDQ (25.0 mg, $1.1 \mathrm{mmol}, 1.1$ equiv) was added before heating at $120{ }^{\circ} \mathrm{C}$ for $6 \mathrm{~h}$ under Ar atmosphere. After completion, purification by flash chromatography on silica gel $($ EtOAc/petroleum ether $=1 / 20)$ gave the product 8: $35.8 \mathrm{mg}$, as a yellow solid, $84 \%$ yield; $\mathrm{mp}=158-160{ }^{\circ} \mathrm{C} ;[\alpha]_{\mathrm{D}}{ }^{25}=-133.7(c=$ 0.70 in $\mathrm{CHCl}_{3}$ ); $95 \%$ ee, determined by HPLC analysis [Daicel Chiralpak IA, $i \mathrm{PrOH} / n \mathrm{Hexane}=20 / 80$, $\left.1.0 \mathrm{~mL} \mathrm{~min}{ }^{-1}, \lambda=254 \mathrm{~nm}\right]: \mathrm{t}$ (major) $=12.29 \mathrm{~min}, \mathrm{t}$ (minor) $=13.98 \mathrm{~min} ;{ }^{1} \mathbf{H ~ N M R}\left(400 \mathrm{MHz}, \mathrm{CDCl}_{3}\right)$ : $\delta(\mathrm{ppm}) 7.59-7.54(\mathrm{~m}, 2 \mathrm{H}), 7.52-7.47(\mathrm{~m}, 2 \mathrm{H}), 7.45-7.31(\mathrm{~m}, 5 \mathrm{H}), 7.30-7.24(\mathrm{~m}, 5 \mathrm{H}), 7.18(\mathrm{~d}, J=$ $8.0 \mathrm{~Hz}, 2 \mathrm{H}), 6.94(\mathrm{~d}, J=8.0 \mathrm{~Hz}, 1 \mathrm{H}), 5.97-5.90(\mathrm{~m}, 1 \mathrm{H}), 4.94-4.84(\mathrm{~m}, 2 \mathrm{H}), 2.36(\mathrm{~s}, 3 \mathrm{H}) ;{ }^{13} \mathbf{C ~ N M R}$ $\left(100 \mathrm{MHz}, \mathrm{CDCl}_{3}\right): \delta$ (ppm) 143.3, 141.8, 141.4, 140.5, 140.0, 135.8, 135.3, 129.5, 128.8, 128.5, 127.8, 127.6, 127.5, 127.4, 127.2, 127.1, 123.9, 121.0, 69.3, 54.0, 21.4; HRMS (ESI-TOF) m/z: [M $+\mathrm{Na}]^{+}$Calcd for $\mathrm{C}_{27} \mathrm{H}_{23} \mathrm{NO}_{2} \mathrm{SNa} 448.1347$; Found 448.1345.

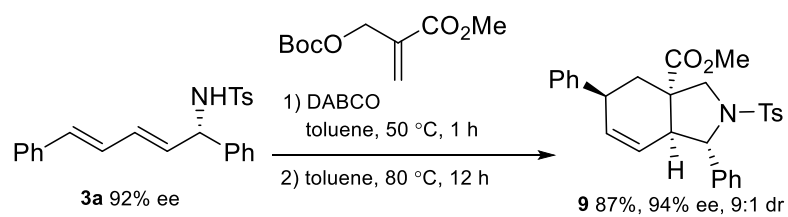

To a mixture of 3a $(0.1 \mathrm{mmol})$, DABCO $(2.2 \mathrm{mg}, 20 \mathrm{~mol} \%)$ was stirred in toluene $(0.5 \mathrm{~mL})$ at $50{ }^{\circ} \mathrm{C}$ was added acrylic ester (43.2 mg, $0.2 \mathrm{mmol}, 2.0$ equiv) slowly, which was stirred for $1 \mathrm{~h}$. Then the reaction mixture was stirred at $80{ }^{\circ} \mathrm{C}$ for $12 \mathrm{~h}$. After completion, purification by flash chromatography on silica gel (EtOAc/petroleum ether =1/20) gave the product 9: $42.5 \mathrm{mg}$, as a white semisolid, 87\% yield; 9:1 dr; $[\alpha]_{\mathrm{D}}{ }^{25}=-59.3\left(c=0.98\right.$ in $\left.\mathrm{CHCl}_{3}\right)$; 94\% ee, determined by HPLC analysis [Daicel Chiralpak IA, $i \mathrm{PrOH} / n \mathrm{Hexane}=20 / 80,1.0 \mathrm{~mL} \mathrm{~min}{ }^{-1}, \lambda=254 \mathrm{~nm}$ ]: $\mathrm{t}$ (major) $=9.01$ min, $\mathrm{t}($ minor $)=9.79 \mathrm{~min} ;{ }^{1} \mathbf{H}$ NMR $\left(400 \mathrm{MHz}, \mathrm{CDCL}_{3}\right): \delta(\mathrm{ppm}) 7.54(\mathrm{~d}, J=8.0 \mathrm{~Hz}, 2 \mathrm{H}), 7.40$ 
$7.12(\mathrm{~m}, 8 \mathrm{H}), 7.13(\mathrm{~d}, J=7.6 \mathrm{~Hz}, 2 \mathrm{H}), 6.97(\mathrm{~d}, J=7.2 \mathrm{~Hz}, 2 \mathrm{H}), 5.71(\mathrm{~d}, J=10.0 \mathrm{~Hz}, 1 \mathrm{H}), 5.66-5.61$ $(\mathrm{m}, 1 \mathrm{H}), 4.28(\mathrm{~d}, J=9.6 \mathrm{~Hz}, 1 \mathrm{H}), 3.92(\mathrm{~d}, J=11.6 \mathrm{~Hz}, 1 \mathrm{H}), 3.74(\mathrm{~s}, 3 \mathrm{H}), 3.67$ (d, $J=11.6 \mathrm{~Hz}, 1 \mathrm{H})$, $3.40-3.22(\mathrm{~m}, 1 \mathrm{H}), 3.20-3.16(\mathrm{~m}, 1 \mathrm{H}), 2.21(\mathrm{~s}, 3 \mathrm{H}), 1.95(\mathrm{dd}, J=13.6,4.8 \mathrm{~Hz}, 1 \mathrm{H}), 0.84-0.78(\mathrm{~m}$, $1 \mathrm{H}) ;{ }^{13} \mathrm{C}$ NMR $\left(100 \mathrm{MHz}, \mathrm{CDCl}_{3}\right): \delta$ (ppm) 173.0, 144.0, 143.7, 141.0, 134.2, 131.8, 129.5, 128.5, $128.5,127.7,127.30,127.26,126.6,126.5,124.1,70.8,58.1,52.6,50.4,50.2,40.0,34.9,21.4$; HRMS (ESI-TOF) m/z: [M + Na $]^{+}$Calcd for $\mathrm{C}_{29} \mathrm{H}_{29} \mathrm{NO}_{4} \mathrm{SNa} 510.1710$; Found 510.1709.

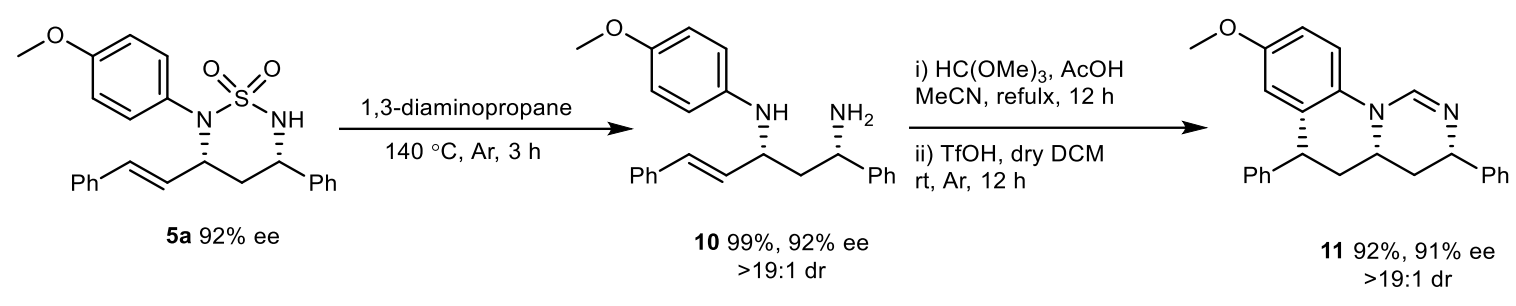

A solution of compound $\mathbf{5 a}(42.1 \mathrm{mg}, 0.1 \mathrm{mmol}, 1.0$ equiv) in 1,3-diaminopropane ( $0.5 \mathrm{~mL})$ was stirred at $140{ }^{\circ} \mathrm{C}$ for $3 \mathrm{~h}$ under Ar atmosphere. After completion, the solution was then cooled to room temperature, quenched with water $(5.0 \mathrm{~mL})$ and extracted with DCM $(5 \times 5 \mathrm{~mL})$. The combined organic phase was dried over $\mathrm{Na}_{2} \mathrm{SO}_{4}$ and concentrated in vacuo to give the crude product, which was further purified by column chromatography on silica gel $(\mathrm{MeOH} / \mathrm{DCM}=1 / 50)$ gave the product 10: $36.0 \mathrm{mg}$, as a yellow oil, $99 \%$ yield; $>19: 1 \mathrm{dr} ;[\alpha]_{\mathrm{D}}{ }^{25}=+48.1\left(c=1.48 \mathrm{in} \mathrm{CHCl}_{3}\right) ; 92 \%$ ee, determined by HPLC analysis [Daicel Chiralpak IG, $i \mathrm{PrOH} / n \mathrm{Hexane}=20 / 80,1.0 \mathrm{~mL} \min -1, \lambda=254 \mathrm{~nm}$ ]: $\mathrm{t}$ $($ minor $)=11.73 \mathrm{~min}, \mathrm{t}($ major $)=13.62 \mathrm{~min} ;{ }^{1} \mathbf{H} \mathbf{~ N M R}\left(400 \mathrm{MHz}, \mathrm{CDCl}_{3}\right): \delta(\mathrm{ppm}) 7.34-7.16(\mathrm{~m}$, $11 \mathrm{H}), 6.73(\mathrm{~d}, J=8.8 \mathrm{~Hz}, 2 \mathrm{H}), 6.58(\mathrm{~d}, J=8.8 \mathrm{~Hz}, 2 \mathrm{H}), 6.52(\mathrm{~d}, J=16.0 \mathrm{~Hz}, 1 \mathrm{H}), 6.11(\mathrm{dd}, J=15.9$, $6.4 \mathrm{~Hz}, 1 \mathrm{H}), 4.13-4.09$ (m, 1H), 3.98-3.93 (m, 1H), 3.71 (s, 3H), 2.64 (brs, 2H), 2.04-1.94 (m, 2H); ${ }^{13} \mathrm{C}$ NMR $\left(100 \mathrm{MHz}, \mathrm{CDCl}_{3}\right): \delta$ (ppm) 152.0, 146.6, 141.8, 136.9, 132.3, 129.9, 128.7, 128.5, 127.3, 127.2, 126.3, 126.1, 115.0, 114.7, 55.7, 55.6, 54.4, 45.2; HRMS (ESI-TOF) m/z: $[\mathrm{M}+\mathrm{Na}]^{+} \mathrm{Calcd}$ for $\mathrm{C}_{24} \mathrm{H}_{26} \mathrm{~N}_{2} \mathrm{ONa} 381.1937$; Found 381.1939.

A solution of compound 10 (35.9 mg, $0.1 \mathrm{mmol}, 1.0$ equiv), trimethoxymethane (31.8 $\mathrm{mg}, 0.3$ mmol, 3.0 equiv) and $\mathrm{AcOH}\left(18.1 \mathrm{mg}, 0.3 \mathrm{mmol}, 3.0\right.$ equiv) in $\mathrm{CH}_{3} \mathrm{CN}$ (1.0 mL) was heated under reflux for $12 \mathrm{~h}$. After completion, the solvent was removed under reduced pressure, and the residue was dissolved in anhydrous DCM (1.0 mL) and followed by the addition of TfOH $(75.6 \mathrm{mg}, 0.5 \mathrm{mmol}$, 5.0 equiv) at $0{ }^{\circ} \mathrm{C}$ under argon atmosphere. The mixture was vigorously stirred at room temperature for $12 \mathrm{~h}$. After completion, the solution was cooled at $0{ }^{\circ} \mathrm{C}$, quenched with aqueous $\mathrm{NaHCO}_{3}$ solution and extracted with DCM $(5 \times 5 \mathrm{~mL})$. The combined organic phase was dried over $\mathrm{Na}_{2} \mathrm{SO}_{4}$ and concentrated in vacuo to give the crude product, which was further purified by column chromatography on neutral aluminium oxide $(\mathrm{MeOH} / \mathrm{DCM}=1 / 50)$ gave the product 11: $34.0 \mathrm{mg}$ $(0.092 \mathrm{mmol})$, as a white solid, $92 \%$ yield; $>19: 1 \mathrm{dr} ; \mathrm{mp}=165-167{ }^{\circ} \mathrm{C} ;[\alpha]_{\mathrm{D}}^{25}=+51.8(c=0.78$ in $\mathrm{CHCl}_{3}$ ); $91 \%$ ee, determined by HPLC analysis [Daicel Chiralpak ID, $i \mathrm{PrOH} / n \mathrm{Hexane}=40 / 60,1.0$ 
$\left.\mathrm{mL} \min ^{-1}, \lambda=254 \mathrm{~nm}\right]: \mathrm{t}$ (minor) $=10.87 \mathrm{~min}, \mathrm{t}($ major $)=19.35 \mathrm{~min} ;{ }^{1} \mathbf{H} \mathbf{N M R}\left(400 \mathrm{MHz}, \mathrm{CDCl}_{3}\right)$ : $\delta(\mathrm{ppm}) 7.94(\mathrm{~d}, J=2.4 \mathrm{~Hz}, 1 \mathrm{H}), 7.44-7.39$ (m, 2H), 7.39-7.30 (m, 4H), 7.29-7.22 (m, 2H), 7.21$7.15(\mathrm{~m}, 2 \mathrm{H}), 7.08(\mathrm{~d}, J=9.2 \mathrm{~Hz}, 1 \mathrm{H}), 6.77(\mathrm{dd}, J=9.0,3.0 \mathrm{~Hz}, 1 \mathrm{H}), 6.37-6.30(\mathrm{~m}, 1 \mathrm{H}), 4.52-4.44$ $(\mathrm{m}, 1 \mathrm{H}), 4.10(\mathrm{dd}, J=12.4,5.6 \mathrm{~Hz}, 1 \mathrm{H}), 4.00-3.90(\mathrm{~m}, 1 \mathrm{H}), 3.61(\mathrm{~s}, 3 \mathrm{H}), 2.42-2.32(\mathrm{~m}, 2 \mathrm{H}), 1.91-$ $1.77(\mathrm{~m}, 1 \mathrm{H}), 1.68-1.56(\mathrm{~m}, 1 \mathrm{H}) ;{ }^{13} \mathrm{C}$ NMR (100 MHz, $\left.\mathrm{CDCl}_{3}\right): \delta(\mathrm{ppm}) 154.8,144.7,144.4,144.3$, 132.5, 129.8, 128.7, 128.5, 128.4, 126.9, 126.8, 126.5, 115.9, 115.7, 113.0, 56.8, 55.4, 52.3, 44.0, 39.1, 38.9; HRMS (ESI-TOF) m/z: [M + H $]^{+}$Calcd for $\mathrm{C}_{25} \mathrm{H}_{25} \mathrm{~N}_{2} \mathrm{O} 369.1967$; Found 369.1963. 


\section{1) Different alkenes in the possible reaction with imine $2 a$}

Several aromatic vinyl compounds and allyl benzene were tested, but they were all inert in the reaction with $2 \mathbf{a}$ under the catalysis of $\mathrm{Pd}\left(\mathrm{PPh}_{3}\right)_{4}$.

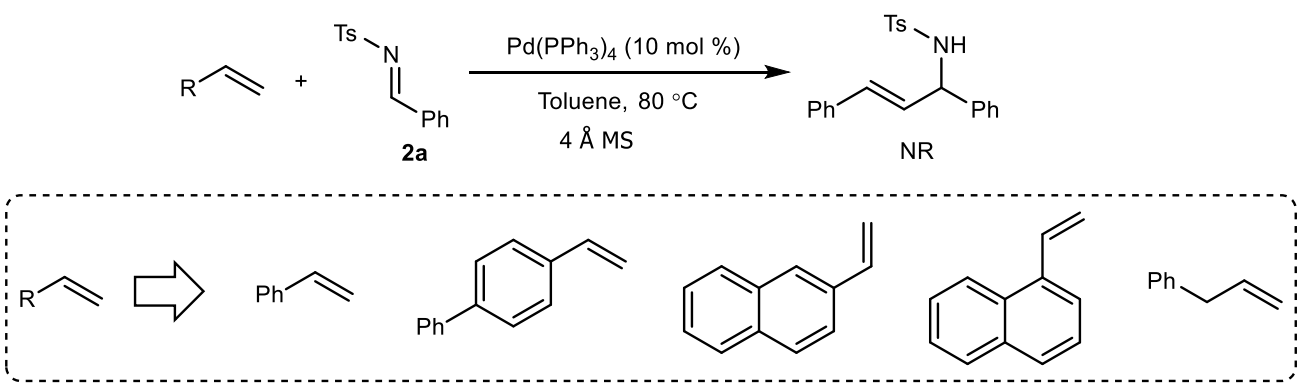

Scheme S2. Unsuccessful attempt for the reaction with alkenes

\section{2) More attempt with 1,3-dienes in the hydrodienylation reaction}

\section{Different 1,3-dienes and imines}

Under the standard conditions, the following 1,3-dienes also were applied to the asymmetric hydrodienylation reaction with imine $\mathbf{2 a}$, but most of them showed low or even no reactivity. The one with a bromobenzene failed probably due the fast oxidative insertion of $\mathrm{Pd}$ to the $\mathrm{C}-\mathrm{Br}$ bond; while 1-phenyl-3-methyl-butadiene afforded the addition products with some inseparable side-products, which might result from a different regioselective $\beta-\mathrm{H}$ elimination at the methyl group after the addition step, together with some isomers in Z-configuration. The internal diene (with a 4-metyl group) and the ones with other substitution patterns were inert in the reaction. On the other hand, an imine with a linear alkyl group also showed very low reactivity.

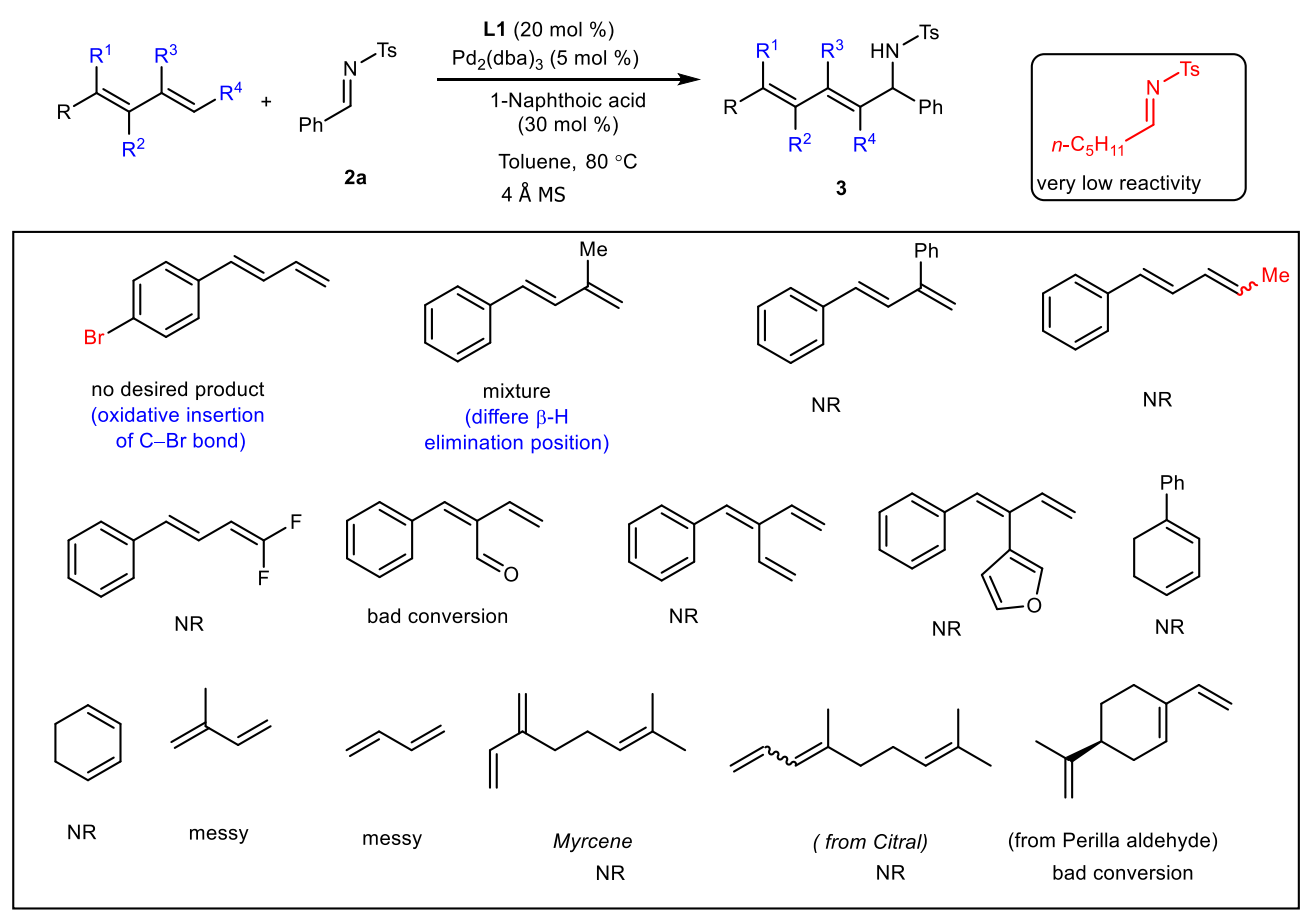

Scheme S3. Unsuccessful attempt for the hydrodienylation reaction 


\section{Different electrophiles}

Besides $N$-sulfonylimines, an $N$-Boc protected imine from benzaldehyde could react with 1e under the asymmetric conditions, giving two products with $\mathrm{C} 4$ - and C1-regioselectivity, respectively, but with lower enantioselectivity.

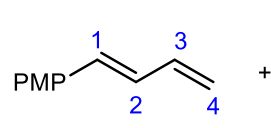

$1 \mathrm{e}$

(1)

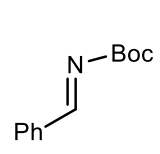$$
\mathrm{Ph}
$$
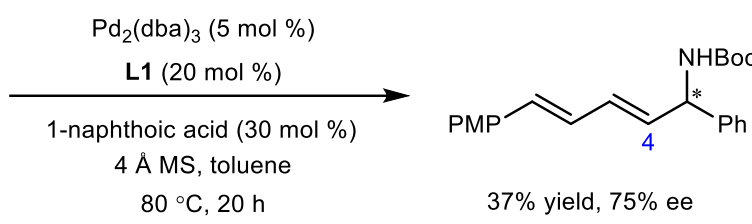

$37 \%$ yield, $75 \%$ ee<smiles>C=C/C=C(\P)C(=NC(=O)c1ccccc1)c1ccccc1</smiles>

$21 \%$ yield, $31 \%$ ee

Scheme S4. The Pd-catalysed reaction with $N$-Boc protected imine

Importantly, some reactive aldehydes and ketones also were compatible in the hydrodienylation reaction, whereas electron-deficient alkenes seemed to be inert at the current stage.

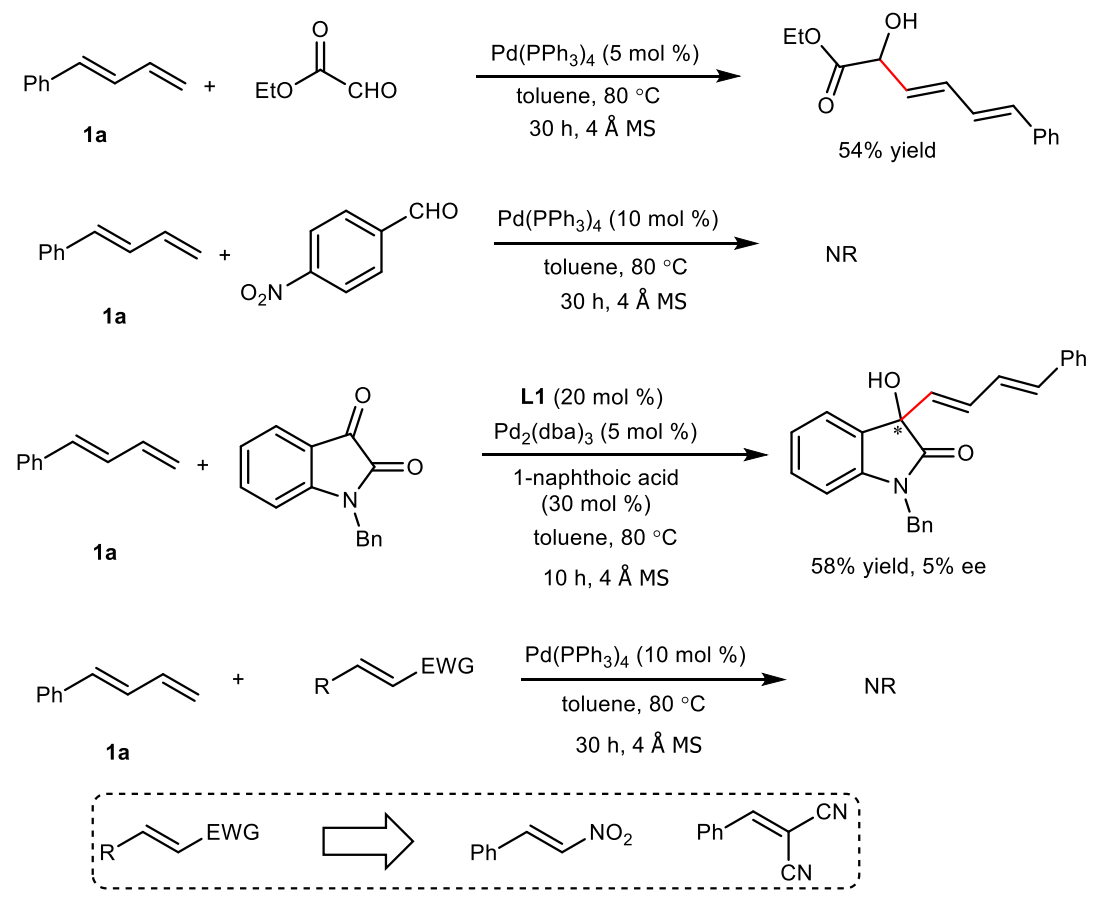

Scheme S5. The hydrodienylation reaction with different carbonyls and activated alkenes

\section{3) More attempt with 1,3-dienes in the cascade reaction for the construction of 1,3-diamines}

\section{Different 1,3-dienes}

Similarly, the following 1,3-dienes were also applied to the asymmetric cascade reaction with the functionalized imine $\mathbf{4 a}$, but disappointing results were obtained. 

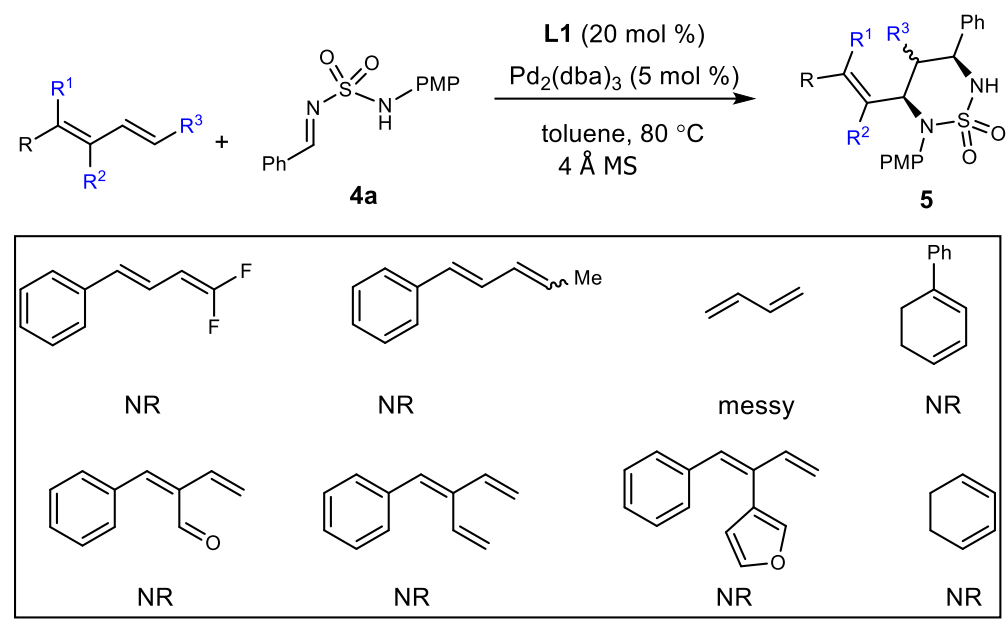

\section{Scheme S6. Unsuccessful attempt for the cascade reaction of 4 a with 1,3-dienes}

In addition, to synthesize the 1,3-diamines $\mathbf{5}$ with a tetrasubstituted stereogenic center, the dienes with a substituent at the C3-position were applied to the asymmetric cascade reaction with $\mathbf{4 a}$. The ones with a methyl group afforded the desired addition products in good conversions, but with some inseparable mixtures, while the others could not work or gave bad converstions.
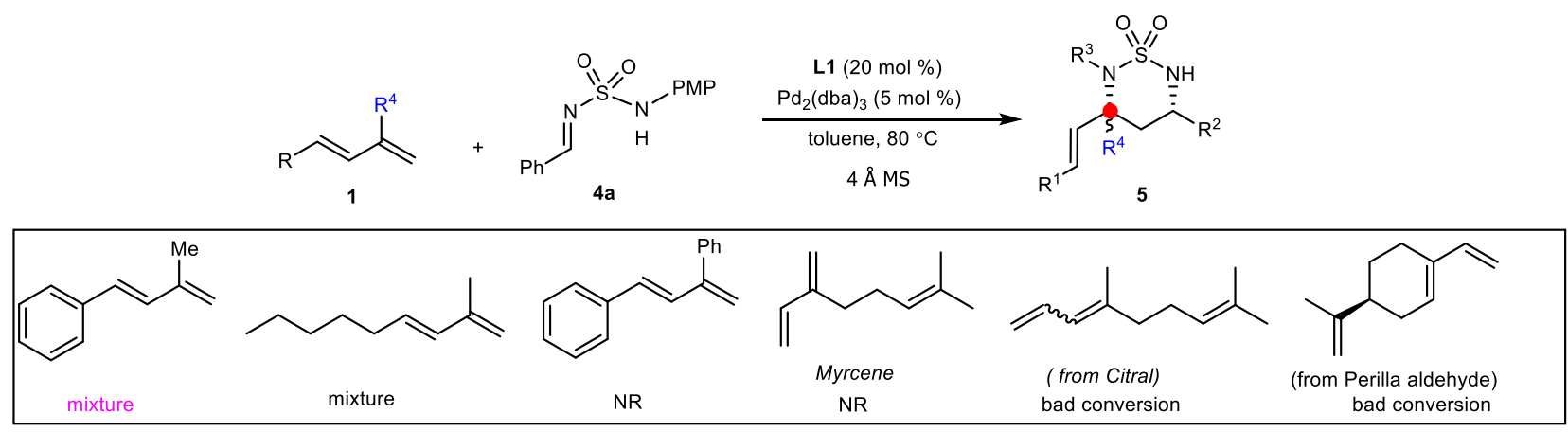

Scheme S7. Unsuccessful attempt for the construction of a tetrasubstituted stereogenic center via the cascade reaction of 1,3 -dienes

\section{Three-component cascade reaction}

An additional nucleophiles were added to the reaction under the catalysis of $\mathrm{Pd}\left(\mathrm{PPh}_{3}\right)_{4}$, and it was found a difuctionalised product was obtained in the presence of piperidine, in good yield and diastereoselectivity. When morpholine was employed as the nucleophile, the diamine and hydroamination product were afford as an inseparable mixture. But no desired cascade reaction occurred when 4-methoxyaniline or dibenzylamine was applied to the reaction. 


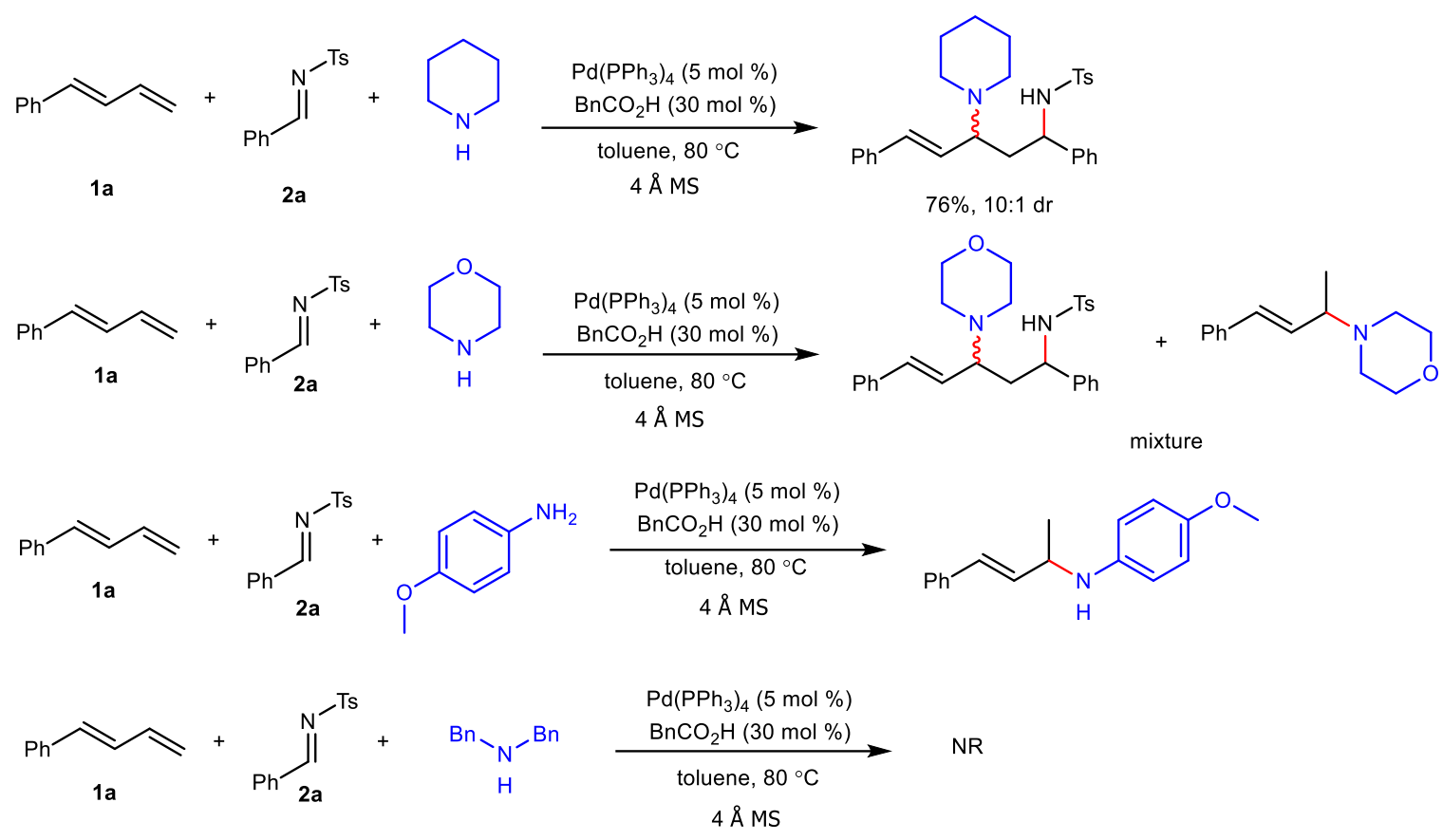

Scheme S8. Three-component cascade reaction for the construction of 1,3-diamines

4) More attempt with 1,3-dienes in the cascade reaction for the construction of chromane derivatives

In addition, a 1,3-diene with an aliphatic chain and a 2-methyl group was applied to the cascade reaction with $\mathbf{6 b}$. Although good converstions were observed, a mixture with the addition products and some inseparable side-products was obtained.

For the cascade reaction with $\mathbf{1 e}$, it was notable that a salicylaldehyde with an electron-withdrawing group showed fair reactivity under the standard conditions, and the desired product was obtained albeit in low yield and stereocontrol, which will be further explored in the future.

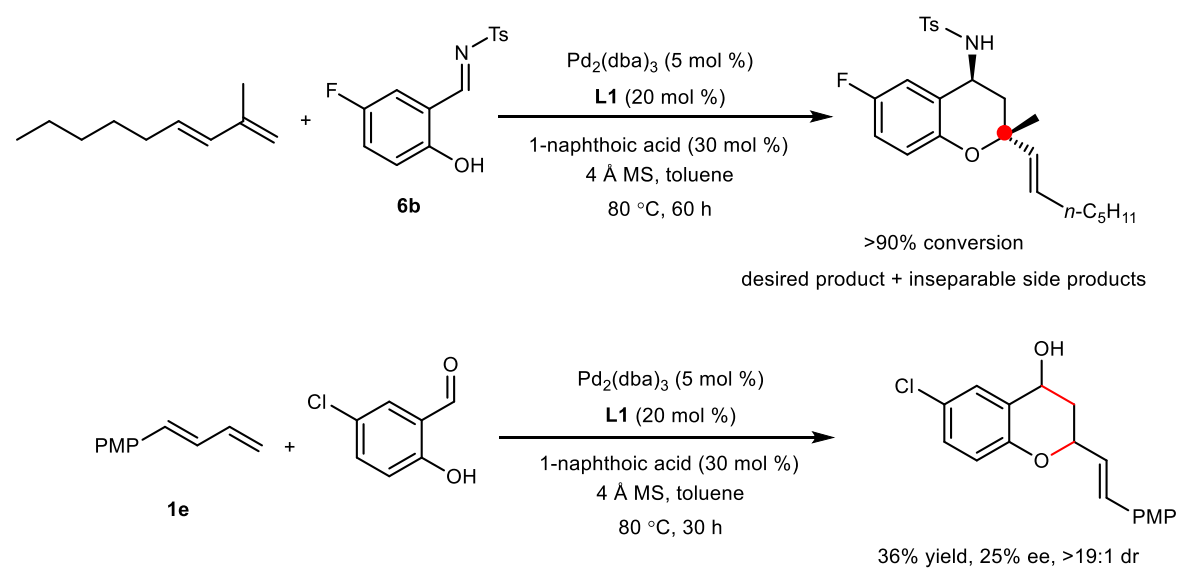

Scheme S9. The asymmetric cascade reaction for the construction of chromane derivatives 


\subsection{Crystal data and structure refinement}

Preparation of the single crystals of enantiopure 3a: Compound 3a (30.0 $\mathrm{mg}, 92 \%$ ee) was dissolved in $i \mathrm{PrOH}(2.0 \mathrm{~mL})$ in a $10 \mathrm{~mL}$ tube and $n$-hexane $(3.0 \mathrm{~mL})$ was added. The tube was sealed by a piece of weighing paper with several tiny holes, thus allowing slow evaporation of the solvents at room temperature. After $72 \mathrm{~h}$, several small particles could be observed at the bottom of the tube. The crystals were chosen and subjected to the single crystal X-ray diffraction analysis for the determination of the absolute configuration of 3a. The data were collected by an Agilent Gemini equipped with a Cu radiation source $(\mathrm{K} \alpha=1.54184 \AA$ ) at 293.8(5) K. CCDC 2031429 (3a) contains the supplementary crystallographic data for this paper. These data can be obtained free of charge via www.ccdc.cam.ac.uk/data_request/cif.
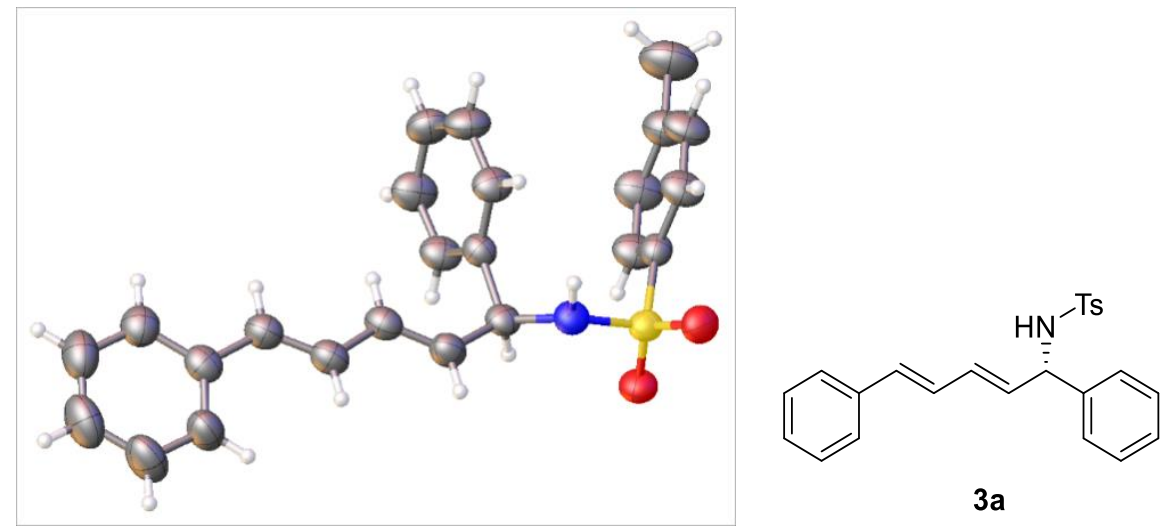

(ellipsoid contour probability 50\%)

Table S4 Crystal data and structure refinement for $3 a$

Identification code

Empirical formula

Formula weight

Temperature/K

Crystal system

Space group

$\mathrm{a} / \AA$

$\mathrm{b} / \AA$

$\mathrm{c} / \AA$

$\alpha /{ }^{\circ}$

$\beta /{ }^{\circ}$

$\gamma /{ }^{\circ}$

Volume $/ \AA^{3}$

Z

$\rho_{\text {calc }} \mathrm{g} / \mathrm{cm}^{3}$

$\mu / \mathrm{mm}^{-1}$
$3 \mathbf{a}$

$\mathrm{C}_{24} \mathrm{H}_{23} \mathrm{NO}_{2} \mathrm{~S}$

389.49

293.8(5)

orthorhombic

P2 $22_{1}{ }_{1}$

8.4491(2)

9.6736(2)

25.4522(7)

90

90

90

2080.29(10)

4

1.244

1.524 
$\mathrm{F}(000)$

Crystal size $/ \mathrm{mm}^{3}$

Radiation

$2 \Theta$ range for data collection/ ${ }^{\circ}$

Index ranges

Reflections collected

Independent reflections

Data/restraints/parameters

Goodness-of-fit on $\mathrm{F}^{2}$

Final $R$ indexes $[\mathrm{I}>=2 \sigma(\mathrm{I})]$

Final R indexes [all data]

Largest diff. peak/hole / e $\AA^{-3}$

Flack parameter
824.0

$0.7 \times 0.4 \times 0.15$

$\mathrm{CuK} \alpha(\lambda=1.54184)$

6.946 to 143.246

$-8 \leq \mathrm{h} \leq 10,-7 \leq \mathrm{k} \leq 11,-30 \leq 1 \leq 31$

11328

$3970\left[\mathrm{R}_{\text {int }}=0.0545, \mathrm{R}_{\text {sigma }}=0.0419\right]$

$3970 / 0 / 258$

1.055

$\mathrm{R}_{1}=0.0587, \mathrm{wR}_{2}=0.1517$

$\mathrm{R}_{1}=0.0610, \mathrm{wR}_{2}=0.1559$

$0.35 /-0.38$

$-0.002(16)$ 
Preparation of the single crystals of enantiopure 5g: Compound 5g (20.0 $\mathrm{mg}$, 94\% ee) was dissolved in DCM $(1.0 \mathrm{~mL})$ in a $10 \mathrm{~mL}$ tube and $n$-hexane $(3.0 \mathrm{~mL})$ was added. The tube was sealed by a piece of weighing paper with several tiny holes, thus allowing slow evaporation of the solvents at room temperature. After $12 \mathrm{~h}$, several small particles could be observed at the bottom of the tube. The crystals were chosen and subjected to the single crystal X-ray diffraction analysis for the determination of the absolute configuration of $\mathbf{5 g}$. The data were collected by an Agilent Gemini equipped with a $\mathrm{Cu}$ radiation source $(\mathrm{K} \alpha=1.54184 \AA$ A) at 298.1(9) K. CCDC 2031430 (5g) contains the supplementary crystallographic data for this paper. These data can be obtained free of charge via www.ccdc.cam.ac.uk/data_request/cif.

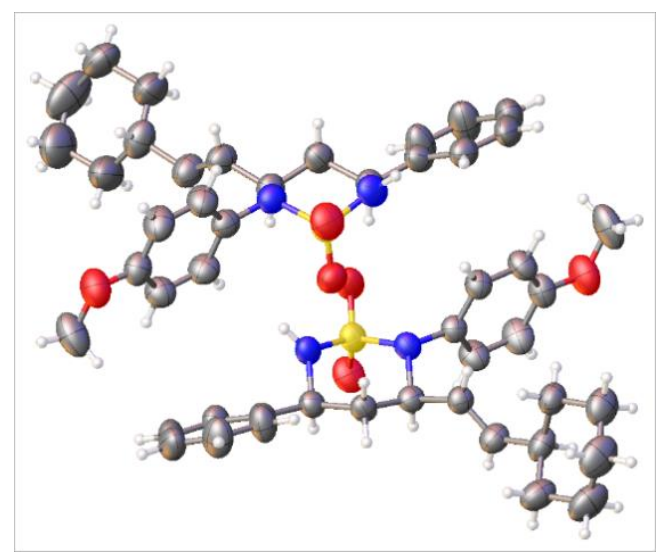<smiles>COc1ccc(N(/C=C/C2CCCCC2)C(C[C@@H](NS(=O)(=O)[O-])c2ccccc2)c2ccccc2)cc1</smiles>

(ellipsoid contour probability 50\%)

\section{Table S5 Crystal data and structure refinement for $\mathbf{5 g}$}

Identification code

Empirical formula

Formula weight

Temperature/K

Crystal system

Space group

$\mathrm{a} / \AA$

b/ $\AA$

$\mathrm{c} / \AA$

$\alpha /^{\circ}$

$\beta /^{\circ}$

$\gamma /{ }^{\circ}$

Volume $/ \AA^{3}$

$\mathrm{Z}$

$\rho_{\text {calc }} \mathrm{g} / \mathrm{cm}^{3}$

$\mu / \mathrm{mm}^{-1}$

$\mathrm{F}(000)$

Crystal size $/ \mathrm{mm}^{3}$
$5 \mathrm{~g}$

$\mathrm{C}_{24} \mathrm{H}_{30} \mathrm{~N}_{2} \mathrm{O}_{3} \mathrm{~S}$

426.56

298.1(9)

monoclinic

$\mathrm{P} 2_{1}$

8.94281(17)

26.4006(5)

$10.57536(19)$

90

112.776(2)

90

2302.10(8)

4

1.231

1.460

912.0

$0.5 \times 0.5 \times 0.15$ 
Radiation

$2 \Theta$ range for data collection/ ${ }^{\circ}$

Index ranges

Reflections collected

Independent reflections

Data/restraints/parameters

Goodness-of-fit on $\mathrm{F}^{2}$

Final R indexes $[\mathrm{I}>=2 \sigma(\mathrm{I})]$

Final $\mathrm{R}$ indexes [all data]

Largest diff. peak/hole / e $\AA^{-3}$

Flack parameter
$\mathrm{CuK} \alpha(\lambda=1.54184)$

6.696 to 142.874

$-10 \leq \mathrm{h} \leq 10,-32 \leq \mathrm{k} \leq 32,-12 \leq 1 \leq 9$

17460

$8225\left[\mathrm{R}_{\text {int }}=0.0391, \mathrm{R}_{\text {sigma }}=0.0418\right]$

$8225 / 1 / 543$

1.064

$\mathrm{R}_{1}=0.0587, \mathrm{wR}_{2}=0.1594$

$\mathrm{R}_{1}=0.0626, \mathrm{wR}_{2}=0.1668$

$0.30 /-0.34$

$-0.014(12)$ 
Preparation of the single crystals of racemic 14: Compound 14 (30.0 $\mathrm{mg}$ ) was dissolved in $\mathrm{CH}_{2} \mathrm{Cl}_{2} / \mathrm{MeOH}(2 \mathrm{~mL} / 0.5 \mathrm{~mL})$ in a $10 \mathrm{~mL}$ tube and $n$-hexane $(2.0 \mathrm{~mL})$ was added. The tube was sealed by a piece of weighing paper with several tiny holes, thus allowing slow evaporation of the solvents at room temperature. After $48 \mathrm{~h}$, several small particles could be observed at the bottom of the tube. The crystals were chosen and subjected to the single crystal X-ray diffraction analysis for the determination of the structure of product 14. The data were collected by a Bruker APEX-II CCD diffractometer equipped with a Mo radiation source $(\mathrm{K} \alpha=0.71073 \AA$ ) at 296.0 K. CCDC 2050827 (15) contains the supplementary crystallographic data for this paper. These data can be obtained free of charge via www.ccdc.cam.ac.uk/data_request/cif.

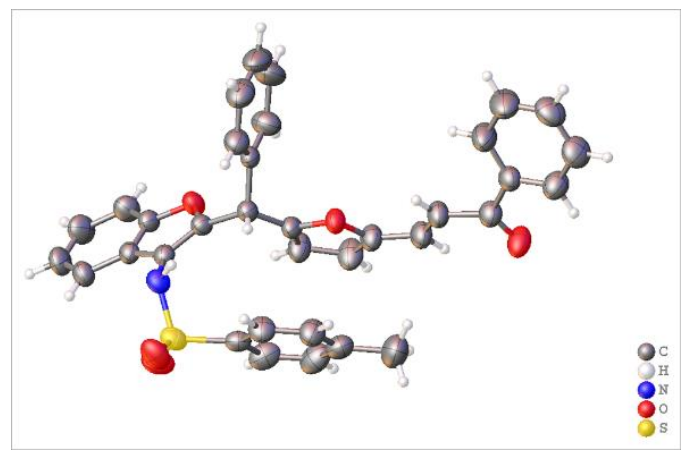

(ellipsoid contour probability 50\%)

\section{Table S10 Crystal data and structure refinement for 14}

Identification code

Empirical formula

Formula weight

Temperature/K

Crystal system

Space group

$\mathrm{a} / \AA$

b/A

$\mathrm{c} / \AA$

$\alpha /^{\circ}$

$\beta /{ }^{\circ}$

$\gamma /{ }^{\circ}$

Volume $/ \AA^{3}$

Z

$\rho_{\text {calc } g / \mathrm{cm}^{3}}$

$\mu / \mathrm{mm}^{-1}$

$\mathrm{F}(000)$

Crystal size $/ \mathrm{mm}^{3}$

Radiation

$2 \Theta$ range for data collection/ ${ }^{\circ}$
14

$\mathrm{C}_{35} \mathrm{H}_{27} \mathrm{NO}_{5} \mathrm{~S}$

573.63

296.0

monoclinic

$\mathrm{P} 2{ }_{1} / \mathrm{c}$

10.2907(6)

$30.0857(19)$

$9.7868(5)$

90

104.909(2)

90

2928.0(3)

4

1.301

0.155

1200.0

$0.3 \times 0.2 \times 0.15$

$\operatorname{MoK} \alpha(\lambda=0.71073)$

4.096 to 54.964 
Index ranges

Reflections collected

Independent reflections

Data/restraints/parameters

Goodness-of-fit on $\mathrm{F}^{2}$

Final $\mathrm{R}$ indexes $[\mathrm{I}>=2 \sigma(\mathrm{I})]$

Final $\mathrm{R}$ indexes [all data]

Largest diff. peak/hole / e $\AA^{-3}$
$-13 \leq \mathrm{h} \leq 13,-39 \leq \mathrm{k} \leq 32,-12 \leq 1 \leq 12$

31470

$6719\left[\mathrm{R}_{\text {int }}=0.1073, \mathrm{R}_{\text {sigma }}=0.0880\right]$

$6719 / 0 / 380$

1.019

$\mathrm{R}_{1}=0.0582, \mathrm{wR}_{2}=0.1178$

$\mathrm{R}_{1}=0.1457, \mathrm{wR}_{2}=0.1524$

$0.20 /-0.25$ 


\subsection{Mechanism study}

\section{(1) Control experiments}

\section{1) Alkenes VS 1,3-dienes}

We have tried several aromatic vinyl compounds and allyl benzene, but they were all inert in the reactions with 2a under the catalysis of $\mathrm{Pd}\left(\mathrm{PPh}_{3}\right)_{4}$, indicating the importance of 1,3-diene structure, and suggesting the potentially different catalytic mechanism from $\mathrm{Ni}(0)$-based catalysis.

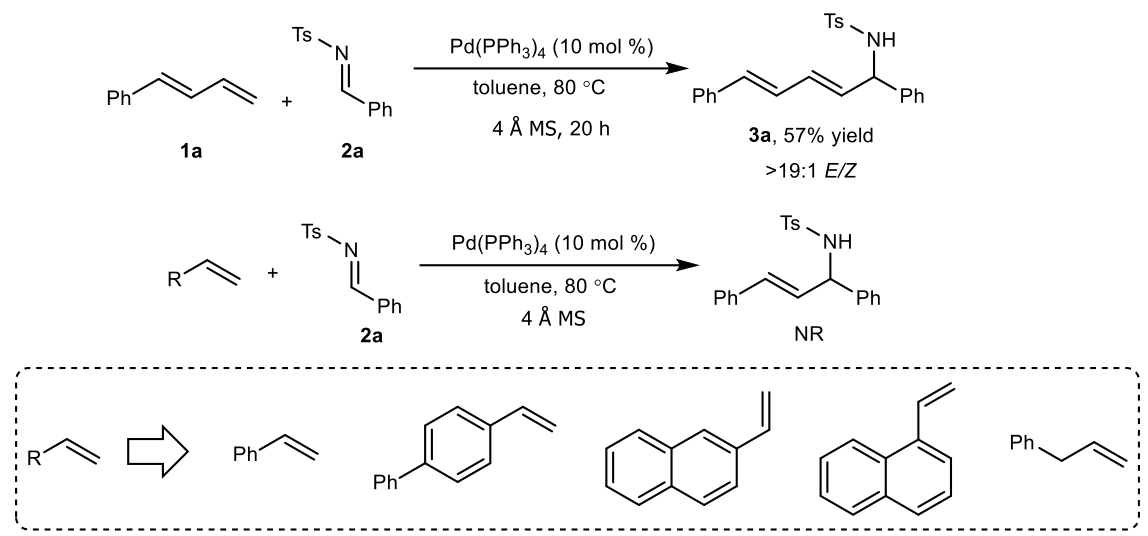

Scheme S10. The comparison between 1,3-dienes and alkenes

\section{2) Acid additives}

The hydrodienylation of $\mathbf{2 a}$ with 1 a could occur under the catalysis of $\mathrm{Pd}\left(\mathrm{PPh}_{3}\right)_{4}$, while the rate could be accelerated by adding catalytic amounts of acid additive, such as 1-naphthoic acid (for more examples, please see Table S1).
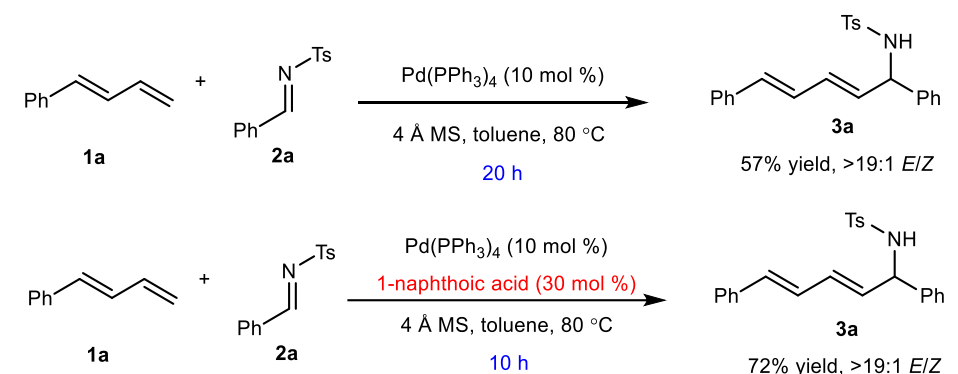

$72 \%$ yield, $>19: 1 E / Z$

Scheme S11. The acid effect on the hydrodienylation reaction

\section{3) The role of Pd catalyst}

The hydrodienylation reaction between $1 \mathbf{a}$ and imine $\mathbf{2 a}$ could proceed smoothly under the catalysis of $\mathrm{Pd}\left(\mathrm{PPh}_{3}\right)_{4}$, while the reaction with some Lewis acids, such as $\mathrm{Cu}(\mathrm{OTf})_{2}$ and $\mathrm{Sc}(\mathrm{OTf})_{2}$, could not work at all, suggesting the Pd catalyst would not serve as a Lewis acid and the reaction likely did not proceed via a simple ene-type addition process.

In addition, we also tried other $\mathrm{Pd}(\mathrm{II})$ salts instead of $\mathrm{Pd}\left(\mathrm{PPh}_{3}\right)_{4}$, including $\mathrm{Pd}(\mathrm{OAc})_{2}, \mathrm{Pd}\left(\mathrm{BF}_{4}\right)_{2}$, $\operatorname{Pd}(\mathrm{TFA})_{2}$ and in-situ formed $\mathrm{Pd}\left(\mathrm{PF}_{6}\right)_{2}$, but they did not work either. However, when phosphine ligands were added, good results were obtained under the catalysis of $\operatorname{Pd}(\mathrm{OAc})_{2}$. Since all the reactions were carried out under Ar, it was speculated that the reaction also started with $P d(0)$, and 
$P d(I I)$ salts would become reactive after the reduction to $P d(0)$ in the presence of phosphine ligands. Nevertheless, without $\mathrm{Pd}$, no reaction occurred even with the addition of acid additives.

Besides, in-situ formed $\mathrm{Ni}(0)$ catalyst was also tested, but no reaction occurred, indicating that different activation mode would exist between $\mathrm{Pd}(0)$ and $\mathrm{Ni}(0)$ complexes.

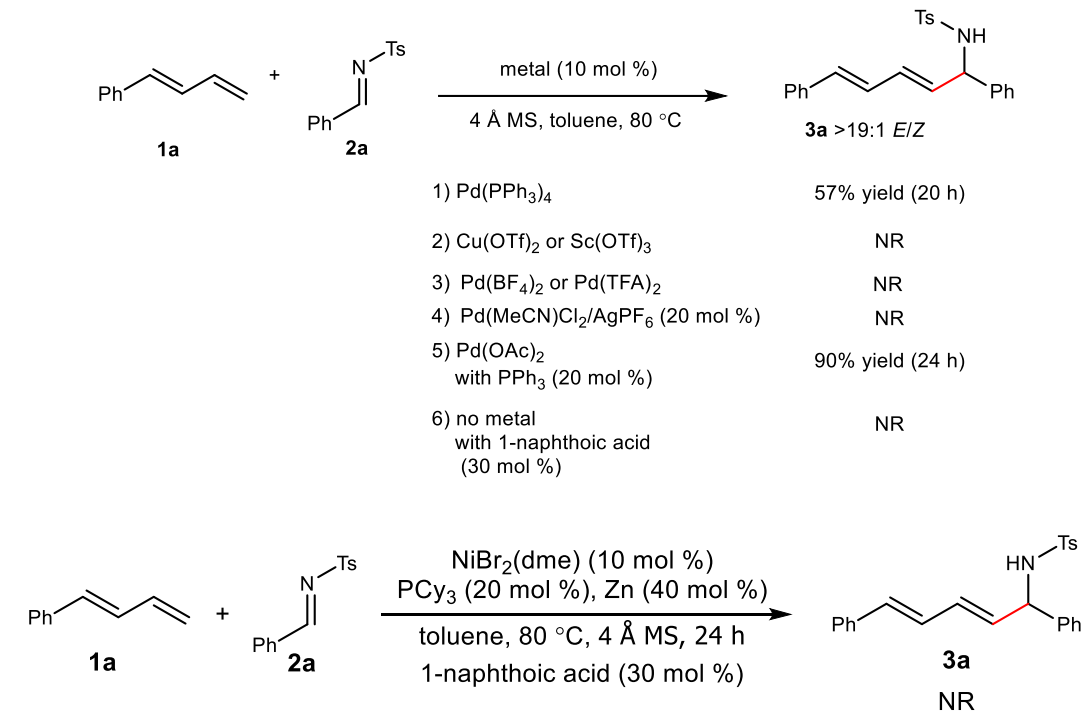

Scheme S12. Different metals in the hydrodienylation reaction

\section{4) Deuterium experiments}

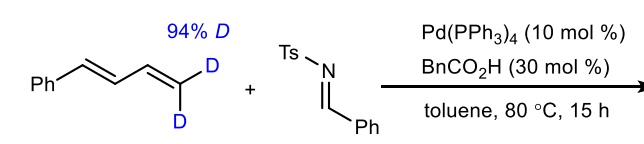

$2 a$

$d-1 \mathrm{a}$

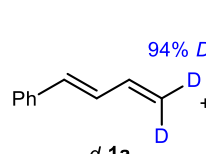

$d-1 \mathbf{a}$
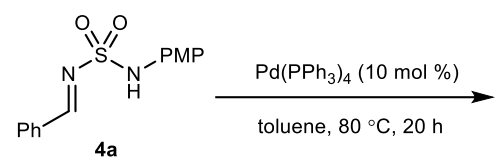

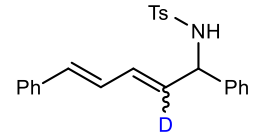

$92 \% D$

$d-3 a, 77 \%$ yield

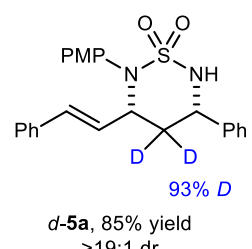

$d-5 a, 85 \%$ yield

Scheme S13. Pd catalysed reaction with deuterated 1,3-dienes

Substrate $d$-1a with $94 \%$ deuterium incorporated into the terminal position was prepared, and it afforded $d \mathbf{- 3 a}$ via hydrodienylation with 2a under the catalysis of $\mathrm{Pd}\left(\mathrm{PPh}_{3}\right)_{4}$ and phenylacetic acid. Only one deuterium atom was kept in the product with $92 \%$ deuterium, suggesting the cleavage of $\mathrm{C}-\mathrm{H}$ at the terminal position of 1,3-diene was involved. Besides, the cascade vinylogous addition/allylic alkylation of $d$-1a with $\mathbf{4 a}$ was carried out in the presence of $\mathrm{Pd}\left(\mathrm{PPh}_{3}\right)_{4}$, and $d$-5a was isolated with the deuteriums distributed at the homoallylic position. In the latter case, almost no C$\mathrm{H}$ cleavage at the terminal position of 1,3-dienes occurred, indicating the $\pi$-allylpalladium intermediate was directly trapped in the reaction. 
5) ${ }^{1}$ H NMR experiments

(i) $\mathbf{1 a}$ in $d$-toluene

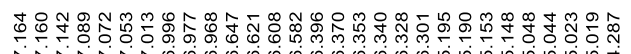

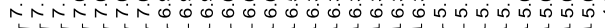
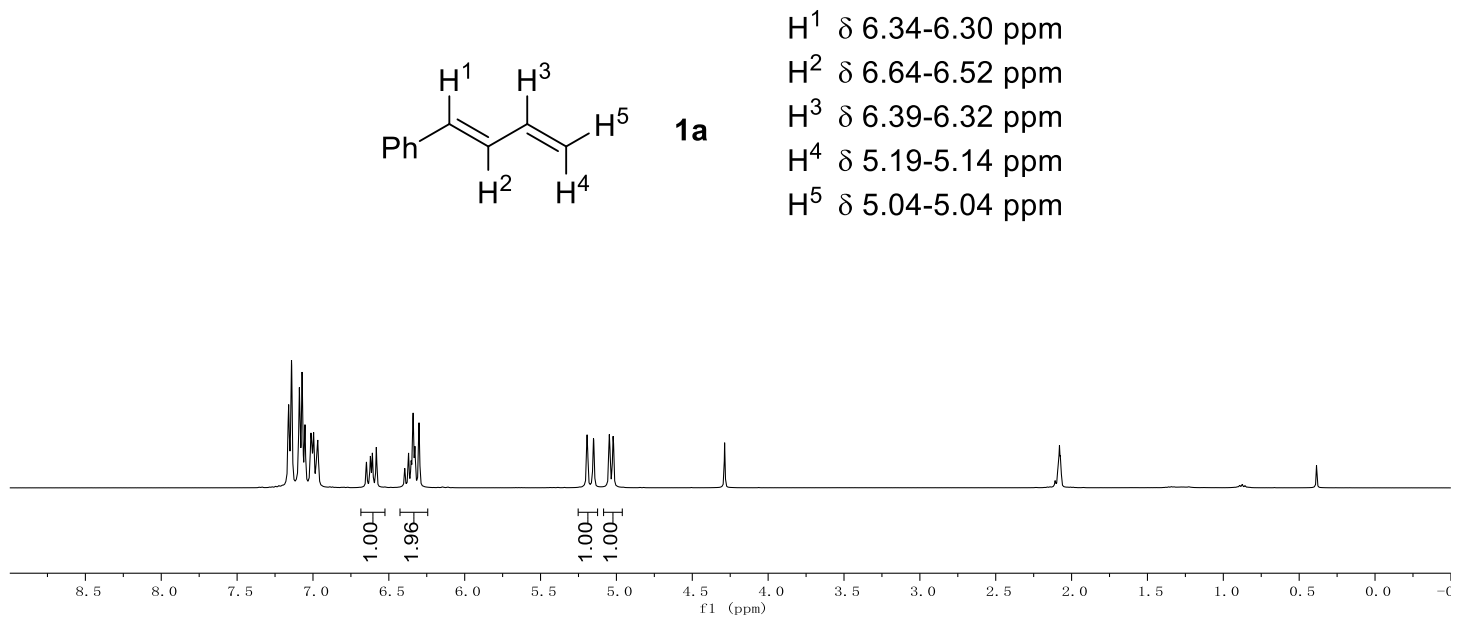

(ii) 1a (1 equiv) and $\mathrm{Pd}\left(\mathrm{PPh}_{3}\right)_{4}$ (1 equiv) in $d$-toluene

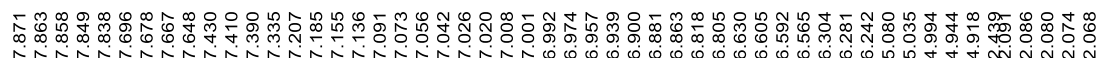

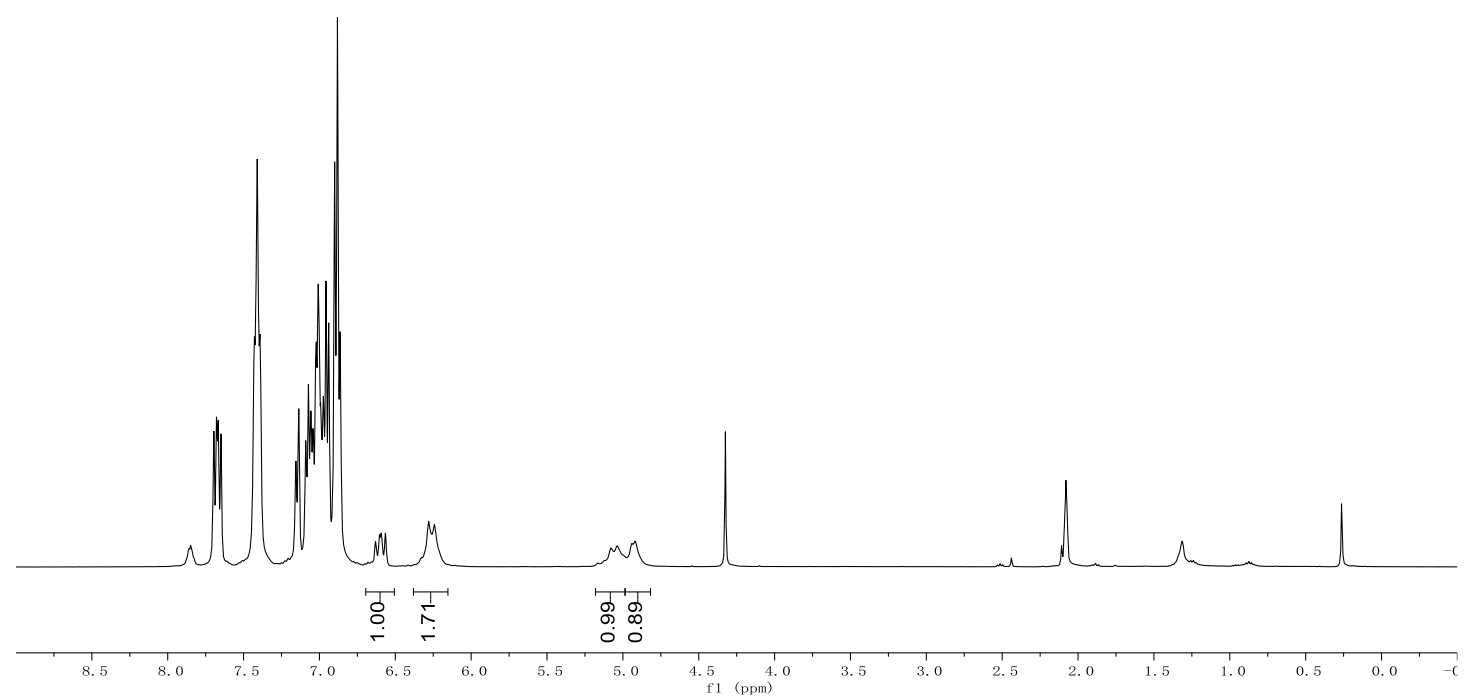


(iii) $1 \mathbf{a}$ (1 equiv), 2a (1 equiv) and $\mathrm{Pd}\left(\mathrm{PPh}_{3}\right)_{4}$ (1 equiv) in $d$-toluene

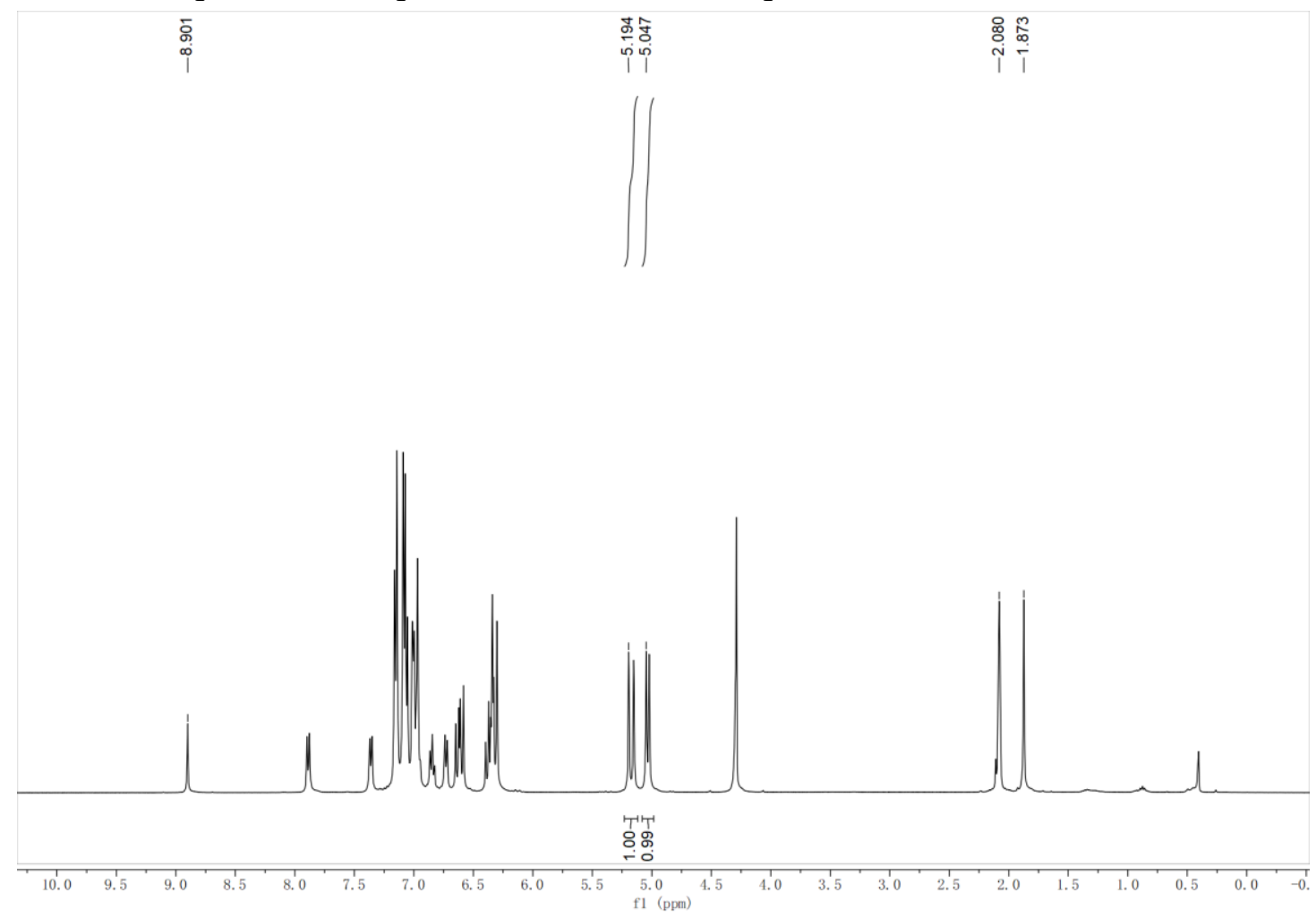

The ${ }^{1} \mathrm{H}$ NMR experiments showed that the diene moiety in 1a experienced apparent high-field shifts when $\mathrm{Pd}\left(\mathrm{PPh}_{3}\right)_{4}$ was added, potentially supporting the electron-donating effect of $\mathrm{Pd}(0)$ on the complexed olefin moiety. Nevertheless, further addition of imine $\mathbf{2 a}$ interfered with the coordination process.<smiles>C=CC=Cc1ccccc1</smiles>
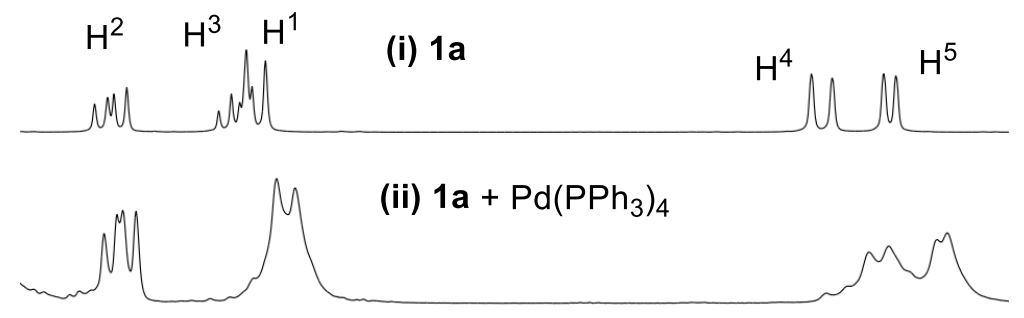

(iii) $1 \mathrm{a}+\mathbf{2 a}+\mathrm{Pd}\left(\mathrm{PPh}_{3}\right)_{4}$

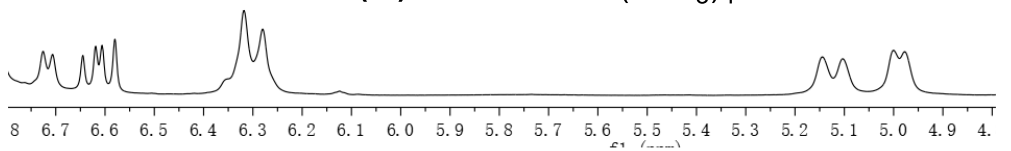


(iv) 2a in $d$-toluene

1
$\infty$
$\infty$
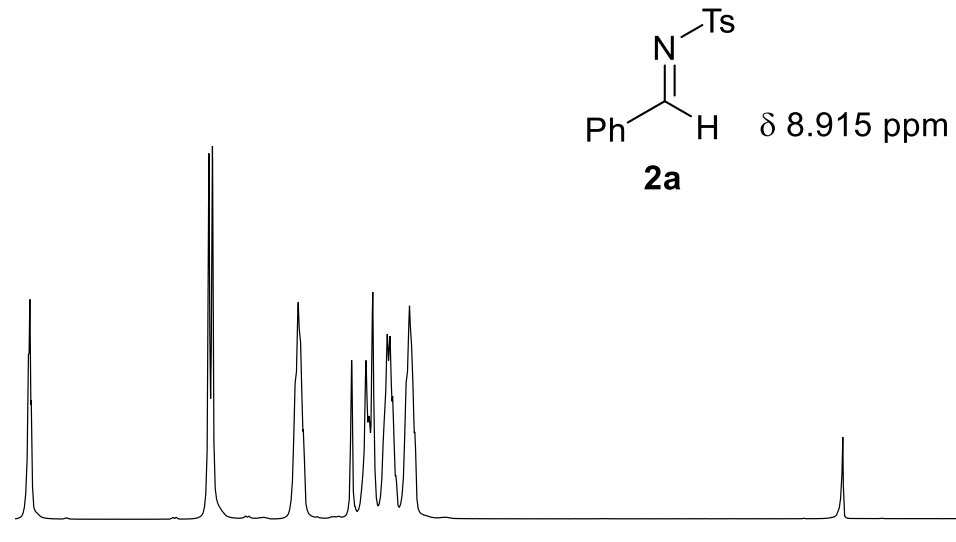

2a

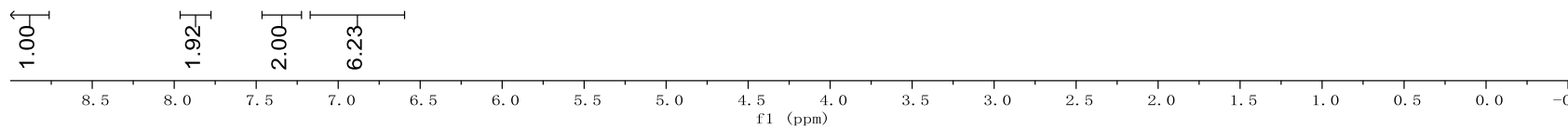

(v) 2a (1 equiv) and $\mathrm{Pd}\left(\mathrm{PPh}_{3}\right)_{4}$ (1 equiv) in $d$-toluene

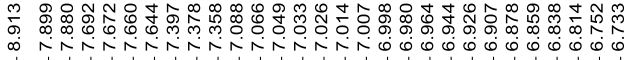
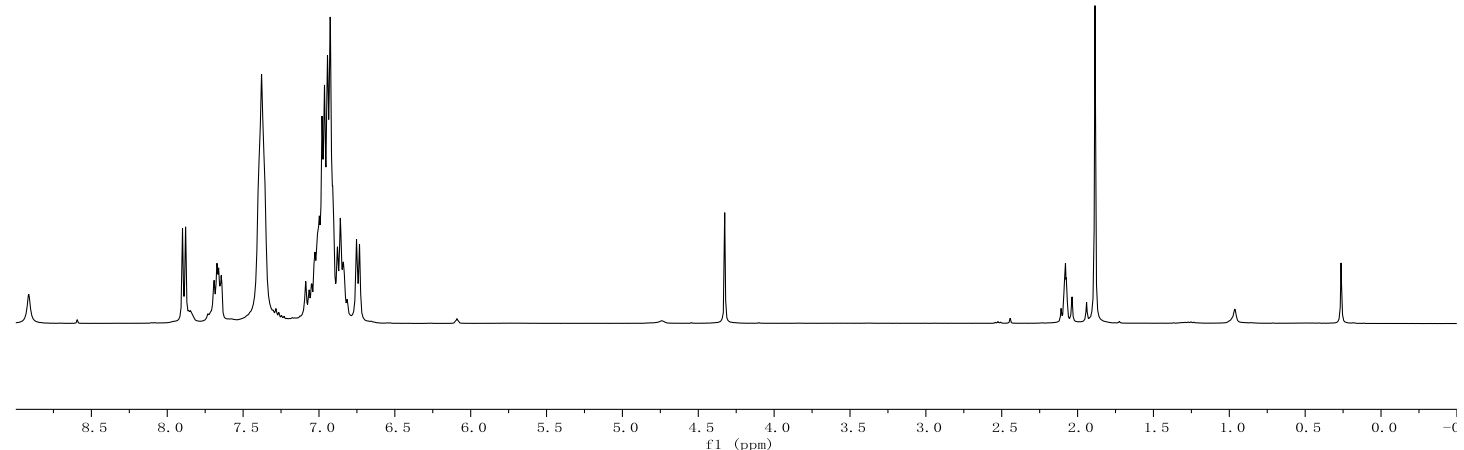

When imine 2a was mixed with $\mathrm{Pd}\left(\mathrm{PPh}_{3}\right)_{4}$, chemical shift of the proton in the imine moiety did not change. It was probably because $\operatorname{Pd}(0)$ is relatively soft, and would not coordinate to imine $\mathbf{2 a}$ tightly. 
(vi) 12 in $d$-toluene

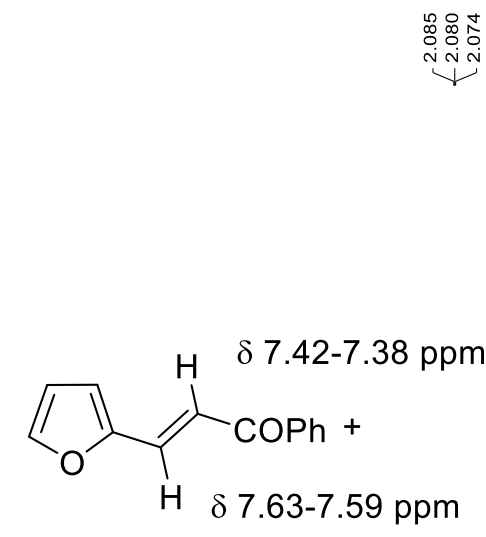

12

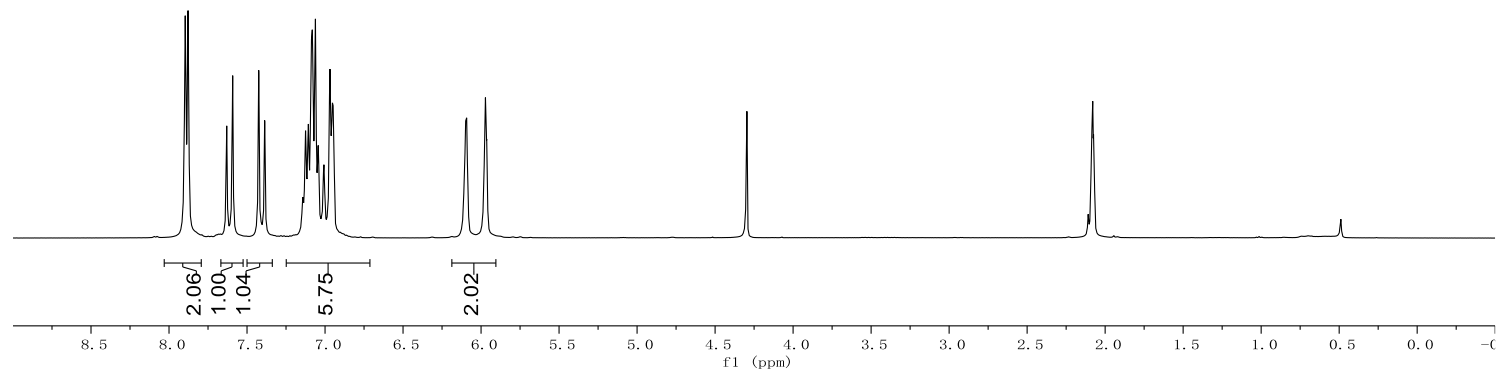

(vii) 12 (1 equiv) and $\mathrm{Pd}\left(\mathrm{PPh}_{3}\right)_{4}$ (1 equiv) in $d$-toluene

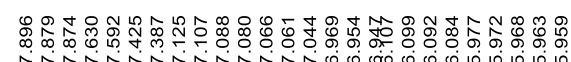

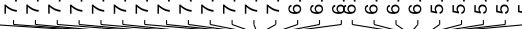

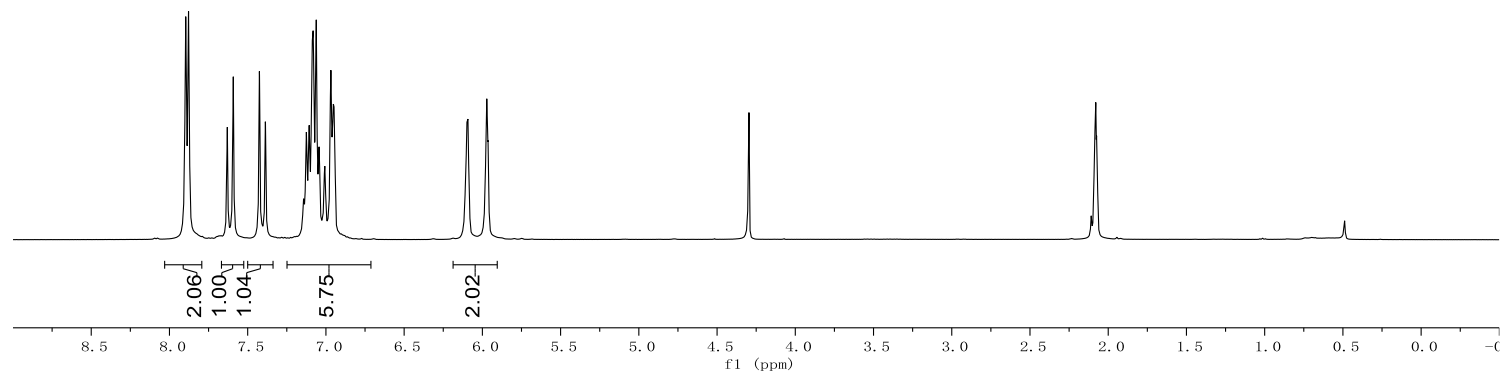

When enone 12 was mixed with $\mathrm{Pd}\left(\mathrm{PPh}_{3}\right)_{4}$, apparent chemical shifts of the internal alkene moiety were not observed. A Lewis acid was previously applied to enhance the formation of stable $\eta^{2}$ enonepalladium(0) complex (see: J. Am. Chem. Soc. 2001, 123, 1944). 


\section{Procedure of the ${ }^{1} \mathrm{H}$ NMR experiments}

(ii) $1 \mathrm{a}+\mathrm{Pd}\left(\mathrm{PPh}_{3}\right)_{4}$ : An oven-dried $5 \mathrm{~mL}$ test-tube equipped with a septum and a magnetic stir bar was charged with $1 \mathrm{a}(3.3 \mathrm{mg}, 0.025 \mathrm{mmol})$ and $\mathrm{Pd}\left(\mathrm{PPh}_{3}\right)_{4}(28.9 \mathrm{mg}, 0.025 \mathrm{mmol}, 1.0$ equiv.). The tube was then evacuated and filled with argon. This cycle was repeated three times, and deuterated toluene $(0.5 \mathrm{~mL})$ was added via syringe. The resulting solution was stirred at $80{ }^{\circ} \mathrm{C}$ for $20 \mathrm{~min}$. Then the resulting solution was directly analyzed via ${ }^{1} \mathrm{H}$ NMR $(400 \mathrm{MHz})$.

(iii) $1 \mathbf{a}+\mathbf{2 a}+\mathrm{Pd}\left(\mathrm{PPh}_{3}\right)_{4}$ : An oven-dried $5 \mathrm{~mL}$ test-tube equipped with a septum and a magnetic stir bar was charged with 1a $(6.5 \mathrm{mg}, 0.025 \mathrm{mmol})$ and $\mathrm{Pd}\left(\mathrm{PPh}_{3}\right)_{4}(28.9 \mathrm{mg}, 0.025 \mathrm{mmol}, 1.0$ equiv.). The tube was then evacuated and filled with argon. This cycle was repeated three times, and deuterated toluene $(0.5 \mathrm{~mL})$ was added via syringe. The resulting solution was stirred at $80{ }^{\circ} \mathrm{C}$ for $20 \mathrm{~min}$, then 2a was added to the resulting solution. The mixture was analyzed via ${ }^{1} \mathrm{H}$ NMR (400 MHz).

(iv) $2 \mathrm{a}+\mathrm{Pd}\left(\mathrm{PPh}_{3}\right)_{4}$ : An oven-dried $5 \mathrm{~mL}$ test-tube equipped with a septum and a magnetic stir bar was charged with $2 \mathbf{a}(6.5 \mathrm{mg}, 0.025 \mathrm{mmol})$ and $\mathrm{Pd}\left(\mathrm{PPh}_{3}\right)_{4}(28.9 \mathrm{mg}, 0.025 \mathrm{mmol}, 1.0$ equiv.). The tube was then evacuated and filled with argon. This cycle was repeated three times, and deuterated toluene $(0.5 \mathrm{~mL})$ was added via syringe. The resulting solution was stirred at $80{ }^{\circ} \mathrm{C}$ for $20 \mathrm{~min}$. Then the resulting solution was analyzed via ${ }^{1} \mathrm{H}$ NMR $(400 \mathrm{MHz})$.

(vii) $12+\mathrm{Pd}\left(\mathrm{PPh}_{3}\right)_{4}$ : An oven-dried $5 \mathrm{~mL}$ test-tube equipped with a septum and a magnetic stir bar was charged with 12 (4.9 mg, $0.025 \mathrm{mmol})$ and $\mathrm{Pd}\left(\mathrm{PPh}_{3}\right)_{4}$ (28.9 mg, $0.025 \mathrm{mmol}, 1.0$ equiv.). The tube was then evacuated and filled with argon. This cycle was repeated three times, and deuterated toluene $(0.5 \mathrm{~mL})$ was added via syringe. The resulting solution was stirred at $80{ }^{\circ} \mathrm{C}$ for $20 \mathrm{~min}$. Then the resulting solution was analyzed via ${ }^{1} \mathrm{H}$ NMR (400 MHz). 
7) The cascade reaction involving an additionl nucleophile

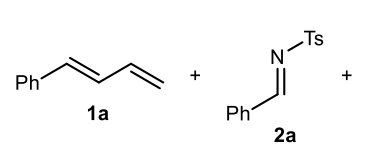<smiles>C1CCNCC1</smiles>
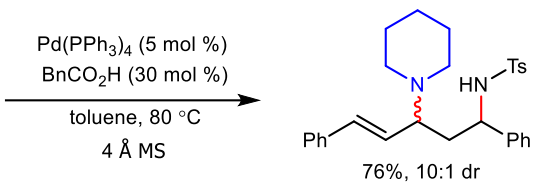

Scheme S14. The cascade reaction with an additional nucleophile

It was found that with an additional nucleophile, such as piperidine, a diamine product was formed in a good yield. The results indicated that a $\pi$-allypalladium complex might be generated as an intermediate after the addition of 1,3-diene $\mathbf{1 a}$ to imine $\mathbf{2 a}$, and then captured by piperidine.

\section{8) Regioselectivity of the hydrodienylation reaction}

The hydrodienylation reaction of $N$-sulfonylimines 2 always took place at the $\mathrm{C} 4$ position of 1,3diene 1. On the other hand, an $N$-Boc protected imine from benzaldehyde, exhibiting good reactivity with 1e, would give two products with $\mathrm{C} 4$ - and $\mathrm{C} 1$-regioselectivity, respectively. The results indicated that the hydrodienylation reaction could occur at both $C 4$ and $C 1$ positions of the 1,3-dienes.

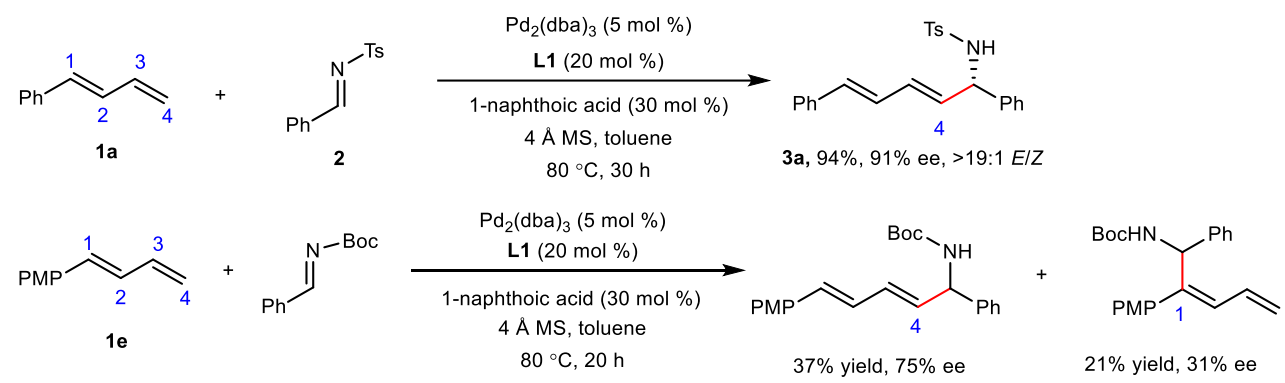

Scheme S15. Different regioselectivity in the reaction between 1,3-dienes and imines

\section{9) Reactions between 1,3-dienes and different electrophiles under the catalysis of palladium}

Beside of $N$-sulfonylimines 2, 4 and 6 listed in the manuscript, some other electrophiles are also applicable in the hydrodienylation reactions.

\section{Aldehydes \& Ketones}

With $\mathrm{Pd}\left(\mathrm{PPh}_{3}\right)_{4}$ as catalyst, the hydrodienylation reaction of ethyl glyoxylate could proceed smoothly with 1a; while oxindole also could participate in the reaction with 1a under the asymmetric conditions. The corresponding hydrodienylated products could be obtained in moderate yields from these highly reactive carbonyl compounds, albeit with moderate stereocontrol after screening more chiral ligands. 


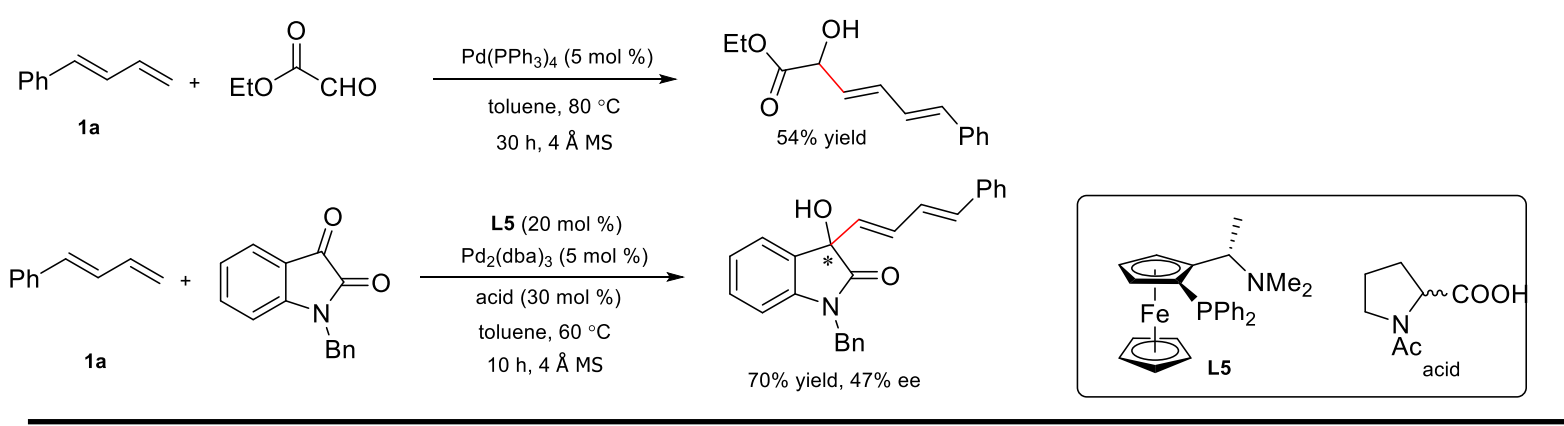

other chiral ligands (additive $=1$-naphthoic acid $)$

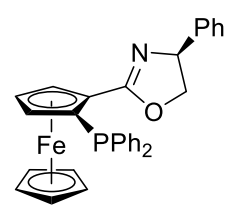

$36 \%, 29 \%$ ee

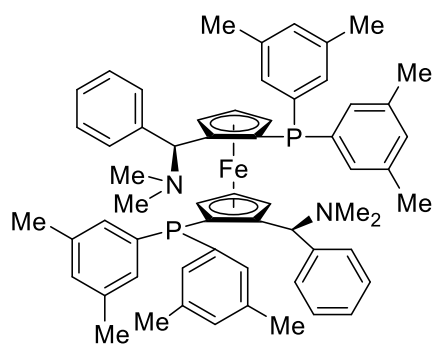

$59 \%, 30 \%$ ee

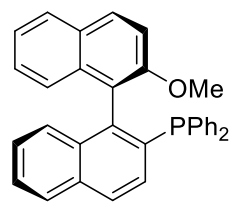

$79 \%, 12 \%$ ee<smiles>CP(c1nc2ccccc2nc1P(C)[13C](C)(C)C)C(C)(C)C</smiles>

trace

Scheme S16. The hydrodienylation reaction with carbonyls

10) Further application of vinylogous activation mode

Friedel-Crafts reaction of deactivated furan 12 with 1 -azadiene 13 


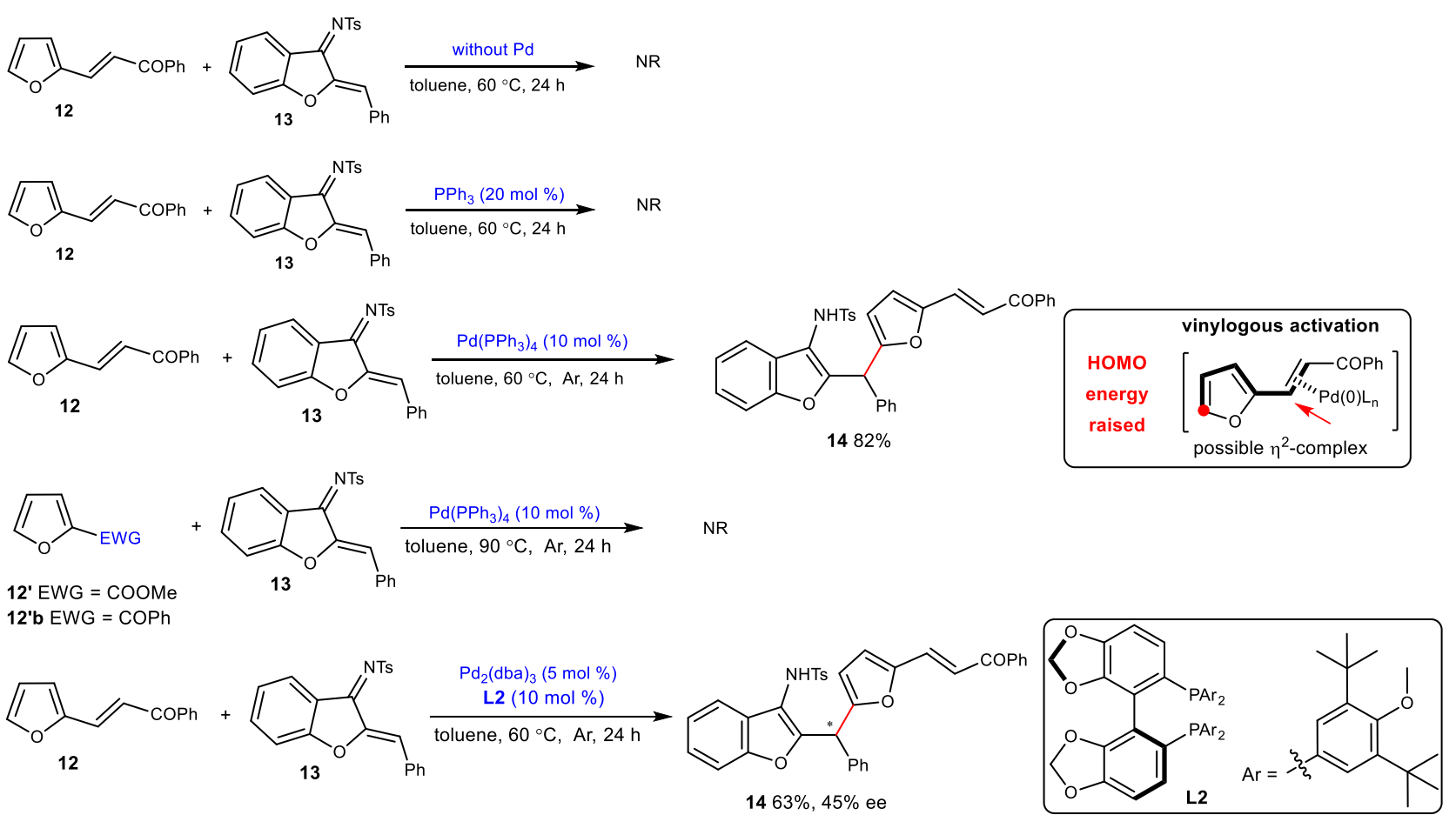

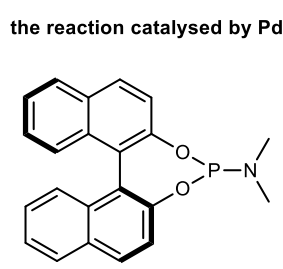

NR

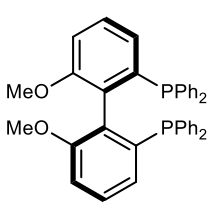

$60 \%$ conv.

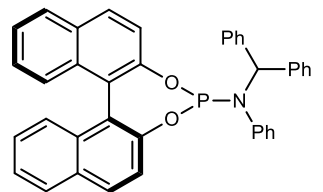

bad conv.

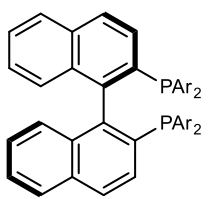

$\mathrm{Ar}=3,5-\mathrm{MeC}_{6} \mathrm{H}_{3}$

$53 \%, 28 \%$ ee
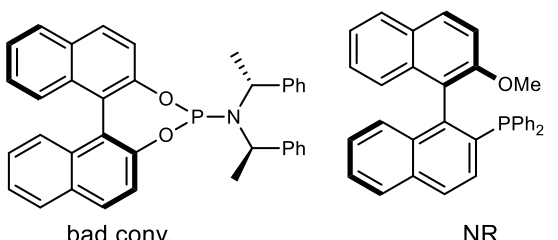

NR
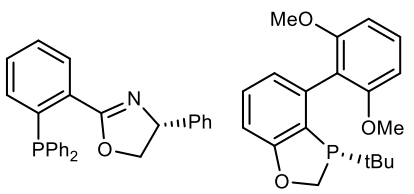

NR

NR

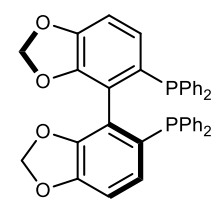

$61 \%,-35 \%$ ee

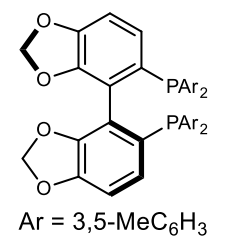

$56 \%, 32 \%$ ee

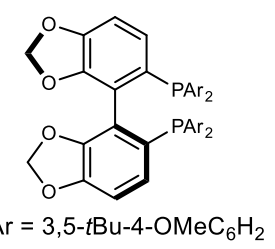

$63 \%, 45 \%$ ee

\section{Scheme S17. Reaction of deactivated furan 12 with 1 -azadiene 13}

No reaction occurred between deactivated furan 12 with 1-azadiene 13 without Pd catalyst or under the catalysis of $\mathrm{PPh}_{3}$, while the Friedel-Crafts reaction at the C5-postion of the furan ring smoothly proceeded in the presence of catalytic amounts of $\mathrm{Pd}\left(\mathrm{PPh}_{3}\right)_{4}$. These results indicated that $\mathrm{Pd}$ catalyst was curcial for the reaction, and the organic $\sigma$-type Lewis base catalysis pathway $\left(\mathrm{PPh}_{3}\right)$ would be precluded. Since the reaction site is at $\mathrm{C} 5$-postion of furan 12, the oxidative cyclization process and $\operatorname{Pd}(0)$ catalyzed $\mathrm{C}-\mathrm{H}$ activation under Ar would not be involved. Therefore, a Pd-coordinated $\eta^{2}$ complex might be the key intermediate with increased nucleophilicity. It should be noticed that ethyl furan-2-carboxylate 12' and furan-2-yl(phenyl)methanone $\mathbf{1 2}^{\prime} \mathbf{b}$ could not undergo similar transformation with 1 -azadiene 13 under the catalysis of $\mathrm{Pd}$ even at $90{ }^{\circ} \mathrm{C}$, demonstrating the importance of the double bond and vinylogous activation. When chiral ligand $\mathbf{L} \mathbf{2}$ was employed, 
moderate enantioselectivity was achieved, further verifying the proposal of the $\eta^{2}$-complex with a chiral ligand.

\section{(2) Computational study on the hydrodienylation reaction}

\section{1) Olefin coordination with metal Pd as $\pi$-Lewis base}

To explain the mechanism for the raised HOMO energies, we calculated the molecular orbitals using simplified modes (ethylene and butadiene) by DFT calculations at B3LYP/def2-TZVPPD level, as shown in Figure S1.

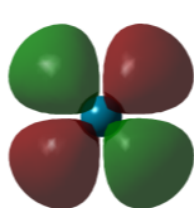

Pd HOMO $\mathrm{dx}^{2}-\mathrm{y}^{2}$

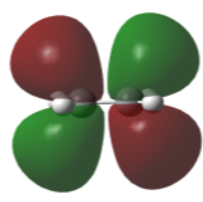

Ethylene LUMO $\pi^{*}$

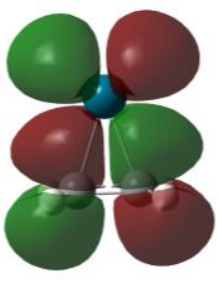

Ethylene-Pd HOMO $\stackrel{P \mathrm{Pd}^{0}}{\underline{\underline{\underline{P}}}}$

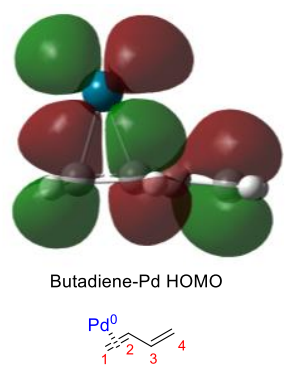

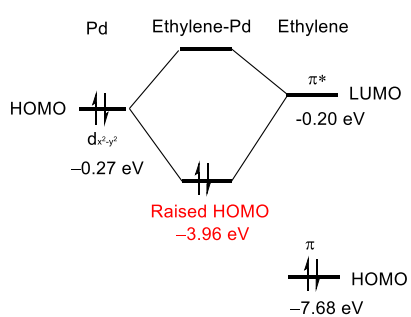

Pd Butadiene-Pd Butadiene

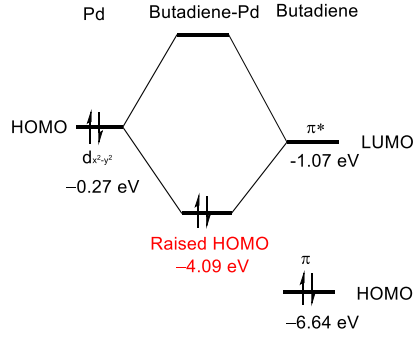

Figure S1. The molecular orbitals of ethylene, butadiene, and the corresponding $\boldsymbol{\eta}^{2}$-coordinated Pdcomplexes

\section{Ethylene}

The atom orbital $d_{\mathrm{x} 2-\mathrm{y} 2}$ of $\mathrm{Pd}$ atom employs a high energy with a value of $-0.27 \mathrm{eV}$. The HOMO ( $\pi$ orbital) and LUMO ( $\pi^{*}$ orbital) of ethylene have energy values of $-7.68 \mathrm{eV}$ and $-0.2 \mathrm{eV}$, respectively. When ethylene coordinates with $\mathrm{Pd}$ atom, the $d_{\mathrm{x} 2 \mathrm{y} 2}$ orbital of Pd overlaps with the $\pi^{*}$ orbital of ethylene to form the $\eta^{2}$-complex. The new HOMO orbital of the complex with an energy value of $3.96 \mathrm{eV}$, which is $3.72 \mathrm{eV}$ higher than that of ethylene, indicating that $\operatorname{Pd}(0)$ could activate ethylene as a $\pi$-Lewis base by increasing the HOMO energy.

\section{Butadiene}

Butadiene, employing a similar LUMO energy as ethylene, could coordinate with Pd as a receptor by offering its LUMO to form coordinating bonds. The HOMO of butadiene-Pd complex is raised to $-5.09 \mathrm{eV}$. Interestingly, when the $d_{\mathrm{x} 2-\mathrm{y} 2}$ orbital of Pd overlaps with the $\pi^{*}$ orbital of butadiene, the $d$ electrons of Pd delocalize to the $\pi^{*}$ orbital. Moreover, it is found the charge density of $\mathrm{C} 4$ atom of the complex is higher than other atoms of butadiene. These results suggest the HOMO of butadiene 
could be activated by $\mathrm{Pd}$ coordination, thus possibly promoting the nucleophilic attack to electrophiles at the uncoordinated double bond.

\section{(E)-1-Phenyl-1,3-diene 1a}

After conformation search and following optimization (Figure S2), two complexes named INT1 and INT1' in which Pd is coordinated to 1,2- or 3,4-double bond were proposed, respectively. We tried but failed to obtain Pd-coordinated $-\eta^{4}$ or $-\eta^{3}$ complexes, and only generate INT1-1 and INT1'1 whose energies were higher than that of INT1, probably due to the $s$-cis-configuration of 1a. The $d$-electrons of Pd delocalise to the $\pi^{*}$ orbital of 1a, and the HOMO energy of $\mathbf{1 a}$ is also raised via the Pd coordination, which is similar to that in the simplified cases. The raised HOMOs of INT1 and INT1' employ values of $-4.88 \mathrm{eV}$ and $-4.71 \mathrm{eV}$, respectively. These theoretical data implied that different regioselectivity in the Pd-catalyzed reaction might be possible due to the small energy difference, as observed in the reaction between 1,3-diene $1 e$ and an N-Boc protected imine.
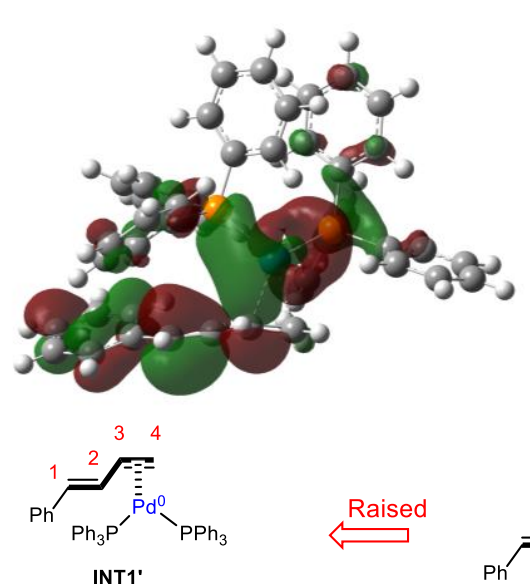

HOMO: $-4.71 \mathrm{eV}$

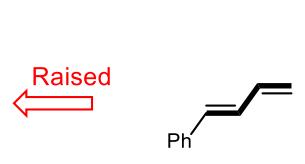

HOMO: $-5.94 \mathrm{eV}$
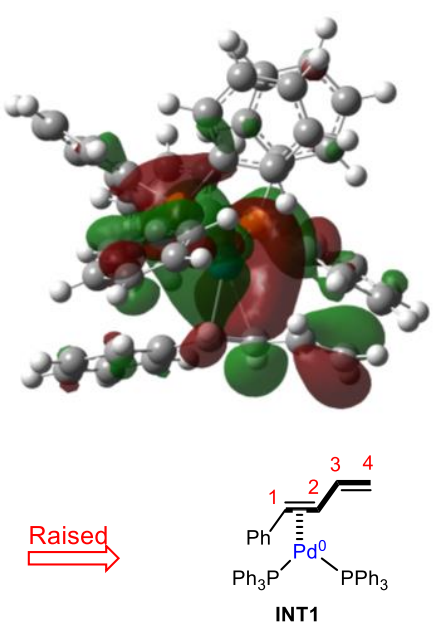

HOMO: $-4.88 \mathrm{eV}$
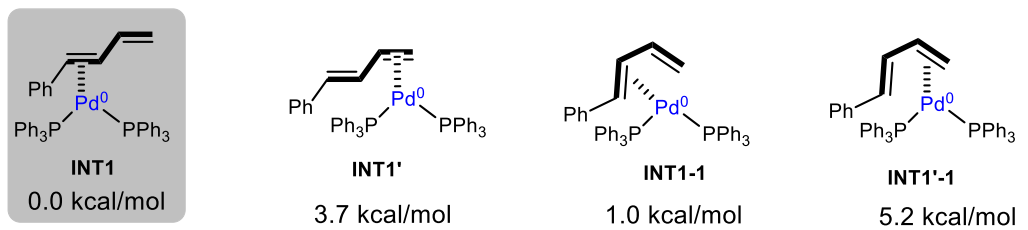

Figure S2. The molecular orbitals and energies of the complexes of $1 \mathrm{a}$ and $\mathbf{P d}\left(\mathrm{PPh}_{3}\right)_{4}$

\section{The reaction process WITHOUT acid additives}

The mechanism for the reaction without acid additive was proposed and calculated as shown in Figure S4. As an electrophile, 2a employs a LUMO energy with a value of $-2.45 \mathrm{eV}$, and would be attacked by HOMO raised INT1 or INT1' at different positions. For the reaction of INT1, 2a attacked INT1 at the $\mathrm{C} 4$ position via TS1A with an energy barrier of $20.3 \mathrm{kcal} / \mathrm{mol}$ to form INT2A. We also proposed some transition states with other reaction sites at INT1, but only TS1B could be generated by forming a C-C bond at the $\mathrm{C} 2$ position. The energy barrier of TS1B with a value of $37.8 \mathrm{kcal} / \mathrm{mol}$ is much higher than that of TS1. On the other side, the possibility for the reaction of INT1' with $\mathbf{2 a}$ 
was also calculated, such as attacking at the $\mathrm{C} 1$ and $\mathrm{C} 4$ positions via $\mathbf{T S 1}^{\prime} \mathbf{A}^{\prime}$ and $\mathbf{T S}^{\prime} \mathbf{B}^{\prime}$. Similar, the energy barriers of $\mathbf{T S 1}^{\prime}{ }^{\prime}$ and $\mathbf{T S 1 B}^{\prime}$ with values of 32.9 and $29.3 \mathrm{kcal} / \mathrm{mol}$, respectively, are much higher than that of TS1A. Among these TSs, TS1A is more favorable, which is consistent with the reaction site at the $\mathrm{C} 4$ position. These results also suggested that the double bond coordinated to Pd might not be reactive towards electrophiles, but in a vinylogous activation pattern.

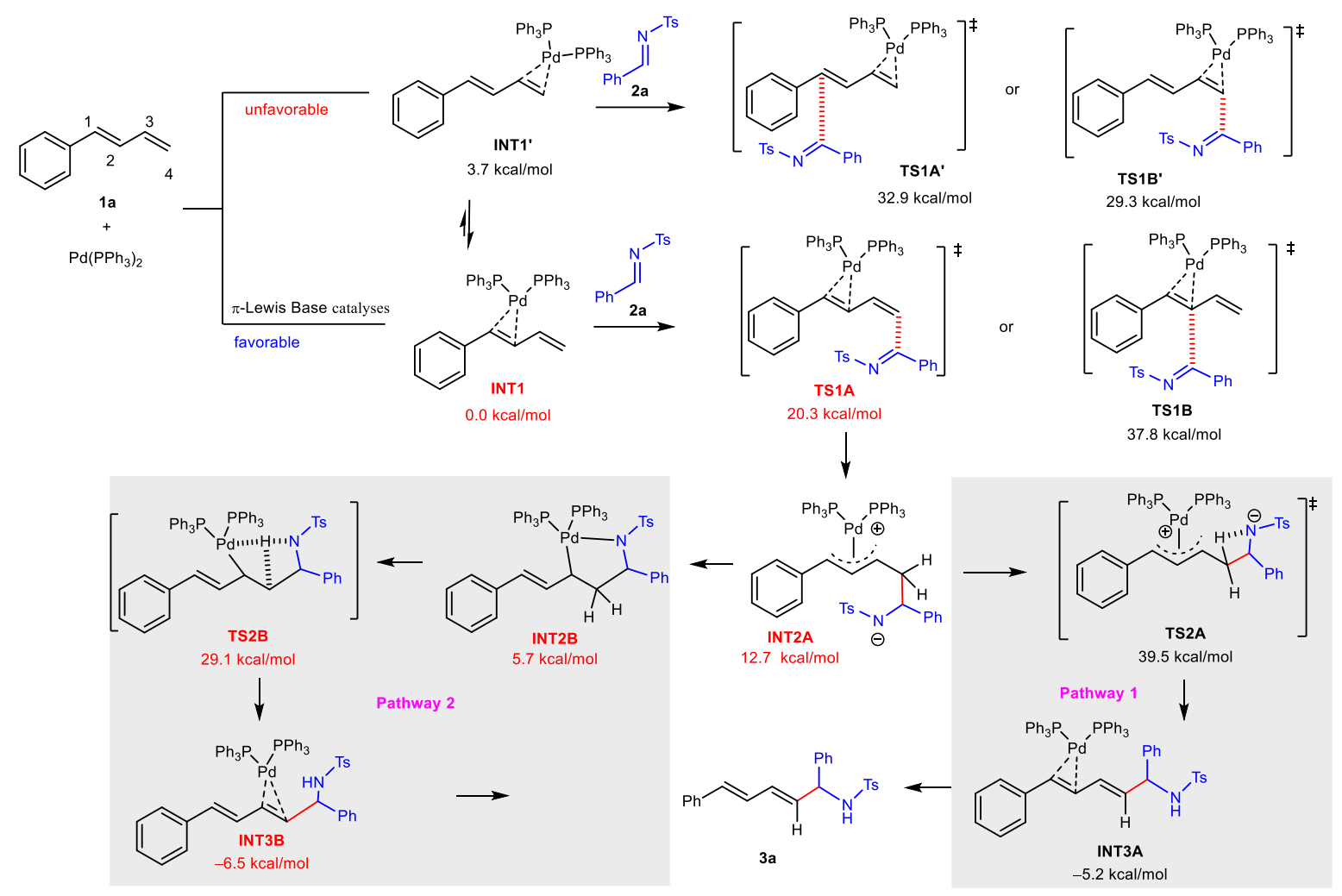

Figure S4. Computed potential energy surface of the reaction of INT1 and 2a at the B3LYP-D3/6311++G(d,p)//B3LYP-D3/6-31G(d) SDD for Pd (toluene) level and is given in kcal/mol relative to energy sum of INT1 and 2a

Since TS1A' and TS1B' via INT1' were not favored, Pathway 1 and Pathway 2 involving the formation of INT2A via TS1A have been calculated accordingly.

Pathway 1: After the nucleophilic attack at the C4 position of INT1, $\operatorname{Pd}(0)$ was converted to $\operatorname{Pd}($ II) with an allyl anion as the ligand, as shown in INT2A. The $\mathrm{H}$ atom at the $\mathrm{C} 4$ position might transfer to the $\mathrm{N}$ anion directly via TS2A with a high energy value of $39.5 \mathrm{kcal} / \mathrm{mol}$.

Pathway 2: INT2A is generated similar as Pathway 1. As the $\mathrm{N}$ anion could coordinate with $\mathrm{Pd}(\mathrm{II})$, another pathway involving $\beta$-H elimination was proposed, and the energy barrier of the $\mathrm{H}$ transfer step for the generation of TS2B is $23.4 \mathrm{kcal} / \mathrm{mol}$ (the energy related to INT1 and $2 \mathbf{a}$ was 29.1 $\mathrm{kcal} / \mathrm{mol})$.

On the other hand, imines could also coordinate to transition metals, thus INT1C and INT1D, with $\mathrm{Pd}$ atom coordinating to with $\mathbf{2 a}$ and $\mathbf{1 a}$ at the $\mathrm{C} 1 / \mathrm{C} 2$ and $\mathrm{C} 3 / \mathrm{C} 4$ position, respectively, have been proposed and calculated. 


\section{Pathway 3:}

For the reaction in INT1C, the C4 of 1 a would attack imine 2a to afford INT2C via TS1C (35.7 $\mathrm{kcal} / \mathrm{mol})$, followed by $\beta$-H elimination via TS2C $(57.6 \mathrm{kcal} / \mathrm{mol})$, finally giving product 3a.

\section{Pathway 4:}

The oxidative cyclization process was also considered via TS1D from INTD to generate INT2D. However, the energy barriers of TS1D was $10.5 \mathrm{kcal} / \mathrm{mol}$ higher than that of TS1C.

Similarly, the oxidative cyclization process started from INT1C by reacting at the $\mathrm{C} 1$ position was also calculated via TS1D'.

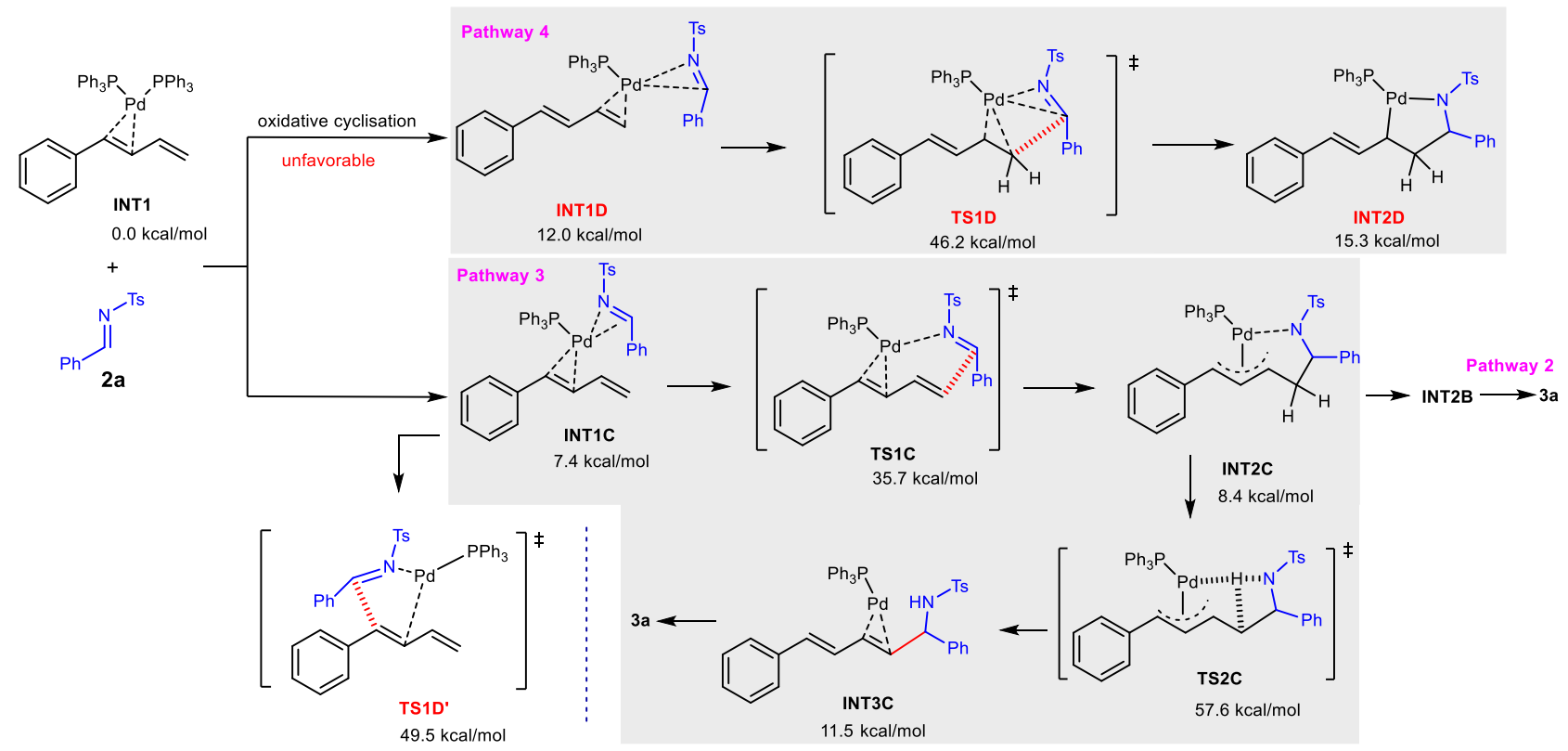

Figure S5. Computed potential energy surface of the reaction of INT1 and 2a at the B3LYP-D3/6311++G(d,p)//B3LYP-D3/6-31G(d) SDD for Pd (toluene) level and is given in kcal/mol relative to energy sum of INT1C

Among the Pathways 1-4, the energy of TS1A (20.3 kcal/mol) is the lowest for the addition step; while TS2B $(29.1 \mathrm{kcal} / \mathrm{mol})$ possesses the lowest energy for the $\mathrm{H}$ atom transfer step. These results suggest Pathway 2 involving TS1A and TS2B is more favorable in the absence of acid additive, but the energy barrier (TS2B, $29.1 \mathrm{kcal} / \mathrm{mol})$ for the H atom transfer step is relatively high, which might be the rate-limiting step.

\section{The reaction process WITH acid additives}

In the presence of carboxylic acids, Pathway 5 and Pathway 6 have been proposed, and the related TSs as well as intermediates were optimised (Figures S6 and S7).

Pathway 5 (NO interaction between acid additive and Pd, Figure S6): The addition step is same to that in the Pathway 1 and Pathway 2. The acid might protonate the $\mathrm{N}$ anion to give INT3D form INT2A. Additionally, we found the acid might promote the addition step to form INT3D, since the energy barrier of Acid-TS1A (20.0 kcal/mol) was slightly lower than that of TS1A $(20.3 \mathrm{kcal} / \mathrm{mol})$. 
Then the $\mathrm{H}$ at the $\mathrm{C} 4$ position in INT3D would be deprotonated by the carboxylate anion, affording INT3A and INT3A' as two $E / Z$ isomers, respectively. The energy barrier of TS3D of the deprotonation step for major product (E-configuration) is $18.1 \mathrm{kcal} / \mathrm{mol}$, which is $7.8 \mathrm{kcal} / \mathrm{mol}$ lower than that of TS3D'. Moreover, the energy of INT3A was also $9.1 \mathrm{kcal} / \mathrm{mol}$ lower than that of INT3A'. These results were consistent with the experimental results that the E-configuration product was the major product.

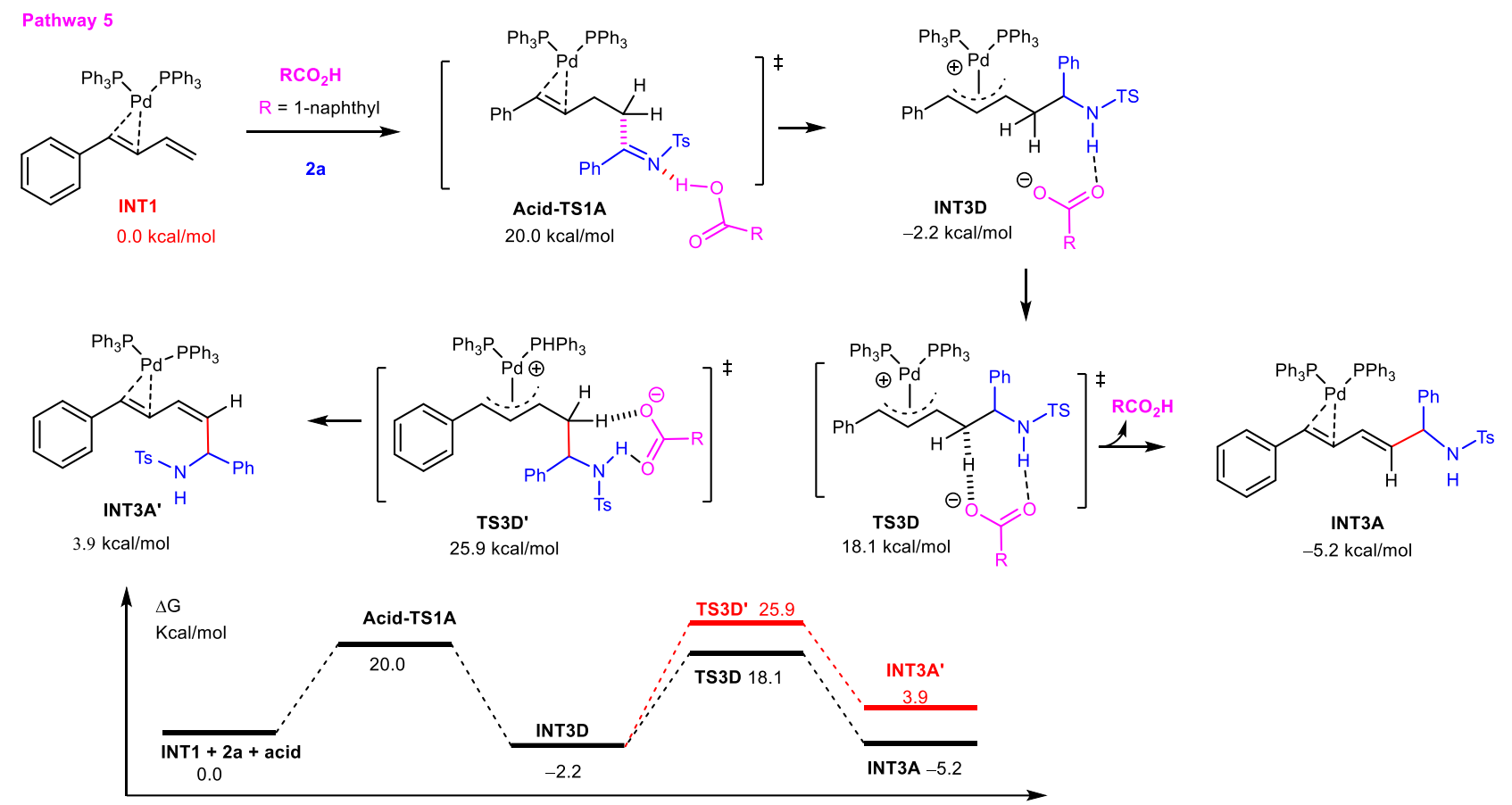

Figure S6. Computed potential energy surface of the hydrogen transfer process promoting by 1naphthoic acid at the B3LYP-D3/6-311++G(d,p)//B3LYP-D3/6-31G(d) SDD for Pd (toluene) level and is given in $\mathrm{kcal} / \mathrm{mol}$ relative to energy sum of INT1 and $2 \mathrm{a}$

Pathway 6 (acid additive coordinates to Pd, Figure S7): Another pathway was proposed with the carboxylic acid ligated to $\mathrm{Pd}$ by replacing a $\mathrm{PPh}_{3}$ ligand, whereas the corresponding INT1E possesses a relatively higher energy $(17.2 \mathrm{kcal} / \mathrm{mol})$. As shown in TS1E $(30.2 \mathrm{kcal} / \mathrm{mol})$, a C-C bond is formed by the addition of INT1E to $\mathbf{2 a}$ with the assistant of $\mathrm{H}$-bonding between the acid and imine $\mathbf{2 a}$, with an energy barrier of $13.0 \mathrm{kcal} / \mathrm{mol}$. Then the deprotonation of INT2E proceeds via TS2E (27.9 $\mathrm{kcal} / \mathrm{mol})$ or TS2E' (28.1 kcal/mol) to give $E / Z$ isomers INT3E and INT3E', respectively. Therefore, this proposed pathway might not be favored for $\mathrm{Pd}\left(\mathrm{PPh}_{3}\right)_{4}$-catalysed reaction in the presence of acid additive, probably because of the unfavored ligand exchange between $\mathrm{PPh}_{3}$ and carboxylic acid. 


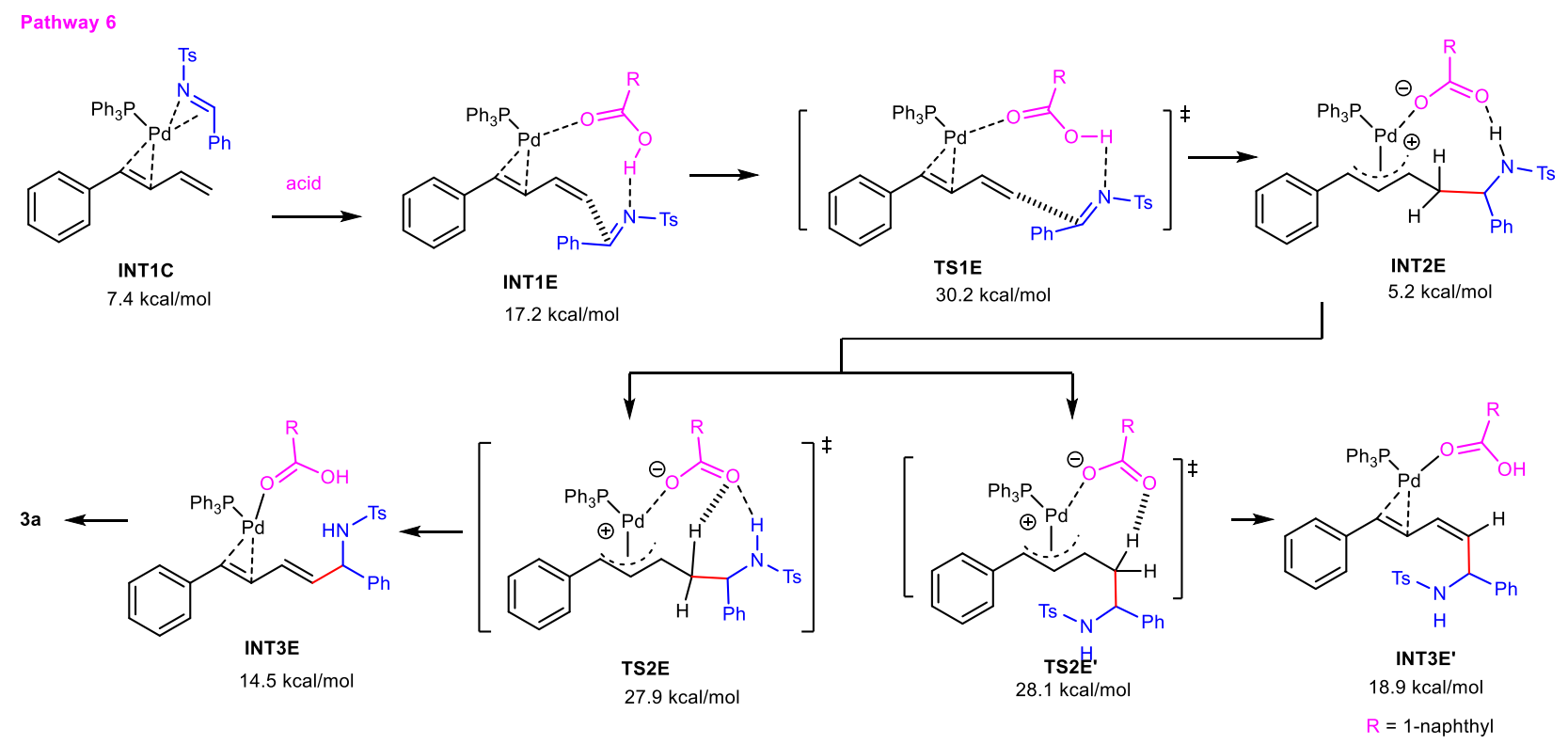

Figure S7. Computed potential energy surface of the hydrogen transfer process promoting by 1naphthoic acid at the B3LYP-D3/6-311++G(d,p)//B3LYP-D3/6-31G(d) SDD for Pd (toluene) level and is given in $\mathrm{kcal} / \mathrm{mol}$ relative to energy sum of INT1E and 2a

\section{(3) The stereoselectivity of the asymmetric hydrodienylation reaction}

It should be noted that the acid additive could enhance the enantioselectivity as well as reactivity. Considering that the addition step is enantiodetermining step, we thought that the acid additive was probably engaged in this process, as proposed in both Pathways 5 and 6. In addition, we assumed that Pathway 6 might also be possible when the reaction was carried out with different ligands, especially in the case that only one phosphine ligand is coordinated to $\operatorname{Pd}(0)$.

\section{1) Linear effect experiments ${ }^{6}$}

Chiral L1 with different ee values was prepared by mixing L1 (99\% ee) and ent-L1 (99\% ee), derived from $(R)$-BINOL/( $R, R)$-bis(1-phenylethyl)amine and $(S)$-BINOL/(S,S)-bis(1-phenylethyl) amine, respectively, in appropriate ratios. The product was separated by preparative TLC. The ee value was then determined by HPLC analysis, which exhibits a linear relationship between ee values of product and the ligand, implying that the ratio of Pd to ligand would be 1:1.
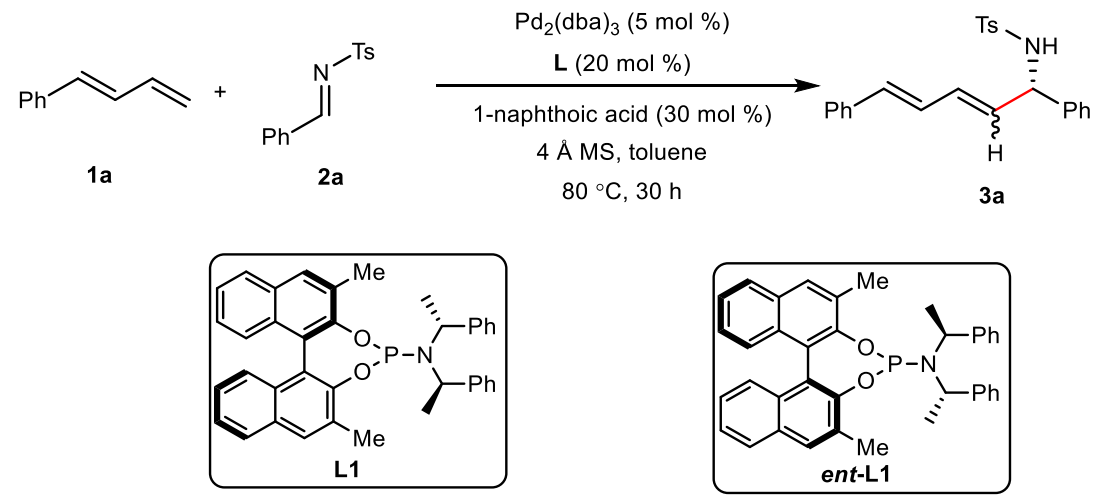


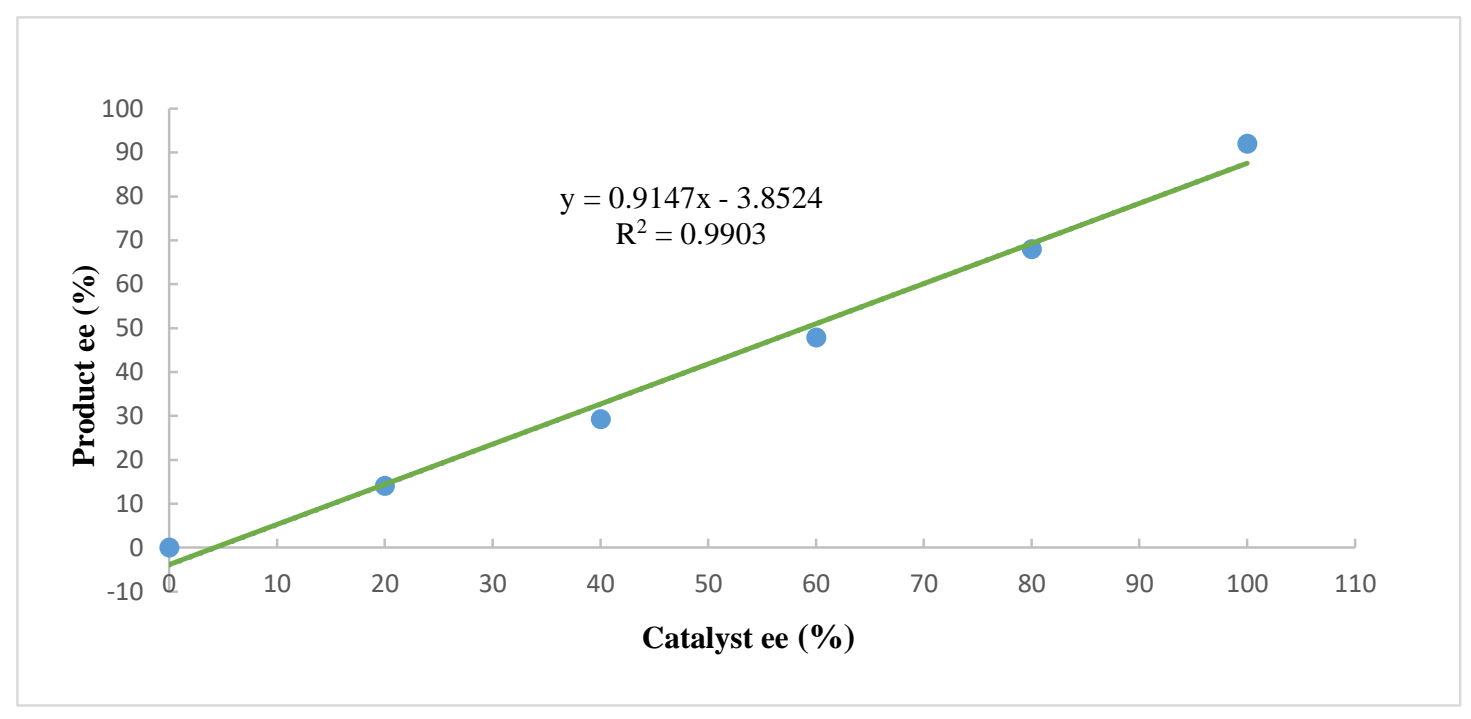

Scheme S18. Linear effect of Pd with L1

Nevertheless, it was found the reaction with $10 \mathrm{~mol} \% \mathbf{L 1}$ gave a lower yield than that with 20 mol \% L1, without ee losses ( $\mathrm{Pd} 10 \mathrm{~mol} \%)$. As a result, $5 \mathrm{~mol} \% \mathrm{Pd}_{2}(\mathrm{dba})_{3}$ together with $20 \mathrm{~mol} \%$ L1 was employed as the optimal catalytic conditions.

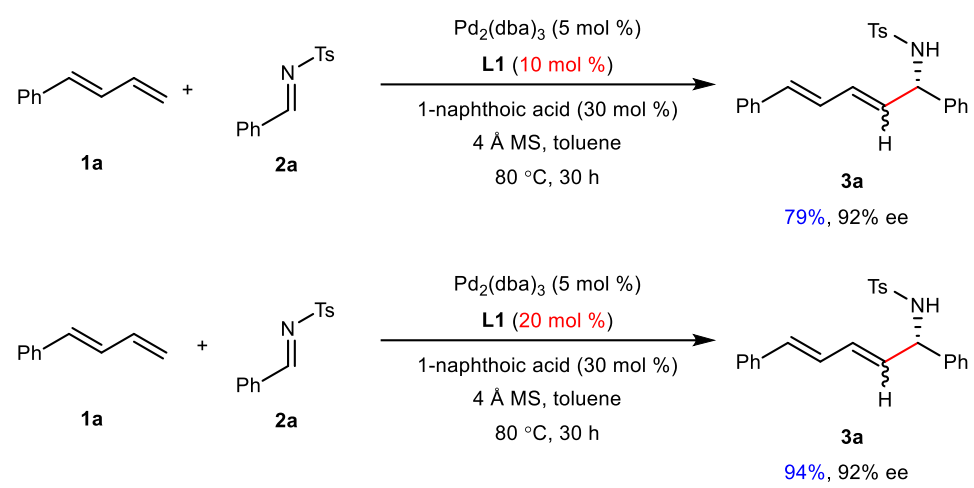

Scheme S19. Screening the ratio of Pd to L1

\section{2) Acid additive}
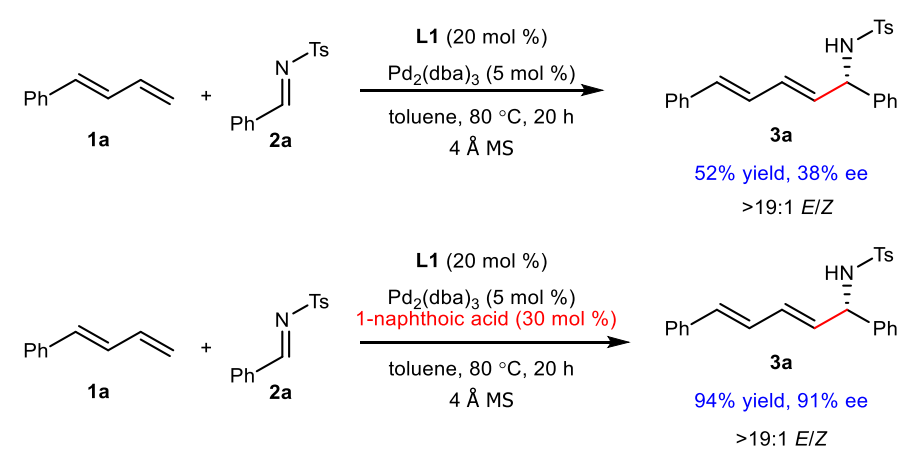

\section{Scheme S20. Acid effect on the asymmetric hydrodienylation reaction}

It was found that acid additive could enhance the reactivity for the hydrodienylation reaction with non-chiral phosphine ligand (control experiments, also see Table S1). For the asymmetric version, 
the stereocontrol could also be significantly improved with L1 as the chiral ligand (38\% ee VS 91\% ee), indicating the acid would be involved in the enantiodetermining step (consistent with the above

\section{Pathway 5 or Pathway 6).}

\section{3) DFT calculations}

According to the above results, we deduced that only one molecular L1 was coordinated to Pd and the acid additive would be engaged in the enantiodetermining step for the asymmetric hydrodienylation reaction. We further conducted comprehensive computational calculations based on the key transition state (Acid-TS1A and TS1E) in this pathway to elucidate the stereoselectivity. Various conformations to form $R$-3a or $S$-3a were generated by SYBYL-X2.0 Ga.conf module and calculated by Gaussian 09 (Figure S8). These results indicate that transition state S1 leading to the final product with $(S)$-configuration is the most favorable one, which is consistent with our experimental results. Looking into the structure of $\mathbf{S 1}, \mathbf{L 1}$, olefin, and the $\mathrm{N}$ atom of imine all coordinated to $\mathrm{Pd}(0)$, and the acid as a proton donor located above 2a with a H-bonding interaction with imine (Scheme $5 \mathrm{~d}$ ). Moreover, a $\pi$ - $\pi$ interaction between the naphthoic acid and phenyl ring of 1a was observed in S1, and it might be helpful for the stereocontrol. Similar conformation to form $R$ 3a was also generated (R6), while its energy is $7.8 \mathrm{kcal} / \mathrm{mol}$ higher than that of $\mathbf{S 1}$.

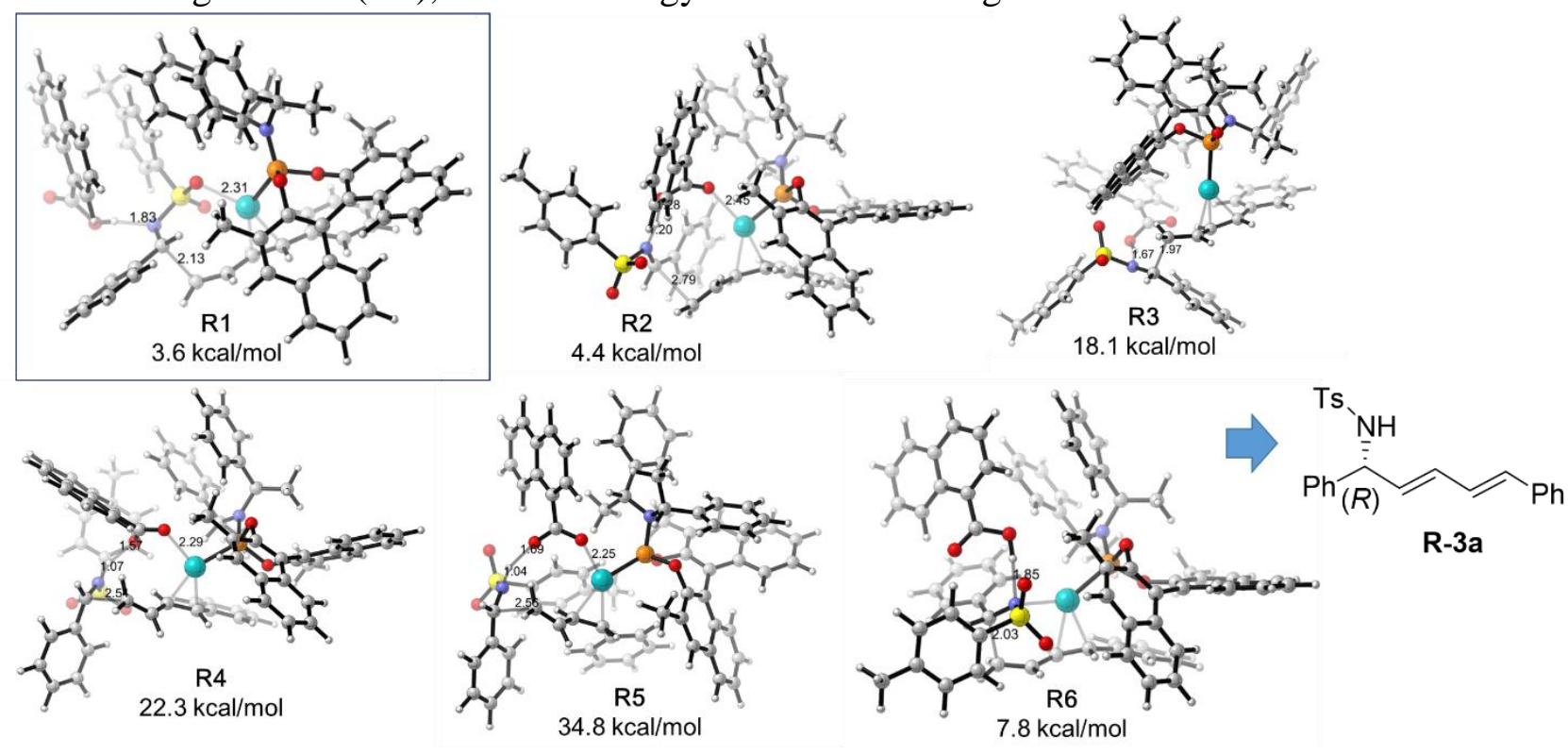



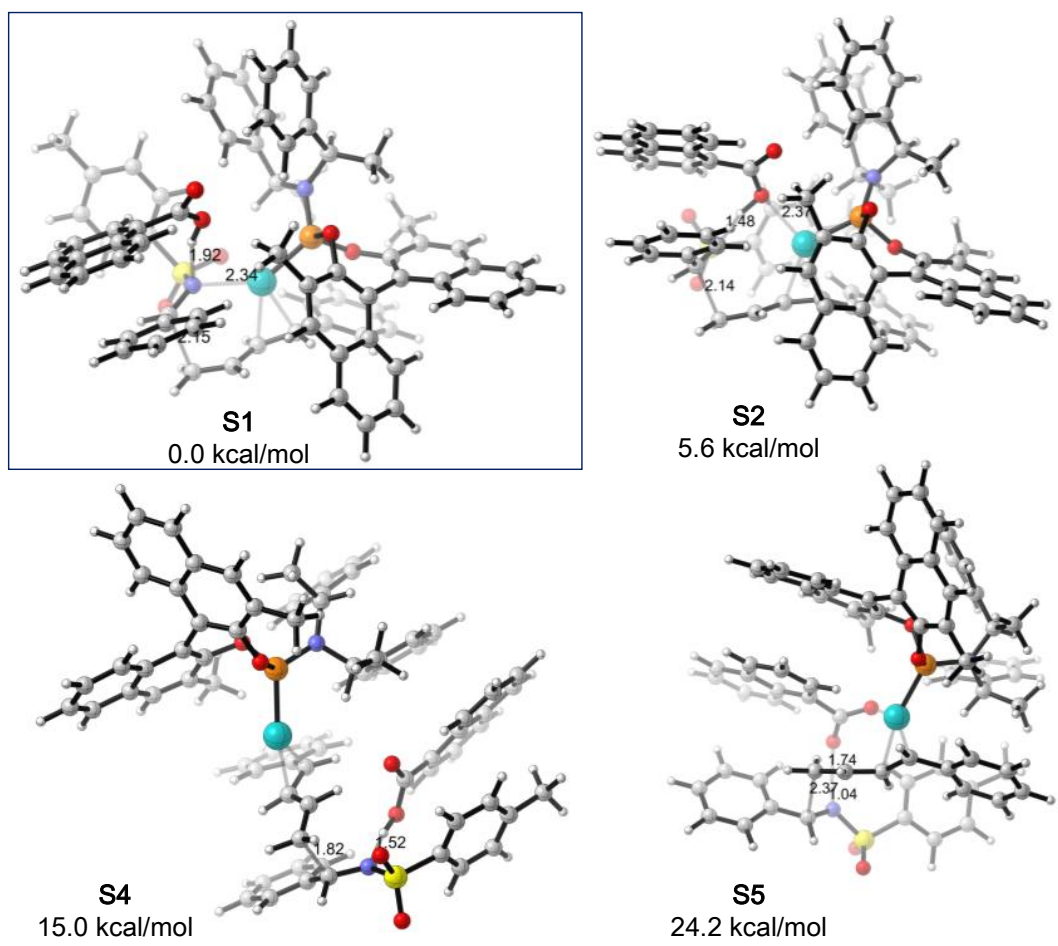

$5.6 \mathrm{kcal} / \mathrm{mol}$

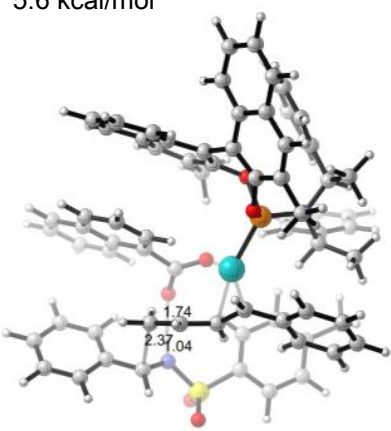

S5

$24.2 \mathrm{kcal} / \mathrm{mol}$
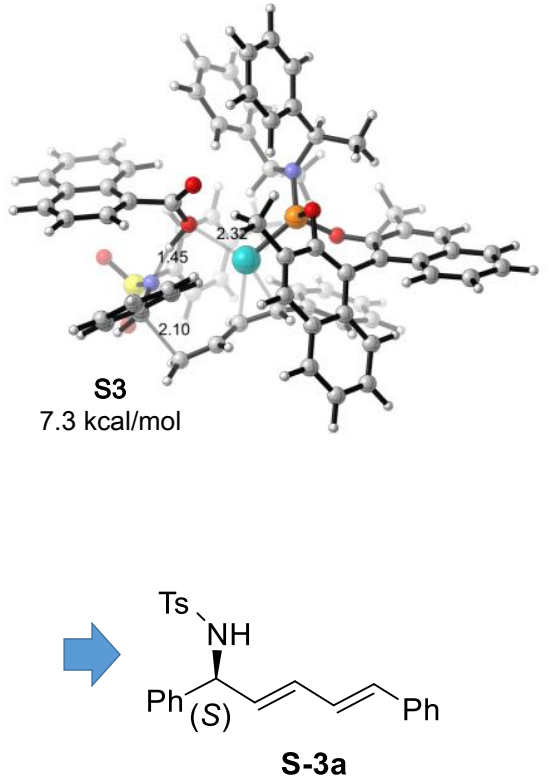

Figure S8. The calculated structures of key TSs for the $\mathrm{C}-\mathrm{C}$ bond formation at the B3LYP-D3/6311++G(d,p)//B3LYP-D3/6-31G(d) SDD for Pd (toluene) level and are given in $\mathrm{kcal} / \mathrm{mol}$ relative to the energy of $S 1$ 
NMR, HRMS Spectra and HPLC Chromatograms

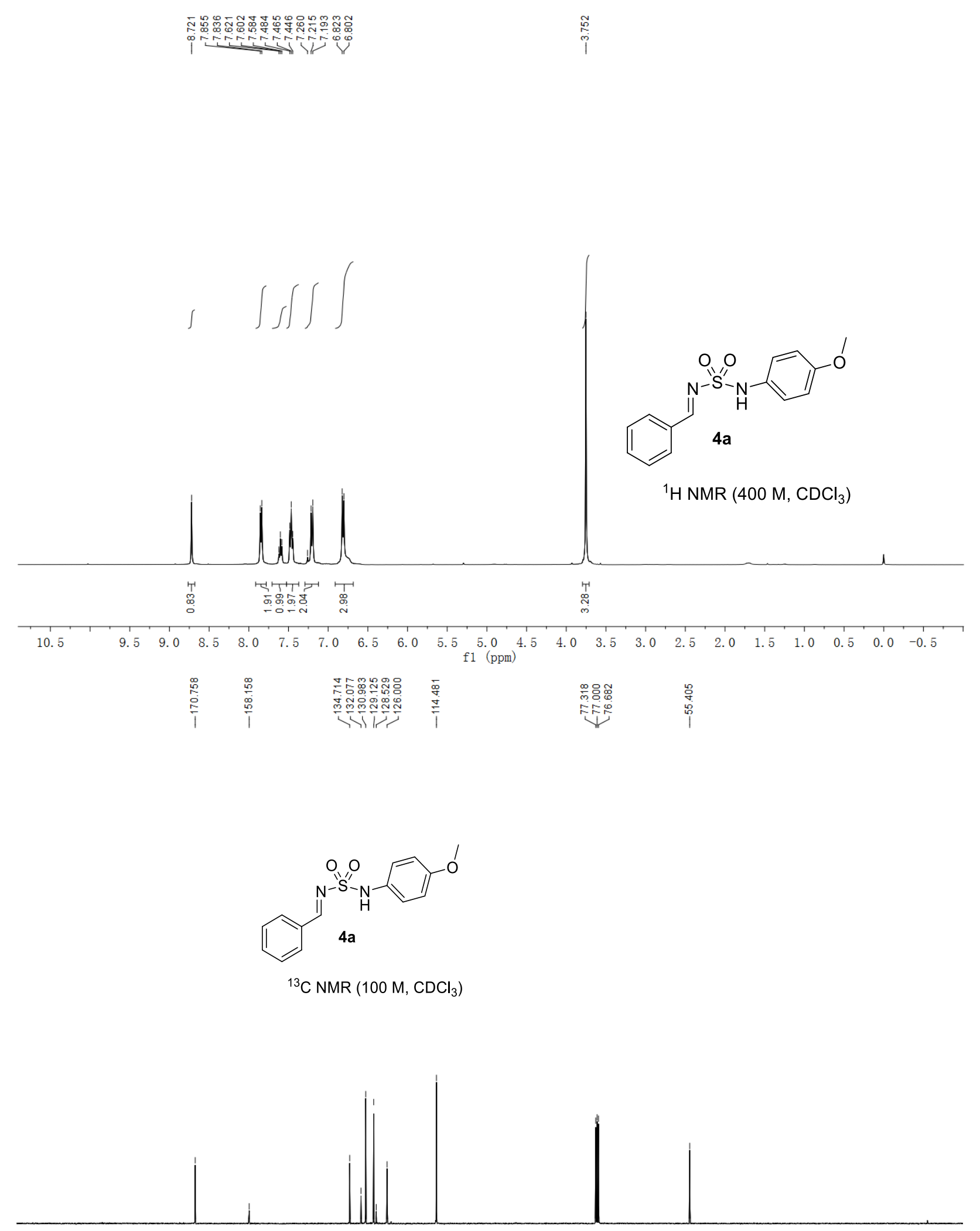

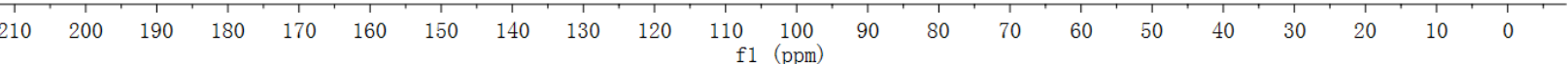




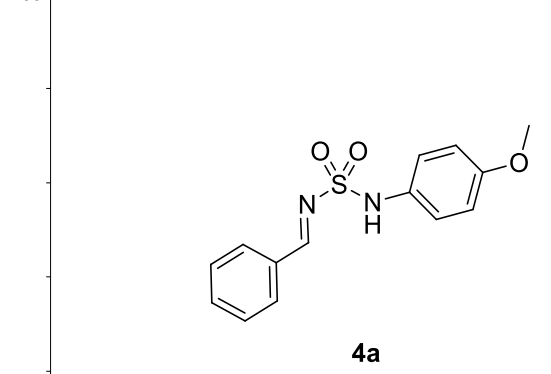

HRMS (ESI-TOF) m/z: [M + Na $]^{+}$

Calcd for $\mathrm{C}_{14} \mathrm{H}_{14} \mathrm{~N}_{2} \mathrm{O}_{3} \mathrm{SNa} 313.0617$
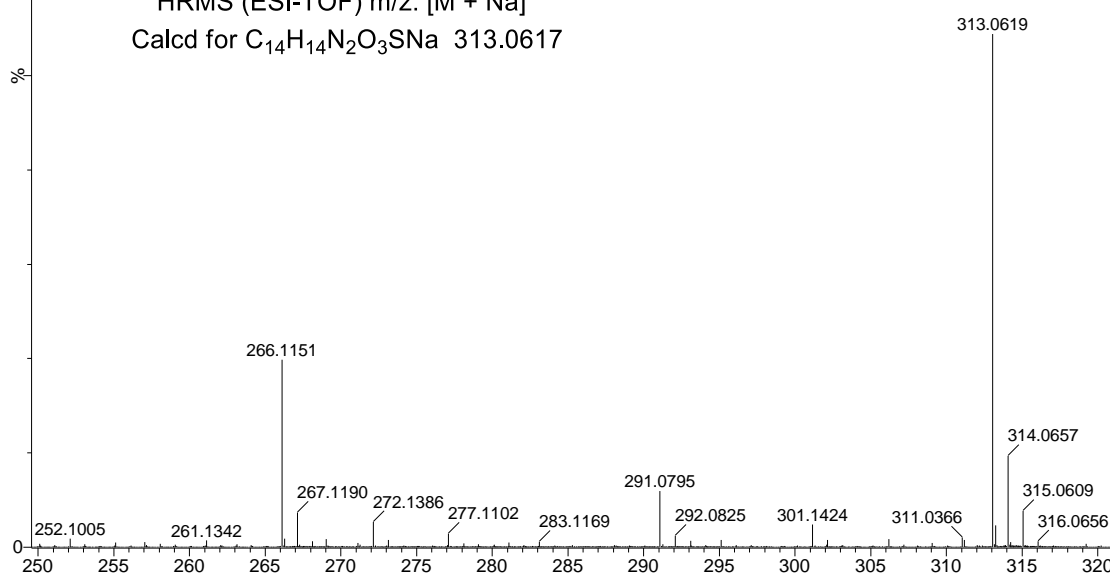

346.0916

347.0863

315.0609

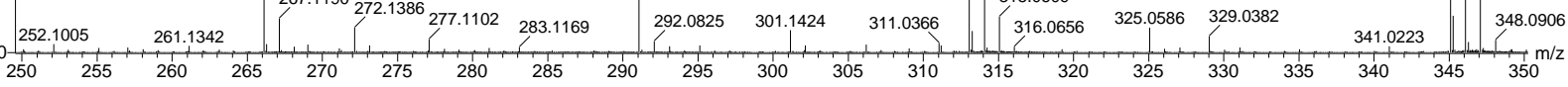




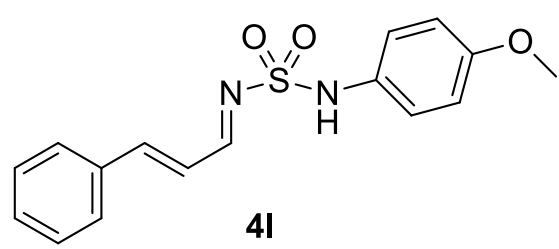

${ }^{1} \mathrm{H}$ NMR (400M, $\mathrm{CDCl}_{3}$ )

$1 \quad 1 / 1 / 15$
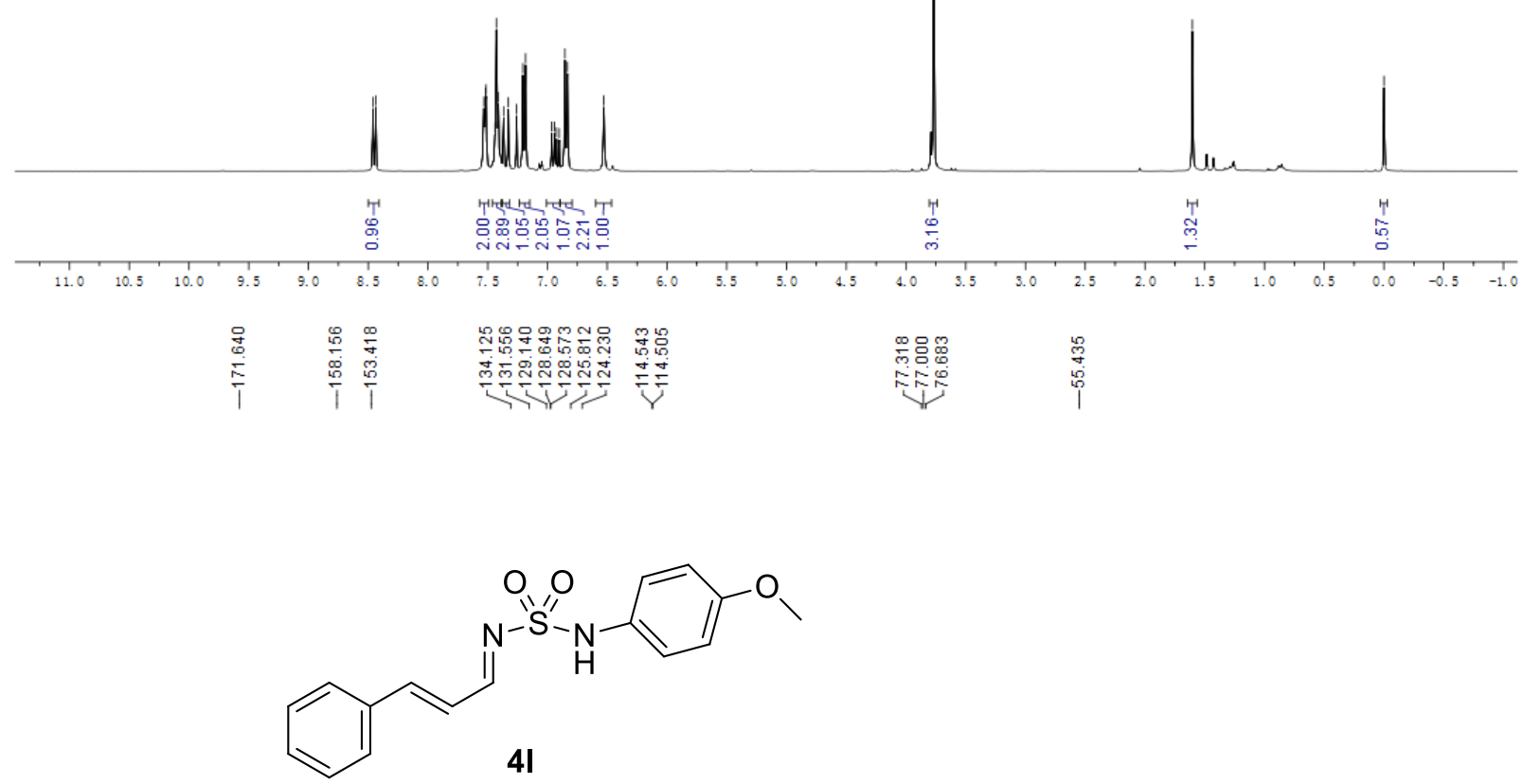

${ }^{13} \mathrm{C}$ NMR (100M, $\mathrm{CDCl}_{3}$ )
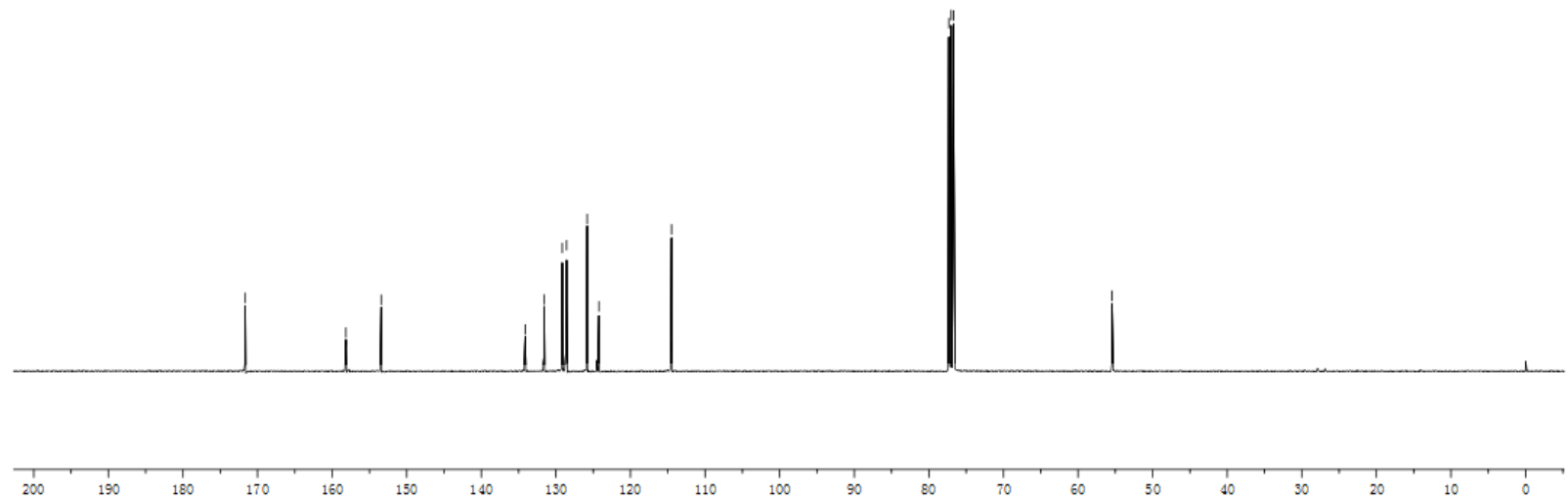
17:04:12

200806_CY_M2 2 (0.034) Cm (1:24)

100

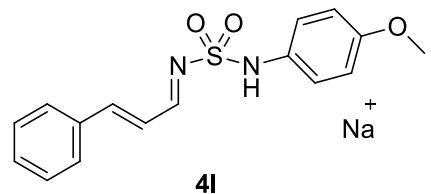

HRMS (ESI-TOF) m/z: [M + Na ${ }^{+}$

Calcd for $\mathrm{C}_{16} \mathrm{H}_{16} \mathrm{~N}_{2} \mathrm{O}_{3} \mathrm{~S} 339.0774$
06-Aug-2020

TOF MS ES+

340.0812

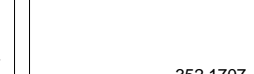




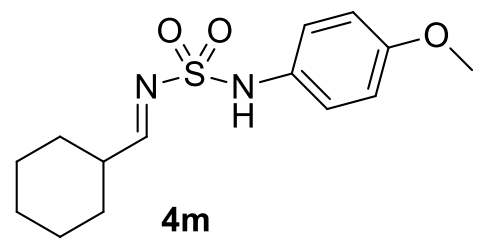

${ }^{1} \mathrm{H}$ NMR (400M, $\mathrm{CDCl}_{3}$ )
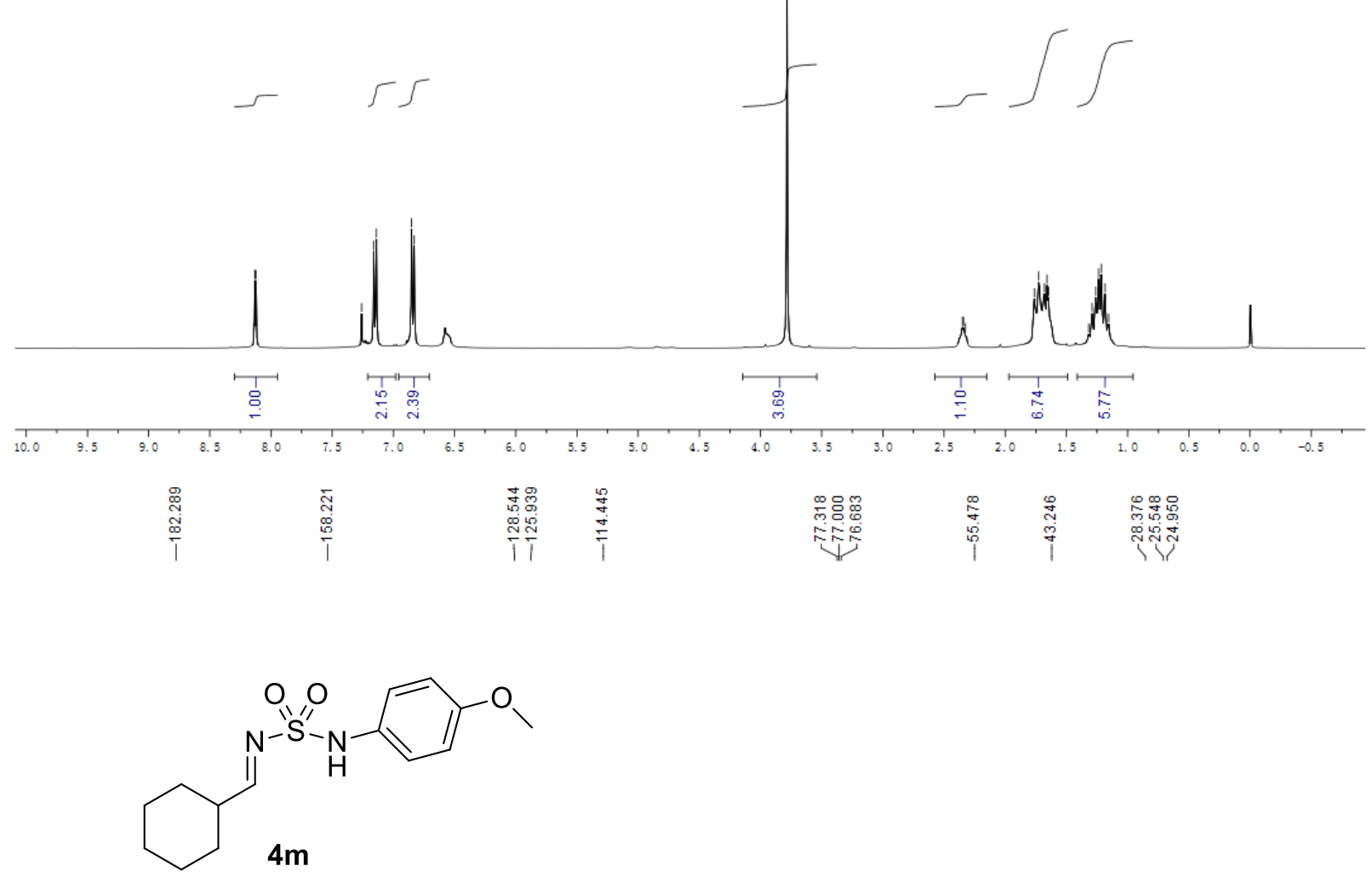

${ }^{13} \mathrm{C}$ NMR $\left(100 \mathrm{M}, \mathrm{CDCl}_{3}\right)$

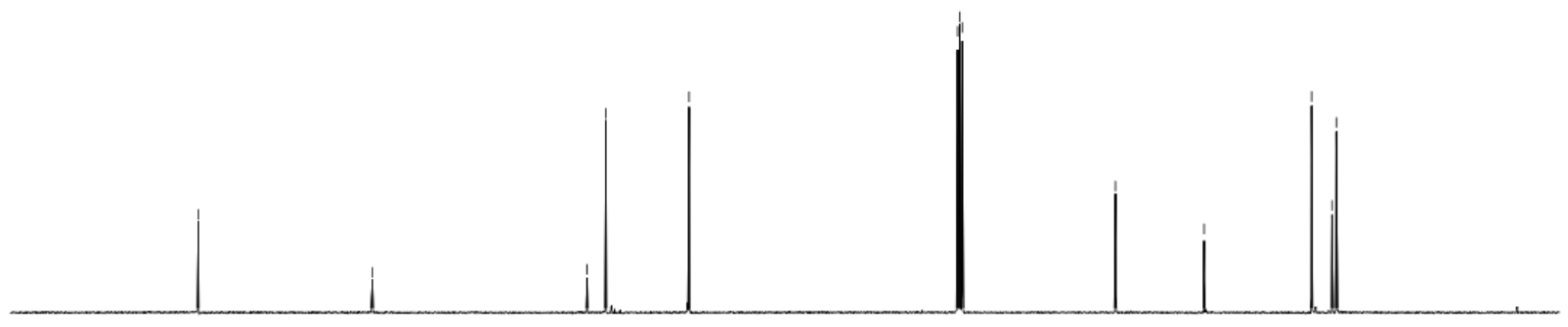




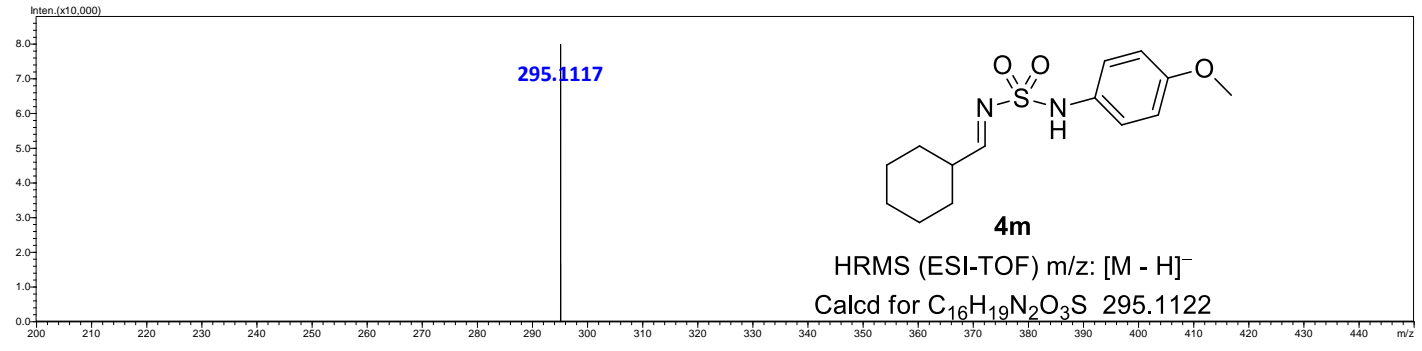




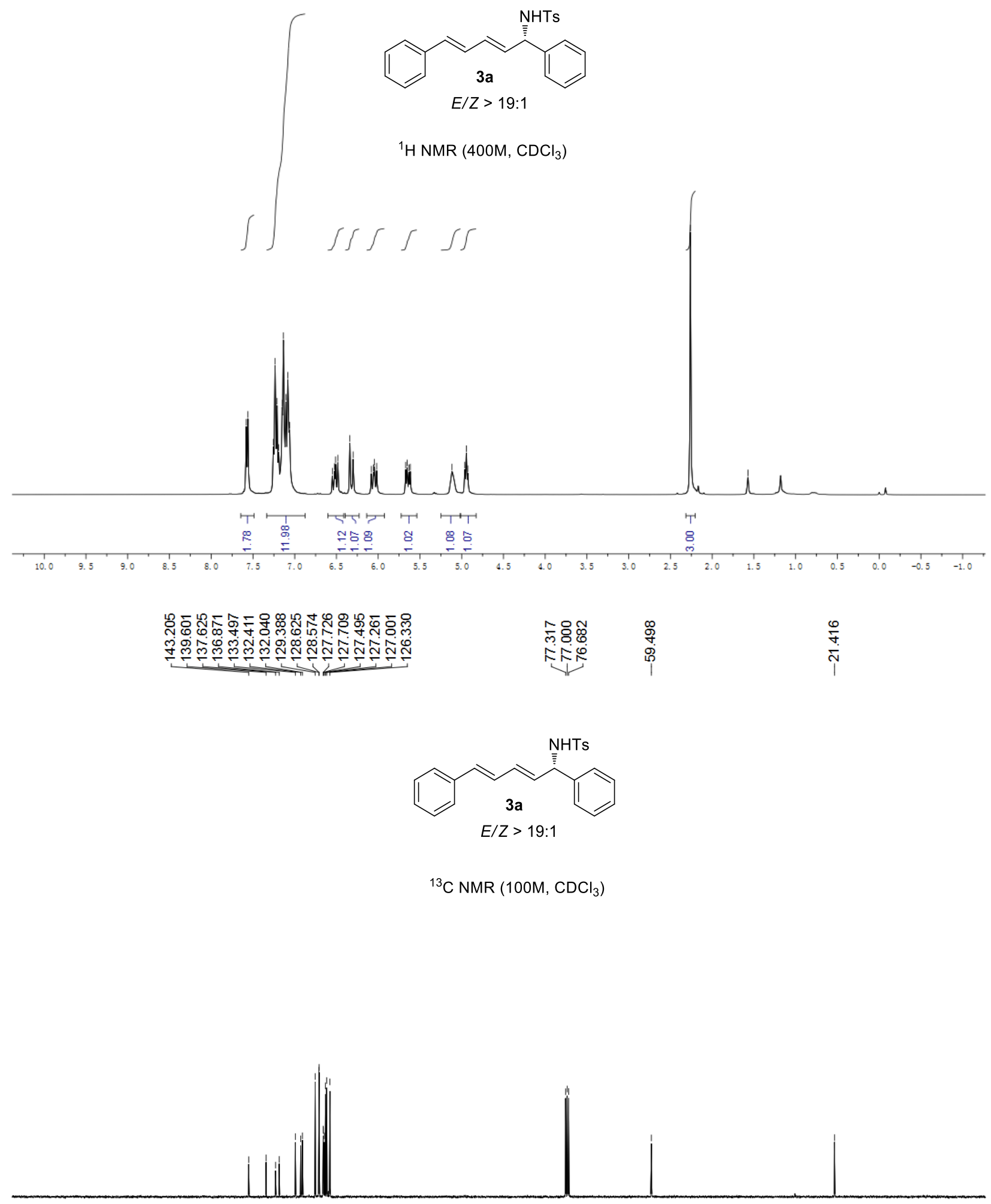

$\begin{array}{rrrrrrrrrrrrrrr}1 & 1 & 1 & 1 & 1 & 1 & 1 & 1 & 1 & 1 & 1 & 1 & 1 & 1 & 1\end{array}$ 

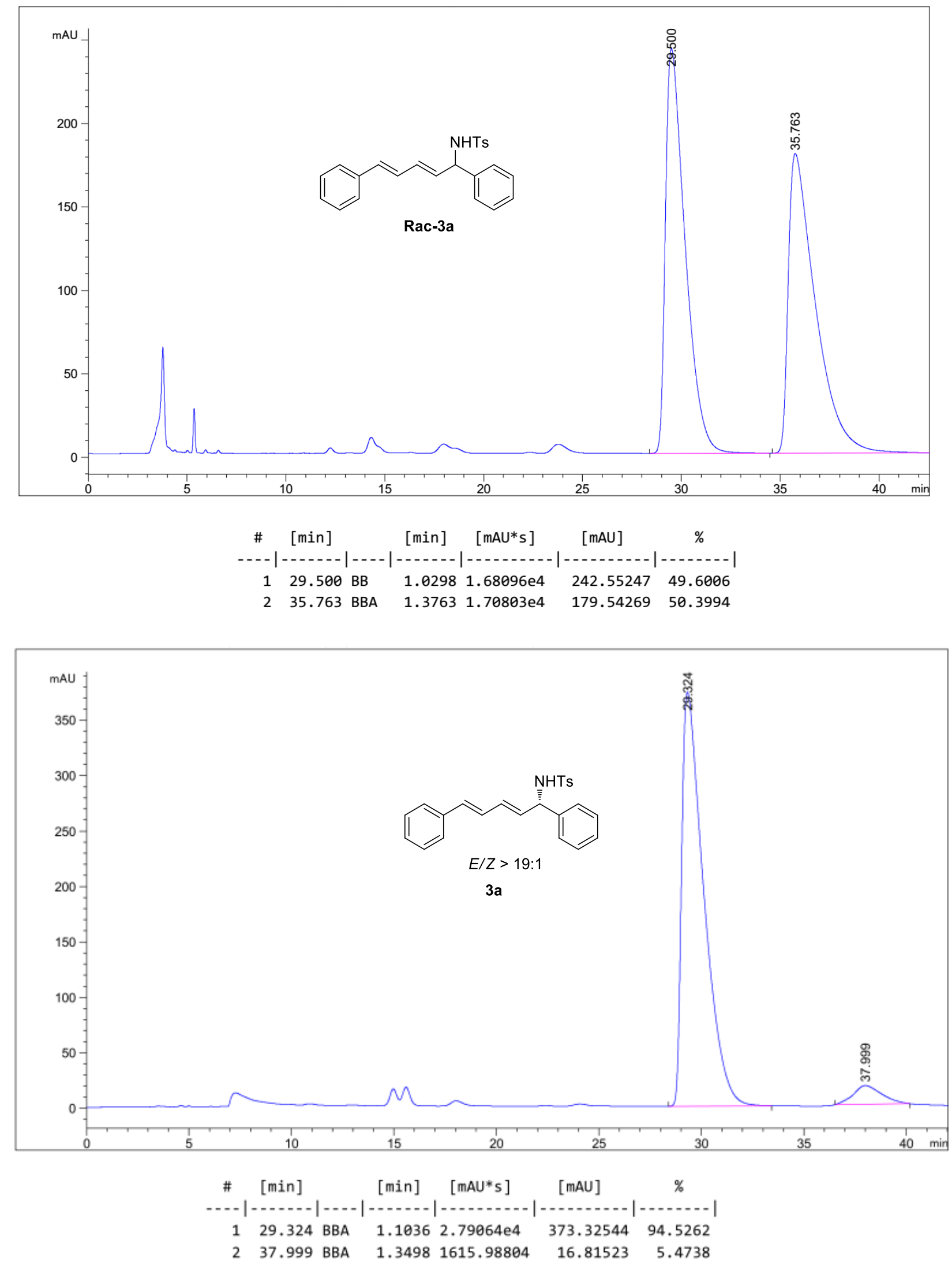
Spectrum Plot Report

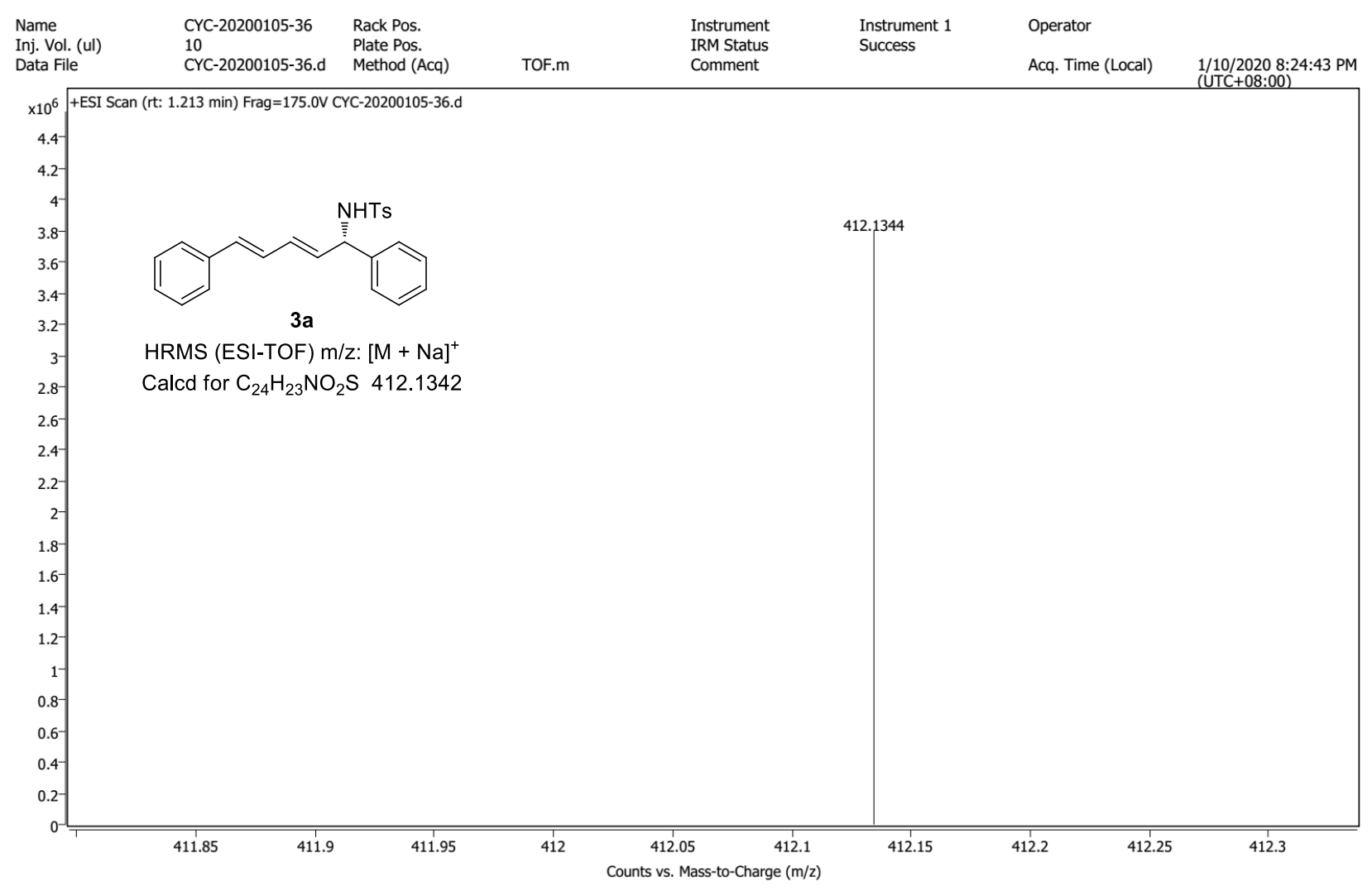




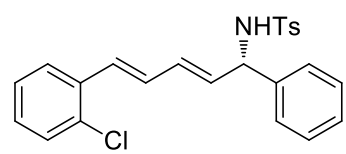

3b

${ }^{1} \mathrm{H}$ NMR (400M, $\mathrm{CDCl}_{3}$ )

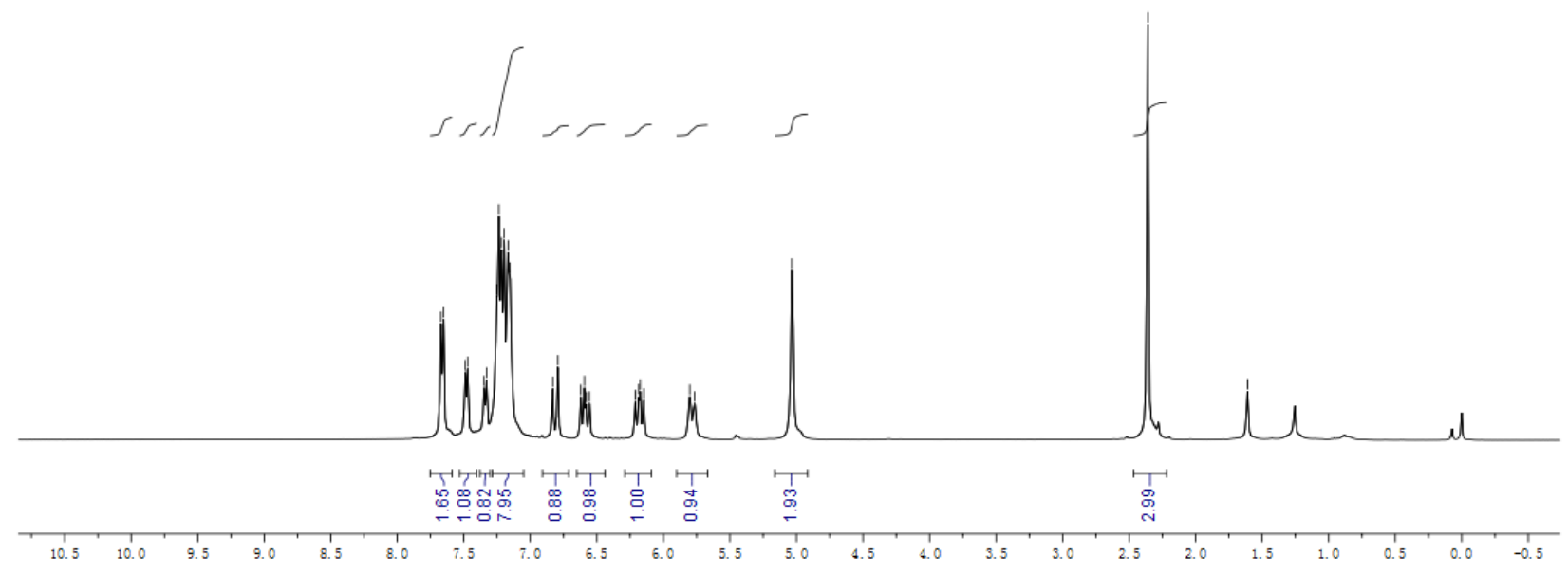

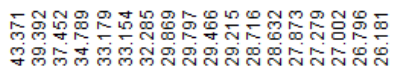

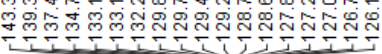

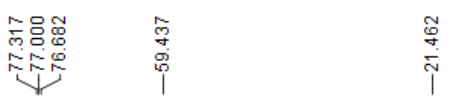

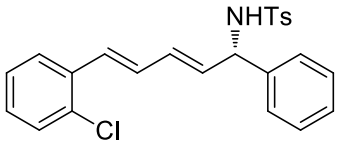

3b

${ }^{13} \mathrm{C} \operatorname{NMR}\left(100 \mathrm{M}, \mathrm{CDCl}_{3}\right)$

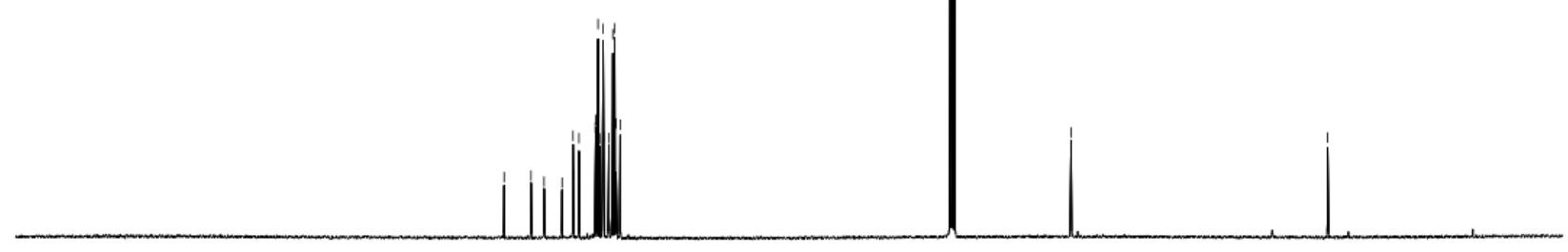



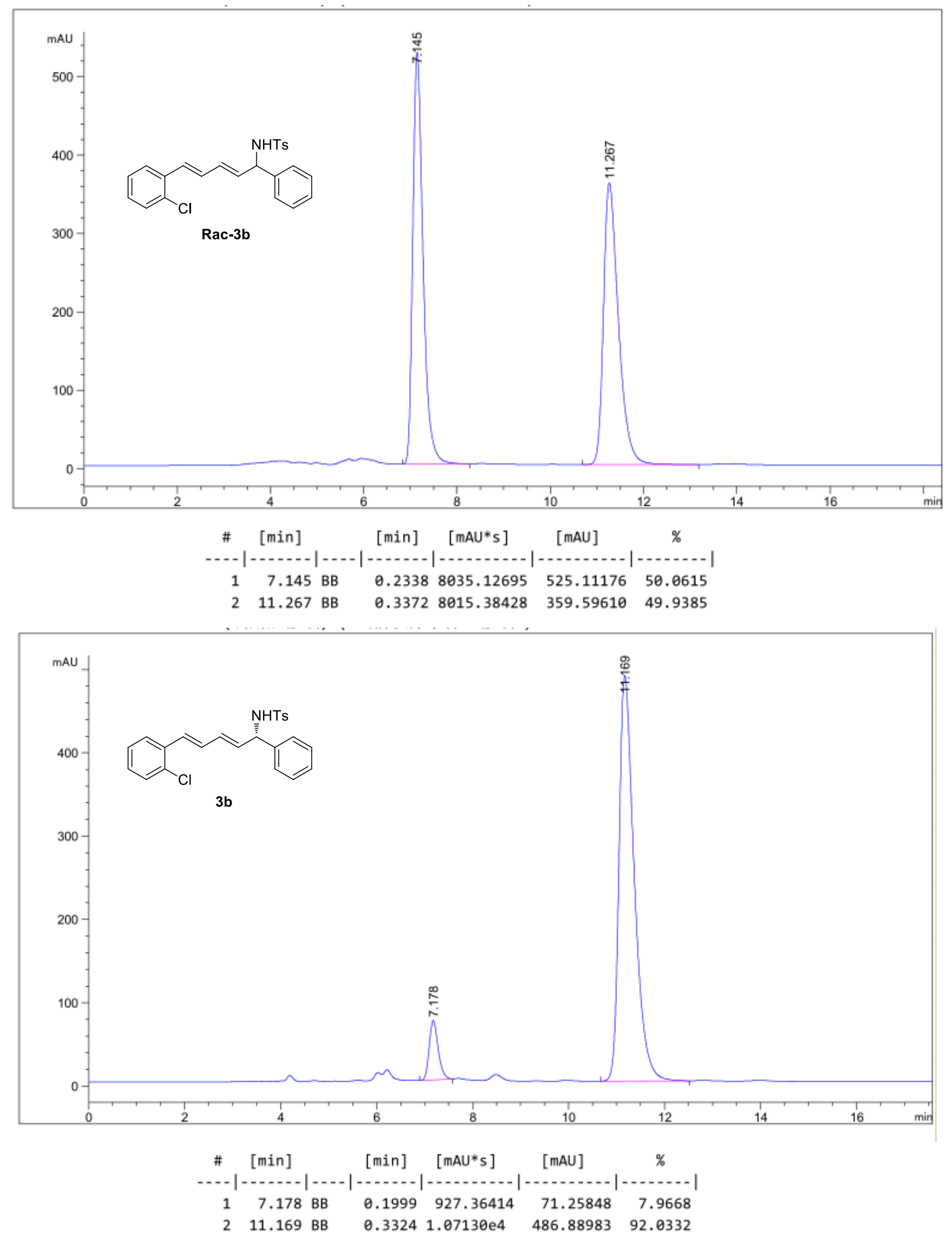
User Spectrum Plot Report

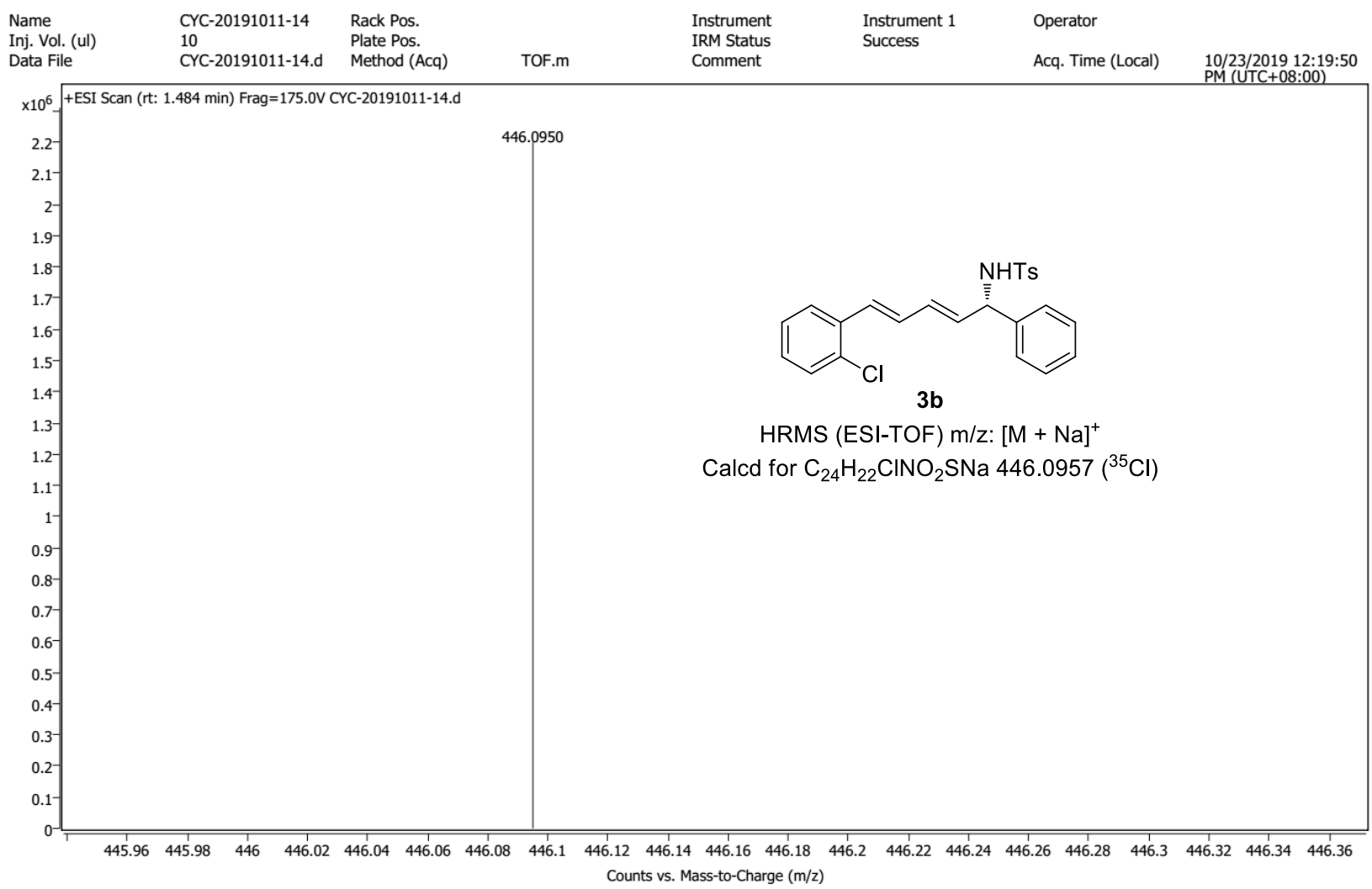

User Spectrum Plot Report

Agilent

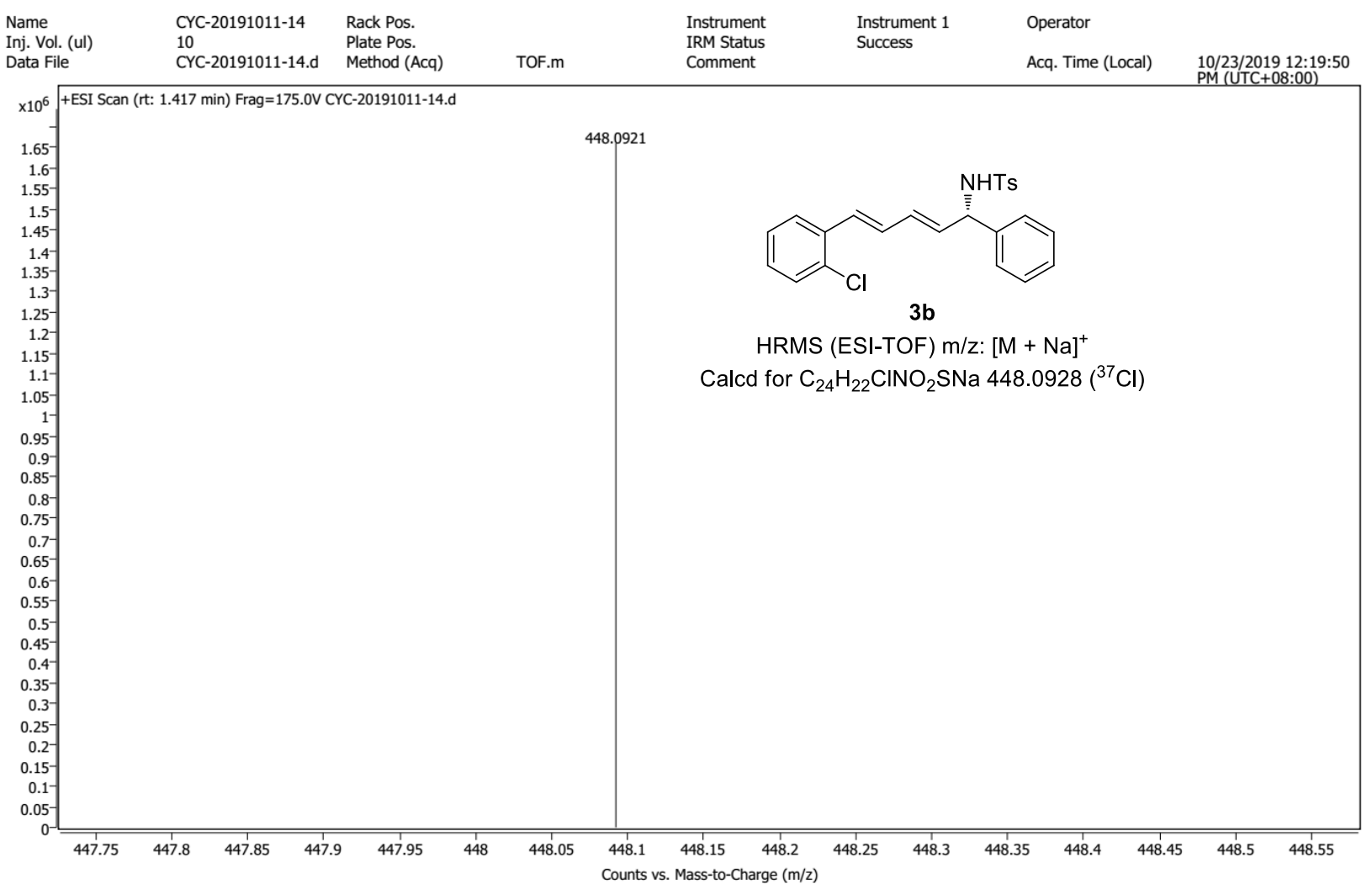



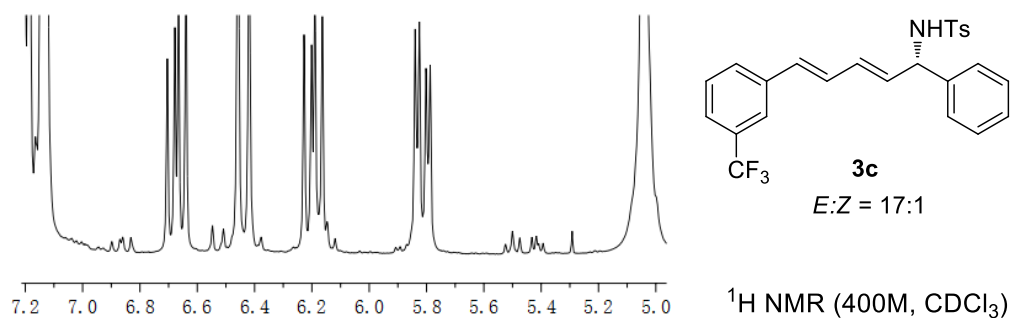

${ }^{1} \mathrm{H}$ NMR (400M, $\left.\mathrm{CDCl}_{3}\right)$

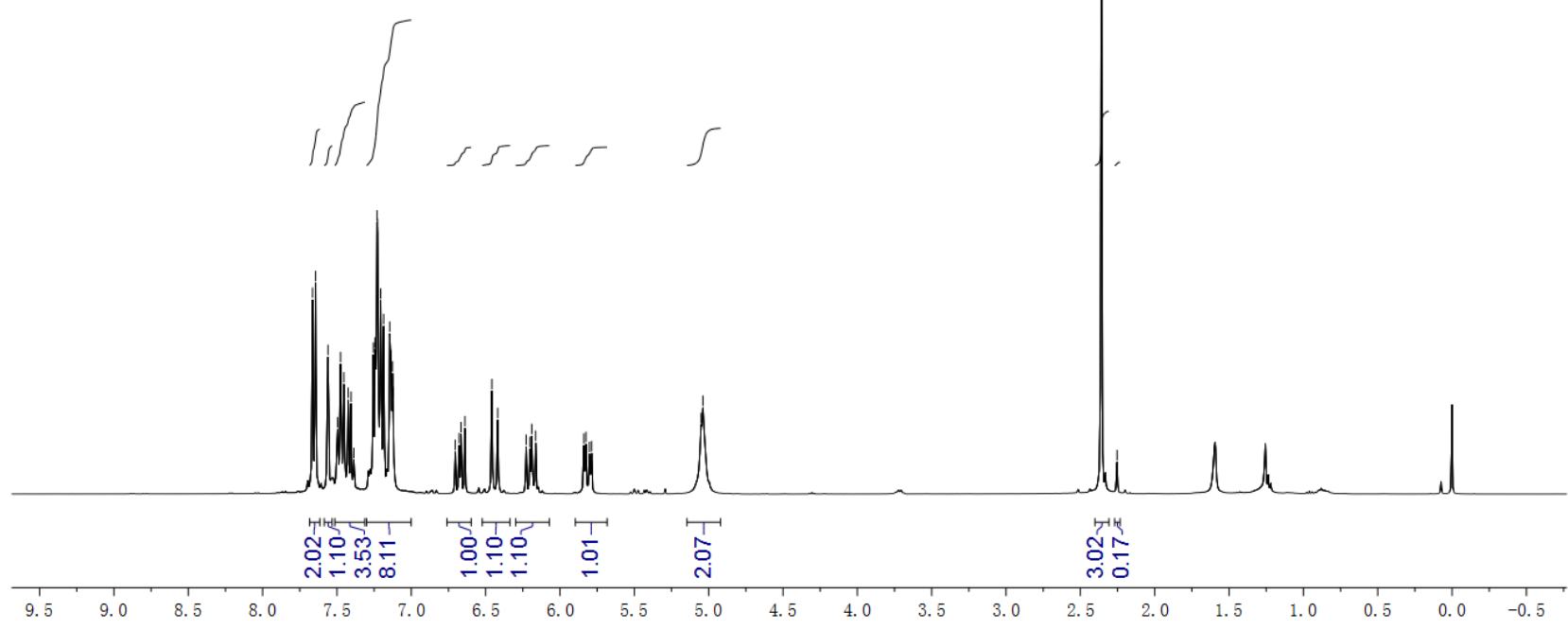

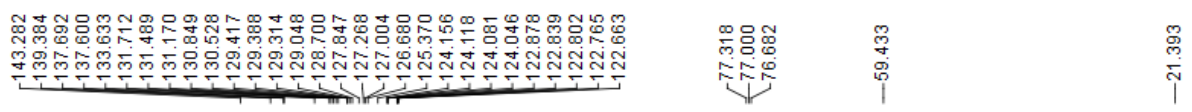

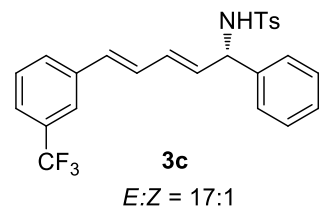

${ }^{13} \mathrm{C}$ NMR (100M, $\left.\mathrm{CDCl}_{3}\right)$

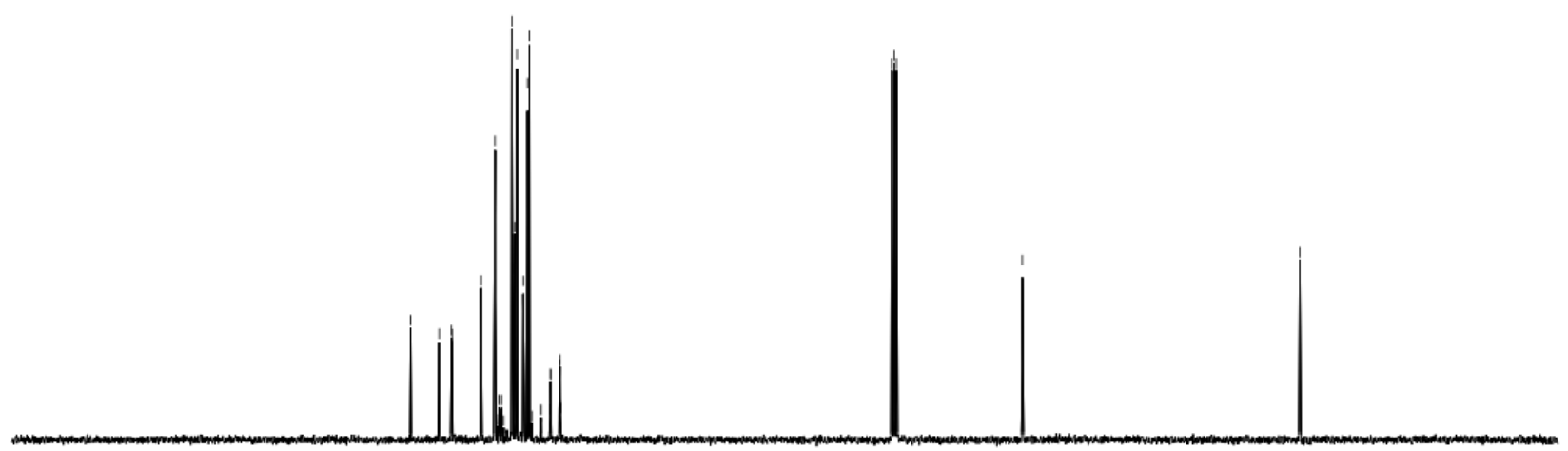



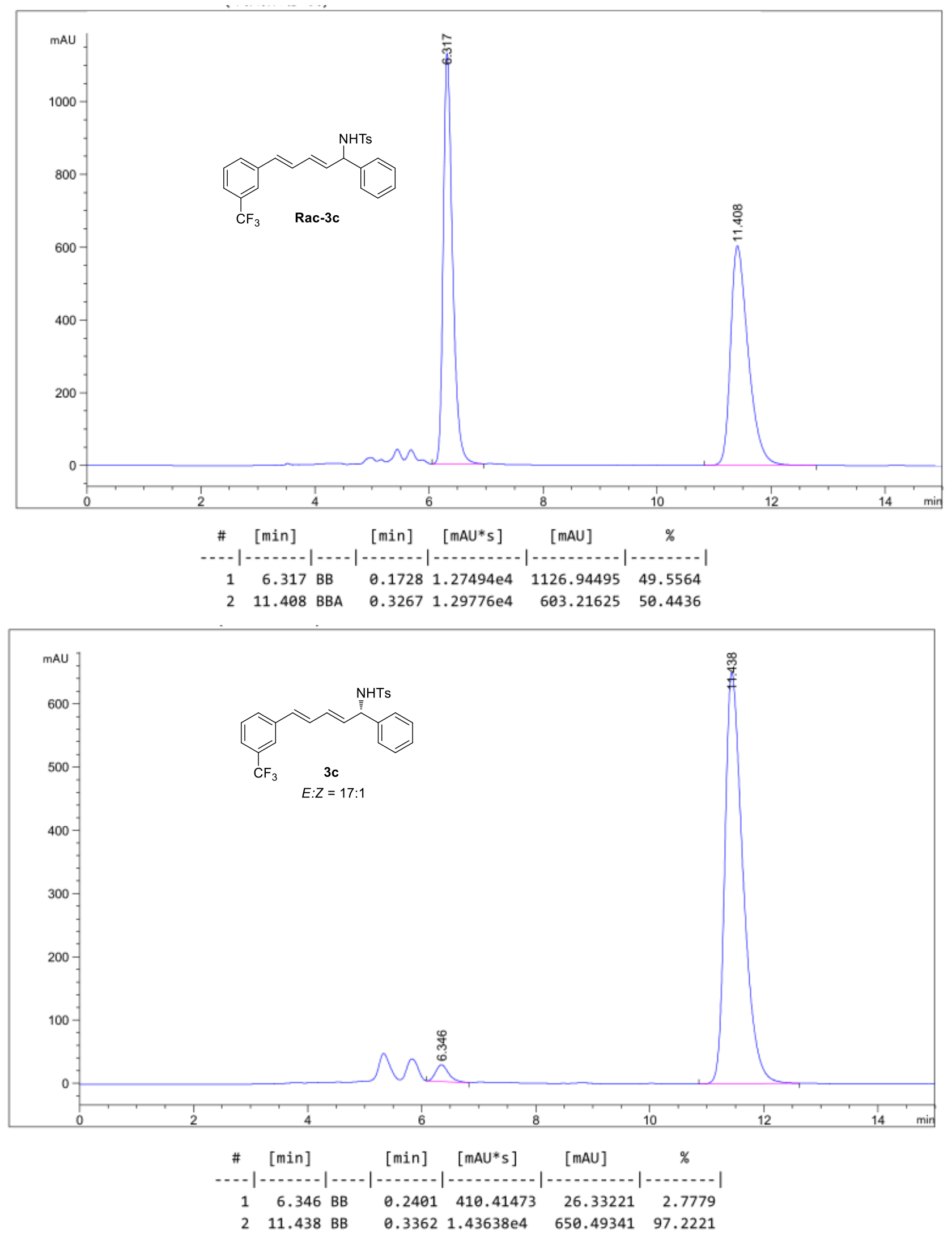
User Spectrum Plot Report

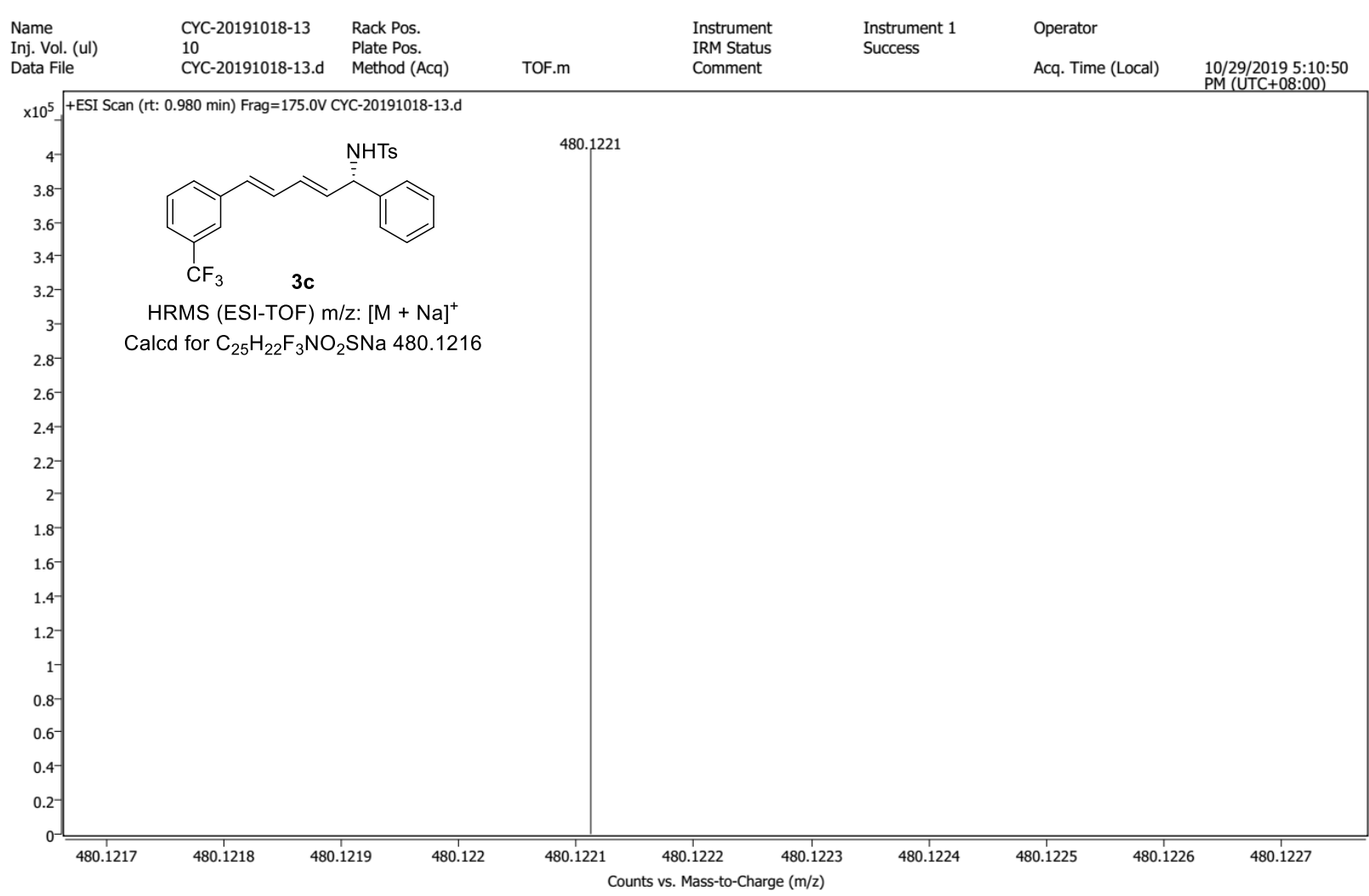




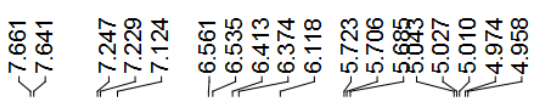

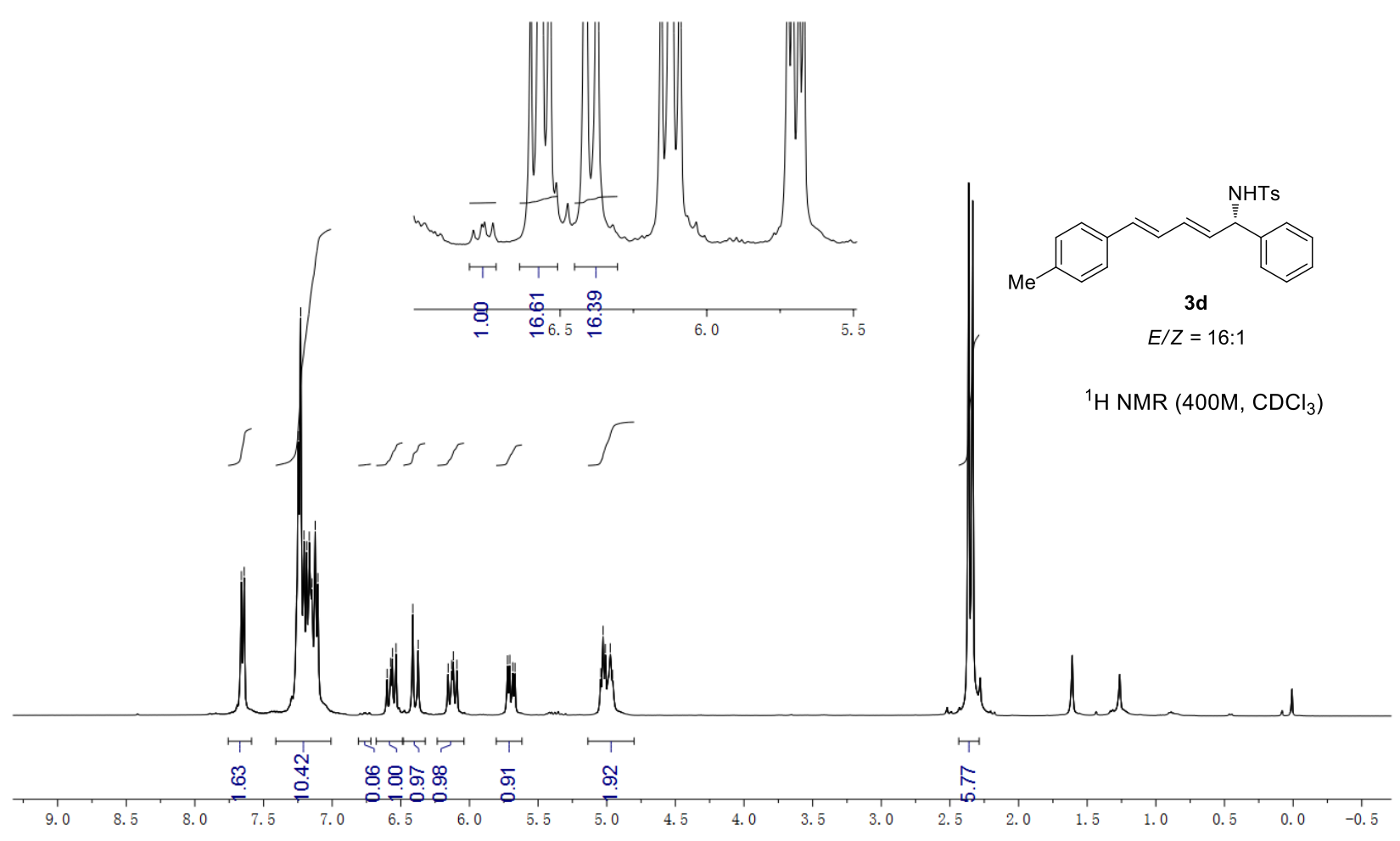

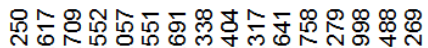

守

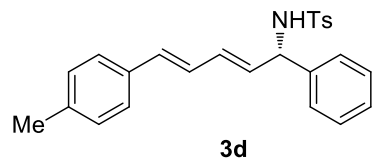

$E / Z=16: 1$

${ }^{13} \mathrm{C}$ NMR (100M, $\left.\mathrm{CDCl}_{3}\right)$

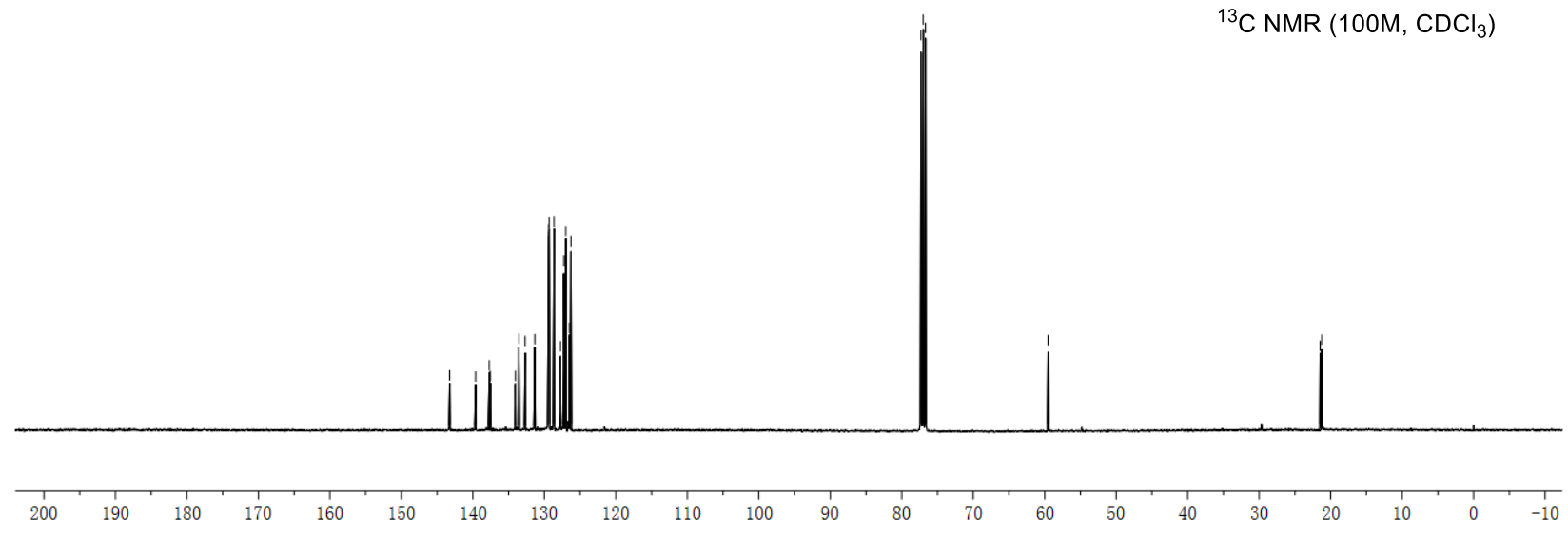



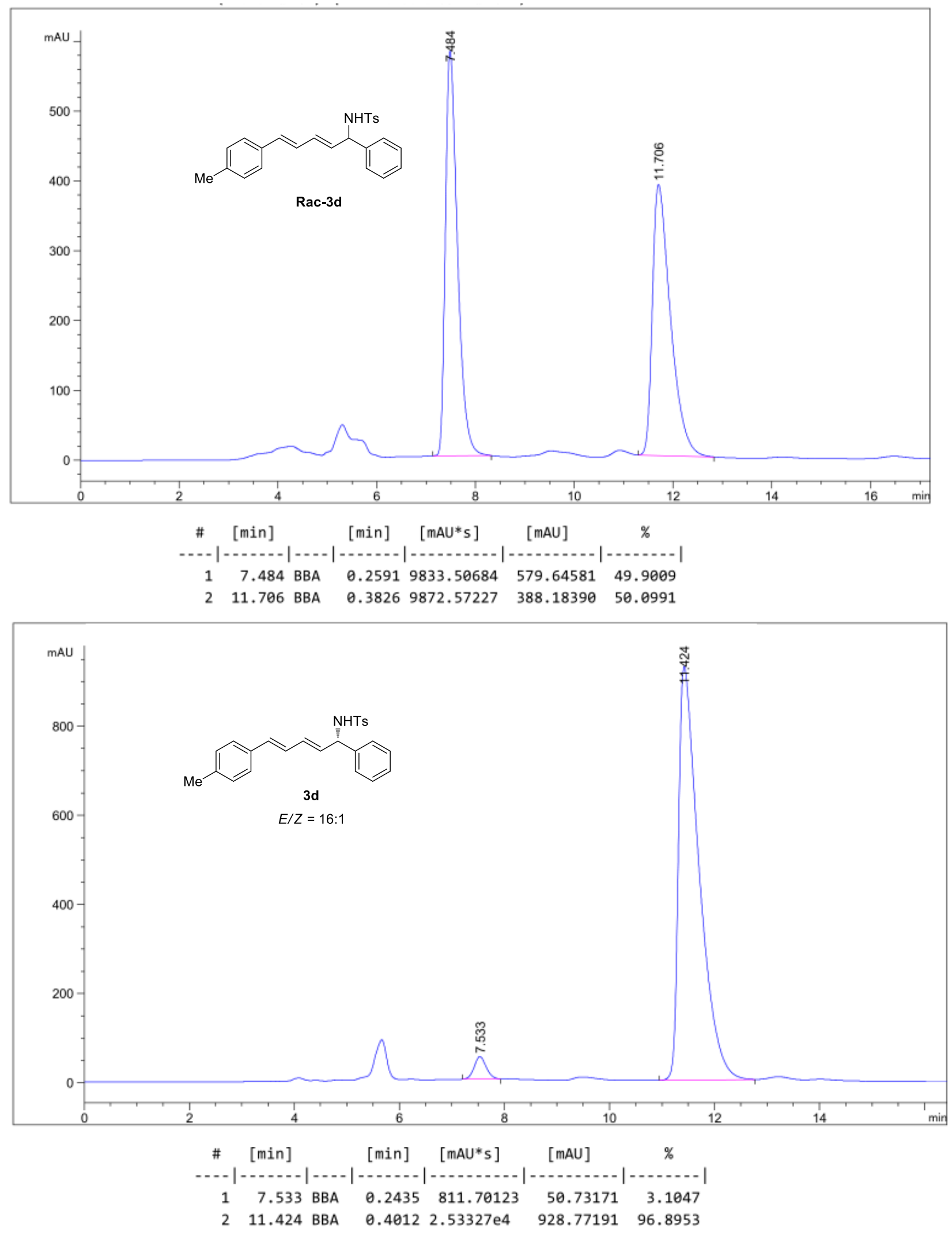
User Spectrum Plot Report

Agilent

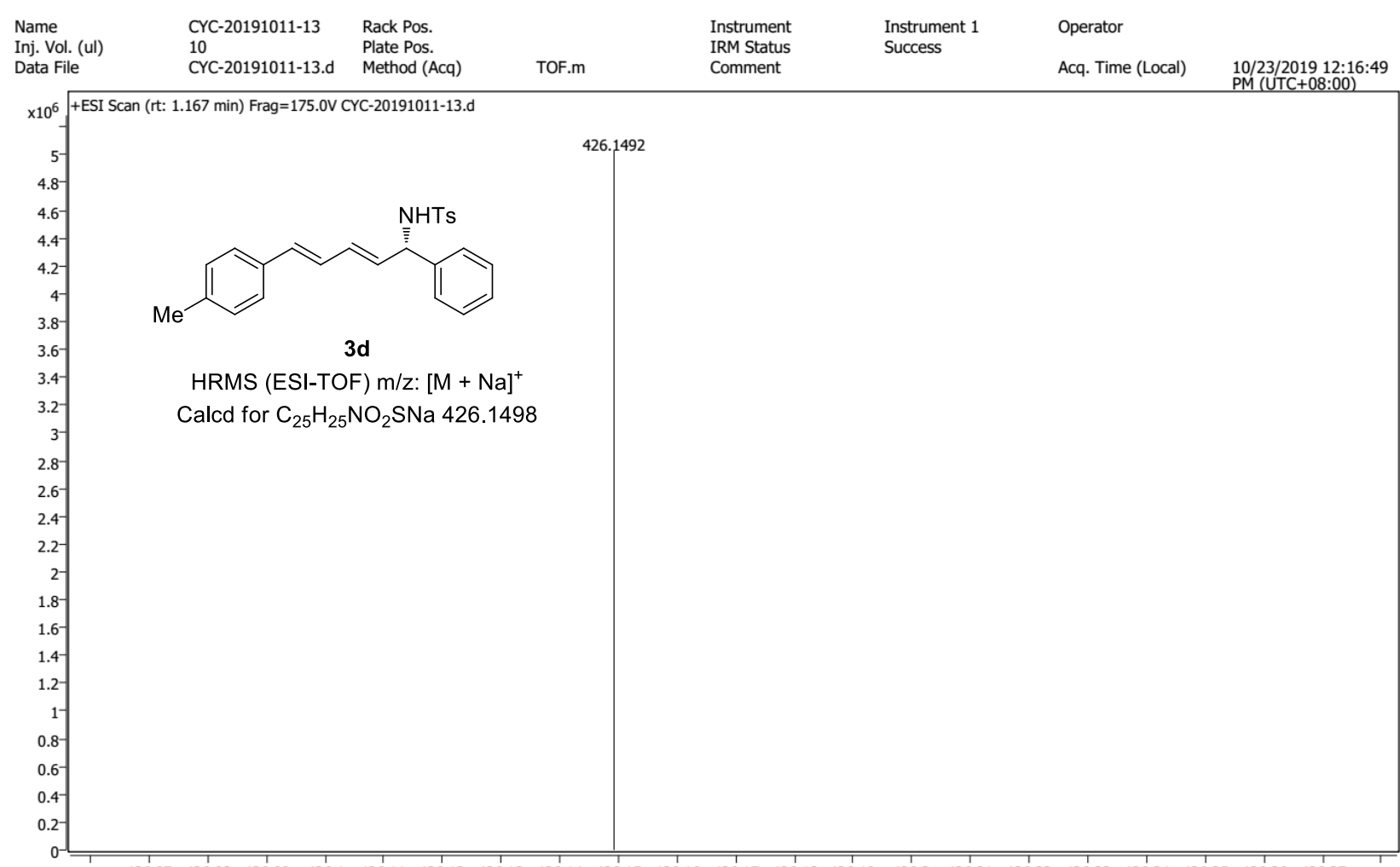

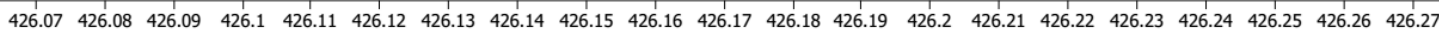
Counts vs. Mass-to-Charge $(\mathrm{m} / \mathrm{z})$ 


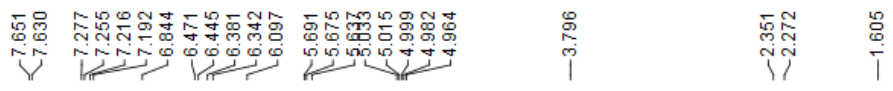

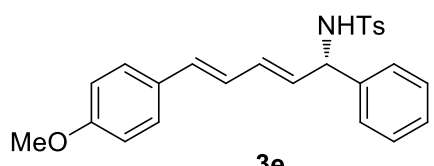

$3 e$

$E / Z>19: 1$
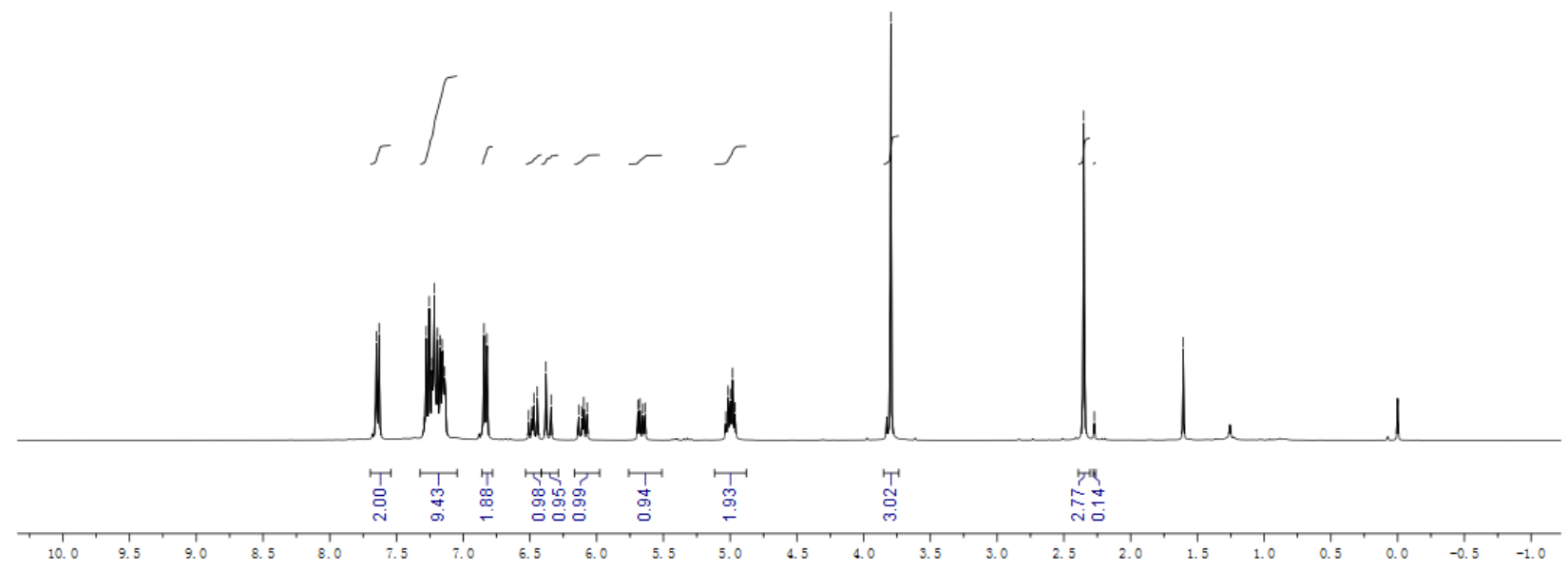

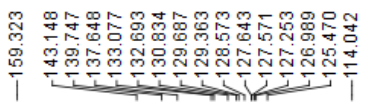

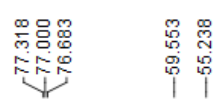

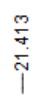
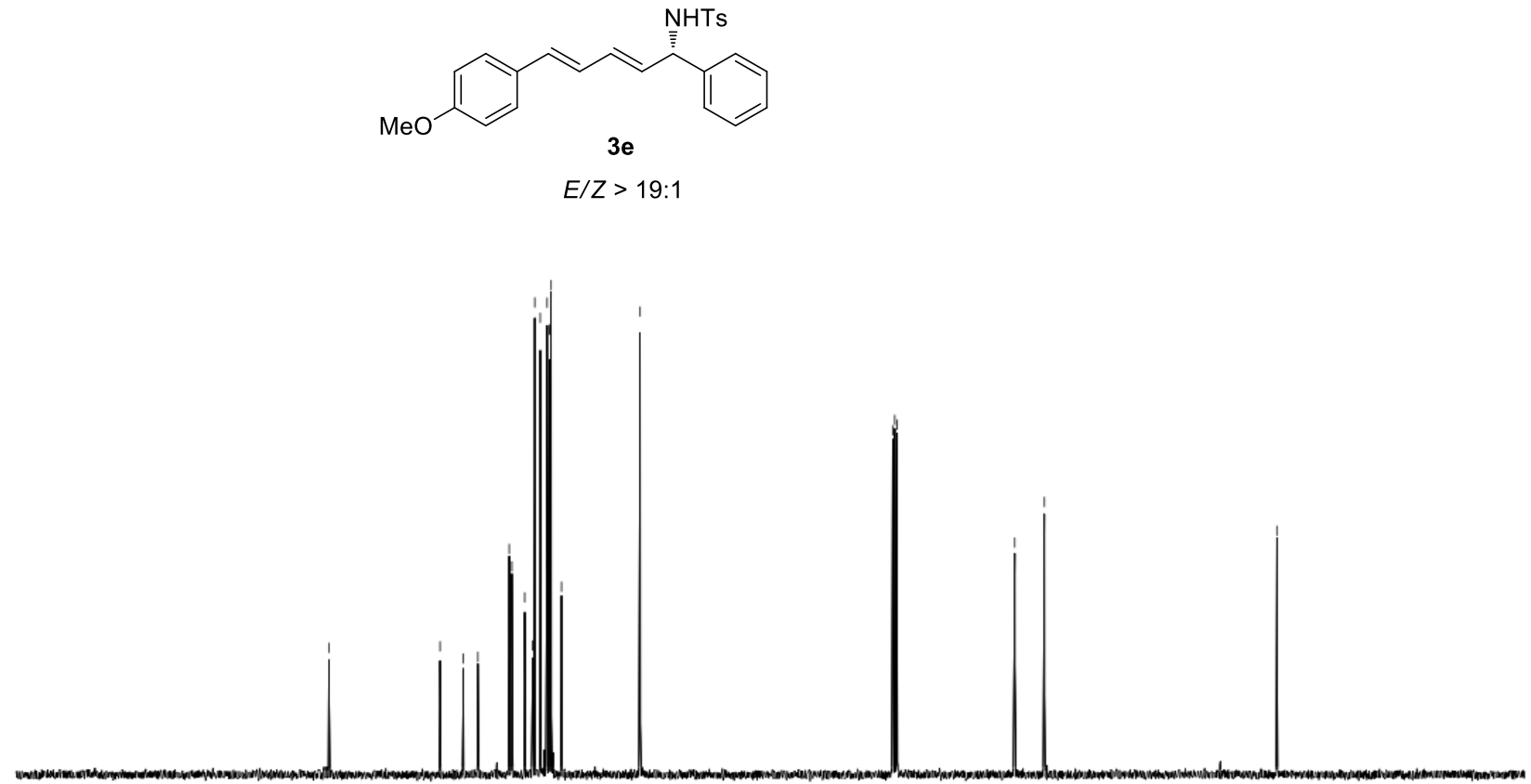

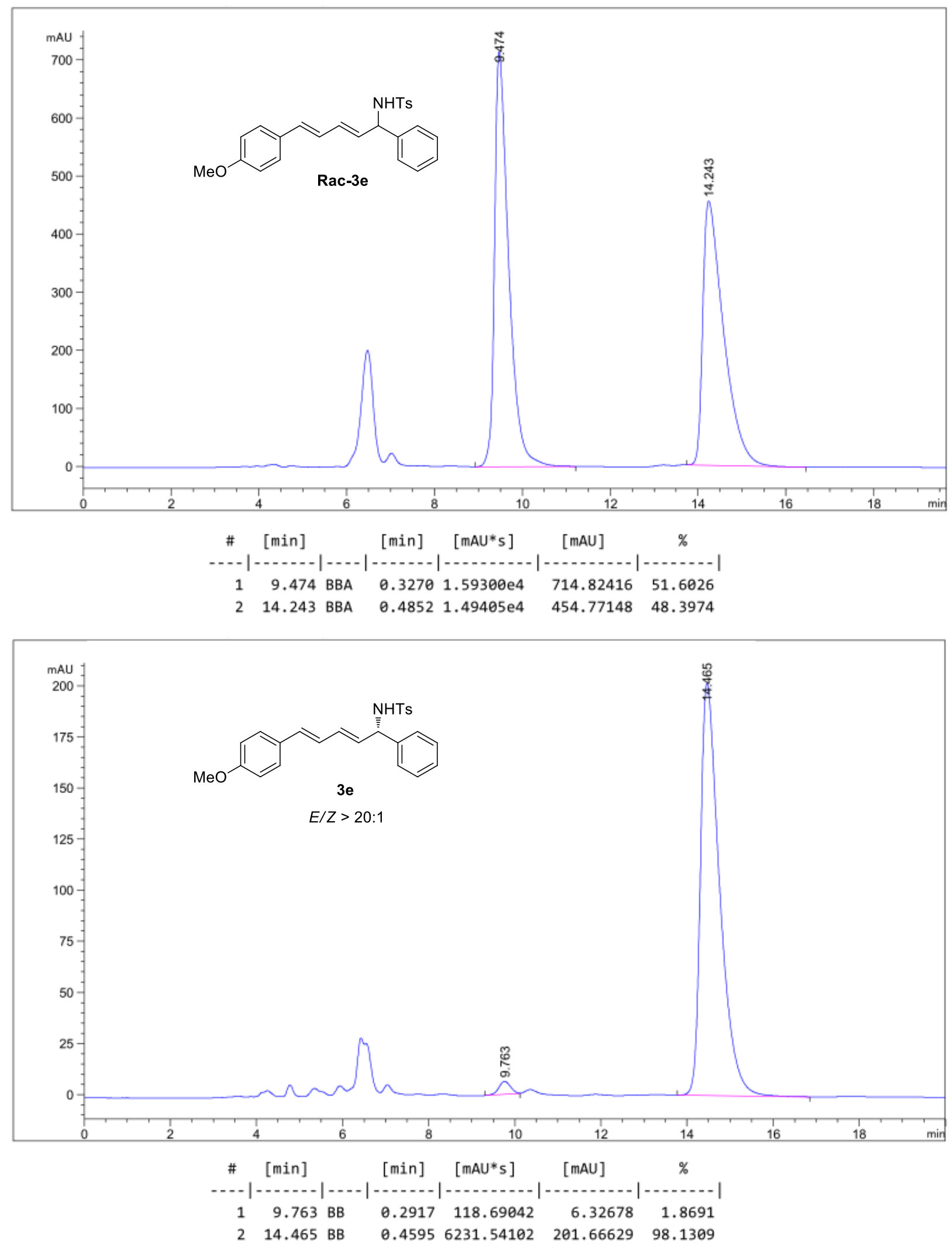
User Spectrum Plot Report

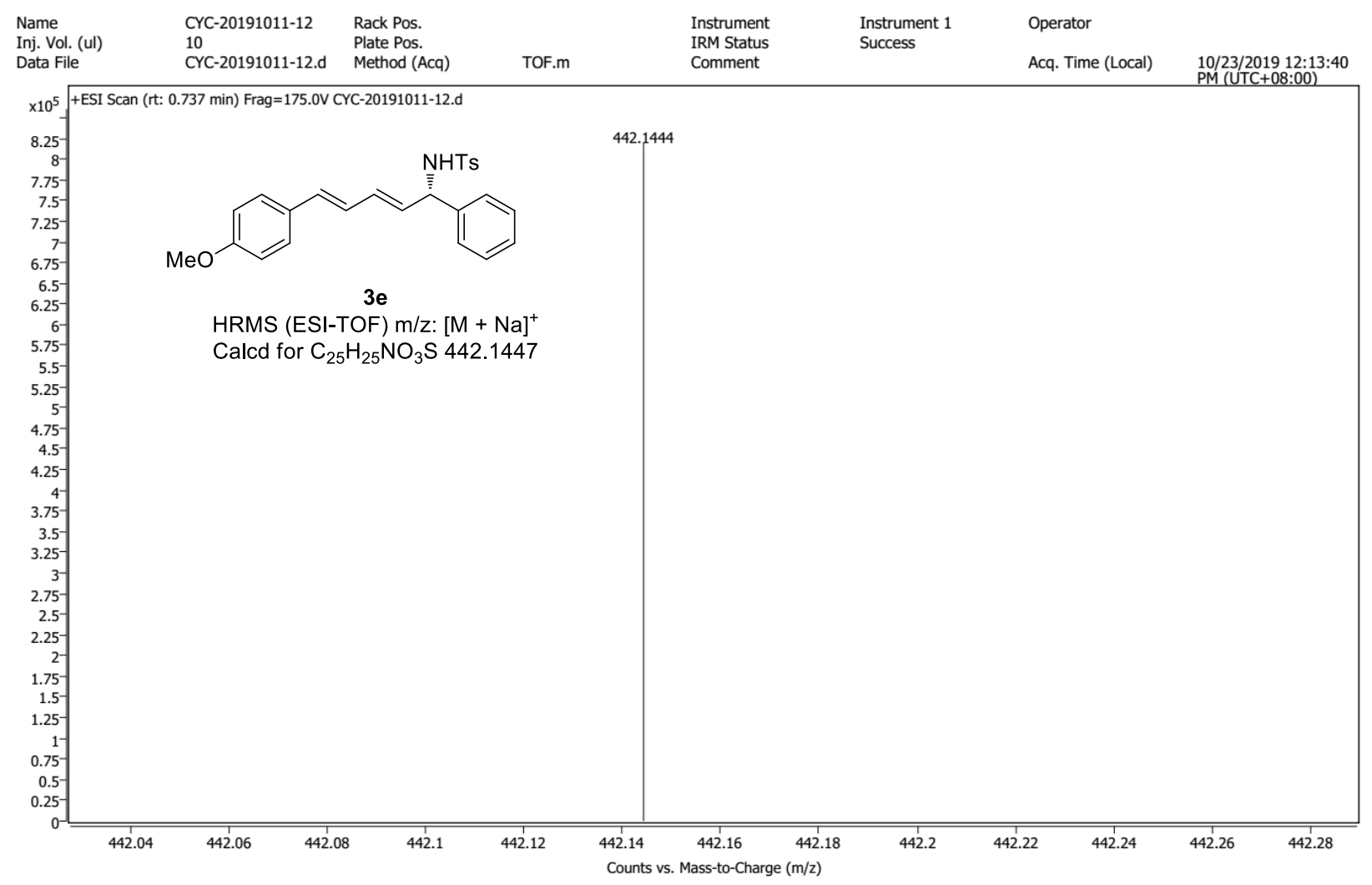



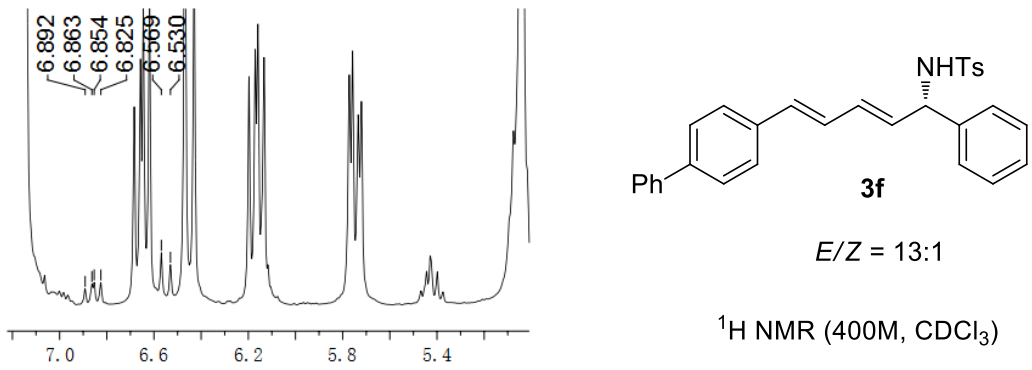

${ }^{1} \mathrm{H}$ NMR (400M, $\left.\mathrm{CDCl}_{3}\right)$
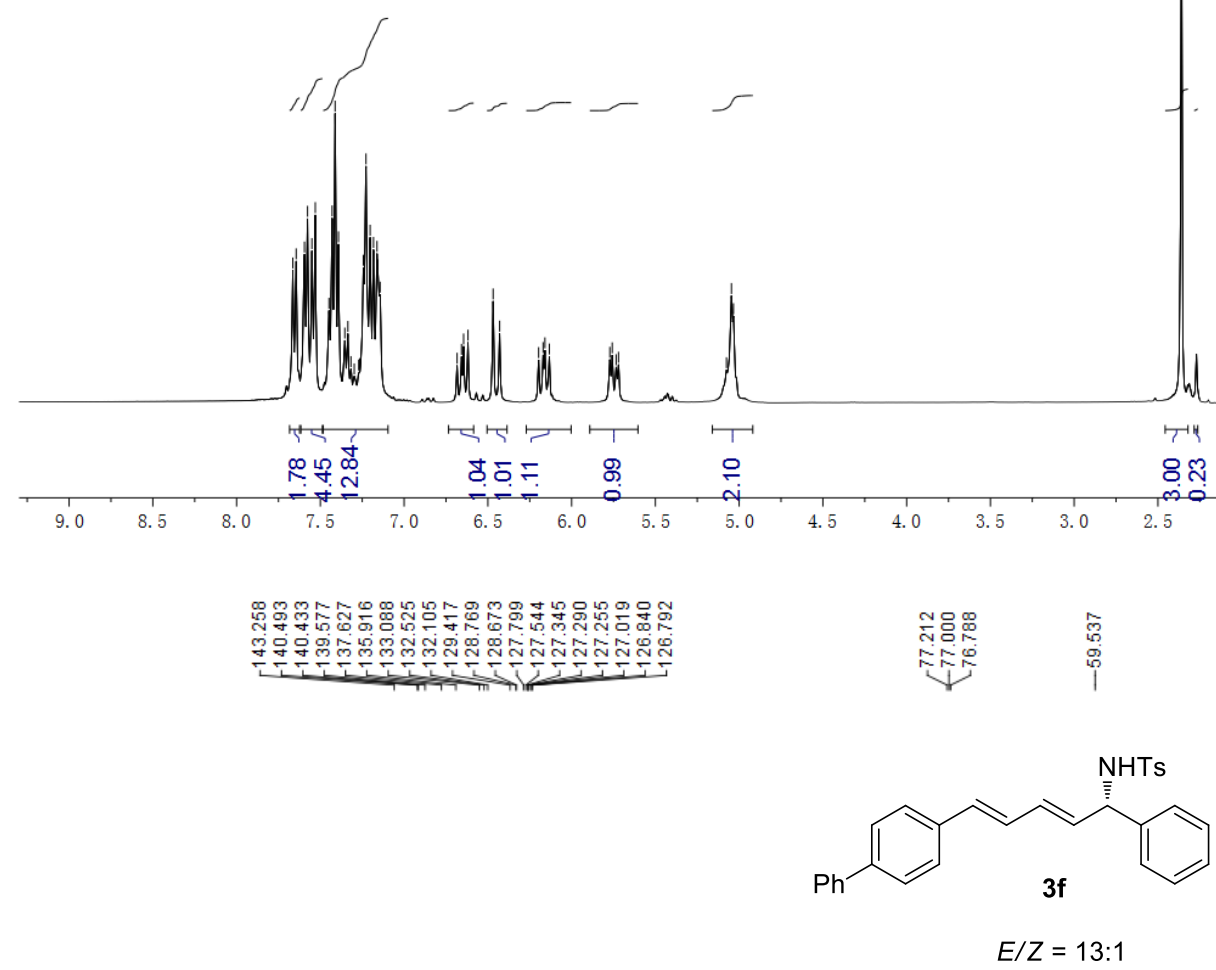

${ }^{13} \mathrm{C}$ NMR (150M, $\mathrm{CDCl}_{3}$ )

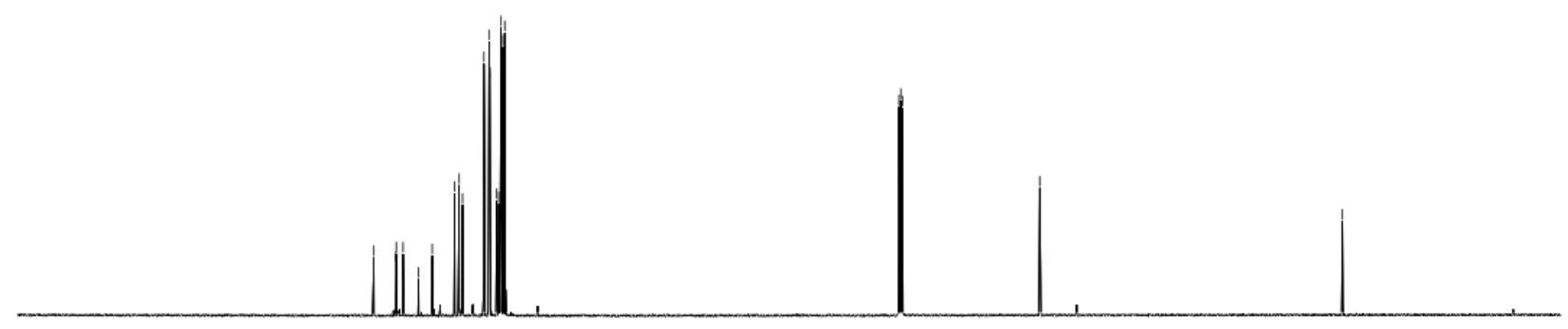



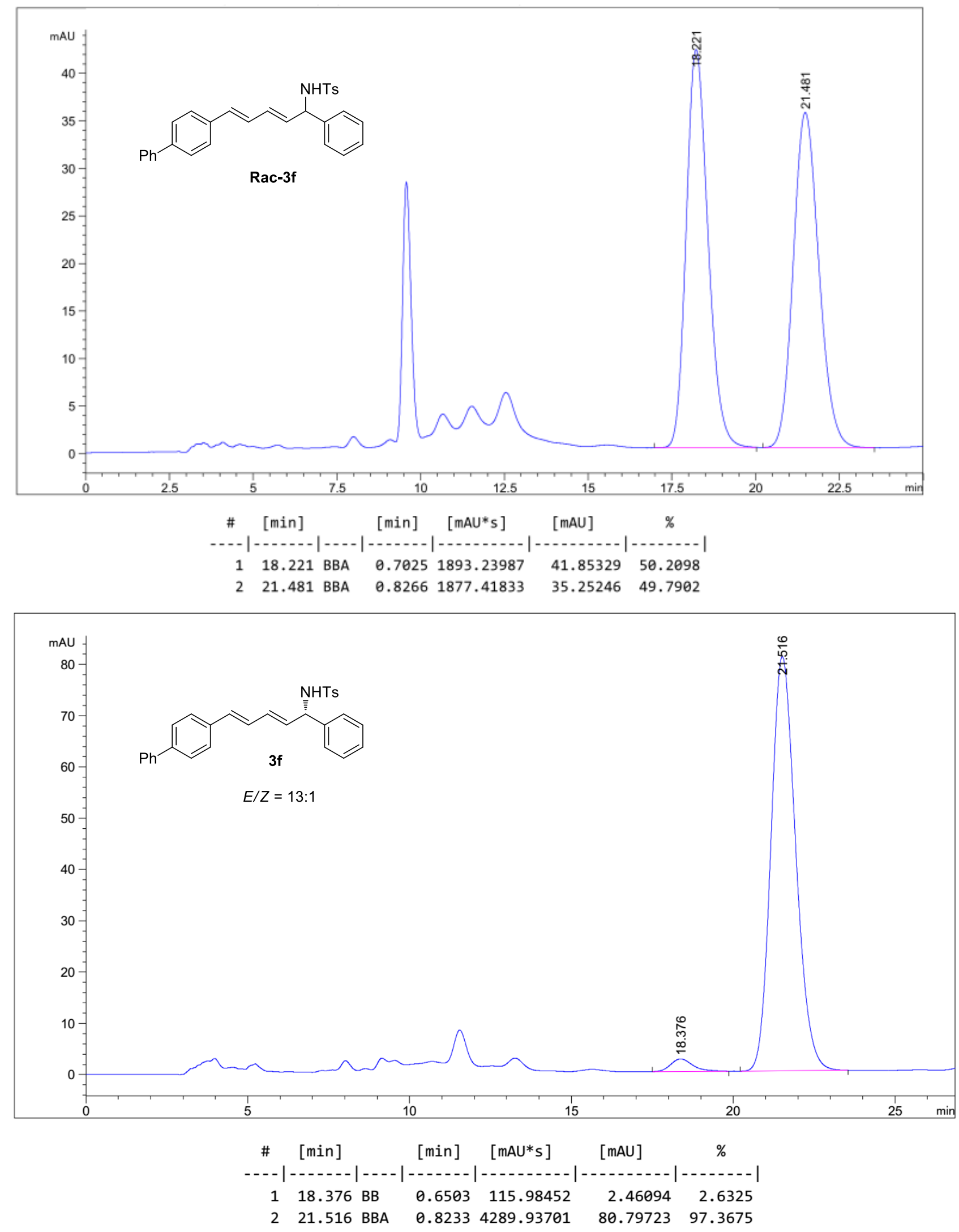


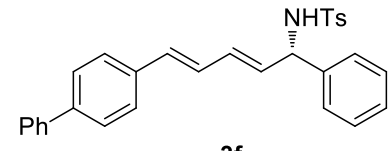

HRMS (ESI-TOF) m/z: [M + Na] $]^{+}$

Calcd for $\mathrm{C}_{30} \mathrm{H}_{27} \mathrm{NO}_{2} \mathrm{SNa} 488.1655$

489.1711

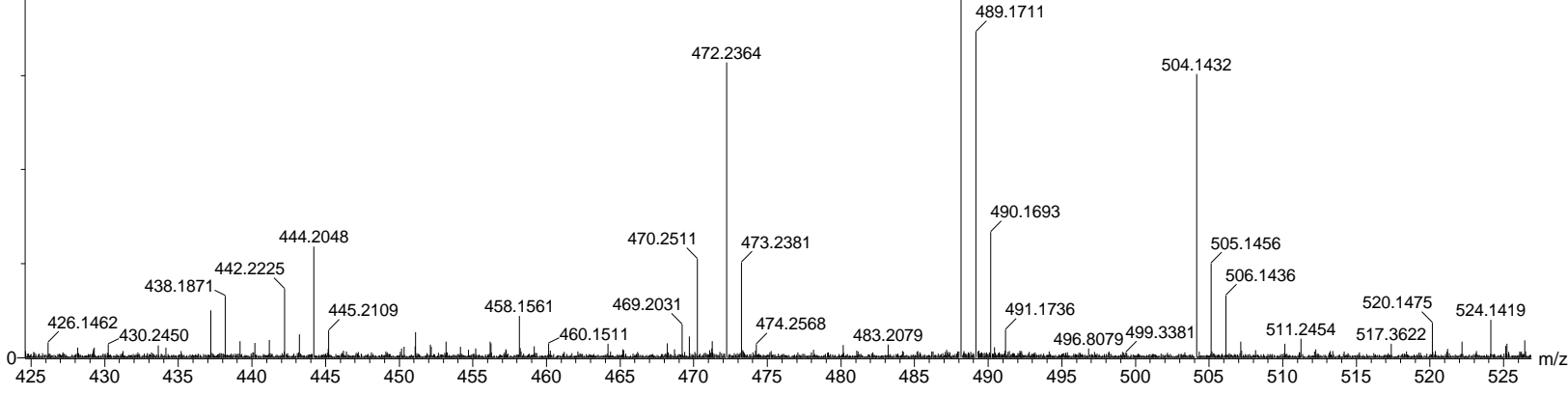




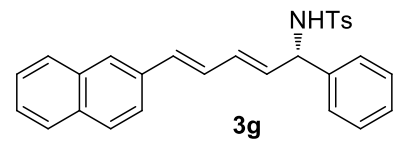

$E / Z=16: 1$

${ }^{1} \mathrm{H}$ NMR (400M, $\mathrm{CDCl}_{3}$ )

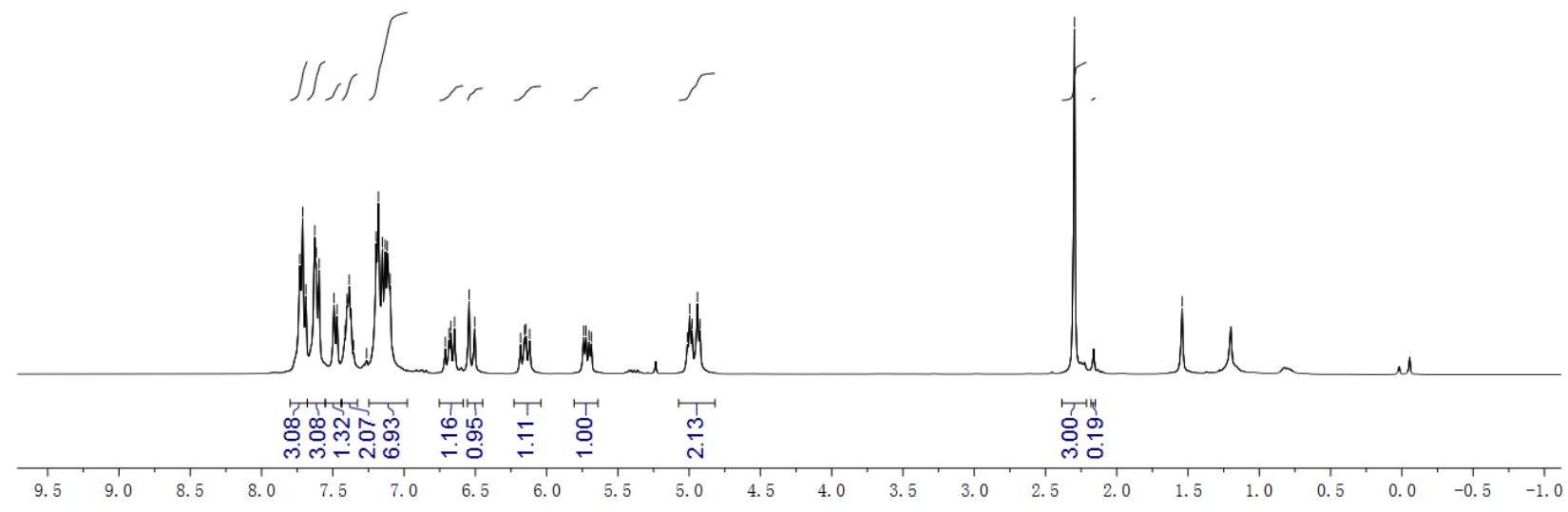

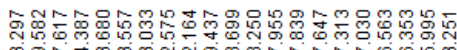

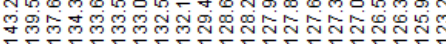

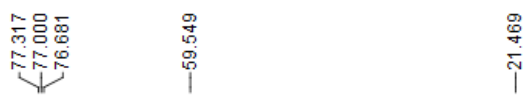

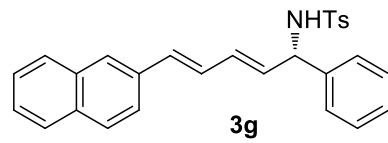

$E / Z=16: 1$

${ }^{13} \mathrm{C}$ NMR $\left(100 \mathrm{M}, \mathrm{CDCl}_{3}\right)$

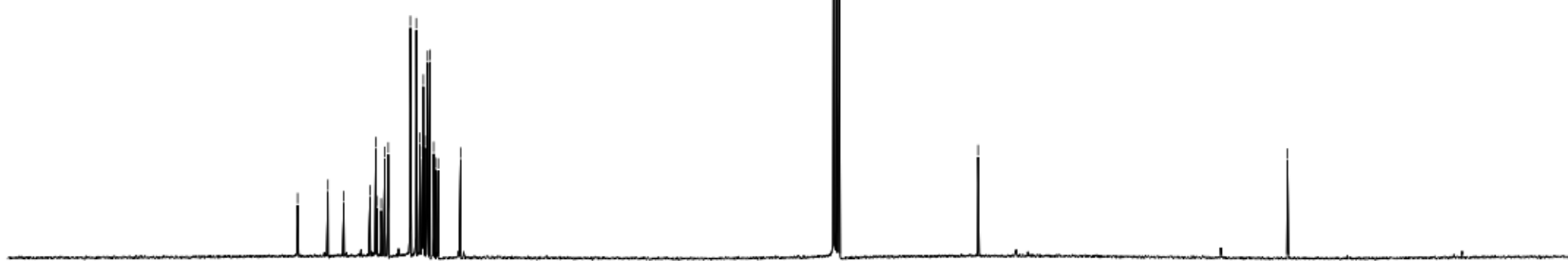

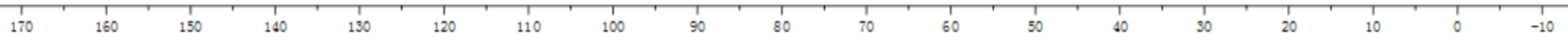



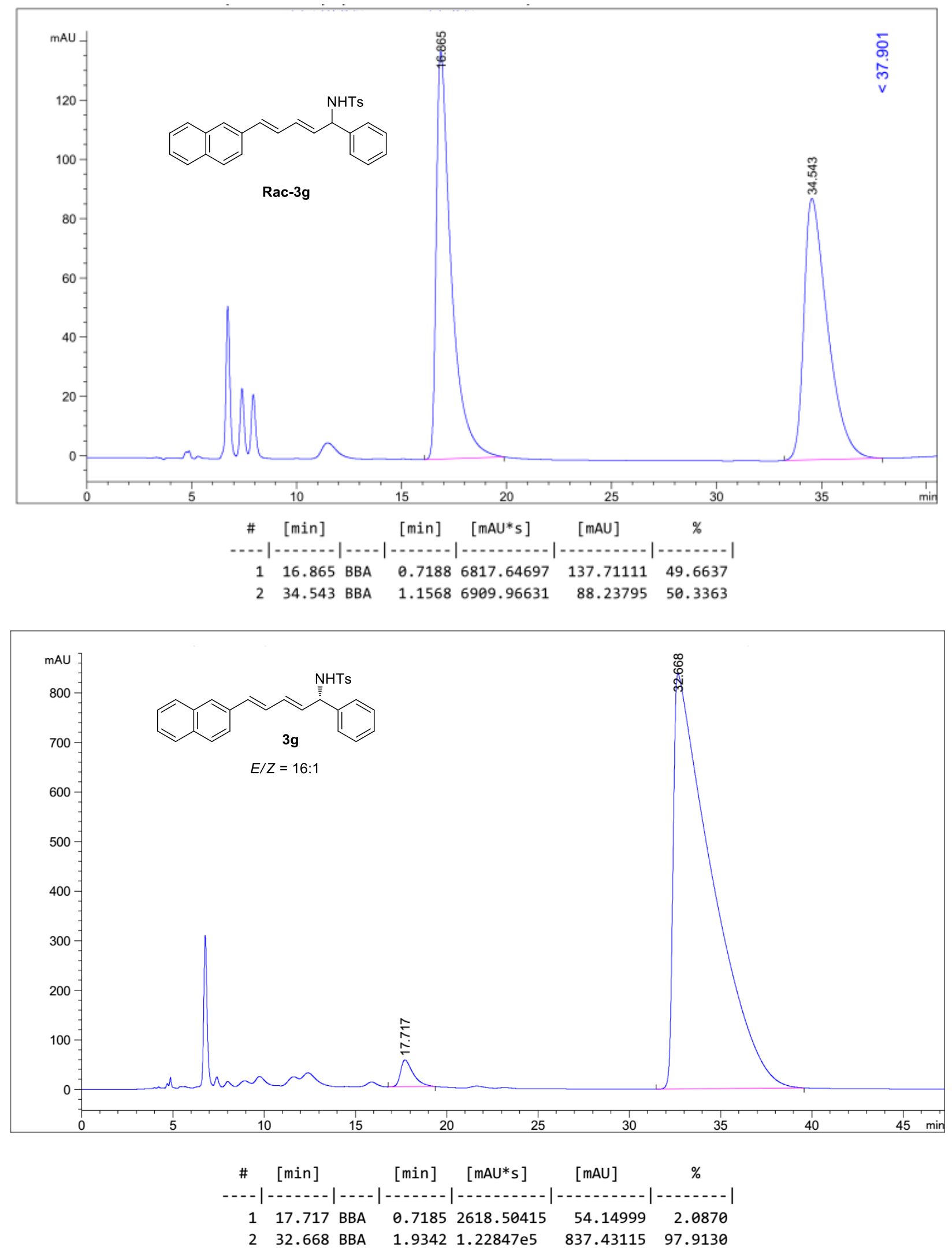
User Spectrum Plot Report

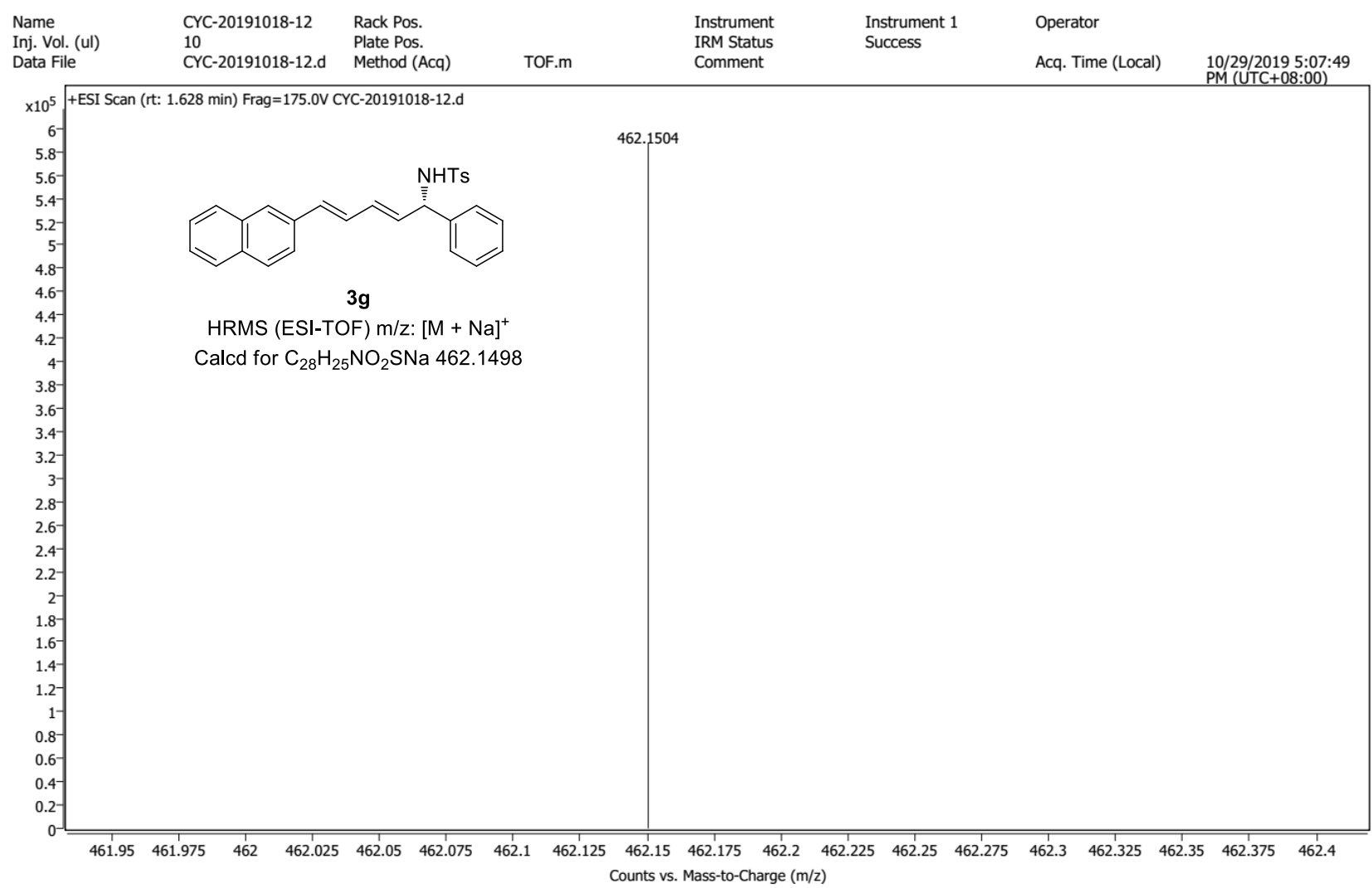



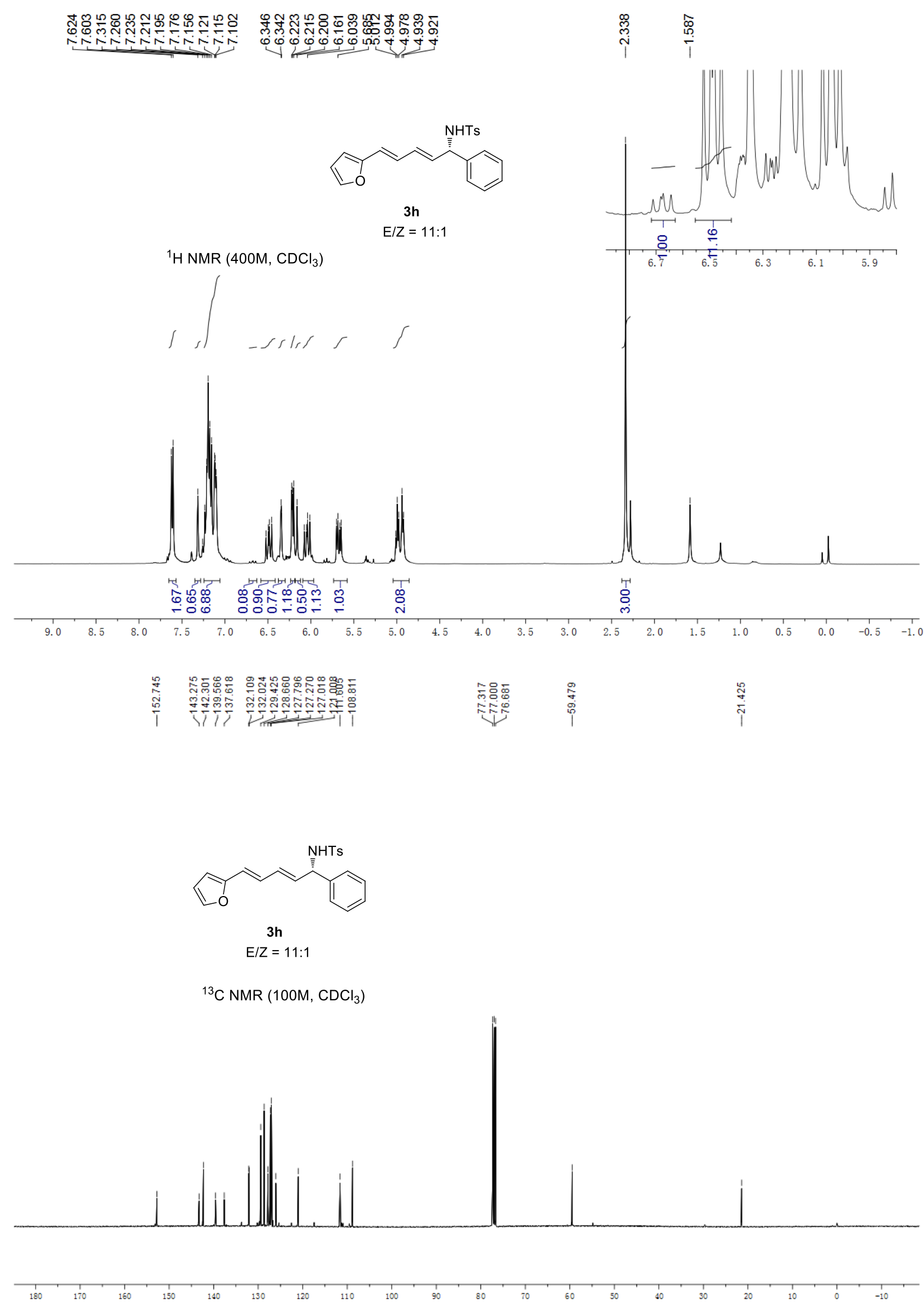

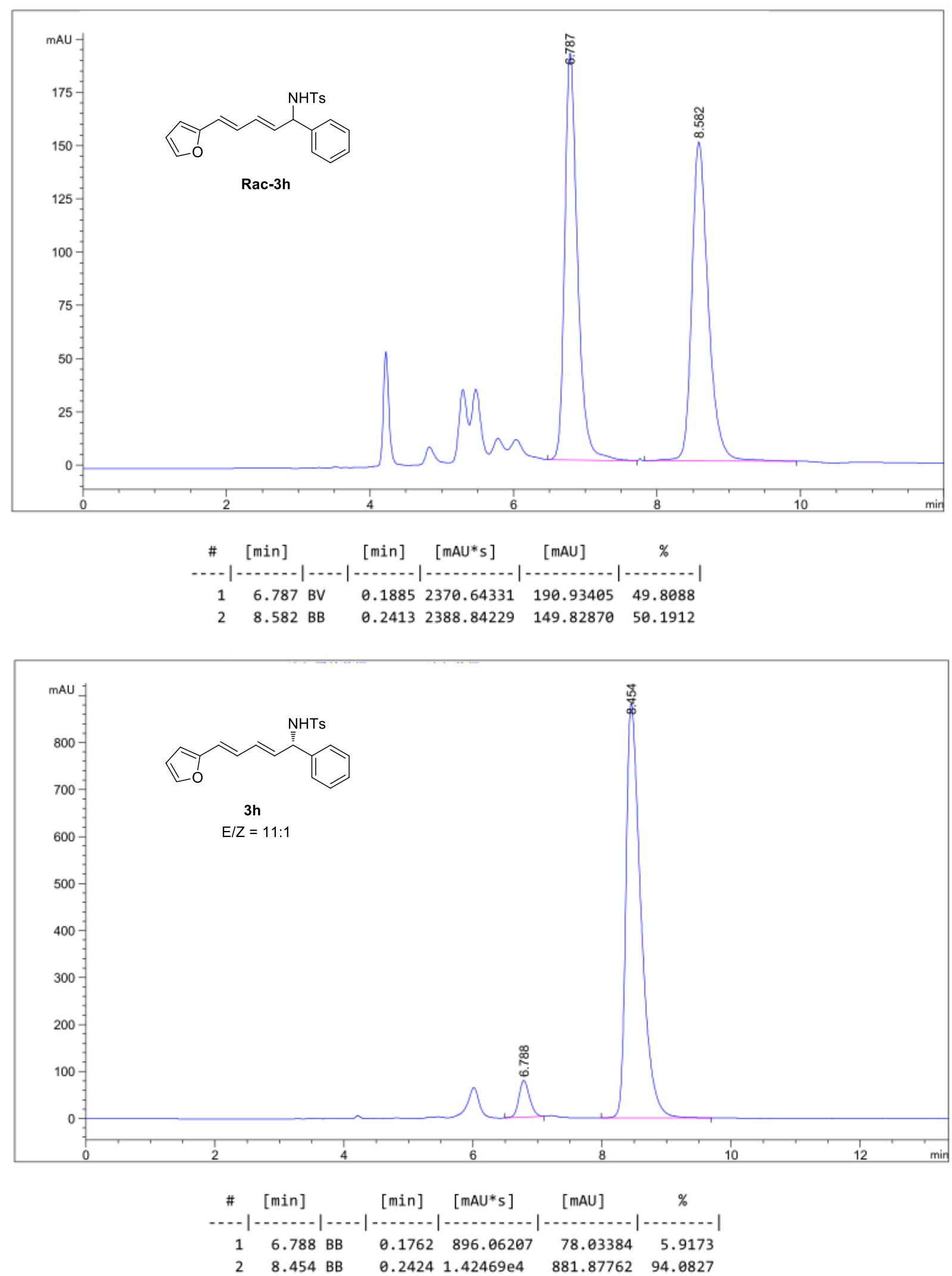
Inten. $(x 10,000,000)$

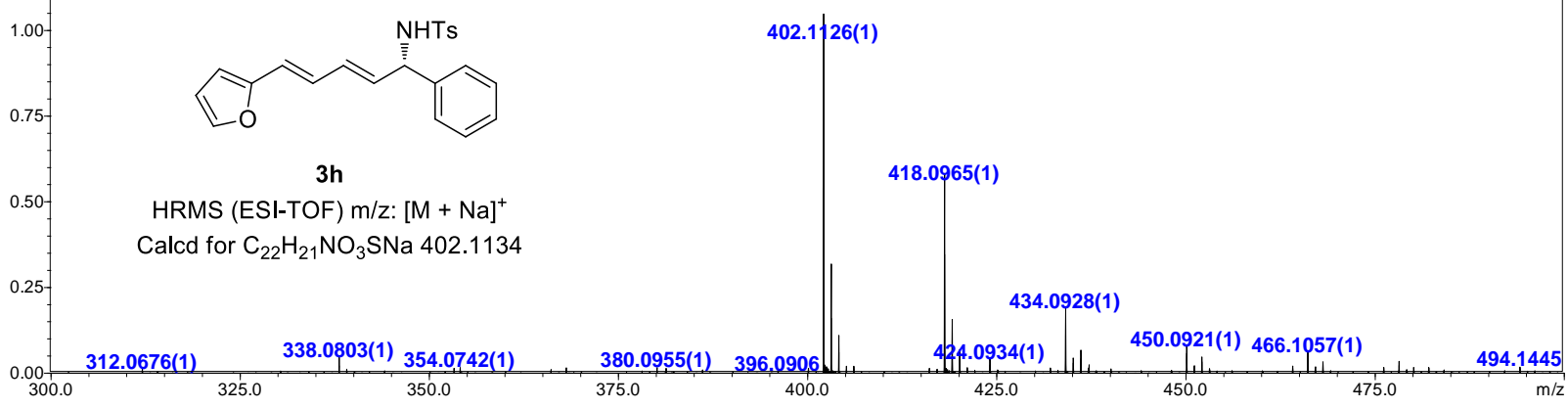




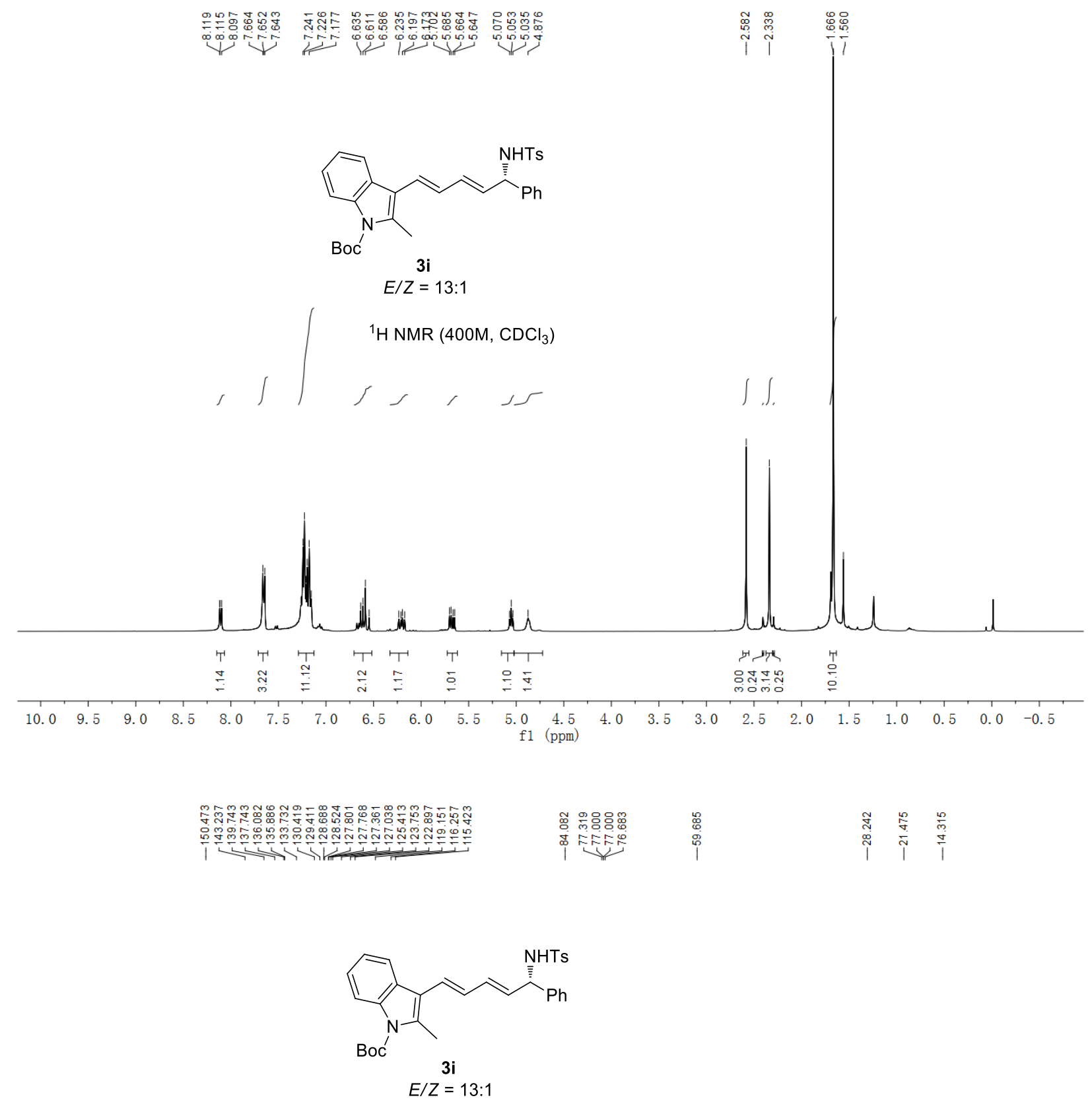

${ }^{13} \mathrm{C} \mathrm{NMR}\left(100 \mathrm{M}, \mathrm{CDCl}_{3}\right)$

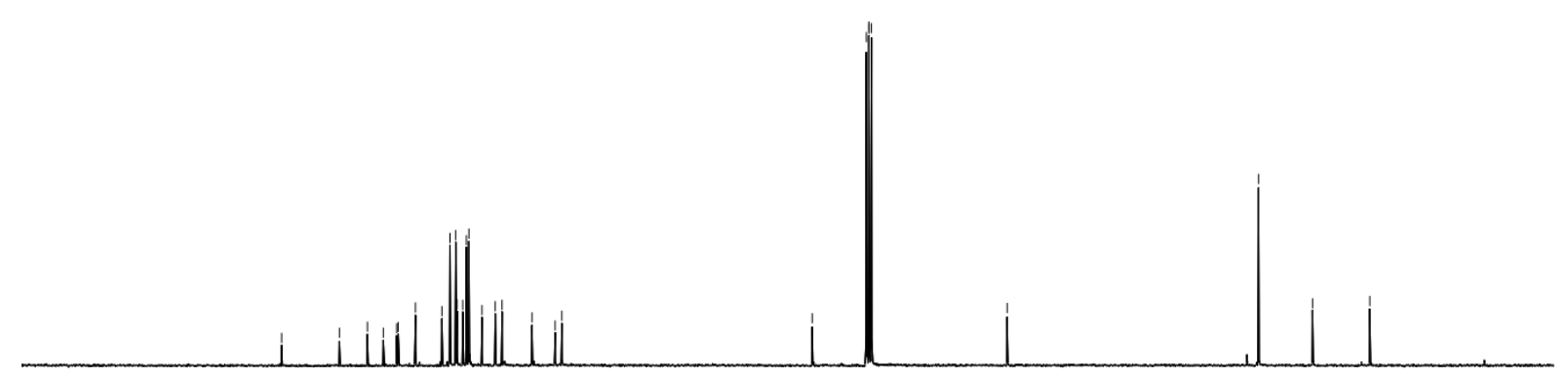

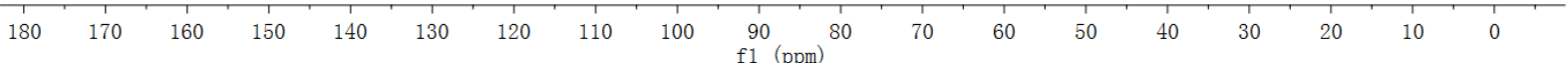




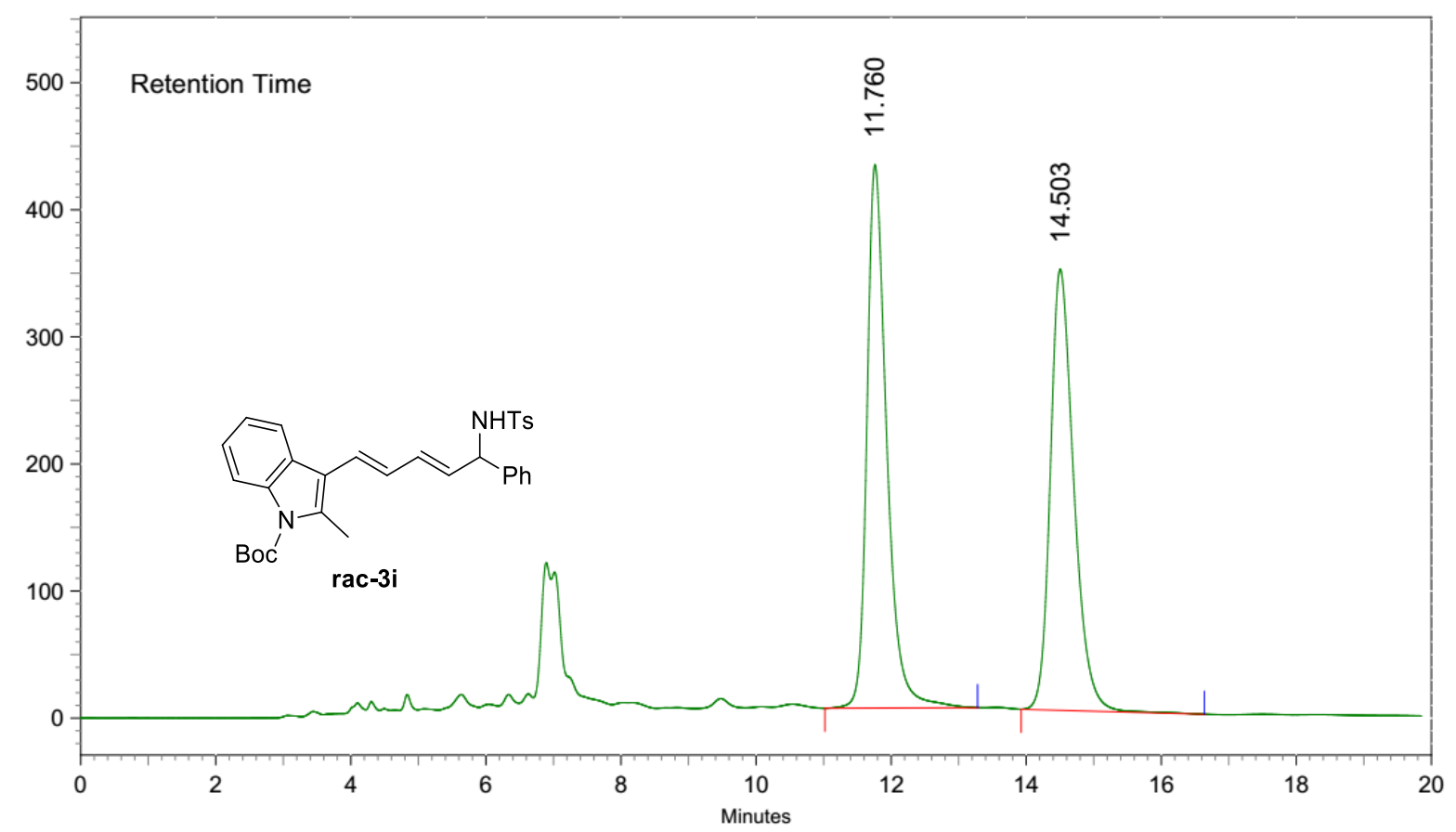

\begin{tabular}{llllll} 
Peak No. & Ret Time & Width & Height & Area & Area [\%] \\
\hline 1 & 11.760 & 2.260 & 7172799 & 146530154 & 50.9889 \\
2 & 14.503 & 2.713 & 5824031 & 140846655 & 49.0111
\end{tabular}

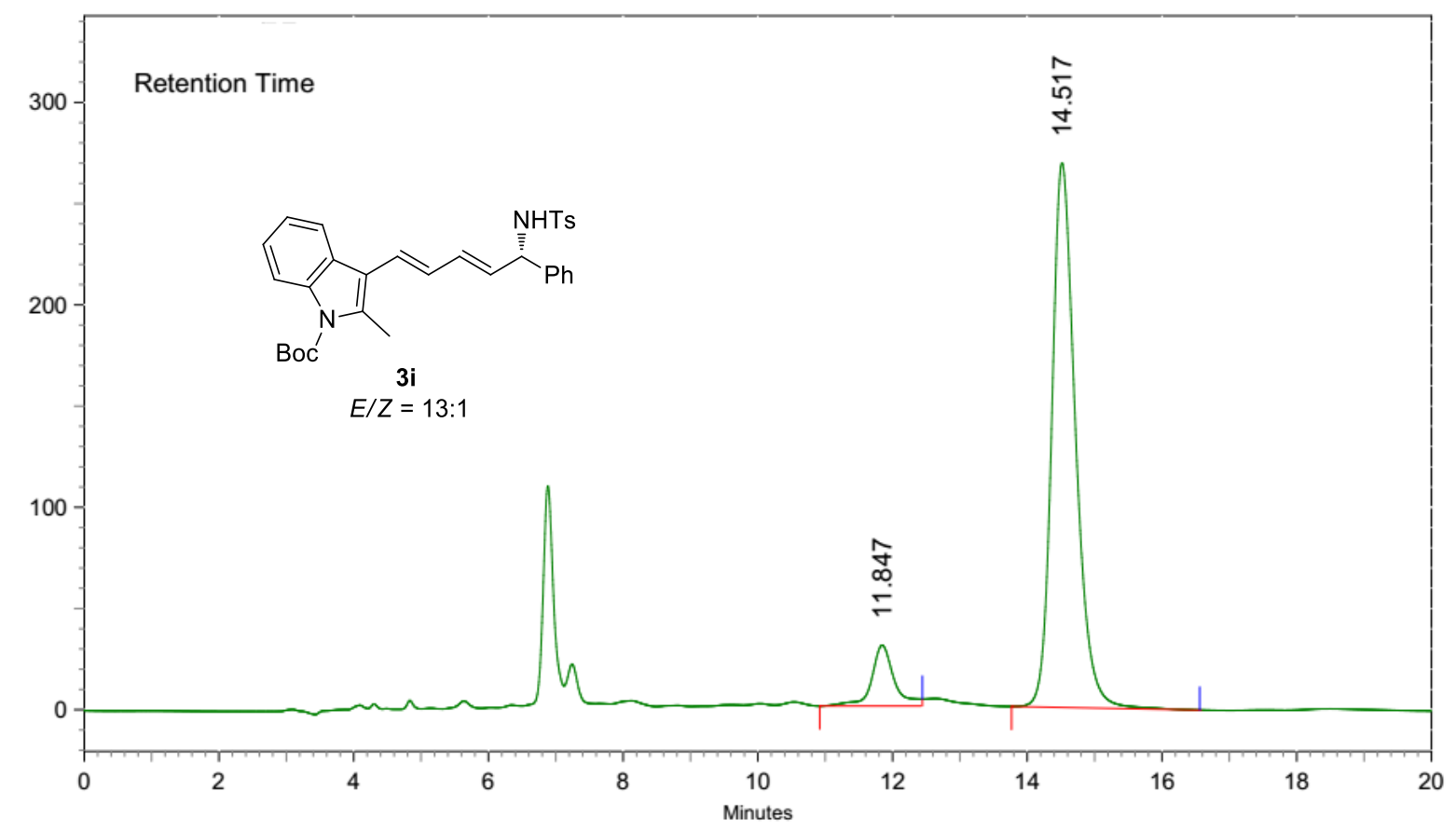

\begin{tabular}{llllll} 
Peak No. & Ret Time & Width & Height & Area & Area [\%] \\
\hline 1 & 11.847 & 1.523 & 504299 & 11766513 & 9.6591 \\
2 & 14.517 & 2.793 & 4513886 & 110051901 & 90.3409
\end{tabular}



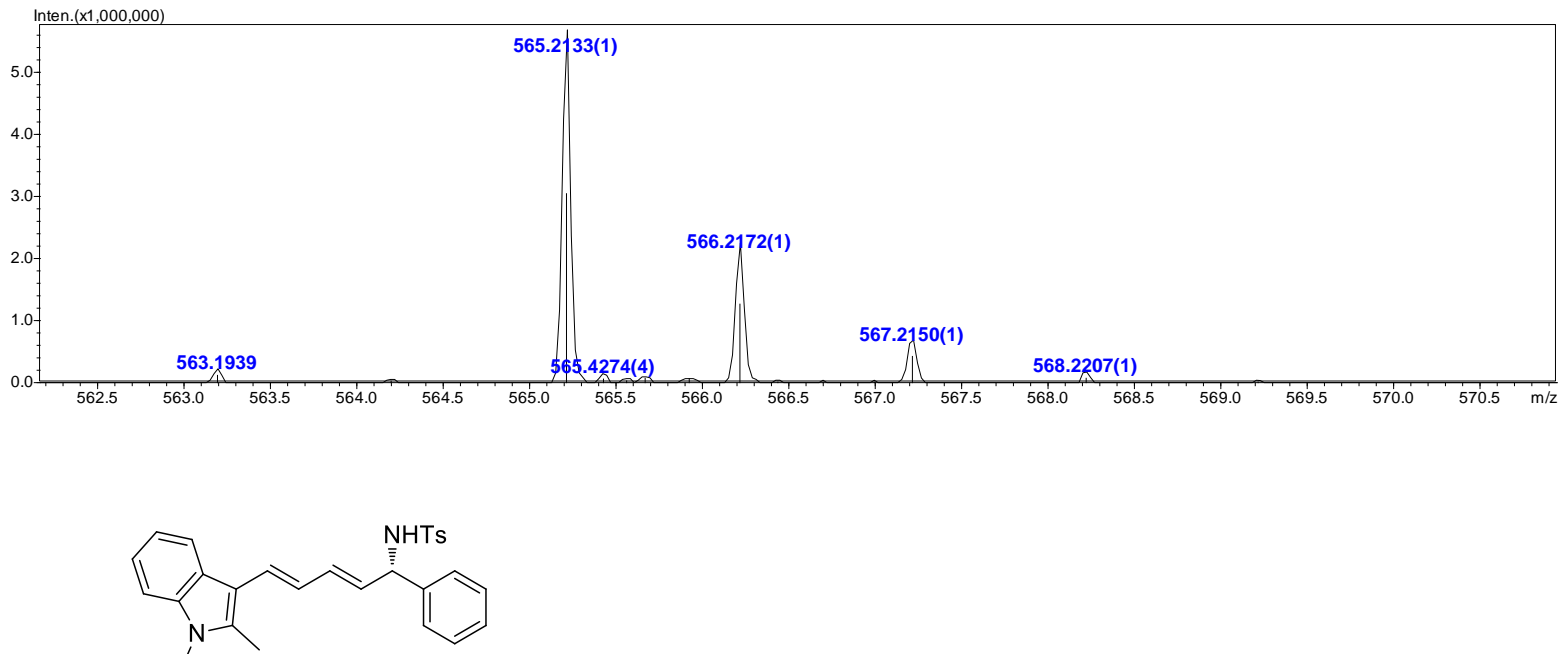

$3 i$

HRMS (ESI-TOF) m/z: [M + Na] ${ }^{+}$

Calcd for $\mathrm{C}_{32} \mathrm{H}_{34} \mathrm{~N}_{2} \mathrm{O}_{4} \mathrm{SNa} 565.2131$ 


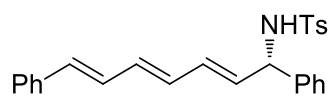

$$
\begin{aligned}
& 3 \mathbf{j} \\
& E / Z \\
& =
\end{aligned}
$$
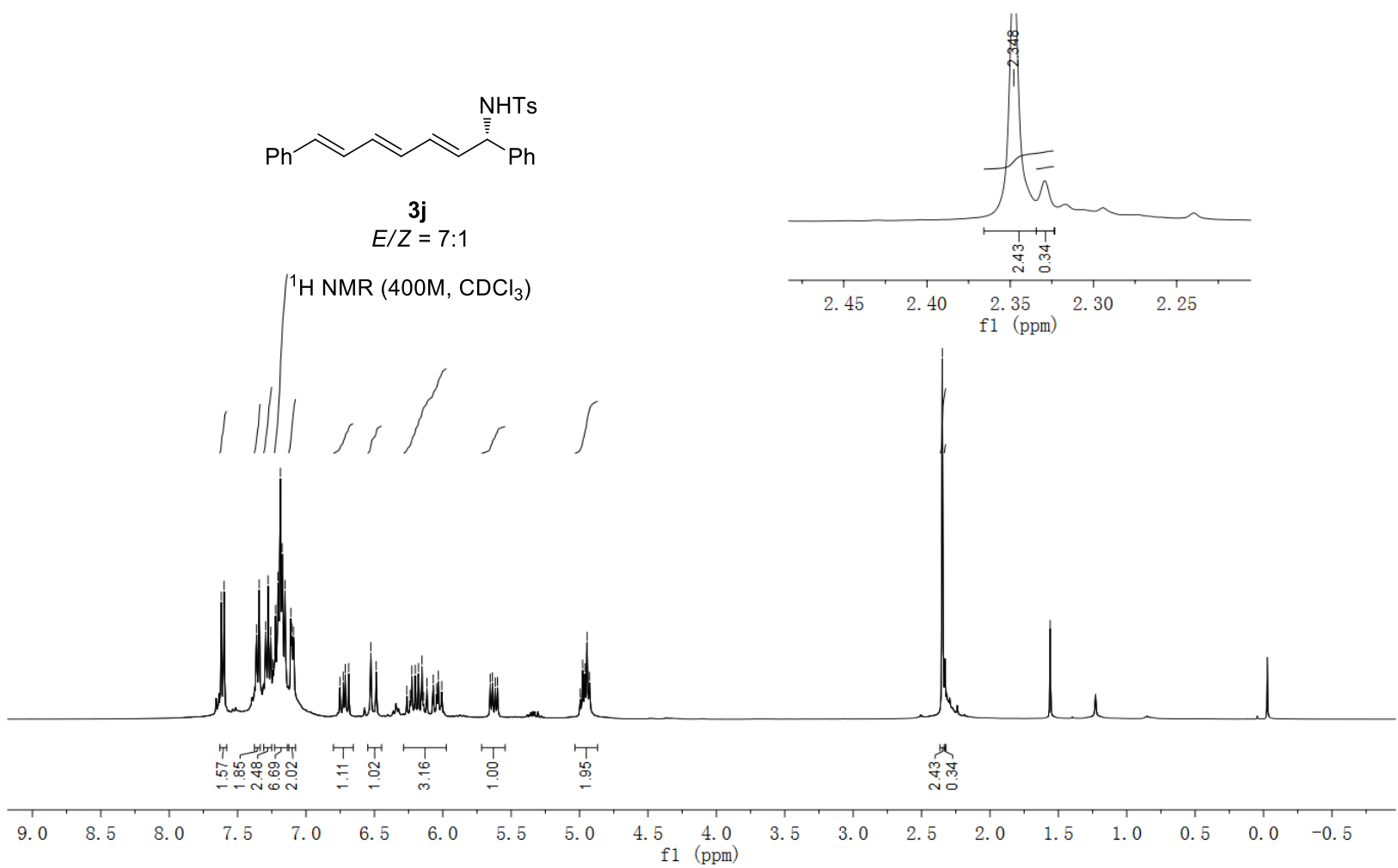

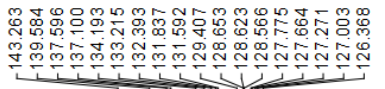

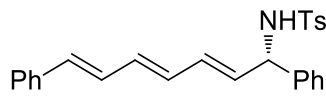

3j

$E / Z=7: 1$

${ }^{13} \mathrm{C}$ NMR $\left(100 \mathrm{M}, \mathrm{CDCl}_{3}\right)$
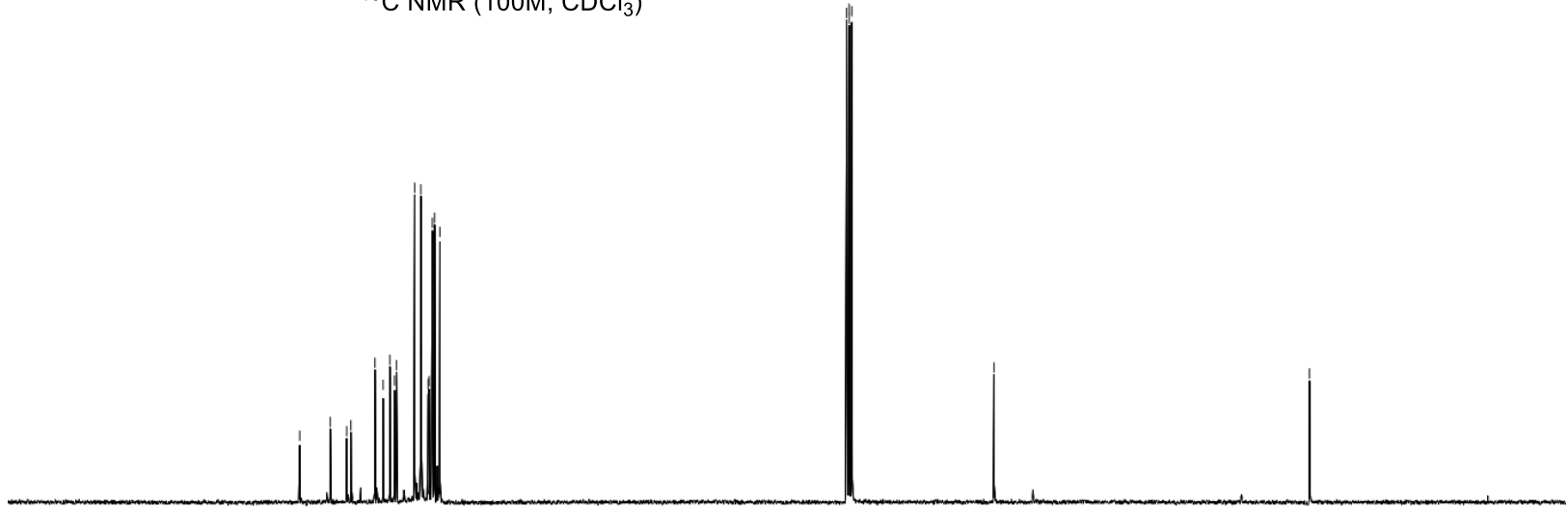

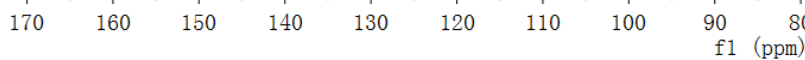




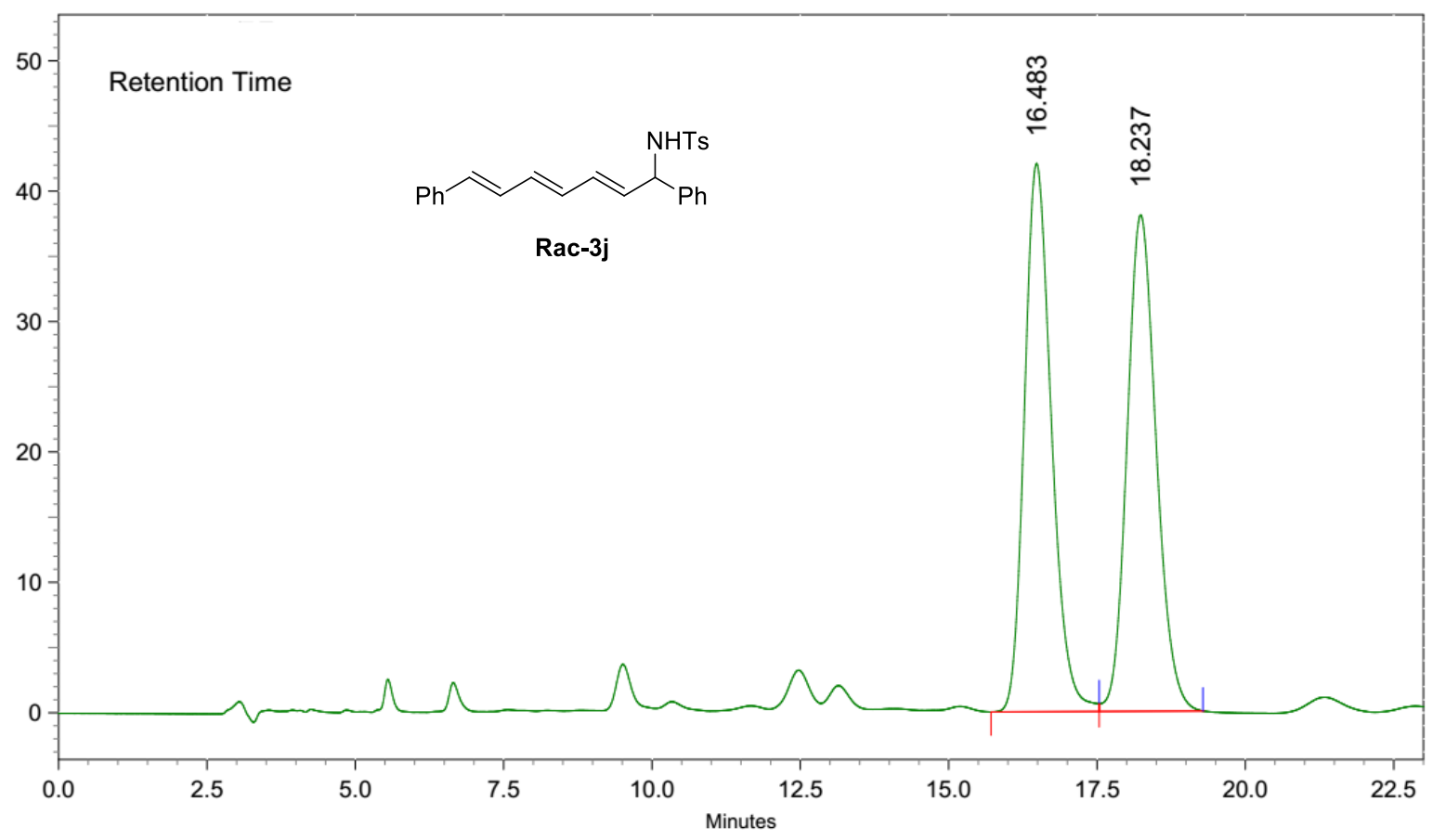

\begin{tabular}{llllll} 
Peak No. & Ret Time & Width & Height & Area & Area [\%] \\
\hline 1 & 16.483 & 1.823 & 705383 & 22337810 & 50.5213 \\
2 & 18.237 & 1.750 & 638762 & 21876804 & 49.4787
\end{tabular}

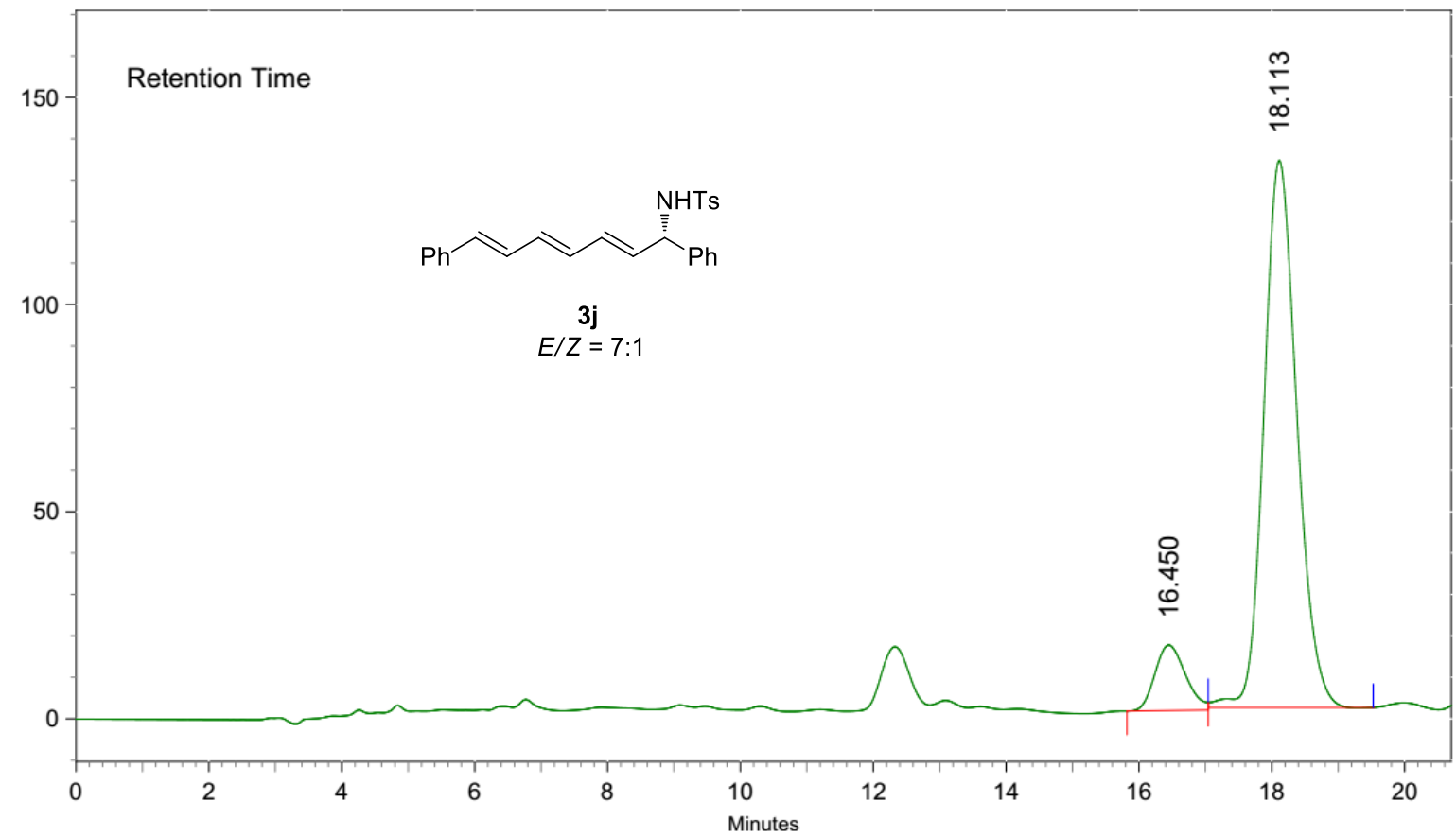

\begin{tabular}{llllll} 
Peak No. & Ret Time & Width & Height & Area & Area [\%] \\
\hline 1 & 16.450 & 1.223 & 265317 & 8471505 & 9.9412 \\
2 & 18.113 & 2.484 & 2216439 & 76744247 & 90.0588
\end{tabular}




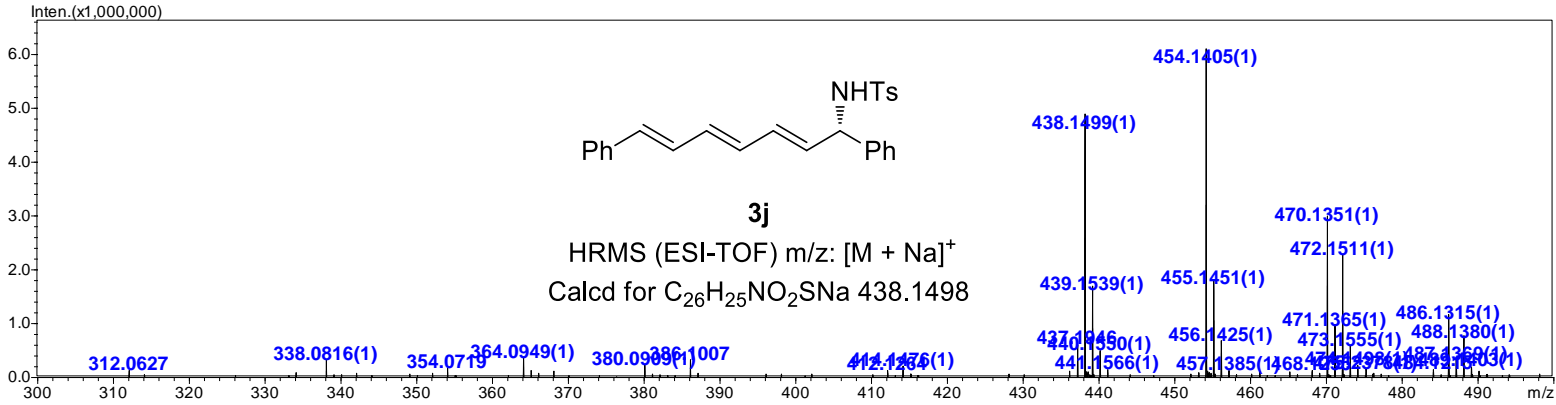




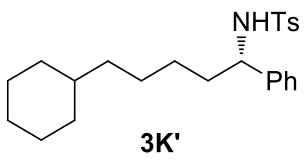

${ }^{1} \mathrm{H}$ NMR (400M, $\mathrm{CDCl}_{3}$ )

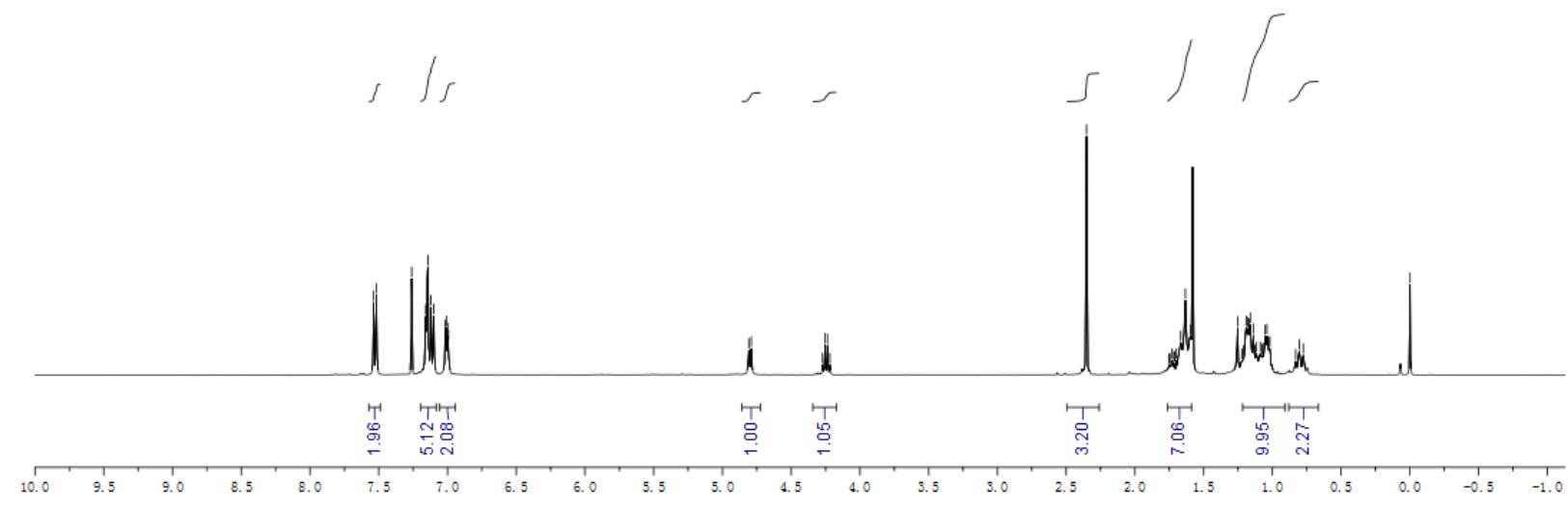

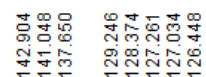

溇选
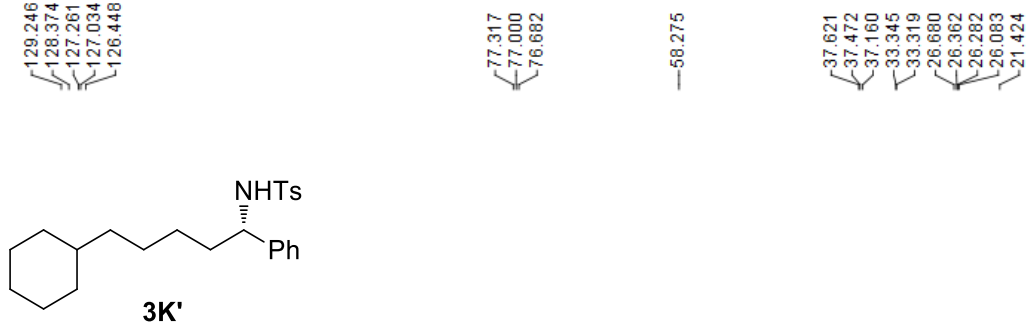

${ }^{13} \mathrm{C}$ NMR (100M, $\mathrm{CDCl}_{3}$ )

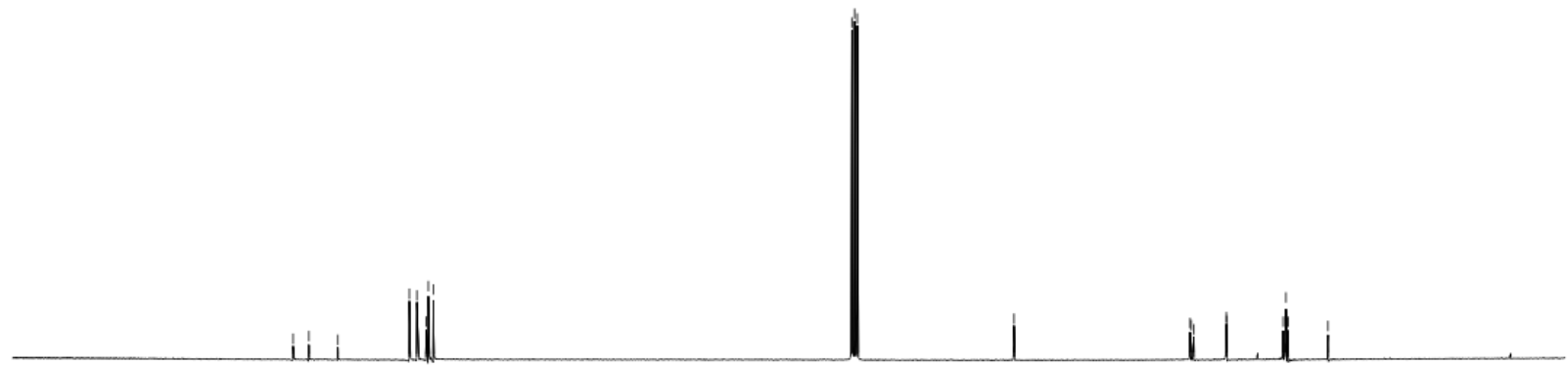




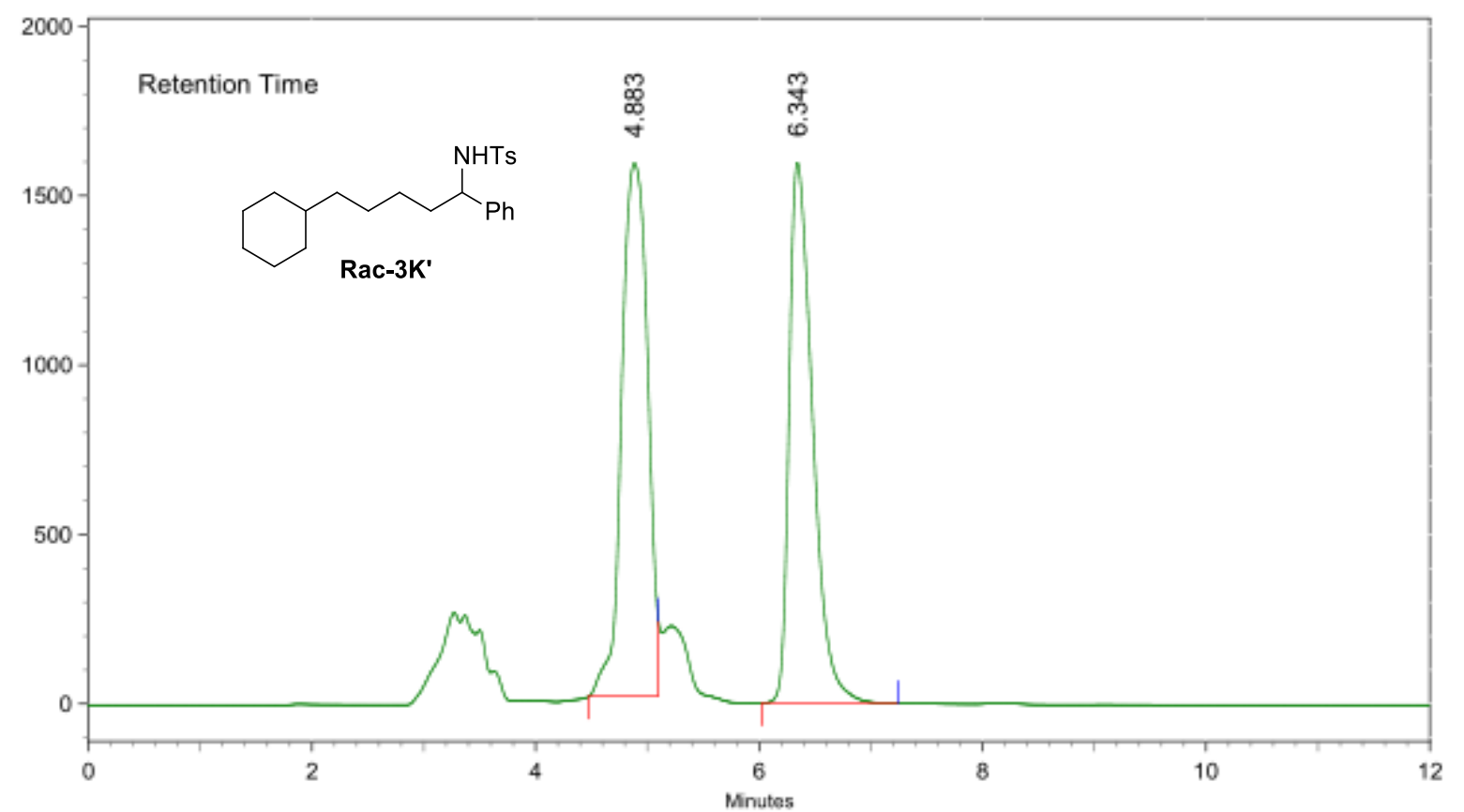

\begin{tabular}{llllll} 
Peak No. & Ret Time & Width & Height & Area & Area [\%] \\
\hline 1 & 4.883 & 0.620 & 26375412 & 426718524 & 52.1314 \\
2 & 6.343 & 1.217 & 26760734 & 391826009 & 47.8686
\end{tabular}

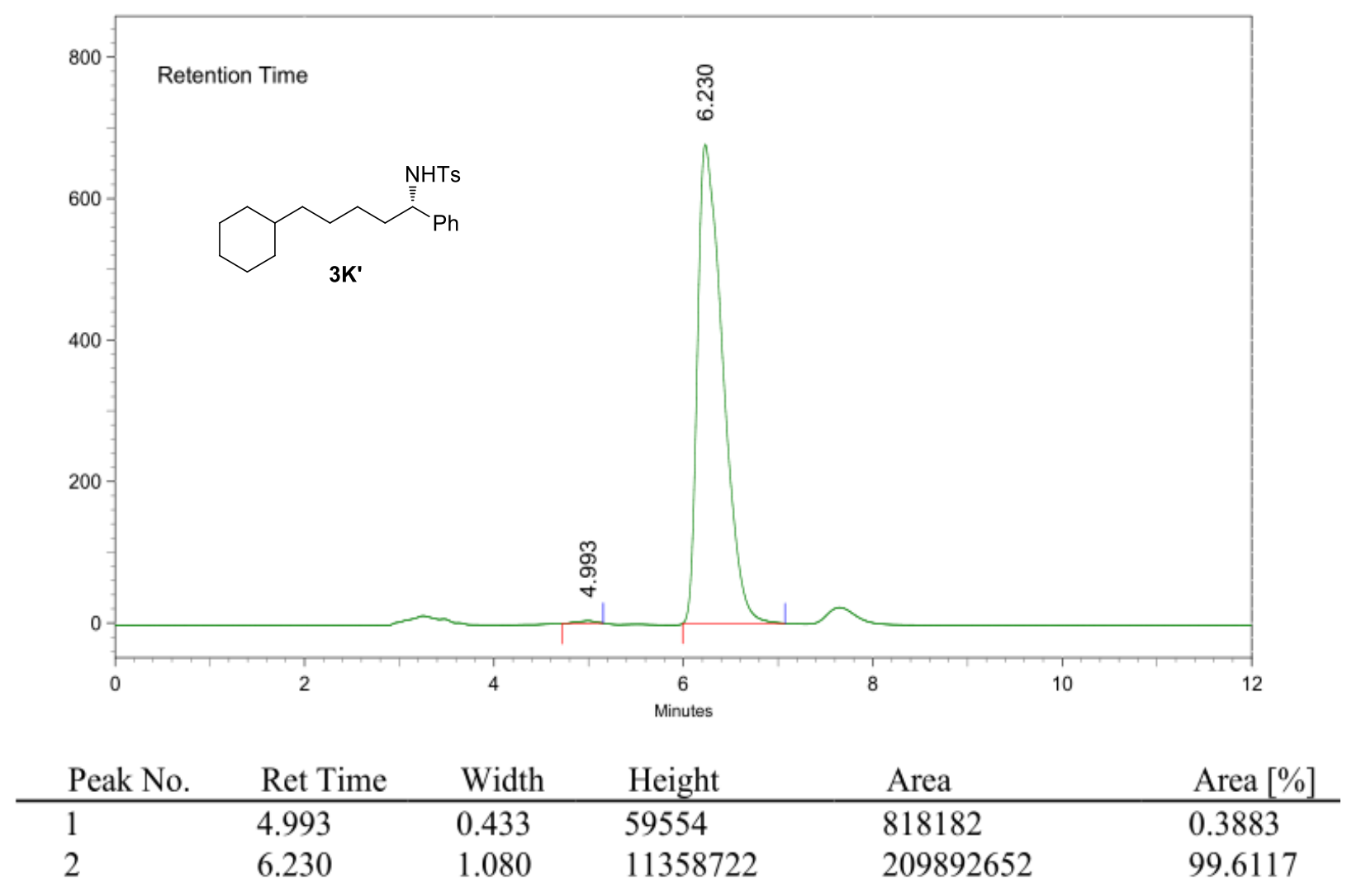




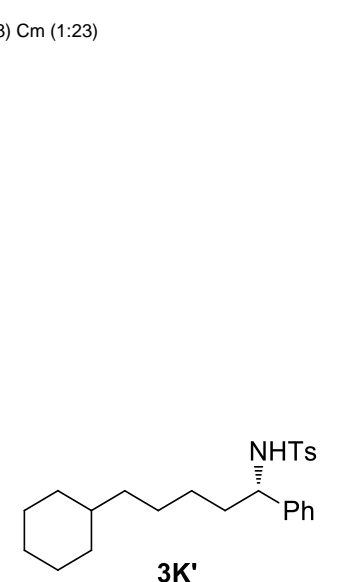

$$
\text { 3K' }
$$

HRMS (ESI-TOF) m/z: [M + Na] ${ }^{+}$ Calcd for $\mathrm{C}_{24} \mathrm{H}_{33} \mathrm{NO}_{2} \mathrm{SNa} 422.2122$

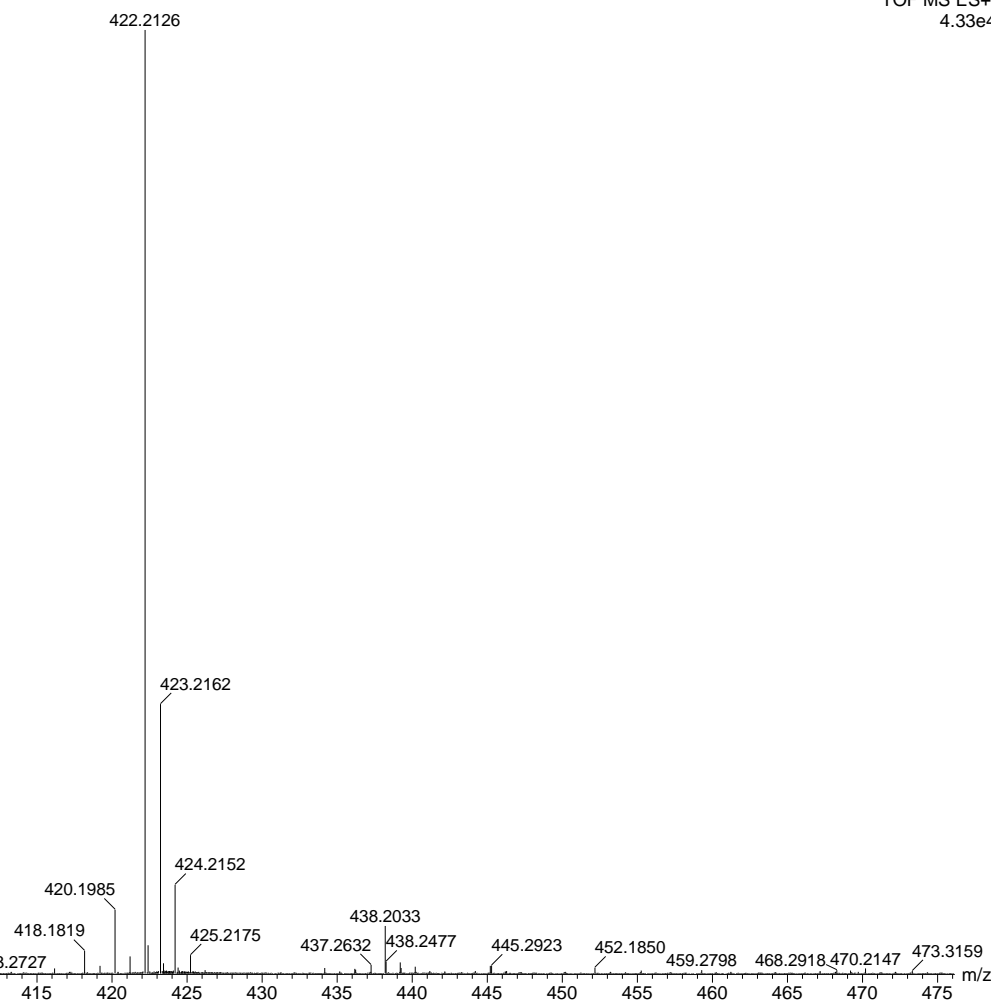




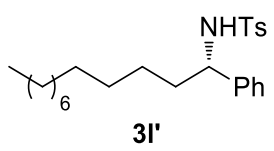

${ }^{1} \mathrm{H}$ NMR (400M, $\mathrm{CDCl}_{3}$ )
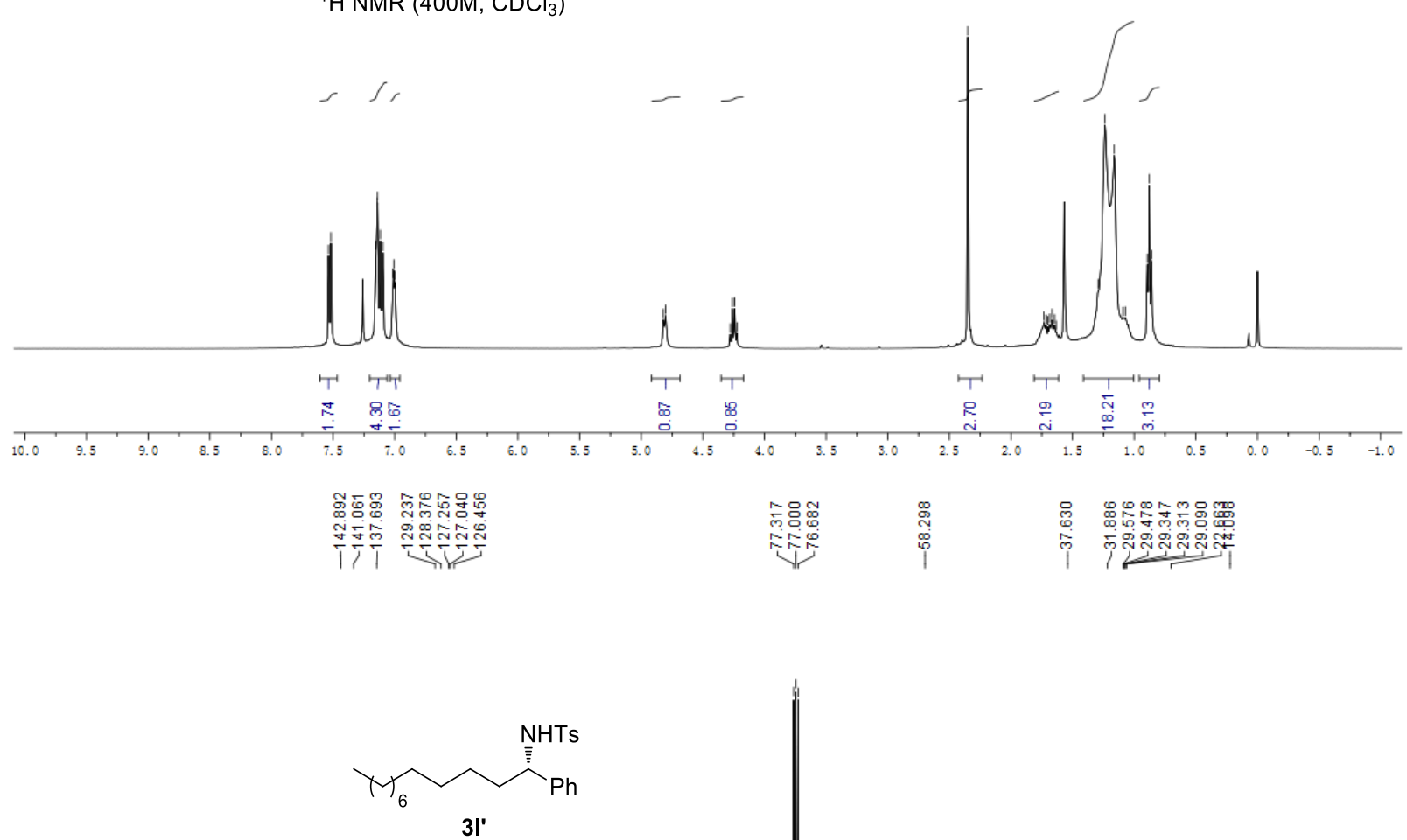

${ }^{13} \mathrm{C}$ NMR (100M, $\left.\mathrm{CDCl}_{3}\right)$

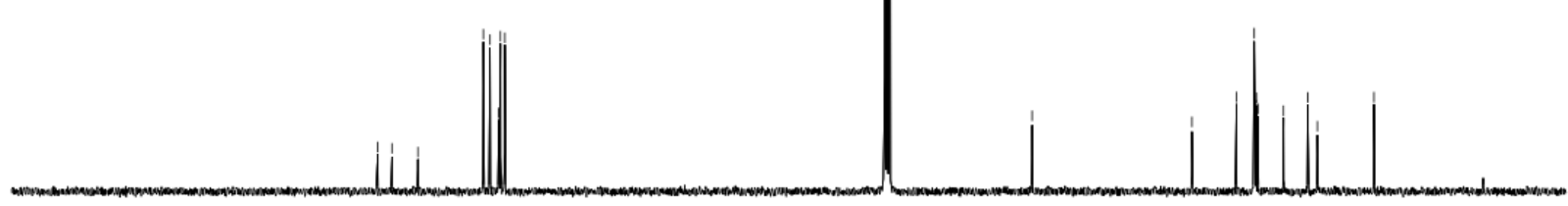




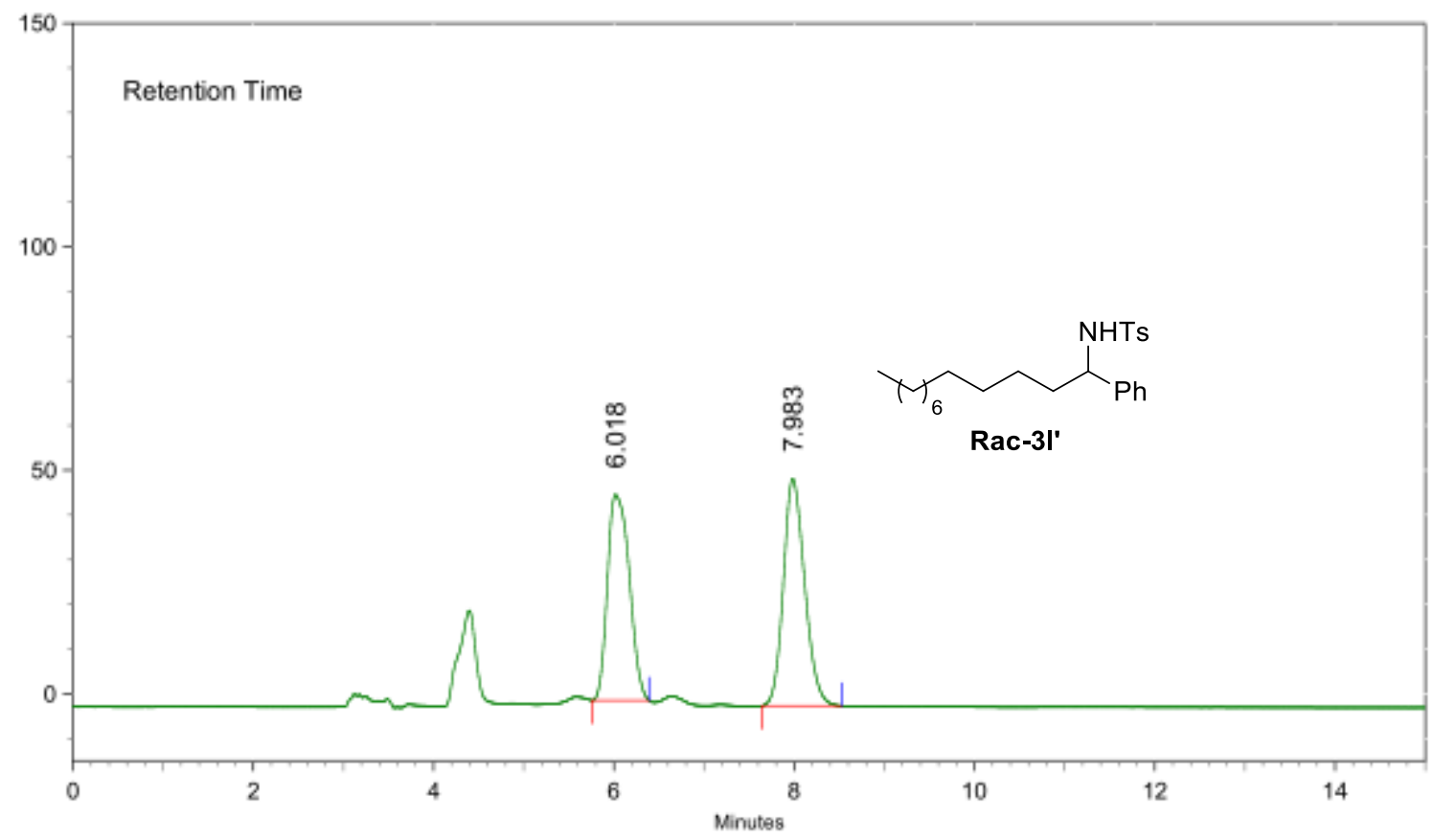

\begin{tabular}{llllll} 
Peak No. & Ret Time & Width & Height & Area & Area [\%] \\
\hline 1 & 6.018 & 0.633 & 771691 & 13006182 & 48.1100 \\
2 & 7.983 & 0.883 & 851808 & 14028089 & 51.8900
\end{tabular}

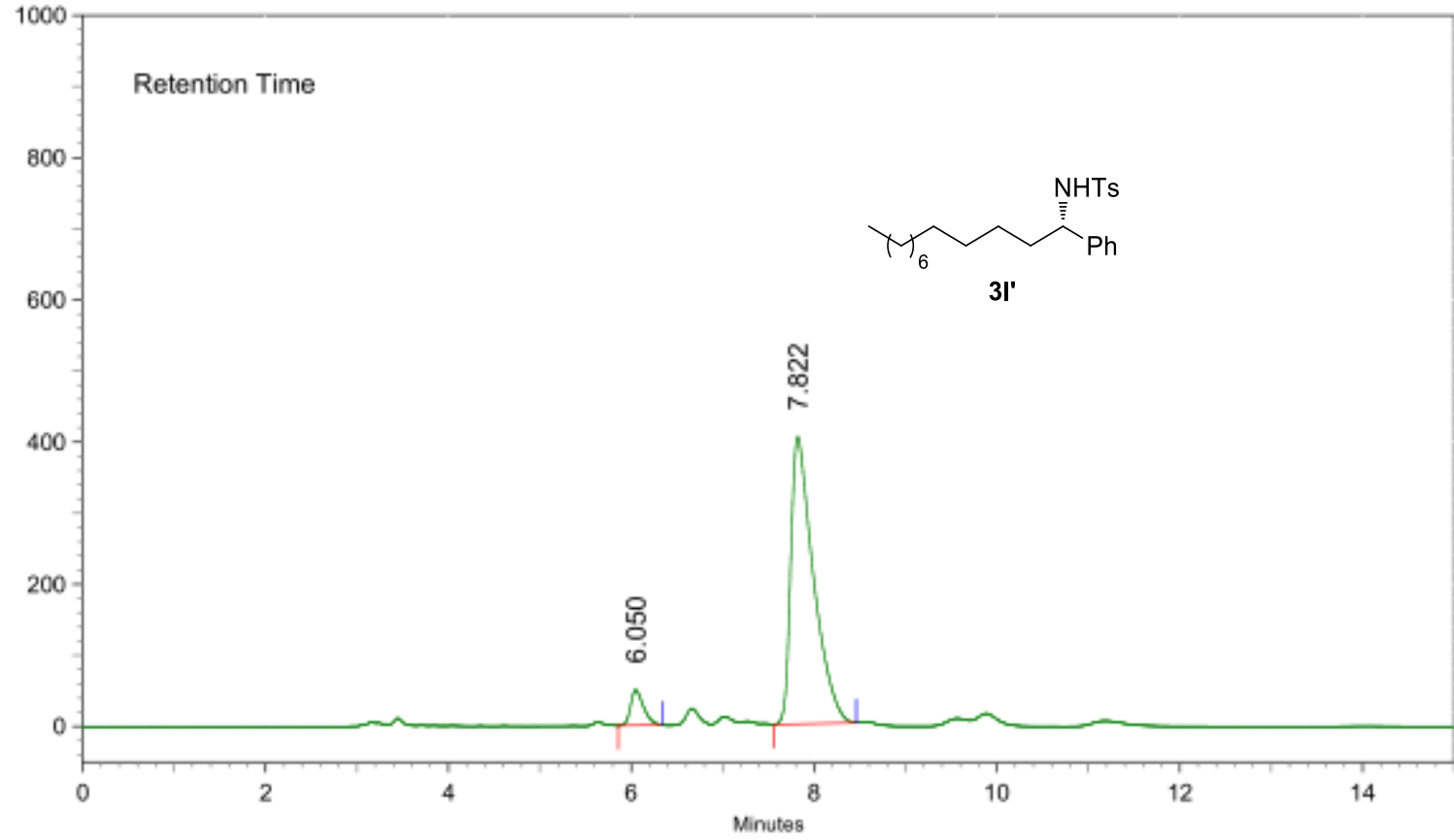

\begin{tabular}{llllll} 
Peak No. & Ret Time & Width & Height & Area & Area [\%] \\
\hline 1 & 6.050 & 0.480 & 832862 & 8281674 & 6.6297 \\
2 & 7.822 & 0.898 & 6765911 & 116635571 & 93.3703
\end{tabular}




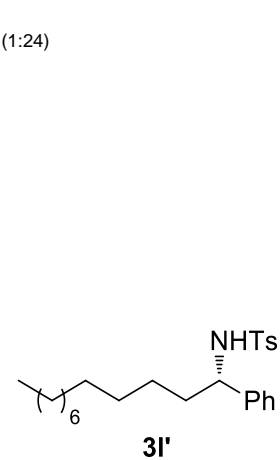

$$
3 I^{\prime}
$$

HRMS (ESI-TOF) m/z: [M + Na] ${ }^{+}$

Calcd for $\mathrm{C}_{25} \mathrm{H}_{37} \mathrm{NO}_{2} \mathrm{SNa} 438.2437$

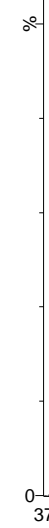

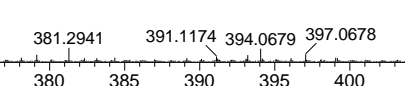

440.2437

439.2469

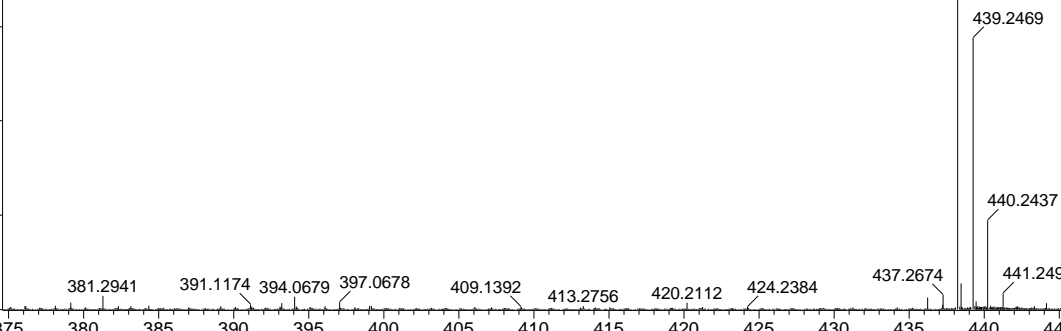

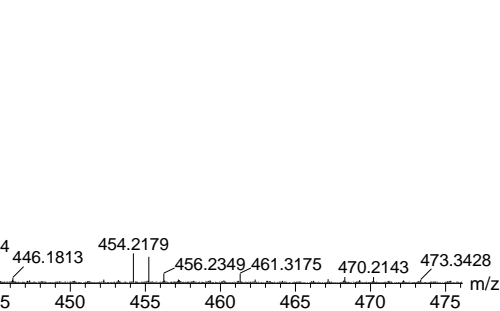




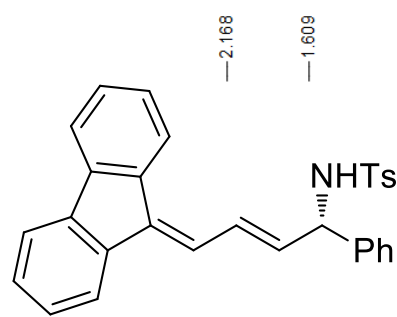

$3 \mathrm{~m}$

$E / Z=15: 1$

${ }^{1} \mathrm{H}$ NMR (400M, $\mathrm{CDCl}_{3}$ )

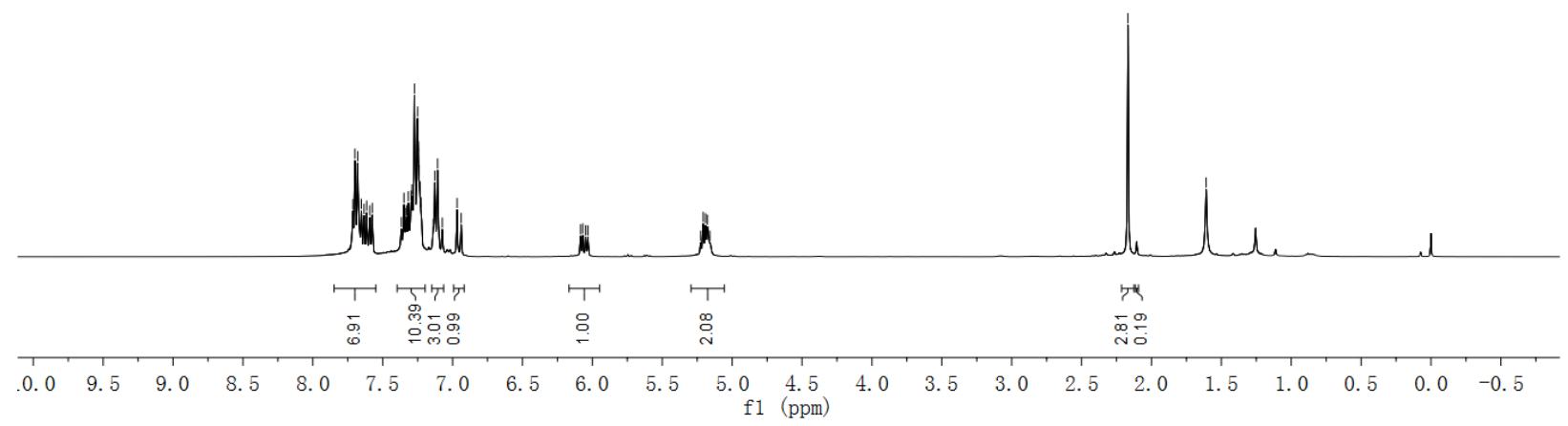

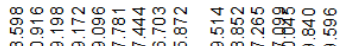

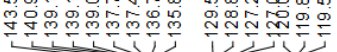

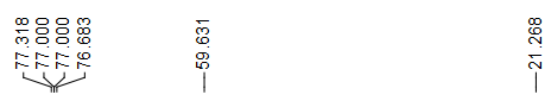

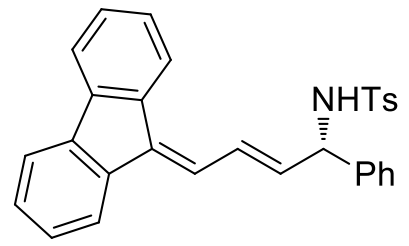

$3 \mathrm{~m}$

$E / Z=15: 1$

${ }^{13} \mathrm{C}$ NMR (100M, $\mathrm{CDCl}_{3}$ )

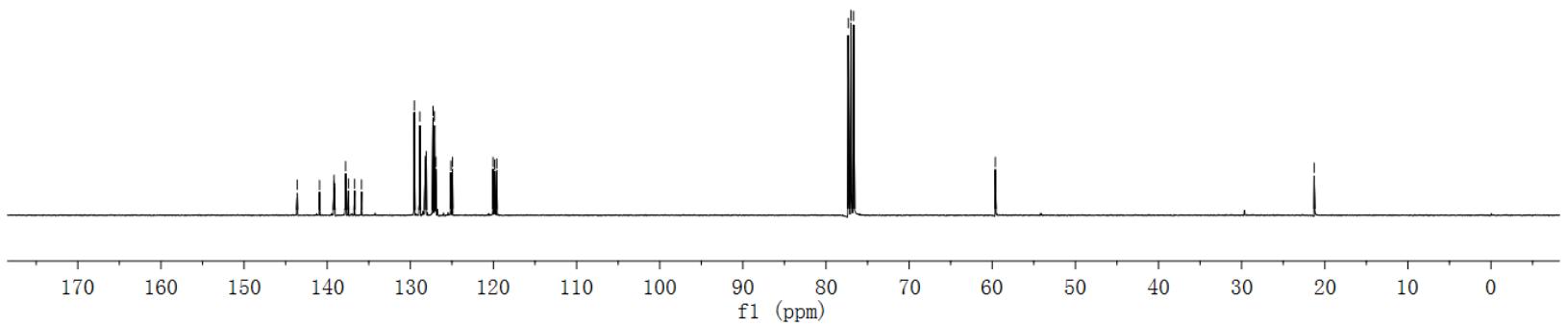




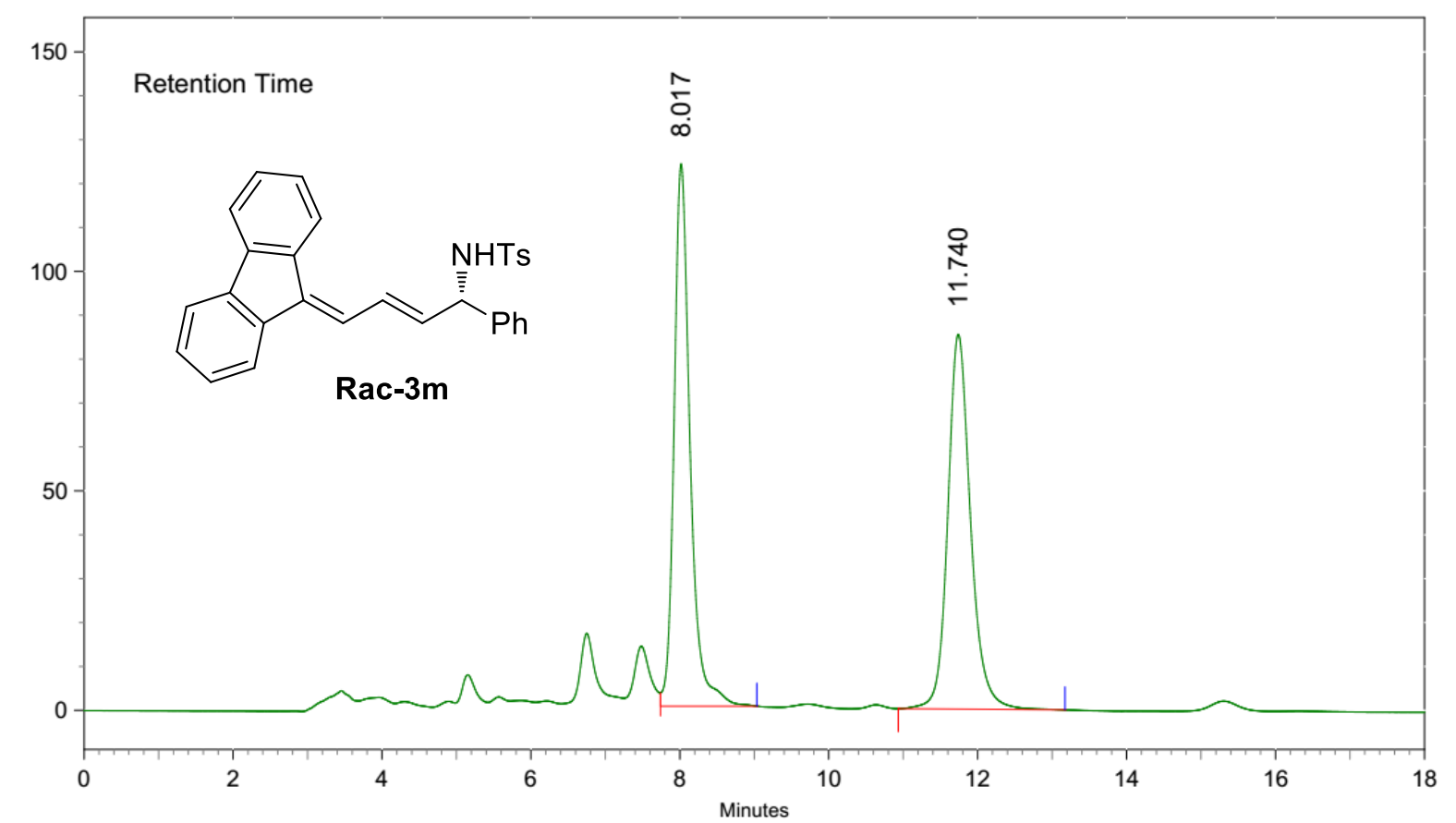

\begin{tabular}{llllll} 
Peak No. & Ret Time & Width & Height & Area & Area [\%] \\
\hline 1 & 8.017 & 1.293 & 2072826 & 31008796 & 50.3957 \\
2 & 11.740 & 2.240 & 1432415 & 30521841 & 49.6043
\end{tabular}

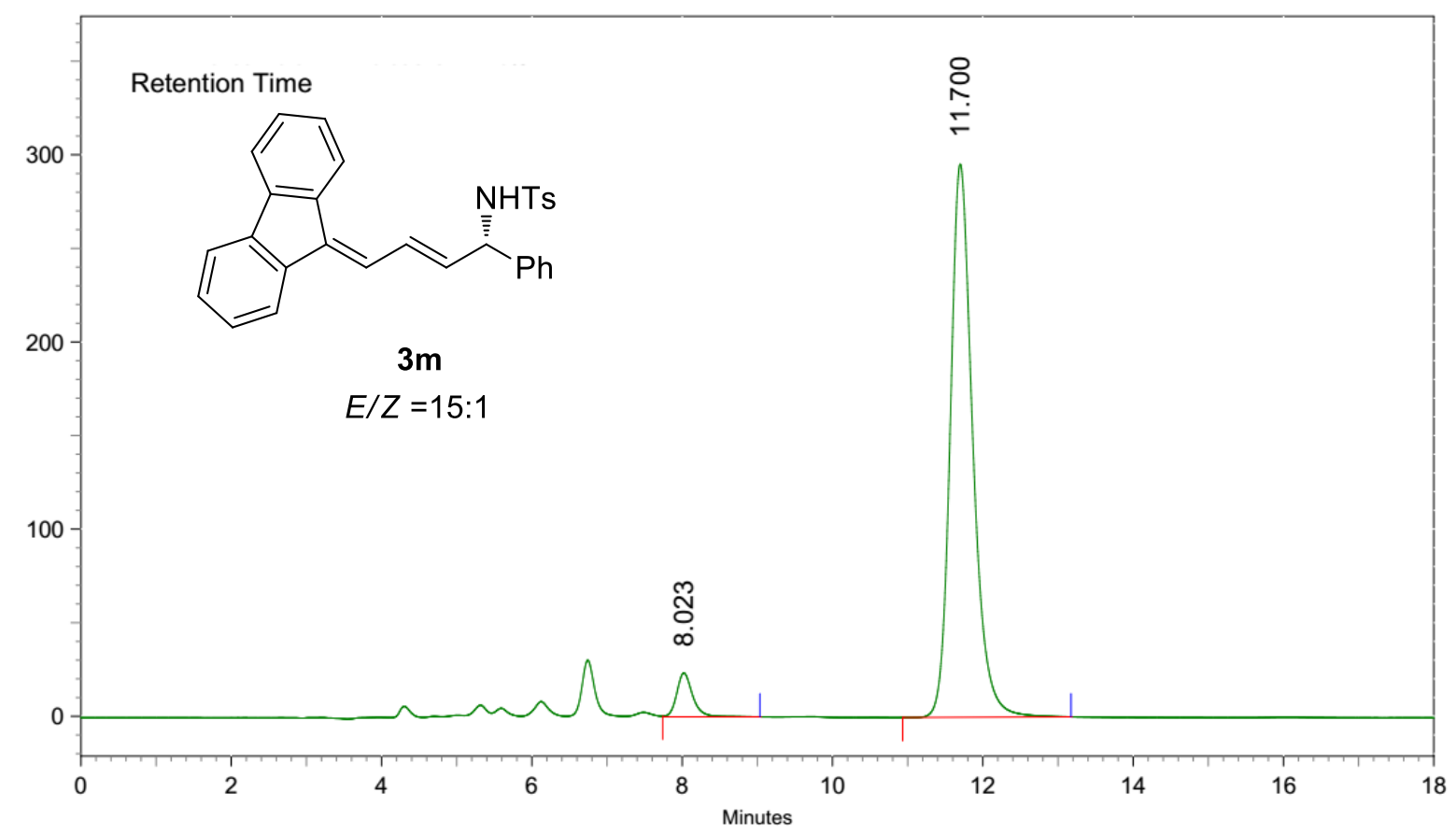

\begin{tabular}{llllll} 
Peak No. & Ret Time & Width & Height & Area & Area [\%] \\
\hline 1 & 8.023 & 1.293 & 393515 & 5804317 & 5.3352 \\
2 & 11.700 & 2.240 & 4958293 & 102988677 & 94.6648
\end{tabular}




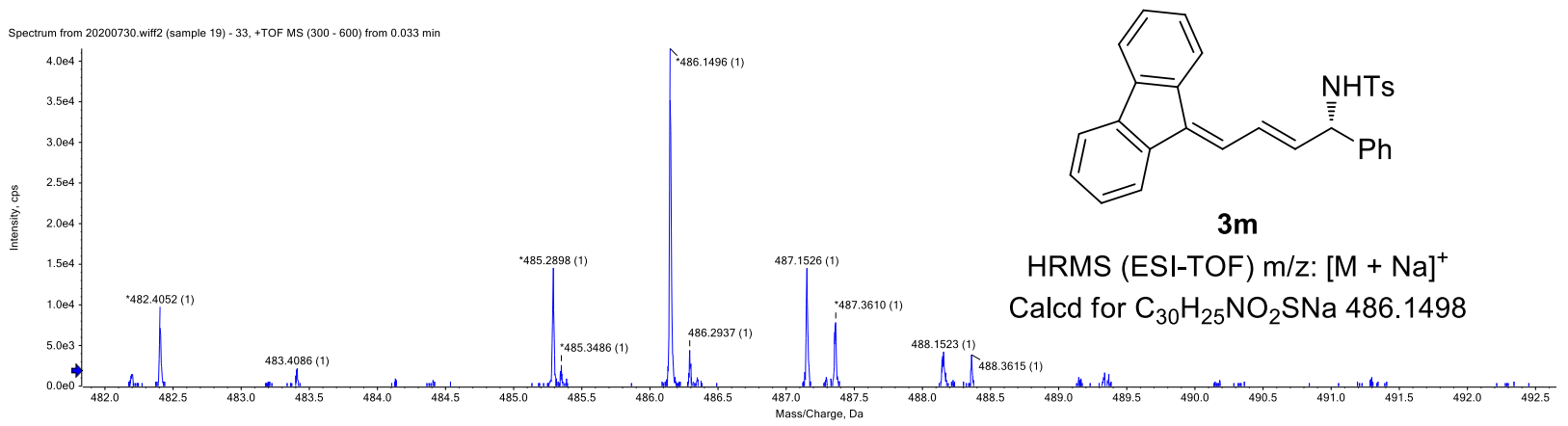




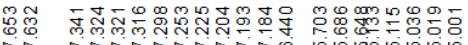

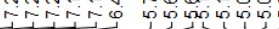

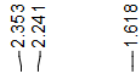

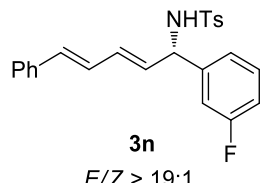

$\mathrm{H} \operatorname{NMR}\left(400 \mathrm{M}, \mathrm{CDCl}_{3}\right.$ )
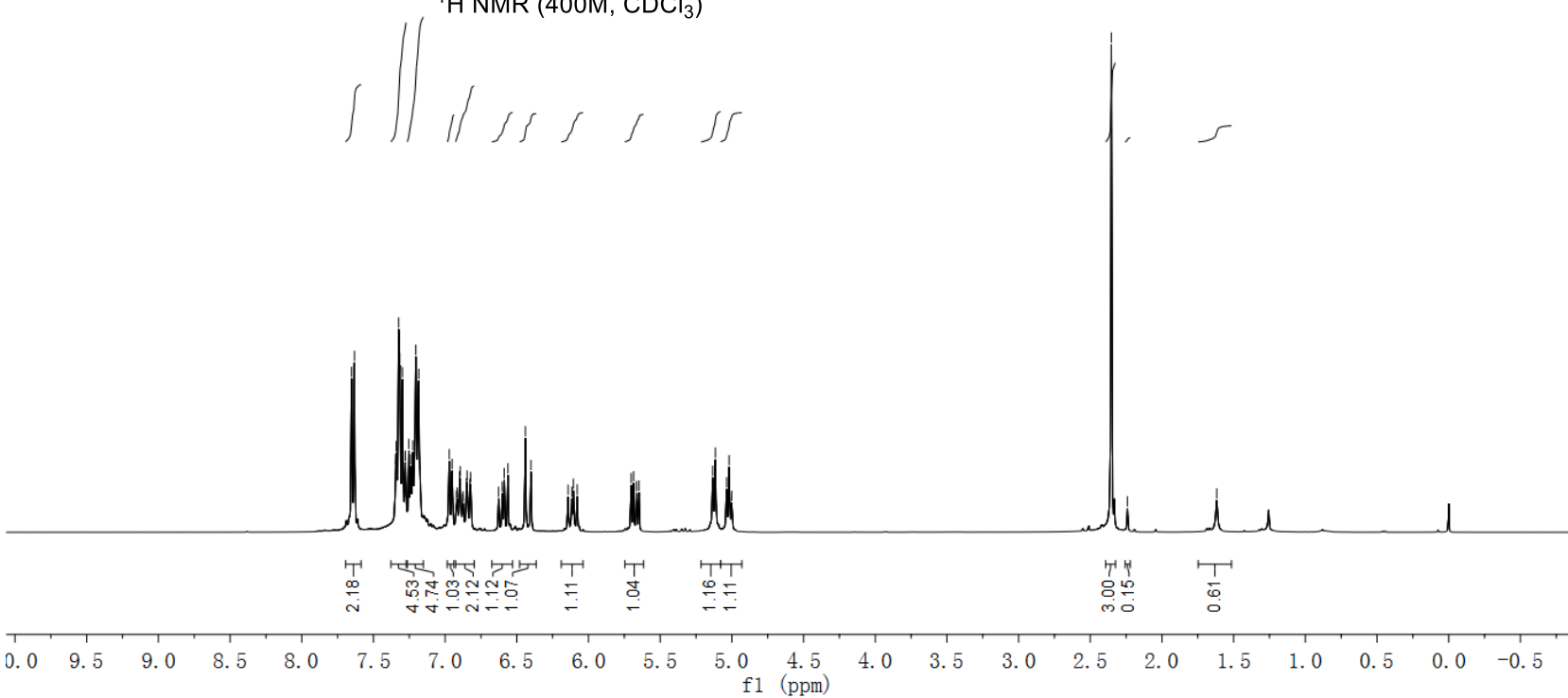

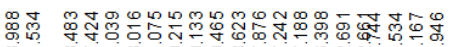

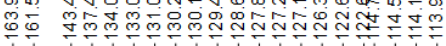

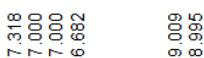

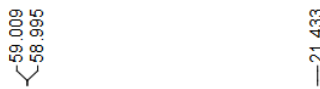

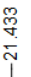

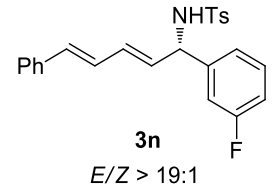

${ }^{13} \mathrm{C}$ NMR (100M, $\mathrm{CDCl}_{3}$ )

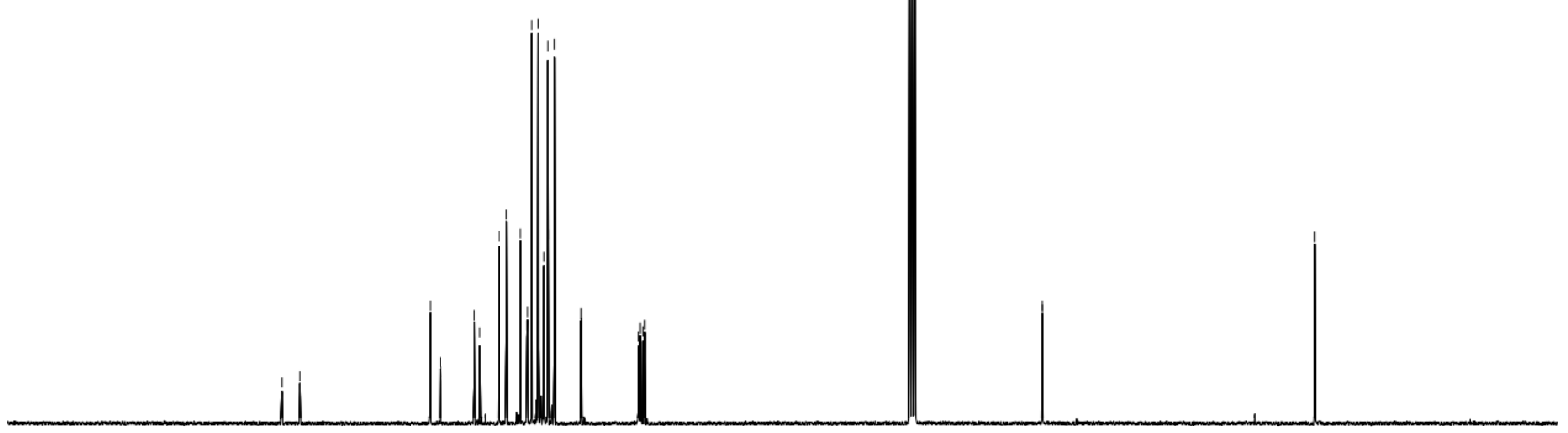

$\begin{array}{llllllllllll}200 & 190 & 180 & 170 & 160 & 150 & 140 & 130 & 120 & 110 & 100 & 90 \\ & & & & & & \end{array}$

$\begin{array}{llllllllll}80 & 70 & 60 & 50 & 40 & 30 & 20 & 10 & 0 & -10\end{array}$ 

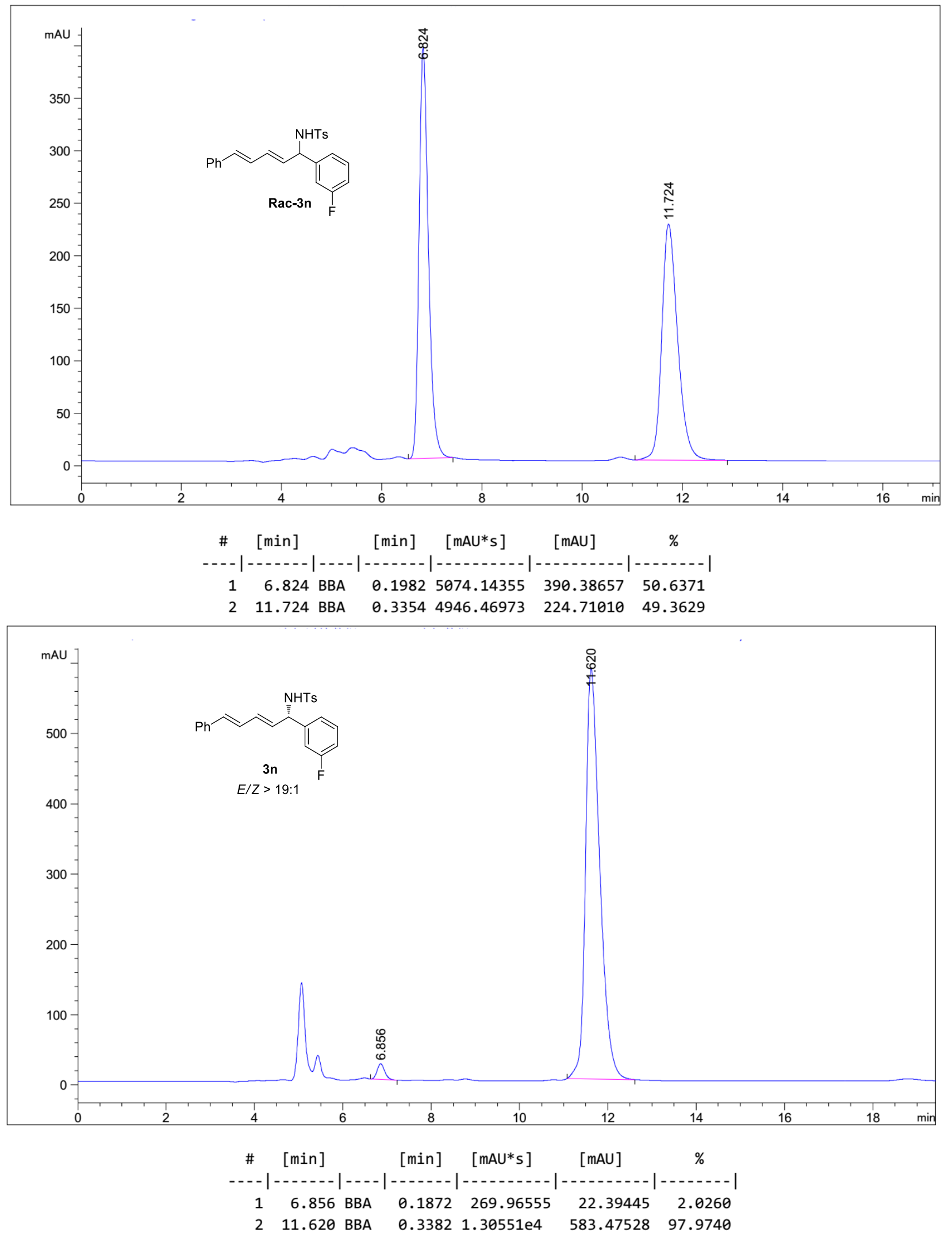


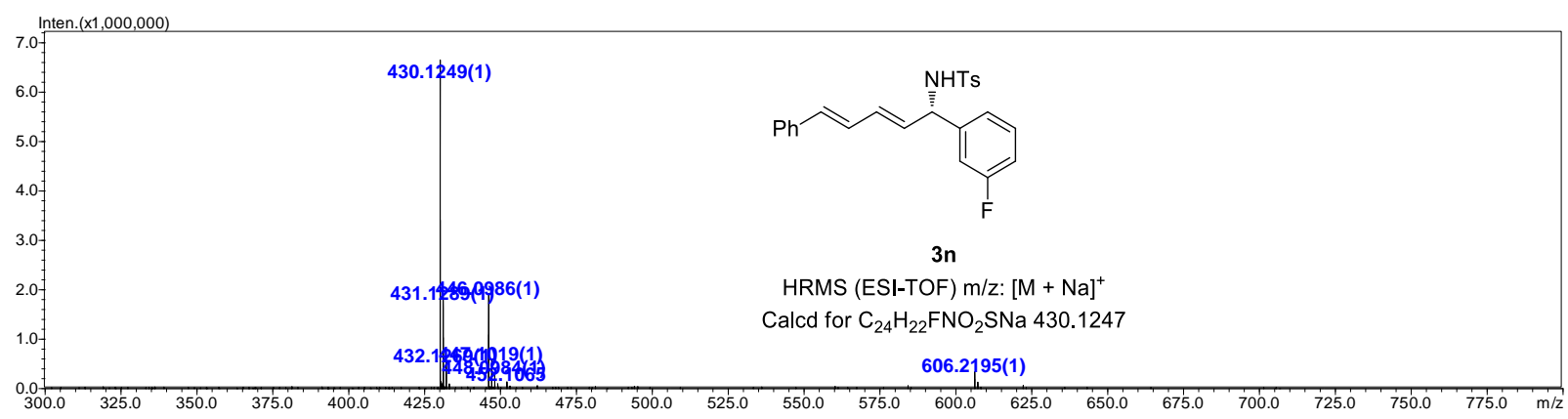


䑻置

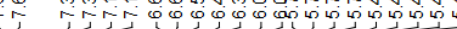

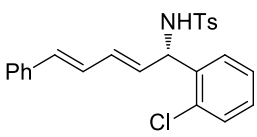

30

${ }^{1} \mathrm{H}$ NMR (400M, $\mathrm{CDCl}_{3}$ )

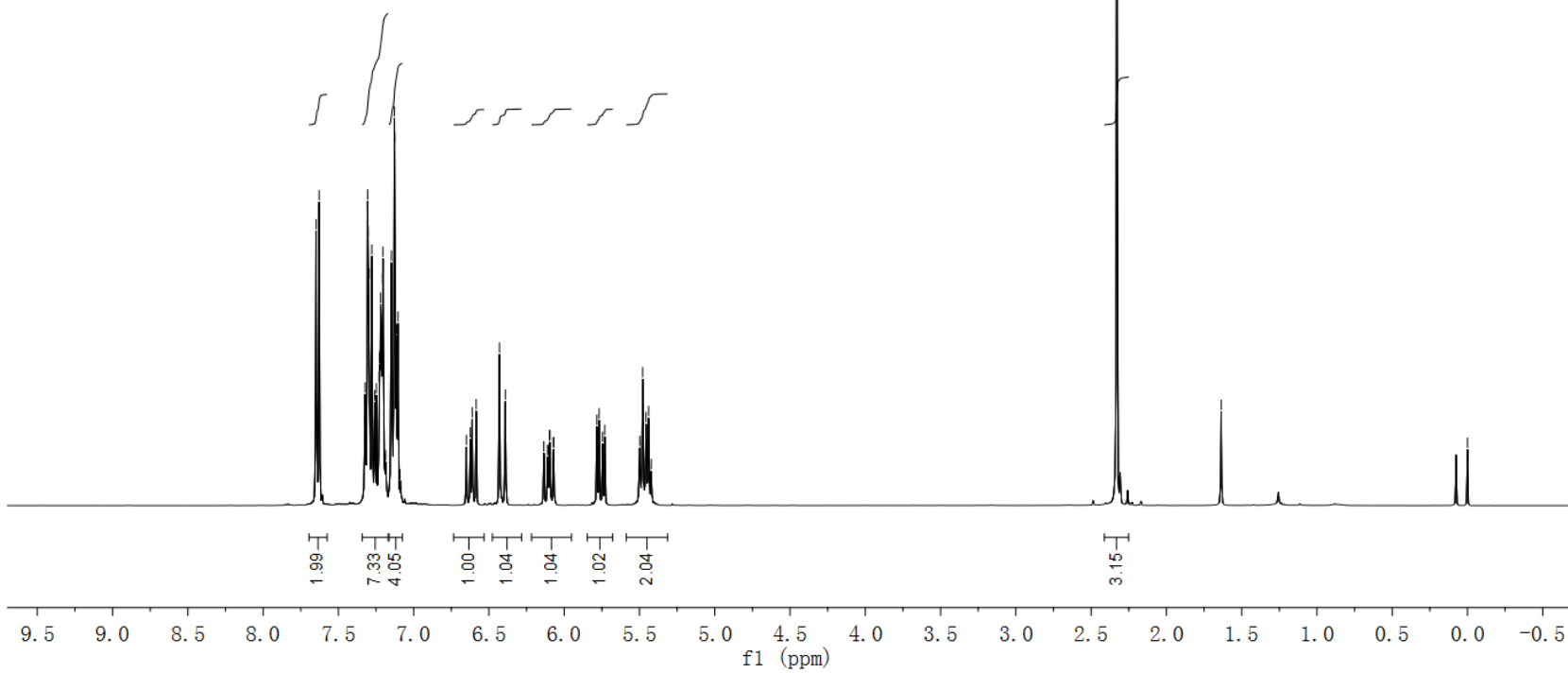

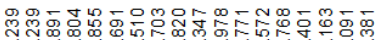

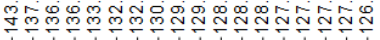

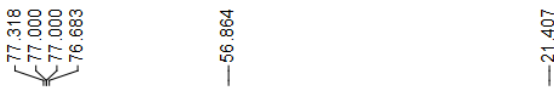

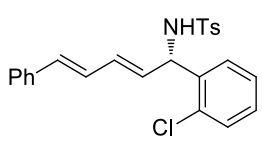

30

${ }^{13} \mathrm{C}$ NMR $\left(100 \mathrm{M}, \mathrm{CDCl}_{3}\right)$

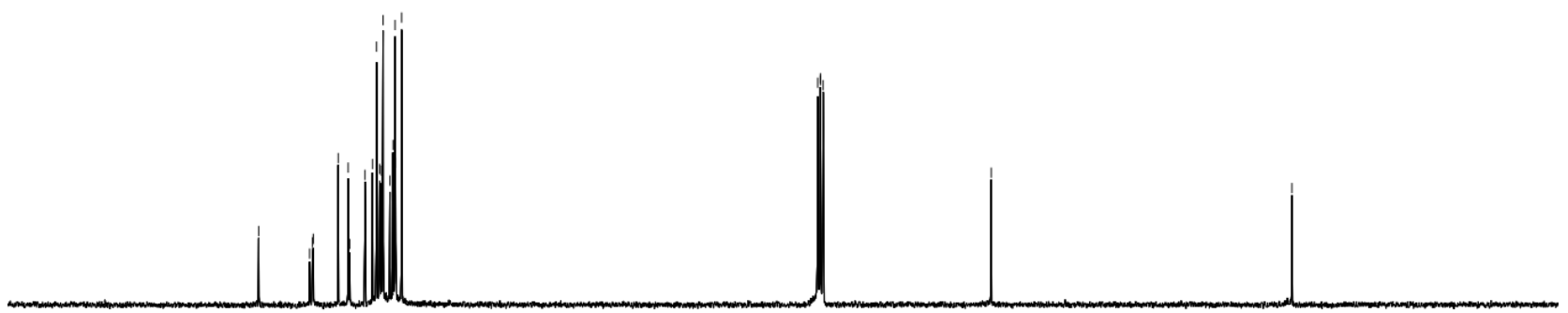

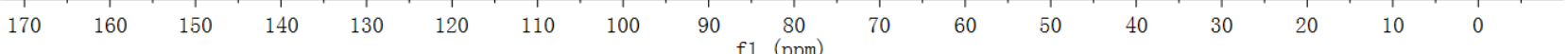




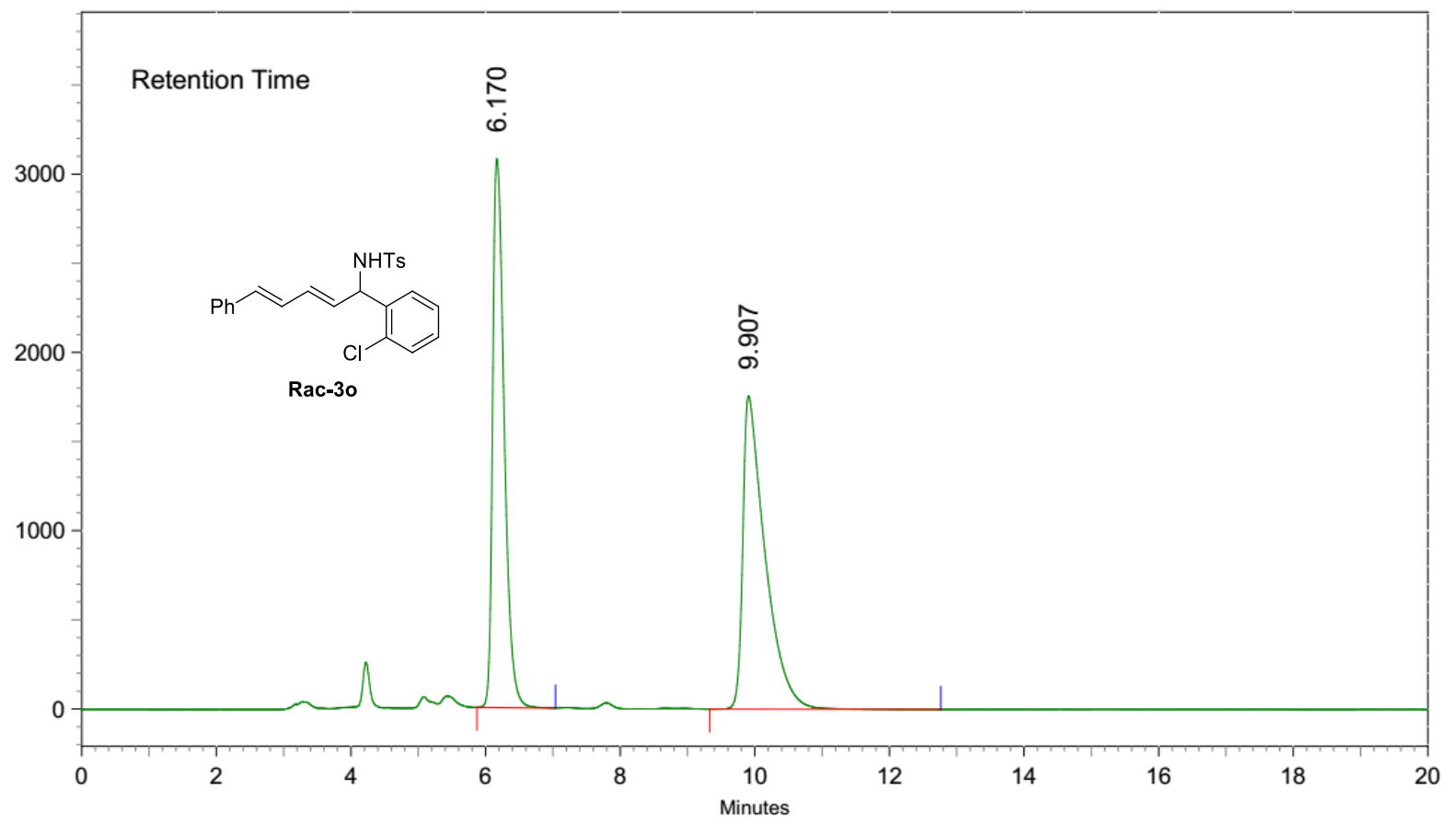

\begin{tabular}{llllll} 
Peak No. & Ret Time & Width & Height & Area & Area [\%] \\
\hline 1 & 6.170 & 1.167 & 51646959 & 617660717 & 47.6660 \\
2 & 9.907 & 3.433 & 29495002 & 678149796 & 52.3340
\end{tabular}

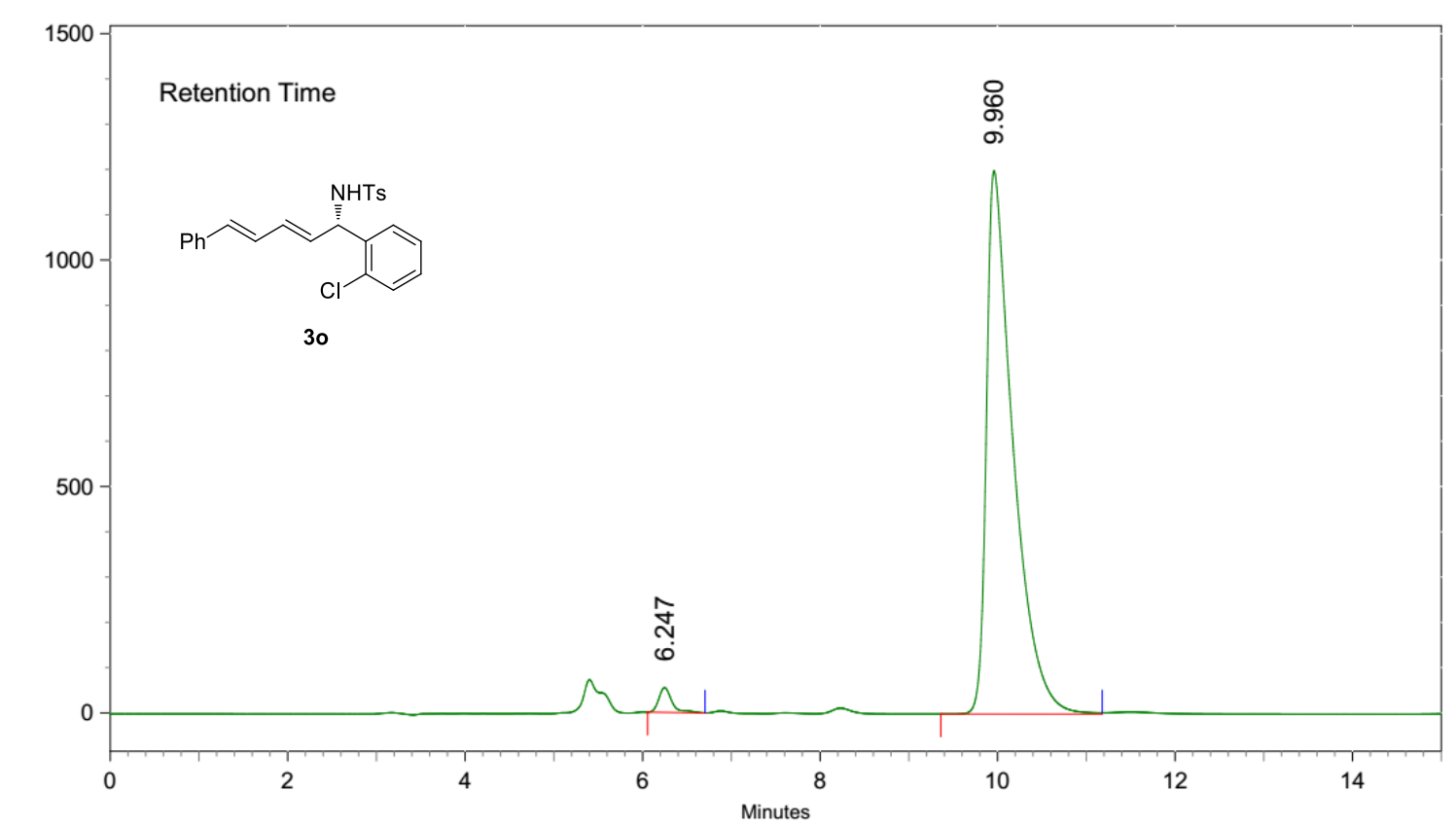

\begin{tabular}{llllll} 
Peak No. & Ret Time & Width & Height & Area & Area [\%] \\
\hline 1 & 6.247 & 0.647 & 918146 & 9413827 & 2.1861 \\
2 & 9.960 & 1.820 & 20128623 & 421204452 & 97.8139
\end{tabular}




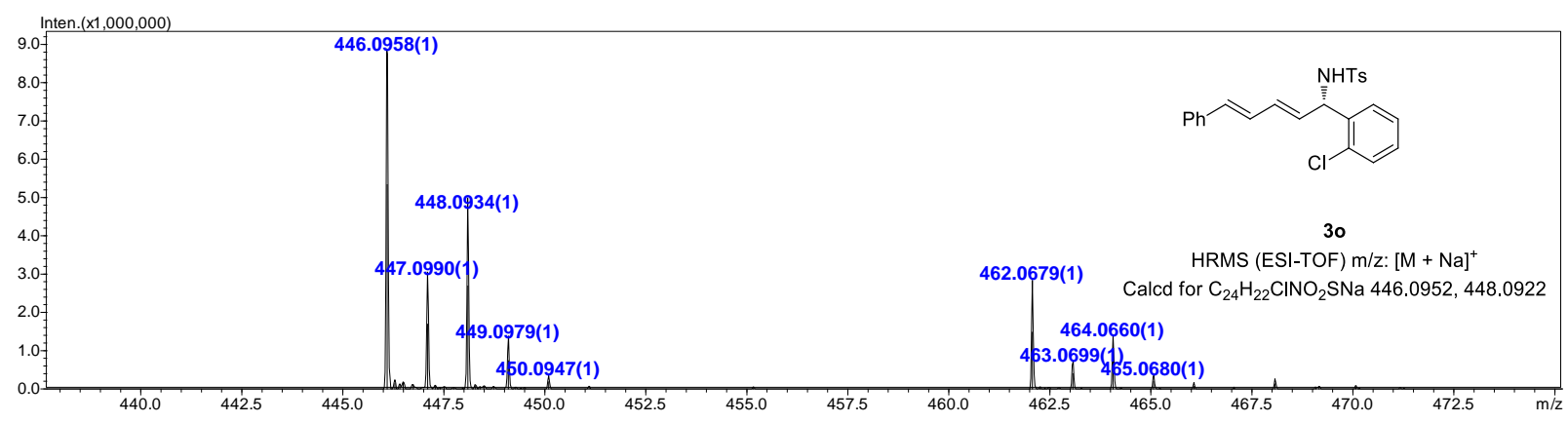




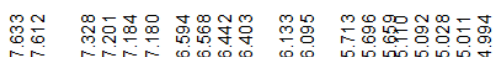

ज证

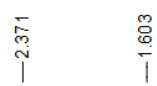

$\stackrel{\circ}{\mathrm{i}}$

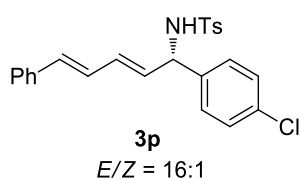

${ }^{1} \mathrm{H} \mathrm{NMR} \mathrm{(400M,} \mathrm{CDCl}_{3}$ )
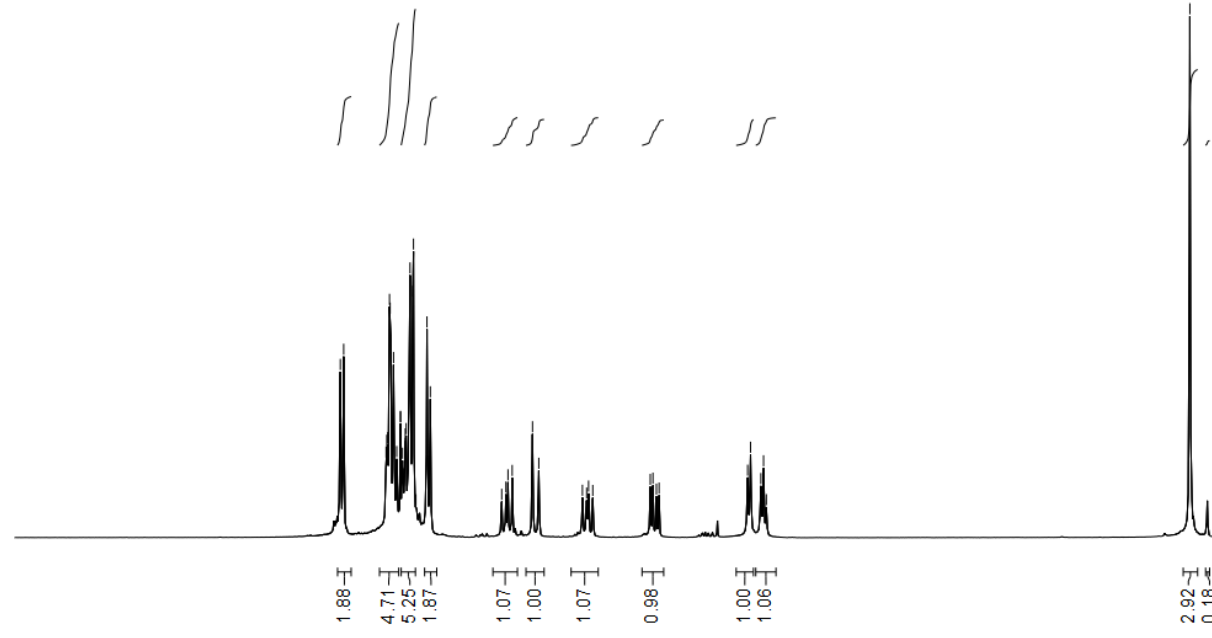

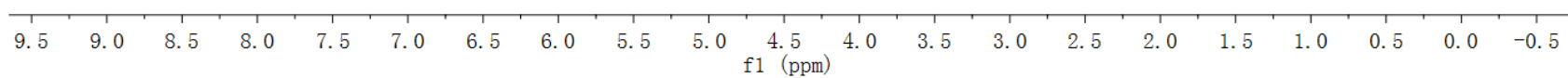

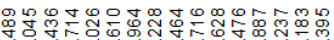

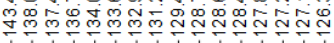

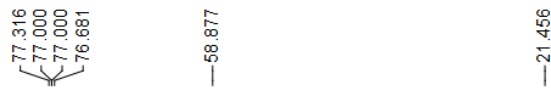

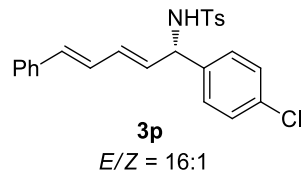

${ }^{13} \mathrm{C}$ NMR (100M, $\mathrm{CDCl}_{3}$ )

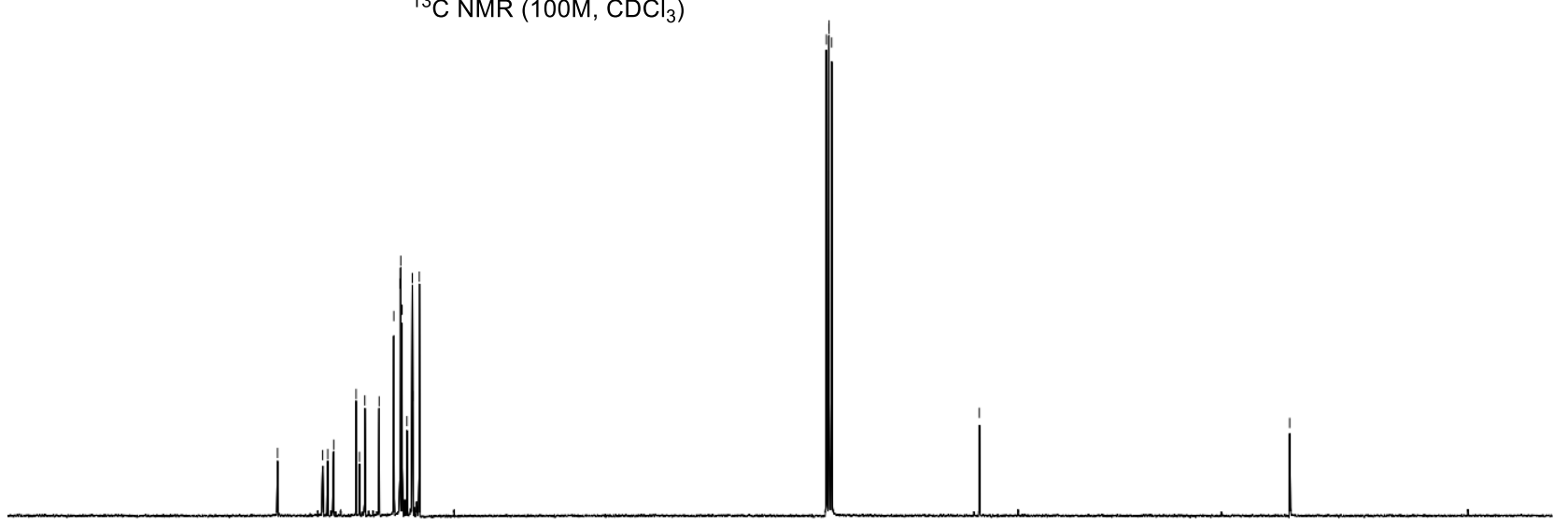

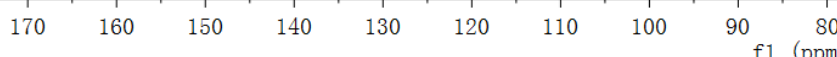




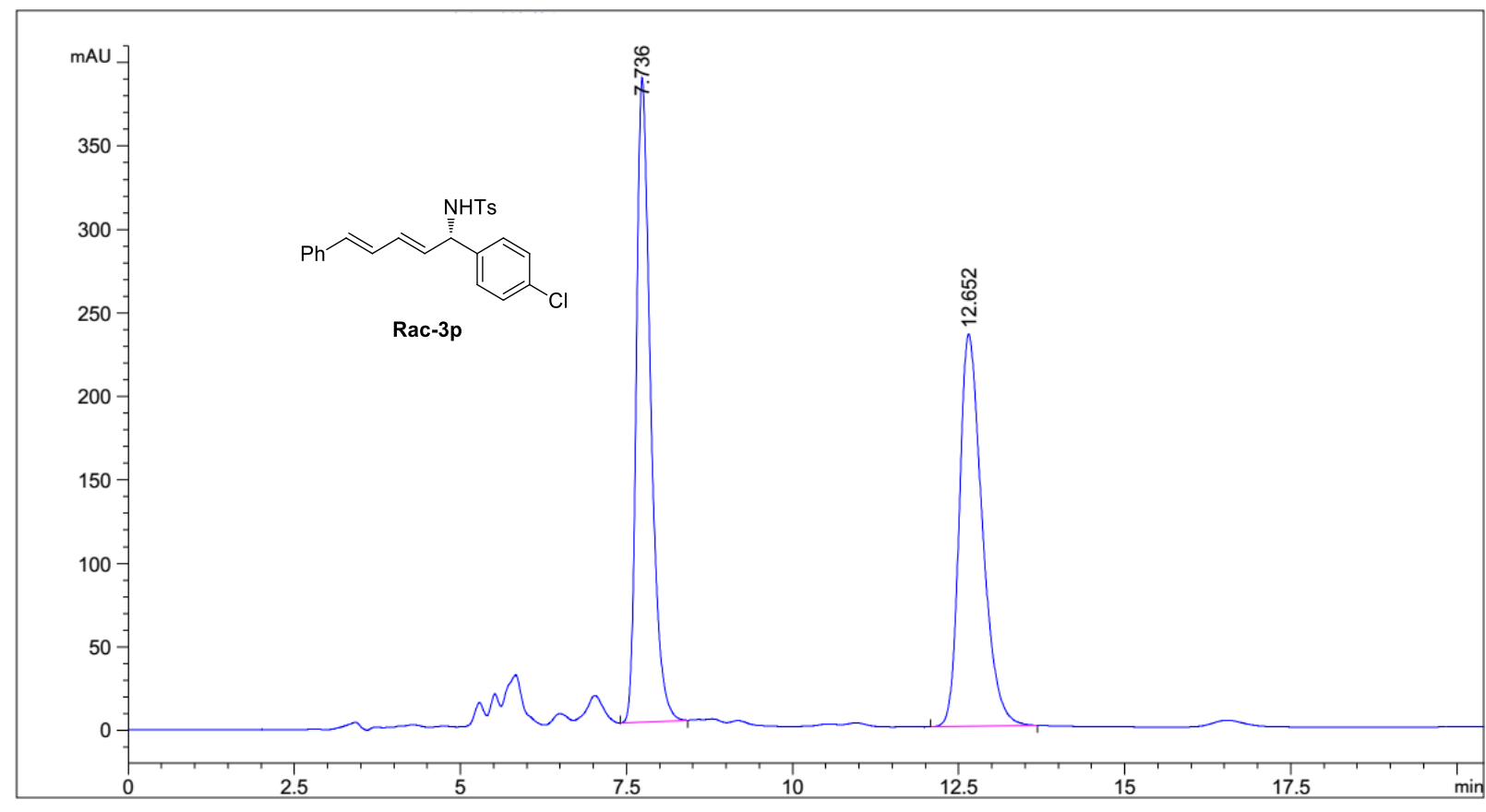

\# $[\mathrm{min}] \quad[\mathrm{min}] \quad[\mathrm{mAU} * \mathrm{~s}] \quad[\mathrm{mAU}] \quad \%$

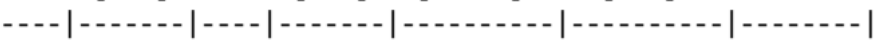

$\begin{array}{lllllll}1 & 7.736 & \text { Bв } & 0.2274 & 5787.72705 & 385.86380 & 50.6597\end{array}$

$\begin{array}{lllllll}2 & 12.652 & \text { BBA } & 0.3657 & 5636.99609 & 235.07689 & 49.3403\end{array}$

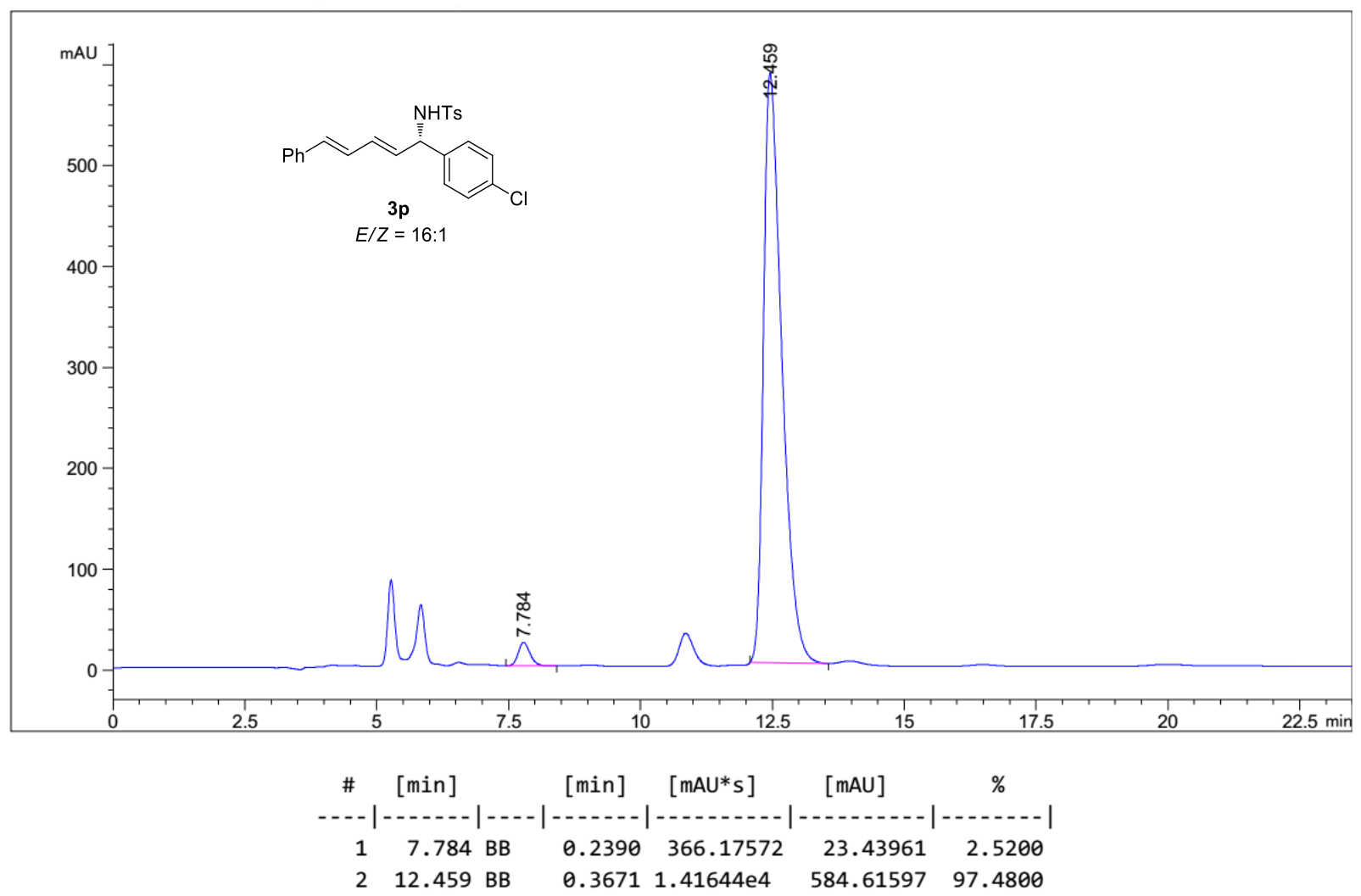




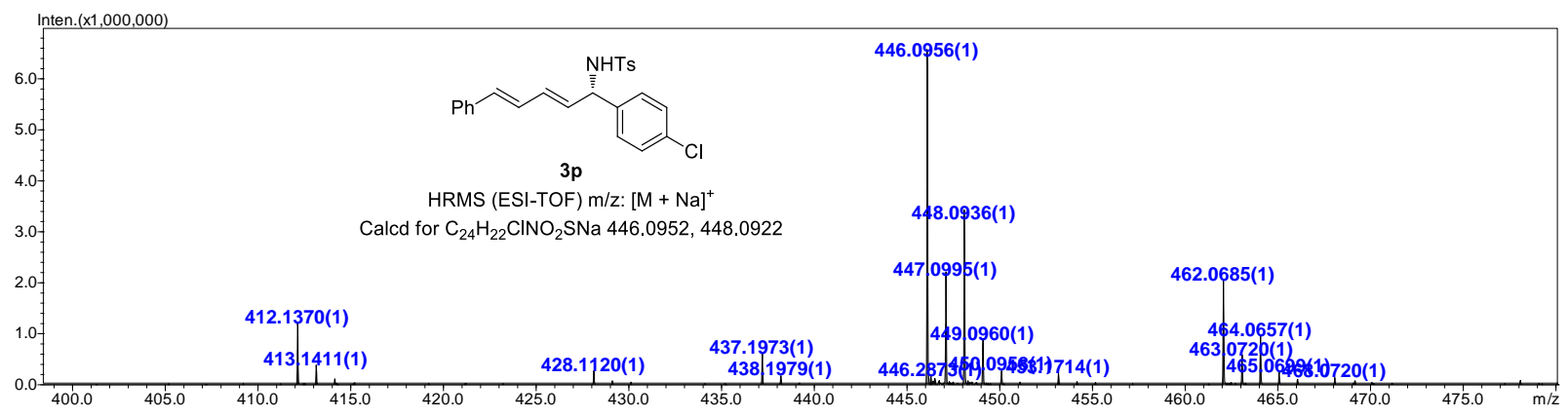




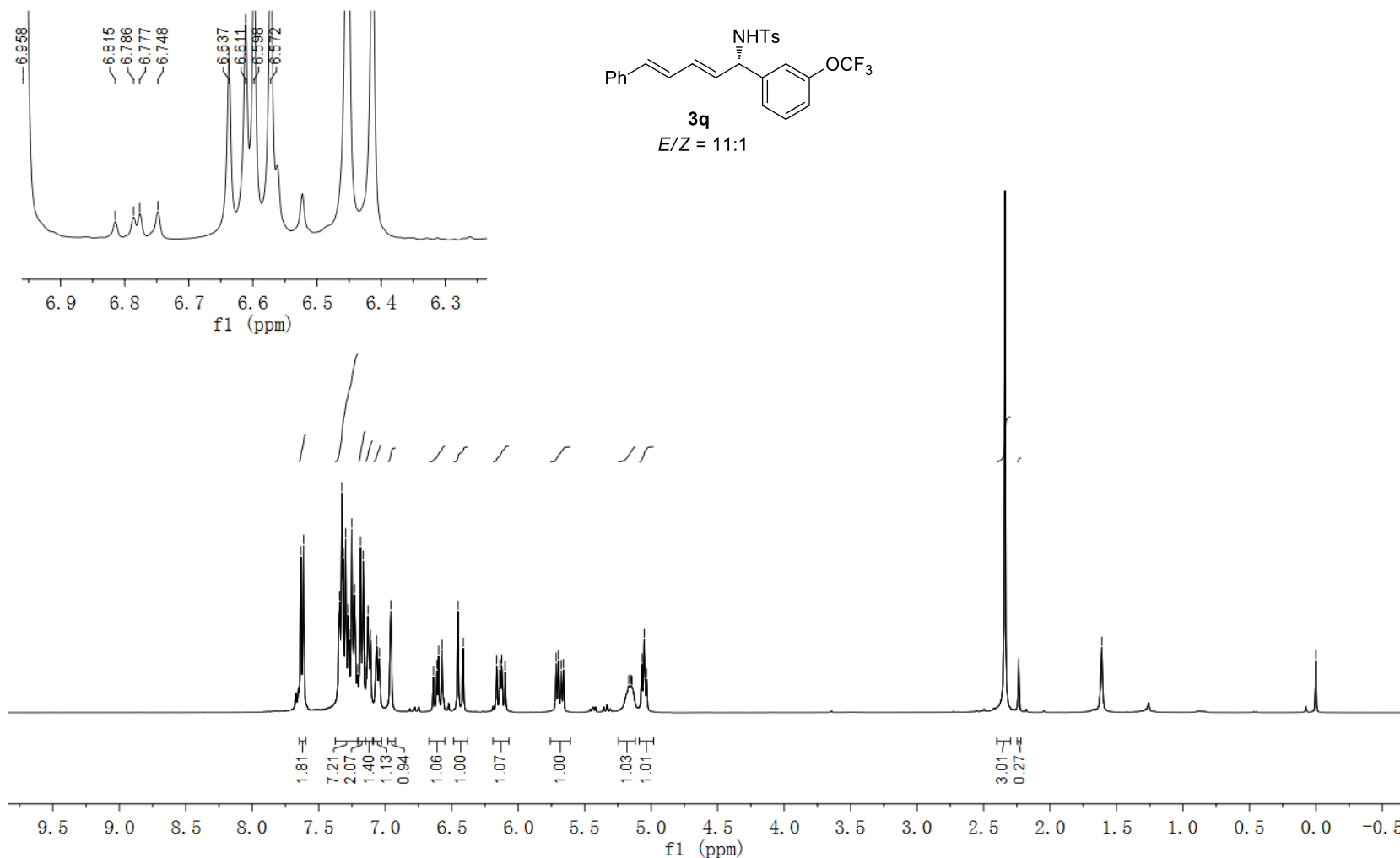

ลิธ

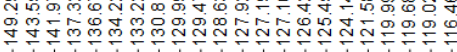

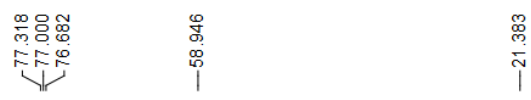<smiles>FC(F)(F)Oc1cccc(C(/C=C/C=C/c2ccccc2)N[In])c1</smiles>

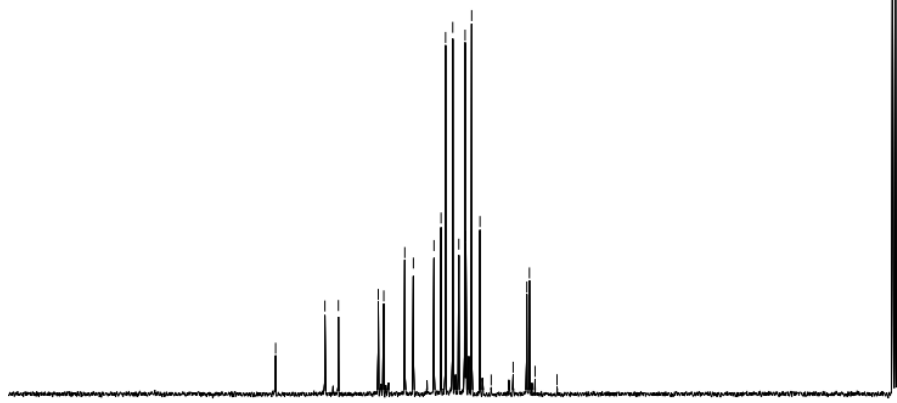

\section{.}




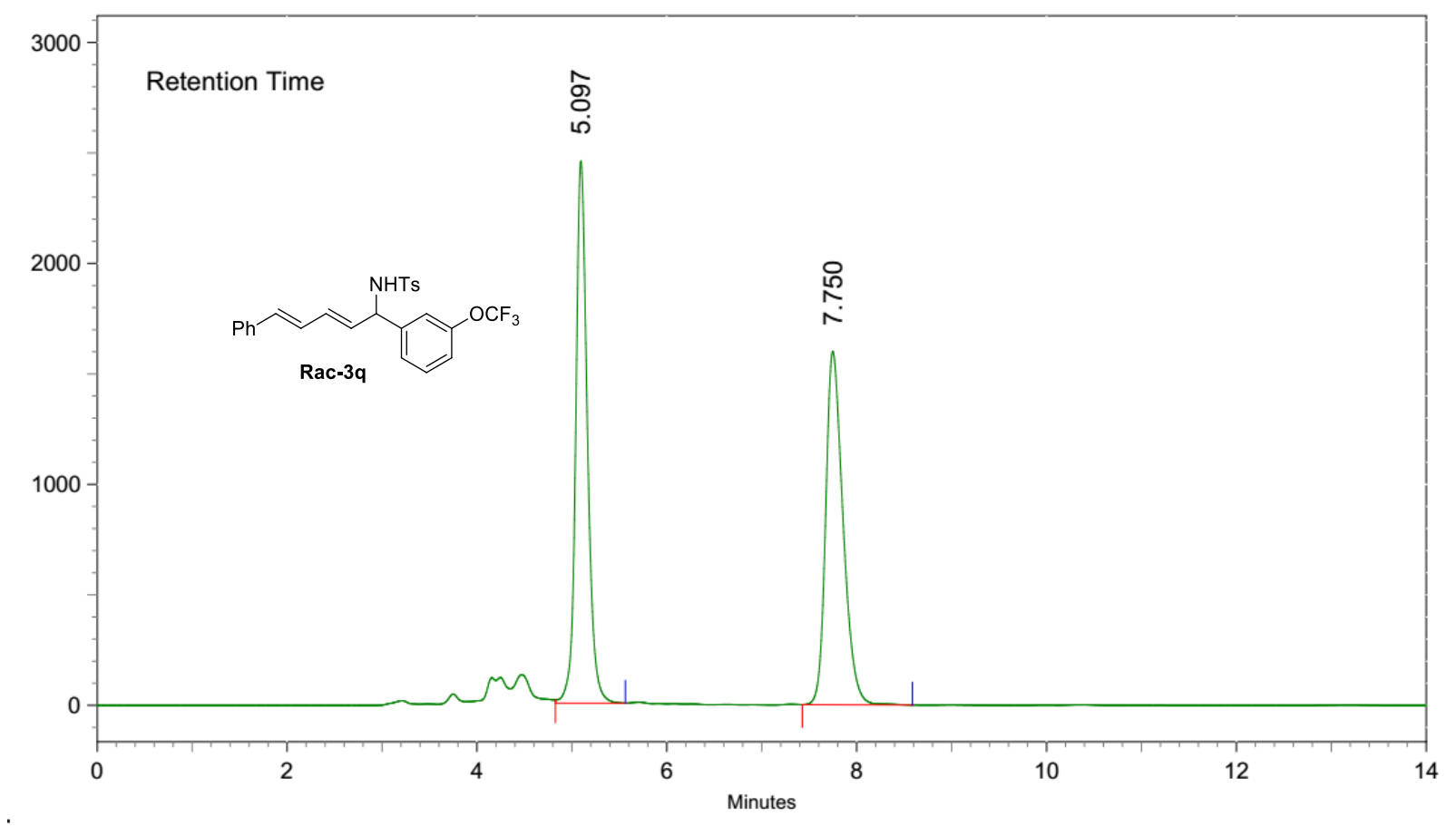

\begin{tabular}{llllll} 
Peak No. & Ret Time & Width & Height & Area & Area [\%] \\
\hline 1 & 5.097 & 0.737 & 41169224 & 349647588 & 50.6625 \\
2 & 7.750 & 1.160 & 26827303 & 340503232 & 49.3375
\end{tabular}

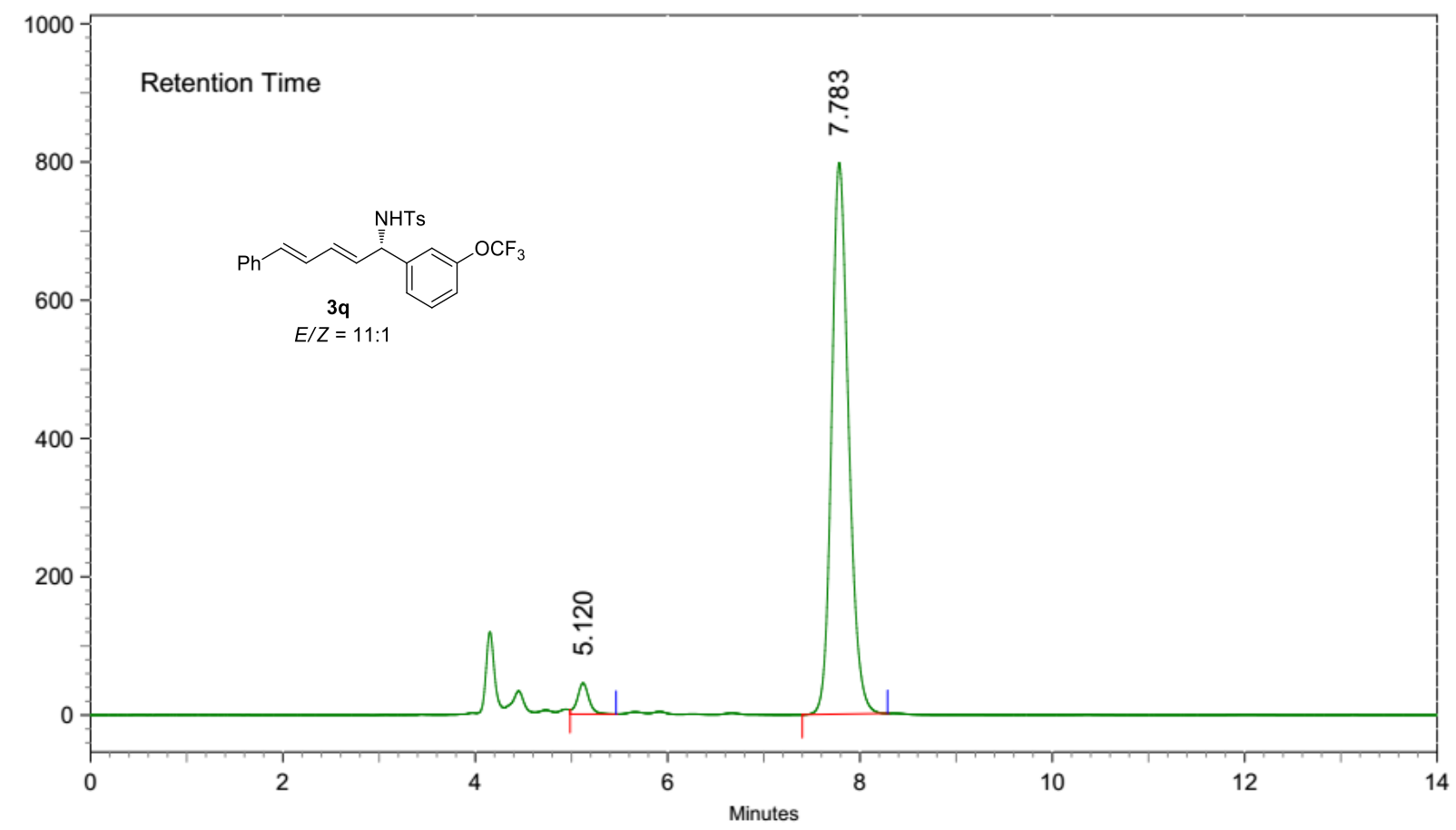

\begin{tabular}{llllll} 
Peak No. & Ret Time & Width & Height & Area & Area [\%] \\
\hline 1 & 5.120 & 0.477 & 752269 & 6226401 & 3.6530 \\
2 & 7.783 & 0.887 & 13386016 & 164220582 & 96.3470
\end{tabular}




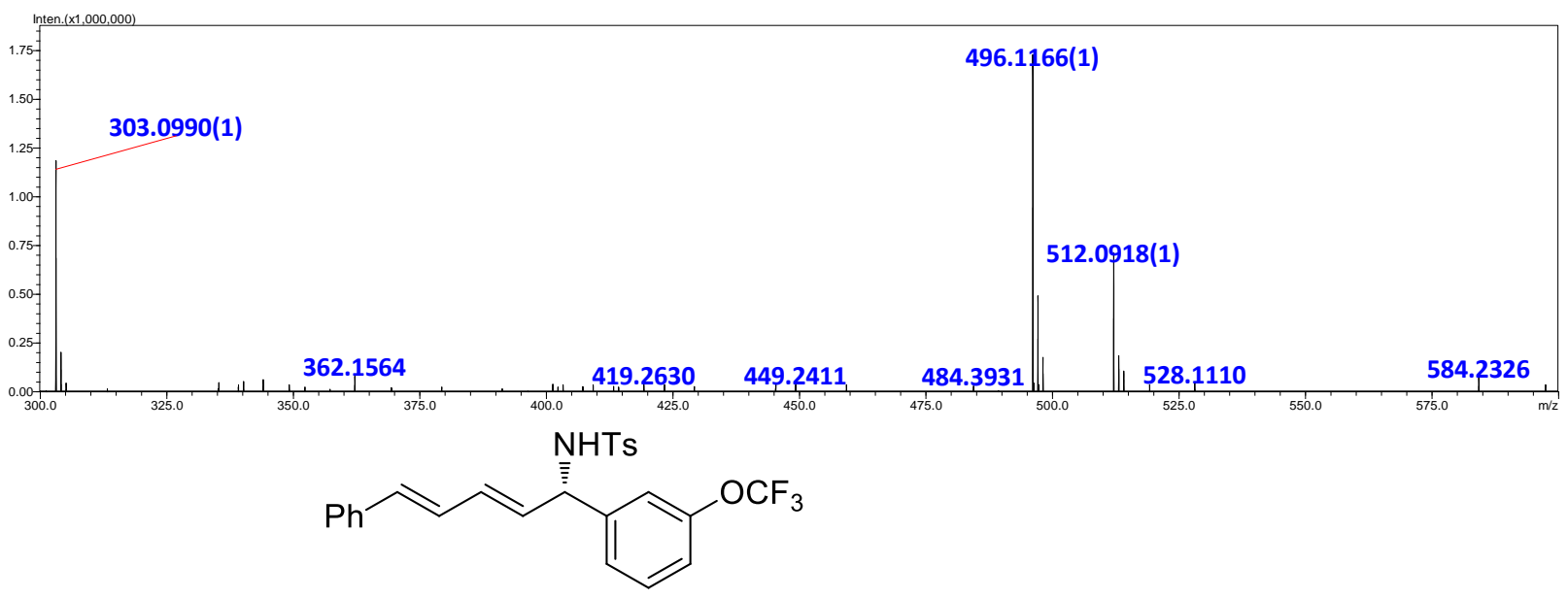

$3 q$

HRMS (ESI-TOF) m/z: [M + Na] ${ }^{+}$

Calcd for $\mathrm{C}_{25} \mathrm{H}_{22} \mathrm{~F}_{3} \mathrm{NO}_{3} \mathrm{SNa} 496.1165$ 


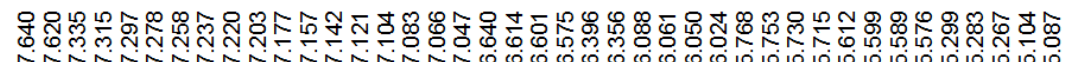
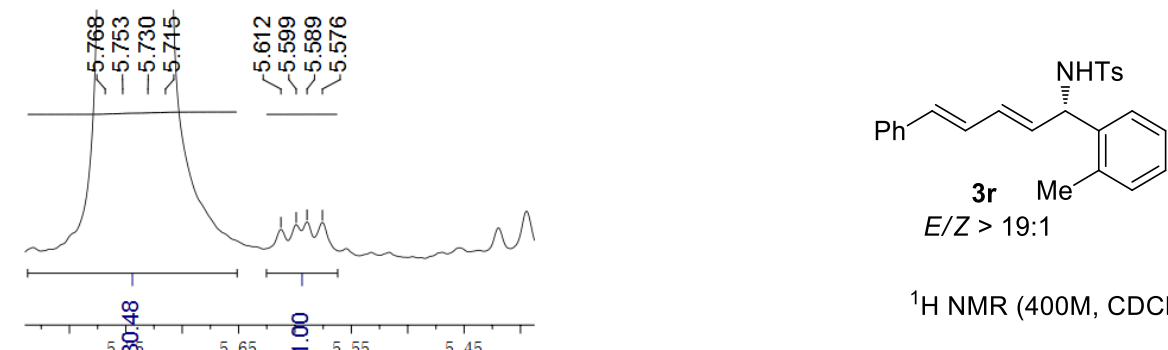

${ }^{1} \mathrm{H}$ NMR $\left(400 \mathrm{M}, \mathrm{CDCl}_{3}\right)$

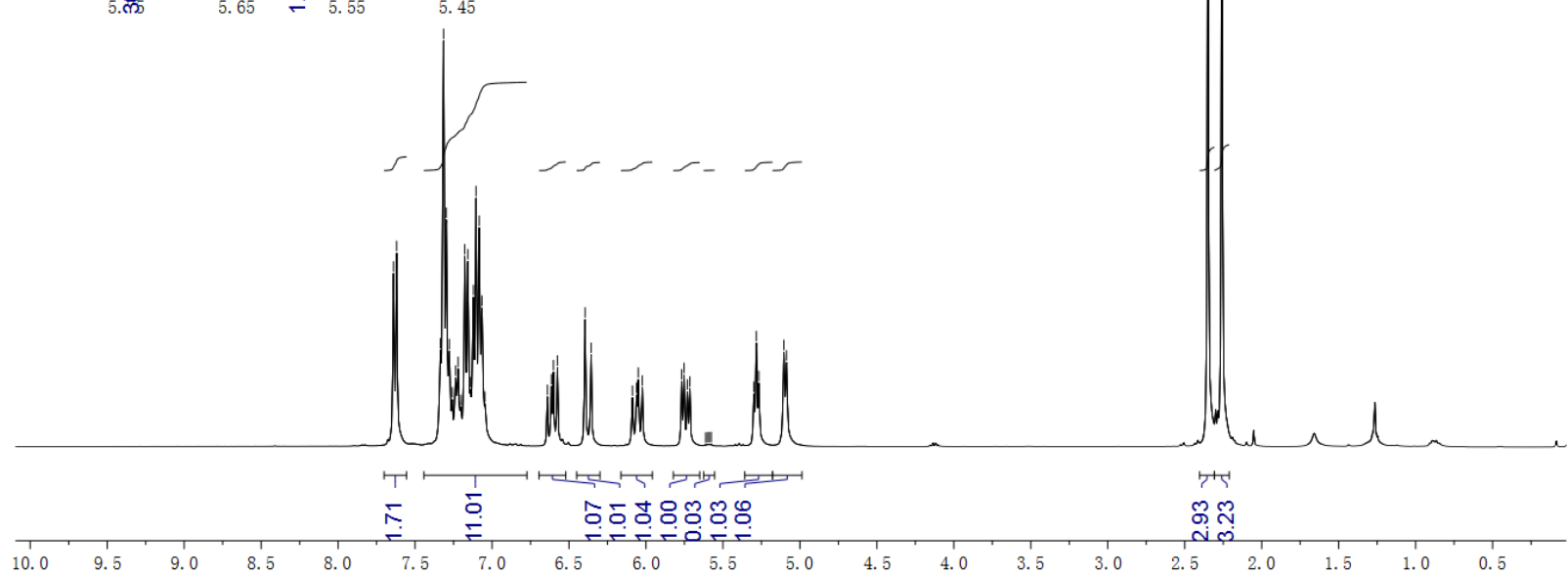

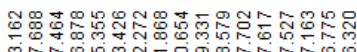

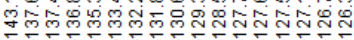

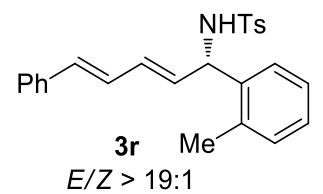

${ }^{13} \mathrm{C}$ NMR $\left(100 \mathrm{M}, \mathrm{CDCl}_{3}\right)$
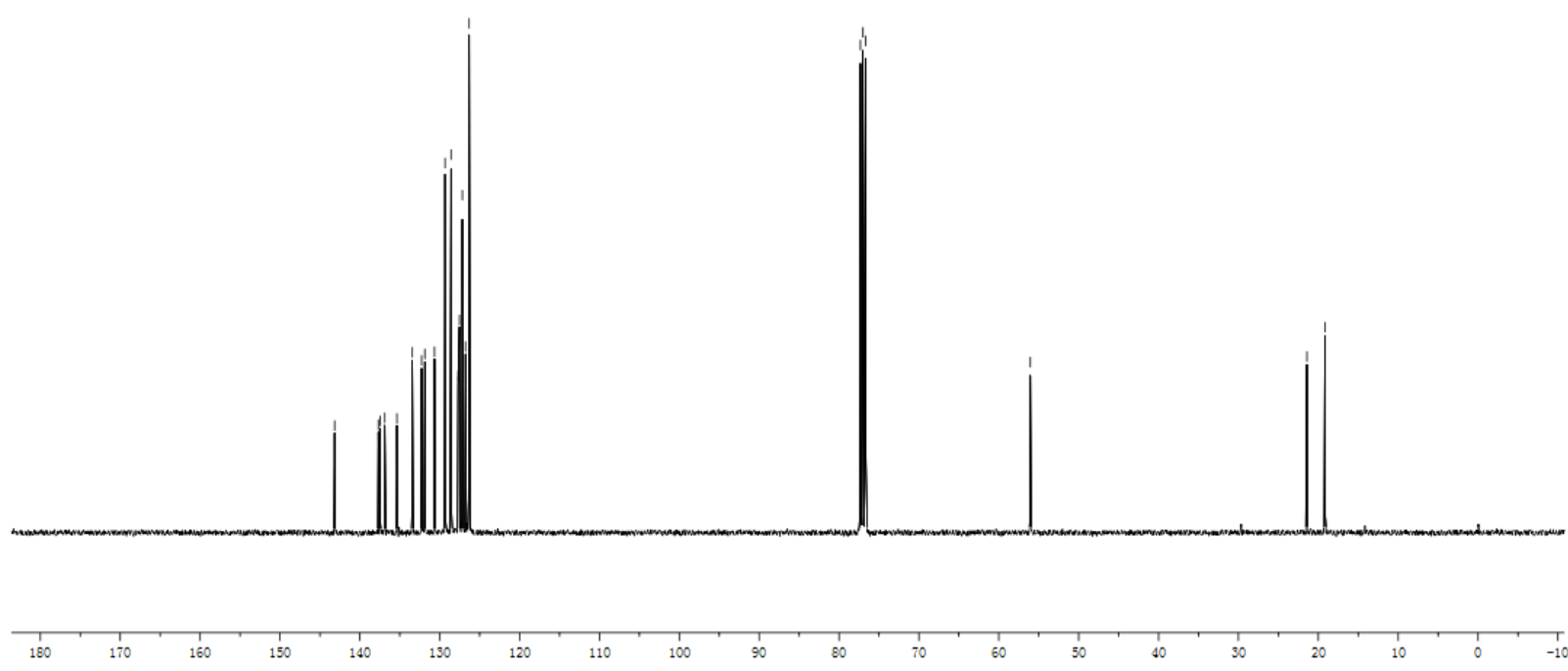

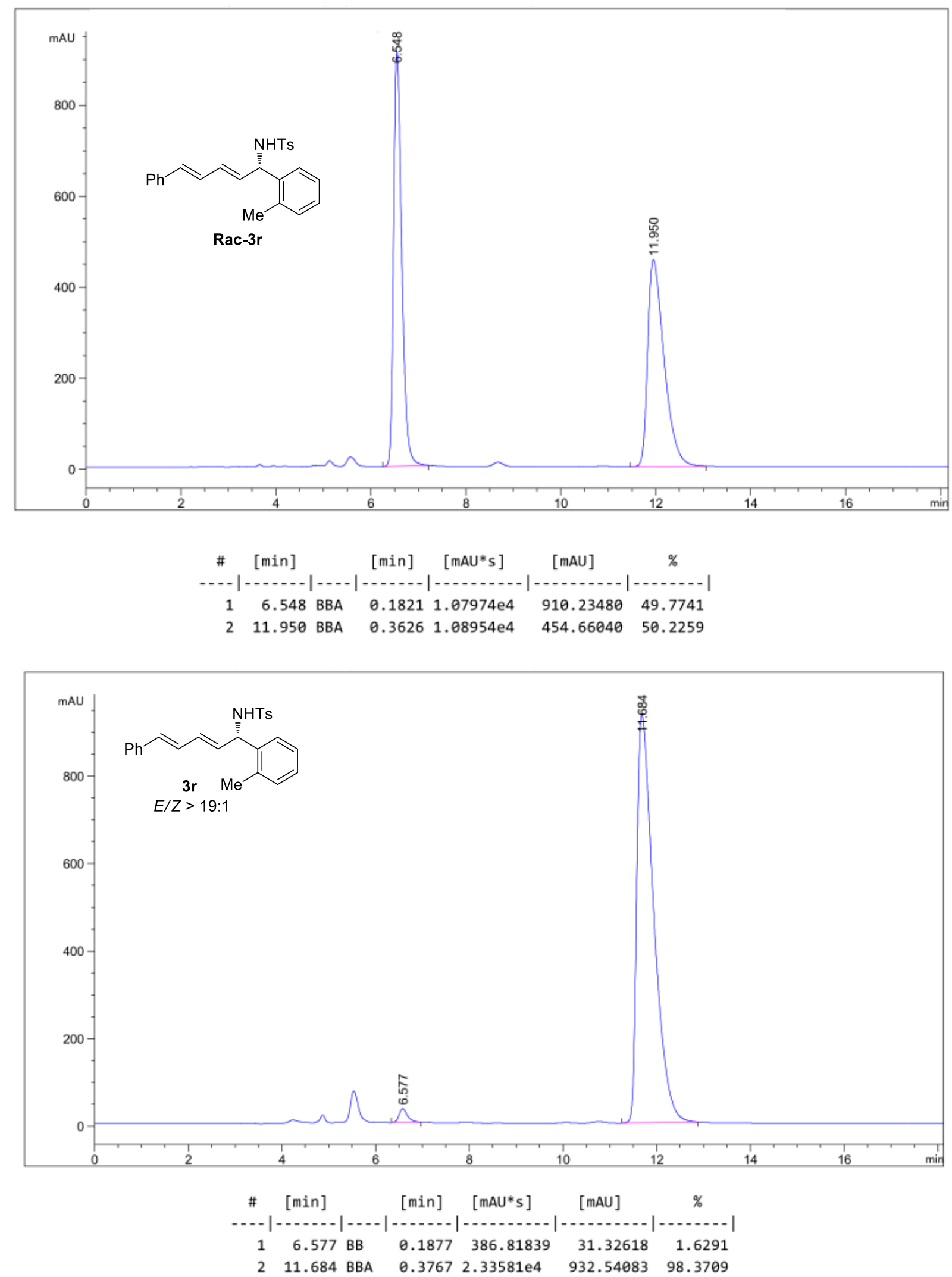
17:39:00

200402_CY_41B $10(0.171) \mathrm{Cm}(1: 24)$

100

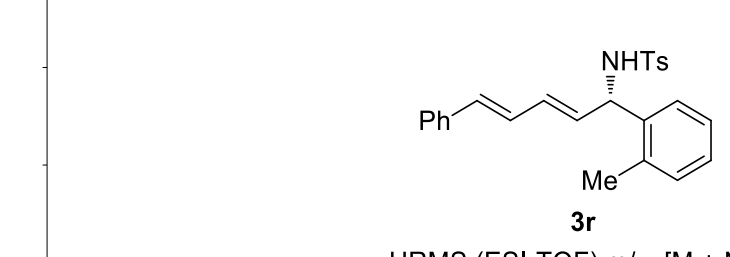

HRMS (ESI-TOF) m/z: [M + Na] $]^{+}$

Calcd for $\mathrm{C}_{25} \mathrm{H}_{25} \mathrm{NO}_{2} \mathrm{SNa} 426.1498$

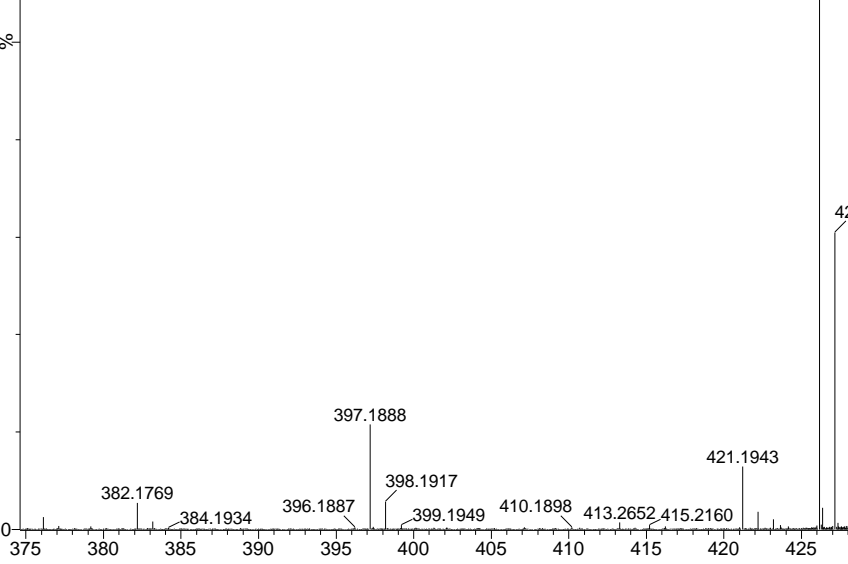

27.1550

\begin{tabular}{ll|l}
429.1532 & 437.1967 & 443.1322
\end{tabular}

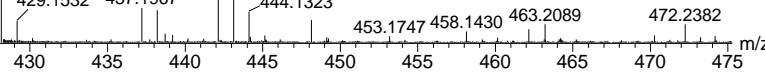




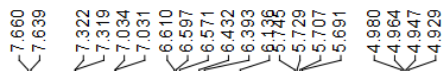

兽<smiles>CCCC(N)c1ccc([N+](=O)[O-])cc1</smiles>

$E / Z=12: 1$

${ }^{1} \mathrm{H}$ NMR (400M, $\mathrm{CDCl}_{3}$ )
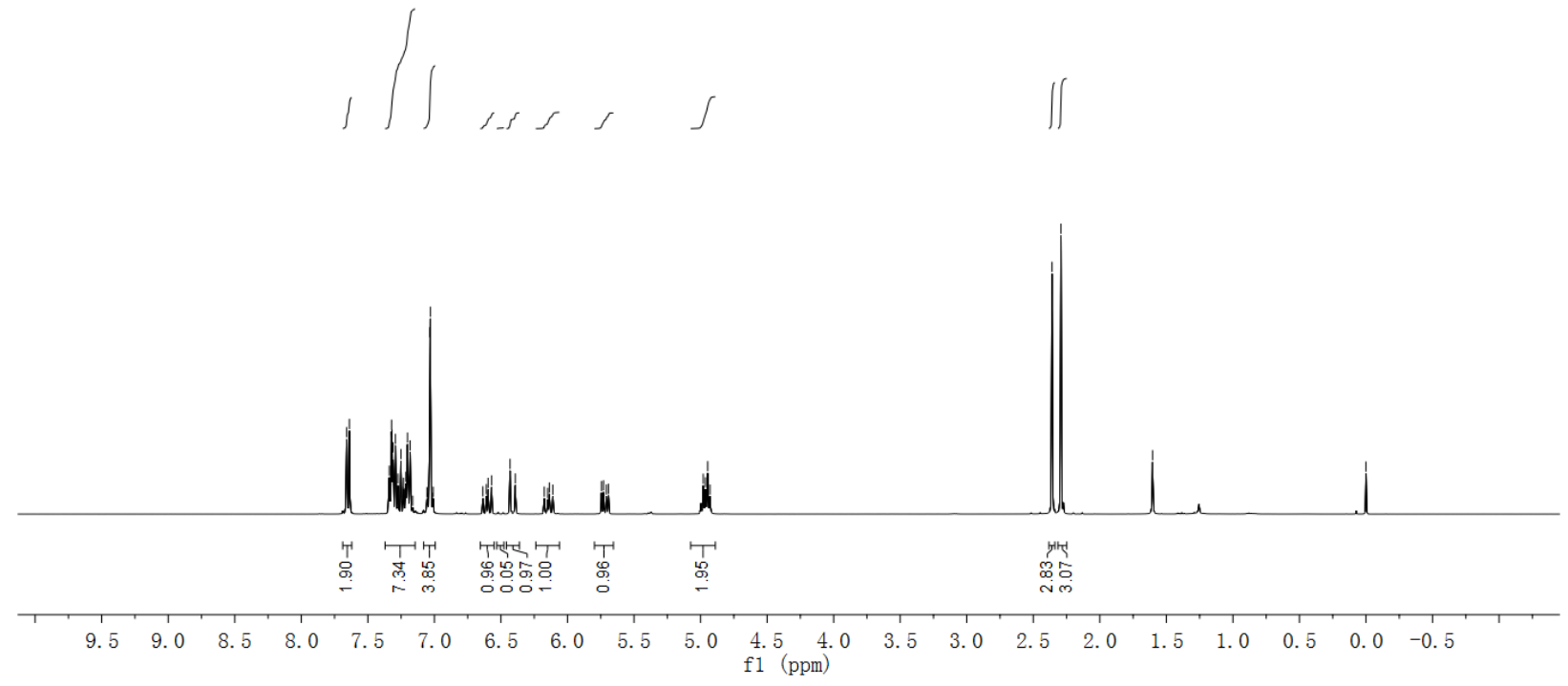

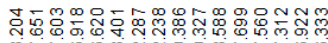

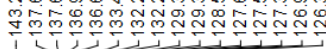

कo

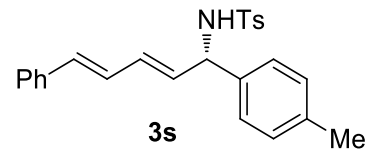

$E / Z=12: 1$

${ }^{13} \mathrm{C} \mathrm{NMR} \mathrm{(100M,} \mathrm{CDCl}_{3}$ )

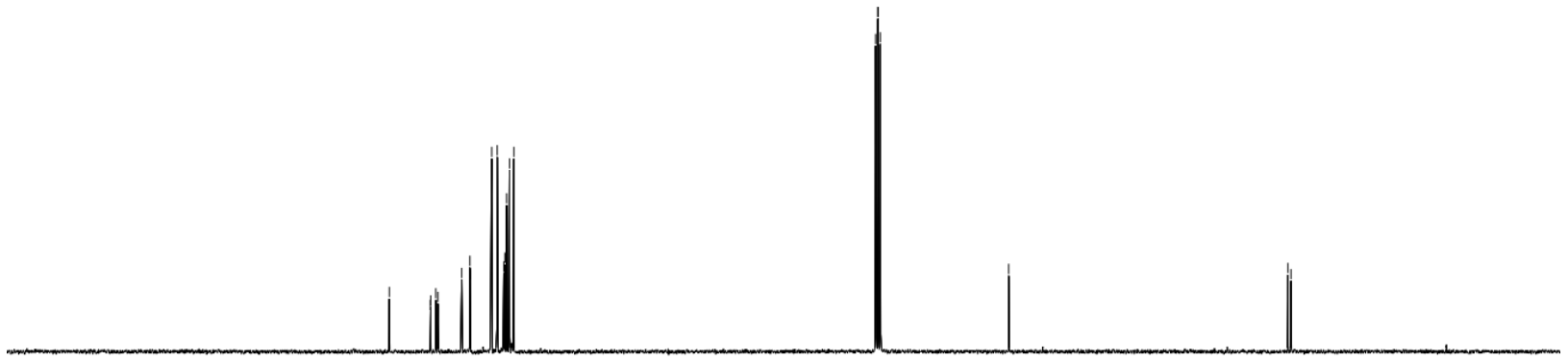

$\begin{array}{lllllllllllllllllllll}190 & 180 & 170 & 160 & 150 & 140 & 130 & 120 & 110 & 100 & \underset{\mathrm{f} 1}{90}(\mathrm{ppm}) & 80 & 70 & 60 & 50 & 40 & 30 & 20 & 10 & 0 & -10\end{array}$ 

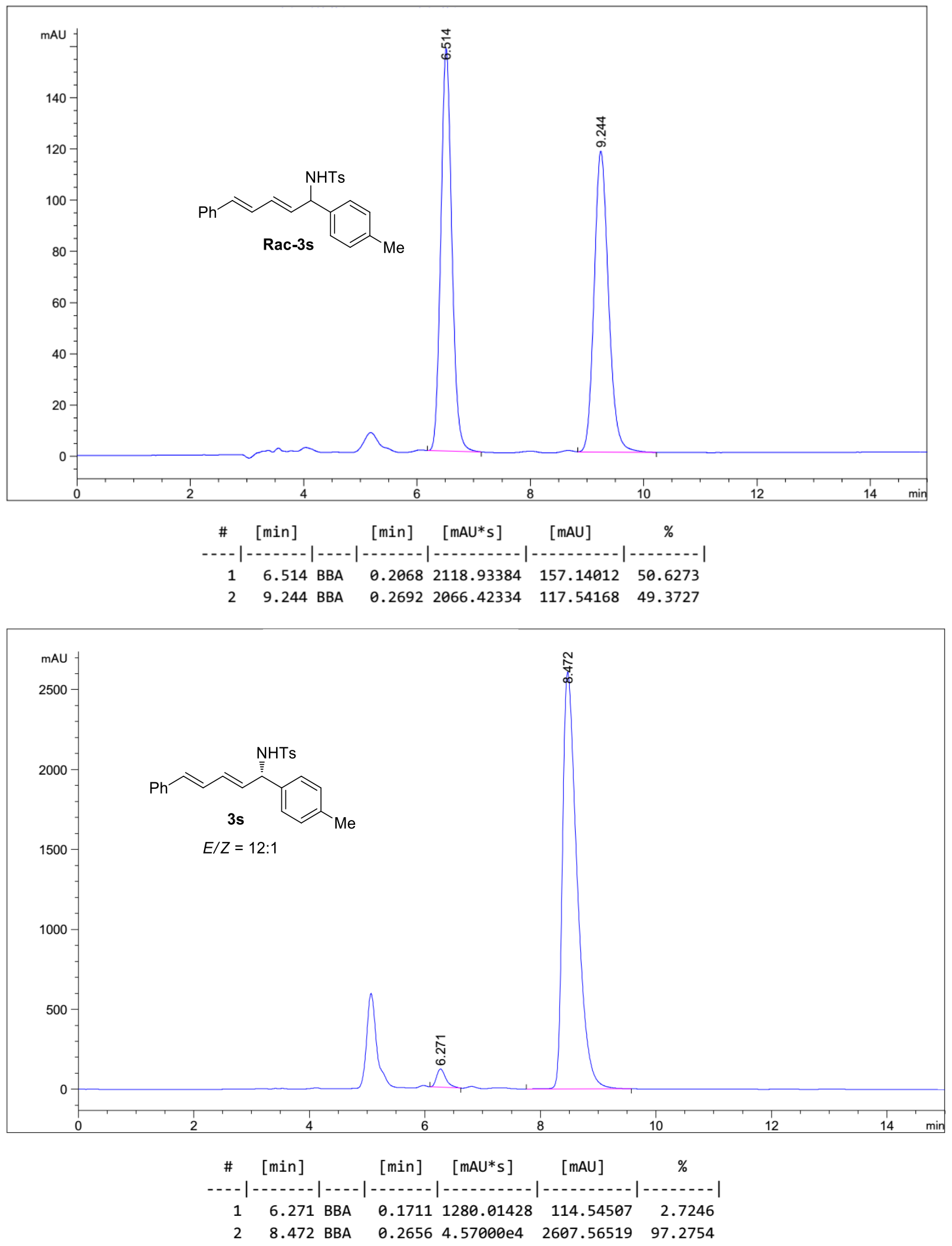
User Spectrum Plot Report

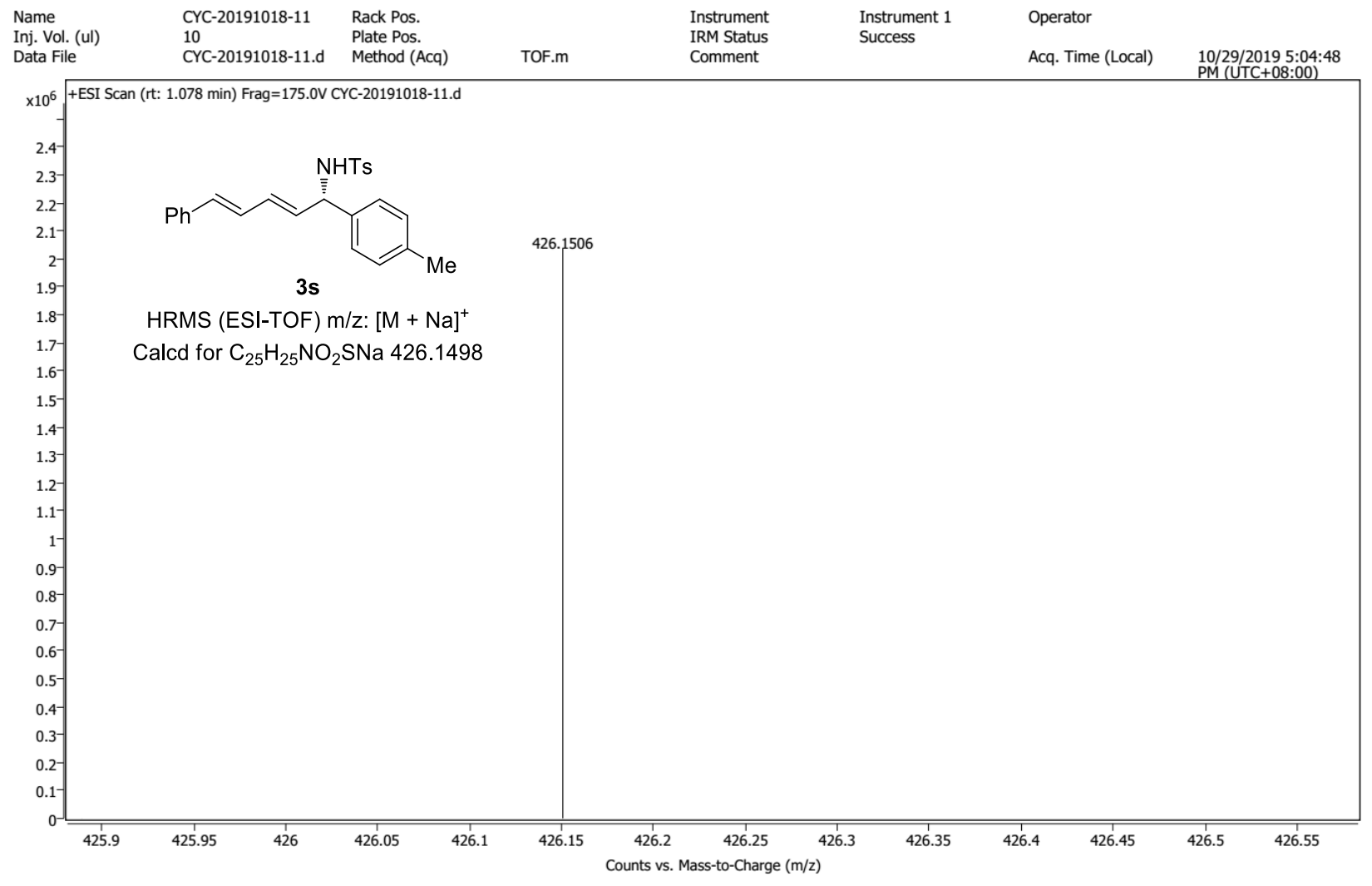




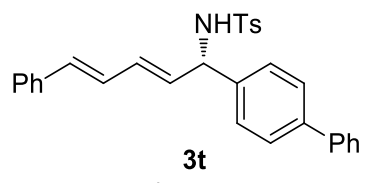

$E / Z=13: 1$

${ }^{1} \mathrm{H}$ NMR (400M, $\left.\mathrm{CDCl}_{3}\right)$

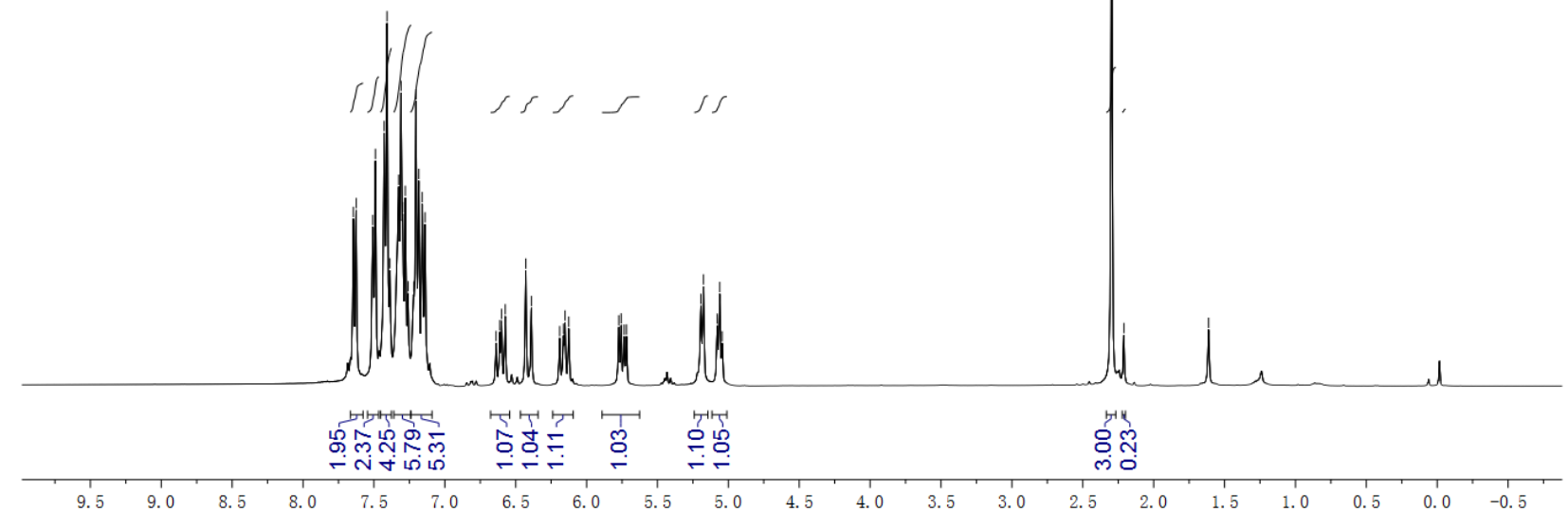

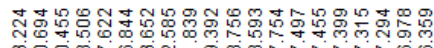

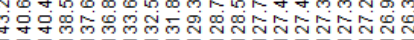

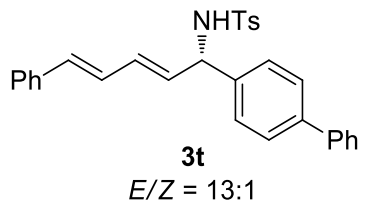

${ }^{13} \mathrm{C}$ NMR $\left(150 \mathrm{M}, \mathrm{CDCl}_{3}\right)$

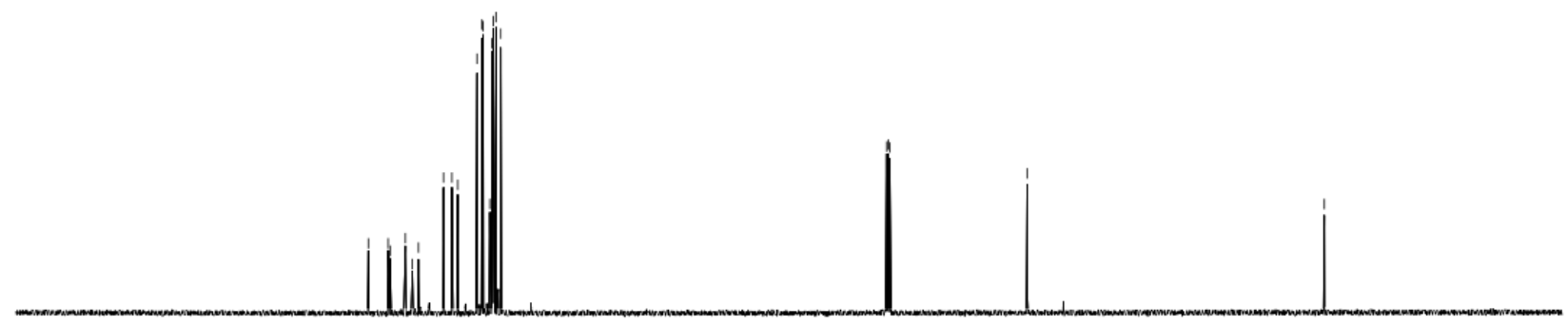



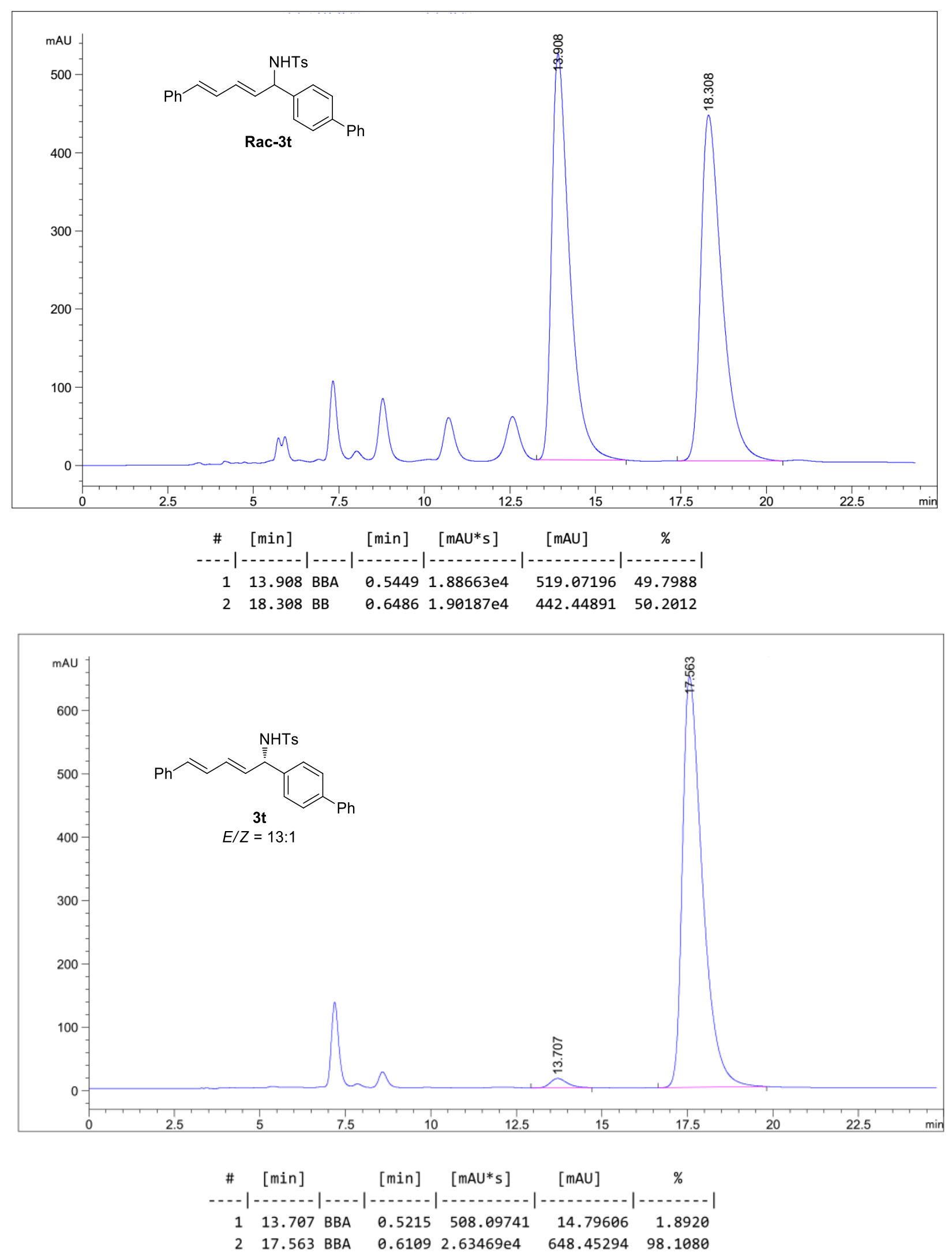


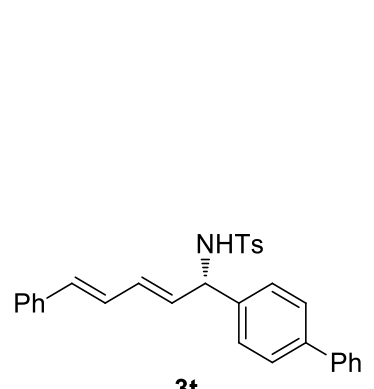

$3 t$

HRMS (ESI-TOF) m/z: [M + Na] ${ }^{+}$

Calcd for $\mathrm{C}_{25} \mathrm{H}_{25} \mathrm{NO}_{2} \mathrm{SNa} 488.1655$

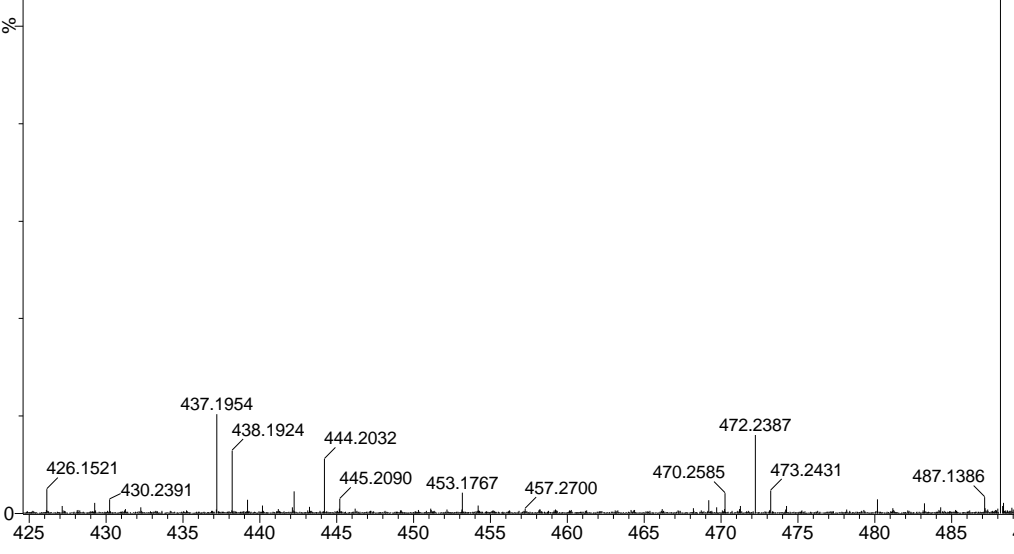

489.1706

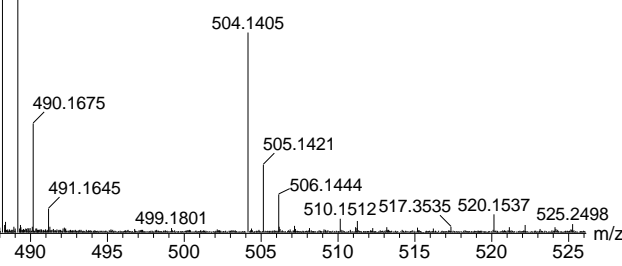




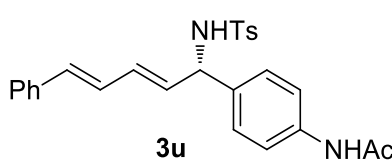

$E / Z=14: 1$

${ }^{1} \mathrm{H}$ NMR (400M, $\left.\mathrm{CDCl}_{3}\right)$

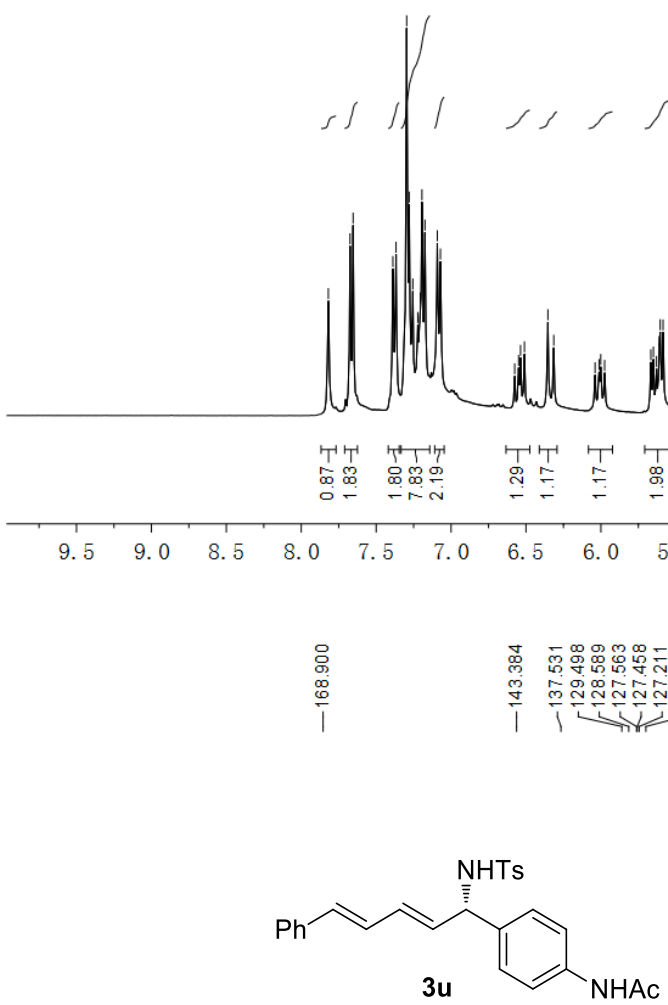

$E / Z=14: 1$

${ }^{13} \mathrm{C}$ NMR $\left(100 \mathrm{M}, \mathrm{CDCl}_{3}\right)$

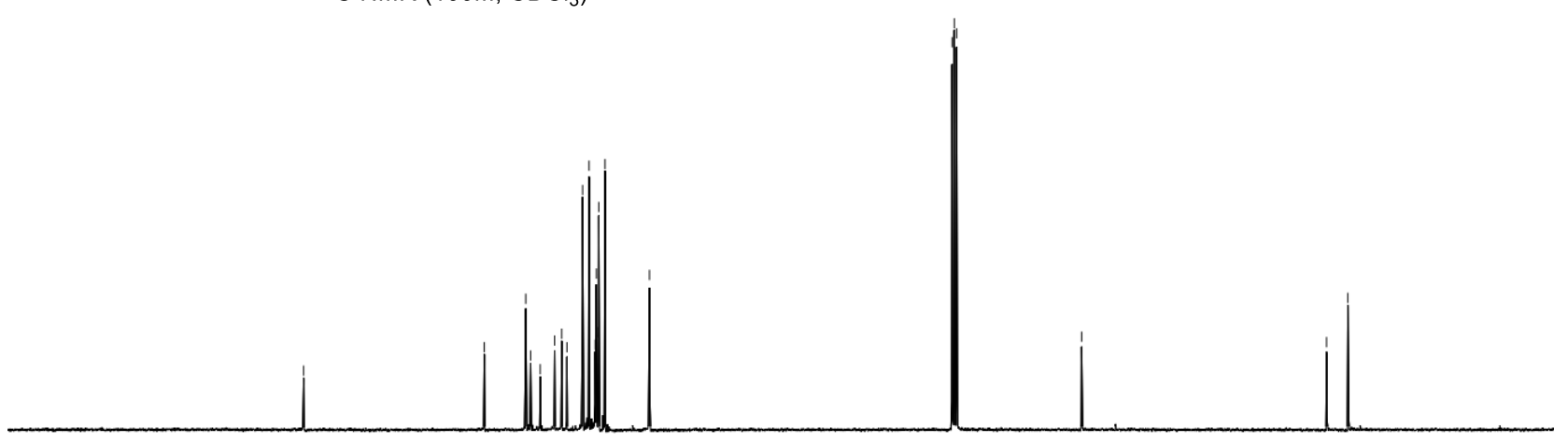

10

f1 (ppm)
䨔

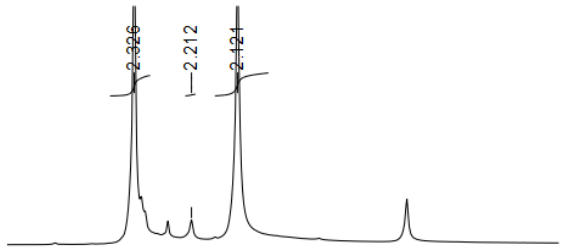

$1+\frac{1}{1}$

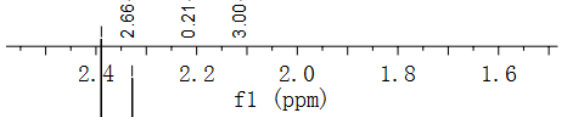

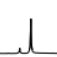

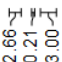

$\mathrm{f} 1_{\mathrm{fpm})}^{4.5} 4$.

赫 


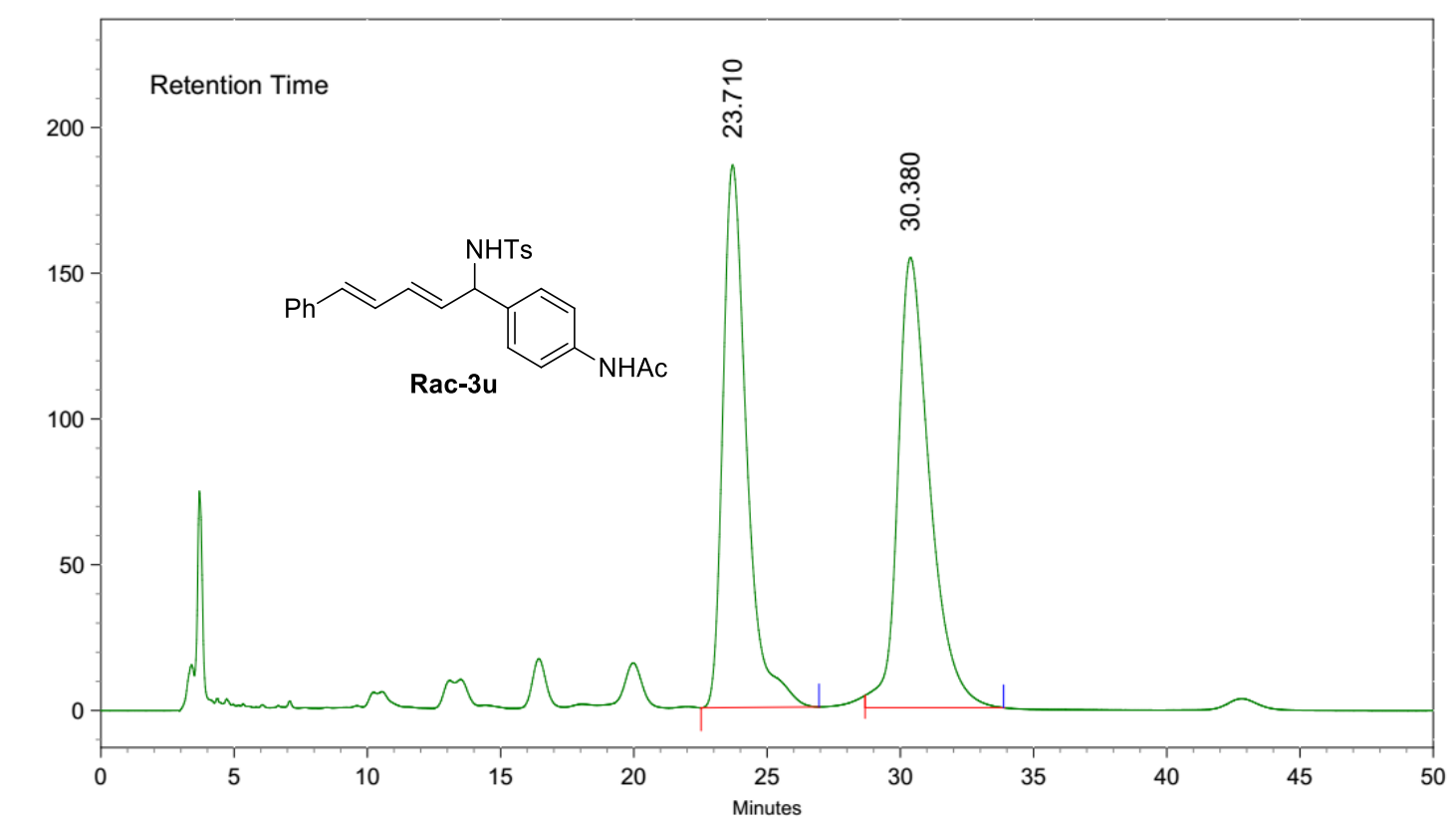

\begin{tabular}{llllll} 
Peak No. & Ret Time & Width & Height & Area & Area [\%] \\
\hline 1 & 23.710 & 4.427 & 3123820 & 194049452 & 47.3075 \\
2 & 30.380 & 5.196 & 2591644 & 216137700 & 52.6925
\end{tabular}

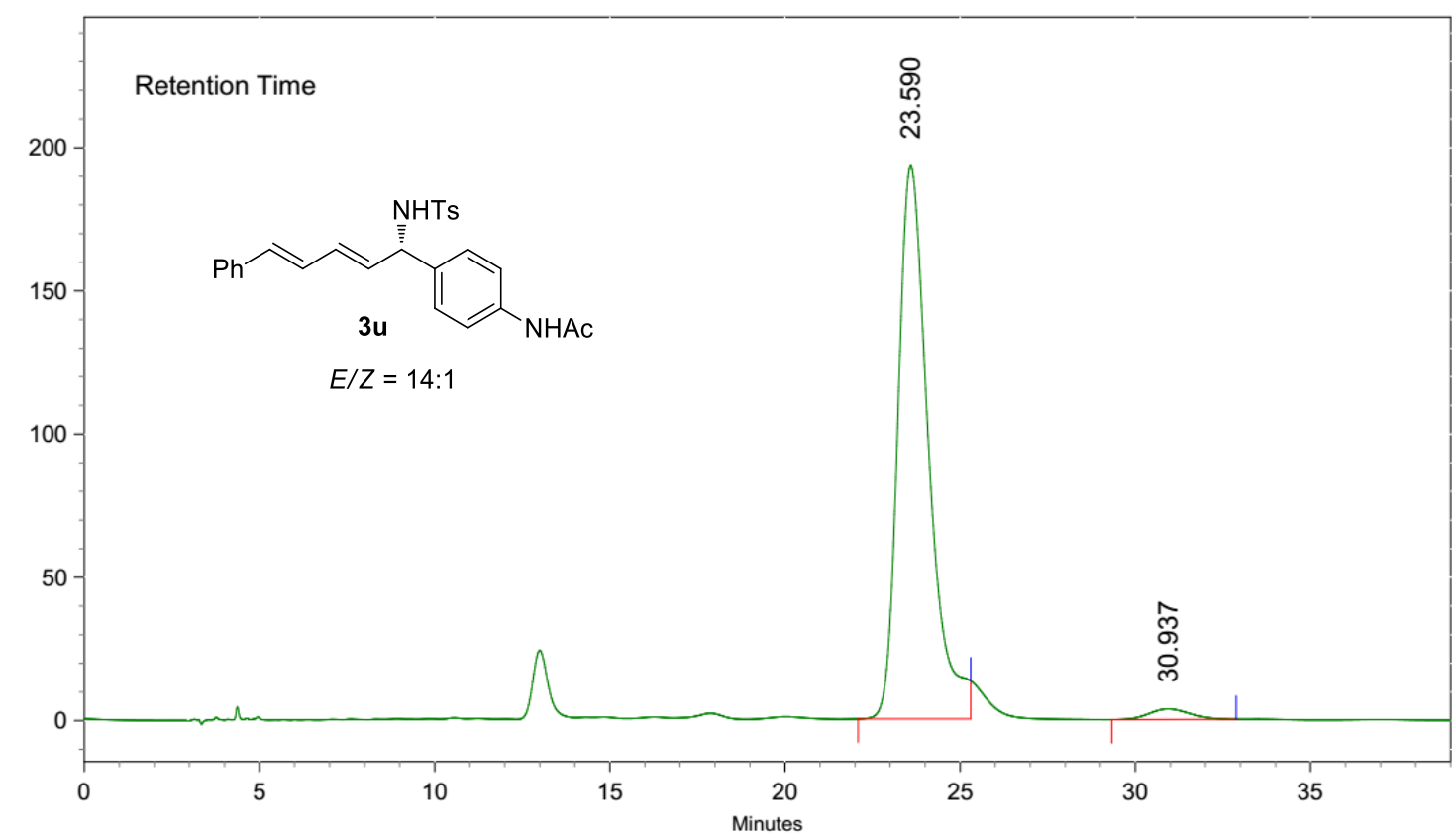

\begin{tabular}{llllll} 
Peak No. & Ret Time & Width & Height & Area & Area [\%] \\
\hline 1 & 23.590 & 3.213 & 3238335 & 195852952 & 97.5046 \\
2 & 30.937 & 3.553 & 61783 & 5012347 & 2.4954
\end{tabular}




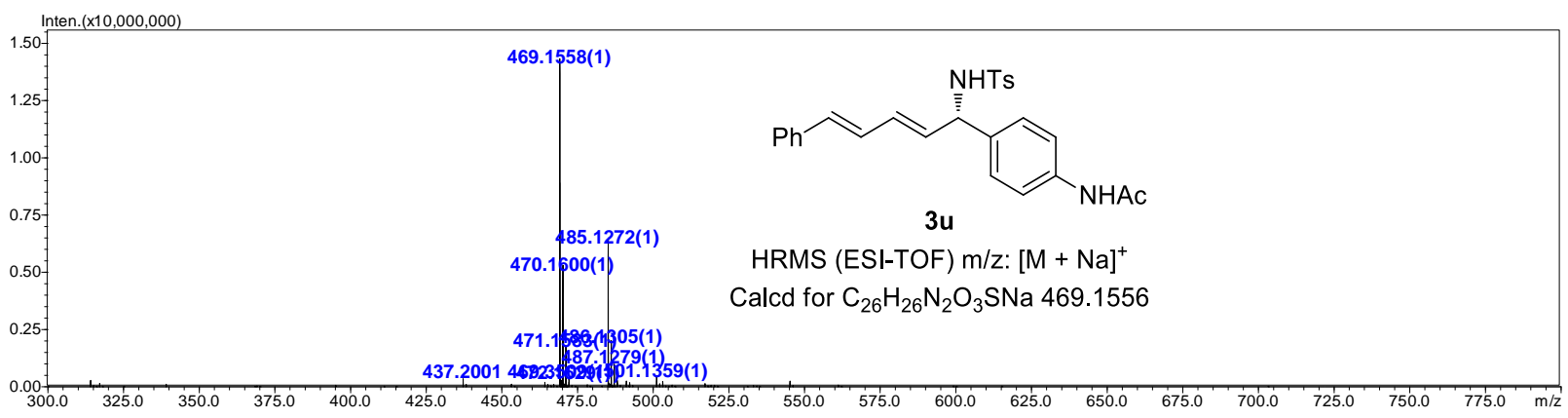




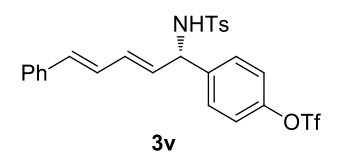

$E / Z>19: 1$

${ }^{1} \mathrm{H}$ NMR (400M, $\mathrm{CDCl}_{3}$ )

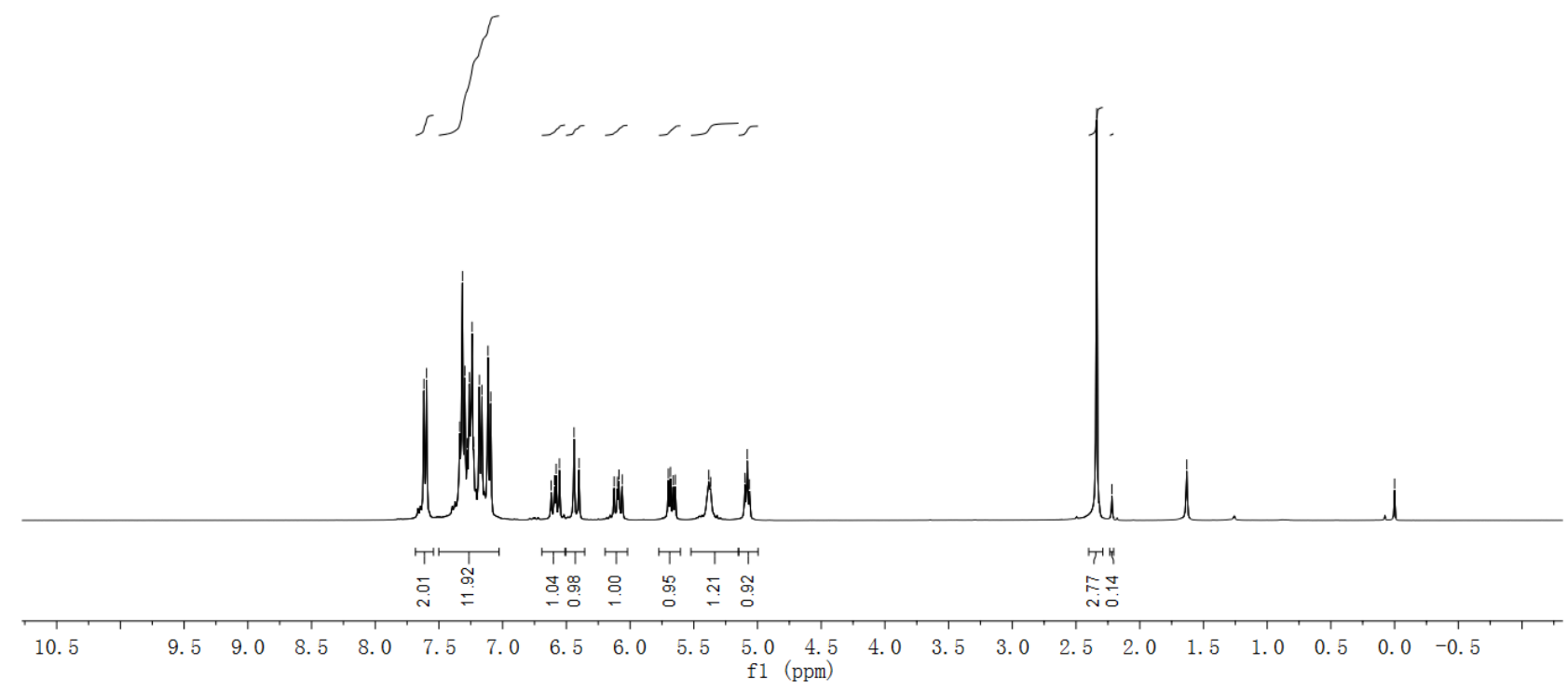

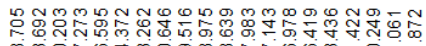

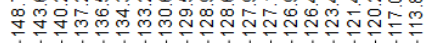

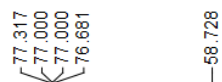

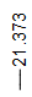

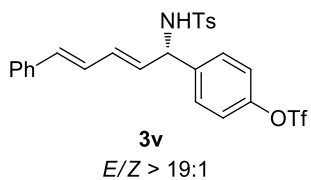

${ }^{13} \mathrm{C}$ NMR $\left(100 \mathrm{M}, \mathrm{CDCl}_{3}\right)$

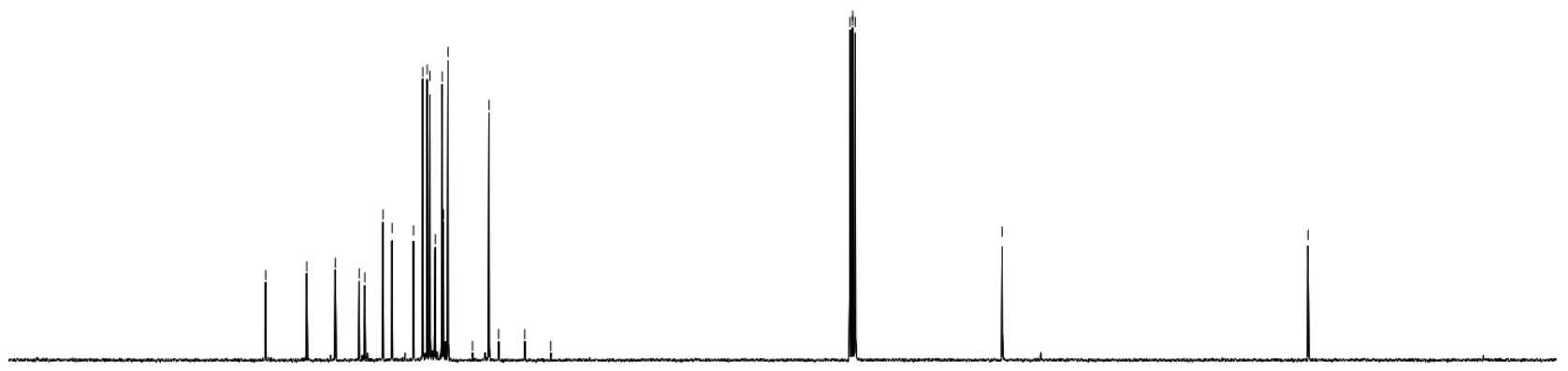

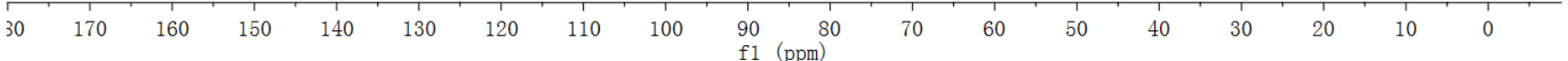




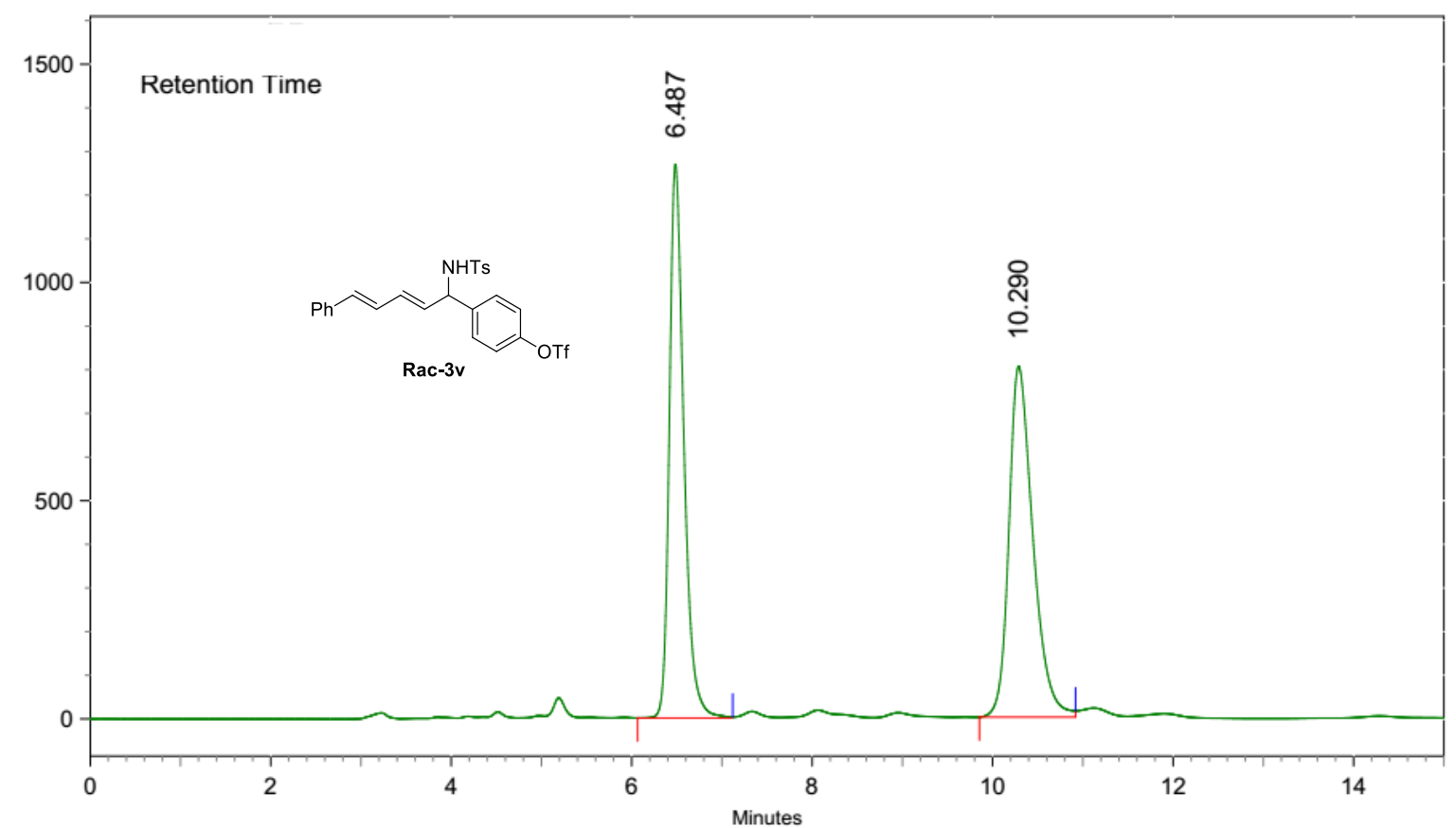

\begin{tabular}{llllll} 
Peak No. & Ret Time & Width & Height & Area & Area [\%] \\
\hline 1 & 6.487 & 1.053 & 21286041 & 237745334 & 48.7712 \\
2 & 10.290 & 1.067 & 13485647 & 249725316 & 51.2288
\end{tabular}

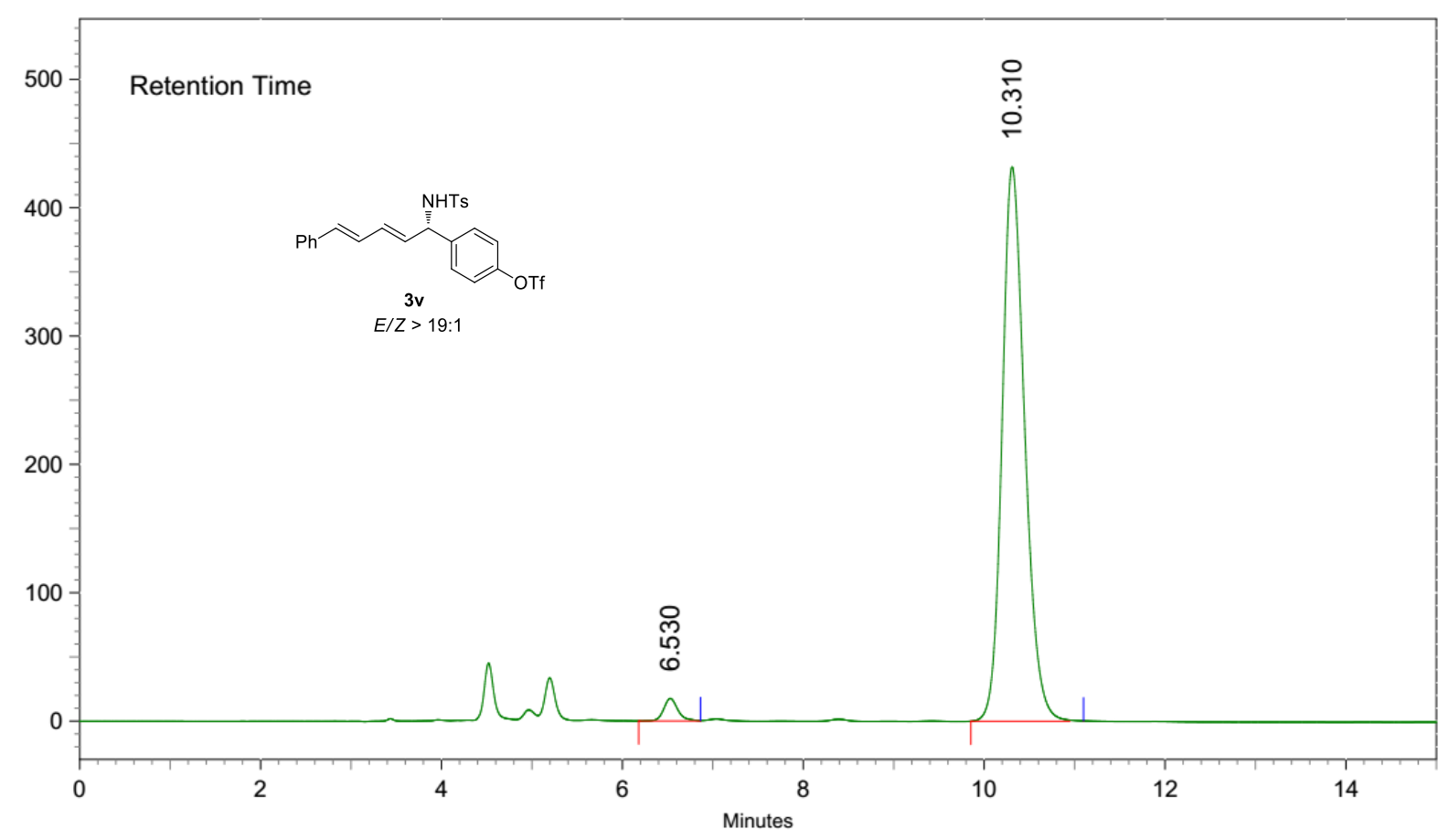

\begin{tabular}{llllll} 
Peak No. & Ret Time & Width & Height & Area & Area [\%] \\
\hline 1 & 6.530 & 0.683 & 294288 & 3242864 & 2.5063 \\
2 & 10.310 & 1.247 & 7246553 & 126146133 & 97.4937
\end{tabular}




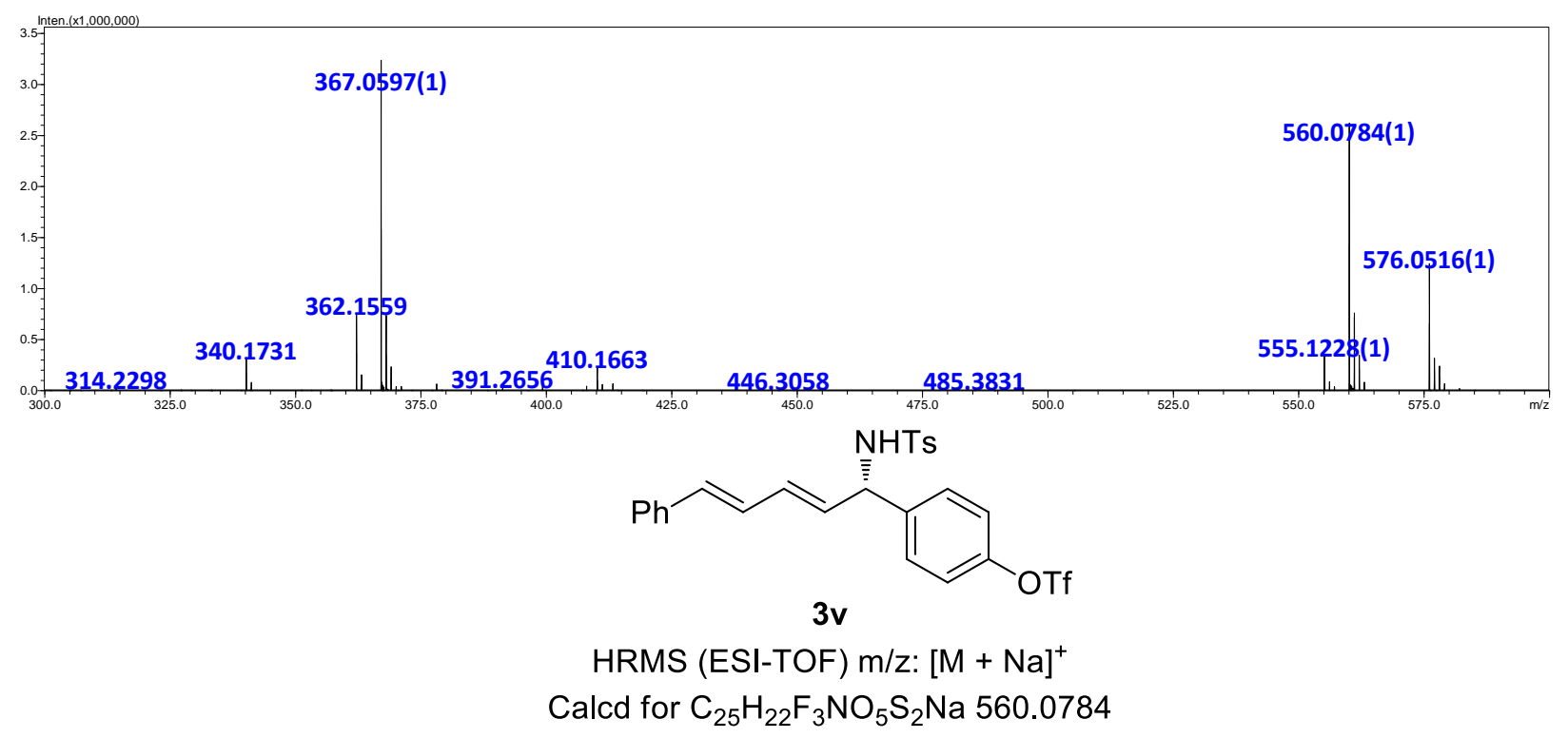




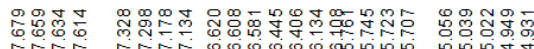

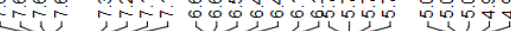

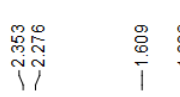

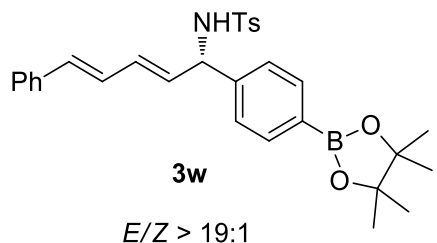

${ }^{1} \mathrm{H} \mathrm{NMR} \mathrm{(400M,} \mathrm{CDCl}_{3}$ )
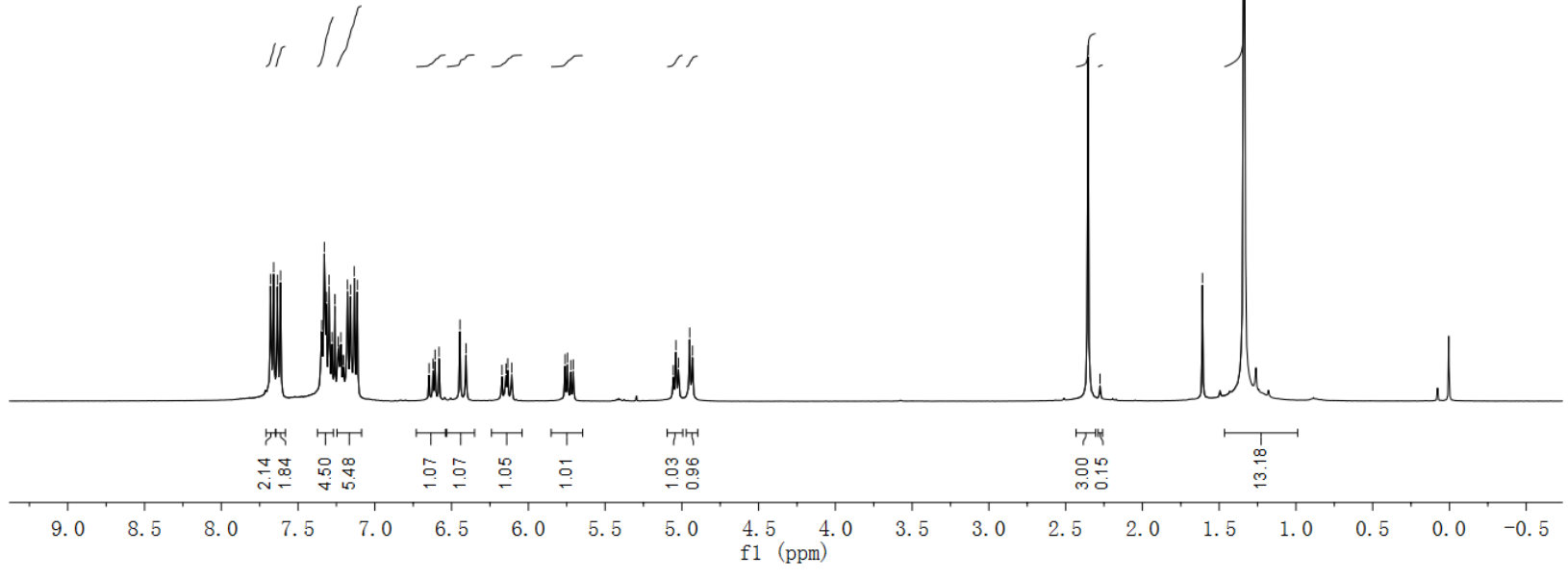

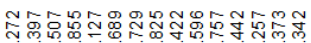

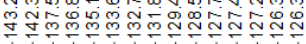

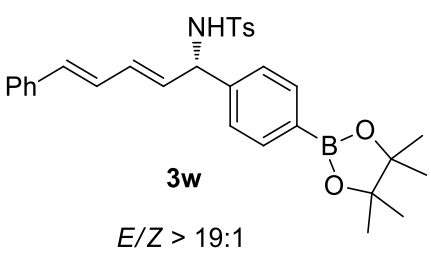

${ }^{13} \mathrm{C}$ NMR $\left(100 \mathrm{M}, \mathrm{CDCl}_{3}\right)$

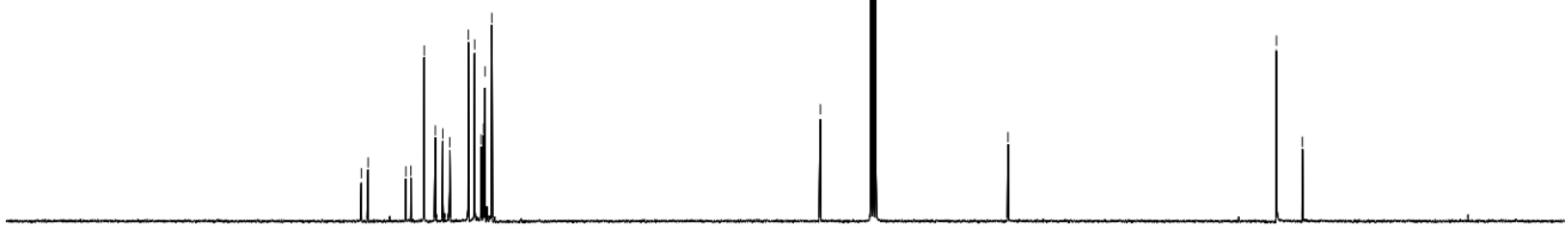




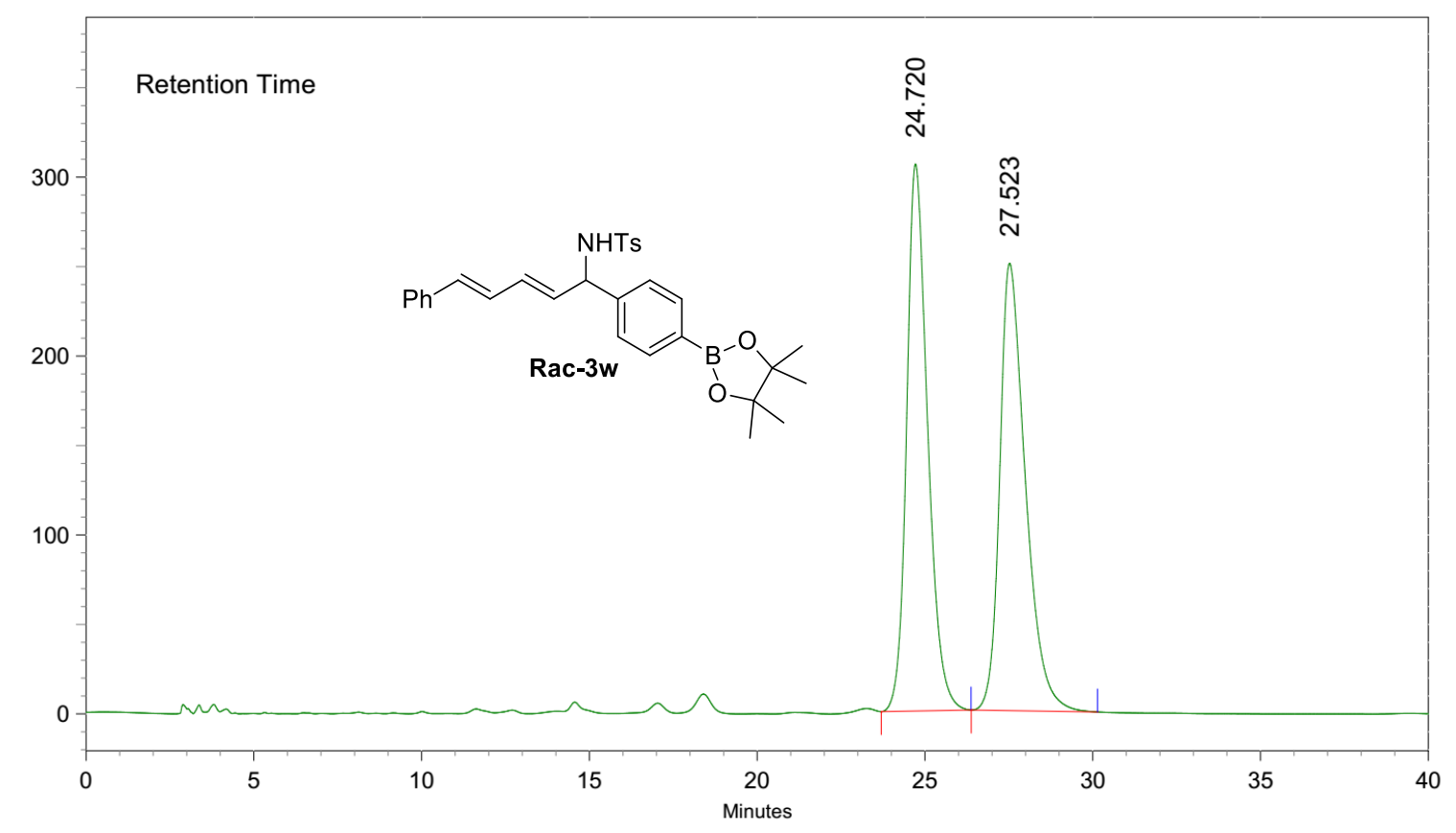

\begin{tabular}{llllll} 
Peak No. & Ret Time & Width & Height & Area & Area [\%] \\
\hline 1 & 24.720 & 2.677 & 5130086 & 222526108 & 49.9531 \\
2 & 27.523 & 3.763 & 4195664 & 222944256 & 50.0469
\end{tabular}

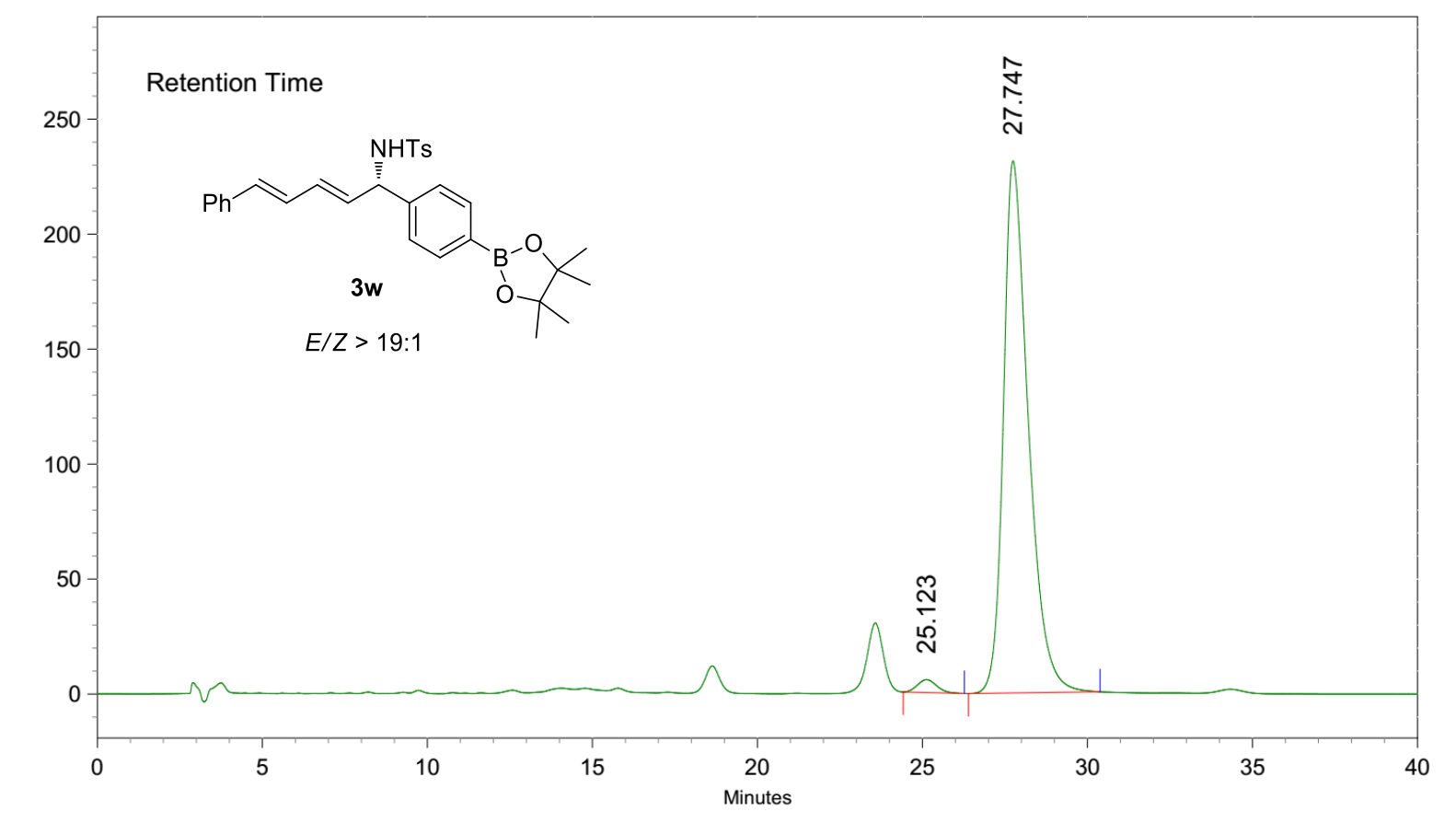

\begin{tabular}{llllll} 
Peak No. & Ret Time & Width & Height & Area & Area [\%] \\
\hline 1 & 25.123 & 1.850 & 94162 & 3799875 & 1.8337 \\
2 & 27.747 & 3.987 & 3881351 & 203428526 & 98.1663
\end{tabular}




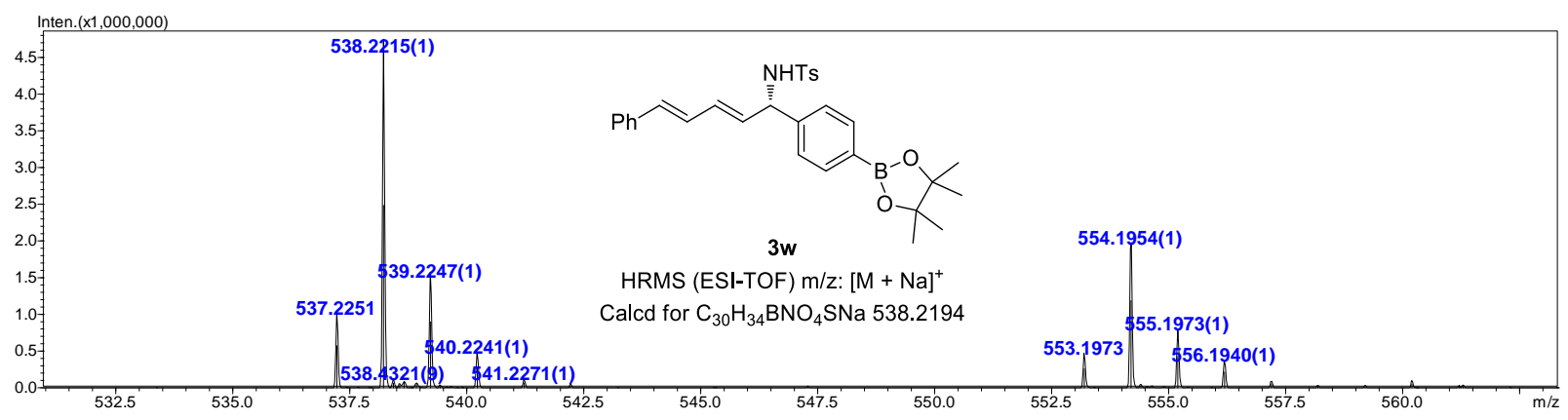




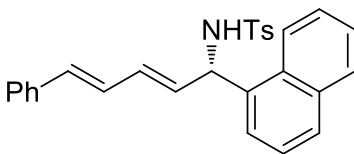

$3 \mathbf{x}$
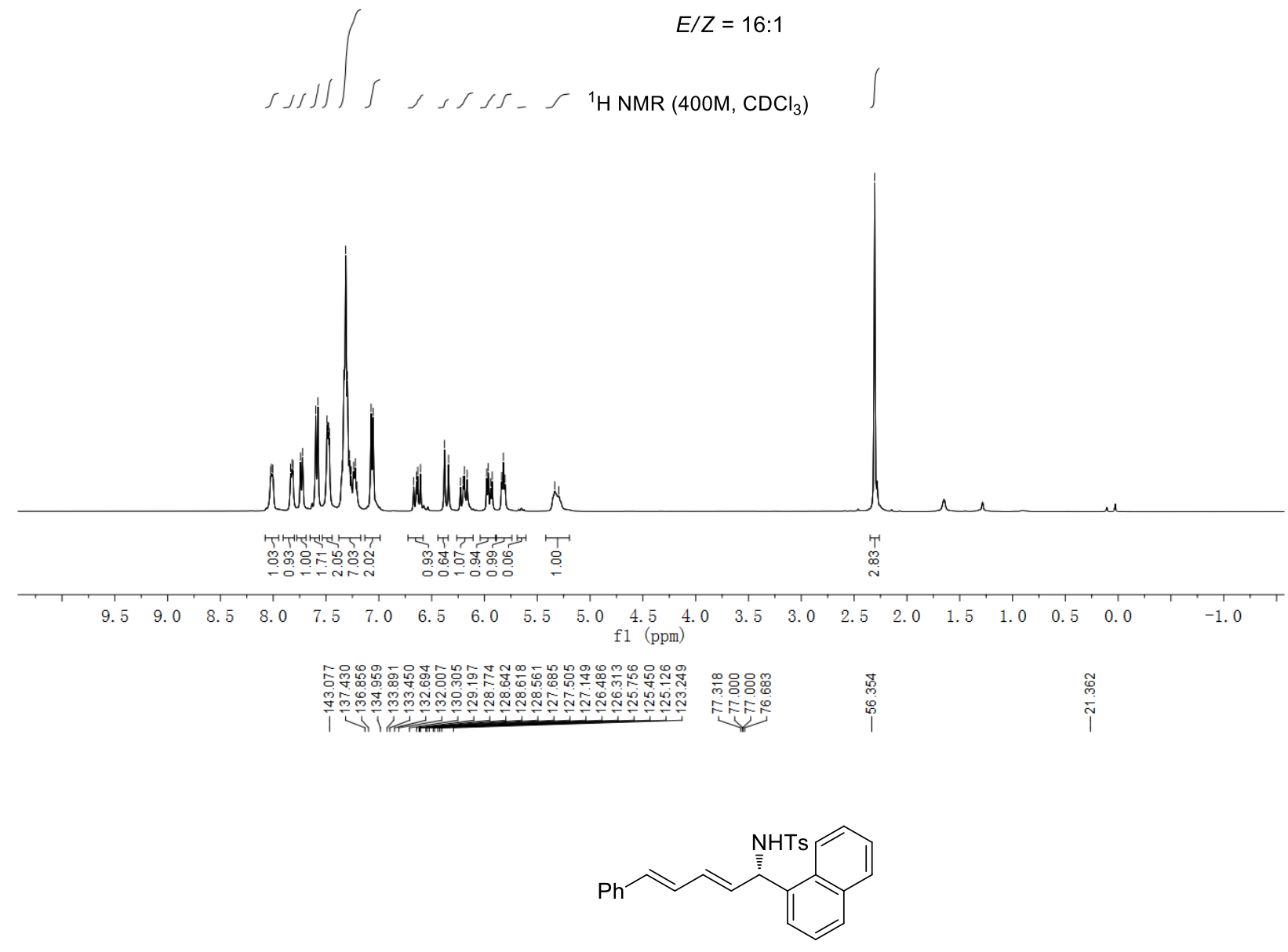

$3 x$

$E / Z=16: 1$

${ }^{13} \mathrm{C}$ NMR $\left(100 \mathrm{M}, \mathrm{CDCl}_{3}\right)$

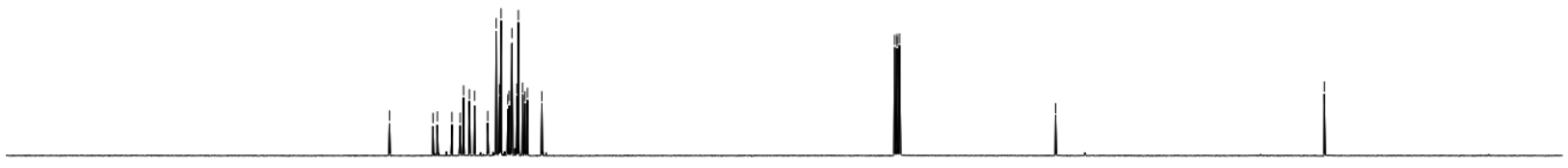



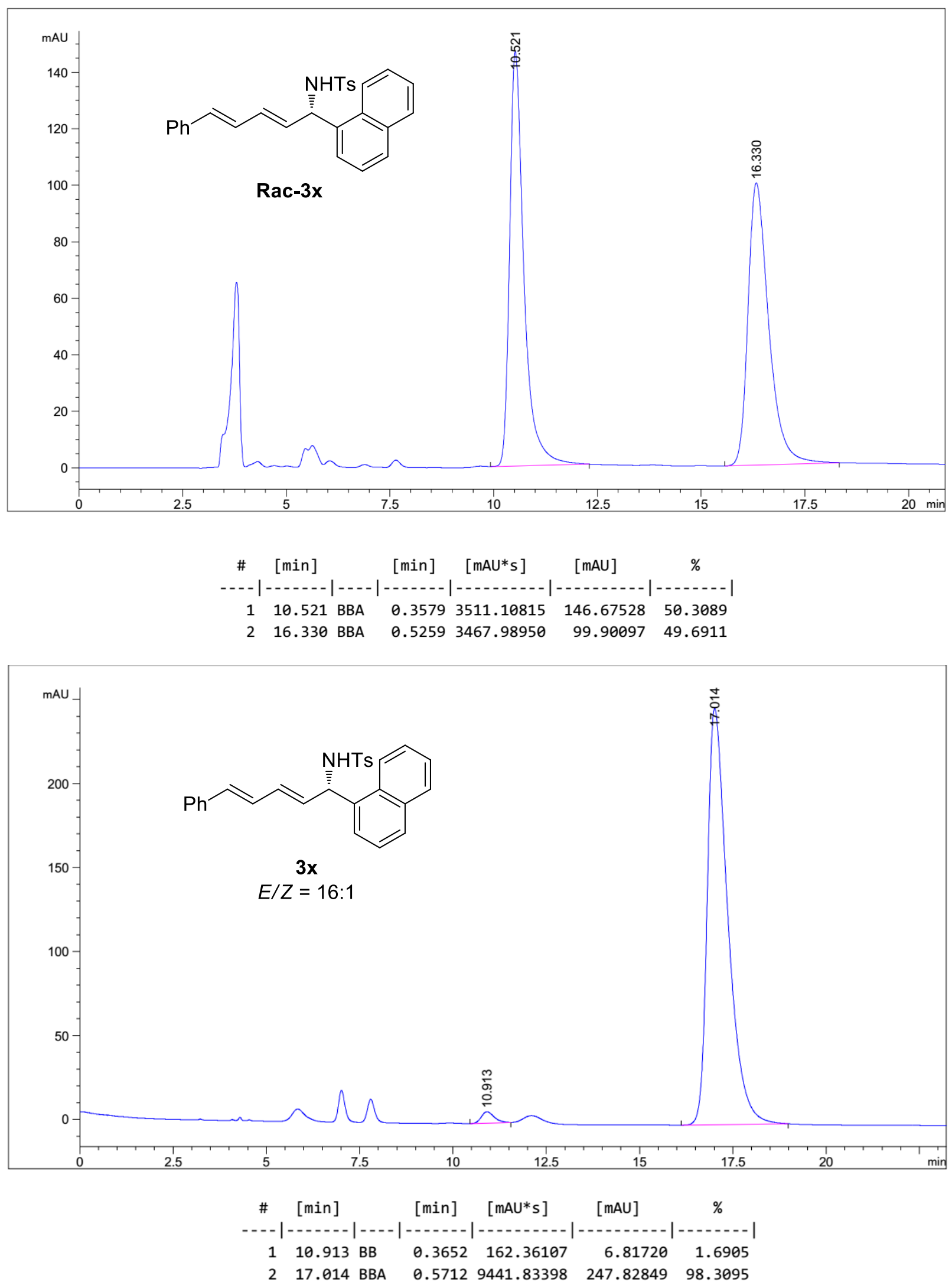
Spectrum Plot Report

Agilent

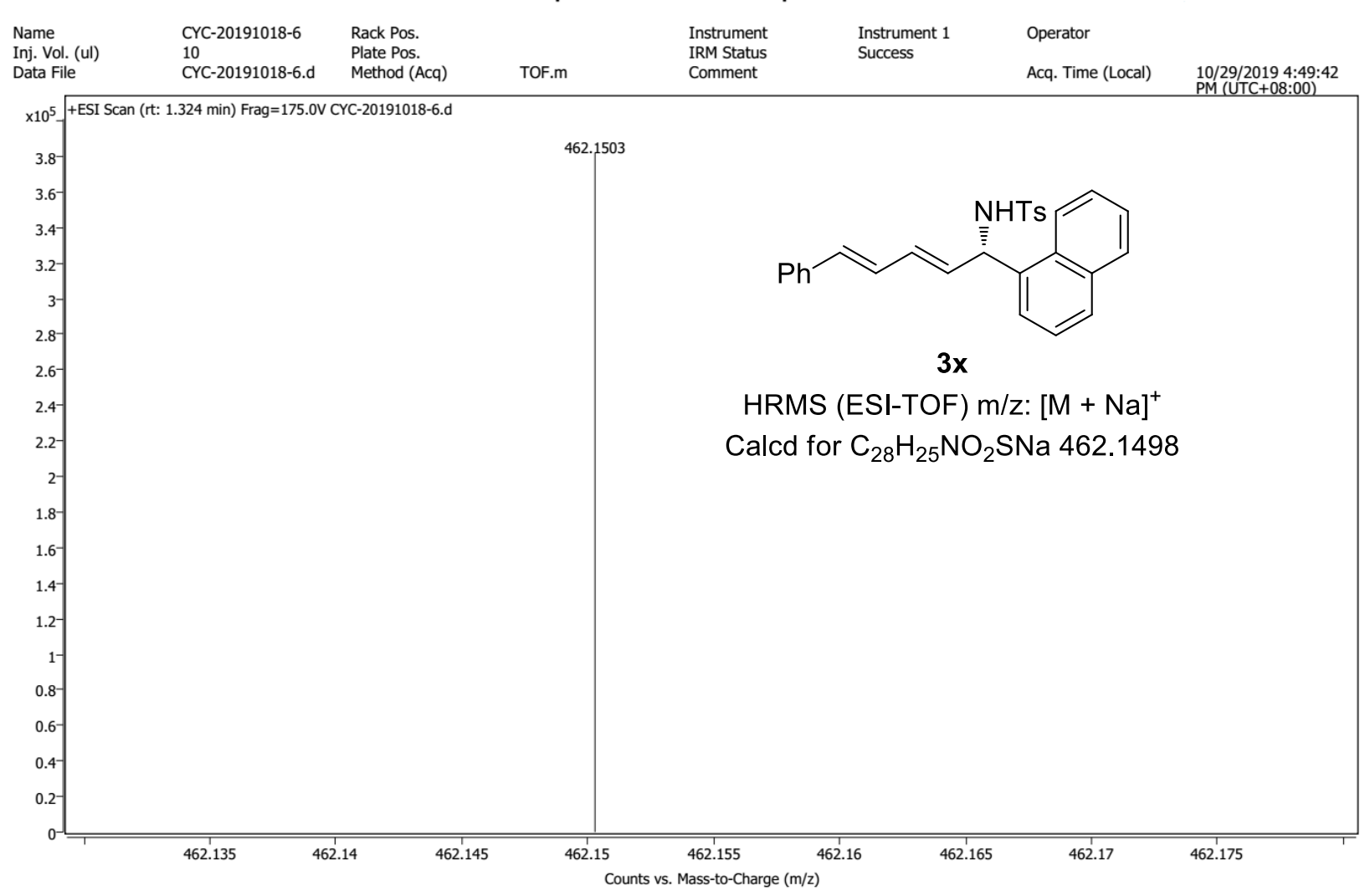




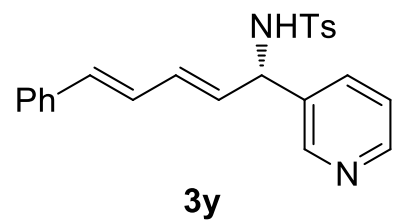

$E / Z>19: 1$

${ }^{1} \mathrm{H}$ NMR (400M, $\mathrm{CDCl}_{3}$ )

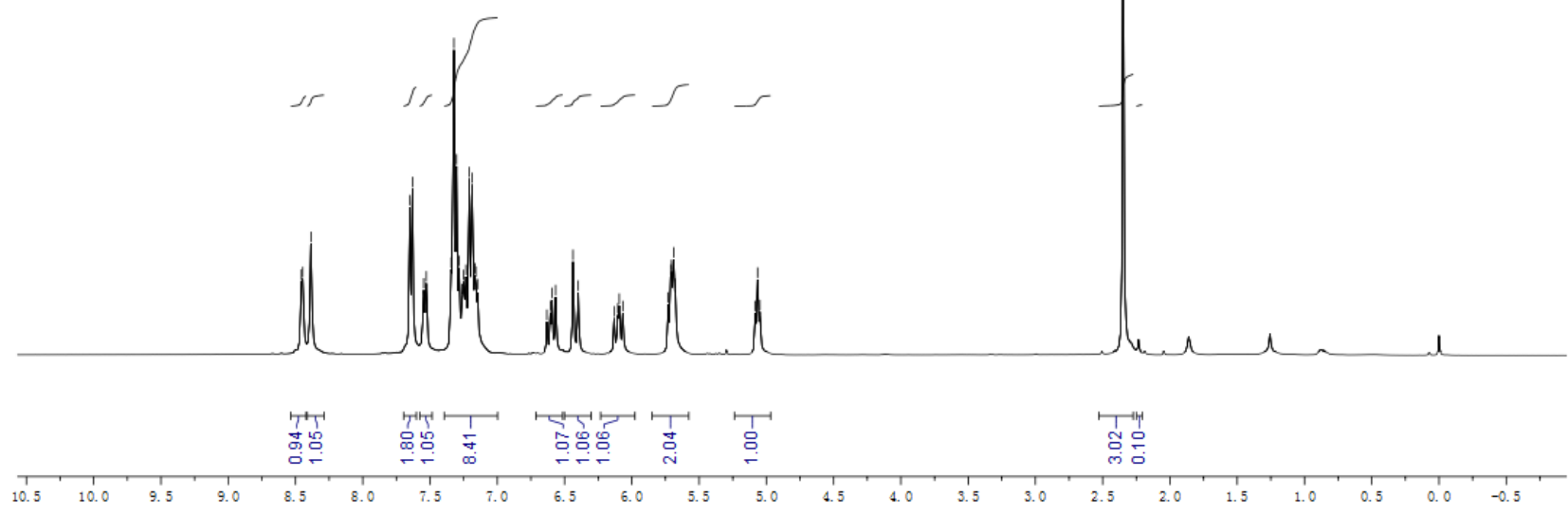

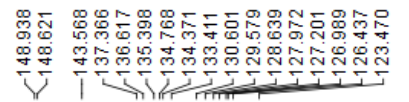<smiles>F[AsH3][C@H](/C=C/C=C/c1ccccc1)c1cccnc1</smiles>

$3 y$

$E / Z>19: 1$

${ }^{13} \mathrm{C}$ NMR $\left(100 \mathrm{M}, \mathrm{CDCl}_{3}\right)$

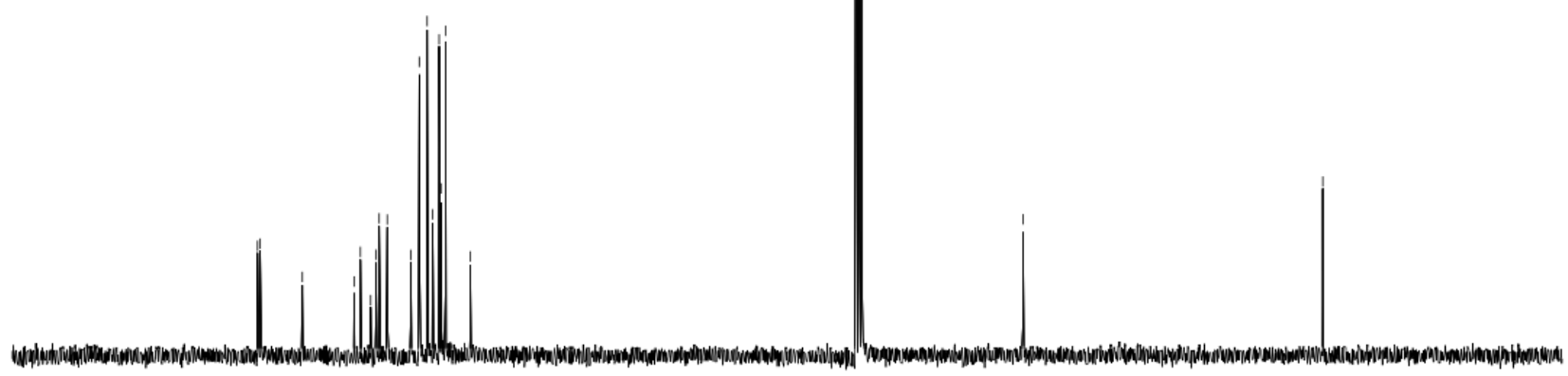



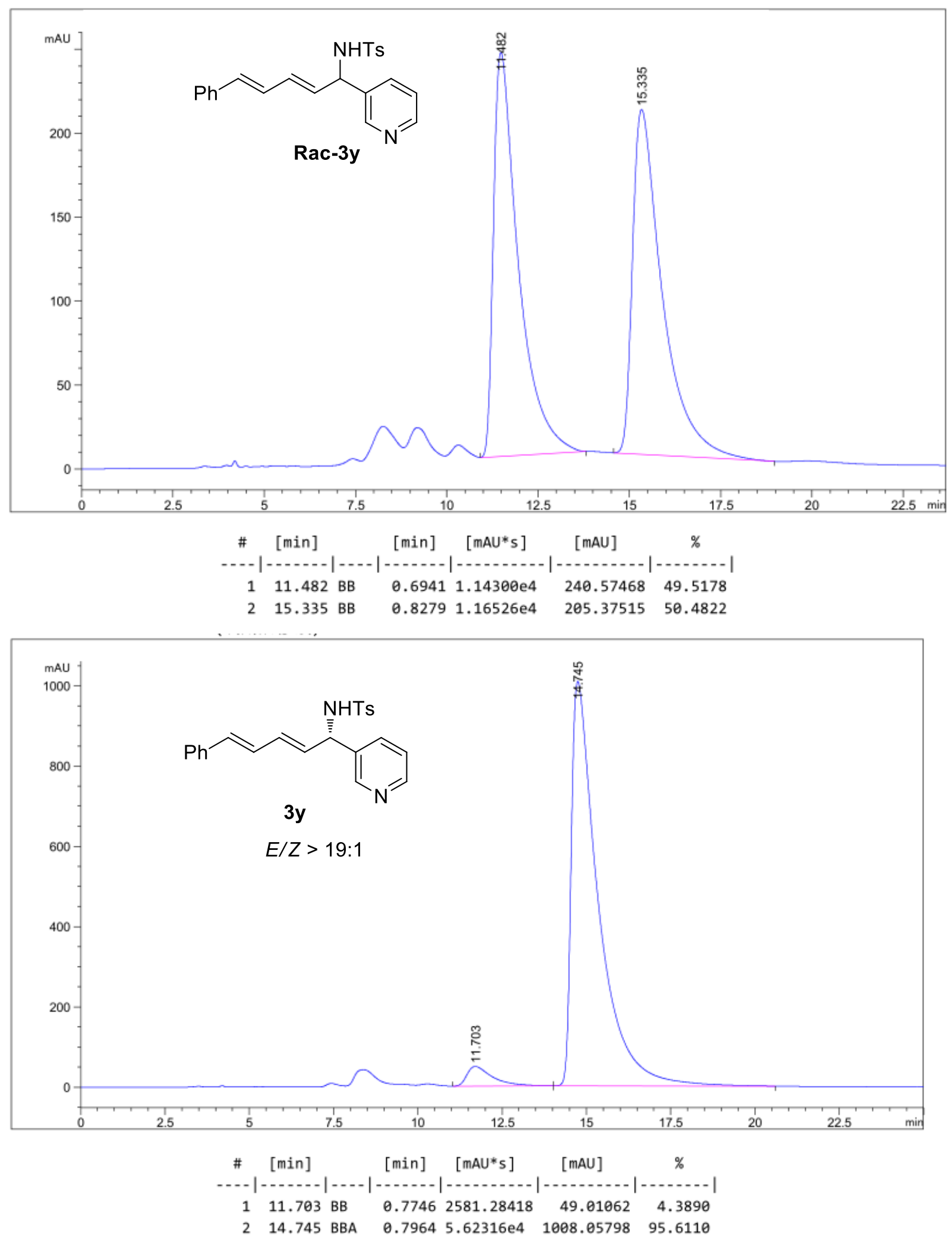


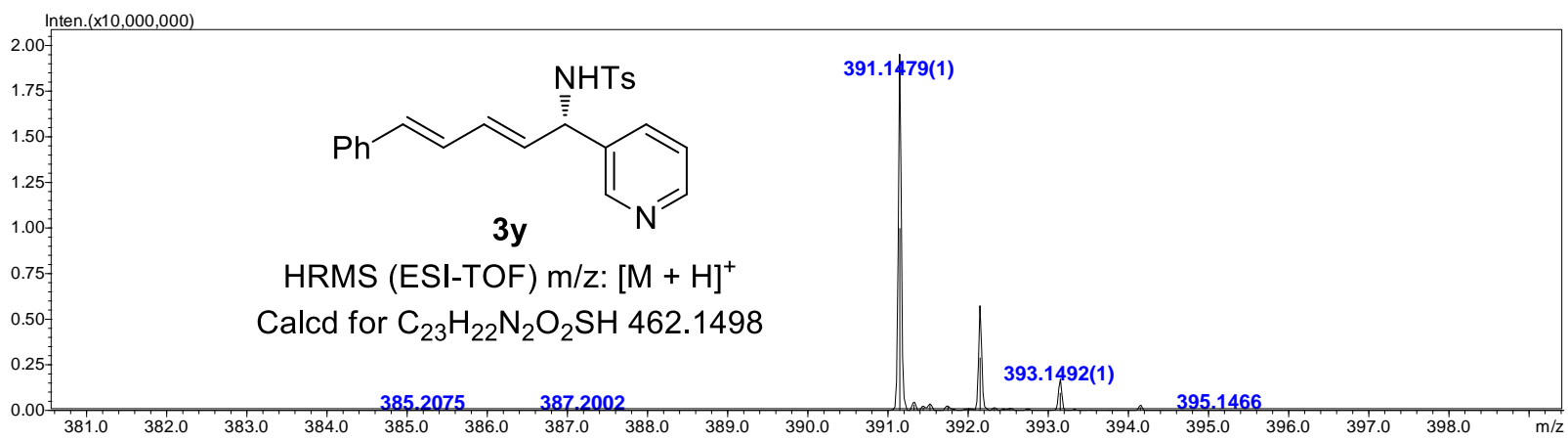




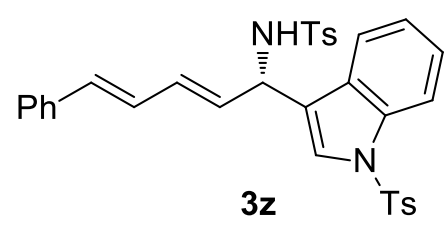

$E / Z>19: 1$

${ }^{1} \mathrm{H} \mathrm{NMR}\left(400 \mathrm{M}, \mathrm{CDCl}_{3}\right)$

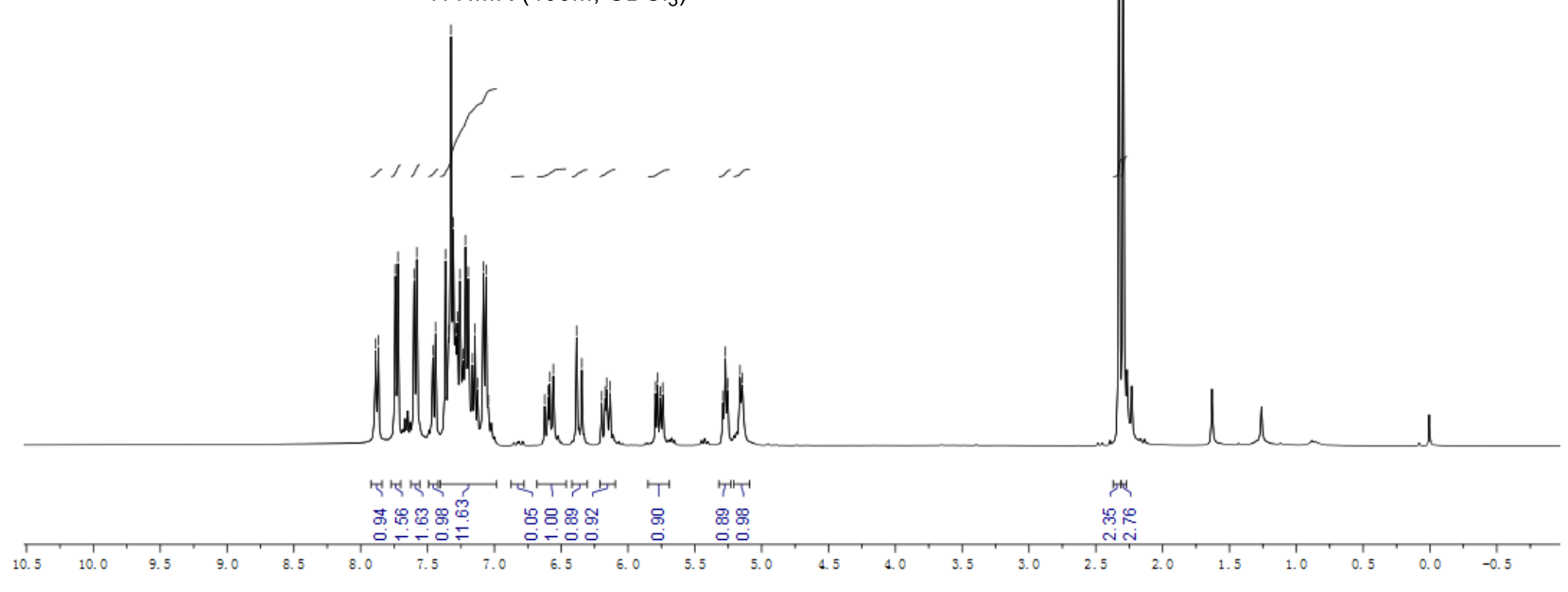

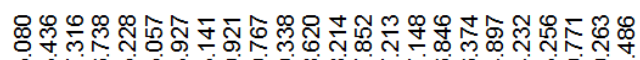

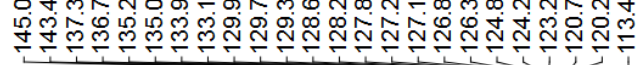

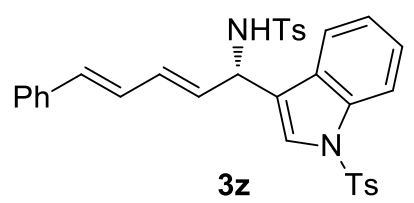

$E / Z>19: 1$

${ }^{13} \mathrm{C}$ NMR (100M, $\mathrm{CDCl}_{3}$ )

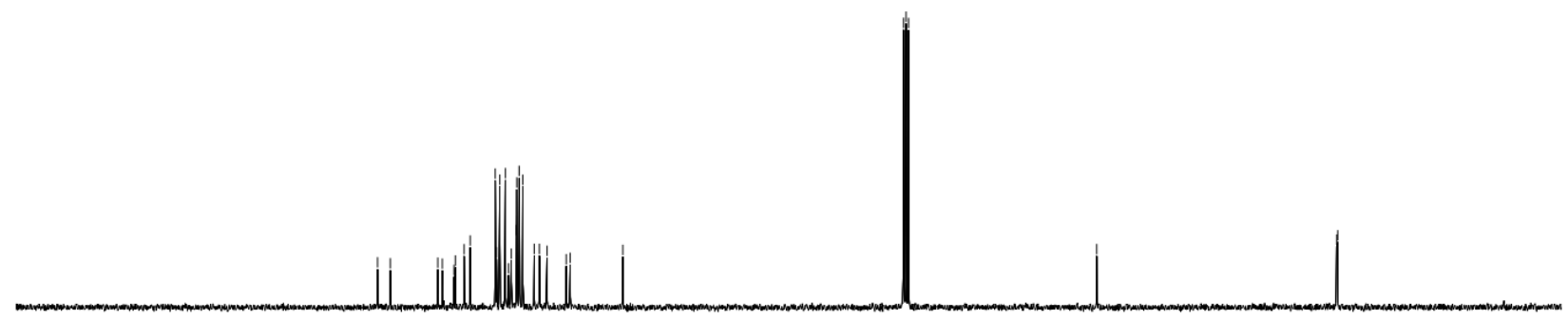

.

190

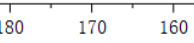

$150 \quad 140$

$130 \quad 120$

$80 \quad 70$

50 

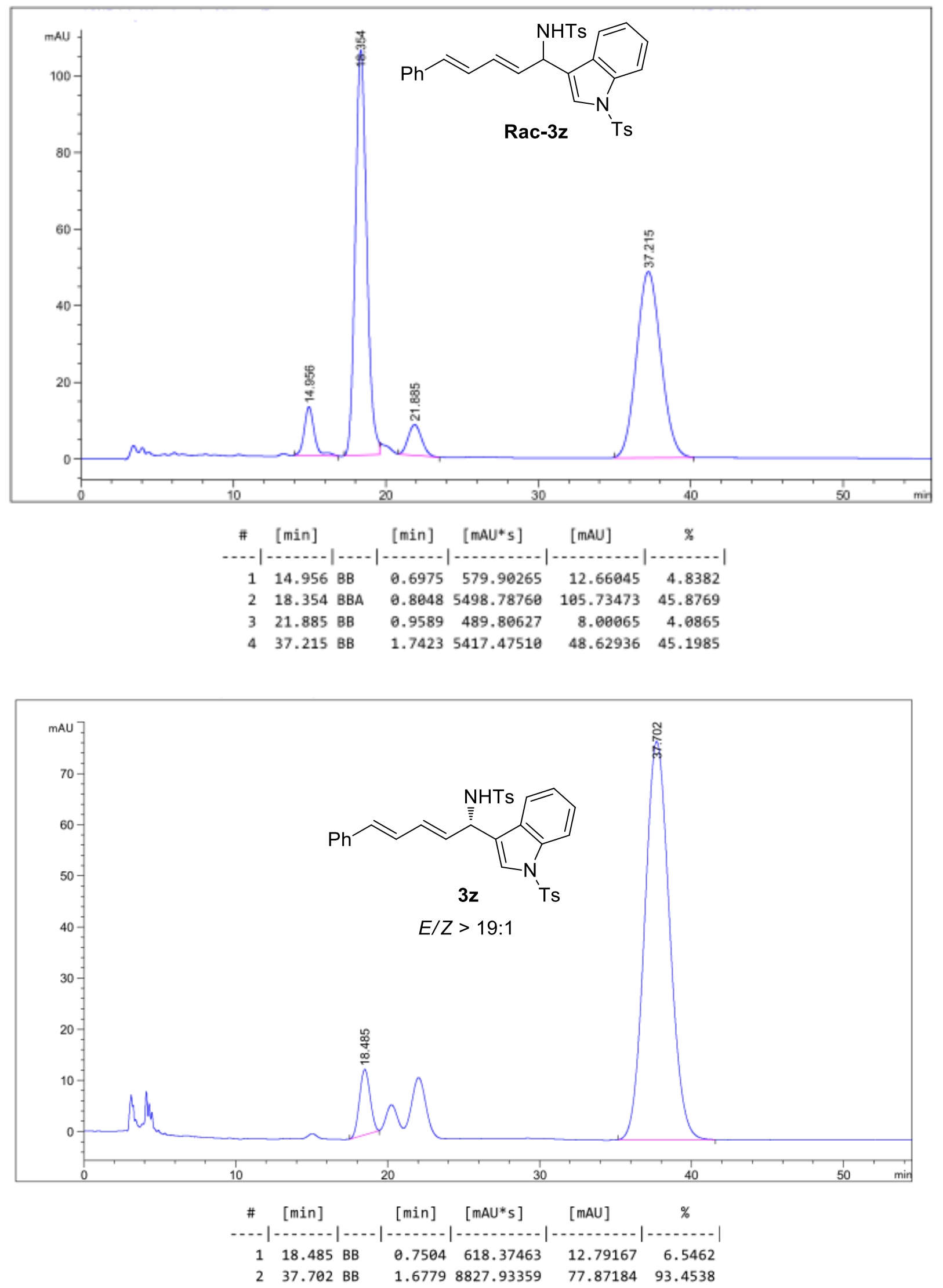


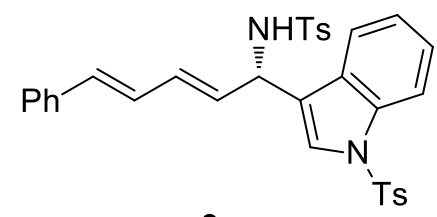

$3 z$

HRMS (ESI-TOF) m/z: [M + Na] $]^{+}$

Calcd for $\mathrm{C}_{33} \mathrm{H}_{30} \mathrm{~N}_{2} \mathrm{O}_{4} \mathrm{~S}_{2} \mathrm{Na} 605.1539$

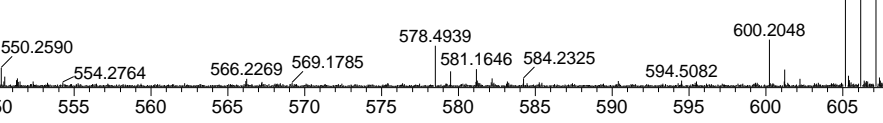

606.1583

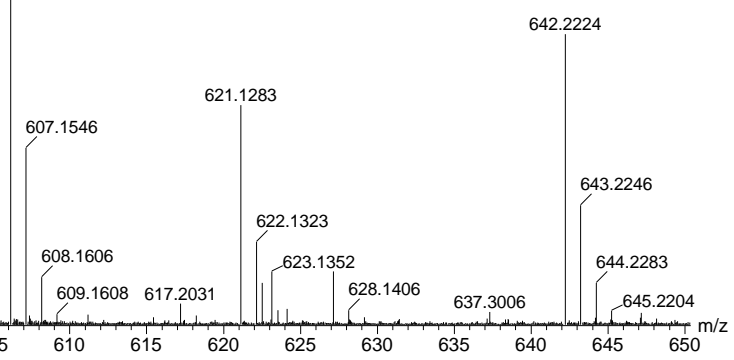




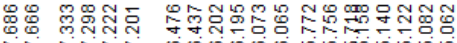

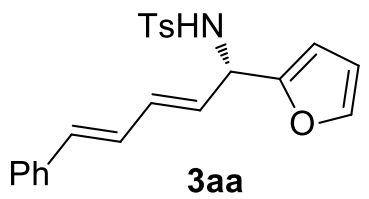

$E / Z=13: 1$

${ }^{1} \mathrm{H}$ NMR (400M, $\mathrm{CDCl}_{3}$ )

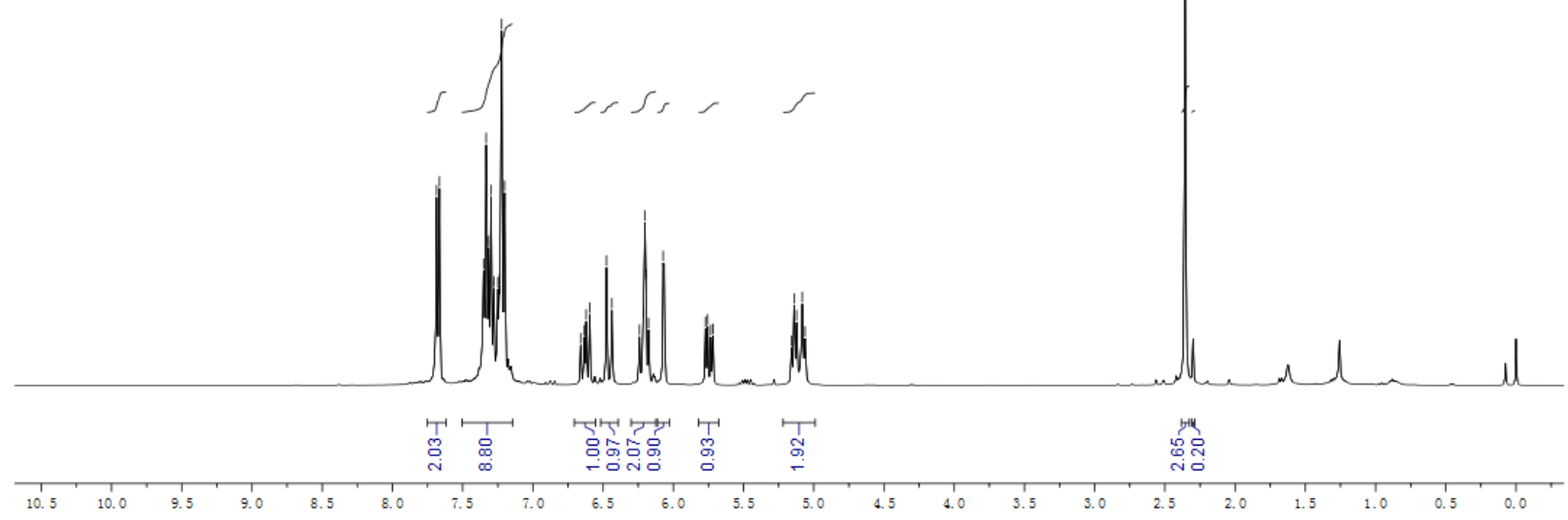

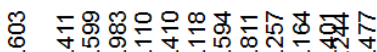

每

ल요용

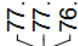

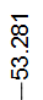

$\frac{\mathfrak{g}}{\stackrel{N}{N}}$

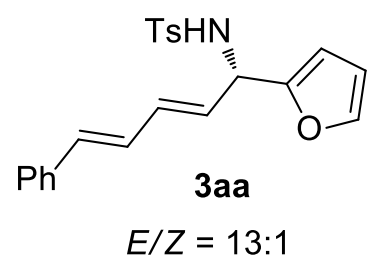

${ }^{13} \mathrm{C}$ NMR (100M, $\left.\mathrm{CDCl}_{3}\right)$

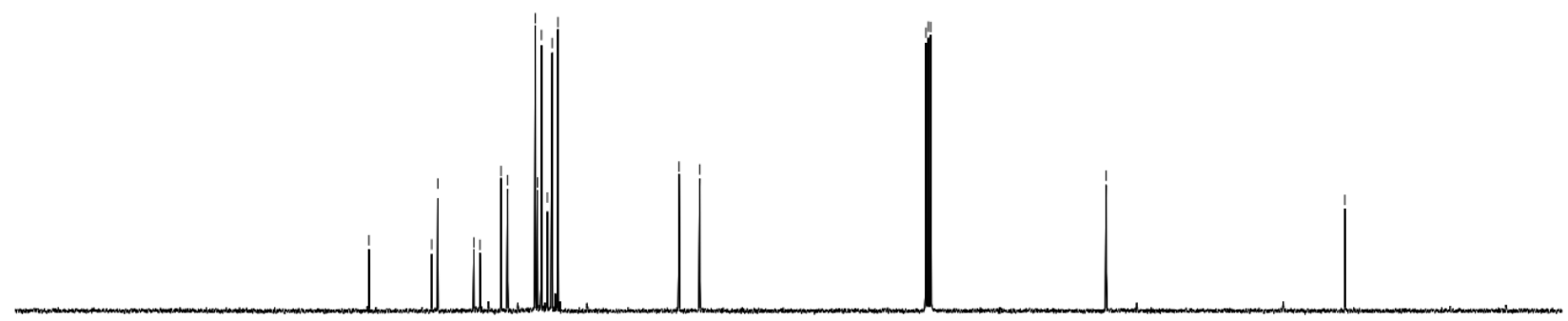



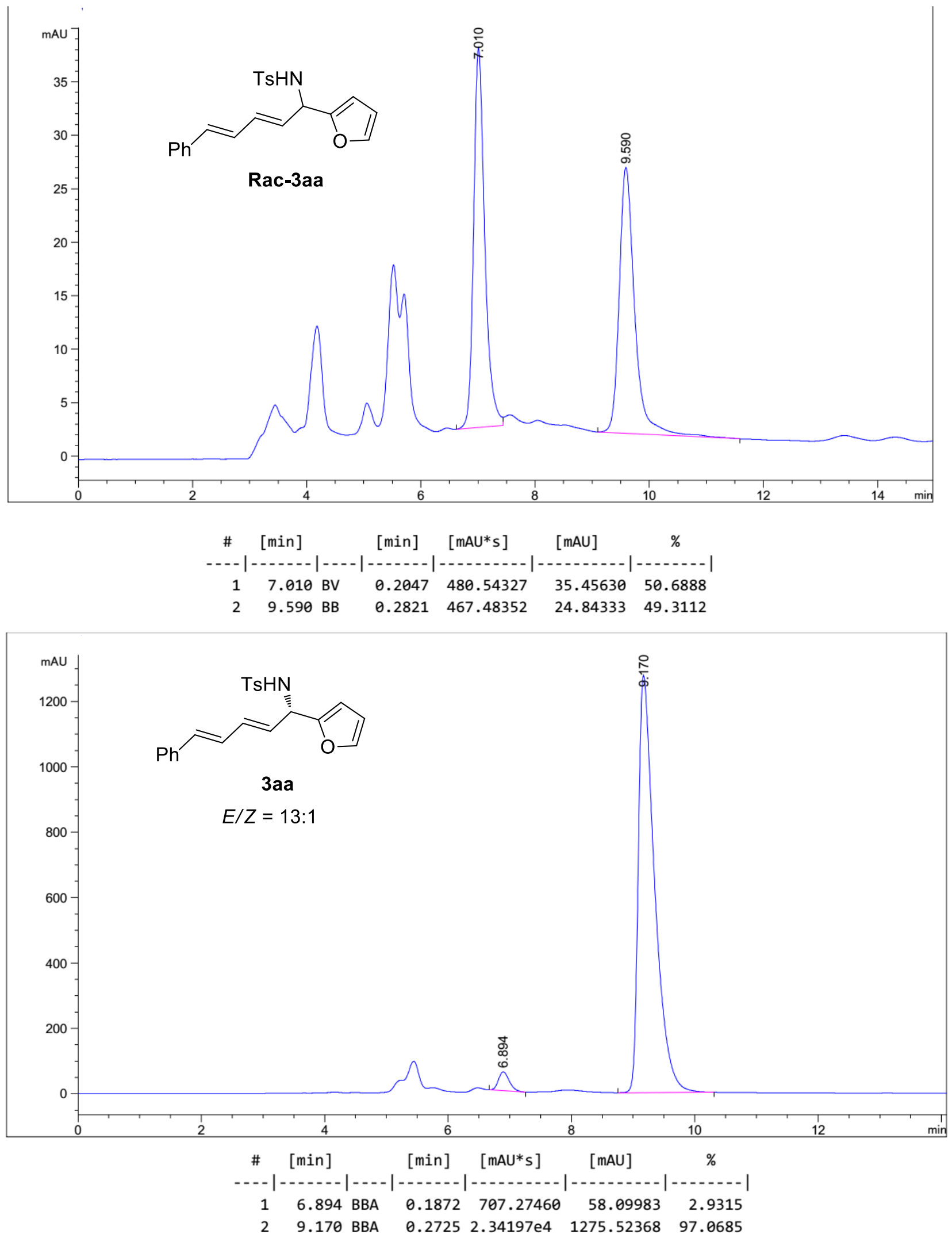
Inten. $(x 1,000,000)$

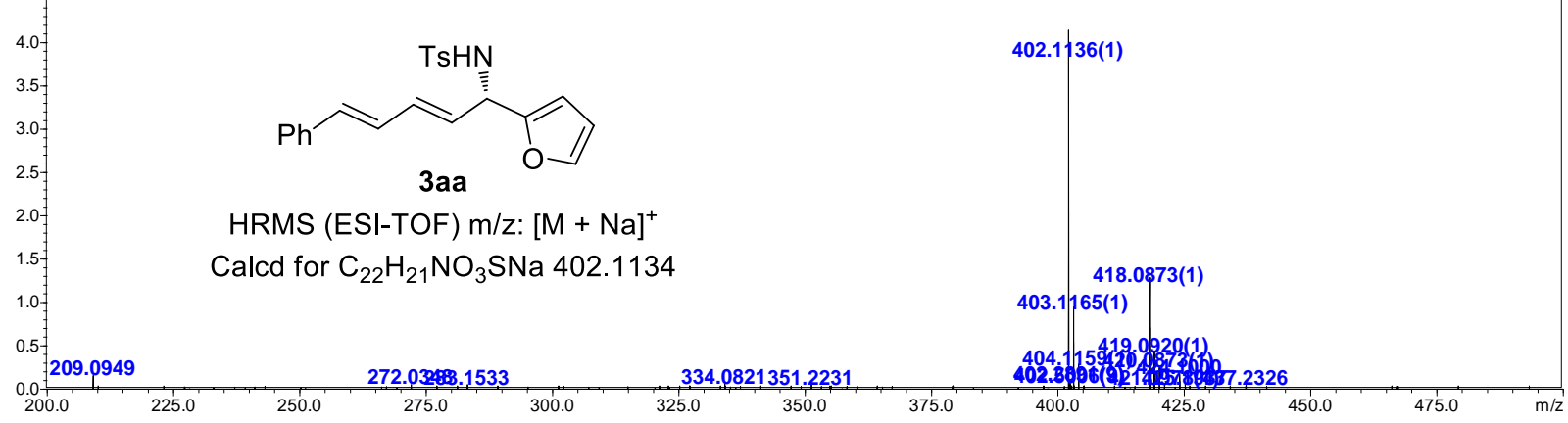




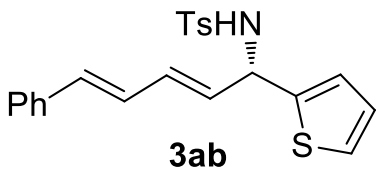

$E / Z=11: 1$

${ }^{1} \mathrm{H}$ NMR (400M, $\left.\mathrm{CDCl}_{3}\right)$

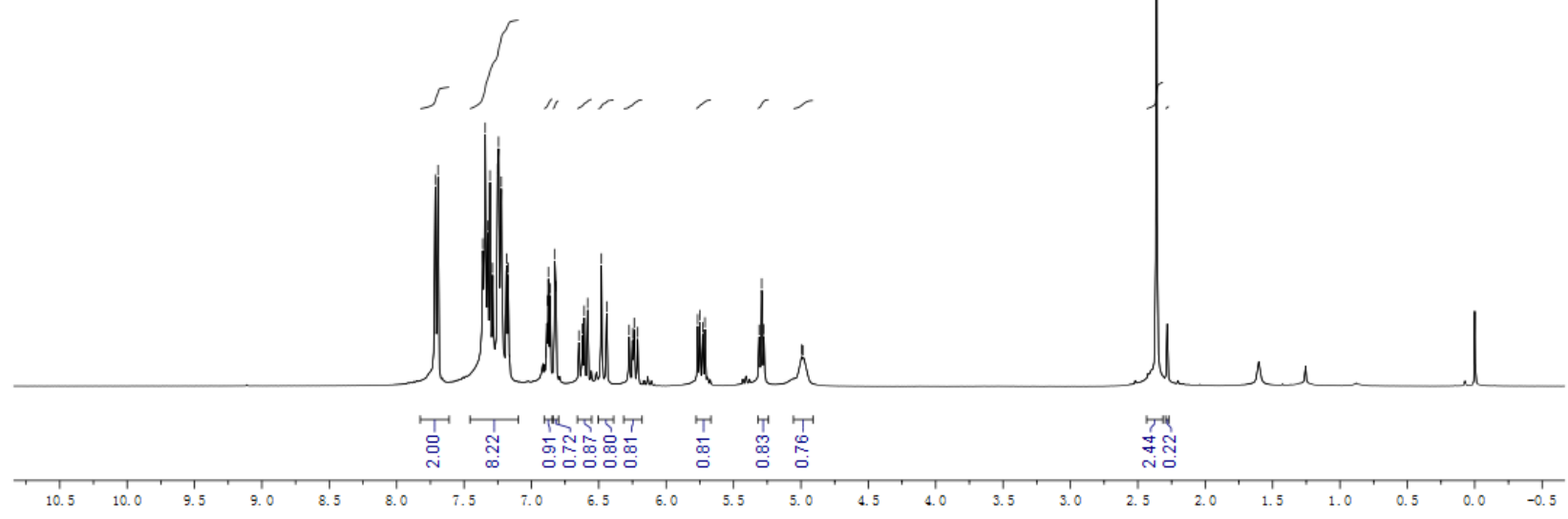

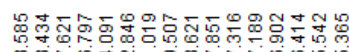

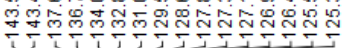

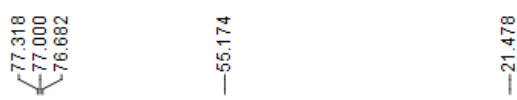

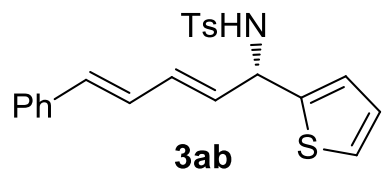

$E / Z=11: 1$

${ }^{13} \mathrm{C}$ NMR (100M, $\left.\mathrm{CDCl}_{3}\right)$

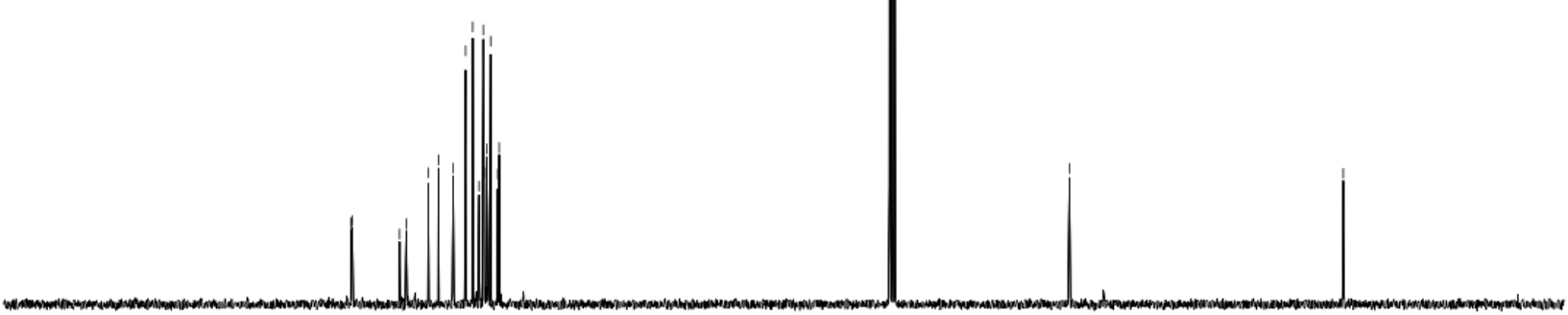




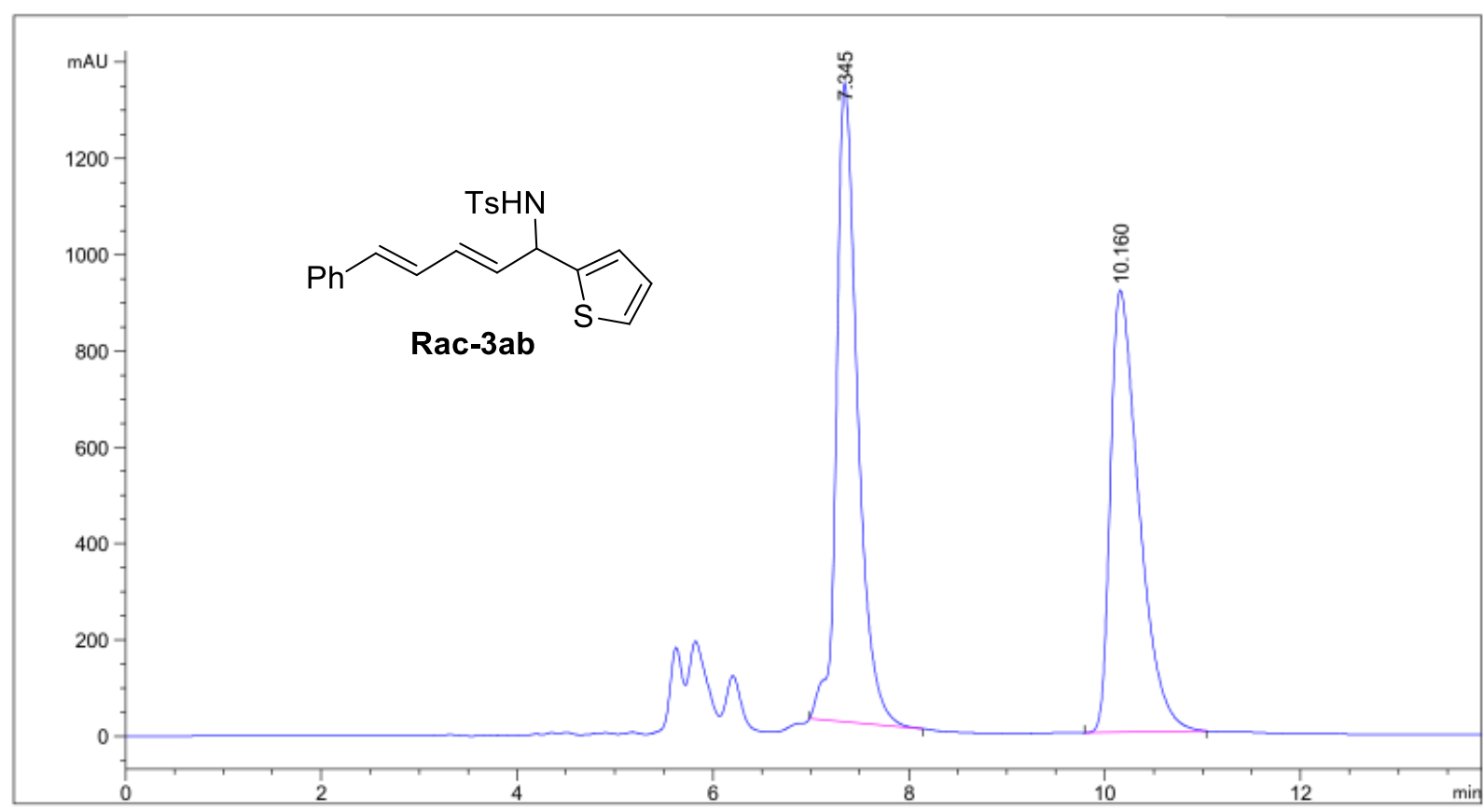

\# $[\mathrm{min}] \quad[\mathrm{min}] \quad[\mathrm{mAU} * \mathrm{~s}] \quad[\mathrm{mAU}] \quad \%$

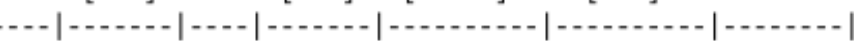

$\begin{array}{lllllll}1 & 7.345 & \text { BвA } & 0.2248 & 1.99341 \mathrm{e} 4 & 1326.85535 & 51.5872\end{array}$

$\begin{array}{lllllll}2 & 10.160 & \text { BBA } & 0.3090 & 1.87074 \mathrm{e} 4 & 918.26581 & 48.4128\end{array}$
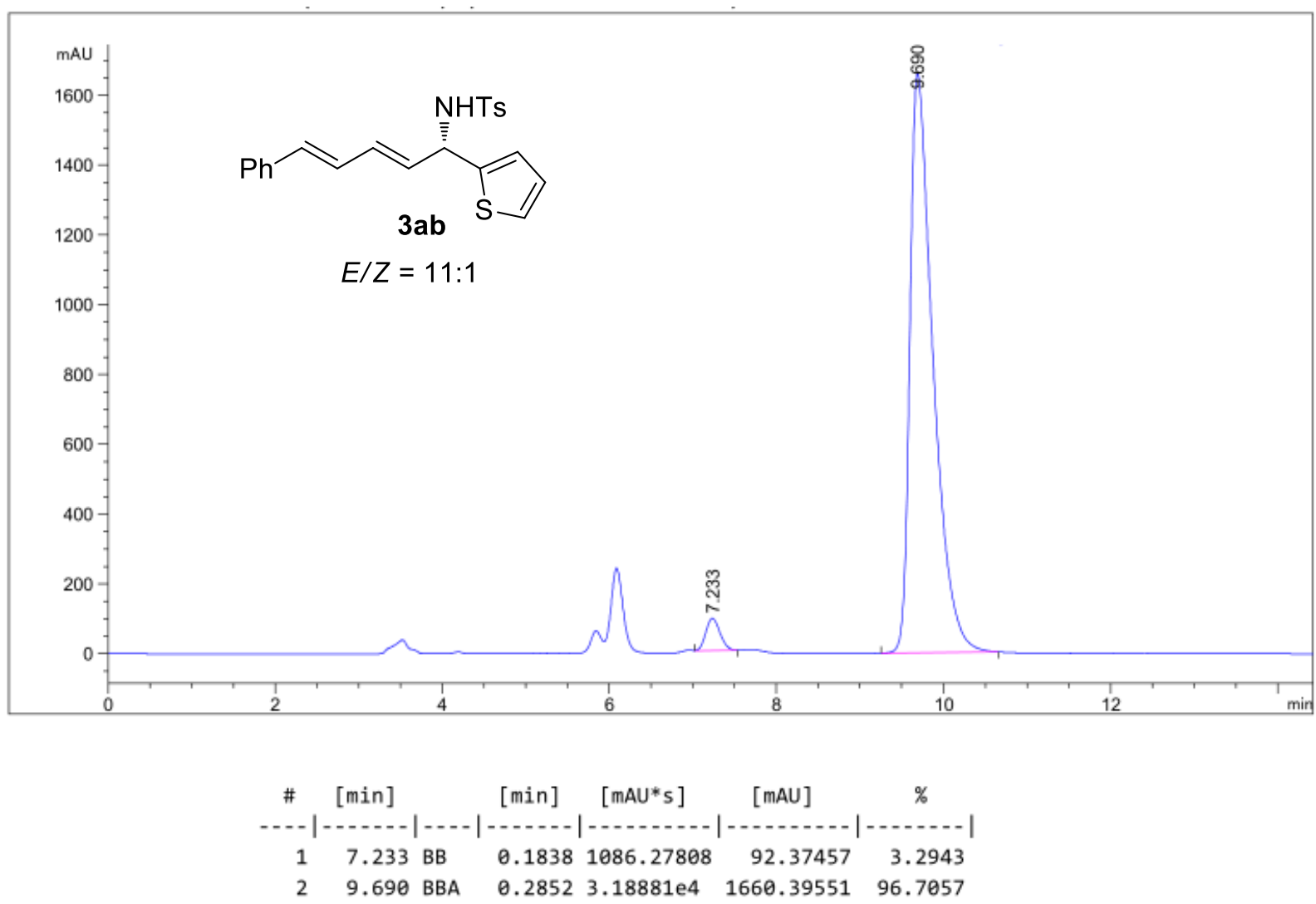
Spectrum Plot Report

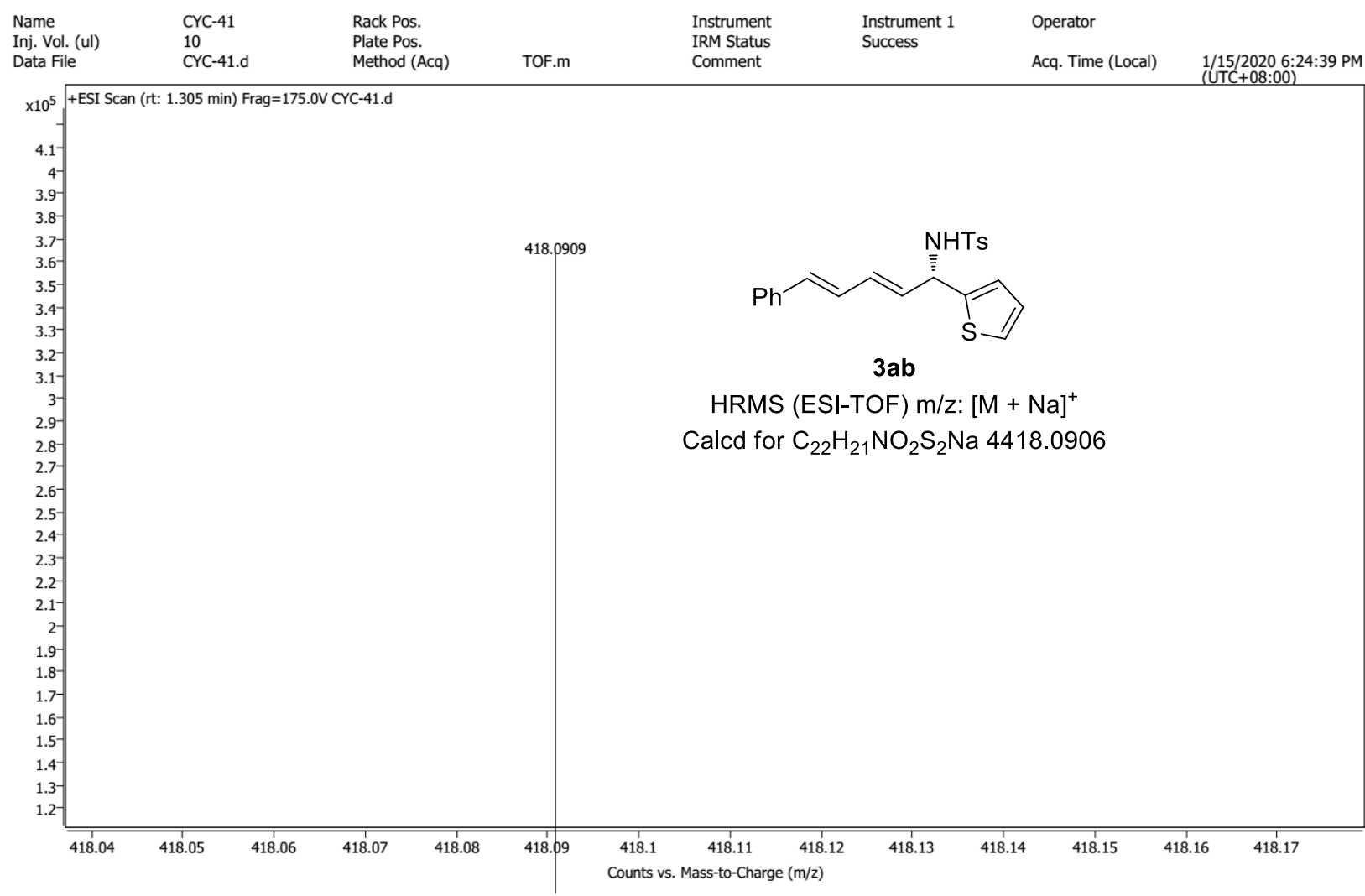


<smiles>O=C(NC(/C=C/c1ccccc1)/C=C/c1ccccc1)c1ccccc1</smiles>

$E / Z=12: 1$

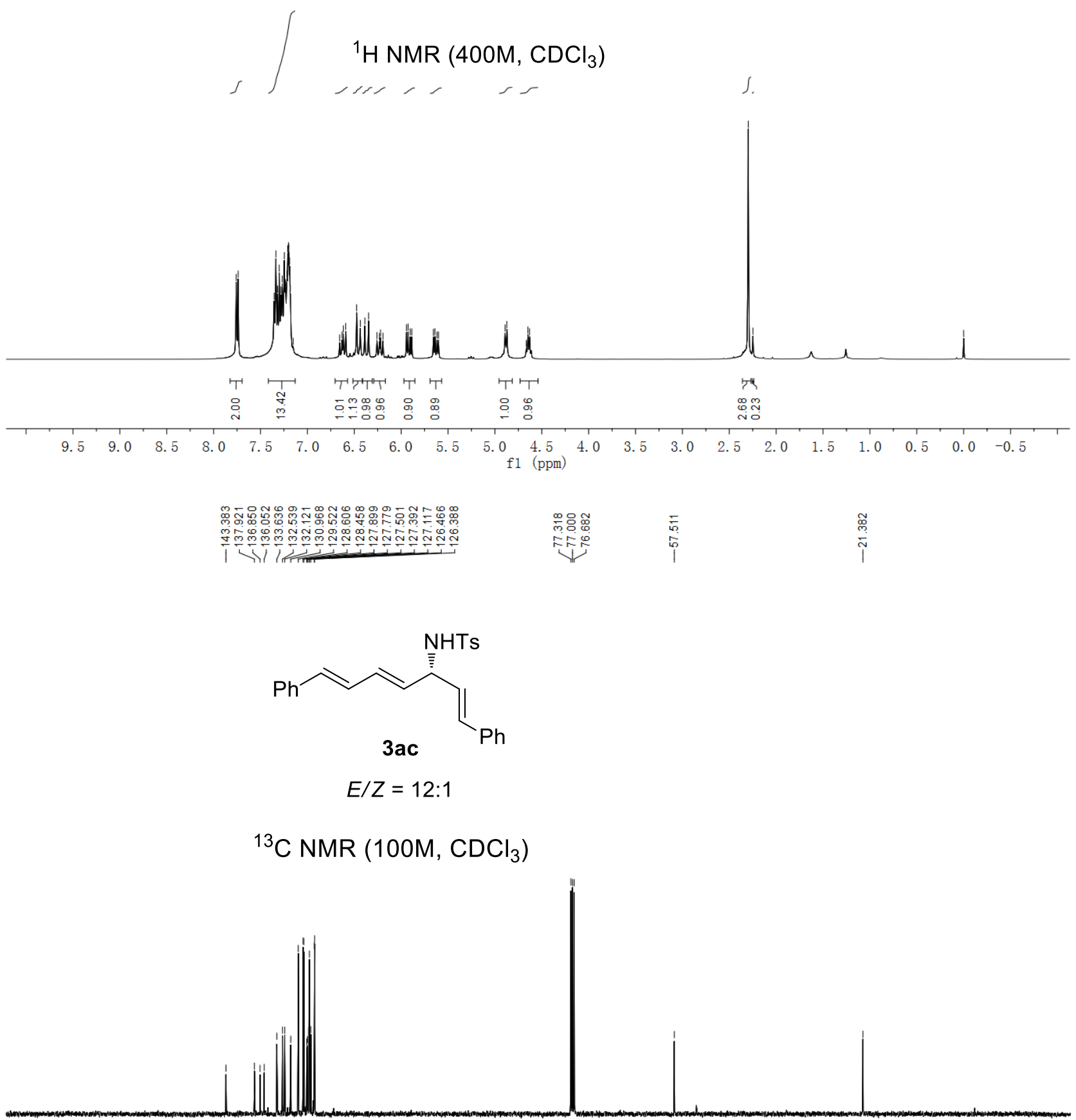

$\begin{array}{llllllllll}180 & 170 & 160 & 150 & 140 & 130 & 120 & 110 & 100 & 90\end{array}$ 

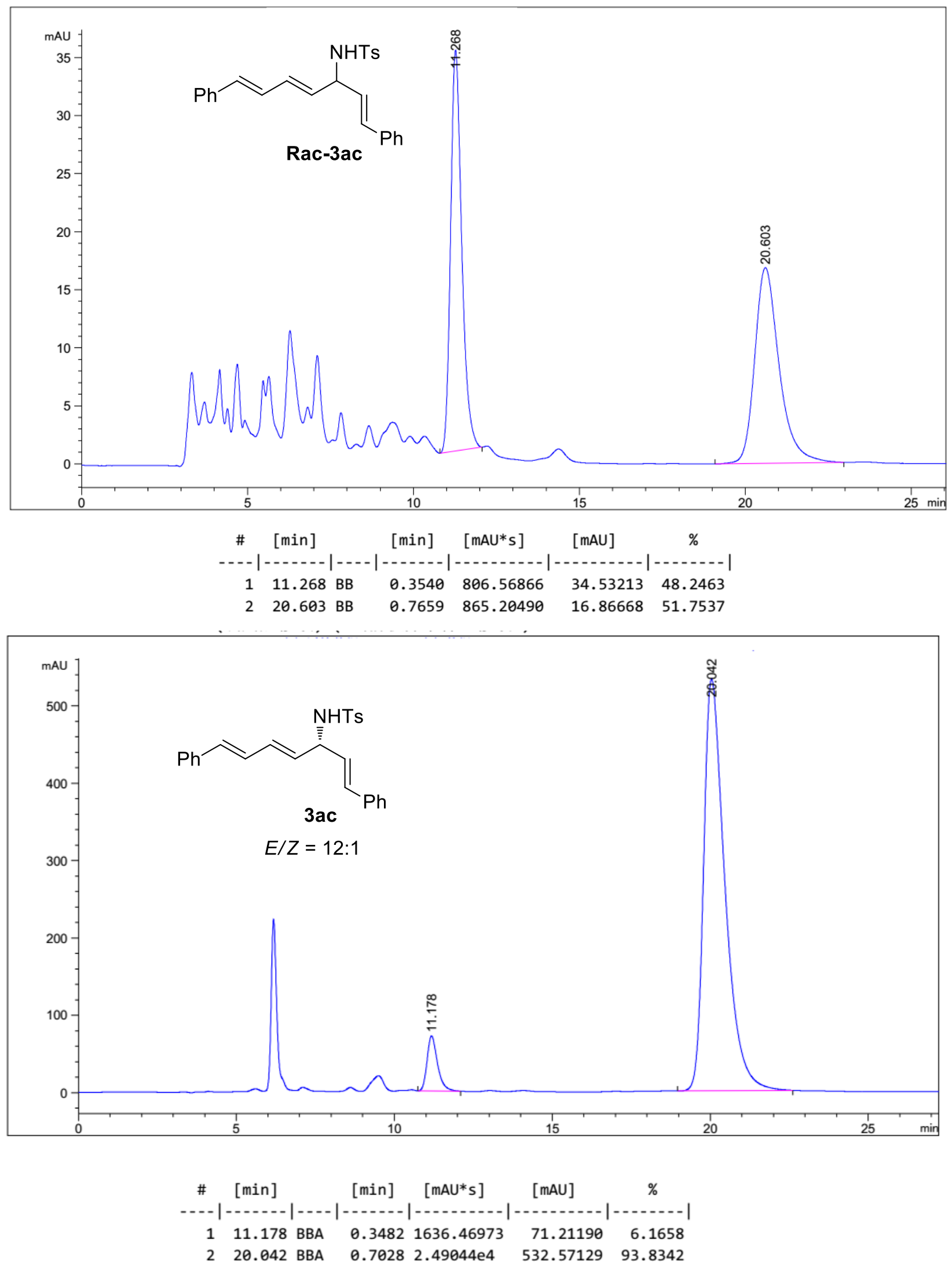


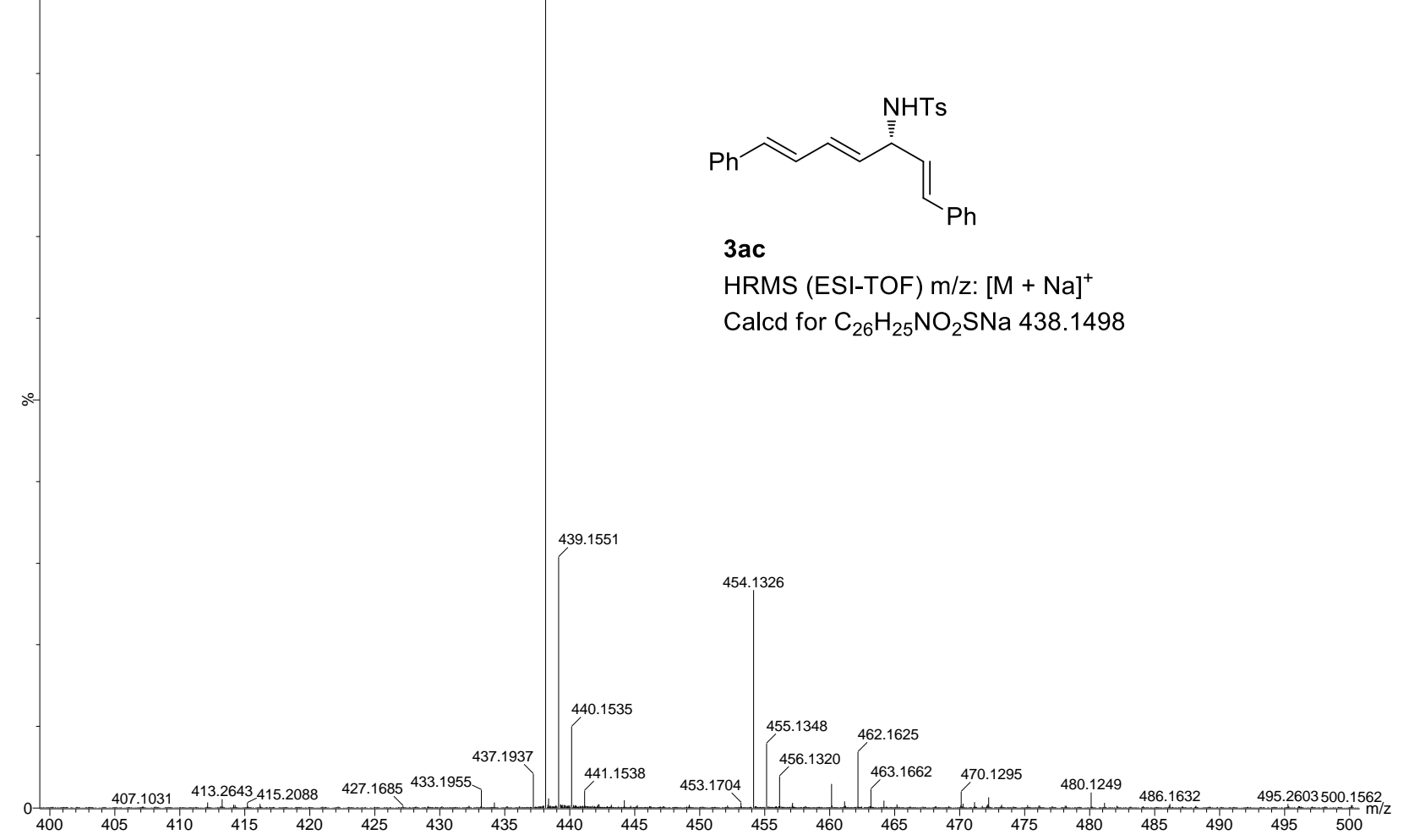




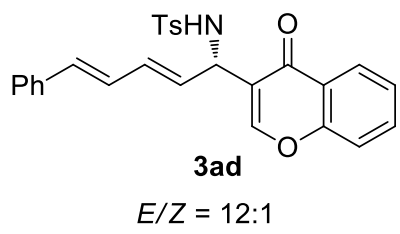

${ }^{1} \mathrm{H}$ NMR (400M, $\mathrm{CDCl}_{3}$ )

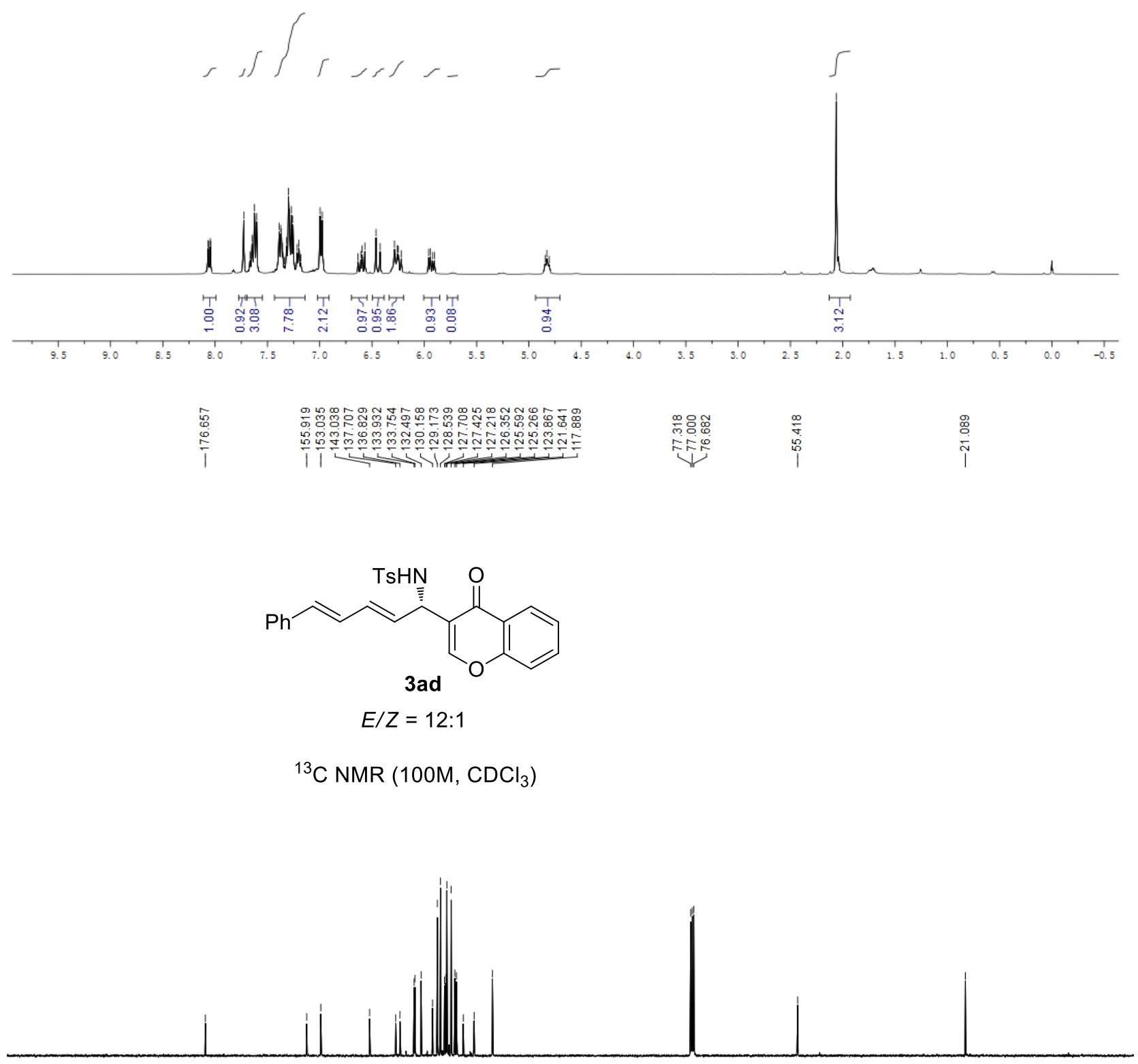

$\begin{array}{lllllllllllllllllllllll}210 & 200 & 190 & 180 & 170 & 160 & 150 & 140 & 130 & 120 & 110 & 100 & 90 & 80 & 70 & 60 & 50 & 40 & 30 & 20 & 10 & 0 & -10\end{array}$ 

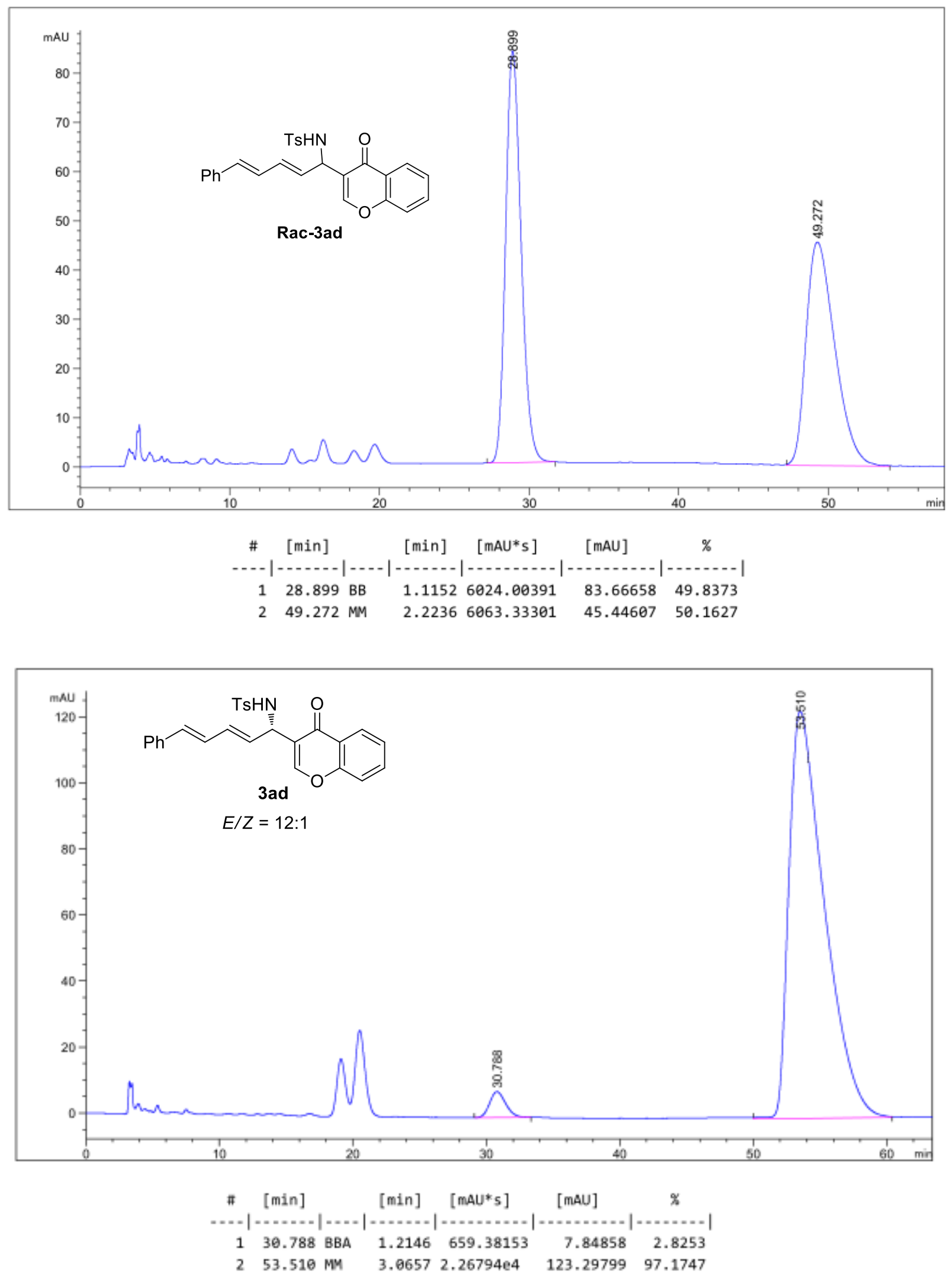
100<smiles>N[C@H](/C=C/C=C/c1ccccc1)c1coc2ccccc2c1=O</smiles>

480.1243

3ad

HRMS (ESI-TOF) m/z: [M + Na] ${ }^{+}$

Calcd for $\mathrm{C}_{27} \mathrm{H}_{23} \mathrm{NO}_{4} \mathrm{SNa} 480.1240$

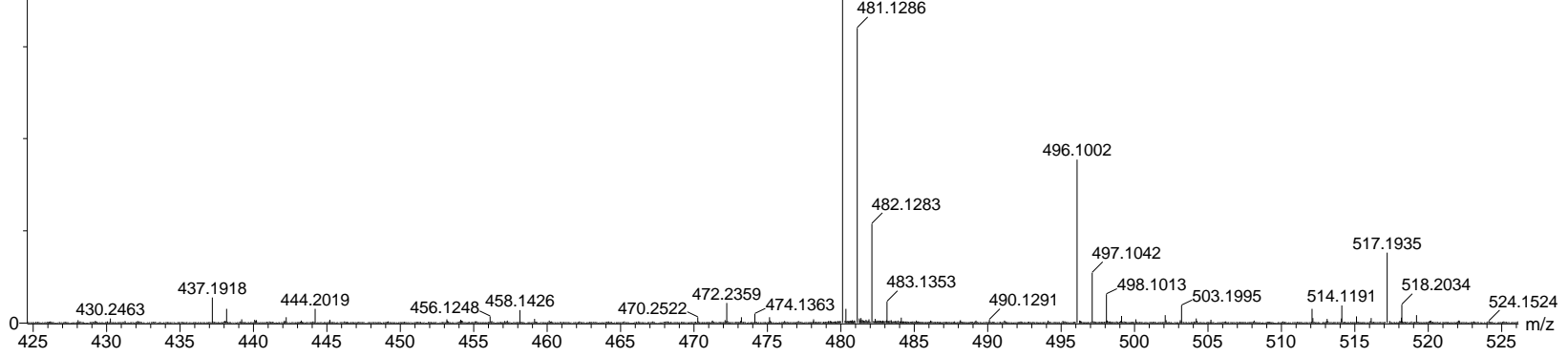




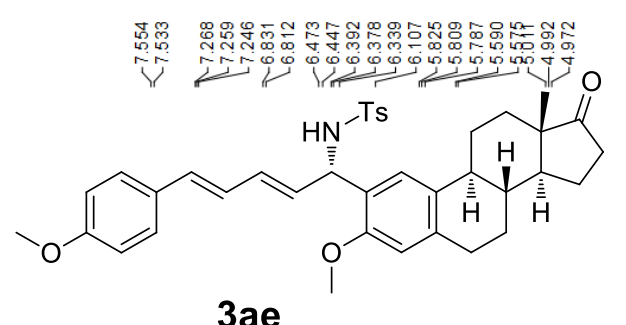

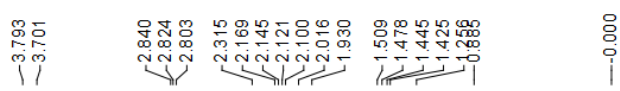

$10: 1 d r$

${ }^{1} \mathrm{H}$ NMR (400M, $\mathrm{CDCl}_{3}$ )

$\iiint \int h, d e r$

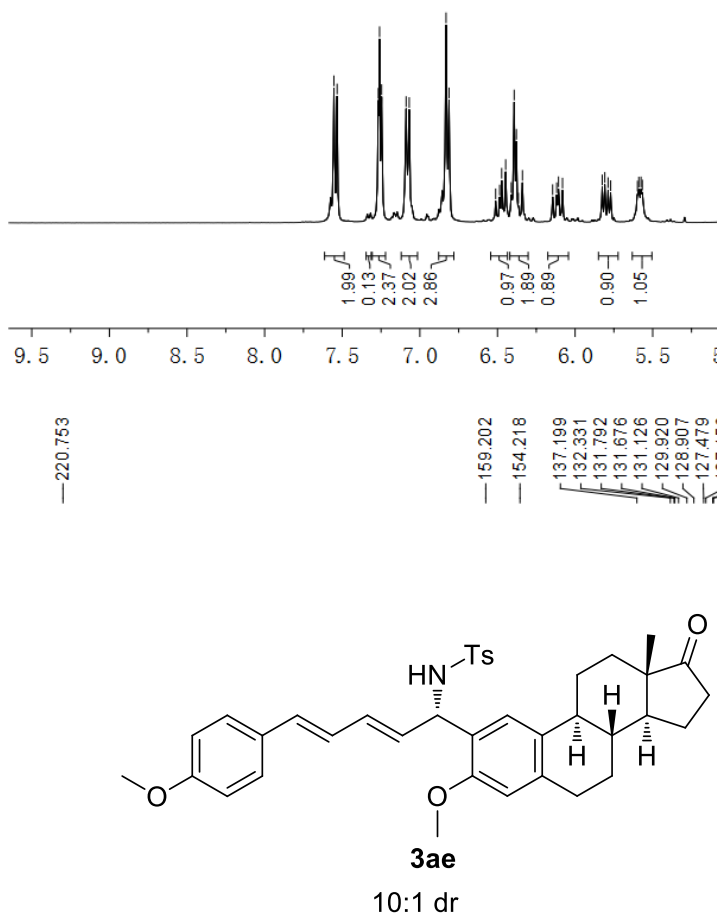

${ }^{13} \mathrm{C} \mathrm{NMR}\left(100 \mathrm{M}, \mathrm{CDCl}_{3}\right)$

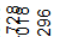

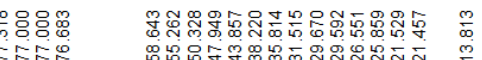

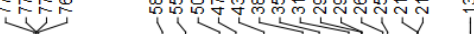

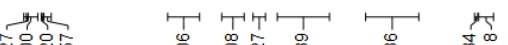 స్ํํำ}


$16: 55: 31$

200806_CY_105 4 (0.068) Cm (1:23)

100

$601.1331 \quad 607.3720$

$601.1804 \quad 608.3303 \quad 618.3690 \quad 6353.3804638 .3590$ 642.3764
06-Aug-2020 TOF MS ES+<smiles></smiles>

$3 a$

HRMS (ESI-TOF) m/z: [M + Na] ${ }^{+}$

Calcd for $\mathrm{C}_{38} \mathrm{H}_{43} \mathrm{NO}_{5} \mathrm{SNa} 648.27554$

667.3263

650.280

668.3266

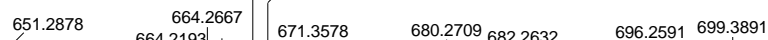

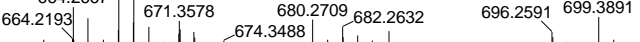

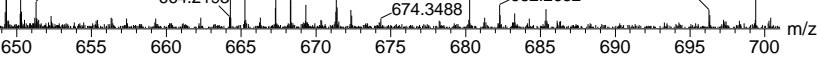




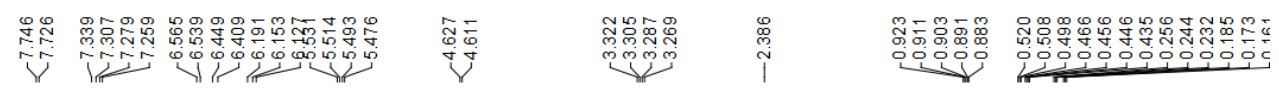<smiles></smiles>

${ }^{1} \mathrm{H}$ NMR $\left(400 \mathrm{M}, \mathrm{CDCl}_{3}\right)$
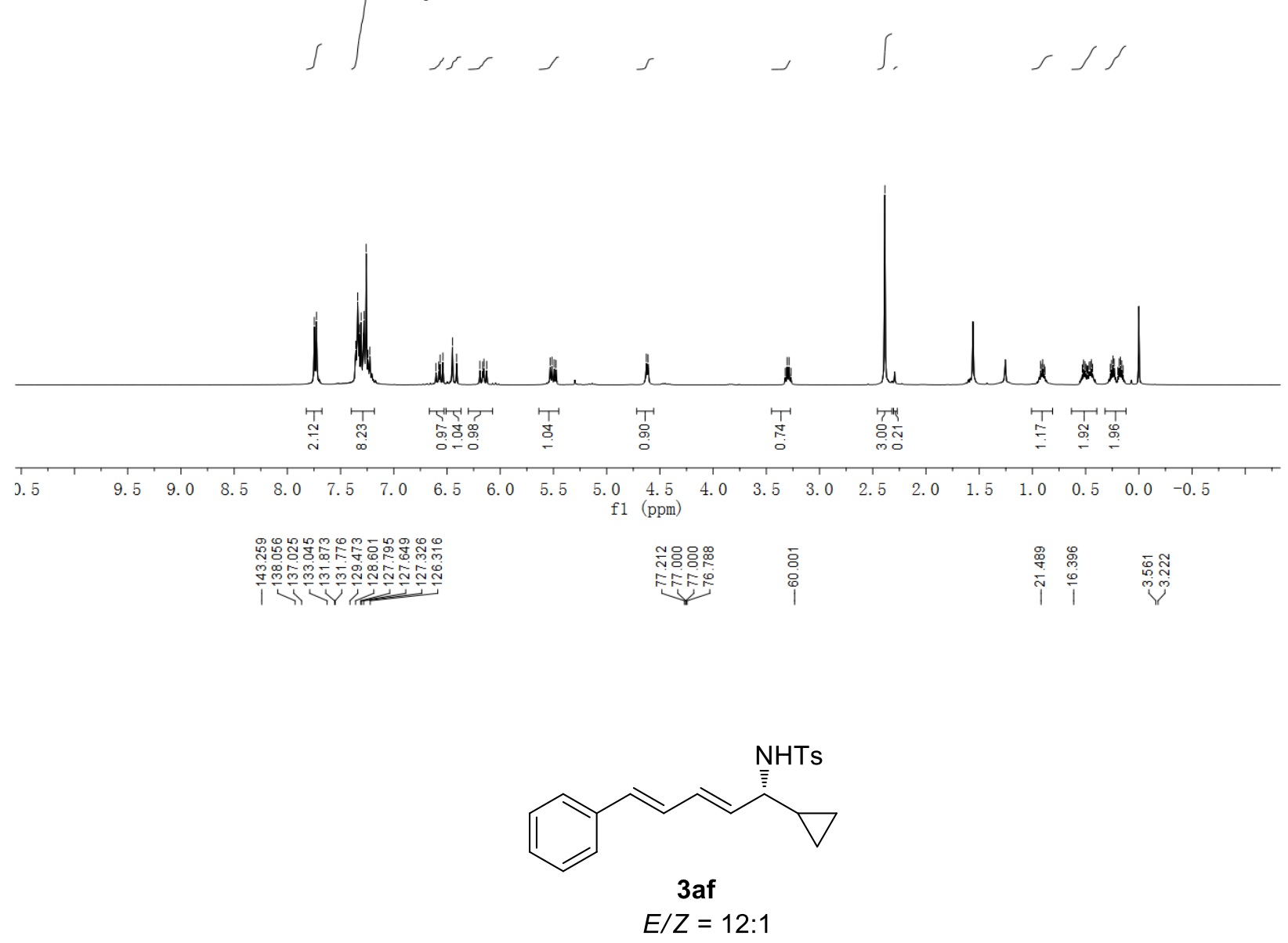

${ }^{13} \mathrm{C}$ NMR (150M, $\left.\mathrm{CDCl}_{3}\right)$

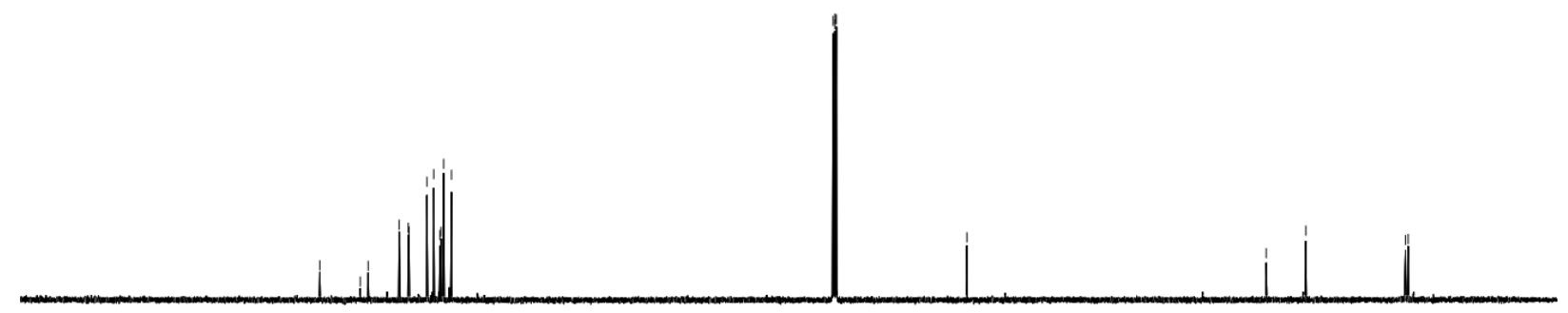

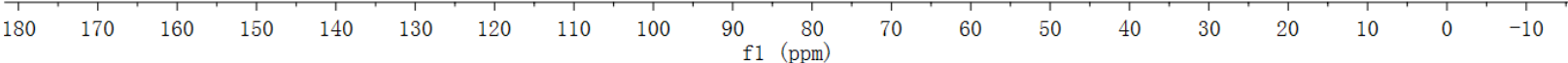



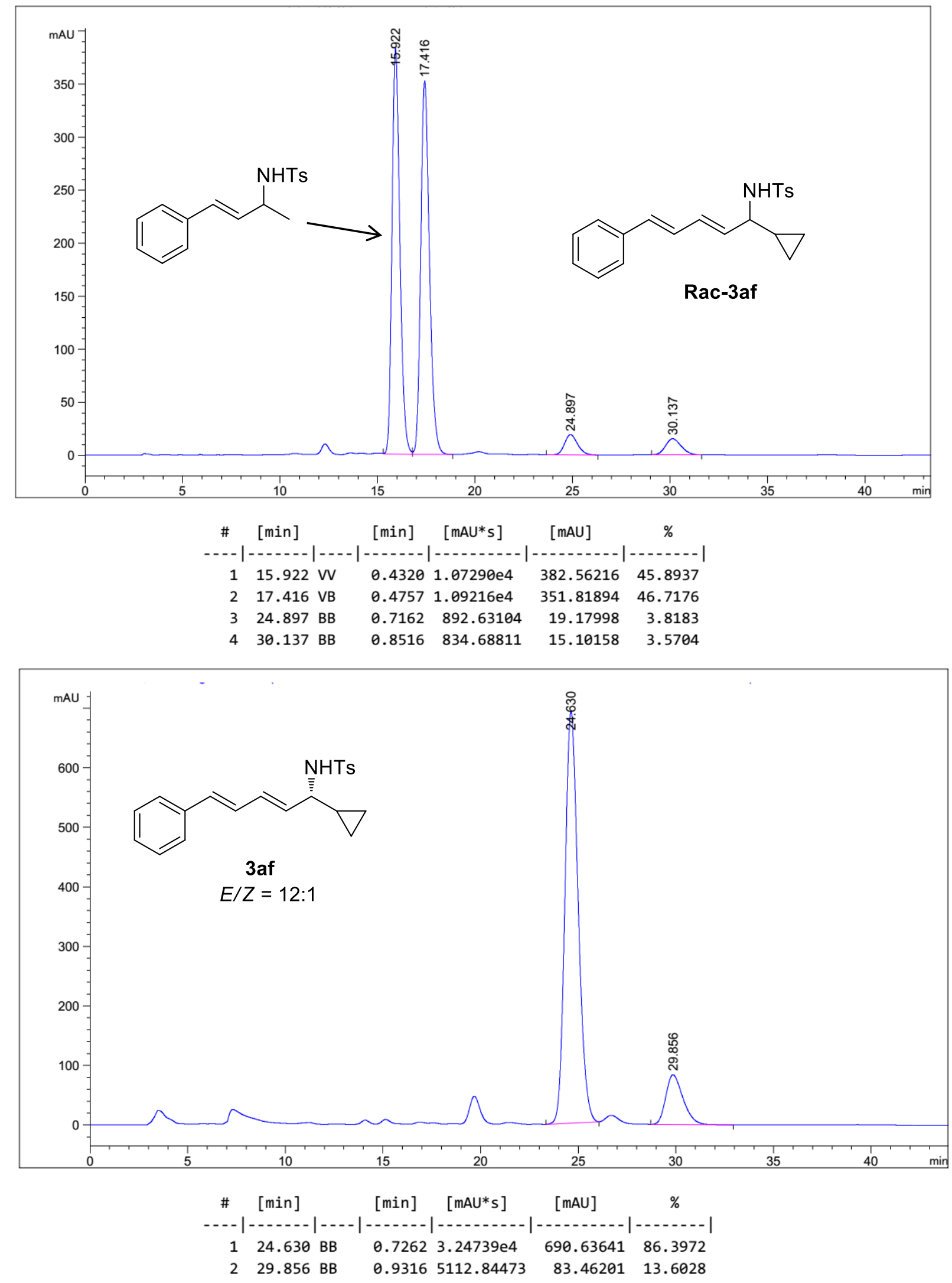

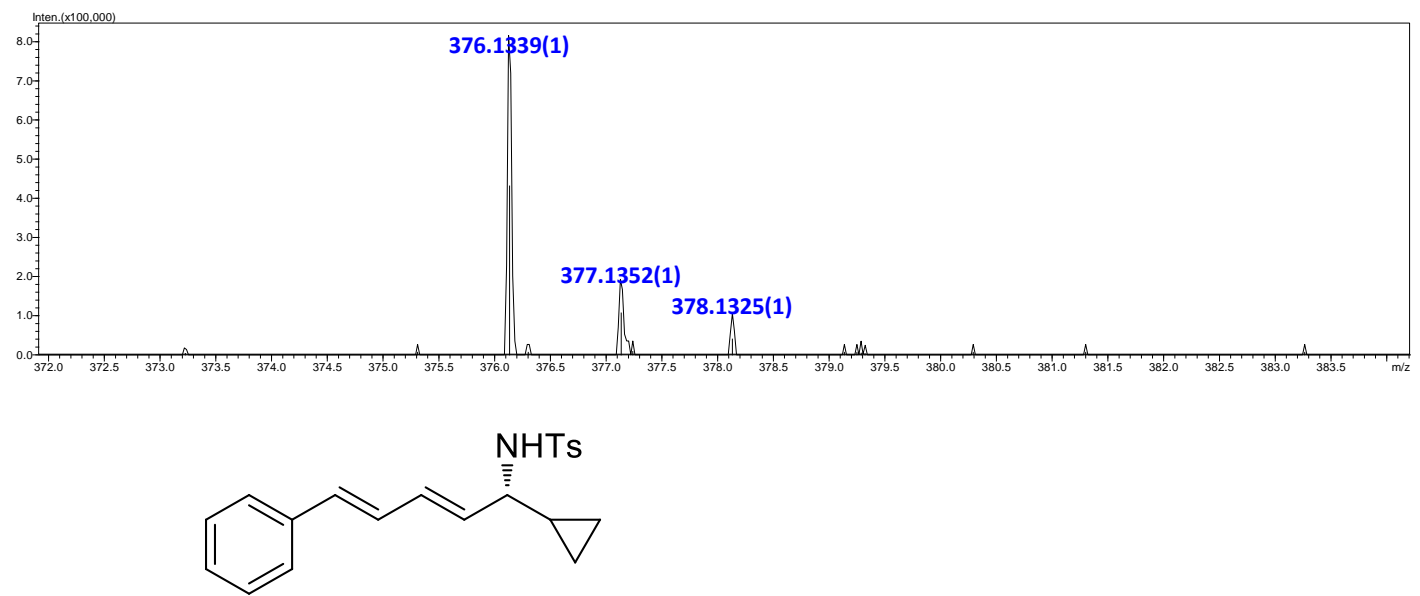

3af

HRMS (ESI-TOF) m/z: [M + Na] ${ }^{+}$

Calcd for $\mathrm{C}_{21} \mathrm{H}_{23} \mathrm{NO}_{2} \mathrm{SNa} 376.1342$ 


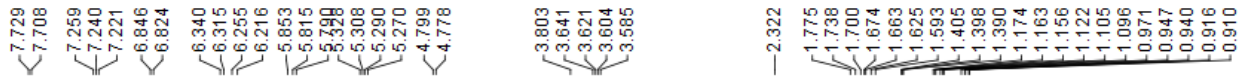<smiles>COc1ccc(/C=C/C=C/[C@H](NS)C2CCCCC2)cc1</smiles>

$E / Z>19: 1$

${ }^{1} \mathrm{H}$ NMR (400M, $\mathrm{CDCl}_{3}$ )

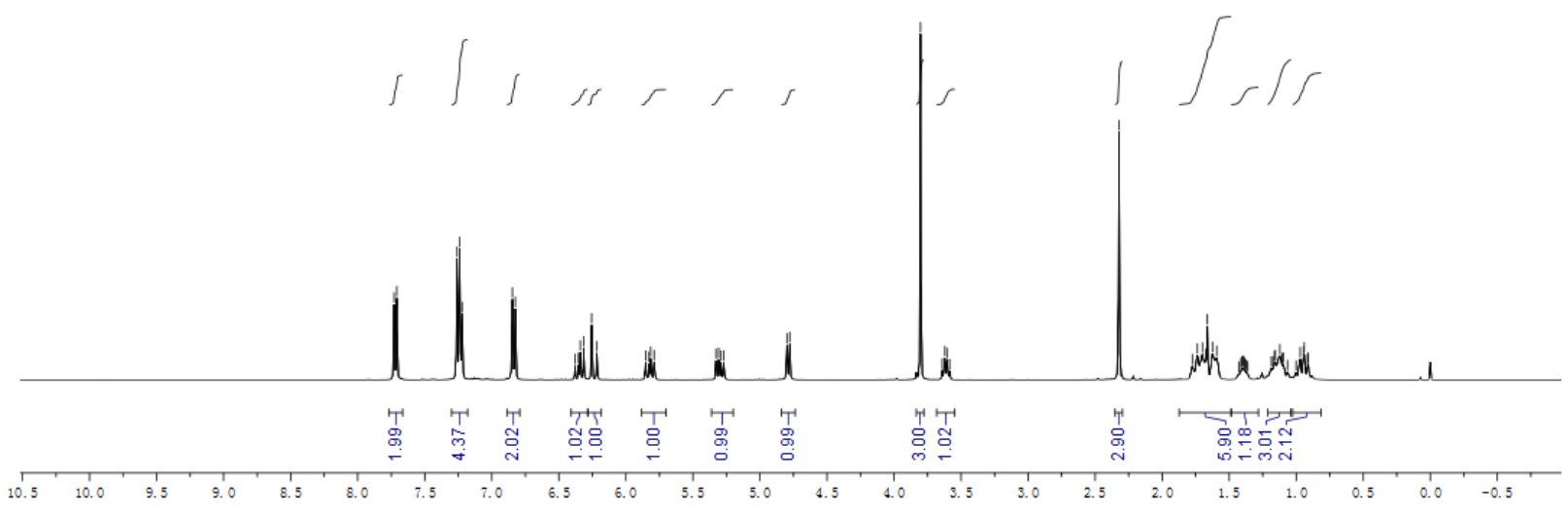

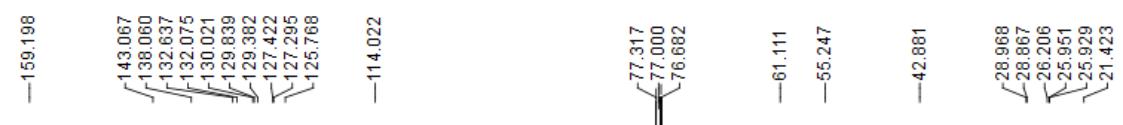<smiles>[Y5]C1CCCCC1[C@@H](N)/C=C/C=C/c1ccc(OC)cc1</smiles>

${ }^{13} \mathrm{C}$ NMR $\left(100 \mathrm{M}, \mathrm{CDCl}_{3}\right)$

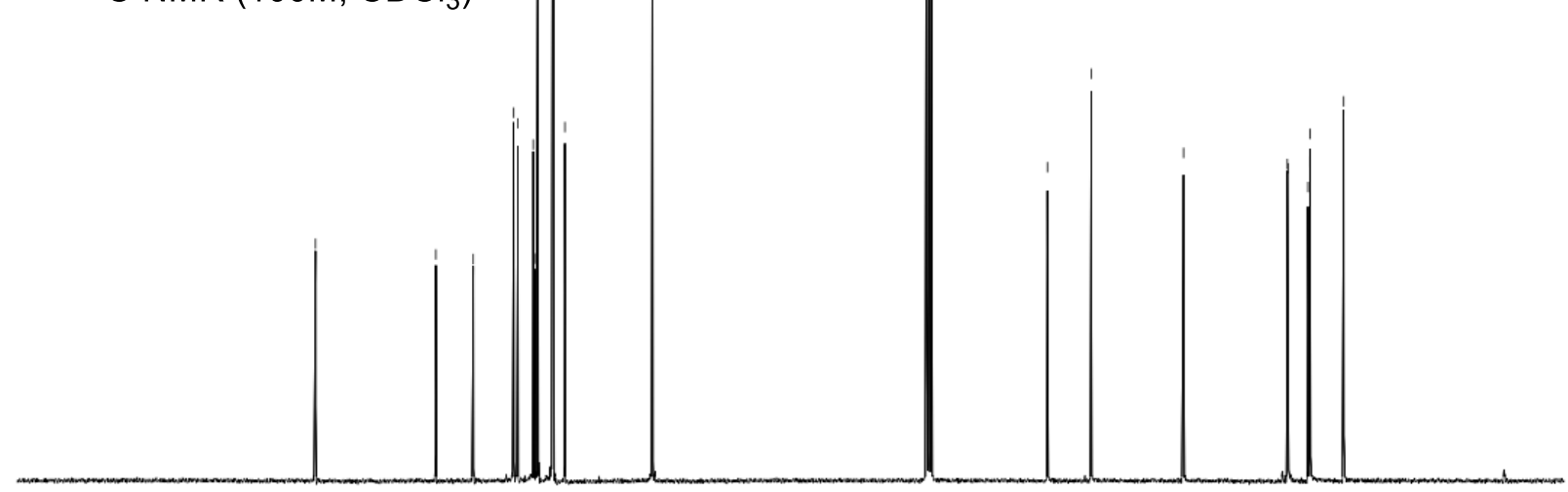



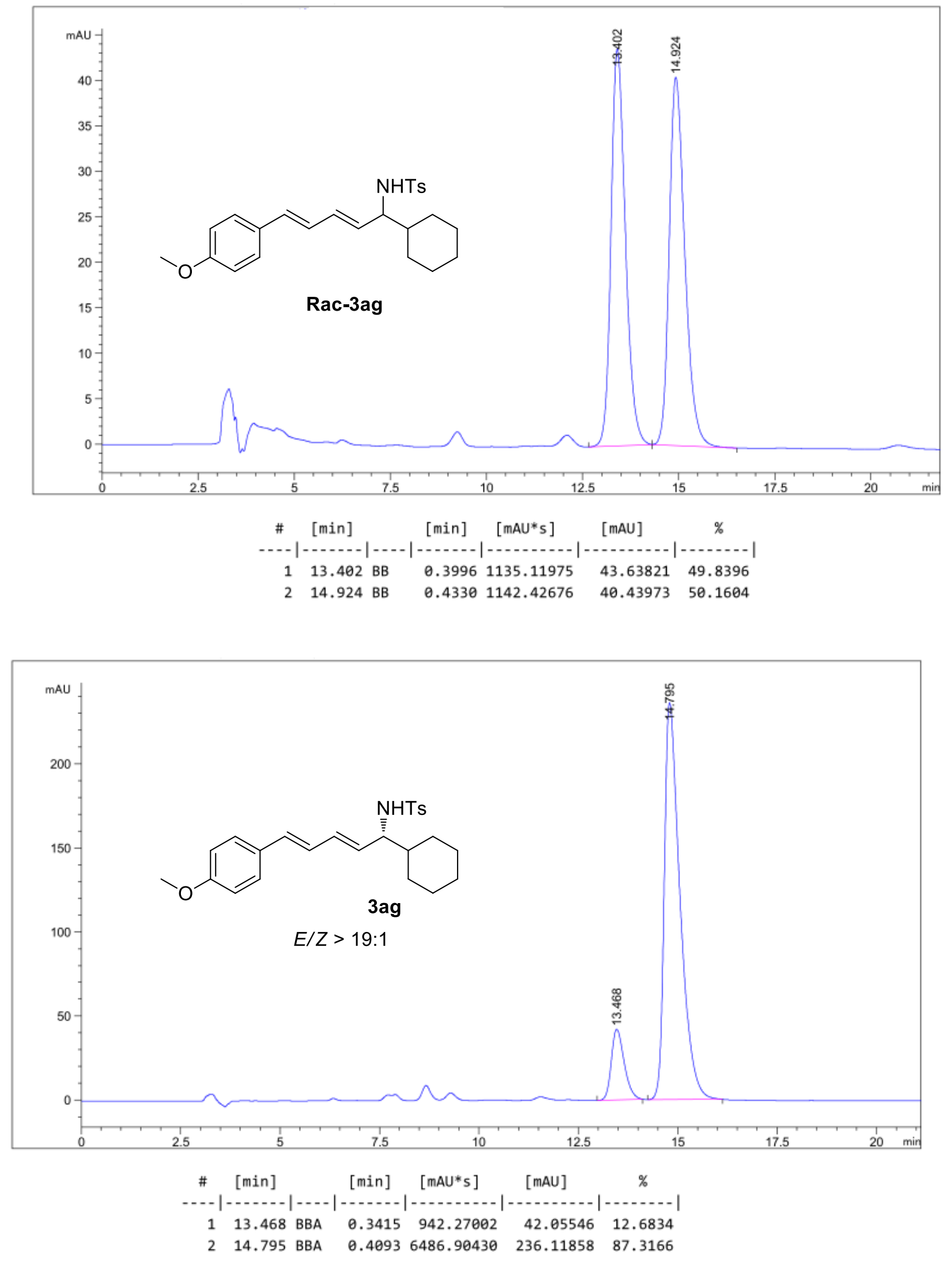


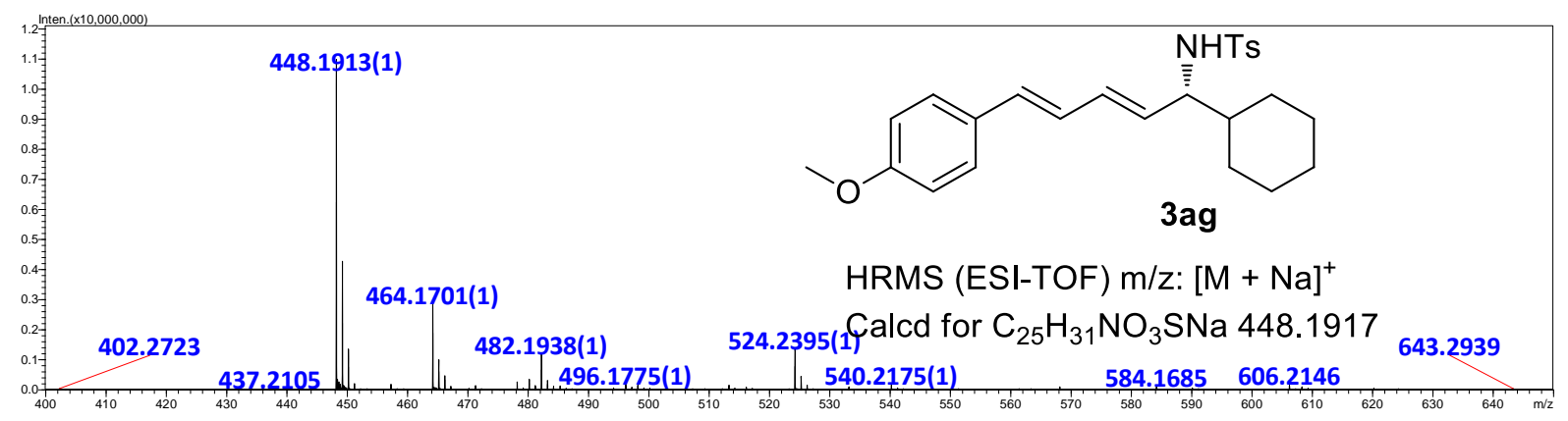




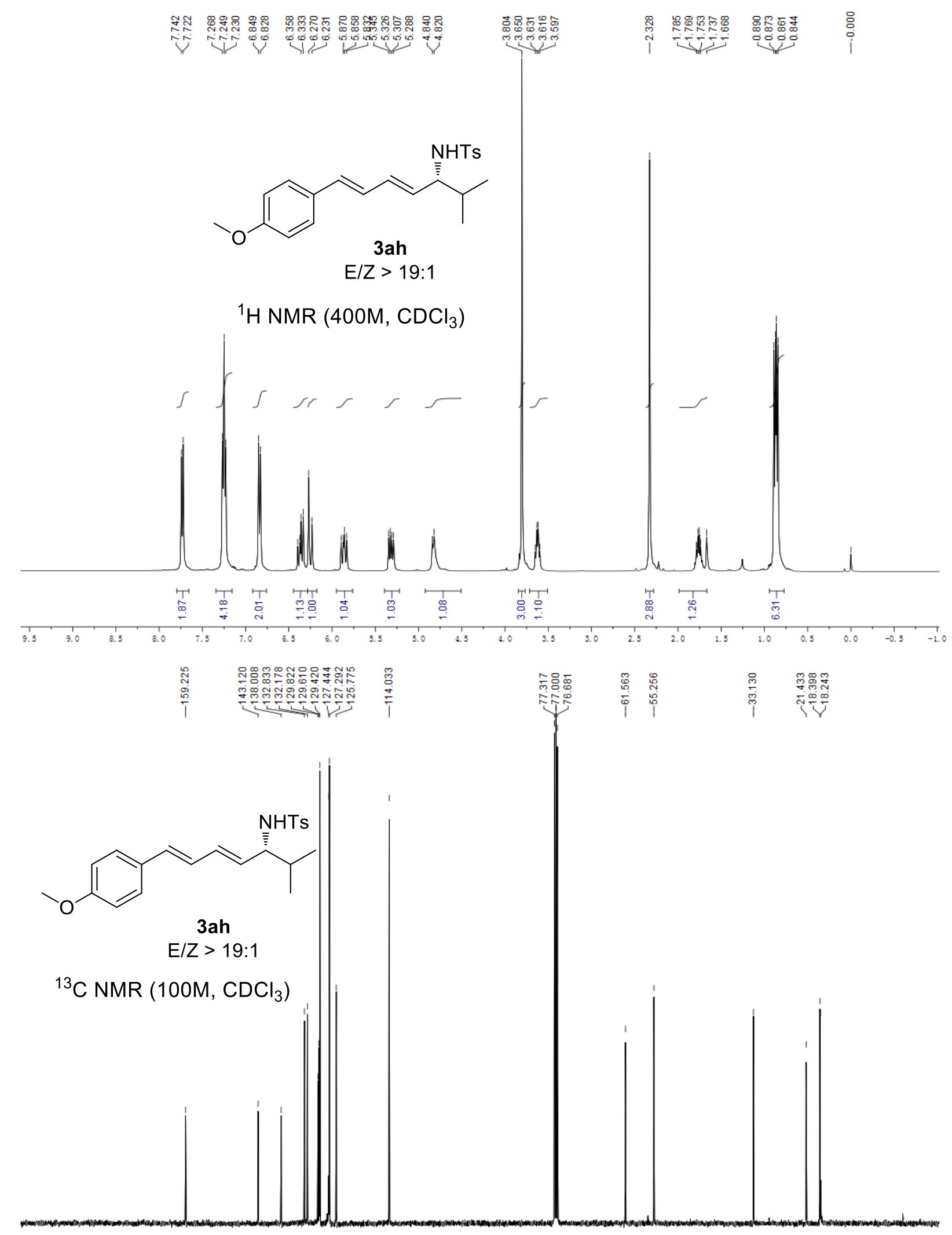



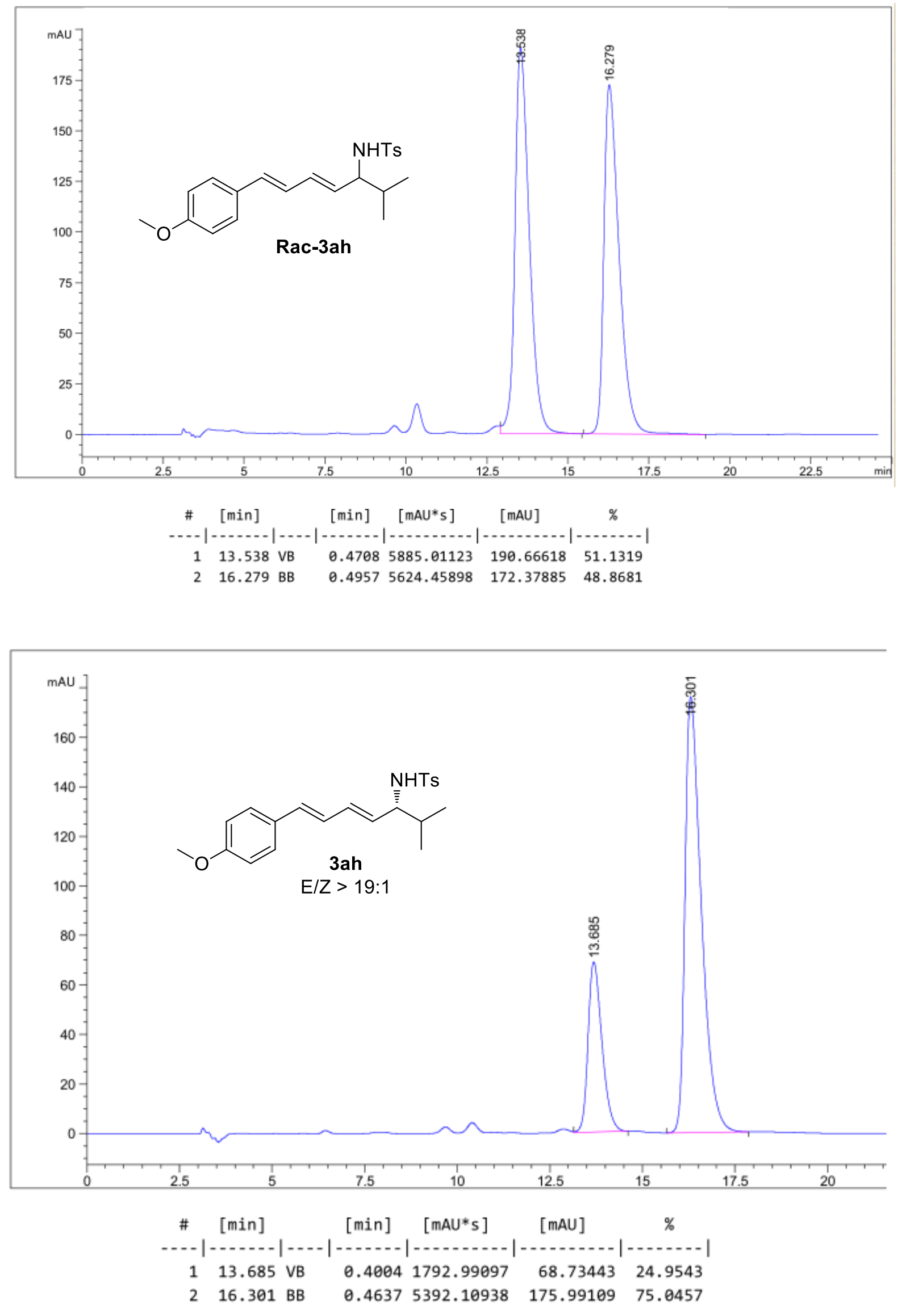


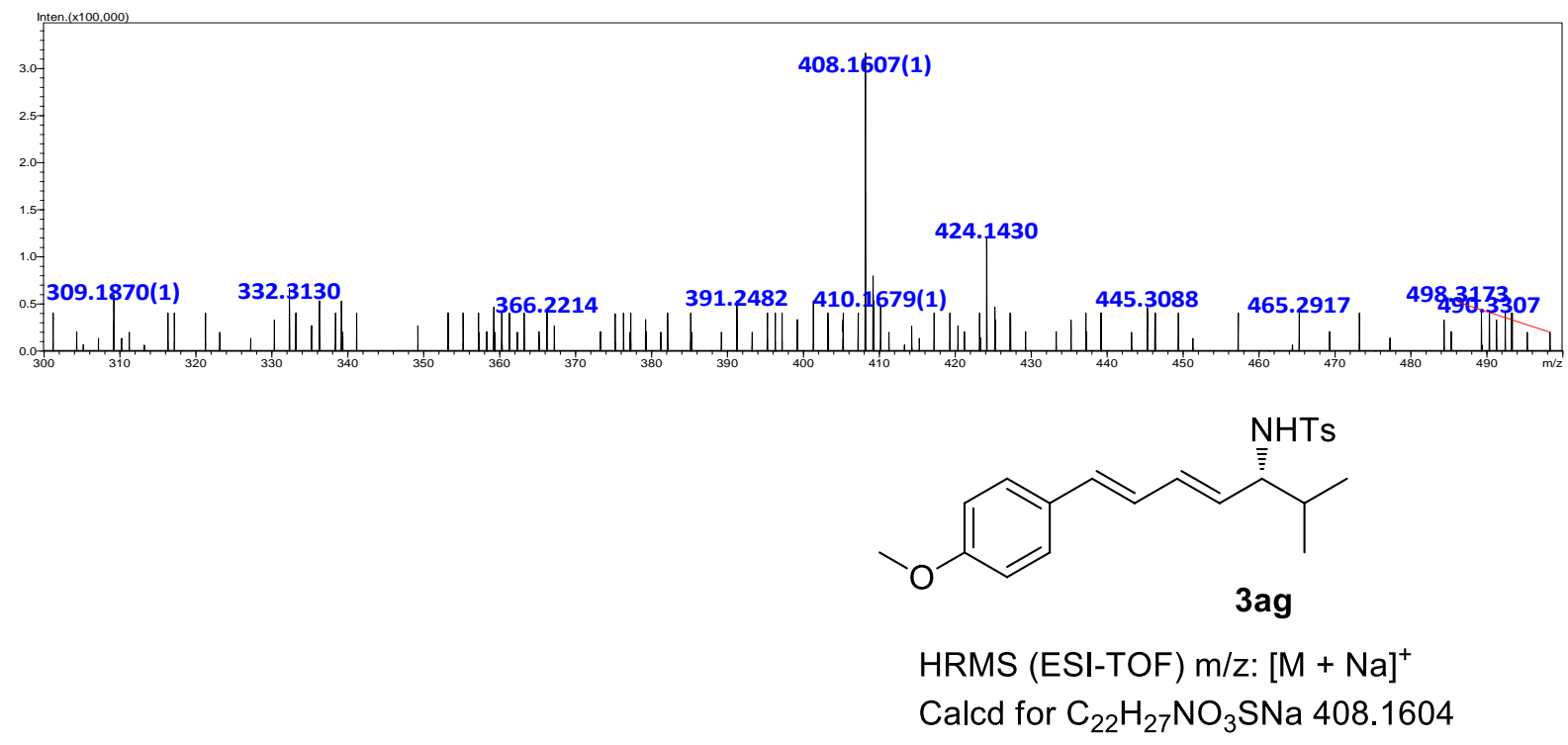



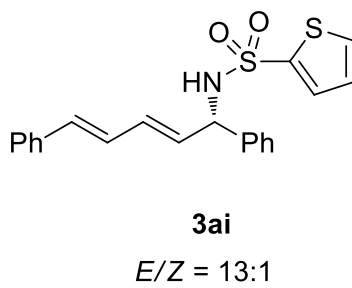

${ }^{1} \mathrm{H}$ NMR (400M, $\mathrm{CDCl}_{3}$ )

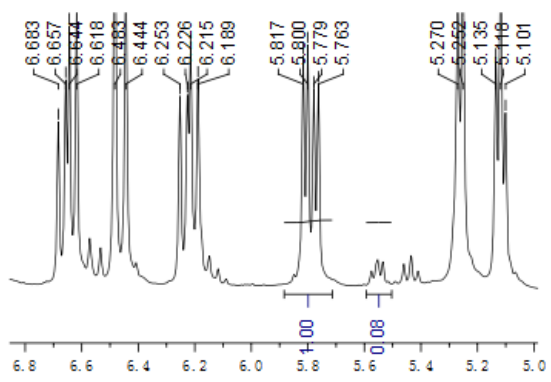

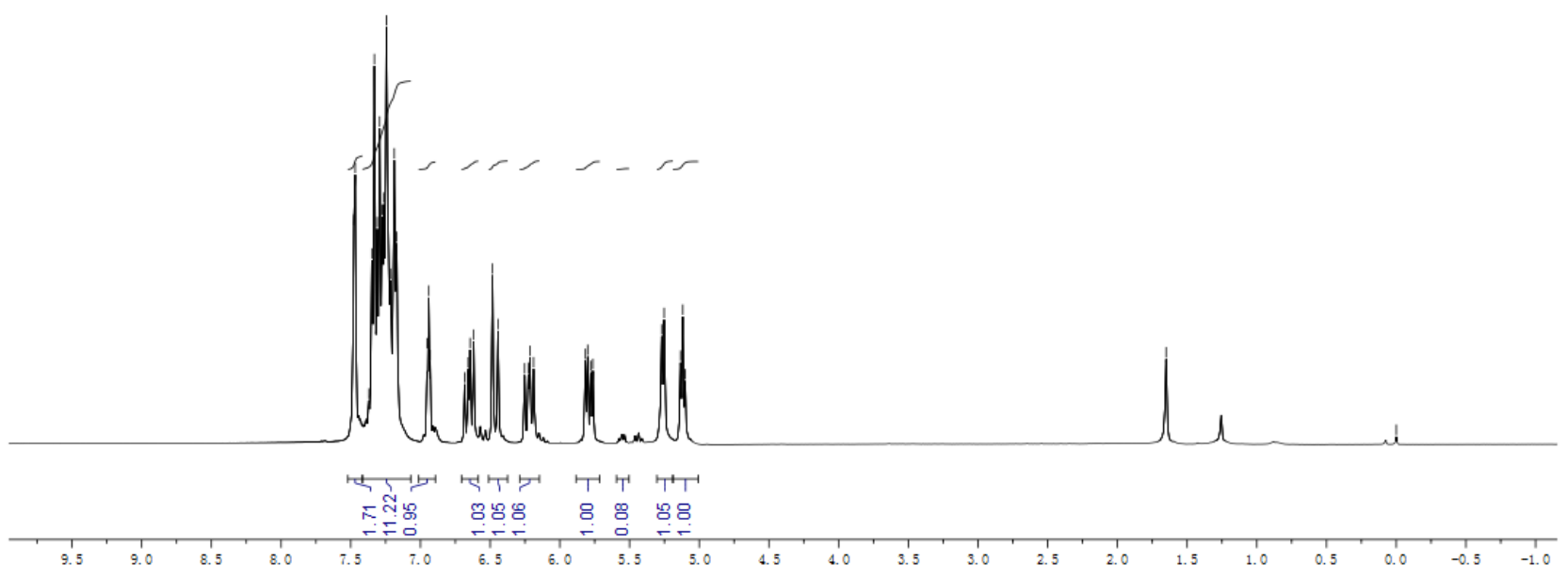

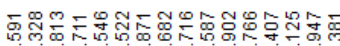
再品

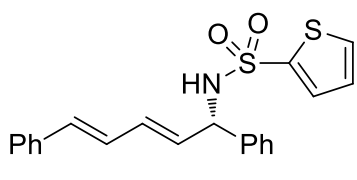

3ai

$E / Z=12: 1$

${ }^{13} \mathrm{C}$ NMR $\left(100 \mathrm{M}, \mathrm{CDCl}_{3}\right)$
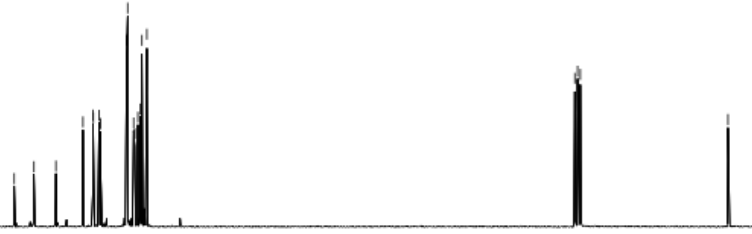

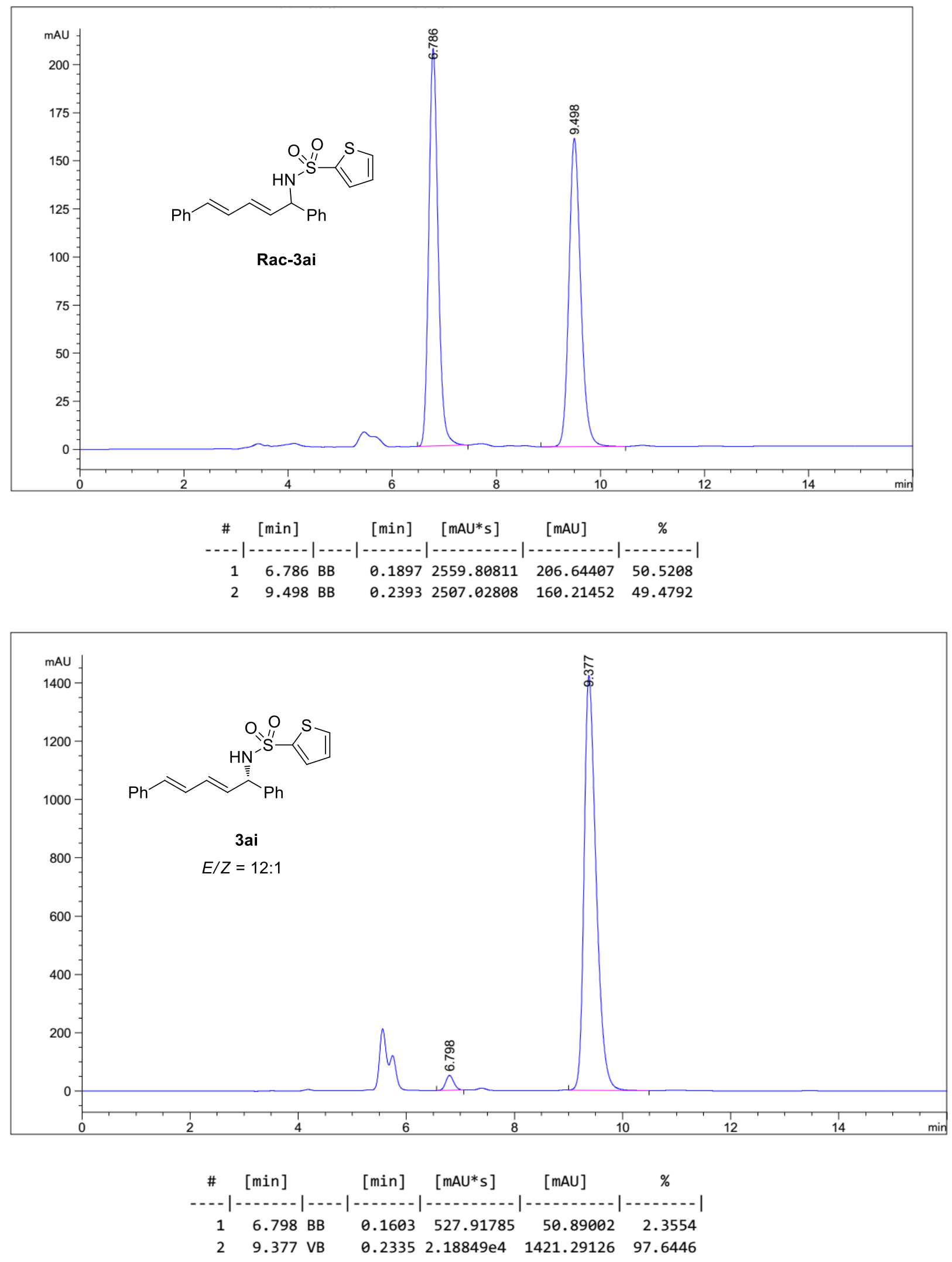


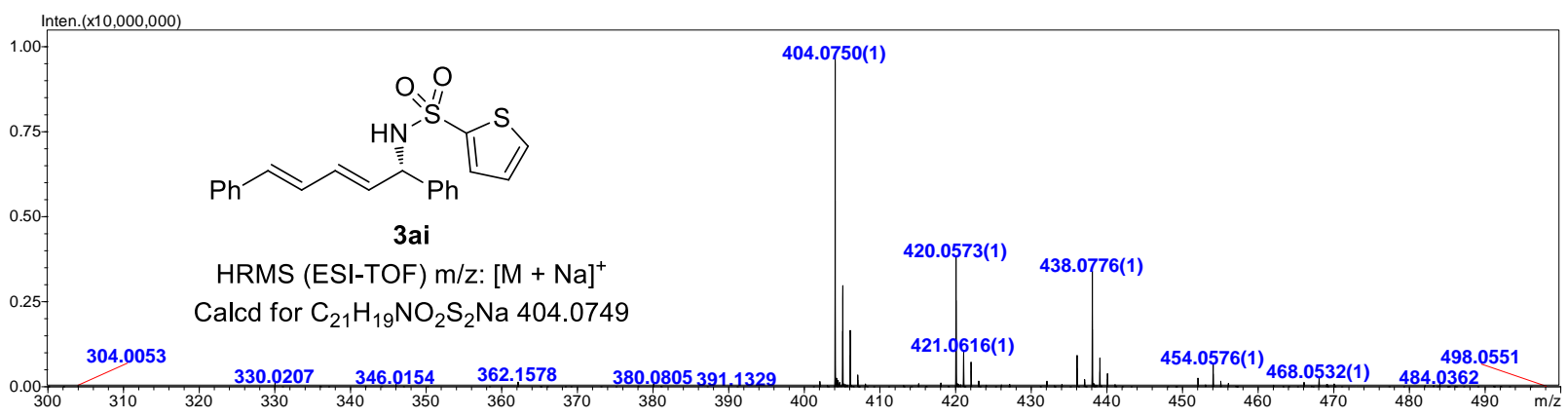




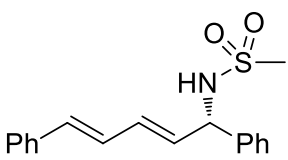

3aj

$E / Z=10: 1$

${ }^{1} \mathrm{H}$ NMR (400M, $\left.\mathrm{CDCl}_{3}\right)$

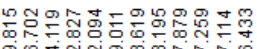

कi

旅雚

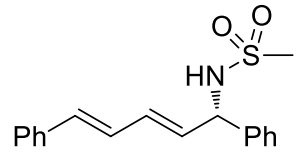

3aj

$E / Z=10: 1$

${ }^{13} \mathrm{C}$ NMR $\left(100 \mathrm{M}, \mathrm{CDCl}_{3}\right)$
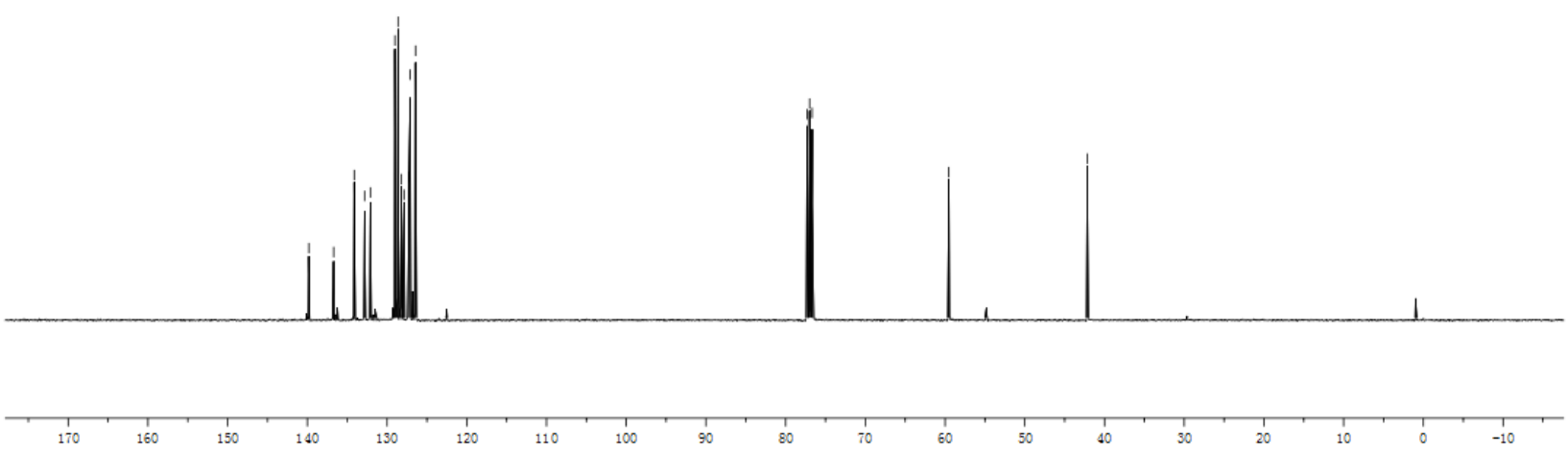

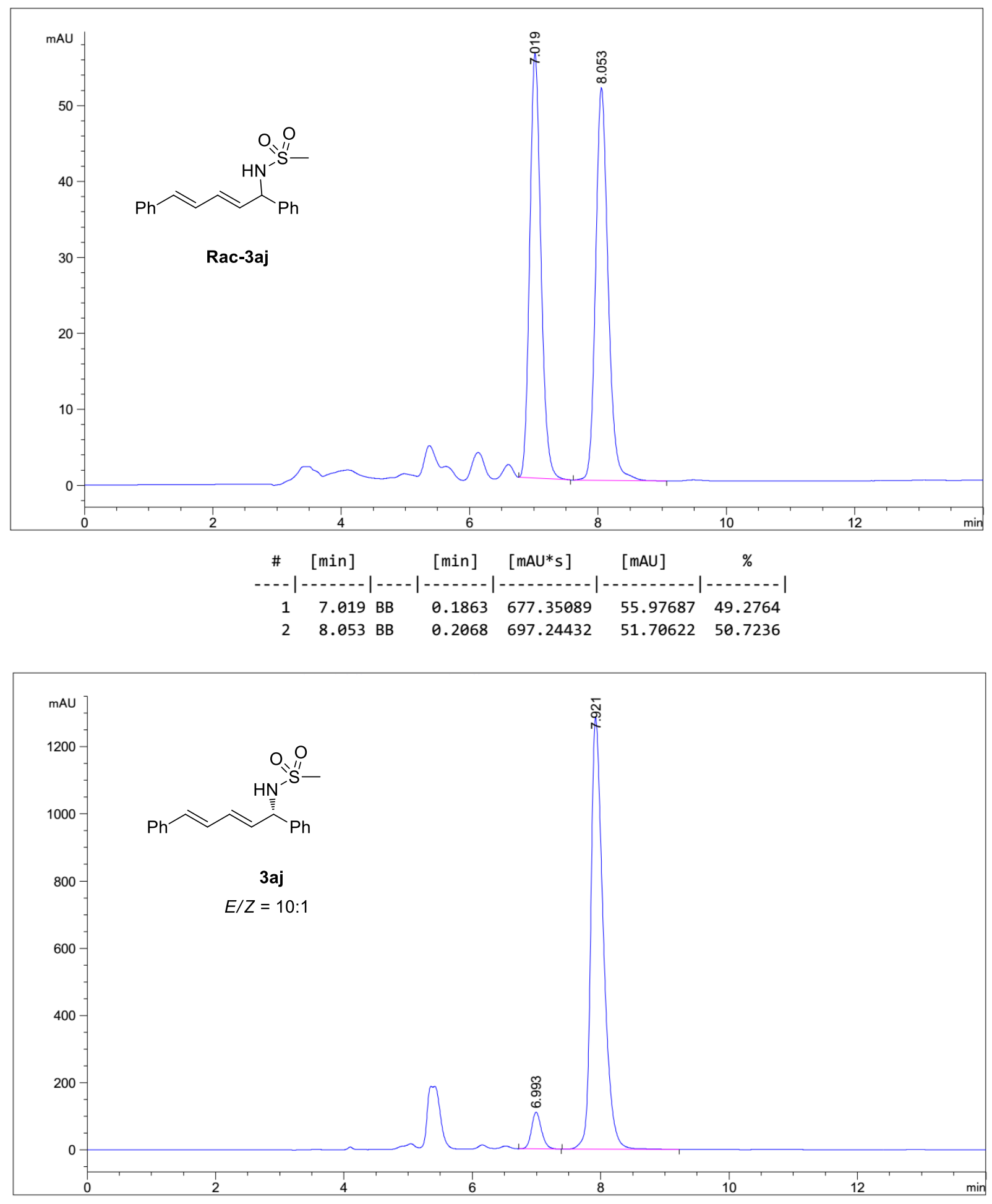


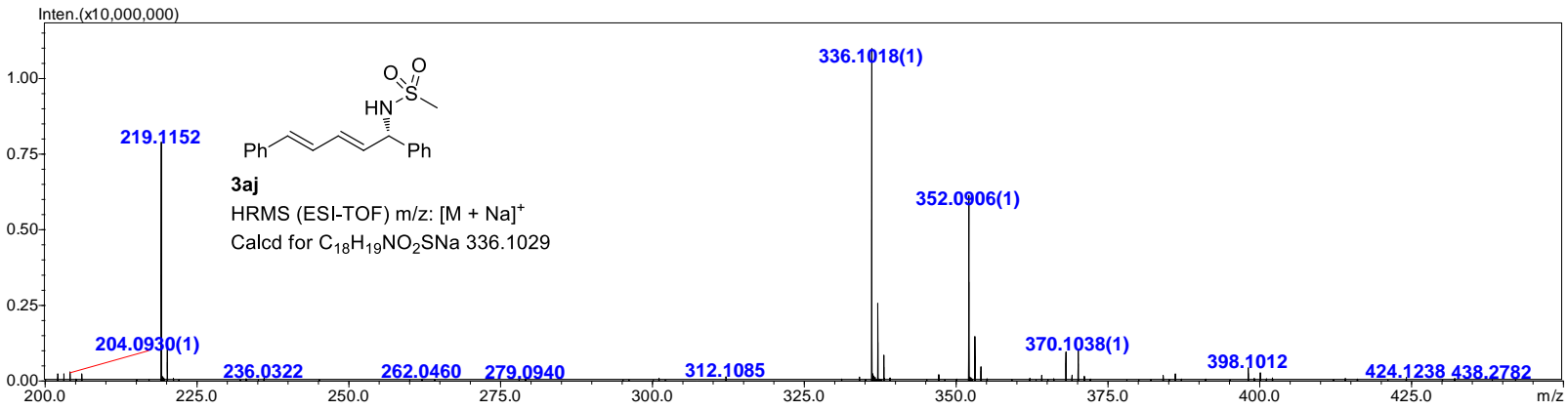


<smiles>N=C1NS(=O)(=O)c2ccccc2C1(/C=C/C=C/c1ccccc1)c1ccccc1</smiles>

$3 a k$

${ }^{1} \mathrm{H}$ NMR $\left(400 \mathrm{M}, \mathrm{CDCl}_{3}\right)$

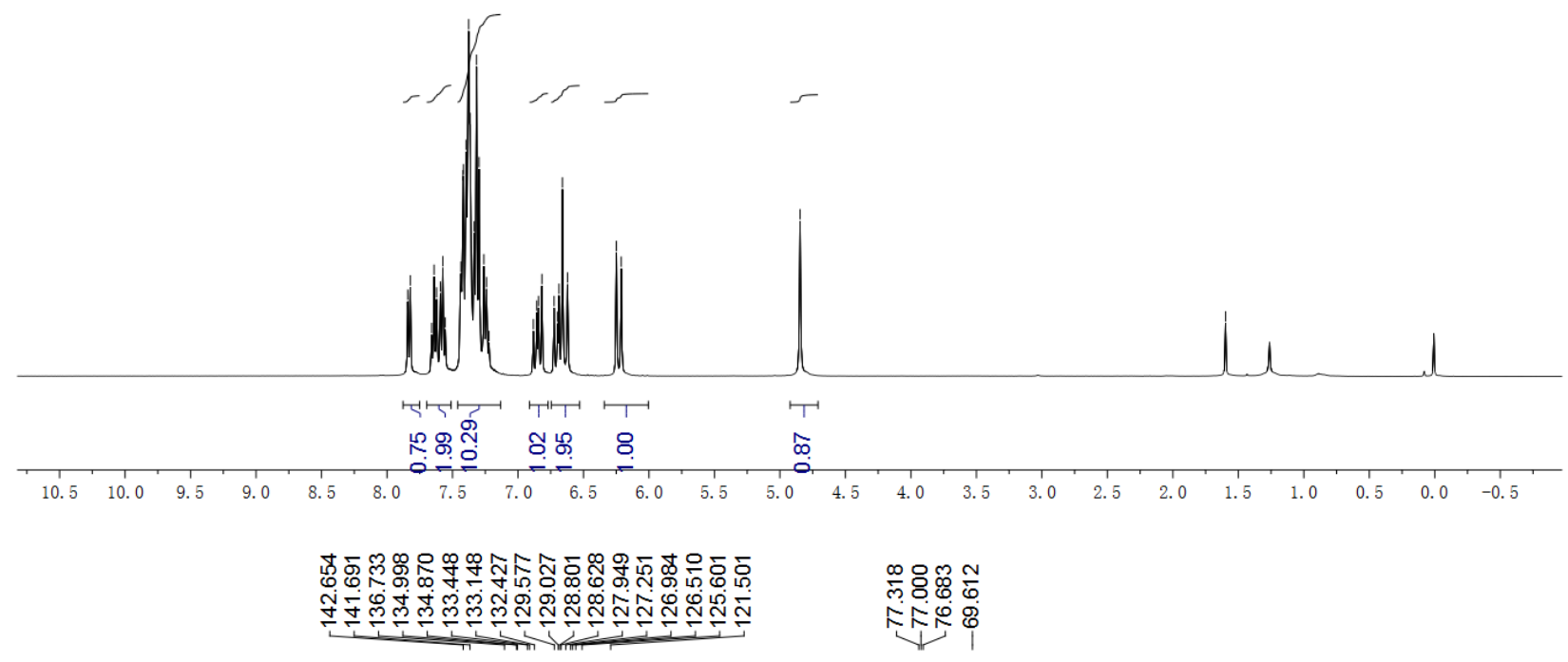<smiles></smiles>

3ak

${ }^{13} \mathrm{C}$ NMR $\left(100 \mathrm{M}, \mathrm{CDCl}_{3}\right)$

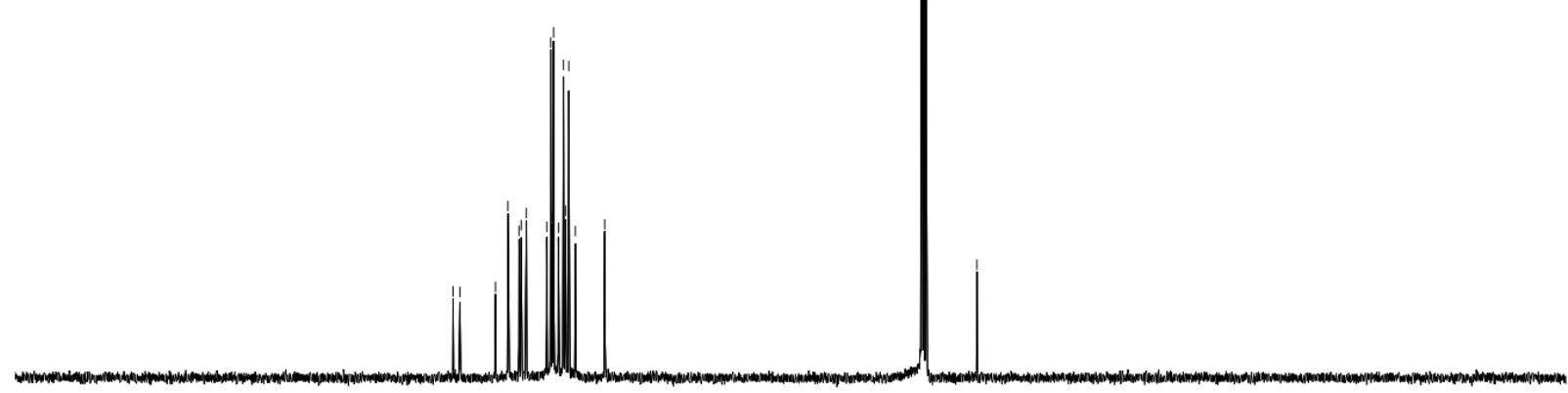

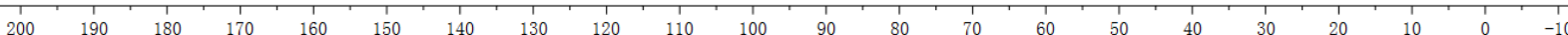



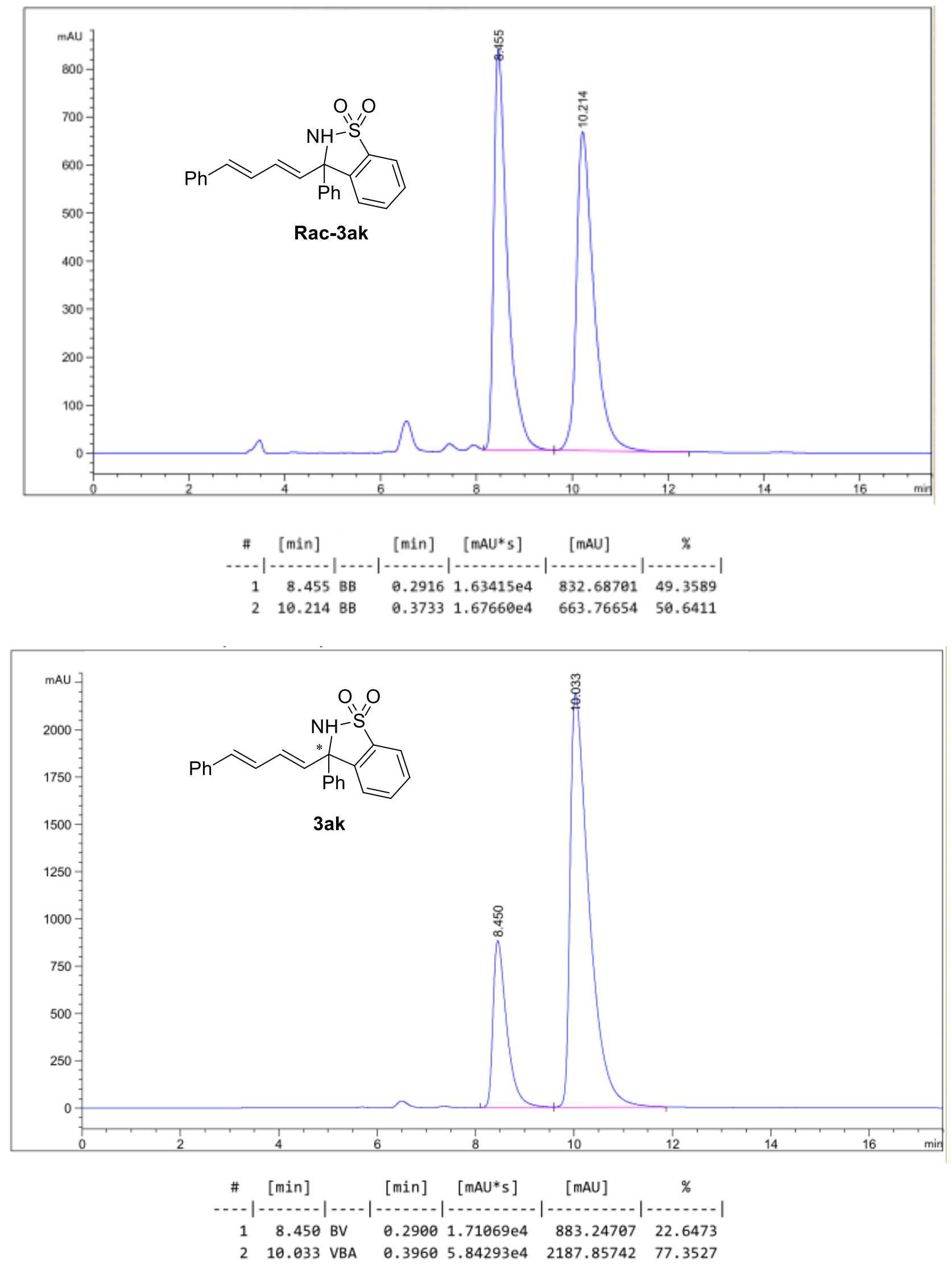


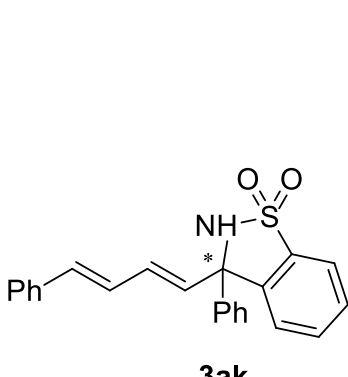

3ak

HRMS (ESI-TOF) m/z: [M + Na]

Calcd for $\mathrm{C}_{23} \mathrm{H}_{19} \mathrm{NO}_{2} \mathrm{SNa} 396.1029$

๙゚

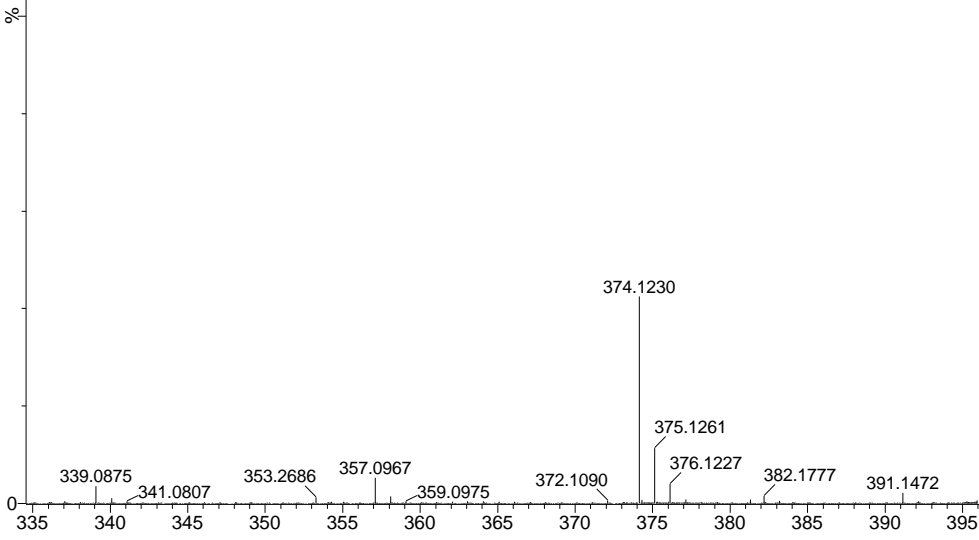

397.1078

$398.1049 \quad 412.0780$

\begin{tabular}{|l|l}
\hline 399.1069 & 418.0875 \\
\hline
\end{tabular}

\begin{tabular}{ll|l|l}
399.1069 & 407.1455 & 418.0875 \\
3 & 419.0913
\end{tabular}

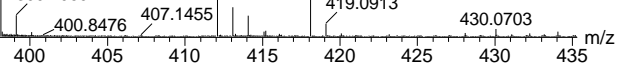




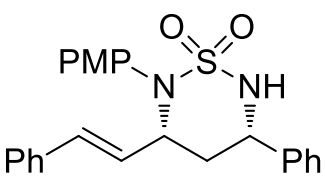

$5 a$

${ }^{1} \mathrm{H}$ NMR (400M, $\left.\mathrm{CDCl}_{3}\right)$
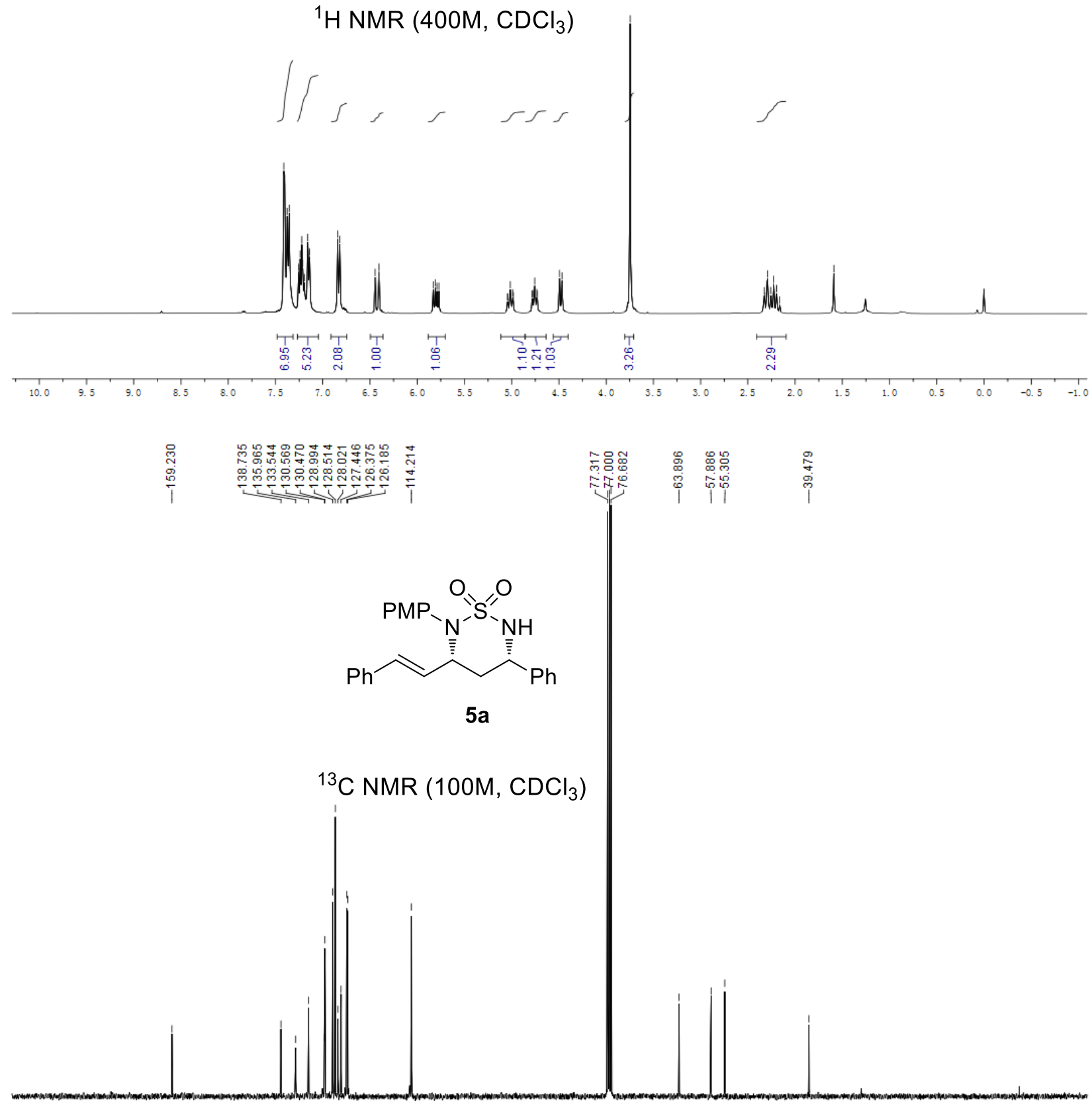

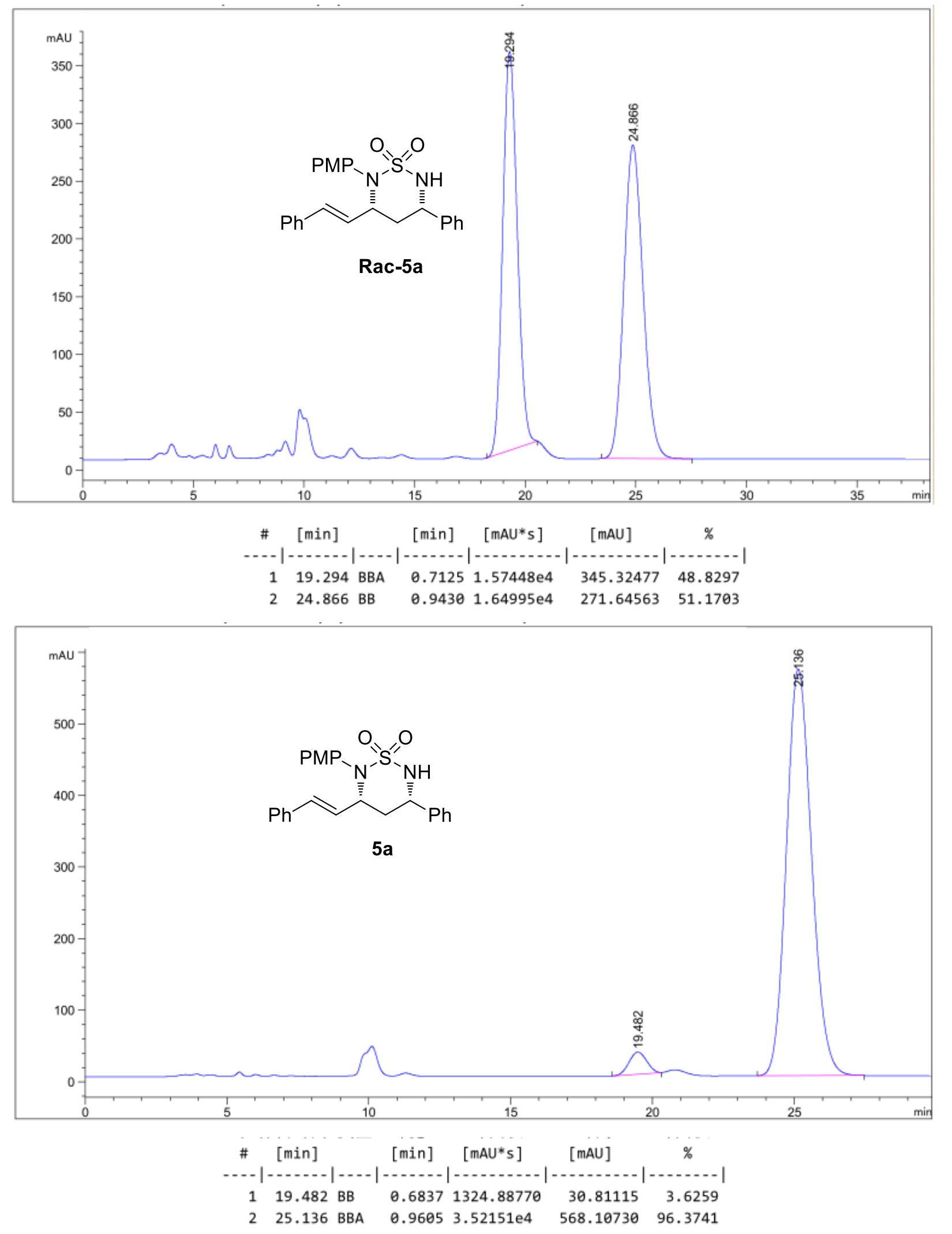
Spectrum Plot Report

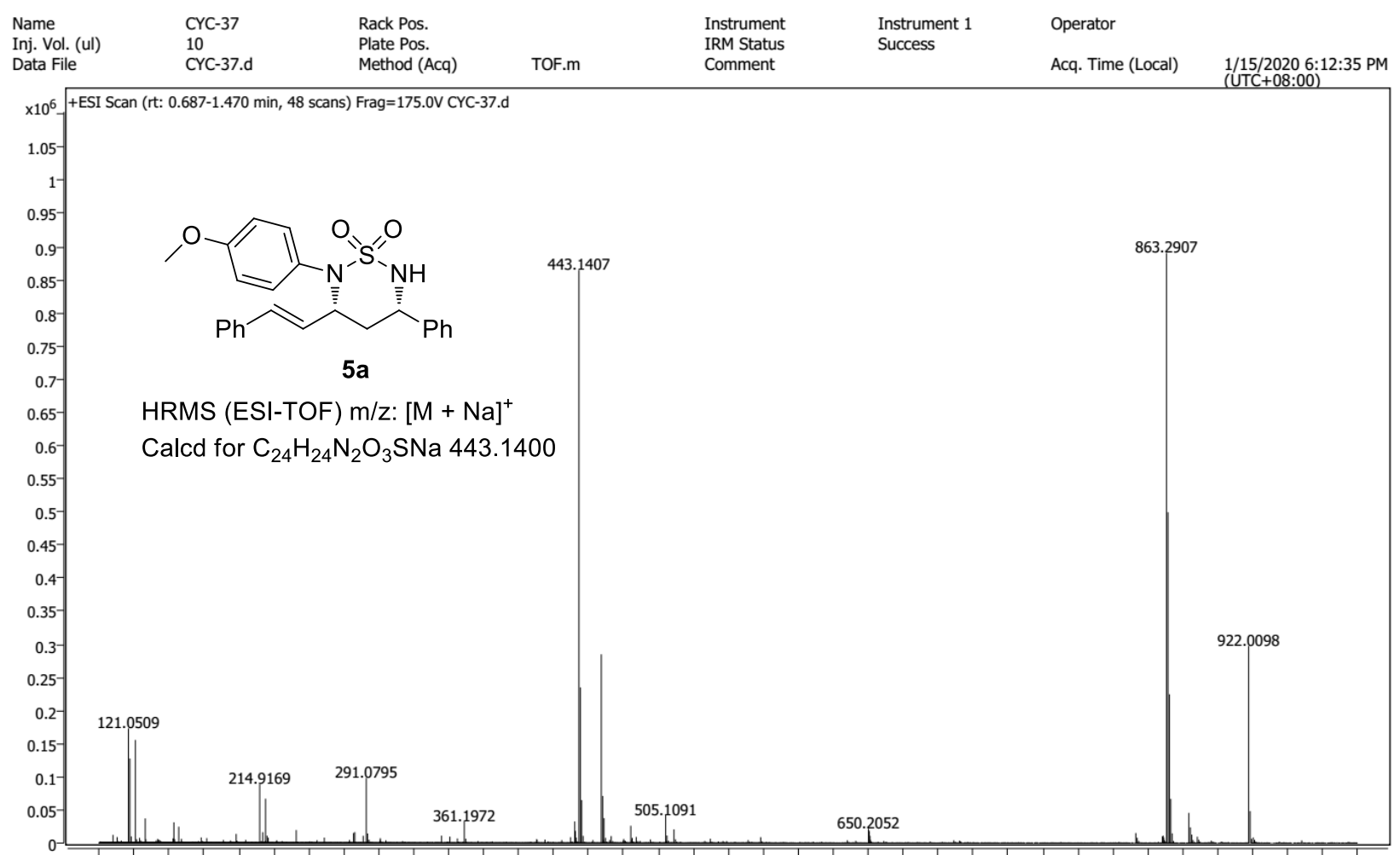

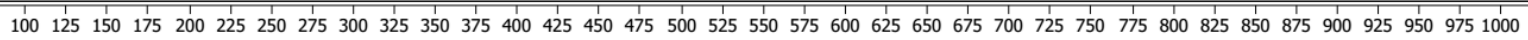
Counts vs. Mass-to-Charge $(\mathrm{m} / \mathrm{z})$ 


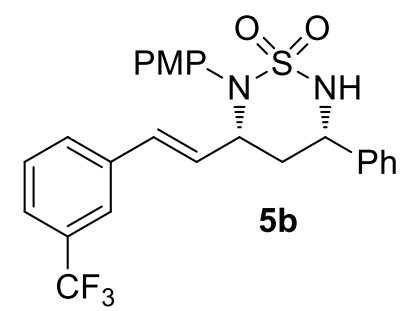

${ }^{1} \mathrm{H}$ NMR $\left(400 \mathrm{M}, \mathrm{CDCl}_{3}\right)$

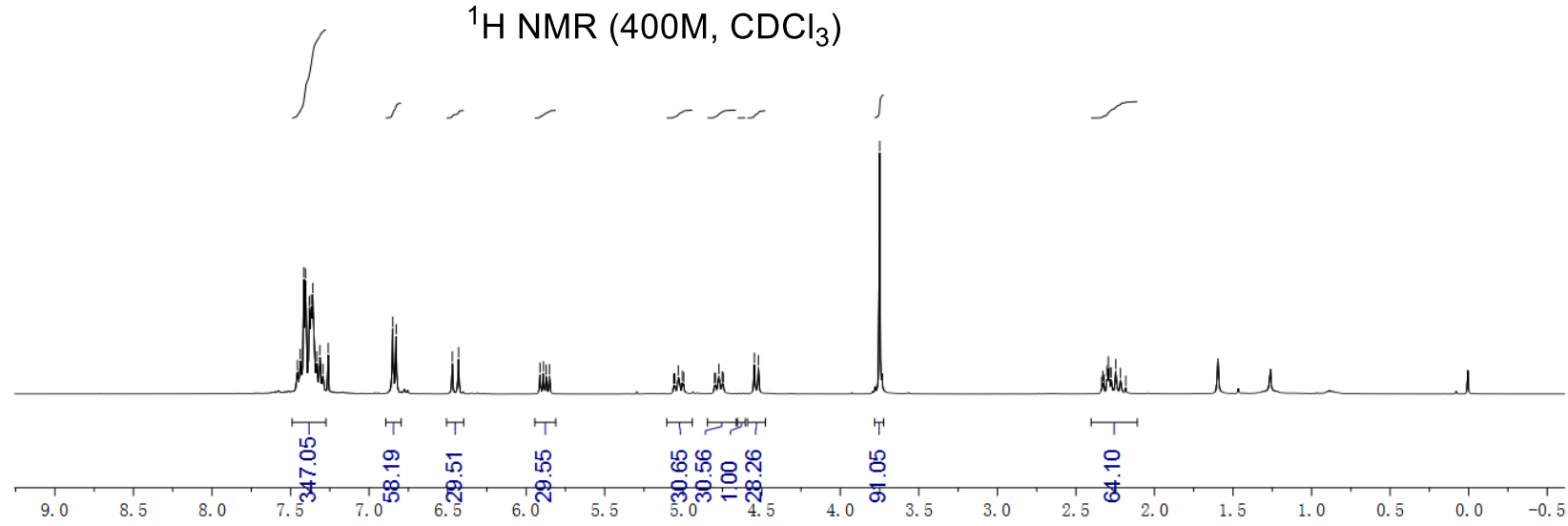

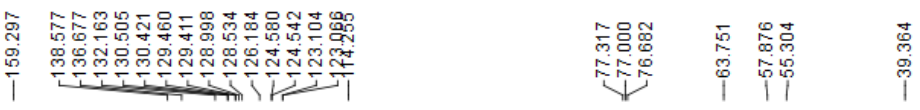

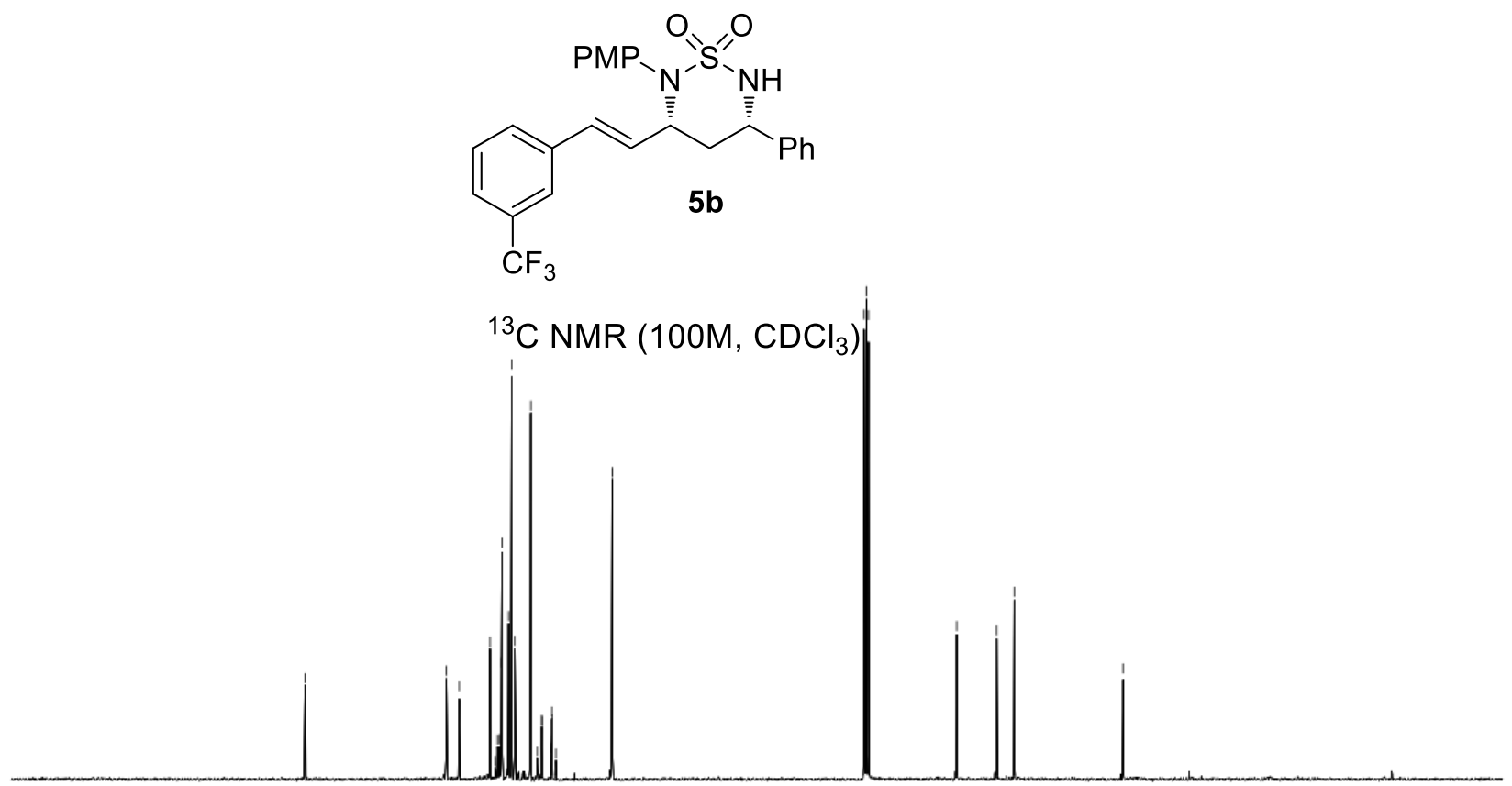

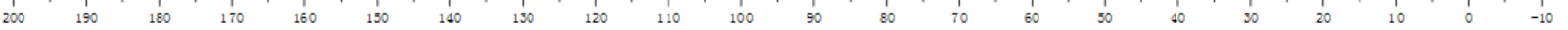



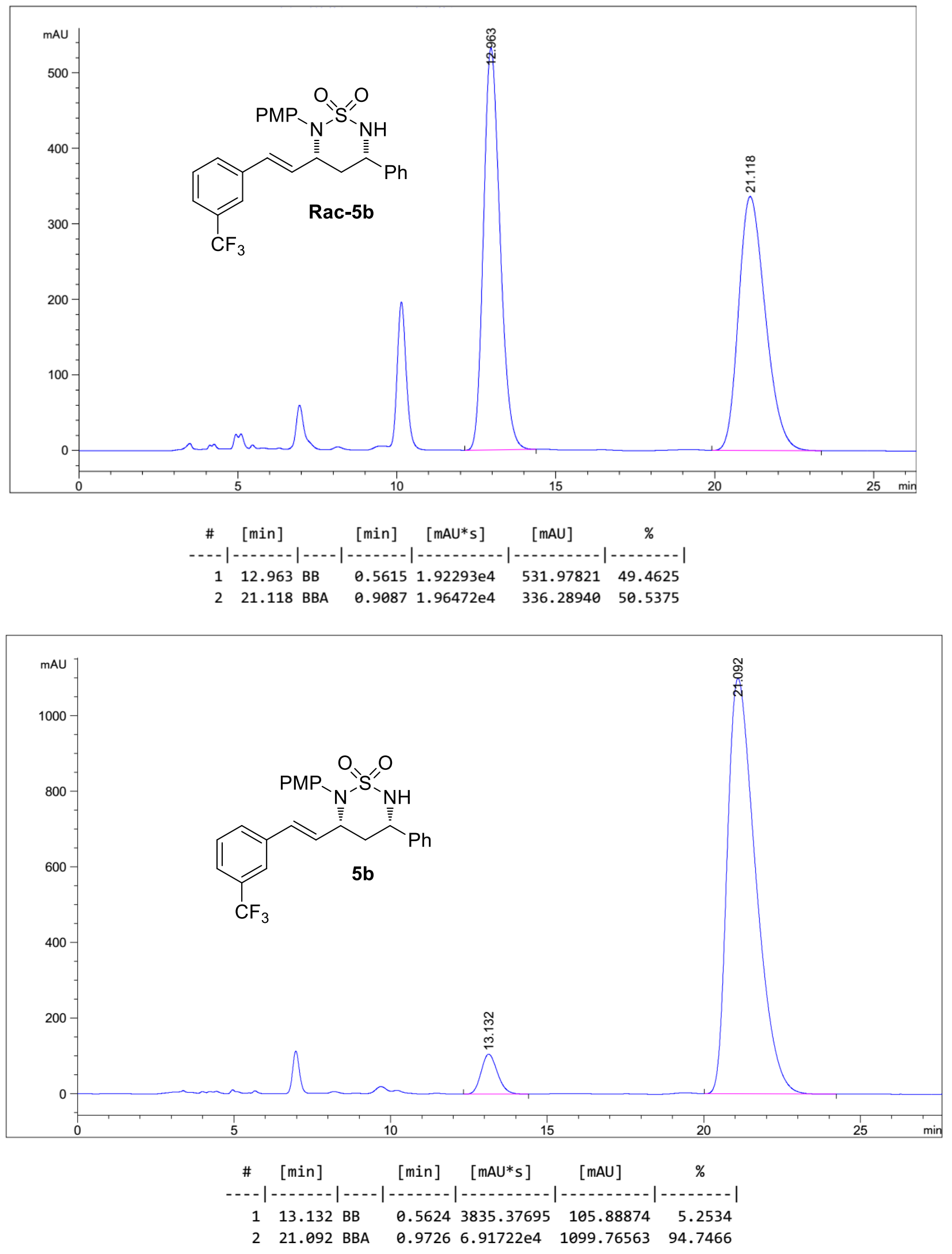


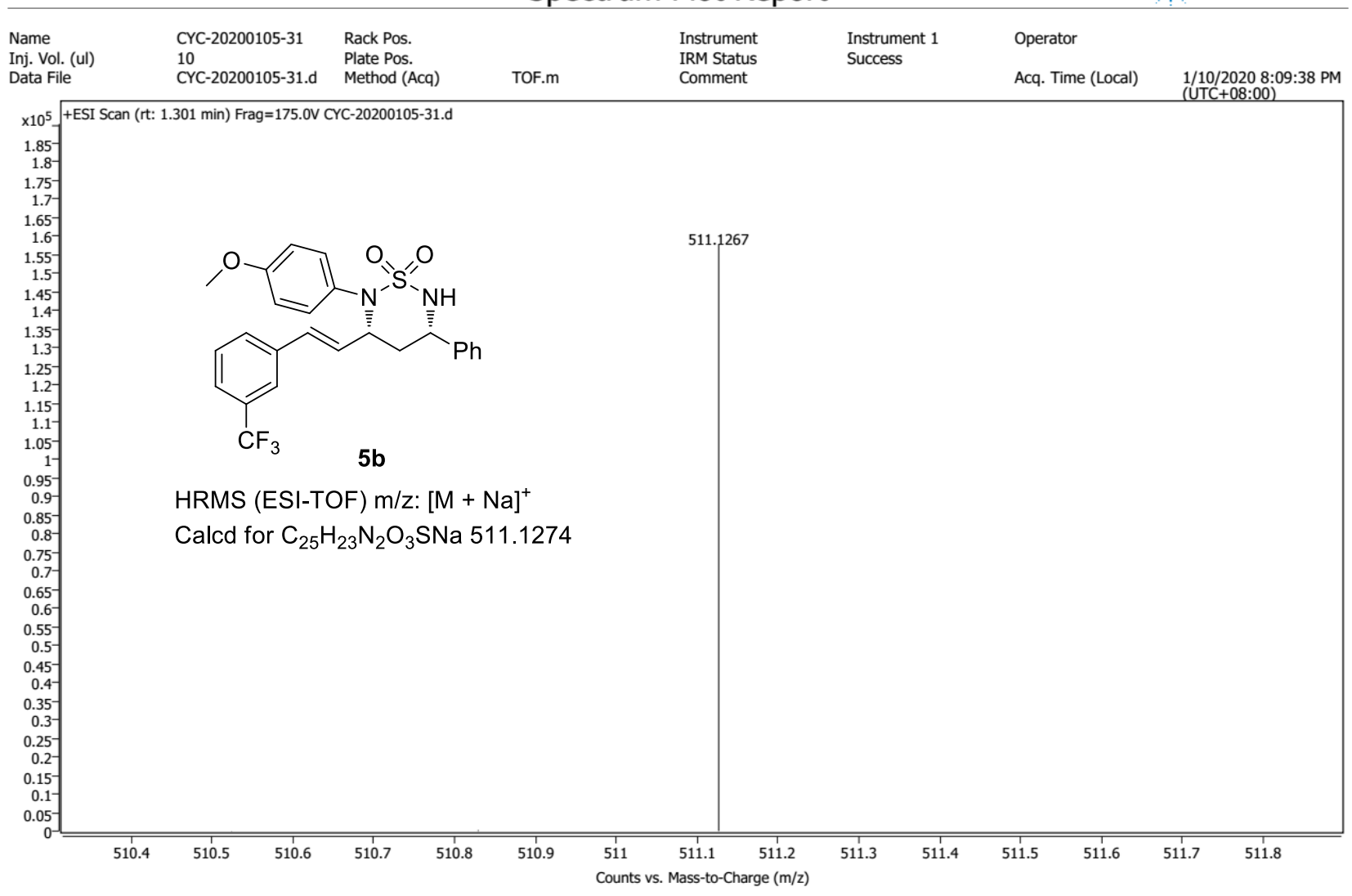




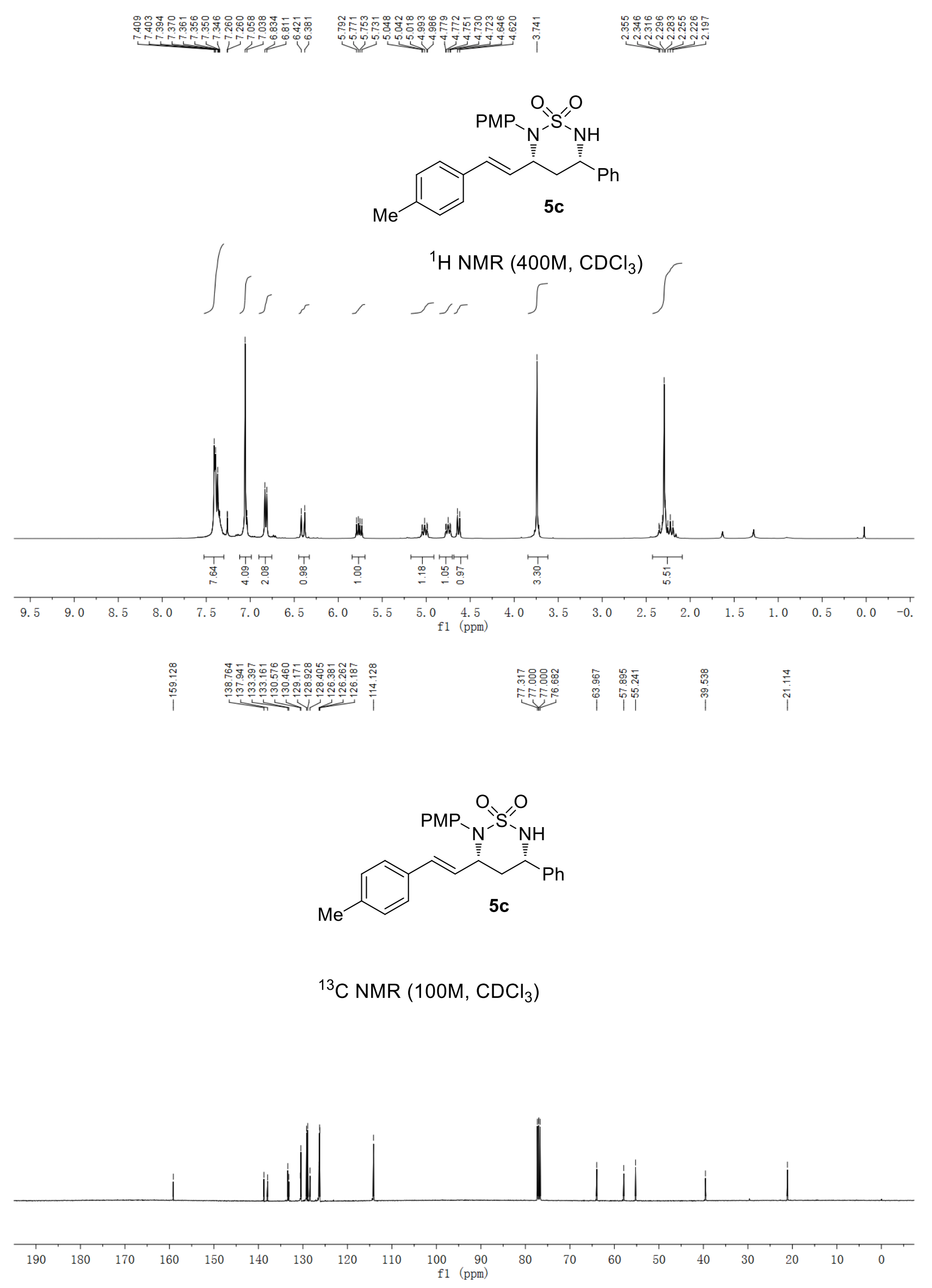



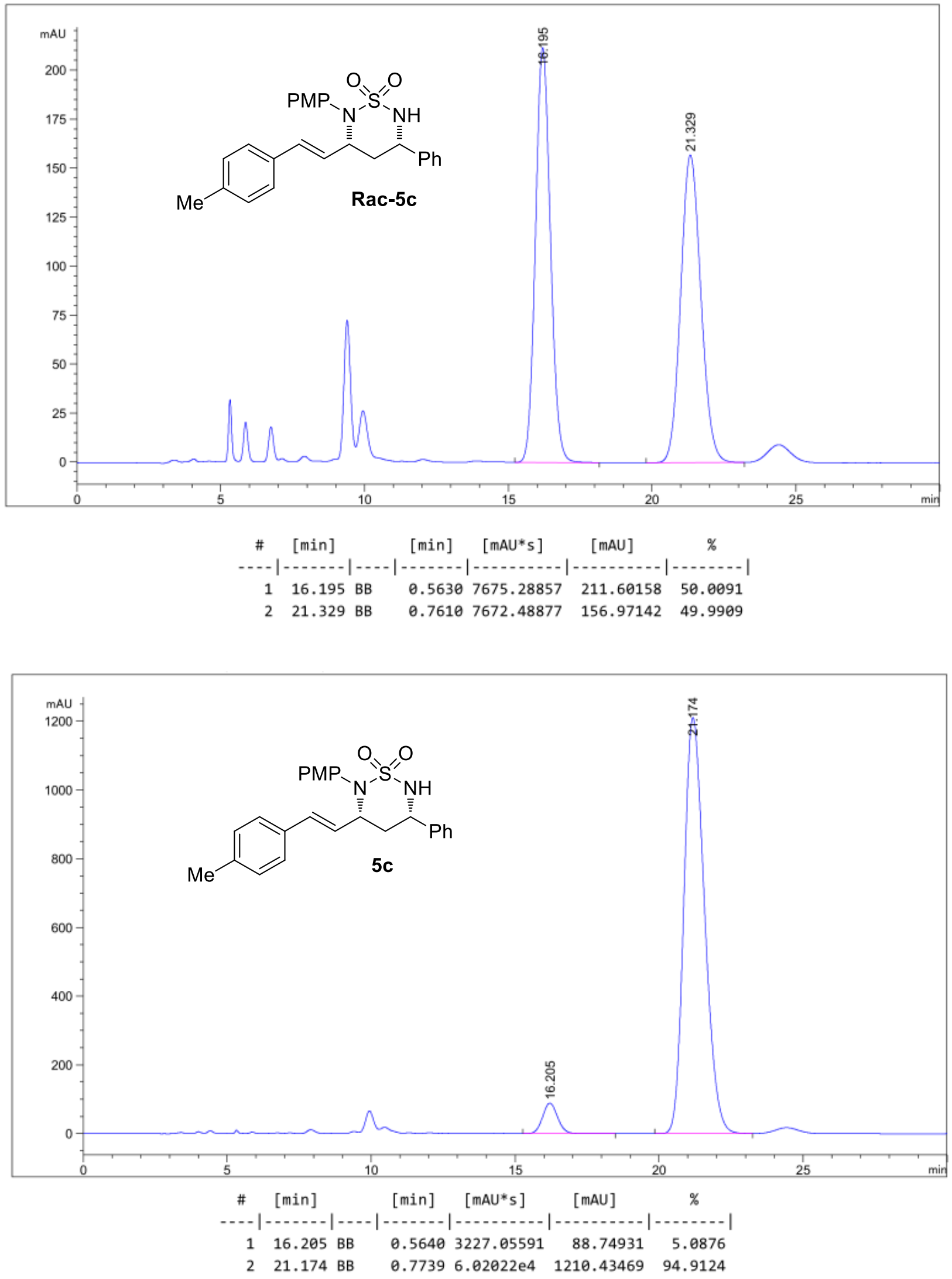


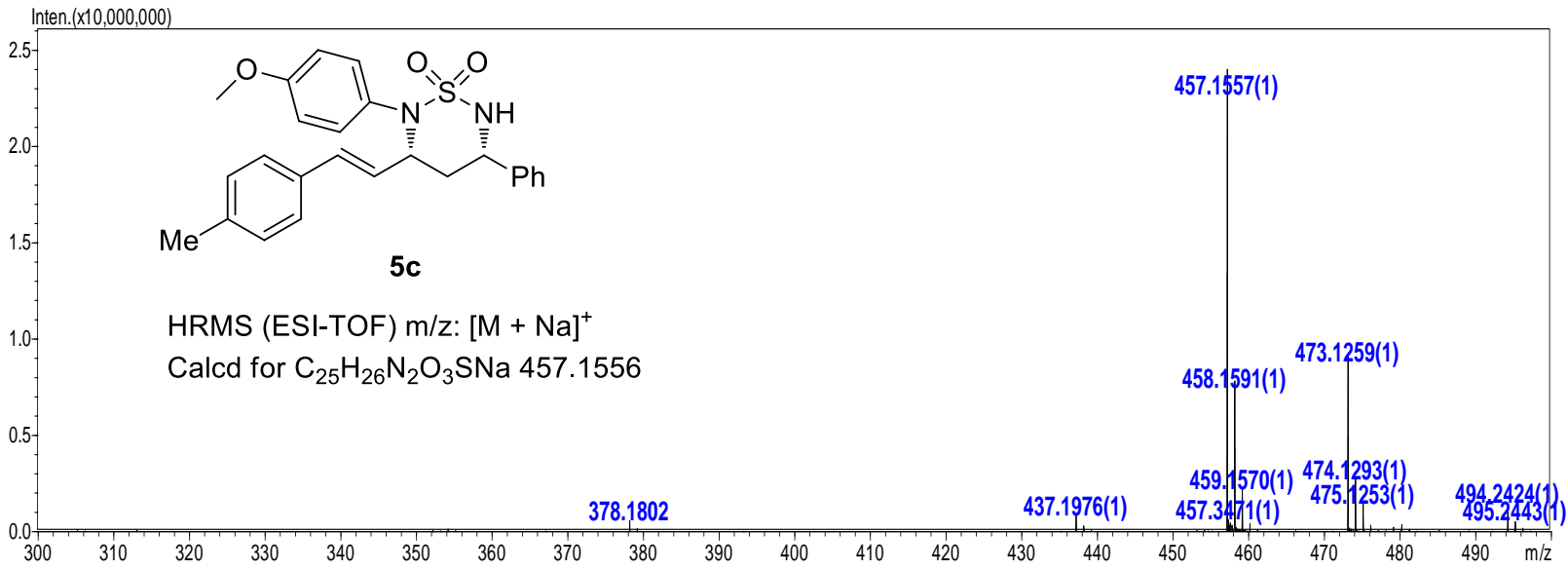




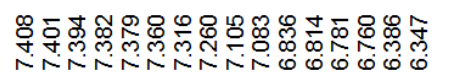

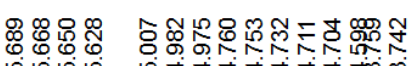

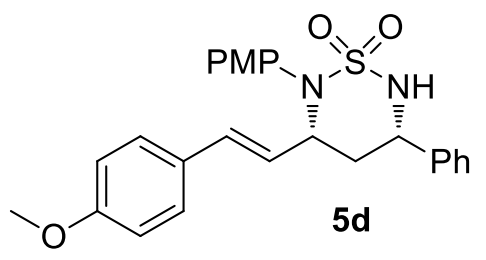

${ }^{1} \mathrm{H}$ NMR (400M, $\mathrm{CDCl}_{3}$ )
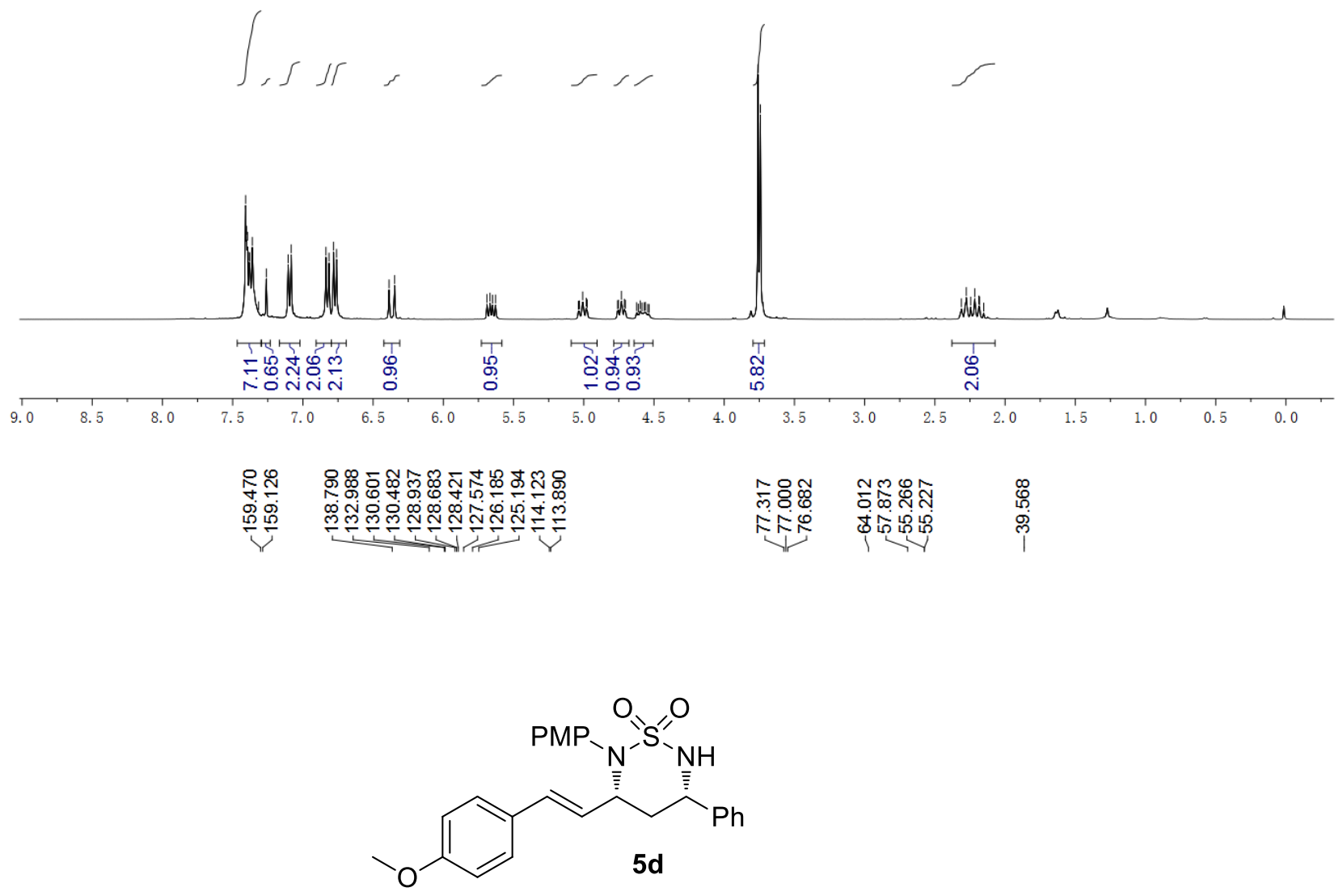

${ }^{13} \mathrm{C}$ NMR (100M, $\mathrm{CDCl}_{3}$ )
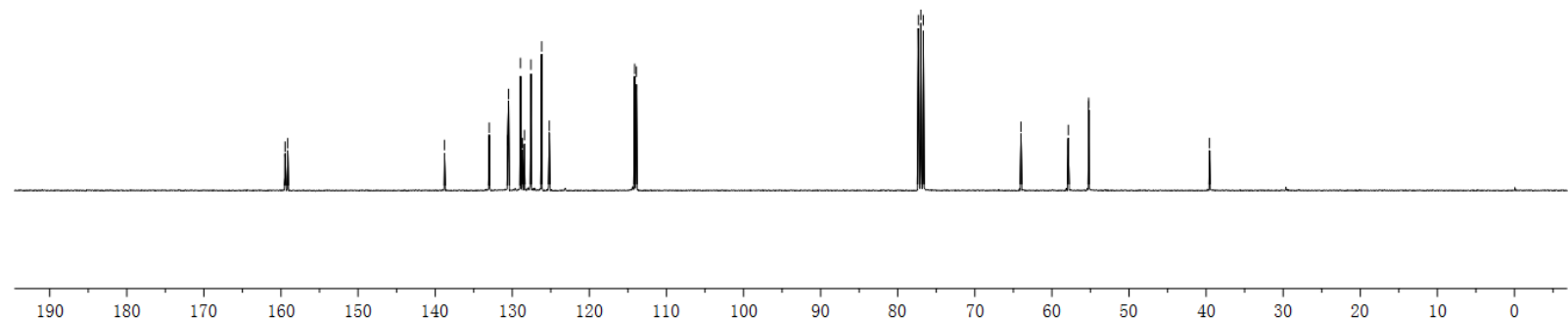


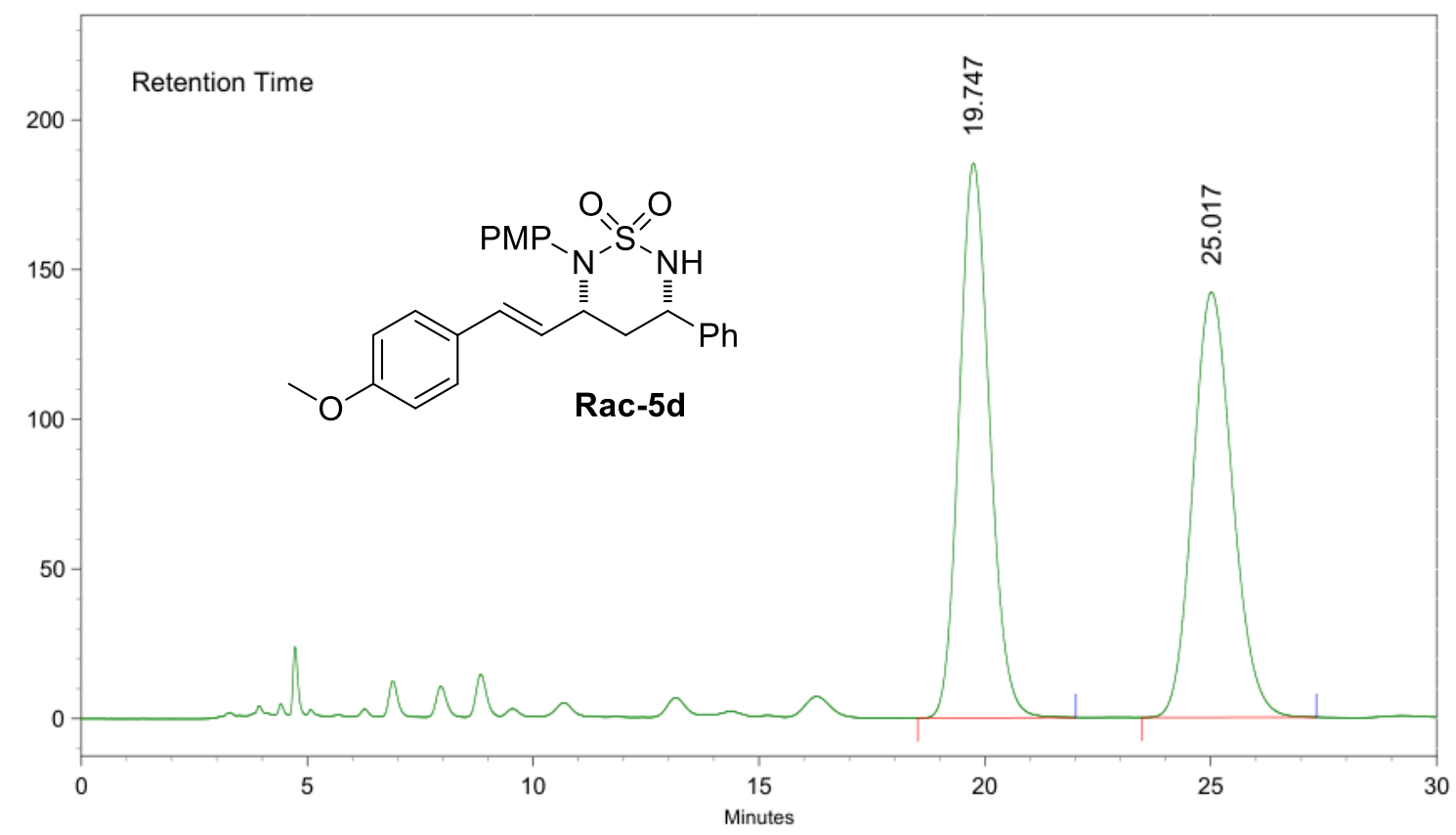

\begin{tabular}{llllll} 
Peak No. & Ret Time & Width & Height & Area & Area [\%] \\
\hline 1 & 19.747 & 3.500 & 3108243 & 145810993 & 50.0639 \\
2 & 25.017 & 3.853 & 2383786 & 145438593 & 49.9361
\end{tabular}

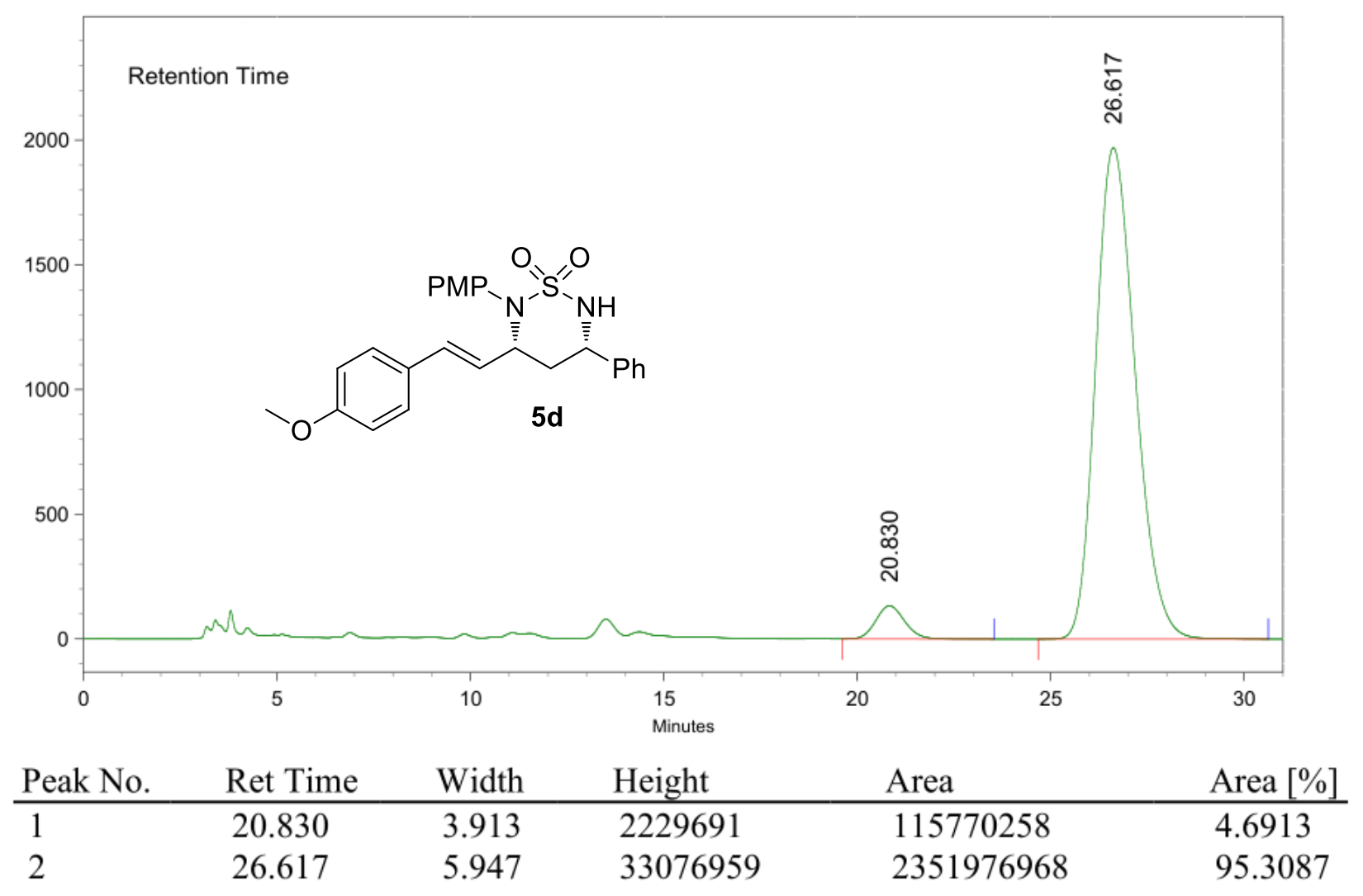


Spectrum Plot Report

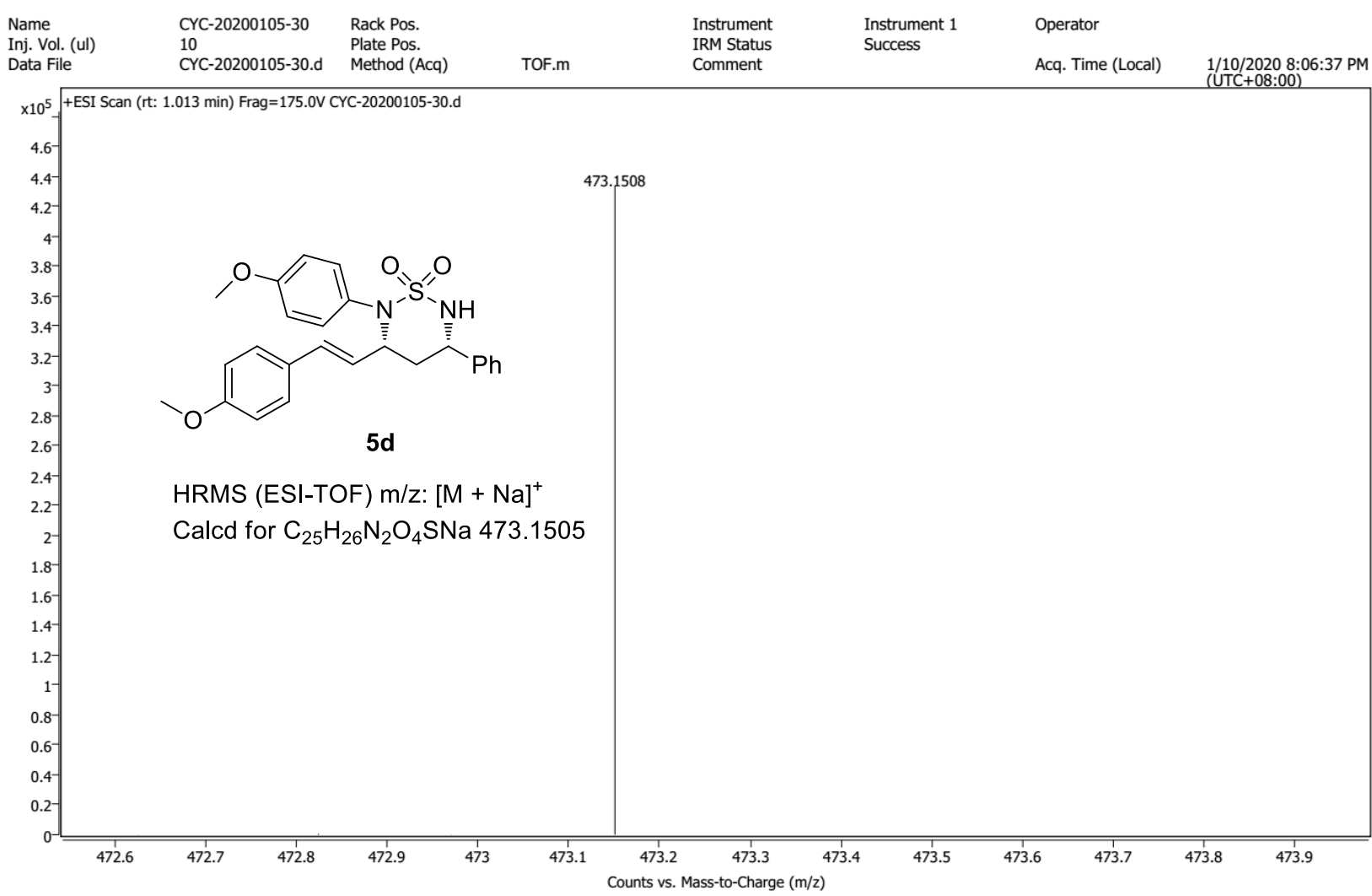


<smiles>O=P(=O)N1[C@@H](/C=C/c2ccc3ccccc3c2)C[C@H](c2ccccc2)N[PH]1(=O)=O</smiles>

$16: 1 \mathrm{dr}$

${ }^{1} \mathrm{H} \operatorname{NMR}\left(400 \mathrm{M}, \mathrm{CDCl}_{3}\right)$
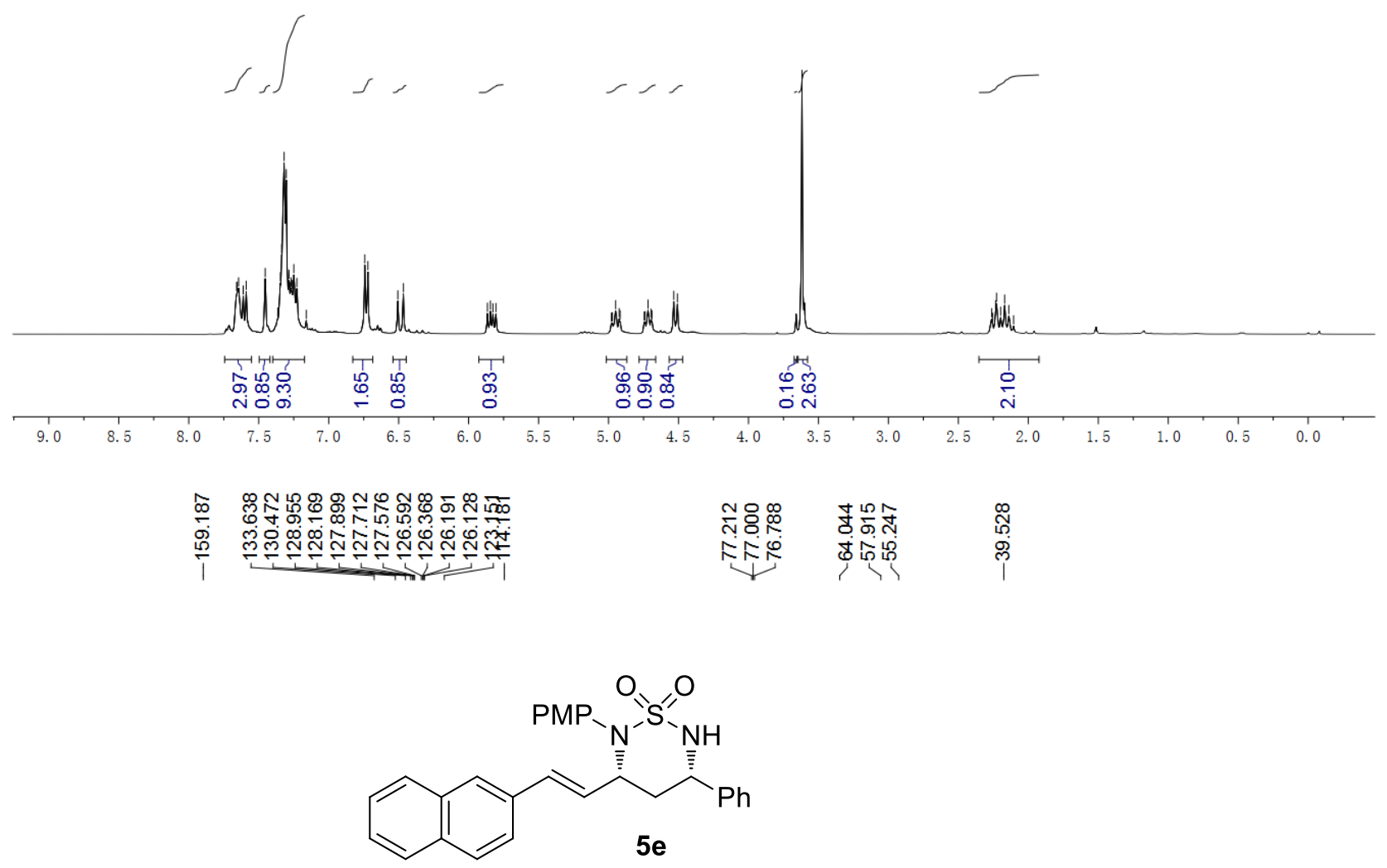

$16: 1 \mathrm{dr}$

${ }^{13} \mathrm{C}$ NMR (150M, $\mathrm{CDCl}_{3}$ )

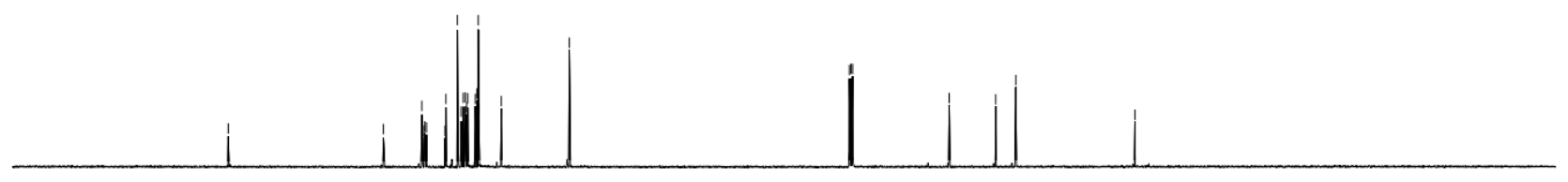

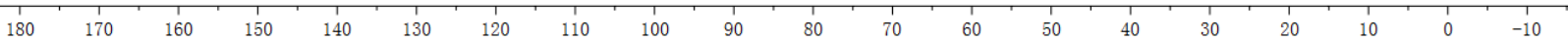




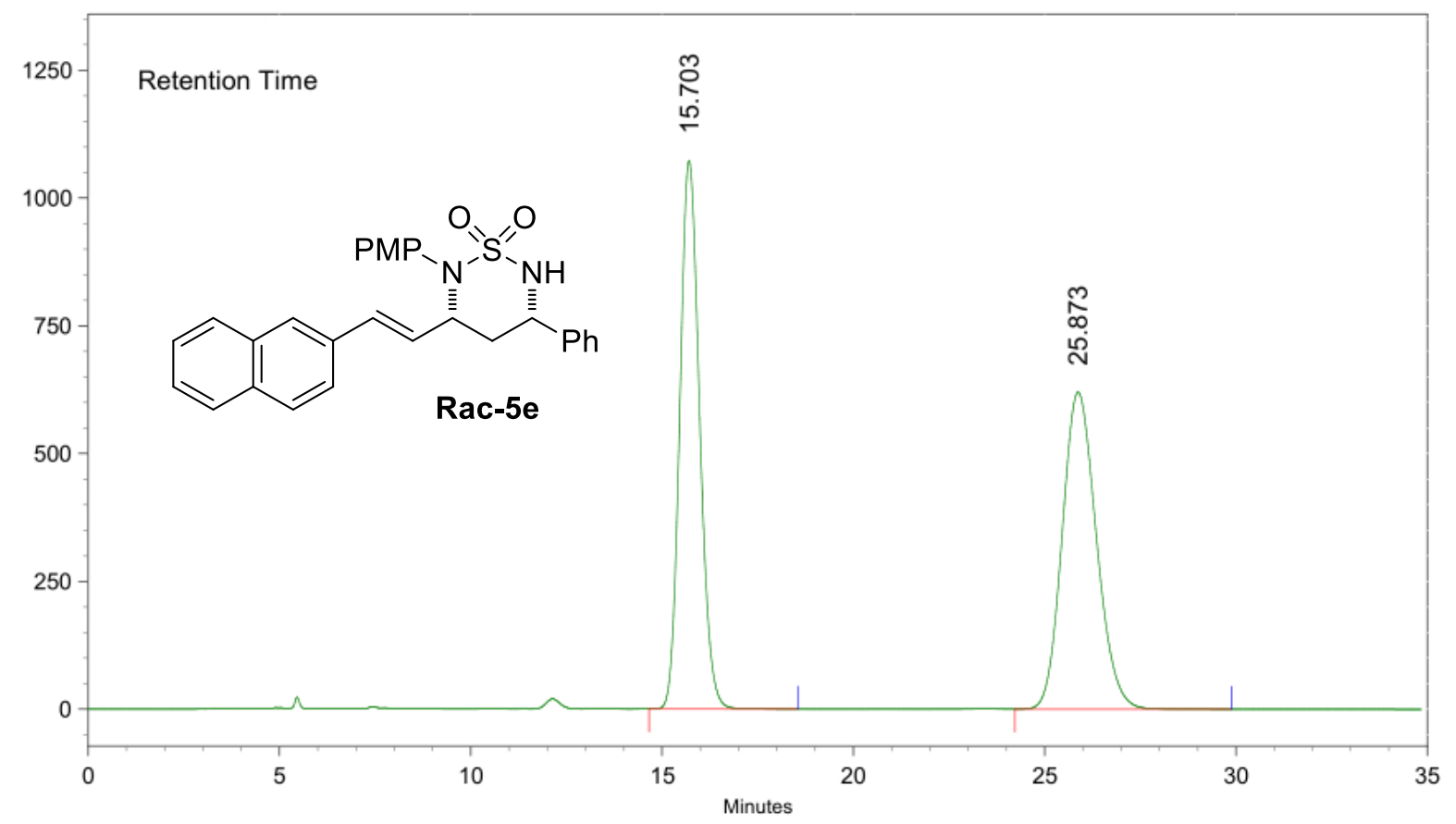

\begin{tabular}{llllll} 
Peak No. & Ret Time & Width & Height & Area & Area [\%] \\
\hline 1 & 15.703 & 3.897 & 18006156 & 644947540 & 49.9329 \\
2 & 25.873 & 5.670 & 10406635 & 646680953 & 50.0671
\end{tabular}

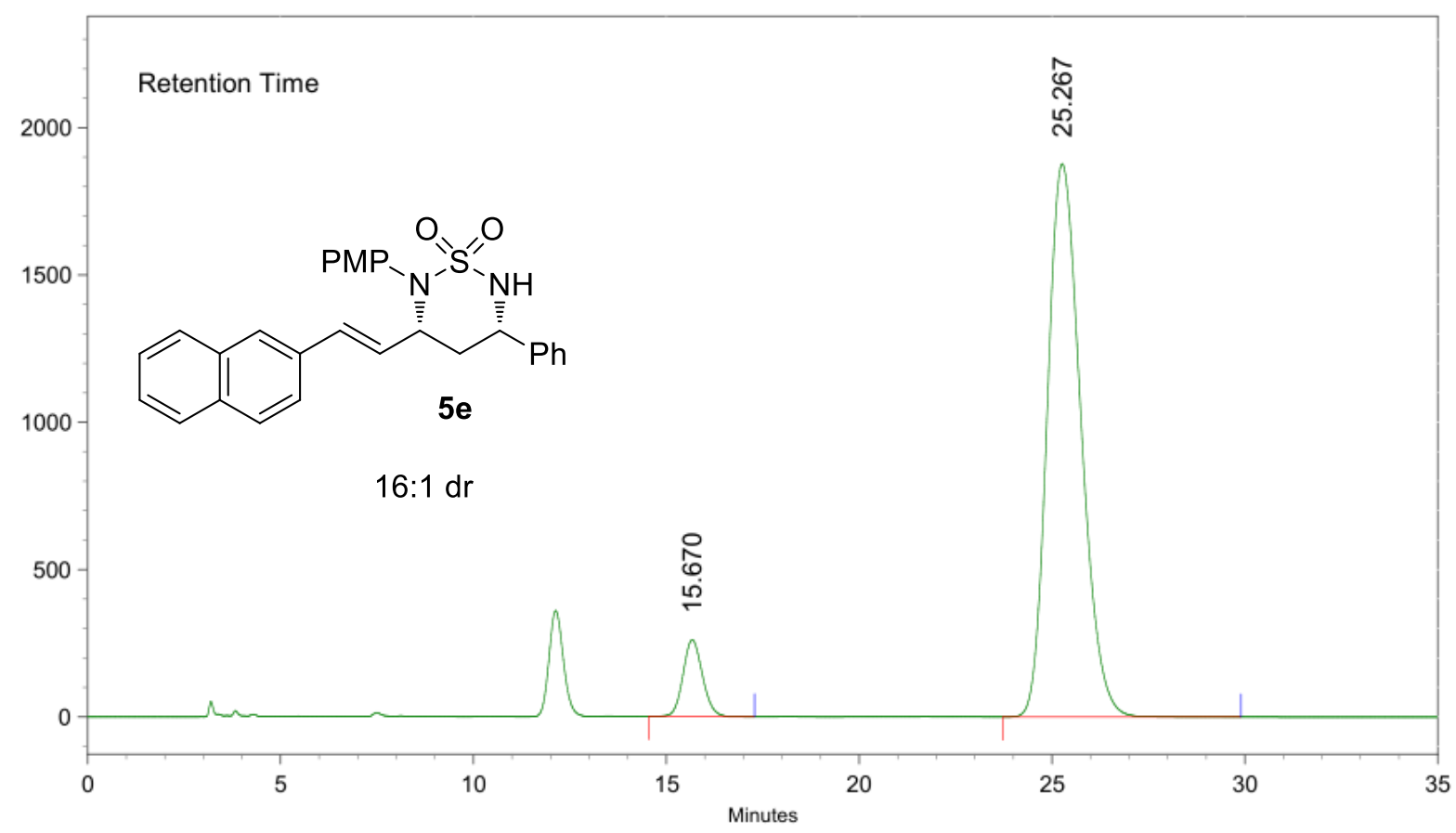

\begin{tabular}{llllll} 
Peak No. & Ret Time & Width & Height & Area & Area [\%] \\
\hline 1 & 15.670 & 2.740 & 4371412 & 153886119 & 7.4887 \\
2 & 25.267 & 6.173 & 31505481 & 1901027924 & 92.5113
\end{tabular}


Spectrum Plot Report

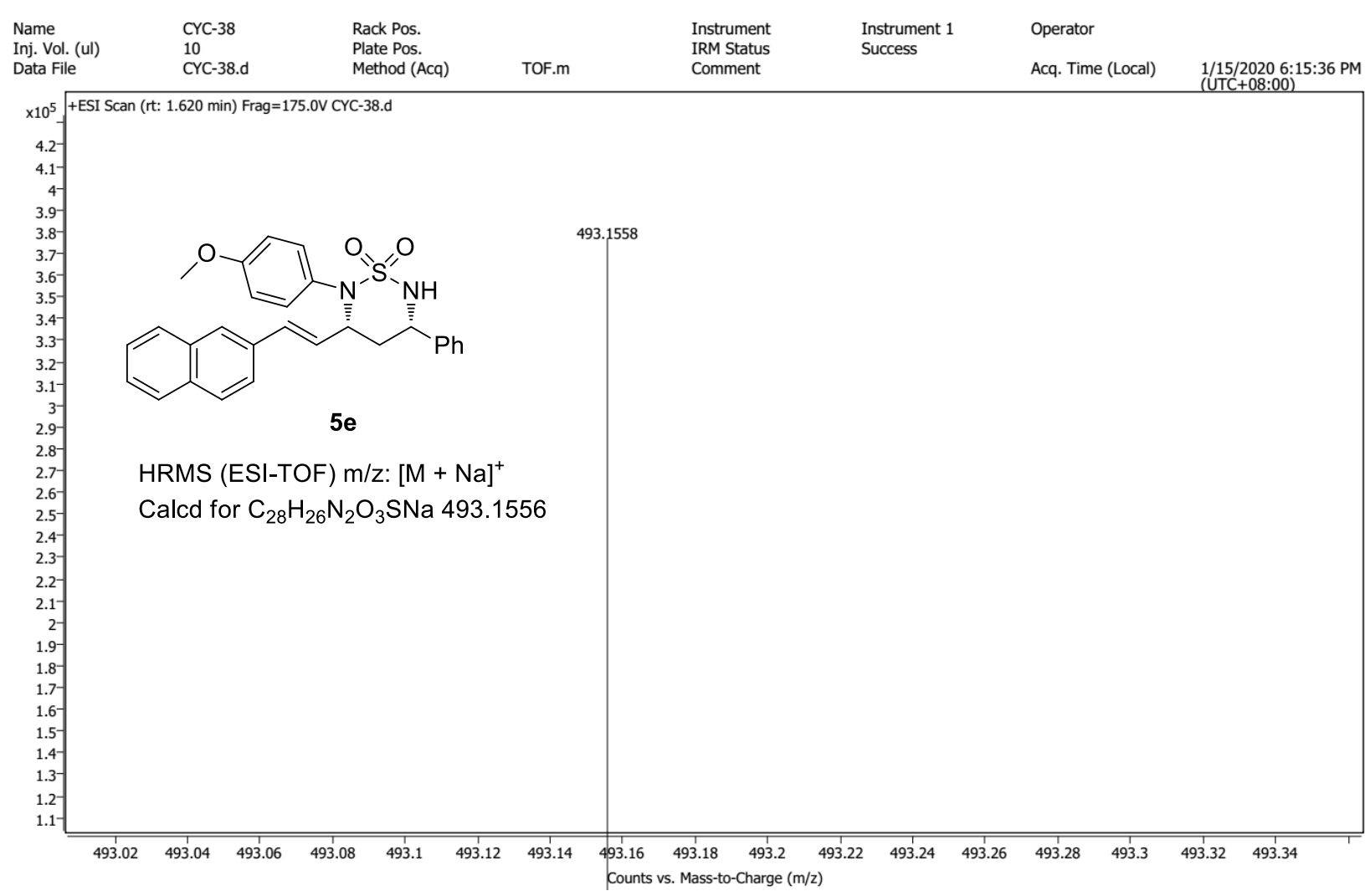




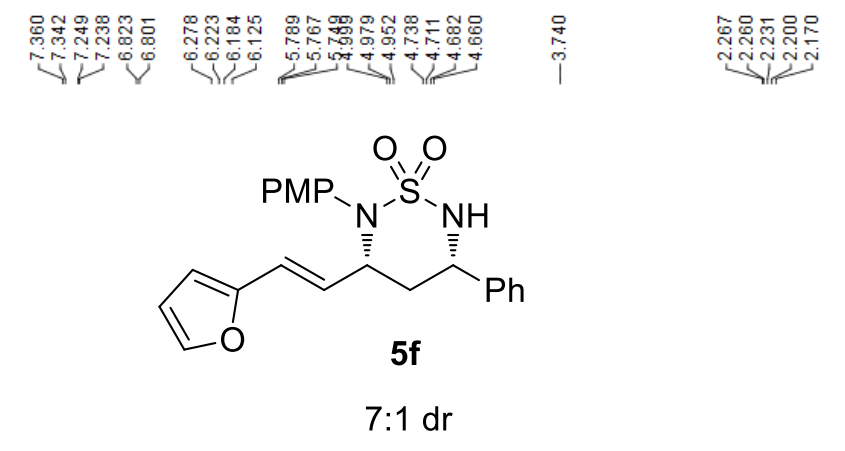

${ }^{1} \mathrm{H}$ NMR (400M, $\mathrm{CDCl}_{3}$ )

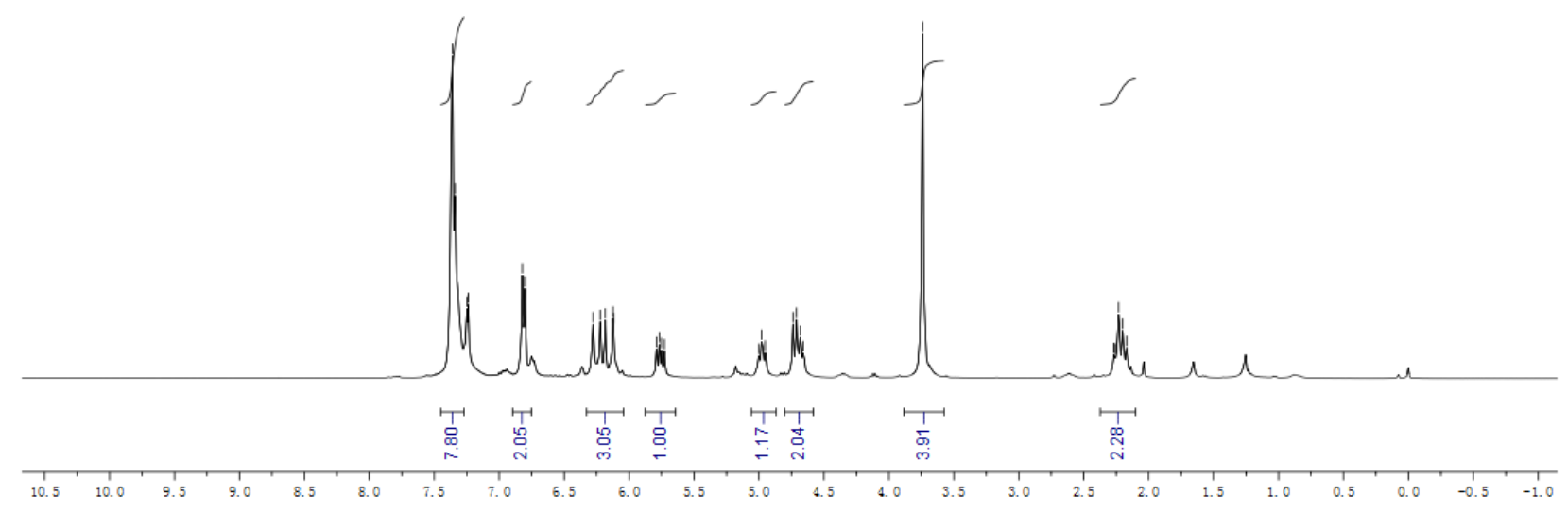

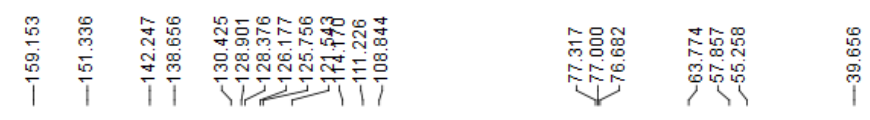

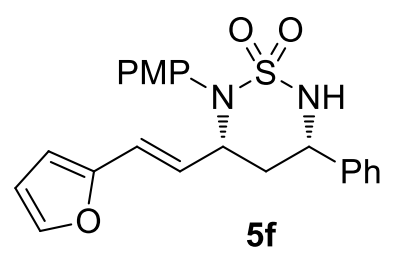

7:1 dr

${ }^{13} \mathrm{C}$ NMR (100M, $\mathrm{CDCl}_{3}$ )

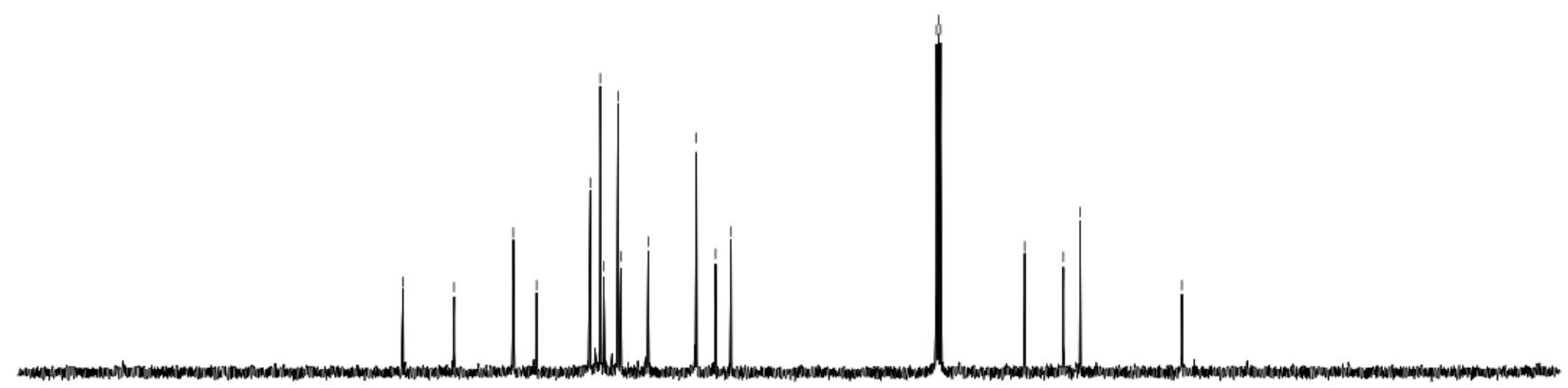



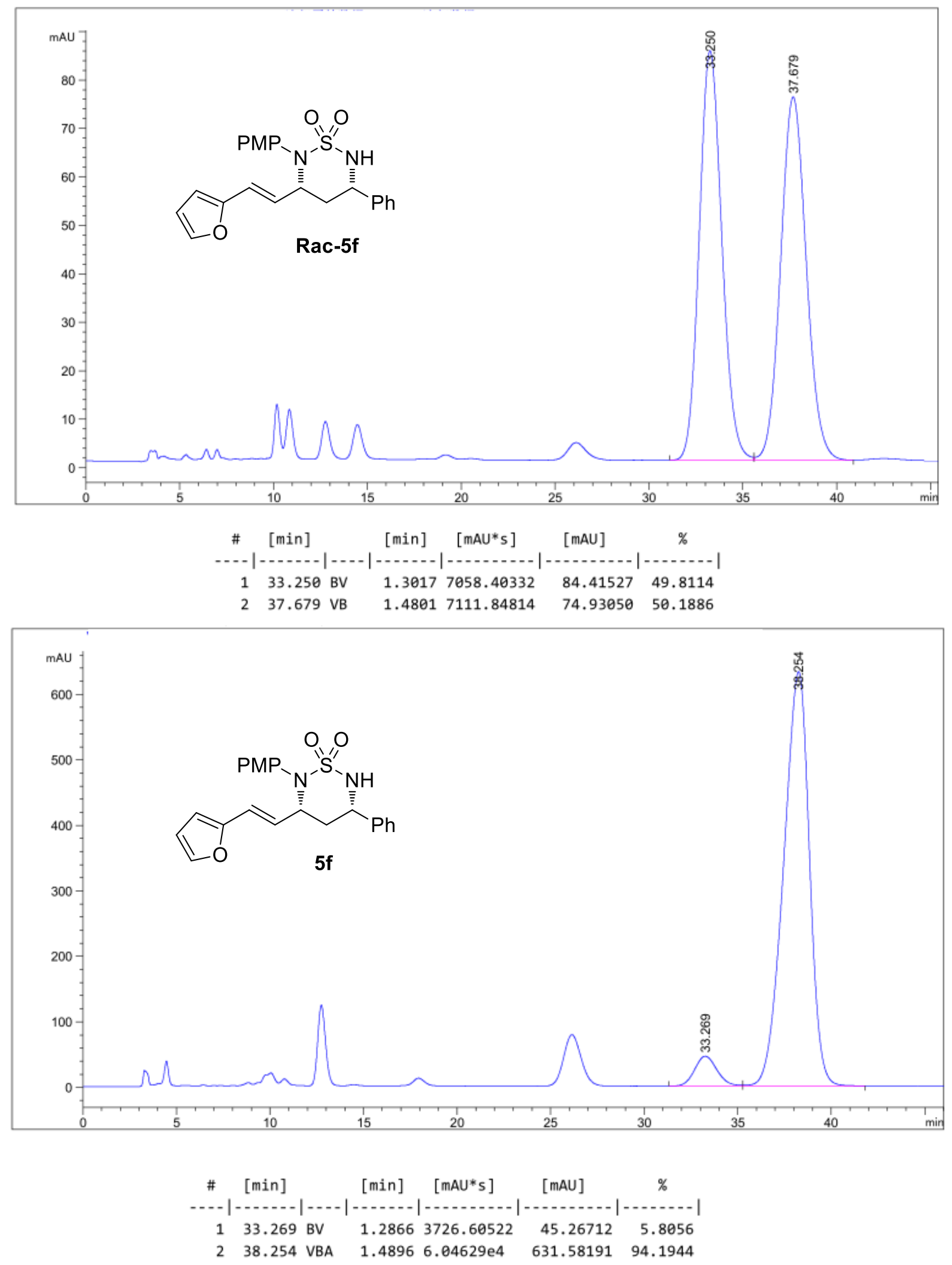


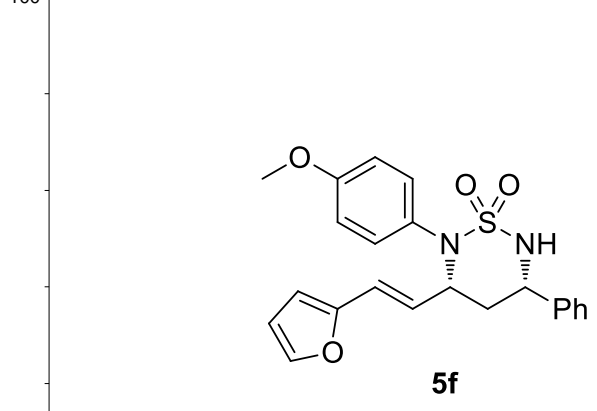

HRMS (ESI-TOF) m/z: [M + Na]

$\circ \quad$ Calcd for $\mathrm{C}_{22} \mathrm{H}_{22} \mathrm{~N}_{2} \mathrm{O}_{4} \mathrm{SNa} 433.1192$

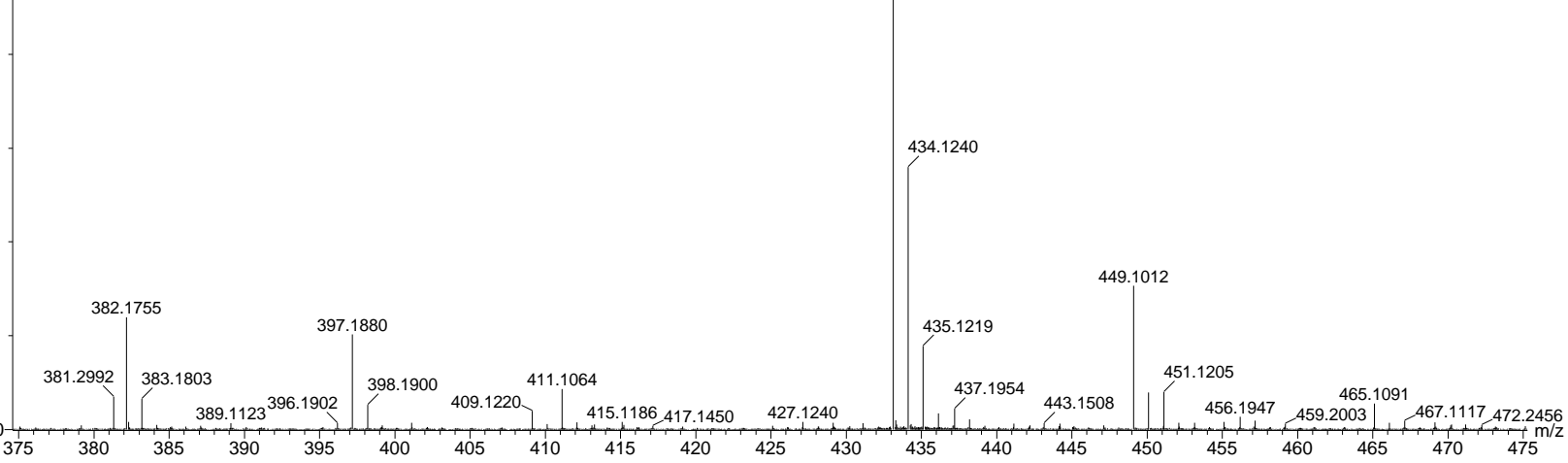




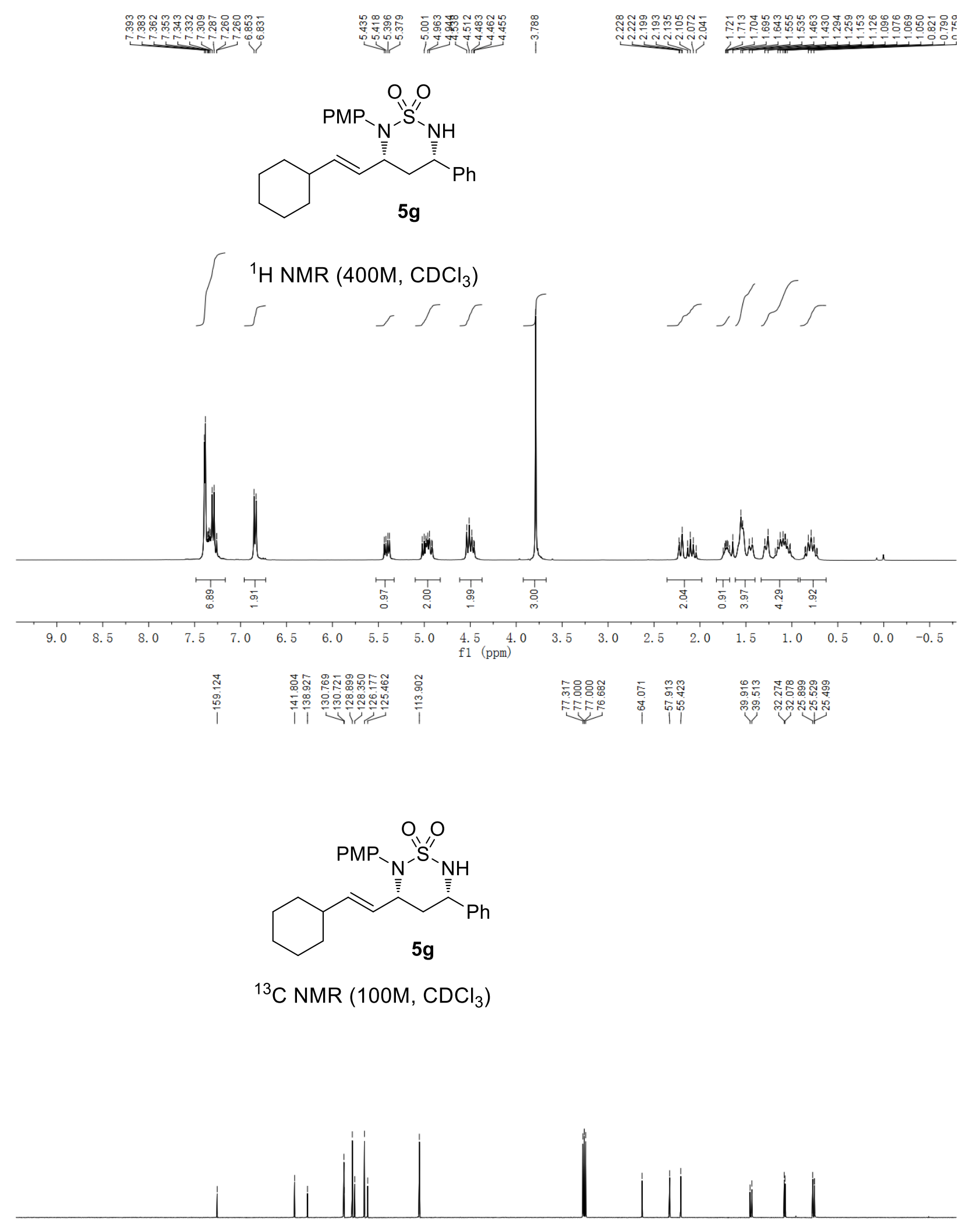

$\begin{array}{llllllllllllllllllllllll}200 & 190 & 180 & 170 & 160 & 150 & 140 & 130 & 120 & 110 & 100 & 90 & 80 & 70 & 60 & 50 & 40 & 30 & 20 & 10 & 0\end{array}$ 


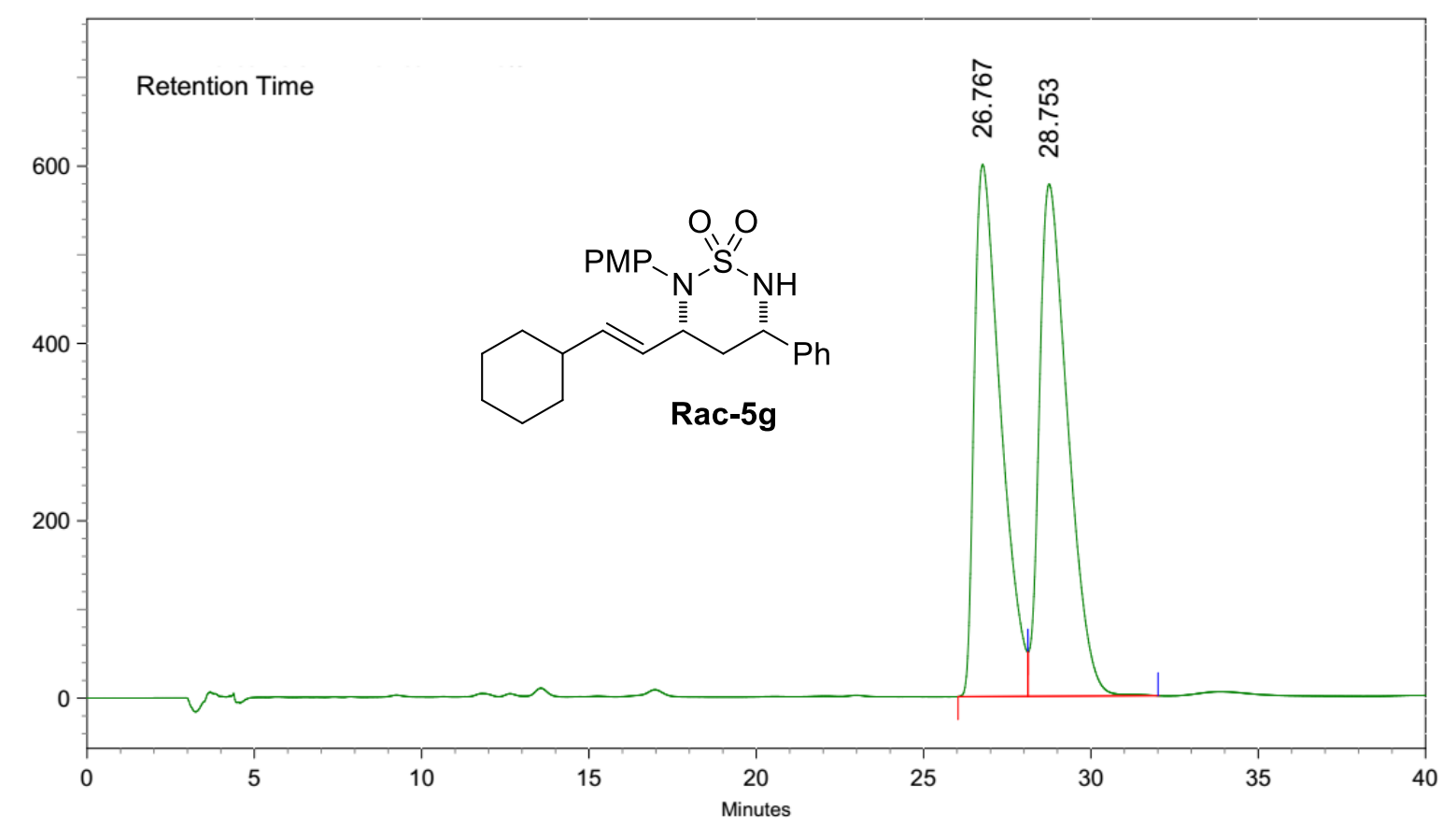

\begin{tabular}{llllll} 
Peak No. & Ret Time & Width & Height & Area & Area [\%] \\
\hline 1 & 26.767 & 2.087 & 10063899 & 554717878 & 49.0729 \\
2 & 28.753 & 3.887 & 9690229 & 575678759 & 50.9271
\end{tabular}

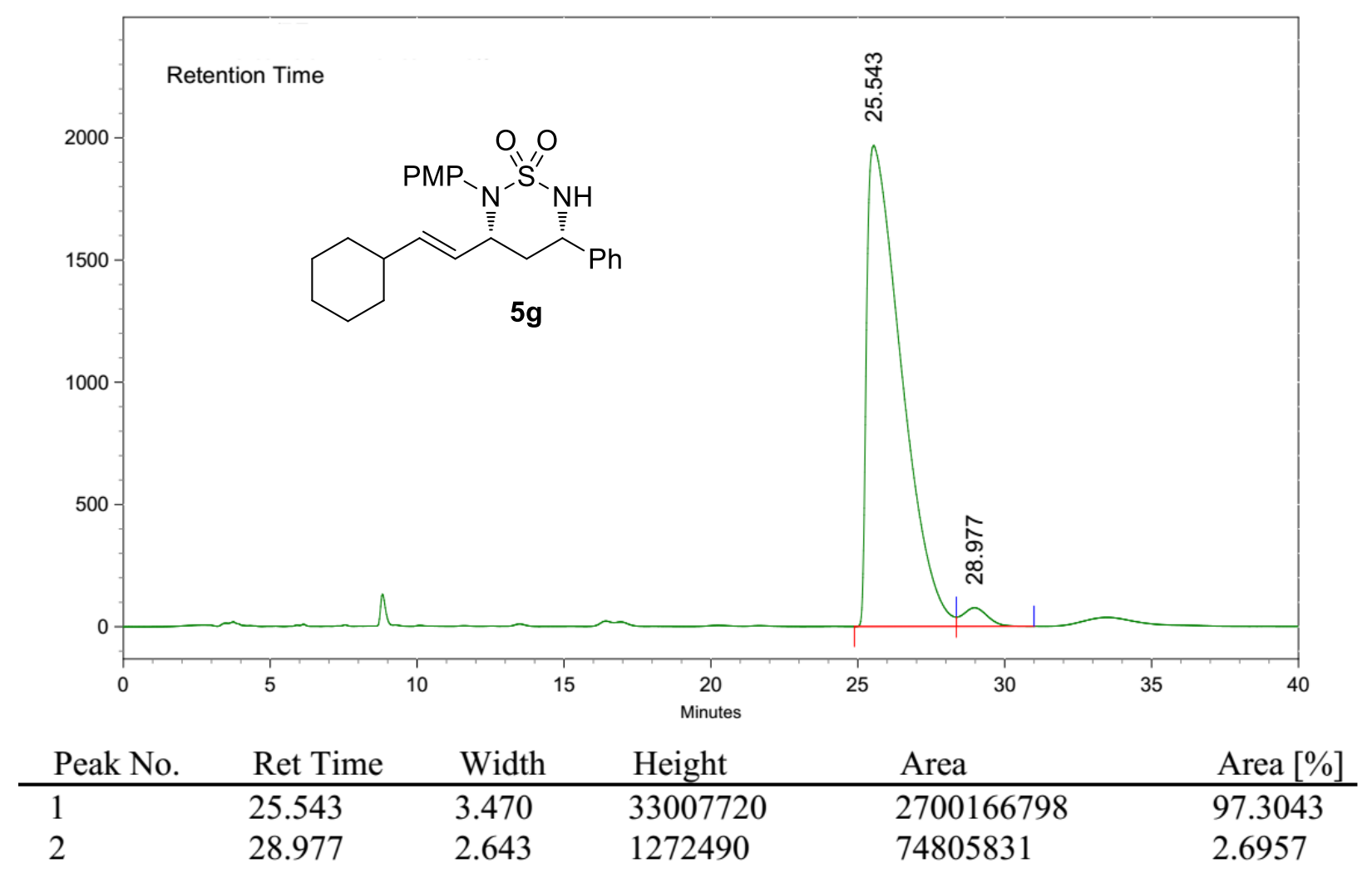




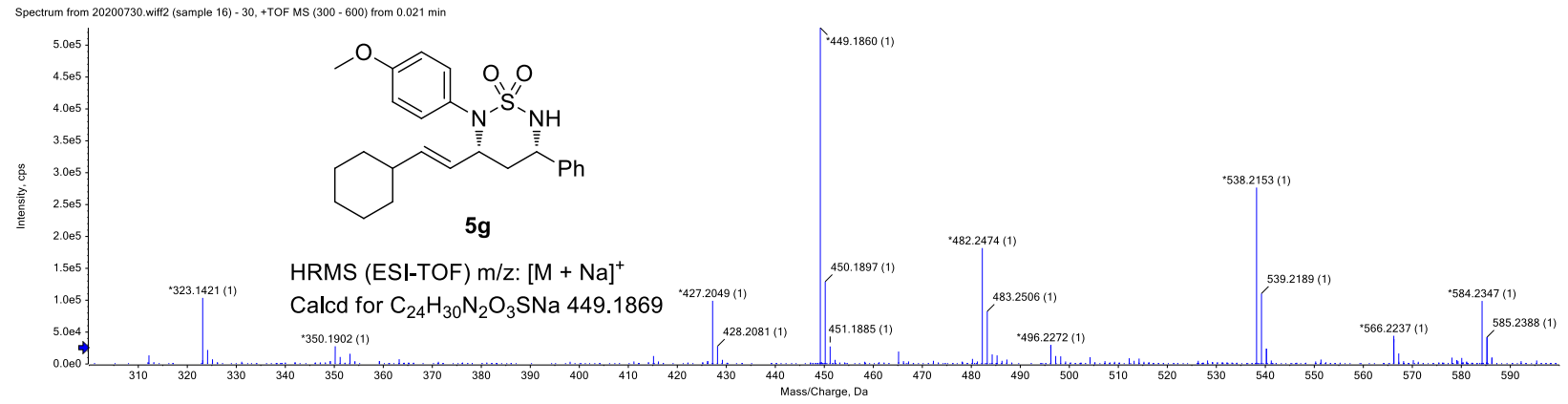




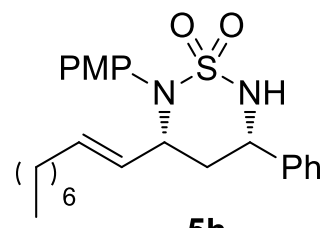

$5 \mathrm{~h}$

$7: 1 \mathrm{dr}$

${ }^{1} \mathrm{H}$ NMR $\left(400 \mathrm{M}, \mathrm{CDCl}_{3}\right)$

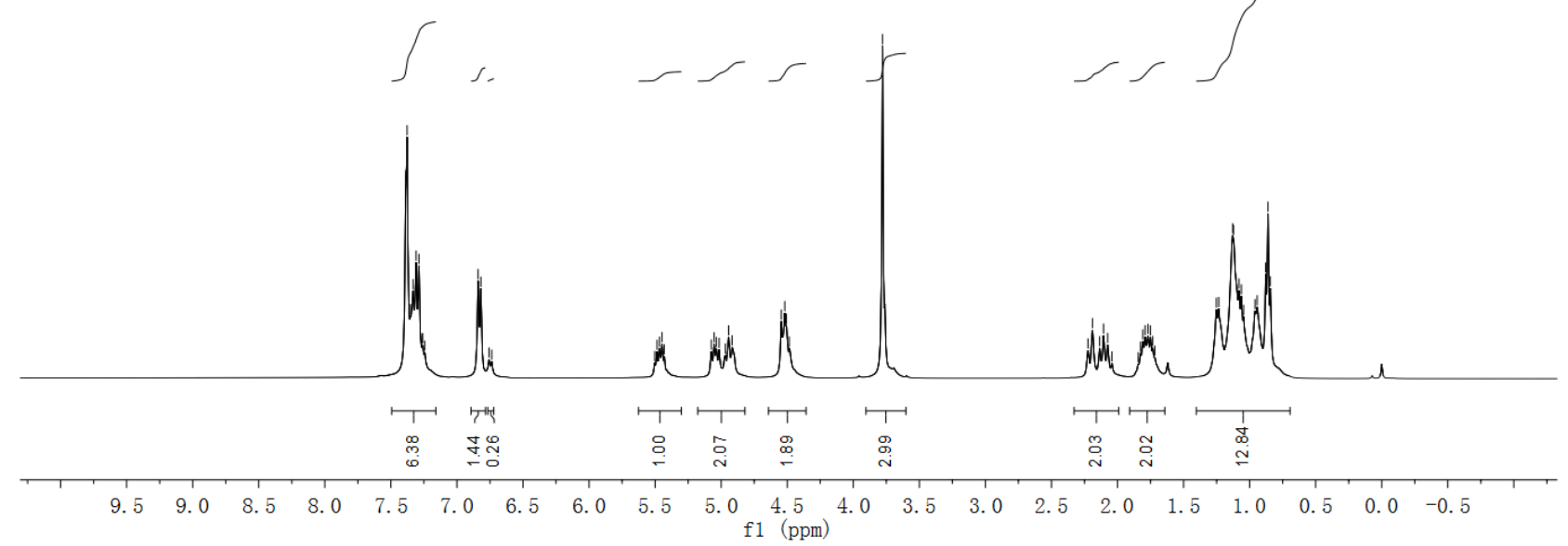

T

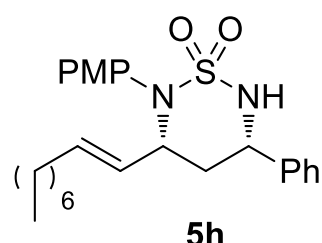

$7: 1 \mathrm{dr}$

${ }^{13} \mathrm{C}$ NMR $\left(100 \mathrm{M}, \mathrm{CDCl}_{3}\right)$

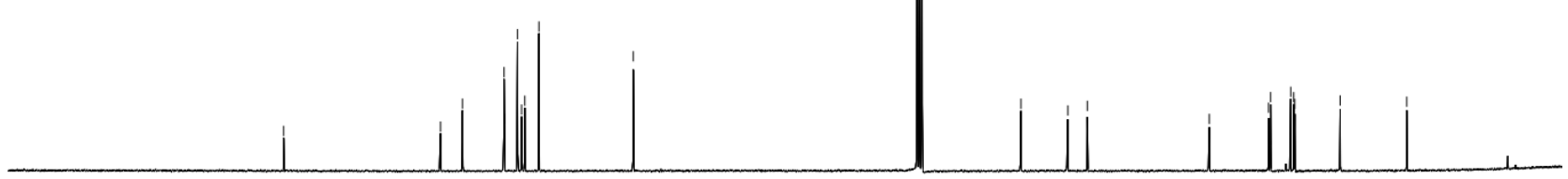

$\begin{array}{rrrrrrrrrrrrrrrrrrrr}190 & 180 & 170 & 160 & 150 & 140 & 130 & 120 & 110 & 100 & \begin{aligned} 90 \\ (100\end{aligned} & 80 & 70 & 60 & 50 & 40 & 30 & 20 & 10 & 0\end{array}$ 

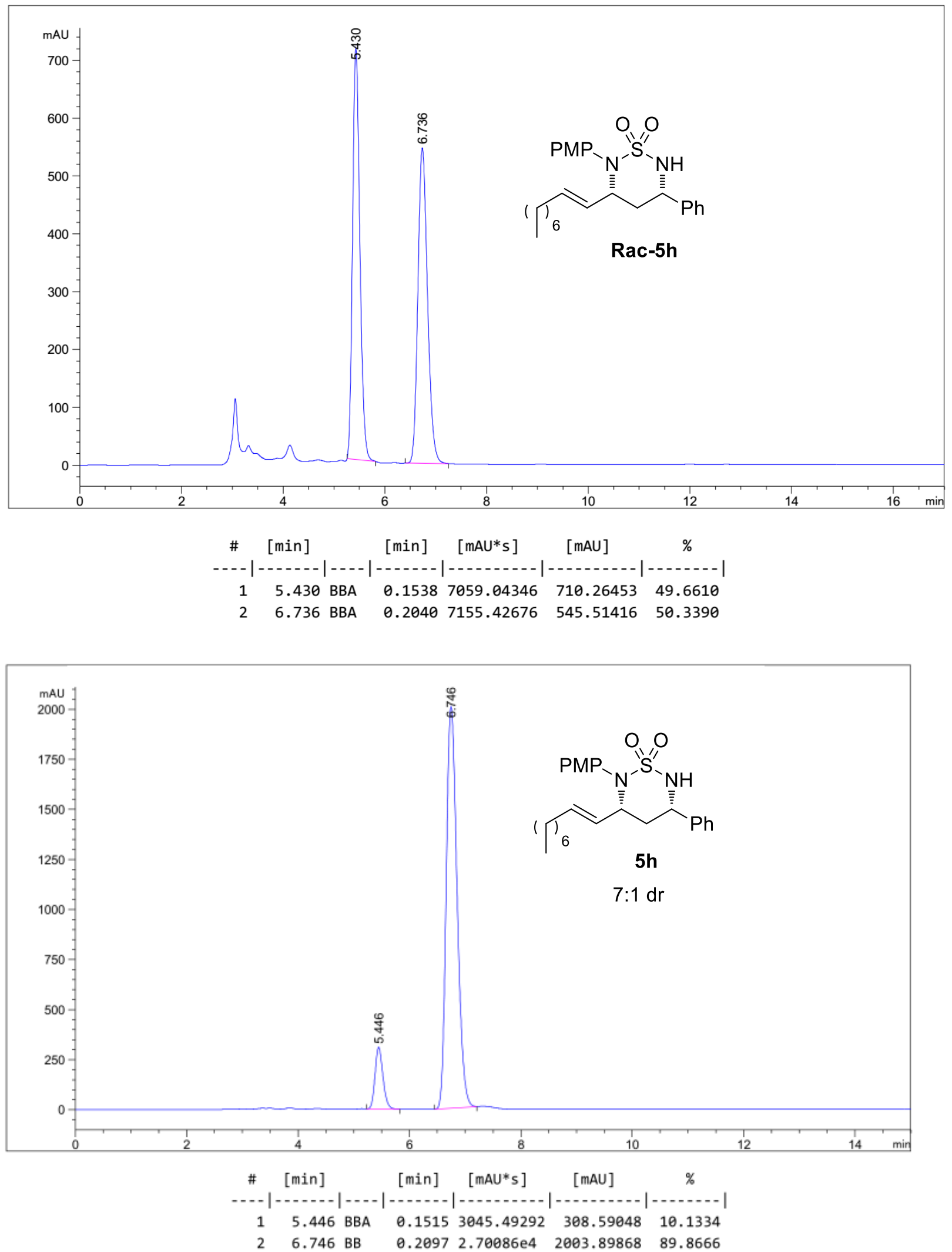


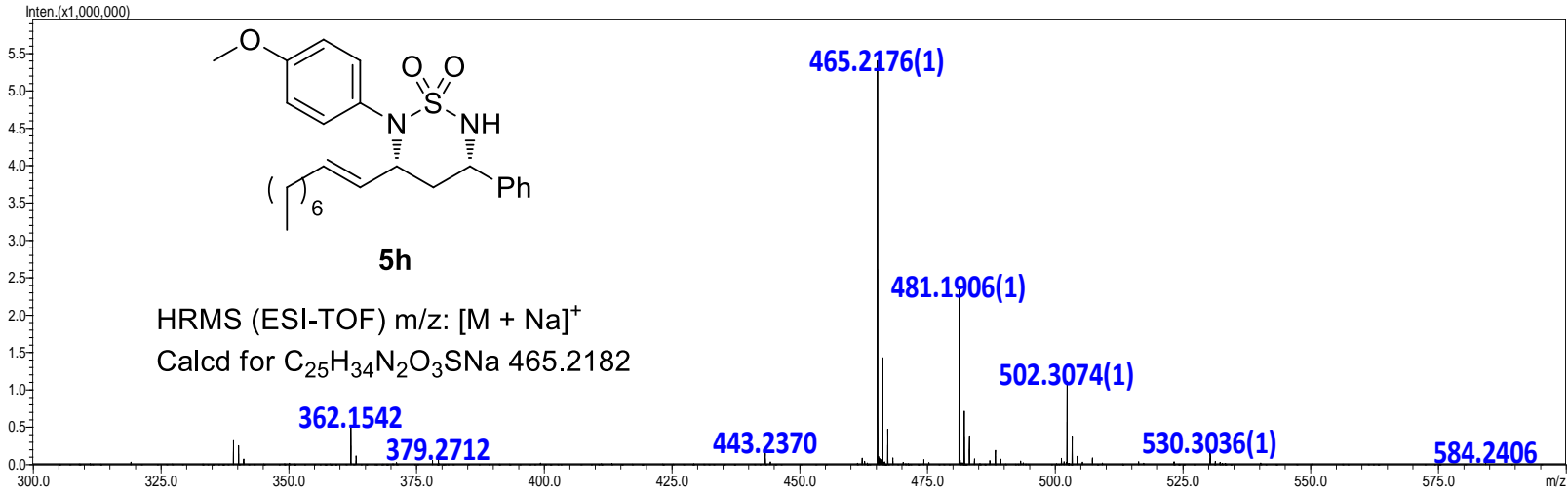




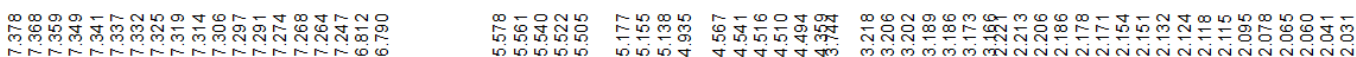<smiles>O=[PH](=O)(N=P)N1C(/C=C/CCOc2ccccc2)C[C@@H](c2ccccc2)NS1(=O)=O</smiles>

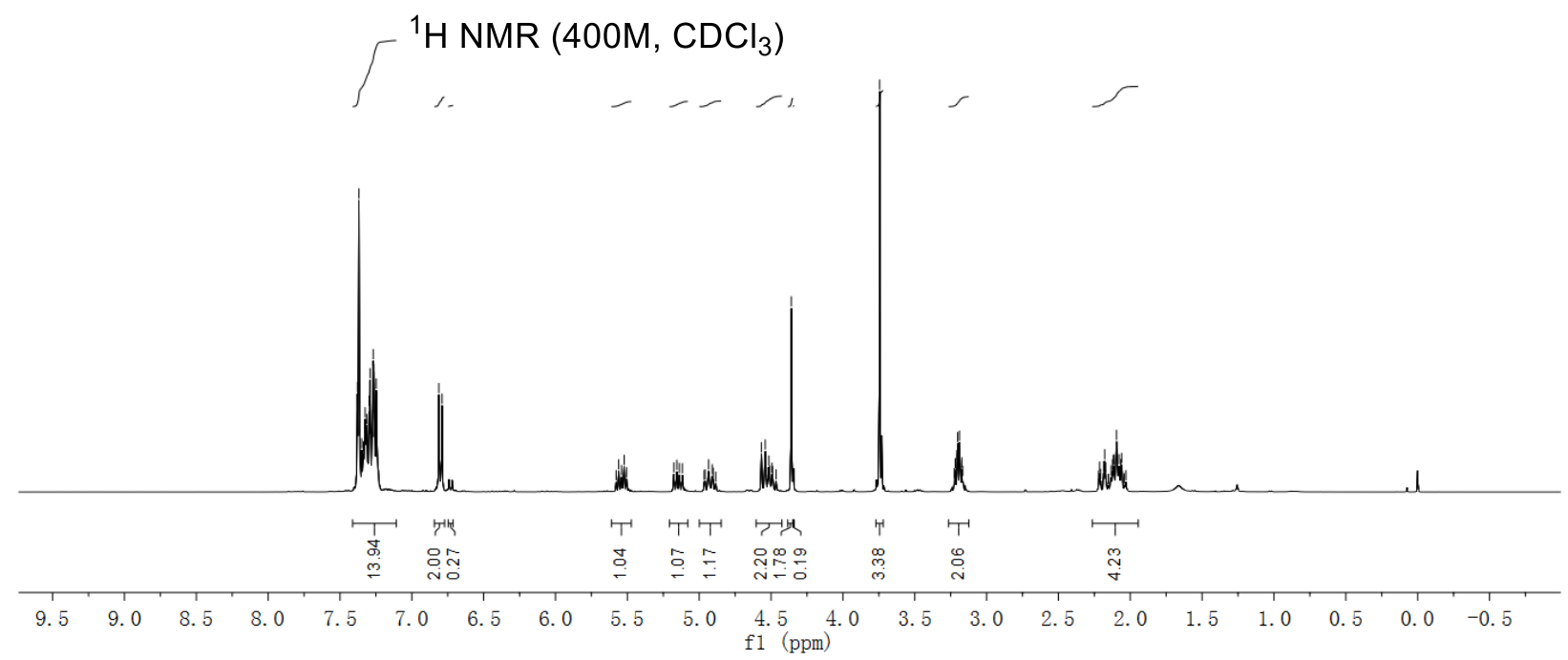

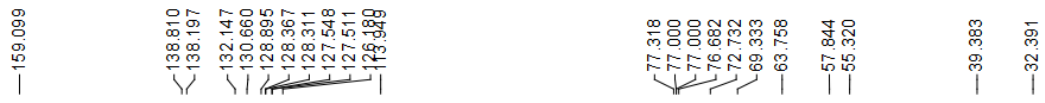

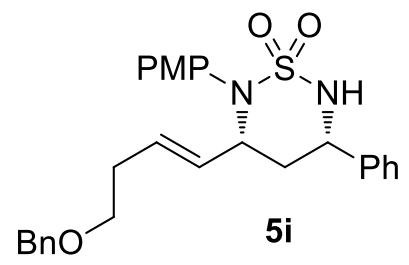

$9: 1 \mathrm{dr}$

${ }^{13} \mathrm{C}$ NMR (100M, $\left.\mathrm{CDCl}_{3}\right)$

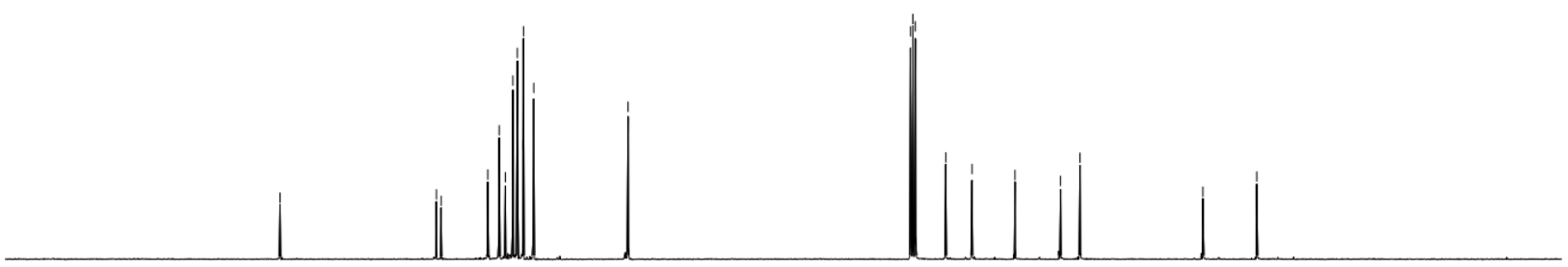

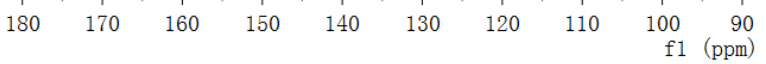



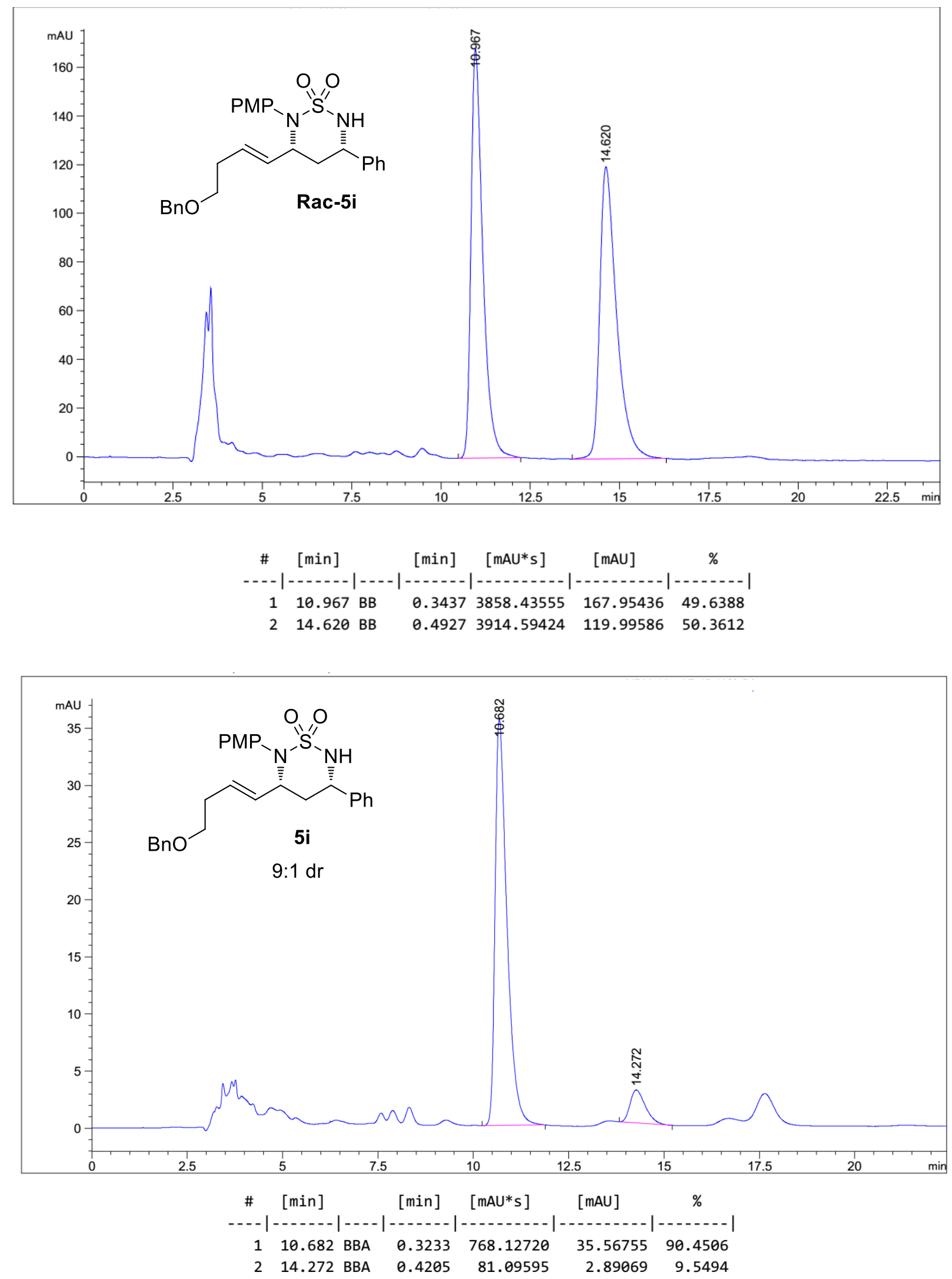


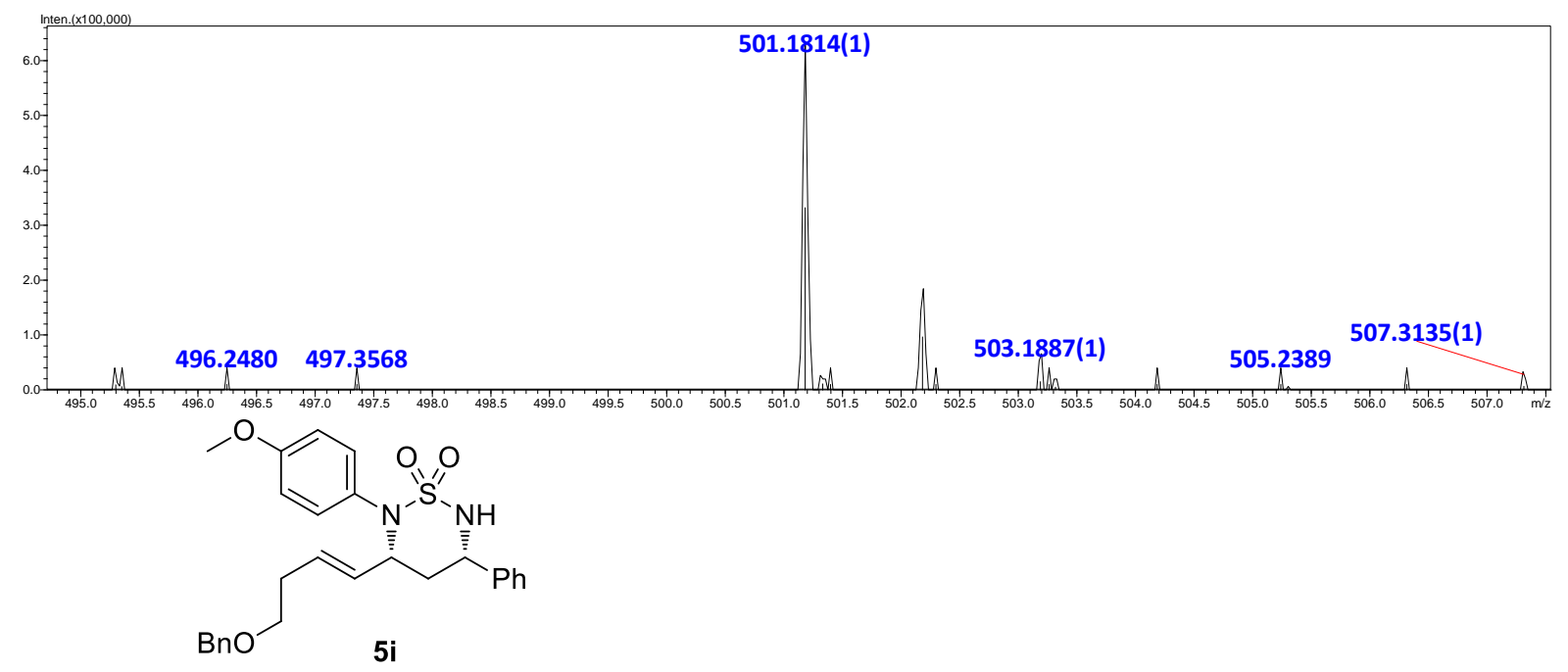

HRMS (ESI-TOF) m/z: [M + Na]

Calcd for $\mathrm{C}_{27} \mathrm{H}_{30} \mathrm{~N}_{2} \mathrm{O}_{4} \mathrm{SNa} 501.1818$ 


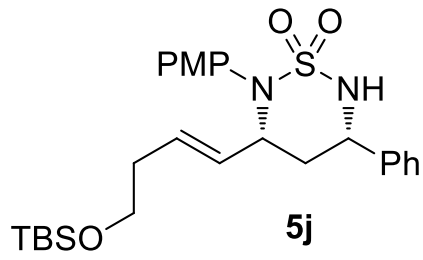

$5: 1 \mathrm{dr}$

${ }^{1} \mathrm{H}$ NMR (400M, $\mathrm{CDCl}_{3}$ )

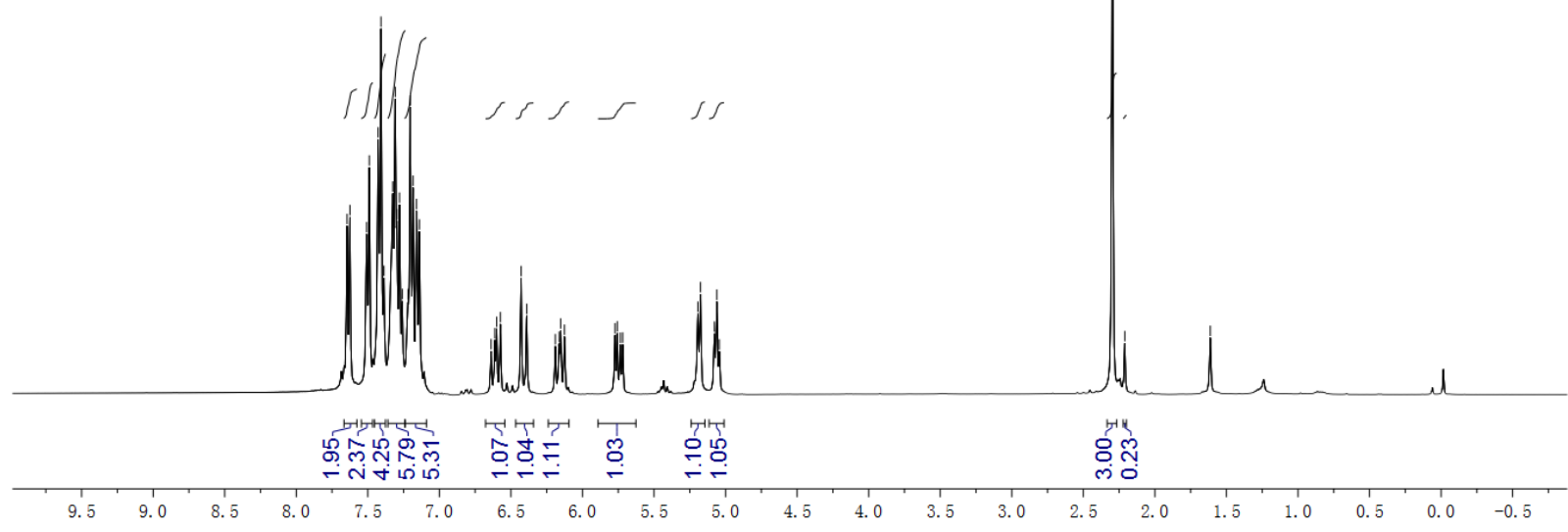

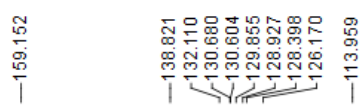

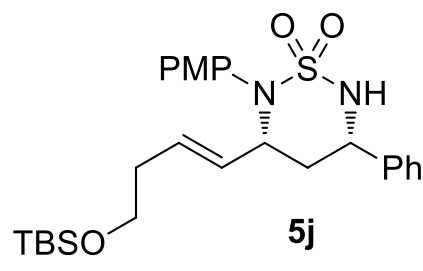

$5: 1 \mathrm{dr}$

${ }^{13} \mathrm{C}$ NMR (100M, $\mathrm{CDCl}_{3}$ )

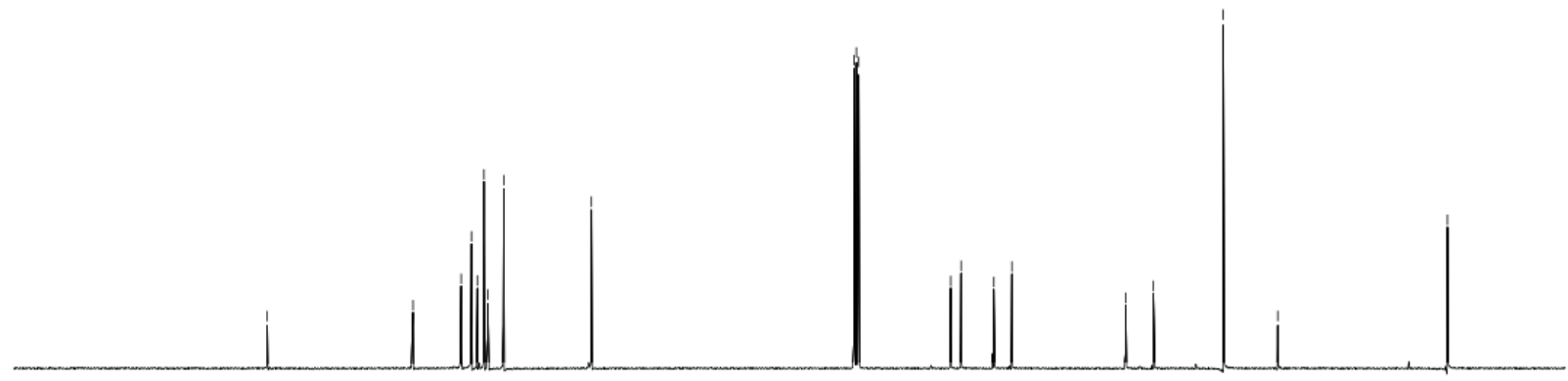



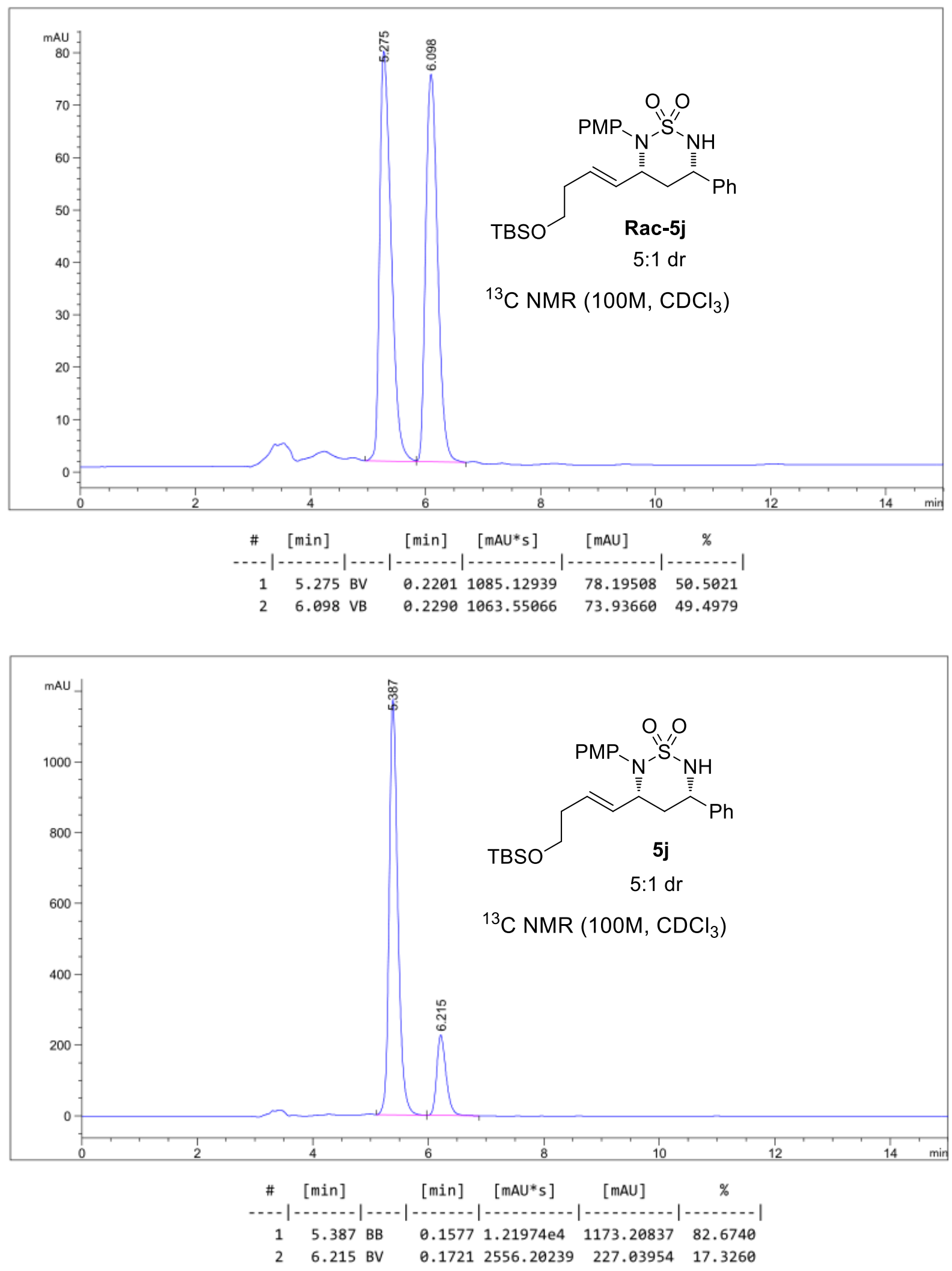


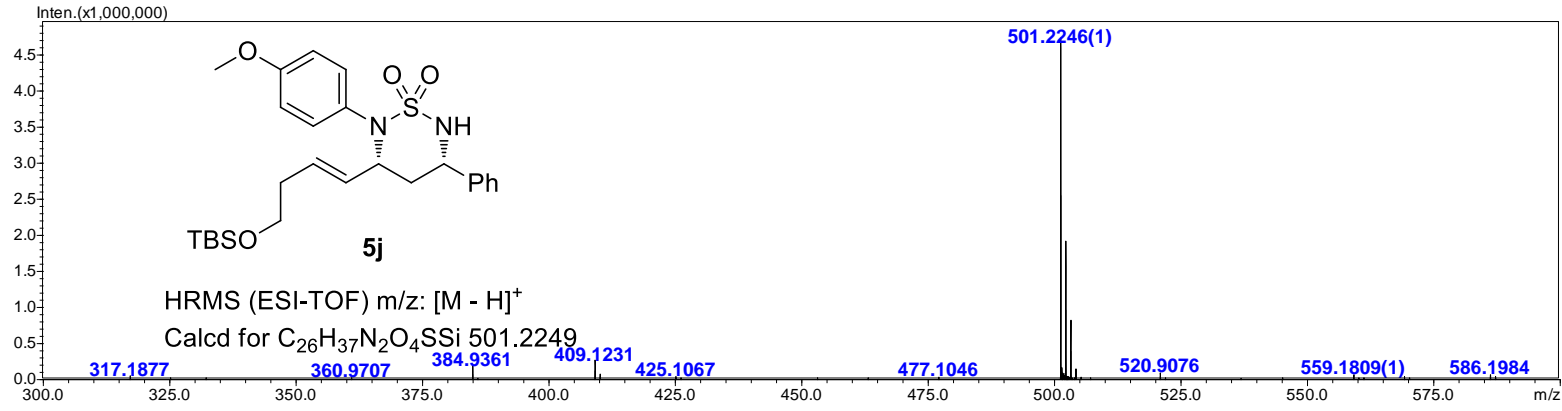




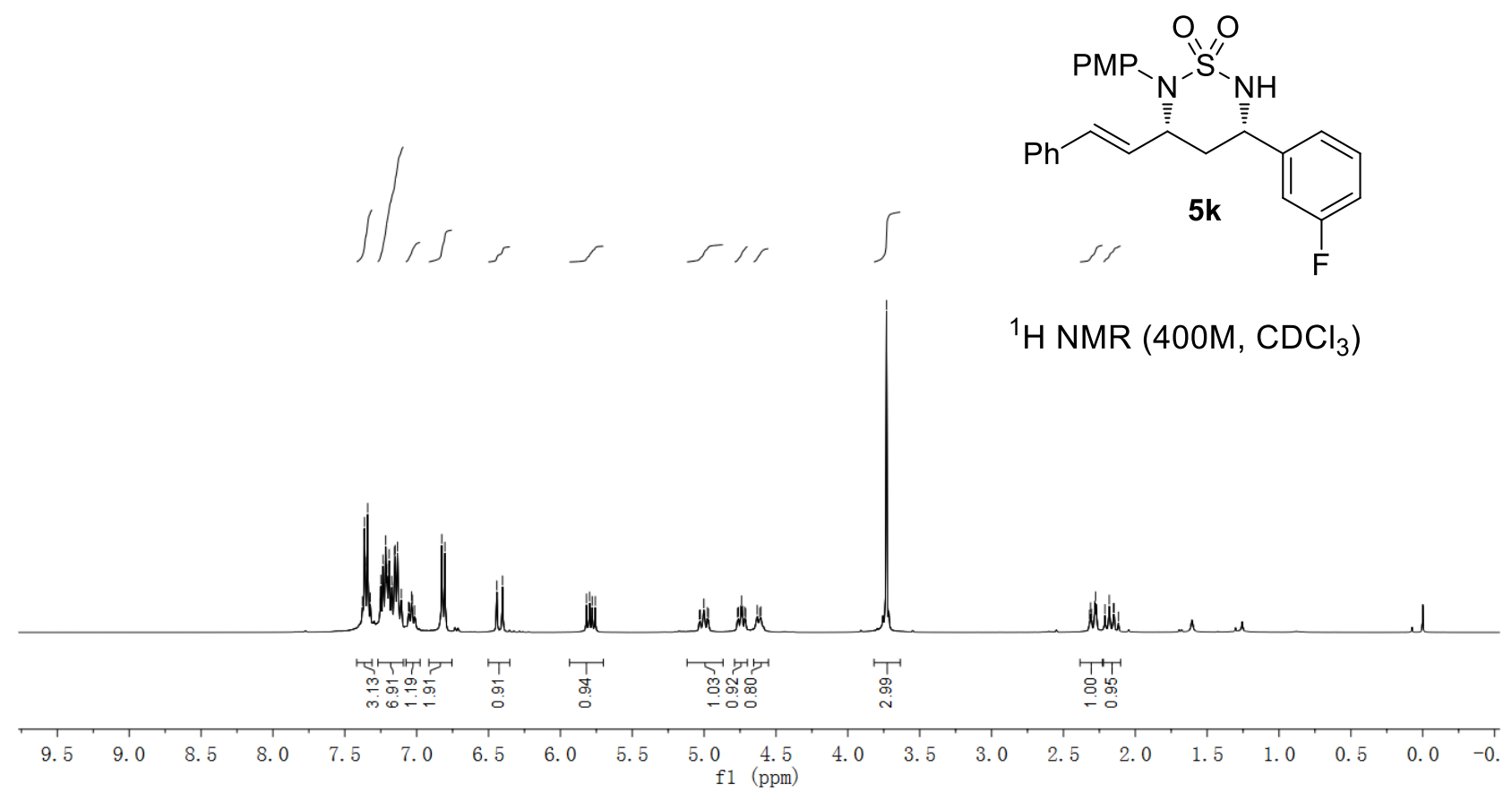

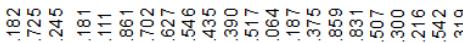

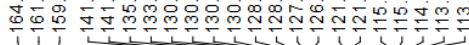

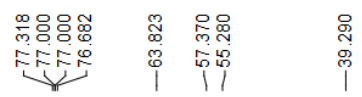

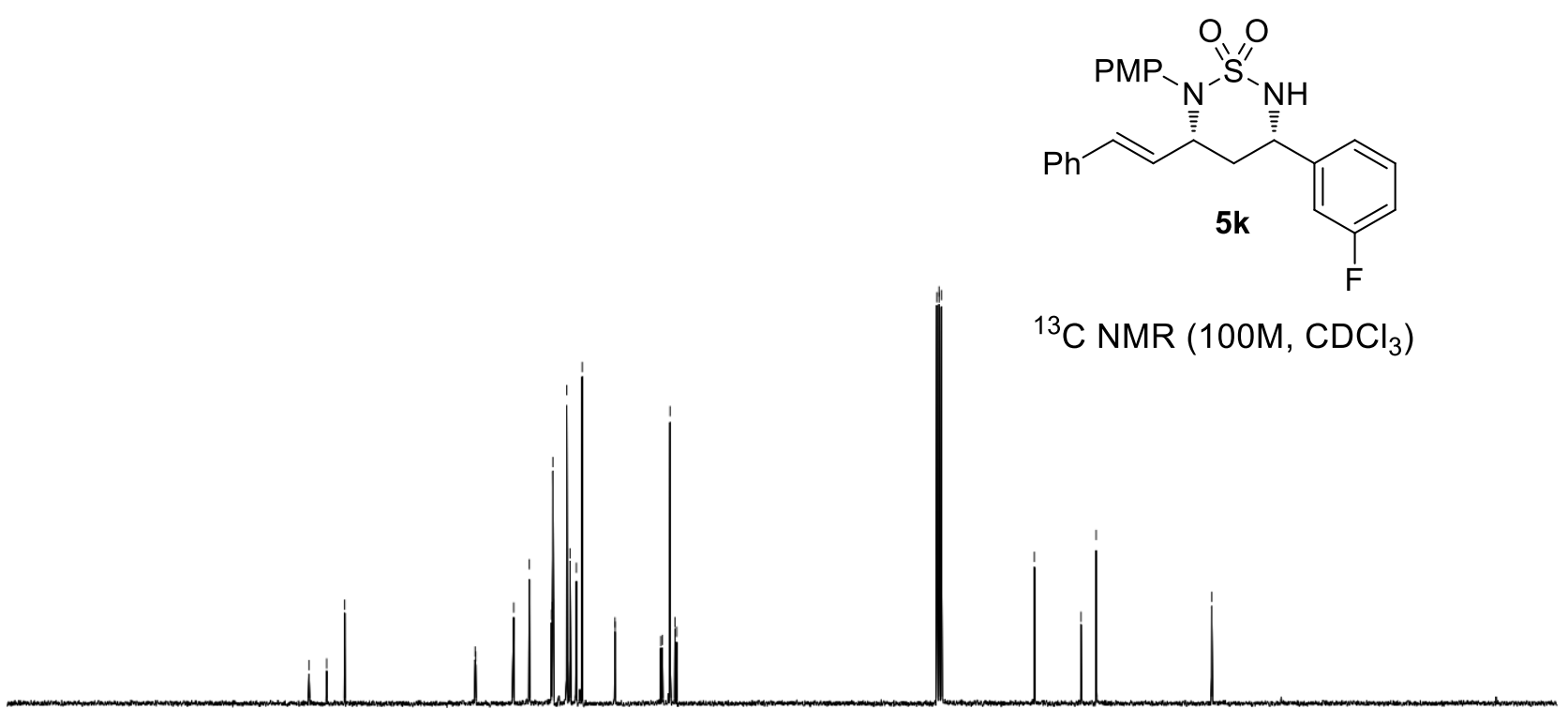

$\begin{array}{lllllllllll}200 & 190 & 180 & 170 & 160 & 150 & 140 & 130 & 120 & 110 & 100 \\ & & & & \end{array}$ 

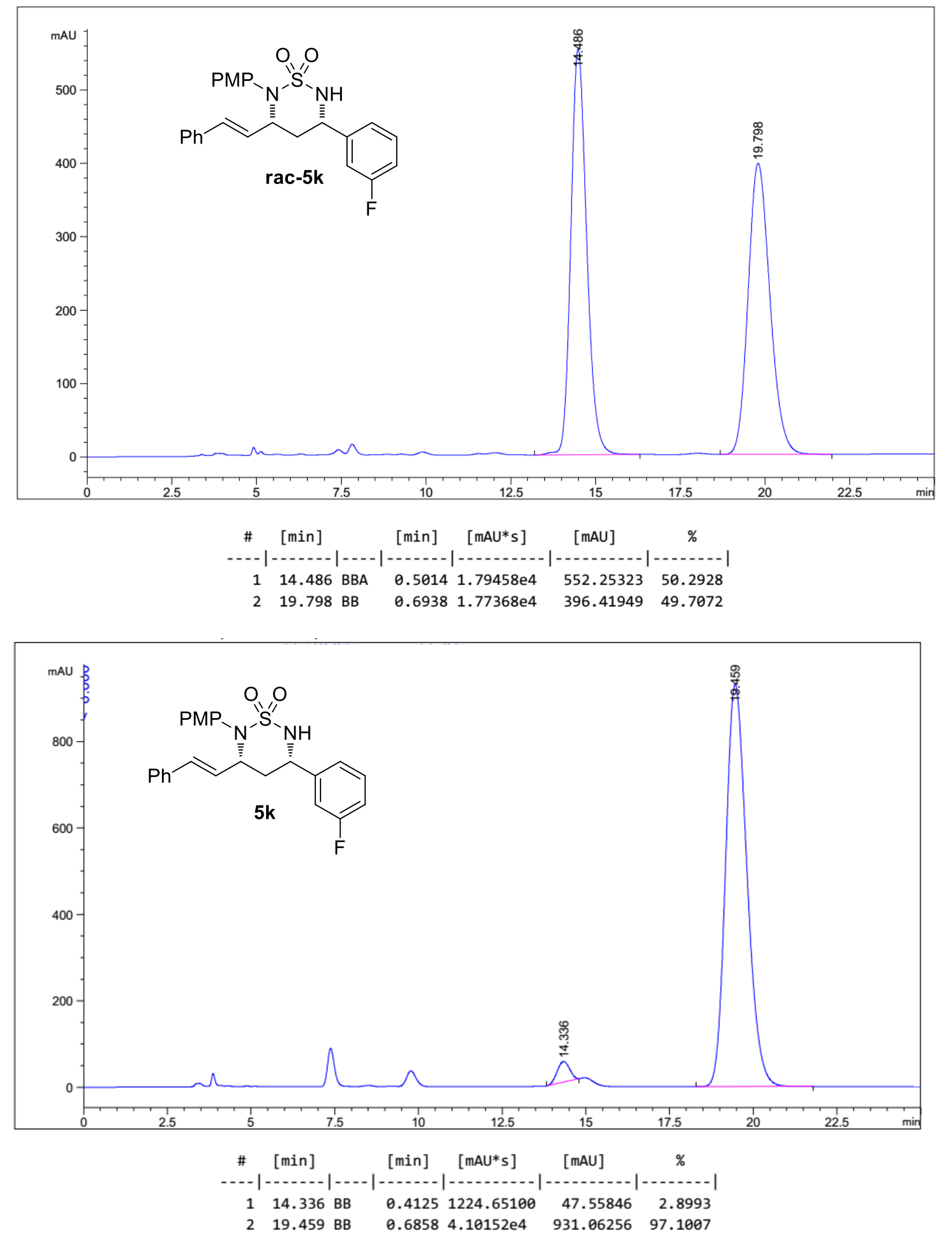
Inten. $(\times 10,000,000)$

2.00

1.75

1.50

1.25

1.00

0.75

0.50

0.25

$0.00 \frac{1}{450.0}$
461.1309(1)

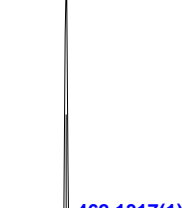

462.1317(1)

463.1313(1)
COP O

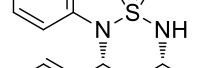

$\mathrm{Ph}$

$5 k$

HRMS (ESI-TOF) m/z: [M + Na] ${ }^{+}$

Calcd for $\mathrm{C}_{24} \mathrm{H}_{23} \mathrm{FN}_{2} \mathrm{O}_{3} \mathrm{SNa} 461.1306$ 

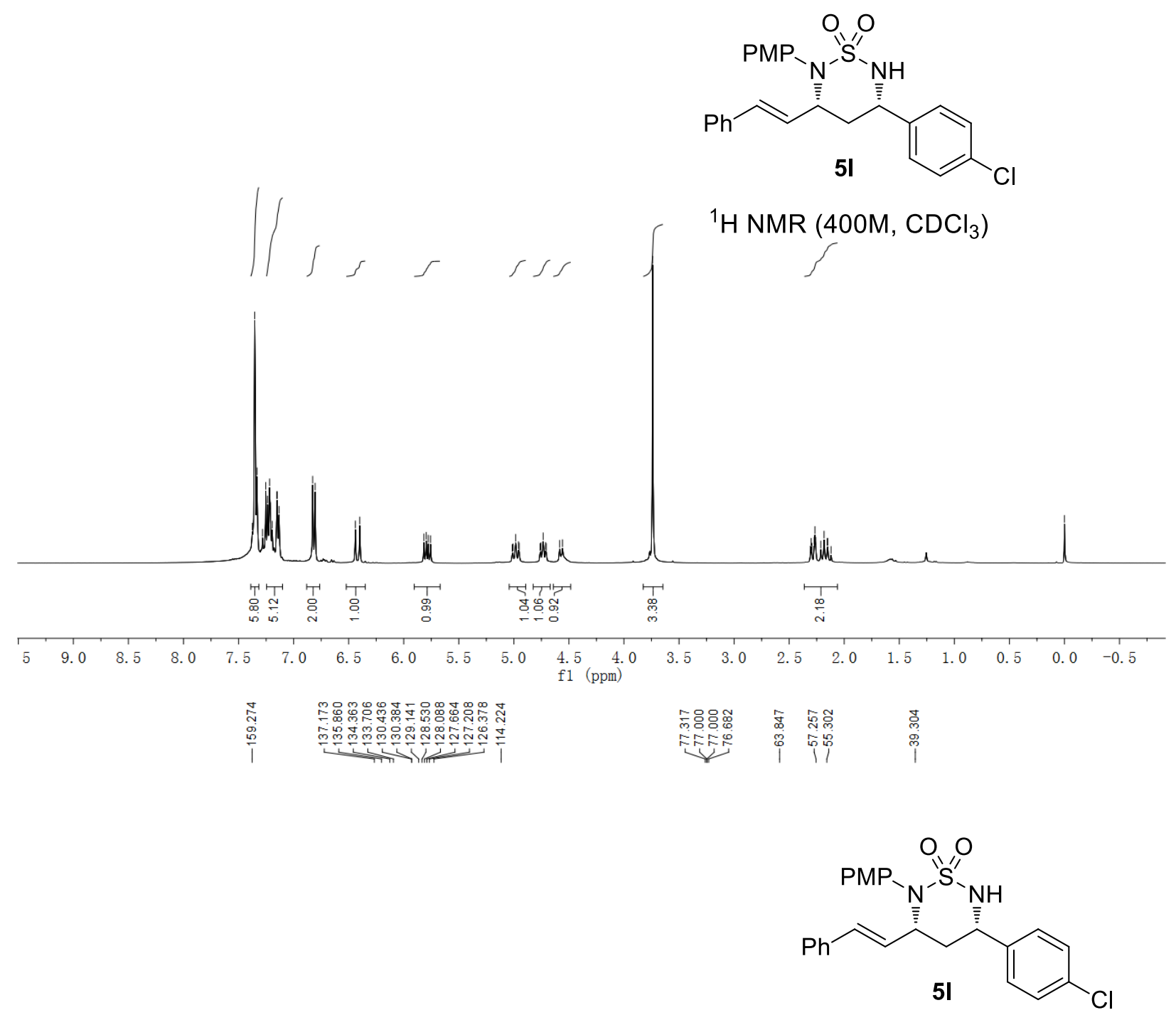

${ }^{13} \mathrm{C}$ NMR $\left(100 \mathrm{M}, \mathrm{CDCl}_{3}\right)$

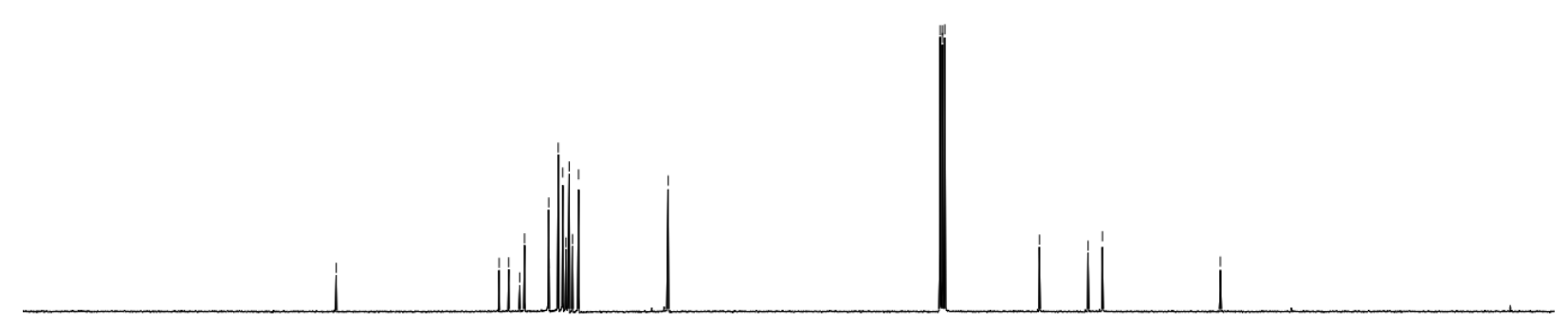

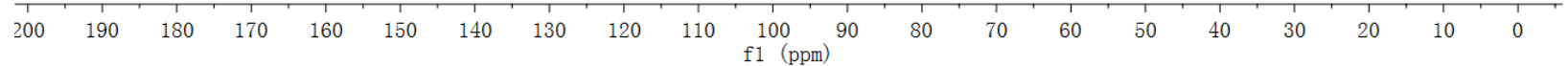



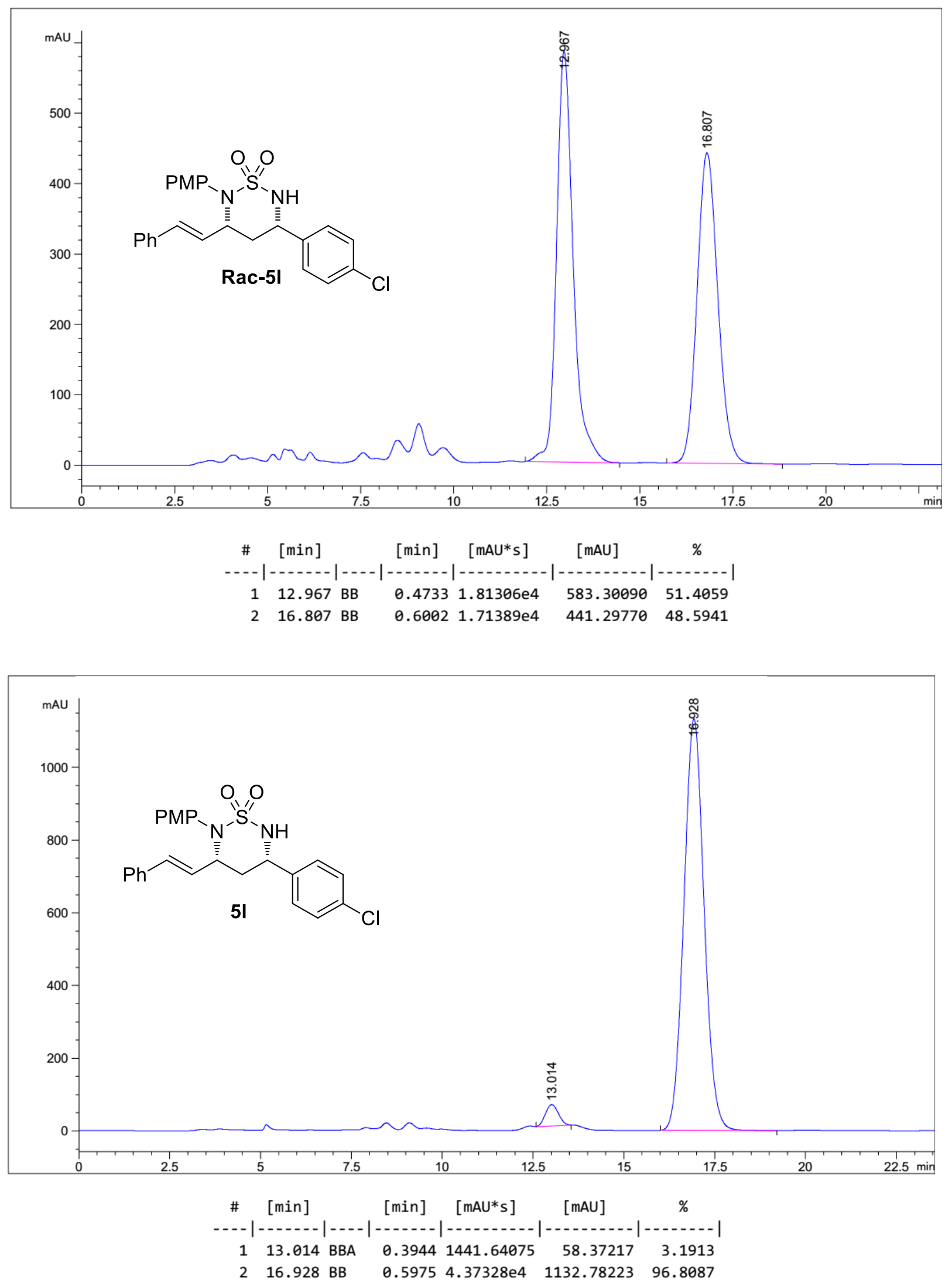


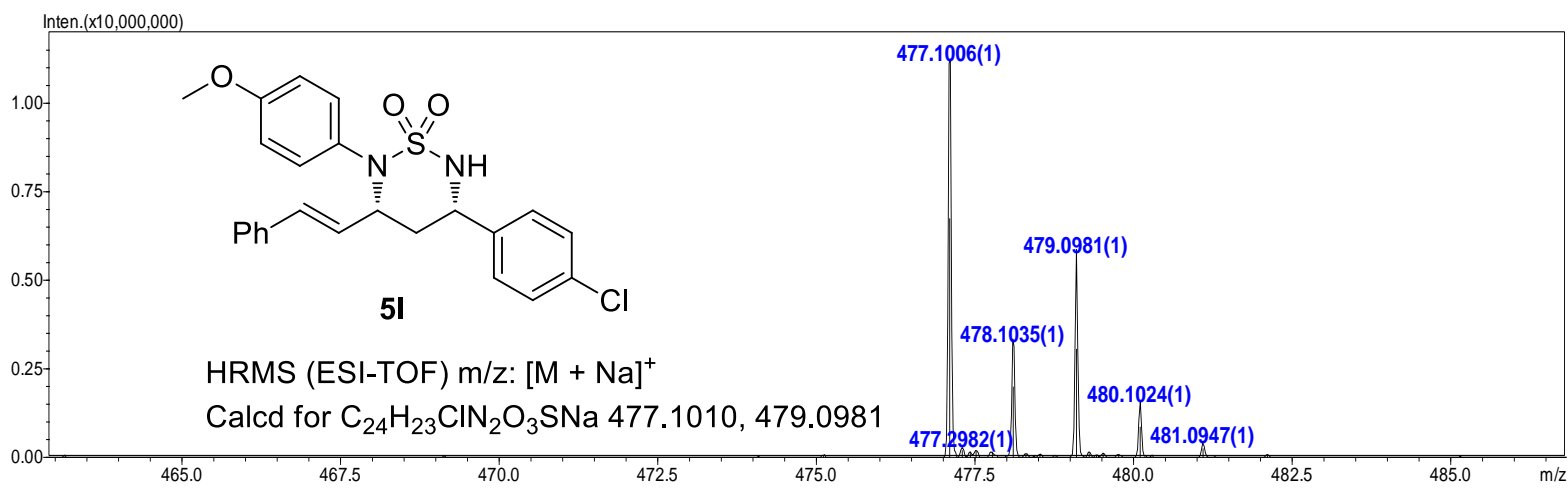



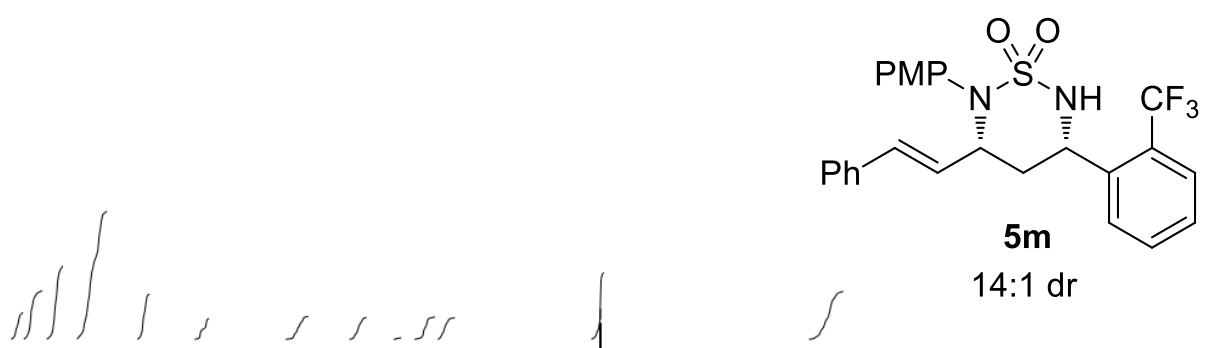

$14: 1 d r$

${ }^{1} \mathrm{H}$ NMR (400M, $\left.\mathrm{CDCl}_{3}\right)$

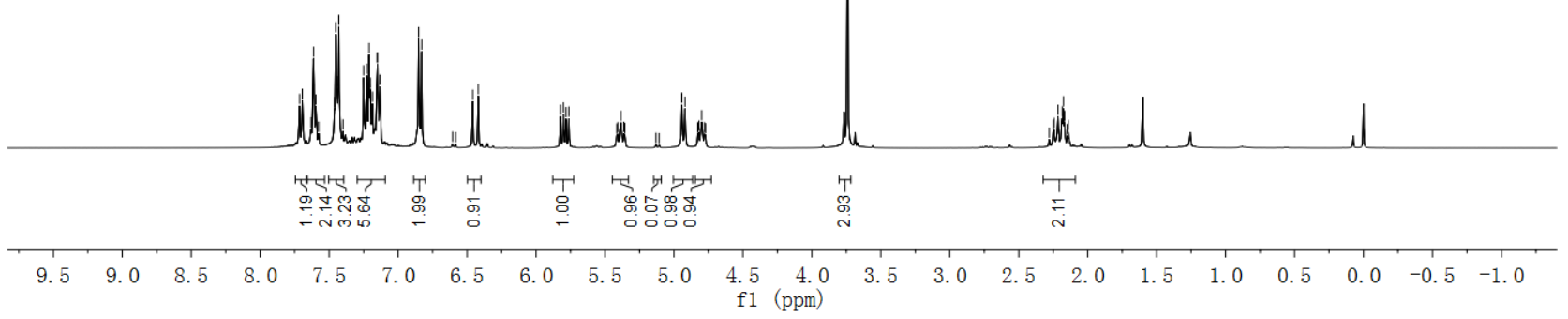
두 年

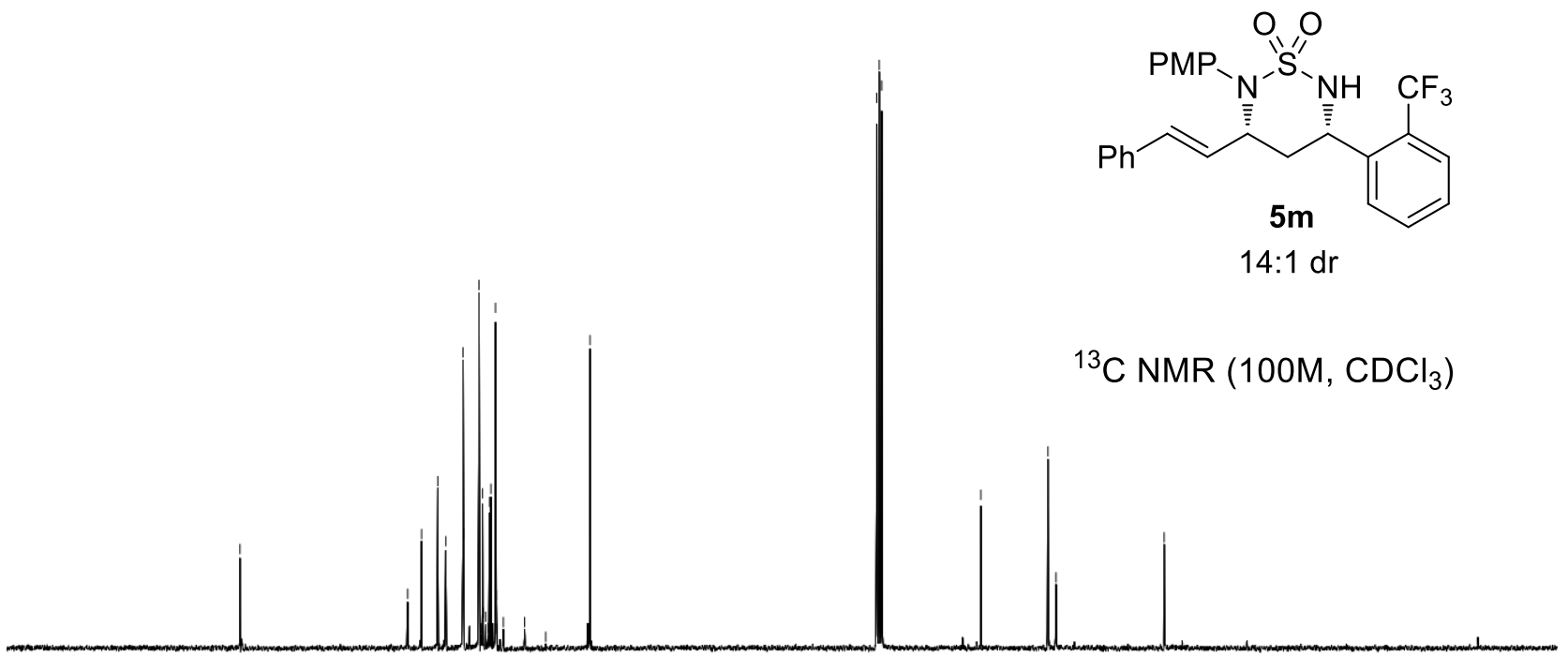



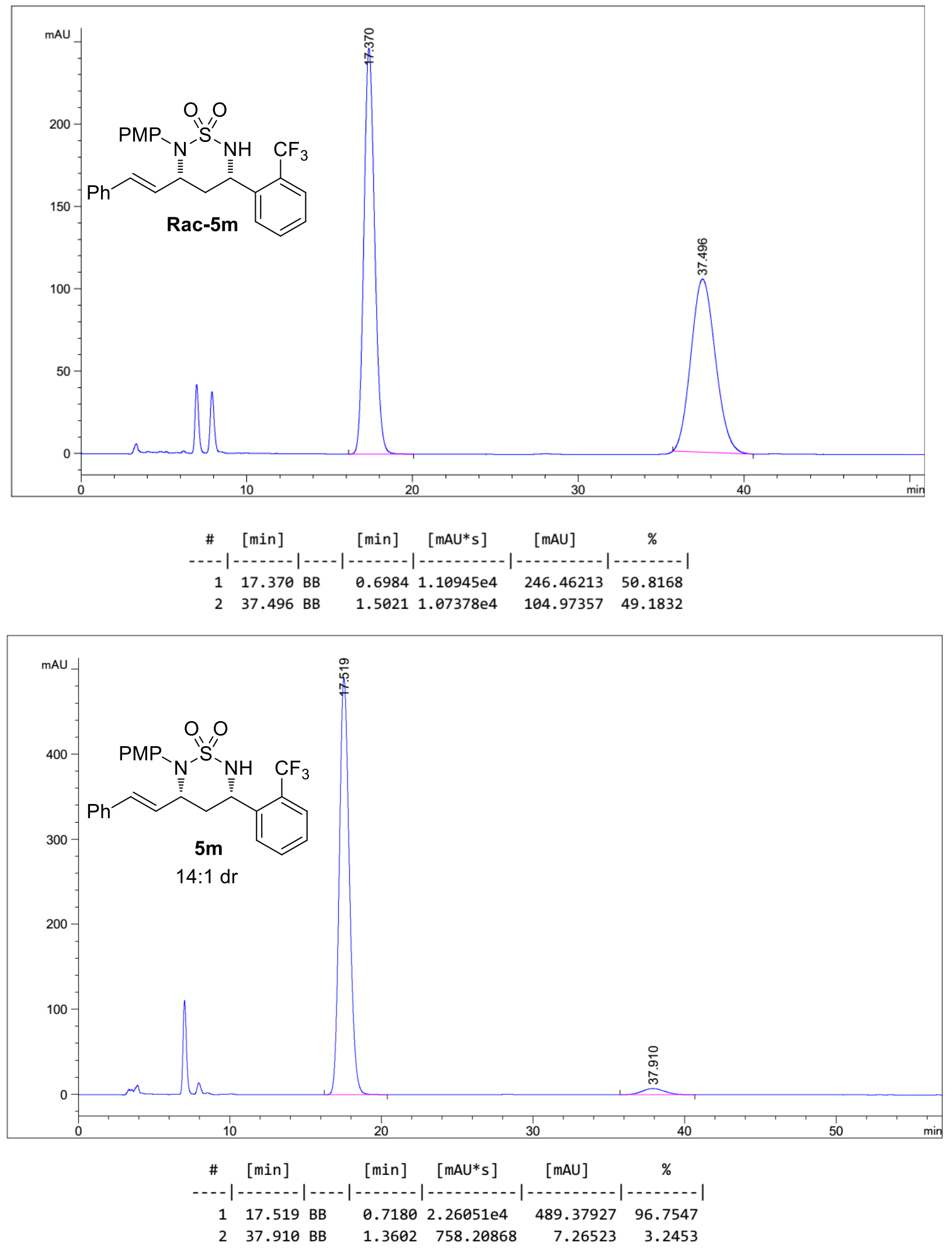
Inten. $(x 10,000,000)$

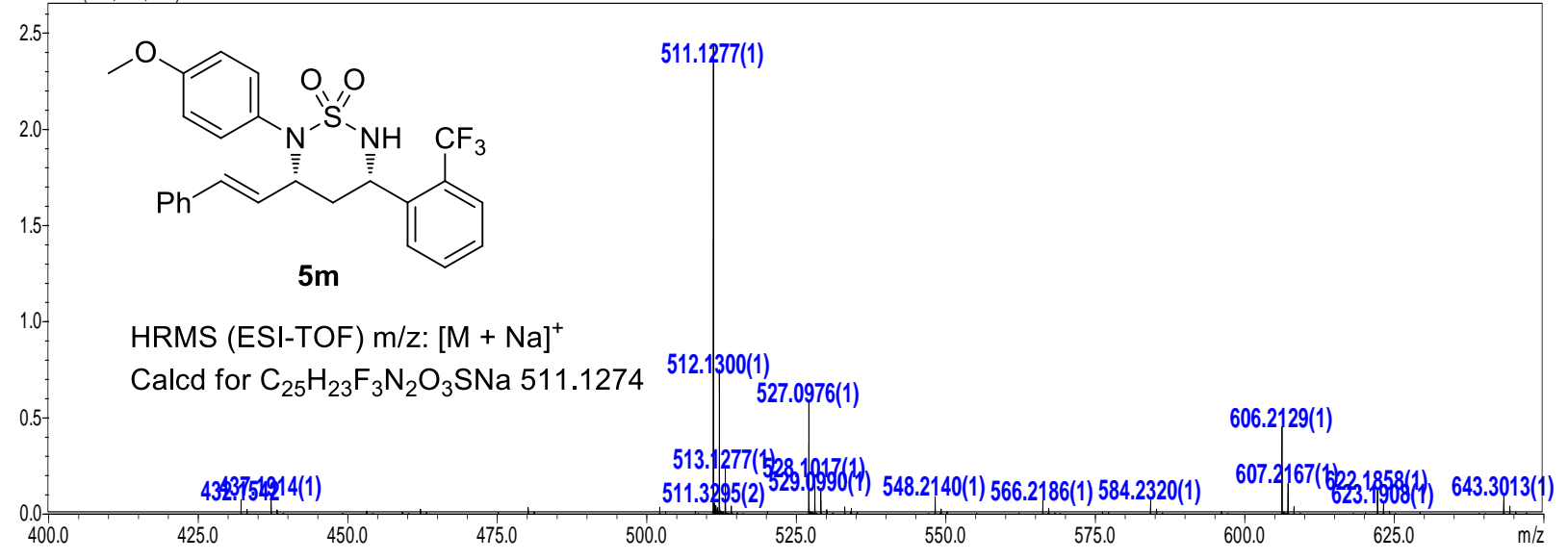




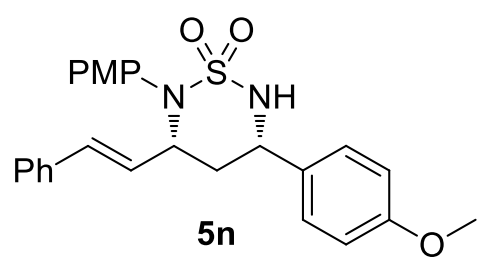

${ }^{1} \mathrm{H}$ NMR (400M, $\mathrm{CDCl}_{3}$ )
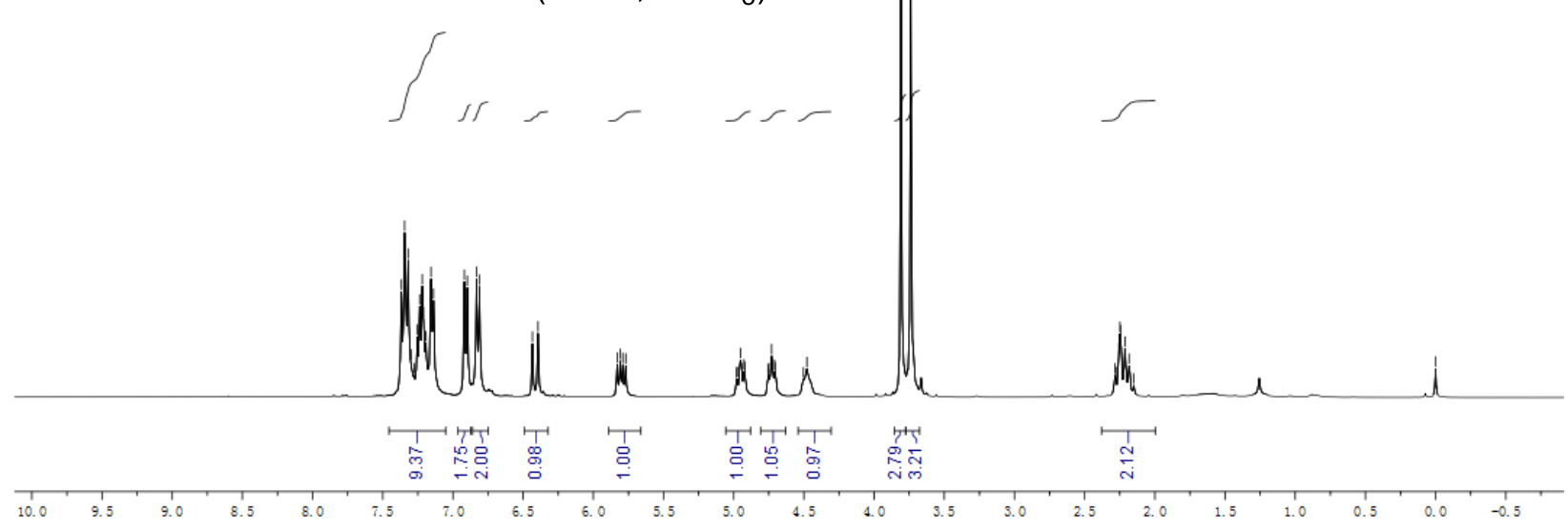
舟

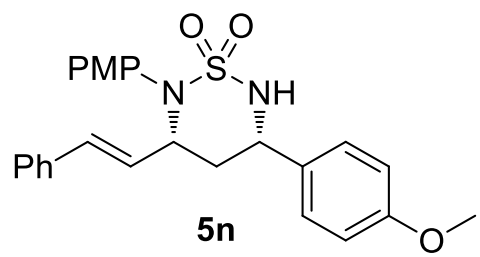

${ }^{13} \mathrm{C}$ NMR (100M, $\left.\mathrm{CDCl}_{3}\right)$
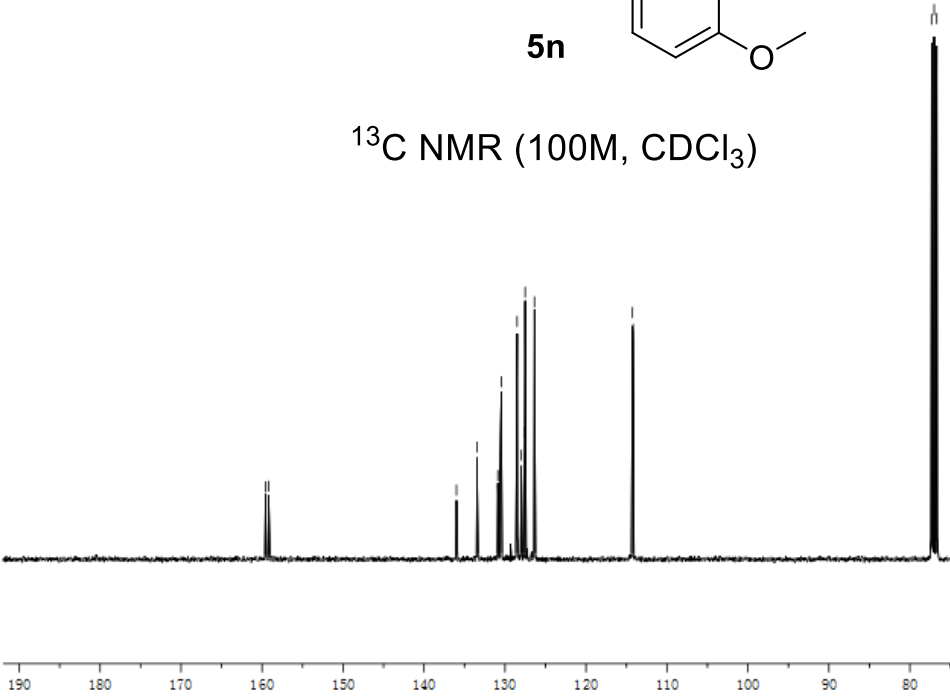

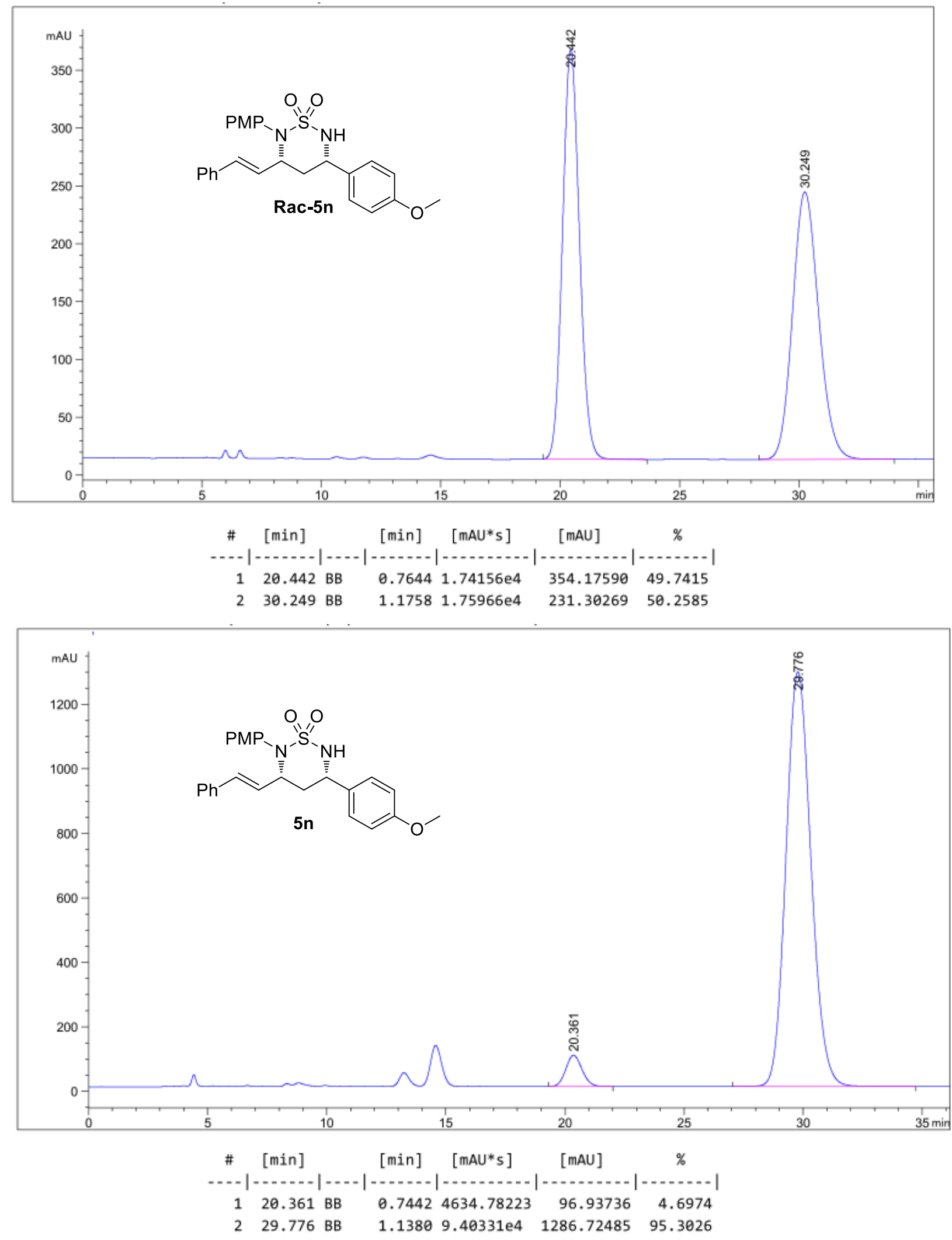
Spectrum Plot Report

Agilent

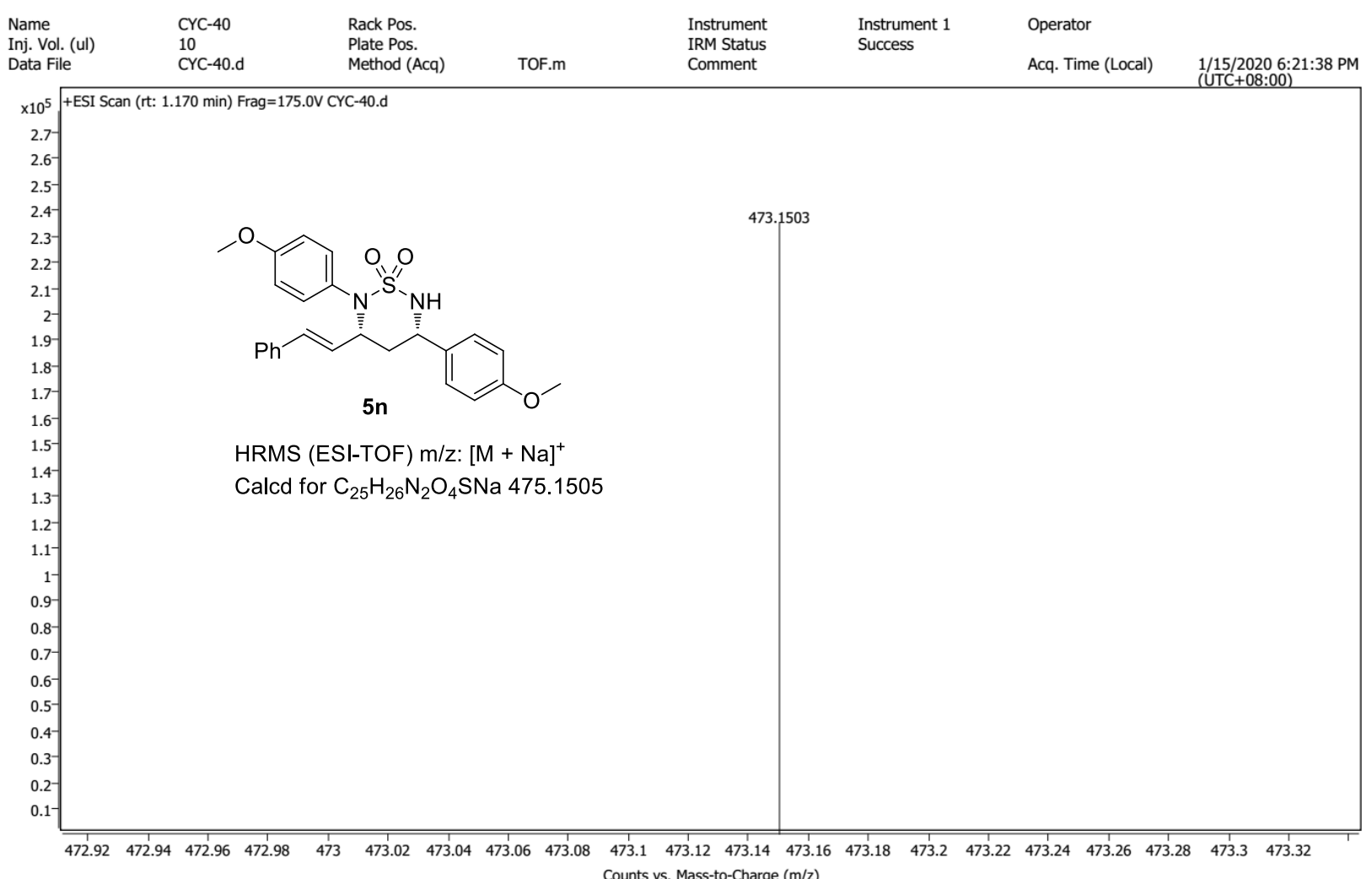




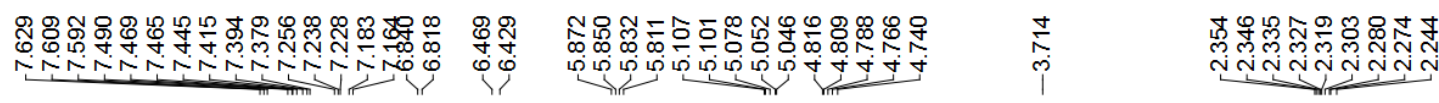

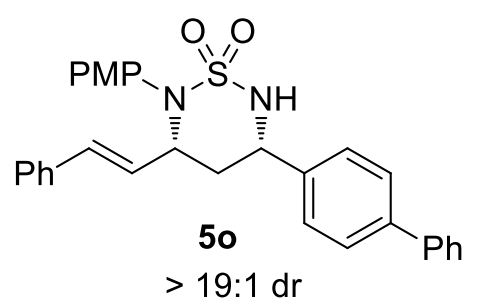

${ }^{1} \mathrm{H}$ NMR $\left(400 \mathrm{M}, \mathrm{CDCl}_{3}\right)$

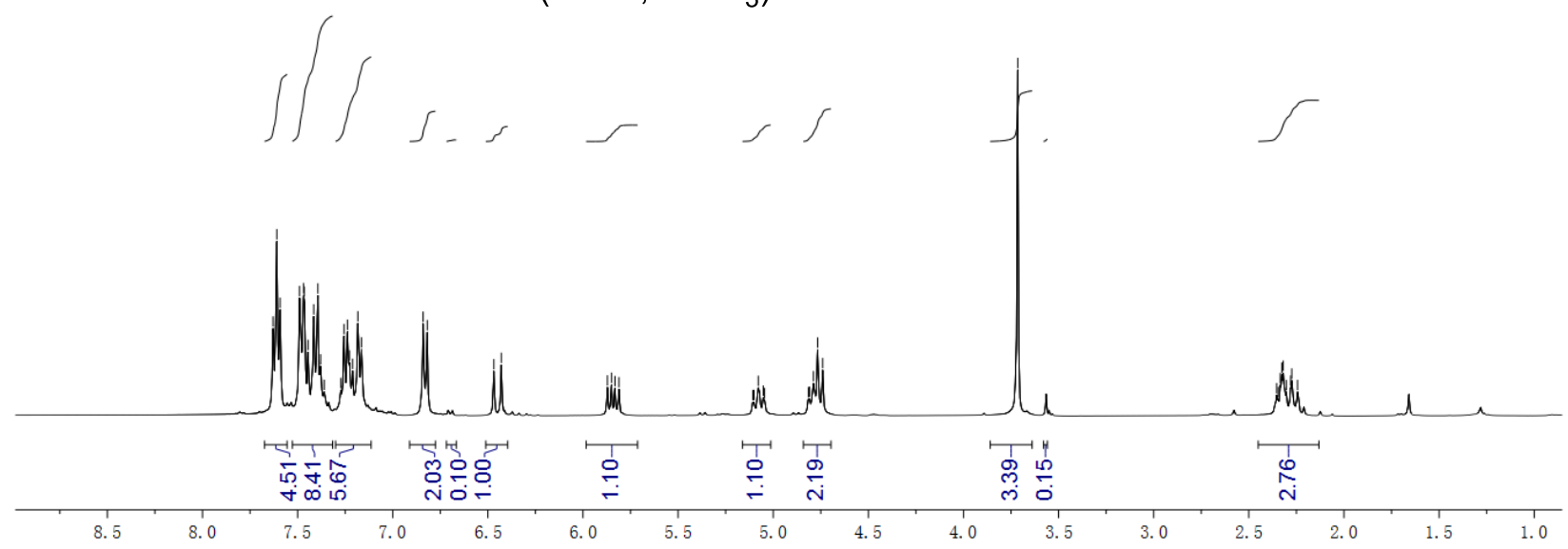

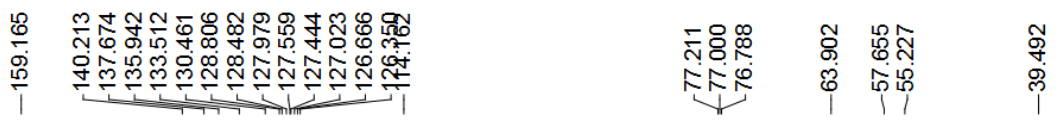

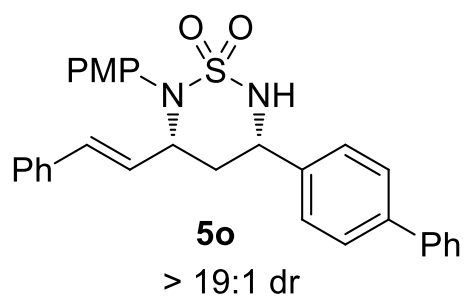

${ }^{13} \mathrm{C}$ NMR (150M, $\mathrm{CDCl}_{3}$ )
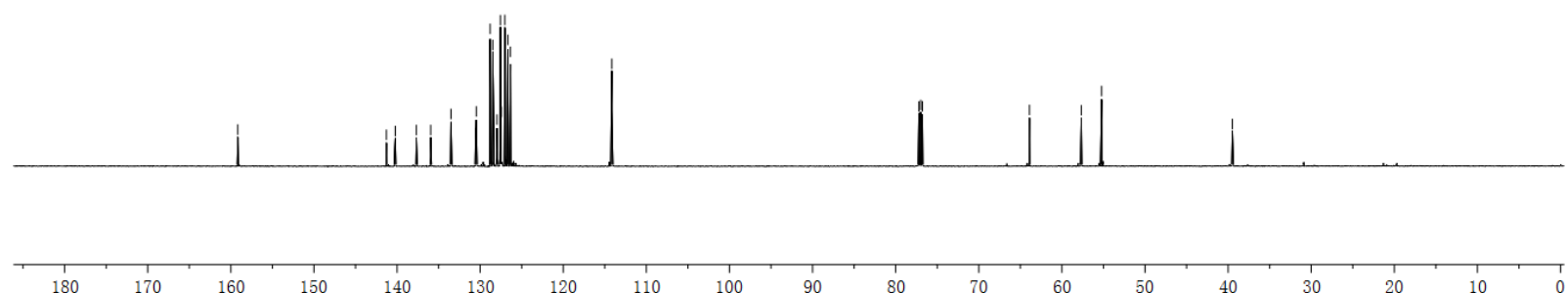

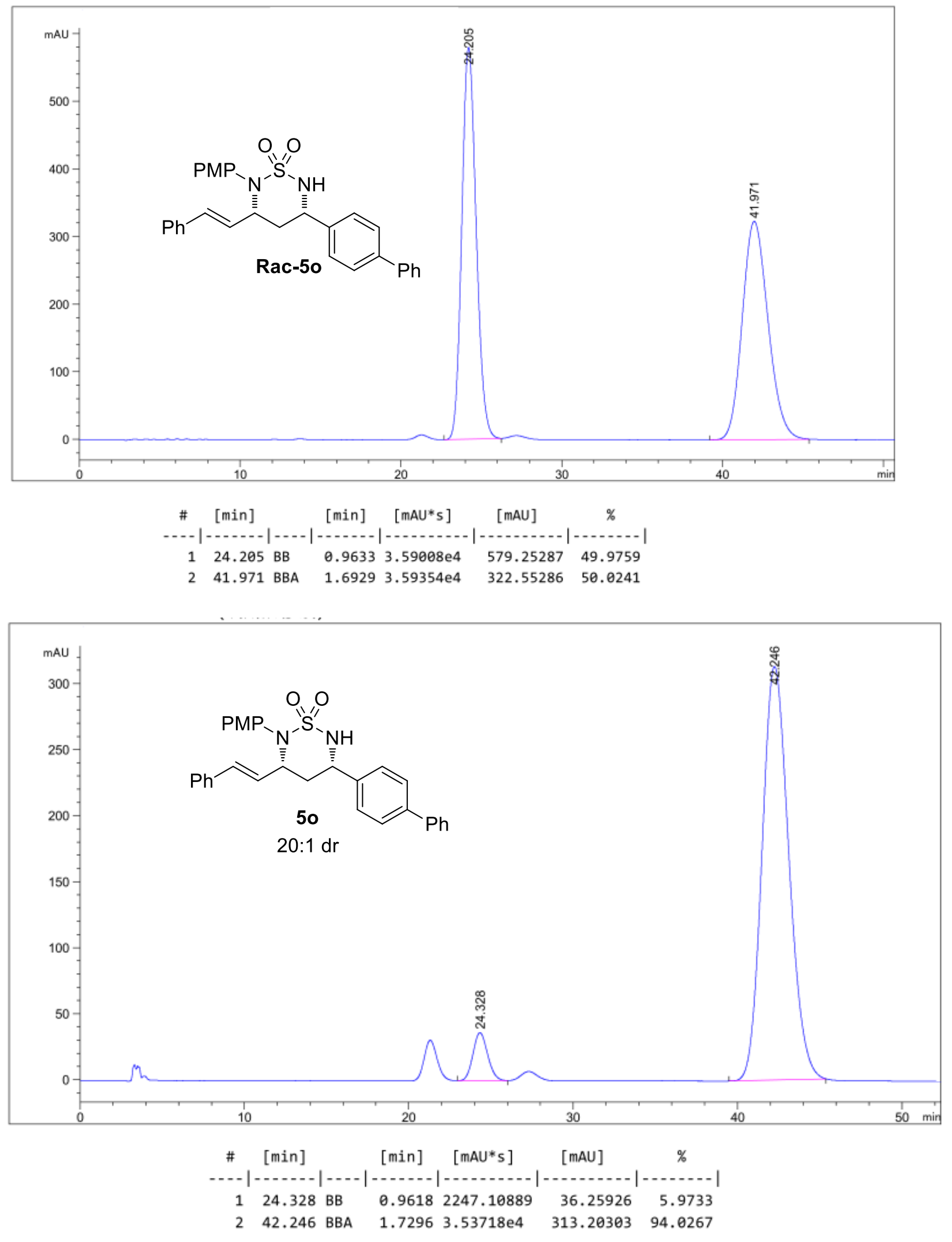
<smiles>COc1ccc(N2C(/C=C/c3ccccc3)C[C@@H](c3ccc(-c4ccccc4)cc3)NS2(=O)=O)cc1</smiles>

HRMS (ESI-TOF) m/z: [M + Na] ${ }^{+}$

Calcd for $\mathrm{C}_{30} \mathrm{H}_{28} \mathrm{~N}_{2} \mathrm{O}_{3} \mathrm{SNa} 459.1713$

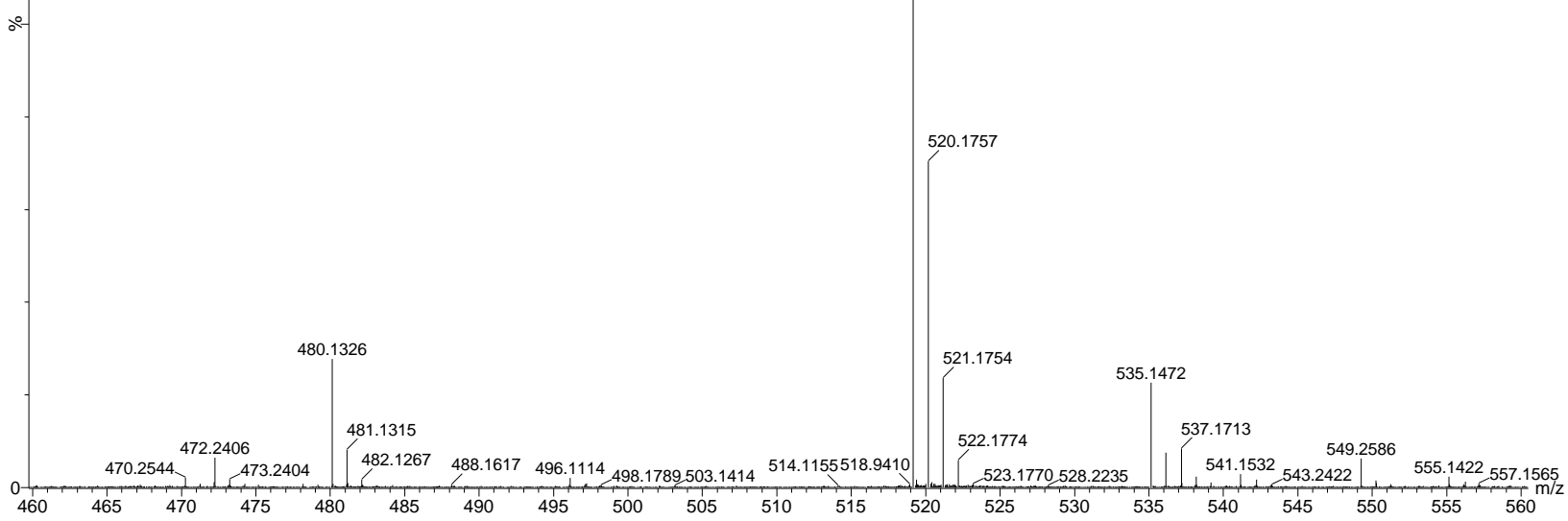



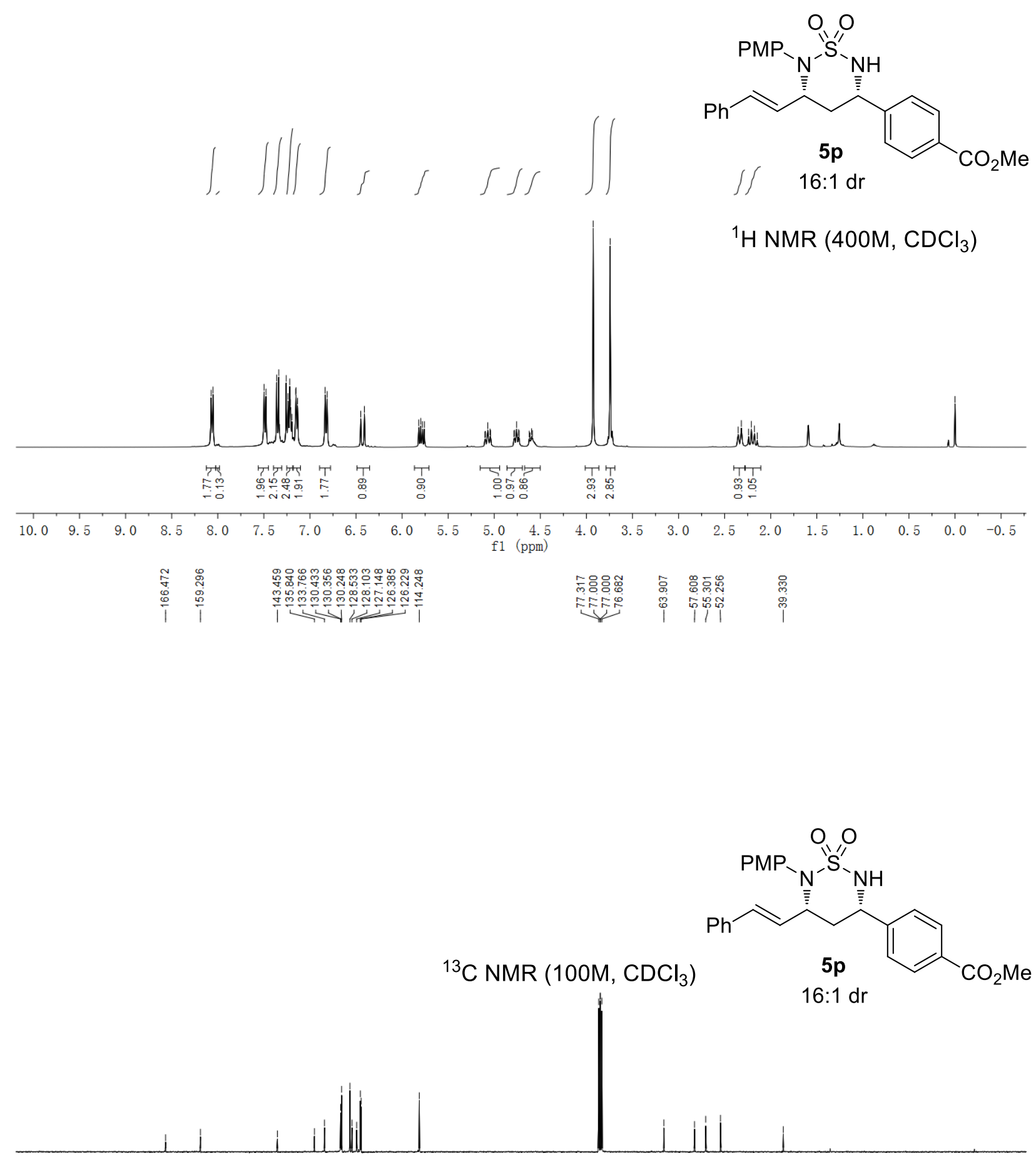

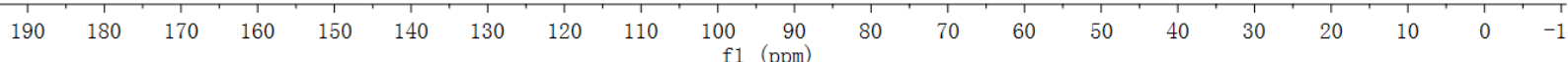



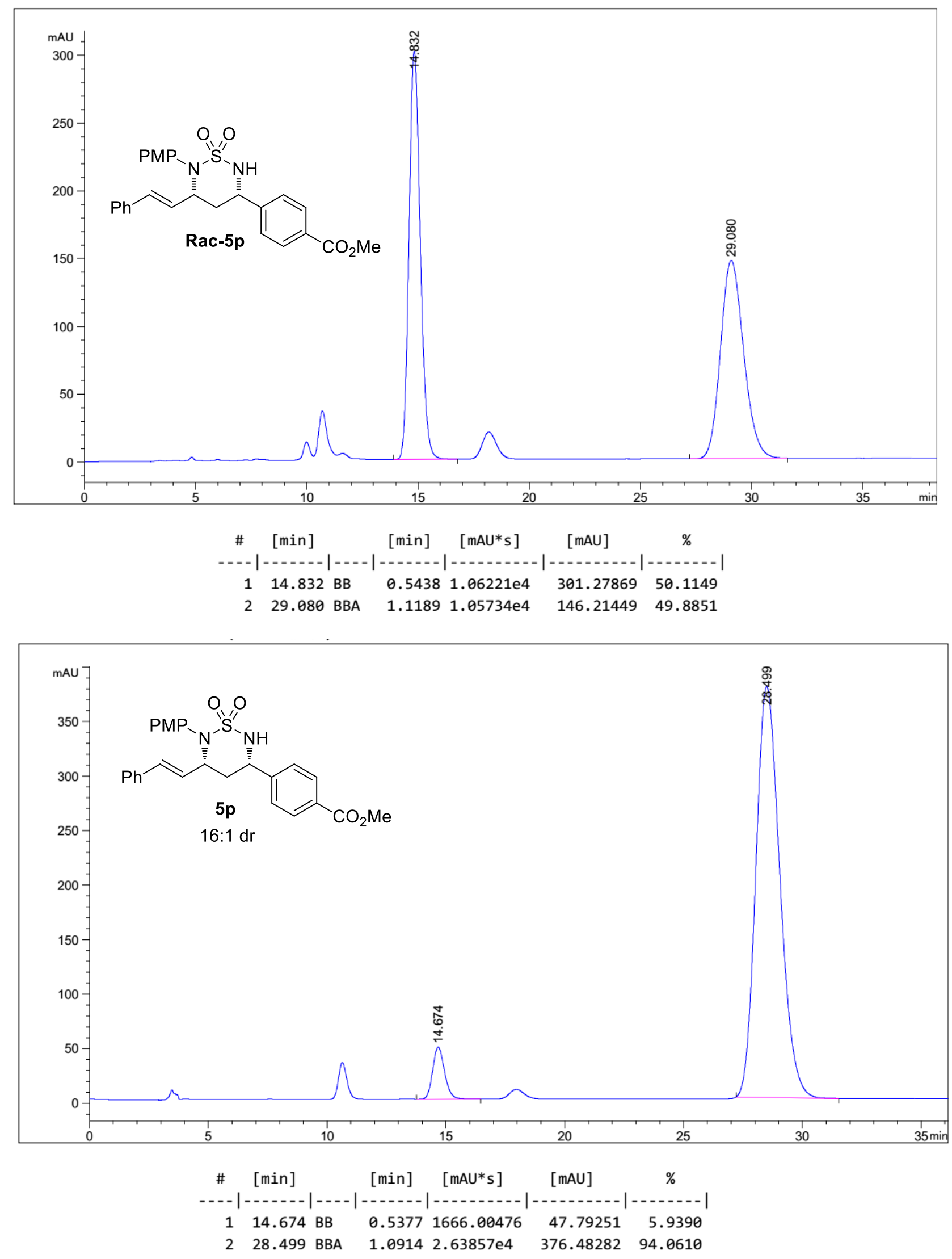


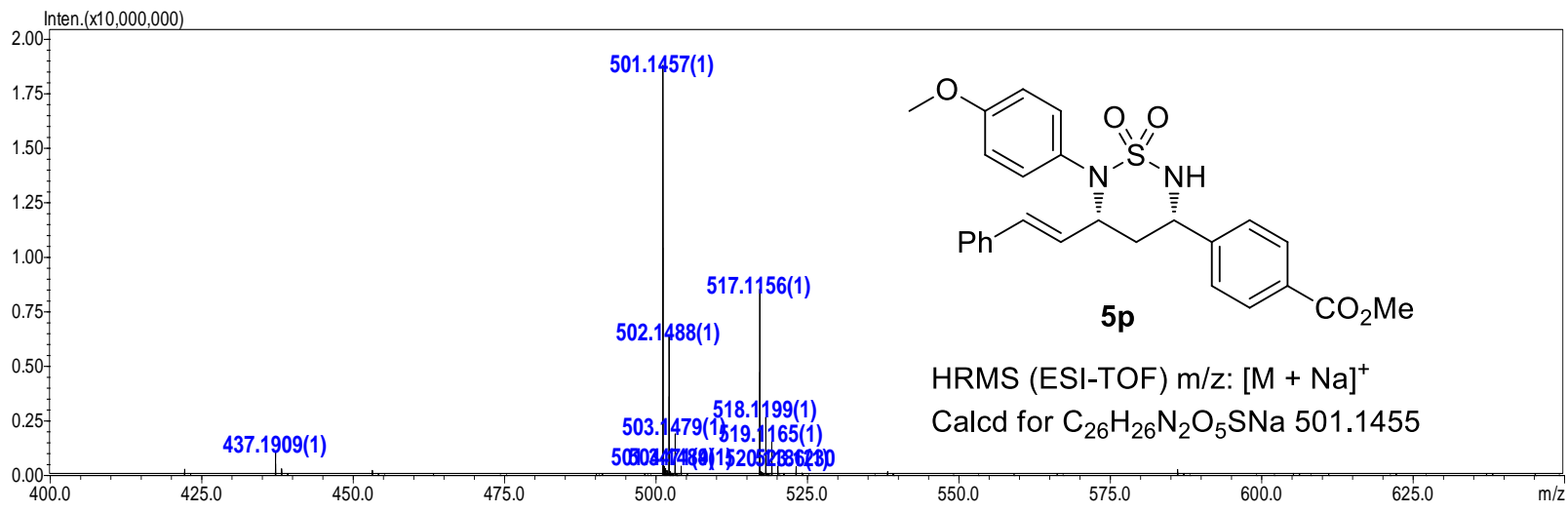




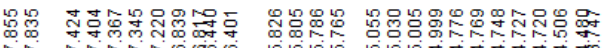

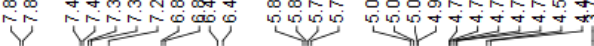<smiles>NP(=O)([GeH3])N1[C@@H](/C=C/c2ccccc2)C[C@H](c2ccc(Cc3ccccc3)cc2)NS1(=O)=O</smiles>

${ }^{1} \mathrm{H}$ NMR $\left(400 \mathrm{M}, \mathrm{CDCl}_{3}\right)$

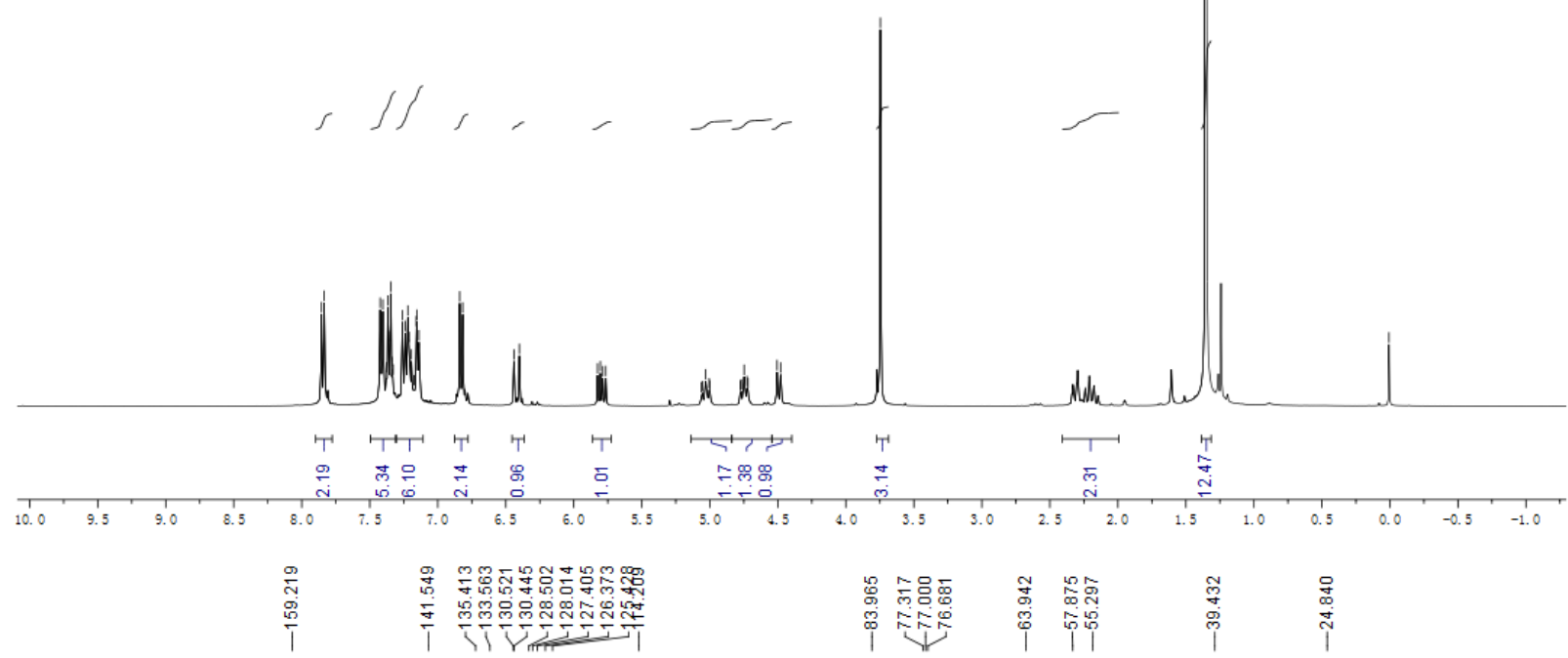<smiles>NP(=O)([O-])N1[C@@H](/C=C/c2ccccc2)C[C@@H](c2ccc(Cc3ccccc3)cc2)NS1(=O)=O</smiles>

$14: 1 \mathrm{dr}$

${ }^{13} \mathrm{C}$ NMR $\left(100 \mathrm{M}, \mathrm{CDCl}_{3}\right)$

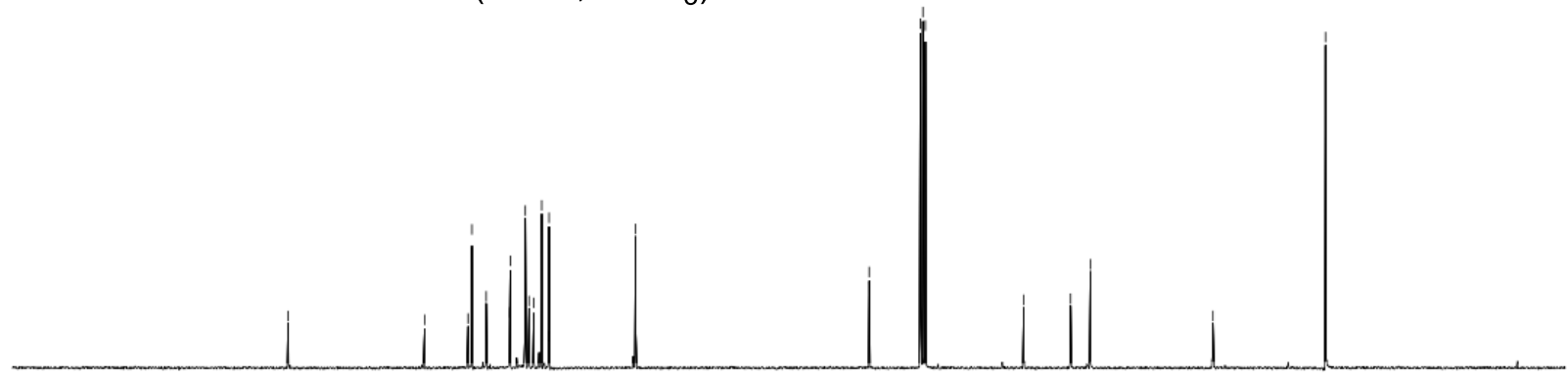



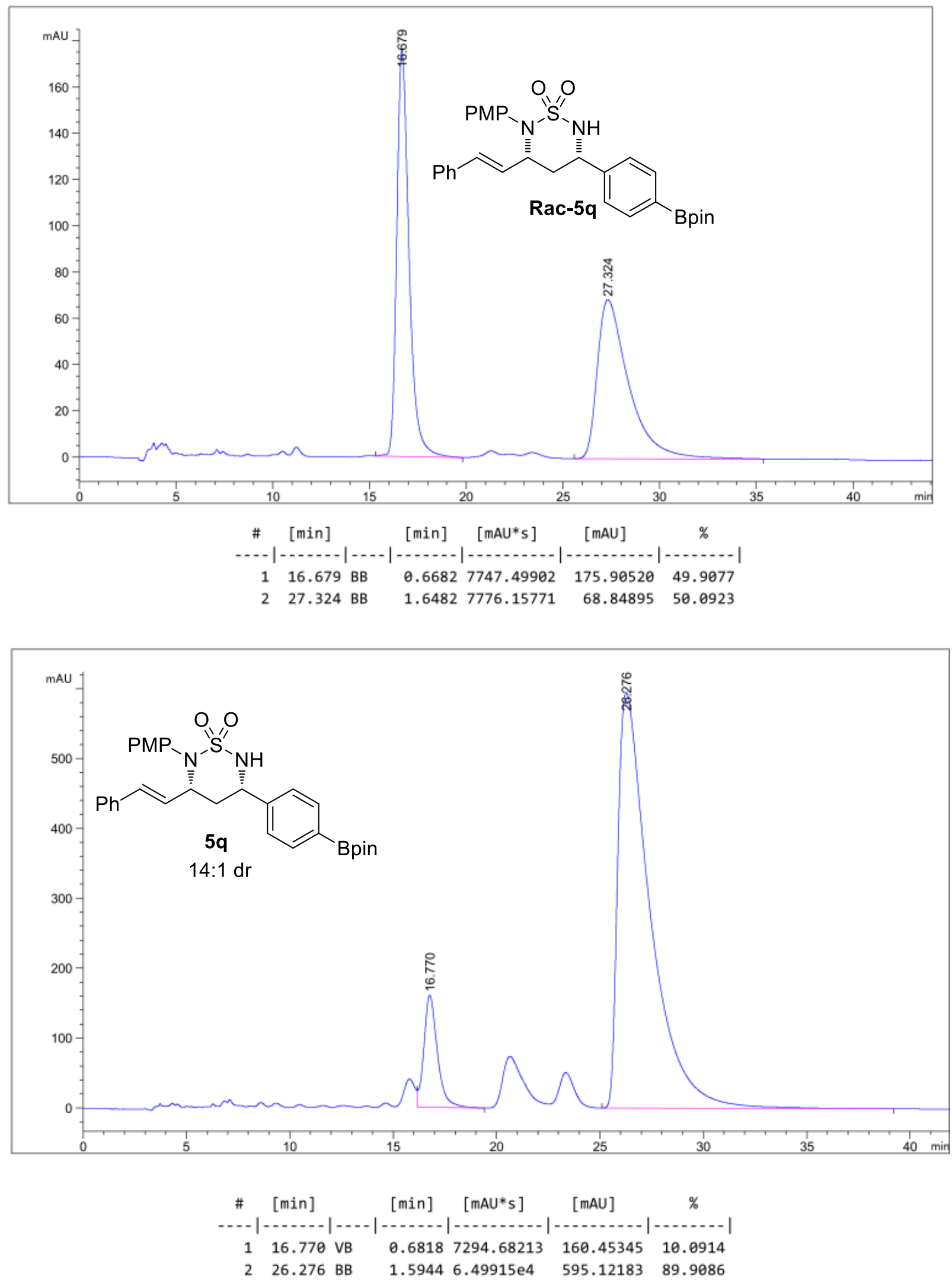


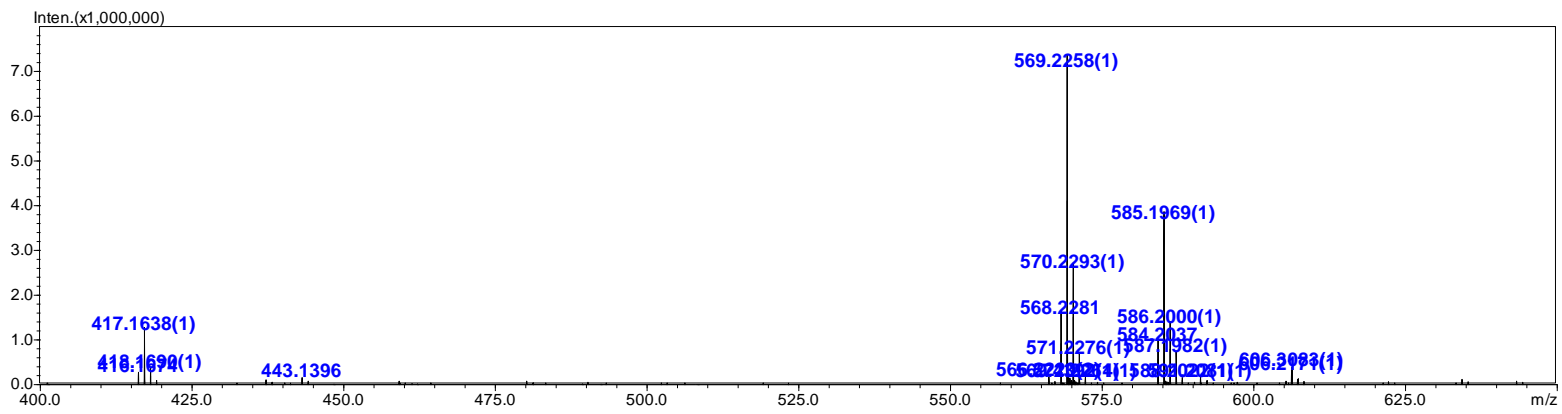<smiles></smiles>

HRMS (ESI-TOF) m/z: [M + Na] ${ }^{+}$

Calcd for $\mathrm{C}_{30} \mathrm{H}_{35} \mathrm{BN}_{2} \mathrm{O}_{5} \mathrm{SNa} 569.2252$ 


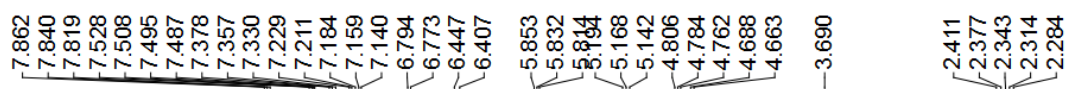

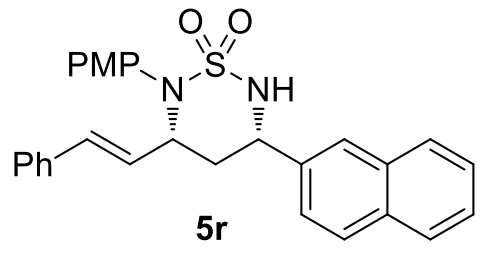

${ }^{1} \mathrm{H}$ NMR (400M, $\mathrm{CDCl}_{3}$ )
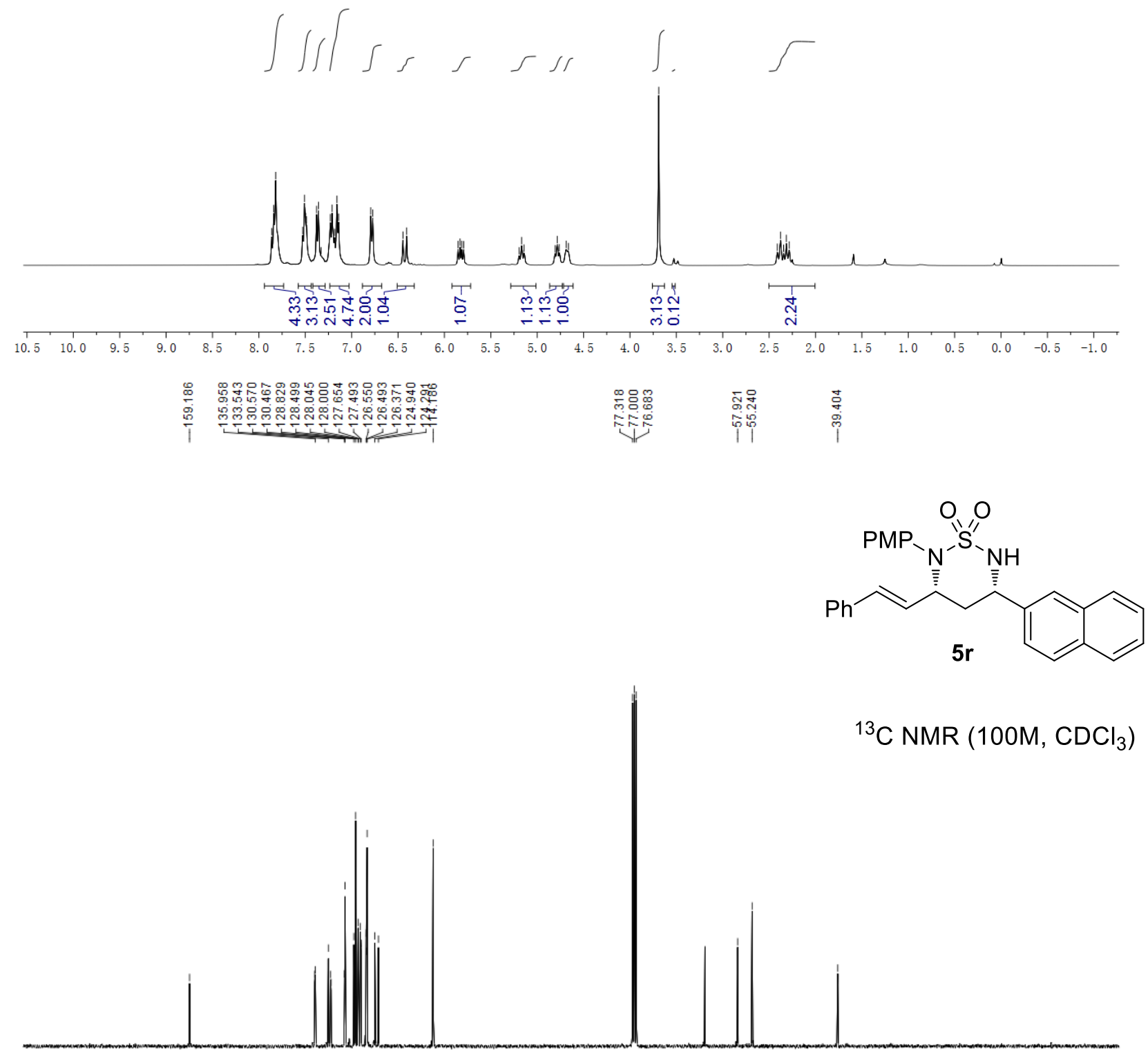

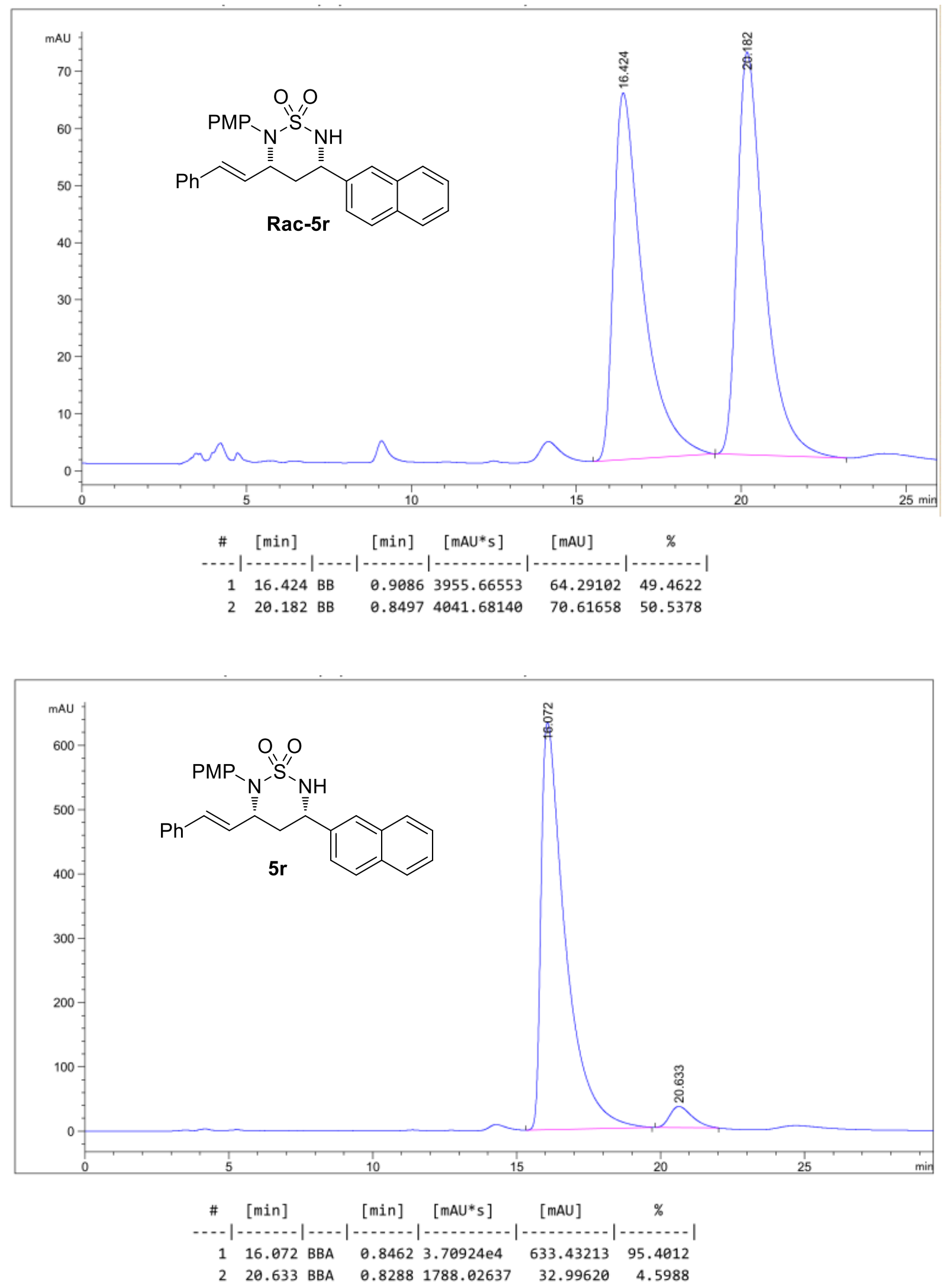


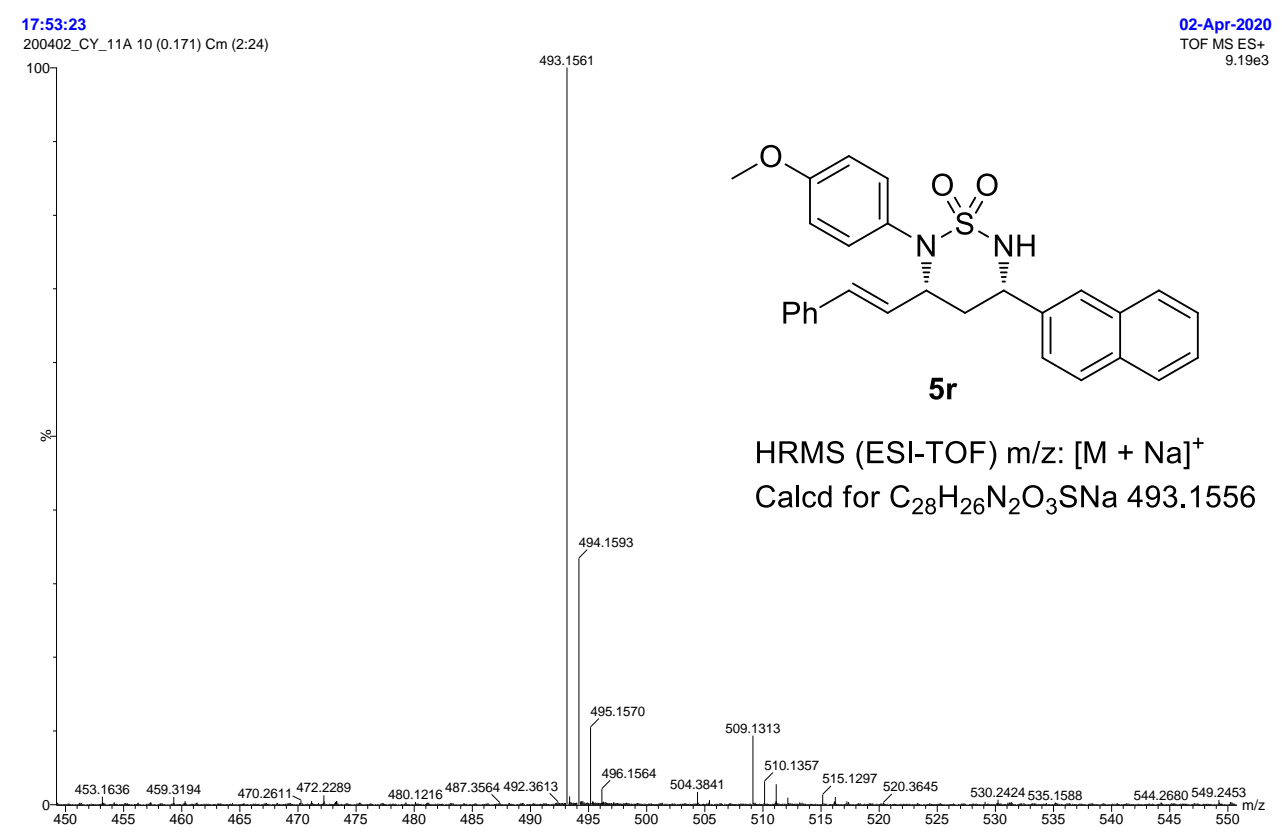



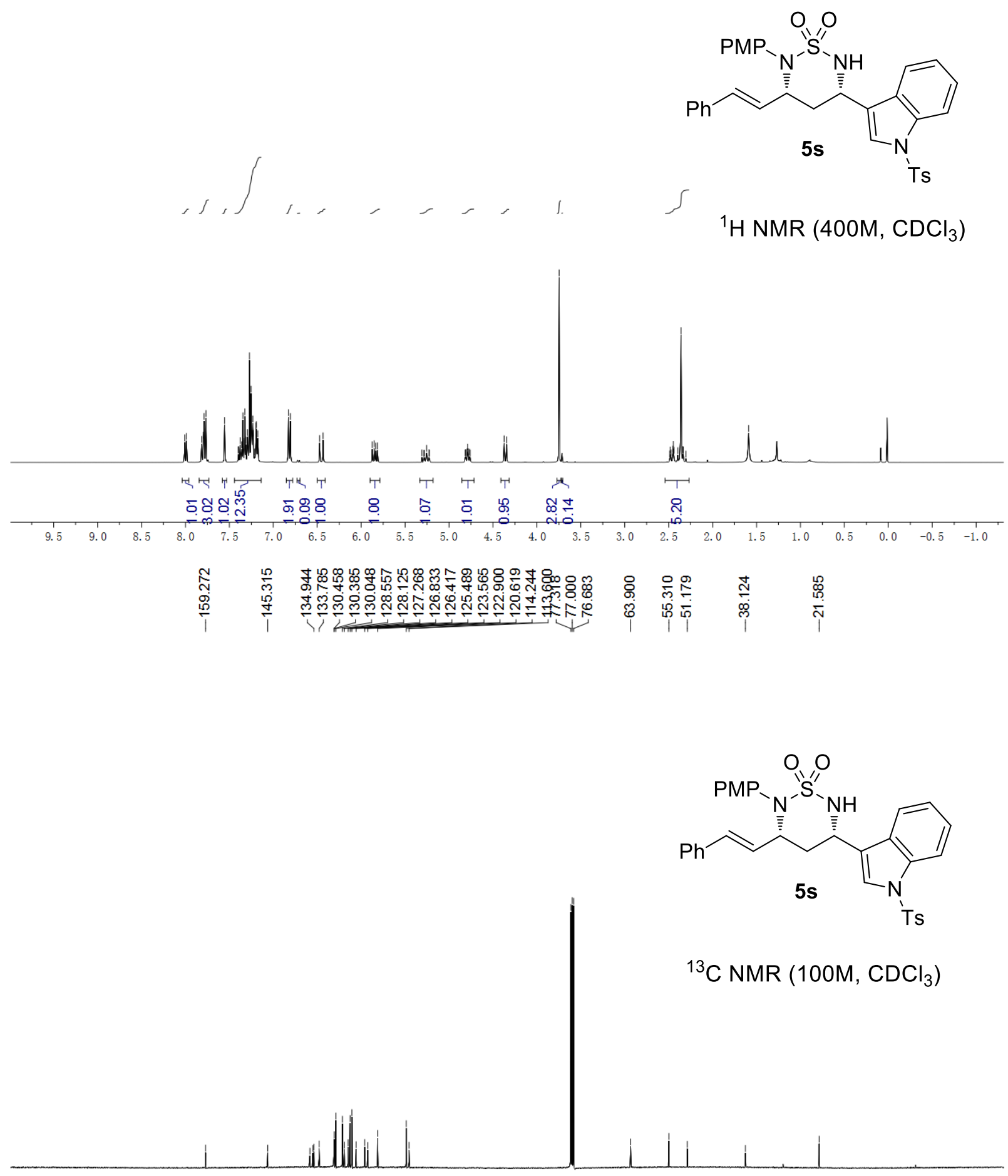

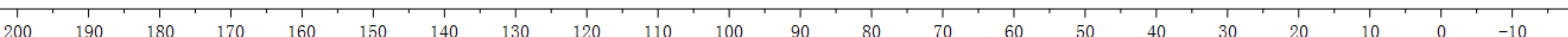



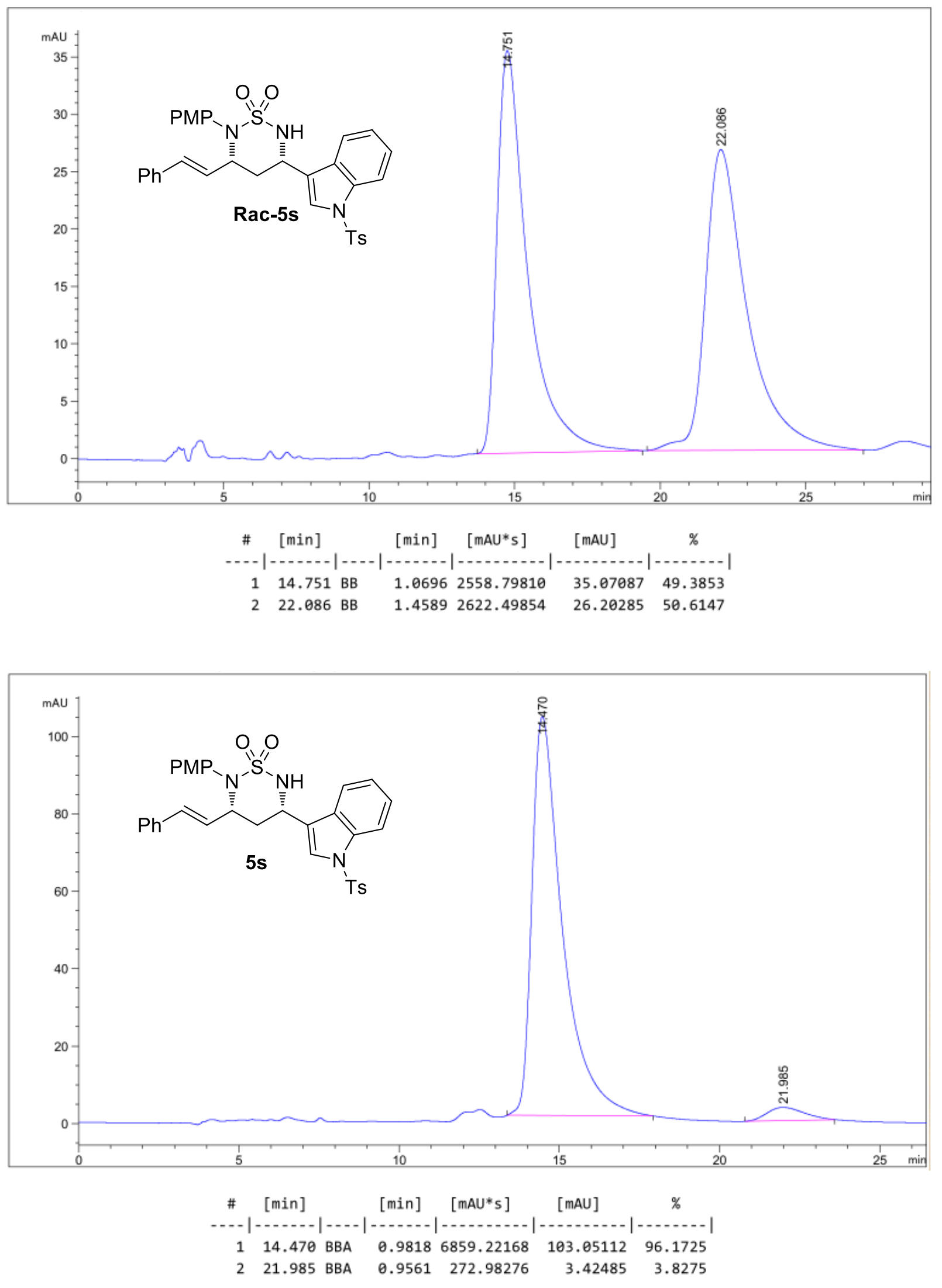


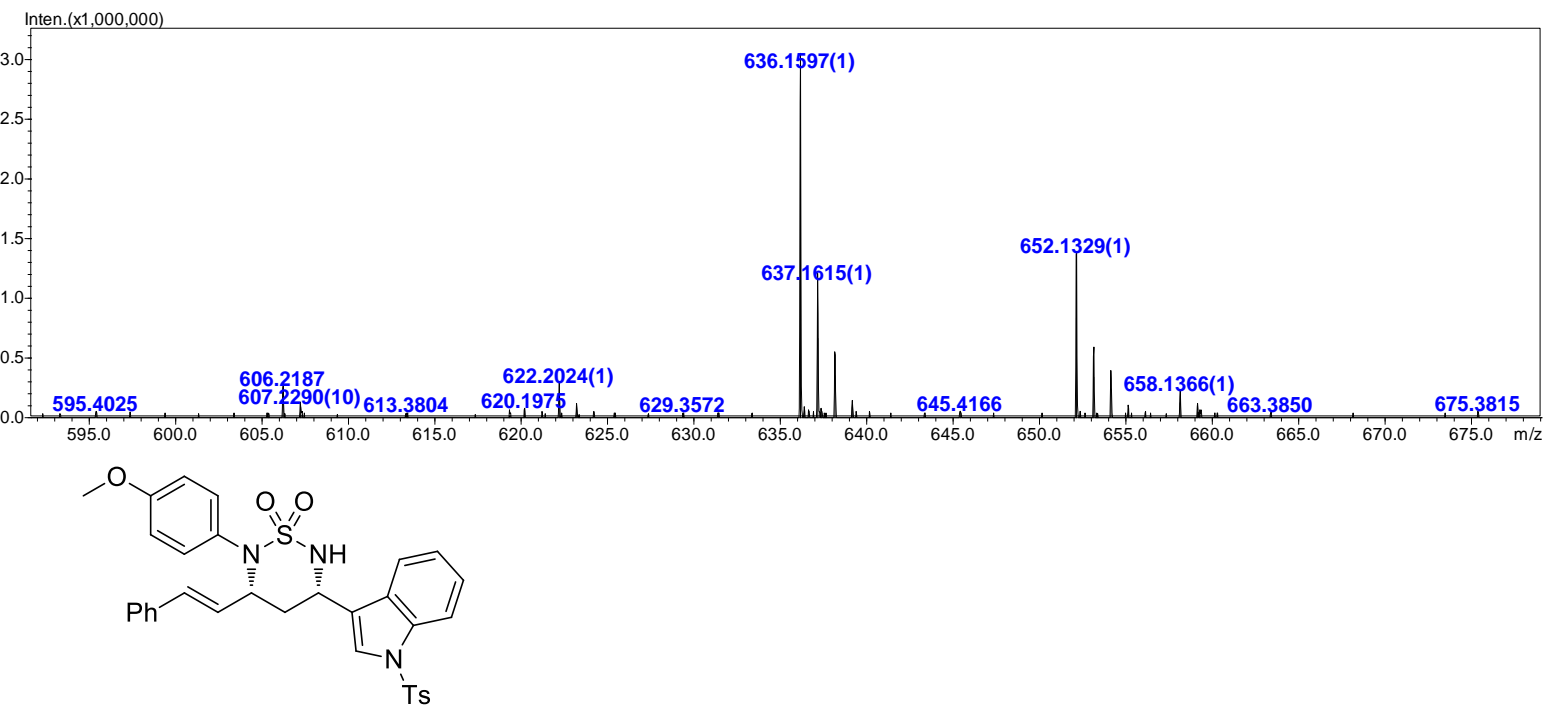

5 s

HRMS (ESI-TOF) m/z: [M + Na]

Calcd for $\mathrm{C}_{33} \mathrm{H}_{31} \mathrm{~N}_{3} \mathrm{O}_{5} \mathrm{~S}_{2} \mathrm{Na} 636.1597$ 

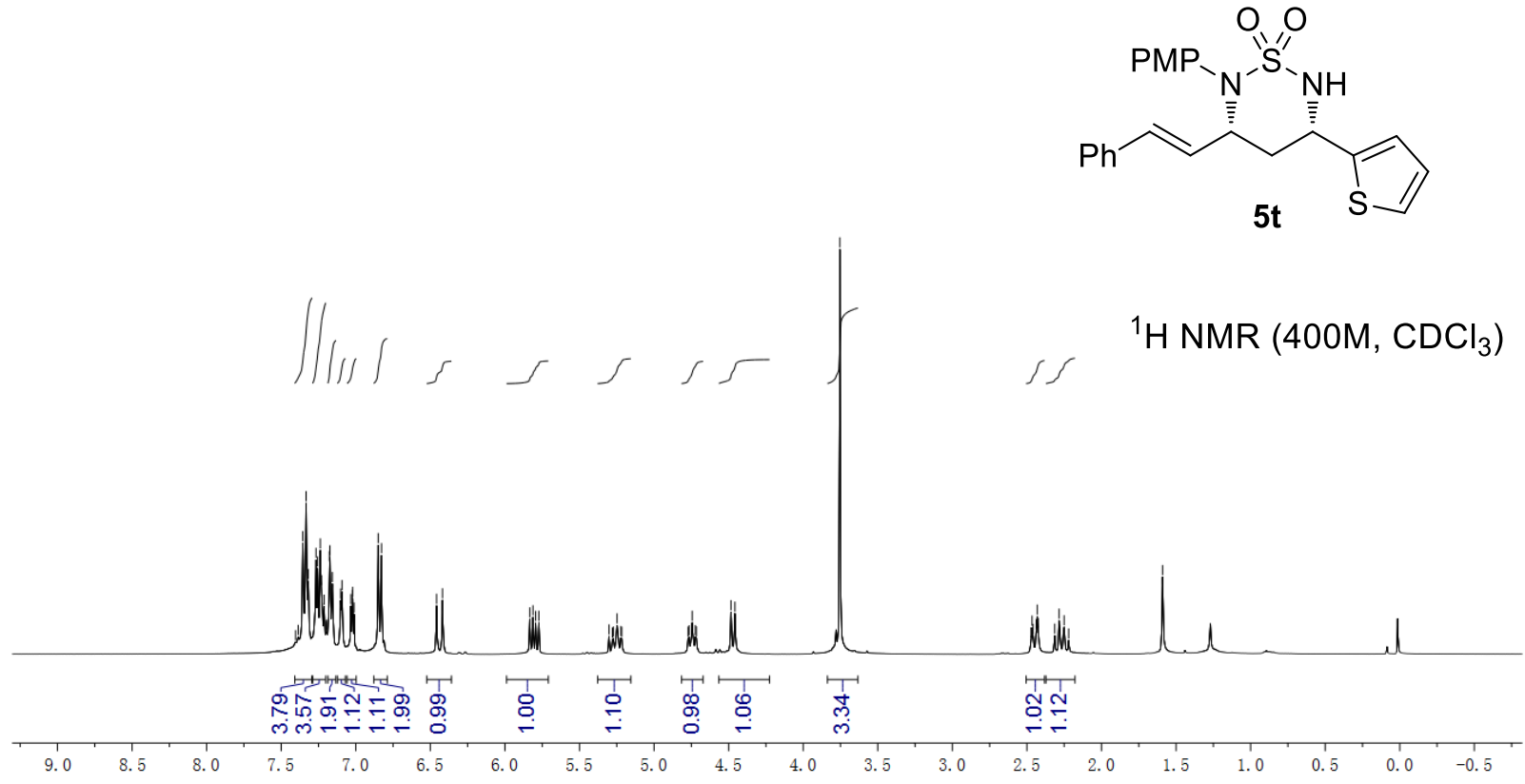

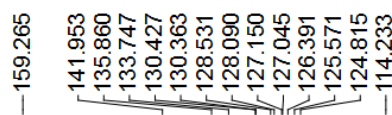

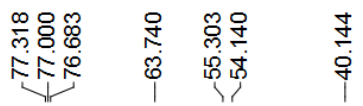

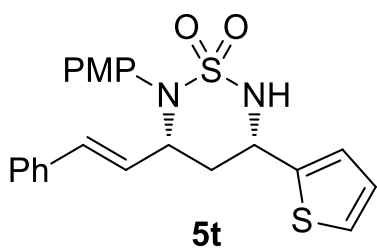

${ }^{13} \mathrm{C}$ NMR $\left(100 \mathrm{M}, \mathrm{CDCl}_{3}\right)$

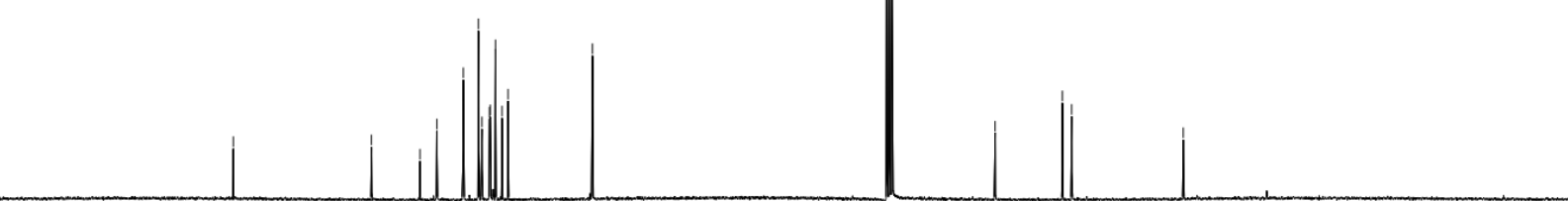

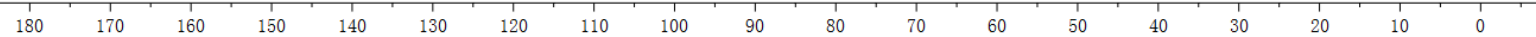



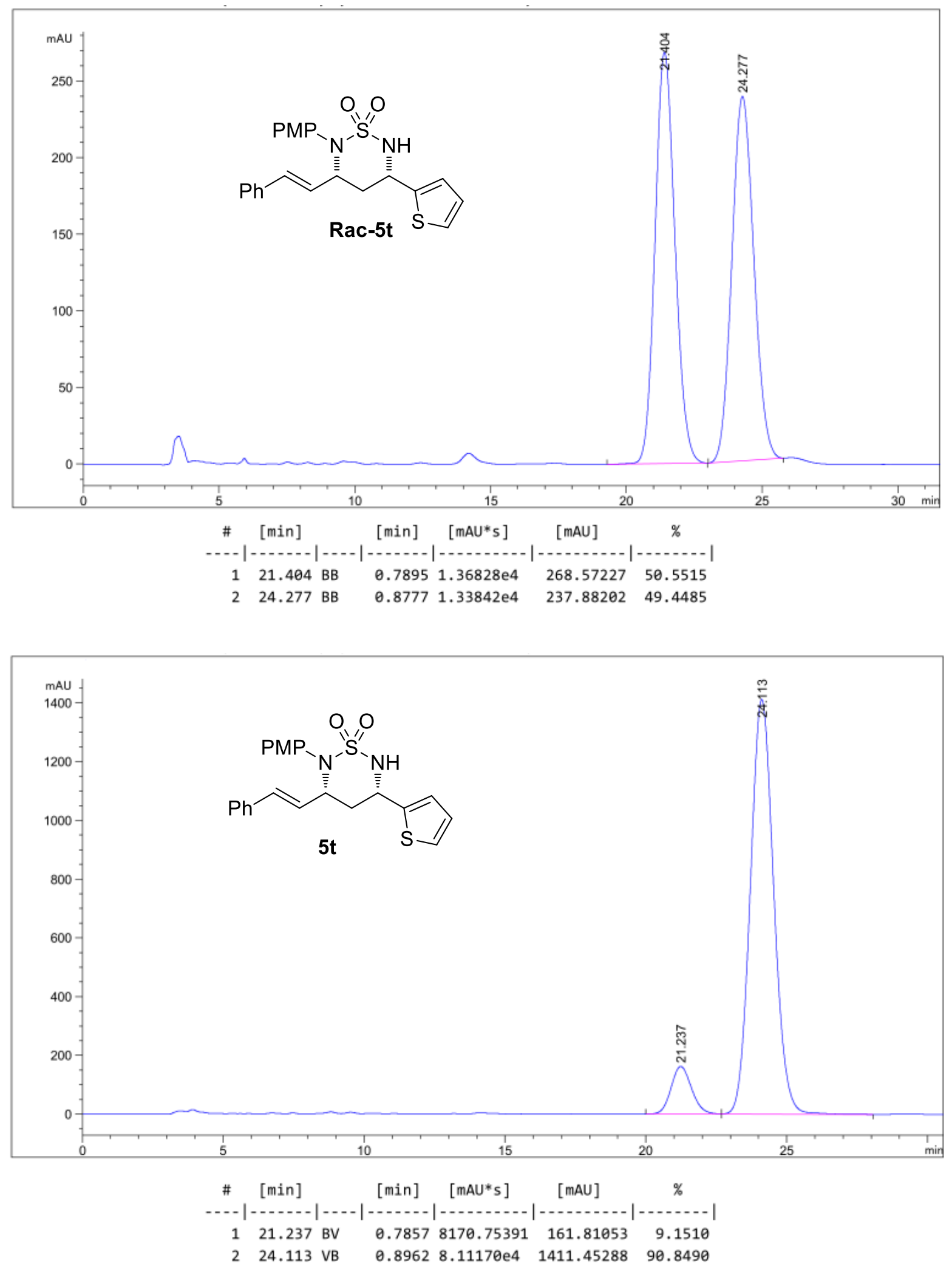
Spectrum Plot Report

Agilent

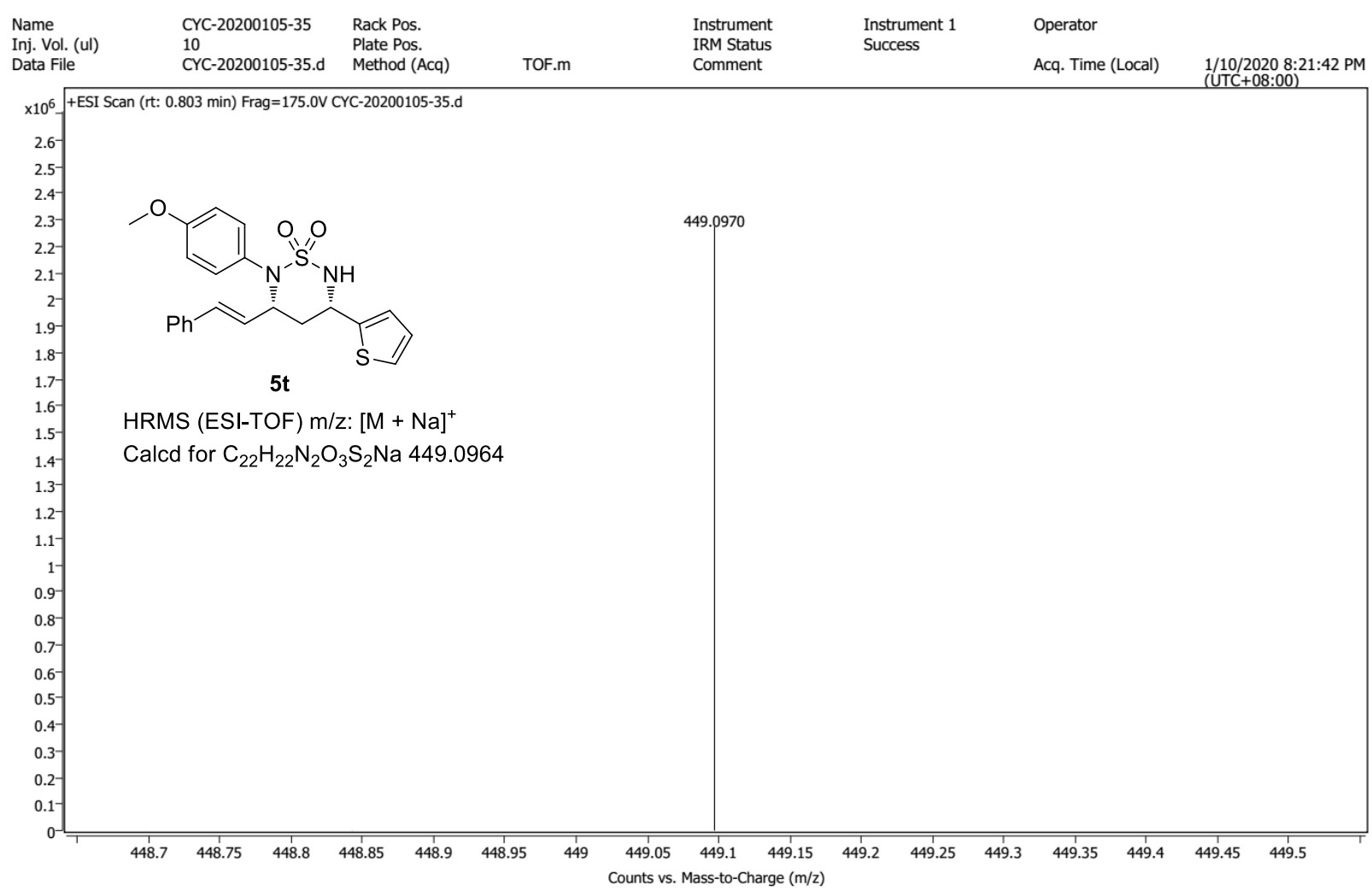




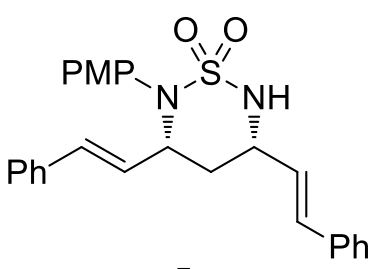

$5 u$

7:1 dr

${ }^{1} \mathrm{H}$ NMR (400M, $\mathrm{CDCl}_{3}$ )
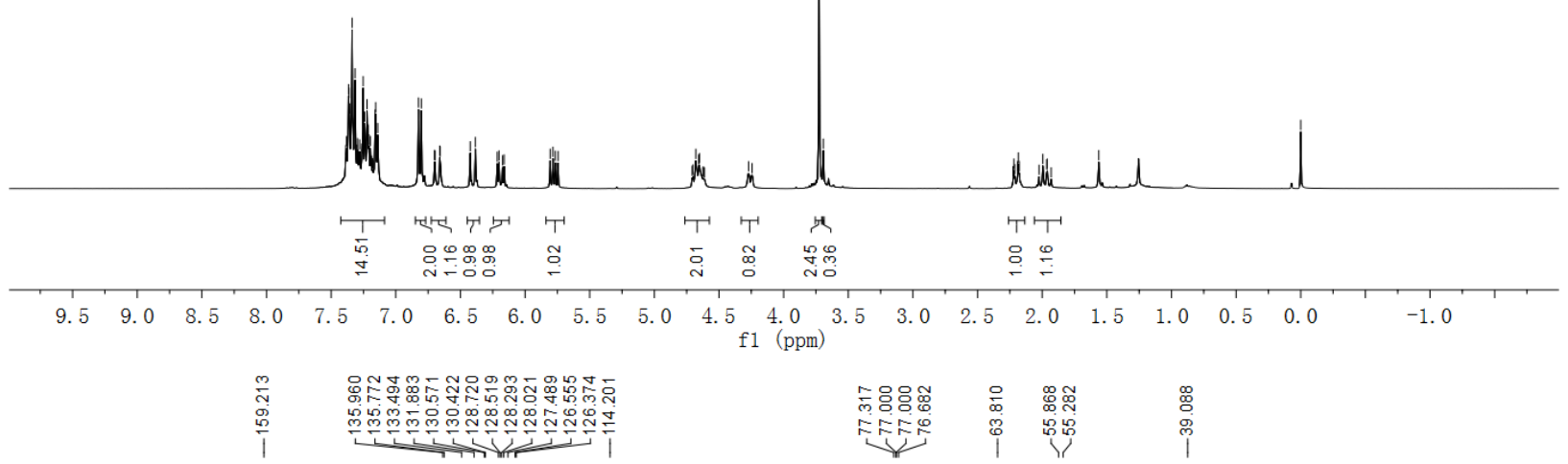

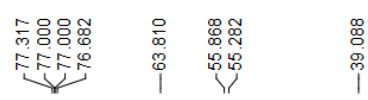

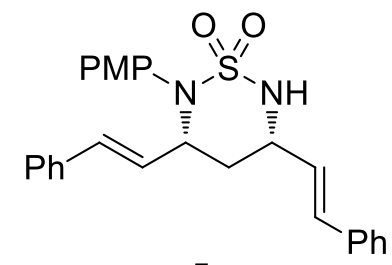

$5 \mathrm{u}$

$7: 1 \mathrm{dr}$

${ }^{13} \mathrm{C}$ NMR (100M, $\mathrm{CDCl}_{3}$ )

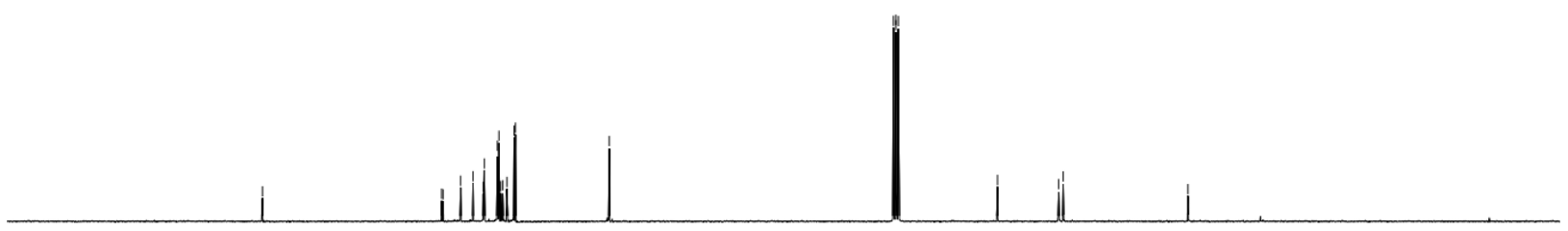

190

$\begin{array}{lllll}160 & 150 & 140 & 130 & 120\end{array}$

10090

$80 \quad 70 \quad 60$

$50 \quad 40$

$30 \quad 20 \quad 10 \quad 0$

0 

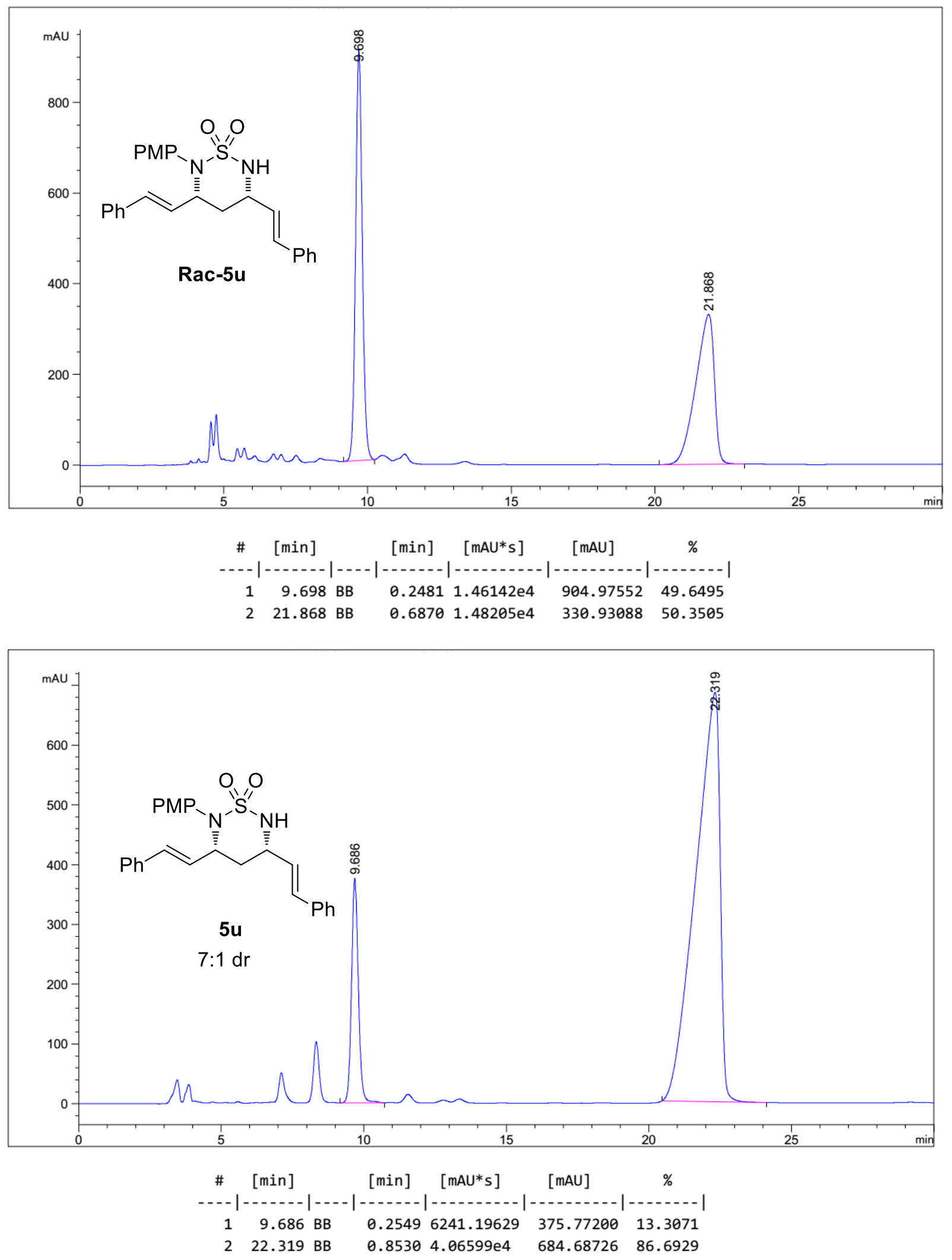
Inten. $(x 10,000,000)$

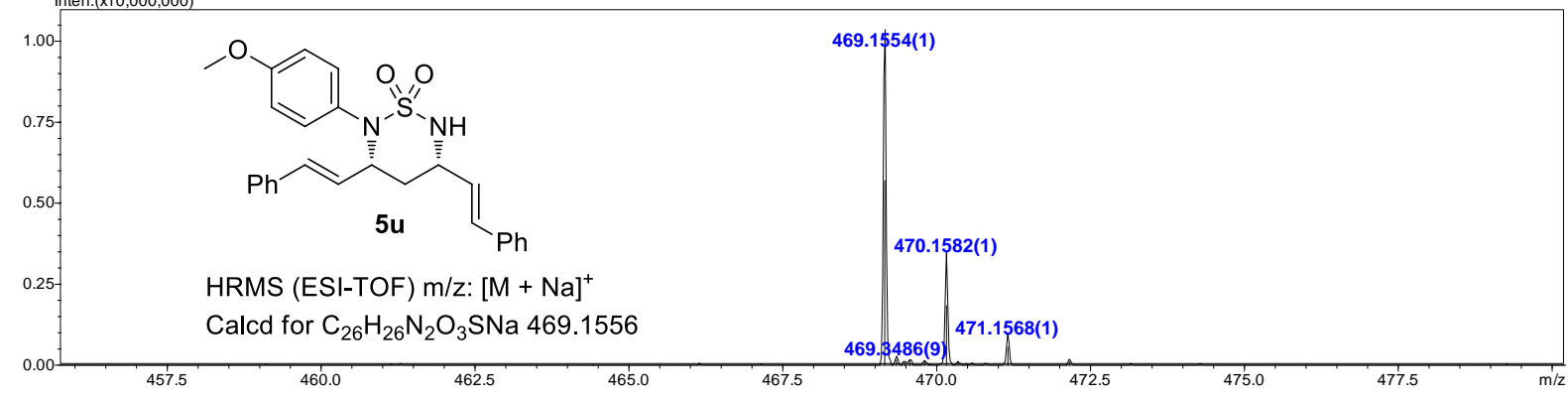


<smiles>O=[R16]N1[C@H](/C=C/c2ccccc2)C[C@@H](C2CCCCC2)NS1(=O)=O</smiles>

$5 v$

${ }^{1} \mathrm{H}$ NMR (400M, $\mathrm{CDCl}_{3}$ )

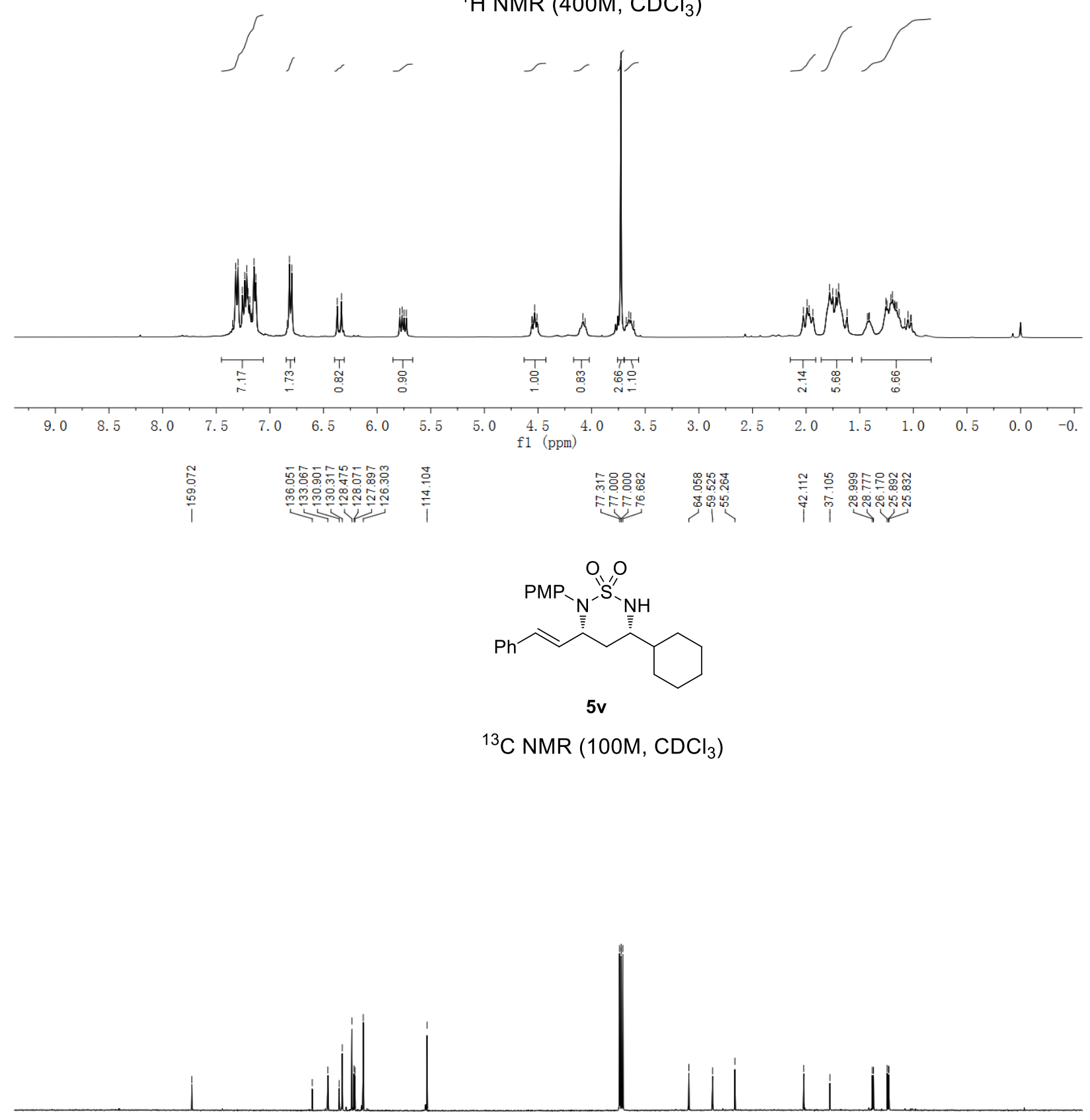

$\begin{array}{llllllllllllllllllllllllllll}1 & 180 & 170 & 160 & 150 & 140 & 130 & 120 & 110 & 100 & 90 & 80 & 70 & 60 & 50 & 40 & 30 & 20 & 10 & 0 & -11\end{array}$ 

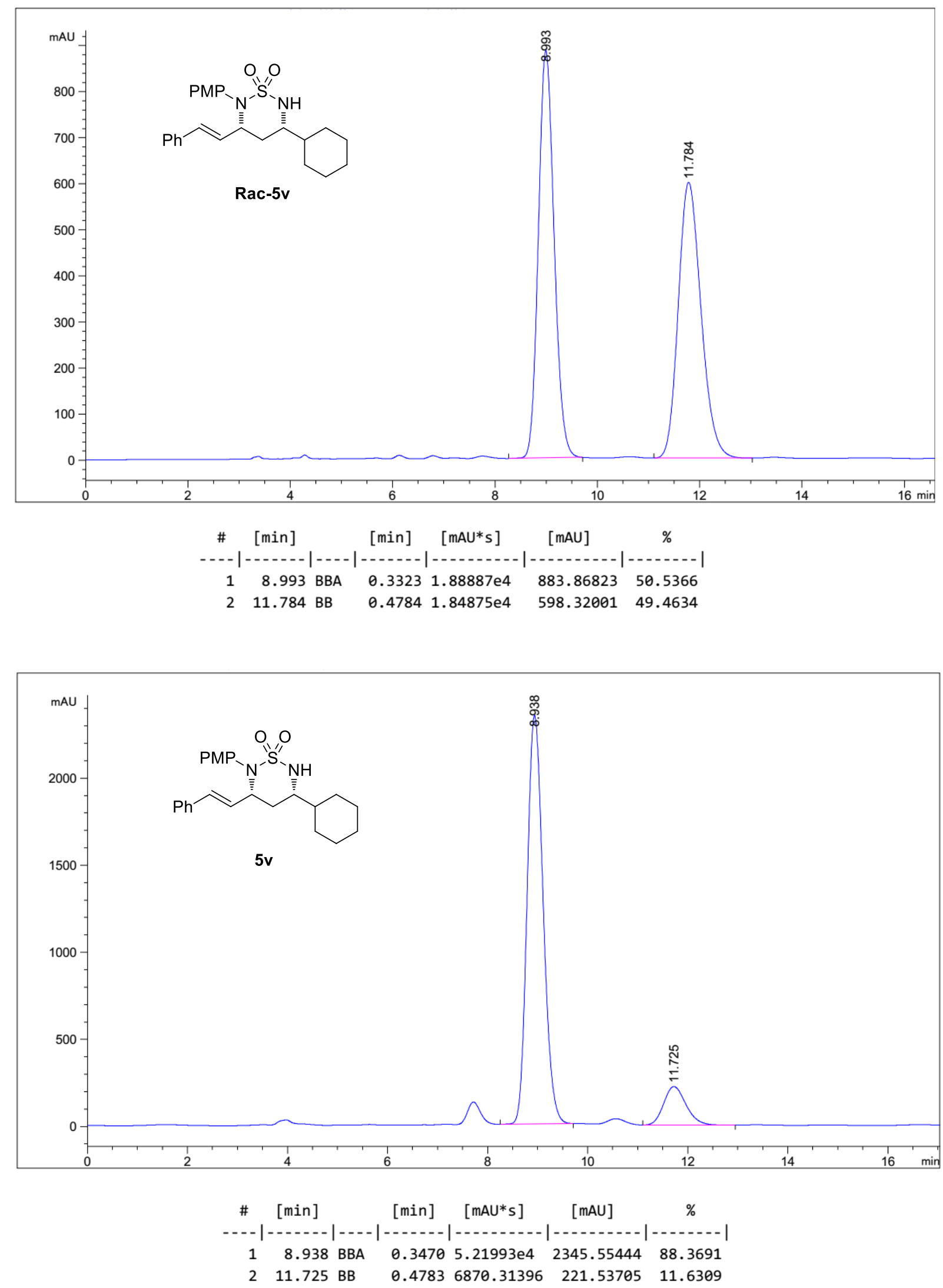


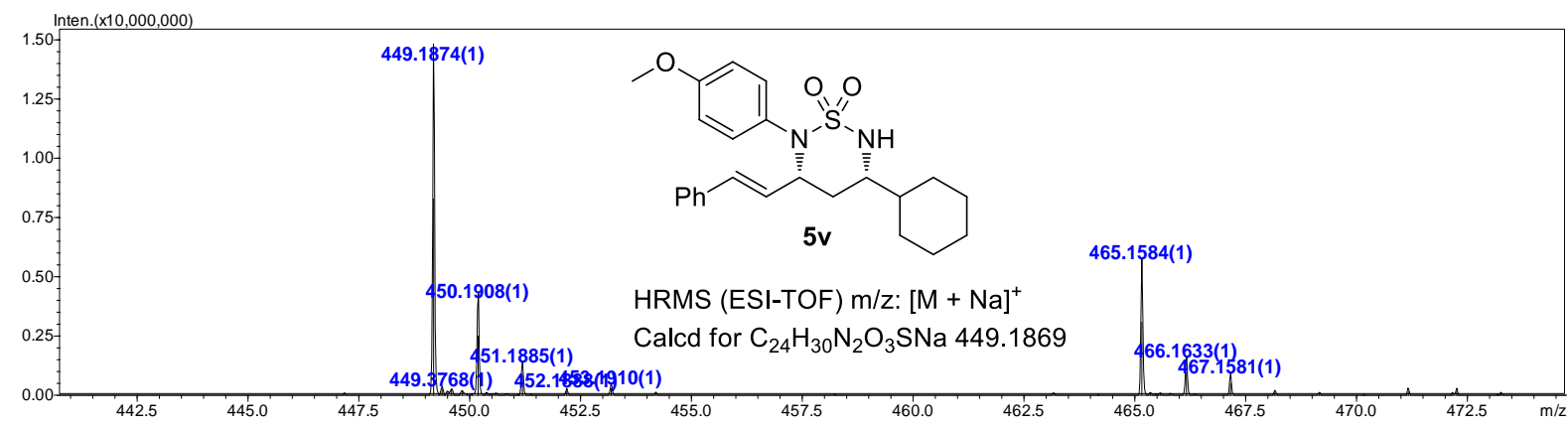


<smiles>O=S1(=O)N[C@@H](c2ccccc2)CC(/C=C/c2ccccc2)N1c1ccccc1</smiles>

${ }^{1} \mathrm{H}$ NMR (400M, $\left.\mathrm{CDCl}_{3}\right)$
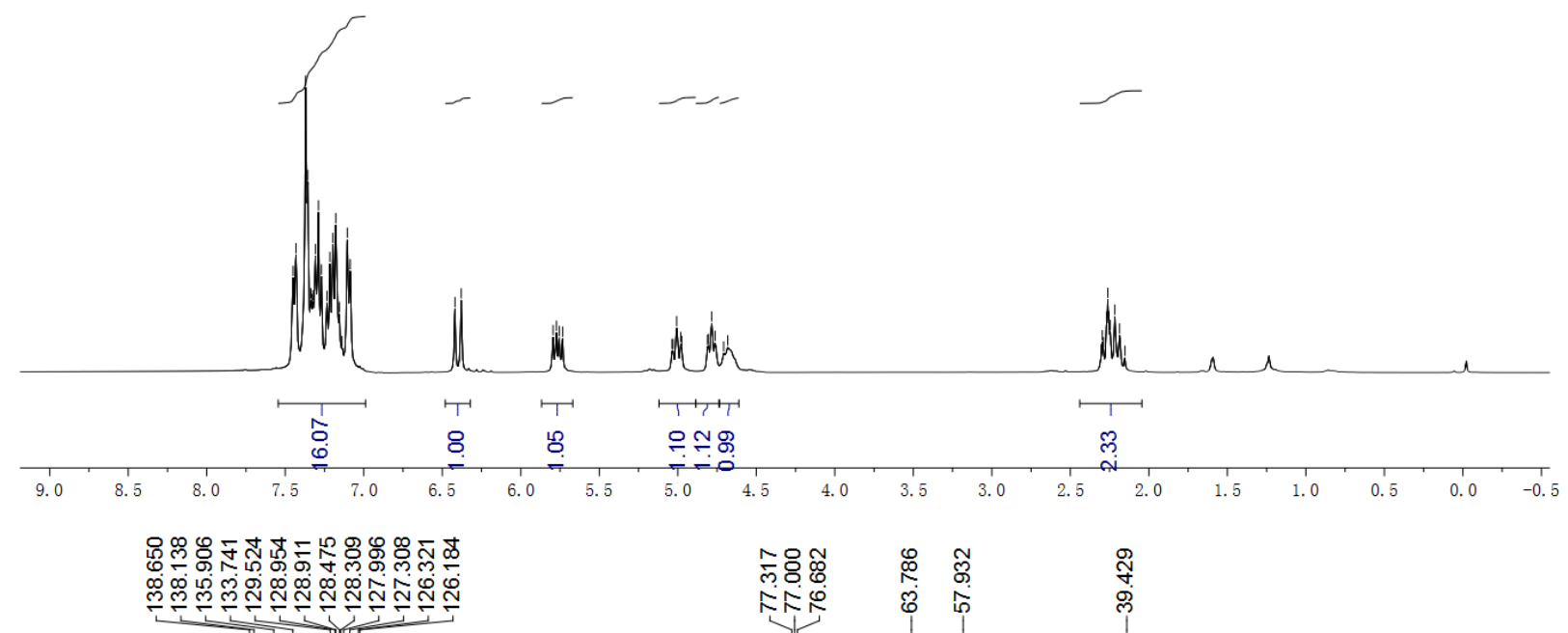

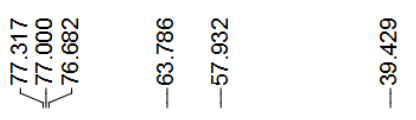
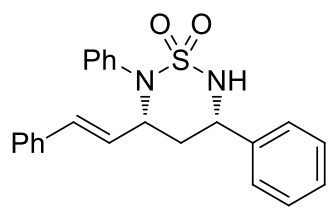

$5 w$

${ }^{13} \mathrm{C}$ NMR (100M, $\mathrm{CDCl}_{3}$ )

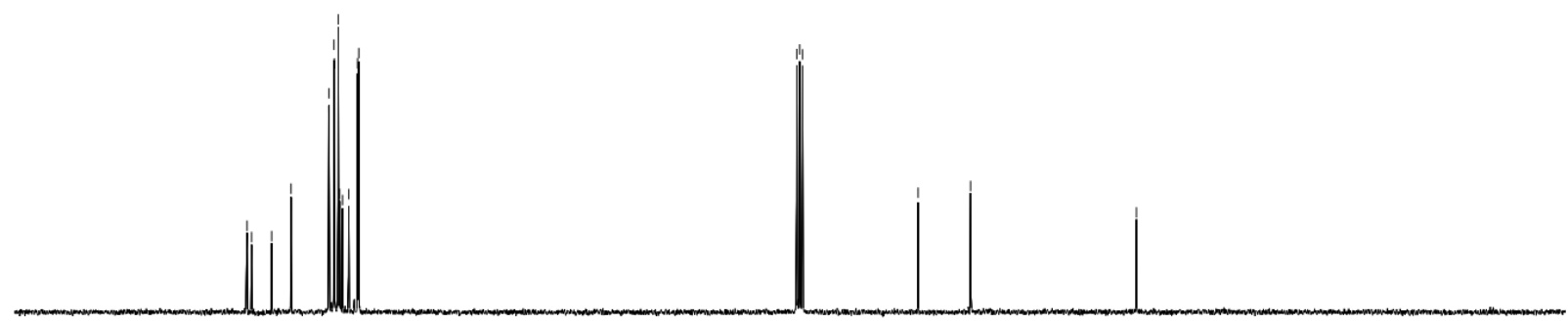



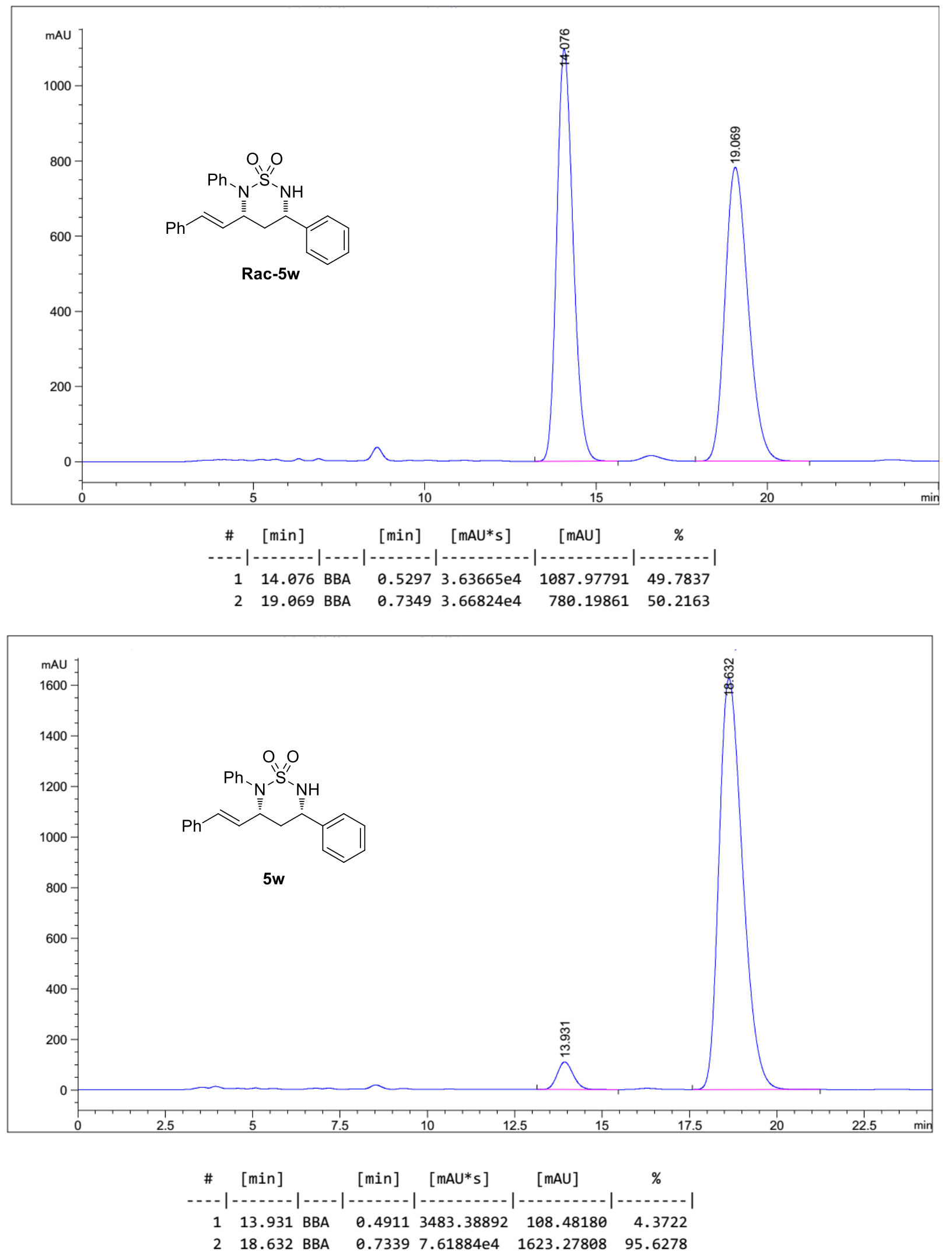


\section{Spectrum Plot Report}

Agilent

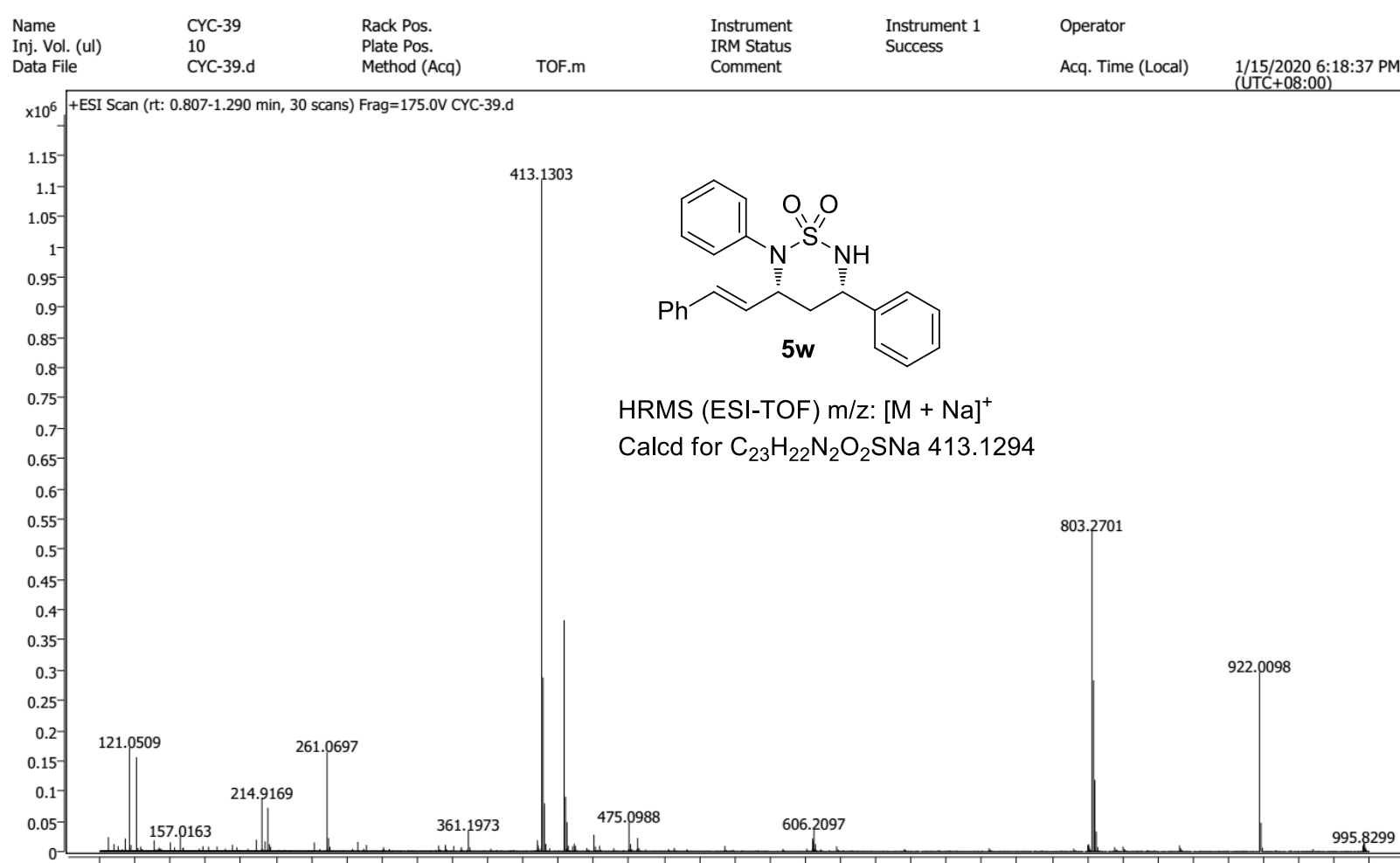

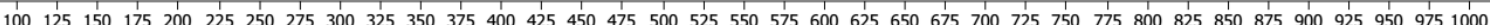
Counts vs. Mass-to-Charge $(\mathrm{m} / \mathrm{z})$ 


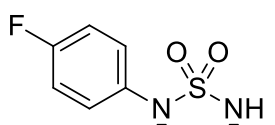<smiles>CC(C/C=C/c1ccccc1)Cc1ccccc1</smiles>

$5 x$

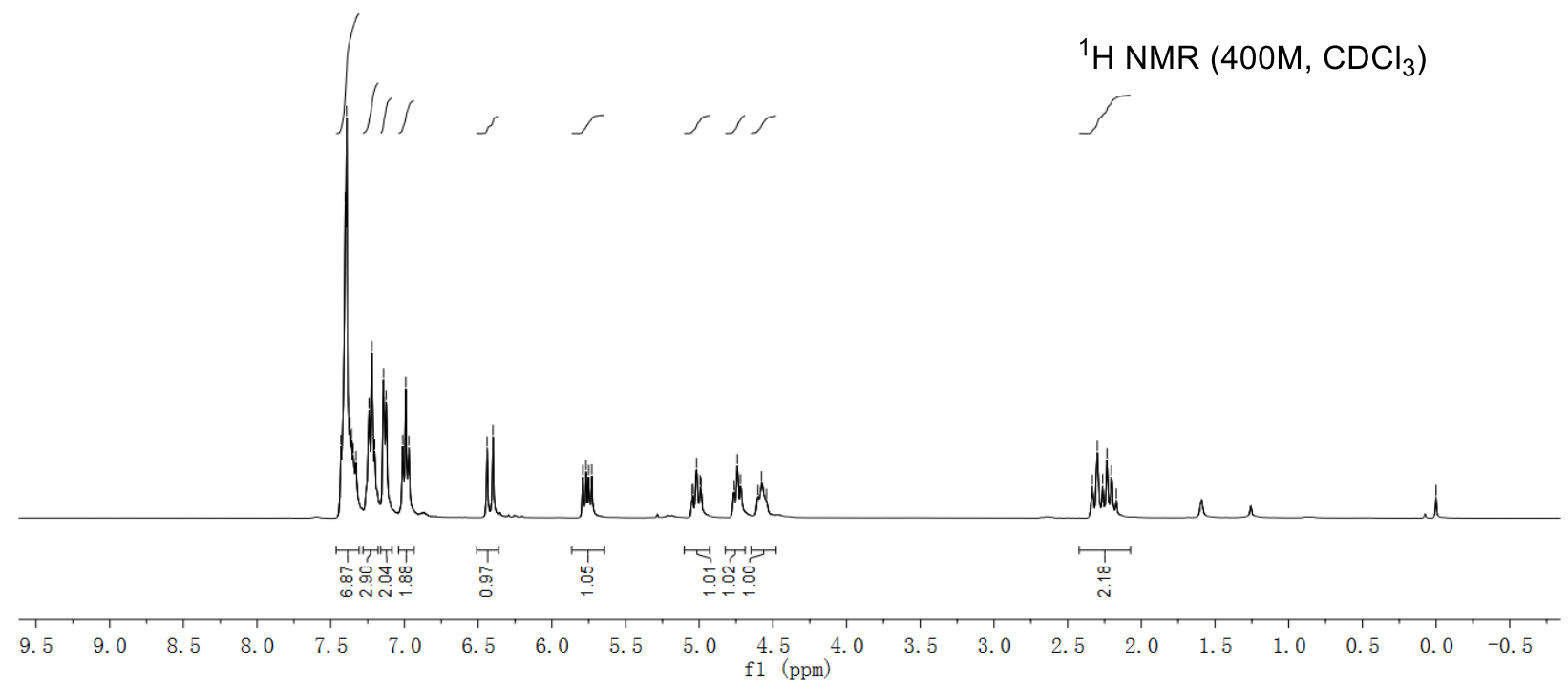

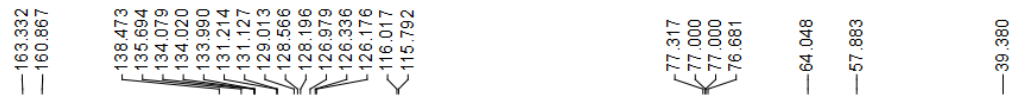

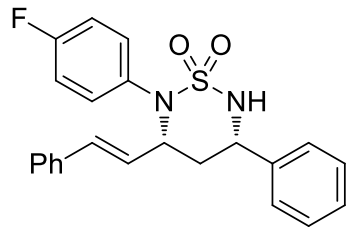

$5 x$

${ }^{13} \mathrm{C}$ NMR (100M, $\left.\mathrm{CDCl}_{3}\right)$

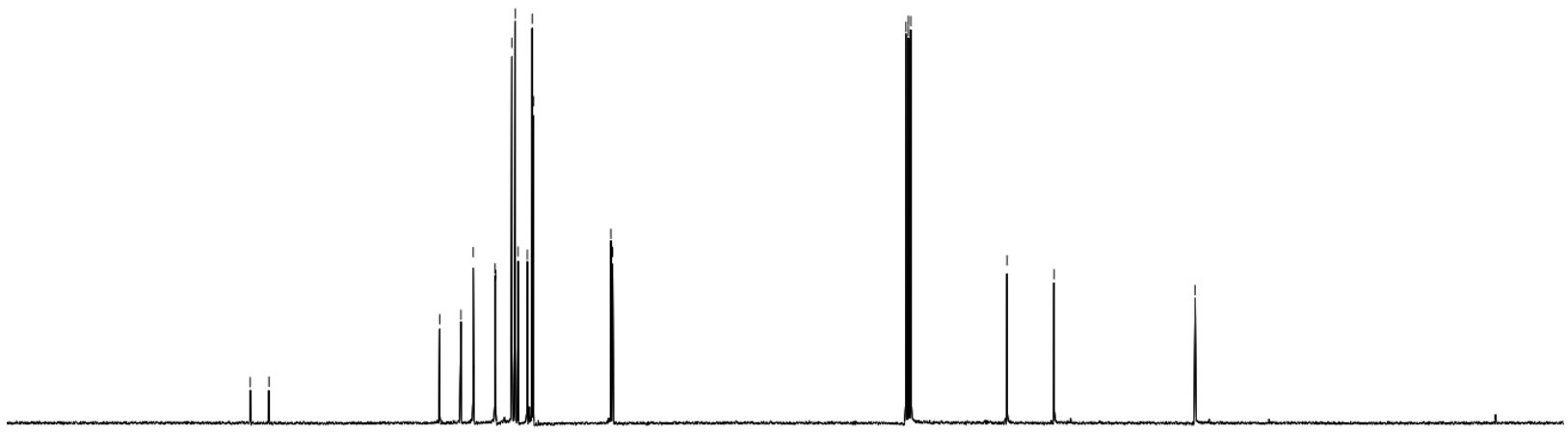

$\begin{array}{rrrrrrrrrr}190 & 180 & 170 & 160 & 150 & 140 & 130 & 120 & 110 & 100 \\ \mathrm{f} 1 & 90 \\ (\mathrm{ppm})\end{array}$ 


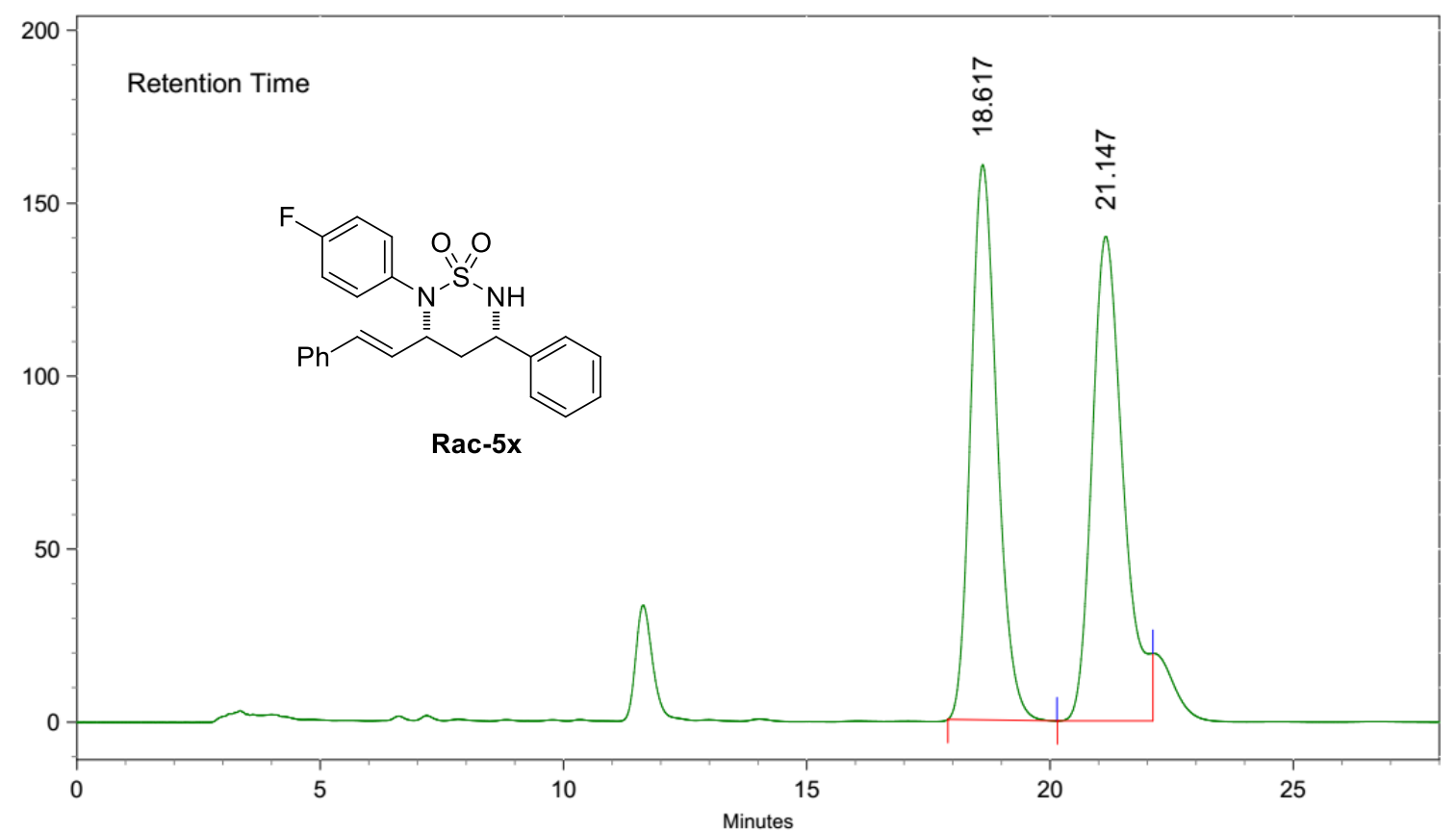

\begin{tabular}{llllll} 
Peak No. & Ret Time & Width & Height & Area & Area [\%] \\
\hline 1 & 18.617 & 2.243 & 2692666 & 101349117 & 49.1098 \\
2 & 21.147 & 1.963 & 2349890 & 105023280 & 50.8902
\end{tabular}

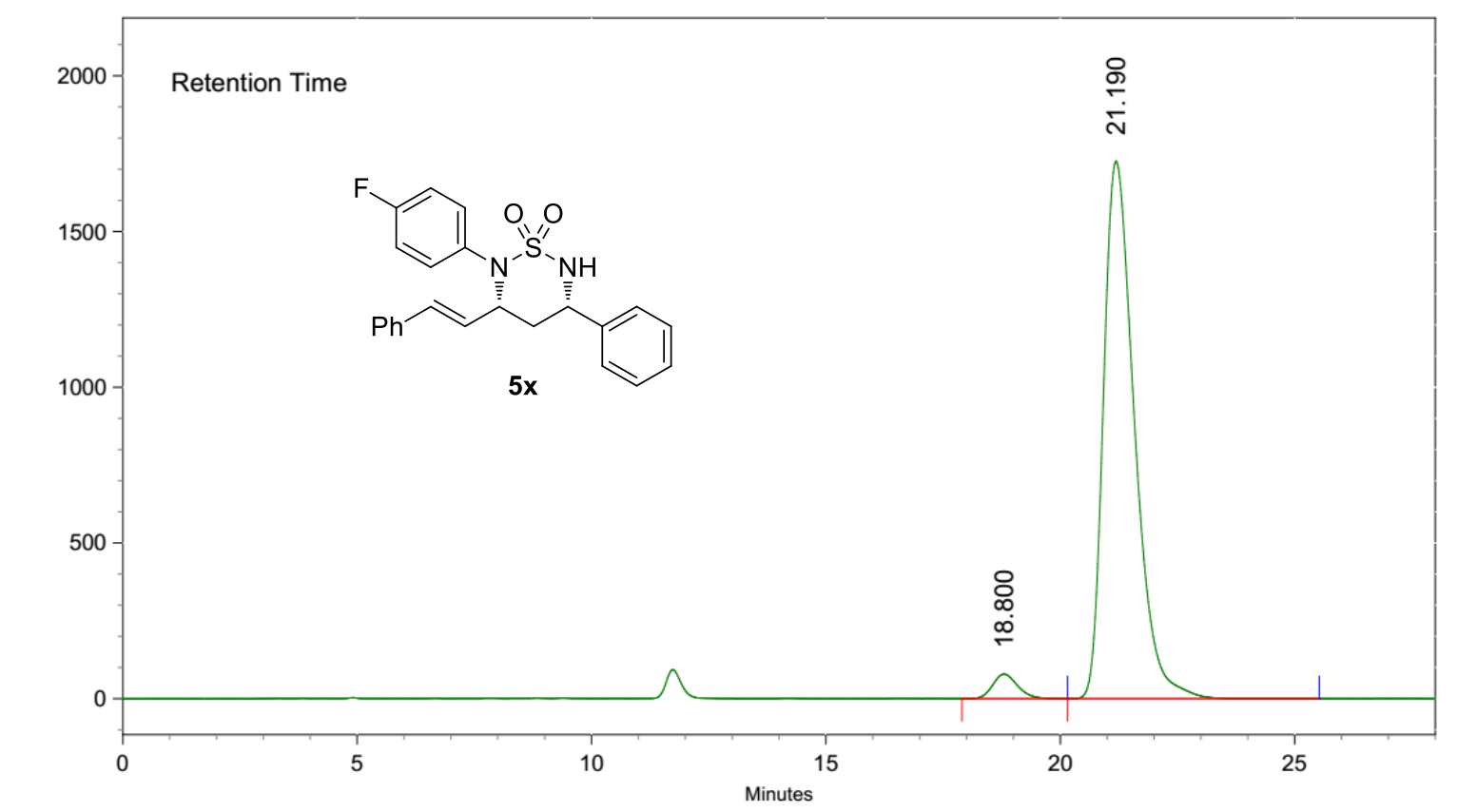

\begin{tabular}{llllll} 
Peak No. & Ret Time & Width & Height & Area & Area [\%] \\
\hline 1 & 18.800 & 2.253 & 1319652 & 48785022 & 3.5500 \\
2 & 21.190 & 5.370 & 28952921 & 1325448014 & 96.4500
\end{tabular}




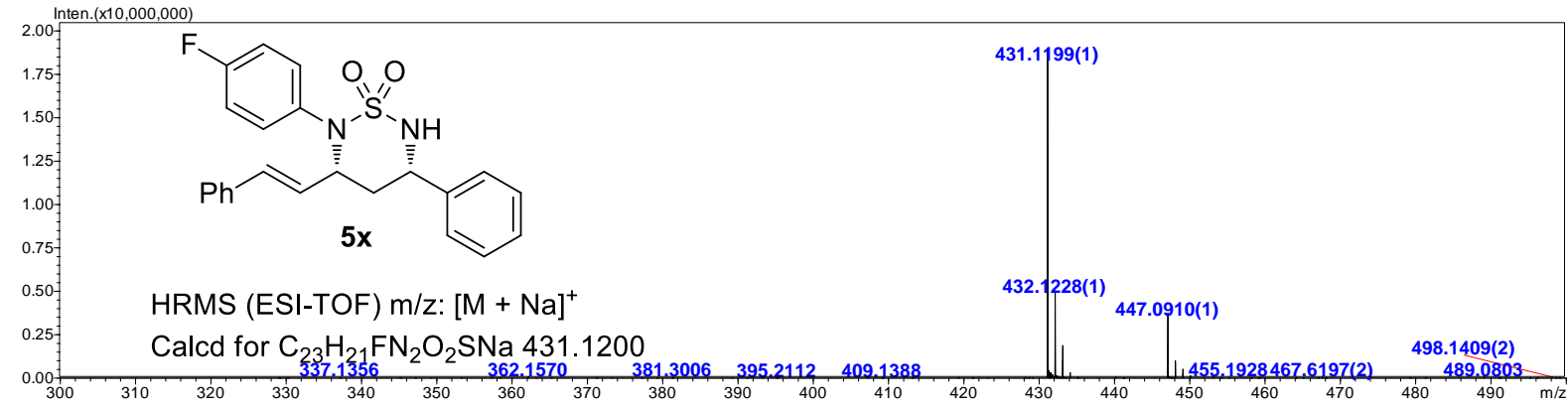


<smiles>[NH3+]NC1C[C@H](/C=C/c2ccccc2)Oc2ccccc21</smiles>

${ }^{1} \mathrm{H}$ NMR (400M, $\mathrm{CDCl}_{3}$ )
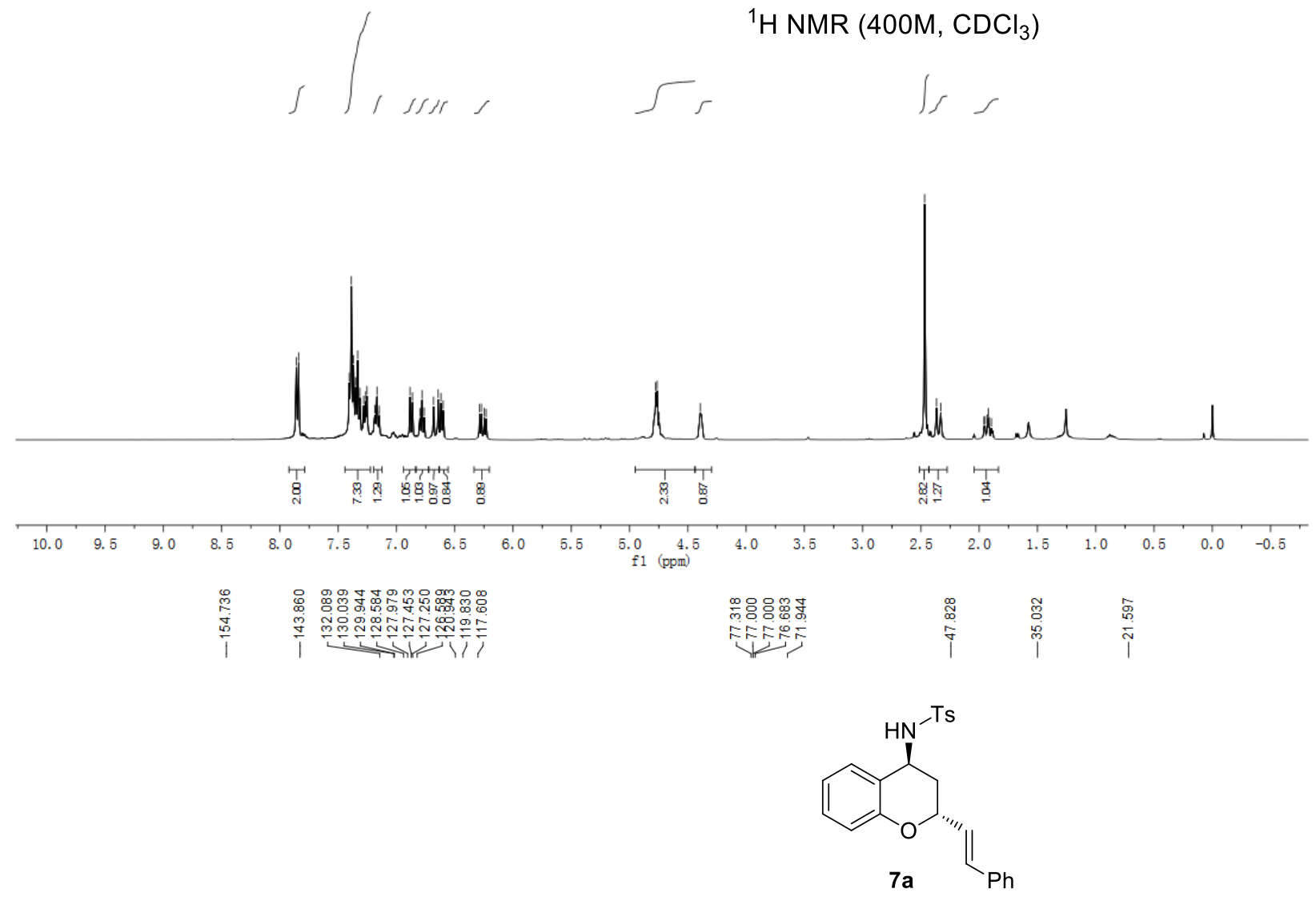

${ }^{13} \mathrm{C}$ NMR (100M, $\mathrm{CDCl}_{3}$ )

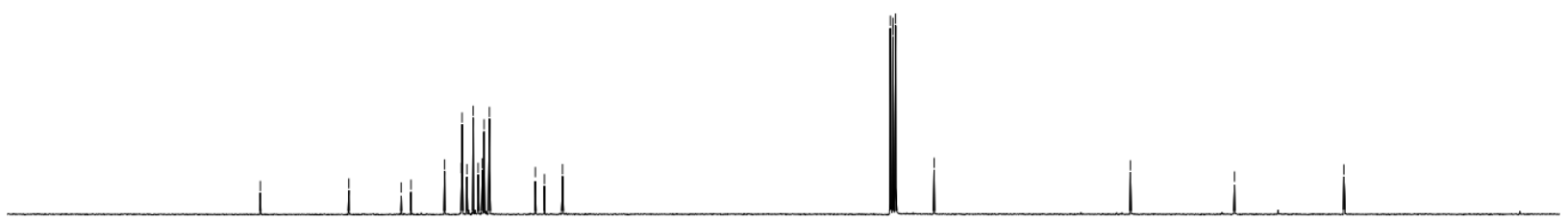

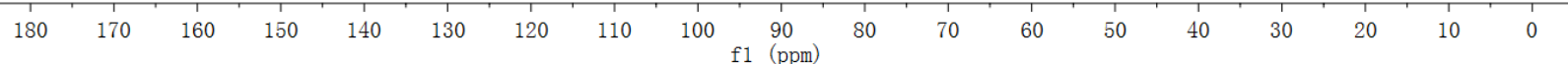



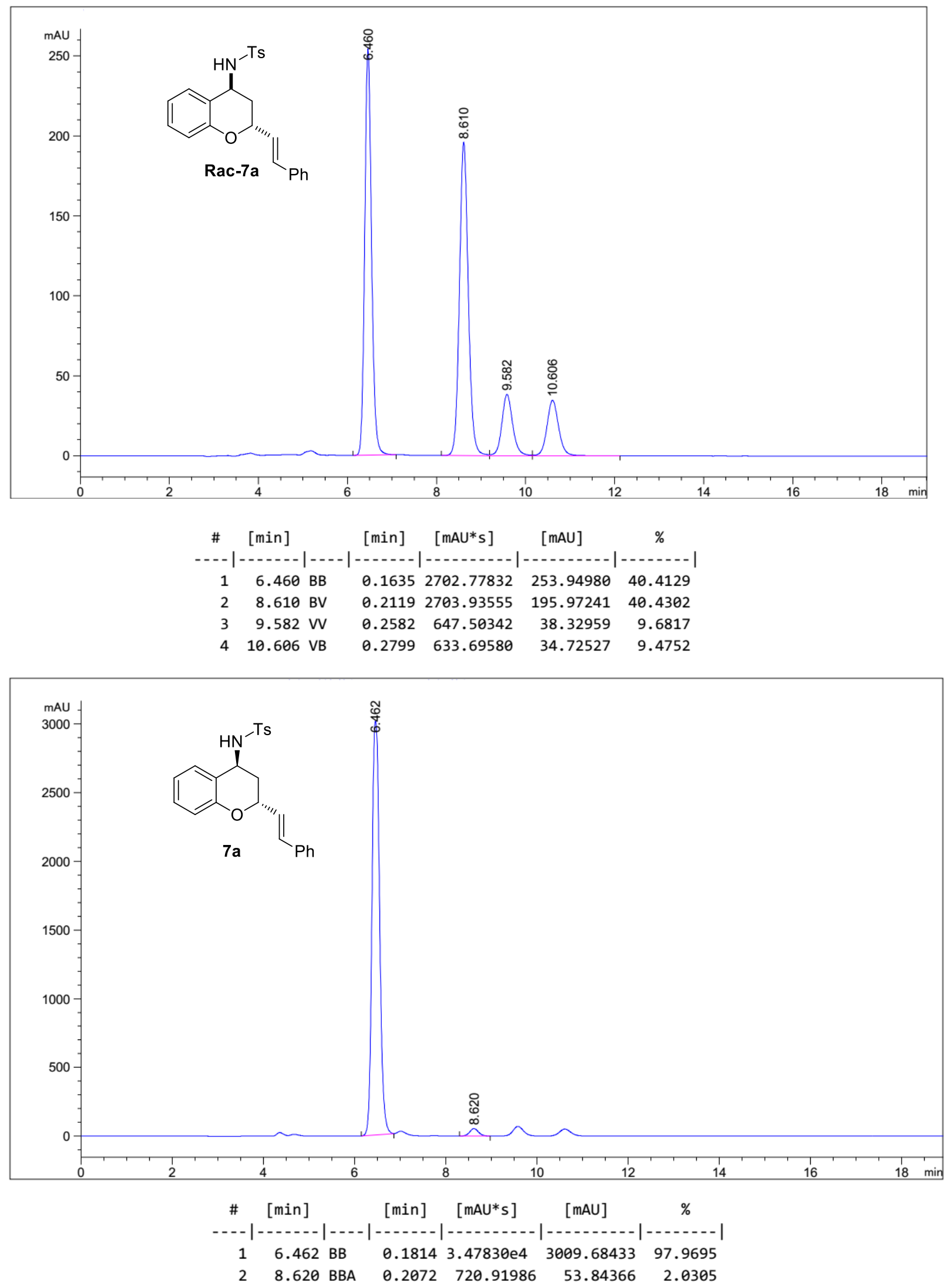


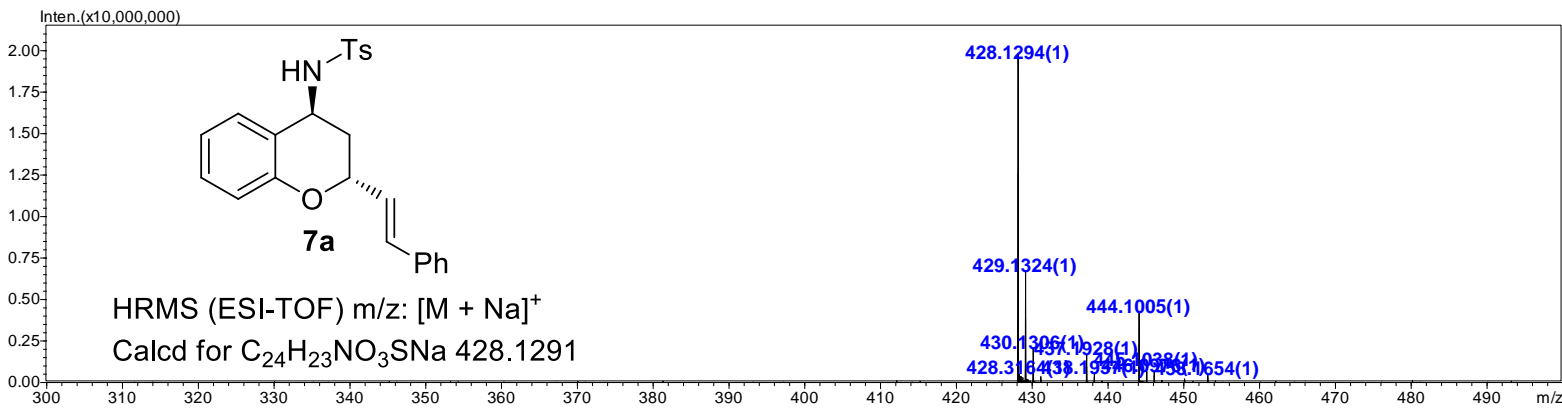




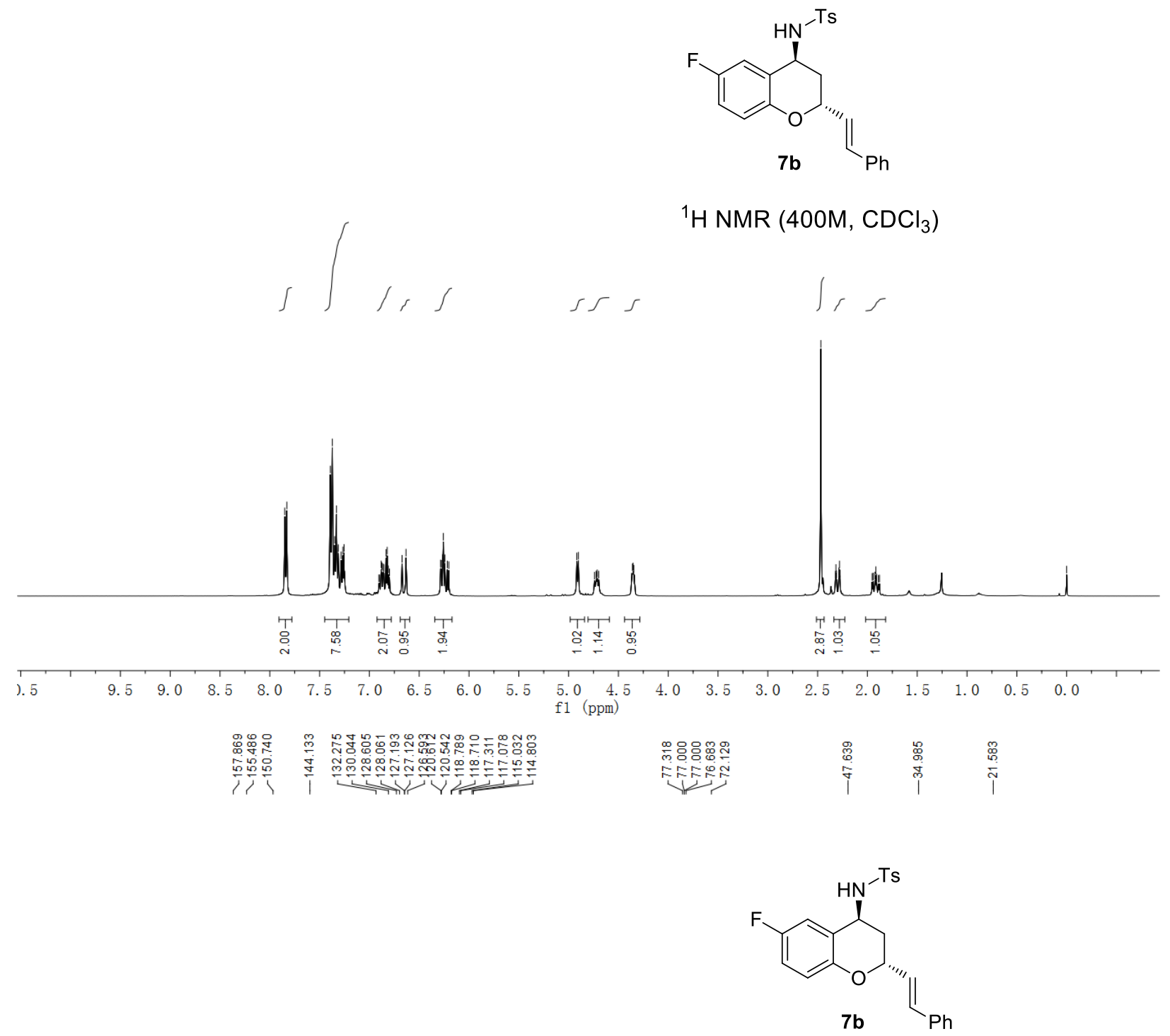

${ }^{13} \mathrm{C} \mathrm{NMR} \mathrm{(100M,} \mathrm{CDCl}_{3}$ )

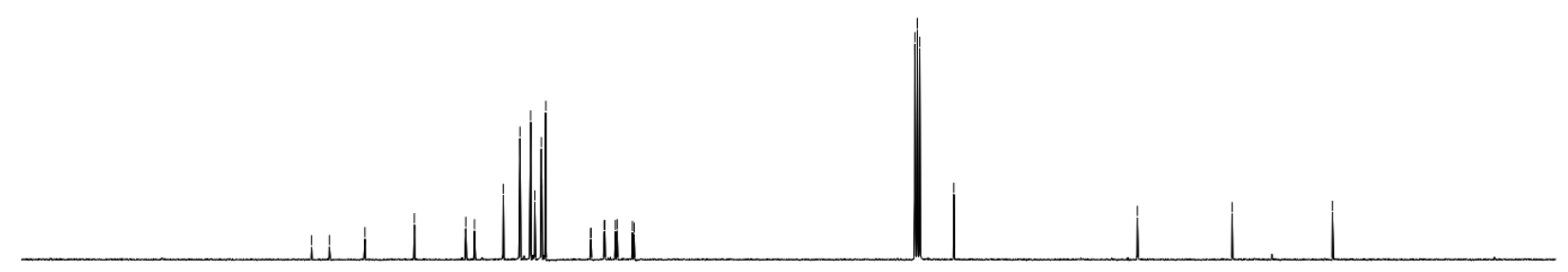

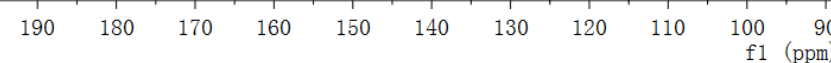




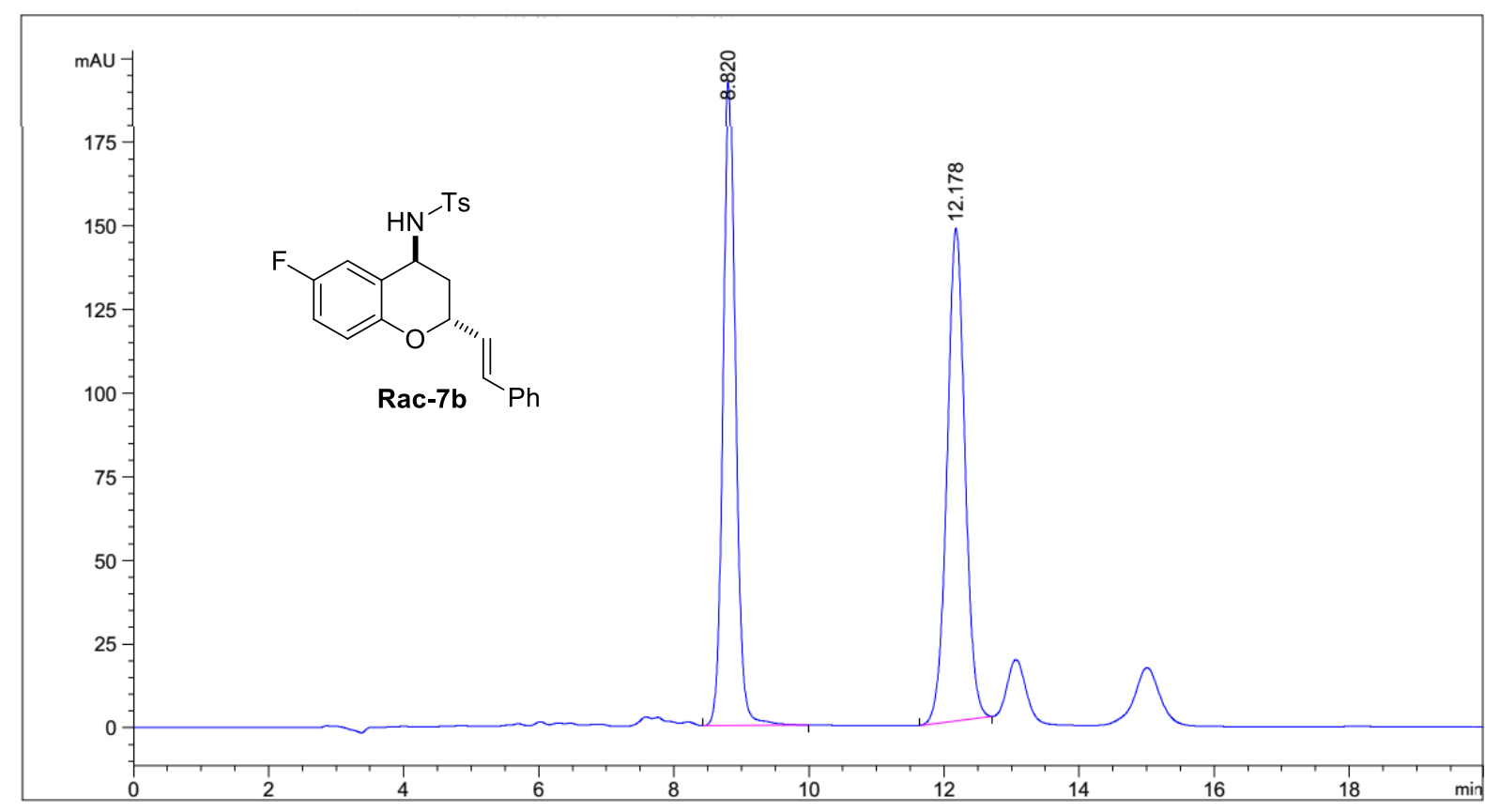

\begin{tabular}{ccccccc}
$\#$ & {$[\mathrm{~min}]$} & {$[\mathrm{min}]$} & {$[\mathrm{mAU} * \mathrm{~s}]$} & {$[\mathrm{mAU}]$} & $\%$ \\
\hline 1 & 8.820 & BB & 0.2010 & 2520.75122 & 192.27597 & 48.1451 \\
2 & 12.178 & BB & 0.2834 & 2714.98779 & 147.38171 & 51.8549
\end{tabular}

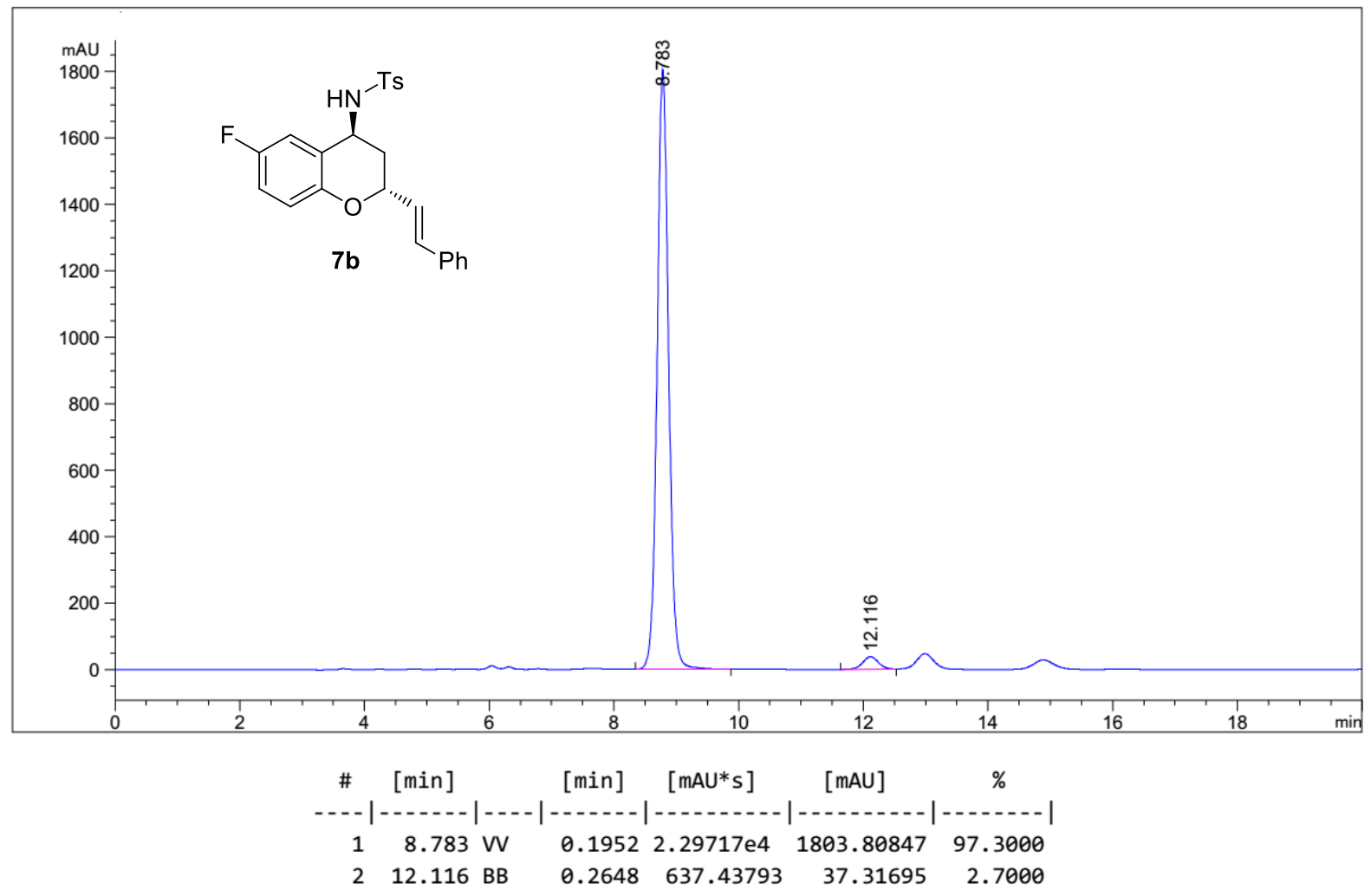




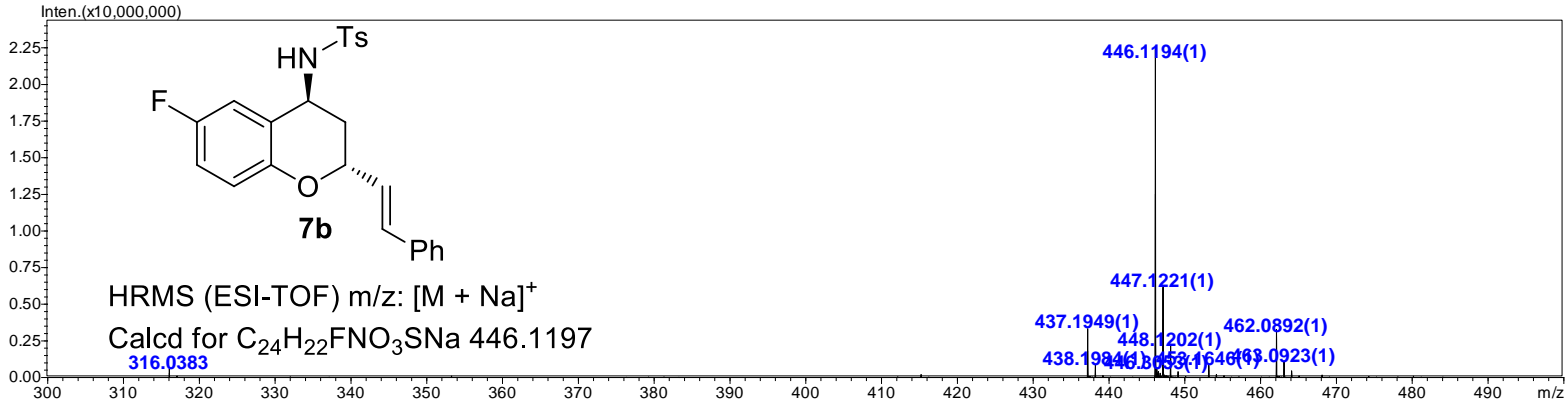



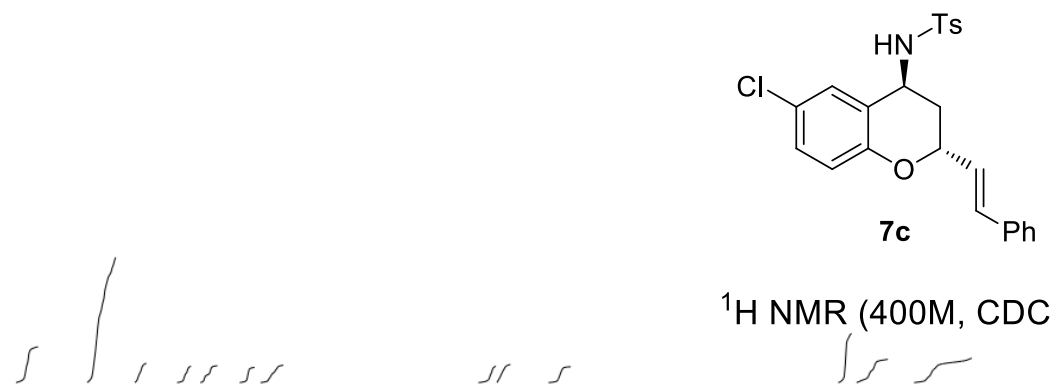

${ }^{1} \mathrm{H}$ NMR $\left(400 \mathrm{M}, \mathrm{CDCl}_{3}\right)$
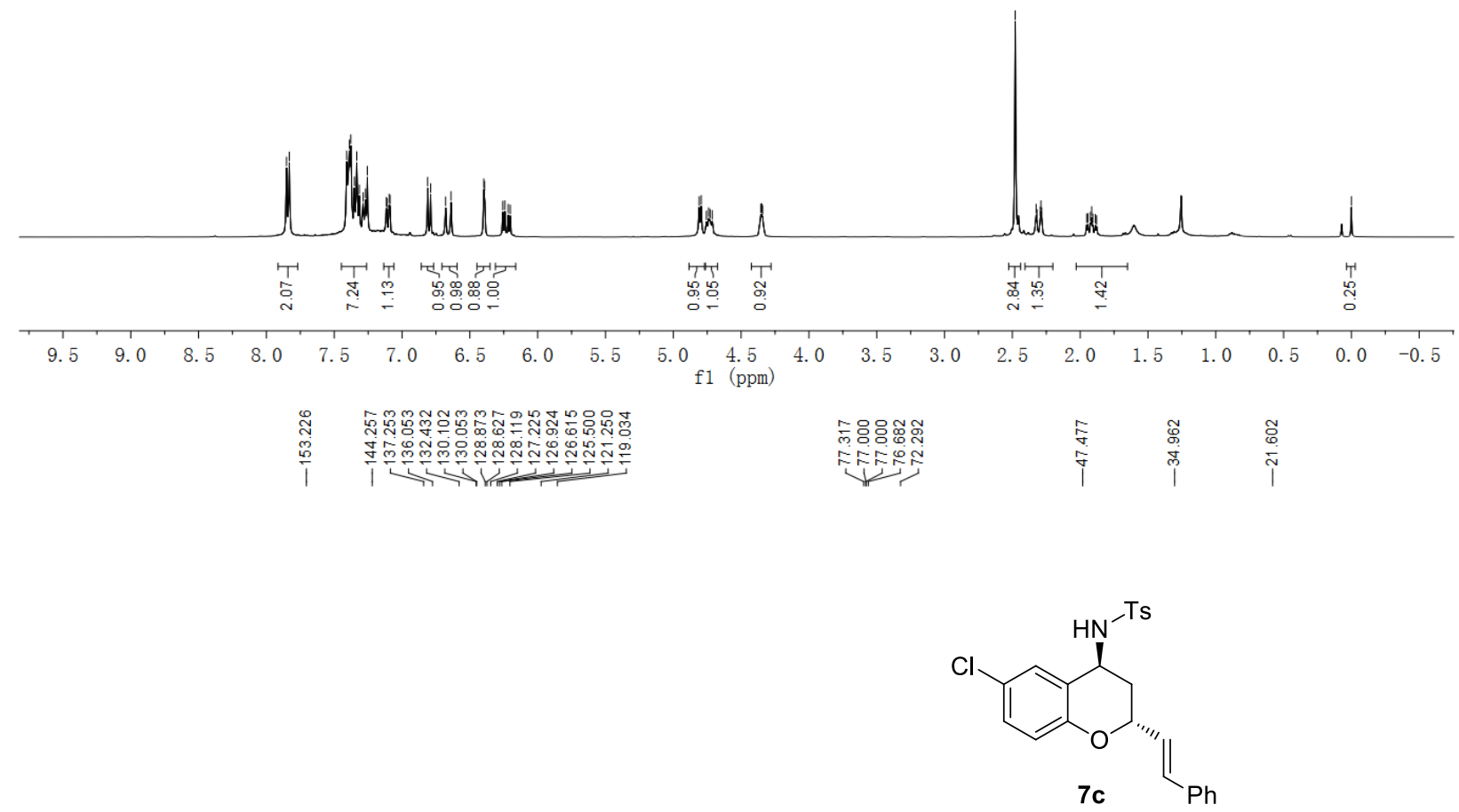

${ }^{13} \mathrm{C}$ NMR $\left.(100 \mathrm{M}, \mathrm{CDCl})_{3}\right)$

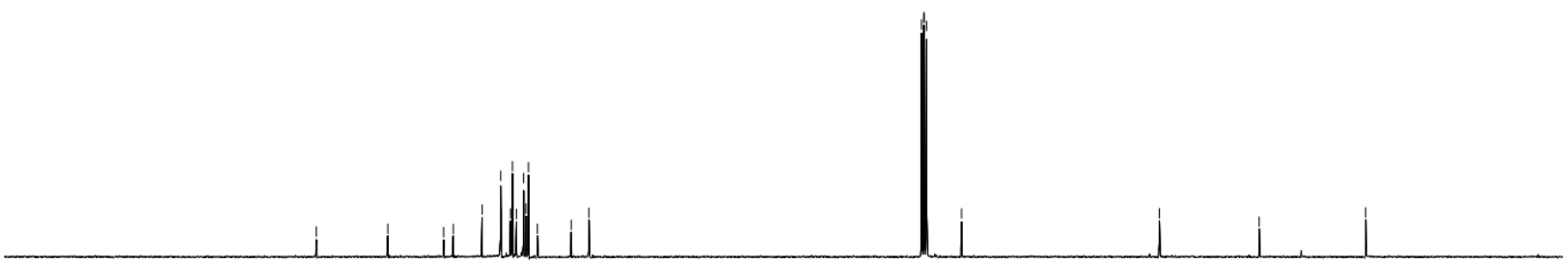

$\begin{array}{rrrrrrrrrr}190 & 180 & 170 & 160 & 150 & 140 & 130 & 120 & 110 & 100 \\ \mathrm{f} 1 & \begin{array}{r}90 \\ (\mathrm{ppm})\end{array}\end{array}$ 

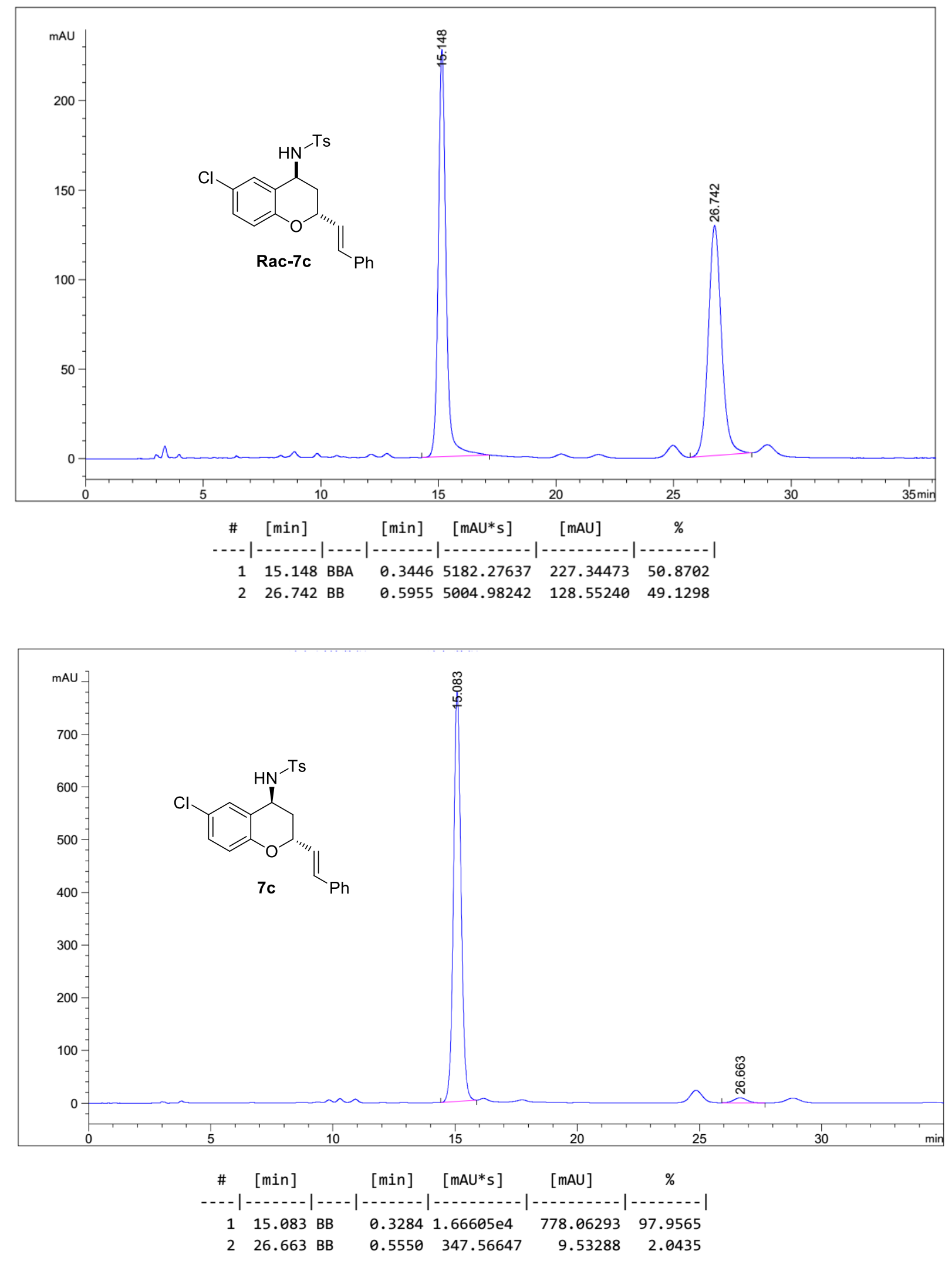

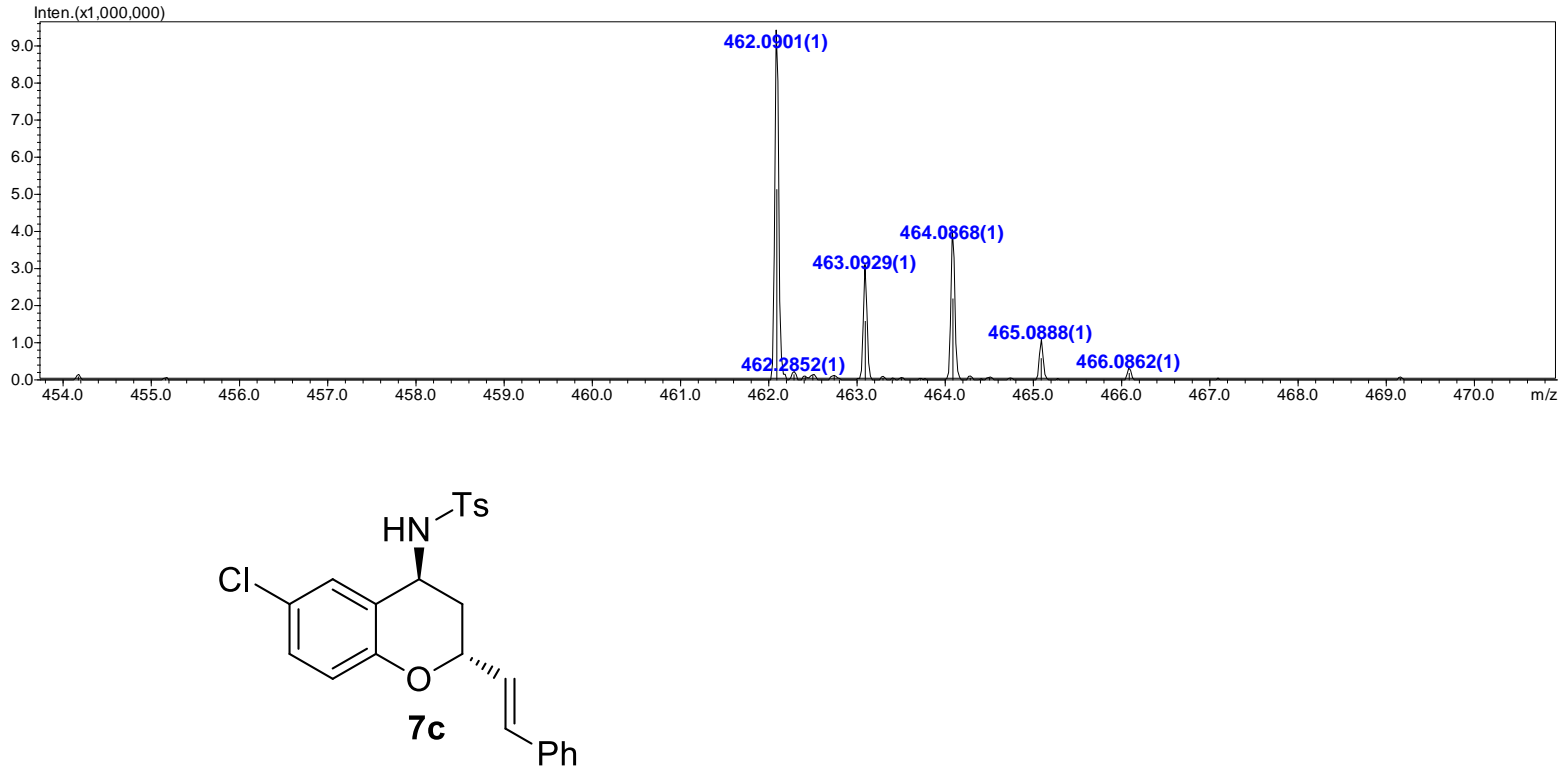

HRMS (ESI-TOF) m/z: [M + Na]

Calcd for $\mathrm{C}_{24} \mathrm{H}_{22} \mathrm{CINO}_{3} \mathrm{SNa} 462.0901\left({ }^{35} \mathrm{Cl}\right), 464.0868\left({ }^{37} \mathrm{Cl}\right)$ 

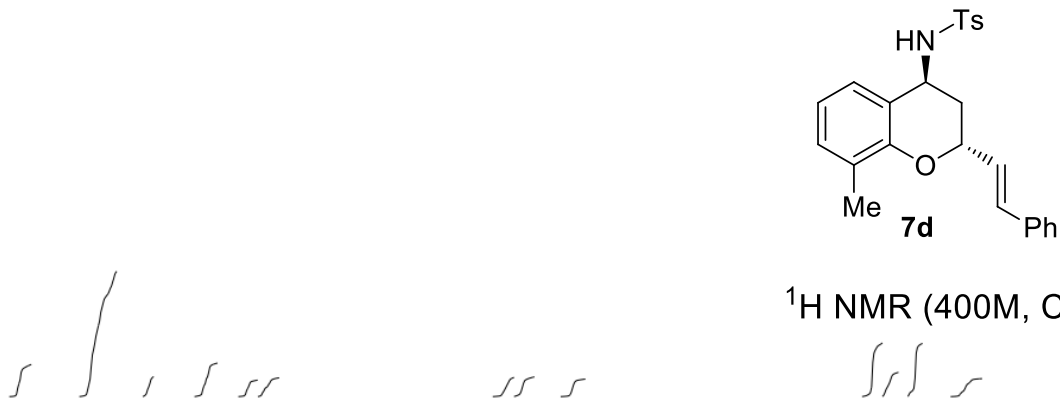

${ }^{1} \mathrm{H}$ NMR (400M, $\left.\mathrm{CDCl}_{3}\right)$
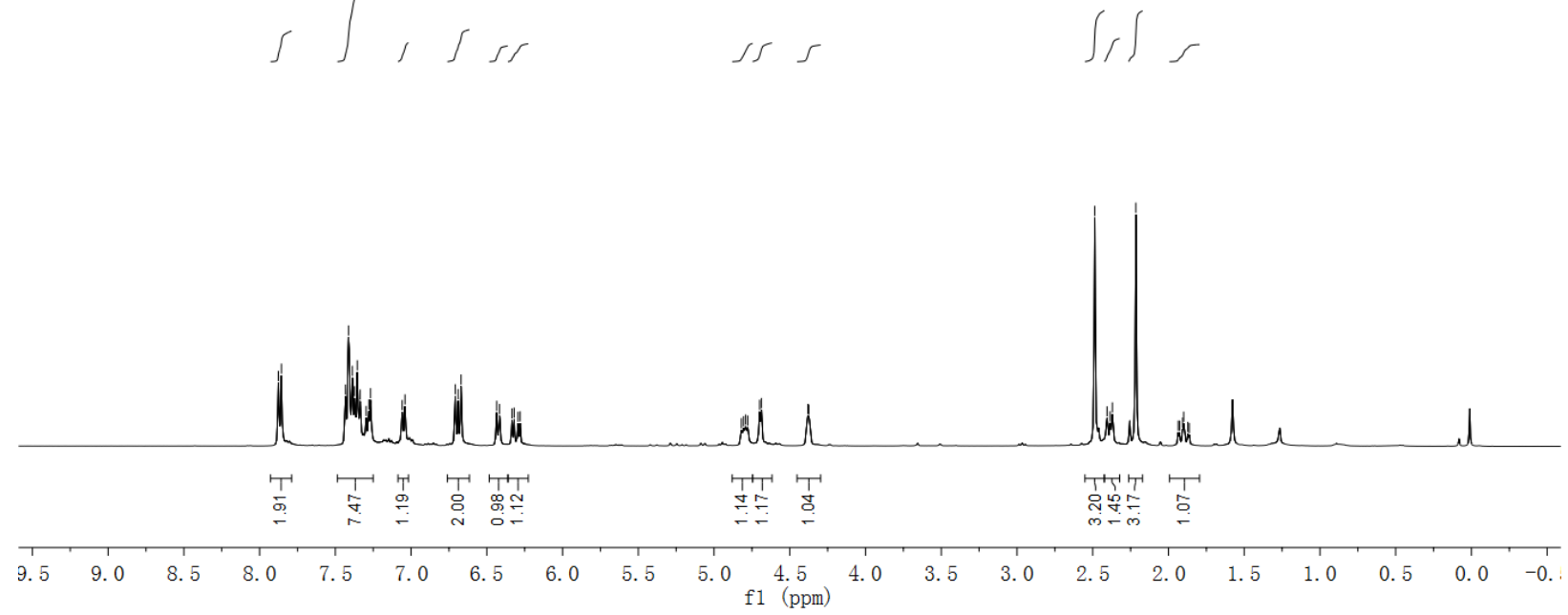

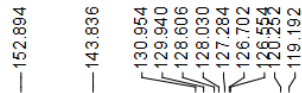

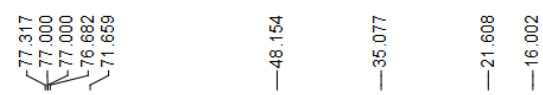

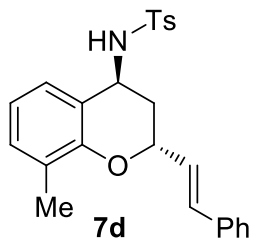

${ }^{13} \mathrm{C}$ NMR $\left(100 \mathrm{M}, \mathrm{CDCl}_{3}\right)$

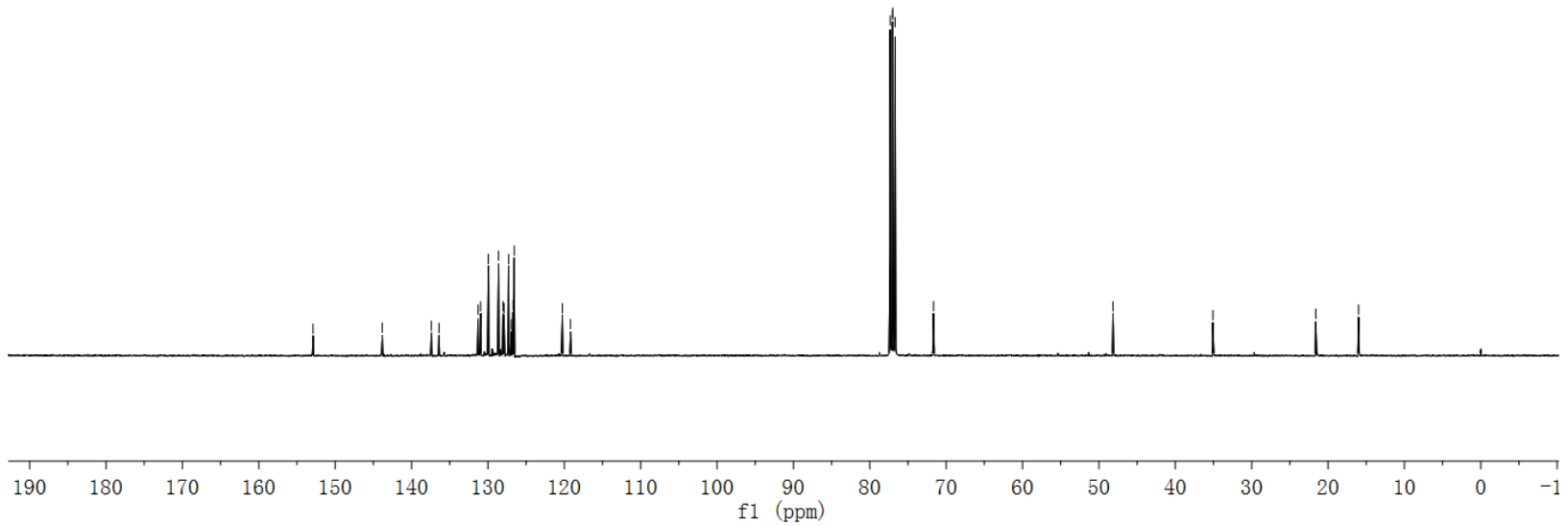



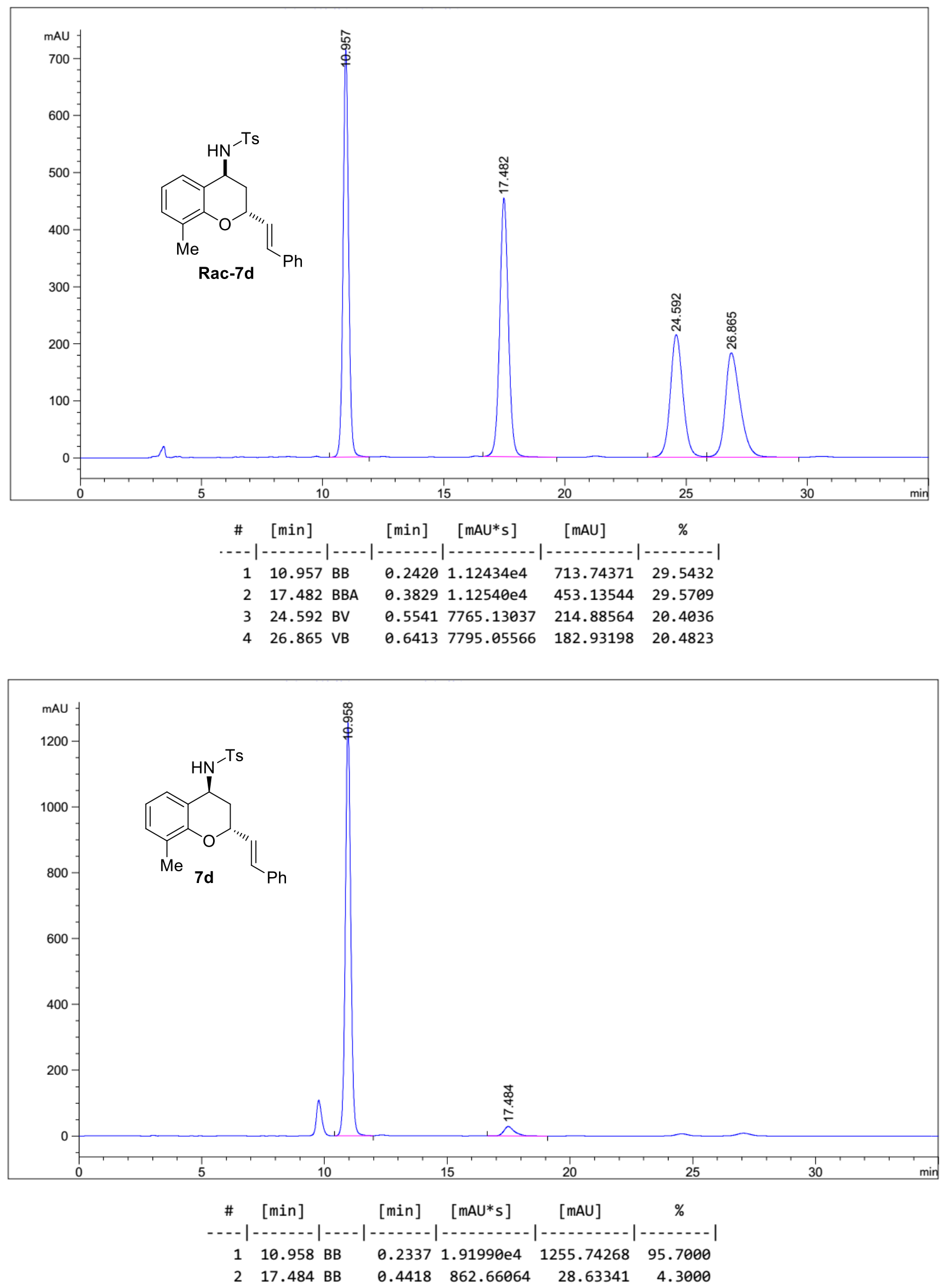


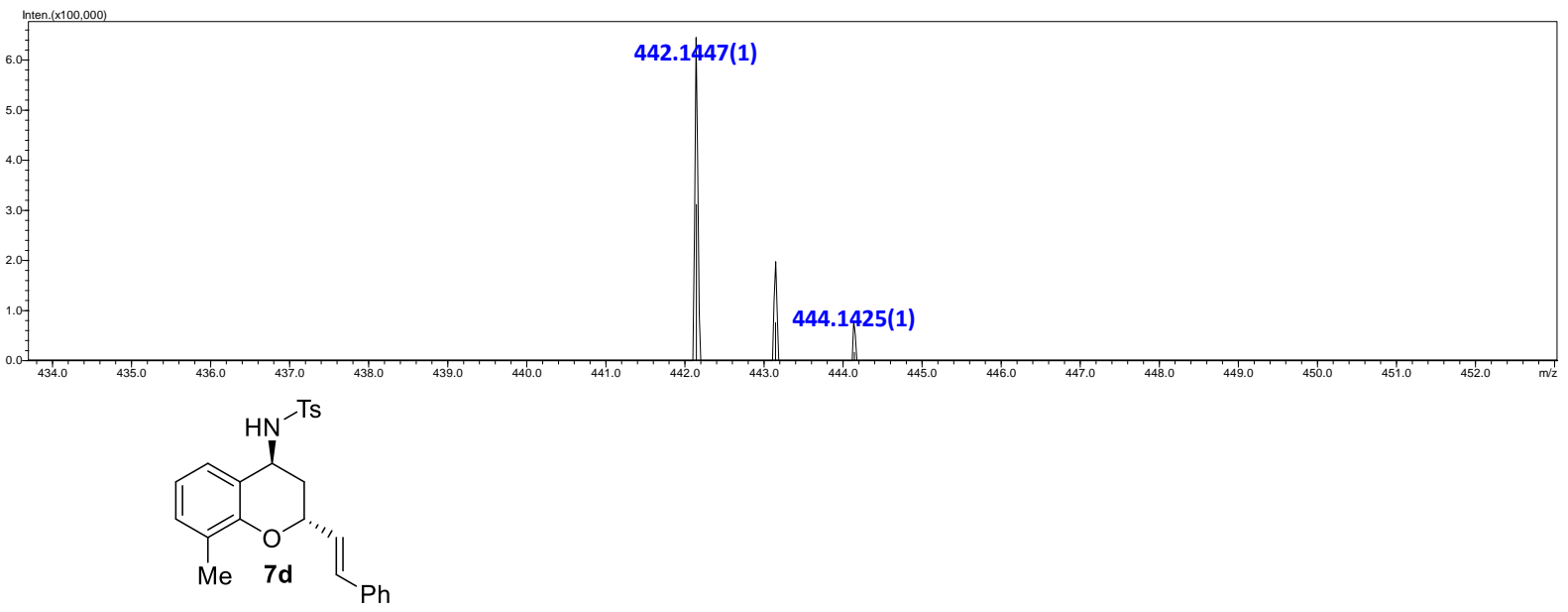

HRMS (ESI-TOF) m/z: [M + Na] ${ }^{+}$

Calcd for $\mathrm{C}_{25} \mathrm{H}_{25} \mathrm{NO}_{3} \mathrm{SNa} 442.1447$ 


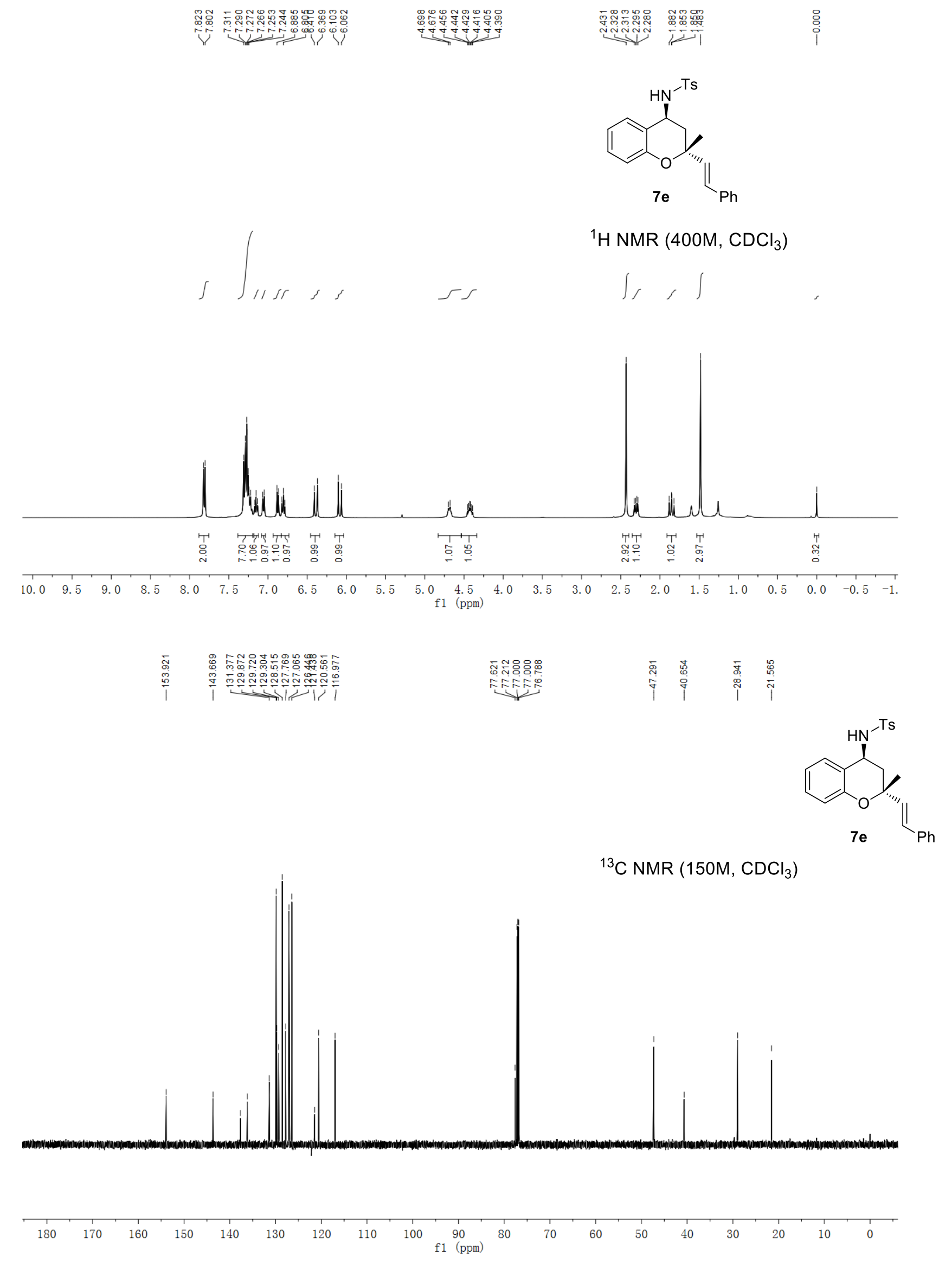



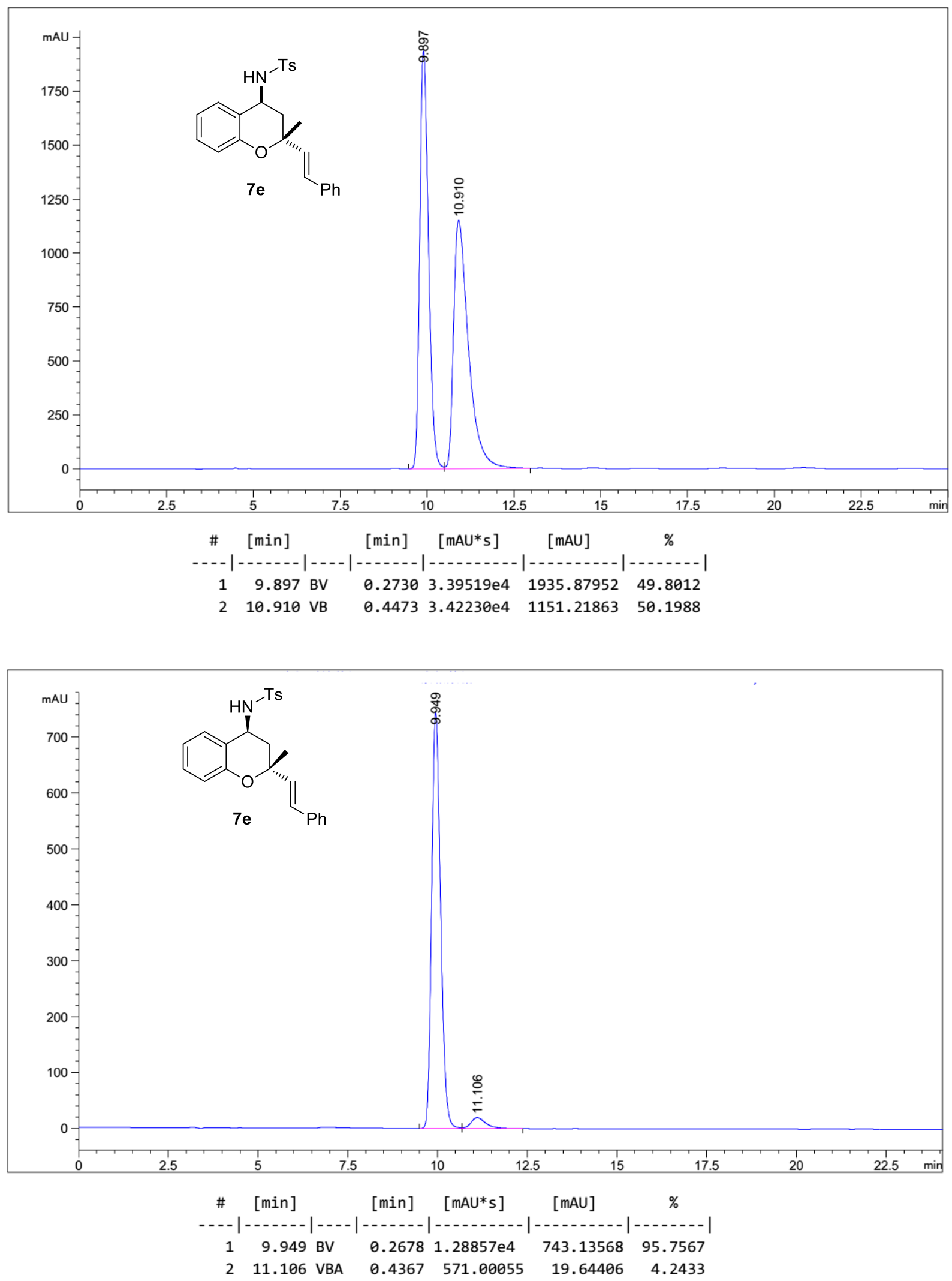


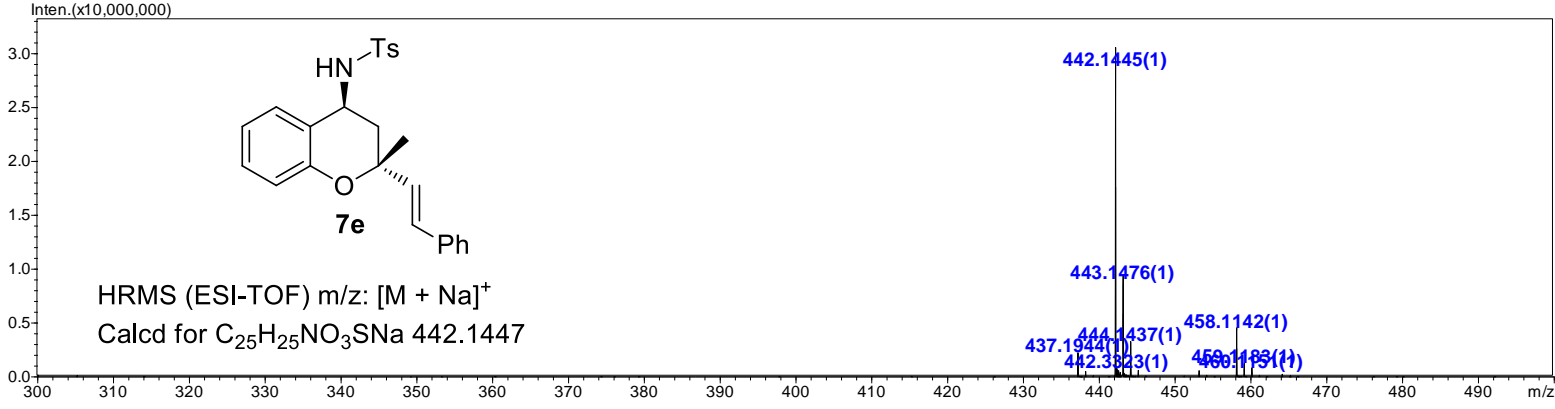




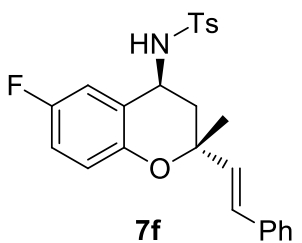

${ }^{1} \mathrm{H}$ NMR (400M, $\left.\mathrm{CDCl}_{3}\right)$

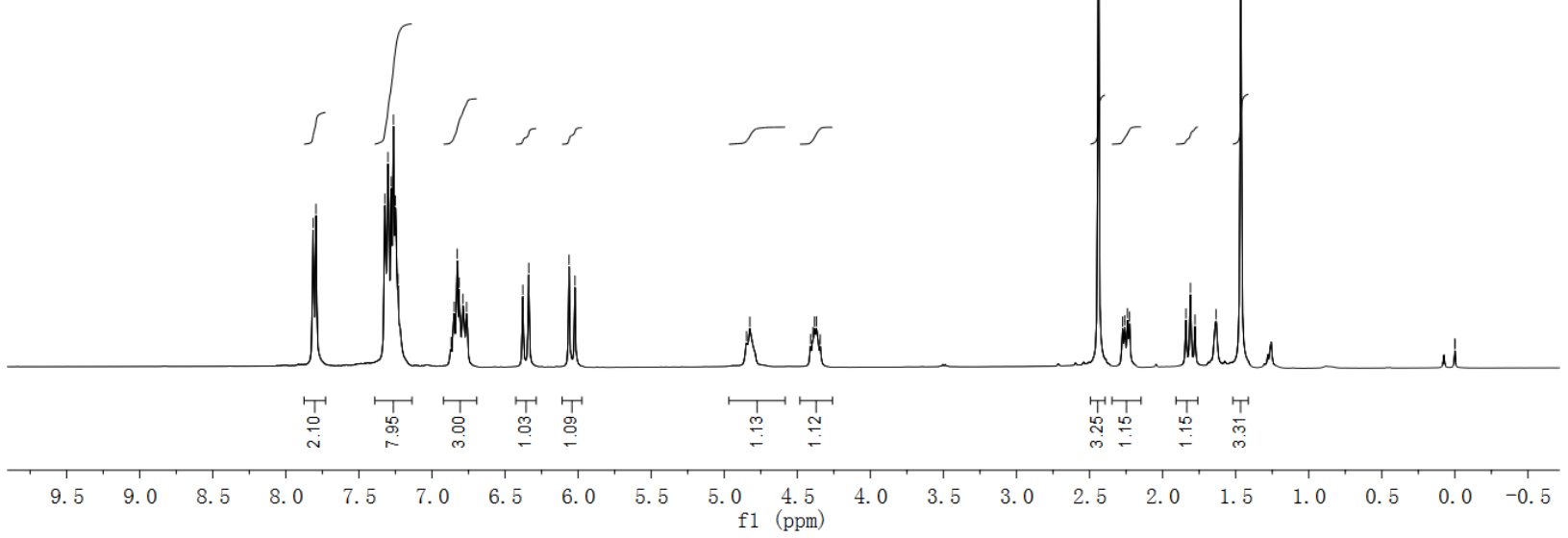

ᄃํ.

L

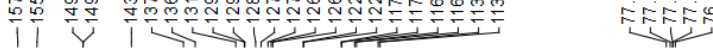

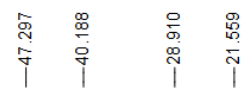

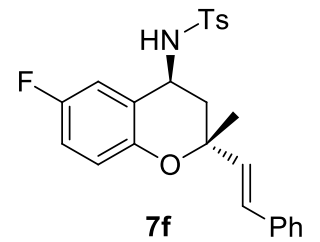

${ }^{13} \mathrm{C}$ NMR $\left(100 \mathrm{M}, \mathrm{CDCl}_{3}\right)$

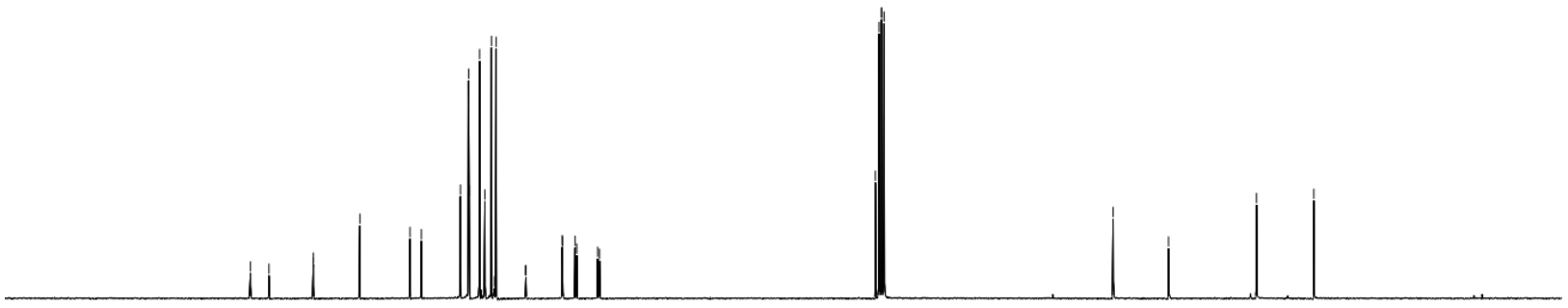

$\begin{array}{llllllllllllllllllllll}180 & 170 & 160 & 150 & 140 & 130 & 120 & 110 & 100 & 90 & 80 & 70 & 60 & 50 & 40 & 30 & 20 & 10 & 0 & -1\end{array}$ f1 (ppm) 

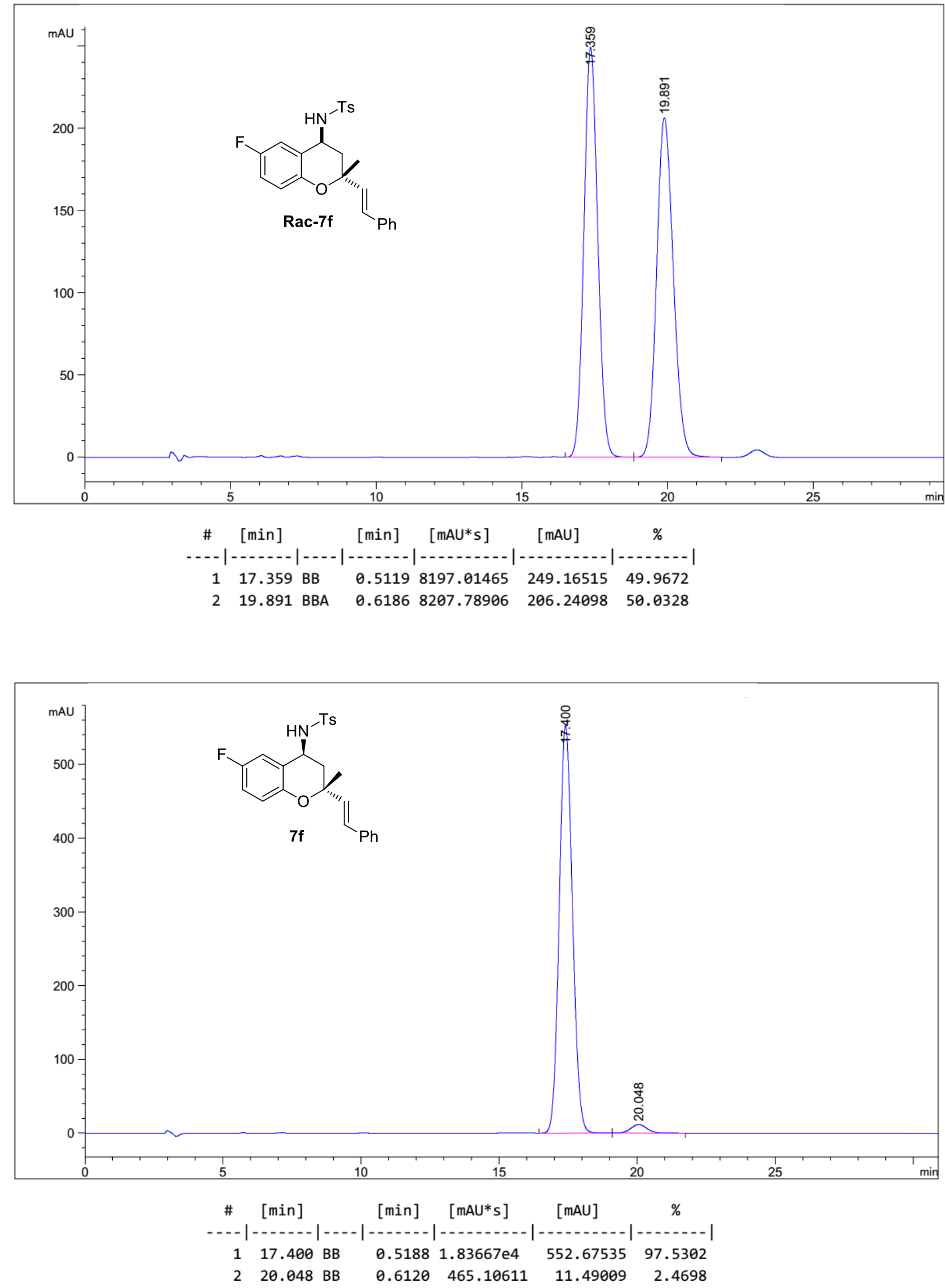

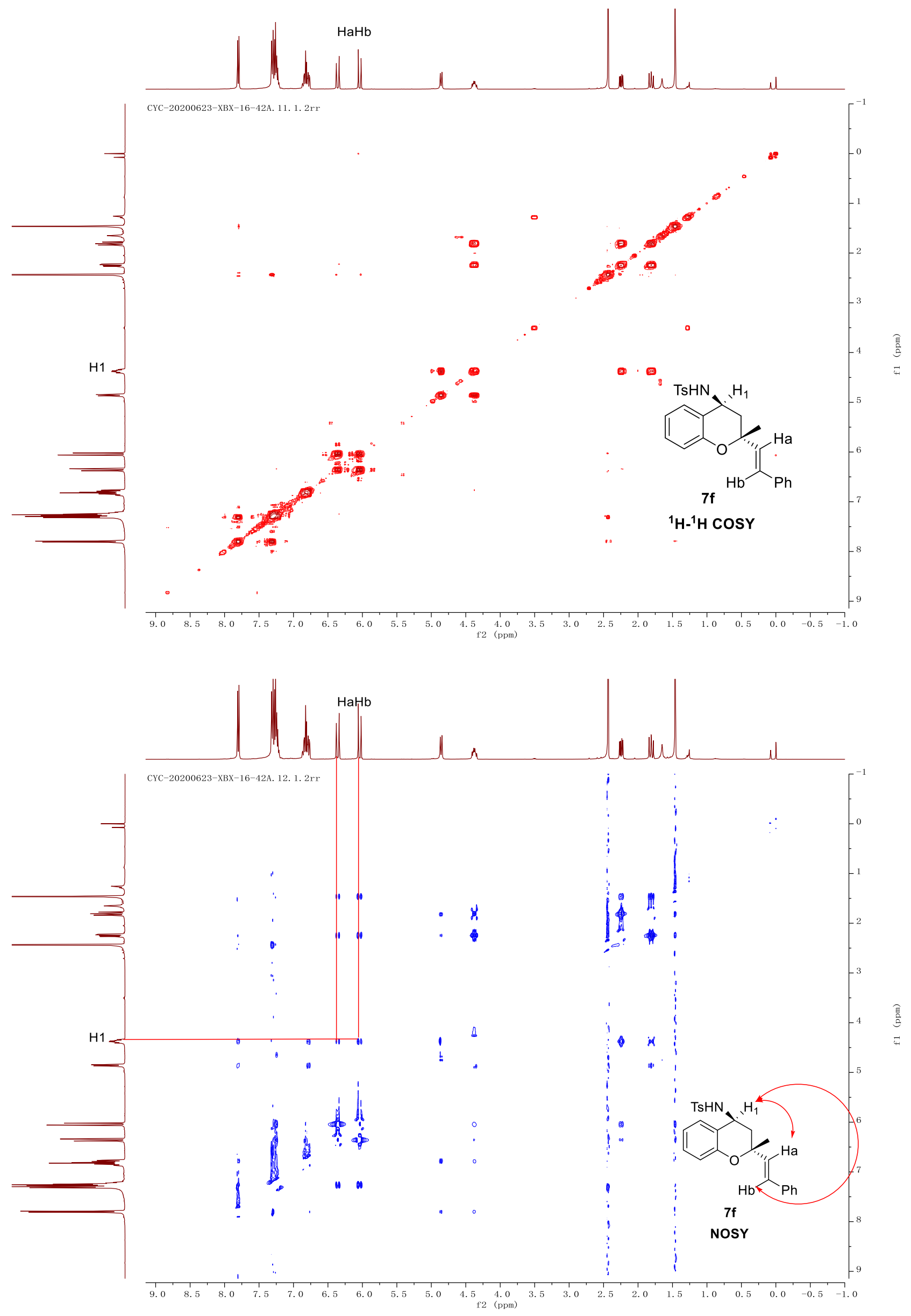


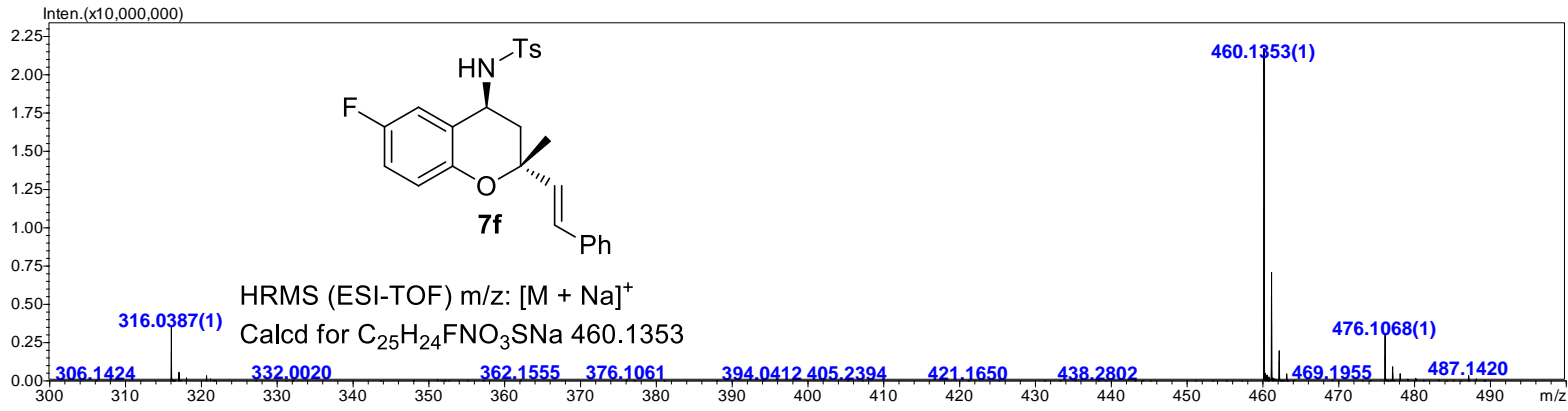




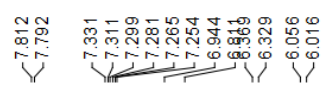

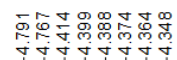

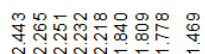

웅<smiles>CC1(C=Cc2ccccc2)CC([NH3+])c2cc(Cl)ccc2O1</smiles>

${ }^{1} \mathrm{H}$ NMR (400M, $\mathrm{CDCl}_{3}$ )
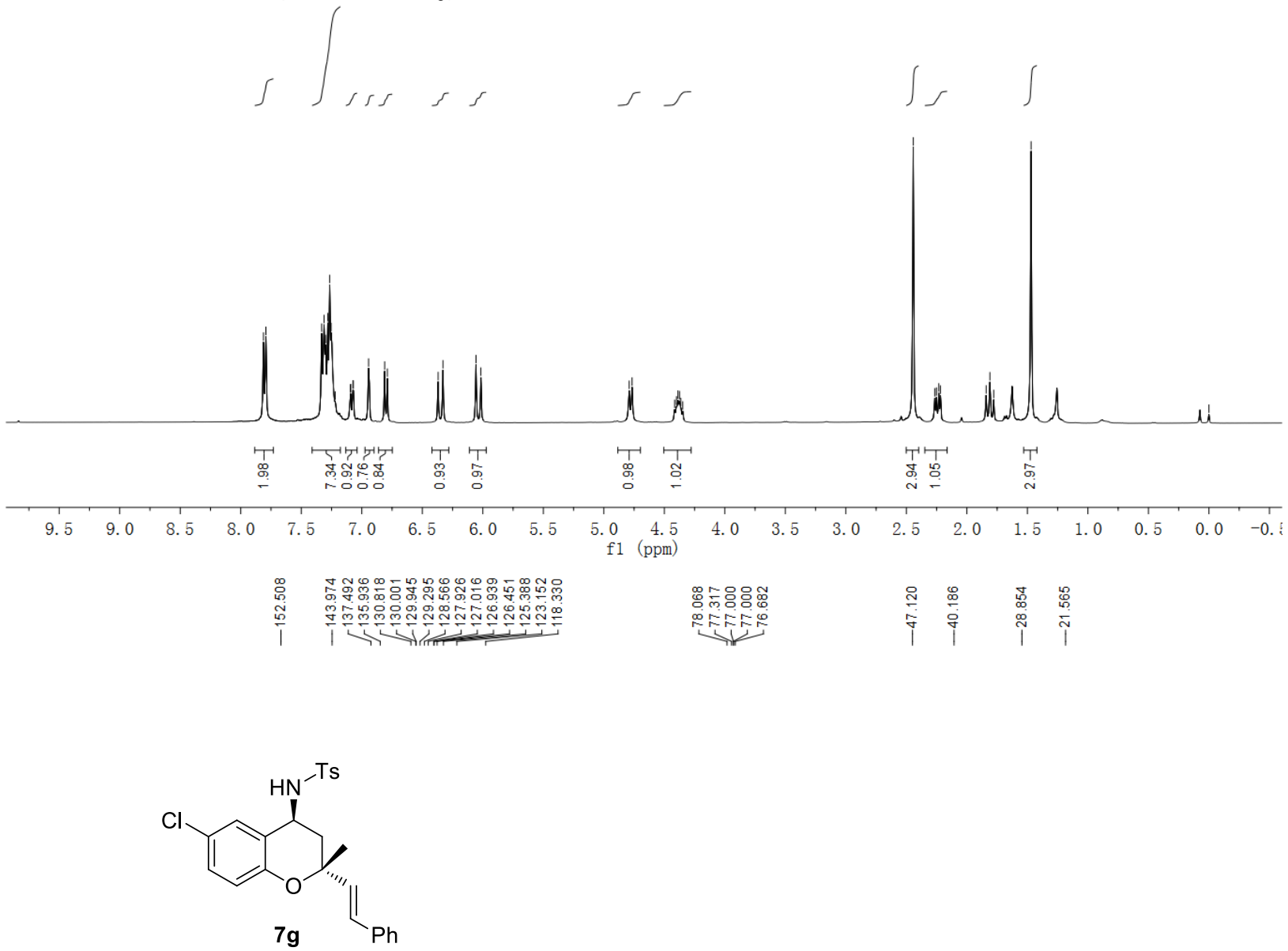

${ }^{13} \mathrm{C}$ NMR (100M, $\left.\mathrm{CDCl}_{3}\right)$

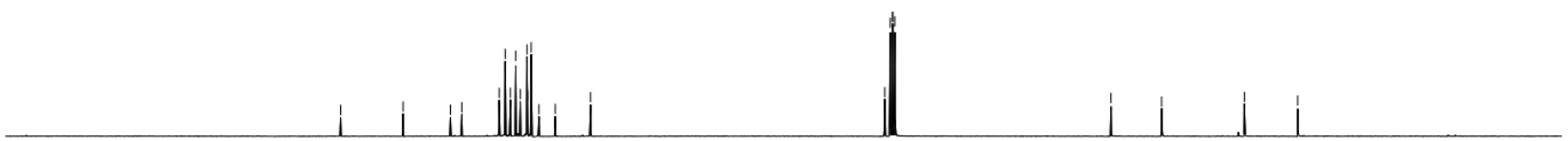

$\begin{array}{rrrrrrrrrr}190 & 180 & 170 & 160 & 150 & 140 & 130 & 120 & 110 & 100 \\ \mathrm{f} 1 & \begin{array}{c}90 \\ (\mathrm{ppm})\end{array}\end{array}$ 

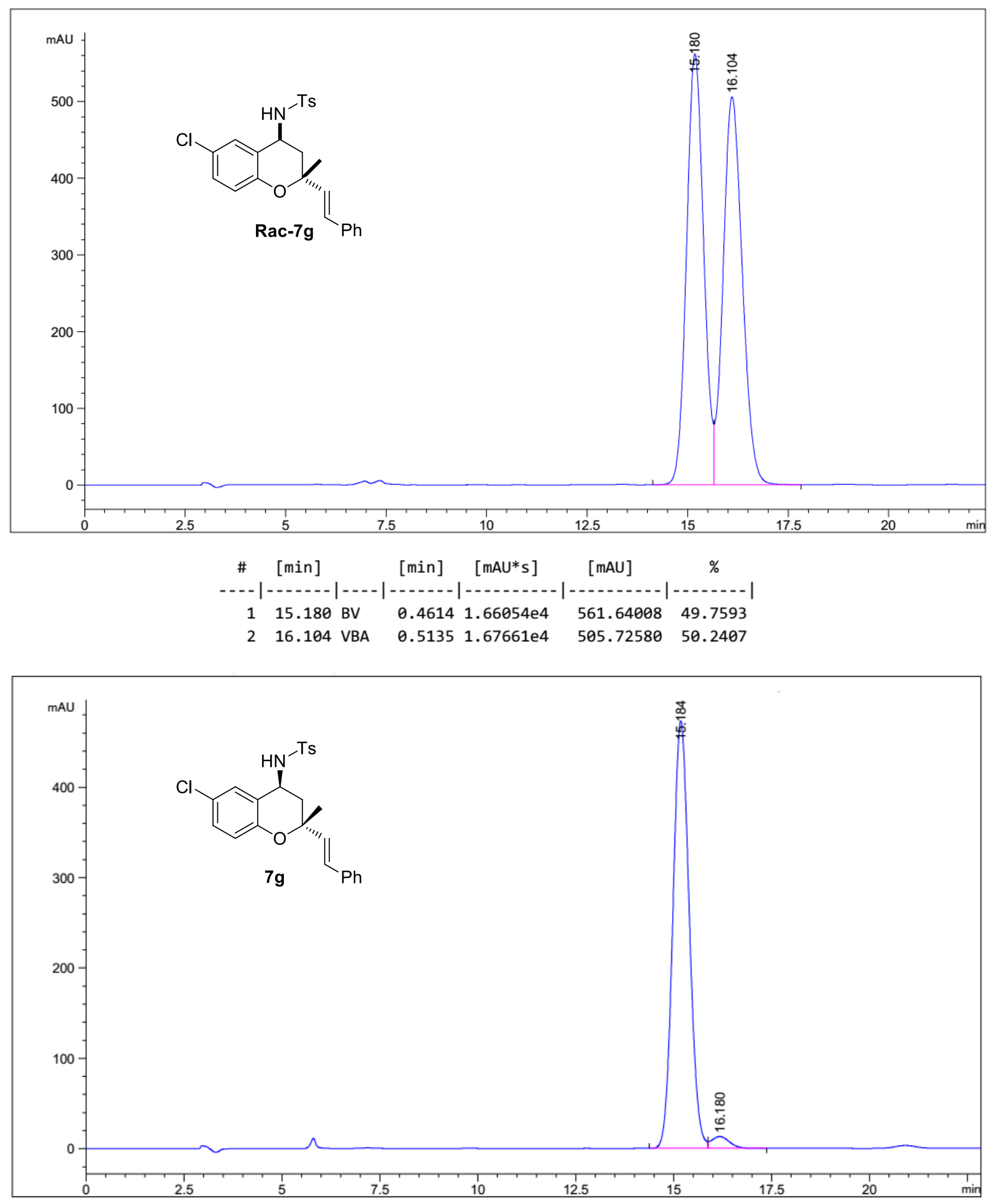

\begin{tabular}{cccccc}
$\#$ & {$[\mathrm{~min}]$} & {$[\mathrm{min}]$} & {$[\mathrm{mAU} * \mathrm{~s}]$} & {$[\mathrm{mAU}]$} & $\%$ \\
\hline 1 & 15.184 BV & 0.4533 & $1.37091 \mathrm{e} 4$ & 472.84387 & 97.0734 \\
2 & 16.180 VB & 0.4865 & 413.29977 & 13.08200 & 2.9266
\end{tabular} 


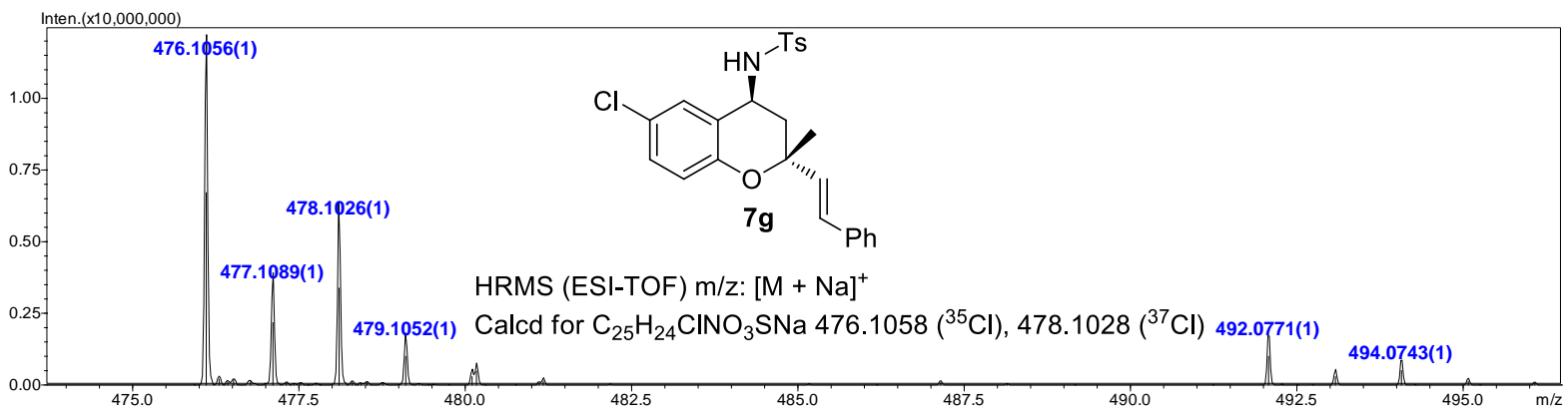




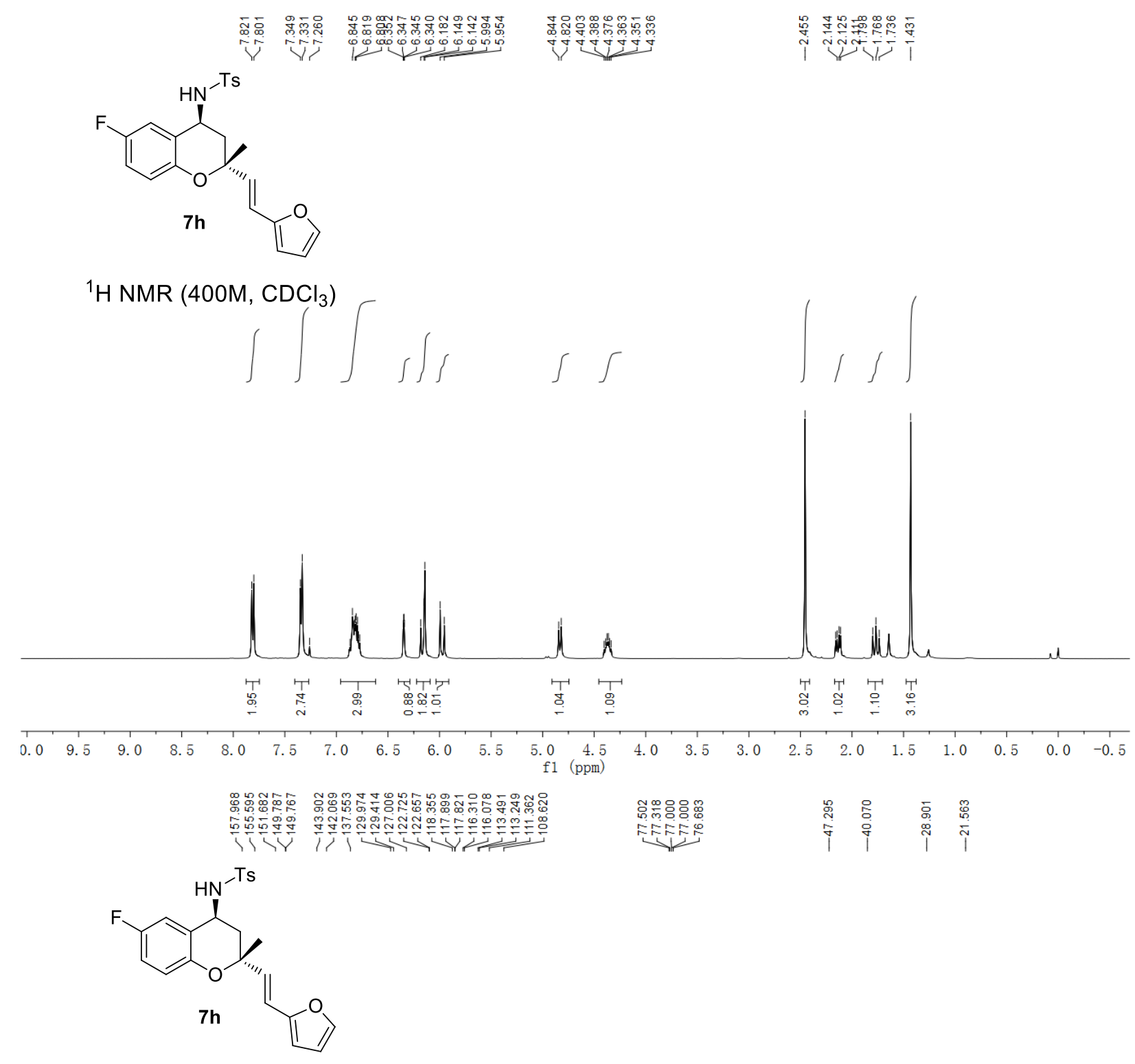

${ }^{13} \mathrm{C}$ NMR $\left(100 \mathrm{M}, \mathrm{CDCl}_{3}\right)$

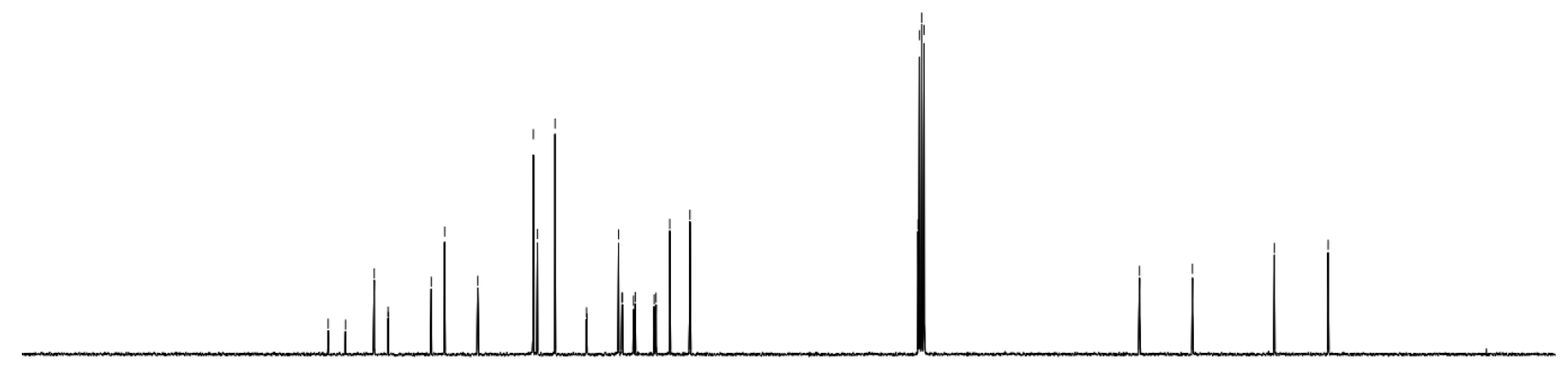

$\begin{array}{lllllllllll}190 & 180 & 170 & 160 & 150 & 140 & 130 & 120 & 110 & \begin{array}{c}100 \\ \mathrm{f} 1\end{array}(\mathrm{ppm})\end{array}$ 

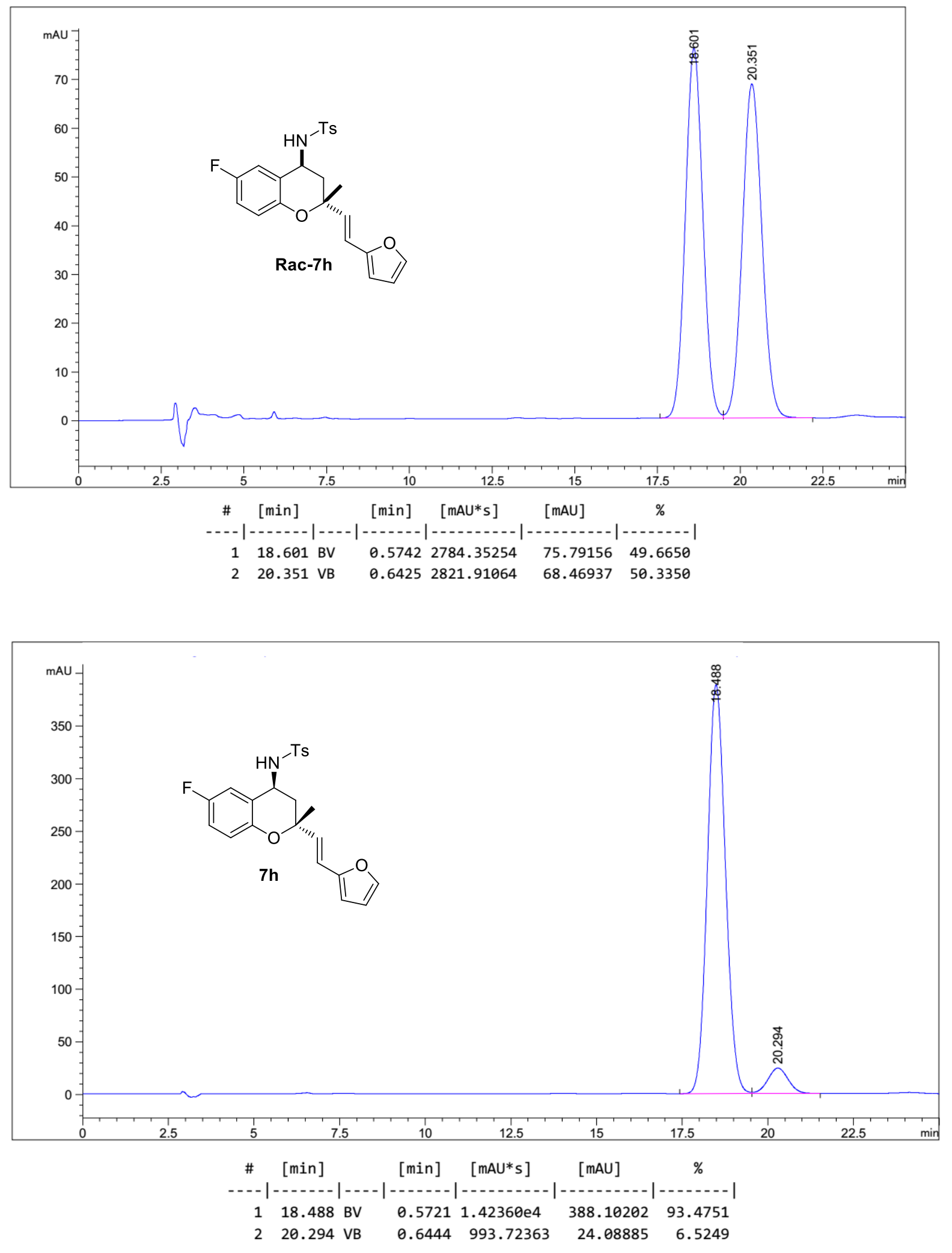


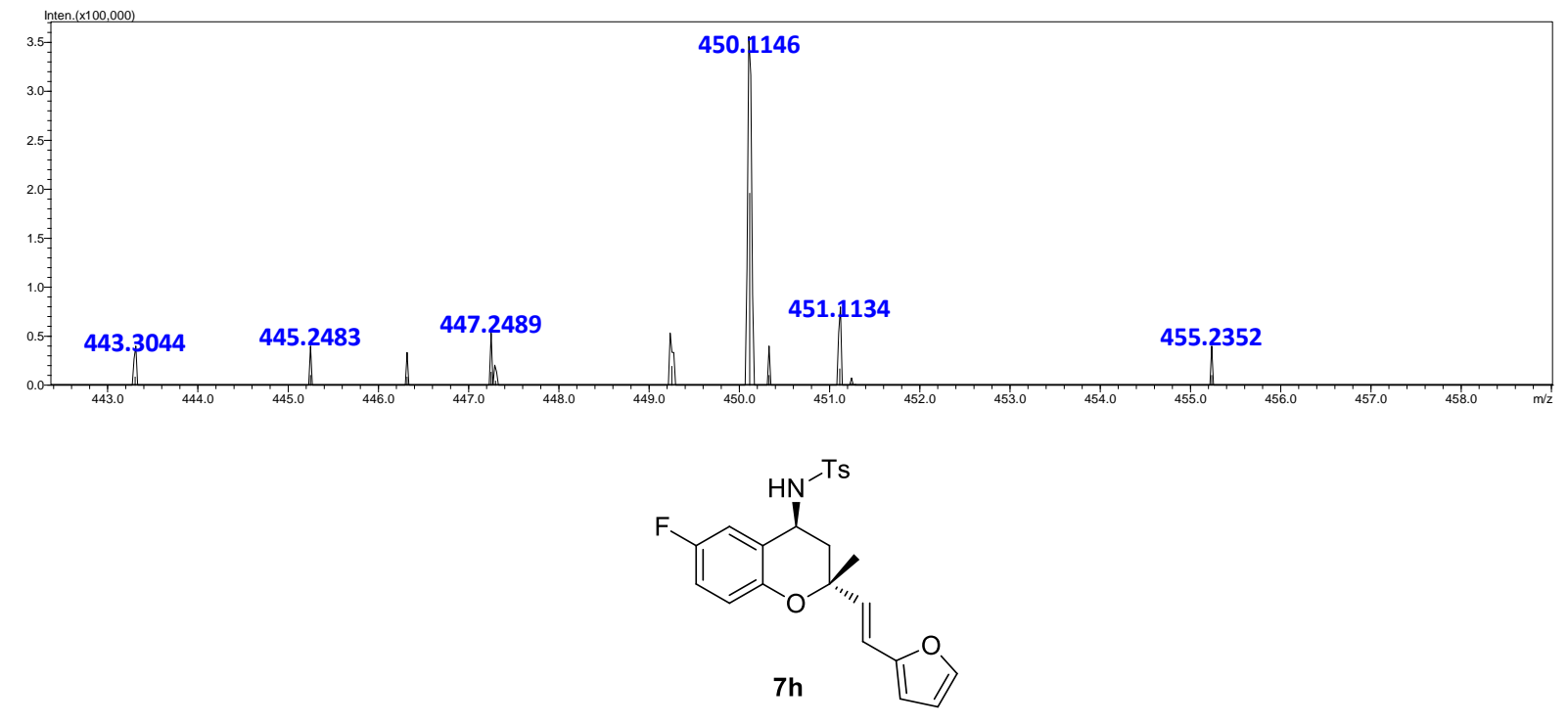

HRMS (ESI-TOF) m/z: [M + Na]

Calcd for $\mathrm{C}_{23} \mathrm{H}_{22} \mathrm{FNO}_{4} \mathrm{SNa} 450.1146$ 


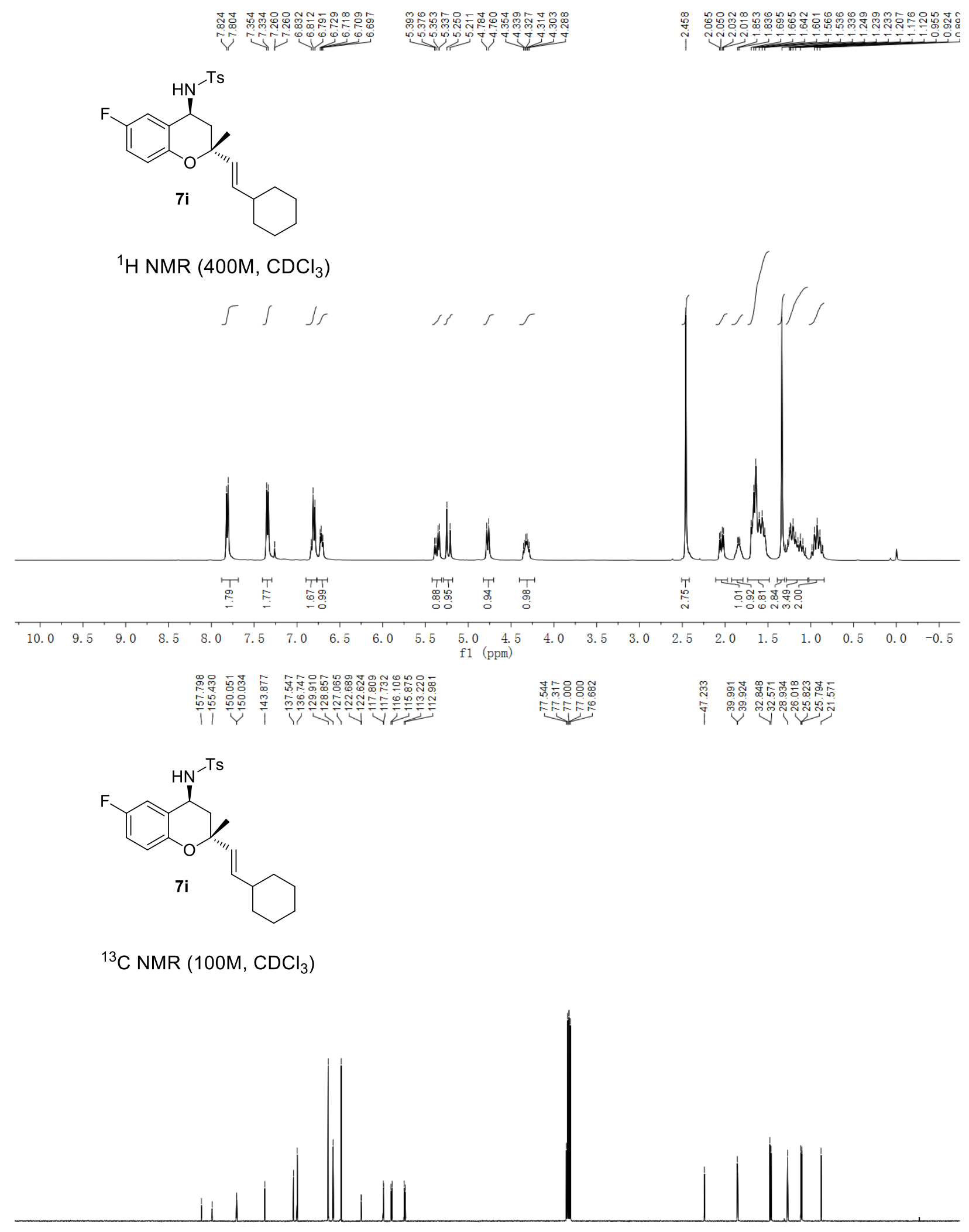

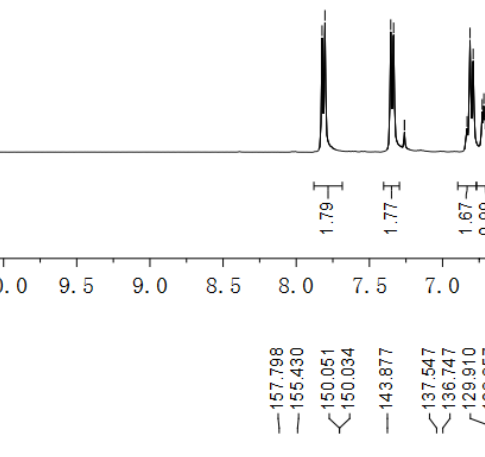

(1)

$\begin{array}{llllllllll}180 & 170 & 160 & 150 & 140 & 130 & 120 & 110 & 100 & 90 \\ \text { f1 } & (\mathrm{ppm})\end{array}$ 

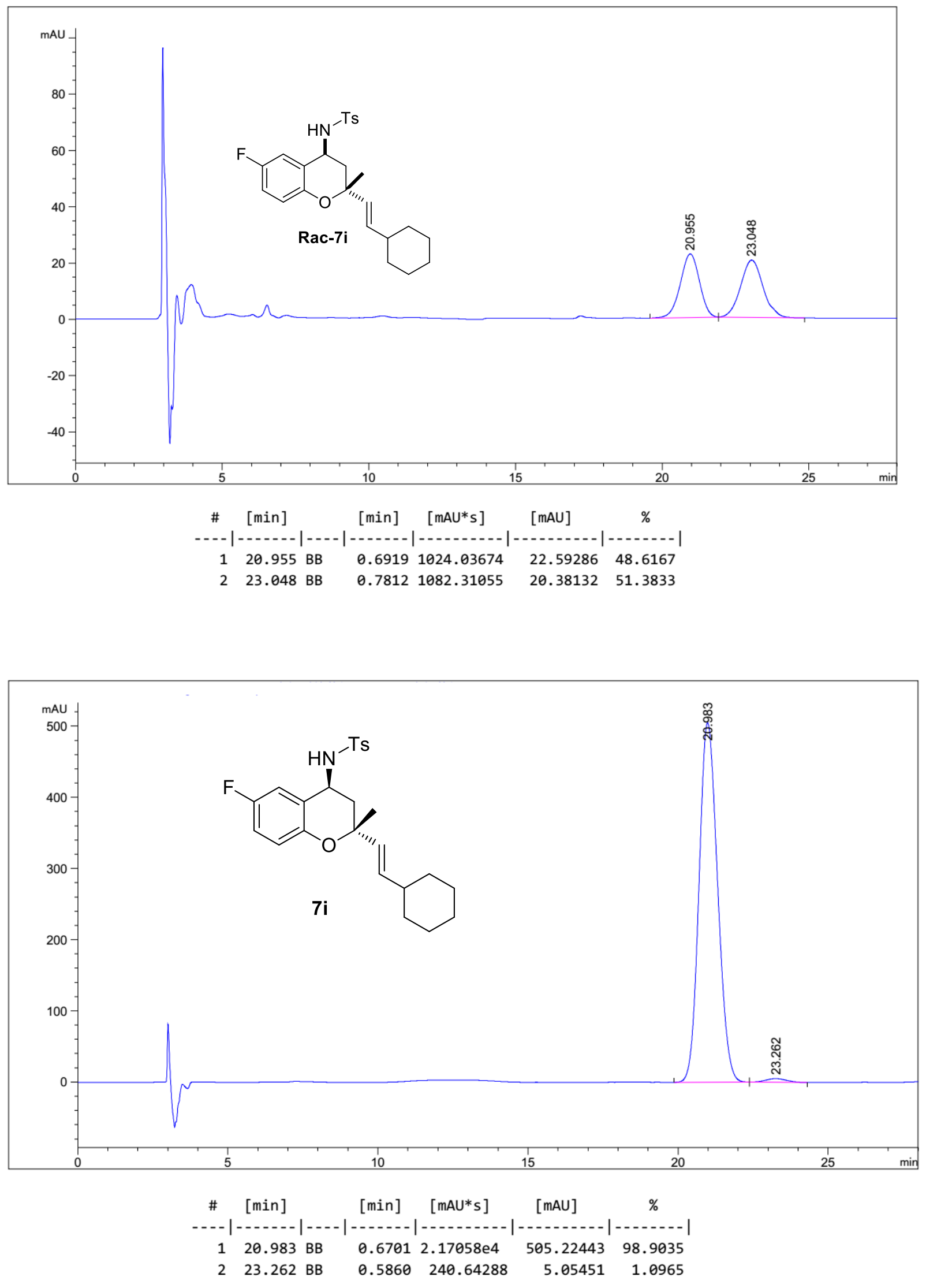

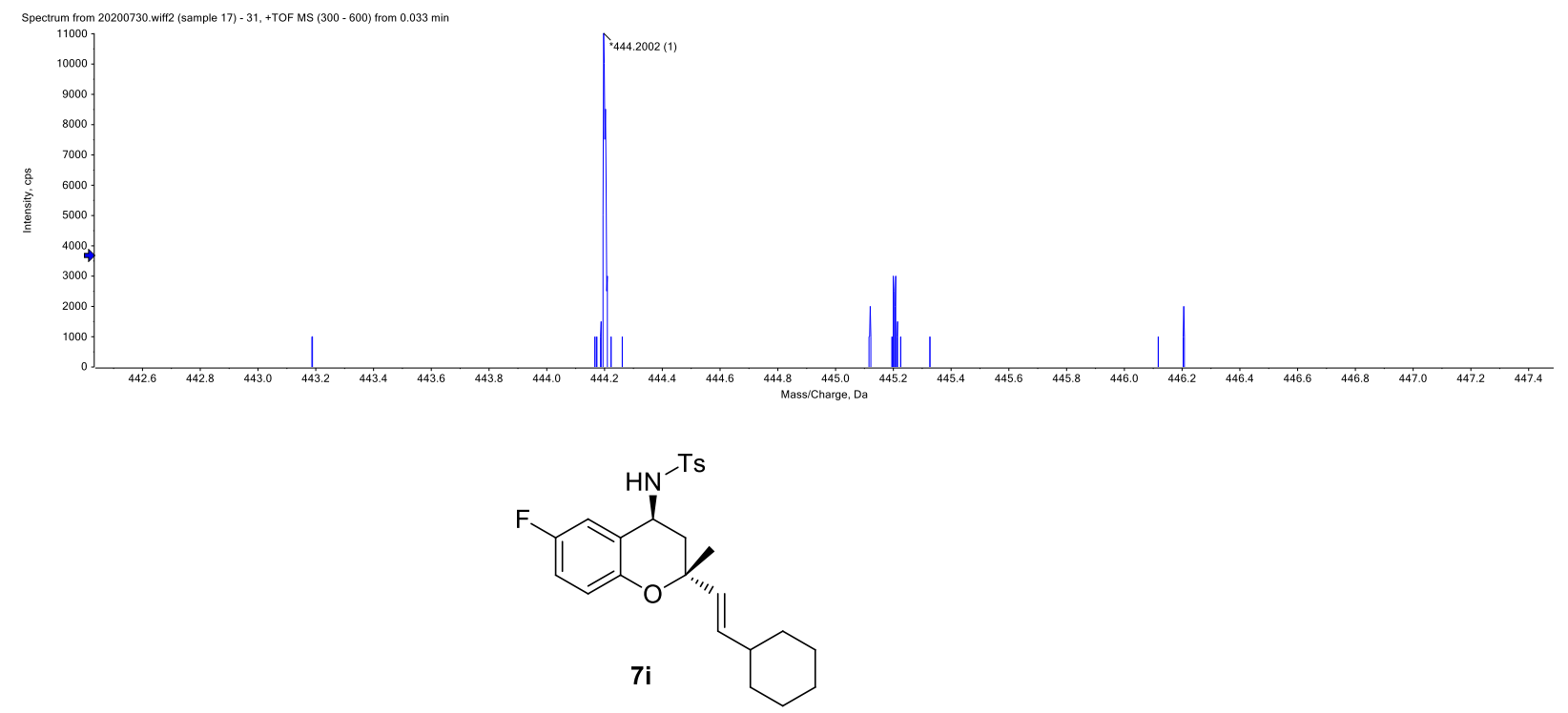

HRMS (ESI-TOF) m/z: [M + H] ${ }^{+}$

Calcd for $\mathrm{C}_{25} \mathrm{H}_{31} \mathrm{FNO}_{3} \mathrm{~S} 444.2003$ 


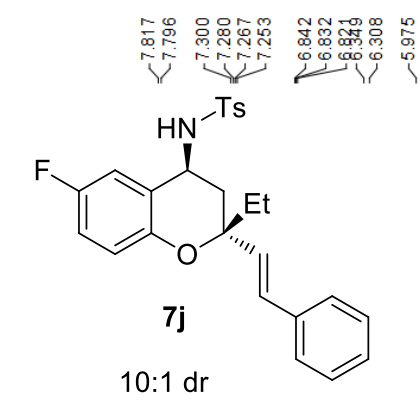

${ }^{1} \mathrm{H}$ NMR $\left(400 \mathrm{M}, \mathrm{CDCl}_{3}\right)$

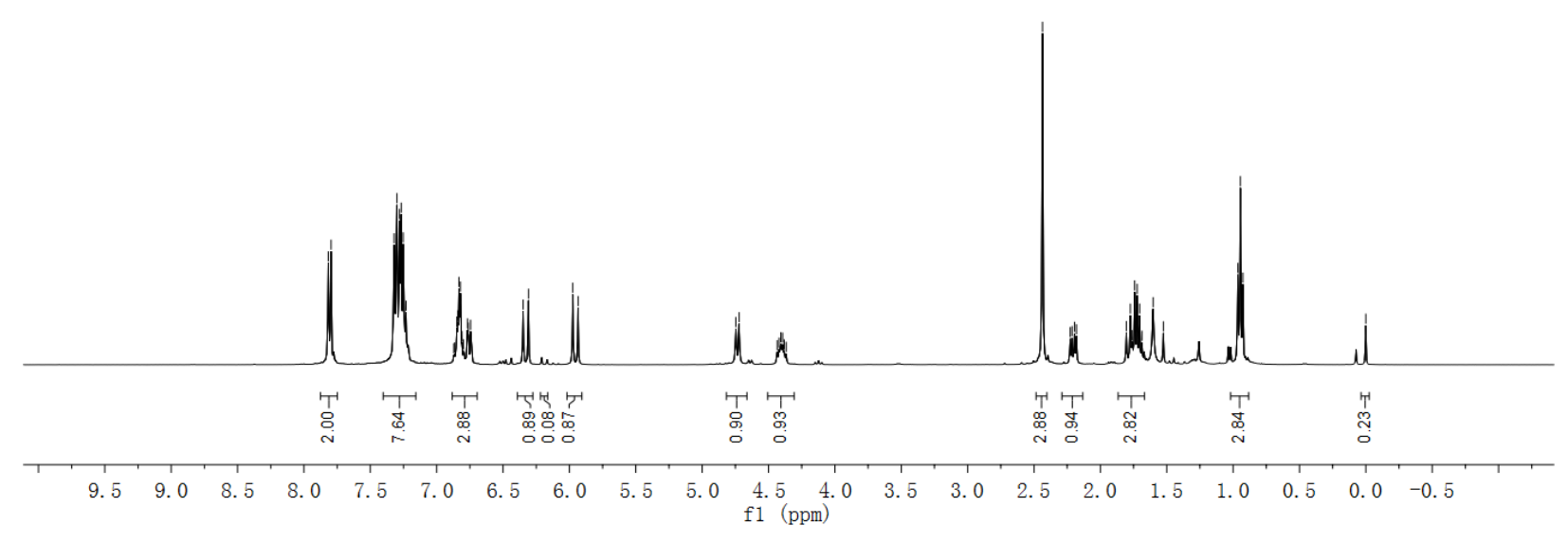

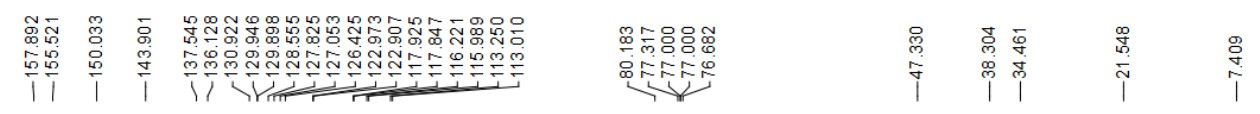<smiles>CCC1(C=Cc2ccccc2)CC(N)c2cc(F)ccc2O1</smiles>

${ }^{13} \mathrm{C}$ NMR $\left(100 \mathrm{M}, \mathrm{CDCl}_{3}\right)$

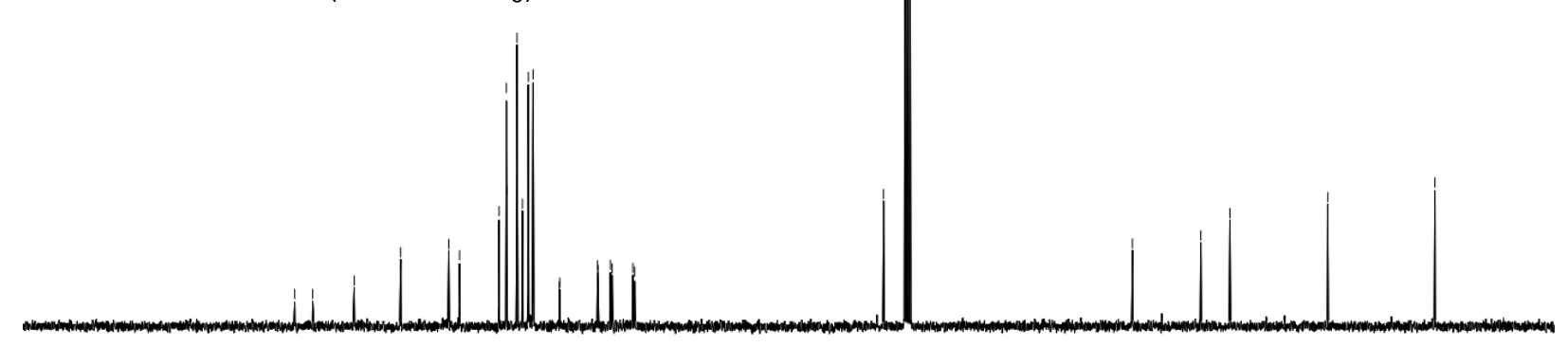

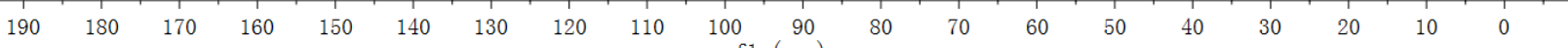




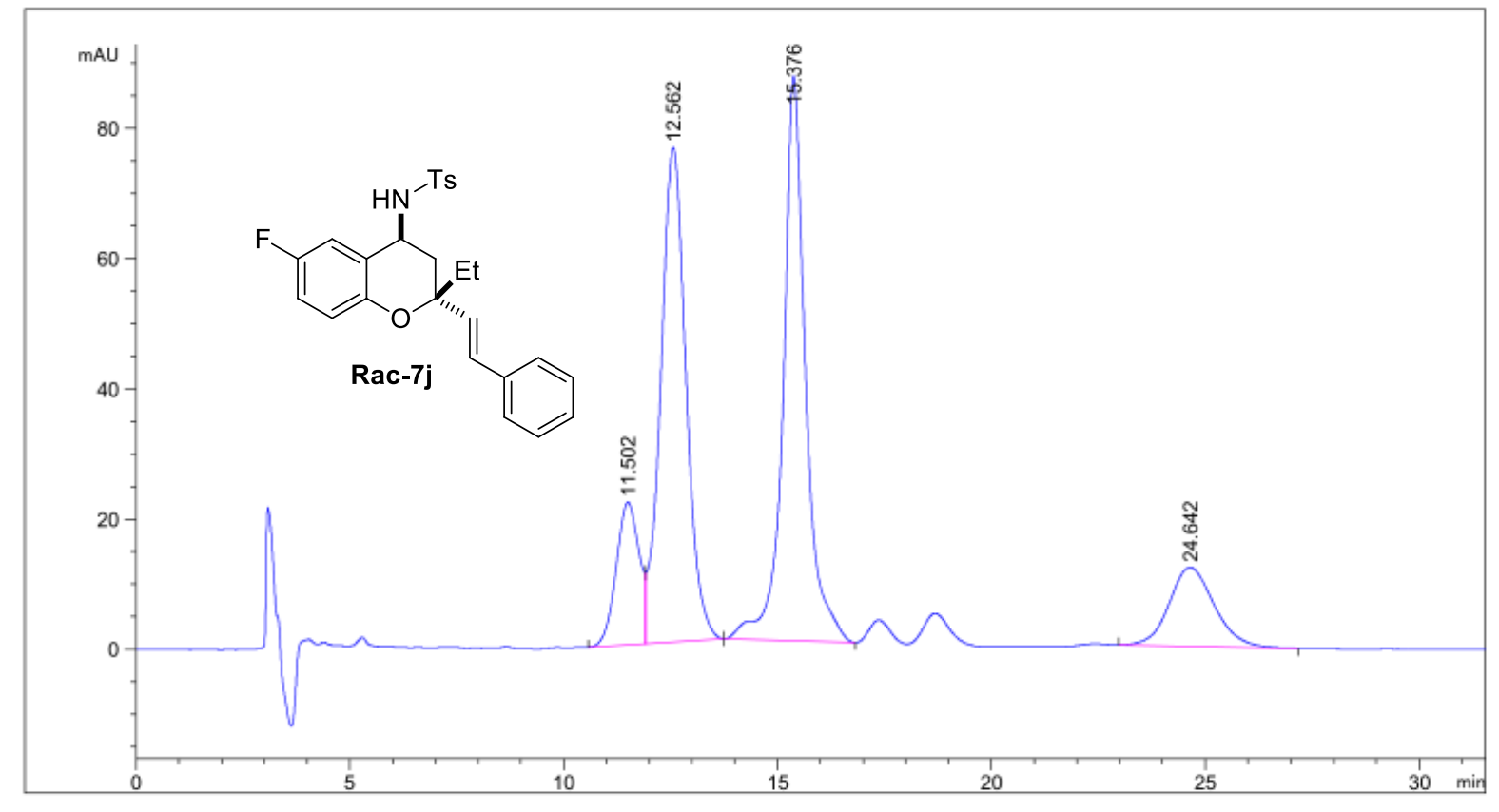

\begin{tabular}{|c|c|c|c|c|c|}
\hline \# & [min] & [min] & {$[\mathrm{mAU} * \mathrm{~s}]$} & {$[\mathrm{mAU}]$} & $\%$ \\
\hline & 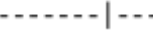 & & 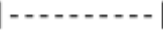 & & \\
\hline 1 & $92 \mathrm{BV}$ & 0.5650 & 813.67938 & 7353 & 724 \\
\hline 2 & $12.562 \mathrm{VB}$ & 0.6247 & 3128.23071 & 75.94551 & 39.1084 \\
\hline 3 & $15.376 \mathrm{BB}$ & 0.5253 & 3168.47241 & 86.71816 & 39.6115 \\
\hline 4 & $24.642 \mathrm{BB}$ & 1.0629 & 888.49634 & 12.12788 & 11.1078 \\
\hline
\end{tabular}

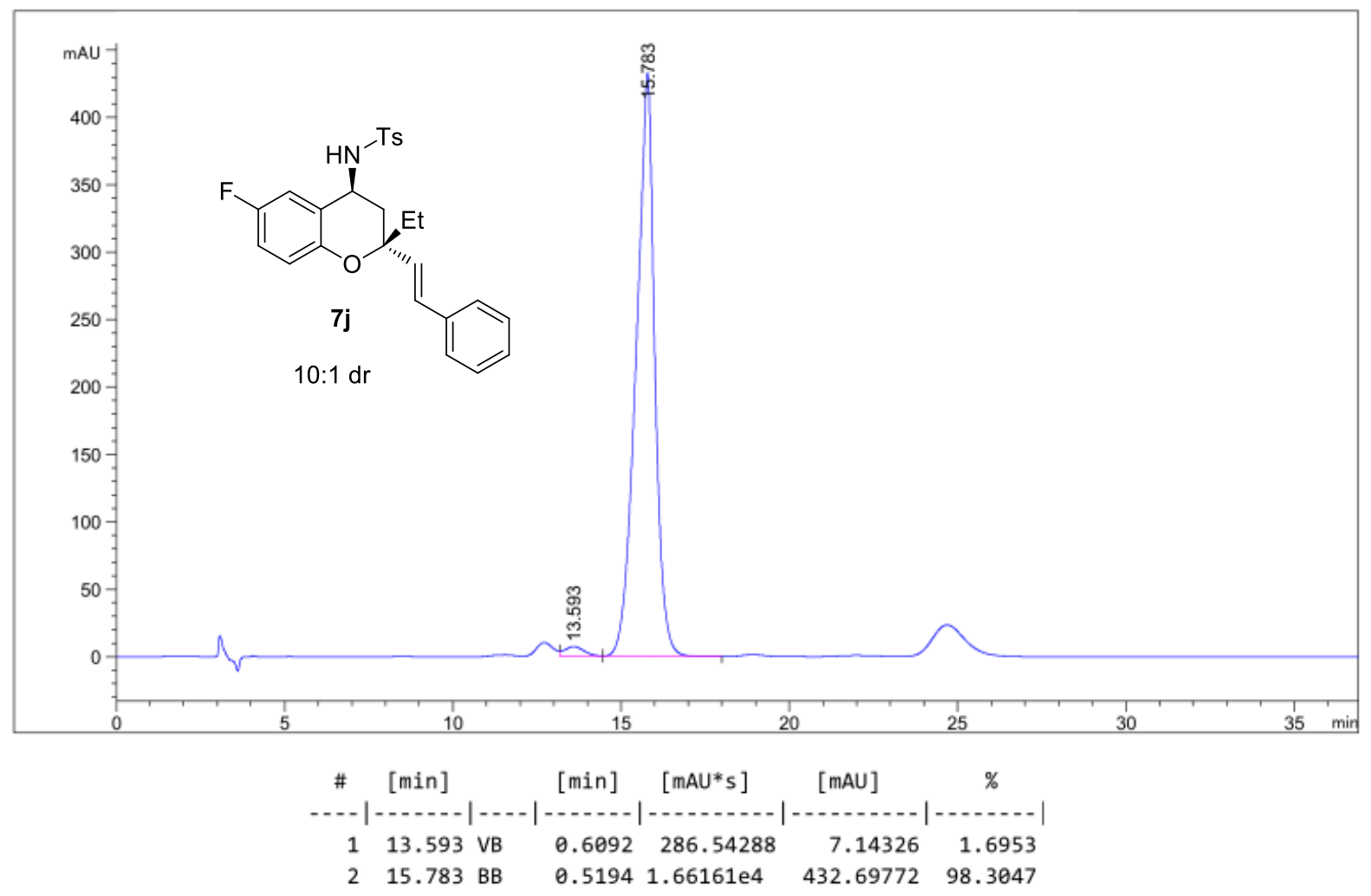




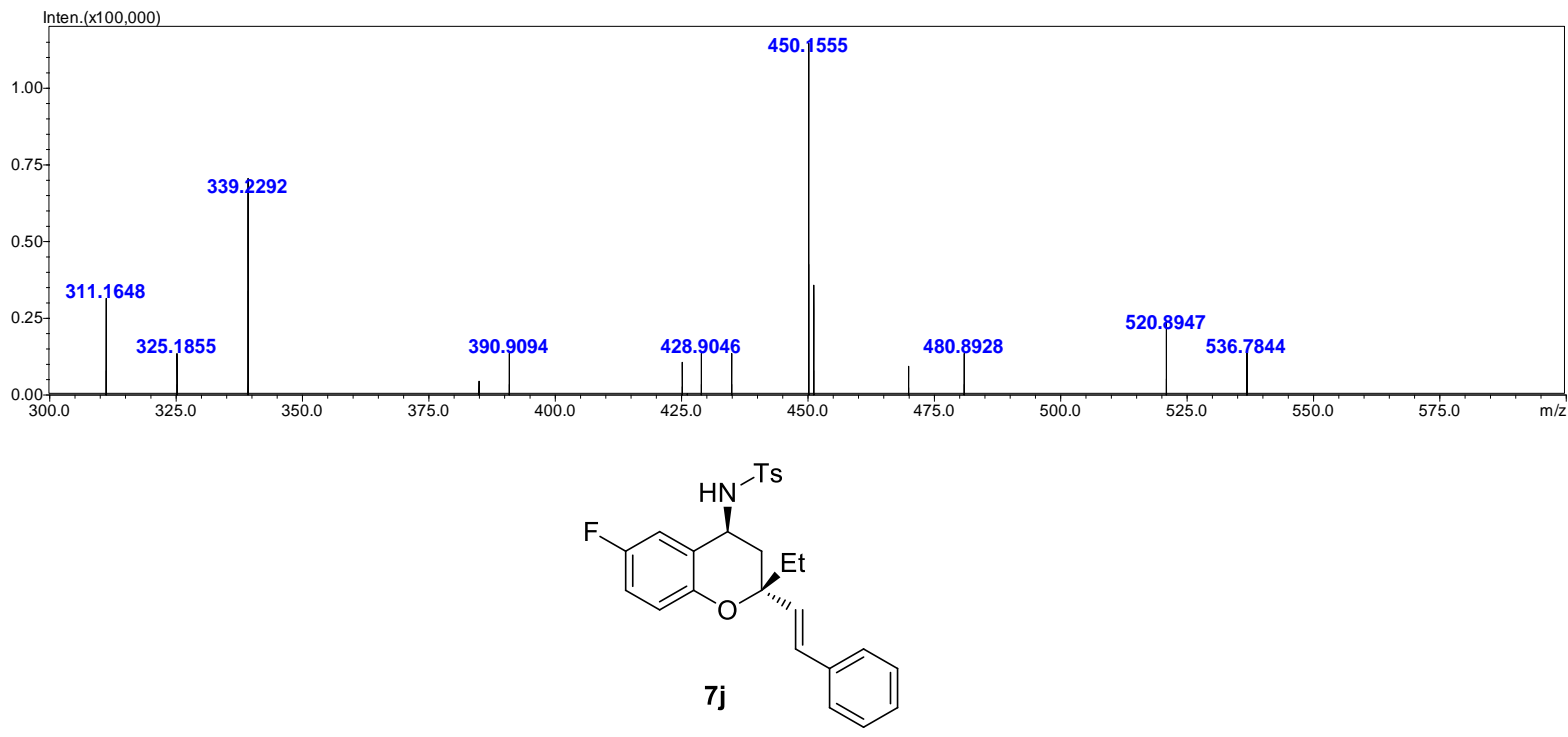

HRMS (ESI-TOF) m/z: [M - H]

Calcd for $\mathrm{C}_{26} \mathrm{H}_{25} \mathrm{FNO}_{3} \mathrm{~S} 444.2003$ 


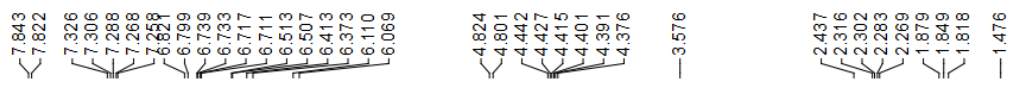

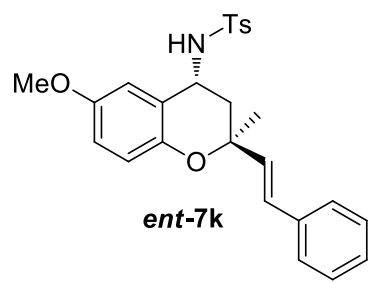

${ }^{1} \mathrm{H}$ NMR (400M, $\mathrm{CDCl}_{3}$ )

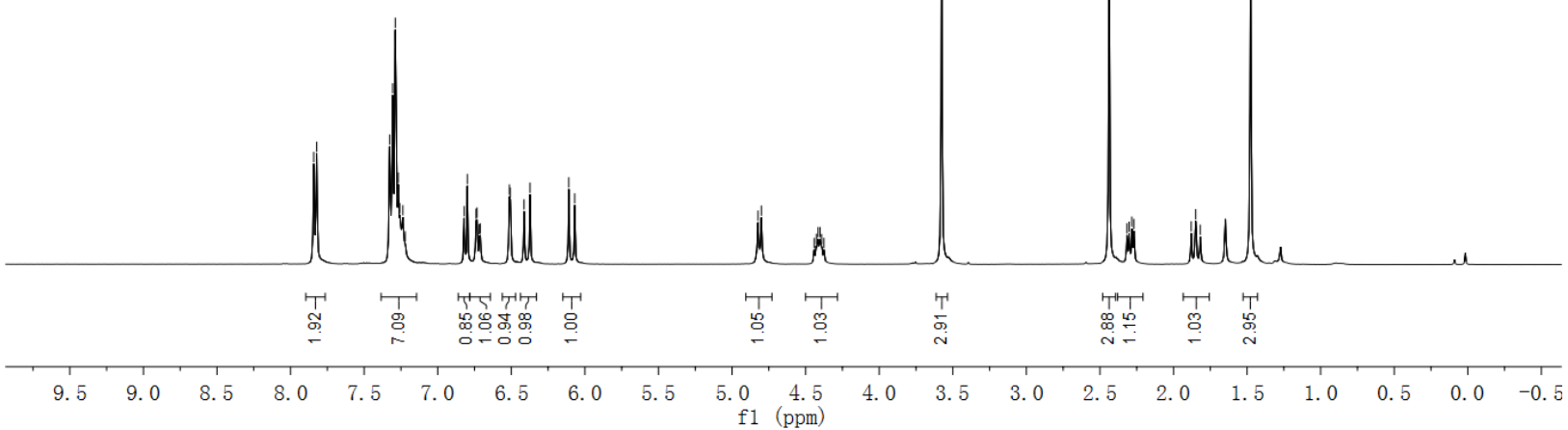

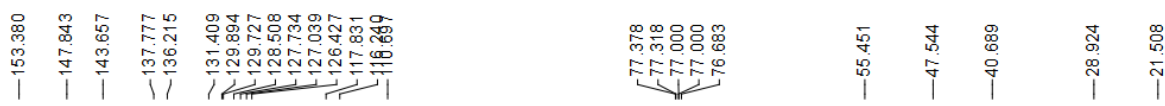
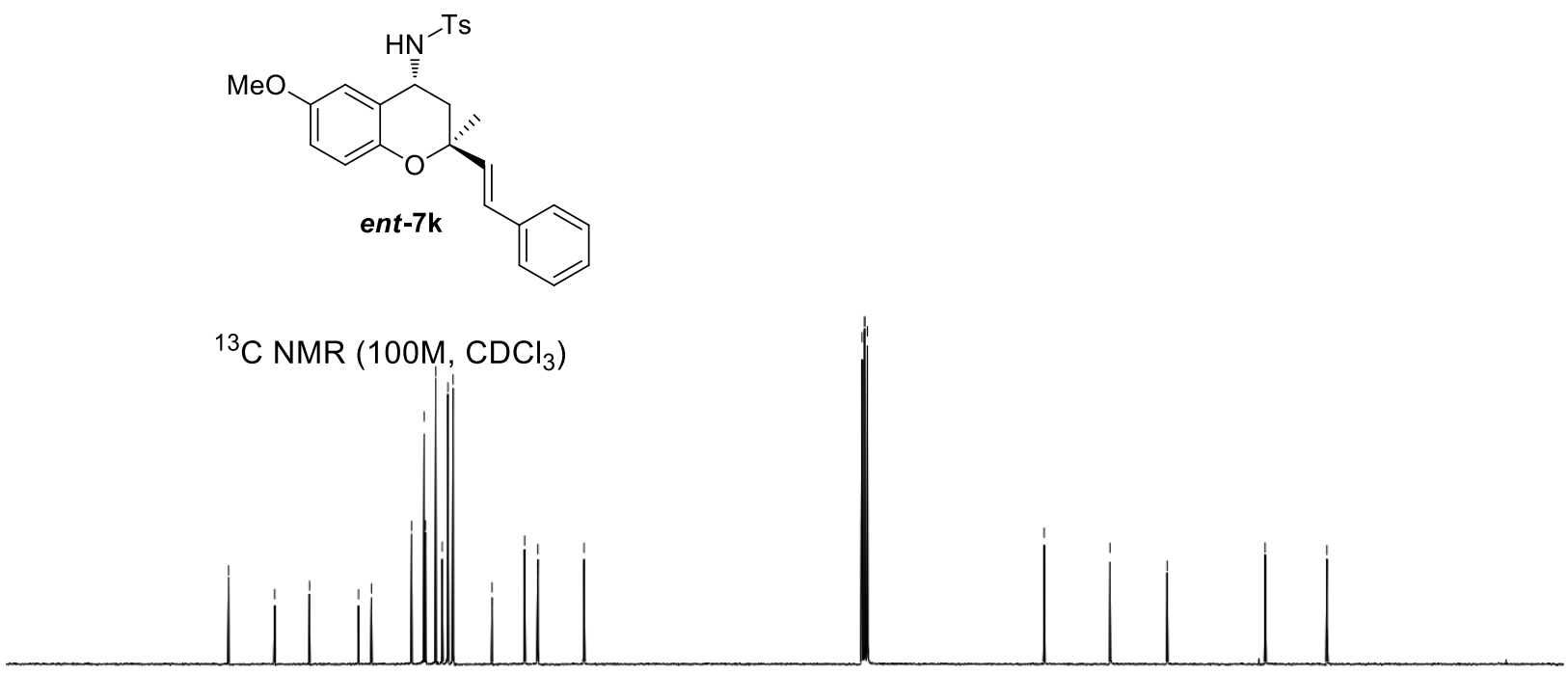

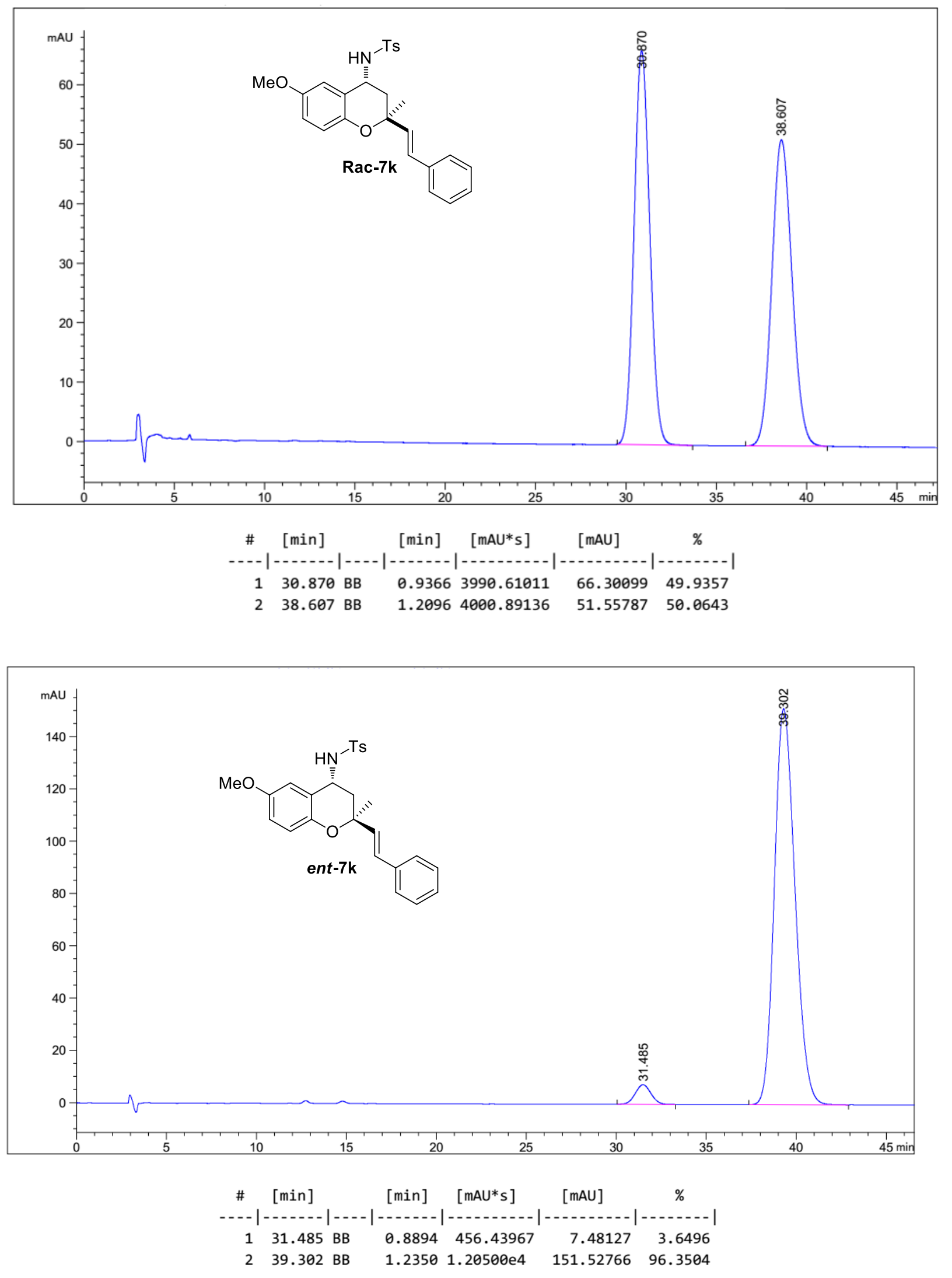


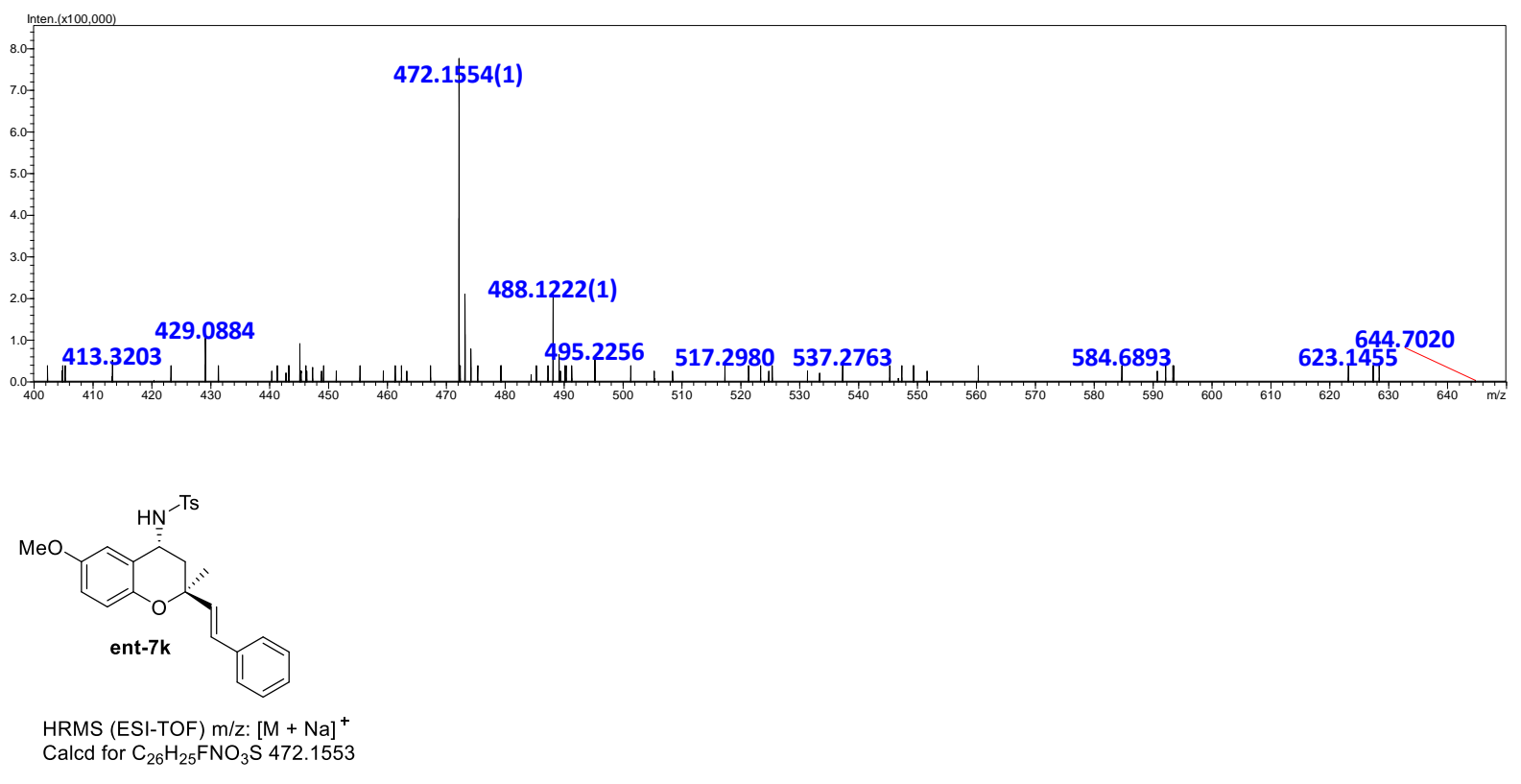




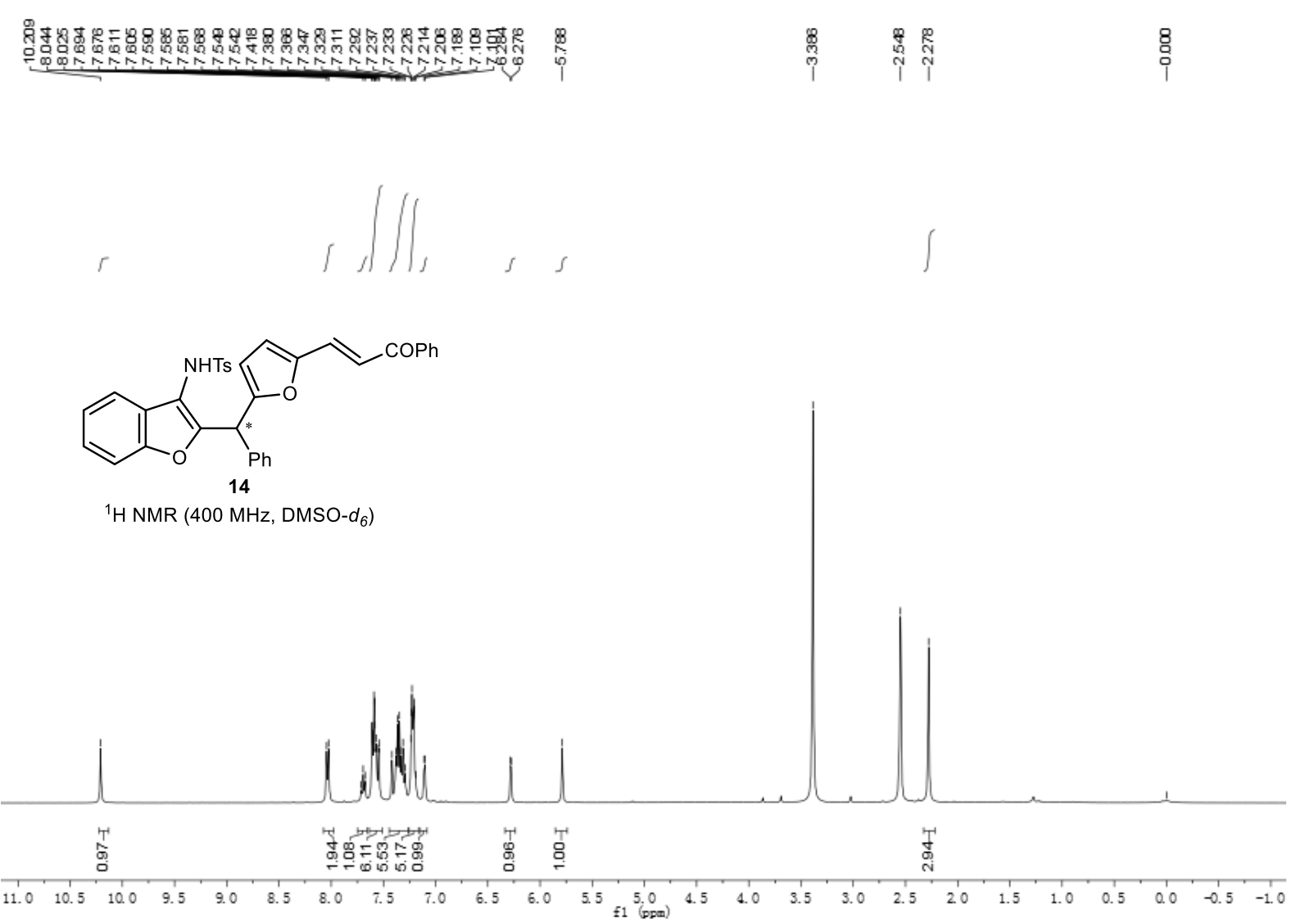

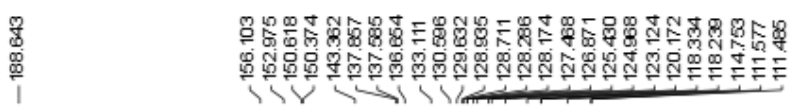
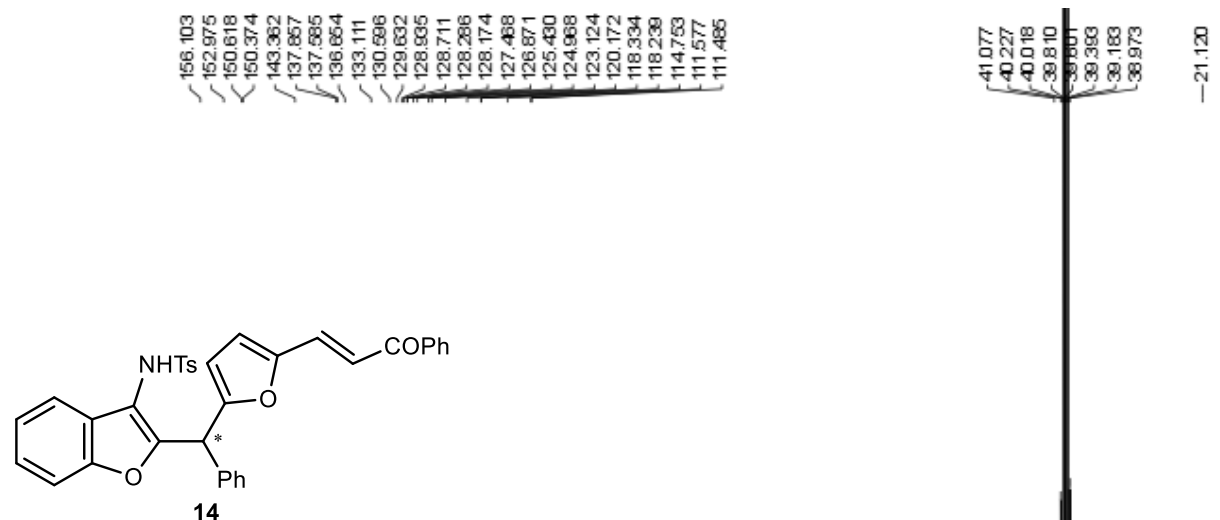

${ }^{13} \mathrm{C}$ NMR $\left(100 \mathrm{MHz}\right.$, DMSO- $\left.d_{6}\right)$
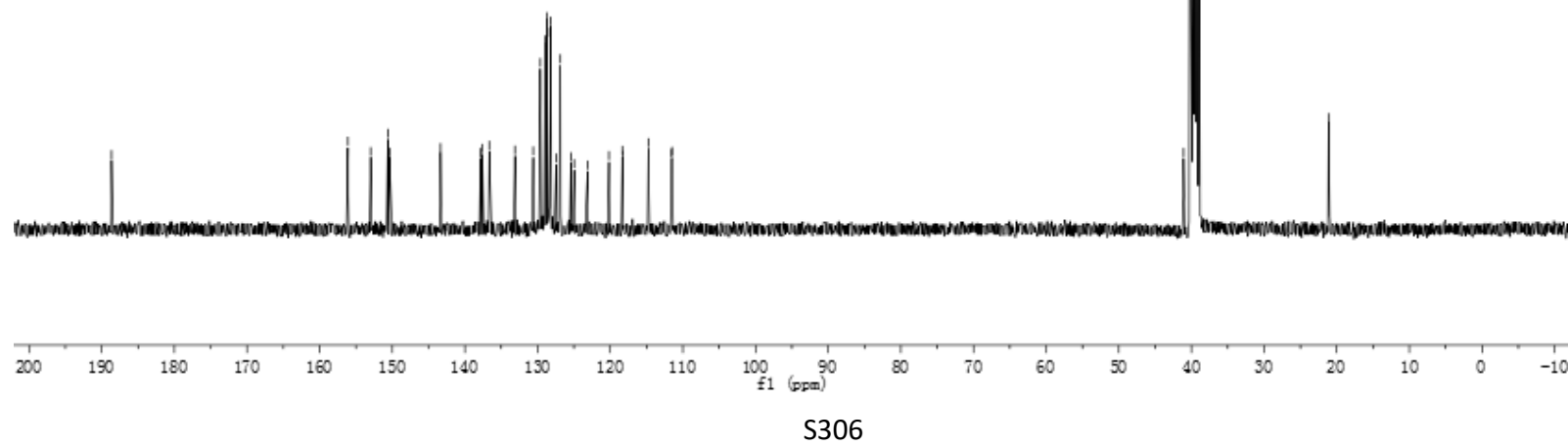


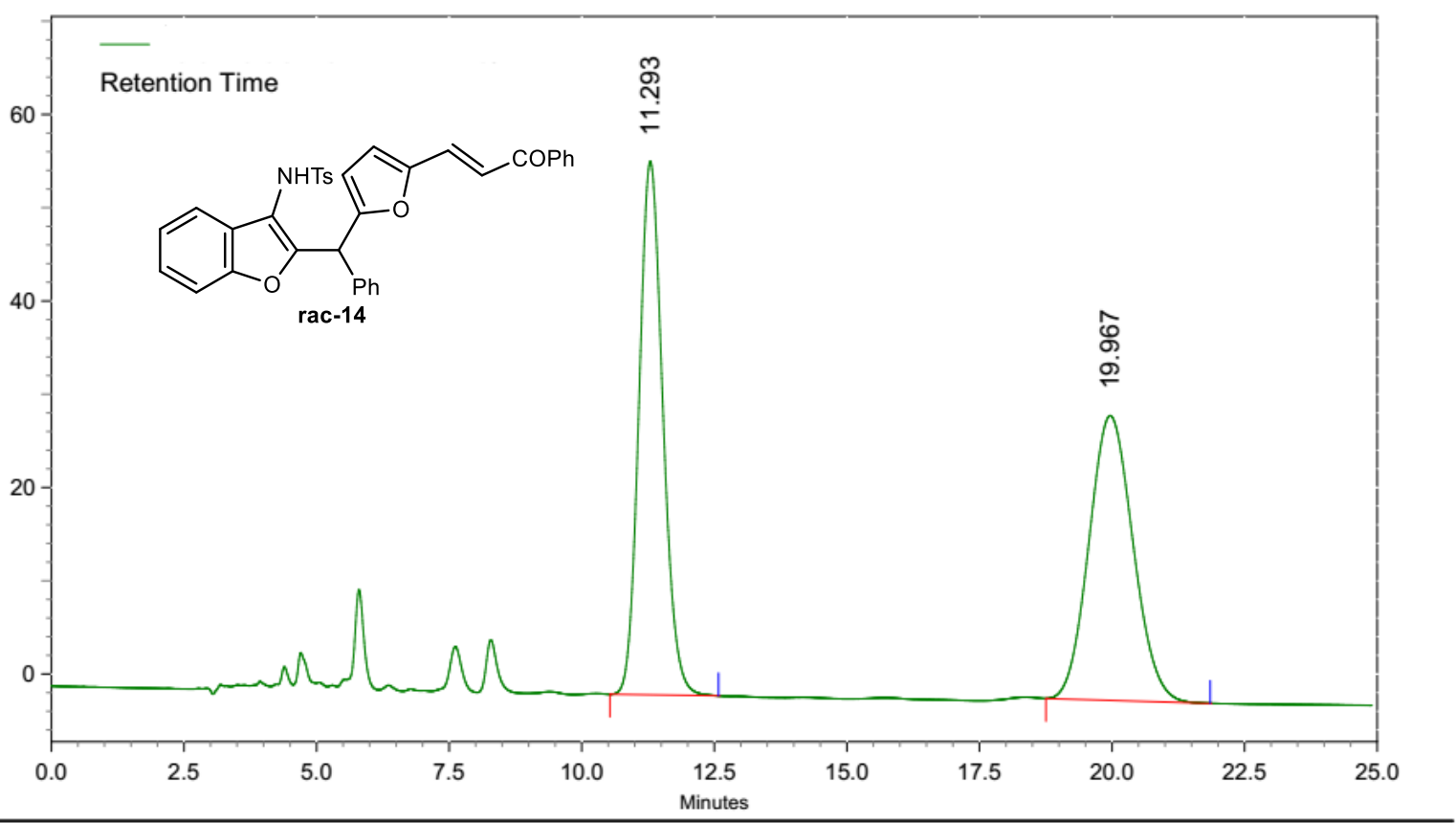

AREA PERCENT REPORT

\begin{tabular}{llllll} 
Peak No. & Ret Time & Width & Height & Area & Area [\%] \\
\hline 1 & 11.293 & 2.047 & 959550 & 29990422 & 50.3035 \\
2 & 19.967 & 3.100 & 512137 & 29628534 & 49.6965
\end{tabular}

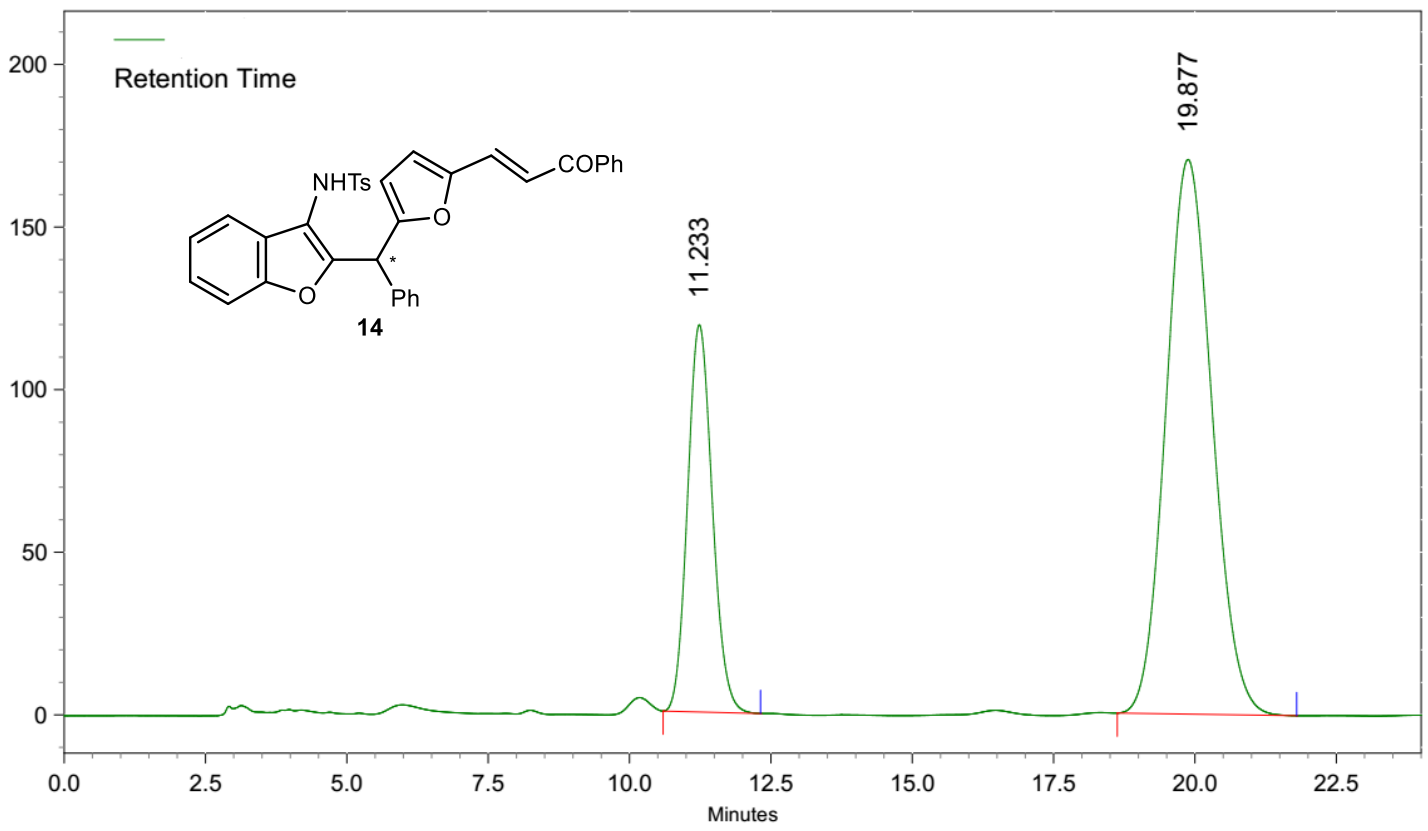

AREA PERCENT REPORT

\begin{tabular}{llllll} 
Peak No. & Ret Time & Width & Height & Area & Area [\%] \\
\hline 1 & 11.233 & 1.723 & 1997383 & 60932170 & 27.3172 \\
2 & 19.877 & 3.173 & 2861377 & 162122117 & 72.6828
\end{tabular}


User Spectrum Plot Report

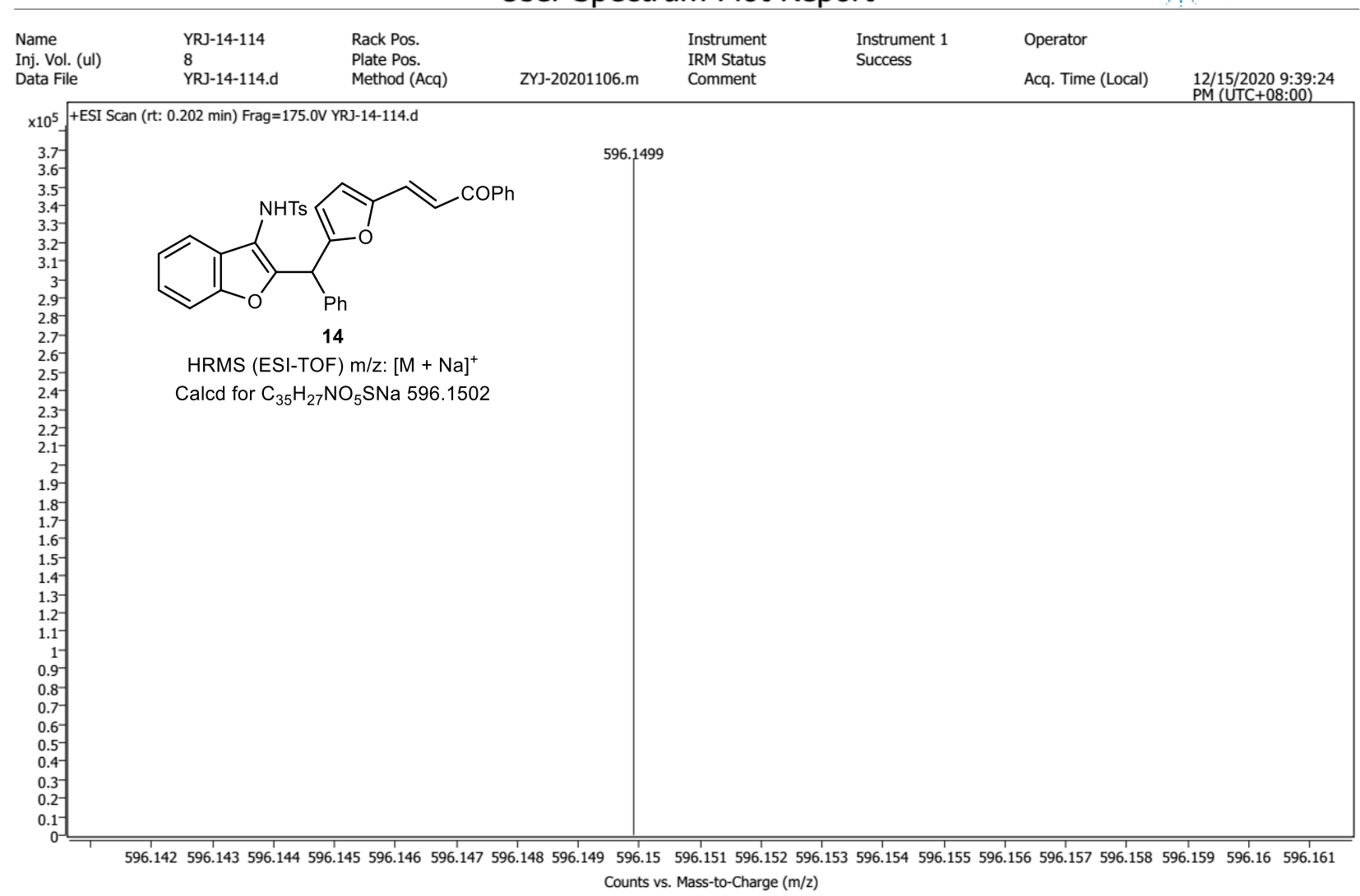




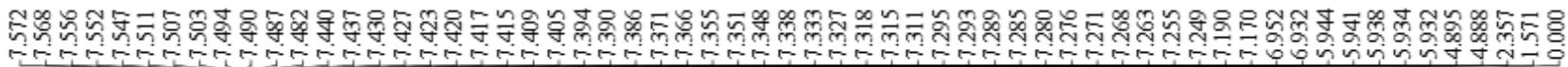

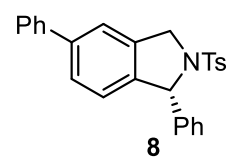

${ }^{1} \mathrm{H} \mathrm{NMR}\left(400 \mathrm{M}, \mathrm{CDCl}_{3}\right)$

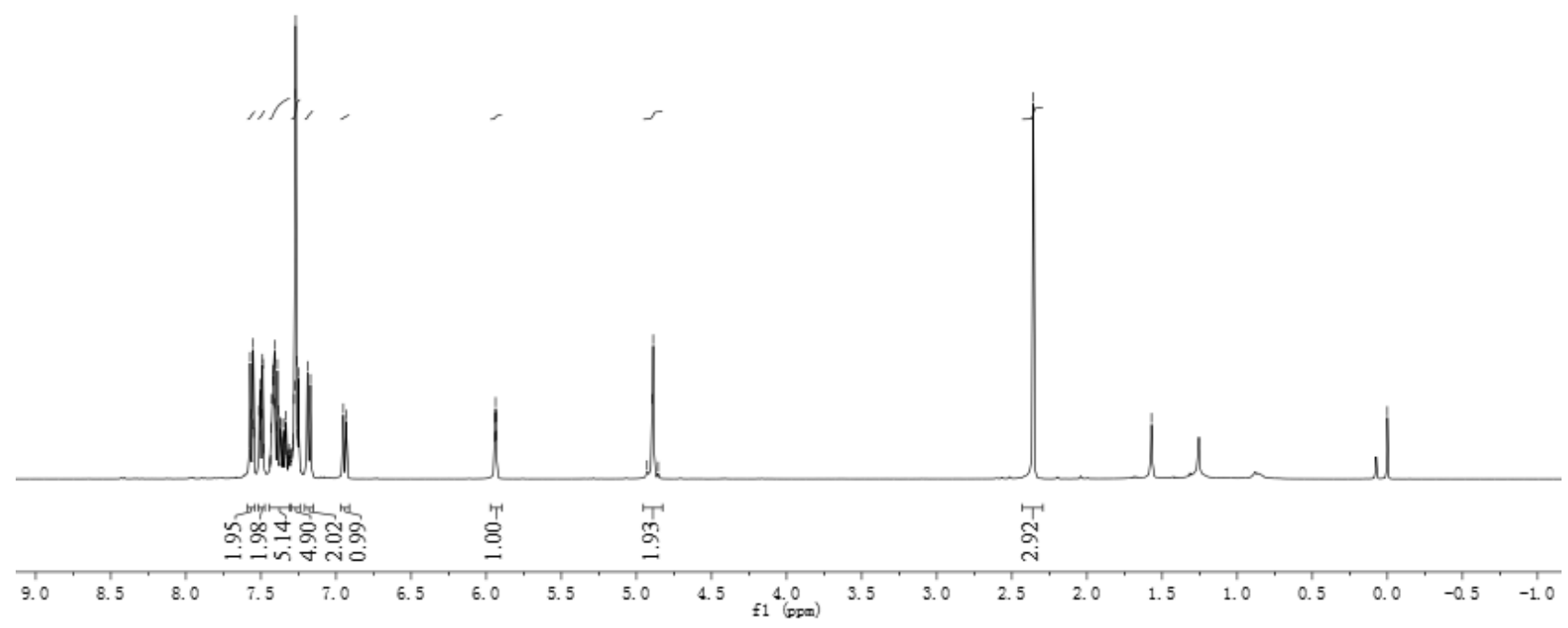

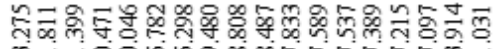

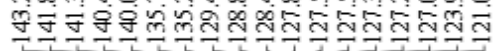

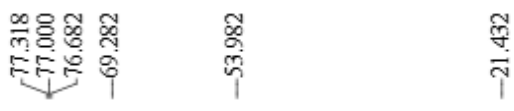

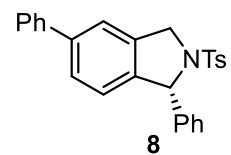

${ }^{1} \mathrm{H}$ NMR (400 M, $\mathrm{CDCl}_{3}$ )

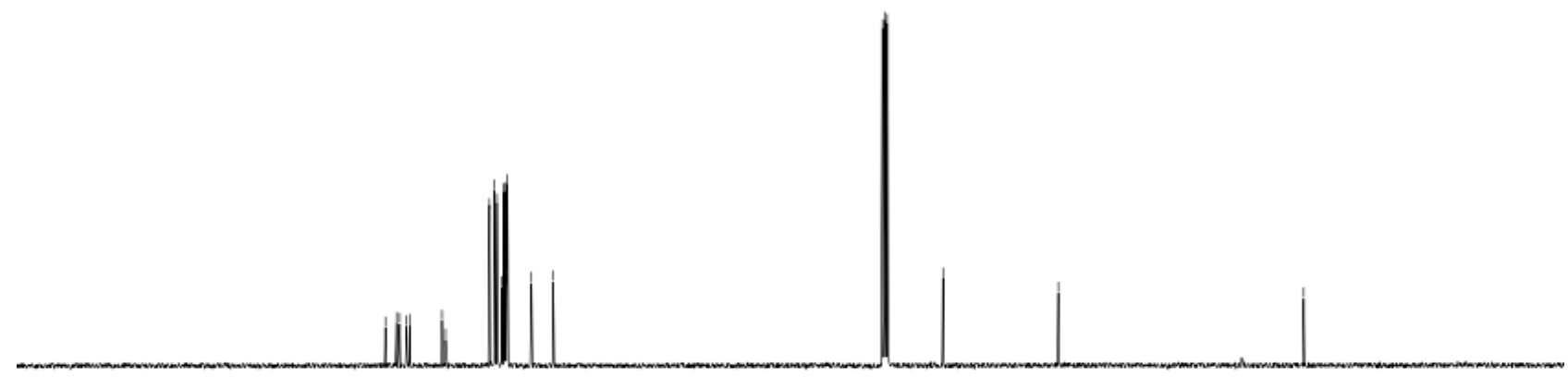

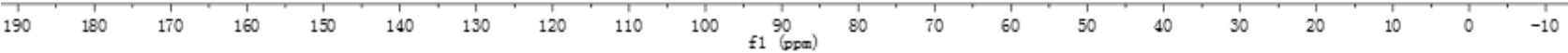




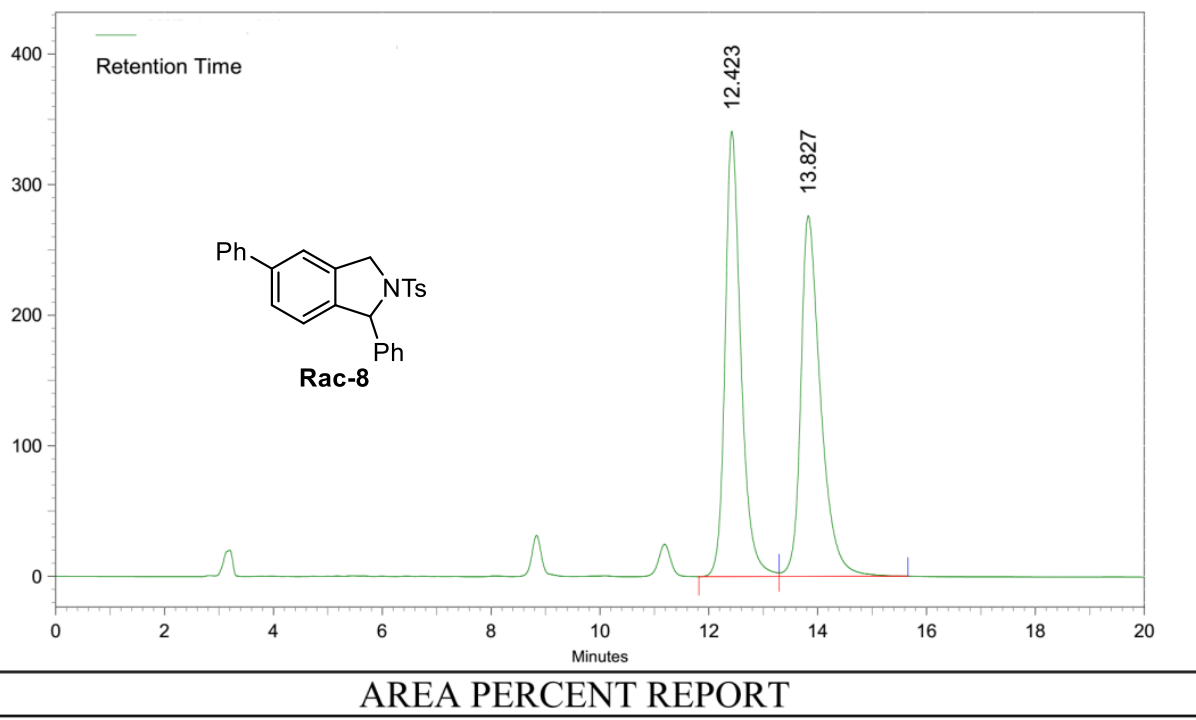

\begin{tabular}{llllll} 
Peak No. & Ret Time & Width & Height & Area & Area [\%] \\
\hline 1 & 12.423 & 1.473 & 5722892 & 115907924 & 49.6292 \\
2 & 13.827 & 2.367 & 4634440 & 117640115 & 50.3708
\end{tabular}

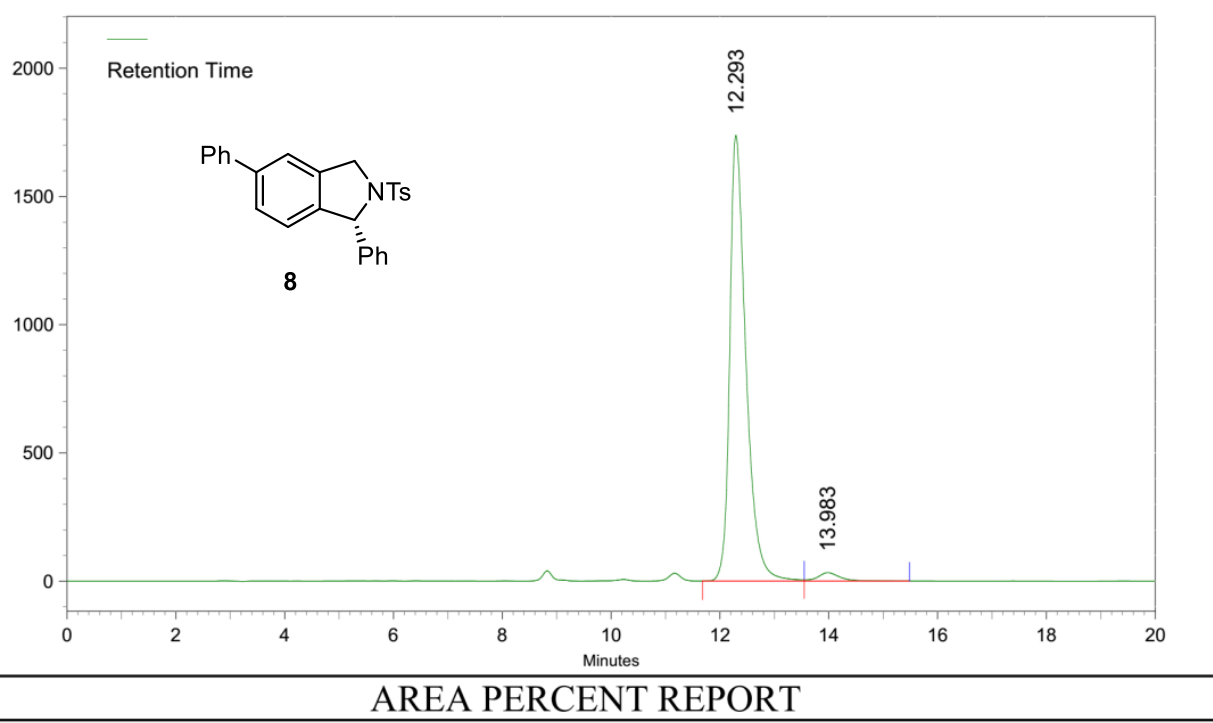

\begin{tabular}{llllll} 
Peak No. & Ret Time & Width & Height & Area & Area [\%] \\
\hline 1 & 12.293 & 1.870 & 29163877 & 602237567 & 97.5327 \\
2 & 13.983 & 1.933 & 543985 & 15235202 & 2.4673
\end{tabular}




\section{ESI+}

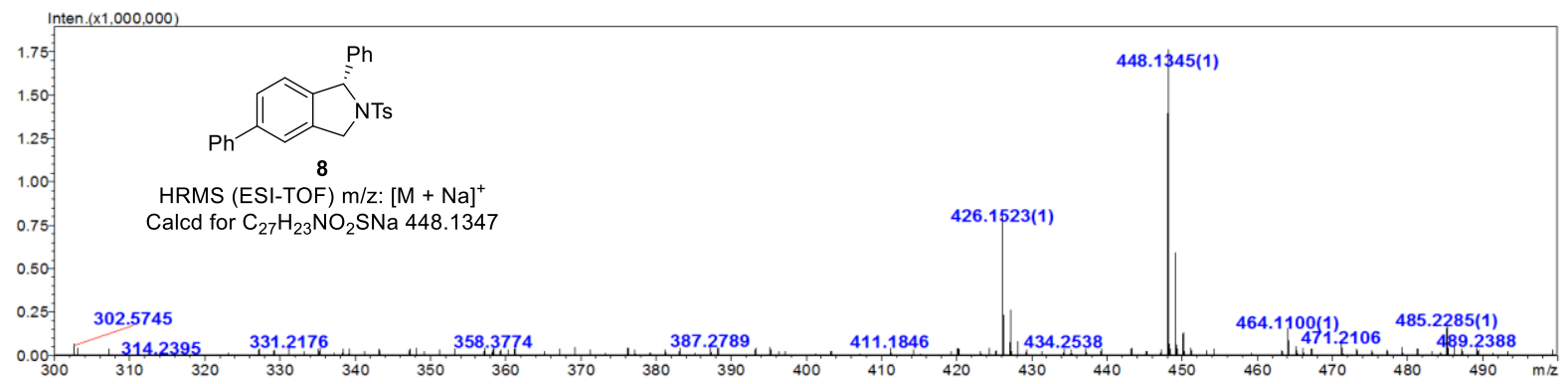



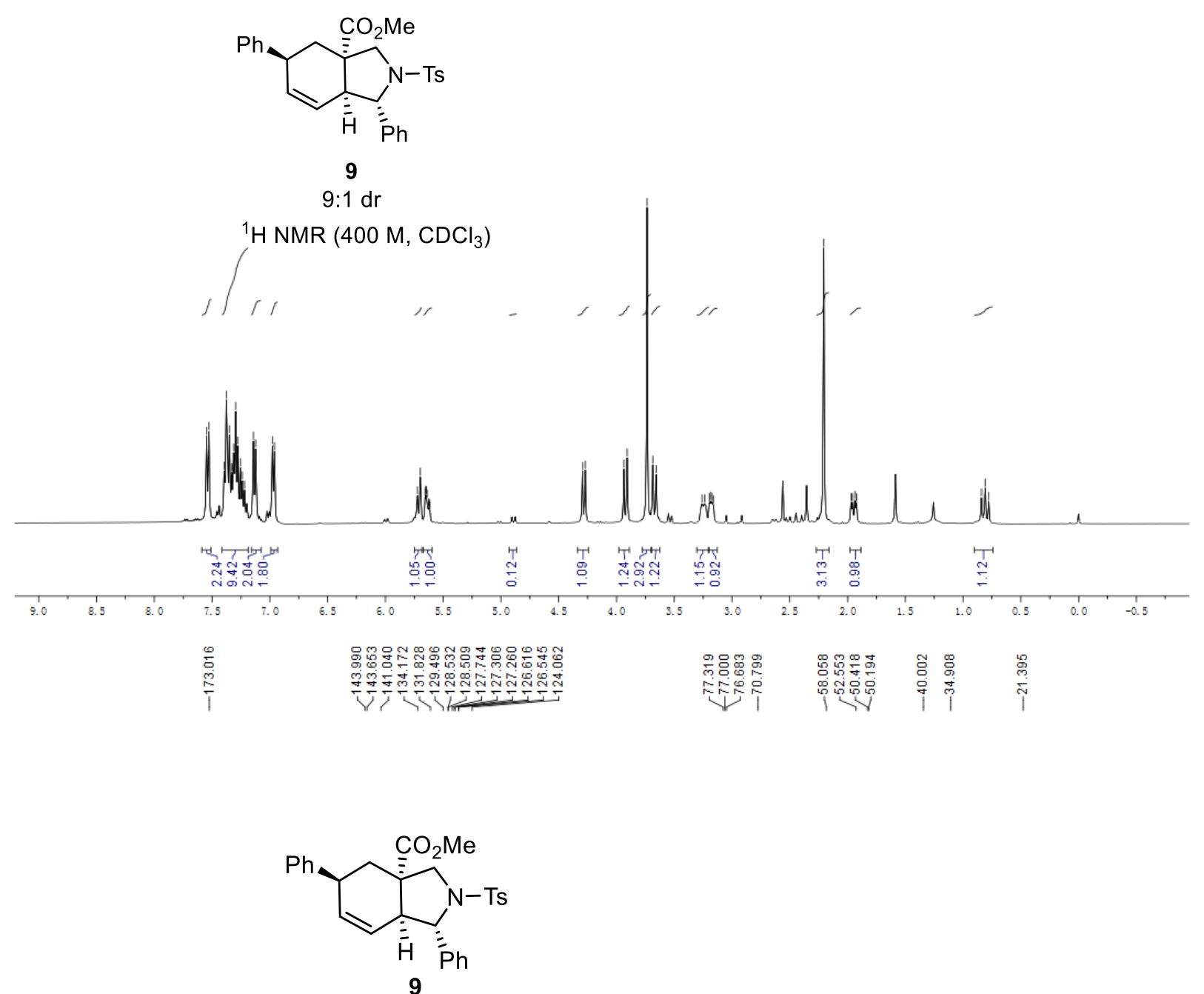

$9: 1 \mathrm{dr}$
${ }^{13} \mathrm{C}$ NMR $\left(100 \mathrm{M}, \mathrm{CDCl}_{3}\right)$

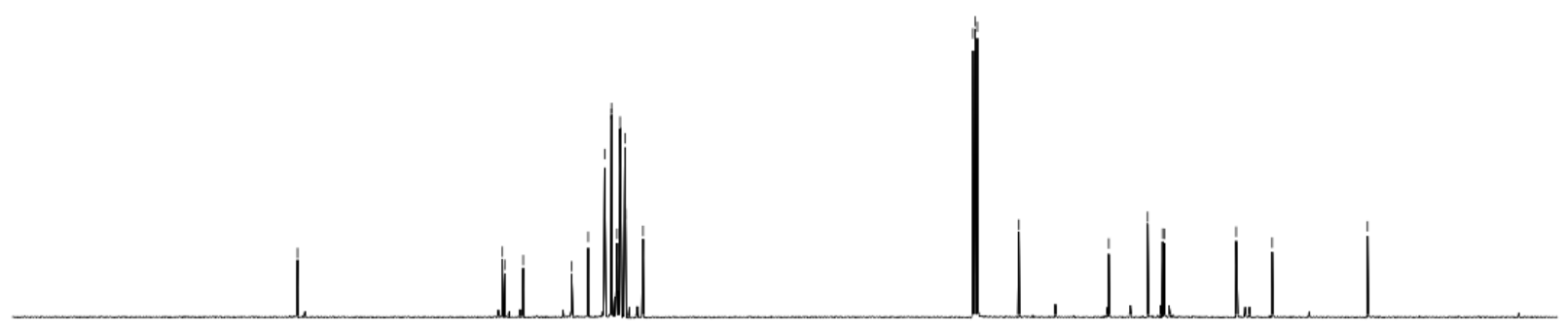

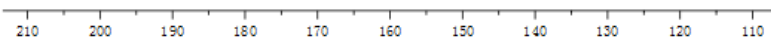




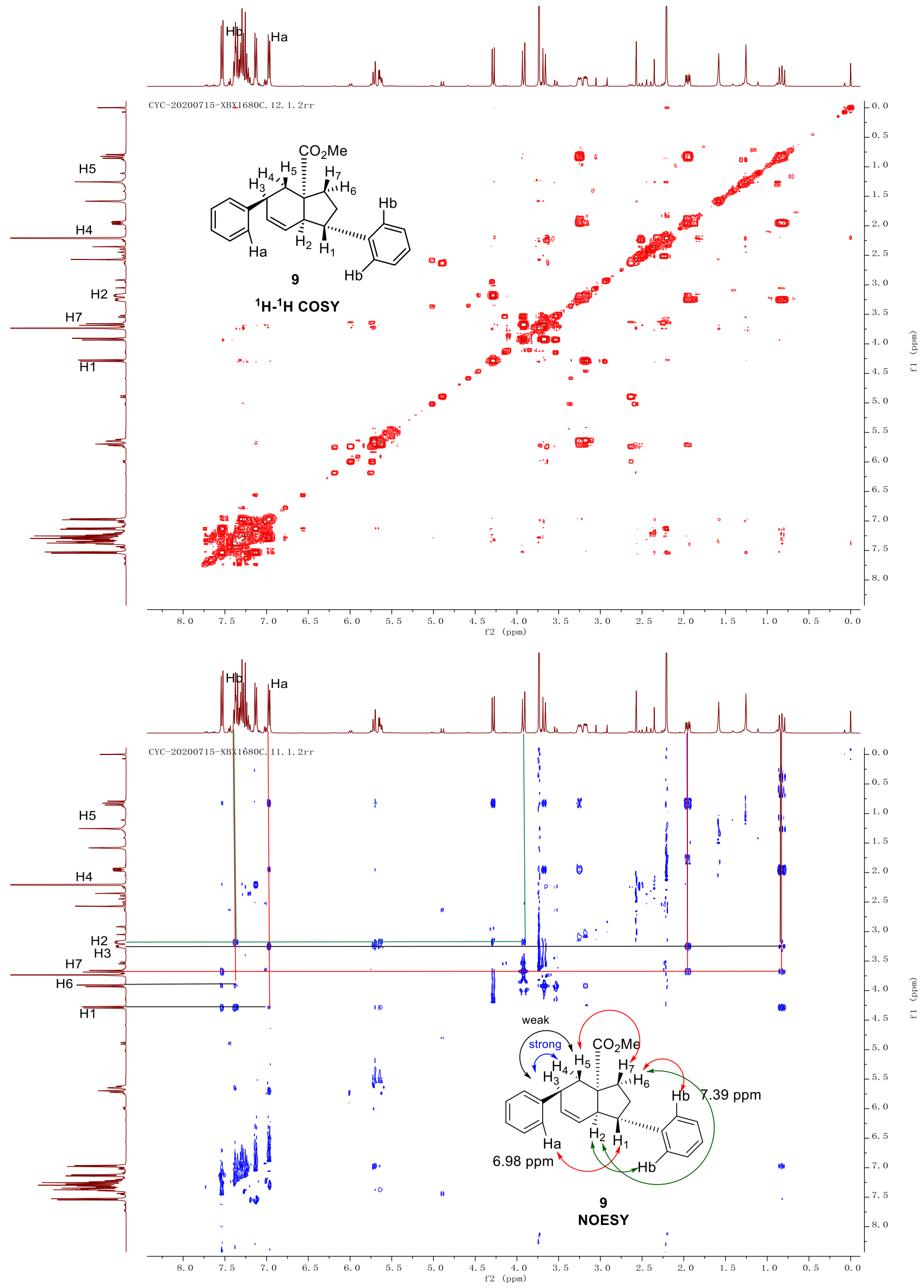



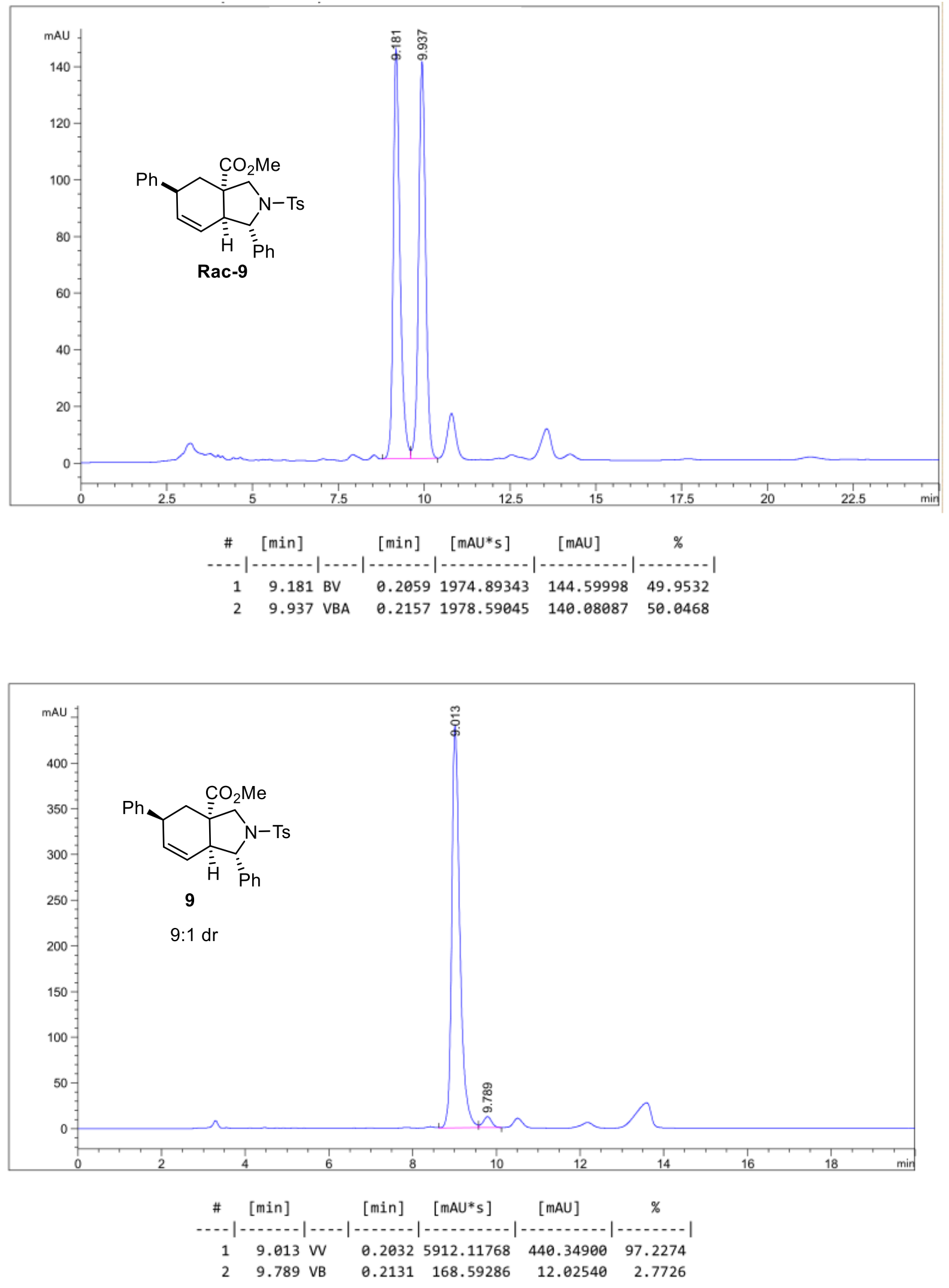

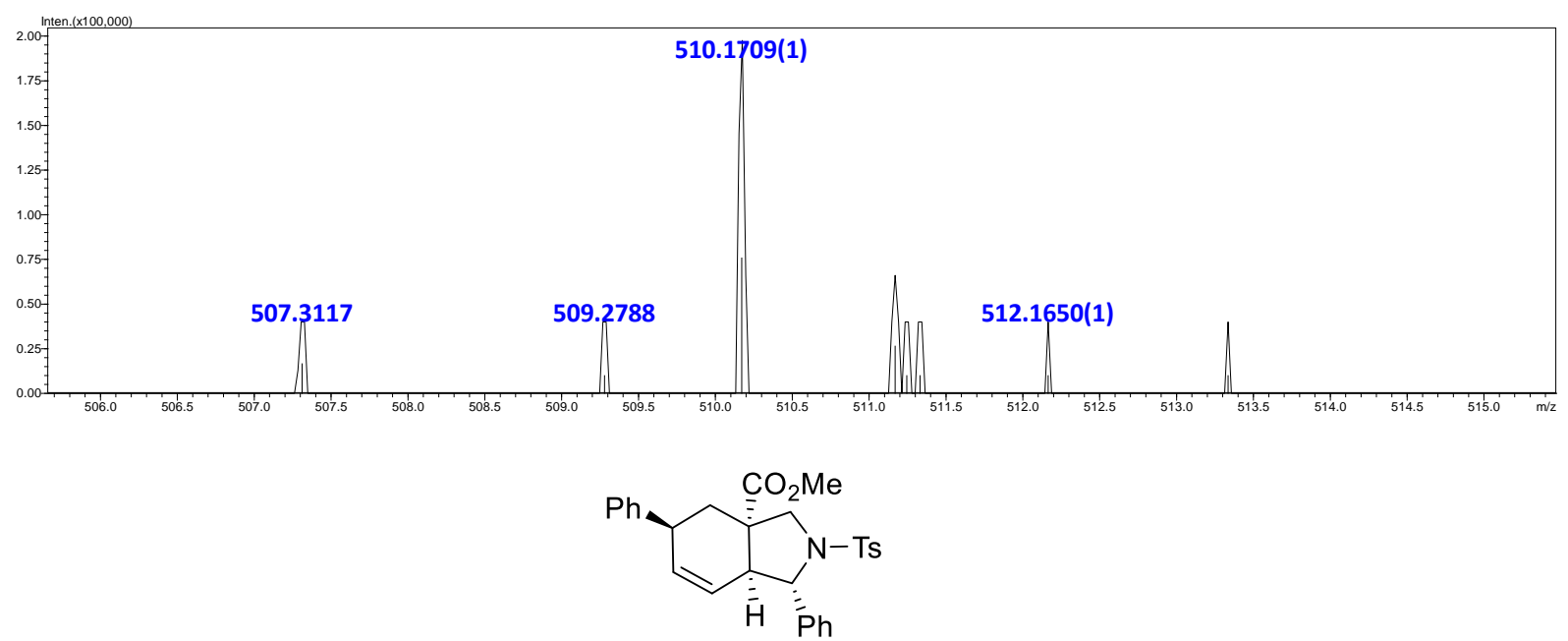

9

HRMS (ESI-TOF) m/z: [M + Na] ${ }^{+}$

Calcd for $\mathrm{C}_{29} \mathrm{H}_{29} \mathrm{NO}_{4} \mathrm{SNa} 510.1710$ 


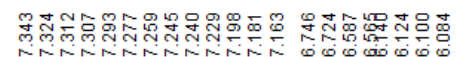

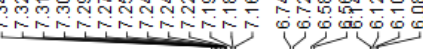

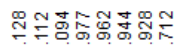

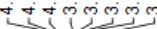

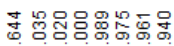

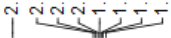

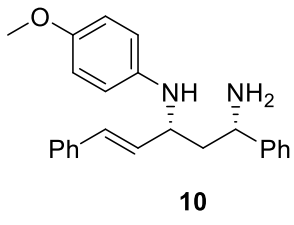

${ }^{1} \mathrm{H}$ NMR (400 M, $\left.\mathrm{CDCl}_{3}\right)$

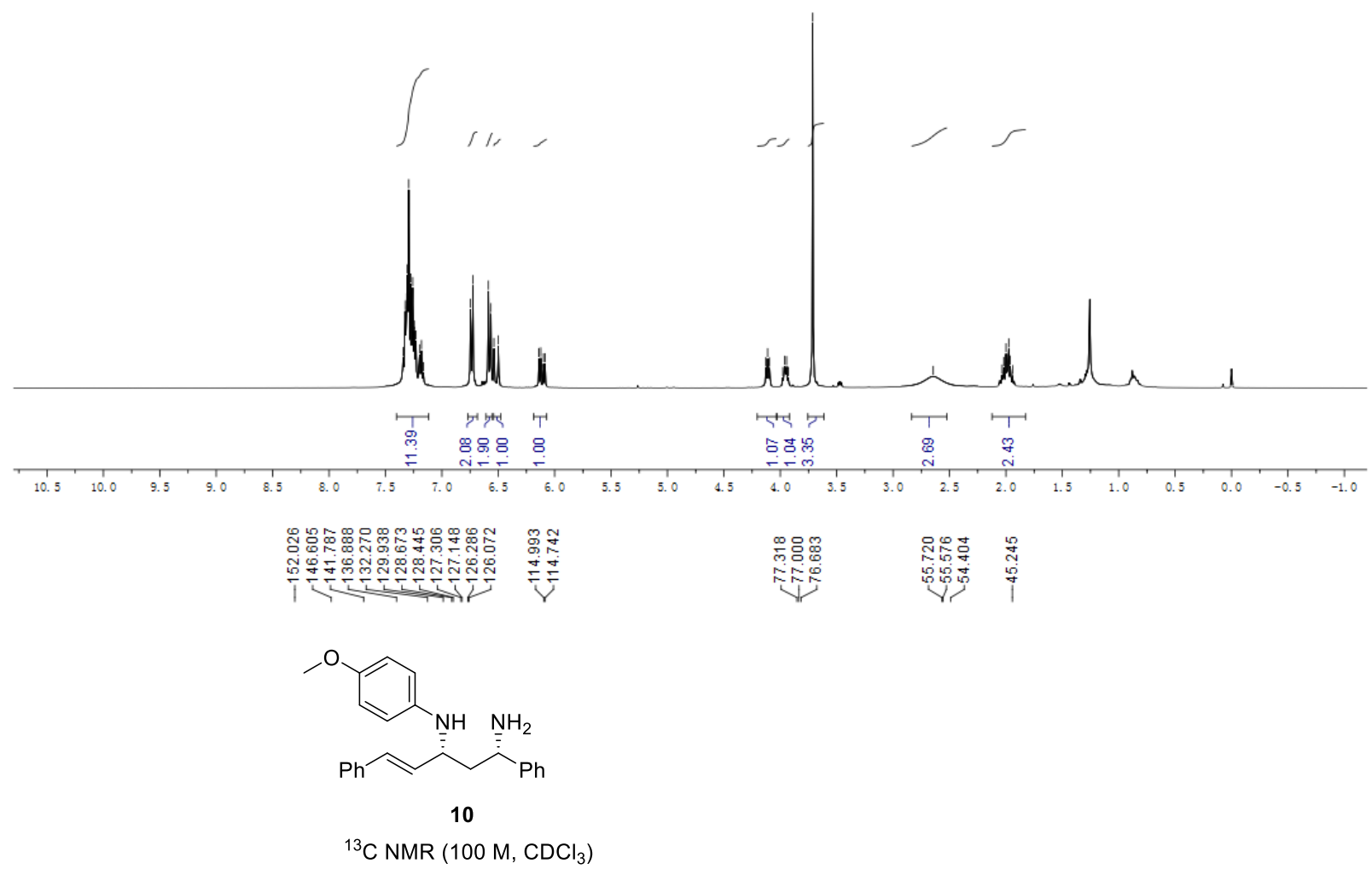



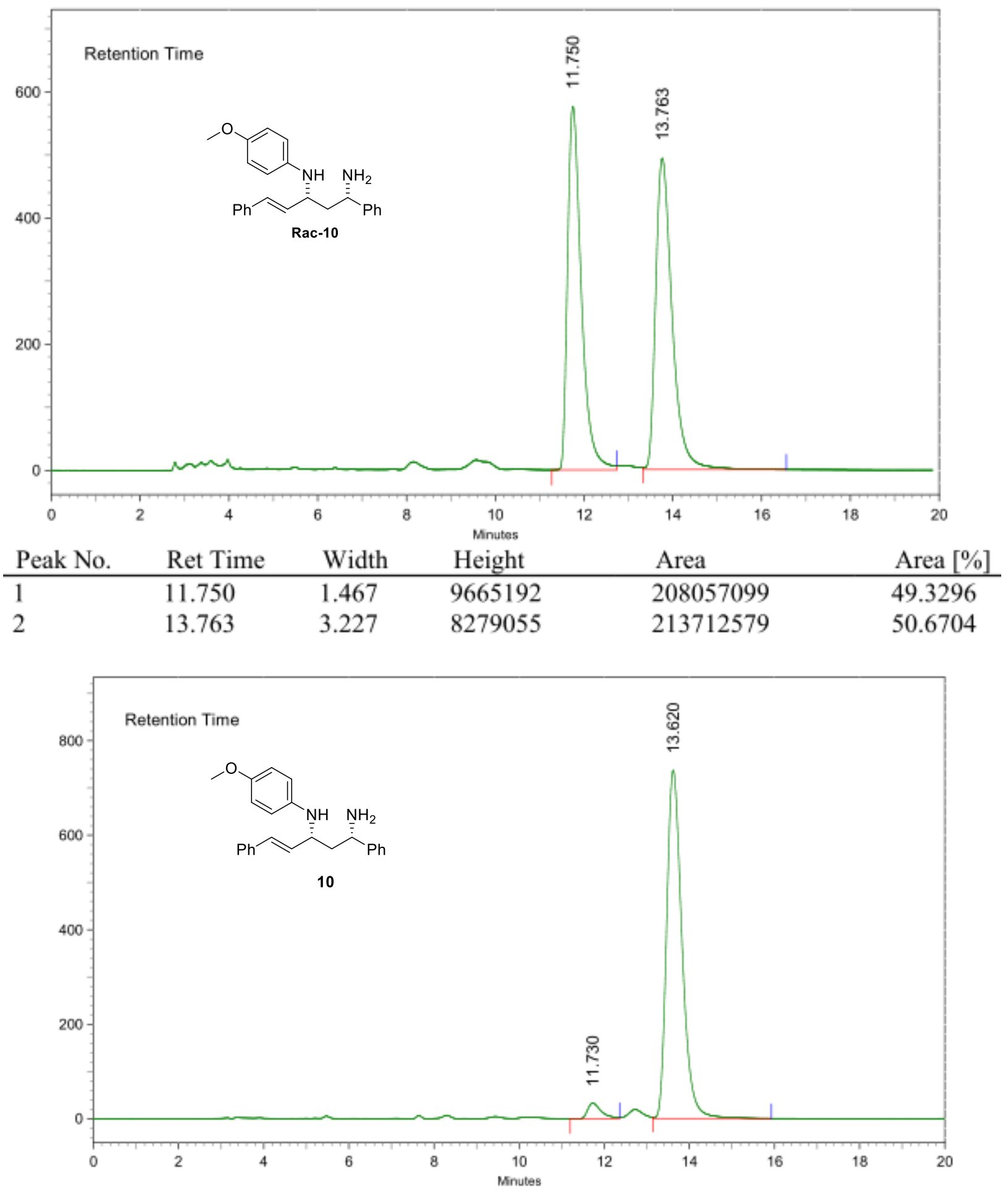

\begin{tabular}{llllll} 
Peak No. & Ret Time & Width & Height & Area & Area [\%] \\
\hline 1 & 11.730 & 1.160 & 550929 & 12434181 & 3.8680 \\
2 & 13.620 & 2.786 & 12356888 & 309030107 & 96.1320
\end{tabular}




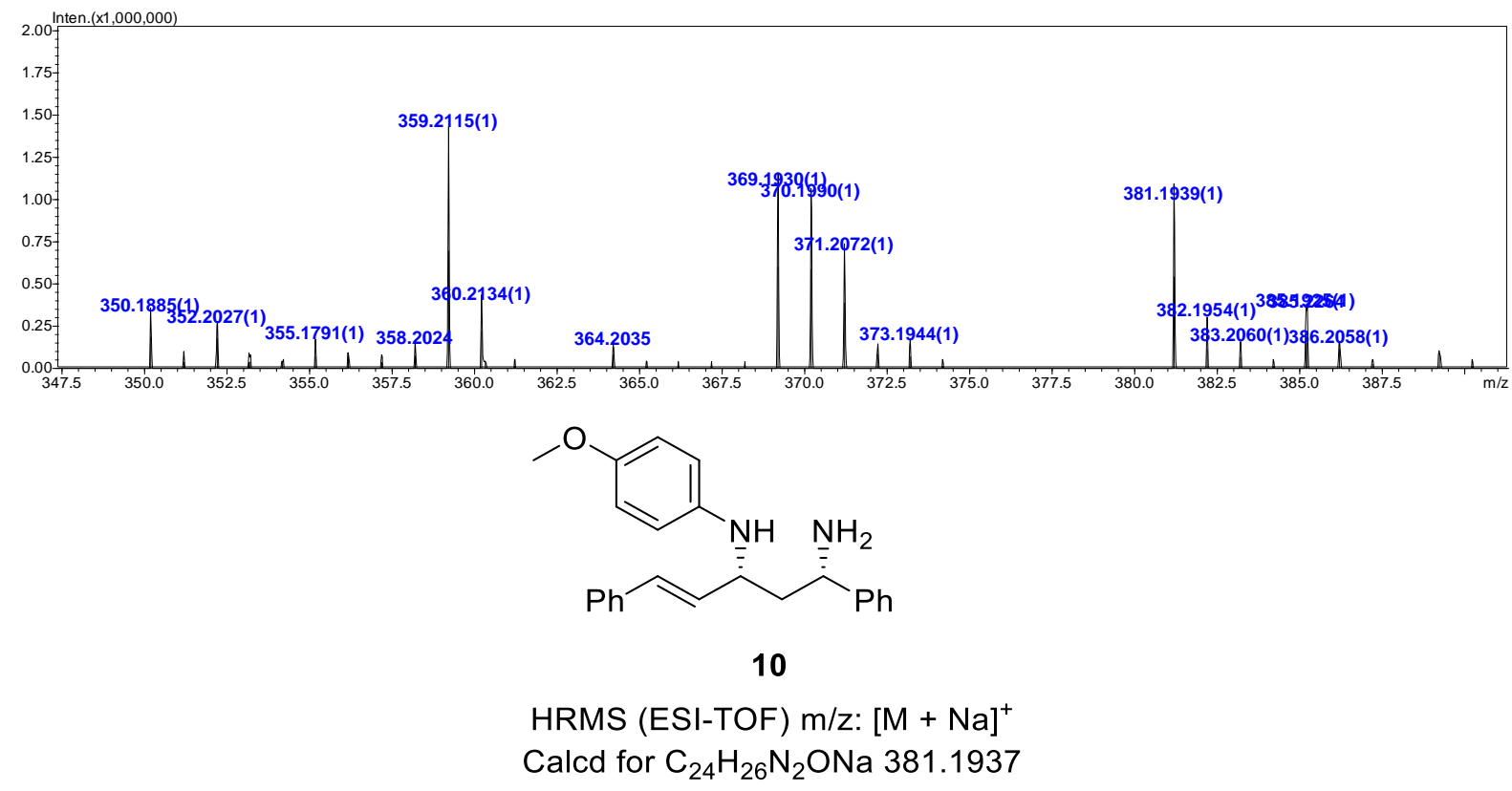




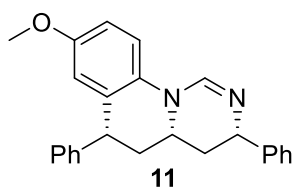

${ }^{1} \mathrm{H}-\mathrm{NMR}\left(400 \mathrm{MHz}, \mathrm{CDCl}_{3}\right)$

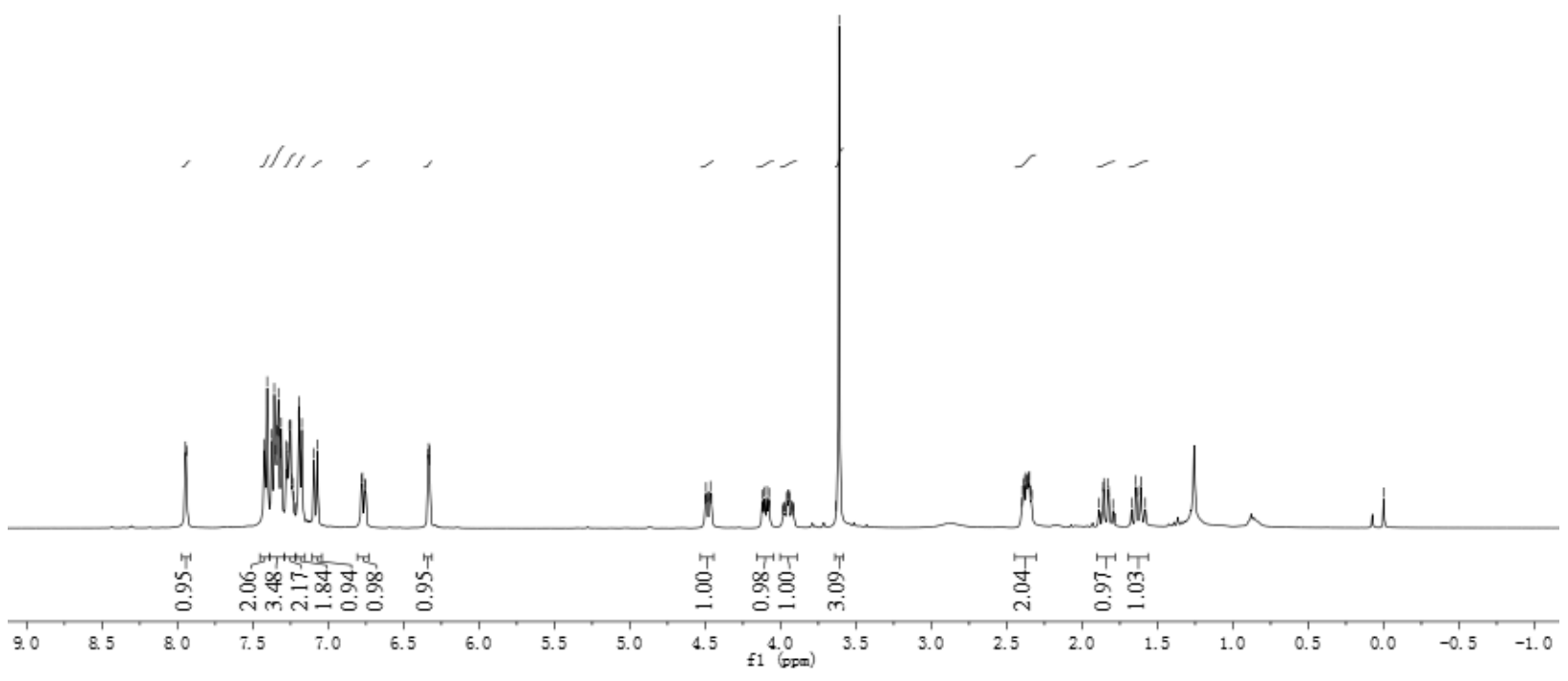

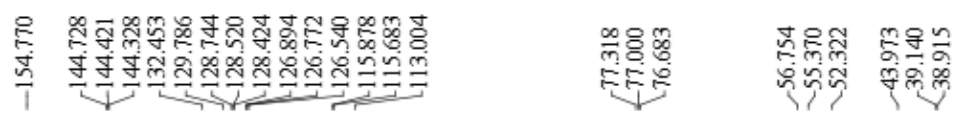
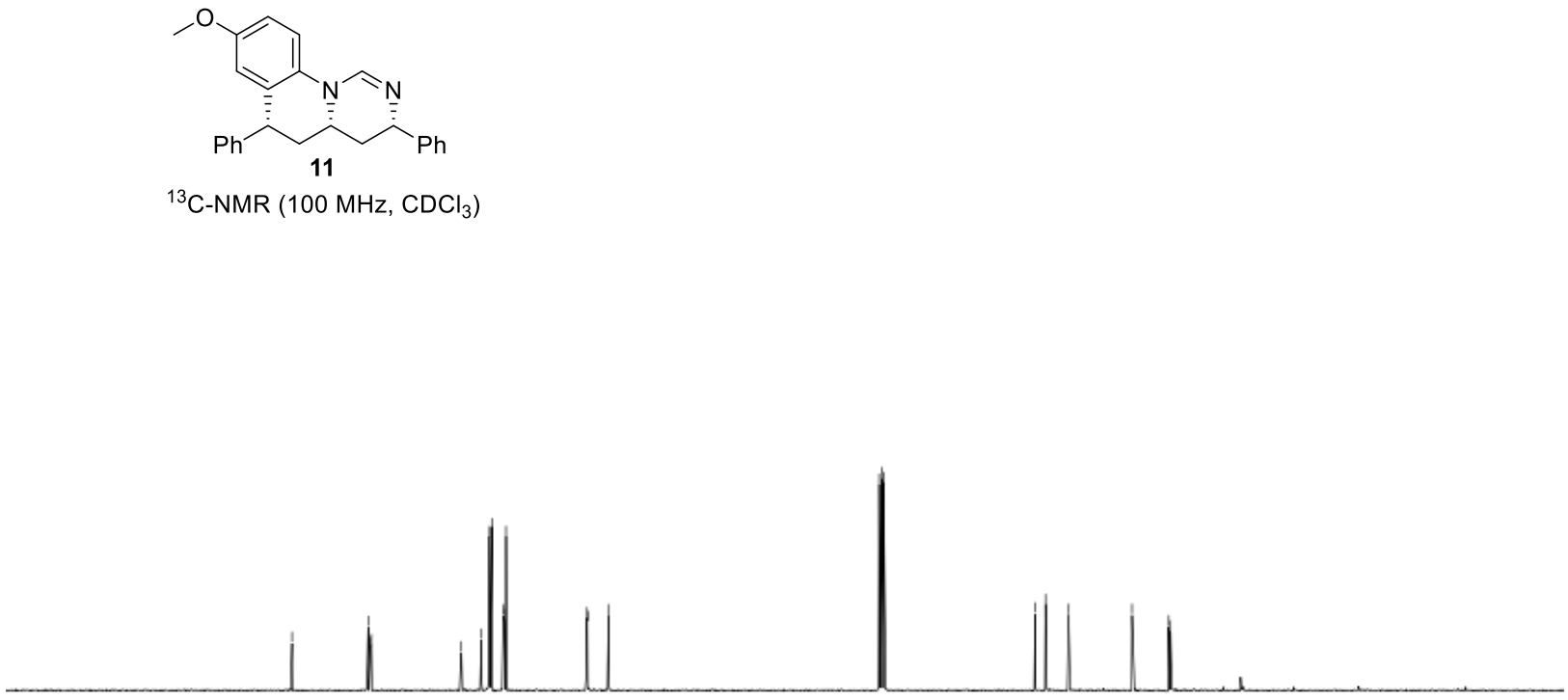

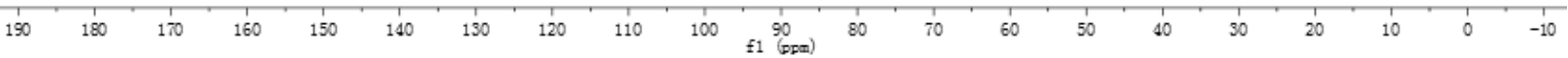




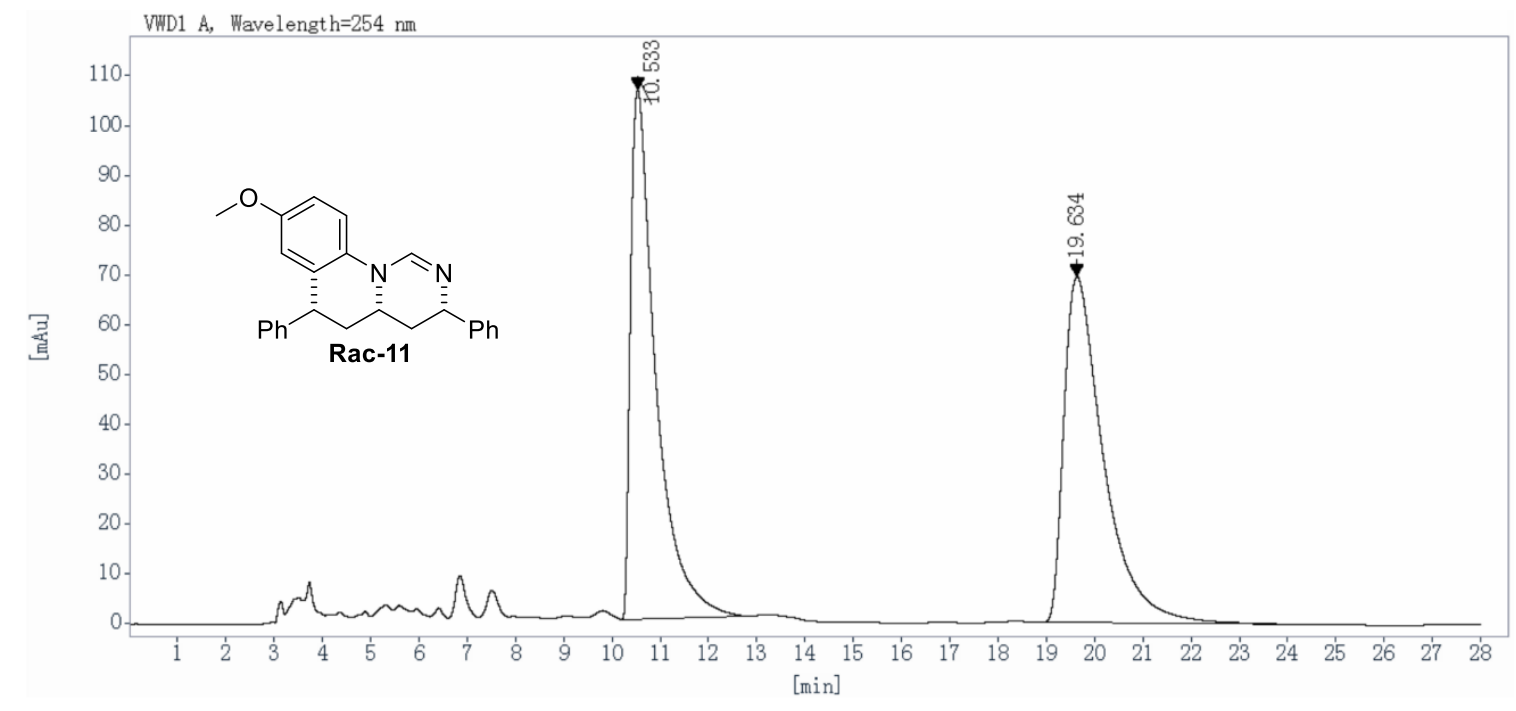

\begin{tabular}{cccccc}
$\begin{array}{c}\text { Ret Time } \\
{[\mathrm{min}]}\end{array}$ & $\begin{array}{c}\text { Peak } \\
\text { Type }\end{array}$ & $\begin{array}{c}\text { Width } \\
{[\mathrm{min}]}\end{array}$ & $\begin{array}{c}\text { Height } \\
{[\mathrm{mAU}]}\end{array}$ & $\begin{array}{c}\text { Area } \\
{[\mathrm{mAU} \text { *s] }}\end{array}$ & $\begin{array}{c}\text { Area } \\
{[\%]}\end{array}$ \\
\hline 10.533 & BB & 0.53 & 106.2691 & 3856.1362 & 49.1962 \\
19.634 & BB & 0.86 & 69.4401 & 3982.1514 & 50.8038
\end{tabular}

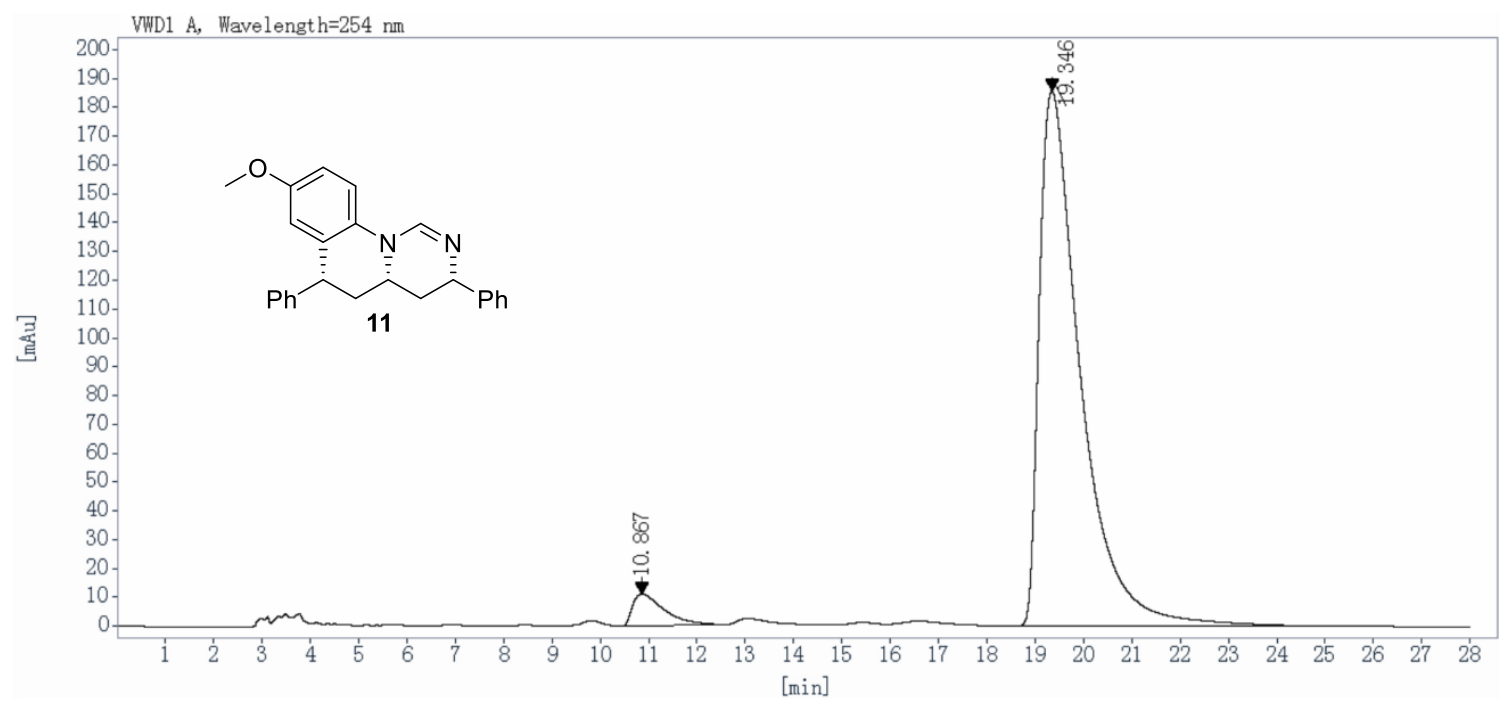

\begin{tabular}{cccccc}
$\begin{array}{c}\text { Ret Time } \\
{[\mathrm{min}]}\end{array}$ & $\begin{array}{c}\text { Peak } \\
\text { Type }\end{array}$ & $\begin{array}{c}\text { Width } \\
{[\mathrm{min}]}\end{array}$ & $\begin{array}{c}\text { Height } \\
{[\mathrm{mAU}]}\end{array}$ & $\begin{array}{c}\text { Area } \\
{[\mathrm{mAU} \text { *s] }}\end{array}$ & $\begin{array}{c}\text { Area } \\
{[\%]}\end{array}$ \\
\hline 10.867 & BBA & 0.66 & 11.0027 & 497.5057 & 4.3127 \\
19.346 & BB & 0.88 & 185.7681 & 11038.3955 & 95.6873
\end{tabular}


ESI+
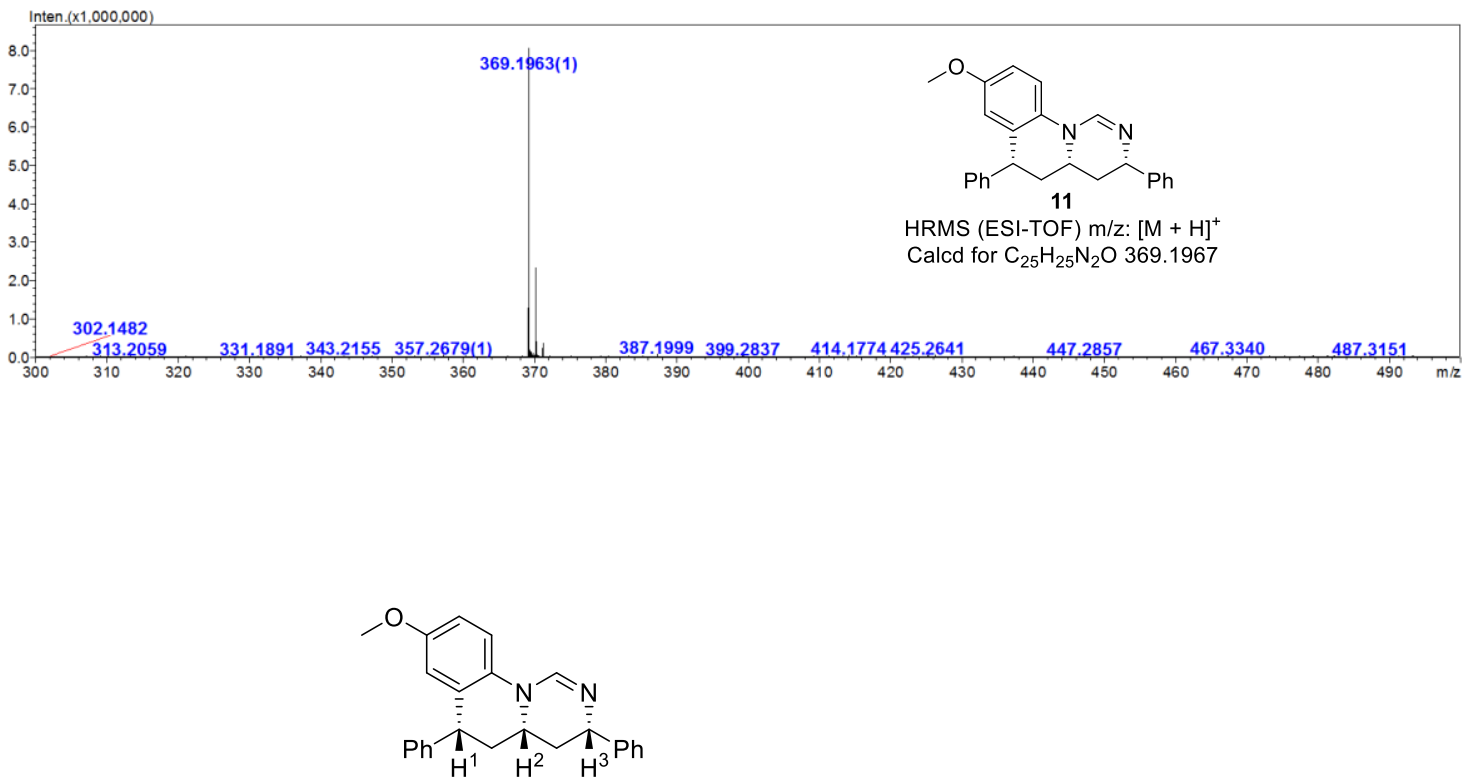

11

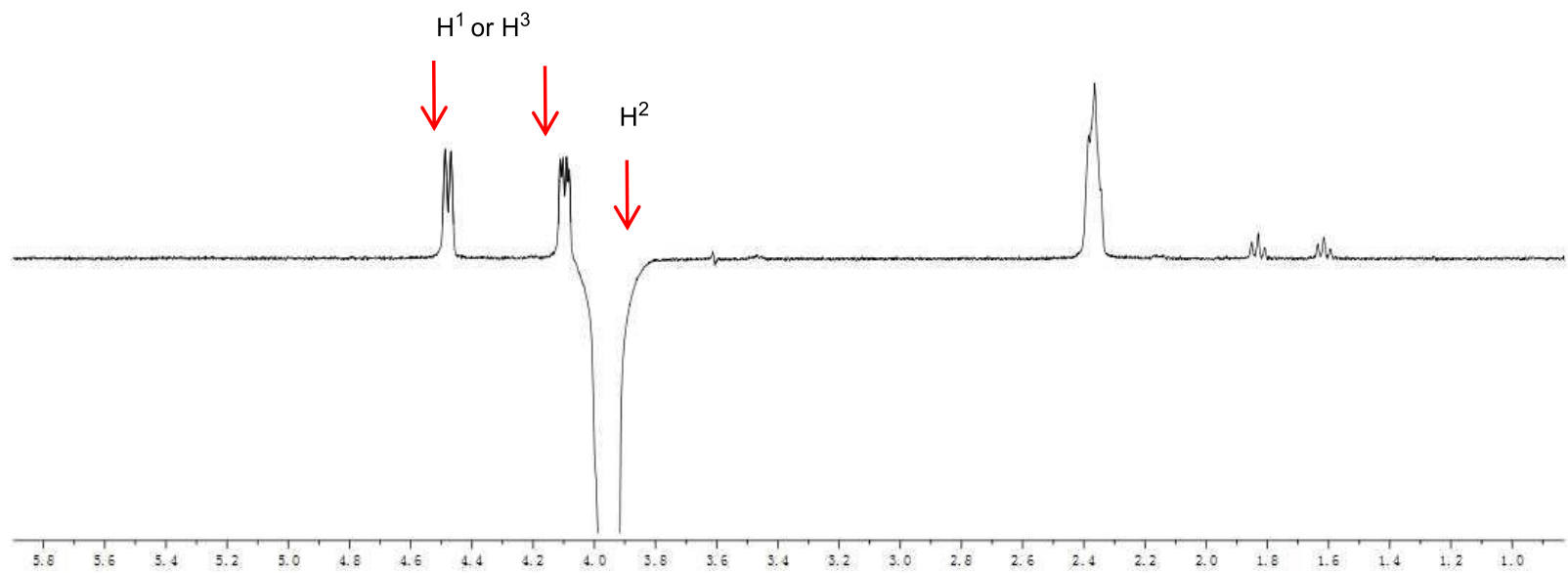




\section{Computational Method}

All calculations were carried out with the GAUSSIAN 09 packages. ${ }^{7}$ The conformations of intermediates were generated by SYBYL-X 2.0 GA Conf. search module and initially optimized and screened by SYBYL-X 2.0. ${ }^{8}$ The structures of ethylene, butadiene, and their Pd-complexes were calculated at $\mathrm{B} 3 \mathrm{LPY}^{9,10} / \mathrm{def} 2-\mathrm{TZVP}^{11}$ level and their molecular orbitals were calculated at B3LYP/def2-TZVPPD. The other geometries of all intermediates and transition states were optimized using B3LYP-D3 ${ }^{12,13}$ functional together with $\mathrm{SDD}^{14}$ basis set for Pd atom and the standard 6-31G(d) basis set for the others ${ }^{15}$ since these classical methods have been frequently used in the optimization of metal-complex. ${ }^{16-19}$ All the optimized structures were calculated after considering various conformations and confirmed by frequency calculations to be either minima or transition states using the same level of theory. For transition states, intrinsic reaction coordinate analysis (IRC) was done to verify that they connect the right reactants. ${ }^{20}$ To take solvent effects into account, solution-phase single-point calculations were performed on the gas-phase geometries. The solution-phase single point energy calculations were done using B3LYP-D3 at SDD for Pd atom and 6-311++G(d,p) ${ }^{21-23}$ level for the others. Solvent effect was accounted for using self-consistent reaction field (SCRF) method, using SMD model. ${ }^{24,25}$ Toluene was used as the solvent. Solution-phase single-point energies corrected by the gas-phase Gibbs free energy corrections were used to describe all the reaction energetics. The molecular orbitals were calculated at B3LYP/def2-TZVPPD. All these energies correspond to the reference state of $1 \mathrm{~mol} / \mathrm{L}, 298 \mathrm{~K}$. All energetics reported throughout the text are in $\mathrm{kcal} / \mathrm{mol}$, and the bond lengths are in angstroms $(\AA)$. Structures were generated using GaussView $6.0^{26}$ and CYLview ${ }^{27}$. 


\section{Computational data:}

2a

Zero-point correction= 0.241944 (Hartree/Particle $)$

Thermal correction to Energy= 0.258097

Thermal correction to Enthalpy $=0.259041$

Thermal correction to Gibbs Free Energy= 0.194941

$\mathrm{E}($ Solv $)=-1144.95239051$

\begin{tabular}{|c|c|c|c|}
\hline $\mathrm{C}$ & 5.084127 & 0.671643 & -0.993637 \\
\hline $\mathrm{C}$ & 3.916629 & -0.081903 & -1.090763 \\
\hline $\mathrm{C}$ & 2.891903 & 0.075468 & -0.145856 \\
\hline $\mathrm{C}$ & 3.050328 & 0.997187 & 0.903112 \\
\hline $\mathrm{C}$ & 4.215707 & 1.747170 & 0.997193 \\
\hline $\mathrm{C}$ & 5.233536 & 1.586169 & 0.049838 \\
\hline $\mathrm{H}$ & 5.874505 & 0.546020 & -1.727455 \\
\hline $\mathrm{H}$ & 3.791822 & -0.797500 & -1.899420 \\
\hline $\mathrm{H}$ & 2.250536 & 1.104141 & 1.628101 \\
\hline $\mathrm{H}$ & 4.337841 & 2.458606 & 1.808568 \\
\hline $\mathrm{H}$ & 6.143592 & 2.174137 & 0.128250 \\
\hline C & 1.678249 & -0.727386 & -0.279668 \\
\hline $\mathrm{N}$ & 0.693651 & -0.615200 & 0.538534 \\
\hline S & -0.626777 & -1.676828 & 0.243520 \\
\hline $\mathrm{O}$ & -0.460443 & -2.389316 & -1.034402 \\
\hline $\mathrm{O}$ & -0.827641 & -2.421292 & 1.488581 \\
\hline C & -1.938877 & -0.486517 & 0.057016 \\
\hline C & -2.438978 & -0.209891 & -1.213347 \\
\hline $\mathrm{C}$ & -2.440990 & 0.153830 & 1.191318 \\
\hline $\mathrm{C}$ & -3.460317 & 0.728730 & -1.344913 \\
\hline $\mathrm{H}$ & -2.034553 & -0.730302 & -2.074312 \\
\hline $\mathrm{C}$ & -3.457513 & 1.090261 & 1.039114 \\
\hline $\mathrm{H}$ & -2.042351 & -0.088897 & 2.170167 \\
\hline $\mathrm{C}$ & -3.983005 & 1.390204 & -0.226856 \\
\hline $\mathrm{H}$ & -3.859631 & 0.949769 & -2.331163 \\
\hline $\mathrm{H}$ & -3.854885 & 1.595820 & 1.915391 \\
\hline $\mathrm{C}$ & -5.107747 & 2.384414 & -0.372172 \\
\hline $\mathrm{H}$ & -5.166829 & 2.776272 & -1.392065 \\
\hline $\mathrm{H}$ & -6.074174 & 1.917734 & -0.141641 \\
\hline $\mathrm{H}$ & -4.984594 & 3.229690 & 0.313069 \\
\hline $\mathrm{H}$ & 1.637951 & -1.431250 & -1.119258 \\
\hline
\end{tabular}

\section{Acid-TS1A}

Zero-point correction= $1.130435($ Hartree/Particle $)$

Thermal correction to Energy $=1.203308$

Thermal correction to Enthalpy= 1.204252

Thermal correction to Gibbs Free Energy= 1.011135

$E($ Solv $)=-4308.12756576$

\begin{tabular}{|c|c|c|c|}
\hline $\mathrm{C}$ & 2.677580 & -4.181070 & -3.175971 \\
\hline $\mathrm{C}$ & 2.320596 & -3.881197 & -1.863380 \\
\hline $\mathrm{C}$ & 1.333117 & -2.921453 & -1.582259 \\
\hline $\mathrm{C}$ & 0.682612 & -2.296580 & -2.666436 \\
\hline $\mathrm{C}$ & 1.032494 & -2.610564 & -3.977292 \\
\hline $\mathrm{C}$ & 2.038813 & -3.544859 & -4.241986 \\
\hline $\mathrm{H}$ & 3.456641 & -4.914829 & -3.364151 \\
\hline $\mathrm{H}$ & 2.819064 & -4.383276 & -1.040631 \\
\hline $\mathrm{H}$ & -0.092595 & -1.560057 & -2.485949 \\
\hline $\mathrm{H}$ & 0.515947 & -2.120098 & -4.798433 \\
\hline $\mathrm{H}$ & 2.314097 & -3.779717 & -5.266265 \\
\hline $\mathrm{C}$ & 1.026241 & -2.588447 & -0.182848 \\
\hline $\mathrm{H}$ & 1.412764 & -3.293933 & 0.550995 \\
\hline $\mathrm{C}$ & -0.099441 & -1.803746 & 0.175698 \\
\hline $\mathrm{H}$ & -0.662596 & -1.280228 & -0.586018 \\
\hline $\mathrm{C}$ & -0.628329 & -1.727486 & 1.478235 \\
\hline $\mathrm{C}$ & -1.823927 & -1.030295 & 1.750089 \\
\hline $\mathrm{H}$ & -2.102331 & -0.860262 & 2.784755 \\
\hline $\mathrm{H}$ & -0.190294 & -2.368752 & 2.245045 \\
\hline $\mathrm{H}$ & -2.017569 & -0.185732 & 1.102797 \\
\hline $\mathrm{Pd}$ & 1.737698 & -0.607955 & 0.472364 \\
\hline $\mathrm{P}$ & 1.330625 & 1.608373 & 1.205636 \\
\hline $\mathrm{P}$ & 4.029750 & -0.812521 & 0.066051 \\
\hline $\mathrm{C}$ & 2.414388 & 2.124379 & 2.598032 \\
\hline $\mathrm{C}$ & 2.709851 & 3.461071 & 2.891713 \\
\hline $\mathrm{C}$ & 2.937921 & 1.113864 & 3.416340 \\
\hline $\mathrm{C}$ & 3.516811 & 3.779176 & 3.983399 \\
\hline $\mathrm{H}$ & 2.321201 & 4.254136 & 2.262165 \\
\hline $\mathrm{C}$ & 3.737185 & 1.432039 & 4.513018 \\
\hline $\mathrm{H}$ & 2.730542 & 0.075853 & 3.169624 \\
\hline $\mathrm{C}$ & 4.029829 & 2.766453 & 4.796549 \\
\hline
\end{tabular}




\begin{tabular}{|c|c|c|c|c|c|c|c|}
\hline $\mathrm{H}$ & 3.745283 & 4.819257 & 4.199696 & $\mathrm{H}$ & 3.349666 & -0.370014 & -4.796929 \\
\hline $\mathrm{H}$ & 4.147831 & 0.639055 & 5.130767 & $\mathrm{H}$ & 7.191694 & 0.962246 & -3.377973 \\
\hline $\mathrm{H}$ & 4.662399 & 3.016767 & 5.643543 & $\mathrm{H}$ & 5.598013 & 0.613858 & -5.253170 \\
\hline $\mathrm{C}$ & 1.586391 & 2.859895 & -0.114570 & $\mathrm{C}$ & 4.609743 & -2.544283 & 0.240300 \\
\hline $\mathrm{C}$ & 1.088186 & 4.170256 & -0.035093 & $\mathrm{C}$ & 5.460373 & -3.157191 & -0.686548 \\
\hline $\mathrm{C}$ & 2.306970 & 2.478919 & -1.254603 & $\mathrm{C}$ & 4.120252 & -3.294998 & 1.320558 \\
\hline $\mathrm{C}$ & 1.336948 & 5.081388 & -1.060262 & $\mathrm{C}$ & 5.808849 & -4.501157 & -0.539407 \\
\hline $\mathrm{H}$ & 0.481642 & 4.467331 & 0.814213 & $\mathrm{H}$ & 5.834749 & -2.591518 & -1.532565 \\
\hline $\mathrm{C}$ & 2.564664 & 3.392734 & -2.276785 & $\mathrm{C}$ & 4.479397 & -4.632488 & 1.474538 \\
\hline $\mathrm{H}$ & 2.656966 & 1.458938 & -1.347091 & $\mathrm{H}$ & 3.438918 & -2.827971 & 2.027431 \\
\hline $\mathrm{C}$ & 2.084554 & 4.698740 & -2.177149 & $\mathrm{C}$ & 5.319983 & -5.241197 & 0.537720 \\
\hline $\mathrm{H}$ & 0.937910 & 6.089250 & -0.991869 & $\mathrm{H}$ & 6.459244 & -4.970731 & -1.272026 \\
\hline $\mathrm{H}$ & 3.130228 & 3.072936 & -3.147662 & $\mathrm{H}$ & 4.093001 & -5.203575 & 2.313995 \\
\hline $\mathrm{H}$ & 2.275659 & 5.413566 & -2.972784 & $\mathrm{H}$ & 5.587748 & -6.288224 & 0.646524 \\
\hline $\mathrm{C}$ & -0.337803 & 2.001453 & 1.848678 & $\mathrm{C}$ & -1.756174 & -5.297599 & 0.015387 \\
\hline $\mathrm{C}$ & -0.634108 & 1.818696 & 3.206660 & $\mathrm{C}$ & -2.338331 & -4.302861 & 0.796679 \\
\hline $\mathrm{C}$ & -1.369695 & 2.348593 & 0.963292 & $\mathrm{C}$ & -2.841034 & -3.131257 & 0.213711 \\
\hline $\mathrm{C}$ & -1.943094 & 1.969551 & 3.666208 & $\mathrm{C}$ & -2.765025 & -2.985542 & -1.176017 \\
\hline $\mathrm{H}$ & 0.155353 & 1.555026 & 3.903919 & $\mathrm{C}$ & -2.179925 & -3.980517 & -1.959832 \\
\hline $\mathrm{C}$ & -2.675146 & 2.498710 & 1.428413 & $\mathrm{C}$ & -1.668672 & -5.135959 & -1.370264 \\
\hline $\mathrm{H}$ & -1.156657 & 2.485626 & -0.089826 & $\mathrm{H}$ & -1.373533 & -6.199925 & 0.485023 \\
\hline $\mathrm{C}$ & -2.967615 & 2.297950 & 2.778785 & $\mathrm{H}$ & -2.392261 & -4.423041 & 1.876717 \\
\hline $\mathrm{H}$ & -2.164079 & 1.812352 & 4.718296 & $\mathrm{H}$ & -3.144914 & -2.085211 & -1.639822 \\
\hline $\mathrm{H}$ & -3.472828 & 2.743435 & 0.738740 & $\mathrm{H}$ & -2.107834 & -3.842545 & -3.034435 \\
\hline $\mathrm{H}$ & -3.993732 & 2.367483 & 3.121069 & $\mathrm{H}$ & -1.202316 & -5.902102 & -1.982782 \\
\hline $\mathrm{C}$ & 5.182176 & 0.134281 & 1.136050 & C & -3.383069 & -2.064935 & 1.098204 \\
\hline $\mathrm{C}$ & 6.033024 & -0.481772 & 2.060808 & $\mathrm{~N}$ & -4.227521 & -1.153801 & 0.518988 \\
\hline $\mathrm{C}$ & 5.164041 & 1.536474 & 1.052840 & $\mathrm{~S}$ & -5.265915 & -0.366859 & 1.529459 \\
\hline $\mathrm{C}$ & 6.849040 & 0.290860 & 2.890464 & $\mathrm{O}$ & -4.915747 & -0.565440 & 2.952271 \\
\hline $\mathrm{H}$ & 6.065440 & -1.563573 & 2.131512 & $\mathrm{O}$ & -5.441051 & 1.004680 & 1.017582 \\
\hline $\mathrm{C}$ & 5.987345 & 2.302487 & 1.871359 & C & -6.819012 & -1.212776 & 1.244606 \\
\hline $\mathrm{H}$ & 4.502588 & 2.029104 & 0.347077 & $\mathrm{C}$ & -7.346077 & -2.048491 & 2.224178 \\
\hline $\mathrm{C}$ & 6.829029 & 1.681437 & 2.797579 & $\mathrm{C}$ & -7.468354 & -1.045011 & 0.018262 \\
\hline $\mathrm{H}$ & 7.504303 & -0.199311 & 3.605296 & $\mathrm{C}$ & -8.540614 & -2.725329 & 1.970539 \\
\hline $\mathrm{H}$ & 5.953871 & 3.385171 & 1.802939 & $\mathrm{H}$ & -6.827722 & -2.150750 & 3.171150 \\
\hline $\mathrm{H}$ & 7.461111 & 2.281276 & 3.446008 & $\mathrm{C}$ & -8.655155 & -1.727787 & -0.219191 \\
\hline $\mathrm{C}$ & 4.578612 & -0.340320 & -1.621946 & $\mathrm{H}$ & -7.044343 & -0.383001 & -0.729553 \\
\hline $\mathrm{C}$ & 3.686385 & -0.538301 & -2.684915 & $\mathrm{C}$ & -9.209098 & -2.579277 & 0.750990 \\
\hline $\mathrm{C}$ & 5.847231 & 0.197663 & -1.883144 & $\mathrm{H}$ & -8.960281 & -3.375143 & 2.734459 \\
\hline $\mathrm{C}$ & 4.052951 & -0.201533 & -3.986900 & $\mathrm{H}$ & -9.165654 & -1.599842 & -1.170851 \\
\hline $\mathrm{H}$ & 2.705155 & -0.954533 & -2.495211 & $\mathrm{C}$ & -10.496910 & -3.315589 & 0.473340 \\
\hline $\mathrm{C}$ & 6.208875 & 0.540668 & -3.186314 & $\mathrm{H}$ & -10.814306 & -3.905701 & 1.338481 \\
\hline $\mathrm{H}$ & 6.546877 & 0.355556 & -1.069485 & $\mathrm{H}$ & -11.307772 & -2.620146 & 0.224479 \\
\hline $\mathrm{C}$ & 5.313016 & 0.344232 & -4.240000 & $\mathrm{H}$ & -10.387838 & -3.999731 & -0.377415 \\
\hline
\end{tabular}




\begin{tabular}{llll}
$\mathrm{H}$ & -3.667816 & -2.427506 & 2.087188 \\
$\mathrm{C}$ & -2.670177 & 0.872910 & -1.792843 \\
$\mathrm{O}$ & -1.694885 & 0.153407 & -1.581176 \\
$\mathrm{O}$ & -3.911171 & 0.510152 & -1.526994 \\
$\mathrm{H}$ & -3.952804 & -0.236766 & -0.816619 \\
$\mathrm{C}$ & -2.501255 & 2.238614 & -2.386188 \\
$\mathrm{C}$ & -3.450272 & 3.312788 & -2.262749 \\
$\mathrm{C}$ & -1.293467 & 2.451193 & -3.032380 \\
$\mathrm{C}$ & -4.684655 & 3.226415 & -1.555599 \\
$\mathrm{C}$ & -3.104150 & 4.579039 & -2.852184 \\
$\mathrm{C}$ & -0.973438 & 3.691317 & -3.614927 \\
$\mathrm{H}$ & -0.585542 & 1.631255 & -3.071181 \\
$\mathrm{C}$ & -5.518536 & 4.318543 & -1.453530 \\
$\mathrm{H}$ & -4.963766 & 2.296548 & -1.081657 \\
$\mathrm{C}$ & -3.998396 & 5.676793 & -2.737049 \\
$\mathrm{C}$ & -1.866997 & 4.732804 & -3.528360 \\
$\mathrm{H}$ & -0.019105 & 3.819209 & -4.114506 \\
$\mathrm{C}$ & -5.183976 & 5.554203 & -2.053010 \\
$\mathrm{H}$ & -6.446617 & 4.222893 & -0.897267 \\
$\mathrm{H}$ & -3.717687 & 6.621626 & -3.196265 \\
$\mathrm{H}$ & -1.634180 & 5.699877 & -3.968037 \\
----------------------------------- & \\
\hline
\end{tabular}

$\mathrm{H}$

C

$\mathrm{H}$

C

C

$\mathrm{H}$

$\mathrm{H}$

$\mathrm{H}$

$\mathrm{Pd}$

$\mathrm{P}$

C

$\mathrm{C}$

C

C

$\mathrm{H}$

$\mathrm{C}$

$\mathrm{H}$

C

$\mathrm{H}$

$\mathrm{H}$

$\mathrm{H}$

$\mathrm{C}$

C

C

\section{INT1C}

Zero-point correction $=0.688486($ Hartree/Particle $)$

Thermal correction to Energy $=0.734089$

Thermal correction to Enthalpy $=0.735033$

Thermal correction to Gibbs Free Energy= 0.601902

$\mathrm{E}($ Solv $)=-2696.81100608$

$\begin{array}{llll}\mathrm{C} & -1.710897 & -3.805456 & -2.433203 \\ \mathrm{C} & -1.929360 & -2.432231 & -2.524385 \\ \mathrm{C} & -0.870619 & -1.547389 & -2.799497 \\ \mathrm{C} & 0.414391 & -2.085897 & -3.008393 \\ \mathrm{C} & 0.627063 & -3.457764 & -2.923871 \\ \mathrm{C} & -0.429966 & -4.324991 & -2.627898 \\ \mathrm{H} & -2.541819 & -4.469689 & -2.211057 \\ \mathrm{H} & -2.924295 & -2.025063 & -2.362903 \\ \mathrm{H} & 1.251439 & -1.423757 & -3.201547 \\ \mathrm{H} & 1.626546 & -3.853866 & -3.081393 \\ \mathrm{H} & -0.256152 & -5.394941 & -2.557810 \\ \mathrm{C} & -1.144186 & -0.103027 & -2.801443\end{array}$

\begin{tabular}{|c|c|c|}
\hline-2.192610 & 0.169500 & -2.700106 \\
\hline-0.237344 & 0.908935 & -3.015333 \\
\hline 0.804825 & 0.694525 & -3.235143 \\
\hline-0.631057 & 2.310876 & -3.068520 \\
\hline 0.237765 & 3.319121 & -3.243535 \\
\hline 1.304328 & 3.131623 & -3.322696 \\
\hline-1.694376 & 2.516994 & -2.950510 \\
\hline-0.095735 & 4.352040 & -3.275747 \\
\hline-0.122350 & 0.278997 & -0.668353 \\
\hline-2.021108 & 0.556692 & 0.568314 \\
\hline-2.012401 & 2.083045 & 1.577799 \\
\hline-3.163370 & 2.541750 & 2.239252 \\
\hline-0.828697 & 2.831293 & 1.655027 \\
\hline-3.120738 & 3.712751 & 2.993135 \\
\hline-4.093519 & 1.987949 & 2.150732 \\
\hline-0.792117 & 4.006688 & 2.408587 \\
\hline 0.051818 & 2.512579 & 1.104604 \\
\hline-1.932488 & 4.444126 & 3.082295 \\
\hline-4.014631 & 4.059988 & 3.503797 \\
\hline 0.127443 & 4.582750 & 2.455028 \\
\hline-1.902650 & 5.360090 & 3.665889 \\
\hline-2.354300 & -0.838331 & 1.706730 \\
\hline-2.175653 & -2.130598 & 1.185325 \\
\hline-2.750680 & -0.686565 & 3.040465 \\
\hline-2.436999 & -3.248503 & 1.972405 \\
\hline-1.812477 & -2.257001 & 0.170019 \\
\hline-2.993107 & -1.810921 & 3.831724 \\
\hline-2.863279 & 0.304758 & 3.465520 \\
\hline-2.848352 & -3.091410 & 3.297522 \\
\hline-2.287016 & -4.240706 & 1.558458 \\
\hline-3.296347 & -1.683370 & 4.867356 \\
\hline-3.037269 & -3.964139 & 3.916370 \\
\hline-3.584326 & 0.725567 & -0.384898 \\
\hline-4.478388 & -0.337536 & -0.561832 \\
\hline-3.811158 & 1.927993 & -1.074576 \\
\hline-5.573220 & -0.203552 & -1.419269 \\
\hline-4.322215 & -1.269681 & -0.029321 \\
\hline-4.906174 & 2.060417 & -1.925739 \\
\hline-3.125297 & 2.759754 & -0.941741 \\
\hline-5.788829 & 0.991670 & -2.105343 \\
\hline-6.260383 & -1.035743 & -1.545322 \\
\hline-5.070056 & 2.997418 & -2.450657 \\
\hline-6.639714 & 1.092991 & -2.772702 \\
\hline 0.644294 & -2.723800 & 3.352631 \\
\hline
\end{tabular}




\begin{tabular}{|c|c|c|c|}
\hline $\mathrm{C}$ & 0.849364 & -1.499851 & 2.721033 \\
\hline $\mathrm{C}$ & 1.195699 & -1.447020 & 1.362922 \\
\hline $\mathrm{C}$ & 1.319531 & -2.646522 & 0.643949 \\
\hline $\mathrm{C}$ & 1.110819 & -3.867924 & 1.276904 \\
\hline $\mathrm{C}$ & 0.777615 & -3.913168 & 2.633896 \\
\hline $\mathrm{H}$ & 0.369109 & -2.748635 & 4.402838 \\
\hline $\mathrm{H}$ & 0.736163 & -0.572610 & 3.277224 \\
\hline $\mathrm{H}$ & 1.573132 & -2.601744 & -0.408499 \\
\hline $\mathrm{H}$ & 1.207015 & -4.788700 & 0.707678 \\
\hline $\mathrm{H}$ & 0.614679 & -4.868240 & 3.125620 \\
\hline $\mathrm{C}$ & 1.411490 & -0.135831 & 0.722141 \\
\hline $\mathrm{N}$ & 2.005133 & -0.082289 & -0.517177 \\
\hline S & 2.834780 & 1.330625 & -0.862675 \\
\hline $\mathrm{O}$ & 2.913702 & 1.423923 & -2.330153 \\
\hline $\mathrm{O}$ & 2.336852 & 2.486880 & -0.086677 \\
\hline $\mathrm{C}$ & 4.471035 & 0.948343 & -0.248587 \\
\hline $\mathrm{C}$ & 5.285540 & 0.076929 & -0.974882 \\
\hline $\mathrm{C}$ & 4.895764 & 1.489828 & 0.961755 \\
\hline $\mathrm{C}$ & 6.539430 & -0.252072 & -0.472320 \\
\hline $\mathrm{H}$ & 4.936257 & -0.323858 & -1.920086 \\
\hline $\mathrm{C}$ & 6.156984 & 1.148970 & 1.450822 \\
\hline $\mathrm{H}$ & 4.248408 & 2.174538 & 1.498238 \\
\hline $\mathrm{C}$ & 6.993903 & 0.276379 & 0.746397 \\
\hline $\mathrm{H}$ & 7.179871 & -0.929191 & -1.032140 \\
\hline $\mathrm{H}$ & 6.496694 & 1.569766 & 2.393628 \\
\hline $\mathrm{C}$ & 8.363919 & -0.079565 & 1.268925 \\
\hline $\mathrm{H}$ & 9.151082 & 0.385575 & 0.661875 \\
\hline $\mathrm{H}$ & 8.498209 & 0.257155 & 2.301282 \\
\hline $\mathrm{H}$ & 8.532009 & -1.162240 & 1.240171 \\
\hline $\mathrm{H}$ & 1.527628 & 0.710017 & 1.401538 \\
\hline
\end{tabular}

\begin{tabular}{|c|c|c|c|}
\hline $\mathrm{C}$ & 6.361054 & -1.715494 & -1.096692 \\
\hline $\mathrm{C}$ & 6.950674 & -0.557741 & -1.612813 \\
\hline $\mathrm{H}$ & 7.015034 & 1.595102 & -1.505222 \\
\hline $\mathrm{H}$ & 5.282115 & 1.744292 & 0.252895 \\
\hline $\mathrm{H}$ & 4.941413 & -2.538849 & 0.285066 \\
\hline $\mathrm{H}$ & 6.670486 & -2.691387 & -1.461741 \\
\hline $\mathrm{H}$ & 7.713334 & -0.627796 & -2.383186 \\
\hline $\mathrm{C}$ & 3.880675 & -0.229577 & 1.354812 \\
\hline $\mathrm{H}$ & 3.789259 & 0.753679 & 1.812823 \\
\hline $\mathrm{C}$ & 2.933175 & -1.148062 & 1.633125 \\
\hline $\mathrm{H}$ & 2.961902 & -2.124479 & 1.150901 \\
\hline $\mathrm{C}$ & 1.786708 & -0.904690 & 2.500882 \\
\hline $\mathrm{C}$ & 0.892981 & -1.910087 & 2.889238 \\
\hline $\mathrm{H}$ & 0.253620 & -1.772942 & 3.756562 \\
\hline $\mathrm{H}$ & 1.788485 & 0.038787 & 3.045412 \\
\hline $\mathrm{H}$ & 1.059884 & -2.937995 & 2.574531 \\
\hline $\mathrm{Pd}$ & -0.021207 & -0.804848 & 1.228993 \\
\hline $\mathrm{P}$ & 0.533325 & 1.106919 & -0.055959 \\
\hline $\mathrm{C}$ & -0.789346 & 1.933842 & -1.028277 \\
\hline $\mathrm{C}$ & -1.197011 & 3.255645 & -0.821624 \\
\hline $\mathrm{C}$ & -1.399496 & 1.180803 & -2.043420 \\
\hline $\mathrm{C}$ & -2.185359 & 3.821371 & -1.631677 \\
\hline $\mathrm{H}$ & -0.743415 & 3.845837 & -0.032920 \\
\hline $\mathrm{C}$ & -2.376529 & 1.748922 & -2.856093 \\
\hline $\mathrm{H}$ & -1.102466 & 0.148148 & -2.195699 \\
\hline $\mathrm{C}$ & -2.769426 & 3.075101 & -2.655652 \\
\hline $\mathrm{H}$ & -2.491187 & 4.850434 & -1.464049 \\
\hline $\mathrm{H}$ & -2.841092 & 1.153828 & -3.636900 \\
\hline $\mathrm{H}$ & -3.531825 & 3.519983 & -3.289200 \\
\hline $\mathrm{C}$ & 1.776298 & 0.813662 & -1.370019 \\
\hline $\mathrm{C}$ & 2.238670 & -0.490429 & -1.580890 \\
\hline $\mathrm{C}$ & 2.241816 & 1.847736 & -2.197985 \\
\hline $\mathrm{C}$ & 3.181904 & -0.753558 & -2.574373 \\
\hline $\mathrm{H}$ & 1.860145 & -1.293244 & -0.957127 \\
\hline $\mathrm{C}$ & 3.193021 & 1.585819 & -3.181518 \\
\hline $\mathrm{H}$ & 1.854143 & 2.854742 & -2.072948 \\
\hline $\mathrm{C}$ & 3.672145 & 0.285482 & -3.363662 \\
\hline $\mathrm{H}$ & 3.549780 & -1.764820 & -2.713163 \\
\hline $\mathrm{H}$ & 3.559071 & 2.394348 & -3.808234 \\
\hline $\mathrm{H}$ & 4.424356 & 0.084123 & -4.120550 \\
\hline $\mathrm{C}$ & 1.209663 & 2.420631 & 1.024078 \\
\hline $\mathrm{C}$ & 2.475566 & 2.995890 & 0.861859 \\
\hline $\mathrm{C}$ & 0.430019 & 2.773957 & 2.140889 \\
\hline $\mathrm{C}$ & 2.954478 & 3.917690 & 1.796010 \\
\hline
\end{tabular}




\begin{tabular}{|c|c|c|c|}
\hline $\mathrm{H}$ & 3.093479 & 2.716625 & 0.016501 \\
\hline $\mathrm{C}$ & 0.907374 & 3.705762 & 3.060757 \\
\hline $\mathrm{H}$ & -0.542845 & 2.310054 & 2.281844 \\
\hline $\mathrm{C}$ & 2.172302 & 4.277041 & 2.893448 \\
\hline $\mathrm{H}$ & 3.941344 & 4.353083 & 1.663617 \\
\hline $\mathrm{H}$ & 0.294769 & 3.977138 & 3.915918 \\
\hline $\mathrm{H}$ & 2.546872 & 4.994052 & 3.618536 \\
\hline C & 0.118593 & -5.436889 & -0.123125 \\
\hline $\mathrm{C}$ & -0.565247 & -4.514355 & 0.665138 \\
\hline $\mathrm{C}$ & -0.974703 & -3.283328 & 0.127943 \\
\hline $\mathrm{C}$ & -0.706439 & -3.005528 & -1.224462 \\
\hline $\mathrm{C}$ & -0.020510 & -3.928560 & -2.008109 \\
\hline C & 0.399864 & -5.144586 & -1.460520 \\
\hline $\mathrm{H}$ & 0.431552 & -6.385153 & 0.304723 \\
\hline $\mathrm{H}$ & -0.777581 & -4.734869 & 1.708287 \\
\hline $\mathrm{H}$ & -1.043413 & -2.061676 & -1.637345 \\
\hline H & 0.186428 & -3.700448 & -3.050044 \\
\hline $\mathrm{H}$ & 0.935284 & -5.863330 & -2.074223 \\
\hline $\mathrm{C}$ & -1.622733 & -2.290349 & 0.998690 \\
\hline $\mathrm{N}$ & -2.051140 & -1.125437 & 0.471211 \\
\hline S & -3.087916 & -0.219658 & 1.491971 \\
\hline $\mathrm{O}$ & -3.496276 & -1.009677 & 2.667449 \\
\hline $\mathrm{O}$ & -2.495077 & 1.113169 & 1.685173 \\
\hline C & -4.495923 & -0.030714 & 0.416284 \\
\hline $\mathrm{C}$ & -5.476353 & -1.023318 & 0.383779 \\
\hline $\mathrm{C}$ & -4.583149 & 1.104543 & -0.385039 \\
\hline $\mathrm{C}$ & -6.556751 & -0.871772 & -0.479756 \\
\hline $\mathrm{H}$ & -5.396312 & -1.885285 & 1.037374 \\
\hline $\mathrm{C}$ & -5.672451 & 1.237316 & -1.243424 \\
\hline $\mathrm{H}$ & -3.816771 & 1.867093 & -0.328429 \\
\hline $\mathrm{C}$ & -6.668163 & 0.255073 & -1.308987 \\
\hline $\mathrm{H}$ & -7.329943 & -1.635269 & -0.508866 \\
\hline $\mathrm{H}$ & -5.746494 & 2.120415 & -1.872217 \\
\hline C & -7.829628 & 0.391385 & -2.262389 \\
\hline $\mathrm{H}$ & -7.963880 & 1.428907 & -2.583511 \\
\hline $\mathrm{H}$ & -7.671439 & -0.215490 & -3.163561 \\
\hline $\mathrm{H}$ & -8.765034 & 0.052025 & -1.804481 \\
\hline $\mathrm{H}$ & -1.990456 & -2.619904 & 1.969535 \\
\hline
\end{tabular}

\section{INT1E}

Zero-point correction= 0.853766 (Hartree/Particle)

Thermal correction to Energy= 0.910514
Thermal correction to Enthalpy $=0.911458$

Thermal correction to Gibbs Free Energy= 0.755502

$\mathrm{E}($ Solv $)=-3271.50196459$

\begin{tabular}{|c|c|c|c|}
\hline $\mathrm{C}$ & -5.342680 & -2.031523 & -1.791178 \\
\hline $\mathrm{C}$ & -4.069402 & -1.553610 & -2.094119 \\
\hline $\mathrm{C}$ & -2.914588 & -2.262521 & -1.717347 \\
\hline $\mathrm{C}$ & -3.088720 & -3.489635 & -1.047171 \\
\hline $\mathrm{C}$ & -4.360793 & -3.976034 & -0.760821 \\
\hline $\mathrm{C}$ & -5.498491 & -3.247314 & -1.122362 \\
\hline $\mathrm{H}$ & -6.212461 & -1.446619 & -2.076329 \\
\hline $\mathrm{H}$ & -3.961299 & -0.604894 & -2.608277 \\
\hline $\mathrm{H}$ & -2.220517 & -4.057549 & -0.730580 \\
\hline $\mathrm{H}$ & -4.465801 & -4.926472 & -0.243836 \\
\hline $\mathrm{H}$ & -6.489986 & -3.624163 & -0.888274 \\
\hline $\mathrm{C}$ & -1.585372 & -1.704744 & -2.037829 \\
\hline $\mathrm{H}$ & -1.598637 & -0.872679 & -2.740705 \\
\hline $\mathrm{C}$ & -0.374615 & -2.390790 & -1.848036 \\
\hline $\mathrm{H}$ & -0.394889 & -3.378192 & -1.390112 \\
\hline $\mathrm{C}$ & 0.832984 & -2.094792 & -2.608347 \\
\hline $\mathrm{C}$ & 1.853836 & -2.948536 & -2.788410 \\
\hline $\mathrm{H}$ & 1.832425 & -3.950750 & -2.364833 \\
\hline H & 0.887802 & -1.112704 & -3.072432 \\
\hline $\mathrm{H}$ & 2.738674 & -2.668025 & -3.349225 \\
\hline Pd & -0.742303 & -0.947658 & -0.164009 \\
\hline$P$ & -2.193206 & 0.525990 & 0.897967 \\
\hline $\mathrm{C}$ & -1.655954 & 1.993716 & 1.869968 \\
\hline $\mathrm{C}$ & -2.433772 & 3.153823 & 1.985283 \\
\hline $\mathrm{C}$ & -0.440885 & 1.918328 & 2.564456 \\
\hline C & -2.009237 & 4.209781 & 2.791596 \\
\hline $\mathrm{H}$ & -3.371128 & 3.230546 & 1.444778 \\
\hline C & -0.027387 & 2.965511 & 3.387174 \\
\hline $\mathrm{H}$ & 0.203651 & 1.057139 & 2.435371 \\
\hline $\mathrm{C}$ & -0.811337 & 4.114475 & 3.503091 \\
\hline $\mathrm{H}$ & -2.618714 & 5.106165 & 2.868485 \\
\hline $\mathrm{H}$ & 0.919733 & 2.889829 & 3.913605 \\
\hline $\mathrm{H}$ & -0.486459 & 4.935547 & 4.136375 \\
\hline C & -3.084365 & -0.467439 & 2.169626 \\
\hline $\mathrm{C}$ & -3.872779 & -1.541423 & 1.724344 \\
\hline C & -2.890276 & -0.294439 & 3.545983 \\
\hline C & -4.451587 & -2.421121 & 2.635030 \\
\hline $\mathrm{H}$ & -4.029342 & -1.690570 & 0.664181 \\
\hline C & -3.460341 & -1.187598 & 4.457601 \\
\hline $\mathrm{H}$ & -2.292935 & 0.534754 & 3.910194 \\
\hline
\end{tabular}




\begin{tabular}{|c|c|c|c|c|c|c|c|}
\hline $\mathrm{C}$ & -4.240174 & -2.253211 & 4.007145 & $\mathrm{H}$ & 7.599828 & 2.867353 & 2.863249 \\
\hline $\mathrm{H}$ & -5.059199 & -3.242248 & 2.265595 & $\mathrm{H}$ & 3.645673 & -3.666566 & -0.211598 \\
\hline $\mathrm{H}$ & -3.297160 & -1.043253 & 5.522229 & C & 1.896057 & 1.518279 & -0.156436 \\
\hline $\mathrm{H}$ & -4.683375 & -2.944721 & 4.718357 & $\mathrm{O}$ & 2.260922 & 1.369508 & 1.004343 \\
\hline $\mathrm{C}$ & -3.555062 & 1.197525 & -0.134729 & $\mathrm{O}$ & 2.243730 & 0.664785 & -1.124578 \\
\hline $\mathrm{C}$ & -4.893618 & 1.218904 & 0.279201 & $\mathrm{H}$ & 2.725345 & -0.112305 & -0.722598 \\
\hline $\mathrm{C}$ & -3.223921 & 1.697633 & -1.401450 & $\mathrm{C}$ & 1.060849 & 2.678200 & -0.593143 \\
\hline $\mathrm{C}$ & -5.885717 & 1.715040 & -0.567496 & $\mathrm{C}$ & 0.302036 & 2.754026 & -1.811945 \\
\hline $\mathrm{H}$ & -5.163341 & 0.834670 & 1.256977 & $\mathrm{C}$ & 1.024003 & 3.730797 & 0.305543 \\
\hline $\mathrm{C}$ & -4.213744 & 2.209224 & -2.239458 & $\mathrm{C}$ & 0.247281 & 1.728338 & -2.796327 \\
\hline $\mathrm{H}$ & -2.194574 & 1.660989 & -1.737513 & $\mathrm{C}$ & -0.483393 & 3.936081 & -2.040877 \\
\hline $\mathrm{C}$ & -5.548516 & 2.210109 & -1.828537 & $\mathrm{C}$ & 0.250533 & 4.882481 & 0.071738 \\
\hline $\mathrm{H}$ & -6.921932 & 1.714831 & -0.240338 & $\mathrm{H}$ & 1.599011 & 3.638294 & 1.217317 \\
\hline $\mathrm{H}$ & -3.937505 & 2.593061 & -3.216013 & $\mathrm{C}$ & -0.515788 & 1.868248 & -3.935134 \\
\hline $\mathrm{H}$ & -6.322194 & 2.594209 & -2.487758 & $\mathrm{H}$ & 0.825147 & 0.833247 & -2.640340 \\
\hline C & 0.001236 & -4.251477 & 1.458943 & $\mathrm{C}$ & -1.255003 & 4.048885 & -3.228356 \\
\hline C & 1.235505 & -4.111604 & 0.819051 & $\mathrm{C}$ & -0.491903 & 4.979662 & -1.080578 \\
\hline $\mathrm{C}$ & 1.860077 & -2.858651 & 0.755377 & $\mathrm{H}$ & 0.235065 & 5.676766 & 0.810591 \\
\hline $\mathrm{C}$ & 1.266468 & -1.740027 & 1.384630 & $\mathrm{C}$ & -1.271546 & 3.040925 & -4.163356 \\
\hline $\mathrm{C}$ & 0.055417 & -1.899950 & 2.070767 & $\mathrm{H}$ & -0.531804 & 1.065288 & -4.666959 \\
\hline C & -0.590488 & -3.153721 & 2.080310 & $\mathrm{H}$ & -1.838147 & 4.953640 & -3.379564 \\
\hline $\mathrm{H}$ & -0.489509 & -5.219901 & 1.483075 & $\mathrm{H}$ & -1.101137 & 5.858661 & -1.275581 \\
\hline $\mathrm{H}$ & 1.708890 & -4.969068 & 0.348375 & $\mathrm{H}$ & -1.861836 & 3.140317 & -5.069916 \\
\hline $\mathrm{H}$ & 1.797569 & -0.793496 & 1.413186 & $-\cdots$ & -.. & --.- & \\
\hline $\mathrm{H}$ & -0.341861 & -1.097887 & 2.678712 & & & & \\
\hline $\mathrm{H}$ & -1.543102 & -3.253218 & 2.590416 & INT1 & & & \\
\hline $\mathrm{C}$ & 3.120568 & -2.740802 & 0.043020 & & & & \\
\hline $\mathrm{N}$ & 3.626105 & -1.600609 & -0.282266 & Zero- 1 & rection $=0.72$ & 2203 (Hartree/) & Particle) \\
\hline $\mathrm{S}$ & 5.099781 & -1.643714 & -1.164227 & Therm & ction to Energ & $y=0.767350$ & \\
\hline $\mathrm{O}$ & 5.711738 & -2.978662 & -1.089680 & Therm & ction to Entha & lpy $=0.768294$ & \\
\hline $\mathrm{O}$ & 4.800331 & -1.030185 & -2.460743 & Therm & ction to Gibbs & Free Energy= & 0.638577 \\
\hline C & 6.047020 & -0.505345 & -0.180810 & $\mathrm{E}(\mathrm{Sol})$ & 88.47681162 & & \\
\hline C & 7.108678 & -0.978018 & 0.585858 & $-\cdots$ & - & ----- & \\
\hline C & 5.688070 & 0.845698 & -0.180615 & $\mathrm{C}$ & -3.967168 & -3.422051 & 0.361866 \\
\hline $\mathrm{C}$ & 7.826059 & -0.073601 & 1.368110 & $\mathrm{C}$ & -3.149805 & -2.927100 & -0.651336 \\
\hline $\mathrm{H}$ & 7.362019 & -2.032011 & 0.562087 & $\mathrm{C}$ & -1.747175 & -3.000761 & -0.557121 \\
\hline C & 6.410545 & 1.728200 & 0.612511 & $\mathrm{C}$ & -1.197812 & -3.618753 & 0.585260 \\
\hline $\mathrm{H}$ & 4.861749 & 1.191891 & -0.790686 & $\mathrm{C}$ & -2.016985 & -4.121174 & 1.592751 \\
\hline C & 7.488130 & 1.284529 & 1.395999 & $\mathrm{C}$ & -3.408323 & -4.020577 & 1.493156 \\
\hline $\mathrm{H}$ & 8.659391 & -0.429217 & 1.967870 & $\mathrm{H}$ & -5.045814 & -3.332213 & 0.266831 \\
\hline $\mathrm{H}$ & 6.134617 & 2.779154 & 0.627487 & $\mathrm{H}$ & -3.595740 & -2.453052 & -1.519828 \\
\hline C & 8.267678 & 2.262330 & 2.239403 & $\mathrm{H}$ & -0.120082 & -3.687622 & 0.691524 \\
\hline $\mathrm{H}$ & 8.975293 & 1.749253 & 2.897216 & $\mathrm{H}$ & -1.566548 & -4.588035 & 2.464949 \\
\hline $\mathrm{H}$ & 8.838562 & 2.956524 & 1.610061 & $\mathrm{H}$ & -4.045209 & -4.406022 & 2.284174 \\
\hline
\end{tabular}




\begin{tabular}{|c|c|c|c|c|c|c|c|}
\hline $\mathrm{C}$ & -0.919000 & -2.398643 & -1.614425 & $\mathrm{H}$ & 5.878308 & -4.353834 & -0.316653 \\
\hline $\mathrm{H}$ & -1.472095 & -2.031976 & -2.477161 & $\mathrm{C}$ & -1.535252 & 2.610191 & -0.556548 \\
\hline $\mathrm{C}$ & 0.471521 & -2.551227 & -1.734891 & $\mathrm{C}$ & -2.634412 & 3.467571 & -0.718651 \\
\hline $\mathrm{H}$ & 1.010876 & -3.196254 & -1.046081 & $\mathrm{C}$ & -0.240379 & 3.130797 & -0.670006 \\
\hline $\mathrm{C}$ & 1.186628 & -2.199871 & -2.958264 & $\mathrm{C}$ & -2.435000 & 4.822769 & -0.979455 \\
\hline $\mathrm{C}$ & 2.465832 & -2.515692 & -3.223444 & $\mathrm{H}$ & -3.642693 & 3.071212 & -0.643964 \\
\hline $\mathrm{H}$ & 2.940048 & -2.223768 & -4.155648 & $\mathrm{C}$ & -0.040737 & 4.487039 & -0.929988 \\
\hline $\mathrm{H}$ & 0.613828 & -1.634887 & -3.695594 & $\mathrm{H}$ & 0.609095 & 2.467679 & -0.568947 \\
\hline $\mathrm{H}$ & 3.074893 & -3.067080 & -2.512726 & $\mathrm{C}$ & -1.138207 & 5.334703 & -1.084639 \\
\hline $\mathrm{Pd}$ & 0.047149 & -0.618418 & -0.629980 & $\mathrm{H}$ & -3.291349 & 5.479956 & -1.104641 \\
\hline $\mathrm{P}$ & 2.150032 & -0.028083 & 0.154386 & $\mathrm{H}$ & 0.972170 & 4.867841 & -1.022303 \\
\hline$P$ & -1.726450 & 0.820690 & -0.185190 & $\mathrm{H}$ & -0.987399 & 6.390422 & -1.293331 \\
\hline $\mathrm{C}$ & 2.880131 & 1.365856 & -0.794235 & $\mathrm{C}$ & -2.220359 & 0.786972 & 1.584740 \\
\hline $\mathrm{C}$ & 3.365648 & 2.535085 & -0.196451 & $\mathrm{C}$ & -2.364639 & -0.476617 & 2.182891 \\
\hline $\mathrm{C}$ & 2.830581 & 1.282843 & -2.196982 & $\mathrm{C}$ & -2.359350 & 1.936007 & 2.372848 \\
\hline $\mathrm{C}$ & 3.790870 & 3.606282 & -0.987082 & $\mathrm{C}$ & -2.655392 & -0.584822 & 3.540033 \\
\hline $\mathrm{H}$ & 3.400631 & 2.615373 & 0.884747 & $\mathrm{H}$ & -2.236456 & -1.372925 & 1.587102 \\
\hline $\mathrm{C}$ & 3.269082 & 2.346760 & -2.981310 & $\mathrm{C}$ & -2.634998 & 1.822913 & 3.738420 \\
\hline $\mathrm{H}$ & 2.443781 & 0.380441 & -2.663108 & $\mathrm{H}$ & -2.241819 & 2.917356 & 1.925603 \\
\hline $\mathrm{C}$ & 3.744271 & 3.515459 & -2.378320 & $\mathrm{C}$ & -2.785094 & 0.565224 & 4.324358 \\
\hline $\mathrm{H}$ & 4.161140 & 4.510647 & -0.511586 & $\mathrm{H}$ & -2.768388 & -1.570101 & 3.983168 \\
\hline $\mathrm{H}$ & 3.229315 & 2.270120 & -4.064442 & $\mathrm{H}$ & -2.735340 & 2.721199 & 4.341899 \\
\hline $\mathrm{H}$ & 4.073562 & 4.349904 & -2.991125 & $\mathrm{H}$ & -2.998922 & 0.480685 & 5.386281 \\
\hline $\mathrm{C}$ & 2.338195 & 0.482727 & 1.909141 & $\mathrm{C}$ & -3.297753 & 0.425194 & -1.058085 \\
\hline $\mathrm{C}$ & 3.578635 & 0.473588 & 2.565841 & $\mathrm{C}$ & -4.515391 & 0.200378 & -0.406662 \\
\hline $\mathrm{C}$ & 1.198689 & 0.902049 & 2.606094 & $\mathrm{C}$ & -3.233738 & 0.272079 & -2.452421 \\
\hline $\mathrm{C}$ & 3.672278 & 0.888021 & 3.893700 & $\mathrm{C}$ & -5.645887 & -0.180430 & -1.134200 \\
\hline $\mathrm{H}$ & 4.465855 & 0.138296 & 2.036822 & $\mathrm{H}$ & -4.578729 & 0.305691 & 0.670860 \\
\hline $\mathrm{C}$ & 1.292365 & 1.316968 & 3.935224 & $\mathrm{C}$ & -4.362836 & -0.097397 & -3.178855 \\
\hline $\mathrm{H}$ & 0.237106 & 0.888018 & 2.109770 & $\mathrm{H}$ & -2.285655 & 0.429306 & -2.961177 \\
\hline $\mathrm{C}$ & 2.529586 & 1.310848 & 4.580007 & C & -5.573398 & -0.332865 & -2.518483 \\
\hline $\mathrm{H}$ & 4.635934 & 0.877512 & 4.395830 & $\mathrm{H}$ & -6.583113 & -0.360922 & -0.614618 \\
\hline $\mathrm{H}$ & 0.394185 & 1.630311 & 4.459239 & $\mathrm{H}$ & -4.298504 & -0.213711 & -4.257277 \\
\hline $\mathrm{H}$ & 2.606334 & 1.627604 & 5.616646 & $\mathrm{H}$ & -6.451803 & -0.634698 & -3.081898 \\
\hline $\mathrm{C}$ & 3.423388 & -1.348363 & 0.016172 & ---- & 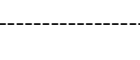 & 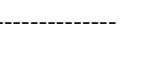 & \\
\hline $\mathrm{C}$ & 4.618303 & -1.211722 & -0.698383 & & & & \\
\hline $\mathrm{C}$ & 3.127557 & -2.583452 & 0.616210 & INT1' & & & \\
\hline $\mathrm{C}$ & 5.498023 & -2.291382 & -0.816512 & & & & \\
\hline $\mathrm{H}$ & 4.861015 & -0.265483 & -1.169641 & \multicolumn{4}{|c|}{ Zero-point correction $=0.722365($ Hartree $/$ Particle $)$} \\
\hline $\mathrm{C}$ & 4.007452 & -3.656369 & 0.505143 & \multicolumn{4}{|c|}{ Thermal correction to Energy $=0.767151$} \\
\hline $\mathrm{H}$ & 2.196084 & -2.697586 & 1.165837 & \multicolumn{4}{|c|}{ Thermal correction to Enthalpy $=0.768095$} \\
\hline $\mathrm{C}$ & 5.195761 & -3.514377 & -0.219265 & \multicolumn{4}{|c|}{ Thermal correction to Gibbs Free Energy= 0.639457} \\
\hline $\mathrm{H}$ & 6.419679 & -2.173498 & -1.379932 & \multicolumn{4}{|c|}{$E($ Solv $)=-2588.47180135$} \\
\hline $\mathrm{H}$ & 3.764996 & -4.605859 & 0.974505 & ----- & & $\cdots$ & \\
\hline
\end{tabular}




\begin{tabular}{|c|c|c|c|c|c|c|c|}
\hline $\mathrm{C}$ & -6.756911 & 2.122158 & -0.172313 & $\mathrm{H}$ & 4.427684 & -3.848577 & 2.669742 \\
\hline $\mathrm{C}$ & -5.781825 & 1.900208 & -1.142506 & $\mathrm{C}$ & 3.765257 & 1.208612 & -1.259743 \\
\hline $\mathrm{C}$ & -4.411082 & 1.966198 & -0.827554 & $\mathrm{C}$ & 4.853780 & 1.947246 & -0.776200 \\
\hline $\mathrm{C}$ & -4.057678 & 2.237997 & 0.510324 & $\mathrm{C}$ & 3.739453 & 0.834214 & -2.610329 \\
\hline $\mathrm{C}$ & -5.032180 & 2.457875 & 1.478905 & $\mathrm{C}$ & 5.897478 & 2.303372 & -1.630161 \\
\hline $\mathrm{C}$ & -6.388358 & 2.405748 & 1.144747 & $\mathrm{H}$ & 4.882112 & 2.247878 & 0.266444 \\
\hline $\mathrm{H}$ & -7.808075 & 2.069867 & -0.443979 & $\mathrm{C}$ & 4.789748 & 1.179773 & -3.461462 \\
\hline $\mathrm{H}$ & -6.075283 & 1.675191 & -2.165116 & $\mathrm{H}$ & 2.882592 & 0.281519 & -2.986401 \\
\hline $\mathrm{H}$ & -3.012483 & 2.226707 & 0.801064 & $\mathrm{C}$ & 5.869258 & 1.917556 & -2.972479 \\
\hline $\mathrm{H}$ & -4.728623 & 2.644849 & 2.505465 & $\mathrm{H}$ & 6.733736 & 2.882316 & -1.247830 \\
\hline $\mathrm{H}$ & -7.148086 & 2.568458 & 1.904030 & $\mathrm{H}$ & 4.759581 & 0.883226 & -4.506253 \\
\hline $\mathrm{C}$ & -3.408765 & 1.662320 & -1.846348 & $\mathrm{H}$ & 6.683260 & 2.196671 & -3.635680 \\
\hline $\mathrm{H}$ & -3.753281 & 1.070947 & -2.693194 & $\mathrm{C}$ & -1.616000 & -0.617165 & 1.463379 \\
\hline $\mathrm{C}$ & -2.097488 & 1.986383 & -1.775918 & $\mathrm{C}$ & -2.683446 & -1.210292 & 2.154825 \\
\hline $\mathrm{H}$ & -1.756642 & 2.631901 & -0.966188 & $\mathrm{C}$ & -1.031838 & 0.541817 & 1.994845 \\
\hline $\mathrm{C}$ & -1.064597 & 1.500760 & -2.676486 & $\mathrm{C}$ & -3.148498 & -0.656463 & 3.347339 \\
\hline $\mathrm{C}$ & 0.231476 & 2.043007 & -2.730825 & $\mathrm{H}$ & -3.173931 & -2.086838 & 1.743957 \\
\hline $\mathrm{H}$ & 0.876370 & 1.820306 & -3.576384 & $\mathrm{C}$ & -1.489422 & 1.092312 & 3.192225 \\
\hline $\mathrm{H}$ & -1.385162 & 0.809084 & -3.454326 & $\mathrm{H}$ & -0.227154 & 1.023897 & 1.452575 \\
\hline $\mathrm{H}$ & 0.458223 & 2.976643 & -2.220282 & $\mathrm{C}$ & -2.552560 & 0.494178 & 3.870140 \\
\hline $\mathrm{Pd}$ & 0.305533 & 0.405722 & -1.241260 & $\mathrm{H}$ & -3.983578 & -1.119632 & 3.865400 \\
\hline$P$ & 2.366721 & 0.665339 & -0.196320 & $\mathrm{H}$ & -1.019644 & 1.992185 & 3.579729 \\
\hline$P$ & -0.954370 & -1.240211 & -0.138466 & $\mathrm{H}$ & -2.921190 & 0.923723 & 4.797584 \\
\hline $\mathrm{C}$ & 2.282663 & 1.976232 & 1.100318 & $\mathrm{C}$ & -0.030139 & -2.775380 & 0.286385 \\
\hline $\mathrm{C}$ & 3.047197 & 1.943568 & 2.275137 & $\mathrm{C}$ & 1.075046 & -3.097243 & -0.513731 \\
\hline $\mathrm{C}$ & 1.381976 & 3.035698 & 0.908969 & $\mathrm{C}$ & -0.377978 & -3.627356 & 1.341765 \\
\hline $\mathrm{C}$ & 2.909551 & 2.945165 & 3.237502 & $\mathrm{C}$ & 1.811757 & -4.255973 & -0.273514 \\
\hline $\mathrm{H}$ & 3.738522 & 1.125009 & 2.445358 & $\mathrm{H}$ & 1.366003 & -2.414074 & -1.307193 \\
\hline $\mathrm{C}$ & 1.245513 & 4.037208 & 1.869475 & $\mathrm{C}$ & 0.366557 & -4.780009 & 1.590466 \\
\hline $\mathrm{H}$ & 0.774241 & 3.054503 & 0.009908 & $\mathrm{H}$ & -1.221346 & -3.384985 & 1.979527 \\
\hline $\mathrm{C}$ & 2.007701 & 3.992078 & 3.039191 & $\mathrm{C}$ & 1.459701 & -5.098039 & 0.781657 \\
\hline $\mathrm{H}$ & 3.505126 & 2.903949 & 4.145365 & $\mathrm{H}$ & 2.674657 & -4.485161 & -0.891487 \\
\hline $\mathrm{H}$ & 0.538927 & 4.846651 & 1.708134 & $\mathrm{H}$ & 0.093253 & -5.430688 & 2.416926 \\
\hline $\mathrm{H}$ & 1.897311 & 4.766901 & 3.792666 & $\mathrm{H}$ & 2.041702 & -5.993553 & 0.981098 \\
\hline $\mathrm{C}$ & 3.106620 & -0.736494 & 0.737463 & $\mathrm{C}$ & -2.444449 & -1.925067 & -0.969904 \\
\hline $\mathrm{C}$ & 4.292496 & -1.366259 & 0.341898 & $\mathrm{C}$ & -2.318299 & -3.013684 & -1.848112 \\
\hline $\mathrm{C}$ & 2.400100 & -1.248578 & 1.839054 & $\mathrm{C}$ & -3.690236 & -1.291331 & -0.853441 \\
\hline $\mathrm{C}$ & 4.764013 & -2.484022 & 1.035319 & $\mathrm{C}$ & -3.413470 & -3.456490 & -2.587525 \\
\hline $\mathrm{H}$ & 4.851859 & -0.983692 & -0.505310 & $\mathrm{H}$ & -1.362843 & -3.520497 & -1.946042 \\
\hline $\mathrm{C}$ & 2.878623 & -2.352643 & 2.536421 & $\mathrm{C}$ & -4.784897 & -1.736629 & -1.595446 \\
\hline $\mathrm{H}$ & 1.468749 & -0.783138 & 2.147351 & $\mathrm{H}$ & -3.814859 & -0.454940 & -0.178894 \\
\hline $\mathrm{C}$ & 4.061950 & -2.977470 & 2.133559 & $\mathrm{C}$ & -4.651030 & -2.817842 & -2.466128 \\
\hline $\mathrm{H}$ & 5.685232 & -2.964018 & 0.715910 & $\mathrm{H}$ & -3.301623 & -4.305540 & -3.256589 \\
\hline $\mathrm{H}$ & 2.314787 & -2.741218 & 3.378839 & $\mathrm{H}$ & -5.737514 & -1.224772 & -1.488948 \\
\hline
\end{tabular}




\begin{tabular}{|c|c|c|c|}
\hline $\mathrm{H}$ & -5.503030 & -3.164709 & -3.044317 \\
\hline INT1-1 & & & \\
\hline Zero-point & correction= & 0.721980 (Ha & rtree/Particle) \\
\hline Thermal co & rrection to En & ergy $=0.767$ & \\
\hline Thermal co & rrection to En & thalpy $=0.76$ & 8109 \\
\hline Thermal co & rrection to Gil & bbs Free Energ & 0.63777 \\
\hline $\mathrm{E}($ Solv $)=$ & -2588.47444 & 344 & \\
\hline $\mathrm{C}$ & 2.820538 & 3.930025 & 1.627568 \\
\hline $\mathrm{C}$ & 1.892107 & 3.604162 & 0.641395 \\
\hline $\mathrm{C}$ & 2.265688 & 2.842819 & -0.484102 \\
\hline $\mathrm{C}$ & 3.612718 & 2.444187 & -0.586617 \\
\hline $\mathrm{C}$ & 4.536762 & 2.762566 & 0.404649 \\
\hline $\mathrm{C}$ & 4.148333 & 3.505495 & 1.522012 \\
\hline $\mathrm{H}$ & 2.503530 & 4.515592 & 2.486902 \\
\hline $\mathrm{H}$ & 0.861546 & 3.925708 & 0.753994 \\
\hline $\mathrm{H}$ & 3.923346 & 1.854752 & -1.443765 \\
\hline $\mathrm{H}$ & 5.564307 & 2.423348 & 0.304434 \\
\hline $\mathrm{H}$ & 4.868547 & 3.754233 & 2.296156 \\
\hline $\mathrm{C}$ & 1.310332 & 2.403824 & -1.514351 \\
\hline $\mathrm{H}$ & 1.771027 & 1.937711 & -2.381164 \\
\hline $\mathrm{C}$ & -0.035972 & 2.793548 & -1.601239 \\
\hline $\mathrm{H}$ & -0.435647 & 3.494451 & -0.872553 \\
\hline $\mathrm{C}$ & -0.883206 & 2.581872 & -2.783561 \\
\hline $\mathrm{C}$ & -0.496313 & 2.165152 & -4.001223 \\
\hline $\mathrm{H}$ & -1.218376 & 2.019398 & -4.798717 \\
\hline $\mathrm{H}$ & -1.940783 & 2.783699 & -2.617817 \\
\hline $\mathrm{H}$ & 0.544285 & 1.963150 & -4.242295 \\
\hline $\mathrm{Pd}$ & 0.027059 & 0.798291 & -0.567609 \\
\hline $\mathrm{P}$ & -2.125484 & 0.308698 & 0.121835 \\
\hline $\mathrm{P}$ & 1.488373 & -0.947736 & -0.119534 \\
\hline $\mathrm{C}$ & -2.745412 & -1.278599 & -0.568722 \\
\hline $\mathrm{C}$ & -3.692673 & -2.090308 & 0.067433 \\
\hline $\mathrm{C}$ & -2.204467 & -1.691520 & -1.795992 \\
\hline $\mathrm{C}$ & -4.091253 & -3.294513 & -0.514529 \\
\hline $\mathrm{H}$ & -4.108406 & -1.790742 & 1.023971 \\
\hline $\mathrm{C}$ & -2.607920 & -2.890785 & -2.379034 \\
\hline $\mathrm{H}$ & -1.447775 & -1.073319 & -2.272276 \\
\hline $\mathrm{C}$ & -3.550200 & -3.696394 & -1.737048 \\
\hline $\mathrm{H}$ & -4.821614 & -3.921298 & -0.009689 \\
\hline $\mathrm{H}$ & -2.170241 & -3.207076 & -3.321281 \\
\hline
\end{tabular}

\begin{tabular}{|c|c|c|c|}
\hline $\mathrm{H}$ & -3.854514 & -4.639205 & -2.183042 \\
\hline $\mathrm{C}$ & -2.389091 & 0.151650 & 1.934745 \\
\hline C & -3.647389 & 0.296697 & 2.538353 \\
\hline C & -1.275457 & -0.125754 & 2.740351 \\
\hline C & -3.788707 & 0.139082 & 3.917251 \\
\hline I & -4.511813 & 0.543974 & 1.929170 \\
\hline C & -1.417647 & -0.287065 & 4.118714 \\
\hline $\mathrm{H}$ & -0.294012 & -0.195495 & 2.286091 \\
\hline C & -2.676040 & -0.158691 & 4.708811 \\
\hline W & -4.767410 & 0.253130 & 4.375479 \\
\hline $\mathrm{H}$ & -0.541122 & -0.499664 & 4.724442 \\
\hline $\mathrm{H}$ & -2.790184 & -0.277983 & 5.782728 \\
\hline C & -3.435443 & 1.508060 & -0.353506 \\
\hline $\mathrm{C}$ & -4.491601 & 1.192999 & -1.216071 \\
\hline 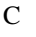 & -3.299520 & 2.830272 & 0.102221 \\
\hline $\mathrm{C}$ & -5.393049 & 2.182704 & -1.618340 \\
\hline 1 & -4.608973 & 0.176531 & -1.576719 \\
\hline C & -4.204934 & 3.812068 & -0.289525 \\
\hline $\mathrm{H}$ & -2.472485 & 3.084304 & 0.760683 \\
\hline 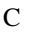 & -5.254078 & 3.491139 & -1.157407 \\
\hline $\mathrm{H}$ & -6.205978 & 1.926171 & -2.292291 \\
\hline 11 & -4.089097 & 4.829894 & 0.072764 \\
\hline $\mathrm{H}$ & -5.954668 & 4.259036 & -1.472887 \\
\hline $\mathrm{C}$ & 0.856649 & -2.662157 & -0.315208 \\
\hline$c$ & 1.324523 & -3.537347 & -1.302243 \\
\hline 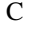 & -0.205551 & -3.071969 & 0.508365 \\
\hline$C$ & 0.737741 & -4.794648 & -1.466981 \\
\hline $\mathrm{H}$ & 2.147564 & -3.239629 & -1.943191 \\
\hline 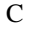 & -0.776647 & -4.330863 & 0.354150 \\
\hline $\mathrm{H}$ & -0.590222 & -2.399716 & 1.268611 \\
\hline $\mathrm{C}$ & -0.311136 & -5.194803 & -0.640621 \\
\hline$\Pi$ & 1.108668 & -5.462256 & -2.240132 \\
\hline $\mathrm{H}$ & -1.603405 & -4.626396 & 0.992329 \\
\hline 11 & -0.768170 & -6.171640 & -0.771566 \\
\hline C & 2.153887 & -0.936500 & 1.592949 \\
\hline $\mathrm{C}$ & 2.387868 & 0.315963 & 2.182247 \\
\hline C & 2.417189 & -2.099756 & 2.328946 \\
\hline 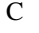 & 2.877482 & 0.402091 & 3.484051 \\
\hline $\mathrm{H}$ & 2.178775 & 1.220793 & 1.623523 \\
\hline 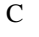 & 2.895441 & -2.010054 & 3.637433 \\
\hline $\mathrm{H}$ & 2.239019 & -3.072919 & 1.883530 \\
\hline $\mathrm{C}$ & 3.125720 & -0.760613 & 4.217598 \\
\hline 11 & 3.058438 & 1.380113 & 3.920038 \\
\hline $\mathrm{H}$ & 3.089566 & -2.917567 & 4.202759 \\
\hline
\end{tabular}




\begin{tabular}{llll}
$\mathrm{H}$ & 3.497760 & -0.694442 & 5.236419 \\
$\mathrm{C}$ & 3.003356 & -0.918600 & -1.158672 \\
$\mathrm{C}$ & 4.293983 & -1.055316 & -0.634590 \\
$\mathrm{C}$ & 2.848533 & -0.648999 & -2.528193 \\
$\mathrm{C}$ & 5.409606 & -0.915767 & -1.462896 \\
$\mathrm{H}$ & 4.428167 & -1.252857 & 0.423471 \\
$\mathrm{C}$ & 3.961394 & -0.529000 & -3.357935 \\
$\mathrm{H}$ & 1.848833 & -0.508668 & -2.932135 \\
$\mathrm{C}$ & 5.247549 & -0.653083 & -2.823605 \\
$\mathrm{H}$ & 6.406870 & -1.010144 & -1.041834 \\
$\mathrm{H}$ & 3.827463 & -0.320289 & -4.415776 \\
$\mathrm{H}$ & 6.116982 & -0.540107 & -3.465047 \\
\hline & & &
\end{tabular}

\section{INT1'-1}

Zero-point correction $=0.722679$ (Hartree/Particle $)$

Thermal correction to Energy $=0.767396$

Thermal correction to Enthalpy $=0.768340$

Thermal correction to Gibbs Free Energy $=0.639886$

$\mathrm{E}($ Solv $)=-2588.46986909$

\begin{tabular}{|c|c|c|c|}
\hline $\mathrm{C}$ & 4.068750 & -3.228994 & 1.184680 \\
\hline $\mathrm{C}$ & 3.403944 & -2.576826 & 0.151289 \\
\hline $\mathrm{C}$ & 2.739412 & -3.301635 & -0.858452 \\
\hline $\mathrm{C}$ & 2.755662 & -4.707563 & -0.774027 \\
\hline $\mathrm{C}$ & 3.421074 & -5.361100 & 0.261110 \\
\hline $\mathrm{C}$ & 4.083438 & -4.625283 & 1.247403 \\
\hline $\mathrm{H}$ & 4.552621 & -2.640710 & 1.959518 \\
\hline $\mathrm{H}$ & 3.351788 & -1.493766 & 0.152367 \\
\hline $\mathrm{H}$ & 2.235141 & -5.286079 & -1.533595 \\
\hline $\mathrm{H}$ & 3.419658 & -6.447280 & 0.300795 \\
\hline $\mathrm{H}$ & 4.594070 & -5.133421 & 2.060538 \\
\hline $\mathrm{C}$ & 1.970446 & -2.639866 & -1.908257 \\
\hline $\mathrm{H}$ & 1.195421 & -3.245161 & -2.376362 \\
\hline $\mathrm{C}$ & 2.094579 & -1.341559 & -2.277429 \\
\hline $\mathrm{H}$ & 2.936368 & -0.767159 & -1.898656 \\
\hline $\mathrm{C}$ & 1.142834 & -0.582173 & -3.087312 \\
\hline $\mathrm{C}$ & -0.158112 & -1.016821 & -3.368193 \\
\hline $\mathrm{H}$ & -0.753086 & -0.481342 & -4.102723 \\
\hline $\mathrm{H}$ & 1.517619 & 0.322147 & -3.561342 \\
\hline $\mathrm{H}$ & -0.478682 & -2.033481 & -3.163849 \\
\hline $\mathrm{Pd}$ & -0.155867 & 0.054959 & -1.362789 \\
\hline $\mathrm{P}$ & -2.022404 & -0.615907 & -0.161047 \\
\hline
\end{tabular}

$\begin{array}{rrr}1.087061 & 1.620383 & -0.152691 \\ -1.548303 & -1.943872 & 1.028638 \\ -2.174603 & -2.118987 & 2.271366 \\ -0.466843 & -2.772452 & 0.691672\end{array}$

$\begin{array}{llll}\mathrm{C} & -1.721925 & -3.098146 & 3.157035 \\ \mathrm{H} & -3.000140 & -1.474871 & 2.556537\end{array}$

$\begin{array}{llll}\text { C } & -0.010418 & -3.748702 & 1.577486\end{array}$

$\begin{array}{llll}\mathrm{H} & 0.048147 & -2.616586 & -0.250840\end{array}$

$\begin{array}{llll}\text { C } & -0.638467 & -3.910668 & 2.814506\end{array}$

$\mathrm{H} \quad-2.211280 \quad-3.219579 \quad 4.119705$

$\begin{array}{llll}\mathrm{H} & 0.845030 & -4.359297 & 1.305595\end{array}$

$\mathrm{H} \quad-0.280468 \quad-4.662507 \quad 3.512646$

$\begin{array}{llll}\text { C } & -2.839480 & 0.616873 & 0.933697\end{array}$

$\begin{array}{llll}\text { C } & -4.116629 & 1.125007 & 0.668213\end{array}$

$\begin{array}{llll}\text { C } & -2.109326 & 1.145456 & 2.011487\end{array}$

$\begin{array}{llll}\text { C } & -4.653284 & 2.139609 & 1.465088\end{array}$

$\begin{array}{llll}\mathrm{H} & -4.695605 & 0.727292 & -0.158837\end{array}$

$\begin{array}{llll}\text { C } & -2.651071 & 2.145259 & 2.812462\end{array}$

$\begin{array}{llll}\mathrm{H} & -1.110225 & 0.775765 & 2.219539\end{array}$

$\begin{array}{llll}\text { C } & -3.925165 & 2.649628 & 2.538792\end{array}$

$\begin{array}{llll}\mathrm{H} & -5.645569 & 2.525132 & 1.246307\end{array}$

$\begin{array}{llll}\mathrm{H} & -2.068550 & 2.548465 & 3.635113\end{array}$

$\begin{array}{llll}\mathrm{H} & -4.342556 & 3.439982 & 3.156299\end{array}$

$\begin{array}{llll}\text { C } & -3.451658 & -1.335663 & -1.070454\end{array}$

C $\quad-4.341280 \quad-2.253176 \quad-0.494425$

$\begin{array}{llll}\text { C } & -3.663109 & -0.916323 & -2.390862\end{array}$

$\begin{array}{llll}\text { C } & -5.423334 & -2.739527 & -1.227474\end{array}$

$\begin{array}{llll}\mathrm{H} & -4.183026 & -2.592541 & 0.524288\end{array}$

$\begin{array}{llll}\text { C } & -4.753523 & -1.393240 & -3.119815\end{array}$

$\begin{array}{llll}\mathrm{H} & -2.956335 & -0.224581 & -2.840727\end{array}$

$\begin{array}{llll}\text { C } & -5.633905 & -2.307676 & -2.539381\end{array}$

$\begin{array}{llll}\mathrm{H} & -6.103305 & -3.456053 & -0.774691\end{array}$

$\begin{array}{llll}\mathrm{H} & -4.908343 & -1.059916 & -4.142317\end{array}$

$\begin{array}{llll}\mathrm{H} & -6.477598 & -2.688363 & -3.108346\end{array}$

$\begin{array}{llll}\text { C } & 1.977835 & 1.020531 & 1.341655\end{array}$

$\begin{array}{llll}\text { C } & 3.073548 & 1.687422 & 1.913628\end{array}$

$\begin{array}{llll}\text { C } & 1.540546 & -0.179071 & 1.918338\end{array}$

$\begin{array}{llll}\text { C } & 3.702889 & 1.167238 & 3.044330\end{array}$

$\begin{array}{llll}\mathrm{H} & 3.446430 & 2.601132 & 1.461377\end{array}$

$\begin{array}{llll}\text { C } & 2.167676 & -0.699494 & 3.050681\end{array}$

$\begin{array}{llll}\mathrm{H} & 0.725061 & -0.722966 & 1.459862\end{array}$

$\begin{array}{llll}\text { C } & 3.249850 & -0.025338 & 3.616892\end{array}$

$\begin{array}{llll}\mathrm{H} & 4.552008 & 1.689872 & 3.476293\end{array}$

$\begin{array}{llll}\mathrm{H} & 1.819171 & -1.640631 & 3.465272\end{array}$ 


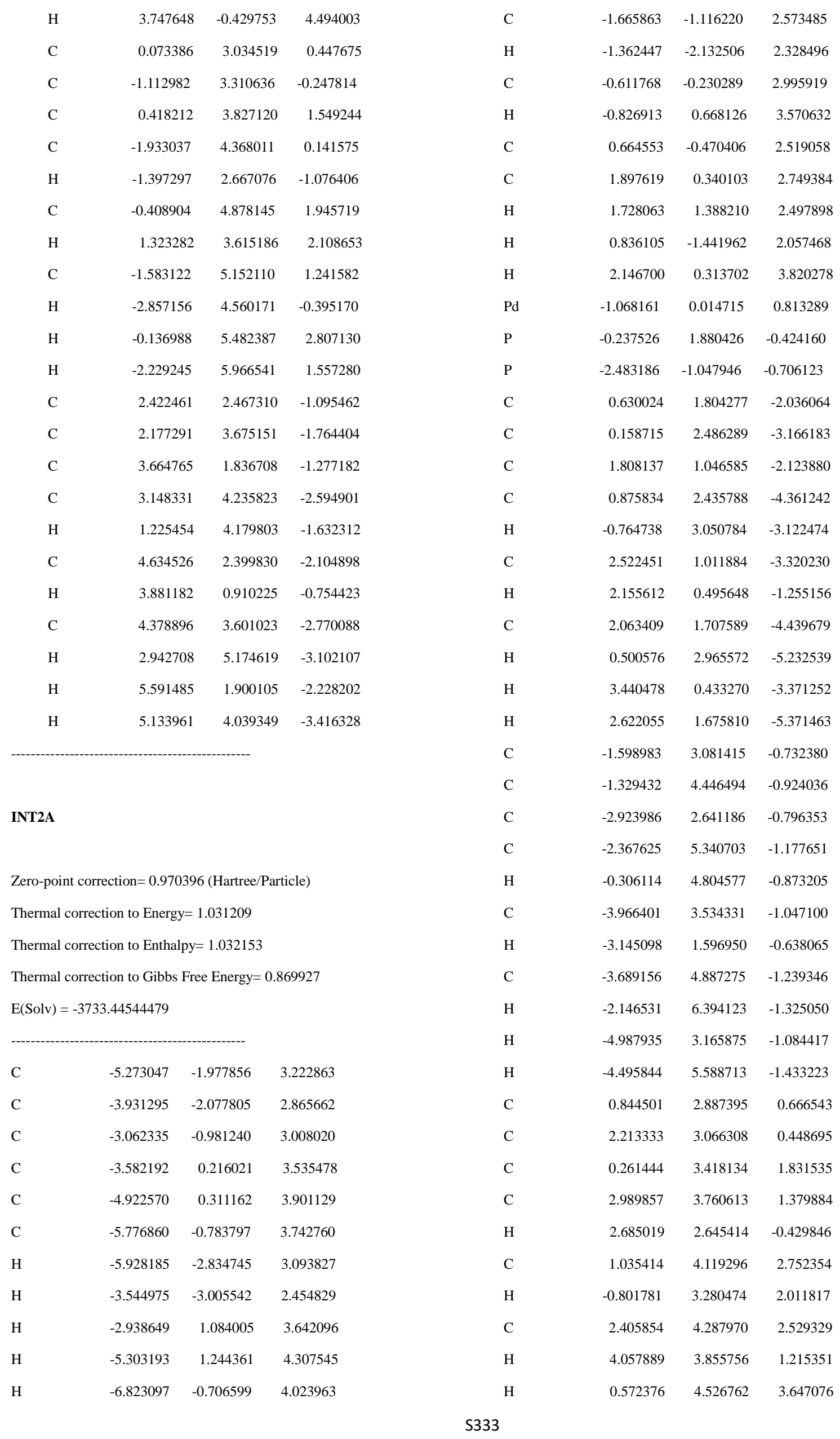




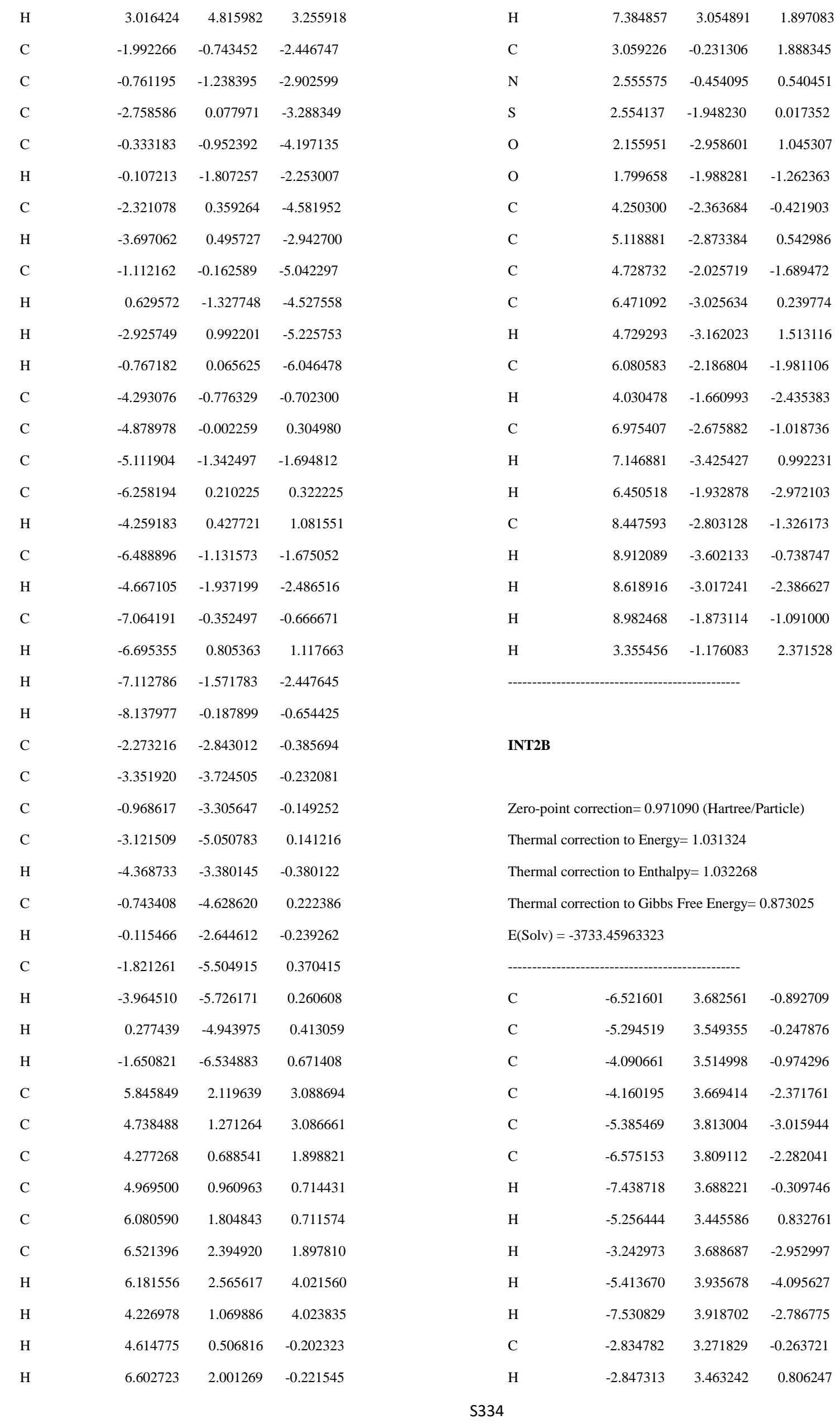




\begin{tabular}{|c|c|c|c|c|c|c|c|}
\hline $\mathrm{C}$ & -1.720679 & 2.736506 & -0.807055 & $\mathrm{C}$ & -2.174704 & -1.876063 & 3.080313 \\
\hline $\mathrm{H}$ & -1.710457 & 2.520512 & -1.876552 & $\mathrm{C}$ & -2.752449 & -2.905279 & 0.972598 \\
\hline $\mathrm{C}$ & -0.489463 & 2.393802 & -0.090482 & $\mathrm{C}$ & -2.320082 & -3.109353 & 3.716339 \\
\hline $\mathrm{C}$ & 0.768130 & 2.887081 & -0.805245 & $\mathrm{H}$ & -1.900034 & -1.004428 & 3.662477 \\
\hline $\mathrm{H}$ & 0.842948 & 2.458132 & -1.814458 & $\mathrm{C}$ & -2.900657 & -4.132963 & 1.610754 \\
\hline $\mathrm{H}$ & -0.509016 & 2.737618 & 0.946279 & $\mathrm{H}$ & -2.922681 & -2.839741 & -0.096639 \\
\hline $\mathrm{H}$ & 0.740813 & 3.980590 & -0.927621 & $\mathrm{C}$ & -2.688243 & -4.238004 & 2.986045 \\
\hline $\mathrm{Pd}$ & -0.144165 & 0.292378 & -0.030863 & $\mathrm{H}$ & -2.146049 & -3.182778 & 4.786152 \\
\hline$P$ & 0.548495 & -1.964763 & -0.724935 & $\mathrm{H}$ & -3.164359 & -5.010802 & 1.029673 \\
\hline$P$ & -2.254550 & -0.141429 & 0.833052 & $\mathrm{H}$ & -2.797561 & -5.198198 & 3.481519 \\
\hline $\mathrm{C}$ & 0.333866 & -3.519886 & 0.235124 & $\mathrm{C}$ & -3.826270 & -0.132321 & -0.122904 \\
\hline $\mathrm{C}$ & -0.018339 & -4.727070 & -0.386375 & $\mathrm{C}$ & -3.858785 & 0.343151 & -1.438526 \\
\hline $\mathrm{C}$ & 0.593718 & -3.507531 & 1.613310 & $\mathrm{C}$ & -5.013842 & -0.617170 & 0.451420 \\
\hline $\mathrm{C}$ & -0.094748 & -5.905588 & 0.355019 & $\mathrm{C}$ & -5.050801 & 0.354447 & -2.162039 \\
\hline $\mathrm{H}$ & -0.229332 & -4.750676 & -1.449549 & $\mathrm{H}$ & -2.948562 & 0.710560 & -1.894142 \\
\hline $\mathrm{C}$ & 0.521620 & -4.690774 & 2.346918 & $\mathrm{C}$ & -6.207535 & -0.593707 & -0.266923 \\
\hline $\mathrm{H}$ & 0.874377 & -2.579623 & 2.094426 & $\mathrm{H}$ & -4.999900 & -1.021376 & 1.458392 \\
\hline $\mathrm{C}$ & 0.180918 & -5.891451 & 1.722903 & $\mathrm{C}$ & -6.229122 & -0.102633 & -1.574396 \\
\hline $\mathrm{H}$ & -0.365636 & -6.834934 & -0.139045 & $\mathrm{H}$ & -5.060238 & 0.749626 & -3.172055 \\
\hline $\mathrm{H}$ & 0.725057 & -4.668704 & 3.413498 & $\mathrm{H}$ & -7.119713 & -0.962663 & 0.193420 \\
\hline $\mathrm{H}$ & 0.124445 & -6.810396 & 2.300244 & $\mathrm{H}$ & -7.160550 & -0.078013 & -2.132377 \\
\hline $\mathrm{C}$ & -0.305745 & -2.360471 & -2.315826 & $\mathrm{C}$ & -2.509227 & 1.048034 & 2.212927 \\
\hline C & 0.317994 & -3.047600 & -3.369280 & $\mathrm{C}$ & -1.383277 & 1.415572 & 2.967823 \\
\hline $\mathrm{C}$ & -1.644461 & -1.979715 & -2.467709 & $\mathrm{C}$ & -3.759897 & 1.582367 & 2.543777 \\
\hline $\mathrm{C}$ & -0.388098 & -3.344469 & -4.535745 & $\mathrm{C}$ & -1.513448 & 2.302229 & 4.036443 \\
\hline $\mathrm{H}$ & 1.355125 & -3.350845 & -3.277718 & $\mathrm{H}$ & -0.396769 & 1.034971 & 2.717027 \\
\hline $\mathrm{C}$ & -2.353864 & -2.276522 & -3.630407 & $\mathrm{C}$ & -3.883679 & 2.476817 & 3.608174 \\
\hline $\mathrm{H}$ & -2.132757 & -1.433259 & -1.675040 & $\mathrm{H}$ & -4.634479 & 1.324118 & 1.958718 \\
\hline $\mathrm{C}$ & -1.724705 & -2.961270 & -4.670697 & $\mathrm{C}$ & -2.762190 & 2.839020 & 4.356370 \\
\hline $\mathrm{H}$ & 0.108961 & -3.876712 & -5.342161 & $\mathrm{H}$ & -0.629741 & 2.576855 & 4.604504 \\
\hline $\mathrm{H}$ & -3.390384 & -1.962274 & -3.717648 & $\mathrm{H}$ & -4.857922 & 2.894466 & 3.847561 \\
\hline $\mathrm{H}$ & -2.268459 & -3.192072 & -5.582490 & $\mathrm{H}$ & -2.860764 & 3.538686 & 5.181600 \\
\hline $\mathrm{C}$ & 2.298011 & -2.075856 & -1.275333 & $\mathrm{C}$ & 4.905393 & 4.753612 & -0.895163 \\
\hline $\mathrm{C}$ & 3.153289 & -3.107716 & -0.873782 & $\mathrm{C}$ & 3.716617 & 4.270495 & -0.349109 \\
\hline $\mathrm{C}$ & 2.763021 & -1.119675 & -2.188433 & $\mathrm{C}$ & 3.286171 & 2.964119 & -0.609096 \\
\hline $\mathrm{C}$ & 4.449468 & -3.189328 & -1.389195 & $\mathrm{C}$ & 4.067144 & 2.152264 & -1.433052 \\
\hline $\mathrm{H}$ & 2.810233 & -3.846292 & -0.158784 & $\mathrm{C}$ & 5.261349 & 2.626529 & -1.973549 \\
\hline $\mathrm{C}$ & 4.052856 & -1.207796 & -2.709115 & $\mathrm{C}$ & 5.686532 & 3.929558 & -1.708587 \\
\hline $\mathrm{H}$ & 2.111108 & -0.306227 & -2.489609 & $\mathrm{H}$ & 5.228433 & 5.768087 & -0.676144 \\
\hline C & 4.900652 & -2.244225 & -2.311218 & $\mathrm{H}$ & 3.122624 & 4.907183 & 0.303652 \\
\hline $\mathrm{H}$ & 5.104098 & -3.994518 & -1.067505 & $\mathrm{H}$ & 3.756176 & 1.130003 & -1.593011 \\
\hline $\mathrm{H}$ & 4.398801 & -0.460415 & -3.417572 & $\mathrm{H}$ & 5.869795 & 1.968774 & -2.589370 \\
\hline $\mathrm{H}$ & 5.907037 & -2.310803 & -2.714901 & $\mathrm{H}$ & 6.619371 & 4.298894 & -2.126547 \\
\hline $\mathrm{C}$ & -2.399855 & -1.759680 & 1.702911 & $\mathrm{C}$ & 1.986809 & 2.470368 & 0.006559 \\
\hline
\end{tabular}




\begin{tabular}{lrrr}
$\mathrm{N}$ & 1.882449 & 0.997179 & 0.136057 \\
$\mathrm{~S}$ & 2.475309 & 0.446123 & 1.568418 \\
$\mathrm{O}$ & 2.032152 & 1.258166 & 2.729089 \\
$\mathrm{O}$ & 2.227396 & -1.011573 & 1.628282 \\
$\mathrm{C}$ & 4.243272 & 0.669962 & 1.424730 \\
$\mathrm{C}$ & 4.835668 & 1.839523 & 1.898130 \\
$\mathrm{C}$ & 4.978409 & -0.265532 & 0.700296 \\
$\mathrm{C}$ & 6.172699 & 2.088471 & 1.604157 \\
$\mathrm{H}$ & 4.245219 & 2.546160 & 2.469876 \\
$\mathrm{C}$ & 6.315981 & -0.004321 & 0.419395 \\
$\mathrm{H}$ & 4.501065 & -1.171299 & 0.350178 \\
$\mathrm{C}$ & 6.924678 & 1.184253 & 0.842926 \\
$\mathrm{H}$ & 6.633549 & 3.010824 & 1.947746 \\
$\mathrm{H}$ & 6.888685 & -0.724915 & -0.159047 \\
$\mathrm{C}$ & 8.341762 & 1.514534 & 0.448099 \\
$\mathrm{H}$ & 8.893333 & 1.980747 & 1.271981 \\
$\mathrm{H}$ & 8.890962 & 0.622393 & 0.130617 \\
$\mathrm{H}$ & 8.346493 & 2.225169 & -0.389209 \\
$\mathrm{H}$ & 1.883648 & 2.945873 & 0.992450 \\
-------------------------------------- & \\
\hline & & & \\
\hline
\end{tabular}

\begin{tabular}{|c|c|c|c|}
\hline $\mathrm{C}$ & -0.102434 & 1.607505 & 2.765010 \\
\hline $\mathrm{C}$ & -1.526745 & 1.255545 & 3.103841 \\
\hline $\mathrm{H}$ & -1.578810 & 0.306587 & 3.642554 \\
\hline $\mathrm{H}$ & 0.043372 & 2.590973 & 2.314293 \\
\hline $\mathrm{H}$ & -1.990635 & 2.037469 & 3.717735 \\
\hline $\mathrm{Pd}$ & 0.481183 & 0.429983 & 1.031811 \\
\hline $\mathrm{P}$ & 1.315633 & -0.130001 & -1.059525 \\
\hline $\mathrm{C}$ & 0.104703 & 0.178785 & -2.397780 \\
\hline $\mathrm{C}$ & 0.244358 & 1.230781 & -3.311970 \\
\hline $\mathrm{C}$ & -1.051488 & -0.619388 & -2.429164 \\
\hline $\mathrm{C}$ & -0.765334 & 1.490669 & -4.241335 \\
\hline $\mathrm{H}$ & 1.135060 & 1.849351 & -3.299658 \\
\hline $\mathrm{C}$ & -2.047990 & -0.361285 & -3.368546 \\
\hline $\mathrm{H}$ & -1.171561 & -1.418549 & -1.703945 \\
\hline $\mathrm{C}$ & -1.912755 & 0.697525 & -4.270522 \\
\hline $\mathrm{H}$ & -0.649273 & 2.310249 & -4.945229 \\
\hline $\mathrm{H}$ & -2.940061 & -0.980918 & -3.380912 \\
\hline $\mathrm{H}$ & -2.697973 & 0.902713 & -4.992799 \\
\hline $\mathrm{C}$ & 1.940925 & -1.815200 & -1.343837 \\
\hline $\mathrm{C}$ & 2.523410 & -2.488279 & -0.262540 \\
\hline $\mathrm{C}$ & 1.909854 & -2.420638 & -2.606855 \\
\hline $\mathrm{C}$ & 3.082813 & -3.750834 & -0.445997 \\
\hline $\mathrm{H}$ & 2.539084 & -2.025051 & 0.716715 \\
\hline $\mathrm{C}$ & 2.460949 & -3.689758 & -2.783018 \\
\hline $\mathrm{H}$ & 1.451369 & -1.904211 & -3.444218 \\
\hline $\mathrm{C}$ & 3.050014 & -4.354130 & -1.704693 \\
\hline $\mathrm{H}$ & 3.535803 & -4.262368 & 0.398035 \\
\hline $\mathrm{H}$ & 2.429130 & -4.160116 & -3.761779 \\
\hline $\mathrm{H}$ & 3.477671 & -5.342894 & -1.845545 \\
\hline $\mathrm{C}$ & 2.739048 & 0.958110 & -1.449520 \\
\hline $\mathrm{C}$ & 3.946229 & 0.469802 & -1.960281 \\
\hline $\mathrm{C}$ & 2.630896 & 2.322374 & -1.131395 \\
\hline C & 5.030605 & 1.330663 & -2.145707 \\
\hline $\mathrm{H}$ & 4.045467 & -0.584135 & -2.195501 \\
\hline $\mathrm{C}$ & 3.708869 & 3.182101 & -1.329424 \\
\hline $\mathrm{H}$ & 1.700326 & 2.702139 & -0.715885 \\
\hline $\mathrm{C}$ & 4.916195 & 2.684609 & -1.830961 \\
\hline $\mathrm{H}$ & 5.966951 & 0.938744 & -2.532926 \\
\hline $\mathrm{H}$ & 3.612821 & 4.235614 & -1.082195 \\
\hline $\mathrm{H}$ & 5.762294 & 3.351053 & -1.971971 \\
\hline $\mathrm{C}$ & -3.046664 & 4.746650 & 0.923323 \\
\hline $\mathrm{C}$ & -2.954894 & 3.535561 & 1.609473 \\
\hline $\mathrm{C}$ & -2.417489 & 2.397801 & 0.992849 \\
\hline $\mathrm{C}$ & -1.990115 & 2.500418 & -0.337076 \\
\hline
\end{tabular}




\begin{tabular}{|c|c|c|c|}
\hline $\mathrm{C}$ & -2.079787 & 3.711585 & -1.026110 \\
\hline $\mathrm{C}$ & -2.602936 & 4.841762 & -0.398005 \\
\hline $\mathrm{H}$ & -3.468269 & 5.616163 & 1.420637 \\
\hline $\mathrm{H}$ & -3.313353 & 3.477484 & 2.634200 \\
\hline $\mathrm{H}$ & -1.595855 & 1.621038 & -0.827998 \\
\hline $\mathrm{H}$ & -1.743706 & 3.759201 & -2.058598 \\
\hline $\mathrm{H}$ & -2.674351 & 5.785460 & -0.931935 \\
\hline $\mathrm{C}$ & -2.305284 & 1.081241 & 1.758993 \\
\hline $\mathrm{N}$ & -1.621944 & 0.091844 & 0.906054 \\
\hline S & -2.000299 & -1.460909 & 1.174074 \\
\hline $\mathrm{O}$ & -1.144565 & -2.279577 & 0.284781 \\
\hline $\mathrm{O}$ & -2.043282 & -1.846811 & 2.605257 \\
\hline $\mathrm{C}$ & -3.696199 & -1.590335 & 0.584144 \\
\hline $\mathrm{C}$ & -4.130724 & -0.802970 & -0.483200 \\
\hline $\mathrm{C}$ & -4.550708 & -2.522689 & 1.172699 \\
\hline $\mathrm{C}$ & -5.428330 & -0.961954 & -0.965258 \\
\hline $\mathrm{H}$ & -3.461419 & -0.068866 & -0.915176 \\
\hline $\mathrm{C}$ & -5.843568 & -2.673660 & 0.676015 \\
\hline $\mathrm{H}$ & -4.198093 & -3.111501 & 2.012446 \\
\hline C & -6.302822 & -1.897705 & -0.396988 \\
\hline $\mathrm{H}$ & -5.769667 & -0.344853 & -1.793126 \\
\hline $\mathrm{H}$ & -6.509975 & -3.402621 & 1.130806 \\
\hline C & -7.717002 & -2.042018 & -0.903734 \\
\hline $\mathrm{H}$ & -7.794868 & -1.759468 & -1.958685 \\
\hline $\mathrm{H}$ & -8.076525 & -3.071031 & -0.798641 \\
\hline $\mathrm{H}$ & -8.405594 & -1.397506 & -0.341313 \\
\hline $\mathrm{H}$ & -3.324719 & 0.742918 & 1.998694 \\
\hline
\end{tabular}

\section{INT2D}

Zero-point correction $=0.693046($ Hartree/Particle $)$

Thermal correction to Energy $=0.736745$

Thermal correction to Enthalpy= 0.737689

Thermal correction to Gibbs Free Energy= 0.609890

INT2D_sov.log

$\mathrm{E}($ Solv $)=-2696.80635448$

$\begin{array}{llll}\mathrm{C} & -3.142959 & -5.318730 & 0.290664 \\ \mathrm{C} & -2.896093 & -4.219693 & -0.529280 \\ \mathrm{C} & -1.626190 & -3.616755 & -0.579971 \\ \mathrm{C} & -0.604339 & -4.166735 & 0.221871 \\ \mathrm{C} & -0.851264 & -5.264834 & 1.038984 \\ \mathrm{C} & -2.122444 & -5.846786 & 1.082275\end{array}$

\begin{tabular}{|c|c|c|}
\hline-4.134436 & -5.763237 & 0.312133 \\
\hline-3.694283 & -3.806251 & -1.138827 \\
\hline 0.391204 & -3.732656 & 0.206753 \\
\hline-0.046938 & -5.672413 & 1.645862 \\
\hline-2.311841 & -6.702097 & 1.724433 \\
\hline-1.434394 & -2.432338 & -1.416383 \\
\hline-2.322527 & -2.070924 & -1.929774 \\
\hline-0.282228 & -1.738113 & -1.543360 \\
\hline 0.613403 & -2.118946 & -1.065262 \\
\hline-0.132410 & -0.475402 & -2.275404 \\
\hline 1.122449 & -0.353354 & -3.183882 \\
\hline 0.899465 & 0.383516 & -3.961988 \\
\hline-1.039498 & -0.241916 & -2.839379 \\
\hline 1.318132 & -1.314763 & -3.677965 \\
\hline 0.030383 & 1.103998 & -0.899444 \\
\hline-2.002905 & 1.005671 & 0.147921 \\
\hline-2.653535 & 2.675675 & 0.517554 \\
\hline-4.024451 & 2.917299 & 0.689439 \\
\hline-1.739757 & 3.733027 & 0.647844 \\
\hline-4.475324 & 4.200425 & 0.997616 \\
\hline-4.735179 & 2.104087 & 0.577800 \\
\hline-2.197692 & 5.013769 & 0.958987 \\
\hline-0.677305 & 3.563891 & 0.493748 \\
\hline-3.562426 & 5.248900 & 1.134386 \\
\hline-5.538569 & 4.382152 & 1.127156 \\
\hline-1.485194 & 5.827939 & 1.052770 \\
\hline-3.916134 & 6.248636 & 1.370239 \\
\hline-1.899767 & 0.173714 & 1.778899 \\
\hline-1.174142 & -1.025346 & 1.862131 \\
\hline-2.531525 & 0.675628 & 2.924212 \\
\hline-1.103027 & -1.723564 & 3.065219 \\
\hline-0.685191 & -1.414464 & 0.978291 \\
\hline-2.442052 & -0.017381 & 4.132859 \\
\hline-3.088555 & 1.605176 & 2.873290 \\
\hline-1.734134 & -1.218329 & 4.204473 \\
\hline-0.563027 & -2.664785 & 3.104700 \\
\hline-2.929820 & 0.381398 & 5.017897 \\
\hline-1.674765 & -1.758863 & 5.144962 \\
\hline-3.392718 & 0.173255 & -0.696994 \\
\hline-4.180267 & -0.802030 & -0.076715 \\
\hline-3.641588 & 0.515665 & -2.035310 \\
\hline-5.205222 & -1.429617 & -0.788128 \\
\hline-3.983289 & -1.085154 & 0.951367 \\
\hline 63363 & -0.113091 & -2 \\
\hline
\end{tabular}




\begin{tabular}{|c|c|c|c|}
\hline $\mathrm{H}$ & -3.024694 & 1.268567 & -2.519121 \\
\hline $\mathrm{C}$ & -5.445682 & -1.090903 & -2.119874 \\
\hline $\mathrm{H}$ & -5.808485 & -2.189952 & -0.300409 \\
\hline $\mathrm{H}$ & -4.846696 & 0.153423 & -3.779507 \\
\hline $\mathrm{H}$ & -6.238306 & -1.586098 & -2.673386 \\
\hline $\mathrm{C}$ & 4.810473 & -2.643565 & -1.471552 \\
\hline $\mathrm{C}$ & 4.154538 & -1.640215 & -2.188217 \\
\hline C & 3.086628 & -0.933693 & -1.627277 \\
\hline C & 2.694602 & -1.249302 & -0.320513 \\
\hline $\mathrm{C}$ & 3.344964 & -2.247342 & 0.400779 \\
\hline $\mathrm{C}$ & 4.406675 & -2.951775 & -0.172631 \\
\hline $\mathrm{H}$ & 5.641015 & -3.176768 & -1.926172 \\
\hline $\mathrm{H}$ & 4.483420 & -1.394645 & -3.195785 \\
\hline $\mathrm{H}$ & 1.892699 & -0.684951 & 0.141543 \\
\hline $\mathrm{H}$ & 3.034946 & -2.458306 & 1.420553 \\
\hline $\mathrm{H}$ & 4.919043 & -3.726442 & 0.391353 \\
\hline C & 2.391064 & 0.145866 & -2.455027 \\
\hline $\mathrm{N}$ & 1.869369 & 1.278279 & -1.702615 \\
\hline$S$ & 2.654525 & 2.390553 & -0.838439 \\
\hline $\mathrm{O}$ & 3.570890 & 3.211671 & -1.640463 \\
\hline $\mathrm{O}$ & 1.509547 & 3.039980 & -0.113298 \\
\hline $\mathrm{C}$ & 3.645781 & 1.602967 & 0.427164 \\
\hline C & 4.928369 & 1.157716 & 0.103314 \\
\hline $\mathrm{C}$ & 3.085819 & 1.326485 & 1.672958 \\
\hline $\mathrm{C}$ & 5.636905 & 0.398219 & 1.027237 \\
\hline $\mathrm{H}$ & 5.354270 & 1.398894 & -0.864218 \\
\hline $\mathrm{C}$ & 3.813142 & 0.572043 & 2.591643 \\
\hline $\mathrm{H}$ & 2.092229 & 1.692373 & 1.906891 \\
\hline C & 5.085517 & 0.080207 & 2.276449 \\
\hline $\mathrm{H}$ & 6.627448 & 0.031643 & 0.771219 \\
\hline $\mathrm{H}$ & 3.380647 & 0.351688 & 3.564438 \\
\hline $\mathrm{C}$ & 5.838676 & -0.803974 & 3.237977 \\
\hline $\mathrm{H}$ & 5.398725 & -0.776422 & 4.239637 \\
\hline $\mathrm{H}$ & 5.822358 & -1.846639 & 2.894059 \\
\hline $\mathrm{H}$ & 6.890356 & -0.507275 & 3.318999 \\
\hline $\mathrm{H}$ & 3.110199 & 0.493672 & -3.209938 \\
\hline
\end{tabular}

\section{INT2E}

Zero-point correction $=0.858530($ Hartree/Particle $)$

Thermal correction to Energy= 0.912924

Thermal correction to Enthalpy= 0.913868

Thermal correction to Gibbs Free Energy $=0.765118$
$\mathrm{E}($ Solv $)=-3271.53037822$

\begin{tabular}{|c|c|c|c|}
\hline $\mathrm{C}$ & -6.591185 & -0.803975 & -1.959773 \\
\hline $\mathrm{C}$ & -5.241302 & -0.812235 & -2.306944 \\
\hline $\mathrm{C}$ & -4.280267 & -1.347402 & -1.439247 \\
\hline $\mathrm{C}$ & -4.702828 & -1.845066 & -0.193575 \\
\hline $\mathrm{C}$ & -6.051134 & -1.836243 & 0.153511 \\
\hline $\mathrm{C}$ & -7.003119 & -1.323007 & -0.732171 \\
\hline $\mathrm{H}$ & -7.319971 & -0.383948 & -2.646886 \\
\hline $\mathrm{H}$ & -4.924789 & -0.402233 & -3.262156 \\
\hline $\mathrm{H}$ & -3.962629 & -2.220685 & 0.508551 \\
\hline $\mathrm{H}$ & -6.360330 & -2.225497 & 1.119820 \\
\hline $\mathrm{H}$ & -8.055036 & -1.317792 & -0.460911 \\
\hline $\mathrm{C}$ & -2.861306 & -1.406919 & -1.850926 \\
\hline $\mathrm{H}$ & -2.636309 & -0.871233 & -2.775148 \\
\hline $\mathrm{C}$ & -2.093890 & -2.595861 & -1.586429 \\
\hline $\mathrm{H}$ & -2.550199 & -3.365718 & -0.967930 \\
\hline $\mathrm{C}$ & -0.743930 & -2.703257 & -1.870334 \\
\hline $\mathrm{C}$ & 0.058920 & -3.920886 & -1.480140 \\
\hline $\mathrm{H}$ & -0.616714 & -4.772650 & -1.347306 \\
\hline $\mathrm{H}$ & -0.284873 & -2.011371 & -2.575273 \\
\hline $\mathrm{H}$ & 0.735080 & -4.177114 & -2.302237 \\
\hline $\mathrm{Pd}$ & -1.321119 & -0.751981 & -0.569634 \\
\hline $\mathrm{P}$ & -2.109017 & 1.306661 & 0.109967 \\
\hline $\mathrm{C}$ & -2.295496 & 1.422596 & 1.923036 \\
\hline $\mathrm{C}$ & -2.942483 & 2.516159 & 2.521015 \\
\hline $\mathrm{C}$ & -1.771576 & 0.404158 & 2.729490 \\
\hline $\mathrm{C}$ & -3.054184 & 2.591941 & 3.908167 \\
\hline $\mathrm{H}$ & -3.364659 & 3.300774 & 1.900958 \\
\hline $\mathrm{C}$ & -1.891589 & 0.482701 & 4.117418 \\
\hline $\mathrm{H}$ & -1.265182 & -0.437997 & 2.275045 \\
\hline $\mathrm{C}$ & -2.528545 & 1.574133 & 4.708466 \\
\hline $\mathrm{H}$ & -3.554647 & 3.441767 & 4.363530 \\
\hline $\mathrm{H}$ & -1.488008 & -0.315432 & 4.732639 \\
\hline $\mathrm{H}$ & -2.620636 & 1.631970 & 5.789484 \\
\hline $\mathrm{C}$ & -3.707528 & 1.937686 & -0.522452 \\
\hline $\mathrm{C}$ & -4.898100 & 1.573866 & 0.124199 \\
\hline $\mathrm{C}$ & -3.771859 & 2.723103 & -1.681453 \\
\hline $\mathrm{C}$ & -6.126426 & 2.009006 & -0.366981 \\
\hline $\mathrm{H}$ & -4.866154 & 0.951128 & 1.010744 \\
\hline $\mathrm{C}$ & -5.005635 & 3.147255 & -2.176044 \\
\hline $\mathrm{H}$ & -2.859410 & 3.023393 & -2.185415 \\
\hline $\mathrm{C}$ & -6.184645 & 2.797047 & -1.517250 \\
\hline $\mathrm{H}$ & -7.038869 & 1.716955 & 0.143016 \\
\hline
\end{tabular}




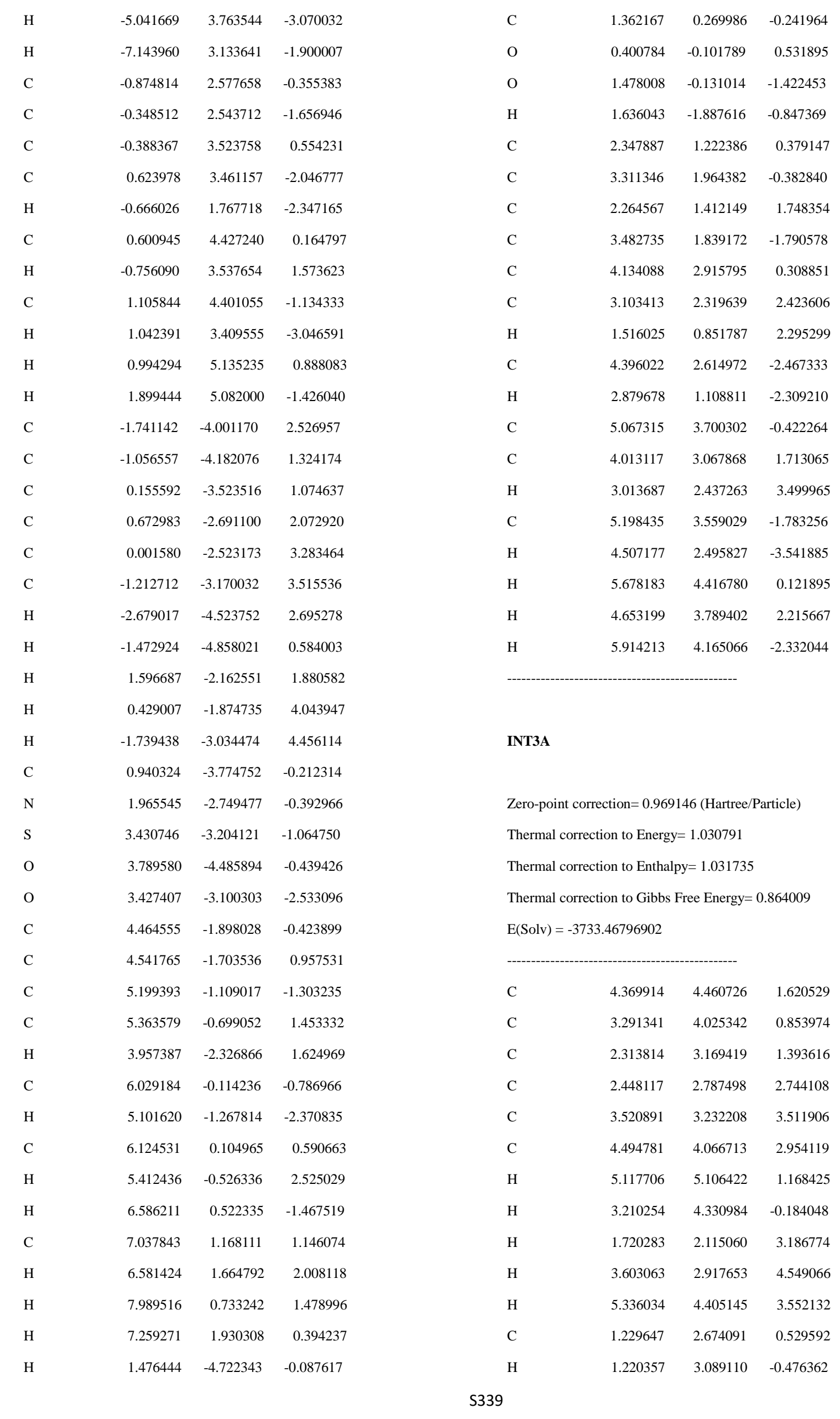




\begin{tabular}{|c|c|c|c|c|c|c|c|}
\hline $\mathrm{C}$ & 0.068012 & 2.033908 & 0.995311 & $\mathrm{C}$ & 4.683803 & -0.602903 & -3.339361 \\
\hline $\mathrm{H}$ & -0.093935 & 1.921815 & 2.063928 & $\mathrm{C}$ & 2.859067 & -1.884469 & -2.406258 \\
\hline $\mathrm{C}$ & -1.102011 & 1.829120 & 0.154038 & $\mathrm{C}$ & 4.787574 & -1.519535 & -4.384736 \\
\hline $\mathrm{C}$ & -2.317809 & 1.461091 & 0.596200 & $\mathrm{H}$ & 5.349031 & 0.254441 & -3.301318 \\
\hline $\mathrm{H}$ & -3.066238 & -0.834551 & 0.244675 & $\mathrm{C}$ & 2.961600 & -2.801458 & -3.452718 \\
\hline $\mathrm{H}$ & -0.951902 & 1.964418 & -0.918499 & $\mathrm{H}$ & 2.098106 & -2.014685 & -1.649352 \\
\hline $\mathrm{H}$ & -2.498569 & 1.318920 & 1.660050 & $\mathrm{C}$ & 3.926786 & -2.619892 & -4.443481 \\
\hline $\mathrm{Pd}$ & 1.542559 & 0.464044 & 0.283506 & $\mathrm{H}$ & 5.537285 & -1.373308 & -5.157650 \\
\hline $\mathrm{P}$ & 0.593435 & -1.589046 & 0.842726 & $\mathrm{H}$ & 2.275358 & -3.642264 & -3.492225 \\
\hline$P$ & 3.546559 & 0.375948 & -0.919873 & $\mathrm{H}$ & 4.006454 & -3.327474 & -5.264262 \\
\hline $\mathrm{C}$ & -0.093902 & -2.458760 & -0.623250 & $\mathrm{C}$ & 4.979201 & -0.036312 & 0.155158 \\
\hline $\mathrm{C}$ & 0.152668 & -3.804655 & -0.918127 & $\mathrm{C}$ & 5.103427 & 0.673818 & 1.361379 \\
\hline $\mathrm{C}$ & -0.887398 & -1.699905 & -1.500106 & $\mathrm{C}$ & 5.879006 & -1.072648 & -0.123580 \\
\hline $\mathrm{C}$ & -0.388484 & -4.380121 & -2.071235 & $\mathrm{C}$ & 6.117331 & 0.360215 & 2.262615 \\
\hline $\mathrm{H}$ & 0.769489 & -4.401122 & -0.254282 & $\mathrm{H}$ & 4.401162 & 1.464988 & 1.596796 \\
\hline $\mathrm{C}$ & -1.447104 & -2.281152 & -2.635002 & $\mathrm{C}$ & 6.885189 & -1.395094 & 0.791148 \\
\hline $\mathrm{H}$ & -1.062520 & -0.649504 & -1.285071 & $\mathrm{H}$ & 5.789182 & -1.634241 & -1.047279 \\
\hline $\mathrm{C}$ & -1.192297 & -3.624315 & -2.924747 & $\mathrm{C}$ & 7.008129 & -0.680280 & 1.983440 \\
\hline $\mathrm{H}$ & -0.190362 & -5.425644 & -2.292202 & $\mathrm{H}$ & 6.201260 & 0.924934 & 3.186738 \\
\hline $\mathrm{H}$ & -2.096773 & -1.692695 & -3.273589 & $\mathrm{H}$ & 7.575181 & -2.204198 & 0.566661 \\
\hline $\mathrm{H}$ & -1.625911 & -4.079268 & -3.810456 & $\mathrm{H}$ & 7.791586 & -0.933414 & 2.692370 \\
\hline $\mathrm{C}$ & 1.655721 & -2.846617 & 1.660457 & $\mathrm{C}$ & 4.052303 & 1.966995 & -1.693462 \\
\hline $\mathrm{C}$ & 1.129575 & -3.941710 & 2.363052 & $\mathrm{C}$ & 5.282409 & 2.590993 & -1.459486 \\
\hline $\mathrm{C}$ & 3.045326 & -2.699320 & 1.567891 & $\mathrm{C}$ & 3.112286 & 2.603891 & -2.519521 \\
\hline $\mathrm{C}$ & 1.983457 & -4.876694 & 2.947163 & $\mathrm{C}$ & 5.564325 & 3.833409 & -2.033123 \\
\hline $\mathrm{H}$ & 0.054026 & -4.058949 & 2.454365 & $\mathrm{H}$ & 6.015303 & 2.115906 & -0.816721 \\
\hline $\mathrm{C}$ & 3.899641 & -3.635124 & 2.151594 & $\mathrm{C}$ & 3.396337 & 3.838515 & -3.097759 \\
\hline $\mathrm{H}$ & 3.455748 & -1.842105 & 1.050381 & $\mathrm{H}$ & 2.150406 & 2.128302 & -2.695747 \\
\hline $\mathrm{C}$ & 3.369299 & -4.726035 & 2.841079 & $\mathrm{C}$ & 4.624032 & 4.461348 & -2.849406 \\
\hline $\mathrm{H}$ & 1.567678 & -5.721755 & 3.489179 & $\mathrm{H}$ & 6.520575 & 4.310752 & -1.836684 \\
\hline $\mathrm{H}$ & 4.973991 & -3.496899 & 2.071920 & $\mathrm{H}$ & 2.659151 & 4.320496 & -3.734008 \\
\hline $\mathrm{H}$ & 4.030717 & -5.454654 & 3.301946 & $\mathrm{H}$ & 4.842686 & 5.429895 & -3.290075 \\
\hline $\mathrm{C}$ & -0.873942 & -1.556409 & 1.959362 & $\mathrm{C}$ & -6.052692 & 3.937066 & -0.728136 \\
\hline $\mathrm{C}$ & -2.042294 & -2.291376 & 1.714251 & $\mathrm{C}$ & -4.955069 & 3.103201 & -0.948782 \\
\hline $\mathrm{C}$ & -0.818543 & -0.727190 & 3.089733 & $\mathrm{C}$ & -4.666603 & 2.060598 & -0.065775 \\
\hline $\mathrm{C}$ & -3.141638 & -2.179272 & 2.570692 & $\mathrm{C}$ & -5.486911 & 1.865645 & 1.051016 \\
\hline $\mathrm{H}$ & -2.112830 & -2.934665 & 0.842932 & $\mathrm{C}$ & -6.584878 & 2.693156 & 1.271513 \\
\hline $\mathrm{C}$ & -1.908839 & -0.627863 & 3.951380 & $\mathrm{C}$ & -6.872956 & 3.731477 & 0.381438 \\
\hline $\mathrm{H}$ & 0.082749 & -0.150174 & 3.279316 & $\mathrm{H}$ & -6.270967 & 4.739709 & -1.427326 \\
\hline $\mathrm{C}$ & -3.078910 & -1.347662 & 3.687871 & $\mathrm{H}$ & -4.324243 & 3.252453 & -1.821787 \\
\hline $\mathrm{H}$ & -4.047386 & -2.733886 & 2.344334 & $\mathrm{H}$ & -5.276742 & 1.036912 & 1.719461 \\
\hline $\mathrm{H}$ & -1.853249 & 0.021509 & 4.820884 & $\mathrm{H}$ & -7.224333 & 2.522144 & 2.133240 \\
\hline $\mathrm{H}$ & -3.935582 & -1.255494 & 4.349503 & $\mathrm{H}$ & -7.732811 & 4.373779 & 0.551398 \\
\hline $\mathrm{C}$ & 3.717549 & -0.781628 & -2.336920 & $\mathrm{C}$ & -3.471556 & 1.153878 & -0.316802 \\
\hline
\end{tabular}




\begin{tabular}{lrrr}
$\mathrm{N}$ & -3.845210 & -0.258560 & -0.069408 \\
$\mathrm{~S}$ & -4.692668 & -1.106765 & -1.244716 \\
$\mathrm{O}$ & -4.354551 & -0.621608 & -2.593334 \\
$\mathrm{O}$ & -4.513069 & -2.519958 & -0.885225 \\
$\mathrm{C}$ & -6.372770 & -0.616007 & -0.910451 \\
$\mathrm{C}$ & -6.964263 & 0.377407 & -1.687840 \\
$\mathrm{C}$ & -7.026930 & -1.157920 & 0.194188 \\
$\mathrm{C}$ & -8.222688 & 0.851052 & -1.331456 \\
$\mathrm{H}$ & -6.430817 & 0.780079 & -2.540087 \\
$\mathrm{C}$ & -8.287992 & -0.675899 & 0.533150 \\
$\mathrm{H}$ & -6.547782 & -1.937281 & 0.776752 \\
$\mathrm{C}$ & -8.894395 & 0.345098 & -0.211156 \\
$\mathrm{H}$ & -8.681861 & 1.640998 & -1.919172 \\
$\mathrm{H}$ & -8.806468 & -1.088503 & 1.394668 \\
$\mathrm{C}$ & -10.224199 & 0.922134 & 0.203419 \\
$\mathrm{H}$ & -10.878389 & 1.085386 & -0.659933 \\
$\mathrm{H}$ & -10.745872 & 0.268353 & 0.908975 \\
$\mathrm{H}$ & -10.083495 & 1.895376 & 0.691907 \\
$\mathrm{H}$ & -3.146135 & 1.281207 & -1.357151 \\
--------------------------------------- & \\
\hline
\end{tabular}

\begin{tabular}{|c|c|c|c|}
\hline $\mathrm{H}$ & -0.621730 & 0.442823 & -2.943266 \\
\hline $\mathrm{C}$ & 0.320062 & -1.519161 & -3.201884 \\
\hline $\mathrm{C}$ & -0.643845 & -2.372268 & -2.813688 \\
\hline $\mathrm{H}$ & -0.538069 & -3.429765 & -3.044798 \\
\hline $\mathrm{H}$ & 1.190337 & -1.937037 & -3.705741 \\
\hline $\mathrm{Pd}$ & 0.809426 & 0.249171 & -0.490282 \\
\hline$P$ & 2.521293 & -1.182547 & 0.126643 \\
\hline $\mathrm{P}$ & -0.394472 & 1.647535 & 0.867230 \\
\hline $\mathrm{C}$ & 4.130267 & -0.336738 & 0.431595 \\
\hline $\mathrm{C}$ & 5.328740 & -1.034243 & 0.647864 \\
\hline $\mathrm{C}$ & 4.139743 & 1.062814 & 0.472442 \\
\hline $\mathrm{C}$ & 6.513016 & -0.341826 & 0.890452 \\
\hline $\mathrm{H}$ & 5.335771 & -2.119534 & 0.617746 \\
\hline $\mathrm{C}$ & 5.325966 & 1.757356 & 0.720291 \\
\hline $\mathrm{H}$ & 3.212797 & 1.602250 & 0.313908 \\
\hline $\mathrm{C}$ & 6.513501 & 1.056752 & 0.926534 \\
\hline $\mathrm{H}$ & 7.436607 & -0.890762 & 1.053275 \\
\hline $\mathrm{H}$ & 5.309402 & 2.842489 & 0.758809 \\
\hline $\mathrm{H}$ & 7.438681 & 1.593818 & 1.117551 \\
\hline $\mathrm{C}$ & 2.290706 & -2.145255 & 1.685246 \\
\hline $\mathrm{C}$ & 2.948916 & -3.350153 & 1.969375 \\
\hline $\mathrm{C}$ & 1.418530 & -1.609341 & 2.641835 \\
\hline $\mathrm{C}$ & 2.738133 & -3.998674 & 3.186414 \\
\hline $\mathrm{H}$ & 3.610778 & -3.793231 & 1.231974 \\
\hline $\mathrm{C}$ & 1.214878 & -2.249181 & 3.864797 \\
\hline $\mathrm{H}$ & 0.874581 & -0.703219 & 2.405189 \\
\hline $\mathrm{C}$ & 1.875052 & -3.448492 & 4.138472 \\
\hline $\mathrm{H}$ & 3.247668 & -4.936230 & 3.392087 \\
\hline $\mathrm{H}$ & 0.523165 & -1.818997 & 4.583181 \\
\hline $\mathrm{H}$ & 1.712524 & -3.959042 & 5.083792 \\
\hline C & 2.959832 & -2.470389 & -1.107617 \\
\hline $\mathrm{C}$ & 3.893600 & -2.194170 & -2.119022 \\
\hline C & 2.203407 & -3.650434 & -1.194745 \\
\hline C & 4.065373 & -3.072873 & -3.189305 \\
\hline $\mathrm{H}$ & 4.490660 & -1.288552 & -2.066827 \\
\hline C & 2.382215 & -4.530796 & -2.259899 \\
\hline $\mathrm{H}$ & 1.457942 & -3.872525 & -0.438538 \\
\hline $\mathrm{C}$ & 3.309033 & -4.244182 & -3.265272 \\
\hline $\mathrm{H}$ & 4.793695 & -2.842705 & -3.962283 \\
\hline $\mathrm{H}$ & 1.788372 & -5.439697 & -2.308166 \\
\hline $\mathrm{H}$ & 3.442781 & -4.928581 & -4.098186 \\
\hline $\mathrm{C}$ & -1.188277 & 0.878171 & 2.344645 \\
\hline C & -1.048158 & 1.343784 & 3.660042 \\
\hline $\mathrm{C}$ & -1.941705 & -0.283347 & 2.118423 \\
\hline
\end{tabular}




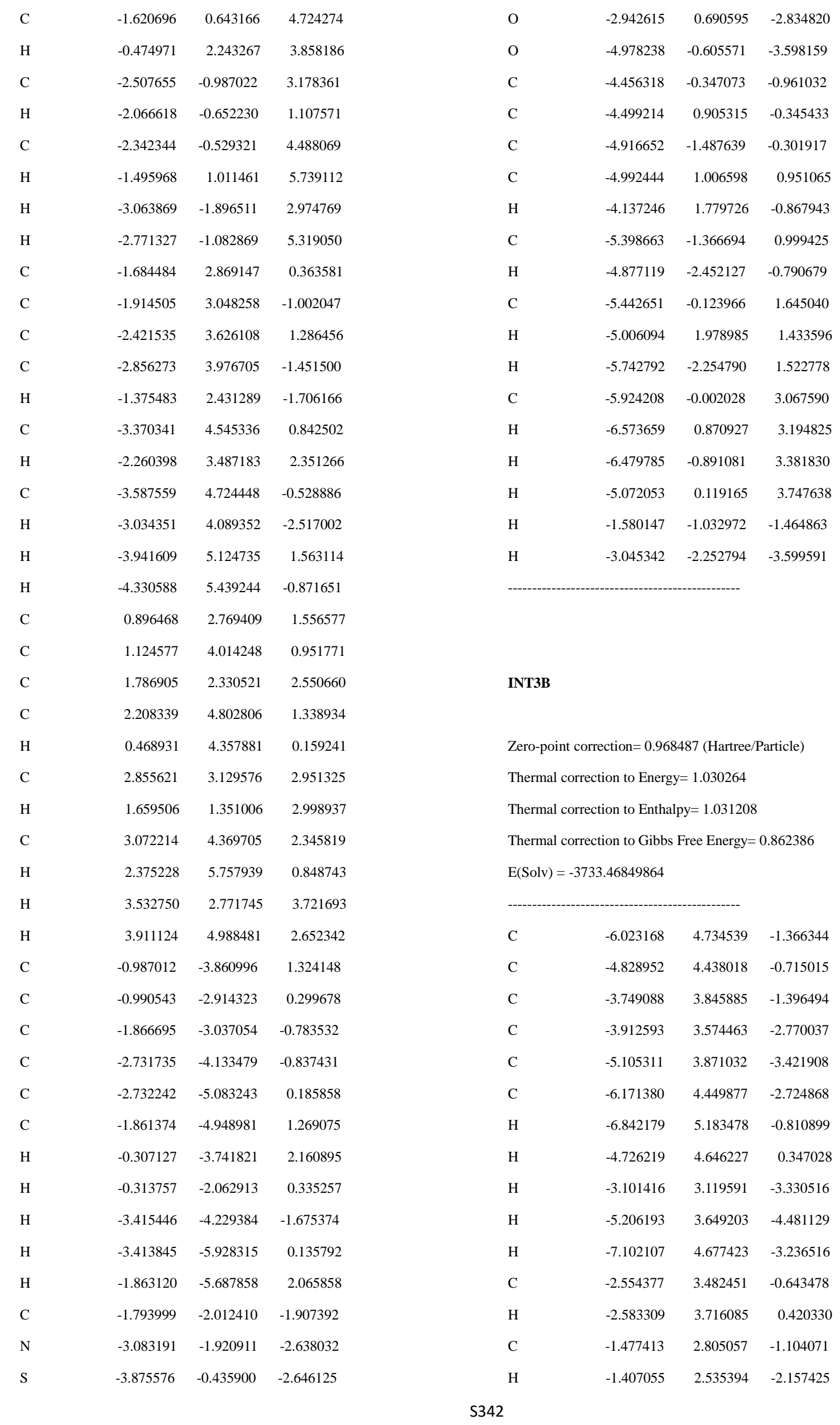




\begin{tabular}{|c|c|c|c|c|c|c|c|}
\hline $\mathrm{C}$ & -0.397239 & 2.346183 & -0.251124 & $\mathrm{C}$ & -2.488012 & -3.194093 & 4.131029 \\
\hline $\mathrm{C}$ & 0.798080 & 1.779967 & -0.716344 & $\mathrm{H}$ & -2.073985 & -1.080687 & 4.129382 \\
\hline $\mathrm{H}$ & 1.638915 & -0.303985 & 0.317162 & $\mathrm{C}$ & -3.052910 & -4.164806 & 1.993168 \\
\hline $\mathrm{H}$ & -0.450232 & 2.650389 & 0.792229 & $\mathrm{H}$ & -3.032015 & -2.824981 & 0.314974 \\
\hline $\mathrm{H}$ & 1.021348 & 1.776020 & -1.780909 & $\mathrm{C}$ & -2.845333 & -4.304548 & 3.368201 \\
\hline $\mathrm{Pd}$ & -0.635226 & 0.077101 & -0.306287 & $\mathrm{H}$ & -2.323946 & -3.296336 & 5.200384 \\
\hline$P$ & 0.070074 & -1.933947 & -1.218492 & $\mathrm{H}$ & -3.310509 & -5.029001 & 1.388991 \\
\hline$P$ & -2.361076 & -0.202403 & 1.258740 & $\mathrm{H}$ & -2.953065 & -5.277922 & 3.838475 \\
\hline $\mathrm{C}$ & 0.038162 & -3.402750 & -0.118834 & $\mathrm{C}$ & -4.100803 & 0.182405 & 0.824925 \\
\hline $\mathrm{C}$ & -0.352519 & -4.675123 & -0.556120 & $\mathrm{C}$ & -4.373544 & 0.718008 & -0.439866 \\
\hline $\mathrm{C}$ & 0.441139 & -3.229584 & 1.213256 & $\mathrm{C}$ & -5.159359 & -0.039370 & 1.719910 \\
\hline $\mathrm{C}$ & -0.336219 & -5.756145 & 0.325956 & $\mathrm{C}$ & -5.679744 & 1.053002 & -0.801552 \\
\hline $\mathrm{H}$ & -0.672934 & -4.820839 & -1.582644 & $\mathrm{H}$ & -3.554281 & 0.893175 & -1.127846 \\
\hline $\mathrm{C}$ & 0.470542 & -4.312640 & 2.088032 & $\mathrm{C}$ & -6.463989 & 0.288842 & 1.356649 \\
\hline $\mathrm{H}$ & 0.745520 & -2.250987 & 1.565654 & $\mathrm{H}$ & -4.956902 & -0.472059 & 2.695437 \\
\hline C & 0.077611 & -5.577299 & 1.647057 & $\mathrm{C}$ & -6.723978 & 0.839761 & 0.097538 \\
\hline H & -0.646289 & -6.738526 & -0.020381 & $\mathrm{H}$ & -5.871658 & 1.498780 & -1.772298 \\
\hline $\mathrm{H}$ & 0.785792 & -4.161626 & 3.115499 & $\mathrm{H}$ & -7.278853 & 0.116455 & 2.054592 \\
\hline $\mathrm{H}$ & 0.086632 & -6.419702 & 2.333146 & $\mathrm{H}$ & -7.740871 & 1.103579 & -0.179683 \\
\hline $\mathrm{C}$ & -1.014601 & -2.415497 & -2.621318 & $\mathrm{C}$ & -1.913363 & 1.006640 & 2.565059 \\
\hline $\mathrm{C}$ & -0.557420 & -3.164475 & -3.714460 & $\mathrm{C}$ & -0.597749 & 0.954255 & 3.063124 \\
\hline C & -2.362539 & -2.025758 & -2.576164 & $\mathrm{C}$ & -2.735755 & 2.076905 & 2.934976 \\
\hline $\mathrm{C}$ & -1.435410 & -3.523450 & -4.738409 & C & -0.115996 & 1.945857 & 3.912208 \\
\hline H & 0.485145 & -3.462916 & -3.763719 & $\mathrm{H}$ & 0.066249 & 0.150203 & 2.758519 \\
\hline $\mathrm{C}$ & -3.239402 & -2.390333 & -3.596939 & $\mathrm{C}$ & -2.249373 & 3.076000 & 3.784595 \\
\hline $\mathrm{H}$ & -2.712796 & -1.425308 & -1.741976 & $\mathrm{H}$ & -3.748166 & 2.139389 & 2.549815 \\
\hline C & -2.777007 & -3.140176 & -4.680672 & $\mathrm{C}$ & -0.944030 & 3.015042 & 4.272328 \\
\hline $\mathrm{H}$ & -1.070772 & -4.103400 & -5.581836 & $\mathrm{H}$ & 0.910670 & 1.887504 & 4.259576 \\
\hline $\mathrm{H}$ & -4.279797 & -2.080849 & -3.548282 & $\mathrm{H}$ & -2.895074 & 3.905727 & 4.060010 \\
\hline $\mathrm{H}$ & -3.457610 & -3.420324 & -5.479797 & $\mathrm{H}$ & -0.568833 & 3.799092 & 4.924092 \\
\hline $\mathrm{C}$ & 1.757602 & -2.018546 & -1.951506 & $\mathrm{C}$ & 4.459558 & 4.618009 & -0.204234 \\
\hline C & 2.726020 & -2.941153 & -1.539768 & $\mathrm{C}$ & 3.393923 & 3.841271 & 0.254843 \\
\hline C & 2.106698 & -1.040694 & -2.896911 & $\mathrm{C}$ & 3.148349 & 2.579960 & -0.292216 \\
\hline C & 4.018530 & -2.889353 & -2.069619 & $\mathrm{C}$ & 3.981895 & 2.101949 & -1.310390 \\
\hline H & 2.477212 & -3.692746 & -0.799046 & $\mathrm{C}$ & 5.047856 & 2.873256 & -1.765997 \\
\hline C & 3.390961 & -0.997101 & -3.432662 & $\mathrm{C}$ & 5.291337 & 4.134295 & -1.214435 \\
\hline $\mathrm{H}$ & 1.366206 & -0.304882 & -3.199699 & $\mathrm{H}$ & 4.644561 & 5.594886 & 0.234278 \\
\hline C & 4.354693 & -1.920752 & -3.014920 & $\mathrm{H}$ & 2.755346 & 4.208545 & 1.054571 \\
\hline $\mathrm{H}$ & 4.761443 & -3.610819 & -1.739783 & $\mathrm{H}$ & 3.811528 & 1.107088 & -1.707397 \\
\hline H & 3.645337 & -0.236196 & -4.165539 & $\mathrm{H}$ & 5.699127 & 2.484314 & -2.544303 \\
\hline H & 5.360938 & -1.880381 & -3.422610 & $\mathrm{H}$ & 6.126667 & 4.733210 & -1.567241 \\
\hline C & -2.547341 & -1.796166 & 2.151006 & $\mathrm{C}$ & 1.996231 & 1.735459 & 0.221354 \\
\hline C & -2.343994 & -1.941955 & 3.527582 & $\mathrm{~N}$ & 2.436002 & 0.334329 & 0.398581 \\
\hline C & -2.895894 & -2.922542 & 1.388268 & $\mathrm{~S}$ & 3.305103 & -0.049226 & 1.781118 \\
\hline
\end{tabular}




\begin{tabular}{|c|c|c|c|}
\hline $\mathrm{O}$ & 2.975282 & 0.859925 & 2.893522 \\
\hline $\mathrm{O}$ & 3.134532 & -1.500967 & 1.942214 \\
\hline $\mathrm{C}$ & 4.967530 & 0.311239 & 1.255608 \\
\hline $\mathrm{C}$ & 5.598275 & 1.468159 & 1.705969 \\
\hline $\mathrm{C}$ & 5.551566 & -0.509967 & 0.292605 \\
\hline $\mathrm{C}$ & 6.833931 & 1.812412 & 1.165836 \\
\hline $\mathrm{H}$ & 5.110302 & 2.092403 & 2.444751 \\
\hline $\mathrm{C}$ & 6.787983 & -0.150730 & -0.233833 \\
\hline $\mathrm{H}$ & 5.030599 & -1.394357 & -0.055132 \\
\hline $\mathrm{C}$ & 7.438209 & 1.019595 & 0.181824 \\
\hline $\mathrm{H}$ & 7.325811 & 2.723388 & 1.495547 \\
\hline $\mathrm{H}$ & 7.248120 & -0.777756 & -0.993282 \\
\hline $\mathrm{C}$ & 8.738531 & 1.448906 & -0.449525 \\
\hline $\mathrm{H}$ & 9.432205 & 1.855540 & 0.294297 \\
\hline $\mathrm{H}$ & 9.233441 & 0.616857 & -0.959850 \\
\hline $\mathrm{H}$ & 8.561786 & 2.237427 & -1.193050 \\
\hline $\mathrm{H}$ & 1.673016 & 2.137301 & 1.188459 \\
\hline
\end{tabular}

\begin{tabular}{|c|c|c|c|}
\hline $\mathrm{H}$ & -1.327171 & -0.389199 & -1.751224 \\
\hline $\mathrm{H}$ & 1.138966 & -2.862904 & 0.527196 \\
\hline $\mathrm{H}$ & 0.883898 & -1.975173 & -2.426144 \\
\hline $\mathrm{Pd}$ & 1.172809 & -0.285431 & -0.308738 \\
\hline $\mathrm{P}$ & 0.687594 & 1.912255 & 0.046475 \\
\hline $\mathrm{C}$ & 0.517339 & 2.324758 & 1.830966 \\
\hline $\mathrm{C}$ & 0.691223 & 3.622478 & 2.331949 \\
\hline $\mathrm{C}$ & 0.176498 & 1.290195 & 2.715360 \\
\hline $\mathrm{C}$ & 0.510180 & 3.882143 & 3.690942 \\
\hline $\mathrm{H}$ & 0.973236 & 4.427542 & 1.660597 \\
\hline $\mathrm{C}$ & -0.009959 & 1.553014 & 4.072333 \\
\hline $\mathrm{H}$ & 0.069606 & 0.279411 & 2.333480 \\
\hline $\mathrm{C}$ & 0.154842 & 2.850047 & 4.562222 \\
\hline $\mathrm{H}$ & 0.650104 & 4.890682 & 4.070013 \\
\hline $\mathrm{H}$ & -0.271318 & 0.742195 & 4.746828 \\
\hline $\mathrm{H}$ & 0.017009 & 3.055035 & 5.620162 \\
\hline $\mathrm{C}$ & -0.912171 & 2.470819 & -0.672216 \\
\hline $\mathrm{C}$ & -1.167015 & 2.149380 & -2.015958 \\
\hline $\mathrm{C}$ & -1.867848 & 3.200569 & 0.046675 \\
\hline $\mathrm{C}$ & -2.345625 & 2.561913 & -2.634646 \\
\hline $\mathrm{H}$ & -0.434320 & 1.573124 & -2.576138 \\
\hline $\mathrm{C}$ & -3.048618 & 3.615049 & -0.575080 \\
\hline $\mathrm{H}$ & -1.688867 & 3.450937 & 1.086729 \\
\hline $\mathrm{C}$ & -3.288178 & 3.300922 & -1.913781 \\
\hline $\mathrm{H}$ & -2.543196 & 2.274566 & -3.660955 \\
\hline $\mathrm{H}$ & -3.782734 & 4.182281 & -0.009137 \\
\hline $\mathrm{H}$ & -4.212801 & 3.612123 & -2.390713 \\
\hline $\mathrm{C}$ & 1.865183 & 3.179962 & -0.567799 \\
\hline $\mathrm{C}$ & 1.463111 & 4.446175 & -1.014199 \\
\hline $\mathrm{C}$ & 3.229250 & 2.853842 & -0.564112 \\
\hline $\mathrm{C}$ & 2.414105 & 5.374803 & -1.440091 \\
\hline $\mathrm{H}$ & 0.408385 & 4.702566 & -1.034495 \\
\hline $\mathrm{C}$ & 4.177896 & 3.786219 & -0.981276 \\
\hline $\mathrm{H}$ & 3.537424 & 1.862138 & -0.241654 \\
\hline $\mathrm{C}$ & 3.771228 & 5.048159 & -1.420935 \\
\hline $\mathrm{H}$ & 2.093898 & 6.352692 & -1.788892 \\
\hline $\mathrm{H}$ & 5.232089 & 3.524015 & -0.974501 \\
\hline $\mathrm{H}$ & 4.509263 & 5.771714 & -1.755472 \\
\hline $\mathrm{C}$ & -2.073610 & -4.085695 & 1.945072 \\
\hline $\mathrm{C}$ & -1.654105 & -3.887162 & 0.626804 \\
\hline $\mathrm{C}$ & -1.421772 & -2.599967 & 0.138499 \\
\hline $\mathrm{C}$ & -1.624070 & -1.507453 & 0.989711 \\
\hline $\mathrm{C}$ & -2.049745 & -1.698548 & 2.301517 \\
\hline $\mathrm{C}$ & -2.272070 & -2.990567 & 2.785733 \\
\hline
\end{tabular}




\begin{tabular}{|c|c|c|c|c|c|c|c|}
\hline $\mathrm{H}$ & -2.251610 & -5.093658 & 2.309187 & $\mathrm{H}$ & -0.492463 & -4.247774 & -3.460665 \\
\hline $\mathrm{H}$ & -1.513258 & -4.740448 & -0.032337 & $\mathrm{H}$ & 1.247718 & -6.025689 & -3.465674 \\
\hline $\mathrm{H}$ & -1.470536 & -0.502390 & 0.614286 & $\mathrm{C}$ & 0.618756 & -2.917641 & 0.868045 \\
\hline $\mathrm{H}$ & -2.220864 & -0.836017 & 2.939477 & $\mathrm{H}$ & 1.222641 & -3.218119 & 1.723793 \\
\hline $\mathrm{H}$ & -2.607149 & -3.140783 & 3.808407 & $\mathrm{C}$ & -0.504820 & -2.099928 & 1.088824 \\
\hline $\mathrm{C}$ & -0.941426 & -2.410213 & -1.293097 & $\mathrm{H}$ & -1.195653 & -1.934786 & 0.273643 \\
\hline $\mathrm{N}$ & -1.600633 & -1.327444 & -2.041354 & $\mathrm{C}$ & -0.600142 & -1.221189 & 2.178539 \\
\hline S & -3.219946 & -1.437191 & -2.450736 & $\mathrm{C}$ & -1.688177 & -0.190939 & 2.227172 \\
\hline $\mathrm{O}$ & -3.475279 & -0.296184 & -3.333461 & $\mathrm{H}$ & -1.507297 & 0.512989 & 3.038429 \\
\hline $\mathrm{O}$ & -3.441598 & -2.822039 & -2.875898 & $\mathrm{H}$ & -0.079000 & -1.474420 & 3.103634 \\
\hline $\mathrm{C}$ & -4.141789 & -1.171939 & -0.942882 & $\mathrm{H}$ & -1.634371 & 0.358818 & 1.288070 \\
\hline $\mathrm{C}$ & -4.229011 & 0.119651 & -0.426582 & $\mathrm{Pd}$ & 1.212287 & -0.708934 & 0.901587 \\
\hline $\mathrm{C}$ & -4.650954 & -2.265780 & -0.244248 & $\mathrm{P}$ & 1.419272 & 1.585351 & 1.393610 \\
\hline $\mathrm{C}$ & -4.814303 & 0.309427 & 0.822389 & $\mathrm{P}$ & 3.237777 & -0.974918 & -0.259915 \\
\hline $\mathrm{H}$ & -3.841521 & 0.960738 & -0.988107 & $\mathrm{C}$ & 2.946327 & 1.816255 & 2.385026 \\
\hline $\mathrm{C}$ & -5.237152 & -2.056764 & 0.999683 & $\mathrm{C}$ & 3.833157 & 2.886434 & 2.233951 \\
\hline $\mathrm{H}$ & -4.567256 & -3.258859 & -0.669058 & $\mathrm{C}$ & 3.200394 & 0.855372 & 3.377903 \\
\hline $\mathrm{C}$ & -5.311957 & -0.773280 & 1.558567 & $\mathrm{C}$ & 4.941222 & 3.003495 & 3.073268 \\
\hline $\mathrm{H}$ & -4.874728 & 1.313751 & 1.233502 & $\mathrm{H}$ & 3.679041 & 3.613409 & 1.446575 \\
\hline $\mathrm{H}$ & -5.622634 & -2.905900 & 1.557481 & $\mathrm{C}$ & 4.303283 & 0.978481 & 4.221114 \\
\hline $\mathrm{C}$ & -5.881461 & -0.577665 & 2.940952 & $\mathrm{H}$ & 2.525577 & 0.009029 & 3.482369 \\
\hline $\mathrm{H}$ & -6.813454 & -1.137835 & 3.075277 & $\mathrm{C}$ & 5.175161 & 2.058731 & 4.072312 \\
\hline $\mathrm{H}$ & -5.176100 & -0.936599 & 3.702089 & $\mathrm{H}$ & 5.629248 & 3.832550 & 2.936573 \\
\hline $\mathrm{H}$ & -6.084658 & 0.477548 & 3.147338 & $\mathrm{H}$ & 4.482988 & 0.231565 & 4.989455 \\
\hline $\mathrm{H}$ & -1.172334 & -3.325110 & -1.851879 & $\mathrm{H}$ & 6.038492 & 2.156349 & 4.724295 \\
\hline \multirow{2}{*}{\multicolumn{4}{|c|}{ - }} & $\mathrm{C}$ & 1.511464 & 2.640551 & -0.093216 \\
\hline & & & & $\mathrm{C}$ & 1.643991 & 4.036300 & 0.001977 \\
\hline \multirow{2}{*}{\multicolumn{4}{|c|}{ INT3D }} & $\mathrm{C}$ & 1.356936 & 2.053519 & -1.353437 \\
\hline & & & & $\mathrm{C}$ & 1.682315 & 4.814311 & -1.152600 \\
\hline \multicolumn{4}{|c|}{ Zero-point correction= 1.136538 (Hartree/Particle) } & $\mathrm{H}$ & 1.682298 & 4.513583 & 0.976073 \\
\hline \multicolumn{4}{|c|}{ Thermal correction to Energy= 1.208466} & $\mathrm{C}$ & 1.394504 & 2.834593 & -2.508490 \\
\hline \multicolumn{4}{|c|}{ Thermal correction to Enthalpy= 1.209410} & $\mathrm{H}$ & 1.151464 & 0.995167 & -1.428801 \\
\hline \multicolumn{4}{|c|}{ Thermal correction to Gibbs Free Energy= 1.022205} & $\mathrm{C}$ & 1.568601 & 4.213581 & -2.409919 \\
\hline \multicolumn{4}{|c|}{$E($ Solv $)=-4308.17406019$} & $\mathrm{H}$ & 1.776924 & 5.893344 & -1.071807 \\
\hline & & ----- & & $\mathrm{H}$ & 1.246076 & 2.364321 & -3.474746 \\
\hline $\mathrm{C}$ & 1.909358 & -5.583083 & -1.460618 & $\mathrm{H}$ & 1.563939 & 4.826161 & -3.305763 \\
\hline $\mathrm{C}$ & 1.732488 & -4.774036 & -0.339917 & $\mathrm{C}$ & 0.133368 & 2.438001 & 2.376943 \\
\hline $\mathrm{C}$ & 0.764270 & -3.758102 & -0.329878 & $\mathrm{C}$ & 0.206996 & 2.535263 & 3.773081 \\
\hline $\mathrm{C}$ & -0.040720 & -3.584238 & -1.472267 & $\mathrm{C}$ & -1.029262 & 2.861677 & 1.714678 \\
\hline $\mathrm{C}$ & 0.133801 & -4.398156 & -2.585645 & $\mathrm{C}$ & -0.877082 & 3.039208 & 4.493419 \\
\hline $\mathrm{C}$ & 1.112241 & -5.397223 & -2.589997 & $\mathrm{H}$ & 1.102565 & 2.218338 & 4.297617 \\
\hline $\mathrm{H}$ & 2.671634 & -6.357123 & -1.448433 & $\mathrm{C}$ & -2.115291 & 3.346167 & 2.440110 \\
\hline $\mathrm{H}$ & 2.353862 & -4.922920 & 0.537666 & $\mathrm{H}$ & -1.111599 & 2.760503 & 0.640107 \\
\hline $\mathrm{H}$ & -0.786546 & -2.796759 & -1.497030 & $\mathrm{C}$ & -2.041924 & 3.433266 & 3.831201 \\
\hline & & & & & & & \\
\hline
\end{tabular}




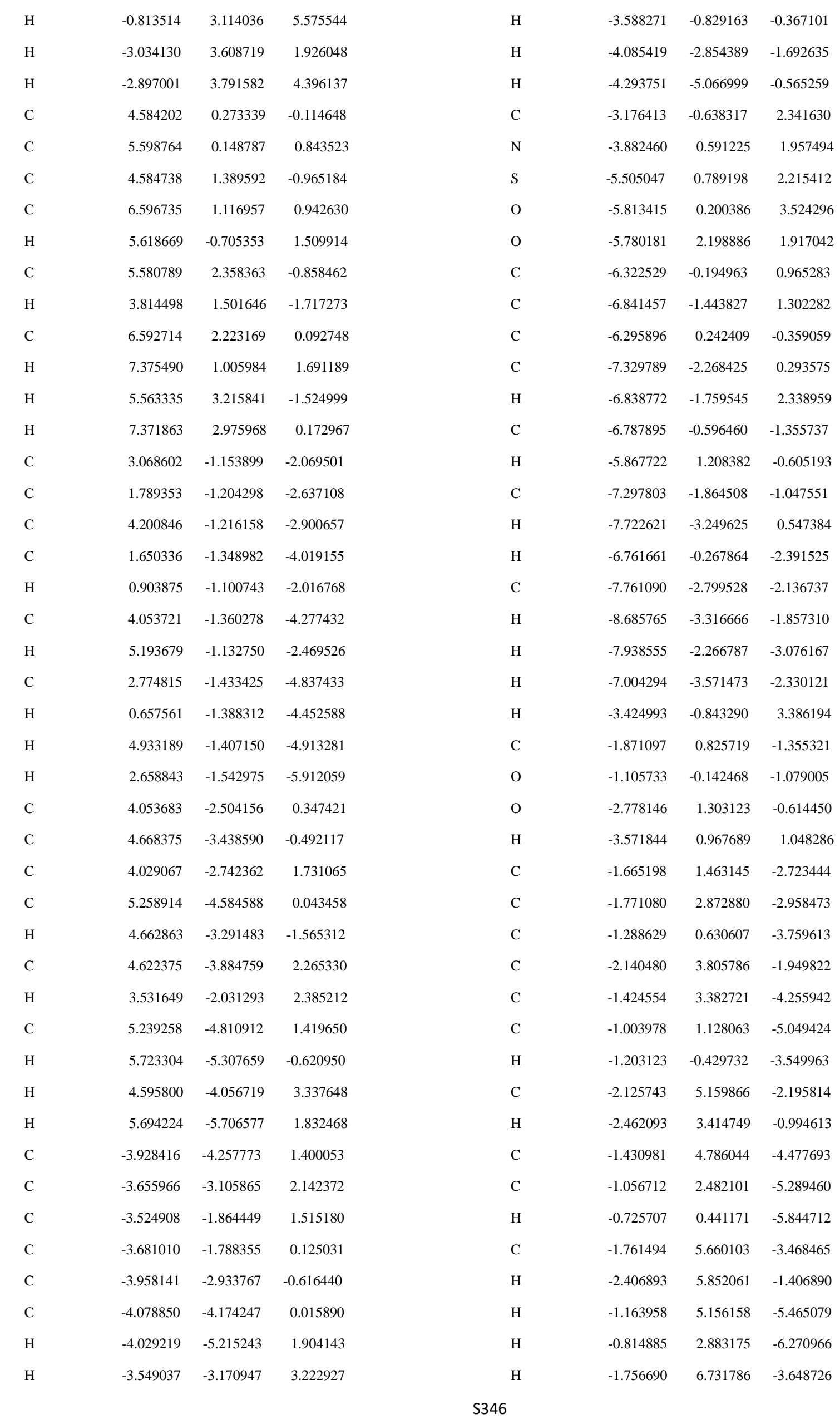




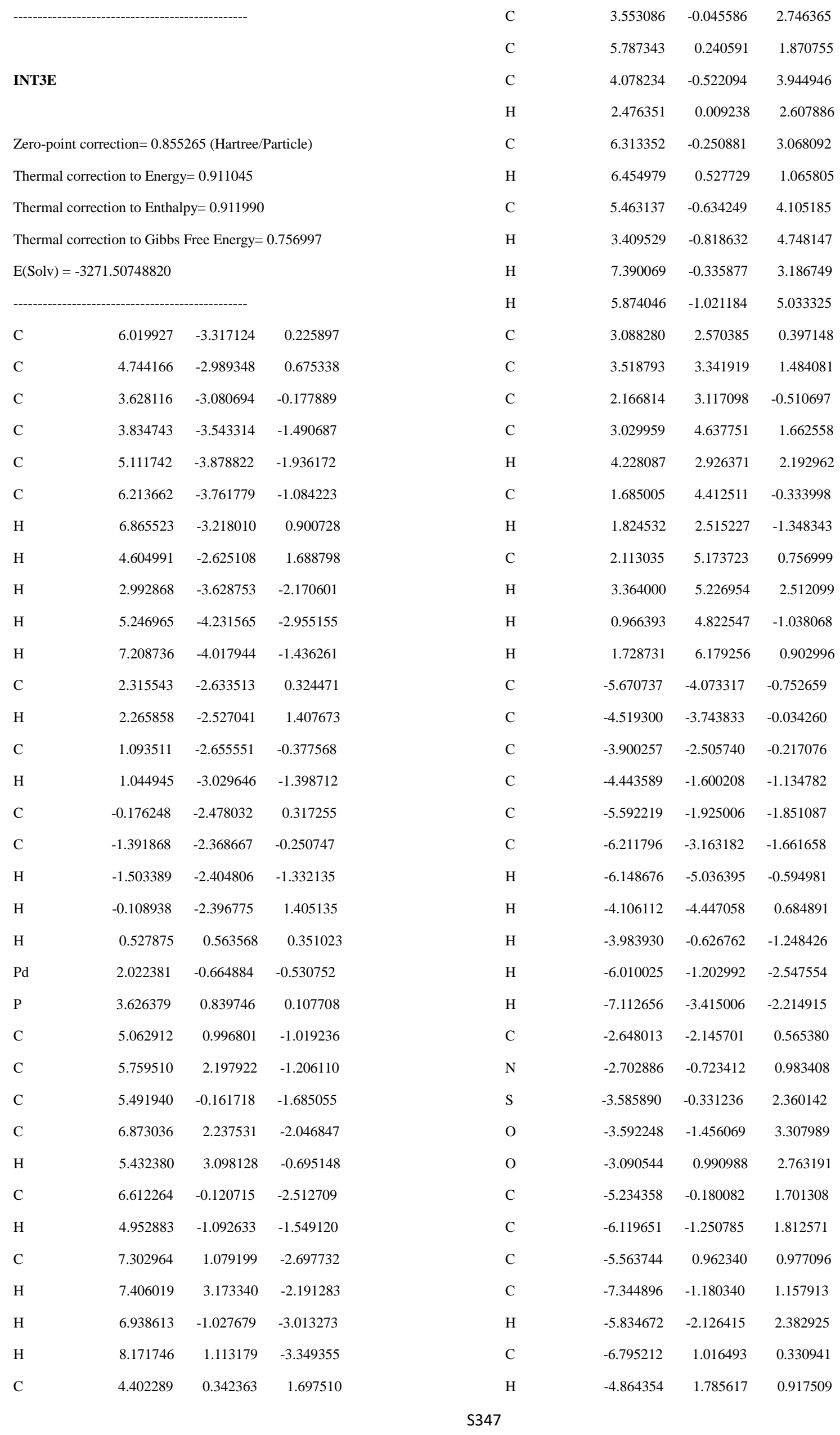




\begin{tabular}{|c|c|c|c|}
\hline $\mathrm{C}$ & -7.692601 & -0.057194 & 0.395239 \\
\hline $\mathrm{H}$ & -8.032511 & -2.019311 & 1.219729 \\
\hline $\mathrm{H}$ & -7.055103 & 1.902239 & -0.242274 \\
\hline $\mathrm{C}$ & -8.990970 & -0.030882 & -0.371155 \\
\hline $\mathrm{H}$ & -8.924985 & -0.672001 & -1.260181 \\
\hline $\mathrm{H}$ & -9.824136 & -0.404138 & 0.234558 \\
\hline $\mathrm{H}$ & -9.240056 & 0.980294 & -0.707682 \\
\hline $\mathrm{H}$ & -2.603150 & -2.776020 & 1.462362 \\
\hline C & -0.558197 & 0.858598 & -1.155103 \\
\hline $\mathrm{O}$ & 0.309430 & 0.356490 & -1.870848 \\
\hline $\mathrm{O}$ & -0.371293 & 0.976936 & 0.168487 \\
\hline $\mathrm{H}$ & -1.804579 & -0.244569 & 1.014898 \\
\hline $\mathrm{C}$ & -1.858534 & 1.327904 & -1.690131 \\
\hline C & -2.703011 & 2.310861 & -1.067566 \\
\hline $\mathrm{C}$ & -2.233846 & 0.739702 & -2.888498 \\
\hline $\mathrm{C}$ & -2.392810 & 2.997275 & 0.137899 \\
\hline $\mathrm{C}$ & -3.932128 & 2.645875 & -1.733986 \\
\hline C & -3.456792 & 1.053427 & -3.510347 \\
\hline $\mathrm{H}$ & -1.568393 & 0.008993 & -3.333042 \\
\hline $\mathrm{C}$ & -3.244268 & 3.953542 & 0.647407 \\
\hline $\mathrm{H}$ & -1.486276 & 2.756031 & 0.671913 \\
\hline $\mathrm{C}$ & -4.780984 & 3.641611 & -1.182939 \\
\hline C & -4.288692 & 1.987877 & -2.939083 \\
\hline $\mathrm{H}$ & -3.734536 & 0.553816 & -4.432896 \\
\hline $\mathrm{C}$ & -4.447475 & 4.286580 & -0.015032 \\
\hline $\mathrm{H}$ & -2.988700 & 4.447487 & 1.579505 \\
\hline $\mathrm{H}$ & -5.701201 & 3.884511 & -1.708055 \\
\hline $\mathrm{H}$ & -5.235000 & 2.245013 & -3.408148 \\
\hline $\mathrm{H}$ & -5.105306 & 5.043038 & 0.402334 \\
\hline
\end{tabular}

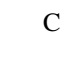

C

C

\section{INT3E'}

Zero-point correction $=0.857452$ (Hartree/Particle)

Thermal correction to Energy $=0.912527$

Thermal correction to Enthalpy= 0.913472

Thermal correction to Gibbs Free Energy $=0.762582$

$\mathrm{E}($ Solv $)=-3271.50606336$

$\begin{array}{llll} & & & \\ \text { C } & 2.879416 & -4.574885 & -1.442039 \\ \mathrm{C} & 2.162316 & -3.506442 & -1.975682 \\ \mathrm{C} & 2.418620 & -2.182993 & -1.571017 \\ \mathrm{C} & 3.433857 & -1.964313 & -0.623186 \\ \mathrm{C} & 4.157903 & -3.032778 & -0.100282\end{array}$

H

H

H

H

H

C

$\mathrm{H}$

C

H

C

C

H

H

H

Pd

$\mathrm{P}$

C

C

C

C

H

H

C

$\mathrm{H}$

$\mathrm{H}$

H

C

C

C

\begin{tabular}{|c|c|c|}
\hline 3.884075 & -4.344206 & -0.498573 \\
\hline 2.655271 & -5.587877 & -1.765146 \\
\hline 1.373247 & -3.692273 & -2.699813 \\
\hline 3.663839 & -0.956806 & -0.295816 \\
\hline 4.947499 & -2.837133 & 0.618630 \\
\hline 4.454606 & -5.173202 & -0.089384 \\
\hline 1.630002 & -1.089282 & -2.166419 \\
\hline 1.162665 & -1.355104 & -3.115795 \\
\hline 1.810215 & 0.291932 & -1.911969 \\
\hline 2.475623 & 0.584324 & -1.106636 \\
\hline 1.538860 & 1.304129 & -2.929160 \\
\hline 1.861033 & 2.613902 & -2.887646 \\
\hline 1.634571 & 3.229111 & -3.753631 \\
\hline 1.053493 & 0.938079 & -3.834508 \\
\hline-1.648604 & 0.409464 & -1.911596 \\
\hline 0.089018 & -0.413119 & -0.828426 \\
\hline-1.300819 & -1.994073 & 0.129163 \\
\hline-0.529989 & -2.844151 & 1.565984 \\
\hline-1.025136 & -2.770317 & 2.873072 \\
\hline 0.650035 & -3.564966 & 1.318655 \\
\hline-0.345562 & -3.402352 & 3.918628 \\
\hline-1.938909 & -2.223612 & 3.079024 \\
\hline 1.309680 & -4.213297 & 2.358622 \\
\hline 1.051861 & -3.618621 & 0.313815 \\
\hline 0.819329 & -4.126563 & 3.665200 \\
\hline-0.735403 & -3.332527 & 4.930350 \\
\hline 2.213882 & -4.774722 & 2.143359 \\
\hline 1.343016 & -4.619858 & 4.479161 \\
\hline-1.744534 & -3.407264 & -0.951952 \\
\hline-1.619979 & -3.254832 & -2.338741 \\
\hline-2.185124 & -4.633642 & -0.433891 \\
\hline-1.938080 & -4.307687 & -3.197876 \\
\hline-1.252391 & -2.310426 & -2.731048 \\
\hline-2.499438 & -5.686643 & -1.291893 \\
\hline-2.271862 & -4.763147 & 0.640775 \\
\hline-2.376726 & -5.525058 & -2.674622 \\
\hline-1.834731 & -4.180544 & -4.271911 \\
\hline-2.836511 & -6.634854 & -0.882565 \\
\hline-2.617376 & -6.348619 & -3.340975 \\
\hline-2.907955 & -1.376903 & 0.767348 \\
\hline-4.145266 & -1.891492 & 0.356472 \\
\hline-2.885772 & -0.267890 & 1.631139 \\
\hline-5.332840 & -1.306199 & 0.798514 \\
\hline-4.181198 & -2.742796 & -0.314933 \\
\hline
\end{tabular}

H 


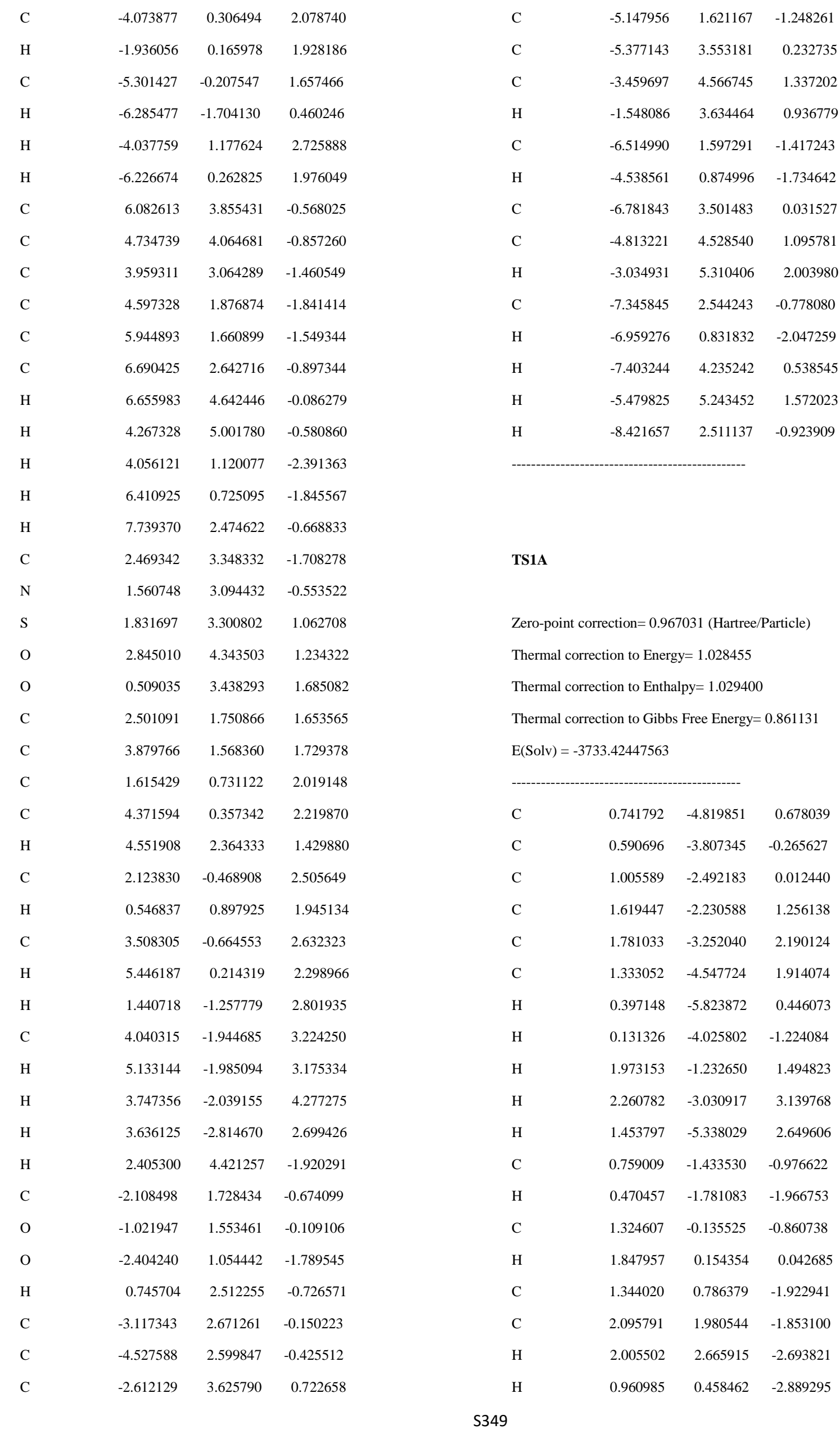




\begin{tabular}{|c|c|c|c|c|c|c|c|}
\hline $\mathrm{H}$ & 2.119523 & 2.483125 & -0.891902 & $\mathrm{C}$ & -6.743652 & 0.446390 & -1.349028 \\
\hline $\mathrm{Pd}$ & -0.876788 & -0.051106 & -0.324781 & $\mathrm{H}$ & -6.698916 & -0.749843 & -3.141576 \\
\hline$P$ & -1.573155 & 2.052252 & 0.485841 & $\mathrm{H}$ & -6.500461 & 1.503662 & 0.516607 \\
\hline $\mathrm{P}$ & -2.680351 & -1.521219 & -0.276410 & $\mathrm{H}$ & -7.678790 & 0.938148 & -1.601039 \\
\hline $\mathrm{C}$ & -3.011329 & 2.742322 & -0.422097 & $\mathrm{C}$ & -2.980341 & -2.339533 & 1.338521 \\
\hline $\mathrm{C}$ & -3.916362 & 3.652022 & 0.138964 & $\mathrm{C}$ & -1.867387 & -2.631426 & 2.141526 \\
\hline $\mathrm{C}$ & -3.200355 & 2.318179 & -1.745483 & $\mathrm{C}$ & -4.263033 & -2.677524 & 1.792592 \\
\hline $\mathrm{C}$ & -4.990235 & 4.129451 & -0.612710 & $\mathrm{C}$ & -2.035174 & -3.250085 & 3.379041 \\
\hline $\mathrm{H}$ & -3.791012 & 3.977807 & 1.166167 & $\mathrm{H}$ & -0.872635 & -2.365992 & 1.804502 \\
\hline $\mathrm{C}$ & -4.270737 & 2.798424 & -2.497076 & $\mathrm{C}$ & -4.427906 & -3.288638 & 3.036243 \\
\hline $\mathrm{H}$ & -2.513750 & 1.589980 & -2.169348 & $\mathrm{H}$ & -5.130374 & -2.454455 & 1.180336 \\
\hline $\mathrm{C}$ & -5.169047 & 3.704141 & -1.930379 & $\mathrm{C}$ & -3.315853 & -3.574892 & 3.831675 \\
\hline $\mathrm{H}$ & -5.689809 & 4.831538 & -0.167250 & $\mathrm{H}$ & -1.161693 & -3.471235 & 3.984797 \\
\hline $\mathrm{H}$ & -4.414950 & 2.450745 & -3.515663 & $\mathrm{H}$ & -5.426030 & -3.540727 & 3.383504 \\
\hline $\mathrm{H}$ & -6.010921 & 4.070878 & -2.510877 & $\mathrm{H}$ & -3.448172 & -4.049115 & 4.800180 \\
\hline $\mathrm{C}$ & -2.071563 & 2.075975 & 2.254350 & $\mathrm{C}$ & -2.497349 & -2.928010 & -1.440341 \\
\hline $\mathrm{C}$ & -2.088488 & 3.260120 & 3.007856 & $\mathrm{C}$ & -2.754012 & -4.253730 & -1.072024 \\
\hline $\mathrm{C}$ & -2.433318 & 0.872226 & 2.872528 & $\mathrm{C}$ & -2.007178 & -2.659273 & -2.728228 \\
\hline $\mathrm{C}$ & -2.495108 & 3.238236 & 4.341475 & $\mathrm{C}$ & -2.515326 & -5.293009 & -1.973403 \\
\hline $\mathrm{H}$ & -1.765604 & 4.192235 & 2.554399 & $\mathrm{H}$ & -3.121797 & -4.476196 & -0.076521 \\
\hline $\mathrm{C}$ & -2.839614 & 0.849789 & 4.207008 & $\mathrm{C}$ & -1.782227 & -3.695645 & -3.631978 \\
\hline $\mathrm{H}$ & -2.376176 & -0.053265 & 2.315070 & $\mathrm{H}$ & -1.784796 & -1.633086 & -3.010015 \\
\hline $\mathrm{C}$ & -2.876917 & 2.035128 & 4.941929 & $\mathrm{C}$ & -2.028519 & -5.018312 & -3.251823 \\
\hline $\mathrm{H}$ & -2.504388 & 4.160010 & 4.916451 & $\mathrm{H}$ & -2.705934 & -6.319332 & -1.672400 \\
\hline $\mathrm{H}$ & -3.110913 & -0.096725 & 4.666010 & $\mathrm{H}$ & -1.400651 & -3.474444 & -4.624681 \\
\hline $\mathrm{H}$ & -3.187015 & 2.022652 & 5.983064 & $\mathrm{H}$ & -1.837232 & -5.829155 & -3.948722 \\
\hline $\mathrm{C}$ & -0.328499 & 3.395171 & 0.433157 & $\mathrm{C}$ & 4.344558 & 1.020413 & -5.598022 \\
\hline $\mathrm{C}$ & -0.503298 & 4.570577 & -0.307320 & $\mathrm{C}$ & 4.212676 & 1.592441 & -4.333047 \\
\hline $\mathrm{C}$ & 0.873706 & 3.188502 & 1.129166 & $\mathrm{C}$ & 4.096341 & 0.787163 & -3.194089 \\
\hline $\mathrm{C}$ & 0.510922 & 5.531158 & -0.341014 & $\mathrm{C}$ & 4.126553 & -0.605337 & -3.339150 \\
\hline $\mathrm{H}$ & -1.425978 & 4.738401 & -0.853092 & $\mathrm{C}$ & 4.253255 & -1.177936 & -4.604064 \\
\hline $\mathrm{C}$ & 1.882380 & 4.149643 & 1.095036 & $\mathrm{C}$ & 4.359842 & -0.368959 & -5.738438 \\
\hline $\mathrm{H}$ & 1.052711 & 2.260412 & 1.662816 & $\mathrm{H}$ & 4.439307 & 1.658314 & -6.472826 \\
\hline $\mathrm{C}$ & 1.699821 & 5.323957 & 0.359066 & $\mathrm{H}$ & 4.199020 & 2.674822 & -4.224696 \\
\hline $\mathrm{H}$ & 0.368773 & 6.440235 & -0.919428 & $\mathrm{H}$ & 4.061458 & -1.216858 & -2.446086 \\
\hline $\mathrm{H}$ & 2.824847 & 3.949474 & 1.592257 & $\mathrm{H}$ & 4.277841 & -2.260055 & -4.704941 \\
\hline $\mathrm{H}$ & 2.492062 & 6.066118 & 0.319212 & $\mathrm{H}$ & 4.463009 & -0.817197 & -6.722986 \\
\hline $\mathrm{C}$ & -4.317549 & -0.809322 & -0.706401 & $\mathrm{C}$ & 3.906023 & 1.408501 & -1.845177 \\
\hline $\mathrm{C}$ & -4.986685 & -1.126191 & -1.894651 & $\mathrm{~N}$ & 4.164538 & 0.567902 & -0.800121 \\
\hline $\mathrm{C}$ & -4.878811 & 0.145717 & 0.157664 & $\mathrm{~S}$ & 4.327551 & 1.222450 & 0.671991 \\
\hline $\mathrm{C}$ & -6.191295 & -0.495942 & -2.215038 & $\mathrm{O}$ & 4.798160 & 2.629868 & 0.665719 \\
\hline $\mathrm{H}$ & -4.572922 & -1.867524 & -2.569463 & $\mathrm{O}$ & 3.120269 & 0.954144 & 1.510086 \\
\hline $\mathrm{C}$ & -6.086378 & 0.760347 & -0.157084 & $\mathrm{C}$ & 5.621319 & 0.196860 & 1.355260 \\
\hline $\mathrm{H}$ & -4.369400 & 0.411616 & 1.077861 & $\mathrm{C}$ & 6.807220 & 0.783483 & 1.781883 \\
\hline
\end{tabular}




$\begin{array}{lrrr}\mathrm{C} & 5.416196 & -1.180917 & 1.475305 \\ \mathrm{C} & 7.804790 & -0.022327 & 2.335135 \\ \mathrm{H} & 6.935704 & 1.855295 & 1.678059 \\ \mathrm{C} & 6.420566 & -1.968800 & 2.024284 \\ \mathrm{H} & 4.485261 & -1.623574 & 1.137750 \\ \mathrm{C} & 7.629636 & -1.403421 & 2.462604 \\ \mathrm{H} & 8.733811 & 0.431140 & 2.671713 \\ \mathrm{H} & 6.268021 & -3.041712 & 2.117300 \\ \mathrm{C} & 8.711212 & -2.276766 & 3.050599 \\ \mathrm{H} & 9.558055 & -1.680412 & 3.403886 \\ \mathrm{H} & 8.334771 & -2.862363 & 3.898221 \\ \mathrm{H} & 9.091555 & -2.991541 & 2.309943 \\ \mathrm{H} & 4.297245 & 2.429736 & -1.774099 \\ --------------------------------------------- & \end{array}$

\section{'TS1A'}

Zero-point correction $=0.967310$ (Hartree/Particle)

Thermal correction to Energy= 1.028337

Thermal correction to Enthalpy= 1.029281

Thermal correction to Gibbs Free Energy= 0.864069

$\mathrm{E}($ Solv $)=-3733.40772557$

\begin{tabular}{|c|c|c|c|}
\hline $\mathrm{C}$ & -1.205352 & -0.290187 & -1.994101 \\
\hline $\mathrm{H}$ & -1.259016 & -0.843101 & -2.930240 \\
\hline $\mathrm{C}$ & -1.621211 & -0.913710 & -0.815079 \\
\hline $\mathrm{C}$ & -2.510675 & -2.046799 & -0.769633 \\
\hline $\mathrm{H}$ & -2.630451 & -2.525970 & -1.741060 \\
\hline $\mathrm{H}$ & -1.545079 & -0.343228 & 0.107207 \\
\hline $\mathrm{Pd}$ & 0.817989 & -0.104539 & -0.920526 \\
\hline $\mathrm{P}$ & 1.921102 & -1.875496 & 0.179040 \\
\hline $\mathrm{P}$ & 2.005417 & 1.859606 & -0.587682 \\
\hline $\mathrm{C}$ & 3.754575 & -1.751858 & 0.209816 \\
\hline $\mathrm{C}$ & 4.537516 & -2.248844 & 1.258456 \\
\hline $\mathrm{C}$ & 4.388110 & -1.160258 & -0.891582 \\
\hline $\mathrm{C}$ & 5.927869 & -2.158569 & 1.201900 \\
\hline $\mathrm{H}$ & 4.063986 & -2.691075 & 2.127599 \\
\hline $\mathrm{C}$ & 5.777584 & -1.076616 & -0.951837 \\
\hline $\mathrm{H}$ & 3.781709 & -0.746556 & -1.692709 \\
\hline $\mathrm{C}$ & 6.550400 & -1.575139 & 0.097496 \\
\hline $\mathrm{H}$ & 6.525365 & -2.540941 & 2.024860 \\
\hline $\mathrm{H}$ & 6.254060 & -0.601794 & -1.804093 \\
\hline $\mathrm{H}$ & 7.633201 & -1.498311 & 0.059627 \\
\hline $\mathrm{C}$ & 1.393222 & -1.990061 & 1.924701 \\
\hline $\mathrm{C}$ & 1.628584 & -3.117031 & 2.728271 \\
\hline
\end{tabular}

\begin{tabular}{|c|c|c|c|}
\hline $\mathrm{C}$ & 0.693199 & -0.901434 & 2.463141 \\
\hline $\mathrm{C}$ & 1.191698 & -3.136000 & 4.051211 \\
\hline $\mathrm{H}$ & 2.125221 & -3.987470 & 2.311263 \\
\hline $\mathrm{C}$ & 0.243096 & -0.926670 & 3.782755 \\
\hline $\mathrm{H}$ & 0.461263 & -0.046746 & 1.838684 \\
\hline $\mathrm{C}$ & 0.501556 & -2.041098 & 4.580831 \\
\hline $\mathrm{H}$ & 1.373350 & -4.013610 & 4.665084 \\
\hline $\mathrm{H}$ & -0.337859 & -0.091448 & 4.159634 \\
\hline $\mathrm{H}$ & 0.147381 & -2.068931 & 5.607470 \\
\hline $\mathrm{C}$ & 1.686097 & -3.563895 & -0.511728 \\
\hline $\mathrm{C}$ & 2.531982 & -4.022418 & -1.533813 \\
\hline $\mathrm{C}$ & 0.620759 & -4.371313 & -0.091361 \\
\hline C & 2.316398 & -5.267659 & -2.121631 \\
\hline $\mathrm{H}$ & 3.368990 & -3.414650 & -1.861736 \\
\hline $\mathrm{C}$ & 0.414705 & -5.620236 & -0.678193 \\
\hline $\mathrm{H}$ & -0.048213 & -4.034372 & 0.689785 \\
\hline $\mathrm{C}$ & 1.257720 & -6.071453 & -1.693712 \\
\hline $\mathrm{H}$ & 2.981505 & -5.612102 & -2.908762 \\
\hline $\mathrm{H}$ & -0.415164 & -6.229231 & -0.335910 \\
\hline $\mathrm{H}$ & 1.092662 & -7.043884 & -2.149172 \\
\hline $\mathrm{C}$ & 3.726681 & 1.864392 & 0.042628 \\
\hline $\mathrm{C}$ & 4.793460 & 2.450261 & -0.647049 \\
\hline C & 3.958345 & 1.263491 & 1.289482 \\
\hline $\mathrm{C}$ & 6.077325 & 2.432031 & -0.097011 \\
\hline $\mathrm{H}$ & 4.625268 & 2.924461 & -1.607803 \\
\hline $\mathrm{C}$ & 5.237658 & 1.248712 & 1.834519 \\
\hline $\mathrm{H}$ & 3.135805 & 0.801232 & 1.828281 \\
\hline $\mathrm{C}$ & 6.301663 & 1.831594 & 1.140536 \\
\hline $\mathrm{H}$ & 6.900019 & 2.890860 & -0.638476 \\
\hline $\mathrm{H}$ & 5.407940 & 0.769437 & 2.793442 \\
\hline $\mathrm{H}$ & 7.301640 & 1.812835 & 1.564254 \\
\hline $\mathrm{C}$ & 1.189917 & 2.970912 & 0.620846 \\
\hline C & -0.052125 & 2.638467 & 1.174590 \\
\hline $\mathrm{C}$ & 1.827773 & 4.158168 & 1.023737 \\
\hline C & -0.660437 & 3.485758 & 2.103612 \\
\hline $\mathrm{H}$ & -0.564380 & 1.720422 & 0.914172 \\
\hline $\mathrm{C}$ & 1.210906 & 5.008697 & 1.937684 \\
\hline $\mathrm{H}$ & 2.806990 & 4.408621 & 0.626426 \\
\hline $\mathrm{C}$ & -0.036147 & 4.673833 & 2.476809 \\
\hline $\mathrm{H}$ & -1.619551 & 3.202978 & 2.520696 \\
\hline $\mathrm{H}$ & 1.707294 & 5.927588 & 2.237425 \\
\hline $\mathrm{H}$ & -0.514380 & 5.335769 & 3.193464 \\
\hline$C$ & 2.111386 & 2.804741 & -2.155381 \\
\hline $\mathrm{C}$ & 1.643993 & 4.115834 & -2.296190 \\
\hline
\end{tabular}




\begin{tabular}{|c|c|c|c|c|c|c|c|}
\hline $\mathrm{C}$ & 2.627365 & 2.137913 & -3.279247 & $\mathrm{H}$ & -1.928818 & -1.698907 & 1.918534 \\
\hline $\mathrm{C}$ & 1.701979 & 4.752620 & -3.538779 & $\mathrm{C}$ & -2.787944 & -5.367686 & 1.012085 \\
\hline $\mathrm{H}$ & 1.228170 & 4.637672 & -1.441641 & $\mathrm{H}$ & -3.048238 & -4.647830 & -1.000329 \\
\hline $\mathrm{C}$ & 2.698031 & 2.779693 & -4.512893 & $\mathrm{C}$ & -2.440579 & -5.038375 & 2.323783 \\
\hline $\mathrm{H}$ & 2.960037 & 1.107179 & -3.185079 & $\mathrm{H}$ & -1.872458 & -3.437248 & 3.653610 \\
\hline $\mathrm{C}$ & 2.232250 & 4.091311 & -4.645679 & $\mathrm{H}$ & -3.048588 & -6.391692 & 0.755839 \\
\hline $\mathrm{H}$ & 1.329363 & 5.768177 & -3.637896 & $\mathrm{H}$ & -2.426238 & -5.802901 & 3.095664 \\
\hline $\mathrm{H}$ & 3.102769 & 2.254083 & -5.373087 & $\mathrm{C}$ & -0.655412 & 1.027670 & -1.969559 \\
\hline $\mathrm{H}$ & 2.275754 & 4.589286 & -5.609895 & $\mathrm{H}$ & -0.325903 & 1.469647 & -2.906202 \\
\hline $\mathrm{C}$ & -5.917932 & -2.660734 & -3.465899 & $\mathrm{H}$ & -1.054972 & 1.741439 & -1.254200 \\
\hline $\mathrm{C}$ & -5.374430 & -2.467577 & -2.197662 & ---- & - & 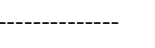 & \\
\hline $\mathrm{C}$ & -4.757273 & -1.254002 & -1.862156 & & & & \\
\hline $\mathrm{C}$ & -4.701627 & -0.232989 & -2.818675 & TS1B & & & \\
\hline $\mathrm{C}$ & -5.239964 & -0.428907 & -4.090749 & & & & \\
\hline $\mathrm{C}$ & -5.847669 & -1.642130 & -4.420410 & Zero-po & rection $=0.963$ & 3477 (Hartree/F & Particle) \\
\hline $\mathrm{H}$ & -6.401529 & -3.603125 & -3.709437 & Therma & tion to Energ & $y=1.026131$ & \\
\hline $\mathrm{H}$ & -5.423505 & -3.260072 & -1.454668 & Therma & tion to Entha & $l p y=1.027075$ & \\
\hline $\mathrm{H}$ & -4.252361 & 0.712059 & -2.535773 & Therma & tion to Gibbs & Free Energy= & 0.854361 \\
\hline $\mathrm{H}$ & -5.193981 & 0.371764 & -4.824712 & $\mathrm{E}($ solv) & 3.40018729 & & \\
\hline $\mathrm{H}$ & -6.272279 & -1.790716 & -5.409605 & --------- & . & -------------- & \\
\hline $\mathrm{C}$ & -4.121112 & -1.084437 & -0.520155 & $\mathrm{C}$ & 1.042447 & 1.241160 & -3.503122 \\
\hline $\mathrm{N}$ & -3.963653 & 0.249522 & -0.157887 & $\mathrm{C}$ & 1.422259 & 0.688933 & -2.297881 \\
\hline S & -3.717240 & 0.619178 & 1.372711 & $\mathrm{C}$ & 0.935757 & 1.226370 & -1.071406 \\
\hline $\mathrm{O}$ & -4.667650 & 0.039951 & 2.349302 & $\mathrm{C}$ & 0.052884 & 2.344725 & -1.123450 \\
\hline $\mathrm{O}$ & -2.272514 & 0.487109 & 1.802238 & $\mathrm{C}$ & -0.332306 & 2.881940 & -2.362292 \\
\hline $\mathrm{C}$ & -3.952016 & 2.394006 & 1.322617 & $\mathrm{C}$ & 0.152168 & 2.335377 & -3.541227 \\
\hline $\mathrm{C}$ & -4.372999 & 3.028539 & 2.489072 & $\mathrm{H}$ & 1.432073 & 0.828835 & -4.429443 \\
\hline C & -3.555896 & 3.142209 & 0.212368 & $\mathrm{H}$ & 2.098734 & -0.156568 & -2.261083 \\
\hline $\mathrm{C}$ & -4.369290 & 4.423035 & 2.550644 & $\mathrm{H}$ & -0.233480 & 2.864951 & -0.219941 \\
\hline $\mathrm{H}$ & -4.695688 & 2.426973 & 3.332467 & $\mathrm{H}$ & -0.990994 & 3.744086 & -2.378108 \\
\hline $\mathrm{C}$ & -3.560816 & 4.531490 & 0.286824 & $\mathrm{H}$ & -0.133708 & 2.763550 & -4.498145 \\
\hline $\mathrm{H}$ & -3.257382 & 2.625521 & -0.692067 & $\mathrm{C}$ & 1.175613 & 0.526425 & 0.172874 \\
\hline C & -3.949445 & 5.193933 & 1.460462 & $\mathrm{H}$ & 1.872801 & -0.300135 & 0.127914 \\
\hline $\mathrm{H}$ & -4.696247 & 4.919906 & 3.460973 & $\mathrm{C}$ & 1.173889 & 1.258918 & 1.491050 \\
\hline $\mathrm{H}$ & -3.249350 & 5.115083 & -0.576667 & $\mathrm{H}$ & 0.367312 & 1.998292 & 1.512183 \\
\hline $\mathrm{C}$ & -3.890792 & 6.699531 & 1.546054 & $\mathrm{C}$ & 0.985313 & 0.357856 & 2.680974 \\
\hline $\mathrm{H}$ & -2.854056 & 7.046525 & 1.651210 & $\mathrm{C}$ & -0.149701 & 0.275499 & 3.378163 \\
\hline $\mathrm{H}$ & -4.297757 & 7.169783 & 0.643652 & $\mathrm{H}$ & -0.249164 & -0.399753 & 4.223356 \\
\hline $\mathrm{H}$ & -4.453680 & 7.074574 & 2.406614 & $\mathrm{H}$ & 1.841232 & -0.246730 & 2.961882 \\
\hline $\mathrm{H}$ & -4.561950 & -1.741086 & 0.236938 & $\mathrm{H}$ & -1.031005 & 0.858143 & 3.117808 \\
\hline $\mathrm{C}$ & -2.458482 & -3.054201 & 0.322301 & $\mathrm{Pd}$ & -0.909912 & 0.076221 & -0.077450 \\
\hline $\mathrm{C}$ & -2.143345 & -2.726214 & 1.651286 & $\mathrm{P}$ & -3.153276 & 0.818104 & 0.034481 \\
\hline C & -2.795767 & -4.384069 & 0.023750 & $\mathrm{P}$ & -0.494326 & -2.214476 & -0.069286 \\
\hline $\mathrm{C}$ & -2.128943 & -3.712890 & 2.635846 & $\mathrm{C}$ & -4.206576 & 0.541288 & -1.436537 \\
\hline
\end{tabular}




\begin{tabular}{|c|c|c|c|c|c|c|c|}
\hline $\mathrm{C}$ & -5.539937 & 0.120702 & -1.371512 & $\mathrm{C}$ & 0.978096 & -2.796755 & 2.272399 \\
\hline $\mathrm{C}$ & -3.586865 & 0.686456 & -2.689776 & $\mathrm{C}$ & -1.236553 & -3.765848 & 2.142378 \\
\hline $\mathrm{C}$ & -6.248106 & -0.143967 & -2.546104 & $\mathrm{C}$ & 1.126345 & -3.328094 & 3.553264 \\
\hline $\mathrm{H}$ & -6.019495 & -0.016793 & -0.408253 & $\mathrm{H}$ & 1.781055 & -2.184047 & 1.883130 \\
\hline $\mathrm{C}$ & -4.302415 & 0.436734 & -3.858590 & $\mathrm{C}$ & -1.088826 & -4.274944 & 3.432324 \\
\hline $\mathrm{H}$ & -2.541190 & 0.980789 & -2.740644 & $\mathrm{H}$ & -2.157935 & -3.950296 & 1.601403 \\
\hline $\mathrm{C}$ & -5.633694 & 0.016802 & -3.788763 & $\mathrm{C}$ & 0.095860 & -4.062590 & 4.140339 \\
\hline $\mathrm{H}$ & -7.281022 & -0.475488 & -2.487661 & $\mathrm{H}$ & 2.051899 & -3.143099 & 4.090372 \\
\hline $\mathrm{H}$ & -3.816773 & 0.554329 & -4.823054 & $\mathrm{H}$ & -1.898036 & -4.847968 & 3.877300 \\
\hline $\mathrm{H}$ & -6.186670 & -0.191739 & -4.700025 & $\mathrm{H}$ & 0.212015 & -4.464567 & 5.142949 \\
\hline $\mathrm{C}$ & -4.077909 & 0.206358 & 1.486080 & $\mathrm{C}$ & 0.878372 & -2.575001 & -1.240893 \\
\hline $\mathrm{C}$ & -5.242711 & 0.835926 & 1.952620 & $\mathrm{C}$ & 2.226686 & -2.659834 & -0.868226 \\
\hline $\mathrm{C}$ & -3.570830 & -0.903260 & 2.176025 & $\mathrm{C}$ & 0.548971 & -2.579758 & -2.609159 \\
\hline $\mathrm{C}$ & -5.901035 & 0.342094 & 3.077492 & $\mathrm{C}$ & 3.222003 & -2.748790 & -1.844746 \\
\hline $\mathrm{H}$ & -5.620970 & 1.717893 & 1.443506 & $\mathrm{H}$ & 2.538880 & -2.611378 & 0.164221 \\
\hline $\mathrm{C}$ & -4.228862 & -1.393063 & 3.304083 & $\mathrm{C}$ & 1.544646 & -2.681256 & -3.577815 \\
\hline $\mathrm{H}$ & -2.646759 & -1.363399 & 1.845448 & $\mathrm{H}$ & -0.488724 & -2.499830 & -2.917041 \\
\hline $\mathrm{C}$ & -5.396262 & -0.774164 & 3.752188 & $\mathrm{C}$ & 2.887276 & -2.760650 & -3.198765 \\
\hline $\mathrm{H}$ & -6.801735 & 0.832694 & 3.435237 & $\mathrm{H}$ & 4.260622 & -2.782079 & -1.532013 \\
\hline $\mathrm{H}$ & -3.815701 & -2.246791 & 3.833253 & $\mathrm{H}$ & 1.270438 & -2.688683 & -4.629223 \\
\hline $\mathrm{H}$ & -5.908038 & -1.150496 & 4.633352 & $\mathrm{H}$ & 3.665813 & -2.825461 & -3.953708 \\
\hline $\mathrm{C}$ & -3.145390 & 2.639712 & 0.264645 & $\mathrm{C}$ & 1.121419 & 5.531218 & 0.858592 \\
\hline $\mathrm{C}$ & -3.573426 & 3.530170 & -0.726236 & $\mathrm{C}$ & 1.442643 & 4.334428 & 1.496483 \\
\hline $\mathrm{C}$ & -2.543696 & 3.146939 & 1.428505 & $\mathrm{C}$ & 2.339270 & 3.426910 & 0.915562 \\
\hline $\mathrm{C}$ & -3.388672 & 4.905373 & -0.560972 & $\mathrm{C}$ & 2.938179 & 3.761273 & -0.301410 \\
\hline $\mathrm{H}$ & -4.042068 & 3.153697 & -1.629142 & $\mathrm{C}$ & 2.616590 & 4.958131 & -0.944984 \\
\hline $\mathrm{C}$ & -2.360702 & 4.517351 & 1.589284 & $\mathrm{C}$ & 1.700976 & 5.843119 & -0.374626 \\
\hline $\mathrm{H}$ & -2.204892 & 2.465144 & 2.202825 & $\mathrm{H}$ & 0.420821 & 6.221108 & 1.321832 \\
\hline $\mathrm{C}$ & -2.777163 & 5.401361 & 0.590007 & $\mathrm{H}$ & 0.983843 & 4.092720 & 2.453450 \\
\hline $\mathrm{H}$ & -3.723764 & 5.587993 & -1.336935 & $\mathrm{H}$ & 3.646825 & 3.059174 & -0.725010 \\
\hline $\mathrm{H}$ & -1.882957 & 4.894211 & 2.488465 & $\mathrm{H}$ & 3.081473 & 5.197816 & -1.898000 \\
\hline $\mathrm{H}$ & -2.623201 & 6.469562 & 0.709403 & $\mathrm{H}$ & 1.449443 & 6.773364 & -0.877294 \\
\hline $\mathrm{C}$ & -1.830243 & -3.248222 & -0.795527 & $\mathrm{C}$ & 2.575599 & 2.070085 & 1.559445 \\
\hline $\mathrm{C}$ & -1.603648 & -4.617456 & -1.017821 & $\mathrm{~N}$ & 3.650950 & 1.394456 & 0.889572 \\
\hline $\mathrm{C}$ & -3.061313 & -2.697335 & -1.152504 & $\mathrm{~S}$ & 4.307910 & 0.149255 & 1.598617 \\
\hline $\mathrm{C}$ & -2.600435 & -5.409999 & -1.579893 & $\mathrm{O}$ & 4.846211 & 0.371290 & 2.965670 \\
\hline $\mathrm{H}$ & -0.647432 & -5.056201 & -0.749591 & $\mathrm{O}$ & 3.486853 & -1.119914 & 1.513092 \\
\hline $\mathrm{C}$ & -4.061163 & -3.489242 & -1.721948 & $\mathrm{C}$ & 5.682603 & -0.152698 & 0.490292 \\
\hline $\mathrm{H}$ & -3.234798 & -1.643402 & -1.003141 & $\mathrm{C}$ & 6.929952 & -0.450899 & 1.027847 \\
\hline $\mathrm{C}$ & -3.831956 & -4.847027 & -1.934328 & $\mathrm{C}$ & 5.483399 & -0.174221 & -0.892604 \\
\hline $\mathrm{H}$ & -2.417517 & -6.467723 & -1.746534 & $\mathrm{C}$ & 7.986329 & -0.775104 & 0.173780 \\
\hline $\mathrm{H}$ & -5.006940 & -3.033866 & -2.000535 & $\mathrm{H}$ & 7.057917 & -0.421860 & 2.104655 \\
\hline $\mathrm{H}$ & -4.604780 & -5.468930 & -2.377325 & $\mathrm{C}$ & 6.543979 & -0.498605 & -1.732450 \\
\hline $\mathrm{C}$ & -0.199253 & -3.026592 & 1.546161 & $\mathrm{H}$ & 4.508022 & 0.076685 & -1.293209 \\
\hline
\end{tabular}




$\begin{array}{llll}\mathrm{C} & 7.812196 & -0.804858 & -1.213483 \\ \mathrm{H} & 8.962469 & -1.007453 & 0.593260 \\ \mathrm{H} & 6.390886 & -0.512033 & -2.809870 \\ \mathrm{C} & 8.961534 & -1.130115 & -2.136866 \\ \mathrm{H} & 9.781809 & -1.615111 & -1.598004 \\ \mathrm{H} & 8.648347 & -1.797499 & -2.948280 \\ \mathrm{H} & 9.366250 & -0.222576 & -2.604363 \\ \mathrm{H} & 2.759385 & 2.220297 & 2.636538\end{array}$

C

TS1B'

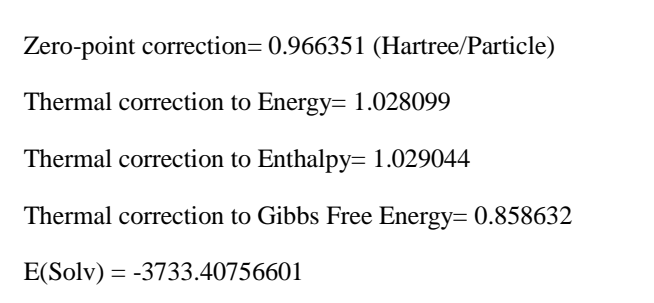

\begin{tabular}{|c|c|c|c|c|c|c|c|}
\hline & & ---- & & $\mathrm{H}$ & -4.322951 & 4.082239 & 1.499836 \\
\hline $\mathrm{C}$ & -5.755215 & -5.037641 & 0.526600 & $\mathrm{H}$ & -3.638773 & 6.185647 & 2.652182 \\
\hline $\mathrm{C}$ & -4.452862 & -4.627765 & 0.252975 & $\mathrm{C}$ & 1.617570 & 2.529599 & 2.074225 \\
\hline $\mathrm{C}$ & -3.801350 & -3.677363 & 1.061751 & $\mathrm{C}$ & 2.875846 & 3.084410 & 1.814008 \\
\hline $\mathrm{C}$ & -4.513454 & -3.149521 & 2.158051 & $\mathrm{C}$ & 1.370393 & 1.951792 & 3.330399 \\
\hline $\mathrm{C}$ & -5.813841 & -3.559768 & 2.432090 & $\mathrm{C}$ & 3.869739 & 3.062399 & 2.796491 \\
\hline $\mathrm{C}$ & -6.444391 & -4.505791 & 1.617931 & $\mathrm{H}$ & 3.082229 & 3.525947 & 0.844990 \\
\hline $\mathrm{H}$ & -6.234346 & -5.773159 & -0.114015 & $\mathrm{C}$ & 2.358805 & 1.937401 & 4.309374 \\
\hline $\mathrm{H}$ & -3.921222 & -5.036210 & -0.601153 & $\mathrm{H}$ & 0.404195 & 1.494362 & 3.528092 \\
\hline $\mathrm{H}$ & -4.046703 & -2.406125 & 2.797313 & $\mathrm{C}$ & 3.613906 & 2.493043 & 4.042948 \\
\hline $\mathrm{H}$ & -6.341760 & -3.138071 & 3.283454 & $\mathrm{H}$ & 4.845212 & 3.490325 & 2.582258 \\
\hline $\mathrm{H}$ & -7.461570 & -4.821171 & 1.831800 & $\mathrm{H}$ & 2.162179 & 1.470234 & 5.269384 \\
\hline $\mathrm{C}$ & -2.449333 & -3.256735 & 0.708984 & $\mathrm{H}$ & 4.390155 & 2.471415 & 4.802595 \\
\hline $\mathrm{H}$ & -2.075490 & -3.625290 & -0.242760 & $\mathrm{C}$ & -1.590616 & 1.360443 & -2.634978 \\
\hline $\mathrm{C}$ & -1.656847 & -2.422232 & 1.422855 & $\mathrm{C}$ & -1.132421 & 0.911090 & -3.879726 \\
\hline $\mathrm{H}$ & -1.985486 & -2.061859 & 2.397753 & $\mathrm{C}$ & -1.657771 & 2.742317 & -2.392591 \\
\hline $\mathrm{C}$ & -0.397651 & -1.883361 & 0.943672 & $\mathrm{C}$ & -0.748110 & 1.826608 & -4.860597 \\
\hline $\mathrm{C}$ & 0.572465 & -1.356189 & 1.877233 & $\mathrm{H}$ & -1.070511 & -0.151696 & -4.083032 \\
\hline $\mathrm{H}$ & 1.199827 & -0.500571 & 1.585950 & $\mathrm{C}$ & -1.290310 & 3.652251 & -3.378950 \\
\hline $\mathrm{H}$ & -0.032988 & -2.310845 & 0.013402 & $\mathrm{H}$ & -1.993934 & 3.109813 & -1.428763 \\
\hline $\mathrm{H}$ & 0.257214 & -1.291696 & 2.919367 & $\mathrm{C}$ & -0.826866 & 3.196647 & -4.614786 \\
\hline $\mathrm{Pd}$ & -0.488410 & 0.243698 & 0.489302 & $\mathrm{H}$ & -0.387691 & 1.463344 & -5.818956 \\
\hline $\mathrm{P}$ & 0.280805 & 2.440295 & 0.814569 & $\mathrm{H}$ & -1.338623 & 4.716405 & -3.171176 \\
\hline $\mathrm{P}$ & -1.982768 & 0.199806 & -1.272401 & $\mathrm{H}$ & -0.522113 & 3.907444 & -5.377507 \\
\hline $\mathrm{C}$ & 1.039153 & 3.204152 & -0.666257 & $\mathrm{C}$ & -3.696437 & 0.621036 & -0.772218 \\
\hline $\mathrm{C}$ & 1.059494 & 4.584235 & -0.903124 & $\mathrm{C}$ & -4.164313 & 0.077459 & 0.435313 \\
\hline $\mathrm{C}$ & 1.631262 & 2.330113 & -1.591758 & $\mathrm{C}$ & -4.547641 & 1.434500 & -1.530814 \\
\hline \multicolumn{8}{|c|}{ S354 } \\
\hline
\end{tabular}




$\begin{array}{lrrrrrrr}\mathrm{C} & -5.460896 & 0.337164 & 0.872160 & \mathrm{H} & 6.678556 & 0.096605 & -2.584729 \\ \mathrm{H} & -3.507343 & -0.556376 & 1.020030 & \mathrm{C} & 8.087823 & -2.033253 & -3.536276 \\ \mathrm{C} & -5.839598 & 1.712068 & -1.079184 & \mathrm{H} & 8.197109 & -1.206242 & -4.247001 \\ \mathrm{H} & -4.200158 & 1.855004 & -2.468205 & \mathrm{H} & 8.874825 & -1.915154 & -2.779953 \\ \mathrm{C} & -6.299052 & 1.164246 & 0.119733 & \mathrm{H} & 8.282521 & -2.968193 & -4.070889 \\ \mathrm{H} & -5.815348 & -0.110037 & 1.796053 & \mathrm{H} & 1.820233 & -3.316046 & 1.393176 \\ \mathrm{H} & -6.489525 & 2.350910 & -1.670785 & - & & & \end{array}$

TS1C

\begin{tabular}{|c|c|c|c|}
\hline \multicolumn{4}{|c|}{ Zero-point correction $=0.689207($ Hartree/Particle $)$} \\
\hline \multicolumn{4}{|c|}{ Thermal correction to Energy $=0.733232$} \\
\hline \multicolumn{4}{|c|}{ Thermal correction to Enthalpy $=0.734177$} \\
\hline \multicolumn{4}{|c|}{ Thermal correction to Gibbs Free Energy $=0.607674$} \\
\hline & 5.77166958 & & \\
\hline $\mathrm{C}$ & 5.621774 & -1.628997 & 0.261104 \\
\hline $\mathrm{C}$ & 4.327900 & -1.784287 & 0.747207 \\
\hline $\mathrm{C}$ & 3.369315 & -2.526567 & 0.033779 \\
\hline $\mathrm{C}$ & 3.768318 & -3.149347 & -1.161266 \\
\hline $\mathrm{C}$ & 5.068326 & -3.001657 & -1.642559 \\
\hline $\mathrm{C}$ & 5.999329 & -2.232216 & -0.941215 \\
\hline $\mathrm{H}$ & 6.334713 & -1.030527 & 0.821208 \\
\hline $\mathrm{H}$ & 4.036116 & -1.299454 & 1.673412 \\
\hline $\mathrm{H}$ & 3.059437 & -3.751693 & -1.720698 \\
\hline $\mathrm{H}$ & 5.355512 & -3.492475 & -2.568805 \\
\hline $\mathrm{H}$ & 7.008969 & -2.111419 & -1.322826 \\
\hline $\mathrm{C}$ & 1.992879 & -2.585404 & 0.558318 \\
\hline $\mathrm{H}$ & 1.928026 & -2.432895 & 1.637598 \\
\hline $\mathrm{C}$ & 0.917584 & -3.298089 & -0.042047 \\
\hline $\mathrm{H}$ & 1.018990 & -3.636663 & -1.072093 \\
\hline $\mathrm{C}$ & -0.308116 & -3.565360 & 0.608828 \\
\hline $\mathrm{C}$ & -1.489591 & -3.952768 & -0.015741 \\
\hline $\mathrm{H}$ & -2.321863 & -4.271491 & 0.599632 \\
\hline $\mathrm{H}$ & -0.369952 & -3.291245 & 1.662227 \\
\hline $\mathrm{H}$ & -1.454187 & -4.380346 & -1.015487 \\
\hline $\mathrm{Pd}$ & 0.618229 & -1.005597 & -0.039135 \\
\hline$P$ & 1.748070 & 0.980120 & -0.065129 \\
\hline $\mathrm{C}$ & 0.665487 & 2.462800 & -0.192054 \\
\hline $\mathrm{C}$ & 0.257810 & 3.197426 & 0.927277 \\
\hline $\mathrm{C}$ & 0.160357 & 2.810386 & -1.456155 \\
\hline $\mathrm{C}$ & -0.625628 & 4.268547 & 0.783223 \\
\hline $\mathrm{H}$ & 0.623032 & 2.931033 & 1.912077 \\
\hline $\mathrm{C}$ & -0.712353 & 3.887705 & -1.599091 \\
\hline
\end{tabular}




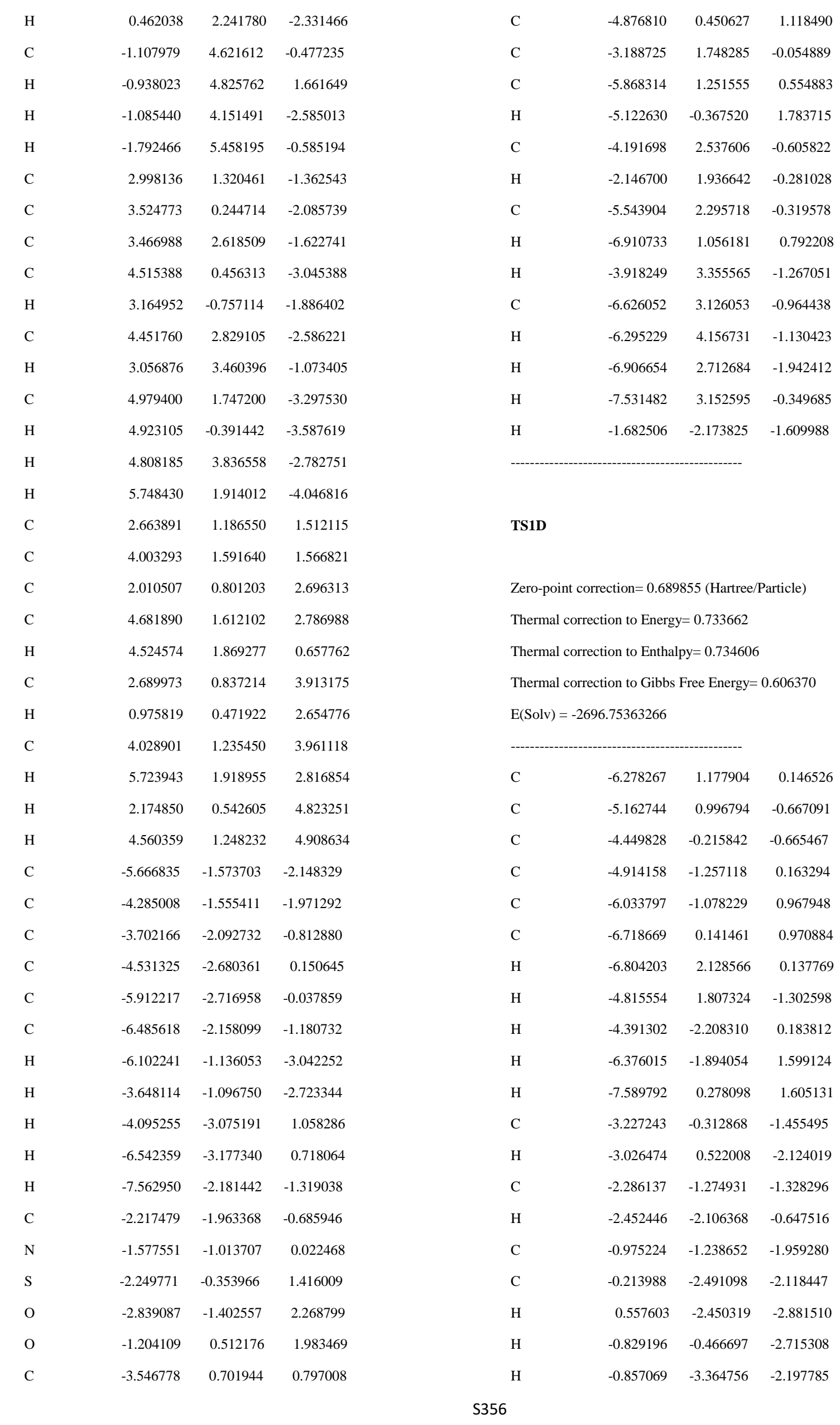




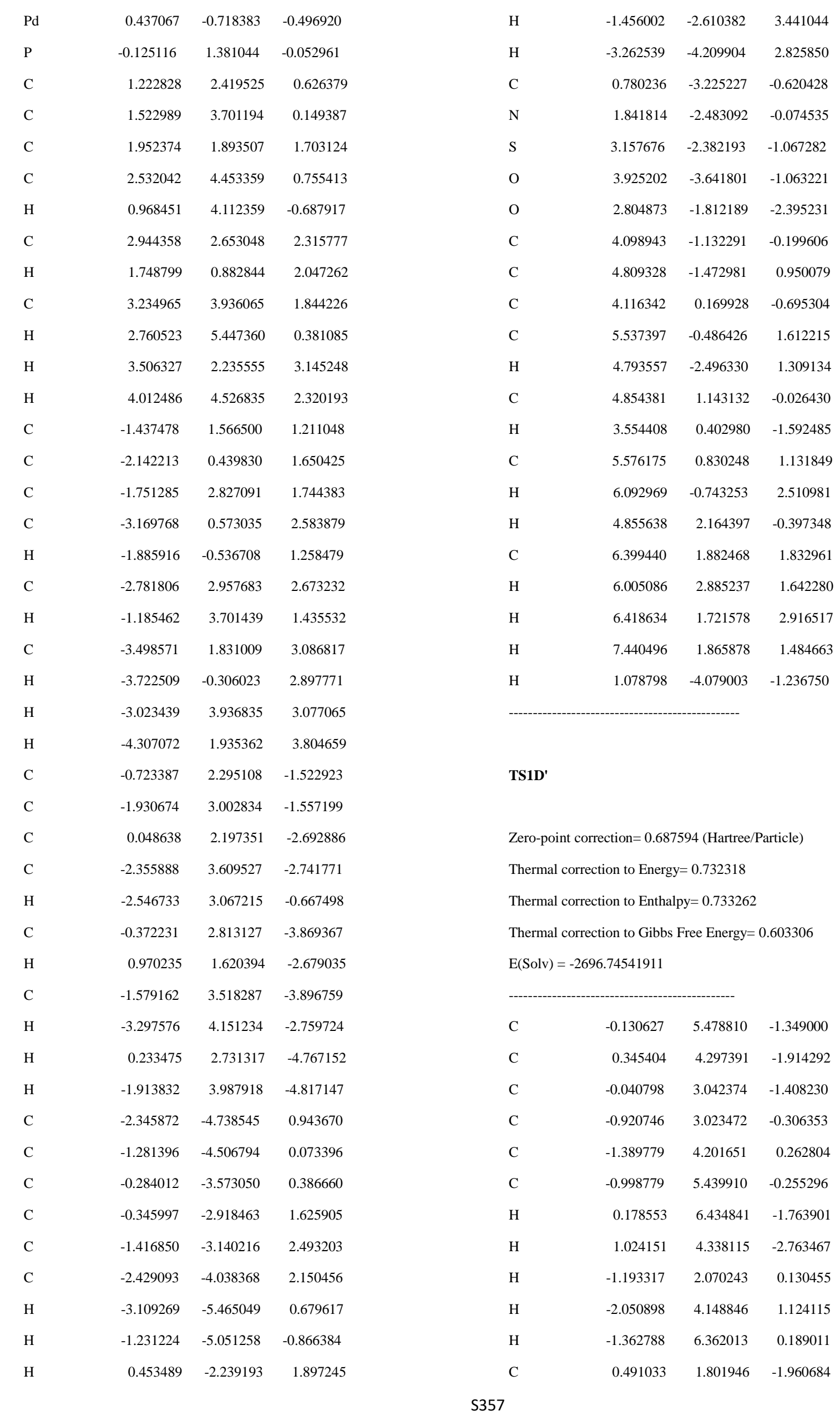




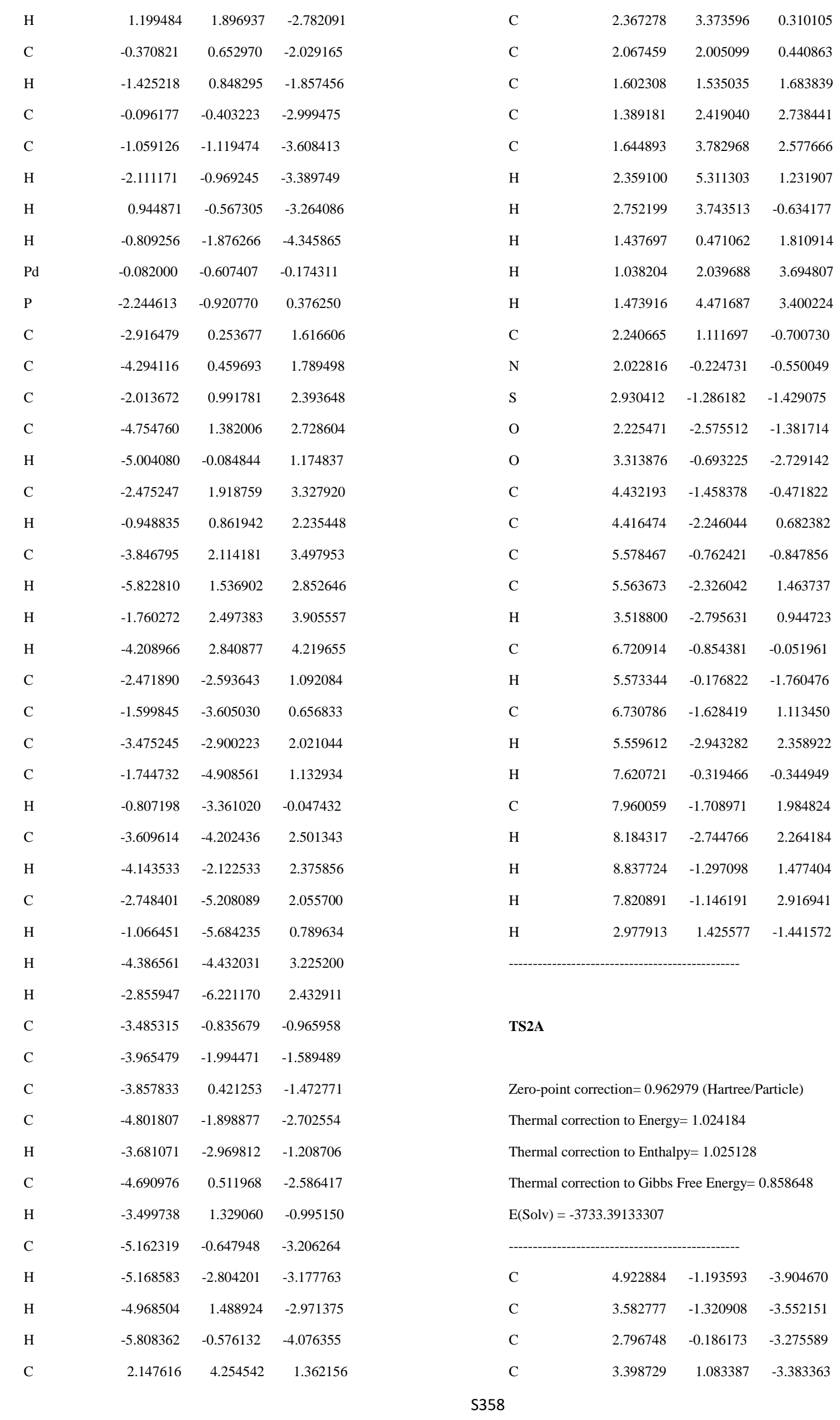




\begin{tabular}{|c|c|c|c|c|c|c|c|}
\hline $\mathrm{C}$ & 4.739462 & 1.207482 & -3.739328 & $\mathrm{C}$ & -2.811126 & 3.895167 & -0.088431 \\
\hline $\mathrm{C}$ & 5.510613 & 0.070584 & -3.998788 & $\mathrm{H}$ & -2.173102 & 2.442413 & 1.363835 \\
\hline $\mathrm{H}$ & 5.513396 & -2.084010 & -4.101596 & $\mathrm{C}$ & -1.087994 & 4.777302 & -1.533618 \\
\hline $\mathrm{H}$ & 3.134451 & -2.305707 & -3.458817 & $\mathrm{H}$ & 0.897938 & 4.014246 & -1.210150 \\
\hline $\mathrm{H}$ & 2.818783 & 1.973861 & -3.161338 & $\mathrm{C}$ & -2.429438 & 4.729010 & -1.137629 \\
\hline $\mathrm{H}$ & 5.185985 & 2.195640 & -3.810857 & $\mathrm{H}$ & -3.854412 & 3.831114 & 0.201860 \\
\hline $\mathrm{H}$ & 6.556795 & 0.169751 & -4.273576 & $\mathrm{H}$ & -0.783271 & 5.425997 & -2.350599 \\
\hline $\mathrm{C}$ & 1.416518 & -0.370150 & -2.816483 & $\mathrm{H}$ & -3.175778 & 5.323584 & -1.655418 \\
\hline $\mathrm{H}$ & 1.062798 & -1.399152 & -2.828286 & $\mathrm{C}$ & 1.902109 & -1.764072 & 2.113141 \\
\hline $\mathrm{C}$ & 0.415333 & 0.648921 & -2.789954 & $\mathrm{C}$ & 1.084810 & -2.829530 & 2.505098 \\
\hline $\mathrm{H}$ & 0.627922 & 1.674474 & -3.078985 & $\mathrm{C}$ & 2.326405 & -0.834078 & 3.076820 \\
\hline $\mathrm{C}$ & -0.909777 & 0.273169 & -2.458937 & $\mathrm{C}$ & 0.707331 & -2.967300 & 3.842317 \\
\hline $\mathrm{C}$ & -1.991717 & 1.087616 & -2.092549 & $\mathrm{H}$ & 0.733392 & -3.545753 & 1.771758 \\
\hline $\mathrm{H}$ & -1.906365 & 0.717038 & -0.802101 & $\mathrm{C}$ & 1.955531 & -0.979625 & 4.409116 \\
\hline $\mathrm{H}$ & -1.081046 & -0.800496 & -2.357150 & $\mathrm{H}$ & 2.943252 & 0.009648 & 2.781090 \\
\hline $\mathrm{H}$ & -1.935673 & 2.162017 & -2.246577 & $\mathrm{C}$ & 1.142276 & -2.048064 & 4.795251 \\
\hline$P d$ & 1.297032 & 0.297265 & -0.692636 & $\mathrm{H}$ & 0.066193 & -3.794571 & 4.133148 \\
\hline $\mathrm{P}$ & 0.722039 & 1.971564 & 0.849183 & $\mathrm{H}$ & 2.279159 & -0.246164 & 5.141147 \\
\hline $\mathrm{P}$ & 2.374045 & -1.459646 & 0.368265 & $\mathrm{H}$ & 0.839256 & -2.152786 & 5.833112 \\
\hline $\mathrm{C}$ & 0.008598 & 1.479656 & 2.458782 & $\mathrm{C}$ & 4.204739 & -1.416434 & 0.450042 \\
\hline $\mathrm{C}$ & 0.200512 & 2.201810 & 3.643968 & $\mathrm{C}$ & 4.900250 & -0.556679 & -0.410717 \\
\hline $\mathrm{C}$ & -0.794236 & 0.331961 & 2.467627 & $\mathrm{C}$ & 4.924229 & -2.219025 & 1.348920 \\
\hline $\mathrm{C}$ & -0.414795 & 1.779707 & 4.822459 & $\mathrm{C}$ & 6.294321 & -0.502085 & -0.377246 \\
\hline $\mathrm{H}$ & 0.832844 & 3.083828 & 3.650206 & $\mathrm{H}$ & 4.351325 & 0.066553 & -1.107333 \\
\hline $\mathrm{C}$ & -1.419600 & -0.077106 & 3.643643 & $\mathrm{C}$ & 6.316562 & -2.161419 & 1.381813 \\
\hline $\mathrm{H}$ & -0.940577 & -0.253943 & 1.568548 & $\mathrm{H}$ & 4.390572 & -2.879238 & 2.025643 \\
\hline $\mathrm{C}$ & -1.229698 & 0.644495 & 4.822777 & $\mathrm{C}$ & 7.003583 & -1.302095 & 0.519209 \\
\hline $\mathrm{H}$ & -0.259163 & 2.339263 & 5.741134 & $\mathrm{H}$ & 6.817920 & 0.162973 & -1.057347 \\
\hline $\mathrm{H}$ & -2.030829 & -0.973549 & 3.623889 & $\mathrm{H}$ & 6.866228 & -2.784438 & 2.081939 \\
\hline $\mathrm{H}$ & -1.705203 & 0.319539 & 5.744486 & $\mathrm{H}$ & 8.088842 & -1.257465 & 0.549009 \\
\hline $\mathrm{C}$ & 2.155401 & 3.037116 & 1.266552 & $\mathrm{C}$ & 1.964027 & -3.003890 & -0.523715 \\
\hline $\mathrm{C}$ & 2.019191 & 4.375949 & 1.662709 & $\mathrm{C}$ & 2.932869 & -3.870835 & -1.042476 \\
\hline $\mathrm{C}$ & 3.438201 & 2.472822 & 1.200013 & $\mathrm{C}$ & 0.606485 & -3.233190 & -0.810327 \\
\hline $\mathrm{C}$ & 3.145300 & 5.128709 & 1.996870 & $\mathrm{C}$ & 2.546201 & -4.952903 & -1.838565 \\
\hline $\mathrm{H}$ & 1.033161 & 4.828775 & 1.698875 & $\mathrm{H}$ & 3.984140 & -3.695883 & -0.838755 \\
\hline $\mathrm{C}$ & 4.562773 & 3.224988 & 1.538442 & $\mathrm{C}$ & 0.224908 & -4.316656 & -1.597069 \\
\hline $\mathrm{H}$ & 3.552835 & 1.446499 & 0.868385 & $\mathrm{H}$ & -0.157795 & -2.565966 & -0.425201 \\
\hline $\mathrm{C}$ & 4.417746 & 4.554522 & 1.938591 & $\mathrm{C}$ & 1.196957 & -5.176733 & -2.118055 \\
\hline $\mathrm{H}$ & 3.029482 & 6.165158 & 2.301639 & $\mathrm{H}$ & 3.303987 & -5.619099 & -2.242506 \\
\hline $\mathrm{H}$ & 5.548200 & 2.771273 & 1.478816 & $\mathrm{H}$ & -0.830109 & -4.460537 & -1.808227 \\
\hline $\mathrm{H}$ & 5.292282 & 5.144609 & 2.198029 & $\mathrm{H}$ & 0.903913 & -6.015496 & -2.743503 \\
\hline $\mathrm{C}$ & -0.516460 & 3.145973 & 0.173110 & C & -5.674785 & 3.288885 & -2.880833 \\
\hline $\mathrm{C}$ & -1.861128 & 3.106985 & 0.566142 & $\mathrm{C}$ & -4.654303 & 2.338676 & -2.886080 \\
\hline C & -0.140484 & 3.985469 & -0.889620 & $\mathrm{C}$ & -4.499518 & 1.450033 & -1.815218 \\
\hline
\end{tabular}




\begin{tabular}{|c|c|c|c|}
\hline $\mathrm{C}$ & -5.396480 & 1.516571 & -0.745801 \\
\hline $\mathrm{C}$ & -6.422365 & 2.463308 & -0.740685 \\
\hline $\mathrm{C}$ & -6.561014 & 3.358232 & -1.803176 \\
\hline $\mathrm{H}$ & -5.776809 & 3.976848 & -3.716066 \\
\hline $\mathrm{H}$ & -3.961444 & 2.295877 & -3.722780 \\
\hline $\mathrm{H}$ & -5.263902 & 0.830742 & 0.082949 \\
\hline $\mathrm{H}$ & -7.111564 & 2.504962 & 0.098852 \\
\hline $\mathrm{H}$ & -7.354498 & 4.100578 & -1.794999 \\
\hline $\mathrm{C}$ & -3.367427 & 0.444387 & -1.816904 \\
\hline $\mathrm{N}$ & -3.078704 & -0.067059 & -0.436645 \\
\hline$S$ & -2.968113 & -1.677472 & -0.264617 \\
\hline $\mathrm{O}$ & -2.625424 & -2.390054 & -1.526125 \\
\hline $\mathrm{O}$ & -2.109747 & -1.950186 & 0.910499 \\
\hline $\mathrm{C}$ & -4.626236 & -2.204211 & 0.168813 \\
\hline $\mathrm{C}$ & -5.394368 & -2.927616 & -0.737846 \\
\hline $\mathrm{C}$ & -5.155141 & -1.813528 & 1.403231 \\
\hline $\mathrm{C}$ & -6.707944 & -3.263910 & -0.403074 \\
\hline $\mathrm{H}$ & -4.958356 & -3.224015 & -1.685284 \\
\hline $\mathrm{C}$ & -6.463186 & -2.156518 & 1.721772 \\
\hline $\mathrm{H}$ & -4.539398 & -1.249406 & 2.096394 \\
\hline $\mathrm{C}$ & -7.261970 & -2.883535 & 0.822751 \\
\hline $\mathrm{H}$ & -7.310754 & -3.831344 & -1.107677 \\
\hline $\mathrm{H}$ & -6.877386 & -1.858839 & 2.682325 \\
\hline $\mathrm{C}$ & -8.686268 & -3.232894 & 1.178505 \\
\hline $\mathrm{H}$ & -9.137860 & -3.884886 & 0.424668 \\
\hline $\mathrm{H}$ & -8.741660 & -3.746154 & 2.145965 \\
\hline $\mathrm{H}$ & -9.308080 & -2.331983 & 1.256092 \\
\hline $\mathrm{H}$ & -3.584153 & -0.367267 & -2.521251 \\
\hline
\end{tabular}

TS2B

Zero-point correction= 0.963005 (Hartree/Particle)

Thermal correction to Energy= 1.024355

Thermal correction to Enthalpy= 1.025299

Thermal correction to Gibbs Free Energy $=0.858661$

$\mathrm{E}($ Solv $)=-3733.40795872$

$\begin{array}{llll}\text { C } & -5.165123 & 5.027204 & -0.981959 \\ \text { C } & -3.913529 & 4.641117 & -0.508714 \\ \text { C } & -2.960496 & 4.058437 & -1.363819 \\ \text { C } & -3.295640 & 3.914813 & -2.724283 \\ \text { C } & -4.544102 & 4.306079 & -3.198194 \\ \text { C } & -5.490839 & 4.856297 & -2.328444\end{array}$

\begin{tabular}{|c|c|c|c|}
\hline $\mathrm{H}$ & -5.888891 & 5.461729 & -0.297737 \\
\hline $\mathrm{H}$ & -3.666337 & 4.764068 & 0.541620 \\
\hline $\mathrm{H}$ & -2.566909 & 3.504721 & -3.417224 \\
\hline $\mathrm{H}$ & -4.778332 & 4.189810 & -4.253131 \\
\hline $\mathrm{H}$ & -6.465286 & 5.159429 & -2.700440 \\
\hline $\mathrm{C}$ & -1.702063 & 3.575714 & -0.798583 \\
\hline $\mathrm{H}$ & -1.466285 & 3.920530 & 0.205614 \\
\hline $\mathrm{C}$ & -0.889769 & 2.659861 & -1.369468 \\
\hline $\mathrm{H}$ & -1.127066 & 2.266367 & -2.357504 \\
\hline $\mathrm{C}$ & 0.292462 & 2.097813 & -0.738481 \\
\hline $\mathrm{C}$ & 1.251363 & 1.351713 & -1.452270 \\
\hline $\mathrm{H}$ & 1.254881 & -0.288830 & -1.076344 \\
\hline $\mathrm{H}$ & 0.583958 & 2.512895 & 0.222677 \\
\hline $\mathrm{H}$ & 1.087158 & 1.218844 & -2.521717 \\
\hline $\mathrm{Pd}$ & -0.192556 & -0.081375 & -0.363211 \\
\hline$P$ & -0.148847 & -2.375365 & -0.827237 \\
\hline$P$ & -2.017405 & 0.259301 & 1.135998 \\
\hline $\mathrm{C}$ & -0.053598 & -3.472956 & 0.632835 \\
\hline $\mathrm{C}$ & -0.487794 & -4.805367 & 0.597116 \\
\hline $\mathrm{C}$ & 0.503918 & -2.953471 & 1.809074 \\
\hline $\mathrm{C}$ & -0.357534 & -5.612051 & 1.725922 \\
\hline $\mathrm{H}$ & -0.935783 & -5.210793 & -0.303768 \\
\hline $\mathrm{C}$ & 0.643605 & -3.770542 & 2.929778 \\
\hline $\mathrm{H}$ & 0.836582 & -1.923136 & 1.847703 \\
\hline $\mathrm{C}$ & 0.213125 & -5.096363 & 2.892194 \\
\hline $\mathrm{H}$ & -0.698965 & -6.643252 & 1.693987 \\
\hline $\mathrm{H}$ & 1.083504 & -3.360587 & 3.833217 \\
\hline $\mathrm{H}$ & 0.316252 & -5.727785 & 3.770609 \\
\hline $\mathrm{C}$ & -1.568889 & -2.991898 & -1.811213 \\
\hline $\mathrm{C}$ & -1.482757 & -4.149866 & -2.600172 \\
\hline $\mathrm{C}$ & -2.781571 & -2.294094 & -1.761041 \\
\hline $\mathrm{C}$ & -2.598954 & -4.606650 & -3.300729 \\
\hline $\mathrm{H}$ & -0.539518 & -4.681215 & -2.677074 \\
\hline $\mathrm{C}$ & -3.898811 & -2.751074 & -2.459843 \\
\hline $\mathrm{H}$ & -2.847603 & -1.381458 & -1.185223 \\
\hline $\mathrm{C}$ & -3.809686 & -3.912121 & -3.228658 \\
\hline $\mathrm{H}$ & -2.521993 & -5.504043 & -3.908262 \\
\hline $\mathrm{H}$ & -4.827570 & -2.189876 & -2.406527 \\
\hline $\mathrm{H}$ & -4.675060 & -4.270900 & -3.778799 \\
\hline C & 1.300855 & -2.830193 & -1.849029 \\
\hline $\mathrm{C}$ & 2.459555 & -3.342648 & -1.259970 \\
\hline $\mathrm{C}$ & 1.308973 & -2.480877 & -3.209024 \\
\hline C & 3.612377 & -3.514187 & -2.028204 \\
\hline $\mathrm{H}$ & 2.485697 & -3.557146 & -0.199433 \\
\hline
\end{tabular}




\begin{tabular}{|c|c|c|c|c|c|c|c|}
\hline $\mathrm{C}$ & 2.458961 & -2.661526 & -3.972330 & $\mathrm{C}$ & 5.180597 & 2.452445 & -4.353613 \\
\hline $\mathrm{H}$ & 0.415896 & -2.064737 & -3.667982 & $\mathrm{H}$ & 4.670315 & 4.472604 & -3.792793 \\
\hline $\mathrm{C}$ & 3.615565 & -3.180109 & -3.381158 & $\mathrm{H}$ & 3.279813 & 3.777010 & -1.860430 \\
\hline $\mathrm{H}$ & 4.513290 & -3.892740 & -1.554984 & $\mathrm{H}$ & 4.131935 & -0.336764 & -2.687893 \\
\hline $\mathrm{H}$ & 2.456441 & -2.391475 & -5.024751 & $\mathrm{H}$ & 5.518409 & 0.343701 & -4.651982 \\
\hline $\mathrm{H}$ & 4.516531 & -3.311466 & -3.974262 & $\mathrm{H}$ & 5.791536 & 2.754655 & -5.200081 \\
\hline $\mathrm{C}$ & -2.227352 & -1.054082 & 2.399909 & $\mathrm{C}$ & 2.723709 & 1.252384 & -1.002463 \\
\hline $\mathrm{C}$ & -1.826793 & -0.878504 & 3.728916 & $\mathrm{~N}$ & 3.034002 & -0.117377 & -0.590007 \\
\hline $\mathrm{C}$ & -2.751588 & -2.294083 & 2.000820 & S & 3.045586 & -0.343205 & 0.983555 \\
\hline $\mathrm{C}$ & -1.952963 & -1.924949 & 4.644455 & $\mathrm{O}$ & 1.808910 & 0.151864 & 1.692087 \\
\hline $\mathrm{H}$ & -1.416403 & 0.071752 & 4.051332 & $\mathrm{O}$ & 3.393562 & -1.749576 & 1.271619 \\
\hline $\mathrm{C}$ & -2.884382 & -3.331567 & 2.918015 & $\mathrm{C}$ & 4.357063 & 0.713310 & 1.635137 \\
\hline $\mathrm{H}$ & -3.055680 & -2.450788 & 0.970855 & $\mathrm{C}$ & 4.215879 & 1.325426 & 2.879519 \\
\hline $\mathrm{C}$ & -2.482148 & -3.150335 & 4.242921 & $\mathrm{C}$ & 5.517495 & 0.899246 & 0.881436 \\
\hline $\mathrm{H}$ & -1.635335 & -1.777758 & 5.672889 & $\mathrm{C}$ & 5.249335 & 2.120155 & 3.373971 \\
\hline $\mathrm{H}$ & -3.277205 & -4.289232 & 2.591635 & $\mathrm{H}$ & 3.298327 & 1.182097 & 3.439656 \\
\hline $\mathrm{H}$ & -2.572386 & -3.965633 & 4.954809 & $\mathrm{C}$ & 6.539848 & 1.698983 & 1.385342 \\
\hline $\mathrm{C}$ & -3.712088 & 0.429691 & 0.449870 & $\mathrm{H}$ & 5.595179 & 0.439685 & -0.098160 \\
\hline $\mathrm{C}$ & -3.872694 & 0.905895 & -0.858144 & $\mathrm{C}$ & 6.425307 & 2.316425 & 2.638891 \\
\hline $\mathrm{C}$ & -4.852200 & 0.139215 & 1.215005 & $\mathrm{H}$ & 5.140087 & 2.601735 & 4.343040 \\
\hline $\mathrm{C}$ & -5.146370 & 1.103218 & -1.390162 & $\mathrm{H}$ & 7.439049 & 1.854074 & 0.793800 \\
\hline $\mathrm{H}$ & -2.995829 & 1.126007 & -1.453922 & $\mathrm{C}$ & 7.551541 & 3.158550 & 3.187208 \\
\hline $\mathrm{C}$ & -6.125649 & 0.328319 & 0.679298 & $\mathrm{H}$ & 7.194544 & 3.857449 & 3.950608 \\
\hline $\mathrm{H}$ & -4.740572 & -0.238109 & 2.226148 & $\mathrm{H}$ & 8.323620 & 2.531253 & 3.652209 \\
\hline $\mathrm{C}$ & -6.274720 & 0.814497 & -0.621948 & $\mathrm{H}$ & 8.039620 & 3.738286 & 2.396134 \\
\hline $\mathrm{H}$ & -5.251263 & 1.501681 & -2.393721 & $\mathrm{H}$ & 2.858401 & 1.984641 & -0.192935 \\
\hline $\mathrm{H}$ & -7.001533 & 0.098998 & 1.279748 & & & --- & \\
\hline
\end{tabular}

TS2C

Zero-point correction= $0.686283($ Hartree/Particle $)$

Thermal correction to Energy $=0.730562$

Thermal correction to Enthalpy $=0.731506$

Thermal correction to Gibbs Free Energy= 0.600579

$\mathrm{E}(\mathrm{Solv})=-2696.72972809$

$\begin{array}{llll}\mathrm{C} & 6.827678 & -1.210921 & -1.363954 \\ \mathrm{C} & 5.716027 & -1.801765 & -0.769526 \\ \mathrm{C} & 4.469544 & -1.824342 & -1.423516 \\ \mathrm{C} & 4.372071 & -1.222521 & -2.694937 \\ \mathrm{C} & 5.484172 & -0.635752 & -3.288215 \\ \mathrm{C} & 6.717032 & -0.626250 & -2.626880 \\ \mathrm{H} & 7.779713 & -1.205291 & -0.841500 \\ \mathrm{H} & 5.803895 & -2.253950 & 0.215080\end{array}$




\begin{tabular}{|c|c|c|c|c|c|c|c|}
\hline $\mathrm{H}$ & 3.418353 & -1.195401 & -3.211533 & $\mathrm{H}$ & 4.135069 & 3.896558 & 3.367608 \\
\hline $\mathrm{H}$ & 5.388809 & -0.177976 & -4.268620 & $\mathrm{H}$ & 2.345842 & 0.379420 & 5.083771 \\
\hline $\mathrm{H}$ & 7.581939 & -0.162964 & -3.092211 & $\mathrm{H}$ & 3.881083 & 2.327669 & 5.276608 \\
\hline $\mathrm{C}$ & 3.325808 & -2.415225 & -0.741359 & $\mathrm{C}$ & -0.378495 & -4.291566 & 3.370999 \\
\hline $\mathrm{H}$ & 3.495609 & -2.725087 & 0.289832 & $\mathrm{C}$ & -0.831772 & -4.240914 & 2.050869 \\
\hline $\mathrm{C}$ & 2.071255 & -2.604004 & -1.262067 & C & -1.297643 & -3.042041 & 1.500514 \\
\hline $\mathrm{H}$ & 1.864157 & -2.344827 & -2.298105 & $\mathrm{C}$ & -1.323603 & -1.892735 & 2.301538 \\
\hline $\mathrm{C}$ & 0.942348 & -2.947955 & -0.427666 & $\mathrm{C}$ & -0.873550 & -1.942469 & 3.622562 \\
\hline $\mathrm{C}$ & -0.404147 & -2.666529 & -0.810677 & $\mathrm{C}$ & -0.391184 & -3.138500 & 4.159085 \\
\hline $\mathrm{H}$ & -0.690356 & -1.270239 & -0.568042 & $\mathrm{H}$ & -0.025193 & -5.231843 & 3.785625 \\
\hline $\mathrm{H}$ & 1.132417 & -3.421086 & 0.534992 & $\mathrm{H}$ & -0.826247 & -5.141533 & 1.440559 \\
\hline $\mathrm{H}$ & -0.618448 & -2.565926 & -1.876635 & $\mathrm{H}$ & -1.725053 & -0.977996 & 1.881822 \\
\hline $\mathrm{Pd}$ & 0.909989 & -0.824558 & 0.021086 & $\mathrm{H}$ & -0.913446 & -1.046574 & 4.237303 \\
\hline $\mathrm{P}$ & 0.876200 & 1.419215 & 0.495284 & $\mathrm{H}$ & -0.045690 & -3.177445 & 5.188584 \\
\hline $\mathrm{C}$ & -0.723904 & 2.225341 & 0.833263 & $\mathrm{C}$ & -1.658547 & -2.956515 & 0.031604 \\
\hline $\mathrm{C}$ & -0.787017 & 3.468225 & 1.485068 & $\mathrm{~N}$ & -2.521409 & -1.798018 & -0.205028 \\
\hline $\mathrm{C}$ & -1.906530 & 1.575026 & 0.454108 & $\mathrm{~S}$ & -3.301482 & -1.862703 & -1.620777 \\
\hline $\mathrm{C}$ & -2.022157 & 4.062394 & 1.733791 & $\mathrm{O}$ & -2.460419 & -1.480683 & -2.793689 \\
\hline $\mathrm{H}$ & 0.126112 & 3.960542 & 1.806832 & $\mathrm{O}$ & -4.068761 & -3.123051 & -1.780668 \\
\hline $\mathrm{C}$ & -3.141555 & 2.172643 & 0.714619 & $\mathrm{C}$ & -4.469104 & -0.521170 & -1.386298 \\
\hline $\mathrm{H}$ & -1.878580 & 0.595787 & -0.015306 & $\mathrm{C}$ & -4.466545 & 0.556400 & -2.266628 \\
\hline $\mathrm{C}$ & -3.199208 & 3.414109 & 1.346894 & $\mathrm{C}$ & -5.401798 & -0.592575 & -0.349594 \\
\hline $\mathrm{H}$ & -2.067194 & 5.024331 & 2.236956 & $\mathrm{C}$ & -5.403852 & 1.577764 & -2.101870 \\
\hline $\mathrm{H}$ & -4.050728 & 1.657822 & 0.430477 & $\mathrm{H}$ & -3.728708 & 0.582903 & -3.060689 \\
\hline $\mathrm{H}$ & -4.162660 & 3.874536 & 1.548243 & $\mathrm{C}$ & -6.332613 & 0.431247 & -0.197584 \\
\hline $\mathrm{C}$ & 1.709130 & 2.400889 & -0.806596 & $\mathrm{H}$ & -5.379895 & -1.436350 & 0.331237 \\
\hline $\mathrm{C}$ & 2.893757 & 1.871122 & -1.345642 & $\mathrm{C}$ & -6.349720 & 1.530306 & -1.071830 \\
\hline $\mathrm{C}$ & 1.220867 & 3.621062 & -1.286573 & $\mathrm{H}$ & -5.397362 & 2.426312 & -2.781658 \\
\hline $\mathrm{C}$ & 3.588936 & 2.562040 & -2.334200 & $\mathrm{H}$ & -7.056578 & 0.383002 & 0.612522 \\
\hline $\mathrm{H}$ & 3.267186 & 0.912684 & -0.993780 & $\mathrm{C}$ & -7.377855 & 2.623626 & -0.910706 \\
\hline $\mathrm{C}$ & 1.915616 & 4.306720 & -2.286330 & $\mathrm{H}$ & -7.584495 & 2.827193 & 0.145905 \\
\hline $\mathrm{H}$ & 0.299209 & 4.030850 & -0.886975 & $\mathrm{H}$ & -7.046717 & 3.555978 & -1.379394 \\
\hline $\mathrm{C}$ & 3.099038 & 3.782925 & -2.807304 & $\mathrm{H}$ & -8.330944 & 2.342230 & -1.377652 \\
\hline $\mathrm{H}$ & 4.502588 & 2.137938 & -2.740143 & $\mathrm{H}$ & -2.107232 & -3.904603 & -0.300054 \\
\hline $\mathrm{H}$ & 1.527558 & 5.250663 & -2.658388 & ----- & 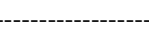 & ---- & \\
\hline $\mathrm{H}$ & 3.634534 & 4.319480 & -3.585395 & & & & \\
\hline $\mathrm{C}$ & 1.872713 & 1.750159 & 1.999308 & TS2E & & & \\
\hline $\mathrm{C}$ & 2.742593 & 2.842289 & 2.109950 & & & & \\
\hline $\mathrm{C}$ & 1.739269 & 0.860801 & 3.077841 & \multicolumn{4}{|c|}{ Zero-point correction $=0.851463$ (Hartree/Particle $)$} \\
\hline $\mathrm{C}$ & 3.461734 & 3.047638 & 3.288763 & \multicolumn{4}{|c|}{ Thermal correction to Energy= 0.906304} \\
\hline $\mathrm{H}$ & 2.861565 & 3.525434 & 1.274766 & \multicolumn{4}{|c|}{ Thermal correction to Enthalpy $=0.907248$} \\
\hline $\mathrm{C}$ & 2.455862 & 1.072261 & 4.254273 & \multicolumn{4}{|c|}{ Thermal correction to Gibbs Free Energy $=0.755004$} \\
\hline $\mathrm{H}$ & 1.075387 & 0.004300 & 2.991000 & \multicolumn{4}{|c|}{$E($ Solv $)=-3271.48411055$} \\
\hline $\mathrm{C}$ & 3.318521 & 2.166122 & 4.361509 & & & 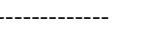 & \\
\hline
\end{tabular}




\begin{tabular}{|c|c|c|c|c|c|c|c|}
\hline $\mathrm{C}$ & 4.726302 & -4.640618 & -0.224160 & $\mathrm{C}$ & 3.534985 & 1.750853 & 1.149009 \\
\hline $\mathrm{C}$ & 3.590316 & -3.971316 & 0.225358 & $\mathrm{C}$ & 4.154032 & 2.045775 & 2.372274 \\
\hline $\mathrm{C}$ & 2.647584 & -3.458820 & -0.681900 & $\mathrm{C}$ & 2.838420 & 2.758207 & 0.465305 \\
\hline C & 2.866892 & -3.664314 & -2.056965 & C & 4.069085 & 3.333290 & 2.903473 \\
\hline $\mathrm{C}$ & 3.995344 & -4.346433 & -2.504569 & $\mathrm{H}$ & 4.694503 & 1.272062 & 2.908222 \\
\hline $\mathrm{C}$ & 4.936689 & -4.830525 & -1.590925 & C & 2.755704 & 4.043483 & 0.999437 \\
\hline $\mathrm{H}$ & 5.449397 & -5.009810 & 0.497502 & $\mathrm{H}$ & 2.349886 & 2.524431 & -0.472900 \\
\hline $\mathrm{H}$ & 3.437855 & -3.821048 & 1.289148 & $\mathrm{C}$ & 3.368802 & 4.330784 & 2.220685 \\
\hline $\mathrm{H}$ & 2.165579 & -3.261895 & -2.780983 & $\mathrm{H}$ & 4.546665 & 3.555764 & 3.853812 \\
\hline $\mathrm{H}$ & 4.147019 & -4.490796 & -3.570730 & $\mathrm{H}$ & 2.201287 & 4.808867 & 0.465329 \\
\hline $\mathrm{H}$ & 5.822233 & -5.352007 & -1.942530 & $\mathrm{H}$ & 3.296780 & 5.328866 & 2.643990 \\
\hline $\mathrm{C}$ & 1.516677 & -2.661680 & -0.168454 & $\mathrm{C}$ & -4.744416 & -3.666600 & 1.777685 \\
\hline $\mathrm{H}$ & 1.412021 & -2.697096 & 0.916132 & $\mathrm{C}$ & -3.954311 & -2.551975 & 1.488006 \\
\hline $\mathrm{C}$ & 0.326235 & -2.388948 & -0.925427 & $\mathrm{C}$ & -3.958832 & -1.990197 & 0.209601 \\
\hline $\mathrm{H}$ & 0.232236 & -2.713642 & -1.958375 & $\mathrm{C}$ & -4.768149 & -2.559089 & -0.780801 \\
\hline $\mathrm{C}$ & -0.751166 & -1.700470 & -0.332821 & $\mathrm{C}$ & -5.563456 & -3.664583 & -0.493202 \\
\hline $\mathrm{C}$ & -1.884808 & -1.165466 & -0.971924 & $\mathrm{C}$ & -5.553481 & -4.223821 & 0.788029 \\
\hline $\mathrm{H}$ & -2.141097 & -1.587030 & -1.948026 & $\mathrm{H}$ & -4.736258 & -4.089279 & 2.778716 \\
\hline $\mathrm{H}$ & -0.659642 & -1.506388 & 0.738433 & $\mathrm{H}$ & -3.344845 & -2.099467 & 2.266431 \\
\hline $\mathrm{H}$ & -1.359957 & 0.097695 & -1.397308 & $\mathrm{H}$ & -4.795674 & -2.103840 & -1.765529 \\
\hline $\mathrm{Pd}$ & 1.653770 & -0.624847 & -0.668082 & $\mathrm{H}$ & -6.200428 & -4.085936 & -1.266001 \\
\hline $\mathrm{P}$ & 3.589925 & 0.087436 & 0.385515 & $\mathrm{H}$ & -6.176889 & -5.084911 & 1.012676 \\
\hline $\mathrm{C}$ & 5.101879 & 0.098000 & -0.647729 & $\mathrm{C}$ & -3.079155 & -0.790795 & -0.100051 \\
\hline $\mathrm{C}$ & 6.058940 & 1.118348 & -0.579947 & $\mathrm{~N}$ & -3.865068 & 0.260970 & -0.789530 \\
\hline $\mathrm{C}$ & 5.304808 & -0.983467 & -1.519355 & $\mathrm{~S}$ & -4.978146 & 1.116043 & 0.154499 \\
\hline $\mathrm{C}$ & 7.205688 & 1.054917 & -1.373681 & $\mathrm{O}$ & -4.534732 & 1.163730 & 1.559741 \\
\hline $\mathrm{H}$ & 5.907837 & 1.958304 & 0.090235 & $\mathrm{O}$ & -5.235904 & 2.352785 & -0.589147 \\
\hline $\mathrm{C}$ & 6.457527 & -1.049293 & -2.298836 & $\mathrm{C}$ & -6.424495 & 0.076645 & 0.089295 \\
\hline $\mathrm{H}$ & 4.564943 & -1.773083 & -1.581960 & $\mathrm{C}$ & -6.803027 & -0.648439 & 1.216187 \\
\hline $\mathrm{C}$ & 7.408938 & -0.028463 & -2.230248 & $\mathrm{C}$ & -7.122233 & -0.031729 & -1.112585 \\
\hline $\mathrm{H}$ & 7.941440 & 1.852588 & -1.320118 & $\mathrm{C}$ & -7.885105 & -1.518244 & 1.124071 \\
\hline $\mathrm{H}$ & 6.605682 & -1.896663 & -2.962140 & $\mathrm{H}$ & -6.238545 & -0.543250 & 2.134266 \\
\hline $\mathrm{H}$ & 8.303913 & -0.075340 & -2.844429 & $\mathrm{C}$ & -8.202709 & -0.905795 & -1.186122 \\
\hline $\mathrm{C}$ & 4.045109 & -1.005353 & 1.788208 & $\mathrm{H}$ & -6.815136 & 0.554359 & -1.971992 \\
\hline $\mathrm{C}$ & 3.050150 & -1.303014 & 2.733304 & $\mathrm{C}$ & -8.589752 & -1.670507 & -0.076829 \\
\hline $\mathrm{C}$ & 5.307028 & -1.594973 & 1.919435 & $\mathrm{H}$ & -8.175965 & -2.102159 & 1.992985 \\
\hline $\mathrm{C}$ & 3.312209 & -2.175364 & 3.786877 & $\mathrm{H}$ & -8.751398 & -1.002675 & -2.119275 \\
\hline $\mathrm{H}$ & 2.066445 & -0.851527 & 2.631387 & $\mathrm{C}$ & -9.717255 & -2.666805 & -0.178783 \\
\hline $\mathrm{C}$ & 5.566905 & -2.475803 & 2.972193 & $\mathrm{H}$ & -9.323093 & -3.685095 & -0.295297 \\
\hline $\mathrm{H}$ & 6.081969 & -1.379576 & 1.192327 & $\mathrm{H}$ & -10.339710 & -2.662341 & 0.722486 \\
\hline $\mathrm{C}$ & 4.572407 & -2.771043 & 3.904322 & $\mathrm{H}$ & -10.360578 & -2.460890 & -1.039741 \\
\hline $\mathrm{H}$ & 2.533972 & -2.397757 & 4.511553 & $\mathrm{H}$ & -2.692806 & -0.400608 & 0.851968 \\
\hline $\mathrm{H}$ & 6.548474 & -2.933401 & 3.059213 & $\mathrm{C}$ & -0.092578 & 1.830179 & -1.621108 \\
\hline $\mathrm{H}$ & 4.774700 & -3.460453 & 4.719007 & $\mathrm{O}$ & 1.003213 & 1.241311 & -1.753730 \\
\hline
\end{tabular}




\begin{tabular}{lrrr}
$\mathrm{O}$ & -1.253521 & 1.258271 & -1.707344 \\
$\mathrm{H}$ & -3.270431 & 0.958497 & -1.244045 \\
$\mathrm{C}$ & -0.094074 & 3.310994 & -1.402983 \\
$\mathrm{C}$ & -0.954327 & 3.952990 & -0.453858 \\
$\mathrm{C}$ & 0.808396 & 4.052932 & -2.144557 \\
$\mathrm{C}$ & -1.859724 & 3.258440 & 0.393120 \\
$\mathrm{C}$ & -0.861031 & 5.379542 & -0.317667 \\
$\mathrm{C}$ & 0.878563 & 5.455845 & -2.016894 \\
$\mathrm{H}$ & 1.464965 & 3.535477 & -2.836396 \\
$\mathrm{C}$ & -2.647959 & 3.930454 & 1.299502 \\
$\mathrm{H}$ & -1.939368 & 2.183447 & 0.316514 \\
$\mathrm{C}$ & -1.687329 & 6.042865 & 0.627899 \\
$\mathrm{C}$ & 0.057671 & 6.103452 & -1.121410 \\
$\mathrm{H}$ & 1.581572 & 6.016355 & -2.625900 \\
$\mathrm{C}$ & -2.565448 & 5.337424 & 1.416597 \\
$\mathrm{H}$ & -3.345724 & 3.366684 & 1.908963 \\
$\mathrm{H}$ & -1.609302 & 7.123861 & 0.714656 \\
$\mathrm{H}$ & 0.103113 & 7.184289 & -1.012462 \\
$\mathrm{H}$ & -3.197367 & 5.856736 & 2.131313 \\
-------------------------------------- & \\
\hline
\end{tabular}

\begin{tabular}{|c|c|c|c|}
\hline $\mathrm{C}$ & -1.258824 & -1.450564 & -2.296882 \\
\hline $\mathrm{C}$ & -1.758329 & -2.698702 & -1.858518 \\
\hline $\mathrm{H}$ & -1.831410 & -3.457674 & -2.640628 \\
\hline $\mathrm{H}$ & -0.577824 & -1.501511 & -3.149486 \\
\hline $\mathrm{H}$ & -0.577040 & -3.231022 & -1.276962 \\
\hline $\mathrm{Pd}$ & 0.382048 & -0.110601 & -0.576909 \\
\hline $\mathrm{P}$ & 2.039673 & 1.217353 & 0.314128 \\
\hline $\mathrm{C}$ & 1.422032 & 2.296467 & 1.662595 \\
\hline $\mathrm{C}$ & 2.221836 & 2.671686 & 2.750704 \\
\hline $\mathrm{C}$ & 0.111710 & 2.787884 & 1.569856 \\
\hline $\mathrm{C}$ & 1.714828 & 3.526178 & 3.730869 \\
\hline $\mathrm{H}$ & 3.235615 & 2.293554 & 2.832337 \\
\hline $\mathrm{C}$ & -0.385552 & 3.652218 & 2.543078 \\
\hline $\mathrm{H}$ & -0.513255 & 2.501454 & 0.733456 \\
\hline $\mathrm{C}$ & 0.412559 & 4.019499 & 3.628378 \\
\hline $\mathrm{H}$ & 2.338686 & 3.806629 & 4.574930 \\
\hline $\mathrm{H}$ & -1.396306 & 4.038106 & 2.447771 \\
\hline $\mathrm{H}$ & 0.021804 & 4.685332 & 4.392723 \\
\hline $\mathrm{C}$ & 2.756795 & 2.399493 & -0.888634 \\
\hline $\mathrm{C}$ & 3.111901 & 1.910145 & -2.154756 \\
\hline $\mathrm{C}$ & 2.893007 & 3.766023 & -0.621293 \\
\hline $\mathrm{C}$ & 3.599211 & 2.771311 & -3.135085 \\
\hline $\mathrm{H}$ & 2.994531 & 0.852410 & -2.363764 \\
\hline $\mathrm{C}$ & 3.369043 & 4.630960 & -1.609137 \\
\hline $\mathrm{H}$ & 2.610462 & 4.157519 & 0.349746 \\
\hline $\mathrm{C}$ & 3.720554 & 4.138288 & -2.866181 \\
\hline $\mathrm{H}$ & 3.872462 & 2.381077 & -4.111687 \\
\hline $\mathrm{H}$ & 3.460732 & 5.692191 & -1.395138 \\
\hline $\mathrm{H}$ & 4.085944 & 4.813984 & -3.634244 \\
\hline $\mathrm{C}$ & 3.486393 & 0.392795 & 1.069087 \\
\hline $\mathrm{C}$ & 4.806911 & 0.703103 & 0.726212 \\
\hline $\mathrm{C}$ & 3.233125 & -0.580910 & 2.049505 \\
\hline $\mathrm{C}$ & 5.865513 & 0.055096 & 1.365693 \\
\hline $\mathrm{H}$ & 5.009372 & 1.448119 & -0.035636 \\
\hline $\mathrm{C}$ & 4.293254 & -1.204726 & 2.700122 \\
\hline $\mathrm{H}$ & 2.209604 & -0.855818 & 2.285510 \\
\hline $\mathrm{C}$ & 5.610793 & -0.889385 & 2.358760 \\
\hline $\mathrm{H}$ & 6.888189 & 0.294164 & 1.087884 \\
\hline $\mathrm{H}$ & 4.091050 & -1.958583 & 3.453679 \\
\hline $\mathrm{H}$ & 6.435080 & -1.392227 & 2.856496 \\
\hline $\mathrm{C}$ & -6.601681 & -3.162425 & -1.149255 \\
\hline $\mathrm{C}$ & -5.274334 & -3.412853 & -0.800150 \\
\hline $\mathrm{C}$ & -4.246612 & -2.553396 & -1.207341 \\
\hline C & -4.579057 & -1.448749 & -2.001368 \\
\hline
\end{tabular}




\begin{tabular}{|c|c|c|c|c|c|c|c|}
\hline $\mathrm{C}$ & -5.904578 & -1.192685 & -2.350867 & $\mathrm{H}$ & 3.977114 & -0.964924 & -3.391518 \\
\hline $\mathrm{C}$ & -6.923000 & -2.044896 & -1.921226 & $\mathrm{H}$ & 6.921444 & -2.824245 & -0.870383 \\
\hline $\mathrm{H}$ & -7.382928 & -3.838499 & -0.813481 & $\mathrm{H}$ & 6.213340 & -4.182702 & 1.052542 \\
\hline $\mathrm{H}$ & -5.032380 & -4.263494 & -0.172500 & $\mathrm{H}$ & 6.358098 & -1.436383 & -2.840334 \\
\hline $\mathrm{H}$ & -3.804936 & -0.781344 & -2.356311 & ----- & & ---- & \\
\hline $\mathrm{H}$ & -6.139737 & -0.324996 & -2.961185 & \multirow{3}{*}{ TS3D } & & & \\
\hline $\mathrm{H}$ & -7.956003 & -1.844816 & -2.192361 & & & & \\
\hline $\mathrm{C}$ & -2.816486 & -2.853860 & -0.763700 & & & & \\
\hline $\mathrm{N}$ & -2.375246 & -2.009268 & 0.369910 & \multicolumn{4}{|c|}{ Zero-point correction $=1.129038($ Hartree/Particle $)$} \\
\hline S & -3.198654 & -1.880511 & 1.814344 & \multicolumn{4}{|c|}{ Thermal correction to Energy $=1.200808$} \\
\hline $\mathrm{O}$ & -4.206668 & -2.939834 & 1.938178 & \multicolumn{4}{|c|}{ Thermal correction to Enthalpy= 1.201752} \\
\hline $\mathrm{O}$ & -2.163096 & -1.691316 & 2.836841 & \multicolumn{4}{|c|}{ Thermal correction to Gibbs Free Energy $=1.014521$} \\
\hline $\mathrm{C}$ & -4.051641 & -0.318103 & 1.631611 & \multicolumn{3}{|c|}{$E($ Solv $)=-4308.13408112$} & \\
\hline $\mathrm{C}$ & -5.328780 & -0.277156 & 1.073040 & -------- & ------- & --------- & \\
\hline $\mathrm{C}$ & -3.394081 & 0.850500 & 2.017333 & $\mathrm{C}$ & 3.175591 & -5.465210 & -2.512340 \\
\hline $\mathrm{C}$ & -5.942335 & 0.958441 & 0.882948 & $\mathrm{C}$ & 2.366322 & -4.857251 & -1.555537 \\
\hline $\mathrm{H}$ & -5.829810 & -1.195684 & 0.792053 & $\mathrm{C}$ & 1.705377 & -3.649650 & -1.827756 \\
\hline C & -4.032068 & 2.075485 & 1.834741 & $\mathrm{C}$ & 1.884875 & -3.067103 & -3.094201 \\
\hline $\mathrm{H}$ & -2.405571 & 0.794340 & 2.460272 & $\mathrm{C}$ & 2.700823 & -3.667840 & -4.047397 \\
\hline $\mathrm{C}$ & -5.306084 & 2.150553 & 1.256425 & $\mathrm{C}$ & 3.349459 & -4.872782 & -3.763093 \\
\hline $\mathrm{H}$ & -6.933725 & 0.995487 & 0.438893 & $\mathrm{H}$ & 3.680705 & -6.396212 & -2.273241 \\
\hline $\mathrm{H}$ & -3.529980 & 2.987519 & 2.144660 & $\mathrm{H}$ & 2.249414 & -5.315472 & -0.578166 \\
\hline C & -5.974182 & 3.483136 & 1.020812 & $\mathrm{H}$ & 1.398144 & -2.126971 & -3.327232 \\
\hline $\mathrm{H}$ & -5.905995 & 3.777402 & -0.034877 & $\mathrm{H}$ & 2.834113 & -3.191253 & -5.014700 \\
\hline $\mathrm{H}$ & -7.038704 & 3.448513 & 1.276830 & $\mathrm{H}$ & 3.986933 & -5.340275 & -4.508118 \\
\hline $\mathrm{H}$ & -5.508254 & 4.275172 & 1.615746 & $\mathrm{C}$ & 0.857257 & -3.043751 & -0.785552 \\
\hline $\mathrm{H}$ & -2.803876 & -3.908204 & -0.455482 & $\mathrm{H}$ & 0.878790 & -3.572612 & 0.169865 \\
\hline C & 1.046162 & -3.021285 & 0.031466 & $\mathrm{C}$ & -0.294984 & -2.278977 & -1.071145 \\
\hline $\mathrm{O}$ & 0.503360 & -2.080303 & 0.664505 & $\mathrm{H}$ & -0.447625 & -1.956472 & -2.099906 \\
\hline $\mathrm{O}$ & 0.455415 & -3.754658 & -0.842667 & $\mathrm{C}$ & -1.268163 & -1.851946 & -0.141117 \\
\hline $\mathrm{H}$ & -1.368994 & -2.075895 & 0.571880 & $\mathrm{C}$ & -2.341144 & -1.007042 & -0.508948 \\
\hline C & 2.487808 & -3.349121 & 0.290436 & $\mathrm{H}$ & -2.634740 & -1.098964 & -1.560269 \\
\hline C & 3.499144 & -2.838330 & -0.575050 & $\mathrm{H}$ & -1.182295 & -2.191388 & 0.891590 \\
\hline C & 2.819922 & -4.130781 & 1.376264 & $\mathrm{H}$ & -1.625943 & 0.186777 & -0.736102 \\
\hline $\mathrm{C}$ & 3.205639 & -2.041953 & -1.714148 & $\mathrm{Pd}$ & 1.327882 & -1.044697 & 0.092322 \\
\hline C & 4.867429 & -3.136866 & -0.276747 & $\mathrm{P}$ & 0.942861 & 0.683767 & 1.664657 \\
\hline C & 4.170589 & -4.447752 & 1.645751 & $\mathrm{P}$ & 3.640100 & -0.959289 & -0.204142 \\
\hline $\mathrm{H}$ & 2.035958 & -4.505653 & 2.027654 & $\mathrm{C}$ & 2.089911 & 0.311310 & 3.060438 \\
\hline C & 4.216805 & -1.558241 & -2.513565 & $\mathrm{C}$ & 2.789831 & 1.280358 & 3.785785 \\
\hline $\mathrm{H}$ & 2.169892 & -1.812094 & -1.948713 & $\mathrm{C}$ & 2.248052 & -1.040259 & 3.411322 \\
\hline C & 5.884588 & -2.604913 & -1.111962 & $\mathrm{C}$ & 3.607355 & 0.908276 & 4.853956 \\
\hline C & 5.171720 & -3.955220 & 0.841437 & $\mathrm{H}$ & 2.713808 & 2.325157 & 3.508902 \\
\hline $\mathrm{H}$ & 4.411271 & -5.075035 & 2.499135 & $\mathrm{C}$ & 3.054309 & -1.410534 & 4.486110 \\
\hline $\mathrm{C}$ & 5.570460 & -1.833024 & -2.206219 & $\mathrm{H}$ & 1.736799 & -1.801856 & 2.826595 \\
\hline
\end{tabular}




\begin{tabular}{|c|c|c|c|c|c|c|c|}
\hline $\mathrm{C}$ & 3.733803 & -0.432815 & 5.214951 & $\mathrm{C}$ & 4.416475 & 0.966347 & -4.352578 \\
\hline $\mathrm{H}$ & 4.152897 & 1.672230 & 5.400392 & $\mathrm{H}$ & 2.269443 & 0.945777 & -4.554698 \\
\hline $\mathrm{H}$ & 3.157340 & -2.460032 & 4.749008 & $\mathrm{H}$ & 6.513185 & 0.886509 & -3.848803 \\
\hline $\mathrm{H}$ & 4.369044 & -0.716518 & 6.049312 & $\mathrm{H}$ & 4.586104 & 1.420970 & -5.324532 \\
\hline C & 1.284231 & 2.412882 & 1.183987 & $\mathrm{C}$ & 4.480158 & -2.589926 & -0.185130 \\
\hline $\mathrm{C}$ & 1.025913 & 3.498085 & 2.037850 & $\mathrm{C}$ & 5.332042 & -3.046828 & -1.195215 \\
\hline $\mathrm{C}$ & 1.814513 & 2.659668 & -0.087064 & $\mathrm{C}$ & 4.222637 & -3.421829 & 0.917494 \\
\hline $\mathrm{C}$ & 1.326971 & 4.797388 & 1.633724 & $\mathrm{C}$ & 5.940192 & -4.298324 & -1.088928 \\
\hline $\mathrm{H}$ & 0.567664 & 3.325155 & 3.006541 & $\mathrm{H}$ & 5.505229 & -2.441430 & -2.076863 \\
\hline $\mathrm{C}$ & 2.127165 & 3.958525 & -0.485631 & $\mathrm{C}$ & 4.844298 & -4.663705 & 1.029684 \\
\hline $\mathrm{H}$ & 1.953040 & 1.833702 & -0.773996 & $\mathrm{H}$ & 3.531910 & -3.089067 & 1.687628 \\
\hline $\mathrm{C}$ & 1.886254 & 5.028593 & 0.374520 & $\mathrm{C}$ & 5.710238 & -5.103699 & 0.025885 \\
\hline $\mathrm{H}$ & 1.109576 & 5.631747 & 2.294223 & $\mathrm{H}$ & 6.589564 & -4.645984 & -1.887249 \\
\hline $\mathrm{H}$ & 2.512113 & 4.133637 & -1.484484 & $\mathrm{H}$ & 4.645007 & -5.291520 & 1.893849 \\
\hline $\mathrm{H}$ & 2.090939 & 6.043651 & 0.048966 & $\mathrm{H}$ & 6.189461 & -6.075219 & 0.106275 \\
\hline $\mathrm{C}$ & -0.677686 & 0.792950 & 2.524669 & $\mathrm{C}$ & -5.325872 & -4.004257 & 1.701257 \\
\hline $\mathrm{C}$ & -0.993094 & -0.078977 & 3.577425 & $\mathrm{C}$ & -4.566332 & -2.832141 & 1.663694 \\
\hline $\mathrm{C}$ & -1.653835 & 1.694491 & 2.073628 & $\mathrm{C}$ & -4.365355 & -2.154691 & 0.459632 \\
\hline $\mathrm{C}$ & -2.255114 & -0.039065 & 4.170816 & $\mathrm{C}$ & -4.941400 & -2.664044 & -0.710715 \\
\hline $\mathrm{H}$ & -0.251113 & -0.778082 & 3.948691 & $\mathrm{C}$ & -5.703706 & -3.827703 & -0.676815 \\
\hline $\mathrm{C}$ & -2.911589 & 1.732775 & 2.671187 & $\mathrm{C}$ & -5.896814 & -4.504258 & 0.531260 \\
\hline $\mathrm{H}$ & -1.441718 & 2.364989 & 1.252838 & $\mathrm{H}$ & -5.479648 & -4.518379 & 2.646436 \\
\hline $\mathrm{C}$ & -3.218083 & 0.865647 & 3.721098 & $\mathrm{H}$ & -4.149210 & -2.417715 & 2.577315 \\
\hline $\mathrm{H}$ & -2.483915 & -0.716922 & 4.988957 & $\mathrm{H}$ & -4.815762 & -2.123006 & -1.643237 \\
\hline $\mathrm{H}$ & -3.663159 & 2.418846 & 2.293533 & $\mathrm{H}$ & -6.160492 & -4.200851 & -1.589233 \\
\hline $\mathrm{H}$ & -4.211470 & 0.875712 & 4.152694 & $\mathrm{H}$ & -6.495164 & -5.411015 & 0.559276 \\
\hline $\mathrm{C}$ & 4.717750 & 0.061462 & 0.889069 & $\mathrm{C}$ & -3.533458 & -0.880620 & 0.429136 \\
\hline $\mathrm{C}$ & 5.432839 & -0.489404 & 1.960350 & $\mathrm{~N}$ & -4.344031 & 0.256877 & -0.048875 \\
\hline $\mathrm{C}$ & 4.770625 & 1.449129 & 0.684634 & $\mathrm{~S}$ & -5.729357 & 0.679695 & 0.783168 \\
\hline $\mathrm{C}$ & 6.183639 & 0.329276 & 2.802877 & $\mathrm{O}$ & -5.705794 & 0.204123 & 2.176578 \\
\hline $\mathrm{H}$ & 5.411913 & -1.557610 & 2.139188 & $\mathrm{O}$ & -5.939068 & 2.104969 & 0.496470 \\
\hline $\mathrm{C}$ & 5.514874 & 2.265645 & 1.532839 & $\mathrm{C}$ & -6.993128 & -0.271563 & -0.049750 \\
\hline $\mathrm{H}$ & 4.234596 & 1.897146 & -0.141300 & $\mathrm{C}$ & -7.703207 & -1.235559 & 0.657051 \\
\hline $\mathrm{C}$ & 6.227442 & 1.707851 & 2.594952 & $\mathrm{C}$ & -7.224824 & -0.054431 & -1.408878 \\
\hline $\mathrm{H}$ & 6.730190 & -0.115472 & 3.629354 & $\mathrm{C}$ & -8.651706 & -2.005249 & -0.014686 \\
\hline $\mathrm{H}$ & 5.534443 & 3.337735 & 1.358564 & $\mathrm{H}$ & -7.481062 & -1.398168 & 1.704534 \\
\hline $\mathrm{H}$ & 6.810165 & 2.342303 & 3.256841 & $\mathrm{C}$ & -8.173460 & -0.831075 & -2.064075 \\
\hline $\mathrm{C}$ & 3.982915 & -0.212468 & -1.845077 & $\mathrm{H}$ & -6.653658 & 0.699046 & -1.940831 \\
\hline $\mathrm{C}$ & 2.901294 & 0.115453 & -2.673469 & $\mathrm{C}$ & -8.898796 & -1.818801 & -1.378520 \\
\hline $\mathrm{C}$ & 5.285950 & 0.084378 & -2.274685 & $\mathrm{H}$ & -9.194656 & -2.775301 & 0.526865 \\
\hline $\mathrm{C}$ & 3.116842 & 0.698799 & -3.922591 & $\mathrm{H}$ & -8.353791 & -0.676252 & -3.125094 \\
\hline $\mathrm{H}$ & 1.889908 & -0.070703 & -2.321271 & $\mathrm{C}$ & -9.933616 & -2.647550 & -2.099159 \\
\hline $\mathrm{C}$ & 5.500797 & 0.663645 & -3.524118 & $\mathrm{H}$ & -10.144823 & -3.577610 & -1.561988 \\
\hline $\mathrm{H}$ & 6.128794 & -0.124686 & -1.623163 & $\mathrm{H}$ & -10.880896 & -2.100820 & -2.195858 \\
\hline \multicolumn{8}{|c|}{ S366 } \\
\hline
\end{tabular}




\begin{tabular}{|c|c|c|c|}
\hline $\mathrm{H}$ & -9.605022 & -2.905599 & -3.111963 \\
\hline $\mathrm{H}$ & -3.169921 & -0.690161 & 1.445304 \\
\hline $\mathrm{C}$ & -1.511556 & 2.189601 & -1.363092 \\
\hline $\mathrm{O}$ & -0.884241 & 1.057245 & -1.219715 \\
\hline $\mathrm{O}$ & -2.637284 & 2.433143 & -0.907353 \\
\hline $\mathrm{H}$ & -3.791981 & 1.099413 & -0.272803 \\
\hline $\mathrm{C}$ & -0.737590 & 3.219015 & -2.141005 \\
\hline $\mathrm{C}$ & -0.808420 & 4.615960 & -1.830860 \\
\hline $\mathrm{C}$ & 0.088742 & 2.778134 & -3.158063 \\
\hline $\mathrm{C}$ & -1.594189 & 5.145715 & -0.770344 \\
\hline $\mathrm{C}$ & 0.006573 & 5.522159 & -2.589591 \\
\hline $\mathrm{C}$ & 0.853679 & 3.679819 & -3.927354 \\
\hline $\mathrm{H}$ & 0.142073 & 1.713274 & -3.354191 \\
\hline $\mathrm{C}$ & -1.557643 & 6.488431 & -0.468382 \\
\hline $\mathrm{H}$ & -2.235953 & 4.474328 & -0.216692 \\
\hline $\mathrm{C}$ & 0.011018 & 6.902598 & -2.255929 \\
\hline $\mathrm{C}$ & 0.814738 & 5.025719 & -3.645166 \\
\hline $\mathrm{H}$ & 1.481881 & 3.305976 & -4.730626 \\
\hline $\mathrm{C}$ & -0.748288 & 7.378525 & -1.212721 \\
\hline $\mathrm{H}$ & -2.163381 & 6.869933 & 0.348733 \\
\hline $\mathrm{H}$ & 0.633241 & 7.575678 & -2.841038 \\
\hline $\mathrm{H}$ & 1.410885 & 5.730156 & -4.220363 \\
\hline $\mathrm{H}$ & -0.734203 & 8.435849 & -0.962842 \\
\hline
\end{tabular}

\section{TS3D'}

Zero-point correction= 1.129988 (Hartree/Particle)

Thermal correction to Energy= 1.202060

Thermal correction to Enthalpy $=1.203004$

Thermal correction to Gibbs Free Energy= 1.014543

$\mathrm{E}(\mathrm{Solv})=-4308.12164691$

$\begin{array}{llll}\mathrm{C} & 4.710704 & -4.601594 & 0.783909 \\ \mathrm{C} & 3.791714 & -3.721562 & 1.349322 \\ \mathrm{C} & 2.535942 & -3.506902 & 0.759327 \\ \mathrm{C} & 2.223388 & -4.212558 & -0.416652 \\ \mathrm{C} & 3.144862 & -5.083903 & -0.986710 \\ \mathrm{C} & 4.393696 & -5.283126 & -0.390737 \\ \mathrm{H} & 5.679362 & -4.740658 & 1.253057 \\ \mathrm{H} & 4.048010 & -3.180299 & 2.254761 \\ \mathrm{H} & 1.253760 & -4.090035 & -0.884513 \\ \mathrm{H} & 2.885100 & -5.614457 & -1.898699 \\ \mathrm{H} & 5.111496 & -5.963708 & -0.839707\end{array}$

\begin{tabular}{|c|c|c|}
\hline 1.600116 & -2.579099 & 1.409850 \\
\hline 1.945239 & -2.195347 & 2.371107 \\
\hline 0.224134 & -2.537824 & 1.145746 \\
\hline-0.141435 & -3.065584 & 0.277385 \\
\hline-0.712876 & -1.839123 & 1.944092 \\
\hline-2.085394 & -1.623500 & 1.683442 \\
\hline-2.595049 & -1.121358 & 2.503216 \\
\hline-0.317017 & -1.355990 & 2.836584 \\
\hline-1.873038 & -0.609187 & 0.699003 \\
\hline 1.235728 & -0.513734 & 0.547433 \\
\hline 1.001831 & 1.515004 & 1.904739 \\
\hline 3.034399 & -0.229407 & -0.893425 \\
\hline 2.689078 & 1.608101 & 2.627365 \\
\hline 3.675104 & 2.451388 & 2.093540 \\
\hline 3.061357 & 0.687441 & 3.623880 \\
\hline 4.986667 & 2.398510 & 2.568255 \\
\hline 3.425540 & 3.142999 & 1.297758 \\
\hline 4.368370 & 0.647166 & 4.107257 \\
\hline 2.320472 & 0.002887 & 4.026556 \\
\hline 5.337016 & 1.508196 & 3.583473 \\
\hline 5.734686 & 3.053864 & 2.131850 \\
\hline 4.631090 & -0.060918 & 4.888460 \\
\hline 6.356805 & 1.475633 & 3.956165 \\
\hline 0.689910 & 3.210813 & 1.254868 \\
\hline 1.134794 & 4.366421 & 1.912851 \\
\hline-0.099646 & 3.341302 & 0.107410 \\
\hline 0.828768 & 5.626674 & 1.402726 \\
\hline 1.722622 & 4.281005 & 2.821444 \\
\hline-0.422750 & 4.603059 & -0.395504 \\
\hline-0.452804 & 2.449208 & -0.392347 \\
\hline 0.051811 & 5.747054 & 0.246913 \\
\hline 1.187201 & 6.516333 & 1.913548 \\
\hline-1.037382 & 4.688264 & -1.285135 \\
\hline-0.190410 & 6.731172 & -0.145015 \\
\hline-0.132516 & 1.557113 & 3.359932 \\
\hline 0.255447 & 1.828392 & 4.678750 \\
\hline-1.491015 & 1.330220 & 3.084467 \\
\hline-0.695402 & 1.839966 & 5.701632 \\
\hline 1.294294 & 2.034848 & 4.912323 \\
\hline-2.440365 & 1.340661 & 4.105096 \\
\hline-1.806683 & 1.140301 & 2.065144 \\
\hline-2.03923 & 1.588660 & 5.420108 \\
\hline-0.381643 & 2.047936 & 6.721350 \\
\hline-3.477516 & 1.129455 & 3.864722 \\
\hline
\end{tabular}

(1) 


\begin{tabular}{|c|c|c|c|c|c|c|c|}
\hline $\mathrm{H}$ & -2.772611 & 1.587533 & 6.221617 & $\mathrm{H}$ & -0.730054 & -5.197921 & -3.158120 \\
\hline $\mathrm{C}$ & 3.575127 & 1.507149 & -1.180646 & $\mathrm{C}$ & -3.063016 & -2.583587 & 0.969322 \\
\hline $\mathrm{C}$ & 4.903626 & 1.936966 & -1.063664 & $\mathrm{~N}$ & -4.292286 & -1.864667 & 0.560415 \\
\hline $\mathrm{C}$ & 2.577784 & 2.448615 & -1.471376 & $\mathrm{~S}$ & -5.341044 & -1.427969 & 1.800609 \\
\hline $\mathrm{C}$ & 5.220616 & 3.288613 & -1.213038 & $\mathrm{O}$ & -5.573813 & -2.625061 & 2.620153 \\
\hline $\mathrm{H}$ & 5.687427 & 1.223170 & -0.837869 & $\mathrm{O}$ & -4.924395 & -0.176401 & 2.461675 \\
\hline $\mathrm{C}$ & 2.894093 & 3.796657 & -1.620763 & $\mathrm{C}$ & -6.798684 & -1.056625 & 0.842904 \\
\hline $\mathrm{H}$ & 1.547525 & 2.126599 & -1.561753 & $\mathrm{C}$ & -7.877057 & -1.935842 & 0.875719 \\
\hline $\mathrm{C}$ & 4.217842 & 4.221672 & -1.483049 & $\mathrm{C}$ & -6.831795 & 0.110340 & 0.076358 \\
\hline $\mathrm{H}$ & 6.253310 & 3.611744 & -1.111497 & $\mathrm{C}$ & -9.013024 & -1.636270 & 0.124746 \\
\hline $\mathrm{H}$ & 2.101339 & 4.512087 & -1.813760 & $\mathrm{H}$ & -7.816991 & -2.833541 & 1.480995 \\
\hline $\mathrm{H}$ & 4.465208 & 5.274797 & -1.583618 & $\mathrm{C}$ & -7.969705 & 0.384312 & -0.675491 \\
\hline $\mathrm{C}$ & 2.726342 & -0.819459 & -2.607070 & $\mathrm{H}$ & -5.976067 & 0.776281 & 0.056609 \\
\hline $\mathrm{C}$ & 1.731067 & -1.782037 & -2.818943 & $\mathrm{C}$ & -9.076630 & -0.477794 & -0.658802 \\
\hline $\mathrm{C}$ & 3.453235 & -0.334609 & -3.704565 & $\mathrm{H}$ & -9.862063 & -2.314608 & 0.145067 \\
\hline $\mathrm{C}$ & 1.471200 & -2.261248 & -4.102621 & $\mathrm{H}$ & -8.001767 & 1.283207 & -1.285957 \\
\hline $\mathrm{H}$ & 1.146568 & -2.134704 & -1.975969 & $\mathrm{C}$ & -10.315793 & -0.147013 & -1.453813 \\
\hline $\mathrm{C}$ & 3.192209 & -0.812708 & -4.988875 & $\mathrm{H}$ & -10.940466 & -1.032279 & -1.608216 \\
\hline $\mathrm{H}$ & 4.215819 & 0.422862 & -3.551268 & $\mathrm{H}$ & -10.927883 & 0.602430 & -0.935124 \\
\hline $\mathrm{C}$ & 2.201003 & -1.777511 & -5.189688 & $\mathrm{H}$ & -10.061243 & 0.266592 & -2.435740 \\
\hline $\mathrm{H}$ & 0.692642 & -3.003214 & -4.249776 & $\mathrm{H}$ & -3.394601 & -3.333083 & 1.696231 \\
\hline $\mathrm{H}$ & 3.757748 & -0.428721 & -5.833379 & $\mathrm{C}$ & -2.354715 & 0.628528 & -1.022125 \\
\hline $\mathrm{H}$ & 1.994223 & -2.143945 & -6.191243 & $\mathrm{O}$ & -1.475747 & 0.137496 & -0.195147 \\
\hline $\mathrm{C}$ & 4.613767 & -1.033186 & -0.425713 & $\mathrm{O}$ & -3.583178 & 0.513850 & -0.907944 \\
\hline $\mathrm{C}$ & 5.374095 & -1.822850 & -1.293934 & $\mathrm{H}$ & -4.078966 & -1.007992 & 0.026085 \\
\hline $\mathrm{C}$ & 5.086716 & -0.789953 & 0.872765 & $\mathrm{C}$ & -1.729107 & 1.335412 & -2.189605 \\
\hline $\mathrm{C}$ & 6.598554 & -2.345805 & -0.873708 & $\mathrm{C}$ & -2.233480 & 2.578218 & -2.685643 \\
\hline $\mathrm{H}$ & 5.013489 & -2.033176 & -2.294586 & $\mathrm{C}$ & -0.609906 & 0.758877 & -2.760237 \\
\hline $\mathrm{C}$ & 6.318218 & -1.294092 & 1.281879 & $\mathrm{C}$ & -3.359823 & 3.241881 & -2.126636 \\
\hline $\mathrm{H}$ & 4.489983 & -0.199269 & 1.556701 & $\mathrm{C}$ & -1.519677 & 3.217858 & -3.754052 \\
\hline C & 7.079268 & -2.074275 & 0.407564 & $\mathrm{C}$ & 0.051805 & 1.363247 & -3.850115 \\
\hline $\mathrm{H}$ & 7.177590 & -2.965704 & -1.552571 & $\mathrm{H}$ & -0.233053 & -0.168079 & -2.348906 \\
\hline $\mathrm{H}$ & 6.675363 & -1.080898 & 2.285390 & $\mathrm{C}$ & -3.749834 & 4.477786 & -2.591714 \\
\hline $\mathrm{H}$ & 8.037662 & -2.475086 & 0.726200 & $\mathrm{H}$ & -3.905934 & 2.755155 & -1.329003 \\
\hline $\mathrm{C}$ & -1.368061 & -5.298650 & -1.098574 & $\mathrm{C}$ & -1.953287 & 4.491682 & -4.208798 \\
\hline $\mathrm{C}$ & -2.004646 & -4.627066 & -0.053659 & $\mathrm{C}$ & -0.387994 & 2.577341 & -4.325166 \\
\hline $\mathrm{C}$ & -2.494559 & -3.327173 & -0.225146 & $\mathrm{H}$ & 0.917426 & 0.875107 & -4.284884 \\
\hline $\mathrm{C}$ & -2.382121 & -2.730023 & -1.486603 & $\mathrm{C}$ & -3.041277 & 5.112707 & -3.639717 \\
\hline $\mathrm{C}$ & -1.747785 & -3.397326 & -2.534372 & $\mathrm{H}$ & -4.608816 & 4.972985 & -2.148009 \\
\hline $\mathrm{C}$ & -1.225464 & -4.678829 & -2.341975 & $\mathrm{H}$ & -1.402510 & 4.969749 & -5.015063 \\
\hline $\mathrm{H}$ & -0.983165 & -6.302052 & -0.940462 & $\mathrm{H}$ & 0.129191 & 3.070285 & -5.144646 \\
\hline $\mathrm{H}$ & -2.097594 & -5.104881 & 0.918106 & $\mathrm{H}$ & -3.360098 & 6.089131 & -3.993353 \\
\hline $\mathrm{H}$ & -2.806599 & -1.750268 & -1.663563 & & & 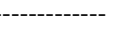 & \\
\hline
\end{tabular}


R1

Zero-point correction= $1.204057($ Hartree/Particle $)$

Thermal correction to Energy $=1.278912$

Thermal correction to Enthalpy=1.279856

Thermal correction to Gibbs Free Energy=1.089020

$\mathrm{E}($ Solv $)=-4250.70974153$

\begin{tabular}{|c|c|c|c|}
\hline $\mathrm{C}$ & 5.133611 & -1.287285 & -3.623733 \\
\hline $\mathrm{C}$ & 4.385791 & -1.801637 & -2.566896 \\
\hline $\mathrm{C}$ & 2.986279 & -1.860599 & -2.637082 \\
\hline $\mathrm{C}$ & 2.350481 & -1.400865 & -3.804862 \\
\hline $\mathrm{C}$ & 3.098105 & -0.892091 & -4.862652 \\
\hline $\mathrm{C}$ & 4.493131 & -0.828490 & -4.776318 \\
\hline $\mathrm{H}$ & 6.216104 & -1.238966 & -3.543754 \\
\hline $\mathrm{H}$ & 4.884729 & -2.153159 & -1.670047 \\
\hline $\mathrm{H}$ & 1.265929 & -1.414910 & -3.866982 \\
\hline $\mathrm{H}$ & 2.591937 & -0.536244 & -5.755868 \\
\hline $\mathrm{H}$ & 5.073568 & -0.425720 & -5.601741 \\
\hline $\mathrm{C}$ & 2.222217 & -2.339147 & -1.469227 \\
\hline $\mathrm{H}$ & 2.832956 & -2.579441 & -0.599876 \\
\hline $\mathrm{C}$ & 0.970142 & -3.024761 & -1.580909 \\
\hline $\mathrm{H}$ & 0.467536 & -3.098276 & -2.540519 \\
\hline $\mathrm{C}$ & 0.398042 & -3.693205 & -0.477368 \\
\hline $\mathrm{C}$ & -0.864658 & -4.295305 & -0.430057 \\
\hline $\mathrm{H}$ & -1.417405 & -4.418290 & -1.357288 \\
\hline $\mathrm{H}$ & 0.993531 & -3.699743 & 0.437938 \\
\hline $\mathrm{H}$ & -1.020295 & -5.066804 & 0.314877 \\
\hline $\mathrm{Pd}$ & 0.760478 & -0.933819 & -0.861480 \\
\hline $\mathrm{C}$ & -2.889269 & -4.490102 & 3.800572 \\
\hline $\mathrm{C}$ & -2.311015 & -3.707459 & 2.802422 \\
\hline $\mathrm{C}$ & -2.800210 & -3.752323 & 1.488943 \\
\hline $\mathrm{C}$ & -3.870464 & -4.608139 & 1.185117 \\
\hline $\mathrm{C}$ & -4.445941 & -5.388430 & 2.184671 \\
\hline $\mathrm{C}$ & -3.959631 & -5.332244 & 3.493959 \\
\hline $\mathrm{H}$ & -2.508770 & -4.438415 & 4.816797 \\
\hline $\mathrm{H}$ & -1.481127 & -3.045466 & 3.038399 \\
\hline $\mathrm{H}$ & -4.257188 & -4.646268 & 0.173100 \\
\hline $\mathrm{H}$ & -5.279867 & -6.040063 & 1.941209 \\
\hline $\mathrm{H}$ & -4.413870 & -5.940902 & 4.270355 \\
\hline $\mathrm{C}$ & -2.176639 & -2.881807 & 0.467586 \\
\hline $\mathrm{N}$ & -2.985172 & -2.360455 & -0.485647 \\
\hline S & -2.335975 & -1.160090 & -1.392463 \\
\hline
\end{tabular}

\begin{tabular}{|c|c|c|c|}
\hline $\mathrm{O}$ & -1.433283 & -0.296417 & -0.545448 \\
\hline $\mathrm{O}$ & -1.719835 & -1.683459 & -2.628909 \\
\hline $\mathrm{C}$ & -3.726578 & -0.158154 & -1.847854 \\
\hline $\mathrm{C}$ & -3.913984 & 1.079330 & -1.234699 \\
\hline $\mathrm{C}$ & -4.576449 & -0.608455 & -2.861659 \\
\hline $\mathrm{C}$ & -4.954937 & 1.893368 & -1.675114 \\
\hline $\mathrm{H}$ & -3.259536 & 1.399165 & -0.435986 \\
\hline $\mathrm{C}$ & -5.621270 & 0.212380 & -3.270331 \\
\hline $\mathrm{H}$ & -4.418604 & -1.578052 & -3.319590 \\
\hline $\mathrm{C}$ & -5.811402 & 1.481323 & -2.701637 \\
\hline $\mathrm{H}$ & -5.106881 & 2.855451 & -1.197230 \\
\hline $\mathrm{H}$ & -6.294123 & -0.131475 & -4.051004 \\
\hline $\mathrm{C}$ & -6.903212 & 2.386639 & -3.211093 \\
\hline $\mathrm{H}$ & -6.618848 & 2.834553 & -4.172378 \\
\hline $\mathrm{H}$ & -7.834736 & 1.833891 & -3.370593 \\
\hline $\mathrm{H}$ & -7.107101 & 3.196417 & -2.506253 \\
\hline $\mathrm{H}$ & -1.372558 & -2.252518 & 0.845713 \\
\hline $\mathrm{C}$ & -6.682752 & -2.015685 & -0.442673 \\
\hline $\mathrm{O}$ & -5.719961 & -2.857772 & -0.864995 \\
\hline $\mathrm{O}$ & -7.824807 & -2.163470 & -0.831516 \\
\hline $\mathrm{H}$ & -4.796060 & -2.568285 & -0.641911 \\
\hline $\mathrm{C}$ & -6.260221 & -0.943865 & 0.522052 \\
\hline $\mathrm{C}$ & -6.895246 & 0.342503 & 0.538887 \\
\hline $\mathrm{C}$ & -5.257824 & -1.224507 & 1.432444 \\
\hline $\mathrm{C}$ & -7.974292 & 0.700071 & -0.314490 \\
\hline $\mathrm{C}$ & -6.401006 & 1.330351 & 1.454765 \\
\hline $\mathrm{C}$ & -4.804588 & -0.260340 & 2.355748 \\
\hline $\mathrm{H}$ & -4.818090 & -2.211253 & 1.458366 \\
\hline $\mathrm{C}$ & -8.519440 & 1.963968 & -0.271118 \\
\hline $\mathrm{H}$ & -8.369611 & -0.049147 & -0.986216 \\
\hline $\mathrm{C}$ & -6.972476 & 2.630255 & 1.455332 \\
\hline $\mathrm{C}$ & -5.355211 & 0.998278 & 2.353720 \\
\hline $\mathrm{H}$ & -4.018431 & -0.524913 & 3.056905 \\
\hline $\mathrm{C}$ & -8.010076 & 2.946264 & 0.609937 \\
\hline $\mathrm{H}$ & -9.349613 & 2.211727 & -0.926549 \\
\hline $\mathrm{H}$ & -6.578837 & 3.368481 & 2.149693 \\
\hline $\mathrm{H}$ & -5.010259 & 1.756808 & 3.050730 \\
\hline $\mathrm{H}$ & -8.446550 & 3.941137 & 0.624639 \\
\hline $\mathrm{P}$ & 1.978501 & 0.892345 & -0.287335 \\
\hline $\mathrm{O}$ & 2.660450 & 0.825200 & 1.244717 \\
\hline $\mathrm{O}$ & 3.385569 & 0.873664 & -1.194493 \\
\hline $\mathrm{N}$ & 1.323298 & 2.429081 & -0.262681 \\
\hline $\mathrm{C}$ & 3.256716 & -0.406191 & 1.498509 \\
\hline $\mathrm{C}$ & 4.684902 & 1.087769 & -0.807787 \\
\hline
\end{tabular}




\begin{tabular}{|c|c|c|c|}
\hline $\mathrm{C}$ & 1.823009 & 3.679920 & 0.379519 \\
\hline $\mathrm{C}$ & 0.087346 & 2.568871 & -1.085884 \\
\hline $\mathrm{C}$ & 4.521670 & -0.643016 & 0.996013 \\
\hline $\mathrm{C}$ & 2.495987 & -1.358160 & 2.229312 \\
\hline $\mathrm{C}$ & 5.255265 & 0.430144 & 0.273120 \\
\hline $\mathrm{C}$ & 5.422176 & 1.962700 & -1.657388 \\
\hline $\mathrm{C}$ & 3.347515 & 3.751007 & 0.499506 \\
\hline $\mathrm{C}$ & 1.148682 & 3.977031 & 1.715027 \\
\hline $\mathrm{H}$ & 1.526442 & 4.482268 & -0.302834 \\
\hline $\mathrm{C}$ & 0.356260 & 3.349285 & -2.374574 \\
\hline $\mathrm{C}$ & -1.080270 & 3.063839 & -0.246067 \\
\hline $\mathrm{H}$ & -0.177814 & 1.550929 & -1.389627 \\
\hline C & 5.065900 & -1.966904 & 1.142906 \\
\hline C & 3.052513 & -2.601735 & 2.419742 \\
\hline $\mathrm{C}$ & 1.124311 & -0.997515 & 2.734982 \\
\hline $\mathrm{C}$ & 6.586799 & 0.806312 & 0.666762 \\
\hline $\mathrm{C}$ & 6.726018 & 2.240927 & -1.325720 \\
\hline $\mathrm{C}$ & 4.761741 & 2.517113 & -2.890858 \\
\hline $\mathrm{H}$ & 3.613938 & 4.719750 & 0.932986 \\
\hline $\mathrm{H}$ & 3.825112 & 3.669948 & -0.478083 \\
\hline $\mathrm{H}$ & 3.746499 & 2.970420 & 1.148237 \\
\hline $\mathrm{C}$ & 0.353390 & 5.119331 & 1.842524 \\
\hline $\mathrm{C}$ & 1.314608 & 3.145260 & 2.828628 \\
\hline $\mathrm{H}$ & -0.520343 & 3.312883 & -3.029190 \\
\hline $\mathrm{H}$ & 1.195923 & 2.889070 & -2.903834 \\
\hline $\mathrm{H}$ & 0.596716 & 4.402627 & -2.194995 \\
\hline $\mathrm{C}$ & -1.364270 & 2.405403 & 0.959146 \\
\hline $\mathrm{C}$ & -1.903953 & 4.118031 & -0.642546 \\
\hline $\mathrm{C}$ & 6.288948 & -2.369558 & 0.540764 \\
\hline C & 4.316460 & -2.946923 & 1.871429 \\
\hline $\mathrm{H}$ & 2.504829 & -3.356348 & 2.979697 \\
\hline $\mathrm{H}$ & 0.430628 & -0.853471 & 1.895804 \\
\hline $\mathrm{H}$ & 0.729848 & -1.792200 & 3.375617 \\
\hline $\mathrm{H}$ & 1.133498 & -0.061160 & 3.302404 \\
\hline $\mathrm{C}$ & 7.329125 & 1.713228 & -0.154242 \\
\hline $\mathrm{C}$ & 7.191743 & 0.338946 & 1.864386 \\
\hline $\mathrm{H}$ & 7.311998 & 2.900138 & -1.961764 \\
\hline $\mathrm{H}$ & 5.450285 & 3.167775 & -3.437804 \\
\hline $\mathrm{H}$ & 4.440292 & 1.705254 & -3.552505 \\
\hline $\mathrm{H}$ & 3.863187 & 3.093362 & -2.641783 \\
\hline $\mathrm{C}$ & -0.295660 & 5.411316 & 3.041517 \\
\hline $\mathrm{H}$ & 0.207666 & 5.766363 & 0.981923 \\
\hline $\mathrm{C}$ & 0.6686688 & 3.434717 & 4.029557 \\
\hline $\mathrm{H}$ & 1.934451 & 2.261650 & 2.744081 \\
\hline
\end{tabular}

\begin{tabular}{|c|c|c|c|}
\hline $\mathrm{C}$ & -2.429888 & 2.803413 & 1.755397 \\
\hline $\mathrm{H}$ & -0.730840 & 1.585141 & 1.273354 \\
\hline $\mathrm{C}$ & -2.985279 & 4.515993 & 0.151704 \\
\hline $\mathrm{H}$ & -1.713362 & 4.639676 & -1.574345 \\
\hline $\mathrm{C}$ & 6.757004 & -3.658047 & 0.674884 \\
\hline $\mathrm{H}$ & 6.851842 & -1.649661 & -0.039872 \\
\hline $\mathrm{C}$ & 4.836568 & -4.261184 & 2.005870 \\
\hline $\mathrm{C}$ & 8.648298 & 2.074414 & 0.223336 \\
\hline $\mathrm{C}$ & 8.469724 & 0.718816 & 2.211228 \\
\hline $\mathrm{H}$ & 6.629690 & -0.318702 & 2.516825 \\
\hline $\mathrm{C}$ & -0.144980 & 4.564411 & 4.139476 \\
\hline $\mathrm{H}$ & -0.927916 & 6.291800 & 3.111965 \\
\hline $\mathrm{H}$ & 0.801014 & 2.775250 & 4.883596 \\
\hline $\mathrm{C}$ & -3.249337 & 3.863080 & 1.352755 \\
\hline $\mathrm{H}$ & -2.621256 & 2.288566 & 2.690829 \\
\hline $\mathrm{H}$ & -3.616114 & 5.339194 & -0.172605 \\
\hline $\mathrm{C}$ & 6.032059 & -4.613561 & 1.423463 \\
\hline $\mathrm{H}$ & 7.689776 & -3.942958 & 0.197195 \\
\hline $\mathrm{H}$ & 4.260186 & -4.990376 & 2.569542 \\
\hline $\mathrm{C}$ & 9.213914 & 1.586108 & 1.379123 \\
\hline $\mathrm{H}$ & 9.201091 & 2.756888 & -0.417411 \\
\hline $\mathrm{H}$ & 8.906623 & 0.353031 & 3.136026 \\
\hline $\mathrm{H}$ & -0.654507 & 4.783511 & 5.073753 \\
\hline $\mathrm{H}$ & -4.086351 & 4.173771 & 1.970946 \\
\hline $\mathrm{H}$ & 6.414327 & -5.624771 & 1.526375 \\
\hline $\mathrm{H}$ & 10.222725 & 1.874057 & 1.660118 \\
\hline
\end{tabular}

$\mathbf{R 2}$
Zero-point correction=1.198741 (Hartree/Particle)
Thermal correction to Energy=1.274897
Thermal correction to Enthalpy=1.275842
Thermal correction to Gibbs Free Energy=1.080394
$\mathrm{E}($ Solv $)=-4250.69991721$

$\begin{array}{llll}\mathrm{C} & -4.692605 & -3.356316 & 1.950821 \\ \mathrm{C} & -3.590563 & -3.242800 & 1.104529 \\ \mathrm{C} & -2.331521 & -2.884279 & 1.604711 \\ \mathrm{C} & -2.202936 & -2.649010 & 2.984410 \\ \mathrm{C} & -3.300007 & -2.769573 & 3.831517 \\ \mathrm{C} & -4.553751 & -3.122491 & 3.319397 \\ \mathrm{H} & -5.661999 & -3.621656 & 1.537765 \\ \mathrm{H} & -3.704529 & -3.421880 & 0.040353 \\ & & & \end{array}$




\begin{tabular}{|c|c|c|c|}
\hline $\mathrm{H}$ & -1.241111 & -2.343667 & 3.383683 \\
\hline $\mathrm{H}$ & -3.182212 & -2.579693 & 4.895690 \\
\hline $\mathrm{H}$ & -5.411198 & -3.209237 & 3.981055 \\
\hline $\mathrm{C}$ & -1.189682 & -2.755896 & 0.671741 \\
\hline $\mathrm{H}$ & -1.465503 & -2.846733 & -0.380062 \\
\hline $\mathrm{C}$ & 0.125675 & -3.138508 & 1.033559 \\
\hline $\mathrm{H}$ & 0.292409 & -3.411157 & 2.073390 \\
\hline $\mathrm{C}$ & 1.114726 & -3.630374 & 0.103076 \\
\hline $\mathrm{C}$ & 2.112853 & -4.479202 & 0.443758 \\
\hline $\mathrm{H}$ & 2.149111 & -4.925928 & 1.433303 \\
\hline $\mathrm{H}$ & 1.041409 & -3.286585 & -0.928888 \\
\hline $\mathrm{H}$ & 2.849320 & -4.817226 & -0.277006 \\
\hline $\mathrm{Pd}$ & -0.178527 & -0.904503 & 0.785932 \\
\hline $\mathrm{C}$ & 1.478621 & -2.849040 & 4.887483 \\
\hline $\mathrm{C}$ & 2.393963 & -3.210212 & 3.909378 \\
\hline $\mathrm{C}$ & 2.746172 & -2.303402 & 2.887475 \\
\hline $\mathrm{C}$ & 2.144976 & -1.028762 & 2.856291 \\
\hline $\mathrm{C}$ & 1.248495 & -0.664375 & 3.861352 \\
\hline $\mathrm{C}$ & 0.912029 & -1.567161 & 4.869732 \\
\hline $\mathrm{H}$ & 1.210470 & -3.553973 & 5.668046 \\
\hline $\mathrm{H}$ & 2.845073 & -4.198722 & 3.921675 \\
\hline $\mathrm{H}$ & 2.388294 & -0.317050 & 2.077944 \\
\hline $\mathrm{H}$ & 0.819145 & 0.330465 & 3.853780 \\
\hline $\mathrm{H}$ & 0.206400 & -1.276771 & 5.642730 \\
\hline $\mathrm{C}$ & 3.715106 & -2.737752 & 1.913249 \\
\hline $\mathrm{N}$ & 4.206659 & -1.989809 & 0.960517 \\
\hline S & 5.294364 & -2.748883 & -0.164057 \\
\hline $\mathrm{O}$ & 5.695364 & -4.031980 & 0.422399 \\
\hline $\mathrm{O}$ & 4.627576 & -2.687538 & -1.464362 \\
\hline $\mathrm{C}$ & 6.672483 & -1.632078 & -0.138419 \\
\hline $\mathrm{C}$ & 7.770618 & -1.940383 & 0.666194 \\
\hline $\mathrm{C}$ & 6.631049 & -0.471452 & -0.914405 \\
\hline $\mathrm{C}$ & 8.847755 & -1.059132 & 0.692503 \\
\hline $\mathrm{H}$ & 7.777442 & -2.856005 & 1.246730 \\
\hline $\mathrm{C}$ & 7.718707 & 0.394211 & -0.868202 \\
\hline $\mathrm{H}$ & 5.768574 & -0.246164 & -1.527145 \\
\hline $\mathrm{C}$ & 8.838444 & 0.116861 & -0.070723 \\
\hline $\mathrm{H}$ & 9.710015 & -1.287763 & 1.312792 \\
\hline $\mathrm{H}$ & 7.695136 & 1.302912 & -1.463328 \\
\hline $\mathrm{C}$ & 10.019585 & 1.054168 & -0.057917 \\
\hline $\mathrm{H}$ & 10.673541 & 0.867935 & -0.919742 \\
\hline $\mathrm{H}$ & 9.698732 & 2.099469 & -0.114039 \\
\hline $\mathrm{H}$ & 10.622634 & 0.927607 & 0.846333 \\
\hline $\mathrm{H}$ & 4.157600 & -3.723204 & 2.039948 \\
\hline
\end{tabular}

\begin{tabular}{|c|c|c|c|}
\hline $\mathrm{C}$ & 2.638121 & 0.831939 & -0.357605 \\
\hline $\mathrm{O}$ & 1.552245 & 0.750730 & 0.260075 \\
\hline $\mathrm{O}$ & 3.707492 & 0.176528 & -0.088087 \\
\hline $\mathrm{H}$ & 3.812216 & -0.922606 & 0.567372 \\
\hline $\mathrm{C}$ & 2.792001 & 1.810728 & -1.493462 \\
\hline $\mathrm{C}$ & 3.402040 & 1.413177 & -2.728003 \\
\hline C & 2.376867 & 3.114767 & -1.317870 \\
\hline $\mathrm{C}$ & 3.746659 & 0.065721 & -3.024665 \\
\hline $\mathrm{C}$ & 3.620852 & 2.408015 & -3.736754 \\
\hline $\mathrm{C}$ & 2.624503 & 4.096369 & -2.304420 \\
\hline $\mathrm{H}$ & 1.859109 & 3.397965 & -0.410618 \\
\hline $\mathrm{C}$ & 4.309647 & -0.272076 & -4.235975 \\
\hline $\mathrm{H}$ & 3.572105 & -0.706489 & -2.287665 \\
\hline $\mathrm{C}$ & 4.208201 & 2.025709 & -4.972263 \\
\hline $\mathrm{C}$ & 3.240700 & 3.752885 & -3.483839 \\
\hline $\mathrm{H}$ & 2.317838 & 5.119917 & -2.117023 \\
\hline $\mathrm{C}$ & 4.551547 & 0.716215 & -5.218949 \\
\hline $\mathrm{H}$ & 4.569300 & -1.308740 & -4.429412 \\
\hline $\mathrm{H}$ & 4.373903 & 2.791906 & -5.725809 \\
\hline $\mathrm{H}$ & 3.434601 & 4.502376 & -4.247317 \\
\hline $\mathrm{H}$ & 4.998564 & 0.437362 & -6.169038 \\
\hline $\mathrm{P}$ & -1.889133 & 0.607383 & 0.512368 \\
\hline $\mathrm{O}$ & -2.287575 & 1.038732 & -1.061394 \\
\hline $\mathrm{O}$ & -3.339758 & -0.139945 & 0.944296 \\
\hline $\mathrm{N}$ & -1.853878 & 2.102553 & 1.273563 \\
\hline $\mathrm{C}$ & -2.365143 & -0.092963 & -1.877369 \\
\hline $\mathrm{C}$ & -4.554662 & -0.112618 & 0.311835 \\
\hline $\mathrm{C}$ & -2.605493 & 3.354306 & 1.003249 \\
\hline $\mathrm{C}$ & -1.140128 & 2.043779 & 2.582132 \\
\hline $\mathrm{C}$ & -3.509556 & -0.866193 & -1.827163 \\
\hline $\mathrm{C}$ & -1.234621 & -0.400608 & -2.681165 \\
\hline $\mathrm{C}$ & -4.683043 & -0.417348 & -1.034734 \\
\hline $\mathrm{C}$ & -5.678708 & 0.157744 & 1.146939 \\
\hline $\mathrm{C}$ & -3.974833 & 3.149522 & 0.344195 \\
\hline $\mathrm{C}$ & -1.774757 & 4.359758 & 0.215642 \\
\hline $\mathrm{H}$ & -2.796325 & 3.798323 & 1.985945 \\
\hline $\mathrm{C}$ & -2.096386 & 1.691216 & 3.725865 \\
\hline $\mathrm{C}$ & -0.292305 & 3.280572 & 2.816298 \\
\hline $\mathrm{H}$ & -0.445578 & 1.203884 & 2.476511 \\
\hline $\mathrm{C}$ & -3.506497 & -2.123053 & -2.524689 \\
\hline $\mathrm{C}$ & -1.263083 & -1.581010 & -3.387925 \\
\hline $\mathrm{C}$ & -0.072752 & 0.549252 & -2.736498 \\
\hline $\mathrm{C}$ & -5.977038 & -0.287976 & -1.646803 \\
\hline $\mathrm{C}$ & -6.924911 & 0.191281 & 0.568585 \\
\hline
\end{tabular}




\begin{tabular}{|c|c|c|c|}
\hline $\mathrm{C}$ & -5.474179 & 0.377876 & 2.620463 \\
\hline $\mathrm{H}$ & -4.452125 & 4.128050 & 0.231308 \\
\hline $\mathrm{H}$ & -4.621677 & 2.520344 & 0.957863 \\
\hline $\mathrm{H}$ & -3.887379 & 2.697514 & -0.643995 \\
\hline $\mathrm{C}$ & -1.599979 & 5.655821 & 0.708725 \\
\hline $\mathrm{C}$ & -1.229304 & 4.032037 & -1.030679 \\
\hline $\mathrm{H}$ & -1.564389 & 1.675219 & 4.684153 \\
\hline $\mathrm{H}$ & -2.530047 & 0.703533 & 3.547795 \\
\hline $\mathrm{H}$ & -2.919113 & 2.409457 & 3.811199 \\
\hline $\mathrm{C}$ & 0.891015 & 3.414742 & 2.079938 \\
\hline $\mathrm{C}$ & -0.646853 & 4.287695 & 3.716904 \\
\hline $\mathrm{C}$ & -4.567514 & -3.063274 & -2.420413 \\
\hline C & -2.360376 & -2.477821 & -3.306383 \\
\hline $\mathrm{H}$ & -0.412882 & -1.851386 & -4.009569 \\
\hline $\mathrm{H}$ & 0.316852 & 0.751623 & -1.736999 \\
\hline $\mathrm{H}$ & 0.736661 & 0.139556 & -3.346013 \\
\hline $\mathrm{H}$ & -0.368147 & 1.514772 & -3.165374 \\
\hline $\mathrm{C}$ & -7.111131 & 0.005436 & -0.825503 \\
\hline $\mathrm{C}$ & -6.179179 & -0.410552 & -3.048081 \\
\hline $\mathrm{H}$ & -7.796801 & 0.387163 & 1.188508 \\
\hline $\mathrm{H}$ & -6.435182 & 0.514319 & 3.125544 \\
\hline $\mathrm{H}$ & -4.955066 & -0.473395 & 3.071414 \\
\hline $\mathrm{H}$ & -4.857491 & 1.262921 & 2.810260 \\
\hline $\mathrm{C}$ & -0.886164 & 6.608152 & -0.019302 \\
\hline $\mathrm{H}$ & -2.004242 & 5.915599 & 1.682613 \\
\hline $\mathrm{C}$ & -0.534914 & 4.987323 & -1.770096 \\
\hline $\mathrm{H}$ & -1.350566 & 3.028974 & -1.419683 \\
\hline C & 1.687224 & 4.548294 & 2.215789 \\
\hline $\mathrm{H}$ & 1.164735 & 2.617315 & 1.396623 \\
\hline C & 0.151352 & 5.426301 & 3.857056 \\
\hline $\mathrm{H}$ & -1.552105 & 4.196209 & 4.309458 \\
\hline C & -4.506851 & -4.277419 & -3.068185 \\
\hline $\mathrm{H}$ & -5.428565 & -2.818464 & -1.810792 \\
\hline $\mathrm{C}$ & -2.337159 & -3.730679 & -3.974399 \\
\hline $\mathrm{C}$ & -8.393808 & 0.116980 & -1.421778 \\
\hline C & -7.435387 & -0.284584 & -3.599292 \\
\hline $\mathrm{H}$ & -5.325837 & -0.597491 & -3.688955 \\
\hline C & -0.354674 & 6.277605 & -1.265943 \\
\hline $\mathrm{H}$ & -0.745650 & 7.605195 & 0.389237 \\
\hline $\mathrm{H}$ & -0.123649 & 4.717306 & -2.737795 \\
\hline $\mathrm{C}$ & 1.314476 & 5.563688 & 3.101025 \\
\hline $\mathrm{H}$ & 2.597580 & 4.641461 & 1.629766 \\
\hline $\mathrm{H}$ & -0.139918 & 6.205667 & 4.556141 \\
\hline $\mathrm{C}$ & -3.385684 & -4.614363 & -3.861445 \\
\hline
\end{tabular}

$\begin{array}{lrrr}\mathrm{H} & -5.325816 & -4.983419 & -2.964942 \\ \mathrm{H} & -1.463095 & -3.982685 & -4.569567 \\ \mathrm{C} & -8.559212 & -0.029143 & -2.780281 \\ \mathrm{H} & -9.245386 & 0.331509 & -0.780639 \\ \mathrm{H} & -7.561991 & -0.376576 & -4.674308 \\ \mathrm{H} & 0.193879 & 7.019005 & -1.841275 \\ \mathrm{H} & 1.931921 & 6.451449 & 3.205879 \\ \mathrm{H} & -3.350932 & -5.573079 & -4.370678 \\ \mathrm{H} & -9.545532 & 0.062954 & -3.225902\end{array}$

$\mathbf{R 3}$
Zero-point correction=1.203122 (Hartree/Particle)
Thermal correction to Energy=1.278892
Thermal correction to Enthalpy=1.279836
Thermal correction to Gibbs Free Energy=1.083447
$\mathrm{E}(\mathrm{Solv})=-4250.68120657$

\begin{tabular}{|c|c|c|c|}
\hline $\mathrm{C}$ & -2.441479 & -5.445192 & -2.219424 \\
\hline $\mathrm{C}$ & -1.922172 & -4.294493 & -2.806816 \\
\hline $\mathrm{C}$ & -0.898958 & -3.561147 & -2.179663 \\
\hline $\mathrm{C}$ & -0.407284 & -4.011283 & -0.937142 \\
\hline C & -0.927248 & -5.163613 & -0.356389 \\
\hline $\mathrm{C}$ & -1.943388 & -5.886071 & -0.991391 \\
\hline $\mathrm{H}$ & -3.235117 & -5.995486 & -2.716022 \\
\hline $\mathrm{H}$ & -2.312112 & -3.948494 & -3.760630 \\
\hline $\mathrm{H}$ & 0.372478 & -3.458641 & -0.424003 \\
\hline $\mathrm{H}$ & -0.543362 & -5.495960 & 0.604001 \\
\hline $\mathrm{H}$ & -2.346815 & -6.782744 & -0.529720 \\
\hline C & -0.400354 & -2.344850 & -2.824254 \\
\hline $\mathrm{H}$ & -0.825330 & -2.121682 & -3.802658 \\
\hline C & 0.739264 & -1.634514 & -2.412052 \\
\hline $\mathrm{H}$ & 1.304964 & -1.952023 & -1.542324 \\
\hline C & 1.020640 & -0.349568 & -2.953551 \\
\hline C & 1.887378 & 0.562971 & -2.292373 \\
\hline $\mathrm{H}$ & 1.834930 & 1.603557 & -2.601440 \\
\hline $\mathrm{H}$ & 0.683739 & -0.125902 & -3.966027 \\
\hline $\mathrm{H}$ & 1.948994 & 0.437599 & -1.217591 \\
\hline C & 3.618924 & -2.186503 & -5.584648 \\
\hline C & 3.662961 & -1.028608 & -4.811766 \\
\hline C & 3.803574 & -1.100425 & -3.418658 \\
\hline $\mathrm{C}$ & 3.904518 & -2.358176 & -2.812537 \\
\hline $\mathrm{C}$ & 3.859312 & -3.518158 & -3.586067 \\
\hline
\end{tabular}




\begin{tabular}{|c|c|c|c|}
\hline $\mathrm{C}$ & 3.715177 & -3.438902 & -4.971864 \\
\hline $\mathrm{H}$ & 3.517020 & -2.112721 & -6.663863 \\
\hline $\mathrm{H}$ & 3.585701 & -0.053990 & -5.288566 \\
\hline $\mathrm{H}$ & 4.000609 & -2.431414 & -1.737489 \\
\hline $\mathrm{H}$ & 3.933816 & -4.487257 & -3.100704 \\
\hline $\mathrm{H}$ & 3.680740 & -4.344277 & -5.571230 \\
\hline C & 3.785125 & 0.163664 & -2.634976 \\
\hline $\mathrm{N}$ & 4.409077 & 0.148095 & -1.419564 \\
\hline S & 4.604539 & 1.627756 & -0.702988 \\
\hline $\mathrm{O}$ & 4.369132 & 2.739436 & -1.649475 \\
\hline $\mathrm{O}$ & 3.836124 & 1.631775 & 0.564051 \\
\hline $\mathrm{C}$ & 6.331371 & 1.622511 & -0.255993 \\
\hline C & 7.218246 & 2.428497 & -0.965159 \\
\hline C & 6.770132 & 0.811451 & 0.794631 \\
\hline $\mathrm{C}$ & 8.568436 & 2.419551 & -0.616018 \\
\hline $\mathrm{H}$ & 6.845925 & 3.053928 & -1.768847 \\
\hline C & 8.121296 & 0.812990 & 1.123369 \\
\hline $\mathrm{H}$ & 6.068099 & 0.188362 & 1.337018 \\
\hline $\mathrm{C}$ & 9.039390 & 1.615606 & 0.428186 \\
\hline $\mathrm{H}$ & 9.266422 & 3.048340 & -1.162714 \\
\hline $\mathrm{H}$ & 8.471880 & 0.182362 & 1.936639 \\
\hline C & 10.497192 & 1.621145 & 0.817382 \\
\hline $\mathrm{H}$ & 11.112853 & 2.110177 & 0.056313 \\
\hline $\mathrm{H}$ & 10.650927 & 2.156531 & 1.763211 \\
\hline $\mathrm{H}$ & 10.875853 & 0.602498 & 0.958516 \\
\hline $\mathrm{H}$ & 3.942415 & 1.056413 & -3.242968 \\
\hline C & 3.147707 & -2.053672 & 1.041313 \\
\hline $\mathrm{O}$ & 2.304533 & -2.375350 & 0.207991 \\
\hline $\mathrm{O}$ & 4.253568 & -1.388312 & 0.757841 \\
\hline $\mathrm{H}$ & 4.235576 & -0.947146 & -0.167787 \\
\hline $\mathrm{C}$ & 3.031570 & -2.446653 & 2.477988 \\
\hline $\mathrm{C}$ & 3.321763 & -1.545148 & 3.552032 \\
\hline C & 2.605993 & -3.735752 & 2.738208 \\
\hline C & 3.678641 & -0.181117 & 3.360124 \\
\hline C & 3.188329 & -2.035026 & 4.895094 \\
\hline C & 2.495169 & -4.213364 & 4.060779 \\
\hline $\mathrm{H}$ & 2.372293 & -4.385991 & 1.901045 \\
\hline $\mathrm{C}$ & 3.905643 & 0.641804 & 4.440583 \\
\hline $\mathrm{H}$ & 3.766187 & 0.221785 & 2.359650 \\
\hline C & 3.440189 & -1.157288 & 5.983164 \\
\hline C & 2.788329 & -3.379238 & 5.115654 \\
\hline $\mathrm{H}$ & 2.178347 & -5.236465 & 4.239506 \\
\hline $\mathrm{C}$ & 3.793413 & 0.153497 & 5.763759 \\
\hline $\mathrm{H}$ & 4.169908 & 1.680955 & 4.267873 \\
\hline
\end{tabular}

\begin{tabular}{|c|c|c|c|}
\hline $\mathrm{H}$ & 3.338284 & -1.542565 & 6.994664 \\
\hline $\mathrm{H}$ & 2.703853 & -3.734733 & 6.139561 \\
\hline $\mathrm{H}$ & 3.978578 & 0.817635 & 6.603271 \\
\hline $\mathrm{Pd}$ & -0.928758 & -0.382186 & -1.781468 \\
\hline $\mathrm{P}$ & -2.796265 & 0.217646 & -0.582143 \\
\hline $\mathrm{O}$ & -2.328423 & 1.054708 & 0.764820 \\
\hline $\mathrm{O}$ & -3.685183 & 1.389024 & -1.382515 \\
\hline $\mathrm{N}$ & -3.889919 & -0.871893 & 0.100213 \\
\hline $\mathrm{C}$ & -1.434059 & 2.083205 & 0.423457 \\
\hline $\mathrm{C}$ & -4.252684 & 2.478400 & -0.733440 \\
\hline $\mathrm{C}$ & -4.095001 & -0.942278 & 1.578715 \\
\hline $\mathrm{C}$ & -4.126883 & -2.106197 & -0.723800 \\
\hline $\mathrm{C}$ & -1.963297 & 3.245800 & -0.108997 \\
\hline $\mathrm{C}$ & -0.048380 & 1.853989 & 0.622406 \\
\hline $\mathrm{C}$ & -3.440447 & 3.421228 & -0.123851 \\
\hline $\mathrm{C}$ & -5.668093 & 2.587798 & -0.788524 \\
\hline $\mathrm{C}$ & -4.907698 & 0.257016 & 2.075168 \\
\hline $\mathrm{C}$ & -2.810039 & -1.239163 & 2.351422 \\
\hline $\mathrm{H}$ & -4.735692 & -1.815447 & 1.713799 \\
\hline $\mathrm{C}$ & -4.481348 & -1.760545 & -2.179149 \\
\hline $\mathrm{C}$ & -5.219337 & -2.970531 & -0.123427 \\
\hline $\mathrm{H}$ & -3.210246 & -2.706609 & -0.752261 \\
\hline $\mathrm{C}$ & -1.053385 & 4.223879 & -0.628998 \\
\hline $\mathrm{C}$ & 0.817745 & 2.828953 & 0.175420 \\
\hline $\mathrm{C}$ & 0.440895 & 0.597523 & 1.290469 \\
\hline C & -4.065004 & 4.523437 & 0.552810 \\
\hline $\mathrm{C}$ & -6.253700 & 3.679998 & -0.191306 \\
\hline $\mathrm{C}$ & -6.472762 & 1.522823 & -1.481770 \\
\hline $\mathrm{H}$ & -5.236081 & 0.092748 & 3.106518 \\
\hline $\mathrm{H}$ & -5.799570 & 0.365848 & 1.452439 \\
\hline $\mathrm{H}$ & -4.340323 & 1.187952 & 2.032079 \\
\hline $\mathrm{C}$ & -2.525131 & -0.639934 & 3.582248 \\
\hline $\mathrm{C}$ & -1.899397 & -2.179554 & 1.848403 \\
\hline $\mathrm{H}$ & -4.666540 & -2.691494 & -2.723432 \\
\hline $\mathrm{H}$ & -3.664556 & -1.235166 & -2.681586 \\
\hline $\mathrm{H}$ & -5.376221 & -1.136978 & -2.234408 \\
\hline $\mathrm{C}$ & -4.908617 & -4.240268 & 0.371705 \\
\hline $\mathrm{C}$ & -6.549102 & -2.530358 & -0.069979 \\
\hline $\mathrm{C}$ & -1.483922 & 5.384575 & -1.325590 \\
\hline $\mathrm{C}$ & 0.353996 & 3.990744 & -0.492287 \\
\hline $\mathrm{H}$ & 1.885967 & 2.686874 & 0.303669 \\
\hline $\mathrm{H}$ & 0.214771 & -0.294862 & 0.693718 \\
\hline $\mathrm{H}$ & 1.522275 & 0.646609 & 1.430356 \\
\hline $\mathrm{F}$ & -0.039673 & 0.4446 & 2.26 \\
\hline
\end{tabular}




\begin{tabular}{|c|c|c|c|}
\hline $\mathrm{C}$ & -5.489634 & 4.656843 & 0.496675 \\
\hline $\mathrm{C}$ & -3.330222 & 5.474532 & 1.311517 \\
\hline $\mathrm{H}$ & -7.334643 & 3.794046 & -0.225854 \\
\hline $\mathrm{H}$ & -7.538797 & 1.767123 & -1.463779 \\
\hline $\mathrm{H}$ & -6.158788 & 1.398976 & -2.524298 \\
\hline $\mathrm{H}$ & -6.327524 & 0.554429 & -0.991540 \\
\hline $\mathrm{C}$ & -1.364891 & -0.969264 & 4.288088 \\
\hline $\mathrm{H}$ & -3.198521 & 0.104391 & 3.992575 \\
\hline $\mathrm{C}$ & -0.739747 & -2.505868 & 2.547063 \\
\hline $\mathrm{H}$ & -2.077441 & -2.652900 & 0.890906 \\
\hline C & -5.905104 & -5.059885 & 0.905711 \\
\hline $\mathrm{H}$ & -3.879647 & -4.583588 & 0.340635 \\
\hline $\mathrm{C}$ & -7.544293 & -3.343612 & 0.467328 \\
\hline $\mathrm{H}$ & -6.802321 & -1.541359 & -0.439118 \\
\hline C & -0.568429 & 6.269502 & -1.851106 \\
\hline $\mathrm{H}$ & -2.546153 & 5.560380 & -1.451601 \\
\hline $\mathrm{C}$ & 1.272493 & 4.921304 & -1.048569 \\
\hline C & -6.112077 & 5.751208 & 1.152572 \\
\hline C & -3.965308 & 6.519287 & 1.945203 \\
\hline $\mathrm{H}$ & -2.255457 & 5.367860 & 1.391645 \\
\hline C & -0.465204 & -1.903328 & 3.775555 \\
\hline $\mathrm{H}$ & -1.159250 & -0.480869 & 5.236905 \\
\hline $\mathrm{H}$ & -0.036091 & -3.213518 & 2.122209 \\
\hline $\mathrm{C}$ & -7.225342 & -4.613993 & 0.955145 \\
\hline $\mathrm{H}$ & -5.646654 & -6.043944 & 1.286941 \\
\hline $\mathrm{H}$ & -8.569905 & -2.987271 & 0.506273 \\
\hline C & 0.821430 & 6.039015 & -1.712842 \\
\hline $\mathrm{H}$ & -0.916953 & 7.147688 & -2.387594 \\
\hline $\mathrm{H}$ & 2.335064 & 4.710307 & -0.961106 \\
\hline C & -5.369070 & 6.668840 & 1.858646 \\
\hline $\mathrm{H}$ & -7.193697 & 5.843503 & 1.092409 \\
\hline $\mathrm{H}$ & -3.382342 & 7.232346 & 2.521040 \\
\hline $\mathrm{H}$ & 0.441973 & -2.150207 & 4.315084 \\
\hline $\mathrm{H}$ & -8.001799 & -5.248101 & 1.373514 \\
\hline $\mathrm{H}$ & 1.529890 & 6.741374 & -2.142780 \\
\hline $\mathrm{H}$ & -5.857085 & 7.500348 & 2.358962 \\
\hline
\end{tabular}

$\mathrm{E}($ Solv $)=-4250.67832011$

\begin{tabular}{|c|c|c|c|}
\hline $\mathrm{C}$ & 1.608915 & 1.532258 & 4.892391 \\
\hline $\mathrm{C}$ & 1.440993 & 2.041790 & 3.606969 \\
\hline $\mathrm{C}$ & 0.214039 & 1.913712 & 2.931246 \\
\hline $\mathrm{C}$ & -0.838971 & 1.258455 & 3.592397 \\
\hline $\mathrm{C}$ & -0.666751 & 0.742018 & 4.875197 \\
\hline $\mathrm{C}$ & 0.557209 & 0.872398 & 5.534151 \\
\hline $\mathrm{H}$ & 2.563138 & 1.654005 & 5.398216 \\
\hline $\mathrm{H}$ & 2.269985 & 2.529645 & 3.102193 \\
\hline $\mathrm{H}$ & -1.811671 & 1.196744 & 3.122658 \\
\hline $\mathrm{H}$ & -1.501882 & 0.249472 & 5.366726 \\
\hline $\mathrm{H}$ & 0.688368 & 0.475158 & 6.536659 \\
\hline $\mathrm{C}$ & 0.075245 & 2.484643 & 1.575748 \\
\hline $\mathrm{H}$ & 0.780953 & 3.287467 & 1.359609 \\
\hline $\mathrm{C}$ & -1.109265 & 2.447036 & 0.783255 \\
\hline $\mathrm{H}$ & -1.919441 & 1.775656 & 1.048284 \\
\hline $\mathrm{C}$ & -1.364182 & 3.386297 & -0.274920 \\
\hline $\mathrm{C}$ & -2.204182 & 3.192243 & -1.339018 \\
\hline $\mathrm{H}$ & -2.361333 & 3.979967 & -2.069449 \\
\hline $\mathrm{H}$ & -0.854644 & 4.349622 & -0.200556 \\
\hline $\mathrm{H}$ & -2.462086 & 2.191467 & -1.647016 \\
\hline $\mathrm{Pd}$ & 0.301772 & 0.983416 & 0.068105 \\
\hline $\mathrm{C}$ & -6.221011 & 6.010736 & -0.804165 \\
\hline $\mathrm{C}$ & -5.730121 & 4.749838 & -1.119856 \\
\hline $\mathrm{C}$ & -5.051657 & 3.983841 & -0.151000 \\
\hline $\mathrm{C}$ & -4.837580 & 4.526531 & 1.131853 \\
\hline $\mathrm{C}$ & -5.320727 & 5.792901 & 1.434722 \\
\hline $\mathrm{C}$ & -6.019207 & 6.533955 & 0.475985 \\
\hline $\mathrm{H}$ & -6.754991 & 6.587417 & -1.553084 \\
\hline $\mathrm{H}$ & -5.877813 & 4.339950 & -2.115379 \\
\hline $\mathrm{H}$ & -4.266723 & 3.975738 & 1.869411 \\
\hline $\mathrm{H}$ & -5.146622 & 6.208217 & 2.422367 \\
\hline $\mathrm{H}$ & -6.395304 & 7.522348 & 0.723035 \\
\hline $\mathrm{C}$ & -4.564262 & 2.682839 & -0.554327 \\
\hline $\mathrm{N}$ & -4.393619 & 1.605427 & 0.213045 \\
\hline $\mathrm{S}$ & -4.949510 & 1.351041 & 1.847318 \\
\hline $\mathrm{O}$ & -3.928714 & 1.844910 & 2.773857 \\
\hline $\mathrm{O}$ & -6.316742 & 1.862721 & 1.910684 \\
\hline $\mathrm{C}$ & -4.947372 & -0.420041 & 1.849456 \\
\hline $\mathrm{C}$ & -3.752144 & -1.113925 & 2.055141 \\
\hline $\mathrm{C}$ & -6.158409 & -1.081722 & 1.642372 \\
\hline $\mathrm{C}$ & -3.778447 & -2.504425 & 2.036516 \\
\hline $\mathrm{H}$ & -2.819474 & -0.585620 & 2.204707 \\
\hline
\end{tabular}




\begin{tabular}{|c|c|c|c|c|c|c|c|}
\hline $\mathrm{C}$ & -6.163345 & -2.472609 & 1.645315 & $\mathrm{C}$ & 2.074003 & -3.904298 & -0.595484 \\
\hline $\mathrm{H}$ & -7.069353 & -0.512858 & 1.495397 & $\mathrm{H}$ & 2.422129 & -3.701560 & 1.484043 \\
\hline $\mathrm{C}$ & -4.978833 & -3.200373 & 1.829103 & $\mathrm{C}$ & 1.037195 & -1.946534 & 3.148693 \\
\hline $\mathrm{H}$ & -2.854529 & -3.055092 & 2.174346 & $\mathrm{C}$ & -0.228346 & -3.118389 & 1.240809 \\
\hline $\mathrm{H}$ & -7.099999 & -3.002740 & 1.496130 & $\mathrm{H}$ & 0.073362 & -1.043611 & 1.484695 \\
\hline $\mathrm{C}$ & -4.988781 & -4.705178 & 1.762482 & $\mathrm{C}$ & 5.150543 & 2.475461 & -1.612300 \\
\hline $\mathrm{H}$ & -4.217727 & -5.138412 & 2.405044 & $\mathrm{C}$ & 3.506737 & 2.179950 & -3.419909 \\
\hline $\mathrm{H}$ & -5.962757 & -5.113308 & 2.050554 & $\mathrm{C}$ & 1.962420 & 0.187220 & -3.513548 \\
\hline $\mathrm{H}$ & -4.771909 & -5.039429 & 0.740180 & $\mathrm{C}$ & 6.817214 & 0.314392 & 0.060975 \\
\hline $\mathrm{H}$ & -4.571205 & 2.465708 & -1.616849 & $\mathrm{C}$ & 6.679344 & -0.432495 & 2.398711 \\
\hline $\mathrm{C}$ & -1.966638 & -0.205114 & -1.553540 & $\mathrm{C}$ & 4.483194 & -0.562422 & 3.625431 \\
\hline $\mathrm{O}$ & -0.715199 & -0.082100 & -1.681012 & $\mathrm{H}$ & 4.577089 & -3.950201 & 0.417566 \\
\hline $\mathrm{O}$ & -2.583428 & -0.133065 & -0.447467 & $\mathrm{H}$ & 4.544045 & -2.461584 & 1.379895 \\
\hline $\mathrm{H}$ & -3.818513 & 0.791514 & -0.176544 & $\mathrm{H}$ & 4.435656 & -2.380027 & -0.387251 \\
\hline $\mathrm{C}$ & -2.768595 & -0.404193 & -2.813088 & $\mathrm{C}$ & 1.854647 & -5.276544 & -0.447621 \\
\hline $\mathrm{C}$ & -3.953235 & -1.211819 & -2.854437 & $\mathrm{C}$ & 1.794737 & -3.307209 & -1.828927 \\
\hline $\mathrm{C}$ & -2.339416 & 0.255909 & -3.950521 & $\mathrm{H}$ & 0.147341 & -2.036693 & 3.779102 \\
\hline $\mathrm{C}$ & -4.427000 & -1.972257 & -1.747481 & $\mathrm{H}$ & 1.520703 & -0.999316 & 3.392140 \\
\hline $\mathrm{C}$ & -4.676450 & -1.292176 & -4.092570 & $\mathrm{H}$ & 1.723088 & -2.762734 & 3.404253 \\
\hline $\mathrm{C}$ & -3.069000 & 0.188687 & -5.156875 & $\mathrm{C}$ & -0.858361 & -3.050857 & -0.008872 \\
\hline $\mathrm{H}$ & -1.425477 & 0.837432 & -3.893645 & $\mathrm{C}$ & -0.424832 & -4.252727 & 2.033178 \\
\hline $\mathrm{C}$ & -5.551916 & -2.758391 & -1.863855 & $\mathrm{C}$ & 6.137930 & 3.288506 & -0.993730 \\
\hline $\mathrm{H}$ & -3.888139 & -1.926738 & -0.811279 & $\mathrm{C}$ & 4.528755 & 2.952327 & -2.810239 \\
\hline $\mathrm{C}$ & -5.839155 & -2.104403 & -4.169268 & $\mathrm{H}$ & 3.071851 & 2.535963 & -4.350931 \\
\hline $\mathrm{C}$ & -4.215799 & -0.567928 & -5.223545 & $\mathrm{H}$ & 1.075946 & 0.086876 & -2.881020 \\
\hline $\mathrm{H}$ & -2.717434 & 0.732467 & -6.029051 & $\mathrm{H}$ & 1.658846 & 0.645658 & -4.460216 \\
\hline $\mathrm{C}$ & -6.270985 & -2.824192 & -3.080015 & $\mathrm{H}$ & 2.325078 & -0.825217 & -3.729073 \\
\hline $\mathrm{H}$ & -5.892130 & -3.329583 & -1.006155 & $\mathrm{C}$ & 7.456451 & -0.191771 & 1.237070 \\
\hline $\mathrm{H}$ & -6.376874 & -2.152220 & -5.113043 & $\mathrm{C}$ & 7.604373 & 0.478665 & -1.110959 \\
\hline $\mathrm{H}$ & -4.783976 & -0.632018 & -6.148351 & $\mathrm{H}$ & 7.178515 & -0.803664 & 3.290892 \\
\hline $\mathrm{H}$ & -7.158758 & -3.446385 & -3.151886 & $\mathrm{H}$ & 5.120372 & -0.777692 & 4.488431 \\
\hline$P$ & 2.172861 & -0.268146 & 0.290405 & $\mathrm{H}$ & 3.808357 & 0.259688 & 3.876269 \\
\hline $\mathrm{O}$ & 3.143507 & -0.571297 & -1.045111 & $\mathrm{H}$ & 3.847362 & -1.436160 & 3.444868 \\
\hline $\mathrm{O}$ & 3.330897 & 0.450992 & 1.290764 & $\mathrm{C}$ & 1.351558 & -6.038748 & -1.502070 \\
\hline $\mathrm{N}$ & 1.866154 & -1.839868 & 0.797543 & $\mathrm{H}$ & 2.047008 & -5.747443 & 0.513381 \\
\hline $\mathrm{C}$ & 3.649335 & 0.577103 & -1.652447 & $\mathrm{C}$ & 1.292839 & -4.065155 & -2.884920 \\
\hline $\mathrm{C}$ & 4.694467 & 0.254982 & 1.235376 & $\mathrm{H}$ & 1.944100 & -2.243737 & -1.951633 \\
\hline C & 2.614951 & -3.103324 & 0.586162 & $\mathrm{C}$ & -1.651249 & -4.098378 & -0.465791 \\
\hline $\mathrm{C}$ & 0.654534 & -1.957105 & 1.669506 & $\mathrm{H}$ & -0.689987 & -2.180726 & -0.630513 \\
\hline $\mathrm{C}$ & 4.724628 & 1.218664 & -1.064596 & $\mathrm{C}$ & -1.218046 & -5.310625 & 1.576303 \\
\hline $\mathrm{C}$ & 3.039855 & 1.010798 & -2.861741 & $\mathrm{H}$ & 0.048944 & -4.325842 & 3.006863 \\
\hline $\mathrm{C}$ & 5.409372 & 0.600784 & 0.100189 & $\mathrm{C}$ & 6.512881 & 4.492467 & -1.547380 \\
\hline $\mathrm{C}$ & 5.317212 & -0.243713 & 2.415424 & $\mathrm{H}$ & 6.591150 & 2.952620 & -0.068770 \\
\hline $\mathrm{C}$ & 4.137723 & -2.950237 & 0.492036 & $\mathrm{C}$ & 4.948977 & 4.190821 & -3.362781 \\
\hline
\end{tabular}




\begin{tabular}{|c|c|c|c|}
\hline $\mathrm{C}$ & 8.849649 & -0.461521 & 1.212249 \\
\hline $\mathrm{C}$ & 8.952120 & 0.194763 & -1.107455 \\
\hline $\mathrm{H}$ & 7.129299 & 0.824749 & -2.020658 \\
\hline $\mathrm{C}$ & 1.065359 & -5.433675 & -2.726043 \\
\hline $\mathrm{H}$ & 1.168301 & -7.100534 & -1.360782 \\
\hline $\mathrm{H}$ & 1.067604 & -3.580558 & -3.831481 \\
\hline $\mathrm{C}$ & -1.826548 & -5.238247 & 0.324083 \\
\hline $\mathrm{H}$ & -2.123494 & -4.029483 & -1.440909 \\
\hline $\mathrm{H}$ & -1.349409 & -6.192745 & 2.197650 \\
\hline $\mathrm{C}$ & 5.922445 & 4.945977 & -2.750111 \\
\hline $\mathrm{H}$ & 7.265470 & 5.100846 & -1.053897 \\
\hline $\mathrm{H}$ & 4.475736 & 4.535925 & -4.278771 \\
\hline $\mathrm{C}$ & 9.588068 & -0.269052 & 0.067295 \\
\hline $\mathrm{H}$ & 9.320280 & -0.835936 & 2.118058 \\
\hline $\mathrm{H}$ & 9.530412 & 0.323260 & -2.018048 \\
\hline $\mathrm{H}$ & 0.662868 & -6.021674 & -3.546435 \\
\hline $\mathrm{H}$ & -2.431084 & -6.065572 & -0.037321 \\
\hline $\mathrm{H}$ & 6.231367 & 5.894337 & -3.180587 \\
\hline $\mathrm{H}$ & 10.652855 & -0.483774 & 0.058855 \\
\hline
\end{tabular}

\section{R5}

\begin{tabular}{|c|c|c|c|}
\hline \multicolumn{4}{|c|}{ Zero-point correction=1.203284 (Hartree/Particle) } \\
\hline \multicolumn{4}{|c|}{ Thermal correction to Energy $=1.278339$} \\
\hline \multicolumn{4}{|c|}{ Thermal correction to Enthalpy $=1.279283$} \\
\hline \multicolumn{4}{|c|}{ Thermal correction to Gibbs Free Energy=1.088935 } \\
\hline $\mathrm{E}($ Solv $)=$ & -4250.6599549 & & \\
\hline $\mathrm{C}$ & 0.638900 & 4.431816 & 1.639487 \\
\hline $\mathrm{C}$ & 0.355506 & 3.581421 & 0.576265 \\
\hline $\mathrm{C}$ & -0.968364 & 3.303752 & 0.198980 \\
\hline $\mathrm{C}$ & -2.002352 & 3.895485 & 0.935513 \\
\hline $\mathrm{C}$ & -1.723850 & 4.738127 & 2.012858 \\
\hline $\mathrm{C}$ & -0.403858 & 5.017110 & 2.366083 \\
\hline $\mathrm{H}$ & 1.674485 & 4.640345 & 1.893094 \\
\hline $\mathrm{H}$ & 1.160531 & 3.118576 & 0.024885 \\
\hline $\mathrm{H}$ & -3.029083 & 3.718109 & 0.638577 \\
\hline $\mathrm{H}$ & -2.543831 & 5.185653 & 2.568985 \\
\hline $\mathrm{H}$ & -0.187332 & 5.685191 & 3.195340 \\
\hline $\mathrm{C}$ & -1.240743 & 2.517007 & -1.028693 \\
\hline $\mathrm{H}$ & -0.717988 & 2.917768 & -1.900720 \\
\hline $\mathrm{C}$ & -2.465937 & 1.839581 & -1.300702 \\
\hline $\mathrm{H}$ & -3.080321 & 1.517096 & -0.463142 \\
\hline
\end{tabular}

\begin{tabular}{|c|c|c|c|}
\hline $\mathrm{C}$ & -3.047476 & 1.713928 & -2.608872 \\
\hline $\mathrm{C}$ & -3.929902 & 0.738294 & -2.984271 \\
\hline $\mathrm{H}$ & -4.392965 & 0.766961 & -3.967173 \\
\hline $\mathrm{H}$ & -2.790731 & 2.489865 & -3.334036 \\
\hline $\mathrm{H}$ & -3.937490 & -0.206439 & -2.457129 \\
\hline $\mathrm{Pd}$ & -0.868032 & 0.414206 & -1.140823 \\
\hline $\mathrm{C}$ & -7.366302 & 4.157451 & -2.044966 \\
\hline $\mathrm{C}$ & -7.052305 & 2.807299 & -2.090155 \\
\hline $\mathrm{C}$ & -6.166183 & 2.246031 & -1.145907 \\
\hline $\mathrm{C}$ & -5.588821 & 3.081062 & -0.167825 \\
\hline $\mathrm{C}$ & -5.895319 & 4.435566 & -0.137707 \\
\hline $\mathrm{C}$ & -6.785816 & 4.976464 & -1.068564 \\
\hline $\mathrm{H}$ & -8.055656 & 4.578588 & -2.770083 \\
\hline $\mathrm{H}$ & -7.485253 & 2.169747 & -2.856132 \\
\hline $\mathrm{H}$ & -4.892713 & 2.675101 & 0.551534 \\
\hline $\mathrm{H}$ & -5.434818 & 5.072183 & 0.611349 \\
\hline $\mathrm{H}$ & -7.024273 & 6.035452 & -1.038966 \\
\hline $\mathrm{C}$ & -5.828545 & 0.854774 & -1.272977 \\
\hline $\mathrm{N}$ & -5.300530 & 0.080281 & -0.308401 \\
\hline S & -5.572033 & 0.090670 & 1.416433 \\
\hline $\mathrm{O}$ & -6.680865 & 1.000860 & 1.686451 \\
\hline $\mathrm{O}$ & -5.656739 & -1.337685 & 1.707202 \\
\hline $\mathrm{C}$ & -4.076526 & 0.736365 & 2.114893 \\
\hline C & -4.172010 & 1.712339 & 3.108878 \\
\hline C & -2.850752 & 0.172231 & 1.749802 \\
\hline $\mathrm{C}$ & -3.006063 & 2.124839 & 3.749437 \\
\hline $\mathrm{H}$ & -5.138855 & 2.128857 & 3.368883 \\
\hline $\mathrm{C}$ & -1.699676 & 0.627559 & 2.381575 \\
\hline $\mathrm{H}$ & -2.803925 & -0.578783 & 0.967092 \\
\hline $\mathrm{C}$ & -1.759757 & 1.591665 & 3.397600 \\
\hline $\mathrm{H}$ & -3.062714 & 2.884454 & 4.523672 \\
\hline $\mathrm{H}$ & -0.734642 & 0.248500 & 2.069185 \\
\hline $\mathrm{C}$ & -0.494366 & 2.027700 & 4.086504 \\
\hline $\mathrm{H}$ & -0.631524 & 2.971235 & 4.620596 \\
\hline $\mathrm{H}$ & -0.169357 & 1.270026 & 4.811659 \\
\hline $\mathrm{H}$ & 0.310052 & 2.164472 & 3.358501 \\
\hline $\mathrm{H}$ & -6.196603 & 0.326623 & -2.144275 \\
\hline $\mathrm{C}$ & -2.426213 & -2.285637 & -1.385787 \\
\hline $\mathrm{O}$ & -1.419748 & -1.651899 & -1.828680 \\
\hline $\mathrm{O}$ & -3.475263 & -1.765638 & -0.916059 \\
\hline $\mathrm{H}$ & -4.829424 & -0.801341 & -0.587988 \\
\hline $\mathrm{C}$ & -2.300241 & -3.793252 & -1.418461 \\
\hline $\mathrm{C}$ & -2.817882 & -4.633284 & -0.378025 \\
\hline $\mathrm{C}$ & & & \\
\hline
\end{tabular}




\begin{tabular}{|c|c|c|c|}
\hline $\mathrm{C}$ & -3.533959 & -4.142304 & 0.750798 \\
\hline $\mathrm{C}$ & -2.557431 & -6.045284 & -0.451073 \\
\hline $\mathrm{C}$ & -1.367971 & -5.743844 & -2.548098 \\
\hline $\mathrm{H}$ & -1.173228 & -3.689573 & -3.216637 \\
\hline $\mathrm{C}$ & -3.952683 & -4.993850 & 1.748172 \\
\hline $\mathrm{H}$ & -3.767474 & -3.088992 & 0.804654 \\
\hline $\mathrm{C}$ & -3.008118 & -6.893000 & 0.596516 \\
\hline $\mathrm{C}$ & -1.841270 & -6.572851 & -1.556861 \\
\hline $\mathrm{H}$ & -0.815286 & -6.151868 & -3.389505 \\
\hline $\mathrm{C}$ & -3.687838 & -6.382012 & 1.676634 \\
\hline $\mathrm{H}$ & -4.500789 & -4.590599 & 2.594848 \\
\hline $\mathrm{H}$ & -2.798942 & -7.957783 & 0.526510 \\
\hline $\mathrm{H}$ & -1.665116 & -7.644897 & -1.599762 \\
\hline $\mathrm{H}$ & -4.024328 & -7.039925 & 2.473058 \\
\hline $\mathrm{P}$ & 1.221704 & -0.250221 & -0.512862 \\
\hline $\mathrm{O}$ & 2.415115 & 0.304405 & -1.524123 \\
\hline $\mathrm{O}$ & 1.717934 & 0.388345 & 0.964353 \\
\hline $\mathrm{N}$ & 1.745660 & -1.891089 & -0.513931 \\
\hline $\mathrm{C}$ & 2.718267 & 1.649885 & -1.498731 \\
\hline $\mathrm{C}$ & 3.057145 & 0.430330 & 1.334774 \\
\hline $\mathrm{C}$ & 2.399172 & -2.321816 & -1.808035 \\
\hline $\mathrm{C}$ & 0.804245 & -2.878528 & 0.095755 \\
\hline $\mathrm{C}$ & 3.474821 & 2.129857 & -0.448686 \\
\hline $\mathrm{C}$ & 2.249395 & 2.453317 & -2.571527 \\
\hline $\mathrm{C}$ & 3.954508 & 1.200497 & 0.609274 \\
\hline $\mathrm{C}$ & 3.433044 & -0.261314 & 2.521405 \\
\hline $\mathrm{C}$ & 2.363555 & -3.837334 & -2.072074 \\
\hline $\mathrm{C}$ & 3.847649 & -1.864307 & -1.917397 \\
\hline $\mathrm{H}$ & 1.844516 & -1.862405 & -2.636755 \\
\hline $\mathrm{C}$ & -0.162447 & -2.280752 & 1.130435 \\
\hline $\mathrm{C}$ & 1.535066 & -4.026149 & 0.774834 \\
\hline $\mathrm{H}$ & 0.172053 & -3.286635 & -0.693462 \\
\hline $\mathrm{C}$ & 3.716190 & 3.542942 & -0.374288 \\
\hline $\mathrm{C}$ & 2.528482 & 3.800335 & -2.520430 \\
\hline $\mathrm{C}$ & 1.486304 & 1.813243 & -3.698869 \\
\hline $\mathrm{C}$ & 5.350974 & 1.095934 & 0.913433 \\
\hline $\mathrm{C}$ & 4.771892 & -0.303705 & 2.843678 \\
\hline $\mathrm{C}$ & 2.394255 & -0.857178 & 3.437083 \\
\hline $\mathrm{H}$ & 2.807148 & -3.995528 & -3.060329 \\
\hline $\mathrm{H}$ & 1.349878 & -4.243296 & -2.091432 \\
\hline $\mathrm{H}$ & 2.958138 & -4.392264 & -1.345337 \\
\hline $\mathrm{C}$ & 4.323709 & -1.419297 & -3.155729 \\
\hline $\mathrm{C}$ & 4.736934 & -1.926795 & -0.842938 \\
\hline $\mathrm{H}$ & -0.785249 & -3.091898 & 1.517281 \\
\hline
\end{tabular}

$\begin{array}{llll}\mathrm{H} & -0.828011 & -1.544264 & 0.682231 \\ \mathrm{H} & 0.364175 & -1.825996 & 1.969046 \\ \mathrm{C} & 0.963333 & -5.303079 & 0.738724\end{array}$

$\begin{array}{llll}\mathrm{C} & 2.710676 & -3.833324 & 1.504328\end{array}$

$\begin{array}{llll}\text { C } & 4.356296 & 4.158250 & 0.735131\end{array}$

$\begin{array}{llll}\text { C } & 3.232986 & 4.380057 & -1.432020\end{array}$

$\begin{array}{llll}\mathrm{H} & 2.184320 & 4.450192 & -3.321450\end{array}$

$\begin{array}{llll}\mathrm{H} & 0.569345 & 1.335438 & -3.330249\end{array}$

$\begin{array}{llll}\mathrm{H} & 1.216337 & 2.555512 & -4.455904\end{array}$

$\begin{array}{llll}\mathrm{H} & 2.082066 & 1.026097 & -4.175048\end{array}$

$\begin{array}{llll}\text { C } & 5.761087 & 0.310801 & 2.035748\end{array}$

$\begin{array}{llll}\mathrm{C} & 6.352953 & 1.695102 & 0.104916\end{array}$

$\begin{array}{llll}\mathrm{H} & 5.085288 & -0.823530 & 3.746136\end{array}$

$\begin{array}{llll}\mathrm{H} & 2.804717 & -0.970146 & 4.445111\end{array}$

$\begin{array}{llll}\mathrm{H} & 1.508311 & -0.218631 & 3.493739\end{array}$

$\begin{array}{llll}\mathrm{H} & 2.065408 & -1.845740 & 3.105589\end{array}$

$\begin{array}{llll}\text { C } & 5.652781 & -1.030263 & -3.315367\end{array}$

$\begin{array}{llll}\mathrm{H} & 3.636578 & -1.357172 & -3.997215\end{array}$

$\begin{array}{lllll}\text { C } & 6.067454 & -1.536777 & -0.995982\end{array}$

$\begin{array}{llll}\mathrm{H} & 4.381885 & -2.256983 & 0.123330\end{array}$

$\begin{array}{llll}\text { C } & 1.560846 & -6.367492 & 1.412031\end{array}$

$\begin{array}{llll}\mathrm{H} & 0.049932 & -5.464162 & 0.174201\end{array}$

$\begin{array}{llll}\text { C } & 3.314162 & -4.897937 & 2.175688\end{array}$

$\begin{array}{llll}\mathrm{H} & 3.157607 & -2.847680 & 1.534068\end{array}$

$\begin{array}{llll}\text { C } & 4.531775 & 5.523862 & 0.783717\end{array}$

$\begin{array}{llll}\mathrm{H} & 4.700328 & 3.538694 & 1.554217\end{array}$

$\begin{array}{llll}\text { C } & 3.442721 & 5.781586 & -1.355750\end{array}$

$\begin{array}{llll}\text { C } & 7.145241 & 0.175720 & 2.320673\end{array}$

$\begin{array}{llll}\text { C } & 7.688296 & 1.535591 & 0.399062\end{array}$

$\begin{array}{llll}\mathrm{H} & 6.053475 & 2.258357 & -0.770573\end{array}$

$\begin{array}{lllll}\text { C } & 6.530621 & -1.083195 & -2.230837\end{array}$

$\begin{array}{llll}\mathrm{H} & 5.998930 & -0.674201 & -4.282248\end{array}$

$\begin{array}{llll}\mathrm{H} & 6.736872 & -1.564470 & -0.141544\end{array}$

$\begin{array}{lllll}\text { C } & 2.742042 & -6.170171 & 2.131138\end{array}$

$\begin{array}{llll}\mathrm{H} & 1.104746 & -7.352960 & 1.369767\end{array}$

$\begin{array}{llll}\mathrm{H} & 4.233473 & -4.731353 & 2.731019\end{array}$

$\begin{array}{llll}\text { C } & 4.079073 & 6.345885 & -0.274287\end{array}$

$\begin{array}{llll}\mathrm{H} & 5.018905 & 5.973331 & 1.644358\end{array}$

$\begin{array}{llll}\mathrm{H} & 3.074658 & 6.404465 & -2.167025\end{array}$

$\begin{array}{llll}\text { C } & 8.092353 & 0.773614 & 1.520586\end{array}$

$\begin{array}{llll}\mathrm{H} & 7.442553 & -0.419363 & 3.180567\end{array}$

$\begin{array}{llll}\mathrm{H} & 8.438703 & 1.989355 & -0.241847\end{array}$

$\begin{array}{llll}\mathrm{H} & 7.562536 & -0.762813 & -2.342386\end{array}$

$\begin{array}{llll}\text { H } & 3.212443 & -7.000622 & 2.650272\end{array}$ 


$\begin{array}{crrr}\mathrm{H} & 4.225456 & 7.420947 & -0.225138 \\ \mathrm{H} & 9.149094 & 0.656885 & 1.743174 \\ & & & \\ - & & \end{array}$

R6

Zero-point correction==1.204482 (Hartree/Particle)

Thermal correction to Energy=1.279477

Thermal correction to Enthalpy=1.280421

Thermal correction to Gibbs Free Energy= 1.090206

$\mathrm{E}($ Solv $)=-4250.70424637$

\begin{tabular}{|c|c|c|c|}
\hline $\mathrm{C}$ & 5.225828 & 0.557648 & 3.140879 \\
\hline $\mathrm{C}$ & 4.178931 & 1.200026 & 2.484786 \\
\hline $\mathrm{C}$ & 2.851689 & 1.024958 & 2.903827 \\
\hline $\mathrm{C}$ & 2.595156 & 0.175882 & 3.993960 \\
\hline $\mathrm{C}$ & 3.640919 & -0.456364 & 4.657490 \\
\hline $\mathrm{C}$ & 4.962076 & -0.264304 & 4.236948 \\
\hline $\mathrm{H}$ & 6.245740 & 0.697438 & 2.795022 \\
\hline $\mathrm{H}$ & 4.382188 & 1.846357 & 1.636553 \\
\hline $\mathrm{H}$ & 1.573830 & 0.012268 & 4.324121 \\
\hline $\mathrm{H}$ & 3.429111 & -1.103605 & 5.504154 \\
\hline $\mathrm{H}$ & 5.776342 & -0.762615 & 4.755113 \\
\hline $\mathrm{C}$ & 1.802710 & 1.812092 & 2.239963 \\
\hline $\mathrm{H}$ & 2.186106 & 2.525274 & 1.507628 \\
\hline $\mathrm{C}$ & 0.594117 & 2.193489 & 2.882278 \\
\hline $\mathrm{H}$ & 0.196181 & 1.581420 & 3.689088 \\
\hline $\mathrm{C}$ & -0.112599 & 3.322456 & 2.483424 \\
\hline $\mathrm{C}$ & -1.477238 & 3.545495 & 2.738365 \\
\hline $\mathrm{H}$ & -1.928889 & 3.047589 & 3.592145 \\
\hline $\mathrm{H}$ & 0.364441 & 3.944179 & 1.730388 \\
\hline $\mathrm{H}$ & -1.870473 & 4.539341 & 2.536647 \\
\hline $\mathrm{Pd}$ & 0.414900 & 0.822498 & 0.838476 \\
\hline $\mathrm{C}$ & -5.036360 & 0.857329 & 3.244776 \\
\hline $\mathrm{C}$ & -4.288842 & 1.799846 & 2.546755 \\
\hline $\mathrm{C}$ & -3.058707 & 1.453223 & 1.961767 \\
\hline $\mathrm{C}$ & -2.600868 & 0.139095 & 2.096983 \\
\hline $\mathrm{C}$ & -3.353228 & -0.812370 & 2.787457 \\
\hline $\mathrm{C}$ & -4.571398 & -0.456286 & 3.365087 \\
\hline $\mathrm{H}$ & -5.989898 & 1.140912 & 3.681837 \\
\hline $\mathrm{H}$ & -4.660924 & 2.815233 & 2.434780 \\
\hline $\mathrm{H}$ & -1.659647 & -0.139866 & 1.633963 \\
\hline $\mathrm{H}$ & -2.994458 & -1.833677 & 2.853931 \\
\hline $\mathrm{H}$ & -5.161443 & -1.198956 & 3.893705 \\
\hline
\end{tabular}

\begin{tabular}{|c|c|c|c|}
\hline $\mathrm{C}$ & -2.323041 & 2.503745 & 1.216525 \\
\hline $\mathrm{N}$ & -1.399455 & 2.105726 & 0.271870 \\
\hline S & -0.944069 & 3.242087 & -0.879553 \\
\hline $\mathrm{O}$ & 0.365401 & 3.827402 & -0.525090 \\
\hline $\mathrm{O}$ & -1.078945 & 2.578935 & -2.185840 \\
\hline $\mathrm{C}$ & -2.136730 & 4.579153 & -0.833650 \\
\hline $\mathrm{C}$ & -3.415674 & 4.376372 & -1.359151 \\
\hline $\mathrm{C}$ & -1.772606 & 5.798550 & -0.265425 \\
\hline $\mathrm{C}$ & -4.334384 & 5.420076 & -1.306469 \\
\hline $\mathrm{H}$ & -3.691798 & 3.408172 & -1.760709 \\
\hline $\mathrm{C}$ & -2.705806 & 6.833963 & -0.228565 \\
\hline $\mathrm{H}$ & -0.769465 & 5.928766 & 0.124844 \\
\hline $\mathrm{C}$ & -3.995281 & 6.661794 & -0.747247 \\
\hline $\mathrm{H}$ & -5.334922 & 5.267679 & -1.702722 \\
\hline $\mathrm{H}$ & -2.427993 & 7.789943 & 0.207660 \\
\hline $\mathrm{C}$ & -4.995018 & 7.791290 & -0.727458 \\
\hline $\mathrm{H}$ & -5.031257 & 8.298624 & -1.700273 \\
\hline $\mathrm{H}$ & -6.005591 & 7.426071 & -0.515573 \\
\hline $\mathrm{H}$ & -4.737026 & 8.542288 & 0.025637 \\
\hline $\mathrm{H}$ & -2.981360 & 3.318005 & 0.928632 \\
\hline $\mathrm{C}$ & -3.824955 & 0.210052 & -1.207364 \\
\hline $\mathrm{O}$ & -2.503822 & -0.010329 & -1.225847 \\
\hline $\mathrm{O}$ & -4.321571 & 1.280734 & -0.892744 \\
\hline $\mathrm{H}$ & -2.050389 & 0.800200 & -0.863839 \\
\hline $\mathrm{C}$ & -4.607811 & -0.991524 & -1.612828 \\
\hline $\mathrm{C}$ & -5.826588 & -1.332522 & -0.942817 \\
\hline $\mathrm{C}$ & -4.132878 & -1.773067 & -2.648883 \\
\hline $\mathrm{C}$ & -6.323751 & -0.621193 & 0.182507 \\
\hline $\mathrm{C}$ & -6.540404 & -2.493630 & -1.389713 \\
\hline $\mathrm{C}$ & -4.859402 & -2.894852 & -3.101762 \\
\hline $\mathrm{H}$ & -3.186584 & -1.518712 & -3.110535 \\
\hline $\mathrm{C}$ & -7.467847 & -1.037065 & 0.824926 \\
\hline $\mathrm{H}$ & -5.789112 & 0.250950 & 0.532103 \\
\hline $\mathrm{C}$ & -7.725191 & -2.883557 & -0.710665 \\
\hline $\mathrm{C}$ & -6.038718 & -3.244265 & -2.486021 \\
\hline $\mathrm{H}$ & -4.470829 & -3.486386 & -3.923882 \\
\hline C & -8.181455 & -2.172854 & 0.374836 \\
\hline $\mathrm{H}$ & -7.823872 & -0.486164 & 1.690950 \\
\hline $\mathrm{H}$ & -8.258842 & -3.763411 & -1.061378 \\
\hline $\mathrm{H}$ & -6.598758 & -4.114373 & -2.819545 \\
\hline $\mathrm{H}$ & -9.085001 & -2.484223 & 0.891494 \\
\hline $\mathrm{P}$ & 1.548010 & -1.043241 & 0.202820 \\
\hline $\mathrm{O}$ & 2.028453 & -1.110377 & -1.394555 \\
\hline c & 3.045767 & -1.190813 & 0.94396 \\
\hline
\end{tabular}




\begin{tabular}{|c|c|c|c|}
\hline $\mathrm{N}$ & 0.799493 & -2.527688 & 0.386596 \\
\hline C & 2.543820 & 0.136283 & -1.769864 \\
\hline C & 4.289638 & -1.302193 & 0.374019 \\
\hline $\mathrm{C}$ & 1.104584 & -3.826128 & -0.278431 \\
\hline $\mathrm{C}$ & -0.004915 & -2.638112 & 1.637867 \\
\hline C & 3.780177 & 0.517618 & -1.280179 \\
\hline $\mathrm{C}$ & 1.720026 & 0.947479 & -2.588758 \\
\hline C & 4.690353 & -0.492781 & -0.677268 \\
\hline C & 5.154308 & -2.249226 & 0.998025 \\
\hline $\mathrm{C}$ & 2.514263 & -3.957021 & -0.870369 \\
\hline C & 0.062666 & -4.173941 & -1.332244 \\
\hline $\mathrm{H}$ & 1.026320 & -4.578897 & 0.512832 \\
\hline C & 0.864627 & -3.078504 & 2.820744 \\
\hline C & -1.256704 & -3.470545 & 1.410425 \\
\hline $\mathrm{H}$ & -0.332274 & -1.615994 & 1.857047 \\
\hline $\mathrm{C}$ & 4.143432 & 1.900114 & -1.415110 \\
\hline C & 2.107669 & 2.255275 & -2.763023 \\
\hline C & 0.502534 & 0.349768 & -3.240119 \\
\hline $\mathrm{C}$ & 6.004409 & -0.695369 & -1.230291 \\
\hline C & 6.435201 & -2.370768 & 0.515861 \\
\hline C & 4.662929 & -3.068849 & 2.160269 \\
\hline $\mathrm{H}$ & 2.634057 & -4.985943 & -1.224060 \\
\hline $\mathrm{H}$ & 3.287826 & -3.756754 & -0.128794 \\
\hline $\mathrm{H}$ & 2.663967 & -3.286761 & -1.715935 \\
\hline C & -0.248980 & -3.277441 & -2.359364 \\
\hline $\mathrm{C}$ & -0.564894 & -5.421311 & -1.306053 \\
\hline $\mathrm{H}$ & 0.270742 & -3.121852 & 3.740172 \\
\hline $\mathrm{H}$ & 1.676388 & -2.361954 & 2.964770 \\
\hline $\mathrm{H}$ & 1.311806 & -4.065370 & 2.659843 \\
\hline C & -2.200866 & -3.034589 & 0.469696 \\
\hline C & -1.495292 & -4.660424 & 2.103991 \\
\hline C & 5.300265 & 2.451277 & -0.800860 \\
\hline C & 3.271242 & 2.775390 & -2.138896 \\
\hline $\mathrm{H}$ & 1.479722 & 2.922908 & -3.343514 \\
\hline $\mathrm{H}$ & -0.238140 & 0.030216 & -2.502249 \\
\hline $\mathrm{H}$ & 0.019782 & 1.080446 & -3.889102 \\
\hline $\mathrm{H}$ & 0.781937 & -0.530418 & -3.832472 \\
\hline $\mathrm{C}$ & 6.891049 & -1.626361 & -0.601630 \\
\hline C & 6.459614 & -0.031141 & -2.401824 \\
\hline $\mathrm{H}$ & 7.117906 & -3.075478 & 0.984926 \\
\hline $\mathrm{H}$ & 5.461460 & -3.715561 & 2.536101 \\
\hline $\mathrm{H}$ & 4.318469 & -2.426883 & 2.974263 \\
\hline $\mathrm{H}$ & 3.814621 & -3.701868 & 1.878545 \\
\hline C & -1.17318 & -3.626172 & -3.341899 \\
\hline
\end{tabular}

$$
\begin{aligned}
& \begin{array}{llll}
\mathrm{H} & 0.227130 & -2.305512 & -2.379640
\end{array} \\
& \begin{array}{llll}
\text { C } & -1.498986 & -5.768905 & -2.281910
\end{array} \\
& \mathrm{H} \quad-0.348300 \quad-6.112050 \quad-0.495513 \\
& \begin{array}{llll}
\text { C } & -3.352633 & -3.776245 & 0.228208
\end{array} \\
& \begin{array}{llll}
\mathrm{H} & -2.021675 & -2.122877 & -0.090170
\end{array} \\
& \begin{array}{llll}
\text { C } & -2.651825 & -5.407521 & 1.861512
\end{array} \\
& \begin{array}{llll}
\mathrm{H} & -0.779958 & -5.018077 & 2.837114
\end{array} \\
& \begin{array}{llll}
\text { C } & 5.574225 & 3.798762 & -0.888102
\end{array} \\
& \begin{array}{llll}
\mathrm{H} & 5.965908 & 1.795334 & -0.251618
\end{array} \\
& \begin{array}{llll}
\text { C } & 3.588320 & 4.156286 & -2.216393
\end{array} \\
& \begin{array}{llll}
\text { C } & 8.197735 & -1.810930 & -1.123362
\end{array} \\
& \begin{array}{llll}
\text { C } & 7.729877 & -0.240761 & -2.892091
\end{array} \\
& \begin{array}{llll}
\mathrm{H} & 5.791369 & 0.645265 & -2.919539
\end{array} \\
& \begin{array}{llll}
\text { C } & -1.803641 & -4.872365 & -3.305951
\end{array} \\
& \begin{array}{llll}
\mathrm{H} & -1.399627 & -2.922828 & -4.139093
\end{array} \\
& \mathrm{H} \quad-1.993562 \quad-6.735131 \quad-2.235972 \\
& \begin{array}{llll}
\text { C } & -3.581300 & -4.966509 & 0.922525
\end{array} \\
& \mathrm{H} \quad-4.067071 \quad-3.433725 \quad-0.507272 \\
& \begin{array}{llll}
\mathrm{H} & -2.819832 & -6.331463 & 2.408485
\end{array} \\
& \begin{array}{llll}
\text { C } & 4.712094 & 4.662211 & -1.603681
\end{array} \\
& \begin{array}{llll}
\mathrm{H} & 6.457606 & 4.201393 & -0.400478
\end{array} \\
& \begin{array}{llll}
\mathrm{H} & 2.908923 & 4.809852 & -2.756264
\end{array} \\
& \begin{array}{llll}
\text { C } & 8.617155 & -1.128705 & -2.242197
\end{array} \\
& \mathrm{H} \quad 8.858592 \quad-2.515507 \quad-0.624289 \\
& \begin{array}{llll}
\mathrm{H} & 8.049351 & 0.277724 & -3.791640
\end{array} \\
& \mathrm{H} \quad \begin{array}{llll}
-2.526734 & -5.142870 & -4.070446
\end{array} \\
& \mathrm{H} \quad-4.481686 \quad-5.543000 \quad 0.728913 \\
& \begin{array}{llll}
\mathrm{H} & 4.936534 & 5.723363 & -1.662214
\end{array} \\
& \begin{array}{llll}
\mathrm{H} & 9.618289 & -1.280804 & -2.635161
\end{array}
\end{aligned}
$$

S1

Zero-point correction=1.204462 (Hartree/Particle)

Thermal correction to Energy=1.279151

Thermal correction to Enthalpy=1.280095

Thermal correction to Gibbs Free Energy=1.092764

$\mathrm{E}($ Solv $)=-4250.71929804$

$\begin{array}{llll}\mathrm{C} & 5.099024 & 0.004374 & -3.540706 \\ \mathrm{C} & 4.157247 & -0.788825 & -2.887732 \\ \mathrm{C} & 2.786089 & -0.538110 & -3.033046 \\ \mathrm{C} & 2.372224 & 0.540413 & -3.835029 \\ \mathrm{C} & 3.313887 & 1.330439 & -4.487241\end{array}$




\begin{tabular}{|c|c|c|c|c|c|c|c|}
\hline $\mathrm{C}$ & 4.680597 & 1.063196 & -4.348002 & $\mathrm{H}$ & -7.511583 & 2.827855 & -0.339363 \\
\hline $\mathrm{H}$ & 6.158108 & -0.200962 & -3.413443 & $\mathrm{H}$ & -6.953290 & 4.358915 & -1.020240 \\
\hline $\mathrm{H}$ & 4.479633 & -1.614976 & -2.261832 & $\mathrm{H}$ & -3.384499 & -2.021544 & -2.729218 \\
\hline $\mathrm{H}$ & 1.314402 & 0.772006 & -3.919931 & $\mathrm{C}$ & -3.036621 & -0.156580 & 1.779127 \\
\hline $\mathrm{H}$ & 2.982328 & 2.163471 & -5.100986 & $\mathrm{O}$ & -2.204629 & 0.271407 & 0.804127 \\
\hline $\mathrm{H}$ & 5.412519 & 1.684077 & -4.857096 & $\mathrm{O}$ & -2.752162 & 0.075756 & 2.937938 \\
\hline $\mathrm{C}$ & 1.816160 & -1.439326 & -2.379319 & $\mathrm{H}$ & -2.349119 & -0.124123 & -0.091860 \\
\hline $\mathrm{H}$ & 2.251572 & -2.141418 & -1.667713 & $\mathrm{C}$ & -4.278658 & -0.865697 & 1.348591 \\
\hline $\mathrm{C}$ & 0.646078 & -1.857902 & -3.066420 & $\mathrm{C}$ & -4.818845 & -1.941240 & 2.127693 \\
\hline $\mathrm{H}$ & 0.347233 & -1.305880 & -3.954342 & $\mathrm{C}$ & -4.929094 & -0.436307 & 0.206323 \\
\hline $\mathrm{C}$ & -0.113668 & -2.987200 & -2.722223 & $\mathrm{C}$ & -4.211802 & -2.436644 & 3.312744 \\
\hline $\mathrm{C}$ & -1.370824 & -3.229648 & -3.263564 & $\mathrm{C}$ & -6.016931 & -2.575518 & 1.661161 \\
\hline $\mathrm{H}$ & -1.654254 & -2.714887 & -4.176265 & $\mathrm{C}$ & -6.125549 & -1.046408 & -0.224320 \\
\hline $\mathrm{H}$ & 0.236355 & -3.601339 & -1.893272 & $\mathrm{H}$ & -4.530407 & 0.396526 & -0.357235 \\
\hline $\mathrm{H}$ & -1.867450 & -4.173796 & -3.065740 & $\mathrm{C}$ & -4.756395 & -3.504907 & 3.989726 \\
\hline $\mathrm{Pd}$ & 0.277813 & -0.428345 & -1.235399 & $\mathrm{H}$ & -3.318887 & -1.952102 & 3.682704 \\
\hline $\mathrm{C}$ & -3.962637 & -4.687020 & 0.173452 & $\mathrm{C}$ & -6.547011 & -3.678286 & 2.381132 \\
\hline $\mathrm{C}$ & -3.809414 & -3.748432 & -0.842879 & $\mathrm{C}$ & -6.651640 & -2.099722 & 0.484972 \\
\hline $\mathrm{C}$ & -2.709199 & -2.877047 & -0.860116 & $\mathrm{H}$ & -6.615584 & -0.676305 & -1.118062 \\
\hline $\mathrm{C}$ & -1.758207 & -2.964973 & 0.168884 & $\mathrm{C}$ & -5.930748 & -4.138291 & 3.521265 \\
\hline $\mathrm{C}$ & -1.915160 & -3.908145 & 1.180882 & $\mathrm{H}$ & -4.276447 & -3.867082 & 4.894582 \\
\hline $\mathrm{C}$ & -3.011555 & -4.772688 & 1.189039 & $\mathrm{H}$ & -7.452969 & -4.151953 & 2.011212 \\
\hline $\mathrm{H}$ & -4.829788 & -5.339733 & 0.181013 & $\mathrm{H}$ & -7.566494 & -2.586274 & 0.155471 \\
\hline $\mathrm{H}$ & -4.553299 & -3.676872 & -1.631502 & $\mathrm{H}$ & -6.345316 & -4.981994 & 4.065677 \\
\hline $\mathrm{H}$ & -0.890424 & -2.309726 & 0.159839 & $\mathrm{P}$ & 1.550160 & 0.857305 & 0.128089 \\
\hline $\mathrm{H}$ & -1.177593 & -3.970503 & 1.971824 & $\mathrm{O}$ & 1.986734 & 0.269832 & 1.635358 \\
\hline $\mathrm{H}$ & -3.133041 & -5.492412 & 1.991962 & $\mathrm{O}$ & 3.076716 & 1.012540 & -0.553874 \\
\hline $\mathrm{C}$ & -2.572899 & -1.958558 & -2.005643 & $\mathrm{~N}$ & 1.052758 & 2.401586 & 0.515715 \\
\hline $\mathrm{N}$ & -1.954189 & -0.752669 & -1.861352 & $\mathrm{C}$ & 2.368344 & -1.066346 & 1.554133 \\
\hline S & -2.208296 & 0.324920 & -3.147133 & $\mathrm{C}$ & 4.315725 & 0.791859 & -0.019438 \\
\hline $\mathrm{O}$ & -1.071028 & 1.261119 & -3.139536 & $\mathrm{C}$ & 1.574758 & 3.321010 & 1.562027 \\
\hline $\mathrm{O}$ & -2.538589 & -0.413297 & -4.380232 & $\mathrm{C}$ & 0.216573 & 3.046008 & -0.547934 \\
\hline $\mathrm{C}$ & -3.654269 & 1.252667 & -2.667423 & $\mathrm{C}$ & 3.590299 & -1.387574 & 0.991643 \\
\hline $\mathrm{C}$ & -4.908400 & 0.868733 & -3.142196 & $\mathrm{C}$ & 1.440728 & -2.024163 & 2.042426 \\
\hline $\mathrm{C}$ & -3.513936 & 2.322292 & -1.783175 & $\mathrm{C}$ & 4.607616 & -0.334309 & 0.734143 \\
\hline $\mathrm{C}$ & -6.035304 & 1.559890 & -2.700635 & $\mathrm{C}$ & 5.299379 & 1.763057 & -0.372753 \\
\hline $\mathrm{H}$ & -4.988852 & 0.056796 & -3.856398 & $\mathrm{C}$ & 2.989646 & 3.020674 & 2.073956 \\
\hline $\mathrm{C}$ & -4.650758 & 2.993331 & -1.343024 & $\mathrm{C}$ & 0.606661 & 3.437929 & 2.732578 \\
\hline $\mathrm{H}$ & -2.534728 & 2.630412 & -1.443980 & $\mathrm{H}$ & 1.621996 & 4.305442 & 1.083117 \\
\hline C & -5.927238 & 2.615382 & -1.783600 & $\mathrm{C}$ & 1.095650 & 3.652530 & -1.644922 \\
\hline $\mathrm{H}$ & -7.016256 & 1.275111 & -3.072806 & $\mathrm{C}$ & -0.795676 & 4.006372 & 0.060813 \\
\hline $\mathrm{H}$ & -4.534993 & 3.815813 & -0.643179 & $\mathrm{H}$ & -0.344317 & 2.229448 & -1.014279 \\
\hline $\mathrm{C}$ & -7.158171 & 3.310881 & -1.259842 & $\mathrm{C}$ & 3.835545 & -2.770137 & 0.682615 \\
\hline $\mathrm{H}$ & -7.978365 & 3.276865 & -1.983978 & $\mathrm{C}$ & 1.725800 & -3.351053 & 1.817375 \\
\hline
\end{tabular}




\begin{tabular}{|c|c|c|c|}
\hline $\mathrm{C}$ & 0.229482 & -1.545223 & 2.794867 \\
\hline $\mathrm{C}$ & 5.924794 & -0.450155 & 1.304675 \\
\hline $\mathrm{C}$ & 6.577104 & 1.592030 & 0.100832 \\
\hline $\mathrm{C}$ & 4.917341 & 2.920143 & -1.254703 \\
\hline $\mathrm{H}$ & 3.263688 & 3.812579 & 2.777946 \\
\hline $\mathrm{H}$ & 3.722419 & 3.014509 & 1.266556 \\
\hline $\mathrm{H}$ & 3.038386 & 2.067734 & 2.599909 \\
\hline $\mathrm{C}$ & -0.074134 & 2.327205 & 3.237167 \\
\hline $\mathrm{C}$ & 0.392771 & 4.688578 & 3.318697 \\
\hline $\mathrm{H}$ & 0.476064 & 4.107801 & -2.424099 \\
\hline $\mathrm{H}$ & 1.700306 & 2.868366 & -2.106640 \\
\hline $\mathrm{H}$ & 1.775738 & 4.415803 & -1.250158 \\
\hline $\mathrm{C}$ & -1.803847 & 3.490439 & 0.886760 \\
\hline $\mathrm{C}$ & -0.747726 & 5.386253 & -0.154549 \\
\hline $\mathrm{C}$ & 4.965822 & -3.198271 & -0.064564 \\
\hline $\mathrm{C}$ & 2.877357 & -3.753618 & 1.089476 \\
\hline $\mathrm{H}$ & 1.049026 & -4.119712 & 2.180356 \\
\hline $\mathrm{H}$ & -0.440577 & -0.965185 & 2.156087 \\
\hline $\mathrm{H}$ & -0.332673 & -2.383852 & 3.212093 \\
\hline $\mathrm{H}$ & 0.519060 & -0.883681 & 3.618123 \\
\hline $\mathrm{C}$ & 6.922206 & 0.515748 & 0.958507 \\
\hline $\mathrm{C}$ & 6.278627 & -1.469575 & 2.229565 \\
\hline $\mathrm{H}$ & 7.346782 & 2.312948 & -0.164731 \\
\hline $\mathrm{H}$ & 5.785817 & 3.556205 & -1.450065 \\
\hline $\mathrm{H}$ & 4.516700 & 2.567673 & -2.208931 \\
\hline $\mathrm{H}$ & 4.136321 & 3.534765 & -0.793716 \\
\hline $\mathrm{C}$ & -0.968920 & 2.466104 & 4.296855 \\
\hline $\mathrm{H}$ & 0.052906 & 1.361642 & 2.766384 \\
\hline $\mathrm{C}$ & -0.495366 & 4.831732 & 4.384052 \\
\hline $\mathrm{H}$ & 0.898706 & 5.562582 & 2.914786 \\
\hline $\mathrm{C}$ & -2.730021 & 4.332451 & 1.495016 \\
\hline $\mathrm{H}$ & -1.836490 & 2.424848 & 1.071940 \\
\hline $\mathrm{C}$ & -1.679034 & 6.235220 & 0.450460 \\
\hline $\mathrm{H}$ & 0.019190 & 5.812254 & -0.792805 \\
\hline $\mathrm{C}$ & 5.140877 & -4.525472 & -0.389962 \\
\hline $\mathrm{H}$ & 5.692598 & -2.461077 & -0.383754 \\
\hline $\mathrm{C}$ & 3.098382 & -5.115526 & 0.755408 \\
\hline $\mathrm{C}$ & 8.229481 & 0.394785 & 1.496289 \\
\hline $\mathrm{C}$ & 7.553438 & -1.553247 & 2.745614 \\
\hline $\mathrm{H}$ & 5.527975 & -2.185585 & 2.540471 \\
\hline $\mathrm{C}$ & -1.181887 & 3.719312 & 4.874238 \\
\hline $\mathrm{H}$ & -1.522177 & 1.597592 & 4.637406 \\
\hline $\mathrm{H}$ & -0.665695 & 5.814092 & 4.816618 \\
\hline $\mathrm{C}$ & -2.669524 & 5.712151 & 1.279989 \\
\hline
\end{tabular}

$\begin{array}{lrrr}\text { H } & -3.482505 & 3.910594 & 2.154795 \\ \text { H } & -1.623648 & 7.306079 & 0.274431 \\ \text { C } & 4.203072 & -5.497534 & 0.028472 \\ \text { H } & 6.006033 & -4.826980 & -0.973193 \\ \text { H } & 2.366472 & -5.852314 & 1.076942 \\ \text { C } & 8.546922 & -0.621764 & 2.367952 \\ \text { H } & 8.975071 & 1.133514 & 1.212568 \\ \text { H } & 7.793991 & -2.339710 & 3.455351 \\ \text { H } & -1.887937 & 3.831165 & 5.692828 \\ \text { H } & -3.384993 & 6.373514 & 1.761070 \\ \text { H } & 4.354020 & -6.541664 & -0.229477 \\ \text { H } & 9.550014 & -0.701520 & 2.776816\end{array}$

\section{S2}

\begin{abstract}
Zero-point correction=1.203761 (Hartree/Particle)
Thermal correction to Energy=1.278066

Thermal correction to Enthalpy $=1.279010$

Thermal correction to Gibbs Free Energy=1.092280

$\mathrm{E}($ Solv $)=-4250.70982272$
\end{abstract}

\begin{tabular}{|c|c|c|c|}
\hline $\mathrm{C}$ & 4.514485 & 0.139503 & 3.547486 \\
\hline $\mathrm{C}$ & 3.545073 & 0.890064 & 2.884134 \\
\hline $\mathrm{C}$ & 2.208730 & 0.468812 & 2.853784 \\
\hline $\mathrm{C}$ & 1.864421 & -0.727147 & 3.502345 \\
\hline $\mathrm{C}$ & 2.829943 & -1.474802 & 4.170035 \\
\hline $\mathrm{C}$ & 4.160998 & -1.045486 & 4.195899 \\
\hline $\mathrm{H}$ & 5.547080 & 0.477094 & 3.551927 \\
\hline $\mathrm{H}$ & 3.819930 & 1.810945 & 2.379223 \\
\hline $\mathrm{H}$ & 0.839635 & -1.076978 & 3.463908 \\
\hline $\mathrm{H}$ & 2.544229 & -2.394742 & 4.672147 \\
\hline $\mathrm{H}$ & 4.914948 & -1.631441 & 4.714124 \\
\hline $\mathrm{C}$ & 1.198727 & 1.297196 & 2.157441 \\
\hline $\mathrm{H}$ & 1.631482 & 2.101345 & 1.561826 \\
\hline $\mathrm{C}$ & -0.065704 & 1.587186 & 2.765229 \\
\hline $\mathrm{H}$ & -0.381501 & 0.996727 & 3.621568 \\
\hline $\mathrm{C}$ & -0.765912 & 2.783352 & 2.500706 \\
\hline $\mathrm{C}$ & -1.930986 & 3.163032 & 3.150840 \\
\hline $\mathrm{H}$ & -2.182455 & 2.690277 & 4.095679 \\
\hline $\mathrm{H}$ & -0.385368 & 3.407088 & 1.691975 \\
\hline $\mathrm{H}$ & -2.319099 & 4.165236 & 2.996592 \\
\hline $\mathrm{Pd}$ & -0.083008 & 0.253238 & 0.873512 \\
\hline $\mathrm{C}$ & -5.031637 & 4.301585 & -0.428459 \\
\hline
\end{tabular}




\begin{tabular}{|c|c|c|c|}
\hline $\mathrm{C}$ & -4.795063 & 3.572691 & 0.736565 \\
\hline $\mathrm{C}$ & -3.620929 & 2.822332 & 0.887959 \\
\hline $\mathrm{C}$ & -2.673498 & 2.828094 & -0.146663 \\
\hline $\mathrm{C}$ & -2.905905 & 3.571635 & -1.299135 \\
\hline $\mathrm{C}$ & -4.084269 & 4.306324 & -1.449092 \\
\hline $\mathrm{H}$ & -5.957003 & 4.859407 & -0.537474 \\
\hline $\mathrm{H}$ & -5.535065 & 3.567273 & 1.532277 \\
\hline $\mathrm{H}$ & -1.751164 & 2.264571 & -0.044172 \\
\hline $\mathrm{H}$ & -2.172622 & 3.563992 & -2.095686 \\
\hline $\mathrm{H}$ & -4.267759 & 4.859176 & -2.364647 \\
\hline $\mathrm{C}$ & -3.459812 & 2.058656 & 2.148600 \\
\hline $\mathrm{N}$ & -3.111207 & 0.759226 & 2.106704 \\
\hline $\mathrm{S}$ & -3.588272 & -0.152973 & 3.434721 \\
\hline $\mathrm{O}$ & -3.593577 & 0.684527 & 4.648049 \\
\hline $\mathrm{O}$ & -4.814134 & -0.884079 & 3.067711 \\
\hline $\mathrm{C}$ & -2.300557 & -1.378982 & 3.610979 \\
\hline $\mathrm{C}$ & -2.121400 & -2.359896 & 2.632140 \\
\hline $\mathrm{C}$ & -1.608747 & -1.436571 & 4.819347 \\
\hline $\mathrm{C}$ & -1.232133 & -3.402568 & 2.873856 \\
\hline $\mathrm{H}$ & -2.671141 & -2.323722 & 1.699532 \\
\hline $\mathrm{C}$ & -0.724821 & -2.491110 & 5.045864 \\
\hline $\mathrm{H}$ & -1.785843 & -0.677265 & 5.572538 \\
\hline $\mathrm{C}$ & -0.525027 & -3.487773 & 4.083061 \\
\hline $\mathrm{H}$ & -1.106458 & -4.172293 & 2.117881 \\
\hline $\mathrm{H}$ & -0.189246 & -2.543347 & 5.989936 \\
\hline $\mathrm{C}$ & 0.439036 & -4.619770 & 4.335271 \\
\hline $\mathrm{H}$ & 0.130352 & -5.530780 & 3.812588 \\
\hline $\mathrm{H}$ & 1.444703 & -4.364075 & 3.978649 \\
\hline $\mathrm{H}$ & 0.516604 & -4.847209 & 5.403217 \\
\hline $\mathrm{H}$ & -4.139396 & 2.365619 & 2.942170 \\
\hline $\mathrm{C}$ & -2.794717 & -0.358086 & -1.132785 \\
\hline $\mathrm{O}$ & -2.199991 & -0.438404 & 0.075371 \\
\hline $\mathrm{O}$ & -2.154570 & -0.611386 & -2.140632 \\
\hline $\mathrm{H}$ & -2.708930 & 0.046696 & 0.870579 \\
\hline $\mathrm{C}$ & -4.231453 & 0.042992 & -1.124312 \\
\hline $\mathrm{C}$ & -4.778780 & 0.859887 & -2.171548 \\
\hline $\mathrm{C}$ & -5.028052 & -0.350315 & -0.063811 \\
\hline $\mathrm{C}$ & -4.053262 & 1.268061 & -3.322721 \\
\hline $\mathrm{C}$ & -6.127687 & 1.324061 & -2.023323 \\
\hline $\mathrm{C}$ & -6.366037 & 0.076728 & 0.049035 \\
\hline $\mathrm{H}$ & -4.634132 & -0.985731 & 0.717173 \\
\hline $\mathrm{C}$ & -4.622760 & 2.101726 & -4.259968 \\
\hline $\mathrm{H}$ & -3.049134 & 0.895257 & -3.463456 \\
\hline $\mathrm{C}$ & -6.677715 & 2.192061 & -3.002569 \\
\hline
\end{tabular}

\begin{tabular}{|c|c|c|c|}
\hline $\mathrm{C}$ & -6.895806 & 0.915683 & -0.901572 \\
\hline $\mathrm{H}$ & -6.947031 & -0.242937 & 0.907672 \\
\hline $\mathrm{C}$ & -5.943206 & 2.579178 & -4.099155 \\
\hline $\mathrm{H}$ & -4.048572 & 2.394767 & -5.134791 \\
\hline $\mathrm{H}$ & -7.698280 & 2.541766 & -2.868333 \\
\hline $\mathrm{H}$ & -7.916674 & 1.279043 & -0.812576 \\
\hline $\mathrm{H}$ & -6.376377 & 3.240652 & -4.844114 \\
\hline $\mathrm{P}$ & 1.469099 & -0.695933 & -0.520346 \\
\hline $\mathrm{O}$ & 1.837777 & 0.137777 & -1.927099 \\
\hline $\mathrm{O}$ & 2.968962 & -0.637620 & 0.236176 \\
\hline $\mathrm{N}$ & 1.291846 & -2.264633 & -1.050221 \\
\hline $\mathrm{C}$ & 1.939910 & 1.505557 & -1.658972 \\
\hline $\mathrm{C}$ & 4.160276 & -0.125901 & -0.203657 \\
\hline $\mathrm{C}$ & 1.940270 & -2.971306 & -2.186798 \\
\hline $\mathrm{C}$ & 0.667314 & -3.148166 & -0.018154 \\
\hline $\mathrm{C}$ & 3.044963 & 1.978752 & -0.975549 \\
\hline $\mathrm{C}$ & 0.865461 & 2.330430 & -2.085798 \\
\hline $\mathrm{C}$ & 4.246221 & 1.123970 & -0.794328 \\
\hline $\mathrm{C}$ & 5.305939 & -0.928189 & 0.074910 \\
\hline $\mathrm{C}$ & 3.255252 & -2.351569 & -2.676219 \\
\hline $\mathrm{C}$ & 0.961089 & -3.161810 & -3.336259 \\
\hline $\mathrm{H}$ & 2.186967 & -3.968041 & -1.805400 \\
\hline $\mathrm{C}$ & 1.734308 & -3.742297 & 0.905837 \\
\hline $\mathrm{C}$ & -0.267691 & -4.162743 & -0.654199 \\
\hline $\mathrm{H}$ & 0.045952 & -2.483152 & 0.593467 \\
\hline $\mathrm{C}$ & 2.984739 & 3.317172 & -0.454766 \\
\hline $\mathrm{C}$ & 0.850237 & 3.632466 & -1.641491 \\
\hline $\mathrm{C}$ & -0.165939 & 1.765405 & -3.021157 \\
\hline $\mathrm{C}$ & 5.528266 & 1.572076 & -1.269543 \\
\hline $\mathrm{C}$ & 6.536839 & -0.453016 & -0.308738 \\
\hline $\mathrm{C}$ & 5.142051 & -2.242373 & 0.787236 \\
\hline $\mathrm{H}$ & 3.676075 & -3.012618 & -3.440340 \\
\hline $\mathrm{H}$ & 3.982755 & -2.254381 & -1.867334 \\
\hline $\mathrm{H}$ & 3.099405 & -1.370334 & -3.123946 \\
\hline $\mathrm{C}$ & 0.167486 & -2.104867 & -3.789644 \\
\hline $\mathrm{C}$ & 0.839665 & -4.413409 & -3.944738 \\
\hline $\mathrm{H}$ & 1.282278 & -4.415428 & 1.638194 \\
\hline $\mathrm{H}$ & 2.244356 & -2.940703 & 1.443998 \\
\hline $\mathrm{H}$ & 2.485630 & -4.311241 & 0.347908 \\
\hline $\mathrm{C}$ & -1.384084 & -3.688463 & -1.355098 \\
\hline $\mathrm{C}$ & -0.048599 & -5.540998 & -0.585191 \\
\hline $\mathrm{C}$ & 3.969681 & 3.837818 & 0.426896 \\
\hline $\mathrm{C}$ & 1.853265 & 4.135295 & -0.770909 \\
\hline $\mathrm{H}$ & 0.041521 & 4.294392 & -1.938834 \\
\hline
\end{tabular}




\begin{tabular}{|c|c|c|c|}
\hline $\mathrm{H}$ & -0.776044 & 0.997397 & -2.539835 \\
\hline $\mathrm{H}$ & -0.825074 & 2.553089 & -3.395264 \\
\hline $\mathrm{H}$ & 0.315555 & 1.287282 & -3.881667 \\
\hline C & 6.686620 & 0.776457 & -1.000174 \\
\hline $\mathrm{C}$ & 5.698482 & 2.764037 & -2.024549 \\
\hline $\mathrm{H}$ & 7.426074 & -1.042196 & -0.097236 \\
\hline $\mathrm{H}$ & 6.116184 & -2.711300 & 0.954804 \\
\hline $\mathrm{H}$ & 4.647525 & -2.104046 & 1.753451 \\
\hline $\mathrm{H}$ & 4.521773 & -2.935361 & 0.209003 \\
\hline $\mathrm{C}$ & -0.749396 & -2.303022 & -4.819393 \\
\hline $\mathrm{H}$ & 0.229507 & -1.141732 & -3.301649 \\
\hline $\mathrm{C}$ & -0.072117 & -4.613774 & -4.980991 \\
\hline $\mathrm{H}$ & 1.434391 & -5.246924 & -3.578982 \\
\hline $\mathrm{C}$ & -2.255625 & -4.570905 & -1.983793 \\
\hline $\mathrm{H}$ & -1.529895 & -2.620520 & -1.452342 \\
\hline $\mathrm{C}$ & -0.925962 & -6.430779 & -1.213067 \\
\hline $\mathrm{H}$ & 0.811351 & -5.933105 & -0.051659 \\
\hline C & 3.839949 & 5.097049 & 0.970761 \\
\hline $\mathrm{H}$ & 4.826309 & 3.223676 & 0.677654 \\
\hline $\mathrm{C}$ & 1.759559 & 5.435544 & -0.209213 \\
\hline $\mathrm{C}$ & 7.958574 & 1.220839 & -1.444947 \\
\hline $\mathrm{C}$ & 6.945548 & 3.163606 & -2.452630 \\
\hline $\mathrm{H}$ & 4.829994 & 3.361318 & -2.273587 \\
\hline $\mathrm{C}$ & -0.873344 & -3.558224 & -5.418680 \\
\hline $\mathrm{H}$ & -1.383280 & -1.479724 & -5.135978 \\
\hline $\mathrm{H}$ & -0.170638 & -5.597938 & -5.430835 \\
\hline $\mathrm{C}$ & -2.028759 & -5.948520 & -1.914965 \\
\hline $\mathrm{H}$ & -3.099861 & -4.180427 & -2.544141 \\
\hline $\mathrm{H}$ & -0.740163 & -7.499900 & -1.155063 \\
\hline C & 2.727532 & 5.908174 & 0.648045 \\
\hline $\mathrm{H}$ & 4.597360 & 5.468585 & 1.654669 \\
\hline $\mathrm{H}$ & 0.897546 & 6.047745 & -0.462037 \\
\hline $\mathrm{C}$ & 8.092289 & 2.393721 & -2.152148 \\
\hline $\mathrm{H}$ & 8.827562 & 0.606421 & -1.222743 \\
\hline $\mathrm{H}$ & 7.046531 & 4.076567 & -3.032566 \\
\hline $\mathrm{H}$ & -1.597073 & -3.715692 & -6.213783 \\
\hline $\mathrm{H}$ & -2.705315 & -6.639628 & -2.409996 \\
\hline $\mathrm{H}$ & 2.637643 & 6.900184 & 1.080744 \\
\hline $\mathrm{H}$ & 9.070607 & 2.722068 & -2.490838 \\
\hline
\end{tabular}

\section{S3}

Zero-point correction= $1.203363($ Hartree/Particle $)$

\begin{abstract}
Thermal correction to Energy=1.277609
Thermal correction to Enthalpy=1.278554

Thermal correction to Gibbs Free Energy= 1.091664

$\mathrm{E}($ Solv $)=-4250.70661030$
\end{abstract}

\begin{tabular}{|c|c|c|c|}
\hline $\mathrm{C}$ & -4.137991 & -0.900731 & 3.770286 \\
\hline $\mathrm{C}$ & -3.398806 & -1.486701 & 2.743785 \\
\hline $\mathrm{C}$ & -2.016663 & -1.278097 & 2.644402 \\
\hline $\mathrm{C}$ & -1.388544 & -0.464249 & 3.600253 \\
\hline $\mathrm{C}$ & -2.123034 & 0.111621 & 4.632734 \\
\hline $\mathrm{C}$ & -3.502731 & -0.102521 & 4.722723 \\
\hline $\mathrm{H}$ & -5.210419 & -1.065906 & 3.823617 \\
\hline $\mathrm{H}$ & -3.891784 & -2.113569 & 2.007808 \\
\hline $\mathrm{H}$ & -0.324556 & -0.274452 & 3.521580 \\
\hline $\mathrm{H}$ & -1.620505 & 0.731016 & 5.369953 \\
\hline $\mathrm{H}$ & -4.075632 & 0.352507 & 5.525867 \\
\hline $\mathrm{C}$ & -1.259549 & -1.936774 & 1.556576 \\
\hline $\mathrm{H}$ & -1.898935 & -2.431427 & 0.823397 \\
\hline $\mathrm{C}$ & -0.000832 & -2.576730 & 1.813374 \\
\hline $\mathrm{H}$ & 0.527386 & -2.342874 & 2.734371 \\
\hline $\mathrm{C}$ & 0.425825 & -3.701639 & 1.080135 \\
\hline $\mathrm{C}$ & 1.584598 & -4.425201 & 1.341289 \\
\hline $\mathrm{H}$ & 2.062300 & -4.321671 & 2.311245 \\
\hline $\mathrm{H}$ & -0.178575 & -3.978787 & 0.216534 \\
\hline $\mathrm{H}$ & 1.718696 & -5.383050 & 0.846981 \\
\hline $\mathrm{Pd}$ & 0.044560 & -0.726874 & 0.453526 \\
\hline $\mathrm{C}$ & 3.509833 & -4.874948 & -3.050039 \\
\hline $\mathrm{C}$ & 3.701911 & -4.548982 & -1.707824 \\
\hline $\mathrm{C}$ & 2.841305 & -3.657485 & -1.052716 \\
\hline $\mathrm{C}$ & 1.767128 & -3.107808 & -1.767375 \\
\hline $\mathrm{C}$ & 1.565456 & -3.448256 & -3.102010 \\
\hline $\mathrm{C}$ & 2.436862 & -4.326449 & -3.752144 \\
\hline $\mathrm{H}$ & 4.194792 & -5.559530 & -3.542115 \\
\hline $\mathrm{H}$ & 4.538100 & -4.977107 & -1.161639 \\
\hline $\mathrm{H}$ & 1.079926 & -2.423400 & -1.280915 \\
\hline $\mathrm{H}$ & 0.730775 & -3.015145 & -3.639021 \\
\hline $\mathrm{H}$ & 2.277477 & -4.580499 & -4.796168 \\
\hline $\mathrm{C}$ & 3.122065 & -3.366921 & 0.377196 \\
\hline $\mathrm{N}$ & 3.155130 & -2.093818 & 0.820514 \\
\hline $\mathrm{S}$ & 4.044519 & -1.841506 & 2.230116 \\
\hline $\mathrm{O}$ & 4.088715 & -3.089213 & 3.016724 \\
\hline $\mathrm{O}$ & 5.297663 & -1.168504 & 1.871007 \\
\hline $\mathrm{C}$ & 3.048429 & -0.662591 & 3.130059 \\
\hline $\mathrm{C}$ & 2.984470 & 0.658010 & 2.683242 \\
\hline
\end{tabular}




\begin{tabular}{|c|c|c|c|}
\hline $\mathrm{C}$ & 2.444920 & -1.047023 & 4.324930 \\
\hline $\mathrm{C}$ & 2.292862 & 1.597727 & 3.439190 \\
\hline $\mathrm{H}$ & 3.497982 & 0.948171 & 1.777256 \\
\hline $\mathrm{C}$ & 1.761405 & -0.089333 & 5.075519 \\
\hline $\mathrm{H}$ & 2.535213 & -2.072411 & 4.665575 \\
\hline $\mathrm{C}$ & 1.672826 & 1.240909 & 4.646787 \\
\hline $\mathrm{H}$ & 2.257899 & 2.628047 & 3.095864 \\
\hline $\mathrm{H}$ & 1.292834 & -0.380745 & 6.011597 \\
\hline $\mathrm{C}$ & 0.920029 & 2.268046 & 5.454807 \\
\hline $\mathrm{H}$ & 1.456144 & 3.222922 & 5.484736 \\
\hline $\mathrm{H}$ & -0.066224 & 2.464291 & 5.016741 \\
\hline $\mathrm{H}$ & 0.765665 & 1.931864 & 6.484719 \\
\hline $\mathrm{H}$ & 3.833112 & -4.056370 & 0.830128 \\
\hline $\mathrm{C}$ & 2.565827 & 0.072163 & -1.664658 \\
\hline $\mathrm{O}$ & 2.119877 & -0.163366 & -0.416279 \\
\hline $\mathrm{O}$ & 1.831262 & 0.508592 & -2.531844 \\
\hline $\mathrm{H}$ & 2.684050 & -0.932907 & 0.097120 \\
\hline $\mathrm{C}$ & 4.010505 & -0.257679 & -1.882345 \\
\hline $\mathrm{C}$ & 5.036343 & 0.358038 & -1.102078 \\
\hline $\mathrm{C}$ & 4.325550 & -1.212137 & -2.827886 \\
\hline C & 4.791133 & 1.450457 & -0.231057 \\
\hline $\mathrm{C}$ & 6.380655 & -0.108922 & -1.254310 \\
\hline $\mathrm{C}$ & 5.654652 & -1.665948 & -2.974122 \\
\hline $\mathrm{H}$ & 3.537274 & -1.652221 & -3.426185 \\
\hline $\mathrm{C}$ & 5.814465 & 2.024661 & 0.487444 \\
\hline $\mathrm{H}$ & 3.789897 & 1.856280 & -0.157026 \\
\hline $\mathrm{C}$ & 7.408530 & 0.489719 & -0.480357 \\
\hline $\mathrm{C}$ & 6.654988 & -1.141143 & -2.188984 \\
\hline $\mathrm{H}$ & 5.871505 & -2.446320 & -3.696979 \\
\hline $\mathrm{C}$ & 7.134053 & 1.528930 & 0.377732 \\
\hline $\mathrm{H}$ & 5.605047 & 2.866887 & 1.140815 \\
\hline $\mathrm{H}$ & 8.423209 & 0.114868 & -0.586957 \\
\hline $\mathrm{H}$ & 7.676936 & -1.499644 & -2.282824 \\
\hline $\mathrm{H}$ & 7.930274 & 1.979232 & 0.963075 \\
\hline $\mathrm{P}$ & -1.407281 & 0.908928 & -0.217404 \\
\hline $\mathrm{O}$ & -2.172416 & 0.723660 & -1.694280 \\
\hline $\mathrm{O}$ & -2.744313 & 0.878136 & 0.797303 \\
\hline $\mathrm{N}$ & -0.898004 & 2.495134 & -0.274409 \\
\hline $\mathrm{C}$ & -2.616671 & -0.590896 & -1.859313 \\
\hline $\mathrm{C}$ & -4.080219 & 0.834821 & 0.496223 \\
\hline $\mathrm{C}$ & -1.511711 & 3.667284 & -0.955133 \\
\hline $\mathrm{C}$ & 0.098350 & 2.821952 & 0.791626 \\
\hline $\mathrm{C}$ & -3.716877 & -1.024847 & -1.142588 \\
\hline $\mathrm{C}$ & -1.884903 & -1.412854 & -2.756889 \\
\hline
\end{tabular}

\begin{tabular}{|c|c|c|c|}
\hline $\mathrm{C}$ & -4.591141 & -0.053659 & -0.435375 \\
\hline $\mathrm{C}$ & -4.906582 & 1.697929 & 1.274250 \\
\hline $\mathrm{C}$ & -3.011797 & 3.542504 & -1.250243 \\
\hline $\mathrm{C}$ & -0.738433 & 4.039636 & -2.212415 \\
\hline $\mathrm{H}$ & -1.399699 & 4.499763 & -0.252122 \\
\hline $\mathrm{C}$ & -0.611105 & 3.237581 & 2.083848 \\
\hline $\mathrm{C}$ & 1.139923 & 3.805850 & 0.284227 \\
\hline $\mathrm{H}$ & 0.624498 & 1.881671 & 0.999517 \\
\hline $\mathrm{C}$ & -3.984607 & -2.437060 & -1.120342 \\
\hline $\mathrm{C}$ & -2.191195 & -2.754409 & -2.777917 \\
\hline $\mathrm{C}$ & -0.862699 & -0.787535 & -3.664291 \\
\hline $\mathrm{C}$ & -5.997407 & 0.002879 & -0.736118 \\
\hline $\mathrm{C}$ & -6.260522 & 1.679851 & 1.041346 \\
\hline $\mathrm{C}$ & -4.287699 & 2.576249 & 2.326363 \\
\hline $\mathrm{H}$ & -3.353068 & 4.493794 & -1.670330 \\
\hline $\mathrm{H}$ & -3.587008 & 3.341783 & -0.344298 \\
\hline $\mathrm{H}$ & -3.218789 & 2.756814 & -1.976540 \\
\hline $\mathrm{C}$ & -0.322785 & 3.066320 & -3.125244 \\
\hline $\mathrm{C}$ & -0.426428 & 5.379136 & -2.457737 \\
\hline $\mathrm{H}$ & 0.111637 & 3.493428 & 2.862602 \\
\hline $\mathrm{H}$ & -1.227826 & 2.411895 & 2.445936 \\
\hline $\mathrm{H}$ & -1.260028 & 4.106394 & 1.929685 \\
\hline $\mathrm{C}$ & 1.912946 & 3.446147 & -0.827264 \\
\hline $\mathrm{C}$ & 1.349313 & 5.056240 & 0.871941 \\
\hline $\mathrm{C}$ & -4.972985 & -3.015319 & -0.279022 \\
\hline $\mathrm{C}$ & -3.185304 & -3.307839 & -1.928304 \\
\hline $\mathrm{H}$ & -1.653175 & -3.418873 & -3.449141 \\
\hline $\mathrm{H}$ & 0.027297 & -0.456393 & -3.121432 \\
\hline $\mathrm{H}$ & -0.558513 & -1.491917 & -4.443695 \\
\hline $\mathrm{H}$ & -1.277474 & 0.099322 & -4.155815 \\
\hline $\mathrm{C}$ & -6.839010 & 0.868295 & 0.031925 \\
\hline $\mathrm{C}$ & -6.592187 & -0.744212 & -1.788638 \\
\hline $\mathrm{H}$ & -6.912219 & 2.321105 & 1.630121 \\
\hline $\mathrm{H}$ & -5.060444 & 3.138992 & 2.858396 \\
\hline $\mathrm{H}$ & -3.725398 & 1.979933 & 3.050929 \\
\hline $\mathrm{H}$ & -3.583490 & 3.290828 & 1.887058 \\
\hline $\mathrm{C}$ & 0.412695 & 3.425477 & -4.252762 \\
\hline $\mathrm{H}$ & -0.528784 & 2.023128 & -2.927124 \\
\hline $\mathrm{C}$ & 0.304382 & 5.743135 & -3.588465 \\
\hline $\mathrm{H}$ & -0.723746 & 6.138715 & -1.738467 \\
\hline $\mathrm{C}$ & 2.872367 & 4.312455 & -1.340550 \\
\hline $\mathrm{H}$ & 1.723175 & 2.500083 & -1.317310 \\
\hline $\mathrm{C}$ & 2.313172 & 5.930049 & 0.358592 \\
\hline $\mathrm{H}$ & 0.758946 & 5.364787 & 1.728494 \\
\hline
\end{tabular}




\begin{tabular}{llll} 
C & -5.157305 & -4.379882 & -0.234783 \\
H & -5.580586 & -2.365800 & 0.339439 \\
C & -3.412955 & -4.708010 & -1.872601 \\
C & -8.230404 & 0.913951 & -0.242103 \\
C & -7.944886 & -0.669066 & -2.038898 \\
H & -5.966986 & -1.374717 & -2.408787 \\
C & 0.730497 & 4.764882 & -4.488137 \\
H & 0.755134 & 2.652645 & -4.934923 \\
H & 0.557518 & 6.786584 & -3.755188 \\
C & 3.076635 & 5.560873 & -0.747148 \\
H & 3.450866 & 4.014376 & -2.209303 \\
H & 2.461441 & 6.900055 & 0.825429 \\
C & -4.374143 & -5.237362 & -1.041637 \\
H & -5.909675 & -4.800131 & 0.426134 \\
H & -2.802557 & -5.356655 & -2.495872 \\
C & -8.779103 & 0.157604 & -1.252342 \\
H & -8.854333 & 1.570941 & 0.358811 \\
H & -8.372522 & -1.246455 & -2.853607 \\
H & 1.314174 & 5.043366 & -5.361308 \\
H & 3.822436 & 6.241438 & -1.148113 \\
H & -4.530935 & -6.311236 & -1.000983 \\
\hline & -9.844947 & 0.203975 & -1.455960 \\
\hline & & & \\
\hline
\end{tabular}

\begin{tabular}{|c|c|c|c|}
\hline $\mathrm{H}$ & -1.530647 & -0.643690 & 0.867497 \\
\hline $\mathrm{C}$ & -0.840164 & -2.638767 & 0.507209 \\
\hline $\mathrm{H}$ & -0.513781 & -3.569024 & 0.962799 \\
\hline $\mathrm{C}$ & -1.342911 & -2.648369 & -0.805106 \\
\hline $\mathrm{C}$ & -1.629775 & -3.828120 & -1.543053 \\
\hline $\mathrm{H}$ & -1.818593 & -3.685553 & -2.604691 \\
\hline $\mathrm{H}$ & -1.790006 & -1.723002 & -1.167837 \\
\hline $\mathrm{H}$ & -1.042903 & -4.712892 & -1.299734 \\
\hline $\mathrm{Pd}$ & 0.778122 & -1.310226 & -0.108836 \\
\hline $\mathrm{C}$ & -3.130834 & -4.250234 & 2.870099 \\
\hline $\mathrm{C}$ & -3.467936 & -3.874378 & 1.568687 \\
\hline $\mathrm{C}$ & -3.095475 & -4.671932 & 0.479122 \\
\hline $\mathrm{C}$ & -2.417234 & -5.873925 & 0.729153 \\
\hline $\mathrm{C}$ & -2.086563 & -6.254611 & 2.027939 \\
\hline $\mathrm{C}$ & -2.430662 & -5.433270 & 3.105643 \\
\hline $\mathrm{H}$ & -3.409855 & -3.607958 & 3.700665 \\
\hline $\mathrm{H}$ & -4.014498 & -2.955945 & 1.401762 \\
\hline $\mathrm{H}$ & -2.136644 & -6.511396 & -0.106157 \\
\hline $\mathrm{H}$ & -1.562325 & -7.190701 & 2.199519 \\
\hline $\mathrm{H}$ & -2.164767 & -5.720252 & 4.119113 \\
\hline $\mathrm{C}$ & -3.301565 & -4.233911 & -0.942873 \\
\hline $\mathrm{N}$ & -4.197589 & -3.189723 & -1.133938 \\
\hline $\mathrm{S}$ & -4.801699 & -3.081015 & -2.665087 \\
\hline $\mathrm{O}$ & -3.718002 & -2.901283 & -3.662547 \\
\hline $\mathrm{O}$ & -5.773235 & -4.159068 & -2.928736 \\
\hline $\mathrm{C}$ & -5.665388 & -1.523609 & -2.523565 \\
\hline $\mathrm{C}$ & -5.247447 & -0.446475 & -3.300289 \\
\hline $\mathrm{C}$ & -6.716234 & -1.390195 & -1.615430 \\
\hline $\mathrm{C}$ & -5.883625 & 0.784433 & -3.151200 \\
\hline $\mathrm{H}$ & -4.425362 & -0.582391 & -3.993349 \\
\hline $\mathrm{C}$ & -7.323730 & -0.147770 & -1.460787 \\
\hline $\mathrm{H}$ & -7.030740 & -2.242517 & -1.023159 \\
\hline $\mathrm{C}$ & -6.910127 & 0.959656 & -2.215326 \\
\hline $\mathrm{H}$ & -5.561960 & 1.631196 & -3.750589 \\
\hline $\mathrm{H}$ & -8.126104 & -0.029889 & -0.737076 \\
\hline $\mathrm{C}$ & -7.522188 & 2.319261 & -1.991508 \\
\hline $\mathrm{H}$ & -6.970217 & 2.866642 & -1.217390 \\
\hline $\mathrm{H}$ & -7.491170 & 2.927180 & -2.901292 \\
\hline $\mathrm{H}$ & -8.564256 & 2.241648 & -1.664153 \\
\hline $\mathrm{H}$ & -3.455409 & -5.097943 & -1.600820 \\
\hline $\mathrm{C}$ & -3.711933 & 0.091781 & -0.198619 \\
\hline $\mathrm{O}$ & -2.830749 & 0.124398 & -1.060989 \\
\hline $\mathrm{O}$ & -4.270626 & -1.014015 & 0.246204 \\
\hline $\mathrm{H}$ & & -1.874367 & \\
\hline
\end{tabular}




\begin{tabular}{|c|c|c|c|c|c|c|c|}
\hline $\mathrm{C}$ & -4.232960 & 1.317993 & 0.487437 & $\mathrm{C}$ & 1.687438 & 5.074043 & 1.608104 \\
\hline $\mathrm{C}$ & -4.293174 & 2.587834 & -0.172555 & $\mathrm{C}$ & 2.434982 & 2.938974 & 2.425310 \\
\hline $\mathrm{C}$ & -4.689403 & 1.191473 & 1.788087 & $\mathrm{H}$ & -1.098994 & 2.568892 & -2.448904 \\
\hline $\mathrm{C}$ & -3.873892 & 2.798014 & -1.514461 & $\mathrm{H}$ & 0.422154 & 1.746564 & -2.843722 \\
\hline $\mathrm{C}$ & -4.858044 & 3.696400 & 0.541570 & $\mathrm{H}$ & 0.430472 & 3.467954 & -2.419275 \\
\hline $\mathrm{C}$ & -5.191873 & 2.299995 & 2.499921 & $\mathrm{C}$ & -0.741762 & 2.475821 & 1.575958 \\
\hline $\mathrm{H}$ & -4.662729 & 0.214146 & 2.256126 & $\mathrm{C}$ & -1.168084 & 4.192762 & -0.053943 \\
\hline $\mathrm{C}$ & -4.019683 & 4.027757 & -2.116647 & $\mathrm{C}$ & 6.623276 & -2.676784 & -1.191737 \\
\hline $\mathrm{H}$ & -3.443575 & 1.966734 & -2.053236 & $\mathrm{C}$ & 5.617494 & -2.955432 & 1.025334 \\
\hline $\mathrm{C}$ & -4.987333 & 4.952166 & -0.109473 & $\mathrm{H}$ & 4.647642 & -3.094601 & 2.952787 \\
\hline $\mathrm{C}$ & -5.282804 & 3.525820 & 1.883623 & $\mathrm{H}$ & 2.152213 & -0.585397 & 2.652411 \\
\hline $\mathrm{H}$ & -5.523421 & 2.173715 & 3.526441 & $\mathrm{H}$ & 3.064968 & -1.528396 & 3.836335 \\
\hline $\mathrm{C}$ & -4.581659 & 5.118236 & -1.412859 & $\mathrm{H}$ & 3.543253 & 0.137182 & 3.459182 \\
\hline $\mathrm{H}$ & -3.700043 & 4.161304 & -3.146708 & $\mathrm{C}$ & 6.871066 & 1.318036 & -2.872880 \\
\hline $\mathrm{H}$ & -5.420401 & 5.780709 & 0.445465 & $\mathrm{C}$ & 7.824064 & 0.242361 & -0.892027 \\
\hline $\mathrm{H}$ & -5.687209 & 4.384217 & 2.414250 & $\mathrm{H}$ & 5.899452 & 2.252136 & -4.562539 \\
\hline $\mathrm{H}$ & -4.692773 & 6.081276 & -1.903173 & $\mathrm{H}$ & 3.564364 & 2.200799 & -5.035084 \\
\hline $\mathrm{P}$ & 2.203175 & 0.462185 & -0.385638 & $\mathrm{H}$ & 2.675639 & 0.790797 & -4.415260 \\
\hline $\mathrm{O}$ & 3.513614 & 0.627878 & 0.627729 & $\mathrm{H}$ & 2.593567 & 2.320431 & -3.555268 \\
\hline $\mathrm{O}$ & 3.009123 & 0.132162 & -1.824824 & $\mathrm{C}$ & 1.560704 & 5.537053 & 2.917621 \\
\hline $\mathrm{N}$ & 1.575612 & 2.000867 & -0.351454 & $\mathrm{H}$ & 1.416959 & 5.723644 & 0.780097 \\
\hline C & 4.262893 & -0.543543 & 0.764466 & $\mathrm{C}$ & 2.308279 & 3.397099 & 3.735070 \\
\hline $\mathrm{C}$ & 4.306792 & 0.492247 & -2.142276 & $\mathrm{H}$ & 2.767270 & 1.928226 & 2.236378 \\
\hline $\mathrm{C}$ & 2.245899 & 3.308591 & -0.102070 & $\mathrm{C}$ & -1.379068 & 3.231342 & 2.553480 \\
\hline $\mathrm{C}$ & 0.126610 & 2.091333 & -0.737195 & $\mathrm{H}$ & -0.287926 & 1.524491 & 1.832893 \\
\hline $\mathrm{C}$ & 5.145688 & -0.887783 & -0.241236 & $\mathrm{C}$ & -1.798962 & 4.961367 & 0.926482 \\
\hline C & 4.065350 & -1.306449 & 1.945711 & $\mathrm{H}$ & -1.107394 & 4.572924 & -1.067295 \\
\hline $\mathrm{C}$ & 5.371334 & 0.039622 & -1.380702 & $\mathrm{C}$ & 7.238502 & -3.902414 & -1.066404 \\
\hline $\mathrm{C}$ & 4.469243 & 1.279037 & -3.316524 & $\mathrm{H}$ & 6.748096 & -2.098489 & -2.099032 \\
\hline $\mathrm{C}$ & 3.700732 & 3.384181 & -0.578942 & $\mathrm{C}$ & 6.278592 & -4.207608 & 1.128492 \\
\hline C & 2.115957 & 3.769865 & 1.346545 & $\mathrm{C}$ & 8.171773 & 1.777412 & -3.206863 \\
\hline $\mathrm{H}$ & 1.684652 & 4.025965 & -0.708733 & $\mathrm{C}$ & 9.071502 & 0.714004 & -1.236130 \\
\hline C & -0.036592 & 2.501315 & -2.199626 & $\mathrm{H}$ & 7.698305 & -0.335504 & 0.015471 \\
\hline $\mathrm{C}$ & -0.626104 & 2.947210 & 0.264071 & $\mathrm{C}$ & 1.867342 & 4.697393 & 3.988132 \\
\hline $\mathrm{H}$ & -0.274289 & 1.075360 & -0.651581 & $\mathrm{H}$ & 1.206535 & 6.547929 & 3.099359 \\
\hline C & 5.811650 & -2.157302 & -0.148100 & $\mathrm{H}$ & 2.550521 & 2.733224 & 4.561223 \\
\hline $\mathrm{C}$ & 4.762100 & -2.488301 & 2.057331 & $\mathrm{C}$ & -1.907043 & 4.483371 & 2.230195 \\
\hline $\mathrm{C}$ & 3.156694 & -0.795563 & 3.030974 & $\mathrm{H}$ & -1.447320 & 2.853156 & 3.569372 \\
\hline $\mathrm{C}$ & 6.689864 & 0.511315 & -1.704555 & $\mathrm{H}$ & -2.218447 & 5.926582 & 0.662350 \\
\hline C & 5.744568 & 1.656791 & -3.665928 & $\mathrm{C}$ & 7.076534 & -4.672535 & 0.108767 \\
\hline C & 3.263388 & 1.669415 & -4.127867 & $\mathrm{H}$ & 7.849610 & -4.283138 & -1.879498 \\
\hline $\mathrm{H}$ & 4.055443 & 4.408541 & -0.428534 & $\mathrm{H}$ & 6.131705 & -4.798334 & 2.029147 \\
\hline $\mathrm{H}$ & 3.783704 & 3.145210 & -1.641414 & $\mathrm{C}$ & 9.253734 & 1.479924 & -2.410666 \\
\hline $\mathrm{H}$ & 4.351797 & 2.714346 & -0.017511 & $\mathrm{H}$ & 8.293395 & 2.380431 & -4.103122 \\
\hline
\end{tabular}




$\begin{array}{rrrr}\mathrm{H} & 9.922222 & 0.499887 & -0.595632 \\ \mathrm{H} & 1.760510 & 5.051594 & 5.009715 \\ \mathrm{H} & -2.403294 & 5.078748 & 2.990918 \\ \mathrm{H} & 7.572653 & -5.634598 & 0.197569 \\ \mathrm{H} & 10.243645 & 1.840868 & -2.673990\end{array}$

S5
Zero-point correction=1.204411 (Hartree/Particle)

Thermal correction to Energy=1.279096

Thermal correction to Enthalpy=1.280040

Thermal correction to Gibbs Free Energy=1.091783

$\mathrm{E}($ Solv $)=-4250.67981695$

$\mathrm{H}$
$\mathrm{H}$
$\mathrm{H}$
$\mathrm{C}$
$\mathrm{N}$
$\mathrm{S}$
$\mathrm{O}$
$\mathrm{O}$

c

C

\section{C}

\begin{tabular}{|c|c|c|c|}
\hline $\mathrm{C}$ & -0.549654 & -4.930811 & -3.482453 \\
\hline $\mathrm{C}$ & -0.342251 & -3.558674 & -3.357186 \\
\hline $\mathrm{C}$ & 0.733451 & -3.052797 & -2.606041 \\
\hline $\mathrm{C}$ & 1.585852 & -3.968459 & -1.968202 \\
\hline $\mathrm{C}$ & 1.377400 & -5.339552 & -2.092206 \\
\hline $\mathrm{C}$ & 0.312692 & -5.830893 & -2.852160 \\
\hline $\mathrm{H}$ & -1.382772 & -5.297535 & -4.076138 \\
\hline $\mathrm{H}$ & -1.012124 & -2.860013 & -3.850750 \\
\hline $\mathrm{H}$ & 2.422487 & -3.605613 & -1.386205 \\
\hline $\mathrm{H}$ & 2.060950 & -6.026671 & -1.601841 \\
\hline $\mathrm{H}$ & 0.156890 & -6.901240 & -2.951244 \\
\hline $\mathrm{C}$ & 0.952916 & -1.596002 & -2.565436 \\
\hline $\mathrm{H}$ & 0.375984 & -1.056934 & -3.317062 \\
\hline C & 2.135040 & -0.936169 & -2.114151 \\
\hline $\mathrm{H}$ & 2.883957 & -1.453509 & -1.518602 \\
\hline $\mathrm{C}$ & 2.466391 & 0.355948 & -2.646201 \\
\hline $\mathrm{C}$ & 3.221716 & 1.335883 & -2.052768 \\
\hline $\mathrm{H}$ & 3.361050 & 2.284869 & -2.556931 \\
\hline $\mathrm{H}$ & 2.049376 & 0.576390 & -3.631889 \\
\hline $\mathrm{H}$ & 3.292179 & 1.365651 & -0.980740 \\
\hline $\mathrm{Pd}$ & 0.544293 & -0.711067 & -0.662689 \\
\hline $\mathrm{C}$ & 5.694139 & 4.319954 & -0.117641 \\
\hline $\mathrm{C}$ & 5.460411 & 2.950289 & -0.160975 \\
\hline $\mathrm{C}$ & 5.727633 & 2.230928 & -1.341019 \\
\hline $\mathrm{C}$ & 6.245179 & 2.905434 & -2.463859 \\
\hline $\mathrm{C}$ & 6.495878 & 4.270811 & -2.405371 \\
\hline $\mathrm{C}$ & 6.213805 & 4.981683 & -1.234024 \\
\hline $\mathrm{H}$ & 5.455929 & 4.869101 & 0.787105 \\
\hline $\mathrm{H}$ & 5.030173 & 2.450638 & 0.699348 \\
\hline
\end{tabular}

\begin{tabular}{|c|c|c|}
\hline 6.442388 & 2.349788 & -3.376828 \\
\hline 6.903507 & 4.783475 & -3.271401 \\
\hline 6.395262 & 6.051806 & -1.193874 \\
\hline 5.460567 & 0.806548 & -1.469136 \\
\hline 5.287869 & -0.014867 & -0.419085 \\
\hline 6.007035 & -1.587584 & -0.403322 \\
\hline 6.367241 & -1.867315 & -1.794882 \\
\hline 7.033235 & -1.527532 & 0.634713 \\
\hline 4.719400 & -2.694582 & 0.123519 \\
\hline 4.883302 & -4.032906 & -0.255253 \\
\hline 3.710733 & -2.299133 & 1.001028 \\
\hline 4.023446 & -4.987097 & 0.275576 \\
\hline 5.670370 & -4.313649 & -0.946536 \\
\hline 2.841278 & -3.274052 & 1.491066 \\
\hline 3.574089 & -1.264692 & 1.302136 \\
\hline 2.988940 & -4.623752 & 1.152824 \\
\hline 4.151406 & -6.030229 & -0.001605 \\
\hline 2.039284 & -2.969723 & 2.153649 \\
\hline 2.045667 & -5.667314 & 1.692547 \\
\hline 1.396977 & -6.046373 & 0.893122 \\
\hline 2.594411 & -6.524542 & 2.099277 \\
\hline 1.402319 & -5.261347 & 2.476403 \\
\hline 5.782335 & 0.306911 & -2.377730 \\
\hline 2.360675 & 1.078536 & 1.254266 \\
\hline 1.502736 & 0.143753 & 1.227325 \\
\hline 3.590851 & 0.901763 & 1.510368 \\
\hline 4.849352 & 0.327276 & 0.461403 \\
\hline 1.908720 & 2.484701 & 0.918479 \\
\hline 2.486684 & 3.644545 & 1.533822 \\
\hline 0.972539 & 2.645930 & -0.086805 \\
\hline 3.397383 & 3.580741 & 2.625603 \\
\hline 2.107909 & 4.940876 & 1.045989 \\
\hline 0.617437 & 3.920919 & -0.574158 \\
\hline 0.521485 & 1.764801 & -0.534472 \\
\hline 3.916612 & 4.727171 & 3.186564 \\
\hline 3.688030 & 2.608119 & 3.000599 \\
\hline 2.669048 & 6.101291 & 1.643100 \\
\hline 1.180596 & 5.046395 & -0.023876 \\
\hline-0.115087 & 4.003608 & -1.370260 \\
\hline 3.556828 & 6.002841 & 2.689448 \\
\hline 4.608293 & 4.651157 & 4.021020 \\
\hline 2.374893 & 7.074575 & 1.257579 \\
\hline 0.909331 & 6.034201 & -0.387563 \\
\hline 3.975170 & 6.898431 & 3.140381 \\
\hline
\end{tabular}




\begin{tabular}{|c|c|c|c|}
\hline $\mathrm{P}$ & -1.677115 & -0.903917 & -0.209453 \\
\hline $\mathrm{O}$ & -2.472193 & 0.095467 & 0.855515 \\
\hline $\mathrm{O}$ & -2.428349 & -0.436536 & -1.637334 \\
\hline $\mathrm{N}$ & -2.265644 & -2.373084 & 0.356805 \\
\hline $\mathrm{C}$ & -2.517511 & 1.460116 & 0.683806 \\
\hline $\mathrm{C}$ & -3.729615 & -0.002287 & -1.782982 \\
\hline $\mathrm{C}$ & -3.626075 & -2.728055 & 0.880943 \\
\hline $\mathrm{C}$ & -1.207251 & -3.384264 & 0.658687 \\
\hline $\mathrm{C}$ & -3.281603 & 2.000321 & -0.335892 \\
\hline $\mathrm{C}$ & -1.824392 & 2.242871 & 1.648571 \\
\hline $\mathrm{C}$ & -4.184840 & 1.140361 & -1.145529 \\
\hline $\mathrm{C}$ & -4.523402 & -0.751963 & -2.698177 \\
\hline $\mathrm{C}$ & -4.549308 & -3.219703 & -0.235606 \\
\hline $\mathrm{C}$ & -4.264379 & -1.676620 & 1.780611 \\
\hline $\mathrm{H}$ & -3.429993 & -3.581858 & 1.535099 \\
\hline $\mathrm{C}$ & -1.521537 & -4.740929 & 0.035205 \\
\hline $\mathrm{C}$ & -0.927142 & -3.410206 & 2.157112 \\
\hline $\mathrm{H}$ & -0.298399 & -3.016352 & 0.169958 \\
\hline $\mathrm{C}$ & -3.203915 & 3.420084 & -0.551770 \\
\hline $\mathrm{C}$ & -1.858315 & 3.608978 & 1.502093 \\
\hline $\mathrm{C}$ & -1.095068 & 1.561926 & 2.772496 \\
\hline $\mathrm{C}$ & -5.586010 & 1.455368 & -1.253246 \\
\hline $\mathrm{C}$ & -5.840322 & -0.391390 & -2.859791 \\
\hline $\mathrm{C}$ & -3.891210 & -1.874706 & -3.478178 \\
\hline $\mathrm{H}$ & -5.476370 & -3.614129 & 0.195207 \\
\hline $\mathrm{H}$ & -4.063857 & -4.013302 & -0.808124 \\
\hline $\mathrm{H}$ & -4.815659 & -2.419351 & -0.925773 \\
\hline $\mathrm{C}$ & -3.792368 & -1.530617 & 3.091204 \\
\hline $\mathrm{C}$ & -5.303412 & -0.850443 & 1.344781 \\
\hline $\mathrm{H}$ & -0.694563 & -5.433076 & 0.213663 \\
\hline $\mathrm{H}$ & -1.636795 & -4.626447 & -1.043166 \\
\hline $\mathrm{H}$ & -2.432802 & -5.194826 & 0.436936 \\
\hline $\mathrm{C}$ & -0.310127 & -2.293054 & 2.741391 \\
\hline $\mathrm{C}$ & -1.295139 & -4.477268 & 2.981778 \\
\hline $\mathrm{C}$ & -3.765042 & 4.056883 & -1.691140 \\
\hline $\mathrm{C}$ & -2.497155 & 4.226663 & 0.396507 \\
\hline $\mathrm{H}$ & -1.332003 & 4.236415 & 2.216556 \\
\hline $\mathrm{H}$ & -0.245896 & 0.988809 & 2.385970 \\
\hline $\mathrm{H}$ & -0.715930 & 2.301980 & 3.483695 \\
\hline $\mathrm{H}$ & -1.754637 & 0.863670 & 3.298375 \\
\hline $\mathrm{C}$ & -6.414233 & 0.678798 & -2.125103 \\
\hline $\mathrm{C}$ & -6.204148 & 2.473860 & -0.478137 \\
\hline $\mathrm{H}$ & -6.468971 & -0.946208 & -3.552214 \\
\hline $\mathrm{H}$ & -4.642700 & -2.405800 & -4.069720 \\
\hline
\end{tabular}

\begin{tabular}{|c|c|c|c|}
\hline $\mathrm{H}$ & -3.126128 & -1.486160 & -4.161784 \\
\hline $\mathrm{H}$ & -3.388922 & -2.590468 & -2.821529 \\
\hline $\mathrm{C}$ & -4.312127 & -0.546791 & 3.928748 \\
\hline $\mathrm{H}$ & -2.995806 & -2.174602 & 3.448022 \\
\hline $\mathrm{C}$ & -5.826163 & 0.137652 & 2.180357 \\
\hline $\mathrm{H}$ & -5.698811 & -0.947983 & 0.340886 \\
\hline $\mathrm{C}$ & -0.076626 & -2.242063 & 4.112924 \\
\hline $\mathrm{H}$ & 0.000786 & -1.458414 & 2.121881 \\
\hline $\mathrm{C}$ & -1.066854 & -4.427450 & 4.361375 \\
\hline $\mathrm{H}$ & -1.762271 & -5.358783 & 2.554528 \\
\hline $\mathrm{C}$ & -3.650570 & 5.417805 & -1.873113 \\
\hline $\mathrm{H}$ & -4.277284 & 3.453387 & -2.431353 \\
\hline $\mathrm{C}$ & -2.412964 & 5.628036 & 0.189106 \\
\hline $\mathrm{C}$ & -7.797169 & 0.981835 & -2.228597 \\
\hline $\mathrm{C}$ & -7.552928 & 2.732774 & -0.587645 \\
\hline $\mathrm{H}$ & -5.603211 & 3.039381 & 0.222153 \\
\hline $\mathrm{C}$ & -5.324665 & 0.300748 & 3.471137 \\
\hline $\mathrm{H}$ & -3.926401 & -0.441721 & 4.939386 \\
\hline $\mathrm{H}$ & -6.615953 & 0.784286 & 1.810474 \\
\hline $\mathrm{C}$ & -0.460190 & -3.309335 & 4.931380 \\
\hline $\mathrm{H}$ & 0.406225 & -1.367524 & 4.539547 \\
\hline $\mathrm{H}$ & -1.366281 & -5.264236 & 4.986436 \\
\hline $\mathrm{C}$ & -2.976731 & 6.216644 & -0.919930 \\
\hline $\mathrm{H}$ & -4.077318 & 5.880052 & -2.758748 \\
\hline $\mathrm{H}$ & -1.871519 & 6.224540 & 0.918757 \\
\hline $\mathrm{C}$ & -8.359423 & 1.990444 & -1.480729 \\
\hline $\mathrm{H}$ & -8.407344 & 0.388069 & -2.904657 \\
\hline $\mathrm{H}$ & -8.001110 & 3.511223 & 0.023207 \\
\hline $\mathrm{H}$ & -5.725080 & 1.074880 & 4.120042 \\
\hline $\mathrm{H}$ & -0.283165 & -3.269653 & 6.002427 \\
\hline $\mathrm{H}$ & -2.897177 & 7.289424 & -1.071563 \\
\hline $\mathrm{H}$ & -9.420236 & 2.208544 & -1.563287 \\
\hline
\end{tabular}

S6

Zero-point correction=1.204005 (Hartree/Particle)

Thermal correction to Energy=1.278923

Thermal correction to Enthalpy= 1.279867

Thermal correction to Gibbs Free Energy=1.089111

$\mathrm{E}(\mathrm{Solv})=-4250.71106337$

$\begin{array}{llll}\text { C } & 4.542166 & -2.953208 & -2.607600 \\ \text { C } & 3.573458 & -2.972651 & -1.605685\end{array}$




\begin{tabular}{|c|c|c|c|}
\hline $\mathrm{C}$ & 2.248407 & -2.612499 & -1.884456 \\
\hline $\mathrm{C}$ & 1.910914 & -2.217501 & -3.191526 \\
\hline C & 2.877809 & -2.204023 & -4.191529 \\
\hline $\mathrm{C}$ & 4.196741 & -2.575363 & -3.905677 \\
\hline $\mathrm{H}$ & 5.566680 & -3.227075 & -2.372329 \\
\hline $\mathrm{H}$ & 3.839039 & -3.268400 & -0.595781 \\
\hline $\mathrm{H}$ & 0.899738 & -1.885301 & -3.407684 \\
\hline $\mathrm{H}$ & 2.607134 & -1.889329 & -5.195718 \\
\hline $\mathrm{H}$ & 4.950209 & -2.557364 & -4.688136 \\
\hline $\mathrm{C}$ & 1.240043 & -2.684367 & -0.809234 \\
\hline $\mathrm{H}$ & 1.644214 & -2.886176 & 0.183383 \\
\hline $\mathrm{C}$ & -0.076914 & -3.153085 & -1.079677 \\
\hline $\mathrm{H}$ & -0.399425 & -3.182099 & -2.117468 \\
\hline $\mathrm{C}$ & -0.978987 & -3.604786 & -0.109320 \\
\hline $\mathrm{C}$ & -2.343702 & -3.767188 & -0.368347 \\
\hline $\mathrm{H}$ & -2.658516 & -3.887794 & -1.401778 \\
\hline $\mathrm{H}$ & -0.629250 & -3.667533 & 0.920269 \\
\hline $\mathrm{H}$ & -2.965760 & -4.269453 & 0.365787 \\
\hline Pd & 0.061726 & -0.891844 & -0.539714 \\
\hline C & -4.474380 & -2.221782 & 3.336672 \\
\hline C & -4.341937 & -2.159952 & 1.952828 \\
\hline $\mathrm{C}$ & -3.125316 & -1.787903 & 1.361032 \\
\hline $\mathrm{C}$ & -2.036594 & -1.475118 & 2.187379 \\
\hline $\mathrm{C}$ & -2.172620 & -1.537792 & 3.572237 \\
\hline $\mathrm{C}$ & -3.386408 & -1.911564 & 4.152187 \\
\hline $\mathrm{H}$ & -5.430745 & -2.483459 & 3.777697 \\
\hline $\mathrm{H}$ & -5.193876 & -2.384233 & 1.316468 \\
\hline $\mathrm{H}$ & -1.084641 & -1.191422 & 1.745011 \\
\hline $\mathrm{H}$ & -1.325295 & -1.292734 & 4.202152 \\
\hline $\mathrm{H}$ & -3.488296 & -1.943044 & 5.232359 \\
\hline C & -3.043237 & -1.803156 & -0.116680 \\
\hline $\mathrm{N}$ & -2.203742 & -0.945292 & -0.766391 \\
\hline$S$ & -2.542455 & -0.510284 & -2.359753 \\
\hline $\mathrm{O}$ & -3.143099 & 0.835236 & -2.334417 \\
\hline $\mathrm{O}$ & -1.316143 & -0.707083 & -3.153895 \\
\hline C & -3.765098 & -1.643331 & -3.008890 \\
\hline C & -3.341669 & -2.779381 & -3.700817 \\
\hline $\mathrm{C}$ & -5.121665 & -1.384330 & -2.815748 \\
\hline C & -4.294175 & -3.670313 & -4.187288 \\
\hline $\mathrm{H}$ & -2.282921 & -2.940778 & -3.869733 \\
\hline C & -6.061672 & -2.288624 & -3.305592 \\
\hline $\mathrm{H}$ & -5.427790 & -0.480854 & -2.301789 \\
\hline 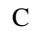 & -5.664701 & -3.445269 & -3.98974 \\
\hline $\mathrm{H}$ & -3 . & -4.55096 & -4.73604 \\
\hline
\end{tabular}

\begin{tabular}{|c|c|c|c|}
\hline $\mathrm{H}$ & -7.120261 & -2.090138 & -3.161281 \\
\hline $\mathrm{C}$ & -6.684652 & -4.435678 & -4.493090 \\
\hline $\mathrm{H}$ & -6.391544 & -4.852501 & -5.462228 \\
\hline $\mathrm{H}$ & -6.789274 & -5.277004 & -3.795718 \\
\hline $\mathrm{H}$ & -7.670712 & -3.974448 & -4.602974 \\
\hline $\mathrm{H}$ & -4.000513 & -2.022185 & -0.586871 \\
\hline $\mathrm{C}$ & -2.679620 & 1.860636 & 1.847542 \\
\hline $\mathrm{O}$ & -1.832628 & 1.469382 & 0.868046 \\
\hline $\mathrm{O}$ & -2.254812 & 2.552633 & 2.751772 \\
\hline $\mathrm{H}$ & -2.178222 & 0.745103 & 0.294626 \\
\hline $\mathrm{C}$ & -4.098833 & 1.413065 & 1.718363 \\
\hline $\mathrm{C}$ & -4.891039 & 1.126302 & 2.879534 \\
\hline $\mathrm{C}$ & -4.645661 & 1.301986 & 0.451889 \\
\hline $\mathrm{C}$ & -4.411179 & 1.243928 & 4.211788 \\
\hline $\mathrm{C}$ & -6.233431 & 0.662067 & 2.680909 \\
\hline $\mathrm{C}$ & -5.982026 & 0.885092 & 0.274743 \\
\hline $\mathrm{H}$ & -4.055044 & 1.541462 & -0.425312 \\
\hline $\mathrm{C}$ & -5.212609 & 0.912605 & 5.281512 \\
\hline $\mathrm{H}$ & -3.406472 & 1.608744 & 4.371632 \\
\hline $\mathrm{C}$ & -7.027478 & 0.319009 & 3.806702 \\
\hline $\mathrm{C}$ & -6.752531 & 0.555469 & 1.364504 \\
\hline $\mathrm{H}$ & -6.396762 & 0.838230 & -0.727728 \\
\hline $\mathrm{C}$ & -6.530056 & 0.439459 & 5.083326 \\
\hline $\mathrm{H}$ & -4.825649 & 1.013406 & 6.291676 \\
\hline $\mathrm{H}$ & -8.041145 & -0.035932 & 3.637711 \\
\hline $\mathrm{H}$ & -7.779301 & 0.221189 & 1.236509 \\
\hline $\mathrm{H}$ & -7.147198 & 0.179170 & 5.938653 \\
\hline$P$ & 1.680377 & 0.684676 & -0.399956 \\
\hline $\mathrm{O}$ & 2.257748 & 1.114703 & 1.111046 \\
\hline $\mathrm{O}$ & 3.096307 & 0.082261 & -1.073654 \\
\hline $\mathrm{N}$ & 1.403818 & 2.167905 & -1.098181 \\
\hline $\mathrm{C}$ & 2.486400 & -0.008806 & 1.901449 \\
\hline $\mathrm{C}$ & 4.370830 & 0.068138 & -0.576707 \\
\hline $\mathrm{C}$ & 2.132729 & 3.447596 & -0.896948 \\
\hline $\mathrm{C}$ & 0.504470 & 2.117001 & -2.305302 \\
\hline $\mathrm{C}$ & 3.578544 & -0.814280 & 1.632678 \\
\hline C & 1.552552 & -0.259073 & 2.942158 \\
\hline $\mathrm{C}$ & 4.651659 & -0.350908 & 0.713958 \\
\hline C & 5.385471 & 0.429610 & -1.511548 \\
\hline C & 3.555365 & 3.326440 & -0.334472 \\
\hline $\mathrm{C}$ & 1.312665 & 4.423399 & -0.062531 \\
\hline $\mathrm{H}$ & 2.233956 & 3.881904 & -1.897298 \\
\hline $\mathrm{C}$ & 1.292204 & 1.697129 & -3.548518 \\
\hline C & -0.280600 & 3.408383 & -2.461690 \\
\hline
\end{tabular}




\begin{tabular}{|c|c|c|c|}
\hline $\mathrm{H}$ & -0.220184 & 1.324550 & -2.104570 \\
\hline $\mathrm{C}$ & 3.640142 & -2.091650 & 2.289319 \\
\hline $\mathrm{C}$ & 1.665214 & -1.452673 & 3.617511 \\
\hline $\mathrm{C}$ & 0.530341 & 0.791602 & 3.275336 \\
\hline $\mathrm{C}$ & 6.015315 & -0.284797 & 1.171367 \\
\hline C & 6.692757 & 0.399744 & -1.091220 \\
\hline $\mathrm{C}$ & 4.999694 & 0.815180 & -2.913091 \\
\hline $\mathrm{H}$ & 3.996139 & 4.328206 & -0.327608 \\
\hline $\mathrm{H}$ & 4.184165 & 2.682306 & -0.951305 \\
\hline $\mathrm{H}$ & 3.559747 & 2.947353 & 0.686853 \\
\hline $\mathrm{C}$ & 0.557451 & 3.999083 & 1.033526 \\
\hline $\mathrm{C}$ & 1.320731 & 5.780503 & -0.396147 \\
\hline $\mathrm{H}$ & 0.633942 & 1.672632 & -4.422483 \\
\hline $\mathrm{H}$ & 1.709619 & 0.697694 & -3.404147 \\
\hline $\mathrm{H}$ & 2.123072 & 2.380659 & -3.755945 \\
\hline $\mathrm{C}$ & -1.327688 & 3.674143 & -1.571689 \\
\hline $\mathrm{C}$ & 0.025895 & 4.356947 & -3.441103 \\
\hline $\mathrm{C}$ & 4.611608 & -3.075077 & 1.959949 \\
\hline $\mathrm{C}$ & 2.653856 & -2.414037 & 3.276410 \\
\hline $\mathrm{H}$ & 0.975494 & -1.682313 & 4.425735 \\
\hline $\mathrm{H}$ & -0.180387 & 0.941884 & 2.460921 \\
\hline $\mathrm{H}$ & -0.029002 & 0.525106 & 4.175168 \\
\hline $\mathrm{H}$ & 1.010238 & 1.761156 & 3.445182 \\
\hline $\mathrm{C}$ & 7.044941 & 0.071074 & 0.243317 \\
\hline $\mathrm{C}$ & 6.390296 & -0.526683 & 2.520729 \\
\hline $\mathrm{H}$ & 7.485079 & 0.659897 & -1.789234 \\
\hline $\mathrm{H}$ & 5.889834 & 1.037799 & -3.508980 \\
\hline $\mathrm{H}$ & 4.440361 & 0.011151 & -3.399122 \\
\hline $\mathrm{H}$ & 4.350222 & 1.697349 & -2.919299 \\
\hline $\mathrm{C}$ & -0.188078 & 4.912735 & 1.777024 \\
\hline $\mathrm{H}$ & 0.510266 & 2.946919 & 1.278720 \\
\hline $\mathrm{C}$ & 0.580178 & 6.699321 & 0.346648 \\
\hline $\mathrm{H}$ & 1.882559 & 6.114831 & -1.265165 \\
\hline $\mathrm{C}$ & -2.034116 & 4.870437 & -1.640031 \\
\hline $\mathrm{H}$ & -1.559123 & 2.949779 & -0.801679 \\
\hline $\mathrm{C}$ & -0.684279 & 5.557672 & -3.516525 \\
\hline $\mathrm{H}$ & 0.825349 & 4.169686 & -4.150995 \\
\hline $\mathrm{C}$ & 4.608791 & -4.309503 & 2.571782 \\
\hline $\mathrm{H}$ & 5.358885 & -2.842433 & 1.210832 \\
\hline $\mathrm{C}$ & 2.690816 & -3.688252 & 3.901261 \\
\hline $\mathrm{C}$ & 8.394524 & 0.114147 & 0.678928 \\
\hline $\mathrm{C}$ & 7.708463 & -0.464120 & 2.916787 \\
\hline $\mathrm{H}$ & 5.622996 & -0.753570 & 3.250464 \\
\hline C & -0.17858 & 6 & 1.435294 \\
\hline
\end{tabular}

\begin{tabular}{lrrr}
$\mathrm{H}$ & -0.797344 & 4.549490 & 2.597716 \\
$\mathrm{H}$ & 0.580690 & 7.748305 & 0.062253 \\
$\mathrm{C}$ & -1.711409 & 5.820312 & -2.611834 \\
$\mathrm{H}$ & -2.827320 & 5.067165 & -0.924623 \\
$\mathrm{H}$ & -0.429652 & 6.286774 & -4.281240 \\
$\mathrm{C}$ & 3.643452 & -4.620405 & 3.557189 \\
$\mathrm{H}$ & 5.354303 & -5.049243 & 2.294911 \\
$\mathrm{H}$ & 1.940736 & -3.916798 & 4.654356 \\
$\mathrm{C}$ & 8.726846 & -0.154090 & 1.987170 \\
$\mathrm{H}$ & 9.162534 & 0.377251 & -0.044314 \\
$\mathrm{H}$ & 7.965307 & -0.647939 & 3.956191 \\
$\mathrm{H}$ & -0.769299 & 6.978308 & 2.005888 \\
$\mathrm{H}$ & -2.257747 & 6.758243 & -2.662689 \\
$\mathrm{H}$ & 3.654038 & -5.594700 & 4.037058 \\
$\mathrm{H}$ & 9.763107 & -0.113244 & 2.310047 \\
\hline
\end{tabular}




\section{References}

(1) Bhowmik, A.; Fernandes, R. A. Iron(III)/O $2-$ Mediated Regioselective Oxidative Cleavage of 1Arylbutadienes to Cinnamaldehydes. Org. Lett. 2019, 21, 9203-9207.

(2) Wang, T. Q.; Hu, Y. Y.; Zhang, S. L. A Novel and Efficient Method for the Olefination of Carbonyl Compounds with Grignard Reagents in the presence of Diethyl Phosphite. Org. Biomol. Chem. 2010, 8, 2312-2315.

(3) Morales, S.; Guijarro, F. G.; García Ruano, J. L.; Cid, M. B. A General Aminocatalytic Method for the Synthesis of Aldimines. J. Am. Chem. Soc. 2014, 136, 1082-1089.

(4) Cai, H.; Zhou, Y.; Zhang, D.; Xu, J.; Liu, H. A Mannich/Cyclization Cascade Process for the Asymmetric Synthesis of Spirocyclic Thioimidazolidineoxindoles. Chem. Commun. 2014, 50, 14771-14774.

(5) Yuan, Q.; Liu, D.; Zhang, W. Iridium-Catalyzed Aymmetric Hydrogenation of $\beta, \gamma$-Unsaturated $\gamma$ Lactams: Scope and Mechanistic Studies. Org. Lett. 2017, 19, 1144-1147.

(6) Guillaneux, D.; Zhao, S.-H.; Samuel, O.; Rainford, D.; Kagan, H. B. Nonlinear Effects in Asymmetric Catalysis. J. Am. Chem. Soc. 1994, 116, 9430-9439.

(7) Frisch, M. J.; Trucks, G. W.; Schlegel, H. B.; Scuseria, G. E.; Robb, M. A.; Cheeseman, J. R.; Scalmani, G.; Barone, V.; Mennucci, B.; Petersson, G. A.; Nakatsuji, H.; Caricato, M.; Li, X.; Hratchian, H. P.; Izmaylov, A. F.; Bloino, J.; Zheng, G.; Sonnenberg, J. L.; Hada, M.; Ehara, M.; Toyota, K.; Fukuda, R.; Hasegawa, J.; Ishida, M.; Nakajima, T.; Honda, Y.; Kitao, O.; Nakai, H.; Vreven, T.; Montgomery Jr. J. A.; Peralta, J. E.; Ogliaro, F.; Bearpark, M. J.; Heyd, J.; Brothers, E. N.; Kudin, K. N.; Staroverov, V. N.; Kobayashi, R.; Normand, J.; Raghavachari, K.; Rendell, A. P.; Burant, J. C.; Iyengar, S. S.; Tomasi, J.; Cossi, M.; Rega, N.; Millam, N. J.; Klene, M.; Knox, J. E.; Cross, J. B.; Bakken, V.; Adamo, C.; Jaramillo, J.; Gomperts, R.; Stratmann, R. E.; Yazyev, O.; Austin, A. J.; Cammi, R.; Pomelli, C.; Ochterski, J. W.; Martin, R. L.; Morokuma, K.; Zakrzewski, V. G.; Voth, G. A.; Salvador, P.; Dannenberg, J. J.; Dapprich, S.; Daniels, A. D.; Farkas, Ö.; Foresman, J. B.; Ortiz, J. V.; Cioslowski, J.; Fox, D. J. Gaussian 09, Gaussian, Inc.: Wallingford, CT, USA, 2009.

(8) Tripos International, Sybyl-X 2.0, Tripos International, St. Louis, MO, USA, 2012.

(9) Giroday, T.; Montero-campillo M. M.; Mora-Diez, N. Thermodynamic Stability of PFOS: M062X and B3LYP Comparison. Comput. Theor. Chem. 2014, 1046, 81-92.

(10)Salvador, M. A.; Antonio, F.C.T.; da Silva, G.D.; Bartoloni, F. H.; Orestes, E.; Coutinho-Neto, M. D.; Homem-de-Mello, P. On the Radicalar Properties of Graphene Fragments: Double-Hybrid DFT and Perturbation Theory Approaches. Theor. Chem. Acc. 2020, 139, 118-129.

(11). Weigend, F.; Ahlrichs, R. Balanced Basis Sets of Split Valence, Triple Zeta Valence and Quadruple Zeta Valence Quality for H to Rn: Design and Assessment of Accuracy. Phys Chem 
Chem Phys. 2005, 7, 3297-3305.

(12)Becke, A. D. Density-Functional Thermochemistry. III. The Role of Exact Exchange. J. Chem. Phys. 1993, 98, 5648-5652.

(13)Lee, C.; Yang, W.; Parr, R. G. Development of the Colle-Salvetti Correlation-Energy Formula into a Functional of the Electron Density. Phys. Rev. B, 1988, 37, 785-789.

(14)Dunning Jr. T. H.; Hay, P. J. in Modern Theoretical Chemistry, Ed. H. F. Schaefer III, Vol. 3 (Plenum, New York, 1977) 1-28.

(15)Rassolov, V. A.; Ratner, M. A.; Pople, J. A.; Redfern, P. C.; Curtiss, L. A. 6-31G* Basis Set for Third-Row Atoms. J. Comp. Chem., 2001, 22, 976-984.

(16)Shranami, D. Computational Study of Enantioselectivity in the Asymmetric Allylation of Aldehydes with Chiral Pt(II) Phosphinite Complexes. J. Org. Chem. 2018, 83, 13911-13921.

(17)Sultan, E.; Duran, K. A Theoretical Study on Cyclometalated Iridium (III) Complexes by Using a Density Functional Theory. J. Theor. Comput. Chem. 2020, 19, 2050006.

(18)Huang, W.; Chang, S. T.; Huang, H.; Wang, C.; Chen, L.; Chen, K.; Lin, M. C. On the Reduction of $\mathrm{O}_{2}$ on Cathode Surfaces of Co-Corrin and Co-Porphyrin: A Computational and Experimental Study on Their Relative Efficiencies in $\mathrm{H}_{2} \mathrm{O} / \mathrm{H}_{2} \mathrm{O}_{2}$ Formation. J. Phys. Chem. C. 2020, 124, 4652-4659.

(19)Hong, X.; Trost, B. M.; Houk, K. N. Mechanism and Origins of Selectivity in Ru(II)-Catalyzed Intramolecular $(5+2)$ Cycloadditions and Ene Reactions of Vinylcyclopropanes and Alkynes From Density Functional Theory. J. Am. Chem. Soc. 2013, 135, 6588-6600.

(20)Fukui, K. The Path of Chemical Reactions-the IRC Approach. Acc. Chem. Res. 1981, 14, 363-368.

(21)Binning Jr. R. C.; Curtiss, L. A. Compact Contracted Basis-Sets for 3rd-Row Atoms: Ga-Kr. J. Comp. Chem. 1990, 11, 1206-1216.

(22)McGrath, M. P.; Radom, L. Extension of Gaussian-1 (G1) Theory to Bromine-Containing Molecules. J. Chem. Phys. 1991, 94, 511-516.

(23)Curtiss, L. A.; McGrath, M. P.; Blaudeau, J.-P.; Davis, N. E.; Binning Jr., R. C.; Radom, L. Extension of Gaussian-2 Theory to Molecules Containing Third-Row Atoms Ga-Kr. J. Chem. Phys. 1995, 103, 6104-6113.

(24)Marenich, A. V.; Cramer, C. J.; Truhlar, D. G. Universal Solvation Model Based on Solute Electron Density and on a Continuum Model of the Solvent Defined by the Bulk Dielectric Constant and Atomic Surface Tensions. J. Phys. Chem. B. 2009, 113, 6378-6396.

(25)Ribeiro, R. F.; Marenich, A. V.; Cramer, C. J.; Truhlar, D. G. Use of Solution-Phase Vibrational Frequencies in Continuum Models for the Free Energy of Solvation. J. Phys. Chem. B. 2011, 115, 14556-14562.

(26)GaussView, Version 6, Dennington, Roy; Keith, Todd A.; Millam, John M. Semichem Inc., S393 
Shawnee Mission, KS, 2016.

(27)Legault, C. Y. CYLview, version 1.0b; Universitéde Sherbrooke: Quebec, Canada, 2009; http://www.cylview.org. 